\title{
Electric Trade in the United States 1994
}

August 1998

Energy Informatlon Adminlstration

Office of Coal, Nuclear, Electric and Alternate Fuels

U.S. Department of Energy

Washington, DC 20585

DASTFISUTTON OF THIS DOCUNENT IS UHARHTED 


\section{Contacts}

Questions regarding the conkents of this report may be directed to:

Electric Power Division

Office of Coal, Naclear, Electric and Alternate Fuels

Energy Information Administuatjon, EI-52

U.S. Deptrtment of Energy

1000 Independence Avenue, S.W.

Washington, DC 20585

FAX (202/254.5765)

Questions of a general nature should be directed to:
Robert Schnapp (202/426-1211),

Difector of the Electric Power Division

Rober,Schnapp@eja.doe.gov

Betsy O'Brien (202/426-1180),

Chief of the Market Assessment Branch

Betsy.OBrien eia doe.gov $^{2}$

John Makens (202/426-] 165),

Project Manager

John.Makens eia.doe.gov

Principal data analyst and author for the Electric Trade in Uniled Srates teport was John W. Makens of the Electric Powe Division. 


\section{DISCLAMER}

This report wes prepared as an account of wort sponsored by en agancy of the Undted Stales Cowernnent. Nether the United Strtes Gowernenent nor any ngecy thereff, nor

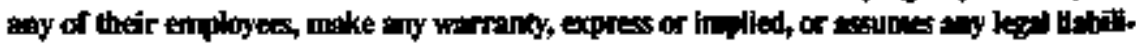

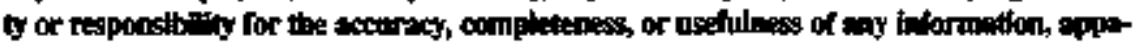

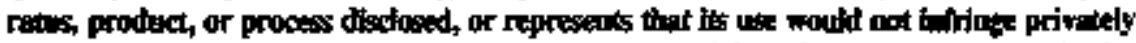
aweed rifit: Refertice berein to any specific commercial peoduct, proces, or servioe by

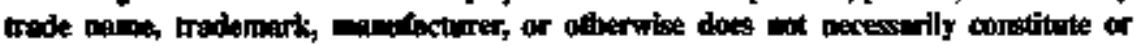

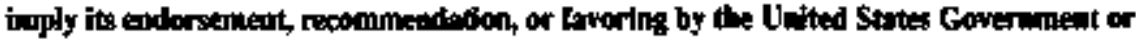

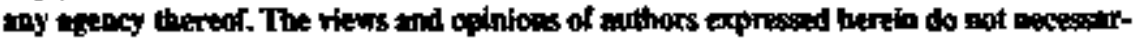

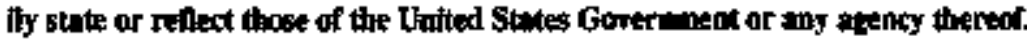




\section{DISCLAIMER}

Portions of this document may be illegible in electronic image products. Images are produced from the best available original document. 


\section{Contents}

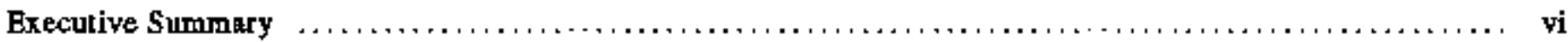

1. Introduction $\ldots, \ldots, \ldots, \ldots, \ldots, \ldots, \ldots, \ldots, \ldots, \ldots, \ldots, \ldots, \ldots, \ldots, \ldots, \ldots, \ldots, \ldots, \ldots, 1$

Background $\ldots \ldots, \ldots, \ldots, \ldots, \ldots, \ldots, \ldots, \ldots, \ldots, \ldots, \ldots, \ldots, \ldots, \ldots, \ldots, \ldots, \ldots, 1$

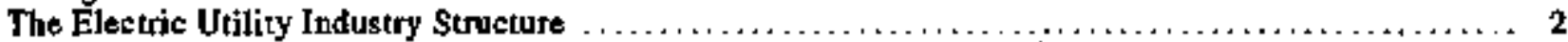

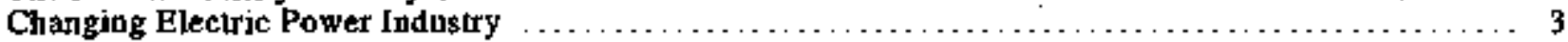

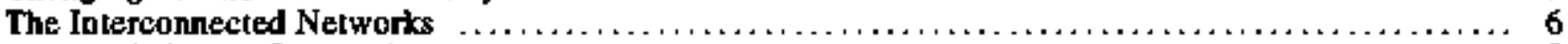

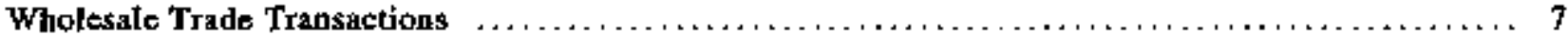

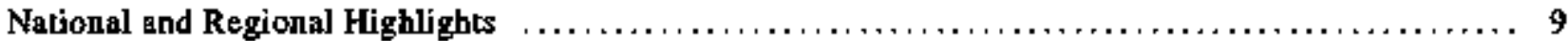

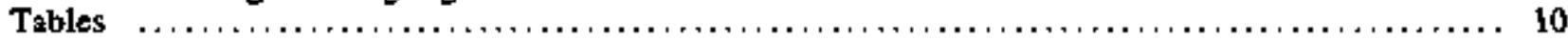

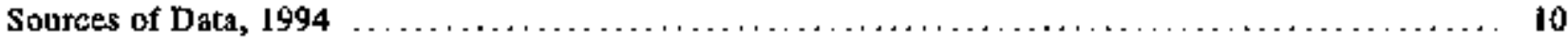

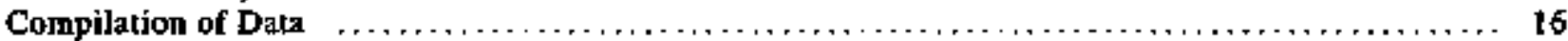

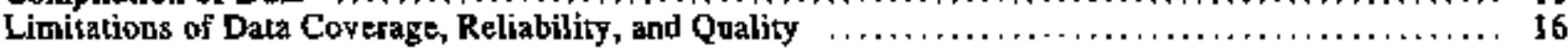

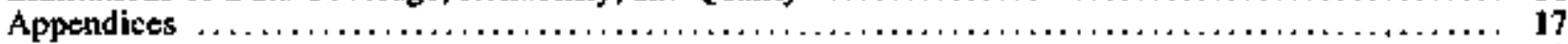

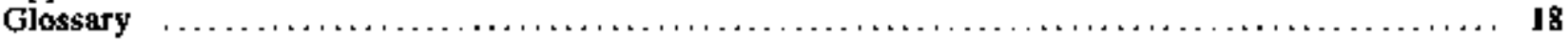

2. Bulk Power Transactions by Investor-Owned Utilities $\ldots \ldots \ldots, \ldots, \ldots, \ldots \ldots \ldots \ldots \ldots \ldots \ldots \ldots, 45$

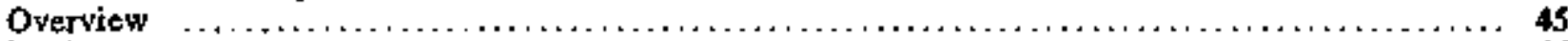

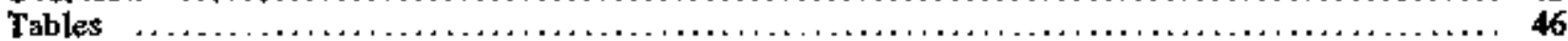

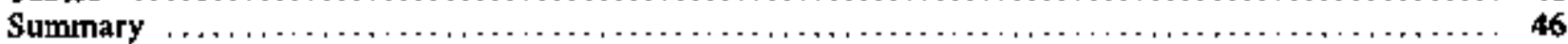

3. Bulk Power Transactions by Federal and Other Publicly Owned Utilities $\ldots \ldots \ldots \ldots \ldots \ldots \ldots \ldots \ldots \ldots, 123$

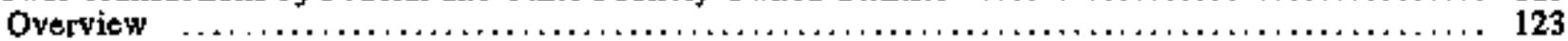

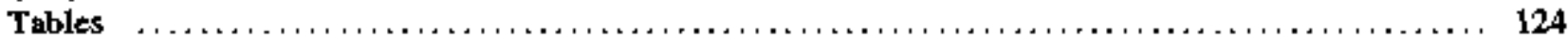

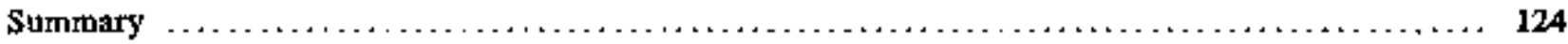

4. Butk Power Transactions by Cooperative Utilities $\ldots \ldots, \ldots, \ldots \ldots \ldots, \ldots \ldots \ldots \ldots, \ldots \ldots \ldots, \ldots, 211$

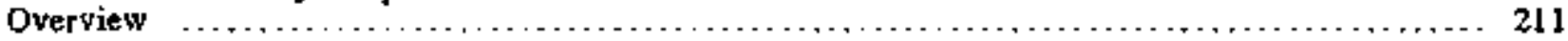

Tables $\ldots \ldots \ldots \ldots \ldots \ldots \ldots \ldots \ldots \ldots \ldots \ldots \ldots \ldots \ldots \ldots \ldots \ldots \ldots \ldots \ldots \ldots \ldots \ldots \ldots \ldots, 213$

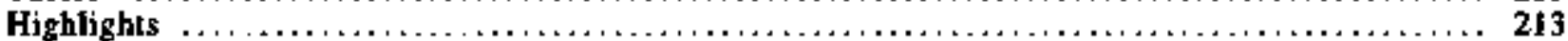

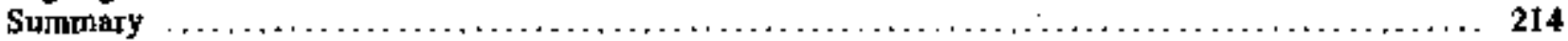

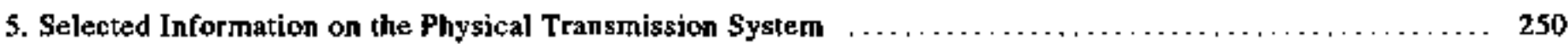

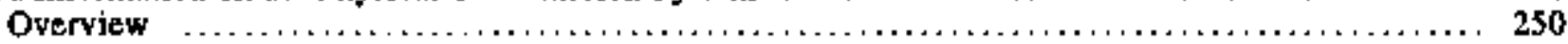

Tables $\ldots \ldots \ldots \ldots \ldots \ldots \ldots \ldots, \ldots \ldots \ldots \ldots \ldots \ldots \ldots \ldots \ldots \ldots \ldots \ldots \ldots \ldots \ldots, 250$

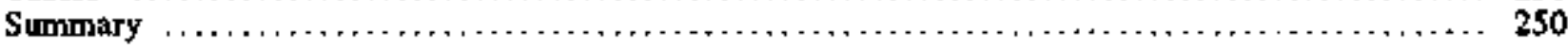

Appendices

A. Foderal Energy Regulatory Comunission Reguiation of the Electric Utility Industry $\ldots \ldots \ldots \ldots \ldots \ldots .288$

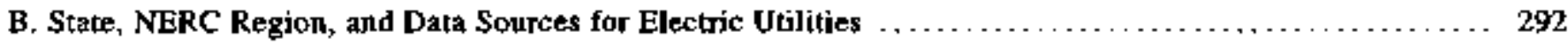

C. Fundanentals of Electric Power Transmission and Integrating Nonutility Generators $\ldots \ldots \ldots \ldots \ldots \ldots .322$

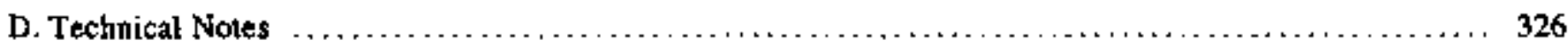

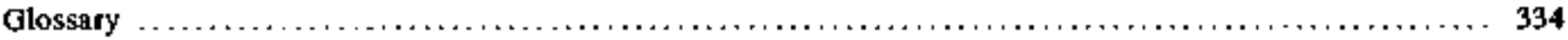


1 Number of Electric Utultues by Class of Ownership Witbun NERC Regjon, 1994

Energy Account Belance by Class of Ownershup, 1994

Components of Source and Drsposition of Electucity by NERC Region, 1994

Electric Trade th the United \$tates by NERC Region, 1987 Through 1994

Intra- and Intertegional Purchases and Sales for Resale by Selected Ownership Classes, 1988, 1990. 1992 , and 1994

6 Firm and Nonftrm Purchases and Sales for Resaie by Investor-Owned U(1)tites, by NERC Region, 1988 , 1990 , 1992, and 1994

7

Wheeling Trade in the United States by NERC Region, 1987 Through 1994

Electricity Purchases by Investor-Owned Uttlttes, by NERC Region, 1994

Electricity Sales for Resale by Investor-Owned Utultues, by NERC Regron, 1994

Investor-Owned Utility Exchanges of Electricity with All Ôwnershtp Classes, by NERC Region 1994

Nonunvestor-Owned Utulty Exchanges of Electricily, by NERC Region, 1994

Electricity Wheelıng for AlI Ownership Classes by Invostor-Owned Utilities, by NERC Reg10n, 1994

Electricity Wheelıng by All Ownershup Classes to Investor-Owned Utılities, by NERC Region, 1994

Purchases by Cooperative Bortowers, by NERC Region, 1994

Receipts by Investor-Owned Utulttes Within and Between NERC Regions, 1994

Deliveries by Investor-Owned Utultues Withn and Between NERC Regions, 1994

Electricity Transachons by Investor-Owned Unlities in Alaska and Hawall, 1994

US Electrucily Trade wilh Canada and Mexico by NERC Regton, 1994

Electricity Putchases by Investor-Owned Utiltues, by \$tate, 1994

Electricity Sales for Resale by Investor-Owned Utuliues, by \$tate, 1994

Electricity Exchanges by Investor-Owned Utilities, by State, 1994

Electricity Purchases by lnvestor-Owned Utulttes Not Reported to the Federal Energy Regulatoly

Commisston, by State, 1994

Electricity Wheelung by Investor-Owned Utilities, by State, 1994

Electricity Wheelıng to Investor-Owned Utılitues, by State, 1994

Electrieity Purchases by Federal Utılities, Fiseal Year Ending September 30. 1994

Electricty Sales for Resale by Federal Utultres, Fiscal Year Ending September 30, 1994

Electricity Purchases by State and Other Government Uulutes, Fiscal Year 1994

Electricity Sales for Resale by State and Other Government Uulutues, Fiscal Year 1994

Electricity Purchases by Municıpal Utilities, Fiscal Year 1994

Electricity Sales for Resale by Municipal Uttlities, Fiscal Year 1994

Electricity Putchases by Publicly Owned Utilities Not Reported on Form EIA-412, by State, 1994

Electrucity Exchanges and Wheelung by Publicly Owned Uulıtes, 1994

Electricty Purchases by the Power Supply Segment of Cooperative Borrowers, by State, 1994

Electructy Purchases by the Distrubution Segment of Cooperatuve Borrowers, by State, 1994

Electricity Purchases by Cooperatuve Utilities Nol Reported to the Rural Utilitjes Service, by state. 1994

Electricity Exchanges and Wheelıng by Cooperative Utılutses, by State, 1994

Transmission Line Stattstics of Investor-Owned Utilities, 1994

Transmission Line Statistics of Public Utilities, by NERC Region, Fiscal Year 1994

252

Transmission Line Statıstıcs of Publıc Utılıtes, Fiscal Year 1994

41 Transmissioa Lunes Added by Investor-Owned Utulutıes, by NERC Region, 1994

Transmission Lines Added by Investor-Owned Utiltties, 1994

Federal Utilities, 1994

State and Other Goversment Utılities, 1994 


\section{Illustrations}

Page

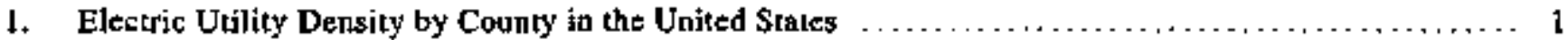

2. North American Electric Reliability Council Region Map for the United States $\ldots \ldots \ldots \ldots \ldots \ldots \ldots, 2$

3. Electriciry Purchases and Costs by Investor-Owned Utilities by NERC Region, $1994 \ldots \ldots \ldots \ldots \ldots$. 5

4. Electricity Sales for Resale and Revente by Investor-Owned Utilities by NERC Region, $1994 \quad \ldots \ldots \ldots .7$

5. U.S. Electricity Trade with Canada and Mexico by NERC Region, $1994, \ldots \ldots \ldots, \ldots \ldots \ldots \ldots, 10$

6. Electricity Purchases and Costs by the Top Ten Investor-Owaed Utilities, $1994 \ldots \ldots \ldots \ldots \ldots \ldots .45$

7. Elestricity Sales for Resale and Revenue by the Top Ten Investor-Owned Urilities, $1994 \ldots \ldots \ldots \ldots .46$

8. Electricity Porchases and Costs by Federal Utilities, Fiscal Year $J 994 \ldots \ldots \ldots \ldots \ldots \ldots \ldots \ldots \ldots, 123$

9. Electricity Sales for Resale and Revenue by Federal Utilities, Fiscal Year $1994 \ldots \ldots \ldots \ldots \ldots \ldots, 124$

10. Electricity Putchases and Costs by the Top Ten State and Othes Governtent Utilities, Fiscal Year

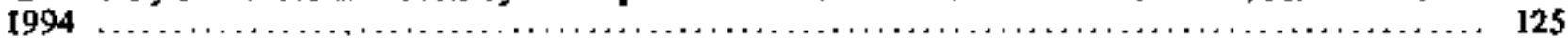

11. Electricity Sales for Resale and Revebue by the Top Ten State and Other Government Utilities, Fiscal Year 1994

Electricicy Purchases and Cosis by the Top Ten Municipal Utilities, Fiscal Year $1994 \ldots 127$

13. Electricity Sales for Resale and Revenue by the Top Ten Mupicipal Utilities. Fiscal Year $1994 \quad \ldots \ldots .128$

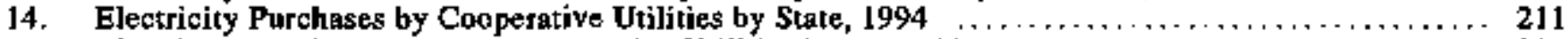

15. Electricity Purchase Costs by Cooperative Utilities by State, $1994 \ldots \ldots \ldots \ldots \ldots \ldots \ldots \ldots \ldots, 212$

I6. Electricity Purchases and Cosis by the Top Ten Cooperarive Utilities, $1994 \ldots \ldots \ldots \ldots \ldots \ldots \ldots \ldots, 2$ I 3

17. Electricity Purcheses by Cooperative Utilities, $1994 \ldots \ldots \ldots \ldots \ldots \ldots \ldots \ldots \ldots \ldots \ldots \ldots \ldots, 214$

18. Selected High-Voltage Transmission Lines in the Contiguous United States $\ldots \ldots \ldots \ldots \ldots \ldots \ldots \ldots \ldots$ 


\section{Executive Summary}

Electricity purchases by jovestor-owned uttities have increased by over 70 percent berwent 1988 and 1994. This is illustrated in a regional comparison of purchases and sales for resale by investor-owned electric nfilitues (Table ES-l) for 1988, 1990, 1992, and 1994. Transactions by investor-owned electric utilities rep. resent almost 55 percent of the transactions in the bulk power market.

Table ES1. Compartson of Electrte Trade Data by North American Electric Reliabillty Council Region Tor Years 1988, 1990, 1992, and 1994

\begin{tabular}{|c|c|c|c|c|c|c|}
\hline \multirow{2}{*}{ NERC Replan } & \multirow{2}{*}{ Geopeplits Ata } & \multicolumn{4}{|c|}{ Dillion Klowptiluent } & \multirow{2}{*}{ 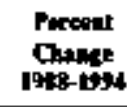 } \\
\hline & & 1)en & $\log$ & 1990 & 1908 & \\
\hline & \multicolumn{6}{|c|}{ 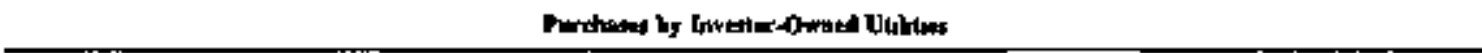 } \\
\hline ECAR & 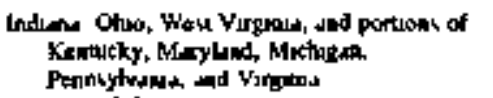 & 91 & 957 & 32 & 347 & 1833 \\
\hline $\begin{array}{l}\text { ERCOT } \\
\text { MAAC }\end{array}$ & 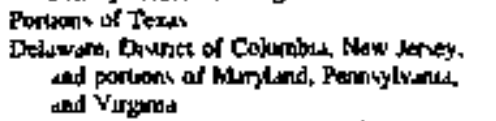 & $\begin{array}{l}172 \\
711\end{array}$ & $\begin{array}{l}280 \\
\text { ग11 } 2\end{array}$ & $\begin{array}{l}2710 \\
413\end{array}$ & $\begin{array}{l}260 \\
3 \geq 5\end{array}$ & $\begin{array}{l}11 \\
1194\end{array}$ \\
\hline MALIN & 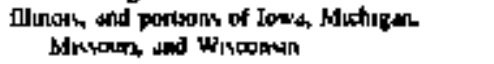 & 758 & 286 & $21 \mathrm{~B}$ & B I & 3420 \\
\hline MAPP(US) & 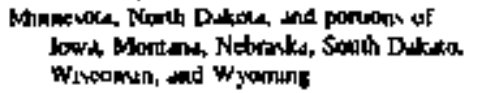 & 310 & 254 & $20 \pi$ & 156 & 的 7 \\
\hline NPCOYUS! & 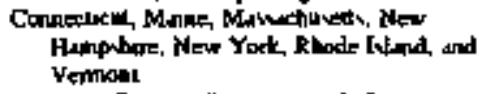 & J69: & 1419 & 2041 & $\operatorname{lot} 3$ & 633 \\
\hline SERC & 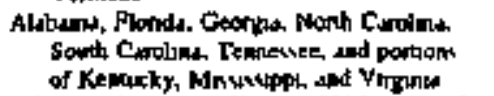 & $\$ 52$ & 868 & B85 & 326 & 6. 2 \\
\hline$S P P$ & 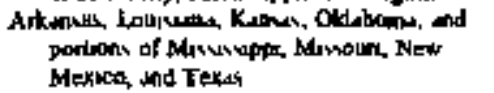 & $\mathbf{s} 1$ & 491 & 337 & 407 & $25 n$ \\
\hline$w s c 0(U s)$ & 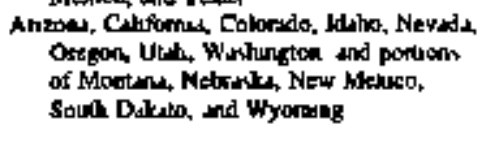 & 1424 & 1320 & 1417 & 1026 & 358 \\
\hline \multirow[t]{2}{*}{ 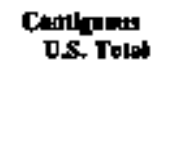 } & & 7125 & 6586 & 5673 & 4158 & TI 4 \\
\hline & \multicolumn{6}{|c|}{ 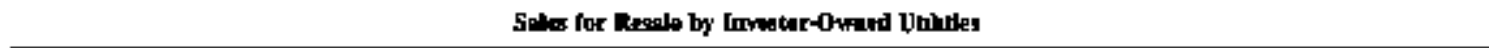 } \\
\hline $\begin{array}{l}\text { PCAR } \\
\text { PRCDT } \\
\text { MAAC } \\
\text { MADN } \\
\text { MAFC } \\
\text { NPCC } \\
\text { SEAC } \\
\text { SPP } \\
\text { WSCC }\end{array}$ & Sume in Abore & $\begin{array}{r}1146 \\
85 \\
461 \\
418 \\
217 \\
3124 \\
655 \\
500 \\
570\end{array}$ & $\begin{array}{r}1320 \\
80 \\
459 \\
263 \\
186 \\
955 \\
893 \\
453 \\
537\end{array}$ & $\begin{array}{r}1176 \\
97 \\
191 \\
292 \\
160 \\
705 \\
935 \\
353 \\
510\end{array}$ & $\begin{array}{r}995 \\
107 \\
147 \\
122 \\
94 \\
648 \\
743 \\
342 \\
402\end{array}$ & $\begin{array}{r}152 \\
-178 \\
2136 \\
2426 \\
539 \\
537 \\
134 \\
391 \\
440\end{array}$ \\
\hline $\begin{array}{l}\text { Contold } \\
\text { DSS, Total }\end{array}$ & & 52.7 & 5164 & 442 & 366,0 & 445 \\
\hline
\end{tabular}

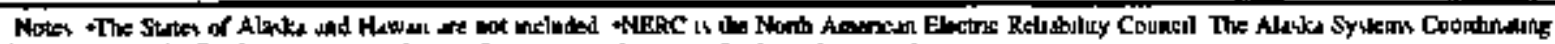

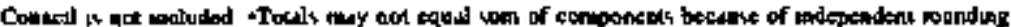

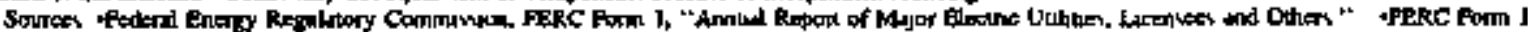

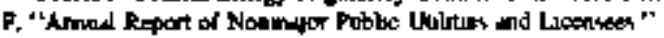


The primary sources of utility data for this publication are shown in Table ES2.

Tabłe ES2. Electricity Data, by Source

\begin{tabular}{|c|c|}
\hline Form & 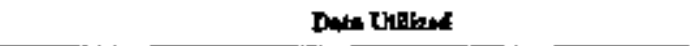 \\
\hline 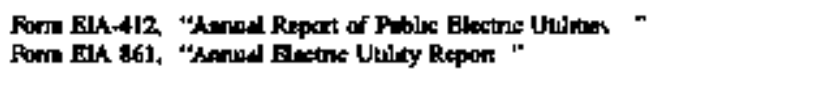 & 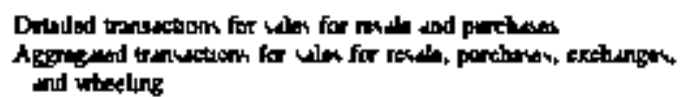 \\
\hline 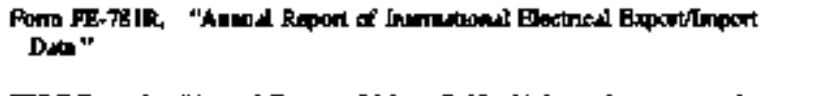 & 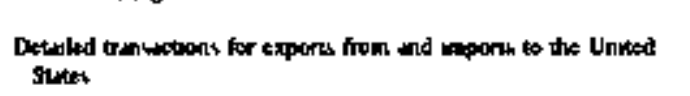 \\
\hline 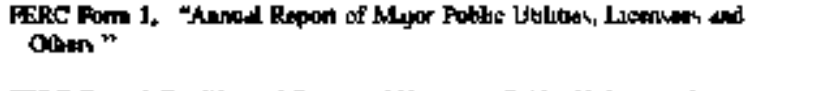 & 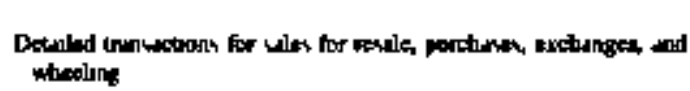 \\
\hline 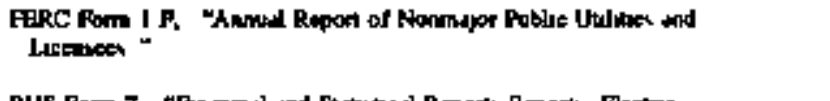 & 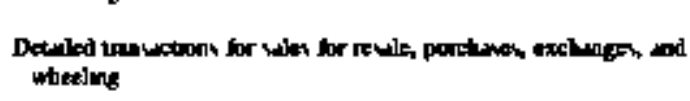 \\
\hline 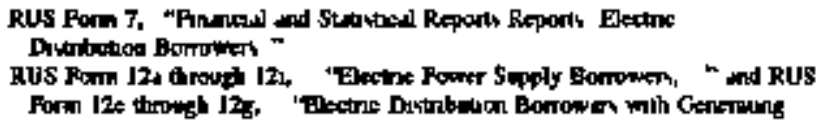 & Detiled trentuctom for proting \\
\hline
\end{tabular}




\section{Introduction}

\section{Background}

Wholesale urade in electricity plays an important role for the U.S. electric utility industry. Wholesale, or bulk power, transactions allow electric urilitics to reduce power costs, jicrease power supply options. and inprove reliability. In 1994, the wholesale trade market totaled 1.9 trillion kilowatthours, about 66 percent of total sales to ultimate constumers.
This publication, Electric Trode in the United States 1994 (ELECTRA), is the fifth in a series of reports on wholesale power transactions prepared by the Office of Coal, Nuclear, Electric and Alternate Fuets, Energy Information Administration (ELA). The electric trade data are published biennially. The first report presented 1986 data, and this report provides information on the electric power industry during 1994.

Figure 1. Electric Utility Density by County in the United States

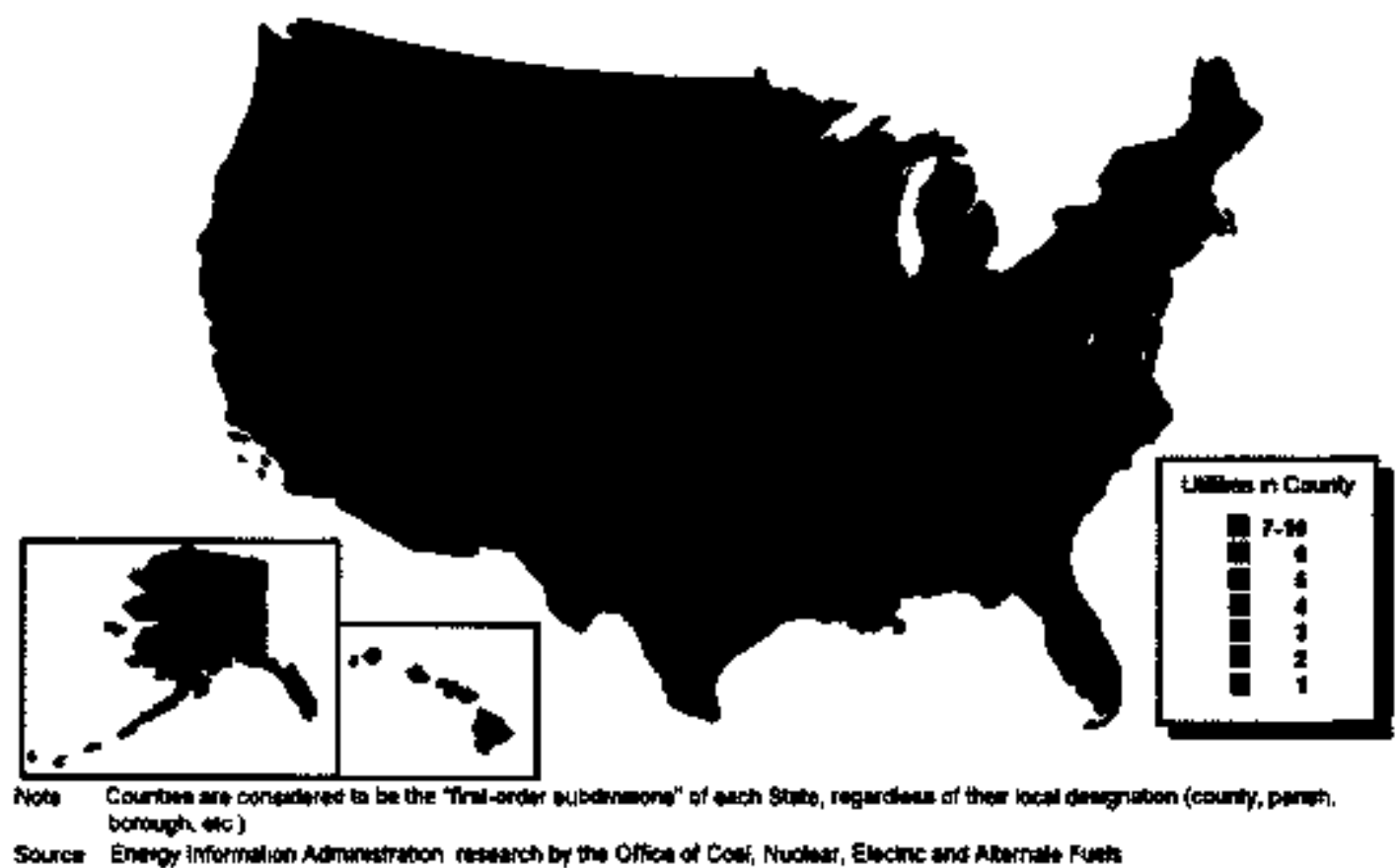

The electric trade data collected and presented in this report furnish important information on the wholesale structure found within the U.S. electric power industry. The pattems of interutility trade in the report support analyses of wholesale power transactions and provide input for a broader understanding of bulk power market issues that define the emerging national electric energy policies. The report includes information on the quantity of power purchased, sold, exchanged, and wheeled; the geographical locations of teansactions and ownership classes involved; and the revenues and costs. 


\section{The Electric Utility Industry Structure}

The utilities covered in this publication include all U.S. electric atjlities in the 50 States and the District of Columbian. Electric utilities fall into three basjc ownership classiffcations: investor-owned (privately owned), publicly awned, and cooperative. Publicly owned utilities may be Federally owned, Stale-owned. or municjpally owned. In 1994, there were 3.204 electric utilities in the United States. Of these, 2,015 were publicly owned (including 10 Foderal utilities), 939 were rural electric cooperatives, and 250 were investor-owned utilities (Tahle I). Some of these elecIric utilities bave service territories extending beyond a single county or parish. Others just serve a munici- pality or part of a county. Most counties in the United States have more than a single urility and some parts of the country have more than I0 electric utilities operating in a county (Figure 1). Many of the Nation's electric utilitics are exclusively distribution utilities, that is, they purchase wholesale power from other uiblities and distribute, on their own distribution lines, the electricity to ultimate consumers. For example, fewer than $1,000^{\prime}$ of the 3,204 utilities in the United States are engaged in power generation. In order to move electricity from utilities with gemernting capability to the distribution utilities, an extensive system of high voltage transmission lines is opejated by the Nation's largest utilities. This transmission network permits the electric trade between utilities, because without it electricity could not be moved from power plants to the thousands of distribution systems and millions of consumers of electric power.

\section{Figure 2. North Amerlcan Electric Reliability Council Region Map for the United States}

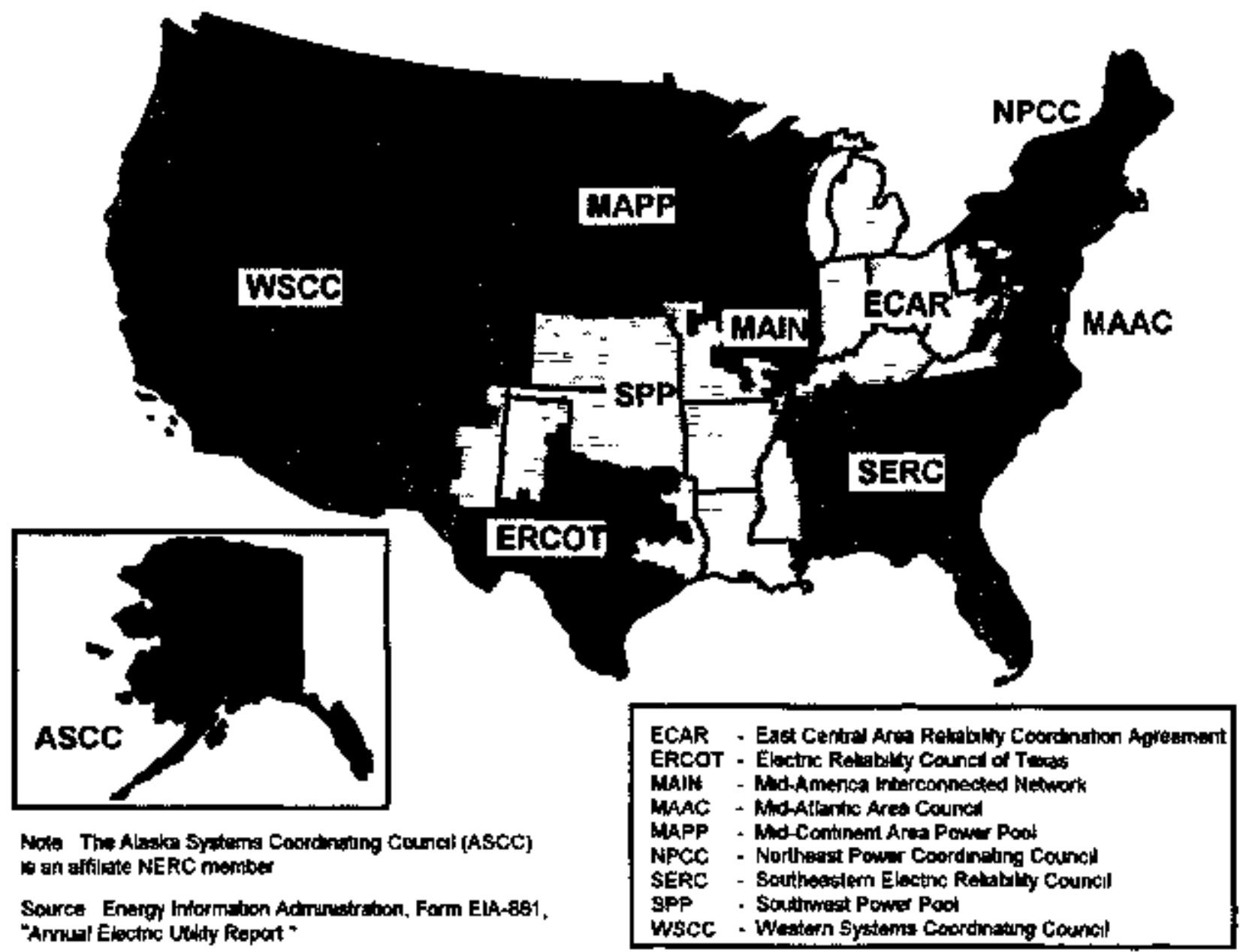

I Eaprgy lajotmolion Administcatsod Form BIA-\$60. "Anmul Electric Generator Repart " 


\section{Investor-Owned Utilities}

There are several basic organizational forms among investor-owned companies. The most prevalent is the single corporation. Another common form is the holding company in which a parent company is established to own one or more operating utility companies which are integrated with one another. The Public Utility Holding Company Act of 1935 requires that the utilities owned by a holding company operate as a single integrared utility system.

Most of the investor-owned utilities sell power at retail rates to several different classes of customers and at wholesale rates to investor-owned, Federal, State, and locil government utilities, public otility districts, and rural electric cooperatives. A wholesale customer can be a distribution utility with litde or no generating capacity of its own or another integrated utility that purchases power to supplement its own generation or for economic reasons.

\section{Publlcly Owned Uthlltes}

Publicly owned electric utilities represent 63 percent of the total pumber of U.S. electric utilities but accounted for only 16 percent of sales to ultimate consumers in 1994. Publicly owned utilities include Federal utilities, State and other government owned vilitites (irrigation distriets, public power districts, and State authorities and other organizations), and municipal utilities.

Federal power plants generate less than 9 percent of the electricity in the United States, primarily from hydroelectric facilities operated by the U. S. Amry Corps of Enginets and the Burean of Reclanation of the U.S. Department of Interior. Electricity is atso generated by the Bureau of Indian Affairs and the International Boundary and Water Commission. Energy generated by these producers is marketed (along with any of their own generation) by the fje Federal power marketing administrations: Bonneville (in the northwestern United States), Southeastern, Southwestern, Western Area, and Alaska. The power marketing administrations are part of the U.S. Department of Energy and operate in all States except those in the Northeast, upper Midwest, and Hawaii. The Tennessee Valley Authority generates electricity from coal and muelear power as well as hydropower. It is the largest Federal power producer and markets eleczicity in both the wholesale and tetajl to arkets. In addition, the U.S. Department of Defense sells a small quantity of power, generated by Federal nonutilities, to the power grid.

Approximately 20 percent of the electricity generated by Federal utilities is sold to retail customers which are usually large industrial customers or Federal installations. Most of the retoaining energy is sold to publicly owned utilities and rural cooperatives for resale. These wholesale customers have a legal prefer. tnce right to Federal electricity. Only the surplus remaining after meeting the energy requirements of preference customers is sold to the investor-owned utilities.
Munjcipal utilitjes accoumt for almost 58 percent of the number of electric utilities in the United States, but produce less than $\$$ percent of total net generation. Municipal utilities tend to be concentrated in cities where the loads are smatl. They exist in every State except Hawaii, but are concentrated, predominately in the Midwest and Southeast, In general, municipal utilities tend to have lower costs than investor-owned utilities becanse they geperally have access to bonds supported by tax based funds and do not pay certain taxes or dividends. They also have high density service areas.

The other publicly owned utilities include Stare avthorities, public power districts, irrigation districts, and other State organizations. State authorities art utilities that function in a manner simitar to Federal utilities. They generate or purchase electricity from other utilities and market large quantities wholesale to groups of utilities within their States at lower prices than the individual utilities would otherwise pay. The public power districts tend to be concentrated in Nebraska, Washington, Oregon, Arizona, and California. Appendix Tables B2 through B4 contain listings of the public power municipals, State authorjties, and Federal atilities used in this publication.

\section{Aural Electric Cooperatives}

Most rural electric cooperacive utilities are formed and owned by groups of residents in rural areas to supply power to those areas. Some cooperatives may be owned by a number of other cooperazives. (There are really three types of cooperatives: (1) distribution only; (2) distribution with power supply; and (3) generation and ranstrission.) Cooperacives currently operate in 46 States, and represent 29 percent of the total utilities in the country. Mfost distribution cooper. atives resemble municipal utilities in that they often do not generate electricity, but purchase it from other utilities. The other type (generating and transmission cooperatives) are usually referred to as power supply cooperatives. These cooperatives are usually owned by the distribution cooperatives to whom they supply wholesale power. Distribution cooperatives resemble Federal utilities by supplying electricity to other utility custorners from thejs generaling capabiltiy. Cooperatives accounted for almost 8 percent of sales to ultimste consumers in $\mathbf{5 9 9 4 .}$

\section{Changing Electric Power Industry}

The electric power industry is undergoing fundamental changes reflected by its evolving structure. For many years, the industry was dominated by locally franchised, vertically integrated utilities, regulated by Scate regulatory agencies. This pieture has been changing rapidly in Jecent years with the passage of new legislation introducing a dew type of business entity, changing regulations, and an increasing reli- 
ance by unlıties on the wholesale purchase of electricity.

\section{Nonutllity Generation in Electric Trade}

The growing role of nonutility generotors (NUG) in electricity supply reflects the acceptance of alternative power supplies in the wholesale electric power market. The near monopoly of electric generation by regulated electric utitities has ended, as many new participants generate electricity and sell power to electric utitities, thus changing long-established instirutional relationships. The outlook is for greater participation by nonutilicy generators in wholesale power supply. The changing roles and relationships between utility and nomutility participants in the wholesale power market are uncertain and have raised several unresolved technical, oconomic, and regulatory issues.

Differences remaln between traditional electric urilities and nonutility power generators for many teasons, including the passage of new tegislation and the application of regutations designed specifically for nonutility generators. For example, a raditional definntion of a urility might simply inclode any busuness entity whose principal business is generaling and selling electricity. This deftaition would exclude industrial generators and cogenerators, the principal nonutility generators organizations producing powet but which arc primanly engaged in business activities other than power sales.

A more common defrtition of an electric utility includes three elements: a franchised service territory: the obligation to serve all customers within this exclusive ares; and the regulations that constitute this compact. This definition, however, ignoses utilitues without retail customers such as generation and transmission companies. All generators of electricity without a service area and with a contractual obligation to serve would be considered nonutilities under this defimtuon. Even with this defunition, there are various classes of nonutility generatous, some of whet reflect regulatory distinctions and otbers, which have evolved over tume and may be less precise and overlapping: independent power producers (IPP), nonutility generators (NUG), ${ }^{2}$ small power producers (SPP), qualifying facilities (QF), and industrial power producers.

Table 1. Number of Electric Utilties by Class of Ownership Within NERC Region, 1994

\begin{tabular}{|c|c|c|c|c|c|c|c|}
\hline \multicolumn{2}{|c|}{ NERC Rezion } & $\begin{array}{l}\text { Inrevar } \\
\text { Oened }\end{array}$ & Eeteratl & $\begin{array}{l}\text { Stur and Wher } \\
\text { Goweminent }\end{array}$ & Maminals & Conopenutuses & US Tاها T) \\
\hline \multicolumn{8}{|c|}{ Norber of Juiluag } \\
\hline ASEC & & 24 & J & 5 & 3] & 22 & 83 \\
\hline ECAR & & 45 & $\mathrm{I}$ & 4 & 232 & $\ln \theta$ & $38 !$ \\
\hline encor & & 6 & 1 & 3 & 67 & no & 133 \\
\hline MAAC & & 19 & 0 & in & 级 & 19 & a \\
\hline MAIN & & If & 0 & $\mathbf{0}$ & 143 & 46 & 207 \\
\hline MAPP & . & 15 & b & 觔 & 458 & 186 & 695 \\
\hline NPCC & . & 56 & $\mathbf{0}$ & 4 & 12] & 12 & נ9נ \\
\hline SERC & & 19 & 2 & 5 & 115 & 189 & 920 \\
\hline SPP & & 16 & i & 8 & 289 & 136 & 470 \\
\hline wsce & & 29 & 4 & 79 & $1 \pi$ & 140 & 421 \\
\hline Hatedu & & 3 & 0 & n & 0 & 0 & 3 \\
\hline U.S. Total & & 250 & 10 & 143 & I. Barz & 939 & 3,204 \\
\hline \multicolumn{8}{|l|}{ Perconet: } \\
\hline ASCC & & 10 & 10 & $\mathbf{y}$ & 2 & 2 & 3 \\
\hline ECAR & & $\mathbf{j}$ & 18 & 3 & 12 & 12 & 12 \\
\hline ERTOT & & 2 & 10 & $\mathbf{2}$ & 1 & $\bar{E}$ & 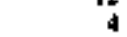 \\
\hline monc & & 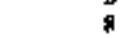 & 0 & 0 & 1 & 2 & 3 \\
\hline MALN & & 7 & 0 & n & 8 & 5 & 6 \\
\hline NAPT & & 6 & 0 & 25 & 25 & 20 & 22 \\
\hline NFC & & 2 & 0 & 1 & 6 & 1 & th \\
\hline SERC & & 8 & 20 & 1 & 17 & $2 B$ & 13 \\
\hline SPP & . & 6 & 30 & 6 & I6 & 17 & 13 \\
\hline wseC & & 12 & 40 & 53 & 9 & 15 & 13 \\
\hline Hawal & & 1 & 0 & 0 & 0 & 0 & $\star$ \\
\hline
\end{tabular}

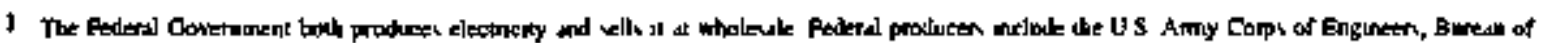

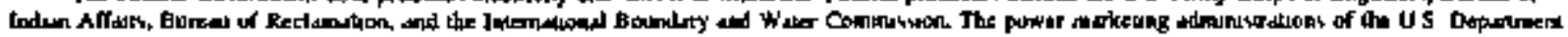

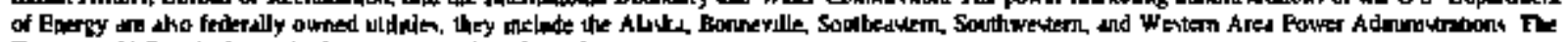

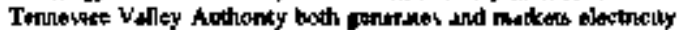

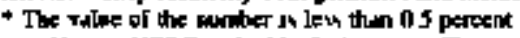

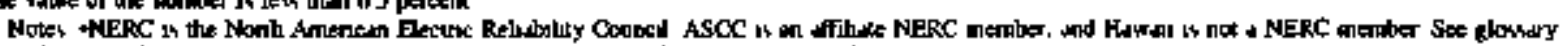

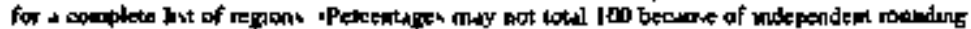

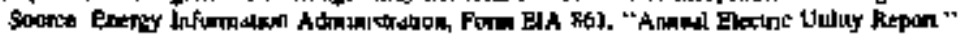

Current legistation and regulations may not always draw clear distinctions. For example, a producer of electricity that woold appear to be a nonutility generator might be considered a utility if it is intercon-

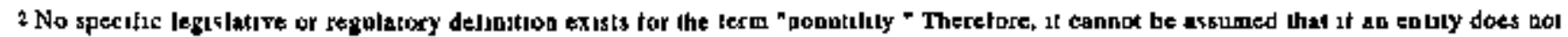
quality as a wtility lhal il is then a doaublity. 
nected to transmission faciltuies in certain ways. Moreover, vazjous laws and regulations may not always draw clear distinctions and may defioe utility broadly to include anyone selling electricity. The clearest exception is for firms, which are not electric utiljties, that sell power from qualifying facilities (QF) as defined under the Public Utility Regulatory Policies Act (PURPA). The Energy Policy Act of 1992 defined new class of nonutility generator, called exempt wholesale generators (EWG), to encourage the development of conventional geperating facilities that will not meet the QF requirements. These EWGs are not considered electric ntibities and are exempted from Public Utility Holding Company Act requirements. Despite the variety of definitions, nonutility power producers are generally considered to be the nontraditional producers of electric energy (i.e., producers that are neither traditional Federal,
State, investor-owned, cooperative, nor mubicipal utilities).

The Federal Government and other usets of electric generation data are interested in this nontraditional generation group because, as nonutility power producers increase their level of produetion of electric energy, the group becomes a more important segment of the U. S. electric power industry. Nonutility power producers sold over 208 billion kijowathours in 1994 (Table 2). As one of the fastest growing segments of the eloctric power industry, nonutility power producers may be responsibte for a significapt portion of new power plant construction. This group is, therefore, of great interest to analysis of the electric power industry. Analysis of the electric utility industry is complicated by the changing structure of the industry. - which now includes a significant number and wide range of nonutility participants--and by the changing mix of transactions that characterize electric trade.

Figure 3. Electricity Purchases and Costs by Investor-Owned Utilitles by NERC Reglon, 1994

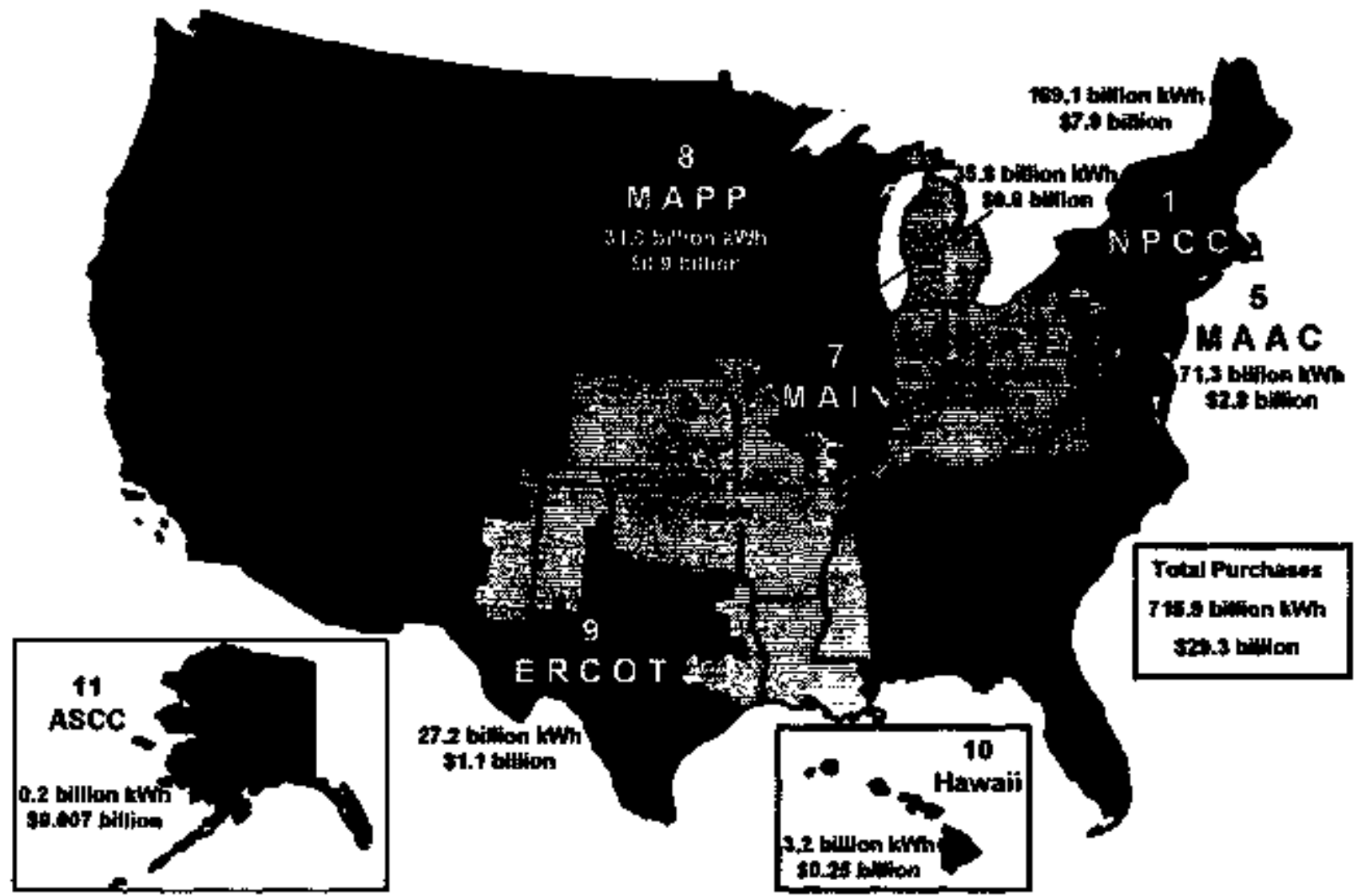

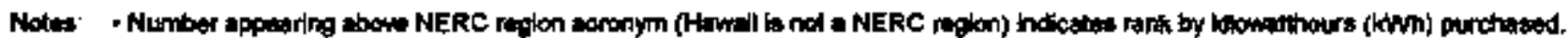

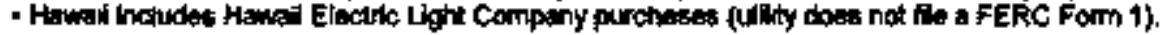

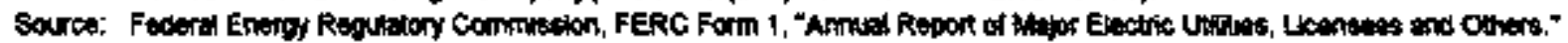

\section{Transmission Services and Energy Broker/Marketers}

The Energy Policy Act of 1992 (EPACT) amended the Federal Power Act (FPA) to allow any electric utility to apply to the Federal Energy Regulatory Commission (FERC) for an order requiring another utility to provide transuission services (wheeljng). Prior to EPACT, FERC conld not mandate an electric utility to provide wheeling services for wholesale electric trade. This change in the law will permit operators of electric generating equiptont to seli wbolesale power (sales for restale) to noncontiguous utilities. The amendment to the FPA will also affect electricity marketers, a relatively new group under FERC's juris. diction. Marketers are business entitios engaged in buying and selling electricity, but with no generating or transmission facilities. Marketers take ownership of 
the electricity and are involved in interstate trade. A number of energy marketers that may be involved in interstate electricity trade have filed with FERC and have had their rates authorized (Appendix Table B7). When ownershjp of the electricity is not taken, then those transactions ate not regulated by FERC (the parties handling the transactions are called brokers).

\section{The Interconnected Networks}

The U.S. bull power system has evolved in to three major nerworks (power grids), which also include smaller groupings or power pools.

Table 2. Energy Account Balance by Class of Ownership, 1994 (Million Kilowatthours)

\begin{tabular}{|c|c|c|c|c|c|c|}
\hline Ite्वाm & $\begin{array}{l}\text { Invevaph } \\
\text { Owead }\end{array}$ & Foderal & $\begin{array}{l}\text { Shlte and odher } \\
\text { Gunerameat }\end{array}$ & Muncepls & Coopetrater & US Total \\
\hline \multicolumn{7}{|l|}{ Soncen 1 Enerty } \\
\hline Nkt Genertios & 2206,045 & 248,946 & 174,786 & J30, 185 & 164,520 & $2,924,961$ \\
\hline Pontuses from Iblukas & 502,165 & 19,933 & B], 939 & 274,054 & 345,722 & $1,226,814$ \\
\hline Pancluses from Plowoulines & 206358 & 124 & 427 & $1,40,5$ & 281 & 203,778 \\
\hline Net Excharge? & 1,012 & $-7,398$ & 1.628 & -102 & -358 & $-3,659$ \\
\hline Recasved & 69211 & 41,060 & T,80.90 & $11,5] 5$ & 25,062 & 154,750 \\
\hline Dollyated & xit.] 40 & $48,9 \sqrt{7}$ & 4,253 & JI, $\mathrm{k}] \mathrm{T}$ & $25,+20$ & 15840 \\
\hline Net Whating & 3,457 & 291 & 210 & 53 & I9I & 4,225 \\
\hline Recesved & 252943 & $47,2,4$ & 12,775 & 16.524 & 7,384 & 33x, Bns \\
\hline Dejเพtred & $245,4 h 5$ & 46,933 & 12,502 & Ifrsitis & 7,193 & 332580 \\
\hline \multicolumn{7}{|l|}{ Dupedifing of Recitr } \\
\hline 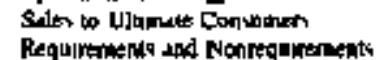 & $2,237,693$ & 46,7013 & 96,656 & 324,9866 & 228,535 & $2,534,563$ \\
\hline Sules for Rosde & 9]7,127 & 198,782 & $19 n_{1}, 262$ & 61,776 & 257,499 & $1,185,352$ \\
\hline Energy Furmbed whowl tharge & 1,976 & 227 & J, 194 & 1,377 & 88 & 4,762 \\
\hline Energy Utud by Nolky & 6571 & 2.305 & 4,999 & $1 . J 67$ & 459 & 15.495 \\
\hline Entrity Lonvest & 158,946 & 13.351 & $\sin \theta$ & Ifig35 & 23,827 & 220,948 \\
\hline
\end{tabular}

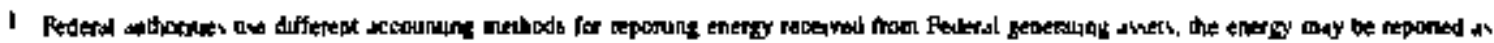
generstan of puretant.

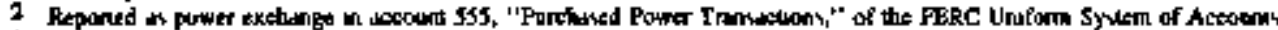

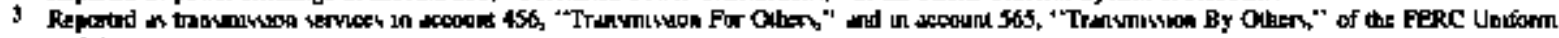
Sychen of heoconas

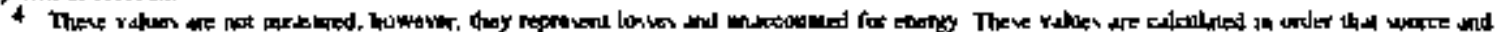
disportion of energy be equivilex.

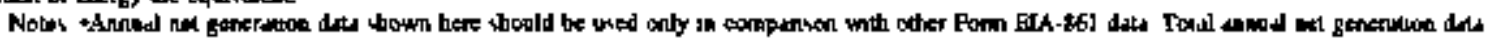

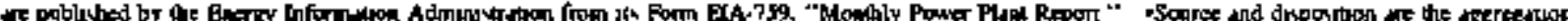

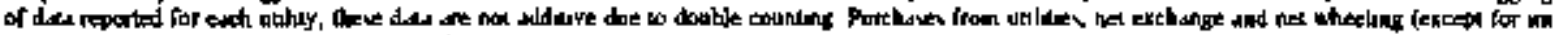

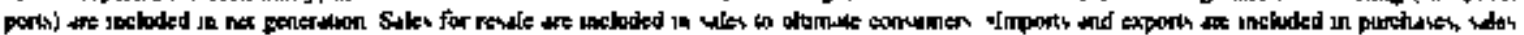

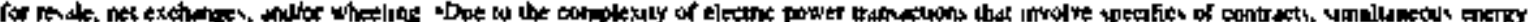

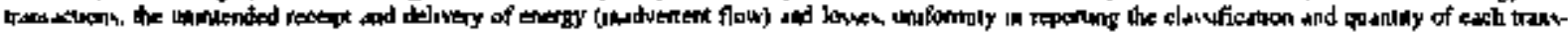

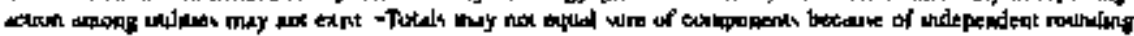

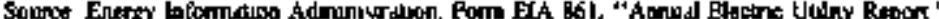

The major networks consist of extra-high voltage connections between individual utilities designed to permit the transfer of electrical energy from one part of the network to another. These transfers are restricted, on occasion, because of a lack of contracrual arrangements or because of inadequate transmission capability (See Appendix C).

The three networks are (1) the Eastern Interconnected System, consisting of the eastern two-thirds of the United Stares; (2) the Western Interconnected System consisting primarily of the Southwest and areas west of the Rocky Mountains; and (3) the Texas lnterconnected System. The latter is not interconnected with the other two networks (except by certain direct current lines). The two other nerworks bave linited interconncctions to each other. Both the Western and Texas interconsect are linked with different parts of Mexico. The Eastern and Western Intereonnects are completely integrated with most of Canads or have links to the Quebec Province power grid. Virtually all U.S. utilities are interconnected with at least onte other utility by these three major gonds. The exceptions are in Alaska and Hawaii. The interconnected utiljties withen each power grid coordinate operations and planning, and buy and sell power among themselves for the benefit of the eustomers.

Overall retiability planning and coordination of the interconnected power systems are the responsibility of the North American Electric Reliability Council (NERC). The NERC was voluntarily formed in 1968 by the electric utulity industry as a result of the 1965 power failure in the Northeast. The regional councils cover the $\mathbf{4 8}$ contuguous States, part of Alaska, ${ }^{3}$ and portions of Canada and Mexico (Figure 2) and are responsible for the overall coordination of bulk power policies that affect the reliability and adequacy of service in their areas. These councils also regularly exchange operacing and planning information among

3 The Alaska System Coordunalung Councl! (ASCC) is an attlluate member of NERC 
their member utilities. The boundaries of the NERC regions follow the service areas of the electric otilitits in the region. The service area of many electric urilities do not foltow State boundaries. Hence, electric trade data are not avallable by State, but are presented by NERC regions, except for Hawaji

\section{Wholesale Trade Transactions}

Wholesale electricity trade has become a major contributor to systems operations in the U.S. electric utility industry. In this publication, wholesale, or bolk powes, transactions are categorized and defined in accordance with FERC reporting requirements.
Wholesale power transactions include purchases, sales for resale, exchanges, and wheeling (i.e., transmission services). Purchase transactions involve buying power from electric utilities and nonutitity producers of electricjty. Sales for resale transactions refer to powtr sold by one electric utility to one or more utilities for distriburion to ultimate consumers. Exchanges involve trading electric epergy when supply and demand conditions are murually advantageous and reversible for the participating parties. Exchanges are often based on time-dependent excess capacity or diversity in load requirements.

\section{Figure 4. Electrlclty Sales for Resale and Revenue by Investor - Owned Utllitles by NERC Region, 1984}

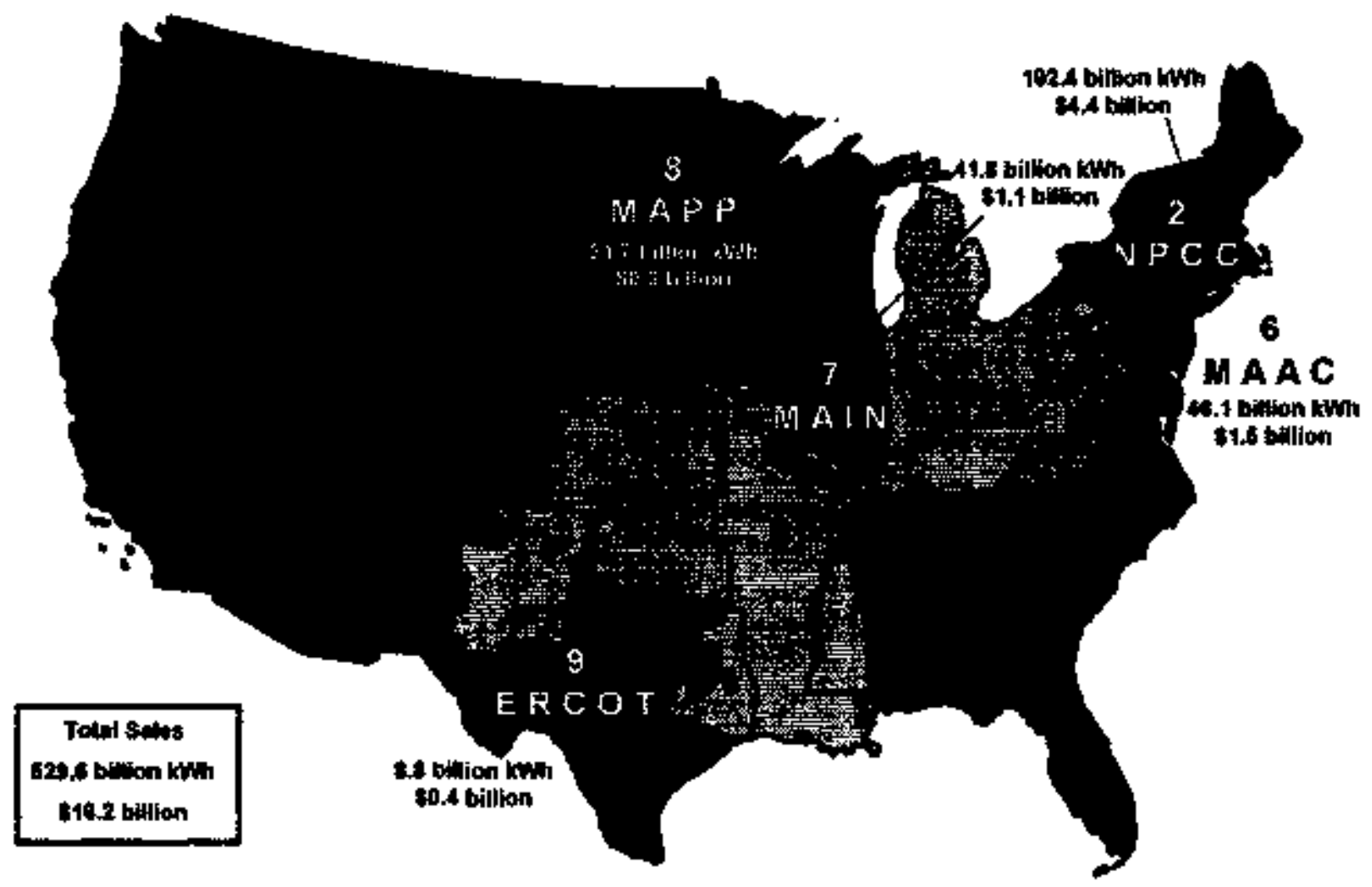

With exchange transactions, monetary repayment or replacement of energy may extend over several years. which is not a member.

Most bulk power transfers involve nejghboring utilities whose systems are directly interconnected. At other times, however, bulk power transfers involve a thitd utility situared belween two unconnected utilities that want to enter into a bulk power transaction. In that transacion, the third (intervening) utility allows its transmission lines to be used for bulk power trans- fers between the two other utilities. This intervening utility is providing a form of transmission service known as wheling. Wheeling is generally defined as the movement of electricity from one system to another over the transmission facilities of an intervening system. (Technically, there is two-party wheeling: (1) seller and whocler and (2) purchaser.) Wheeling is conducted under terms and conditions that ensure safety and reliability and that prevent the wheeling uility from being unfatrly burdened with added costs. 
Table 3 provides an overview of wholesale and retai] tansactions in the electic urjluty sysiem by NERC region. The table includes data on net generation, purchases, energy losses, sales to ultimate consurvers, and electric trade transactions. Electricity is purchased by all classes of electric ubilities. Purchases from nonutilitjes accounted for 7 percent of sales to consumers in 1994. These purchases are made generally by utilities in whose service territories the nonvtilities are located. Losses represent energy lost in transmission, distribution, and power maccounted for, which amounted to 7.5 percent of sales to consumers in 1994. Electricity inflows--purchases, exchanges-1n, and wheeling-in--represent almost 66 percent of sales to consurters. Table 4 presents a bistorical view of electric trade flows (including net generation) and how components of electric trade changed for years 1987 through 1994. Chapters I through 4 of this publication focus on purchases, sales for resale, exchanges, and wheeling of electricity (columins 4 and
5 of Table 3) Drata for energy flows tepresent the summations of nows of energy between utilities in accordance with the provjsıons of their service agreements. These flows represent accounting transacijons rather than meiered flows. Detaited covernge of wholesale trade activities by NERC region for each class of owmership is presented in Chapters 2 through 4. The electricity transactions reported in the remaning chapters do not include transmission and distribution losses. Chapter 5 presents selected data on the physical transmission systems.

\section{Categories of Wholesale Trade Transactions}

There are two main types of bulk power transactions: coordination transacions and requirement transactions capacity sale usually has a separate cost from the demand charge.

Table 3. Components of Source and Disposition of Electrikity by NERC Region, 1994

\begin{tabular}{|c|c|c|c|c|c|c|c|c|}
\hline & & $\begin{array}{c}\text { NEt } \\
\text { Ceneration }\end{array}$ & $\begin{array}{l}\text { Enerpy } \\
\text { Fumulued } \\
\text { dod Uved } \\
\text { by Uwity }\end{array}$ & $\begin{array}{l}\text { Nonululty } \\
\text { Purchdites }\end{array}$ & 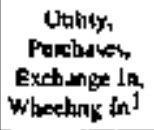 & $\begin{array}{l}\text { Sales for } \\
\text { Resule } \\
\text { Exchenge Ous } \\
\text { Wheding Out }\end{array}$ & $\begin{array}{l}\text { Totel Regood } \\
\text { Exety Lan }\end{array}$ & 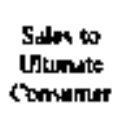 \\
\hline \multicolumn{9}{|c|}{ Taersy (nnion kWh) } \\
\hline ASCC & & 4,913 & 37 & 4 & 3,948 & 3,945 & 350 & 4533 \\
\hline ECAR & & 492014 & I.J8? & J2,659 & 186.341 & 199,188 & 20,959 & 459,74 \\
\hline ERCOYT & & 210,256 & tôs & 23,264 & 117.828 & 112,9895 & 13,278 & 218,781 \\
\hline MAAC & & 206,221 & 2841 & 2n,911 & 73,999 & 6in,206 & 14,450 & 273,635 \\
\hline$\triangle A D N$ & & 22],730 & 78 & 392 & 66,145 & 58,584 & 14,492 & 214,204 \\
\hline MAPP & , & I3A, 607 & 624 & 583 & $108,4 \pi 2$ & 92834 & |1.2] & $12,3,935$ \\
\hline MPCC & & 189,546 & סות & 19,348 & 218,003 & 198,490 & Is,707 & 201,6 , \\
\hline SERC & & ถิ78, 423 & 2,542 & $24,0 \mathrm{AN}$ & 373,641 & Jตร.M. & 49,982 & 6ك6, \\
\hline SPP & & 260,125 & $1,6,77$ & 6,854 & 165,263 & 153,9 99 & 19,299 & 27,183 \\
\hline wscec & . & 537,399 & $9,23 t$ & 63,297 & 404.728 & $\$ 29,0,34$ & 47.4]5 & 527,696 \\
\hline HNata & & 5,728 & 20 & 3,42 & 1 & 6 & 552 & 6,593 \\
\hline Us, Total & & 2524961 & 20,256 & 205,738 & $1,718,469$ & $1,575,14]$ & 220.948 & $2.934,563$ \\
\hline \multicolumn{9}{|l|}{ Prepent } \\
\hline AsCC & . & " & - & 4 & * & $*$ & $\bullet$ & $\bullet$ \\
\hline ECAR & . & 17 & 6 & 6 & (1) & 12 & 14 & 16 \\
\hline ERCOT & & 7 & 2 & נג & 7 & 7 & 6 & 3 \\
\hline MAAC & & $?$ & 14 & 10 & 4 & 4 & 7 & $g$ \\
\hline MALIN & & 8 & 4 & $*$ & 4 & 7 & 7 & 7 \\
\hline MAFP & & 4 & 3 & $=$ & 6 & 6 & 5 & 4 \\
\hline NPCC & & 6 & 5 & 24 & 13 & 12 & 8 & 8 \\
\hline SBRC & & 27 & 11 & 12 & 22 & 23 & 23 & 28 \\
\hline SPP & & 9 & 6 & 3 & ID & 9 & 9 & 9 \\
\hline wSC: & & 18 & $\$ 6$ & 12 & 24 & 26 & 21 & I8 \\
\hline 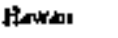 & & \pm & $\bullet$ & 2 & $\star$ & $\star$ & $\star$ & 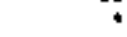 \\
\hline
\end{tabular}

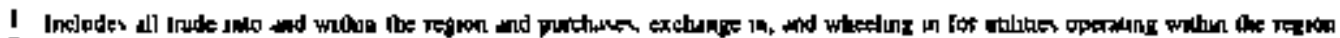

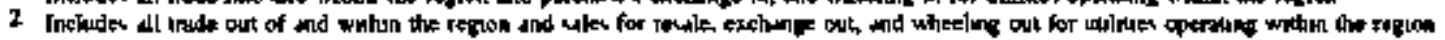

- The ralue of ube nomber wlesi then 05 pareent

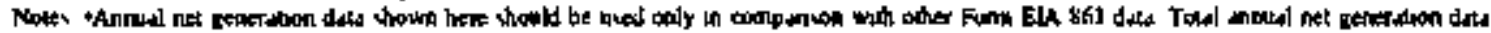

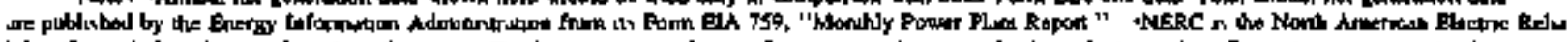

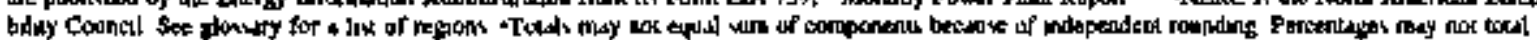
jou betale of independent pounting

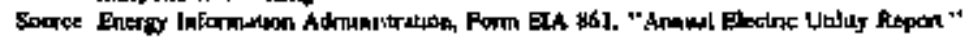

Coordunation transactuons are unterruptible and generally represent short term sales as contrasted to requirerrent transactions which usually are established for longer periods of time. Requirement transactions cover power supply coniracts in which furm service equales to the energy needs required by a designaled customer base plus coverage of associated electrical losses and the electrical energy is supplied by other utilities. The duration of the sale and the type of capacity sold are key factors in determining the type and value of the transaction. The length of the sale can be 1 hour, several months, a year, or longer. Another type of transaction is an exchange in which the energy usually is returned in kind at a date agreed upon by both utrlitues.

Frrm sales usually Involve the safe of capactiy, meaning that etther the associated energy will be 
taken by the purchaser or the purchaser will pay for the capacity during those periods when the energy is not taken. This payment is called a demand charge. The dolivery is scheduled as past of the nomal operaring condjitions of the affected electic systems. Firm capacity may be mate avajtable from the selling utitily's entire pool of generating facilities or from a speciffc generating unit. The capacicy offered in these trassactions is usnally for a specified period of time and negotiated in advance of the trade. The associated energy purchased through a capacity sale usually has a separate cost from the demand charge.

One of the most common types of firm power transactions involves the transfer of full or partial requirements power. Requirement contraets involve electric utilities that bave either insufficient of no generating capability to satisfy their customer load. These utilities negotiate Jong-term, firm power contracts in which the terms and conditions obligate the selling electric vtility to provide the buying electric vitility a level of service equivalent to the seller's requirement to support its retail customers. An electric utility purchasing requirement power may have contrats with more than one electric ubility in order to cover their customer base.

Nonfirm sales, sometimes called energy, economy, or interrugtible sales, rarely have a demand or capacity charge included in the price of the transaction. These rransactions are typically for short periods and strbject to curtailment or cessation of delivery by the supplier in accordance with prior agreements or under specified conditions. Electric utilities engage in these rraasactions in order to gain operational savings, such as avoiding the use of more expensive fuels and selling electricity generated by the spillage of excess reservoir water through a water-driven turbinegenerator.

In addition to domestic wholesale transactions, U.S. utilities have engaged in international trade agreements with utilities from Canada and Mexico that cover a nuraber of transaction options. These options include purchasing nonfirm energy from relatively inexpensive renewable resources, snch as hydroelectric facilities in Canada and geothermal facilities in Mexico; acquiring additional generating capability to support the requirements for supply at U.S. electric utilities; the holding of reservoir water to be turned into electricity; and sharing the benefits of coordinated planing between the systems.

\section{Regional Recelpts and Deliveries of Wholesale Power}

A 1arge mount of the electricity generated in the United States is traded under wholesale putchases and sales for resale contracts. The vast majority of wholesale transactions for investor-owati, Fedetal, and cooperative utilites involve utilities within the same NERC regional boundaries. Many of the differences between intra- and interregional wholesale power transactions result from the historical development of multiple transmission links among clusters of beighboring utifities. In addition, the development of shared interconnection and coordination agreements among these groups of utilities help delineate the regional operating boundaries of electric utilities.

Investor-owned electric utilities have led the other ownership classes in both intra- and interregional purchases and sales for resale. Interregional soutres provided 10 perceat of investor-owned purchases in 1994. Cooperatives made intertegional purchases of 5 percent while Federal utilities purchased 8 percent (Table 5).

As shown in Table 6, both firm purchases and sales for resalc represented almost 52 percent of investorowned utilitjes transactions (miscellaneous purchases and sales for resale are incloded in the total). Utilities in the WSCC region purchased the most firm elecsricity, while the utjlitjes in the ECAR sold the most in 1994. Utilities in NPCC purchased the most nonfirm energy, and SERC utilities sold the most nonfirm power.

Table 7 shows wheeling trade in the U.S. by NERC Region for the years 1987 through 1994.

\section{National and Regional Highlights}

The FERC Form 1, "Annual Report of Major Electric Utilities, Licensees and Others," is used to collect annual data on electricity trade by quantity, cost, and revenue from invescor-owned utilities. These data were used to construct the tables in Chapters 1 and 2 and show the quanticy and value of firm and nonfirm electricity trade reported.

Purchases from all sources by major investor-0wned utilities toraled 361.7 billion kilowatthours of firm power for $\$ 18$ bitlion and 326.5 billion kilowathours of nonfirm energy for $\$ 10.5$ billion in 1994 (Table B).

Investor-owned utilities exchanged approximately 63 biltion kitowatthours of electricity with other utilities for both receipts and deliveries (Table 10). The most exchange activity oceurred in ERCOT, with 3.3 billion ktlowatthours each in receipts and deliveries.

The excbanges reported with noninveslor-owned utilities in 1994 (Table II), were mostly with Federal utilities, acconnting for hatf of the exchanges. 


\section{Tables}

National and regional aggregates are presented at the end of this chapter for data covering:

- Purchases, sales for resale, and exchanges by investor-owned utilities with all ownership classes (Tables 8 through 10 )

- Exchanges by noninvestor-owned utilutes (Table (1)

- Wheeling transactions of investor-owned utilities (Tables 12 and 13)

- Regional purchases by cooperatıve bortowers (Table 14)

- Trading whthin and berween regions by investorowned utilities (Tab]es 15 and 16 )
- Transactuons of tovestor-owned utilitues in Alaska and Hawai (Table 17)

- U.S electricity trade with Canada and Mexico (Table 18).

\section{Sources of Data, 1994}

Summaty information for the United States are provided in Tables 1 through 4 and are obtained from the Form ElA-861 "Annual Electric Utility Report," which is a mandarory report ftled by all U.S. electric utilues. The survey represents the collection of data from the complete universe of electric utilities. All other electric utiluty surveys ctted in this publication represent subsets from

\section{Flgure 5. U.S. Electriclty Trade with Canada and Mexico by NERC Region, 1994}

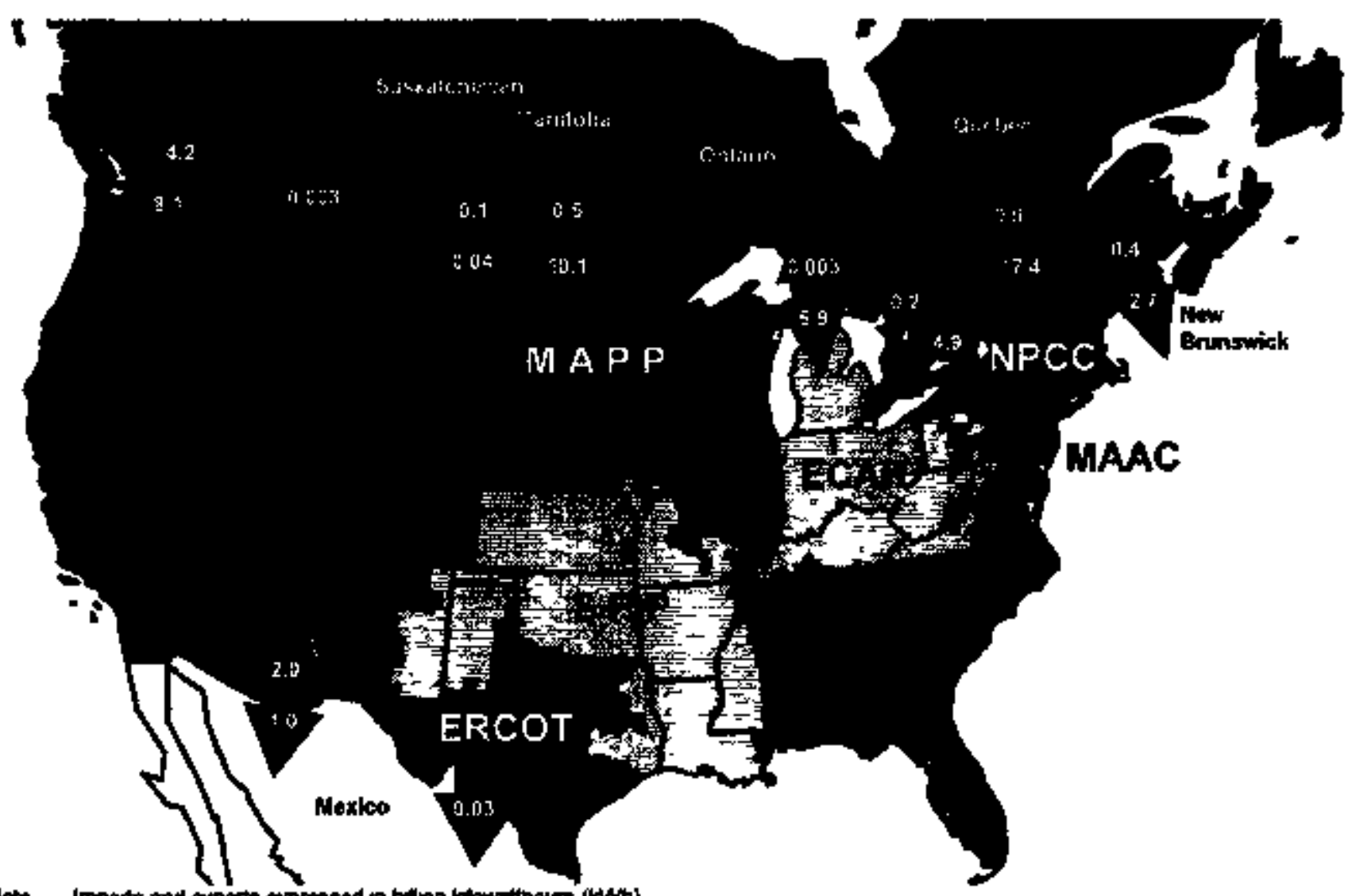

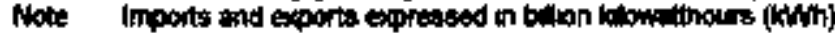

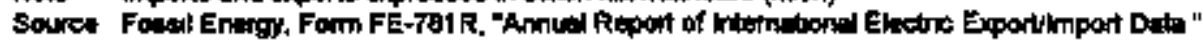


Table 4. Electric Trade in the United States by NERC Region, 1987 Throngh 1994 (Million Kilowatthours)

\begin{tabular}{|c|c|c|c|c|c|c|c|c|c|c|}
\hline Yer & $\underset{\text { Cesmation }}{\text { Nes }}$ & $\begin{array}{l}\text { Purtota: } \\
\text { Chunge } \\
\text { from } \\
\text { Prior } \\
\text { Yor }\end{array}$ & $\begin{array}{l}\text { Pucluases } \\
\text { de Excking pes } \\
\text { Pabsintd }\end{array}$ & 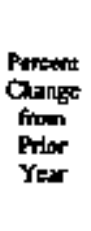 & 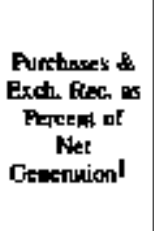 & $\begin{array}{l}\text { Soles for } \\
\text { Uhinote } \\
\text { Contumers }\end{array}$ & $\begin{array}{l}\text { Parient } \\
\text { Qmange } \\
\text { firow } \\
\text { Drier } \\
\text { Yens }\end{array}$ & $\begin{array}{c}\text { Sider } \\
\text { for Reale } \\
\text { de Exchnigen } \\
\text { Detivered? }\end{array}$ & 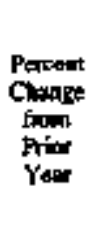 & 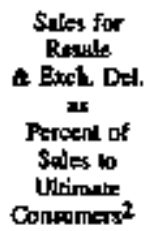 \\
\hline
\end{tabular}

\begin{tabular}{|c|c|c|c|c|c|c|c|c|c|c|}
\hline \multicolumn{11}{|c|}{ An thrles in ASCC } \\
\hline 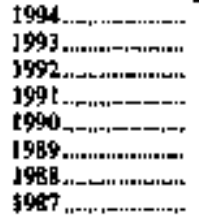 & $\begin{array}{l}4,913 \\
4,650 \\
4,735 \\
4,654 \\
4,6601 \\
4,575 \\
4,401 \\
4,41 ?\end{array}$ & $\begin{array}{r}5.4 \\
-1.6 \\
1.8 \\
-.1 \\
1.8 \\
2.6 \\
1.1 \\
-7\end{array}$ & $\begin{array}{l}3,251 \\
2,562 \\
2,549 \\
2,361 \\
2,343 \\
2,281 \\
2,183 \\
2,1381\end{array}$ & $\begin{array}{r}26.1 \\
1.6 \\
7.6 \\
.6 \\
2.4 \\
4.5 \\
1.9 \\
1.1\end{array}$ & $\begin{array}{l}56.7 \\
55.4 \\
53.7 \\
50.8 \\
50.4 \\
49.4 \\
48.9 \\
4.2\end{array}$ & $\begin{array}{l}4,5,33 \\
4,374 \\
4,338 \\
4,245 \\
4,253 \\
4,142 \\
4,149 \\
3,041\end{array}$ & $\begin{array}{r}3.6 \\
.8 \\
2.0 \\
0 \\
2.7 \\
2.4 \\
2.6 \\
-2.2\end{array}$ & $\begin{array}{l}3,251 \\
2,400 \\
2,542 \\
2,336 \\
2,3301 \\
2,244 \\
2,177 \\
2,097\end{array}$ & $\begin{array}{r}71.1 \\
-2.4 \\
8.5 \\
7 \\
3.8 \\
3.1 \\
3.8 \\
1.4\end{array}$ & $\begin{array}{l}71.7 \\
56.7\end{array}$ \\
\hline
\end{tabular}

\begin{tabular}{|c|c|c|c|c|c|c|c|c|c|c|}
\hline \multirow[b]{2}{*}{ 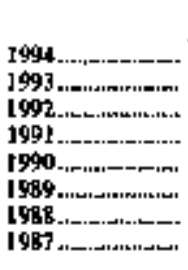 } & \multicolumn{10}{|c|}{ AD Uthile in EChe } \\
\hline & $\begin{array}{l}492,674 \\
494,512 \\
483,530 \\
488,100 \\
485,128 \\
487,659 \\
487,040 \\
465,569\end{array}$ & $\begin{array}{r}-0.5 \\
2.5 \\
-.9 \\
.6 \\
-.5 \\
.1 \\
5.1 \\
5.6\end{array}$ & $\begin{array}{l}161 ., 139 \\
170,205 \\
150,417 \\
165,313 \\
210,122 \\
184,902 \\
174,991 \\
150,752\end{array}$ & $\begin{array}{r}-1.2 \\
7.4 \\
4.2 \\
-21.4 \\
13.8 \\
8.1 \\
13.4 \\
-14.5\end{array}$ & $\begin{array}{l}34.7 \\
34.4 \\
32.6 \\
33.9 \\
43.7 \\
37.9 \\
35.1 \\
32.5\end{array}$ & $\begin{array}{l}459,747 \\
447,062 \\
429,591 \\
430,311 \\
413,732 \\
414,97 ? \\
408,171 \\
389,799\end{array}$ & $\begin{array}{r}2.8 \\
4.1 \\
-7 \\
2.8 \\
.9 \\
1.7 \\
4.7 \\
4.6\end{array}$ & $\begin{array}{l}168,558 \\
185,300 \\
181,111 \\
191,274 \\
245,897 \\
224,860 \\
217,724 \\
193,641\end{array}$ & $\begin{array}{r}-9,6 \\
2.3 \\
-53 \\
-22.2 \\
9.3 \\
3.3 \\
124 \\
-9.0\end{array}$ & $\begin{array}{l}35.7 \\
41.4 \\
42.3 \\
44.4 \\
93.3 \\
54.2 \\
53.3 \\
19.7\end{array}$ \\
\hline & \multicolumn{10}{|c|}{ Al Uthlaina h ERCOT } \\
\hline \multirow[t]{2}{*}{ 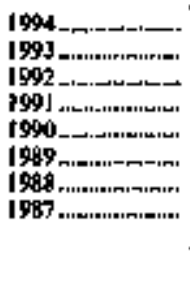 } & $\begin{array}{l}204,256 \\
198,167 \\
198,442 \\
191,040 \\
188,516 \\
185,011 \\
179,442 \\
j 73,645\end{array}$ & $\begin{array}{r}3.1 \\
4.1 \\
-.8 \\
1.8 \\
1.9 \\
3.1 \\
3.7 \\
.1\end{array}$ & $\begin{array}{r}117,0033 \\
117,775 \\
10,972 \\
196,408 \\
94,902 \\
93,067 \\
90,782 \\
90,193\end{array}$ & $\begin{array}{r}-0.6 \\
13.1 \\
-4 \\
12.1 \\
2.0 \\
-.7 \\
4.0 \\
34.7\end{array}$ & $\begin{array}{l}57.3 \\
59.4 \\
55.6 \\
55.4 \\
50.3 \\
51.3 \\
52.3 \\
51.9\end{array}$ & $\begin{array}{l}215,781 \\
212,182 \\
203,2016 \\
244,319 \\
202,694 \\
195,648 \\
192,36 ? \\
184,494\end{array}$ & $\begin{array}{l}3.1 \\
4.4 \\
-5 \\
1.1 \\
3.3 \\
1.7 \\
4.3 \\
1.5\end{array}$ & $\begin{array}{l}88,496 \\
88,169 \\
78,876 \\
81,853 \\
68,589 \\
63,772 \\
63,269 \\
66,139\end{array}$ & $\begin{array}{r}128 \\
11.8 \\
-2.4 \\
17.9 \\
1.8 \\
.1 \\
1.7 \\
4.7\end{array}$ & $\begin{array}{l}4.6 \\
4 J .5 \\
38.8 \\
39.6 \\
33.9 \\
34.4 \\
35.0 \\
35.8\end{array}$ \\
\hline & \multicolumn{10}{|c|}{ 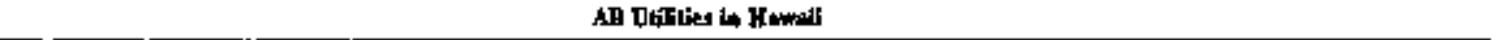 } \\
\hline \multirow[t]{2}{*}{ 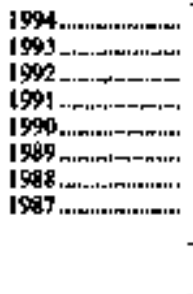 } & $\begin{array}{l}5,728 \\
5,790 \\
6,551 \\
6,991 \\
7,746 \\
7,692 \\
7,499 \\
7,1102\end{array}$ & $\begin{array}{r}-1.1 \\
-11.7 \\
-6.2 \\
-9.8 \\
.7 \\
2.6 \\
5.6 \\
4.9\end{array}$ & $\begin{array}{l}3,444 \\
3,106 \\
2,328 \\
1,405 \\
314 \\
48 \\
497 \\
484\end{array}$ & $\begin{array}{r}10.9 \\
33.4 \\
45.1 \\
124.8 \\
57.9 \\
-9.1 \\
3.3 \\
-0.9\end{array}$ & $\begin{array}{r}\text { fan.7 } \\
53.6 \\
35.5 \\
22.9 \\
4.2 \\
5.9 \\
6.6 \\
6.8\end{array}$ & $\begin{array}{l}8,393 \\
8,525 \\
8,112 \\
8,154 \\
7,948 \\
7,619 \\
7,411 \\
7,028\end{array}$ & $\begin{array}{l}3.2 \\
-.1 \\
2.2 \\
2.3 \\
4.2 \\
3.2 \\
5.4 \\
3.7\end{array}$ & $\begin{array}{r}6 \\
5 \\
3 \\
5 \\
5 \\
5 \\
89 \\
83\end{array}$ & $\begin{array}{r}26.9 \\
65.5 \\
-39.2 \\
10.4 \\
38.6 \\
-94.6 \\
6.6 \\
2.7\end{array}$ & $\begin{array}{l}125 \\
.1 \\
01 \\
.1 \\
1 \\
.0 \\
1.2 \\
1.2\end{array}$ \\
\hline & & & & & Artive & $A C$ & & & & \\
\hline \multirow[t]{2}{*}{ 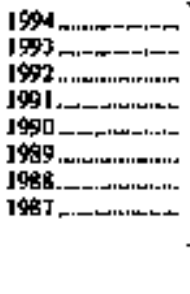 } & $\begin{array}{l}206,211 \\
205,552 \\
193,3318 \\
197,235 \\
103,393 \\
193,898 \\
191,874 \\
165,541\end{array}$ & $\begin{array}{r}6.3 \\
6.3 \\
-2.0 \\
2.0 \\
-3 \\
1.0 \\
3.4 \\
2.9\end{array}$ & 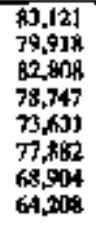 & $\begin{array}{r}10 \\
-315 \\
5.2 \\
6.9 \\
-53 \\
13.5 \\
7.3 \\
130\end{array}$ & $\begin{array}{l}40.3 \\
38.9 \\
42.8 \\
39.9 \\
38.1 \\
40.2 \\
75.9 \\
34.6\end{array}$ & $\begin{array}{l}223,695 \\
220,037 \\
210,799 \\
212,725 \\
217,770 \\
217,325 \\
212, \pi 15 \\
194,677\end{array}$ & $\begin{array}{l}1.6 \\
4.4 \\
-.9 \\
2.4 \\
.2 \\
2.7 \\
5.8 \\
4.5\end{array}$ & $\begin{array}{l}48,445 \\
47,536 \\
49,6,37 \\
45,447 \\
43,84 ? \\
46,950 \\
41,141 \\
41,120\end{array}$ & $\begin{array}{r}2.0 \\
-4.3 \\
9.2 \\
3.6 \\
-6.6 \\
16.9 \\
-2.4 \\
5.4\end{array}$ & $\begin{array}{l}21.3 \\
21.6 \\
21.5 \\
21.4 \\
21.1 \\
22.6 \\
14.8 \\
21.4\end{array}$ \\
\hline & \multicolumn{10}{|c|}{ 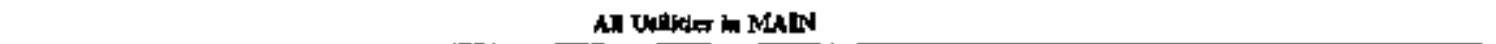 } \\
\hline 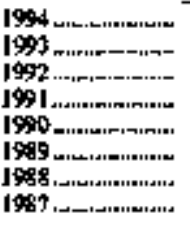 & $\begin{array}{l}221,770 \\
217,284 \\
201,288 \\
206,996 \\
291,553 \\
290,660 \\
198,666 \\
185,240\end{array}$ & $\begin{array}{r}2.1 \\
8.5 \\
-3.2 \\
2.6 \\
.5 \\
1.0 \\
1.3 \\
2.7\end{array}$ & $\begin{array}{l}61,661 \\
52,911 \\
52,121 \\
54,585 \\
6 \$ 2,7188 \\
62,755 \\
56,241 \\
54,6015\end{array}$ & $\begin{array}{r}-2.01 \\
20.2 \\
-4.1 \\
-20.1 \\
9.7 \\
110.1 \\
4.1 \\
0.2\end{array}$ & $\begin{array}{l}27.8 \\
28.9 \\
26.1 \\
26.4 \\
33.9 \\
31.2 \\
28.6 \\
29.5\end{array}$ & $\begin{array}{l}214,304 \\
207,004 \\
2100,571 \\
201,815 \\
193,0 \times 48 \\
136,405 \\
185,400 \\
176,801\end{array}$ & $\begin{array}{l}3.5 \\
3.2 \\
-6 \\
4.5 \\
2.5 \\
1.6 \\
4.9 \\
2.9\end{array}$ & $\begin{array}{l}53,77 d \\
57,590 \\
57,312 \\
44,517 \\
62,174 \\
59,407 \\
54,9,57 \\
40,517\end{array}$ & $\begin{array}{r}-6.6 \\
54.4 \\
-16.2 \\
-28.4 \\
4.7 \\
8.1 \\
13.3 \\
6.5\end{array}$ & $\begin{array}{l}25.1 \\
27.8 \\
18.6 \\
22.1 \\
32.2 \\
31.5 \\
29.6 \\
27.4\end{array}$ \\
\hline
\end{tabular}

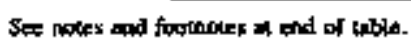


Table 4. Electric Trade in the United States by NERC Reglon, 1987 Through 1994 (Million Kilowarthours) (Continued)

\begin{tabular}{|c|c|c|c|c|c|c|c|c|c|c|}
\hline Yar & Centation & $\begin{array}{l}\text { Percest } \\
\text { Cluninte } \\
\text { from } \\
\text { Pror } \\
\text { Yex }\end{array}$ & $\begin{array}{l}\text { Pechass } \\
\text { Rexbersen } \\
\text { Recerved }\end{array}$ & $\begin{array}{l}\text { Percenx } \\
\text { Chunte } \\
\text { finm } \\
\text { Pres: } \\
\text { Yen }\end{array}$ & 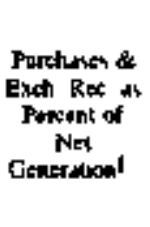 & 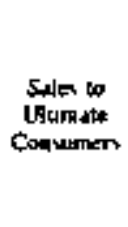 & $\begin{array}{l}\text { Percenr } \\
\text { Chinge } \\
\text { Inow } \\
\text { Ponp } \\
\text { Yer }\end{array}$ & 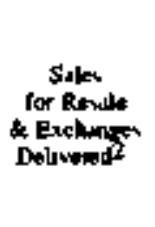 & $\begin{array}{l}\text { Petrenl } \\
\text { Ching } \\
\text { from } \\
\text { Pror } \\
\text { Ytedr }\end{array}$ & 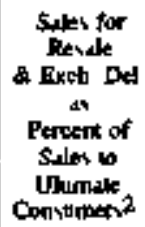 \\
\hline
\end{tabular}

\begin{tabular}{|c|c|c|c|c|c|c|c|c|c|c|}
\hline & \multicolumn{10}{|c|}{ AD Udives in Mift } \\
\hline $\begin{array}{l}1994 \\
1993 \\
1992 \\
1991 \\
1996 \\
1989 \\
1988 \\
1987\end{array}$ & $\begin{array}{l}124,607 \\
124,898 \\
124,053 \\
122,991 \\
129,750 \\
116,410 \\
113,238 \\
122,38]\end{array}$ & $\begin{array}{r}412 \\
10 \\
-24 \\
19 \\
37 \\
-15 \\
-14 \\
10\end{array}$ & $\begin{array}{c}90,020 \\
92,44] \\
119,672 \\
196,175 \\
197,416 \\
105,828 \\
106,950 \\
5 T, 815\end{array}$ & $\begin{array}{r}-26 \\
-367 \\
81 \\
-21 \\
21 \\
-18 \\
97 \\
-100\end{array}$ & $\begin{array}{l}722 \\
741 \\
947 \\
555 \\
8901 \\
902 \\
904 \\
799\end{array}$ & $\begin{array}{l}128,935 \\
124,142 \\
117,281 \\
120,5,22 \\
115,912 \\
113,692 \\
11,136 \\
104,657\end{array}$ & $\begin{array}{r}79 \\
58 \\
-27 \\
40 \\
79 \\
5 \\
81 \\
1\end{array}$ & $\begin{array}{r}74,417 \\
82,194 \\
108,352 \\
98,6194 \\
101,829 \\
9,309 \\
101,646 \\
101,044\end{array}$ & $\begin{array}{r}-95 \\
-226 \\
74 \\
-37 \\
46 \\
-43 \\
6 \\
-56\end{array}$ & $\begin{array}{l}577 \\
661 \\
898 \\
814 \\
878 \\
1564 \\
699 \\
915\end{array}$ \\
\hline
\end{tabular}

\begin{tabular}{|c|c|c|c|c|c|c|c|c|c|c|}
\hline \multirow[b]{2}{*}{$\begin{array}{l}1994 \\
1993 \\
1992 \\
1991 \\
1990 \\
1989 \\
1989 \\
1987\end{array}$} & \multicolumn{10}{|c|}{ All Itilutea in NrCC } \\
\hline & $\begin{array}{l}189,546 \\
195,1401 \\
202,978 \\
218,053 \\
223,866 \\
233,604 \\
223,996 \\
212,165\end{array}$ & $\begin{array}{r}-29 \\
-39 \\
-69 \\
-43 \\
-25 \\
43 \\
56 \\
-41\end{array}$ & $\begin{array}{l}198,467 \\
181,770 \\
166,884 \\
154,206 \\
141,784 \\
141,102 \\
140,796 \\
179,060\end{array}$ & $\begin{array}{r}92 \\
89 \\
82 \\
88 \\
5 \\
6 \\
9 \\
-5\end{array}$ & $\begin{array}{l}1047 \\
931 \\
822 \\
717 \\
622 \\
604 \\
676 \\
653\end{array}$ & $\begin{array}{l}235,679 \\
236,012 \\
233,395 \\
233,443 \\
234542 \\
231,573 \\
2210,453 \\
216,979\end{array}$ & $\begin{array}{r}11 \\
11 \\
-1 \\
-4 \\
4 \\
20 \\
55 \\
45\end{array}$ & $\begin{array}{l}129,903 \\
121556 \\
118,108 \\
129,117 \\
118,194 \\
122,257 \\
116,647 \\
113,161\end{array}$ & $\begin{array}{r}69 \\
29 \\
-17 \\
16 \\
-93 \\
48 \\
13 \\
-148\end{array}$ & $\begin{array}{l}544 \\
515 \\
506 \\
514 \\
504 \\
524 \\
510 \\
531\end{array}$ \\
\hline
\end{tabular}

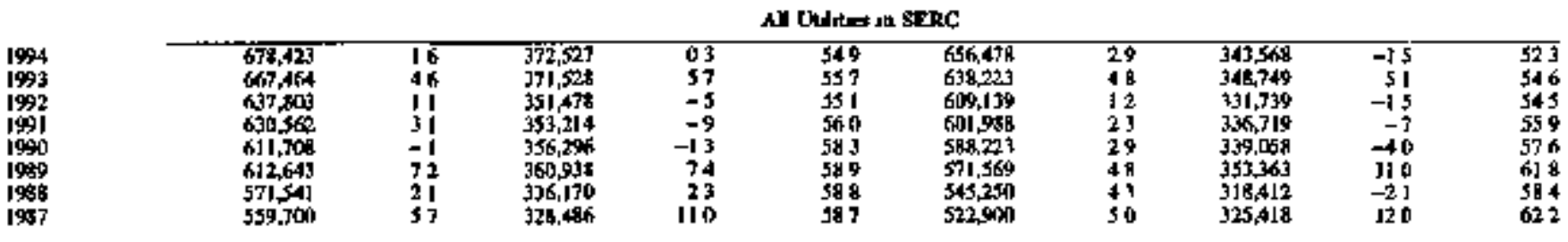

\begin{tabular}{|c|c|c|c|c|c|c|c|c|c|c|}
\hline \multirow[b]{2}{*}{$\begin{array}{l}1994 \\
1993 \\
1992 \\
1991 \\
19901 \\
1999 \\
1998 \\
1987\end{array}$} & \multicolumn{10}{|c|}{ Al Ubluts a STt } \\
\hline & $\begin{array}{l}2610,1025 \\
256,901 \\
242,514 \\
244,415 \\
24,764 \\
229,032 \\
223,346 \\
201,465\end{array}$ & $\begin{array}{r}12 \\
59 \\
-8 \\
-1 \\
24 \\
24 \\
7 \\
16\end{array}$ & $\begin{array}{l}148,574 \\
141,319 \\
128,586 \\
127,896 \\
120,583 \\
120,646 \\
110,226 \\
105,972\end{array}$ & $\begin{array}{r}51 \\
99 \\
5 \\
61 \\
0 \\
94 \\
40 \\
-87\end{array}$ & $\begin{array}{l}571 \\
550 \\
530 \\
523 \\
493 \\
505 \\
472 \\
457\end{array}$ & $\begin{array}{l}257,163 \\
249,691 \\
235,320 \\
218,328 \\
236,253 \\
233,134 \\
218,281 \\
211,806\end{array}$ & $\begin{array}{r}25 \\
62 \\
-13 \\
9 \\
59 \\
22 \\
31 \\
17\end{array}$ & $\begin{array}{l}130,540 \\
126,397 \\
115,901 \\
114,484 \\
199,1] 3 \\
114,7601 \\
105,777 \\
107,226\end{array}$ & $\begin{array}{r}17 \\
91 \\
12 \\
45 \\
-19 \\
85 \\
-15 \\
-81\end{array}$ & $\begin{array}{l}308 \\
506 \\
493 \\
481 \\
462 \\
514 \\
485 \\
506\end{array}$ \\
\hline
\end{tabular}

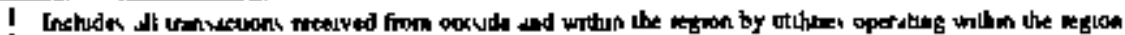

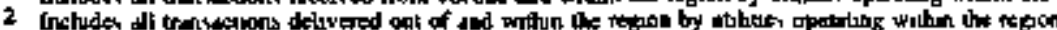

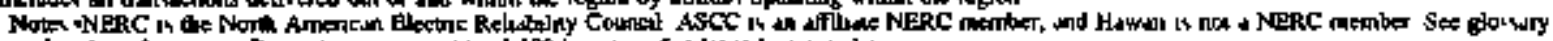

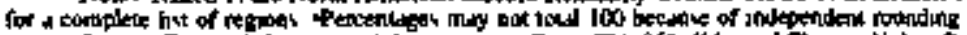

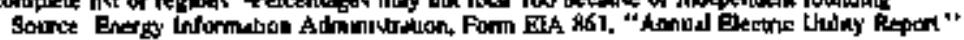


Table 5. Intra- and Interrejional Purchases and Sales for Resale by Selected Ownership Classes, 1988, 1990, 1992, and 1994

(Million Kilowatthours)

\begin{tabular}{|c|c|c|c|c|c|c|c|c|c|c|}
\hline \multirow[b]{2}{*}{$\begin{array}{l}\text { Type of Traburan } \\
\text { TOwnershop Clavs }\end{array}$} & \multicolumn{5}{|c|}{ Inrifetrond } & \multicolumn{5}{|c|}{ Intepresiolil } \\
\hline & I994 & 1992 & 3990 & I过: & $\begin{array}{c}\text { Tord } \\
\text { Percent } \\
\text { Deffermes } \\
\text { 1968 } 1994\end{array}$ & 1994 & 3992 & 1990 & 1988 & $\begin{array}{l}\text { Toul } \\
\text { Pencend } \\
\text { Dufforince } \\
\text { J988 1994 }\end{array}$ \\
\hline \multicolumn{11}{|l|}{ Purdeson } \\
\hline $\begin{array}{l}\text { Inventor Dwhed } \\
\text { Fodetall } \\
\text { Cooptrilypen }\end{array}$ & $\begin{array}{r}6,1,1,893 \\
17,827 \\
289,444\end{array}$ & $\begin{array}{r}598.671 \\
17.948 \\
266.844\end{array}$ & $\begin{array}{r}521,524 \\
8,476 \\
257,467\end{array}$ & $\begin{array}{r}394,594 \\
74,217 \\
254,766\end{array}$ & $\begin{array}{r}681 \\
-760 \\
116\end{array}$ & $\begin{array}{r}75,597 \\
1576 \\
16,766\end{array}$ & $\begin{array}{r}59,927 \\
1,795 \\
14,234\end{array}$ & $\begin{array}{r}4,1,27 \\
2,637 \\
15,505\end{array}$ & $\begin{array}{r}20,509 \\
19,644 \\
7,573\end{array}$ & $\begin{array}{r}2633 \\
-920 \\
1214\end{array}$ \\
\hline \multicolumn{11}{|l|}{ Solve far Restil? } \\
\hline $\begin{array}{l}\text { Investor-Dwined } \\
\text { Fedeplal] }\end{array}$ & $\begin{array}{l}467,479 \\
184,993\end{array}$ & $\begin{array}{l}152,42 \\
174,156\end{array}$ & $\begin{array}{l}77,8901 \\
135,800\end{array}$ & $\begin{array}{l}320,739 \\
174,239\end{array}$ & $\begin{array}{r}45 \% \\
61\end{array}$ & $\begin{array}{l}\text { 62.205 } \\
13,255\end{array}$ & $\begin{array}{l}61.928 \\
12.973\end{array}$ & $\begin{array}{l}70,294 \\
11,764\end{array}$ & $\begin{array}{r}4,5,275 \\
2,918\end{array}$ & $\begin{array}{l}375 \\
3543\end{array}$ \\
\hline
\end{tabular}

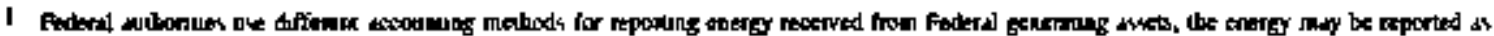

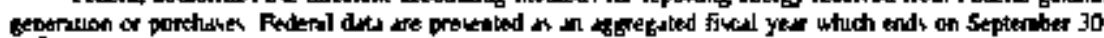

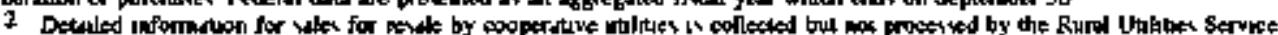

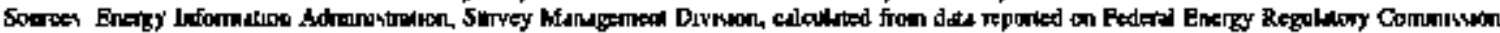

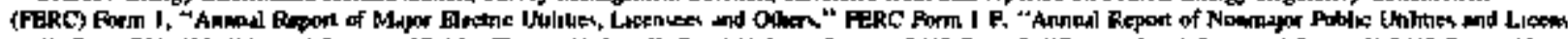

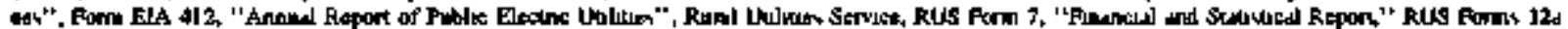

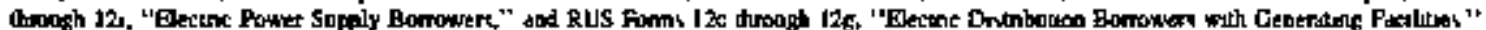

Table 6. Firm and Nonflrm Purchases and Sales Ior Resale by Investor-Owned Utillithes, by NERC Region, 1989, 1990, 1992, and 1994

(Mtllion Kilowatthours)

\begin{tabular}{|c|c|c|c|c|c|c|c|c|c|c|}
\hline \multirow[b]{2}{*}{ NERC Rrgan } & \multicolumn{5}{|c|}{ Prinn } & \multicolumn{5}{|c|}{ Nontion } \\
\hline & 1094 & 1992 & 1990 & 1998 & $\begin{array}{l}\text { Percent } \\
\text { Ch-ilst } \\
\text { josis } 1594\end{array}$ & 1904 & 1902 & 1990 & 1989 & $\begin{array}{c}\text { Periend } \\
\text { Codnge } \\
1988-1994\end{array}$ \\
\hline & \multicolumn{10}{|c|}{ 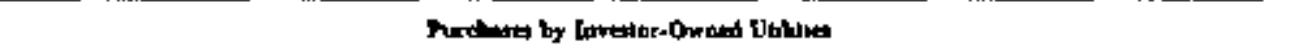 } \\
\hline $\begin{array}{l}\text { ECAR } \\
\text { ERCOT } \\
\text { MAAC } \\
\text { MAIN } \\
\text { MAPP } \\
\text { NPCC } \\
\text { SPRC } \\
\text { SPP } \\
\text { WSCC }\end{array}$ & 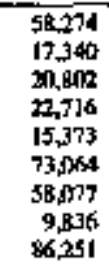 & $\begin{array}{r}46,741 \\
2,716 \\
28,244 \\
11,547 \\
13,652 \\
74,925 \\
57,671 \\
2,941 \\
13,037\end{array}$ & $\begin{array}{r}47,191 \\
22,285 \\
25,025 \\
12,463 \\
6,193 \\
65,349 \\
59,1934 \\
6,466 \\
78,1977\end{array}$ & $\begin{array}{r}13,77 \\
21,427 \\
23,26,0 \\
3,970 \\
5,485 \\
73,573 \\
44,418 \\
13,161 \\
40,094\end{array}$ & $\begin{array}{r}3210 \\
-191 \\
-106 \\
1810 \\
1803 \\
-7 \\
108 \\
-253 \\
757\end{array}$ & $\begin{array}{r}70,778 \\
9,574 \\
47,185 \\
53,124 \\
10,107 \\
99,672 \\
27,079 \\
40,683 \\
52,576\end{array}$ & 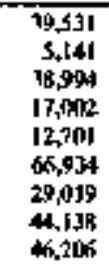 & $\begin{array}{r}22,196 \\
3,399 \\
14,3 \% 0 \\
9,307 \\
13,744 \\
31,642 \\
28,642 \\
30,227 \\
59,169\end{array}$ & $\begin{array}{r}10,358 \\
2,919 \\
7,256 \\
4,218 \\
10,117 \\
23,732 \\
8,1517 \\
26,777 \\
50,878\end{array}$ & $\begin{array}{r}1923 \\
2279 \\
5531 \\
2110 \\
-1 \\
2328 \\
2323 \\
515 \\
33\end{array}$ \\
\hline \multirow[t]{2}{*}{ Condugrom US Tuld } & 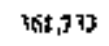 & 342,472 & 724,524 & $24 \mathbb{R}, 104$ & $45 \mathrm{~B}$ & 326,490 & 299,6076 & 219.300 & 149.406 & 1155 \\
\hline & \multicolumn{10}{|c|}{ 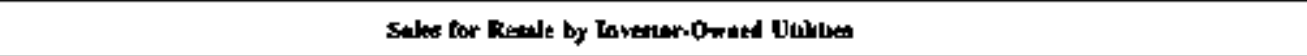 } \\
\hline $\begin{array}{l}\text { ECAR } \\
\text { ERCOT } \\
\text { BLAAC } \\
\text { MANN } \\
\text { MAFP } \\
\text { NPCC } \\
\text { SERC } \\
\text { SPP } \\
\text { WSOC }\end{array}$ & $\begin{array}{r}7,199 \\
5,941 \\
9,494 \\
2,743 \\
7,909 \\
52,9: 55 \\
7,5,234 \\
18,645 \\
27,894\end{array}$ & $\begin{array}{r}74.719 \\
6,599 \\
13,978 \\
16,775 \\
9,828 \\
57,816 \\
4.940 \\
17.119 \\
29,962\end{array}$ & $\begin{array}{r}75,759 \\
7,232 \\
6,712 \\
22,125 \\
6,769 \\
47,578 \\
4,551 \\
15,506 \\
27516\end{array}$ & $\begin{array}{r}27,31 \\
5,657 \\
6,24 \\
10,890 \\
4,198 \\
49,477 \\
19,759 \\
14,800 \\
19,220\end{array}$ & $\begin{array}{r}-61 \\
-119 \\
604 \\
3452 \\
807 \\
71 \\
-114 \\
259 \\
44\end{array}$ & $\begin{array}{r}17,319 \\
2,916 \\
52,141 \\
15,426 \\
8,216 \\
13,3] 1 \\
50,839 \\
31,148 \\
29,955\end{array}$ & $\begin{array}{r}51,251 \\
1,392 \\
28,411 \\
10,170 \\
2,728 \\
37,964 \\
46,339 \\
27,944 \\
23,210\end{array}$ & $\begin{array}{r}42,064 \\
1,415 \\
9,106 \\
6,854 \\
11,175 \\
19,917 \\
49,424 \\
19,960 \\
23,360\end{array}$ & $\begin{array}{r}22, J 03 \\
2,065 \\
6,323 \\
1,236 \\
5,002 \\
1,3,164 \\
36,568 \\
21,377 \\
39,351\end{array}$ & $\begin{array}{r}689 \\
412 \\
4183 \\
14899 \\
641 \\
2391 \\
390 \\
457 \\
548\end{array}$ \\
\hline Contupaces U S Total & 258.984 & 264.376 & 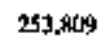 & 214,952 & I: T & $25,2,279$ & 237,49 & 18289 & 127,180 & 1007 \\
\hline
\end{tabular}

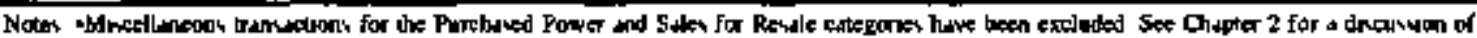

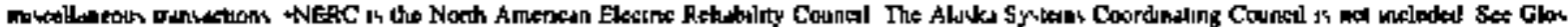
wry por a bus of regrons

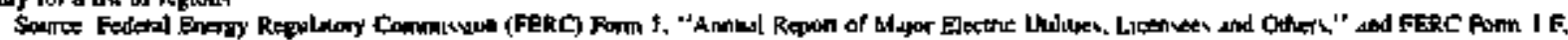

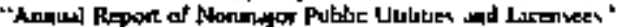

the Form EIA-861 unuverse Tables 5 through 44 jaclude detajled information developed from direct fulings of different surveys by 198 of the 250 investorowned electric utilities; 502 of the 2,005 State and 
municipal electric utilities; 857 of the 939 cooperative electric utilities, and 8 of the 10 Federal utilities.* The detailed data represent subsets of the universe data provided in Tables 1 through 4 . (The requirements for filing are discussed below.) of the total number of electric utilities identified, but not required to file a detailed tata collection form with a Federal agency, toost have little geotrating capability and usually purchase part or all of their electrical energy from one or more of the electric utilities who did file. However, some do buy from cogenerators, cooperatives, or other utilities not required to file. The summary quantity of purchases made by these utilities are included in this publication (Tables 22, 31, and 35). These utilities were identified by matching the names of filing utilities and all the utilities listed on these filjngs with the universe frame of Form EIA-861, "Annual Electric Utility Report." Chapters 2 through 4 provide detailed data on individual electric utilities developed from the direct filings. However, if the direct ibling indicates no wholesale trade transactions, then those efectric utilities were not shown in the respective lables. Tables 37 through 44 provide information on portions of the physical transmission sysicas.

Some data for this publication come from sources outside of EIA. These include: (1) the Federal Energy Regulatory Commission (FERC), (2) the Rural Utilicies Service (RUS) in the Department of Agriculture, and (3) the Office of Fossil Energy (FE) in the Department of Energy (see Table ES2). These agencies manage all aspects of their respective surveys and data base maintenance including quality concrol, frame maintenance, formatting of survey information, survey forms development, handling of nonresponse, and data imputation.

Chapter 1 gives an overview of bulk power trade and provides national and regional electric trade data by all ownership classes. These data come from several forms, as no form was specifically designed to colfect electricity trade information from all ownership classes. International trade data are from Form FE-781R, "Annual Report of International Electrical Export/Import Data," (Table 18).

Chapter 2 presents data on bulk power transactions of "Major" and "Minor" investor-owned utilities. Major investor-owned electric utilities are defined as those private utilities that have had, in the past 3 consecutive calendar years, sales or transmission services that exceeded one of the following: l billion kilowatthours of total annual sales, 100,000 kilowatthours of annual sales for resale, 500,000 kilowatthours of annual gross interchange-out or 500,000 kilowatthours of transmission for others (wheeling). The criteria for classification as a minor utility are: (I) total anntal sales of 10 million kilowatthours or more in the previous calendar ycar, and (2) not being classified as a major utility.

The investor-owned utilities shown in this publication report on FERC Form 1, "Annual Report of Major Electric Utilities, Licensees and Others" and FERC Form 1-F, "Annual Report of Nonmajor Public Utilities and Licensees."

The FERC forms are primarily financial reporting forms. The wholesale data are collected on 4 of the approxinatcly 75 survey schedules of the FERC Form 1. The wholesale electricity trade schedules are sa]es for resale, purchased power, summary of interchange, and transmission of electricity for or by others. These electricity transactions are shown by utility class of ownership in Tables 19 throngh 24.

The investor-owned electric utilities that fall below the filing criteria for the FERC Forms $t$ and J-F are not required to fie. Appendjx Table B 1 lists the major and minor utilities that file FERC Forms 1 and 1-F.

Data in Chapter 3 cover wholesale electricity trade for the 502 public uftlites thar report on Form EIA-412, "Annual Report of Poblic Electric Utilities," which is used to collect data on purchased power and sales for resale transactions. The public utilities report information by fiscal year; therefore, consistent torals cannot be provided for these classes of ownership. Public electric ntjlities are required to submit Form EIA-412 when their generation, transmission, or distribution of electricity results in 120 million kilowathours of sales to ultimate consomers and/or 120 million kjlowatthours of sales for resale. Data on exchanges and wheeling in this chapter are reported on Form EIA.861, "Annus] Electric Utility Report."

Chapter 4 presents data submitied to the Rural Utilities Service (RUS) of the U.S. Department of Agriculture by cooperative bocrowers. The RUS collects information from all utilities that have "borrowed" under the RUS loan guarante programs and uses the information to verify and administer its loan programs. Appendix Table B5 lists cooperative borrowers. Data covering electricity trade for cooperative borrowers are reported for a calendar-year basis on several closely related RUS forms: RUS Form 7 , "Financial and Statistical Report," RUS Forms 12a through 12i, "Electric Power Supply Borrowers," and RUS Forms 12e through I2g, "Electric Distribution Borrowers with Generating Facilities." These forms are used to collect data on purchased power. Sales for resale transactions are nol ayailable in a macbinereadable format from the RUS.

Chapres 5 presents data on the physical transmission systems. In addition, transmission line statistics are being provided for publicly owned utilities from data reported on form $\mathrm{ElA}-412$.

\footnotetext{
1 The two Federal utilities that do and tile the Form ELA-4/2 do nol tell wholesale powes. Electedcliy produced by these utilities is marketed by oute or more of the Pederal power marketing adminisicalions.
} 
Tabłe 7. Wheding Trade in the United States by NERC Region, 1987 Through 1994 (Million Kilowarthours)

\begin{tabular}{|c|c|c|c|c|c|c|c|c|}
\hline Whaciling & 1994 & 1993 & 1992 & | & 1950 & 1909 & 1988 & 1965 \\
\hline & \multicolumn{8}{|c|}{ AlUtities in ICAR } \\
\hline \multirow[t]{2}{*}{ 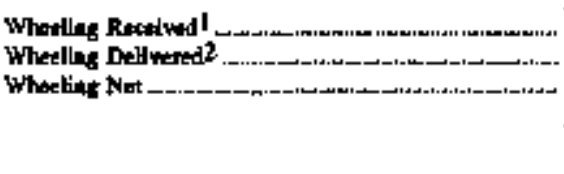 } & $\begin{array}{r}30,861 \\
30,639 \\
211\end{array}$ & $\begin{array}{r}31,191 \\
30,994 \\
197\end{array}$ & $\begin{array}{r}31,303 \\
31.618 \\
186\end{array}$ & $\begin{array}{r}30,293 \\
30,161 \\
\text { I32 }\end{array}$ & $\begin{array}{r}32,391 \\
22,157 \\
58\end{array}$ & $\begin{array}{r}21,199 \\
\text { 20994 } \\
\text { lif4 }\end{array}$ & $\begin{array}{r}15,121 \\
15,036 \\
\text { BS }\end{array}$ & $\begin{array}{r}10,347 \\
10,285 \\
65\end{array}$ \\
\hline & \multicolumn{8}{|c|}{ All Utilta in ERCOT } \\
\hline \multirow[t]{2}{*}{ 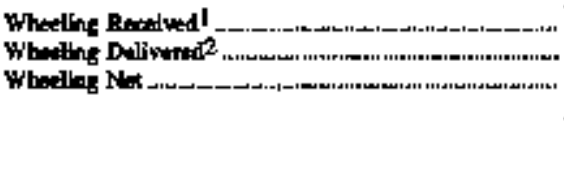 } & $\begin{array}{r}24,0109 \\
24,089 \\
-19\end{array}$ & $\begin{array}{r}26,716 \\
26,686 \\
31\end{array}$ & $\begin{array}{r}24,0 \pi 7 \\
240907 \\
-13\end{array}$ & $\begin{array}{r}20,934 \\
20,957 \\
-2\end{array}$ & $\begin{array}{r}16,481 \\
16,496 \\
-14\end{array}$ & $\begin{array}{r}9.414 \\
9,97 \\
117\end{array}$ & $\begin{array}{r}7,861 \\
7,913 \\
-53\end{array}$ & $\begin{array}{r}6,532 \\
6,543 \\
-11\end{array}$ \\
\hline & \multicolumn{8}{|c|}{ 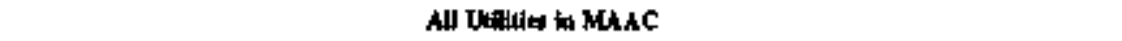 } \\
\hline \multirow[t]{2}{*}{ 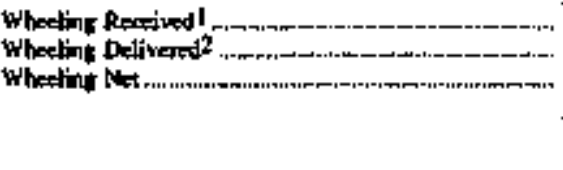 } & $\begin{array}{r}11.759 \\
11.730 \\
69\end{array}$ & $\begin{array}{r}13,132 \\
13,030 \\
100\end{array}$ & $\begin{array}{r}9,868 \\
9.779 \\
88\end{array}$ & $\begin{array}{r}5,842 \\
5,842 \\
-\end{array}$ & $\begin{array}{r}5,774 \\
5,774 \\
-\end{array}$ & $\begin{array}{r}8,385 \\
8,3,60 \\
24\end{array}$ & $\begin{array}{r}7,482 \\
7,465 \\
16\end{array}$ & $\begin{array}{l}6,6165 \\
7,226 \\
-5610\end{array}$ \\
\hline & \multicolumn{8}{|c|}{ Al Ut'tikes in MAN } \\
\hline \multirow[t]{2}{*}{ 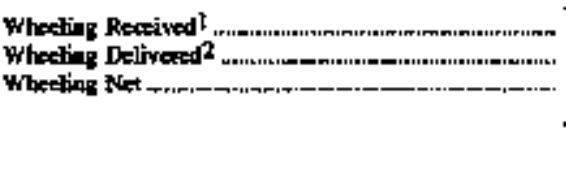 } & $\begin{array}{r}4,877 \\
4,810 \\
67\end{array}$ & $\begin{array}{r}5,015 \\
4,951 \\
6 ?\end{array}$ & $\begin{array}{r}3,499 \\
j, 104 \\
85\end{array}$ & $\begin{array}{r}3,819 \\
3,739 \\
8,9\end{array}$ & $\begin{array}{r}1,45] \\
1,414 \\
37\end{array}$ & $\begin{array}{l}1 \text { 年15 } \\
1,215 \\
10\end{array}$ & $\begin{array}{r}1,915 \\
1,906 \\
10\end{array}$ & $\begin{array}{r}2,071 \\
2,017 \\
54\end{array}$ \\
\hline & \multicolumn{8}{|c|}{ Al Dtilike : MLPP } \\
\hline \multirow[t]{2}{*}{ 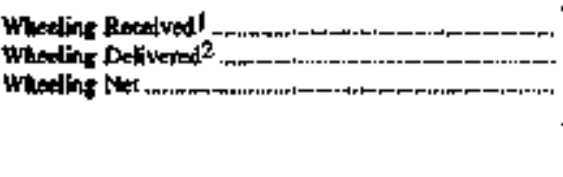 } & $\begin{array}{r}19,008 \\
18,4] 7 \\
620\end{array}$ & $\begin{array}{r}16,781 \\
16,130 \\
691\end{array}$ & $\begin{array}{r}19661 \\
10,860 \\
114\end{array}$ & $\begin{array}{l}9,484 \\
7,935 \\
1,549\end{array}$ & $\begin{array}{l}8,477 \\
7,656 \\
821\end{array}$ & $\begin{array}{l}9,2018 \\
8,197 \\
1,012\end{array}$ & $\begin{array}{l}7,804 \\
6,705 \\
1,0,08\end{array}$ & $\begin{array}{r}1,174 \\
6,727 \\
647\end{array}$ \\
\hline & \multicolumn{8}{|c|}{ ADVtilles In NRCC } \\
\hline \multirow[t]{2}{*}{ 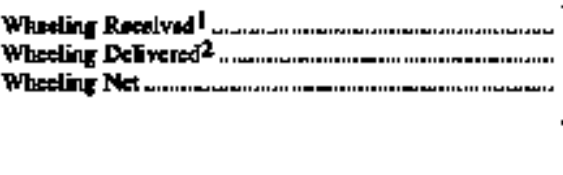 } & $\begin{array}{r}69,885 \\
68,587 \\
296\end{array}$ & $\begin{array}{r}67,815 \\
67,553 \\
26,2\end{array}$ & $\begin{array}{r}60,687 \\
60,495 \\
191\end{array}$ & $\begin{array}{r}57,173 \\
96,523 \\
248\end{array}$ & $\begin{array}{r}48,613 \\
48,399 \\
214\end{array}$ & $\begin{array}{r}42,897 \\
42,351 \\
546\end{array}$ & $\begin{array}{r}38,365 \\
38,0989 \\
2,36\end{array}$ & $\begin{array}{r}23,4 B 6 \\
2 \pi, 611 \\
475\end{array}$ \\
\hline & \multicolumn{8}{|c|}{ An Ut'inda is \$ECC } \\
\hline \multirow[t]{2}{*}{ 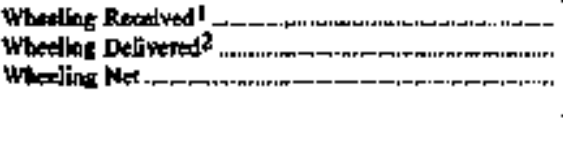 } & $\begin{array}{r}25,134 \\
27,514 \\
1,62011\end{array}$ & $\begin{array}{r}27,132 \\
25,304 \\
1,809\end{array}$ & $\begin{array}{l}27,211 \\
25,406 \\
1,803\end{array}$ & $\begin{array}{r}28,457 \\
27,447 \\
1,050\end{array}$ & $\begin{array}{r}29,291 \\
20,174 \\
1,117\end{array}$ & $\begin{array}{r}27,326 \\
21,865 \\
841\end{array}$ & $\begin{array}{r}12,354 \\
11,749 \\
6 ! 1\end{array}$ & $\begin{array}{r}11,099 \\
9966 \\
1,015\end{array}$ \\
\hline & \multicolumn{8}{|c|}{ AD biviles in STP } \\
\hline \multirow[t]{2}{*}{ 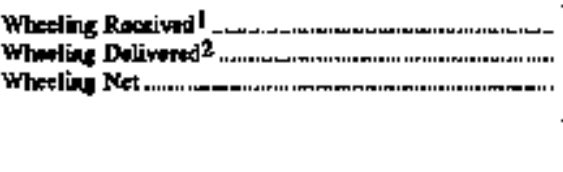 } & $\begin{array}{r}23,543 \\
23,418 \\
97\end{array}$ & $\begin{array}{r}25,529 \\
25,4] 9 \\
109\end{array}$ & $\begin{array}{r}21,749 \\
21,670 \\
1100\end{array}$ & $\begin{array}{r}22,150 \\
23,078 \\
72\end{array}$ & $\begin{array}{l}27.09 ! \\
26,0 \% 5 \\
\text { IOS }\end{array}$ & $\begin{array}{r}19,59] \\
19,924 \\
-3,33\end{array}$ & $\begin{array}{r}20,0589 \\
19,956 \\
133\end{array}$ & $\begin{array}{r}10,136 \\
9,818 \\
330\end{array}$ \\
\hline & \multicolumn{8}{|c|}{ Al UInited In FSCC } \\
\hline \multirow[t]{2}{*}{ 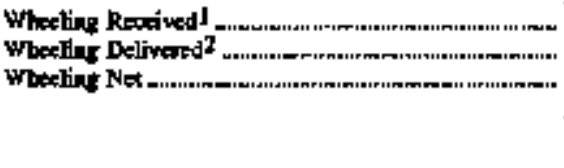 } & $\begin{array}{r}127,915 \\
126,672 \\
1,243\end{array}$ & $\begin{array}{r}137.931 \\
136499 \\
1.441\end{array}$ & $\begin{array}{r}127,5] 4 \\
125,521 \\
2,492\end{array}$ & $\begin{array}{r}144,465 \\
142,502 \\
1,874\end{array}$ & $\begin{array}{r}152,097 \\
148,7188 \\
3,708\end{array}$ & $\begin{array}{r}164,824 \\
163,627 \\
1,2011\end{array}$ & $\begin{array}{r}157,755 \\
157,687 \\
74\end{array}$ & $\begin{array}{r}118,925 \\
118,995 \\
-69\end{array}$ \\
\hline & \multicolumn{7}{|c|}{ Contipuous U.S. Totgl } & $\cdot$ \\
\hline 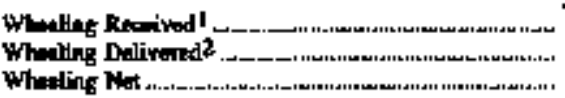 & $\begin{array}{r}336,109 \\
312,480 \\
4,225\end{array}$ & $\begin{array}{r}351,701 \\
347,012 \\
4,660\end{array}$ & $\begin{array}{r}318,538 \\
312,788 \\
5,756\end{array}$ & $\begin{array}{r}323_{4} 133 \\
318,179 \\
4,560\end{array}$ & $\begin{array}{r}322,667 \\
316,591 \\
6,077\end{array}$ & $\begin{array}{r}299,027 \\
206,297 \\
3,525\end{array}$ & $\begin{array}{r}200,170 \\
266,309 \\
2,262\end{array}$ & $\begin{array}{r}190,049 \\
192,020 \\
2,019\end{array}$ \\
\hline
\end{tabular}

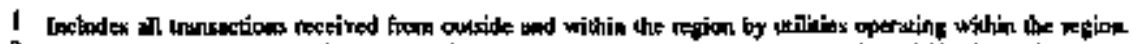

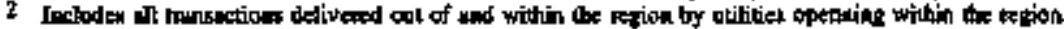

$\rightarrow$ bat Apolicolo ghoms

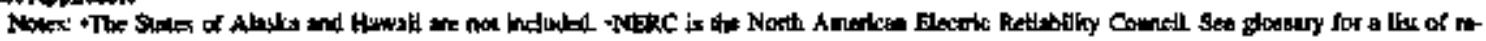

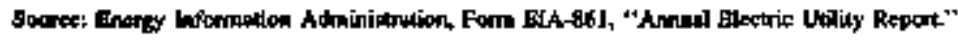




\section{Compllation of Data}

The Federal Government recognizes that wholesale trade data needs to be consolidated and automated. The EIA examined the data and initiated a process with the FERC to correct defjejencies. Responsibility for correcting errors and making contact with the FERC Form I respondents rests with the FERC. Principal among the problens with existing wholesale trade data are: (1) ibconsistencies in reporting mutual rransactions by buyers and sellers, (2) lack of unjformity and standardization in teporting, (3) lack of uniformity in reporting requirements among various forms including reporting periods, and (4) multiple and sometimes overlapping reporting and aggregation of individual transactions.

Many of these errors are identified by automated intraform range, arithmeuc and logic checks, and interform consistency edits (for example, crosschecking between submissions of the respondents). The Technical Notes provide more information on data linkages, revisions, and correction procedures used in editing data on wholesale electricity trade. In addition, the corrections to these problems also serve as the foundation for future aralytical work to improve reporting on ELA forms.

\section{Limitations of Data Coverage, Reliability, and Quality}

Electricity transactions have a wide range of terms and conditions designed to accommodate prevailing cireumstances. Because of the lack of standardization in contract terminology, reporting of each transaction is not consistent across the industry. The lack of uniformity, combined with the complexity of the transactions, affects the accuracy of the data. This is further complicated by the accounting of simultaneous energy transactions, the receipt and delivery of energy caused by inadvertent flow, and the losses associated with transmission.

In developing the data base for ELECTRA, ElA bas mande a major effort to detect and correct respondent reporting errors data processing issucs, and problems with data interpretation. However, some emor correction issues cannot be addressed by EIA since they involve survey design, operations decisions, and quality control procedures beyond ELA s control. A discussion of the error detection methods and the correction process used by ElA is deseribed in the Techpical Notes to this publication. The data within ELECTRA come from survey filings that arc available to the public from the respective agency that is responsible for the survey form.

The major sources of incompatibility between data collection forms include: ( $\$$ different reporting years, (2) different reporting requirements, (3) different reporting of murual transactions by purchasers and sellers, (4) lack of uniformity and standsurdization in the definitions of terms used in the industry, and (5) multiple reporting of individual transactions. The single greatest source of incompatibility is the different "reporting year" reçuirements of Form EIA-412 compared with the other forms. The innual data reported on Form EIA-412 by a public utility are based on that utilizy's fiscal year. The annual data reported on Form EIA-861, FERC Form ], RUS Form 7 , and RUS Form 12 are based on the calendar year.

The second largest source of incompatibility is the difference in reporting requirements among the forms. The fout rypes of electric trade transactions and the forms used to collect them are as follows:

- Purchases-aEkC Forms $I$ and $1-F$, Form ElA.4I2, RUS Form 7, RUS Form 12

- Sales for Resale--FERC Forms J and 1-F, Form ElA-4I2

- Exchanges--FERC Forms I and 1-F

- Wheeling-FERC Forms I and I-F

Form ELA-861 is also used to gather information on all four types of transactions; the date, however, ate submitted on energy flows rather than funancial performance. ElA-861 data ate at an aggregare level and do not identify buyers and sellers. These differences make it impossible to disaggregate the types of electric trade transactions across oweership classes for all forms. This publication provides significantly more complete infortration on the investor owned utilixies than other ownership classes because of the greater detail collected on FERC Fom 1 .

Dala teceived from FERC, RUS, and FE can be incorsistent with similat data collected by EIA. Some data tements from the survey forms used are not consistent within the same respondent filing aggregations of detailed information within individual schedules aceounts do not equal summary schedule accounts). Therefore, the detailed transaction information was used for the basis of aggregation and cons. parison. lo other cases, megawatthours or revenue data may not sum to the totals reported to EIA. EIA atcempts to resolve such inconsistencies, which often are related on how accounting adjustments (both positive and negative for dollars and megawathours) are treated or accepted under the regulatory oversight When cross form consistency cannot be achieved, the data used in ELECTRA is as "reported."

The data on wholestle tectricity trade are collected on Federal survey data forms that are accountingsystem based. These forms were designed for regulatory parposes to meet administrative and rate case needs of individuat uritities as opposed to overall aggregate industry statistical and analytical purposes.

Data for the Commonwealth of Pyerto Rico and the U.S. Trust Territories are excluded in this publication. Geographical location and electrical system isolation limil the usefulness of any wholesale trade data from these areas. 


\section{A verage Unt Cost and Unt Revenue}

This publication presents annual surnmations of electricity transactions (energy) with the corresponding revenue and cose information. Dividing cost or revenue by the transaction quantity gives the arerage cost or average revenue per unit of energy. However, il does not necessarily indicate the price specified in contracis.

Wholesale sales are transaction-specific and lose relevance when aggregated to ctasses of ownership and geographic regions. The price that any one utility charges another for wholesale energy comprises numerous sysrem-spectfic facrors. For example, the price of purchased power can consist of demand charges, energy charges, and facility charges. A demand charge is a fixed charge determined according to the maximum capacity demanded by the purcbasing utility during a specifited period of time. Demand charges, being fixed, are unaffected by the number of kilowatthours of energy purchased; therefore, the average charge per kilowattbour decreases as the volume of energy sales increascs.

In addition to demand charges, wholesale electricity transactions are frequently subject to facility charges that include transmission, generation, and other system operating charges. These charges typically vary by ownership class, system characteristics, and region.

Furthermore, demand charges and energy charges are affected by time of delivery, quantity of energy, and reliability of supply. Typically, energy delivered during peak periods costs more than energy delivered during off-peak periods. Firm power, which can have a level of reliability similar to that given to a suppli. er's customers, costs more than econorny energy. Economy energy is intenuptible on short notice and replaces the more expensive firm power energy that is available to the purchaset.

For electric trade transactions, the average revenue or cost per unit of energy, calculated by dividing the revenue or cost by the associated energy, is not a substitute for total price. Electric trade transactions are comprised of various energy transactions of different value. Differences in average revenue or cost per unit of energy may represent a difference in the operating conditions rather than a difference in price structure. The quantity and value numbers presented represent the summation of all trade in that category for a year. Individual prices associated with a particular trade, the impact of seasonal and peak usage, and contract specific conditions cannot be separated from the aggregate. The reader is cautioned against using unit revenue or cost as a substitute for trute price.

\section{Account Adjustments}

Utilities must adjust the current year data to reflect not only the corrections is atcounting for physical flows but also any regulatory and court decisions. Rate case rebates, meter adjustments, and ingdvertent power flow corrections are examples of these changes.

Both uegative and positive adjustments are present in the revenue, cost, and quantity of trade numbers. Negative and positive numbers in the interchange tables represent a direction of flow--positive is a receipt and negative is a delivery. In the purchased power and sales for resale tables, data (either positive or nega(jve) represent either the booking of accounting adjus [ments of reversal in payments and energy flows.

Transmission of power from generating facilities under joint ownership can be reported in several ways. Typically, joint owners operate with interconnected transmission networks under mumally beneficial service agreements. The actual transfer of energy actoss transmission systerns is usually considered a bonrnonetary transaction and not a wholesale transgction. When a joint owner lacks an interconnected path to the generaling facility, the actual flow of energy is subject to the transmission service of the wheeling third party, accounted for as a monetary transaction, and reported, as "Transmission For Others."

In somc of the tables, data caregorized as "Orher" include power pool data, international trade trapsactions, and any nomutility generated electricity puschased. This information is ofren reported in a manner that prevents the identification of the buyer and/or seller.

\section{Appendlces}

Appendix A identifies the tegalatory gujdance provided by FERC through its Uniform System of Accounts and selected instructions provided on FERC Form 1 .

Appepdix B contains the location, NERC tegion, and data source of each utility for the 1994 filings used in this report. The intormation is presented by the urility forms: (I) FERC Form I and 1-F (Investor-Owned); (2) Form ELA-412 (Federal, State and Other Government Utilities, and Municipalities); (3) RUS Form 7 and RUS Form 12 (Cooperatives); (4) Form EIA-861 (Whesling and Exchanges for Noninvestor-Owned Utilities); and (5) Energy Marketing (brokering-where ownership of power is transferred) Groups under FERC jutrisdictional anthority.

Appendix C contains a description of the fundamentals of electric power transmission, the control and operation of electric systems, and an overview of integrating NUGs into the power grid.

Technical Notes (Appendix D) describe the linkages and revisions made to the various data collection 
systems used in preparing this publication. The Notes also explain data quality and correction procedures used. Copies of the collected data are available to the public. See "Obtaining Copies of the Data" in the Notes for further information.

\section{Glossary}

A glossary of terms bas been inchuded to assist nontechnical as well as technical users in understanding the statistical data in this publication. 
Table 8. Electricily Purchases by Investor-Owned Utlitites, by NERC Region, 1994

\begin{tabular}{|c|c|c|c|c|c|c|}
\hline \multirow[b]{2}{*}{$\begin{array}{c}\text { \$elket by } \\
\text { Dumarstip Ollaxs }\end{array}$} & \multirow{2}{*}{$\begin{array}{l}\text { Deanand } \\
\text { Cherge } \\
\text { Onbyl } \\
\text { (dodlers) }\end{array}$} & \multicolumn{5}{|c|}{ Eim } \\
\hline & & $\begin{array}{c}\text { Derangid } \\
\text { Cost } \\
(\text { Wollinis) }\end{array}$ & $\begin{array}{l}\text { Bnergy } \\
\text { Cont } \\
\text { [dollont] }\end{array}$ & $\begin{array}{l}\text { Oller } \\
\text { Cend } \\
\text { (dojlers) }\end{array}$ & $\begin{array}{c}\text { Total } \\
\text { Cosd } \\
\text { (dwilat })\end{array}$ & $\begin{array}{c}\text { Pentheres } \\
\text { (thouch l lWhy }\end{array}$ \\
\hline \multicolumn{7}{|c|}{ Lavester-0wnet Utilikep in ECAR } \\
\hline 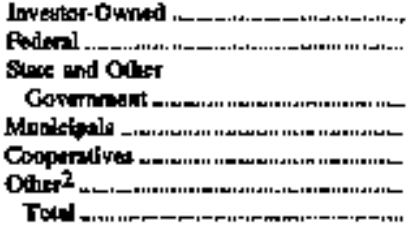 & $\begin{array}{l}- \\
- \\
-\end{array}$ & $\begin{array}{r}472,937,049 \\
22,086 \\
- \\
29,050,041 \\
2,167,114 \\
461,773,414 \\
925,900,715\end{array}$ & $\begin{array}{r}698,917,606 \\
2,843,622 \\
- \\
26,745,952 \\
16,951,9740 \\
313,495,422 \\
1,057,953,402\end{array}$ & 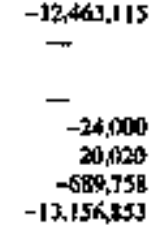 & $\begin{array}{r}1,119,39,1,500 \\
2,666,708 \\
- \\
55,711,994 \\
19,138,134 \\
814,579,078 \\
2,011,747,454\end{array}$ & $\begin{array}{r}35,853,473 \\
117,044 \\
- \\
1,94,949 \\
1912,489 \\
19,541,191 \\
38,274,086\end{array}$ \\
\hline \multicolumn{7}{|c|}{ Invectox-Owned Uetrita in ERCOT } \\
\hline 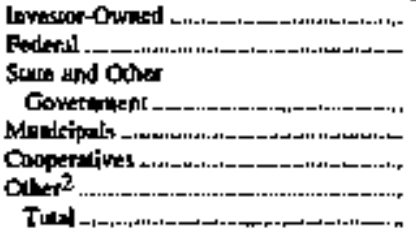 & $\begin{array}{l}- \\
- \\
-\end{array}$ & $\begin{array}{l}64,447,048 \\
- \\
6,854,754 \\
- \\
\overline{7} 7,79,752,766 \\
447,689,572\end{array}$ & 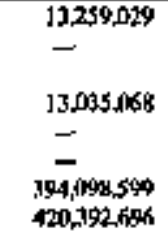 & $\begin{array}{l}26,368,056 \\
= \\
3,610,374 \\
- \\
-\quad 52,259 \\
29,022,371\end{array}$ & $\begin{array}{l}104074932 \\
- \\
23.505 .6010 \\
= \\
7 \pi 1,424,106 \\
998,004,639\end{array}$ & $\begin{array}{l}3,812,569 \\
- \\
-692,604 \\
- \\
14,834,819 \\
17,339,992\end{array}$ \\
\hline \multicolumn{7}{|c|}{ Inweslow-Owned Ftities in MAL } \\
\hline 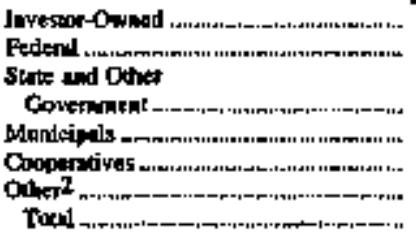 & $\begin{array}{l}12,137,639 \\
- \\
- \\
- \\
-\quad 00,944 \\
12,207,543\end{array}$ & $\begin{array}{r}232,859,913 \\
- \\
324,746 \\
1,522 \\
-\quad 81,550,182 \\
334,736,417\end{array}$ & 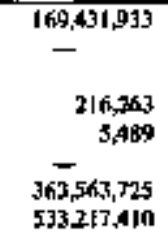 & $\begin{array}{l}1,822,954 \\
- \\
- \\
- \\
- \\
1,859,635 \\
3,6 \times 2,599\end{array}$ & $\begin{array}{l}424,114,6010 \\
- \\
541,0159 \\
7,011 \\
-\quad 4673,42 \\
871,63,64,412\end{array}$ & $\begin{array}{r}9,158,318 \\
- \\
43529 \\
71 \\
11,599,397 \\
20,391,715\end{array}$ \\
\hline \multicolumn{7}{|c|}{ 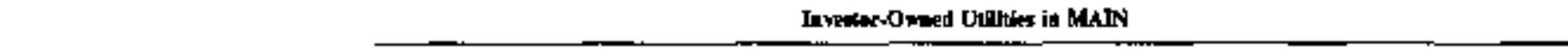 } \\
\hline 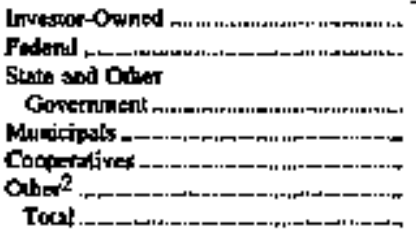 & 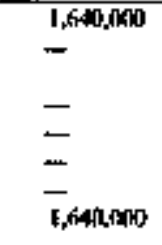 & $\begin{array}{r}113,657,945 \\
789,000 \\
-\quad 29,400 \\
3.674,100 \\
797,428 \\
118,047,634\end{array}$ & $\begin{array}{r}370,833,621 \\
19,802,198 \\
- \\
10,1,1,314 \\
10,613,949 \\
11,013,104 \\
4[2,914,124\end{array}$ & $\begin{array}{l}-70,378 \\
- \\
- \\
= \\
- \\
-71,373\end{array}$ & $\begin{array}{r}484,42 J, 189 \\
20,671.196 \\
- \\
592,714 \\
14,292,049 \\
11.814,534 \\
331.393,684\end{array}$ & $\begin{array}{r}20,706,981 \\
847,865 \\
-\quad \\
29,313 \\
711,637 \\
421216 \\
22,716,412\end{array}$ \\
\hline \multicolumn{7}{|c|}{ Inwestor-0wed Ullktes in MLAF } \\
\hline 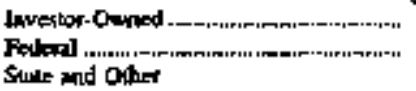 & - & $\begin{array}{l}38,073,463 \\
-\end{array}$ & $\begin{array}{l}\text { 49,nath, R36 } \\
-\end{array}$ & 1.02tsil & $\begin{array}{l}\text { J08,7999. } \\
-\end{array}$ & $\begin{array}{l}3,248,699 \\
-\end{array}$ \\
\hline 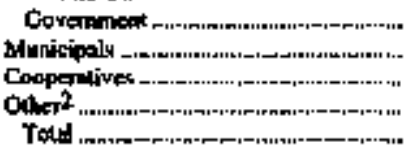 & $\begin{array}{r}1,780,050) \\
979,950 \\
2 \mathrm{R}, 3,974 \\
50,075 \\
2,695,899\end{array}$ & $\begin{array}{r}30,500,000 \\
1,415,652 \\
69,231,5801 \\
9,239,246 \\
234,469,561\end{array}$ & 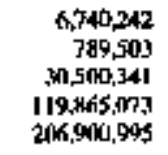 & $\begin{array}{l}- \\
-455,528 \\
-{ }_{1,476,539}\end{array}$ & $\begin{array}{r}1,241,242 \\
2,195,155 \\
99,187,449 \\
216,104,319 \\
442,827,099\end{array}$ & $\begin{array}{r}6018,261 \\
28,497 ? \\
3,580,410 \\
7,5016,897 \\
15,372,954\end{array}$ \\
\hline
\end{tabular}

Sec nores and footustes in and of tublo. 
Table 8. Electricity Purchases by Investor-Ownext Utilities, by NERC Region, 1994 (Continued)

\begin{tabular}{|c|c|c|c|c|c|c|c|}
\hline \multirow[b]{2}{*}{$\begin{array}{c}\text { sillexs by } \\
\text { Onicenhup Clas }\end{array}$} & \multicolumn{3}{|c|}{ Nonfinew } & \multicolumn{2}{|c|}{ Mreedlanopin? } & \multicolumn{2}{|c|}{ Region Iutul } \\
\hline & 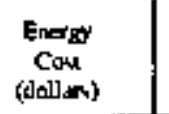 & $\begin{array}{c}\text { Onher } \\
\text { Cout } \\
\text { (dollun) }\end{array}$ & $\begin{array}{l}\text { Purchuses } \\
\text { (thanumal } \neq w h \text { ) }\end{array}$ & $\underset{\text { (Bolunt }}{\text { Cont }}$ & 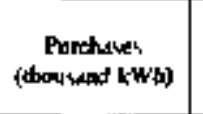 & $\begin{array}{c}\text { Cont } \\
\text { (doptans })\end{array}$ & Purthns \\
\hline \multirow{9}{*}{ 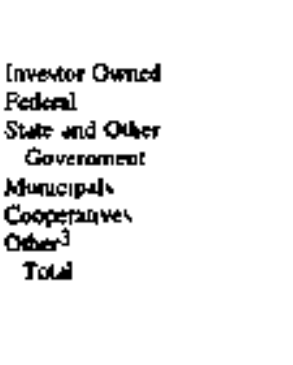 } & \multicolumn{7}{|c|}{ 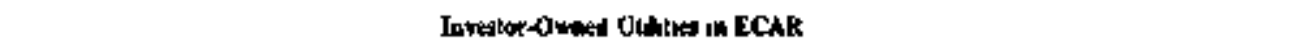 } \\
\hline & $219,(134,463$ & $13.318,354$ & II.27ka.211! & $259.213,638$ & 9.390 .747 & $1, \overline{111,437995}$ & 55314,430 \\
\hline & $9,832 \times 24$ & & 326,484 & - & & 12.699 .332 & 443,528 \\
\hline & 296,728 & $5,971,263$ & 191,197 & - & - & $\$, 931,981$ & $51,39 ?$ \\
\hline & $2,603,069$ & - & Im,kBs & $-2,4|k|$ & $7 G B$ & $58,172,663$ & 2054,395 \\
\hline & $12,377,8112$ & 274,989 & $7 m, 542$ & - & & $32,194,925$ & 1519,971 \\
\hline & $519,891,246$ & $-B, 07$ A & $18,778,535$ & 1.349897 & 195.894 & {$[.326,81], 061$} & $38,515,760$ \\
\hline & $798,039,922$ & 20,0 thins36 & $70,377.994$ & $260,5 \sin$ ues & ולה.4.587 & $7,050,447,957$ & $99,2,39,491$ \\
\hline & \multicolumn{7}{|c|}{ Inreator-0 wnad Chinles ua ERCOT } \\
\hline Invertor Owhed & 7.1000 .850 & $-73,176$ & 2989286 & 4,867 & 286,184 & J11,016,874 & $2,597.959$ \\
\hline Fedenul & BIs,sing & - & 56,352 & - & - & 815,505 & 56,352 \\
\hline State and Other & & & & & & & \\
\hline Govanomedt & 311,849 & $3,3,60$ & 16,691 & 6.176 & - & $20,827,185$ & 309,205 \\
\hline Marroc口quk & $3,376,340$ & 80,815 & 141653 & 6,698 & - & $3,469,853$ & 141,659 \\
\hline Cocpersuter & 513,506 & $-97,064$ & 35,J9: & $1, \operatorname{fg}^{2} ?$ & - & 418,124 & 35,194 \\
\hline Oter $^{3}$ & 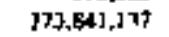 & 489,47 & $9.002,102$ & 361,143 & -141 & $945,315,893$ & $21,859,747$ \\
\hline \multirow[t]{2}{*}{ Tat } & $175.259 .1 \% 4$ & $410 \mathrm{j}+\mathrm{tat}$ & $9,533,841$ & 5001067 & 286.343 & $1,034,847,432$ & $27,200,176$ \\
\hline & \multicolumn{7}{|c|}{ 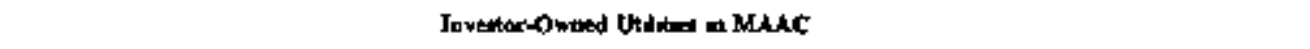 } \\
\hline Inveruar Oninal & $419,651,511$ & 1742807,894 & 17525 3748 & 59159213 & $7.136,658$ & $1089.324,077$ & $29,829,685$ \\
\hline Feterd & - & - & - & - & & - & - \\
\hline State dnd Olhe & & & & & & & \\
\hline Govermanept & $1,565,229$ & - & $221,8 \%$ & - & - & $4,106,289$ & $26.5,425$ \\
\hline Momerpels & $116,561,404$ & 2,974 & $1,945,354$ & - & - & $116,574,389$ & $1,945,475$ \\
\hline Conpesatives & 7. 血7.750 & & 191,872 & s,5tg,9ns & - & $8,564,655$ & 191,832 \\
\hline Other? & $1.024,478,313$ & $80,6158,99 B$ & $27,5400,500$ & $18,251,309$ & - & $1, \sin , 082,066$ & $39,099, \$ 97$ \\
\hline \multirow[t]{2}{*}{ Iold } & $1,361,787,297$ & $254,892,8,66$ & $47,384,991$ & $81,929,447$ & $3,136,658$ & $2,78 B, 453,479$ & $71,323,264$ \\
\hline & \multicolumn{7}{|c|}{ 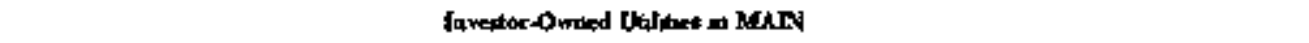 } \\
\hline Invertax Onved & $186,574,493$ & 3293,359 & $11,1850,609$ & $-200,040$ & 71 & $675.225,491$ & $32,565,661$ \\
\hline Petieral & $8,832,287$ & - & $481,86,3$ & - & $\rightarrow$ & $29,503,485$ & $1,329,728$ \\
\hline Stan wad Ober & & & & & & & \\
\hline Goyermatent & - & - & - & - & - & - & - \\
\hline Mulucipal & $1,270,9 \% 6$ & - & 37,293 & - & - & $1,80,680$ & 66,570 \\
\hline Coopersurver & $7.916,01012$ & $6, \mathbb{D B 1}, 0 \times 11$ & 301,527 & - & ang & 28289051 & $3,108,673$ \\
\hline Odutu ${ }^{3}$ & $8,424,788$ & - & 244,292 & 215,488 & -799 & 201430,350 & 365,129 \\
\hline \multirow[t]{2}{*}{ Told } & $212,44,936$ & $9,574,469$ & $13,119,6028$ & 31,428 & -59 & $755,292,457$ & $35,825,961$ \\
\hline & \multicolumn{7}{|c|}{ Itwetar-Owned Udt $\Rightarrow$ MLAFP } \\
\hline Invedor Owned & $57,478,390$ & - & 3,265, , 14 & $221,74 \beta_{1}, 663$ & $5,602,051$ & $387,326,983$ & $12,115,964$ \\
\hline Fodard] & $13.576,1082$ & - & 791,061 & -164 , 0158 & $-1,172$ & $13,4]+2,24$ & 789.869 \\
\hline Sute Jod Other & & & & & & & \\
\hline Govemenentic & $26,391,791$ & $93,5 \pi 7.19$ & $2,536,714$ & - & - & $1.98,389,213$ & $3, \sqrt{44,975}$ \\
\hline Muterpals & $3,610,485$ & - & 465,719 & - & - & $10,779,590$ & 40,306 \\
\hline Cooper unver & $33,701,340$ & $-2,2,14$ & $2,40]$ A 358 & I & - & 137,170, ing & $5,982,248$ \\
\hline Whest & $10,390,146$ & - & 642,844 & - & 4h.720 & 236,752540 & $8,590,511$ \\
\hline Tucd] & $153,748,234$ & $91,374, y d 6$ & $10.103,240$ & 271 SR2,785 & $5,541,599$ & $911 \times 28.959$ & $31.113,793$ \\
\hline
\end{tabular}

See nuter and finotrotes a end of wble 
Table 8. Electricity Purchases by Investor-Owned Utiltites, by NERC Region, 1994 (Continued)

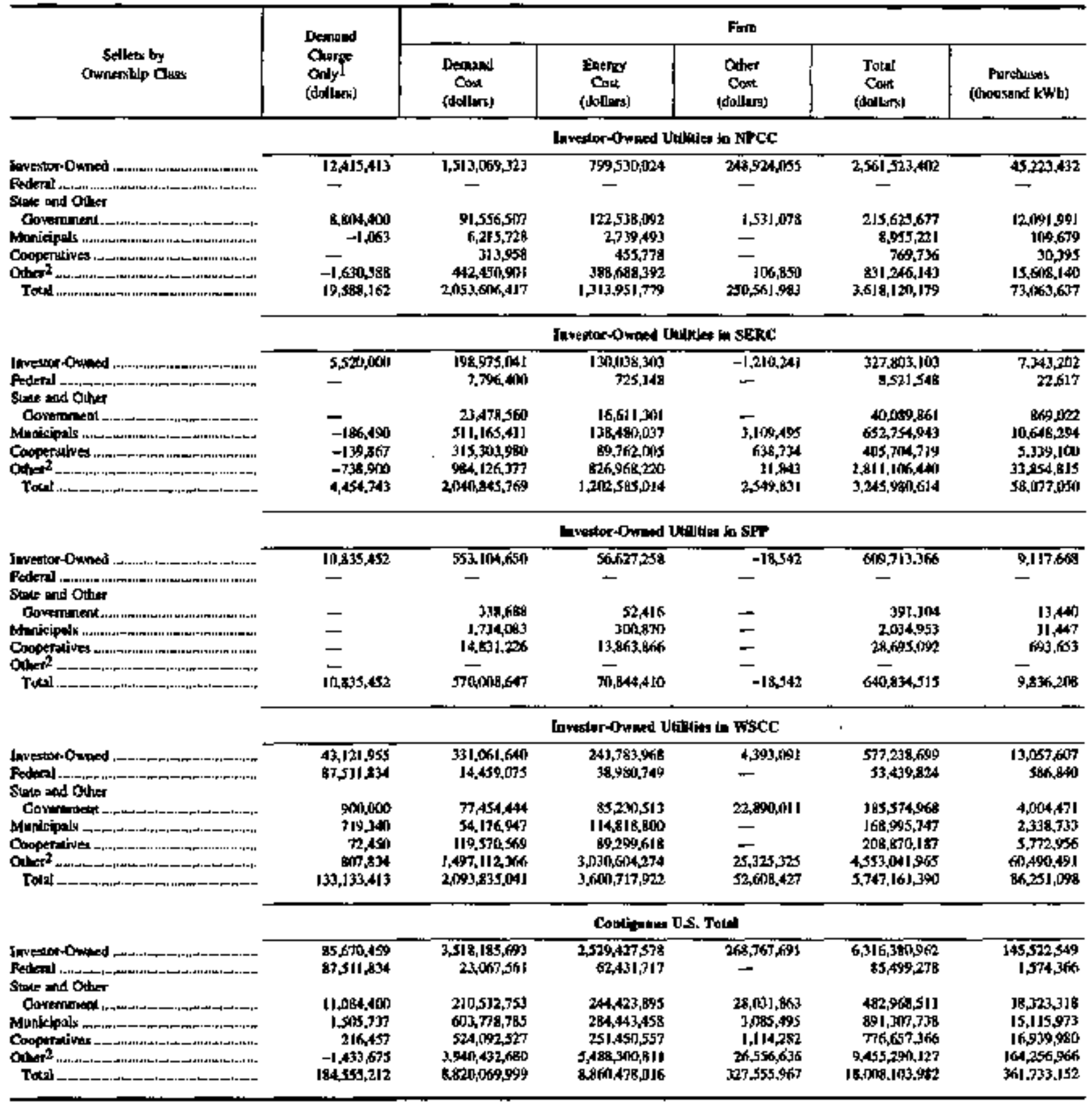

See noter and fooderien an end of toble 
Table 8. Electricity Purchases by Inwestor-0wned Utilities, by NERC Region, 1994 (Continued)

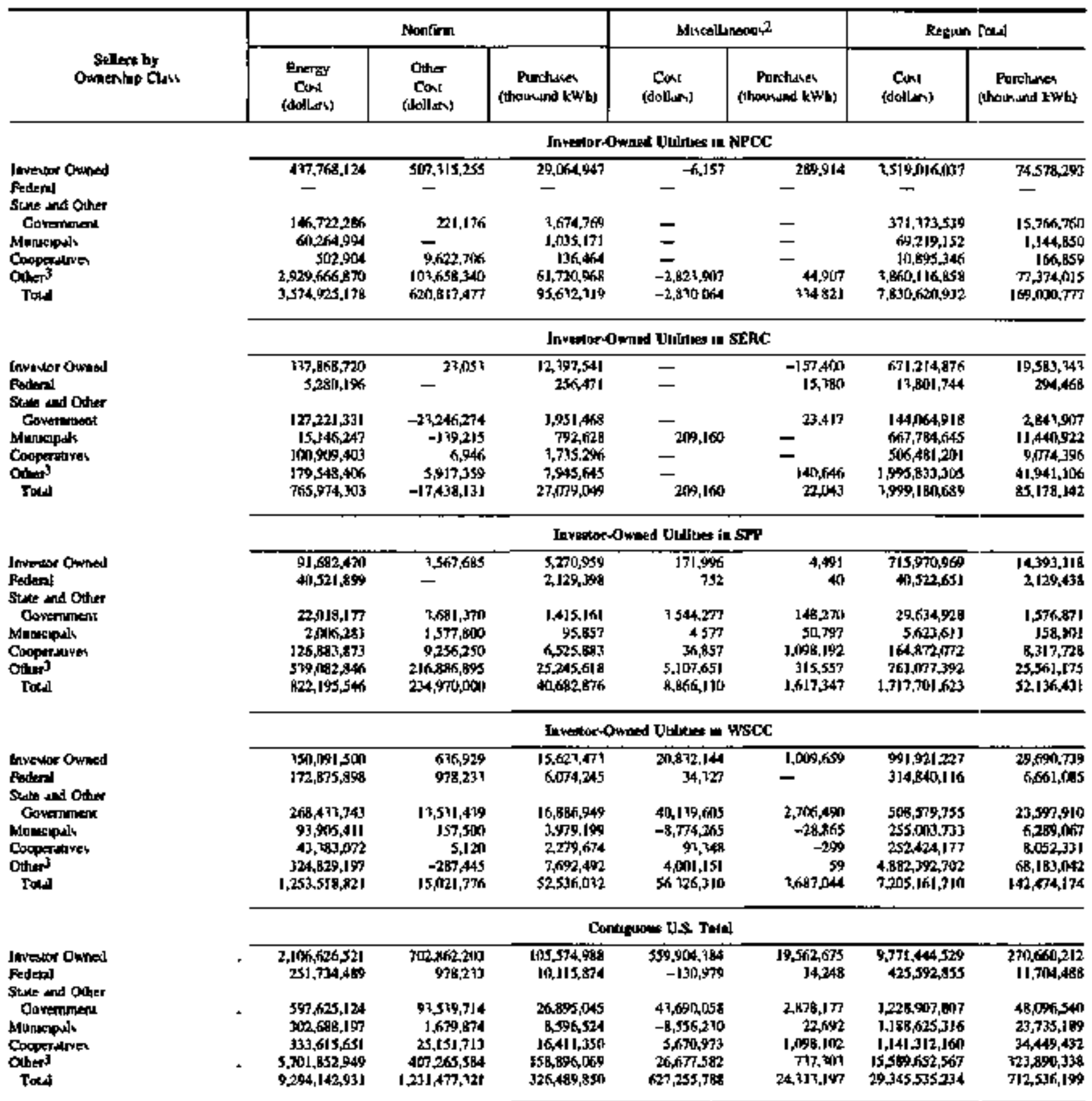

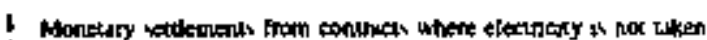

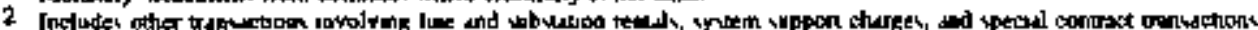

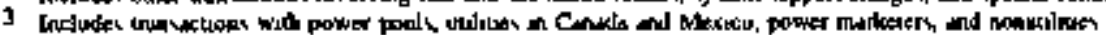

- Non Apolitate

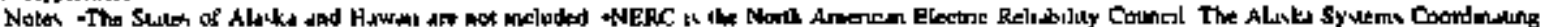

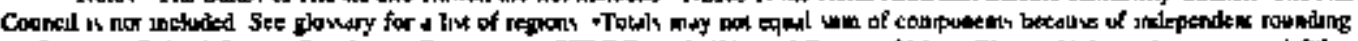

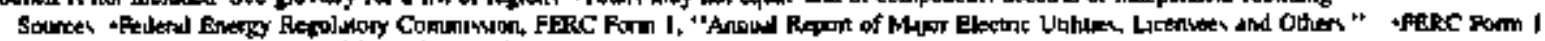

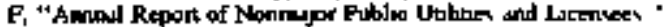


Table 9. Electricity Sales for Resale by Investor-Owned Utillities, by NERC Region, 1994

\begin{tabular}{|c|c|c|c|c|c|c|}
\hline \multirow[b]{2}{*}{$\begin{array}{l}\text { Poxluxat by } \\
\text { Owneship Cuak }\end{array}$} & \multirow{2}{*}{$\begin{array}{l}\text { Demund } \\
\text { Changt } \\
\text { Dnly' } \\
\text { (dodturs) }\end{array}$} & \multicolumn{5}{|c|}{ Fwn } \\
\hline & & $\begin{array}{l}\text { Demand } \\
\text { Revenot } \\
\text { (dedlursit }\end{array}$ & 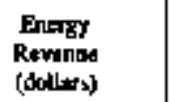 & $\begin{array}{l}\text { Other } \\
\text { Reweate } \\
\text { (lolysis) }\end{array}$ & 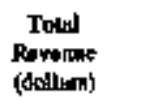 & $\begin{array}{c}\text { Sulkx } \\
\text { (thoongond } k \text { W/a) }\end{array}$ \\
\hline \multirow{8}{*}{ 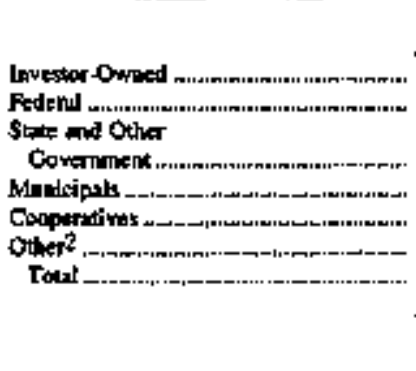 } & \multicolumn{6}{|c|}{ 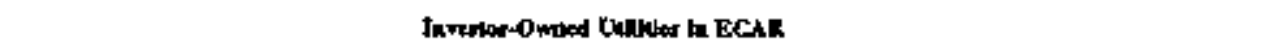 } \\
\hline & $2^{5.815,729}$ & 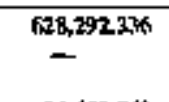 & $888,332,584$ & $-14,1,1,664$ & $1,302,543,236$ & $\begin{array}{l}55,731,460 \\
-\end{array}$ \\
\hline & - & $24,6,2,543$ & $31,028,016$ & $-1,034,59]$ & $54,625, \$ 65$ & $1,875,185$ \\
\hline & $1,844,027$ & 207,958551 & $246,969,029$ & $-11,823,109$ & $499,034,270$ & $12,629,583$ \\
\hline & 7,500 & $69, B \mid 4,621$ & $60,85 t, 195$ & $-3,44], 974$ & $196,239,842$ & $0,741,275$ \\
\hline & & $21,925,328$ & $\$ 5395063$ & -54587 & $77,465,8,4$ & $3,181,228$ \\
\hline & $7.667,256$ & $452,622,378$ & $1,291.755 .887$ & $-14,366_{1}, 125$ & $2,210,013,140$ & $77,159,001$ \\
\hline & \multicolumn{6}{|c|}{ Investor-Omend Uk"lithe is ERCOT } \\
\hline \multirow{8}{*}{ 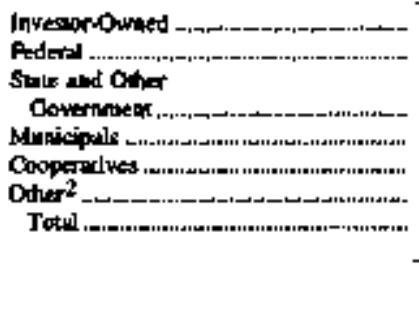 } & - & 77268.764 & $14,77,641$ & $26,698,319$ & $120,344,724$ & $1,751,591$ \\
\hline & - & & & - & - & - \\
\hline & - & - & - & - & - & - \\
\hline & - & $2,692,445$ & $3,606,671$ & 233,510 & 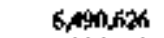 & ] 64,550 \\
\hline & - & $100,892,236$ & $59,505.994$ & $38,(187,213$ & $201,085,413$ & $\$ .982 .455$ \\
\hline & - & $36,4 T 7$ & 41,532 & 5,066 & 80,035 & 1,601 \\
\hline & - & $185,849,922$ & $77,529,3 \times 8$ & $67,624,1108$ & $\$ 31,003,068$ & $5.9010,53$ \\
\hline & \multicolumn{6}{|c|}{ Investor-Owned Utride b MAtC } \\
\hline \multirow{8}{*}{ 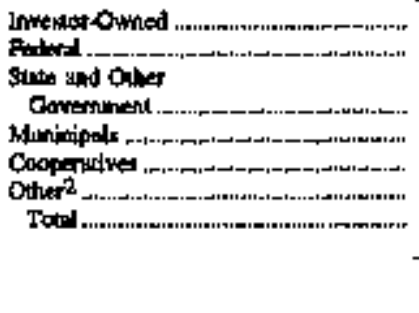 } & $\overline{-}$ & $47,105,907$ & $23,896,719$ & $-1.525,000$ & $69,817,618$ & $J, 146,572$ \\
\hline & - & - & - & - & - & - \\
\hline & - & - & - & - & - & - \\
\hline & - & $37,414,554$ & $49.115,201$ & $-1,009,1,857$ & 85,500,6伯 & $1,9 F 0,486$ \\
\hline & - & $1893,182,066$ & $109,367,6012$ & $-3,336,441$ & $209,213,517$ & $4,268,355$ \\
\hline & - & $12.094,969$ & $5], 625,773$ & - & $64,320,762$ & $2,668,568$ \\
\hline & - & $200,397,506$ & 203.945 .25 & $-5,490,236$ & $42,242,565$ & $9,994,481$ \\
\hline & \multicolumn{6}{|c|}{ 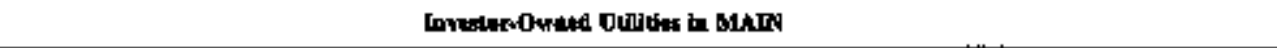 } \\
\hline Invessinmoumed & - & $128,486,992$ & 255,792828 & $-1,760,850$ & $382,538,341$ & $12,6 \pi 7.264$ \\
\hline 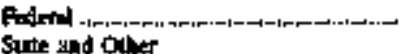 & - & - & - & - & - & - \\
\hline 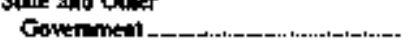 & - & - & - & - & - & - \\
\hline 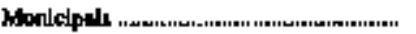 & - & $97.197,772$ & $164,958,906$ & $-14,033,566$ & $248,112,712$ & $6,6010,948$ \\
\hline 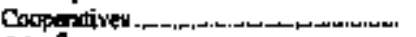 & - & $65,1770,1] 7$ & $54,228,298$ & 517,754 & $119,846,169$ & $\$, 021,138$ \\
\hline \multirow{3}{*}{ 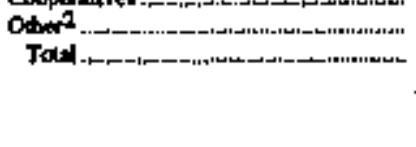 } & 180,000 & $334,0 \mathrm{mon}$ & 925,1717 & - & $1,259.131$ & 40,597 \\
\hline & 180,000 & $291,9 \pi, 251$ & 479,905,ine & $-15,246,992$ & $751,736,391$ & $22,140,991$ \\
\hline & \multicolumn{6}{|c|}{ 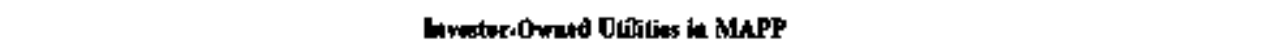 } \\
\hline 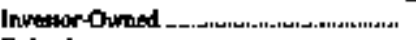 & $5,0,0$ & $45.586,950$ & $65,935,921$ & -536516 & $111, \overline{206,363}$ & $1,174,699$ \\
\hline 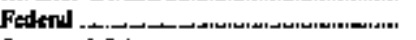 & - & 153,669 & 335,634 & 86,391 & 75,687 & 22,948 \\
\hline Starta and Other & & & & & & \\
\hline 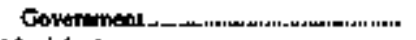 & - & - & - & - & - & - \\
\hline 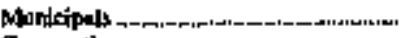 & - & $52555,12]$ & $61,914,953$ & $3,857,772$ & $118,327,448$ & $3,262,904$ \\
\hline Cooperatives. & $12,6-40$ & 612002 & 207694 & 203,287 & $1,023,5 \pm 8$ & 21,183 \\
\hline Other2 2 & & $3,4,736$ & 762.625 & 792 & 698,JS9 & 27,532 \\
\hline 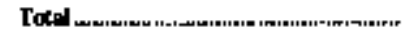 & 17,640 & 992013,984 & 125.356 .20 & $3,611,728$ & $201,631,639$ & $7,909,396$ \\
\hline
\end{tabular}

See nater and footrinates $*$ end of whle. 
Tahle 9. Electricity Sales for Resale by Investor-Omned Ut:lities, by NERC Region, 1994 (Continued)

\begin{tabular}{|c|c|c|c|c|c|c|c|}
\hline \multirow[b]{2}{*}{ 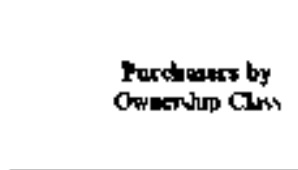 } & \multicolumn{3}{|c|}{ Nonfirm } & \multicolumn{2}{|c|}{ 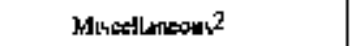 } & \multicolumn{2}{|c|}{ Regon 1004 } \\
\hline & 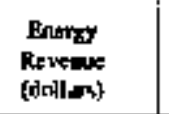 & $\begin{array}{l}\text { Other } \\
\text { Revenve } \\
\text { (dollam) }\end{array}$ & Sules & $\begin{array}{l}\text { Ravenewe } \\
\text { (dallen) }\end{array}$ & $\begin{array}{c}\text { Saleh } \\
\text { (llow wend klah) }\end{array}$ & $\begin{array}{l}\text { Ravenne } \\
\text { (dollan) }\end{array}$ & 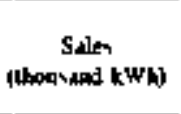 \\
\hline & \multicolumn{7}{|c|}{ Inwegtoc-Onned Uculter in ECAR } \\
\hline Invever Orined & $469,822,706$ & $42,641,6 \mid 4$ & $15.94 k .769$ & $90,293,375$ & $\overline{-}$ & 2,111,196,6stid & 31280,429 \\
\hline Federol & $14,1 \leq t 2 \pi$ & - & 759.508 & - & - & 14.157235 & 759508 \\
\hline Stine and Outher & $1,751,465$ & - & & & & & \\
\hline $\begin{array}{l}\text { Contertintent } \\
\text { Moruapuls }\end{array}$ & $\begin{array}{r}2,3,31,565 \\
24,279,352\end{array}$ & $\overline{I . \mid 04431}$ & $\begin{array}{r}\$ 9,551 \\
\end{array}$ & $\bar{z}$ & $\overline{-}$ & $\begin{aligned} 53,977,993 \\
46,316,150\end{aligned}$ & $\begin{aligned} 1,924,738 \\
17,644,657\end{aligned}$ \\
\hline Coopersives & $211,575, \sqrt{7} 7$ & $1,899,457$ & $I_{1}, 41,43 j$ & $\bar{z}$ & - & $158,663,976$ & 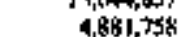 \\
\hline OAber 3 & 497045,193 & - & $15, m i s, 301$ & $-4,302$ & -51 & $50,5 m, 315$ & 21965,978 \\
\hline \multirow{2}{*}{ Total } & $1,027.278,346$ & $45,609, \sin$ & $37, J 36,48 B$ & 90,284.,453 & -51 & $3,380,8: \% 31\}$ & $114,497,868$ \\
\hline & \multicolumn{7}{|c|}{ Invertor-Onned Uidkies in ERCOT } \\
\hline Invertor-Dened & 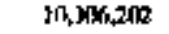 & 987,344 & 491,059 & 1T म4: & - & ] $31,695,313$ & 2243,486 \\
\hline Fadral & & - & - & - & - & - & - \\
\hline State and Doler & & & & & & & \\
\hline Caramprenl & $1,4,33,351$ & $m$ & 71,766 & 3,716 & - & $1,64], \Omega 67$ & 7,266 \\
\hline BSenecppeds & $23,674,852$ & 260052 & $1.113,5600$ & 141,684 & - & $31,167,194$ & $1.274,510$ \\
\hline Cooptraclyes & $9.998,716$ & - & 503,341 & 289,824 & - & 214371,980 & $4,516,198$ \\
\hline Others & $16,1<\leqslant, 025$ & 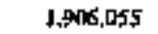 & 701.874 & 1,89 ,.272 & - & $18,049,378$ & 705.475 \\
\hline \multirow[t]{2}{*}{ Tond } & $59,775,[47$ & $3,651,431$ & 29]67, 399 & $2,754,489$ & - & $1 \times 6,746,935$ & $8,8] 6,935$ \\
\hline & \multicolumn{7}{|c|}{ Innegtoc-Omoed Ckulutes = MANC } \\
\hline Invevlot Ounned & $767,398,310$ & $229,480,568$ & $17,957,543$ & 34939706 & $1,115.152$ & $201,655,822$ & $22,219,249$ \\
\hline Poderal & - & - & - & - & - & - & - \\
\hline Slate and Qher & & & & & & & \\
\hline Dotamment & $7,372,494$ & - & 147,746 & - & - & $3,302,494$ & 147.756 \\
\hline Morucepals & $22,215,254$ & $5,549,272$ & 929,850 & $-28,6 ; f$ & - & $113,261,578$ & $2,84 \pi, 336$ \\
\hline Coxprastiven & $3,438,007$ & - & $173,45,3$ & - & - & 212 R5, 51,574 & $4,442,3918$ \\
\hline \multirow{3}{*}{$\begin{array}{l}\text { Othes } \\
\text { Tald }\end{array}$} & $316,454,6,36$ & 73,097 & $12,932,15 \mathrm{fi}$ & $57.679,9008$ & 799,837 & $226,538,4063$ & {$[6,400,57]$} \\
\hline & 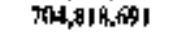 & $235.122,957$ & .32 .140 .770 & R2.615.196 & $3,9] 4,989$ & 1,$4 ;, 409,4[1$ & $\left.\phi_{6},(1 \leq t), 240\right)$ \\
\hline & \multicolumn{7}{|c|}{ 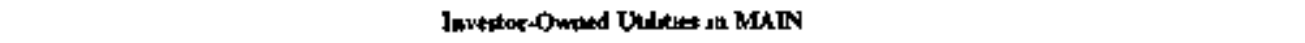 } \\
\hline Forester Onned & $248,537,49]$ & $1,670,6(x)$ & 12879,960 & - & 14,42 & $592,225,821$ & 25,571670 \\
\hline Fedetal & $25, \$ 11,5,30$ & - & 1,893,941 & - & - & $25,811,570$ & 1.8539.94] \\
\hline Sulte ded Other & & & & & & & \\
\hline Oonton-meat & $\rightarrow$ & $\overline{-}$ & - & - & - & - & - \\
\hline Momapul & 48,300, Sist & $1,325,01010$ & 1,561.184 & - & - & $297,752,219$ & 8.165 .852 \\
\hline Coopessuver & $20,356,527$ & - & 1,MmLSit & - & - & $150,200.696$ & 4,051,654 \\
\hline \multirow{3}{*}{$\begin{array}{l}\text { Oula. } \\
\text { Totd }\end{array}$} & $35,912,451$ & - & 2070,134 & 271064 & -162 & $37,374,621$ & 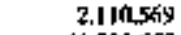 \\
\hline & $7 d B, 4+8,496$ & $2,995,000$ & $19,426,397$ & 27,800 & 14280 & 1.10L,1SLRA] & 41.313,6fig \\
\hline & \multicolumn{7}{|c|}{ Imvelor-Owaed Dulutes a MAPT } \\
\hline Inestor Dwned & $85,334,98 \mathrm{BI}$ & - & $1,200,578$ & $221,8 B_{0}, 292$ & $5,602, \sqrt{51}$ & $418,226,646$ & $13,283,328$ \\
\hline $\begin{array}{l}\text { Federpal } \\
\text { Stute ofd Oher }\end{array}$ & $1,966,553$ & - & 283,684 & - & & $4,539,2,10$ & 306.872 \\
\hline Dovemment & $4,612,447$ & - & $2 \leftrightarrow 27$ & - & - & $4,612,443$ & 257271 \\
\hline Munucipuls & I5,890,6\% & - & 964,303 & - & - & $134,218,544$ & 4.317.207 \\
\hline Cospatitries & $J 8,539,458$ & - & $1.244,923$ & - & - & $19,599,6866$ & 1,266,10K \\
\hline 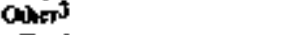 & $3,498,231$ & - & $2 \$ 6,47 y$ & - & - & $4,196,384$ & $2+6011$ \\
\hline Totdl & $131,859,266$ & - & 2.205,438 & $22\rfloor, 880,192$ & $5,6 n 2,1051$ & $385,388,937$ & 24.716,795 \\
\hline
\end{tabular}

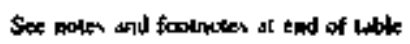


Table 9. Electricity Sales for Resale by Investor.0wned Utilties, by NERC Region, 1994 (Continaed)

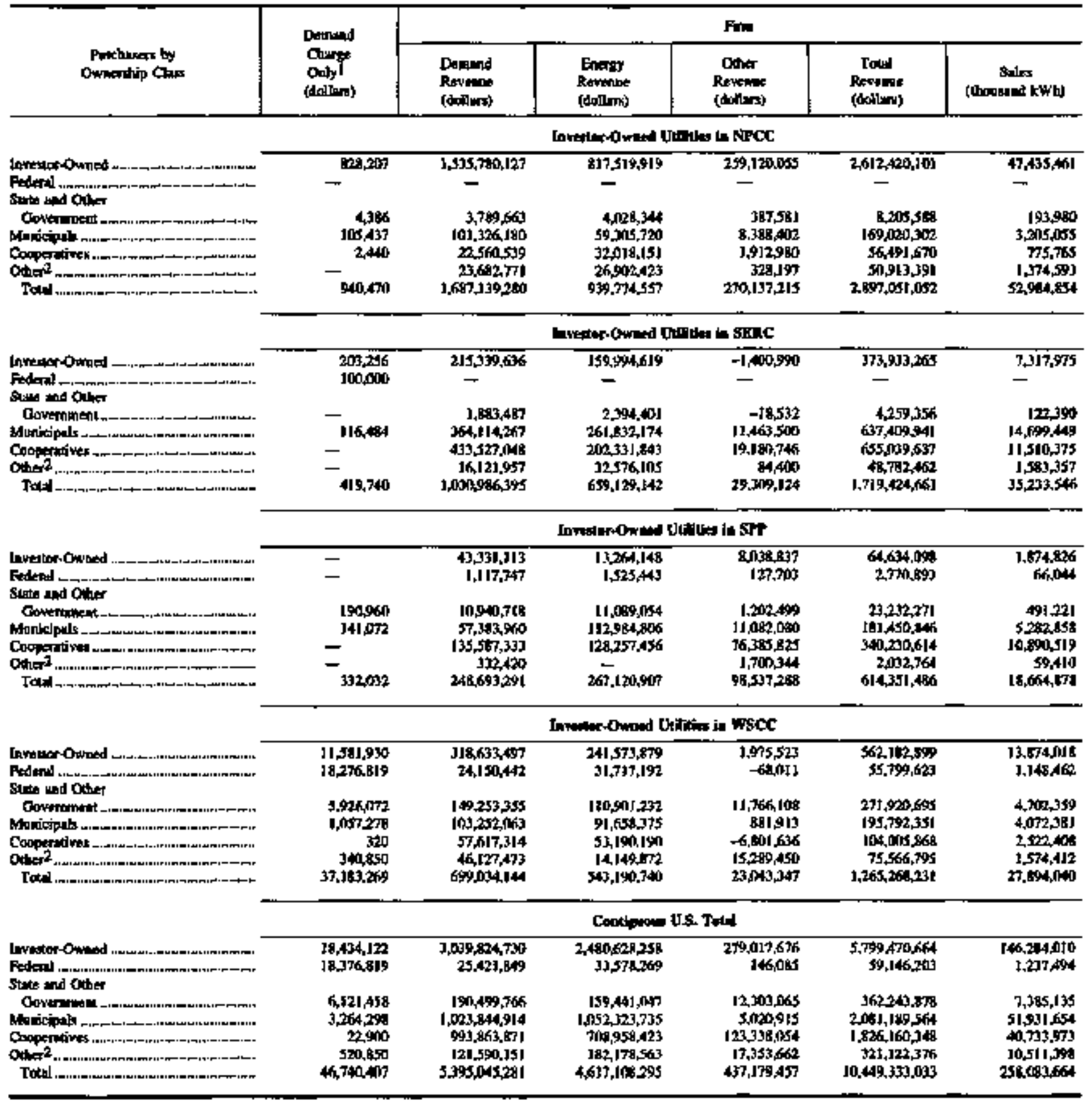

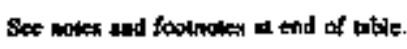


Table 9. Eloctricity Sales for Resale by Investor-Owned Utijities, by NERC Region, 1994 (Continoed)

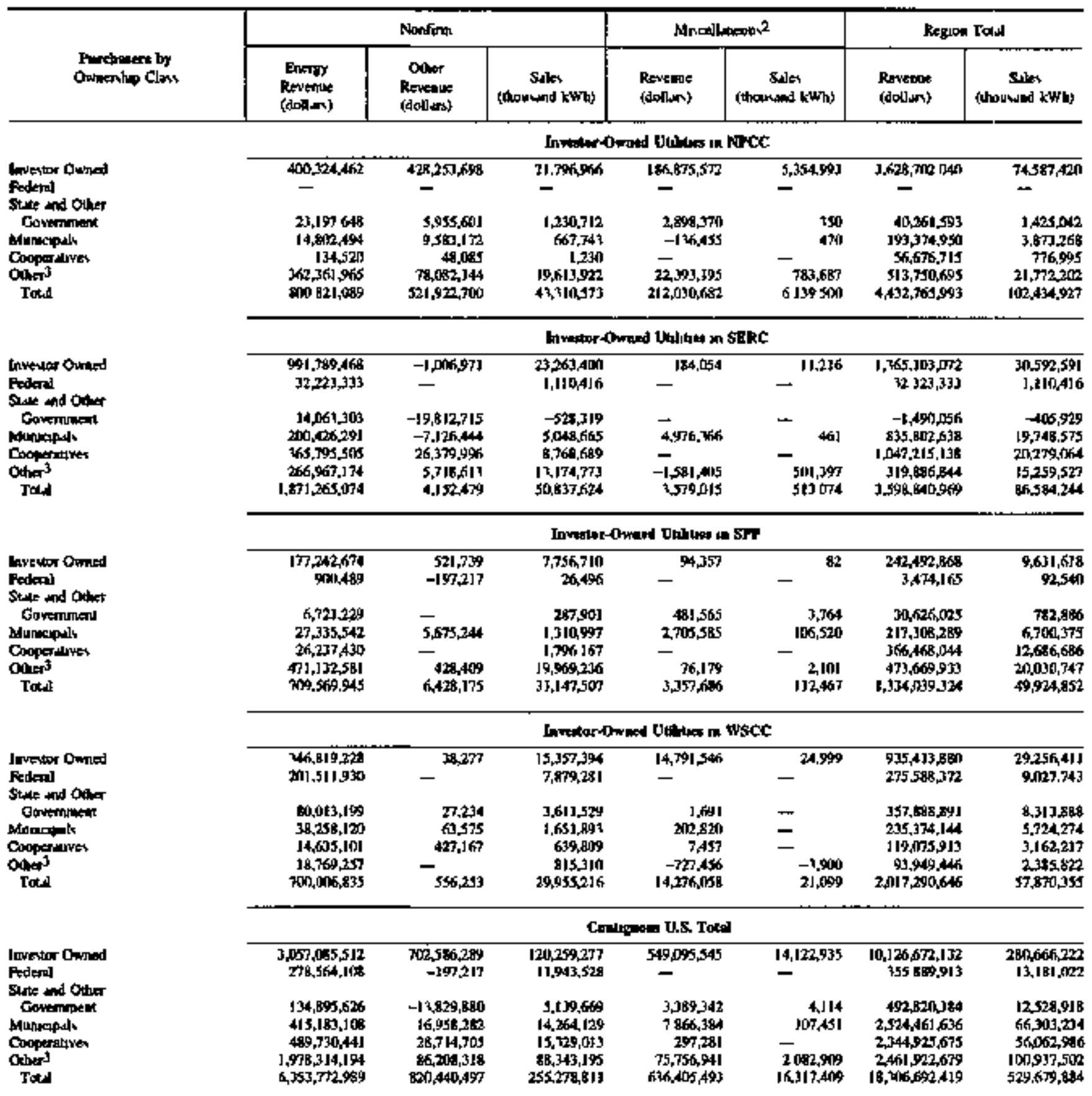

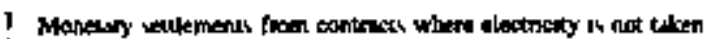

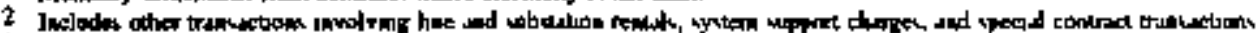

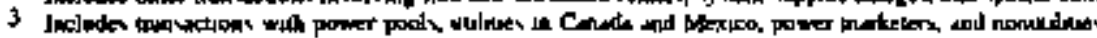

Hital Applicabie

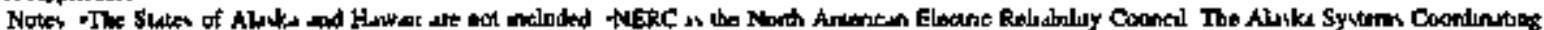

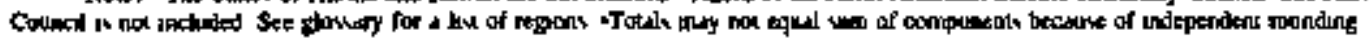

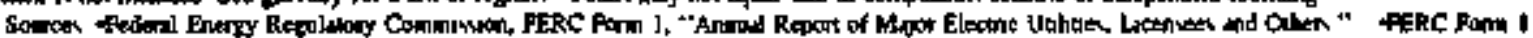

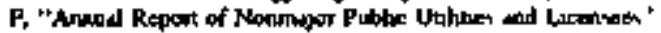


Table 10. Imvestor-Owned Utility Exchanges of Electricity with Al Ownership Classes, by NERC Region, 1994

\begin{tabular}{|c|c|c|c|c|c|c|}
\hline 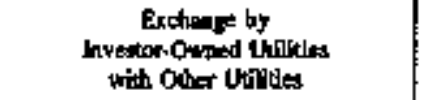 & $\begin{array}{c}\text { Roceivetl } \\
\text { (thourend I'th) }\end{array}$ & $\begin{array}{c}\text { Detlveredz } \\
\text { (fhoucid kWhy }\end{array}$ & 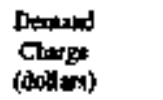 & $\begin{array}{l}\text { Eurty } \\
\text { Cherp: } \\
\text { fdollurs }\end{array}$ & $\begin{array}{l}\text { Duher } \\
\text { Ornge } \\
\text { (dollest) }\end{array}$ & $\begin{array}{l}\text { Sotilemenl } \\
\text { (doHers) }\end{array}$ \\
\hline \multirow{4}{*}{ 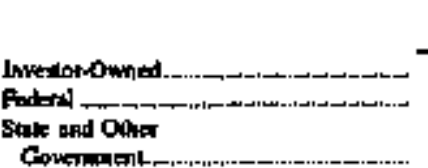 } & \multicolumn{6}{|c|}{ Inwe:eronmed Utilitis la ECAR } \\
\hline & \multirow{3}{*}{$\begin{array}{r}1,0+4,821 \\
210,649\end{array}$} & \multirow{3}{*}{$\begin{array}{l}1,2+2,260 \\
266,107\end{array}$} & 949,666 & 227,726 & 60,759 & 938,151 \\
\hline & & & \multirow[t]{2}{*}{ 一 } & - & - & \\
\hline & & & & - & -7 & \\
\hline \multirow{5}{*}{ 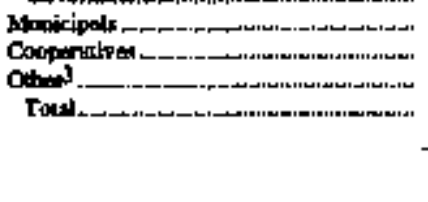 } & \multirow{3}{*}{$\begin{array}{r}21,122 \\
144,123 \\
152720\end{array}$} & 32148 & \multirow[b]{4}{*}{ 469, } & \multirow{4}{*}{$\begin{array}{l}-6,979 \\
-4,071, A 00 \\
-3,779,775\end{array}$} & \multirow{3}{*}{$\begin{array}{r}7,110 \\
14,004\end{array}$} & \multirow{4}{*}{$\begin{array}{r}-7,069 \\
18,094 \\
-4,191,190 \\
-3, \sqrt{144,236}\end{array}$} \\
\hline & & 233,435 & & & & \\
\hline & & 287,903 & & & & \\
\hline & $1,073,645$ & $2,0601,883$ & & & 85,873 & \\
\hline & & & emor-0wared Ut & in ERCOT & & \\
\hline 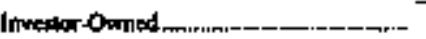 & $20,286,106$ & 21035,992 & - & - & $-5,564$ & $-5,568$ \\
\hline 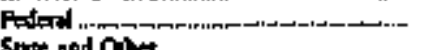 & - & - & - & - & - & - \\
\hline 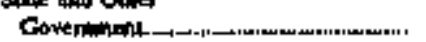 & I 16,146 & $1.838,013$ & - & - & 一 & - \\
\hline 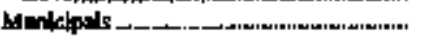 & $8,833,5.83$ & $10,236,932$ & - & - & - & 一 \\
\hline 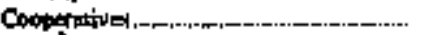 & 64,416 & (63,267 & - & - & 36,359 & 36,25 \\
\hline 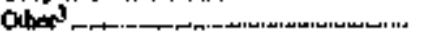 & $3,877,016$ & - & - & $-15,6$ ifi, $57 ?$ & 一 & -15, , 值, $7 T$ \\
\hline 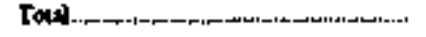 & $33,177,297$ & $30.176,144$ & - & -15 , & $3 J, 191$ & -15.531586 \\
\hline & & & thandent U & In MAdC & & \\
\hline 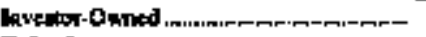 & + & $\rightarrow$ & - & - & - & - \\
\hline 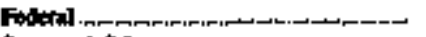 & $\rightarrow$ & - & - & - & - & - \\
\hline Sule wnd baher & & & & & & \\
\hline Dovanמmerh & $\rightarrow$ & - & - & - & - & - \\
\hline 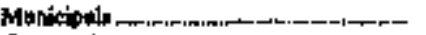 & $\rightarrow$ & - & - & - & - & - \\
\hline Conpedilingr. & $\rightarrow$ & - & - & - & - & $\leftarrow$ \\
\hline Olhth & - & - & - & - & - & - \\
\hline 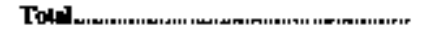 & - & - & - & $\rightarrow$ & $\rightarrow$ & $\rightarrow$ \\
\hline & & & Hexporowned $t$ & la Mats & & \\
\hline 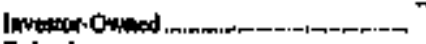 & 191.158 & 8,629 & - & $3,769, \mathrm{BS7}$ & 12,236 & $3,782,093$ \\
\hline 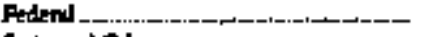 & - & 一 & 一 & - & - & - \\
\hline \$lath and Othat & & & & & & \\
\hline 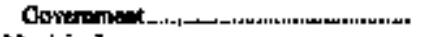 & - & 一 & - & 一 & 一 & \\
\hline 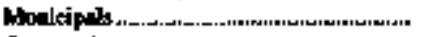 & 8,38 & 17,036 & - & $-120,00 \mid$ & 24,748 & -95253 \\
\hline 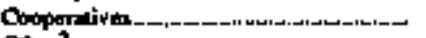 & & & - & & - & \\
\hline 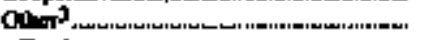 & 278 & כ202 & - & 4,958 & 一 & 4,988 \\
\hline Teth & J99,984 & 26,580 & - & $3,654,844$ & $3,5,944$ & 3,491828 \\
\hline & & & nettor-Omised D & h Martr & & \\
\hline Gestordund & 31,30 & 27,954 & $=$ & - & - & - \\
\hline 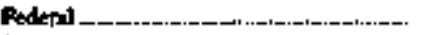 & 11,000 & 21,387 & - & - & - & - \\
\hline Sinc and Other & & & & & & \\
\hline 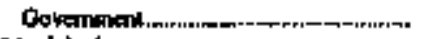 & 7292 & 一 & - & - & - & - \\
\hline 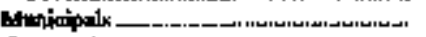 & - & - & - & - & - & - \\
\hline 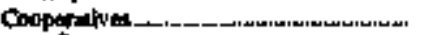 & - & - & - & - & - & ع \\
\hline 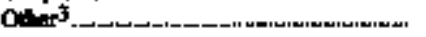 & - & 102,532 & - & 一 & 一 & 一 \\
\hline 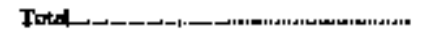 & 51,624 & $151, \mathrm{BT3}$ & - & - & - & - \\
\hline
\end{tabular}

Set notas and footonles as end of toble. 
Table 10. Investor-Owned Utilty Exchanges of Electricity with All Ownerstip Cbassea, by NERC Region, 1994 (Continued)

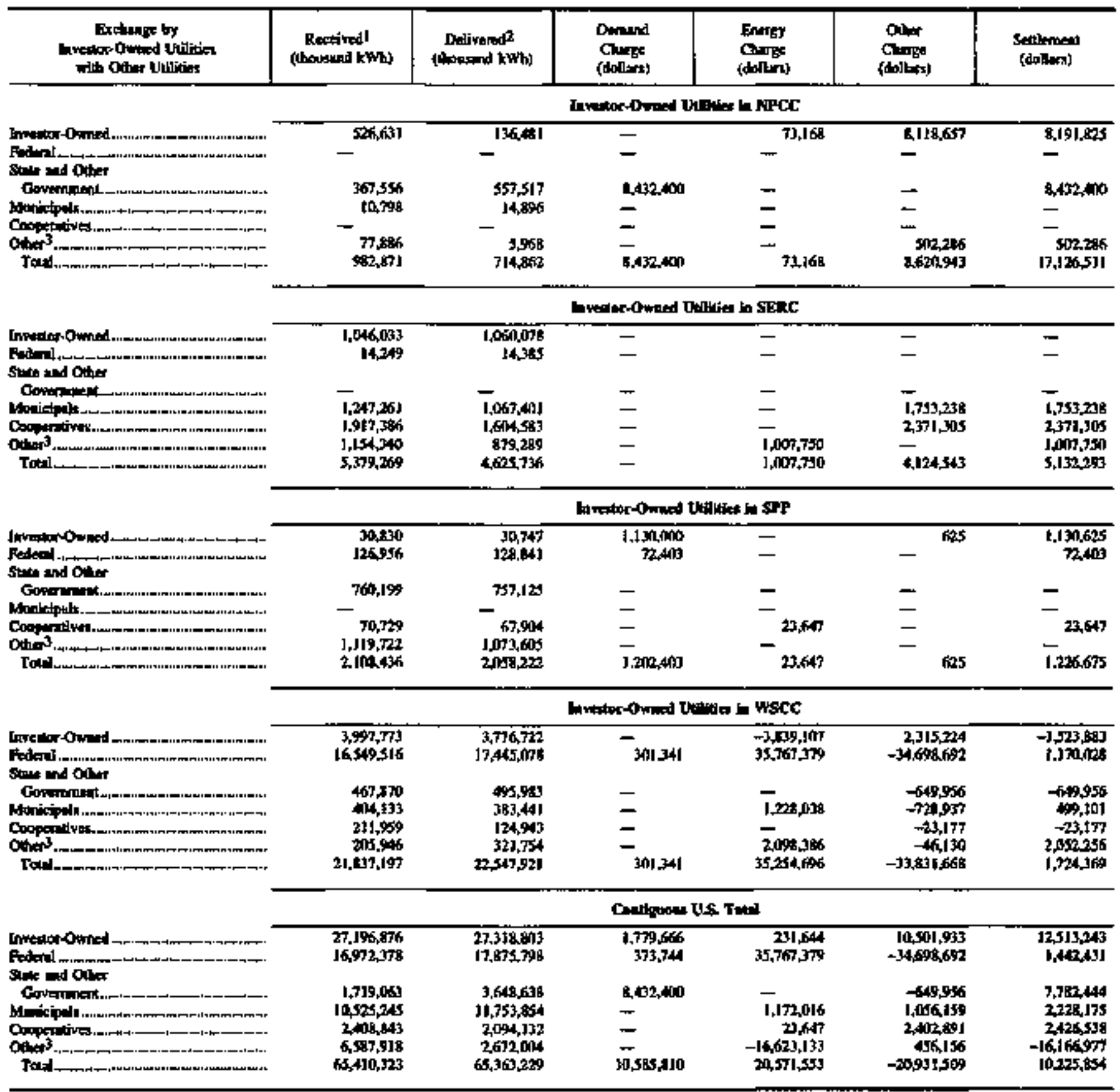

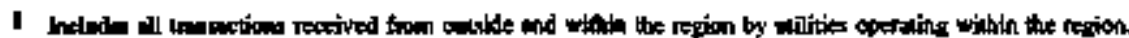

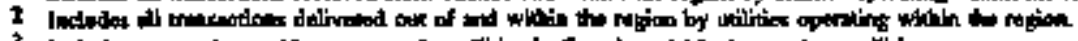

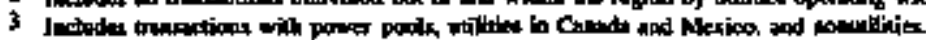

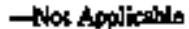

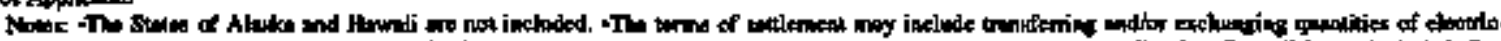

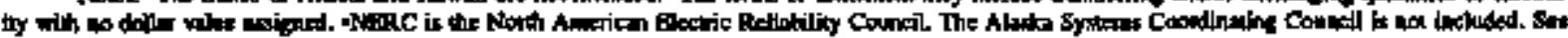

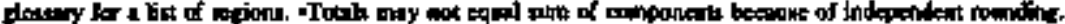

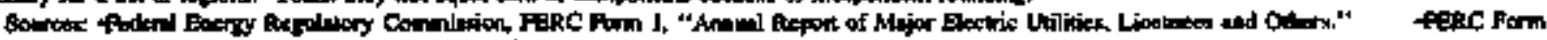

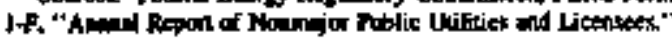


Table 11. Noninvestor-Owned Utilty Ercharges of Electricity, by NERC Region, 1994 (Milfion Kilowathours)

\begin{tabular}{|c|c|c|c|c|c|c|c|c|c|c|}
\hline \multirow{2}{*}{ 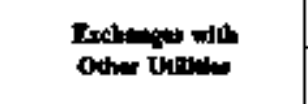 } & \multicolumn{9}{|c|}{ NDRC Rugion of Narimestion.Owned Utilly } & \multirow{2}{*}{$\begin{array}{l}\text { Comipactis } \\
\text { US. }\end{array}$} \\
\hline & ECAR & ERCYT & MARC & MaIN & MLAPP & NTCC & SERC & sPP & WSCE & \\
\hline \multicolumn{11}{|l|}{ fenderal } \\
\hline Recintd 1 & - & سمد & - & - & - & - & 25,937 & 535 & 14: & $41,0,2$ \\
\hline 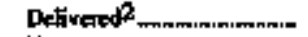 & - & - & - & - & - & 一 & 26,706 & 502 & 21,772 & 48979 \\
\hline 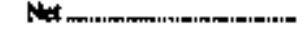 & 一 & - & 一 & - & 一 & 一 & -749 & 33 & $-7,1+1$ & -7, 察的 \\
\hline \multicolumn{11}{|l|}{ 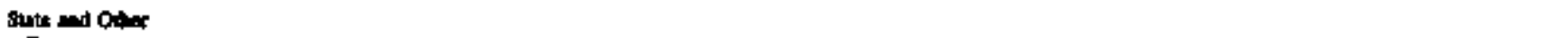 } \\
\hline Acochedil & - & - & - & - & I & 1,094 & 16 & 135 & 3.692 & 4,918 \\
\hline 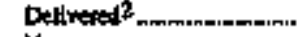 & 10 & ع & - & - & 19 & 放1 & 43 & 157 & $1,7 \pi$ & 20206 \\
\hline 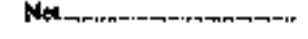 & $-1 \sqrt{1}$ & - & 一 & 一 & -19 & $\mathbf{8 1 4}$ & $-\mathbf{t}$ & -22 & $1.1 \%$ & 2,632 \\
\hline \multicolumn{11}{|l|}{ Mrrielpoly. } \\
\hline Rondved I & 13 & $1,4,0$ & 181 & - & In & 1,539 & 891 & $\mathbf{1 , 3 0 0}$ & 6,017 & 11,515 \\
\hline Delivand & 119 & 1,174 & 2 & - & 15 & 4in & 315 & 1,113 & 2,15 & 11,617 \\
\hline Nef & -16 & J6 & 179 & - & -4 & 1,136 & 357 & 201 & $-2,0 \mathrm{IL}$ & $-\operatorname{In}$ \\
\hline \multicolumn{11}{|l|}{ Cocpertipan } \\
\hline 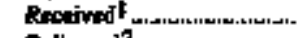 & 256 & 20,155 & - & 触 & 5,966 & - & 10 & 39t & 1,549 & $24,9 \%$ \\
\hline 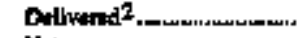 & 321 & 20,310 & - & 258 & 2,137 & - & 42 & 339 & 1537 & 25,342 \\
\hline 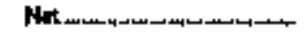 & -25 & -205 & - & 4 & -171 & - & -11 & $\$ 2$ & 12 & $-\mathrm{J53}$ \\
\hline \multicolumn{11}{|l|}{ Retion Tot } \\
\hline 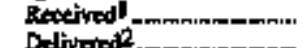 & 308 & 23,945 & 181 & 30 . & 1,576 & 2609 & 26,924 & 2,379 & 25,868 & $8 x \operatorname{seg}$ \\
\hline 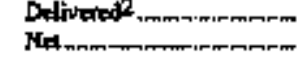 & $\begin{array}{r}409 \\
-241\end{array}$ & $\begin{array}{r}22,184 \\
-239\end{array}$ & 139 & $\frac{258}{44}$ & $\begin{array}{l}2,171 \\
-194\end{array}$ & $\begin{array}{r}640 \\
1,949\end{array}$ & $\begin{array}{r}27.126 \\
-212\end{array}$ & $\begin{array}{r}2,112 \\
267\end{array}$ & $\begin{array}{l}33,242 \\
-7,374\end{array}$ & $\begin{array}{l}88,225 \\
-4,721\end{array}$ \\
\hline
\end{tabular}

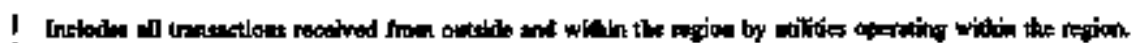

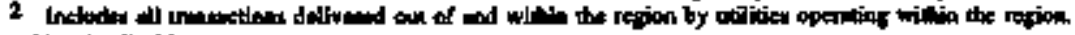

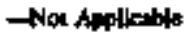

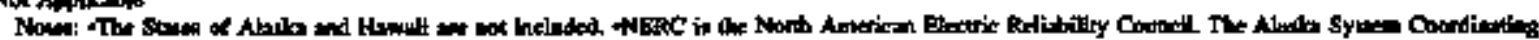

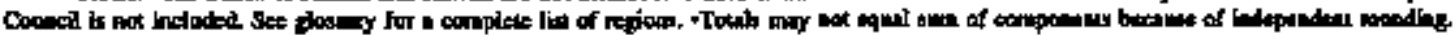

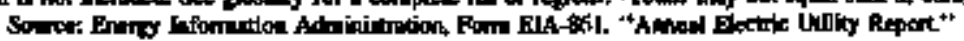


Table 12. Clectricity Wheding for Al Ownership Clases by Investor-Owned Utaities, by NERC Region, 1994

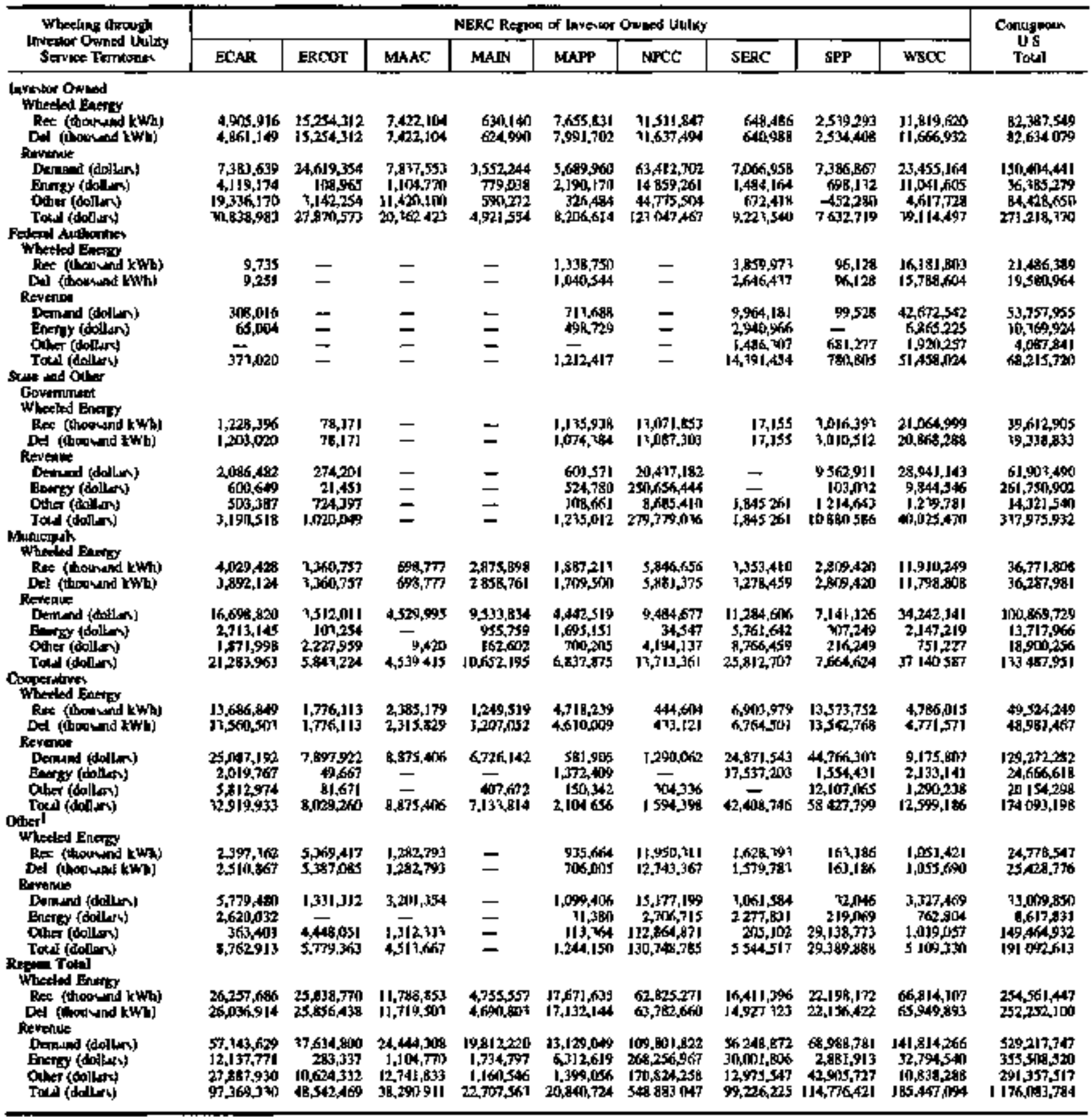

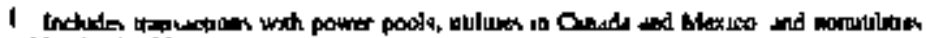

$\rightarrow$ Mou Applieste

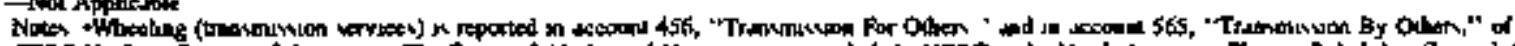

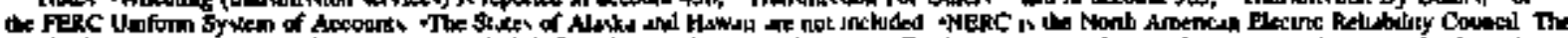

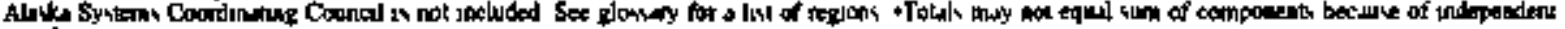
touding

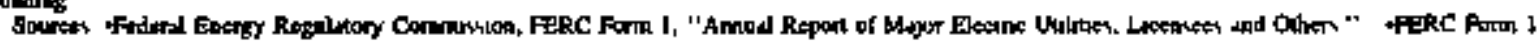

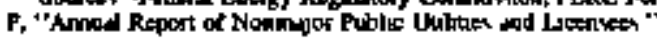


Table 13. Electricity Wheeling by All Ownership Classes to Inwestor-Owned Utilitis, by NERC Region, 1994

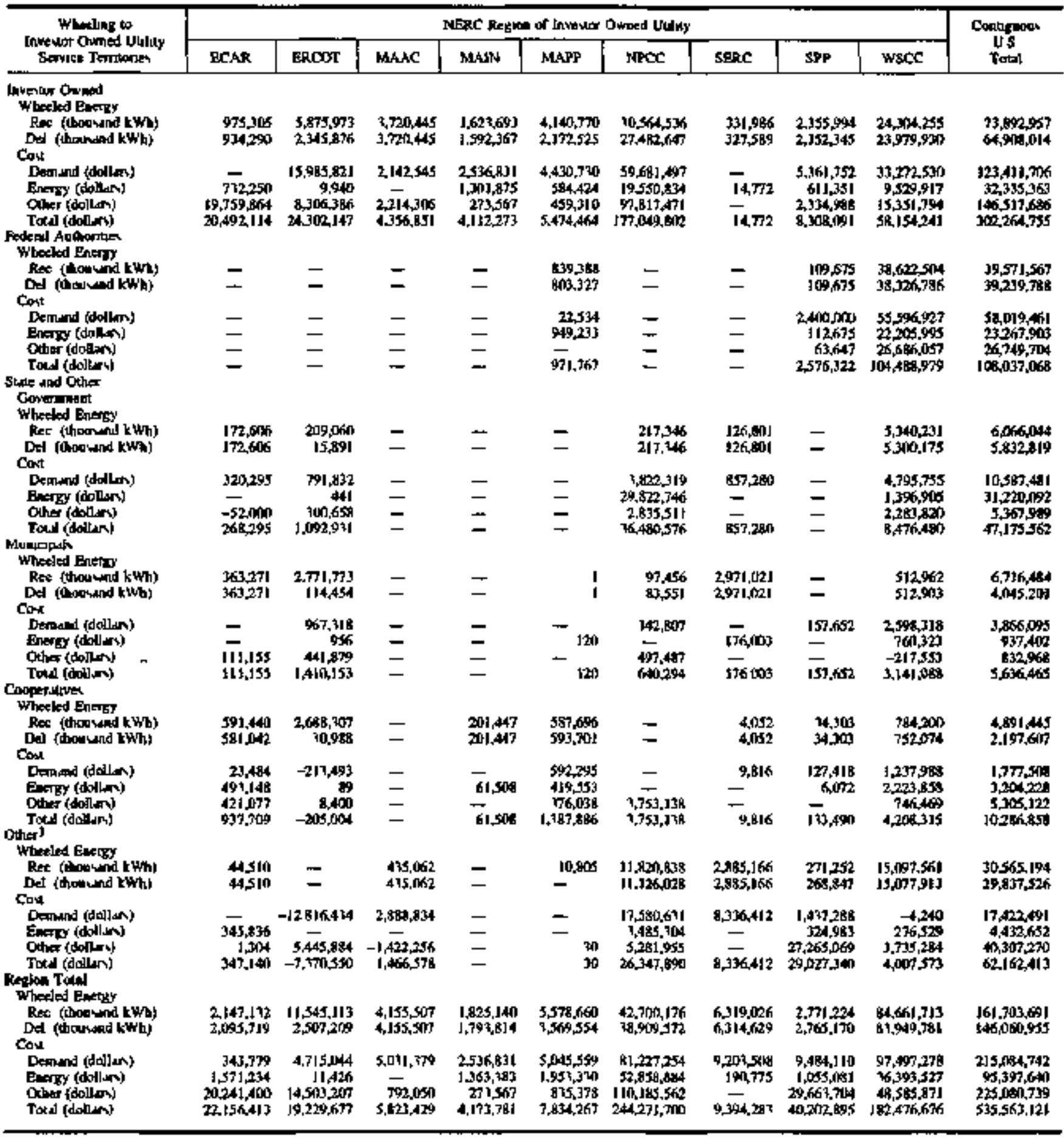

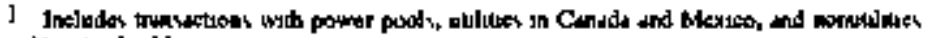

Thon Appicabde

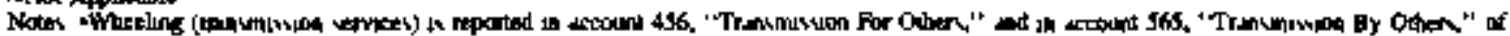

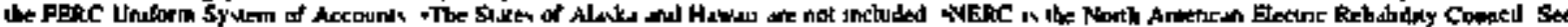

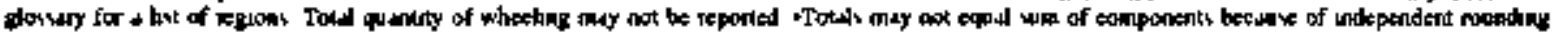

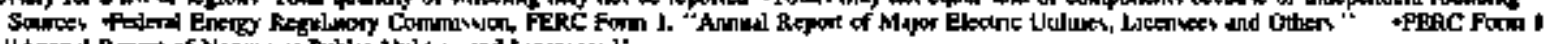

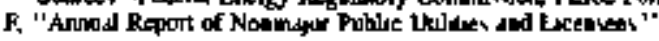


Table 14. Purchases by Cooperative Borrowers, by NERC Reqlon, 1994

\begin{tabular}{|c|c|c|c|c|c|c|}
\hline \multirow{2}{*}{ sedler by } & & \multicolumn{5}{|c|}{ NERC Regon of Cosperditer } \\
\hline & & ECAR & ERACOT & MAAC & MLAIN & MAPP \\
\hline \multicolumn{2}{|l|}{ 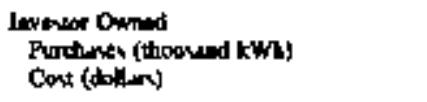 } & $\begin{array}{c}2,761,376 \\
99,533,407\end{array}$ & $\begin{array}{r}3,906,640 \\
153,492,190\end{array}$ & $\begin{array}{r}3,295,869 \\
19,629,443\end{array}$ & $\begin{array}{r}2,127,368 \\
94,494,990\end{array}$ & $\begin{array}{r}4,212, \mathrm{MK} \\
\mathrm{J}, 1,269,973\end{array}$ \\
\hline \multicolumn{2}{|l|}{ 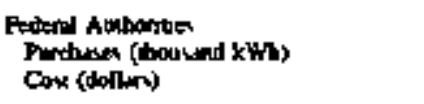 } & 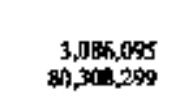 & $\begin{array}{r}316,353 \\
5, a 40,904\end{array}$ & $\overline{-}$ & $\begin{array}{l}40,940 \\
85,0 B\end{array}$ & $\begin{array}{r}1,79,191 \\
40,706,031\end{array}$ \\
\hline 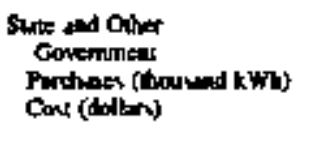 & - & $\begin{array}{r}136,525 \\
4,276,489\end{array}$ & $\begin{array}{r}79,616 \\
1,936,777\end{array}$ & $\begin{array}{r}267,359 \\
1,138,763\end{array}$ & $\bar{z}$ & $\begin{array}{r}2,862,468 \\
8 ., 156,779\end{array}$ \\
\hline 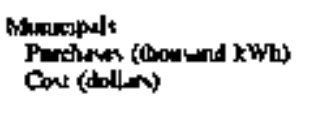 & $\dot{-}$ & $\begin{array}{l}1,361.267 \\
n, 98 \%, 97 t\end{array}$ & $\begin{array}{r}171,2 \pm 8 \\
2660,45\end{array}$ & $\underline{-}$ & $\overline{-}$ & $\begin{array}{r}31,900 \\
52 x, 611\end{array}$ \\
\hline \multicolumn{2}{|l|}{ 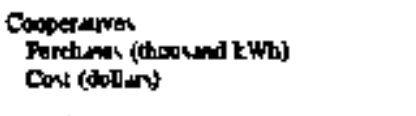 } & $\begin{array}{r}29,253,357 \\
908,47,912\end{array}$ & $\begin{array}{r}9,798,137 \\
40.351,1875\end{array}$ & $\begin{array}{r}3,676,336 \\
207,200,847\end{array}$ & $\begin{array}{r}8,065,528 \\
376,720287\end{array}$ & $\begin{array}{r}21,688,308 \\
819,7158,813\end{array}$ \\
\hline 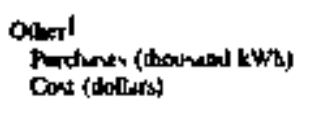 & - & $\begin{array}{r}1,219,055 \\
65,3130.224\end{array}$ & $\begin{array}{r}760,377 \\
39,137,189\end{array}$ & $\begin{array}{r}8,4122 \\
1,973,98617\end{array}$ & $\begin{array}{r}62,160 \\
7,450,427\end{array}$ & $\begin{array}{r}f, 505,395 \\
16,305,312\end{array}$ \\
\hline \multirow[t]{3}{*}{ 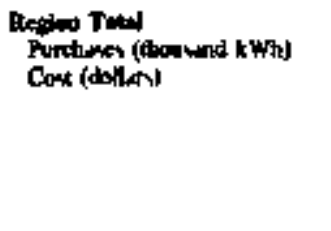 } & & $\begin{array}{r}33,536,6515 \\
1,2 N 3,924,302\end{array}$ & $\begin{array}{r}15,734,249 \\
6,3,436,976\end{array}$ & $\begin{array}{r}7,247,466 \\
360,742.939\end{array}$ & $\begin{array}{r}10,315,991 \\
41,47,292\end{array}$ & \multirow{3}{*}{$\begin{array}{c}\text { Conaguons } \\
\text { us } \\
\text { 1othl }\end{array}$} \\
\hline & & \multicolumn{4}{|c|}{ Nenc Rogero of Cooperpintes } & \\
\hline & & NFC & SERC & $\mathbf{s W P}$ & wsoc & \\
\hline \multicolumn{2}{|l|}{ 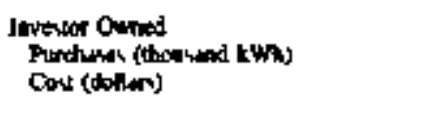 } & $\begin{array}{r}79 \%, 471 \\
57,00 \%, 006\end{array}$ & $\begin{array}{r}17,146,960 \\
893,912,2344\end{array}$ & $\begin{array}{r}8,133,291 \\
259,457,305\end{array}$ & $\begin{array}{r}2,434,931 \\
99,931,369\end{array}$ & 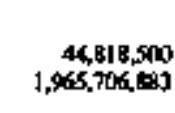 \\
\hline \multicolumn{2}{|l|}{ 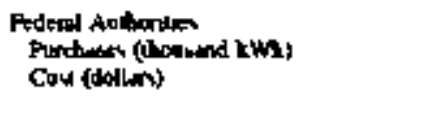 } & $\overline{-}$ & 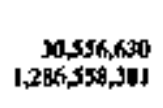 & $\begin{array}{r}3,526,78 \\
60,006,6919\end{array}$ & $\begin{array}{r}11,065,424 \\
2(1,4,5), 726\end{array}$ & $\begin{array}{r}53,390,191 \\
1,745,657,720\end{array}$ \\
\hline \multicolumn{2}{|l|}{ 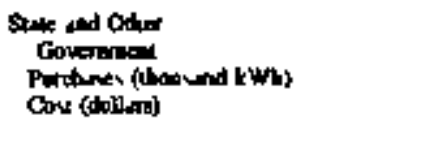 } & $\begin{array}{r}194,577 \\
7,070,859\end{array}$ & $\begin{array}{r}7,076,597 \\
273,050 \leq 588\end{array}$ & $\begin{array}{r}4.114,9017 \\
98.614,273\end{array}$ & $\begin{array}{r}529.799 \\
99243.009\end{array}$ & $\begin{array}{r}16,201,788 \\
512,247,069\end{array}$ \\
\hline \multicolumn{2}{|l|}{ 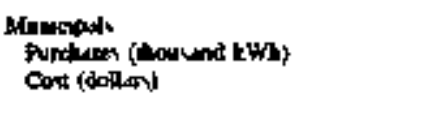 } & $\begin{array}{r}2,150 \\
991,329\end{array}$ & $\begin{array}{r}189,485 \\
4,821,010\end{array}$ & $\begin{array}{r}1,707, A 21 \\
37,04,371\end{array}$ & $\begin{array}{r}15,205 \\
317,170\end{array}$ & $\begin{array}{r}3,47,256 \\
80,730,64 \pi\end{array}$ \\
\hline \multicolumn{2}{|l|}{ 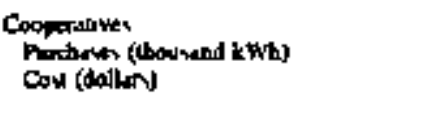 } & חוננ11, & $\begin{array}{r}59,779,799 \\
2913,269,459\end{array}$ & $\begin{array}{r}41,5196,752 \\
1,693,396,451\end{array}$ & $\begin{array}{r}14,996,406 \\
646,600,658\end{array}$ & $\begin{array}{r}184,5] 2,748 \\
8,161,704,246\end{array}$ \\
\hline \multicolumn{2}{|l|}{ 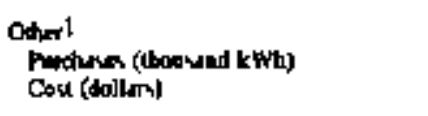 } & $\begin{array}{r}1,02,996 \\
5,461,411\end{array}$ & $\begin{array}{r}251,800 \\
9,062,420\end{array}$ & $\begin{array}{r}52780 \\
4,206,749\end{array}$ & 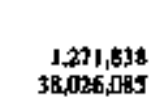 & $\begin{array}{r}4, \pm 1], 364 \\
182,292,307\end{array}$ \\
\hline 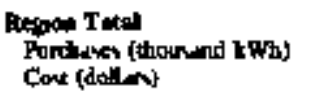 & & $\begin{array}{r}1,210,304 \\
78925,540\end{array}$ & $\begin{array}{l}|14,910,13| \\
3,386,874,194\end{array}$ & $\begin{array}{r}59,321,759 \\
2,151,74,135\end{array}$ & $\begin{array}{r}30,304,647 \\
1,061,180,017\end{array}$ & $\begin{array}{r}306,210,236 \\
12.548,519,534\end{array}$ \\
\hline
\end{tabular}

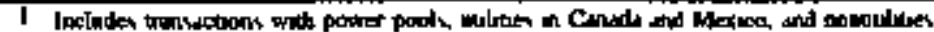

$\rightarrow$ Net Appestolin.

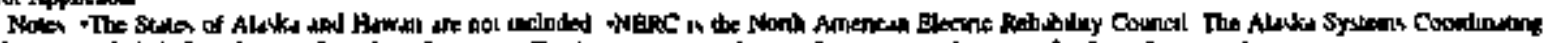

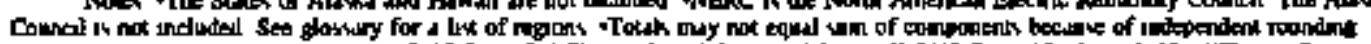

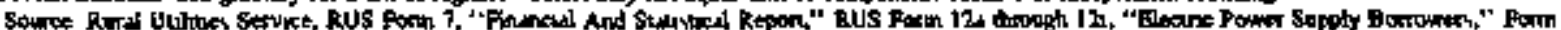

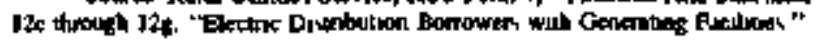


Table 15. Recejpls by Investor-Owned Utilities Within and Between NERC Regions, 1994

\begin{tabular}{|c|c|c|c|c|}
\hline \multirow{3}{*}{ 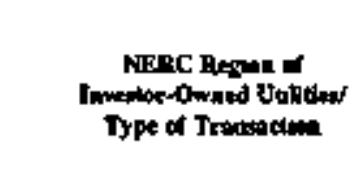 } & \multicolumn{4}{|c|}{ 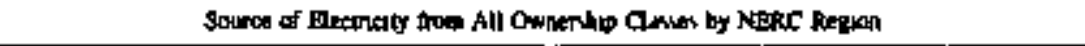 } \\
\hline & \multicolumn{2}{|c|}{ ECAR } & \multicolumn{2}{|c|}{ ERCOT } \\
\hline & (Whound kWh) & Cont & 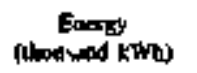 & Coder \\
\hline \multicolumn{5}{|l|}{ ECAR } \\
\hline 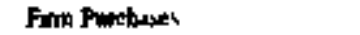 & $49.145,951$ & $1,817,019,175$ & - & - \\
\hline Nooflim Pwecheyteh & $28,434,668$ & $720,185,060$ & - & - \\
\hline 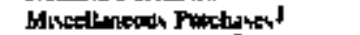 & $9,5 \$ 7,401$ & $2601,362,445$ & - & - \\
\hline Exchugen Rexerret? & $7,031,213$ & $-2,496,402$ & - & - \\
\hline Wheeling By Othen ${ }^{3}$ & $2,140,596$ & $20,294,841$ & - & - \\
\hline \multicolumn{5}{|l|}{ ERCOT } \\
\hline Pintin Purchises & - & - & $14,528,795$ & 713 ,A90,75s \\
\hline Moning Pordersen & - & - & $9,210,450$ & $180,6+2,448$ \\
\hline 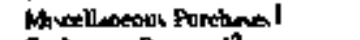 & - & - & 286,343 & 500,567 \\
\hline Exeldingas Reverved 2 & - & - & $33,001,33 t$ & $-15,631,586$ \\
\hline Whonling By Othen' & - & - & $11,545, J 13$ & $29,524,64 \mathrm{~J}$ \\
\hline \multicolumn{5}{|l|}{ MANC } \\
\hline Fin Purcherses & 250,034 & $6,252,911$ & - & - \\
\hline Monfinm PVIritung & $6,501,784$ & $250,571,572$ & - & - \\
\hline Wherlappom funturel & - & 51,453 & - & - \\
\hline Exchanger Retred? & - & - & - & - \\
\hline Wheabr By Oahen? & - & - & - & $\rightarrow$ \\
\hline \multicolumn{5}{|l|}{ MAN } \\
\hline Fotm Purathen & $2,092, \sqrt{166}$ & $52,508,597$ & - & - \\
\hline Nonfinen Ponting & $1,578,245$ & $28,831,606$ & - & - \\
\hline 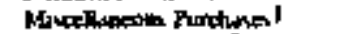 & - & - & - & - \\
\hline Exchandersed? & 450 & 10,302 & - & - \\
\hline Whechap By Oaver ${ }^{3}$ & - & - & - & - \\
\hline \multicolumn{5}{|l|}{ MAYP } \\
\hline Fina PWhather & - & - & - & . \\
\hline Nominime Poratumery & 12,460 & Mx.991 & - & - \\
\hline 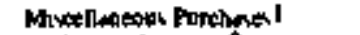 & - & - & - & - \\
\hline 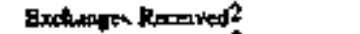 & - & - & - & - \\
\hline Wheting By Oben ${ }^{3}$ & - & - & - & - \\
\hline \multicolumn{5}{|l|}{ NAPCC } \\
\hline Fim Prechones & - & - & - & - \\
\hline Nowing Porthiven & - & - & $\mathbf{I}$ & BS \\
\hline 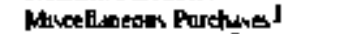 & 18 & - & - & - \\
\hline 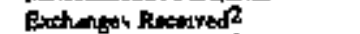 & - & - & - & - \\
\hline \multirow{2}{*}{\multicolumn{5}{|c|}{ SERC }} \\
\hline & & & & \\
\hline funt Purcherses & $2,004,849$ & $258,78,5,852$ & - & - \\
\hline Monfinm Purctures & 65,742 & 2,1562019 & - & - \\
\hline Mhootlmeous Pardhat I & & - & - & - \\
\hline Exchungen Receaved? & $245 n$ & - & - & - \\
\hline Wherting By Oten ${ }^{3}$ & - & - & - & - \\
\hline \multicolumn{5}{|l|}{ SPP } \\
\hline Fifm Pontiner & - & - & - & - \\
\hline Monfien Portumey & - & - & - & - \\
\hline 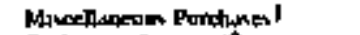 & - & - & 2010 & - \\
\hline Brechngat Recetredi2 & 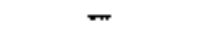 & - & - & - \\
\hline Arwing By O0kn ${ }^{3}$ & - & - & 139,643 & $1,789,591$ \\
\hline \multicolumn{5}{|l|}{ wsce } \\
\hline Finm Ponthases & $-\pi$ & - & $1,13], 985$ & 65,817990 \\
\hline Sonffin Pardinger & - & - & - & - \\
\hline Haweellwareons Porchneal & - & - & - & - \\
\hline 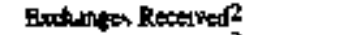 & ــ & - & - & - \\
\hline Wbetimg By O4hes J & - & - & - & - \\
\hline \multicolumn{5}{|l|}{ Betion T Toll } \\
\hline 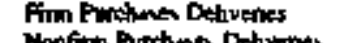 & 58.550920 & $2,134,186,535$ & 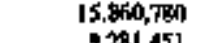 & 783.568 .735 \\
\hline Nonsilitin Purchenen Dehromos & $36,593,115$ & $1, \sqrt{4}+1,051,568$ & $9,281,45]$ & 186, 642,513 \\
\hline 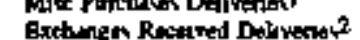 & $\begin{array}{l}9,587,419 \\
1056,270\end{array}$ & 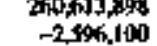 & $\begin{array}{r}208,353 \\
15,000,138\end{array}$ & $\begin{array}{r}300,367 \\
-15,631,396\end{array}$ \\
\hline 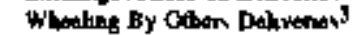 & 2140596 & 20298,341 & $11,564,756$ & $2 t .314 .224$ \\
\hline
\end{tabular}

See noter 4nd fouthowe nt and of who 
Table 15. Receipts by Investor-Owned Utilities Within and Between NERC Regions, 1994 (Continued)

\begin{tabular}{|c|c|c|c|c|c|}
\hline \multirow{3}{*}{ 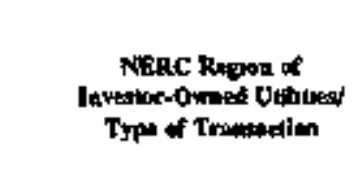 } & & \multicolumn{4}{|c|}{ 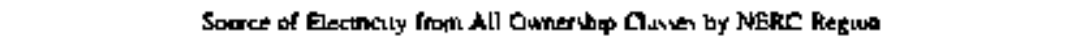 } \\
\hline & & \multicolumn{2}{|c|}{ MAAC } & \multicolumn{2}{|c|}{ MAIN } \\
\hline & & 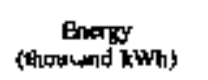 & $\begin{array}{c}\text { Cou } \\
\text { (dollons) }\end{array}$ & $\begin{array}{c}\text { Energy } \\
\text { (Itwedel twh) }\end{array}$ & $\begin{array}{c}\text { Cou } \\
{[\mathrm{d} / \mathrm{ln} \text { ) }}\end{array}$ \\
\hline \multicolumn{6}{|l|}{ ECAR } \\
\hline Fint Porthuxer & & 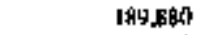 & $5,655,6004$ & $1,726,385$ & $4 \leqslant, 630,3 \leqslant 2$ \\
\hline Nonfiron Punturen & & $x+, 49$ & $1,008,6646$ & I $(\pi+2,28 \mathrm{~L}$ & $17,897,815$ \\
\hline Musetilacood Purch pal & & - & - & - & $-2,400$ \\
\hline Exchanges Recesved? & . & - & - & - & $-5,4,52$ \\
\hline \multirow{2}{*}{\multicolumn{6}{|c|}{ ARCOT }} \\
\hline & & & & & \\
\hline Fen Puntuses & & - & - & - & - \\
\hline Nomfingh Putrouk & & - & - & - & $\rightarrow$ \\
\hline Bturethemenh Rurchent & & - & - & - & - \\
\hline Exchungsos Rectived & & - & - & - & - \\
\hline Wholung By Cabon ${ }^{3}$ & & - & - & - & - \\
\hline \multicolumn{6}{|l|}{ MAAC } \\
\hline Fith Punchust & & IB,A34,6I7 & $816,629,1107$ & - & - \\
\hline Nomfon Puctiona & & 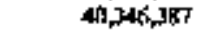 & $1.564,745,812$ & $=$ & $=$ \\
\hline Mnotheroon Pordunel & & 3,1 16, & $81,925,511$ & - & - \\
\hline Exchunger Reopred? & & - & $=$ & - & - \\
\hline Wheclung By Dlbern ${ }^{3}$ & & $\$, 155,507$ & $5,(52), 429$ & - & - \\
\hline \multicolumn{6}{|l|}{ DIARN } \\
\hline Ferm Pprtuser & & - & - & $12,9606.709$ & $37,720,59$ \\
\hline 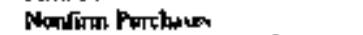 & & - & - & $8,729,402$ & $139.824,724$ \\
\hline 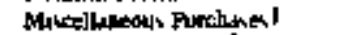 & & - & - & -59 & 31,428 \\
\hline 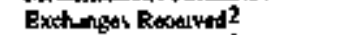 & & - & - & JRA / $\mathbf{R}$ & 746,45 \\
\hline Whoting by Other, 3 & & - & - & fil8,9t0 & $1,257,670$ \\
\hline \multicolumn{6}{|l|}{ MAPP } \\
\hline Fum Punchesus: & & - & - & fi45 17s & $29,859, \mathrm{k2} 7$ \\
\hline Monfirm Ponctures & & - & - & $1.216 \times 41$ & $27,453,9016$ \\
\hline 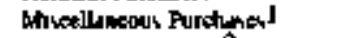 & & - & - & - & - \\
\hline Exchingev, Receyved? & & - & - & - & - \\
\hline \multirow{2}{*}{\multicolumn{6}{|c|}{ NACC }} \\
\hline & & & & & \\
\hline Frem Purchares & & $3,578,4 \mathrm{RB}$ & 266.927 .347 & $=$ & - \\
\hline Nonfin Purdaks & & 3.129 .273 & $88,999,419$ & - & - \\
\hline 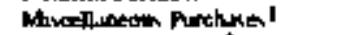 & & - & - & - & - \\
\hline 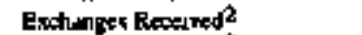 & & - & - & - & - \\
\hline Whethig Gy Ohen ${ }^{3}$ & & 47.nSS & 116,289 & - & - \\
\hline \multicolumn{6}{|l|}{ sercs } \\
\hline Foth Puxtheser & & 6.1655 & 269,142 & $\$ 32,517$ & $12,13,9,920$ \\
\hline 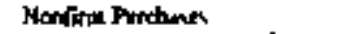 & & - & - & 4,890 & 87.217 \\
\hline 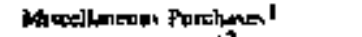 & & - & - & - & - \\
\hline Exchunts Recelved? & & - & - & - & $\rightarrow$ \\
\hline Wheldof By Oben 3 & & - & - & - & $\rightarrow$ \\
\hline \multicolumn{6}{|l|}{$\$ p$} \\
\hline Firm Purchner & & - & - & 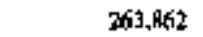 & 11, ,646, (19t5 \\
\hline Nonfan Purture & & 18.575 & 317,916 & 2180,728 & $38,522,352$ \\
\hline 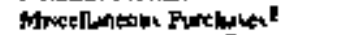 & & - & - & - & - \\
\hline 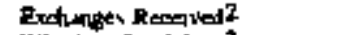 & & - & - & - & - \\
\hline \multirow{2}{*}{\multicolumn{6}{|c|}{ wsec }} \\
\hline & & & & & \\
\hline Firn Purthines & & 2,1455 & 197.717 & - & - \\
\hline Monfm Aurchores & & - & $=$ & - & - \\
\hline Brucellineoss Purthes I & & - & - & - & - \\
\hline Bxhanger Rexelved? & & - & - & - & - \\
\hline Wheeting By Othen? 3 & & 22,0093 & $508, \sqrt{6} 61$ & - & - \\
\hline \multicolumn{6}{|l|}{ Reprom Totat } \\
\hline 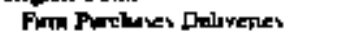 & & $22,631,135$ & $1,089,737,8097$ & $15,934,644$ & $428,929,593$ \\
\hline Noption Punclumen Delivenes & & $49,568,734$ & 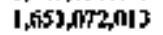 & $13,130,242$ & $219,102,014$ \\
\hline 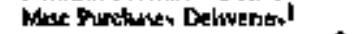 & & $3,33,6,65 \pi$ & RE, $, 25,514$ & -59 & 29,028 \\
\hline Exchanges Rocolyed Deluvencl? & & - & - & 184,134 & $3,431 \leq 13$ \\
\hline Whetung By Other Deliveres? & & 4224,645 & $8,281,809$ & 605743 & $1,641,905$ \\
\hline
\end{tabular}

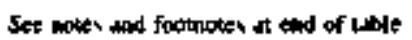


Table 15. Receipts by Invesior-Owned Uttlities Within and Between NERC Regions, 19\%4 (Continued)

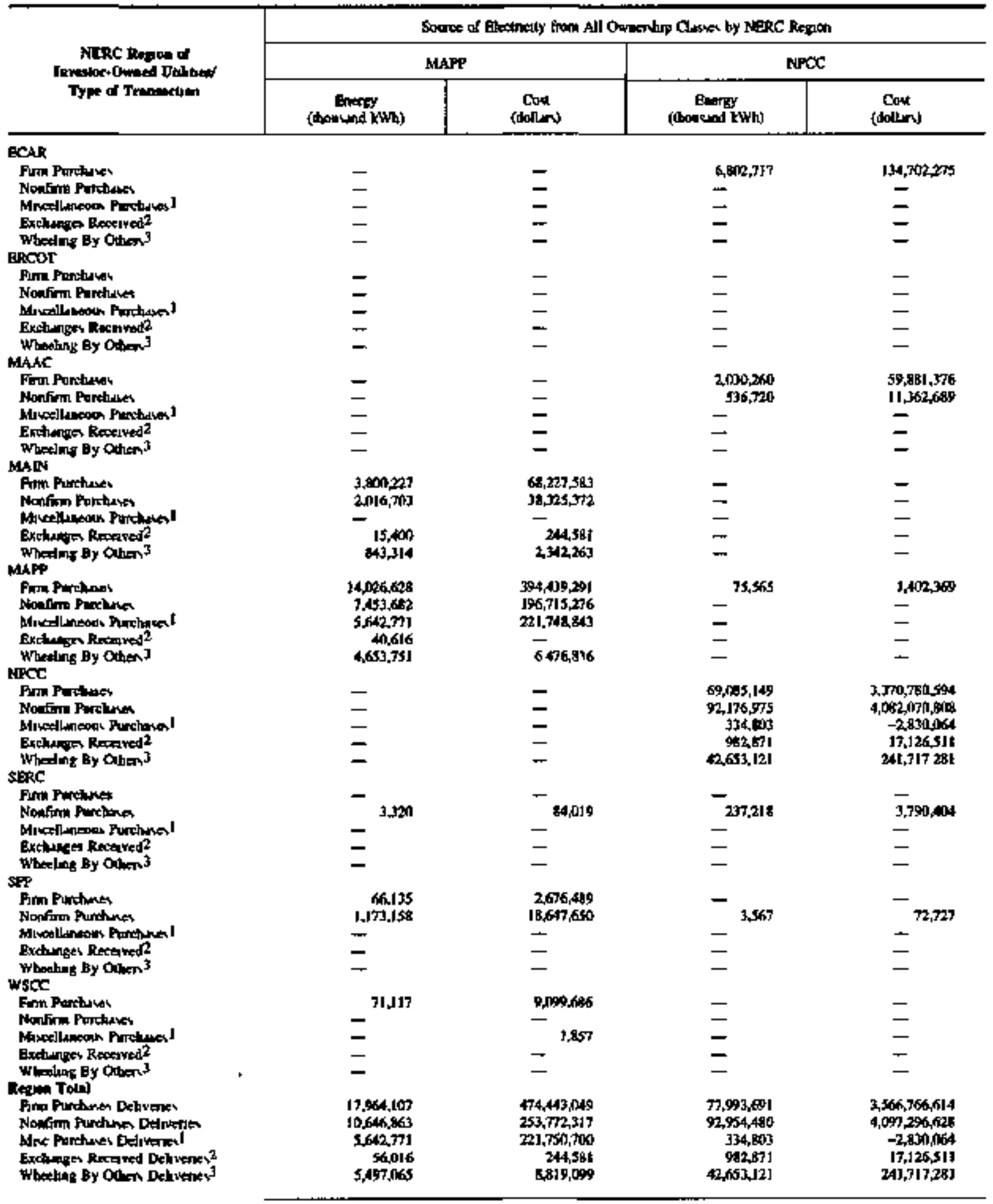

See noles and loolnowes at end of toble 
Table 15. Recelpts by Jnvestor-Owned Utilities within and Between NERC Reglons, 1994 (Continned)

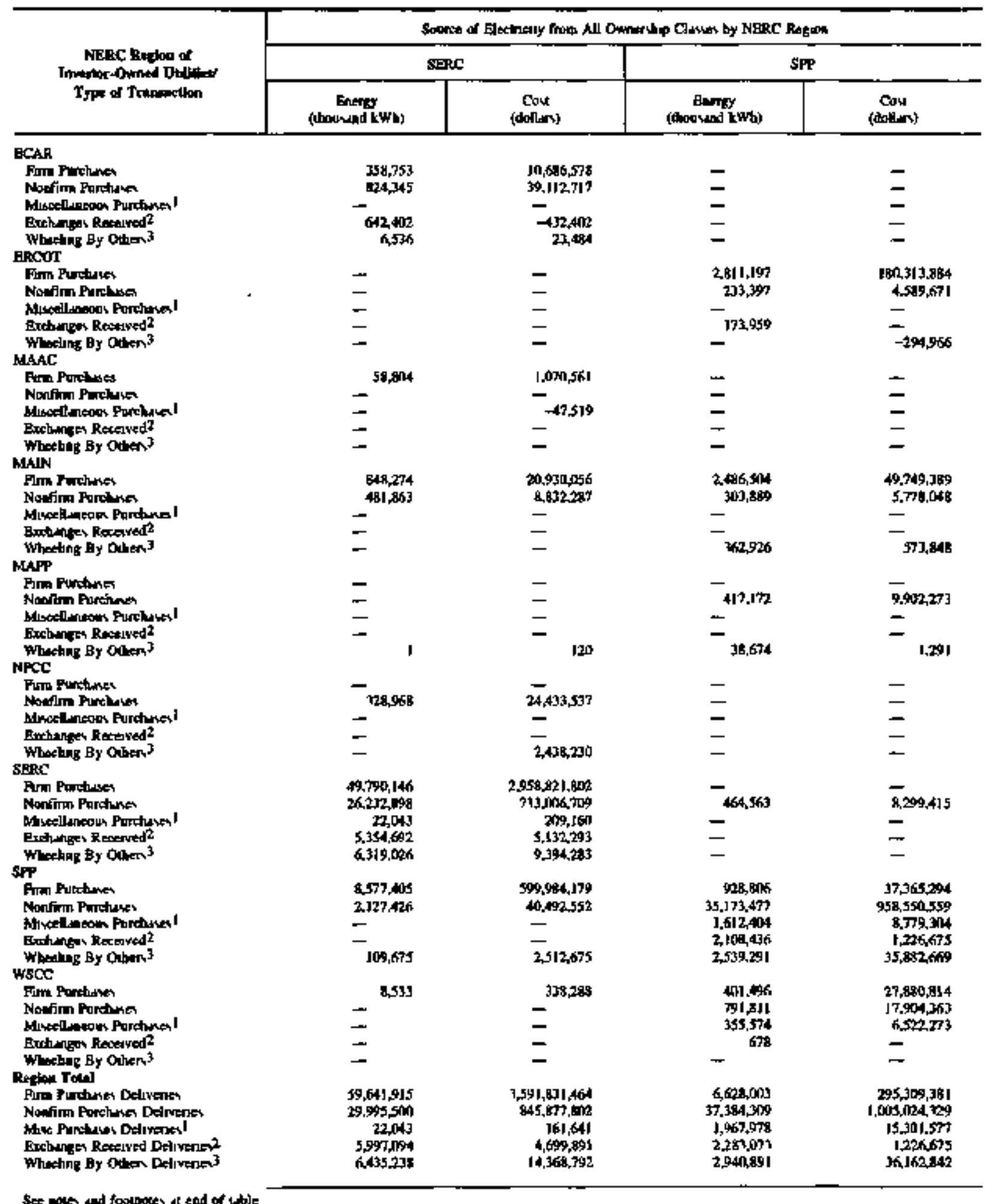


Table 15. Recejpts by Investor-Owned Utilitles Whithin and Between NERC Regions, 1994 (Continued)

\begin{tabular}{|c|c|c|c|c|}
\hline \multirow{3}{*}{ 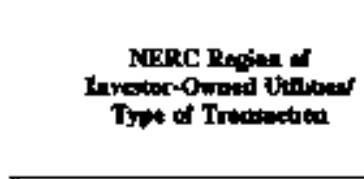 } & \multicolumn{4}{|c|}{ 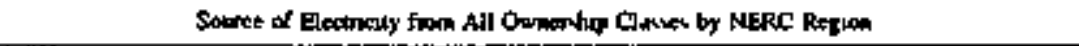 } \\
\hline & \multicolumn{2}{|c|}{ wsce } & \multicolumn{2}{|c|}{ Contugmans } \\
\hline & 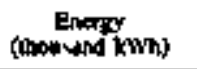 & Cort & 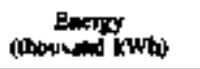 & $\underset{(\phi N n)}{C o 4}$ \\
\hline \multicolumn{5}{|l|}{$\operatorname{meds}$} \\
\hline imin 1 & - & - & $58,274,466$ & $2,011.347 .454$ \\
\hline 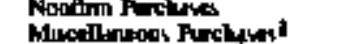 & - & - & $\begin{aligned} 30,377,994 \\
9,97,4 \text { at }\end{aligned}$ & T7d,140,458 \\
\hline Bxchumes Reoc|ued? & - & - & $1,673,645$ & $=7,044,236$ \\
\hline \multicolumn{5}{|l|}{ ERCOT } \\
\hline Finn Punchass & - & - & $17,3,99,998$ & $898,004,639$ \\
\hline Nonfin Pasher & 58,904 & $1,030,107$ & $9,973, B 41$ & $186,262,226$ \\
\hline 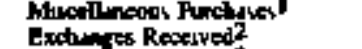 & - & - & 236,343 & 5812567 \\
\hline Whexht By Ohers? & $\overline{-}$ & $\overline{-}$ & $11,545,119$ & $\begin{aligned}-13,021,986 \\
18,229,6] 1\end{aligned}$ \\
\hline \multirow{5}{*}{ 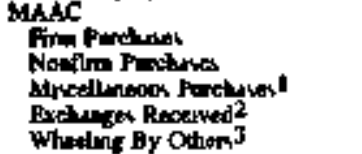 } & - & - & & \\
\hline & - & - & $20,001,715$ & $\log _{0 \rightarrow 2} 8043,955$ \\
\hline & - & - & $3,136,648$ & $81,929,47$ \\
\hline & - & - & - & - \\
\hline & - & 一 & $4,155,50 \mathrm{H}$ & $5,823,429$ \\
\hline \multicolumn{5}{|l|}{ BAAIN } \\
\hline 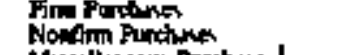 & $\begin{array}{r}323,012 \\
9,496\end{array}$ & $\begin{array}{l}111295.550 \\
257218\end{array}$ & 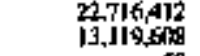 & $\begin{array}{l}5,3,431,684 \\
2,1,879,345\end{array}$ \\
\hline 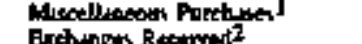 & - & $\overline{-}$ & -59 & 31, 020 \\
\hline Buchungth Retervatis & - & - & 199,984 & $3,691,1008$ \\
\hline \multicolumn{5}{|l|}{ MAPP } \\
\hline Pron Puchum & 625,565 & $|9, \mathrm{~B} 2|, \sin$ & $15,772,954$ & $445,522,994$ \\
\hline Momita Puthers & $\begin{array}{r}1,012,960 \\
-1,172\end{array}$ & $\begin{array}{l}17,484,734 \\
-145,1958\end{array}$ & $10.103,240$ & $246,721, \sqrt{16}$ \\
\hline Ekchinger Rocenred? & 11,008 & -1 - & 51,624 & 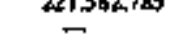 \\
\hline Wheling By Othess? & 839,N99 & 981,785 & $5,578,660$ & $7,834,257$ \\
\hline \multicolumn{5}{|l|}{ NPOC } \\
\hline Fith Porlinues & - & 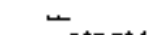 & 79063,697 & $3,637,7,1,3,41$ \\
\hline Manfirim Parduks & 3.110 & $238,8=6$ & $95,632,319$ & 4,I95, 742,655 \\
\hline 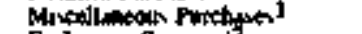 & $=$ & - & $\$ 34,1,121$ & $-2830,064$ \\
\hline Erithinger Recurred? & - & - & 982,871 & $17,126,511$ \\
\hline Wheling By Others' & - & - & $42,70,0,176$ & סט, 244,271, \\
\hline \multicolumn{5}{|l|}{ seinc } \\
\hline Fim Porchave & 5470173 & $20,016,547$ & $58,07, n 50$ & $3,25,4,435,357$ \\
\hline Monting Porches & 70418 & $1,2] 2,159$ & $27 \operatorname{son}, 149$ & 746536.172 \\
\hline 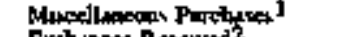 & 一 & - & n, & 209160 \\
\hline Exchingts & - & - & $5,179,269$ & $5,132,293$ \\
\hline Whesule By Ohan? & - & - & 6,319,0Bs & $9.194,283$ \\
\hline \multicolumn{5}{|l|}{ SPP } \\
\hline Prim Purlukes & - sose & & 9.936200 & $651,669,967$ \\
\hline 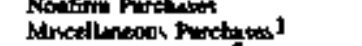 & $\begin{array}{l}3,945 \\
2,933\end{array}$ & $\begin{array}{l}161,790 \\
86,806\end{array}$ & $40,682,8 \%$ & 1,067.165,546 \\
\hline Excluabsor Recented2 & & - & $2,108,436$ & $1,226,675$ \\
\hline Whed on By that 3 & 2615 & $37.5 \mathrm{mp}$ & 271.224 & 412012805 \\
\hline \multicolumn{5}{|l|}{ wsct } \\
\hline Partives & $44,435,532$ & $5,776,960,318$ & $\operatorname{grn} 251,490$ & $5,830,294,893$ \\
\hline Mreflime004 Frobues] & $30,74,221$ & $\begin{array}{r}1,250 ; 036,234 \\
09,000,180\end{array}$ & 32,3301012 & J,20x,941,997 \\
\hline Exchungen Roserwed? & $29,896,599$ & 1,721369 & $2,8,837,197$ & $1,724,369$ \\
\hline Whedint By Ohlach] & $84,699,630$ & 181.965513 & $84,661.713$ & 182476,676 \\
\hline Fin Paches Dajneases & $84,127,953$ & $5, B 27, B 86, D 16$ & $361,733,152$ & $18,192,659,194$ \\
\hline Natim Rritures Delurames & $52, B 95,154$ & 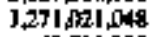 & $326,489,850$ & $30,525,620,252$ \\
\hline 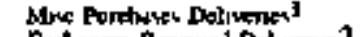 & $3,333,231$ & $49,722,928$ & 24,113197 & $67,245,798$ \\
\hline 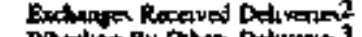 & $21,847,527$ & 172469 & $65,410,323$ & $\ln 22 \times 5,85 \$$ \\
\hline Mhedint By Duber Detivents ${ }^{3}$ & BS.491,636 & 182958264 & $161,303,691$ & $535 \$ 6,121$ \\
\hline
\end{tabular}

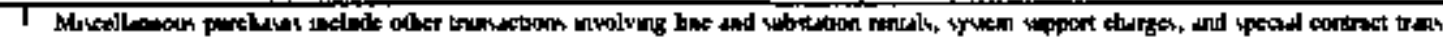
stopans

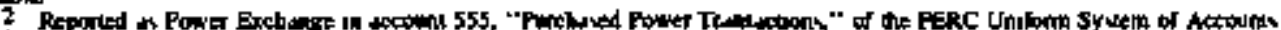

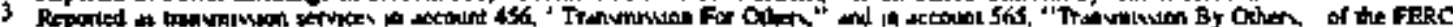

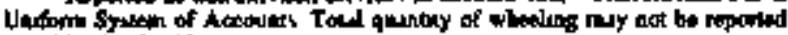

- Hot Appintele

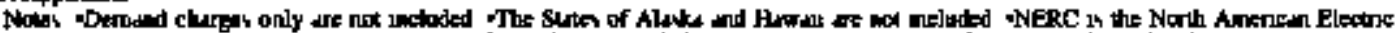

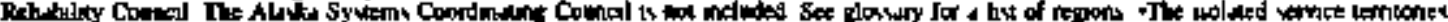

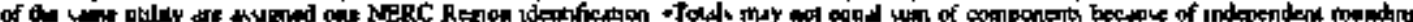

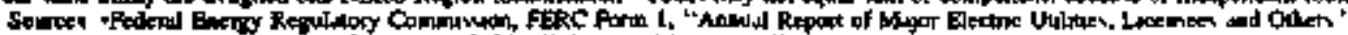

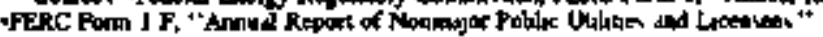


Table 16. Deliveries by Investor-Ommed Utilities Within and Between NERC Regions, 1994

\begin{tabular}{|c|c|c|c|c|}
\hline \multirow{3}{*}{ 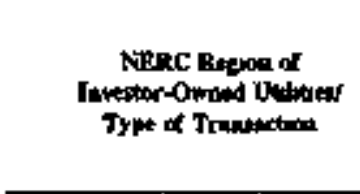 } & \multicolumn{4}{|c|}{ 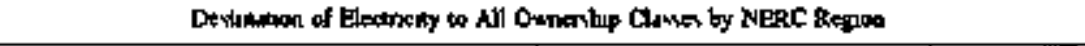 } \\
\hline & \multicolumn{2}{|c|}{ echis } & \multicolumn{2}{|c|}{ RRCOT } \\
\hline & (0movend kw) & $\begin{array}{l}\text { Revenot } \\
\text { (dolury) }\end{array}$ & $\begin{array}{c}\text { Enefgy } \\
\text { (llowher kWh) }\end{array}$ & $\begin{array}{l}\text { Ravenot } \\
\text { (dolldary) }\end{array}$ \\
\hline \multicolumn{5}{|l|}{ BCAR } \\
\hline Pim Sular & 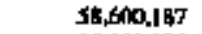 & $1,634,386,593$ & $=$ & - \\
\hline Nompition Sisten & 36,159 ant & $0,047,342,913$ & - & - \\
\hline Bdretllneoos SAles I & -51 & $90,289,073$ & - & - \\
\hline Bxtheres Deltreced? & 1,395,94: & - & - & - \\
\hline Wheding For Oulm 3 & $21,939.782$ & $79,611,923$ & - & - \\
\hline \multicolumn{5}{|l|}{ ERCOr } \\
\hline Fim Sdev & - & - & $5,1776,589$ & 299,998,015 \\
\hline Monfirfa SAk & - & - & 2649.509 & $56, m g, 424$ \\
\hline 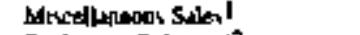 & - & - & - & $2,354,489$ \\
\hline Exthutigen Delyerat? & - & - & $13,176,144$ & - \\
\hline Whetding For OAms & - & - & $25,697, A 93$ & $4 \pi, 219,820$ \\
\hline \multicolumn{5}{|l|}{ MAAC } \\
\hline Finf Salex & B2.J17 & 3.609 .924 & - & - \\
\hline 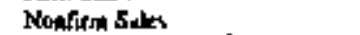 & 3,696 & 317,116 & - & - \\
\hline 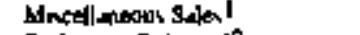 & - & - & - & - \\
\hline 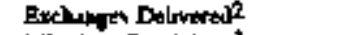 & - & - & - & - \\
\hline \multirow{2}{*}{\multicolumn{5}{|c|}{ MARS }} \\
\hline & & & & \\
\hline Firm Sular & $2,176,736$ & $59,037,815$ & - & - \\
\hline Noufins Salor & 964,619 & $14,954,093$ & - & - \\
\hline Blscel|meo0s Sdeal & & - & - & - \\
\hline Exclunges Delswercod? & 11 & - & - & - \\
\hline Whesing Fot Dother & - & - & - & - \\
\hline \multicolumn{5}{|l|}{ MAPP } \\
\hline Fmm SAle & - & - & - & - \\
\hline Nonflme Sdks & - & - & - & - \\
\hline 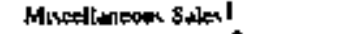 & - & - & - & - \\
\hline Exchunges Detwerdi? & - & - & - & - \\
\hline Wheelun For Olhers? & - & - & - & - \\
\hline \multicolumn{5}{|l|}{ NPCC } \\
\hline Finm Sden & - & - & - & - \\
\hline Nonfimn Salk & - & - & - & $\rightarrow$ \\
\hline 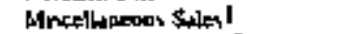 & - & - & - & - \\
\hline Exhangen Detiverod? & - & - & - & - \\
\hline \multirow{2}{*}{\multicolumn{5}{|c|}{ sere }} \\
\hline & & & & \\
\hline Fime Saldev & 54,697 & $25,502,369$ & - & - \\
\hline Montivin Sake & 4575 & $1,009,80 \%$ & - & - \\
\hline Mroctlateotw Salal & - & - & - & - \\
\hline Exchusgen Dehrapd? & 27,161 & - & - & - \\
\hline \multirow{2}{*}{\multicolumn{5}{|c|}{ SPP }} \\
\hline & & & & \\
\hline Furm Stw & 30,272 & 1,406,973 & $1,490,271$ & SB,075,908 \\
\hline Monfirm Sakv & - & - & 355,574 & $6,522,279$ \\
\hline Burefllapeow، Salg' & - & - & - & - \\
\hline Enchungen Delinefed ${ }^{2}$ & - & - & - & - \\
\hline Wheelnas For Olwerw & - & - & 28,745 & 5253 \\
\hline \multicolumn{5}{|l|}{ wsoc } \\
\hline Frtm Saks & - & - & - & - \\
\hline Nonition Ster & - & - & 27,051 & $1,341 ., 026$ \\
\hline 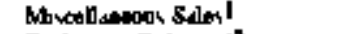 & - & - & - & - \\
\hline Exchinuet Deliveral? & $=$ & $=$ & - & - \\
\hline Whechar for Octhen 3 & - & - & - & - \\
\hline \multicolumn{5}{|l|}{ Detion Told } \\
\hline Pirm Salev Rerenps & $61,464,1105$ & $1,727,964,394$ & $6,566,859$ & $328,073,913$ \\
\hline Nonfirm Saler Renom & $37,19 t, 906$ & $1, \sqrt{164,421,983}$ & $3,293,004$ & $66,572,323$ \\
\hline 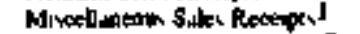 & $-\mathbf{s I}$ & $90,280,073$ & - & 2., 54,489 \\
\hline Exchange Deflvered Recripts 2 & $1,419.141$ & - & $33,176,144$ & - \\
\hline Whesling Res Oner Rectipu ${ }^{3}$ & $24,601,532$ & $85, \sqrt{106}, 392$ & $25.726,278$ & $47.272,398$ \\
\hline
\end{tabular}

Set noter and footwoles at end of tublo 
Table 16. Deliveries by Investor-Owned Uttlities Within and Between NERC Regions, 1994 (Conlinued)

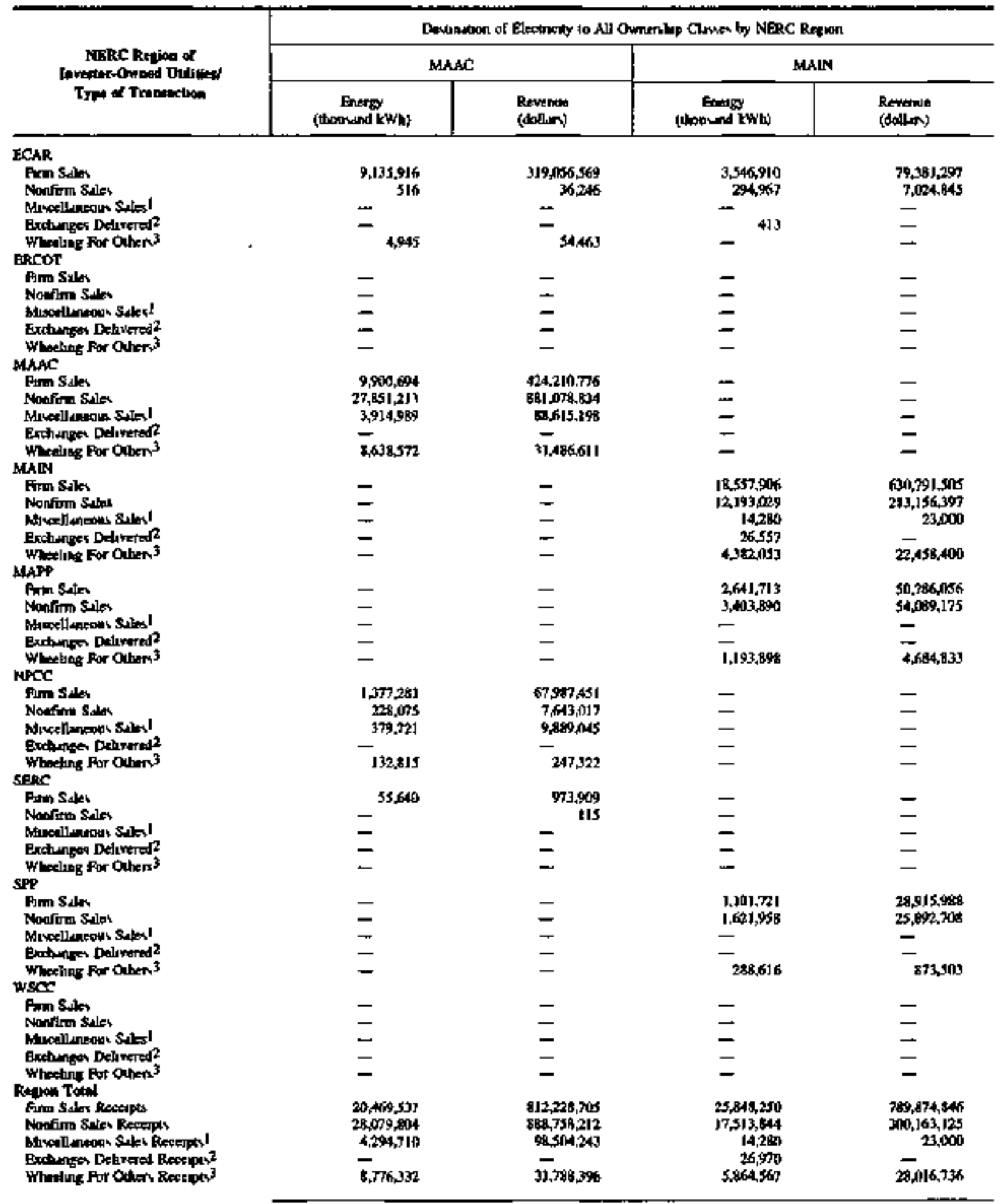

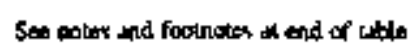


Table 16. Deliveries by Investor-Owned Utilities Witbin and Between NERC Repions, 1994 (Contimued)

\begin{tabular}{|c|c|c|c|c|}
\hline \multirow{3}{*}{ 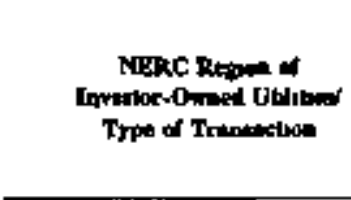 } & \multicolumn{4}{|c|}{ 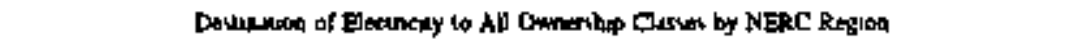 } \\
\hline & \multicolumn{2}{|c|}{ MAPP } & \multicolumn{2}{|c|}{ NPCC } \\
\hline & 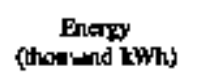 & $\begin{array}{l}\text { Renenue } \\
\text { (dotisint) }\end{array}$ & $\begin{array}{l}\text { Energy } \\
\text { (thoosend } k \text { tha) }\end{array}$ & $\begin{array}{l}\text { Rewemide } \\
\text { (dollerkt }\end{array}$ \\
\hline \multicolumn{5}{|l|}{ ECAR } \\
\hline Fm SAler & - & - & 2,524 & 49,237 \\
\hline Nonflm Saks & - & - & - & - \\
\hline 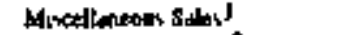 & - & - & - & - \\
\hline 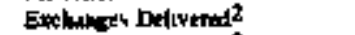 & - & - & - & — \\
\hline \multirow{2}{*}{\multicolumn{5}{|c|}{ excot }} \\
\hline & & & & \\
\hline Fint Salos & - & - & - & - \\
\hline Norring Salm & - & - & - & - \\
\hline Mrentanterim Swas! & - & - & - & - \\
\hline Exclubest Delwered2 & - & - & - & - \\
\hline Whoting For Othery 3 & - & - & - & - \\
\hline \multicolumn{5}{|l|}{ MANC } \\
\hline Pinn S.tar & - & - & 5,099 & 311244 \\
\hline Nonfirm Sules & - & - & $4.285,021$ & $58,545.698$ \\
\hline 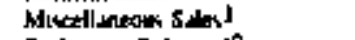 & - & - & - & - \\
\hline Exthingen Delwered ${ }^{2}$ & - & - & - & - \\
\hline Wheting For Colens & - & - & $4[9,18]$ & $1,4[2.53]$ \\
\hline \multicolumn{5}{|l|}{ MAON } \\
\hline Fons solar & $1,731, \mathrm{mr}$ & 90,38B,BA9 & - & - \\
\hline Nomform Sales & $0,197,612$ & $20 \Omega 03,150$ & - & - \\
\hline Blisedimeows Suld & - & - & - & - \\
\hline Exclupes Delryered" & - & - & - & - \\
\hline Wheeling For Orher" 3 & 1.547 & 1,960 & - & - \\
\hline \multicolumn{5}{|l|}{ MASP } \\
\hline Fum SAles & S,Als.989 & $169,094,696$ & - & - \\
\hline Nonforie Saliter & $3,197,463$ & $54,392,61$ & - & $=$ \\
\hline Mdncallmeos SAld & 5, Ging, os! & $271.890,292$ & - & - \\
\hline Exctulegen Delinered2 & 130,486 & - & - & - \\
\hline Wheting For oulen ${ }^{3}$ & $14 A B 7,6 M B$ & $14,819,829$ & - & - \\
\hline \multicolumn{5}{|l|}{ NPCC } \\
\hline Purin Salen & - & - & 51,691573 & 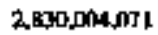 \\
\hline Noufirm Salex & - & - & $43,082,498$ & $3,315,100,722$ \\
\hline Murealiancoun Sales I & - & - & 5.T59.119 & $202,141,617$ \\
\hline Exchinges Delivered? & - & . & 714,862 & - \\
\hline Wheaing For Othern 3 & - & 248,320 & $63,649,845$ & $548,207,405$ \\
\hline \multicolumn{5}{|l|}{ SERC } \\
\hline Fmm Sales & - & - & - & - \\
\hline Noning Sten & - & - & - & - \\
\hline Arrcallineorth SalesI & - & - & $=$ & - \\
\hline Euthanges Dehwered" & - & - & - & - \\
\hline Wheelary for Ochers ${ }^{3}$ & - & - & - & - \\
\hline \multicolumn{5}{|l|}{ spp } \\
\hline Finm sules & - & - & 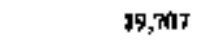 & 375,453 \\
\hline Nominn Sesten & 299,922 & $5.004,259$ & $m$ & - \\
\hline 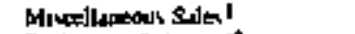 & - & - & - & - \\
\hline 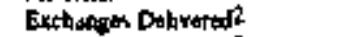 & - & - & - & - \\
\hline \multirow{2}{*}{\multicolumn{5}{|c|}{ wsec }} \\
\hline & & & & \\
\hline Foth Sales & 293,063 & $9,111,095$ & - & $=$ \\
\hline Monfing Sar & 970 & 25,953 & - & - \\
\hline Mexcelluapoor Sules I & - & $1,3,3$ & - & $\pi$ \\
\hline Exchinges Deltwered2 & - & - & - & - \\
\hline Whecling For Othery & 3 & 297,972 & - & - \\
\hline \multicolumn{5}{|l|}{ Recom Tatd } \\
\hline frn Sul, Rexuls & $6,630,0799$ & $286,396,580$ & $31,675,303$ & $2,831,140,007$ \\
\hline Monfirm Ses Rreepts & 4535,967 & $6,731,033$ & $47,368,419$ & $1,371,646,470$ \\
\hline Mhreflowean Sales Restipesl & $5,602 n 51$ & $221,8 \mathrm{BB}, 63 \mathrm{BB}$ & $5,159.779$ & $202,141,617$ \\
\hline 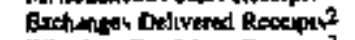 & 130,486 & - & J14, thit & - \\
\hline Wheting For Othen Recerpis 3 & $14,010,537$ & $15,628,406$ & 64,$069 ; 023$ & $549,800,236$ \\
\hline
\end{tabular}

See noter and frotwoter an end af twble 
Table 16. Deliveries by Investor-Owned Utilities Within and Between NERC Regions, 1994 (Continued)

\begin{tabular}{|c|c|c|c|c|}
\hline \multirow{3}{*}{ 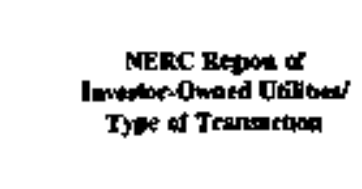 } & \multicolumn{4}{|c|}{ 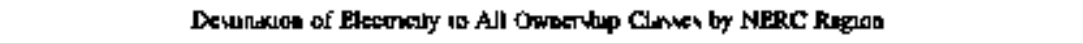 } \\
\hline & \multicolumn{2}{|c|}{ SERC } & \multicolumn{2}{|c|}{$\mathbf{S P P}$} \\
\hline & Eecty & $\begin{array}{l}\text { Rewetwue } \\
\text { (dolon) }\end{array}$ & $\begin{array}{c}\text { Energy } \\
\text { (dholond Ywh) }\end{array}$ & $\begin{array}{l}\text { Reventer } \\
\text { (dollot) }\end{array}$ \\
\hline \multicolumn{5}{|l|}{ ECAR } \\
\hline ماندS & $5,873,094$ & JBA,BO6, 410 & - & - \\
\hline Monfitul Sales & 804329 & $18,445,784$ & - & - \\
\hline Mretellincaos SalesI & - & - & - & - \\
\hline Exth.ipss Delmered? & 665,322 & - & - & - \\
\hline Whation For Oxbers & $4,092,187$ & 17,300944 & - & - \\
\hline \multicolumn{5}{|l|}{ ERCOT } \\
\hline Fan Sales & - & - & 823,349 & 61,905, \\
\hline Nonfom Salex & - & - & 266,889 & $4,720,154$ \\
\hline 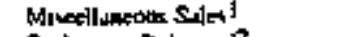 & - & - & - & - \\
\hline 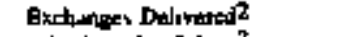 & - & - & - & - \\
\hline Whecluag For Oaben 3 & - & 196 (5) & 158,945 & $1,126,0898$ \\
\hline \multicolumn{5}{|l|}{ MAAC } \\
\hline Prm \$aln & 6,sis & 380,619 & 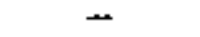 & $\rightarrow$ \\
\hline Nunforth Siles & - & - & - & - \\
\hline 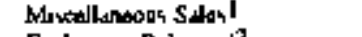 & - & - & - & - \\
\hline Exchingos Delwered2 & 一 & - & $\Rightarrow$ & 一 \\
\hline Wheding Fir Othand & - & - & - & - \\
\hline \multicolumn{5}{|l|}{ Malw } \\
\hline Fim \$시내 & - & - & $2 \pi 32$ & $11,906,222$ \\
\hline Nonfm Sales & $1,103,94,1$ & 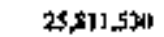 & $3,247,194$ & $76,988,326$ \\
\hline 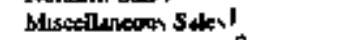 & 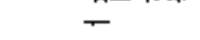 & - & - & - \\
\hline Exclunger Delıtwered? & - & - & - & 一 \\
\hline Whesing Fon other 3 & $m$ & $\longrightarrow$ & 307,203 & 247,200 \\
\hline \multicolumn{5}{|l|}{ MAPP } \\
\hline FMm Sales & - & - & 201,504 & $11.768,587$ \\
\hline Nhonfinen Sules & - & - & 1376,205 & $19,360,731$ \\
\hline Mhrix|llaneour SditeI & - & - & - & - \\
\hline Exchuagu Delmented? & - & - & - & - \\
\hline Whecling For Othat & - & - & - & - \\
\hline \multicolumn{5}{|l|}{ NPPCS } \\
\hline Fitin Sallex & - & - & - & - \\
\hline Nontim sales & - & - & - & - \\
\hline Mixceldungour Siles I & - & - & - & $m$ \\
\hline Exch inger Delivaned? & - & - & - & - \\
\hline 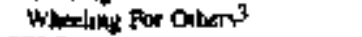 & - & - & - & - \\
\hline \multicolumn{5}{|l|}{ SERC } \\
\hline Fon & $34,603,200$ & $1,599,367,723$ & - & - \\
\hline Nondinth Sales & $41,831,499$ & $1,792,764,576$ & B,941,550 & $480,043,061$ \\
\hline 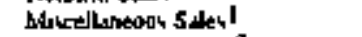 & 513074 & $3,5 \pi$ ats & - & - \\
\hline Evctunges Dellvered? & $4,602,575$ & - & - & 一 \\
\hline Wheling For Oders & $14,3,37042$ & $94,513,555$ & 46.637 & 975,434 \\
\hline \multicolumn{5}{|l|}{$\operatorname{sip}$} \\
\hline Purm Sales & 200,599 & $9,054,84]$ & 15,641,ing & sin3,726,001 \\
\hline Nonflum Stes & - & - & $28,112.126$ & $662,814,254$ \\
\hline Mriedleneons Sall & - & - & 112,467 & 1,357.686 \\
\hline Exctunger Delıpal' & - & 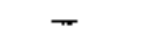 & $2, \sqrt{58,2212}$ & - \\
\hline Whecling for others? & 2.261 .750 & $7,178,382$ & $19,381,964$ & $106,223,650$ \\
\hline \multicolumn{5}{|l|}{ wsec } \\
\hline Fim Stles & - & 一 & - & - \\
\hline Norfing Salev & - & - & $\mathbf{k}, 247$ & 233,478 \\
\hline Mivedluteous Salas & - & 一 & - & - \\
\hline Exisbutis Deturened? & - & - & 654 & - \\
\hline Whething Res Gther 3 & - & 一 & 5,115 & 3d,48I \\
\hline \multicolumn{5}{|l|}{ 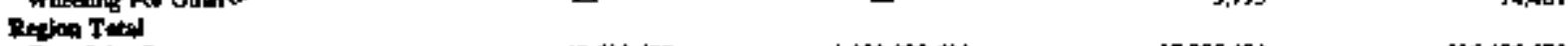 } \\
\hline Frm Sdes Resaph & $40,483,477$ & $1,883,429,591$ & $17,006,494$ & $586,496,673$ \\
\hline Nonfurn Suls Recents & $44,499,71$ & $1,437,021,890$ & $4,057,211$ & $1,244,950,104$ \\
\hline 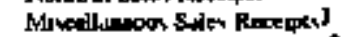 & 513,074 & $5,579,015$ & 112,467 & $3,357,686$ \\
\hline 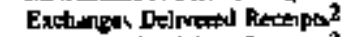 & $5,2 \times 18,097$ & - & $2, \sqrt{153}, 876$ & - \\
\hline Whecling For Oulatin Ratenps] & $20,690,979$ & $122,080,434$ & $19,899,9 \times 4$ & $108,646,853$ \\
\hline
\end{tabular}

See notes and foctrotes 4 ind of lable 
Tahle 16. Deliveries by Investor-Owned Utillites Whin and Between NERC Regions, 1994 (Conthued)

\begin{tabular}{|c|c|c|c|c|}
\hline \multirow{3}{*}{ 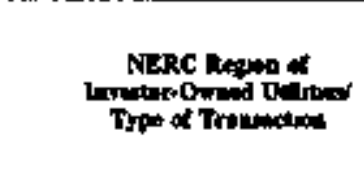 } & \multicolumn{4}{|c|}{ 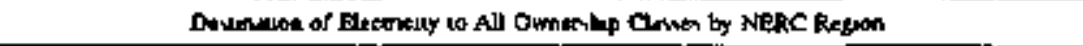 } \\
\hline & \multicolumn{2}{|c|}{$\mathbf{w s c t}$} & \multicolumn{2}{|c|}{ 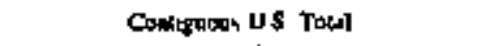 } \\
\hline & (thoorengl & $\begin{array}{l}\text { Revenle } \\
\text { (dantur) }\end{array}$ & (Atoower kw) & $\begin{array}{l}\text { Revoripe } \\
\text { (dotur) }\end{array}$ \\
\hline \multicolumn{5}{|l|}{ EChR } \\
\hline & عa & - & $77,199,039$ & $2,247,600,396$ \\
\hline Nowfm Sules & - & - & $57,338, \mathrm{BSA}$ & $1,[1,2,847,848$ \\
\hline 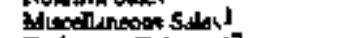 & - & - & -51 & $90,289.073$ \\
\hline Exchengev Delrraod? & - & - & 201,89 & - \\
\hline Whoching For Othan & - & - & $26,036,9] 4$ & $97,30,30$ \\
\hline \multicolumn{5}{|l|}{ ERCOT } \\
\hline Firm saled & - & 一 & $5,900,537$ & $331,003,868$ \\
\hline Norfin Salat & - & $\overline{-}$ & 2516,398 & $60,42,578$ \\
\hline 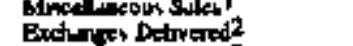 & $\overline{-}$ & $\bar{F}$ & $\overline{33.136,144}$ & $=$ \\
\hline Whecting For Ditan & 一 & - & 25856478 & $4,502,4$ 的 \\
\hline Fing sales & - & - & $9,904, A B I$ & $\$ 28,852,565$ \\
\hline Noufirm sulsa & - & - & $72,140,770$ & 90,9,941,648 \\
\hline 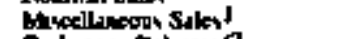 & - & - & 3914989 & $88,615,19 B$ \\
\hline 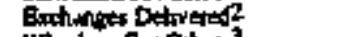 & - & 一 & $=$ & \\
\hline Whecloos for Dethen & 一 & - & $10,719,500$ & 38290911 \\
\hline Find Siln & - & - & $22,342,991$ & $751,916,-395$ \\
\hline Madifn Sth & - & - & 19,426397 & 351413,496 \\
\hline 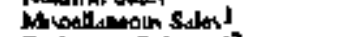 & 一 & - & 14,290 & $\mathbf{2 3}, 000$ \\
\hline Exchenger Deluwered2 & - & - & 26,583 & - \\
\hline Wholug For Others & 一 & 一 & 4,6901803 & 22707,563 \\
\hline \multicolumn{5}{|l|}{ MAPP } \\
\hline 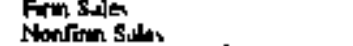 & - 287,880 & $\overline{4,025,799}$ & $\begin{array}{l}7,909,306 \\
8,295,4+8\end{array}$ & $231,649,279$ \\
\hline 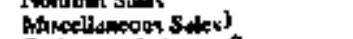 & & - & $5,642,051$ & 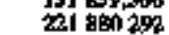 \\
\hline Exelinger Deiperes? & 21,387 & $\ddot{-}$ & 151,815 & - \\
\hline Whond Fir Ohers? & 1,330606 & 1324062 & $17,132,144$ & 20840724 \\
\hline \multicolumn{5}{|l|}{ NPCC } \\
\hline 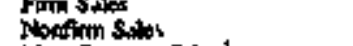 & - & 二 & 41210533 & $1,322,74,769$ \\
\hline Breflimeod Sulesd & $\overline{-}$ & & $6,139,500$ & 212 031,6062 \\
\hline Wheclers Por Others & $=$ & $=$ & 63782,660 & $\operatorname{sis} 1004$ \\
\hline \multicolumn{5}{|l|}{ sepc } \\
\hline finn sus & - & - & $35,233,446$ & I,719,84d, \\
\hline Nopofintin salek & - & 一 & $50,837,624$ & I,875,41],153 \\
\hline 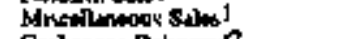 & عبد & - & 513,074 & $3 \operatorname{sig}, 013$ \\
\hline Exchunges Dehrated? & 一 & - & $4,1,25,736$ & \\
\hline Whenling For Olhmin & 543,644 & $1,437,236$ & 14927,323 & 9922623 \\
\hline \multirow{5}{*}{ 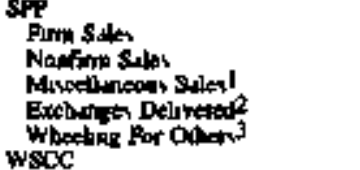 } & 179.699 & $14,728,354$ & I8,864,878 & 61468318 \\
\hline & 192,927 & 14962,526 & $31 / 47507$ & J15 998, I20 \\
\hline & - & - & 112467 & $\$ 357,686$ \\
\hline & - & - & $2,154,222$ & - \\
\hline & - & 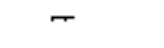 & $22,156,422$ & 1/47Jfithl \\
\hline \multirow{3}{*}{ 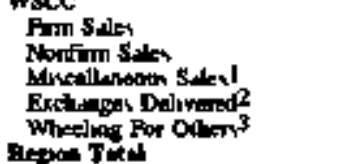 } & $27, \sin , 9 \pi$ & $1,291,340,405$ & $27,894,0+10$ & $6,302,451,300$ \\
\hline & $29,918,0$ & $698,959,101$ & 29955216 & 700563,008 \\
\hline & $22,547,267$ & $\begin{array}{l}14,274,730 \\
+\end{array}$ & $22, \begin{array}{r}21,099 \\
47,92]\end{array}$ & $\begin{array}{l}14276058 \\
-\end{array}$ \\
\hline Fum silies becests & $27,779,676$ & $1,7015,068,759$ & 25B, 1203,664 & 10,496005440 \\
\hline Hanfin Sulas Rectipte & $30,990,855$ & $713,048,346$ & 255278,811 & $7,174213,486$ \\
\hline 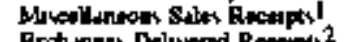 & 21, n99 & $14,274,326$ & 16,317459 & $x, 16415,493$ \\
\hline Exchingen Delivered Receipdn? & $22,5 \times 6,454$ & 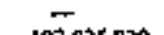 & $65,367,229$ & - \\
\hline Wheding For Othars Recaper & $68,6] 9,025$ & $187,835,979$ & $252,252,100$ & 1,776083734 \\
\hline
\end{tabular}

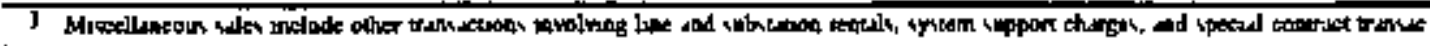
ang

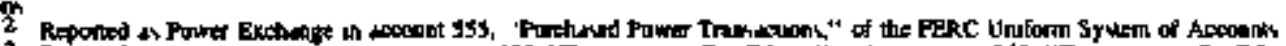

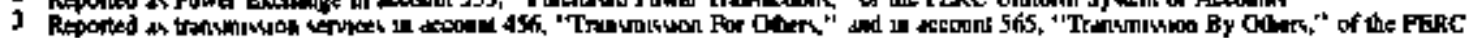

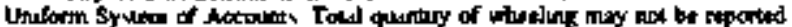

-Not Apphitito

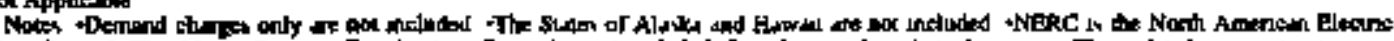

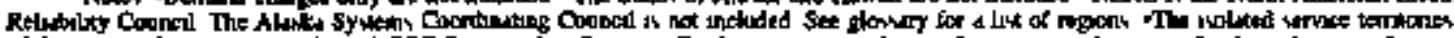

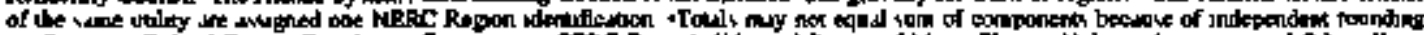

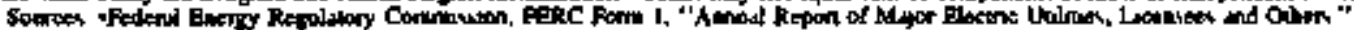

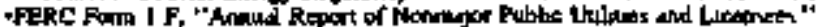


Table 17. Klectricity Transactions by lnvestor-Owned Utilities in Alaska and Hawaii, 1994

\begin{tabular}{|c|c|c|c|c|}
\hline \multirow[b]{2}{*}{ Type ar Trenesctiva } & \multicolumn{2}{|c|}{ Alukt } & \multicolumn{2}{|c|}{ Hinfal } \\
\hline & (thouland rwh) & Cont & 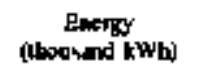 & $\underset{\text { (dollurs) }}{\text { Cous }}$ \\
\hline \multicolumn{5}{|l|}{ Tecelvei } \\
\hline \multirow{6}{*}{ 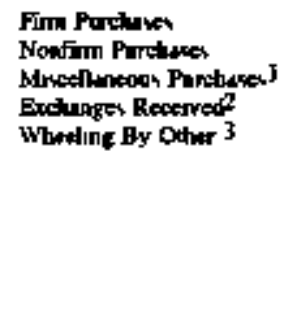 } & \multirow{4}{*}{$-^{235,555}$} & \multirow{2}{*}{$\overline{7,333,606}$} & \multirow{2}{*}{$\begin{array}{r}150,548 \\
1,000,193\end{array}$} & \multirow{2}{*}{$\begin{array}{r}12,275,282 \\
214,497,868\end{array}$} \\
\hline & & & & \\
\hline & & - & \multirow{2}{*}{ 2,fíg } & $\bar{z}$ \\
\hline & & - & & ت \\
\hline & \multicolumn{2}{|c|}{ Alated } & \multicolumn{2}{|c|}{ 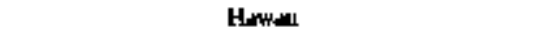 } \\
\hline & $\begin{array}{c}\text { Encrgy } \\
\text { (thoumd twh) }\end{array}$ & $\begin{array}{l}\text { Revenpe } \\
\text { (didnin) }\end{array}$ & $\begin{array}{c}\text { Energy } \\
\text { (thoulwhd kWM) }\end{array}$ & $\begin{array}{l}\text { Rerenoe } \\
\text { (dollmb) }\end{array}$ \\
\hline \multicolumn{5}{|l|}{ Delinted } \\
\hline Frn Sales & - & - & - & - \\
\hline Nontrin Sules & - & - & - & - \\
\hline Mivedilanocon S.letI & - & 一 & - & - \\
\hline Exchnagzes Dalwoted2 & - & - & 6,415 & $-171,862$ \\
\hline 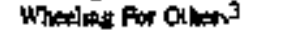 & - & - & - & 一 \\
\hline
\end{tabular}

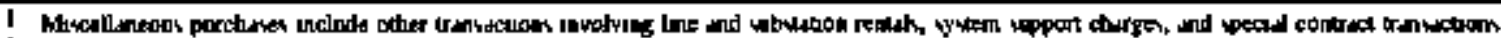

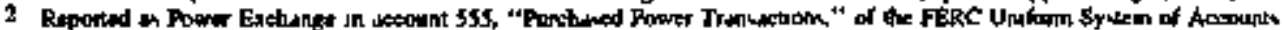

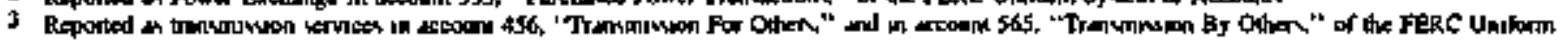

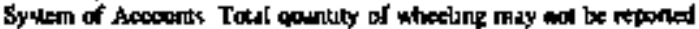

-Not Apphesble

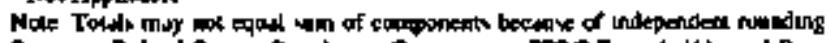

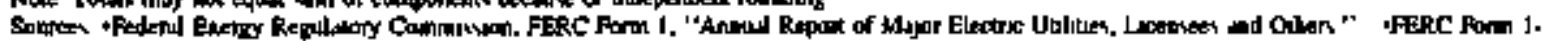

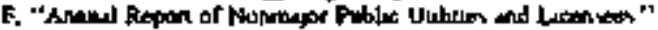


Table 18. US. Electricity Trade with Canada and Mexico by NERC Region, 1994

\begin{tabular}{|c|c|c|c|c|c|c|}
\hline \multirow{2}{*}{$\begin{array}{l}\text { Ptownes, Compicyt } \\
\text { Typo of Tranimetion }\end{array}$} & \multicolumn{5}{|c|}{ IS NERC Regton } & \multirow{2}{*}{ Bepon That } \\
\hline & NPCS & PCAR & ERCOT & MAPP & wscc & \\
\hline 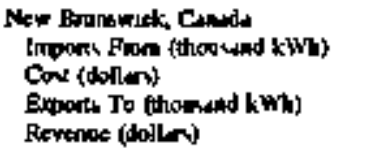 & $\begin{array}{r}2,749,547 \\
55,384,321 \\
418,647 \\
1,069,606\end{array}$ & $\begin{array}{l}= \\
z \\
=\end{array}$ & $\begin{array}{l}z \\
\bar{z} \\
=\end{array}$ & $\begin{array}{l}z \\
z \\
z\end{array}$ & $\begin{array}{l}\ddot{z} \\
= \\
-\end{array}$ & $\begin{array}{r}2,749,547 \\
-3,384,723 \\
418,896 \\
1,049,6016\end{array}$ \\
\hline 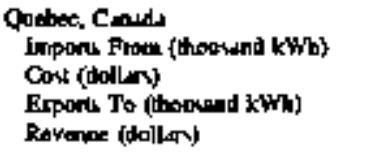 & $\begin{array}{r}17,447,541 \\
330,966,819 \\
945,0541 \\
20,414,900\end{array}$ & $\begin{array}{l}= \\
= \\
-\end{array}$ & $\begin{array}{l}= \\
= \\
z\end{array}$ & $\begin{array}{l}= \\
=\end{array}$ & $\begin{array}{l}\bar{z} \\
z \\
-\end{array}$ & $\begin{array}{r}17,443,541 \\
330,966,519 \\
9,45,056 \\
20,41,400\end{array}$ \\
\hline 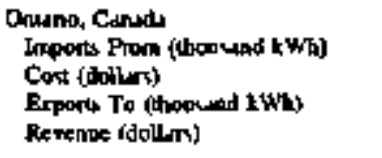 & $\begin{array}{r}4,887,413 \\
76,491,844 \\
180,619 \\
5,195,543\end{array}$ & $\begin{array}{r}6,919,598 \\
1 \times 8,420,6901 \\
2,925 \\
45,475\end{array}$ & $\begin{array}{l}- \\
- \\
-\end{array}$ & $\begin{array}{l}- \\
- \\
-\end{array}$ & $\begin{array}{l}- \\
- \\
-\end{array}$ & $\begin{array}{r}11,797,015 \\
214,912,444 \\
180,544 \\
5,94,068\end{array}$ \\
\hline 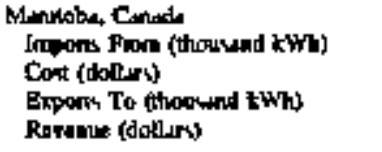 & $\begin{array}{l}= \\
=\end{array}$ & $\begin{array}{l}= \\
z \\
=\end{array}$ & $\begin{array}{l}z \\
z \\
z\end{array}$ & $\begin{array}{r}10,085,923 \\
213.194,733 \\
651996 \\
1,995,281\end{array}$ & $\begin{array}{l}z \\
z \\
z\end{array}$ & $\begin{array}{r}10,0085,923 \\
213.694,733 \\
657,594 \\
1,505,281\end{array}$ \\
\hline 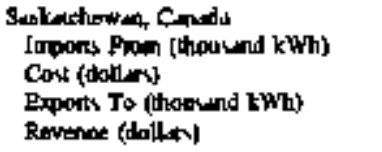 & $\begin{array}{l}\bar{z} \\
\bar{z}\end{array}$ & $\begin{array}{l}= \\
= \\
=\end{array}$ & $\begin{array}{l}\overline{-} \\
\overline{-}\end{array}$ & $\begin{array}{r}44,293 \\
314,932 \\
91,963 \\
1455,293\end{array}$ & $\begin{array}{l}\bar{z} \\
=\end{array}$ & $\begin{array}{r}44,293 \\
344,932 \\
91,963 \\
1,055,298\end{array}$ \\
\hline 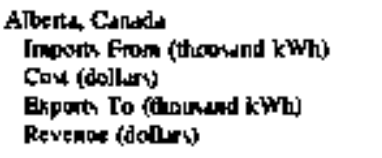 & $\begin{array}{l}I \\
I \\
=\end{array}$ & $\begin{array}{l}- \\
- \\
-\end{array}$ & $\begin{array}{l}- \\
- \\
-\end{array}$ & $\underset{113}{7,552}$ & $\begin{array}{r}- \\
2.974 \\
109,974\end{array}$ & a \\
\hline 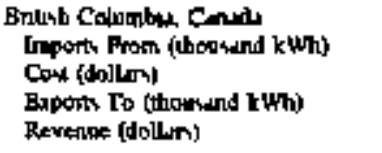 & $\begin{array}{l}z \\
z \\
-\end{array}$ & $\begin{array}{l}= \\
z \\
=\end{array}$ & $\begin{array}{l}z \\
z \\
z\end{array}$ & $\begin{array}{l}= \\
z \\
=\end{array}$ & 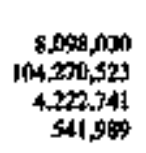 & $\begin{array}{r}3.099,0311 \\
104,57,523 \\
4,222,741 \\
541,949\end{array}$ \\
\hline 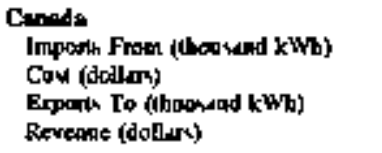 & $\begin{array}{r}25,000,505 \\
472,8+2,983 \\
1,54,511 \\
27,780,099\end{array}$ & $\begin{array}{r}6,909,598 \\
138,426,6900 \\
2,925 \\
48,475\end{array}$ & $\begin{array}{l}- \\
- \\
-\end{array}$ & $\begin{array}{r}10,120,216 \\
217,504,665 \\
750,022 \\
2963,631\end{array}$ & $\begin{array}{r}8,098,030 \\
104,270,523 \\
4,226,715 \\
661.968\end{array}$ & $\begin{array}{r}54,210,349 \\
929,063,731 \\
6,521,293 \\
31,058,133\end{array}$ \\
\hline 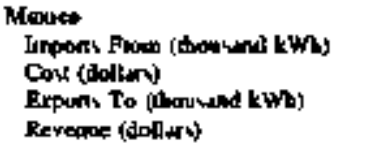 & $\begin{array}{l}- \\
- \\
-\end{array}$ & $\begin{array}{l}= \\
z \\
-\end{array}$ & $\begin{array}{r}30 \\
25,261 \\
2,\{17,712\end{array}$ & $\begin{array}{l}- \\
= \\
z\end{array}$ & $\begin{array}{r}2,011,249 \\
\$, 4612,70 \% \\
1,043,407 \\
49,639,294\end{array}$ & $\begin{array}{r}2011,319 \\
89,4(6,719 \\
1,068,6618 \\
50,677,0+18\end{array}$ \\
\hline 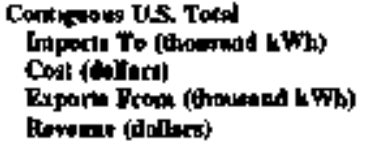 & $\begin{array}{r}25,410,505 \\
97,94,983 \\
1,544,51 \\
27,3+2,099\end{array}$ & $\begin{array}{r}6,4,4,988 \\
138,42,0,014 \\
2,025 \\
48,675\end{array}$ & $\begin{array}{r}70 \\
2+361 \\
2,017,712\end{array}$ & 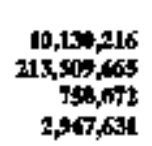 & $\begin{array}{r}16,10 \times, 279 \\
187,731,232 \\
5,269,122 \\
49,321,266\end{array}$ & $\begin{array}{r}52,2,9,641 \\
1,012,504,490 \\
1,591,481 \\
01,735,183\end{array}$ \\
\hline
\end{tabular}

\section{-Nex inphablite}

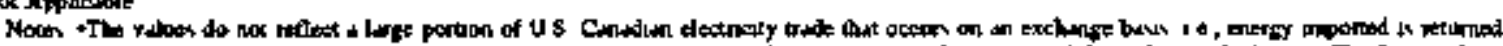

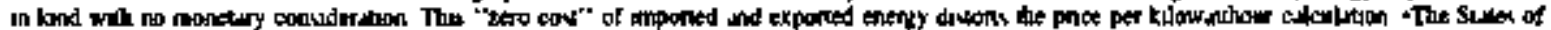

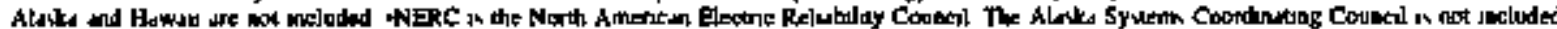

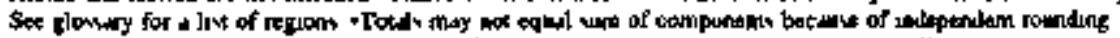

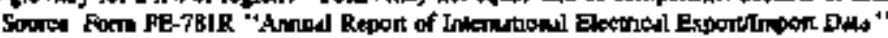




\section{Bulk Power Transactions by Investor-Owned Utilities}

\section{Overview}

This chapter presents data for todjvidual investorowned utilities on purchased power, sales for resale, exchanges, and wheeling. Sales for resale are generally sales to other investor-owned utilitjes, public power municipals, and cooperatives under obligations to support wholesale requifement custotners. These sales represent one of the roost active parts of the electricity trade market. Purchases are generally made from other investor-0wned uttlities and Federal power marketing authoritics. These purchases are made in order to take advantage of cheaper rates generally coning fron hydroelectric generation, to supplement or replace internal power supplies, and for handling longer term energy emergencies. Exchanges cover in-kind transfers, and wheeling (transmission for and by others) describes transmission services provided by utilities to other utilities. The tables present data for indjviduat utilities aggregaced to show cade with different classes of ownership.

Data are from the FERC Form 1, which is the only dati source that identifies individual utilities involved in exchanges and wheeling. 5

Figure 6. Electricity Purchases and Costs by the Top Ten Investor-Owned Utilitles, 1994

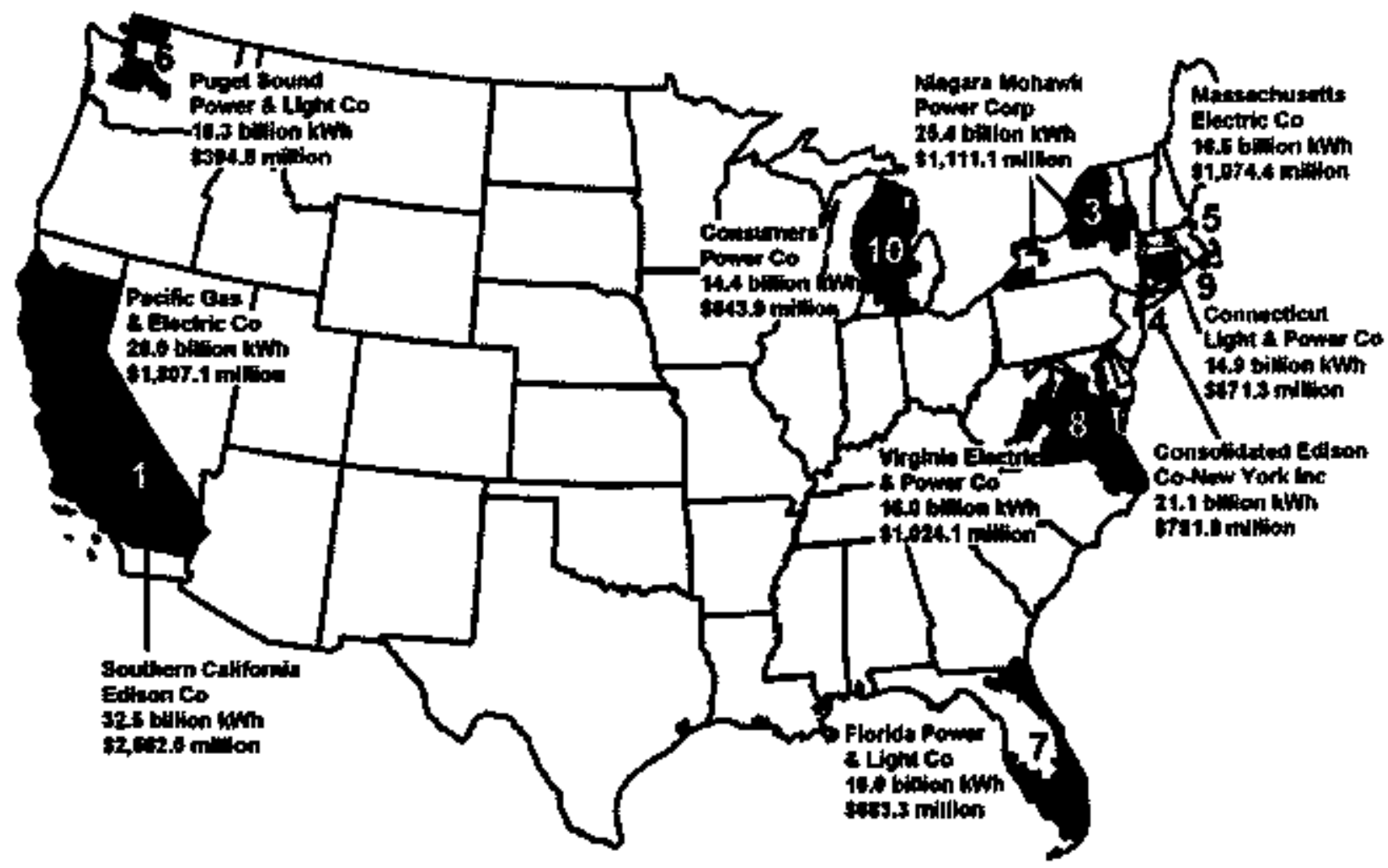

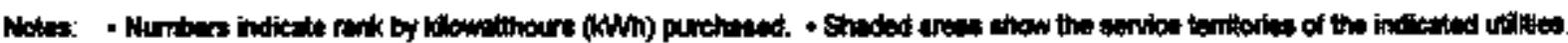

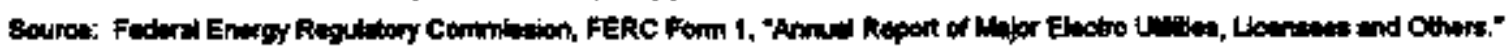

5 This is wat the cuse for othor dala collected on axchages and wheliog. Oofy aggregated data are collected op exchapges and whtelias

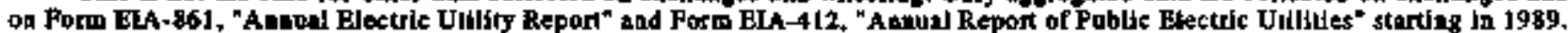




\section{Tables}

This chapter presents data or:

- Energy purchases and cost, with cost data shown for demand, energy, and other (Table 19).

- Sales for resale and revenue, with revenue data shown for demand, energy, and other (Table 20).

- Exchanges of power by ownership elass, identifying quantities transfered with any monetary settlement (Table 21), Nonmonetary settlements that may have occured are not shown. Exchanges are transactions where electricity provided to one utility is retomed in kind at a later date. In these transactions, the value of the electricity may or may not have been priced at the same level. Addjtional money or electricity could have been involved in the exchanges, but the details of the transactions are not collected on the FERC fonms.

- Electricity purchases by investor-owned utilities not listed elsewhere (Table 22). (These utilities are not listed elsewhere because of their small size and/or their power was purchased from cooperarives.)

- Wheeling through and to service territories of investor-owned utilities (Tables 23 and 24).
In each table, the investor-owned utilities are listed by State aceording to their corporate matiling addresses.

\section{Summary}

Differences are present in all wholesale trade categories. Some are based an the diversity of the cost of energy coming from the individual mix of generating facilities that developed over time as the electric utility industry met local and regional cbanges in load. This includes development of inexpensive local natural resources. In other cases, differences can be explained because some electric utilities are requirement customers of others and lack generation capability so they cannot sel] electricily.

The data indicate a great diversiry in wholesale e]ectricity trade and a complex market for electricity. The diversity represent differences in the influence of geographical location; the ability to access multiple parts of the transmission grid; the support of reliability and stability standards; and the load requirements coming from varied customer compositions of service territories.

\section{FIgure 7. Electriclty Sales for Resale and Revenue by the Top Ten Investor-Owned Utillties, 1994}

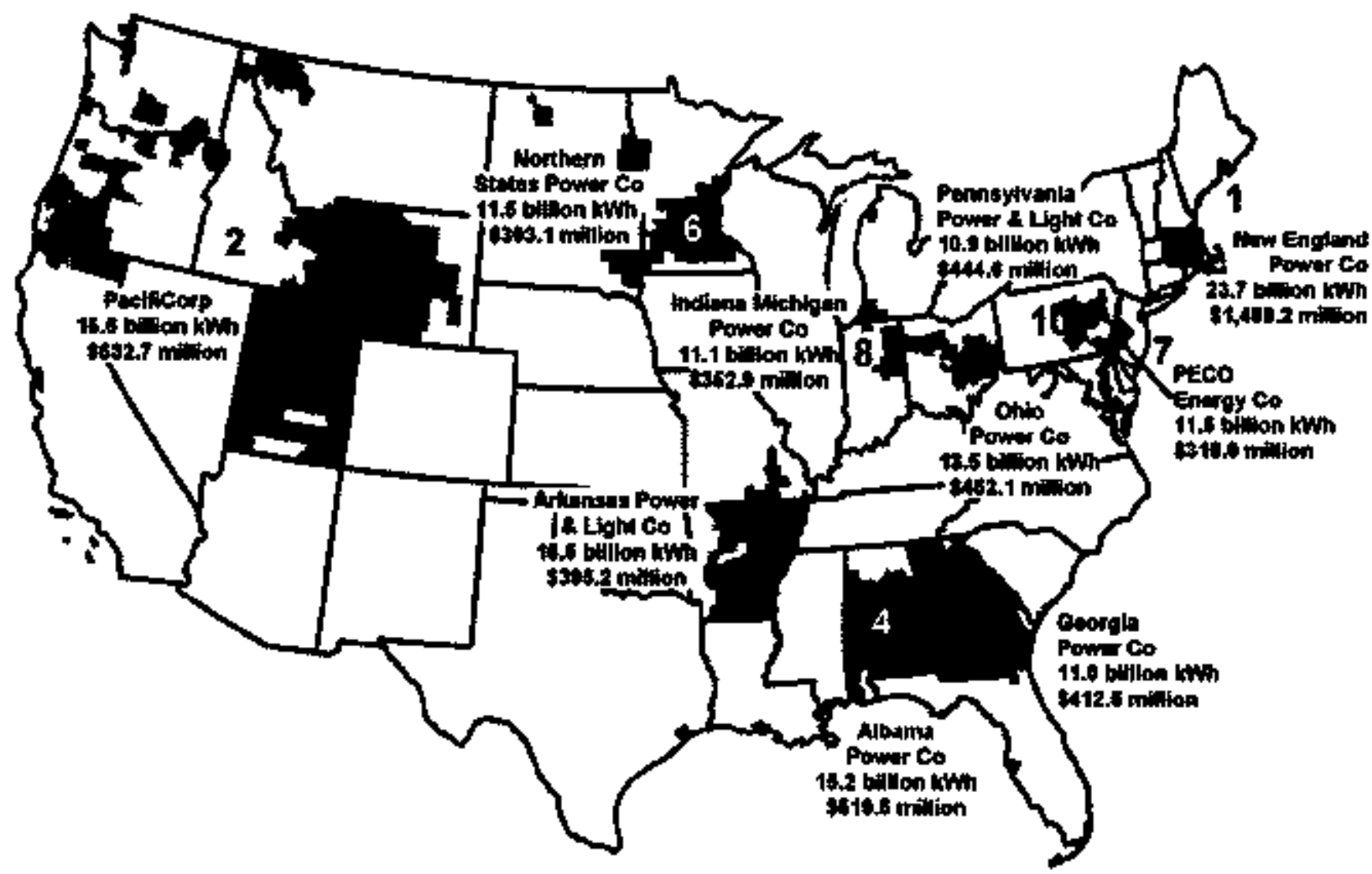

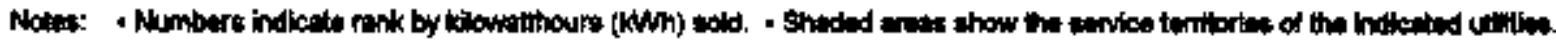

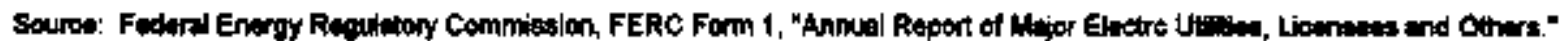


Table 19. Electriclity Purchases by Investor-Owned Utilities, by State, 1994

\begin{tabular}{|c|c|c|c|c|c|c|c|}
\hline 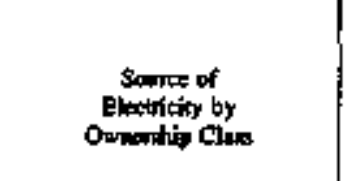 & 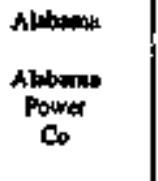 & 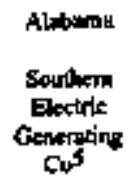 & 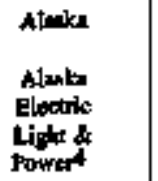 & $\begin{array}{l}\text { Arlzana } \\
\text { Arizans } \\
\text { Publit } \\
\text { Service } \\
\text { Co }\end{array}$ & 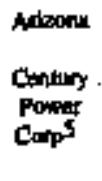 & 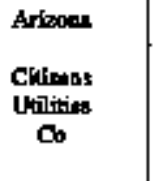 & 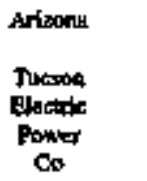 \\
\hline \multicolumn{8}{|l|}{ Inveitoflownd } \\
\hline Prichenek (thoulnoud KWh) & $2,866,035$ & - & - & 614.941 & - & 98,780 & 302,631 \\
\hline Com (dollens) & 85.716280 & - & - & $23,763,728$ & - & $46,144,380$ & $8,764,240$ \\
\hline Denetid Cherge Oalyl & - & - & - & - & - & - & - \\
\hline Denand & - & - & - & $11,129,980$ & - & $24,604,-113$ & - \\
\hline 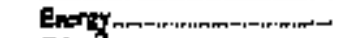 & $85,716,280$ & - & - & $12,694,348$ & - & $21,179,56]$ & $8,764,240$ \\
\hline Other & - & - & - & - & - & I3A,40 & - \\
\hline \multicolumn{8}{|l|}{ 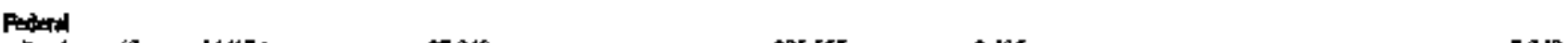 } \\
\hline Puthinges (lloosulal kWb) & 37,040 & - & 235,555 & 3,485 & - & - & 7,340 \\
\hline Coll (dollkny) - & 802485 & - & $7,333,8[6$ & 61, 31, 3 & - & - & J45.86\$ \\
\hline 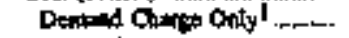 & $\rightarrow$ & - & - & - & - & - & - \\
\hline 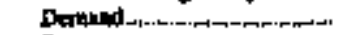 & 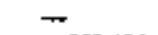 & - & - & - & - & - & - \\
\hline Energy_-_- س & 202485 & - & 7,331,80 & 61,913 & - & - & 145,865 \\
\hline Ohher & - & - & - & - & - & - & - \\
\hline \multirow{2}{*}{\multicolumn{8}{|c|}{ Sinte and Olher }} \\
\hline & & & & & & & \\
\hline horingues (throtand kwh) & 1,408 & - & - & 891,232 & - & - & 20,180 \\
\hline 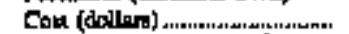 & 31,713 & - & - & $\$ 9,062,564$ & - & - & $40,1 \mathrm{~m}$ \\
\hline Derned Cherge Only 1 ......... & - & - & - & - & - & - & - \\
\hline 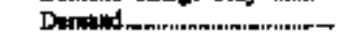 & - & - & - & $17,632,400$ & - & - & عـ \\
\hline 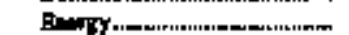 & 3],713 & $\rightarrow$ & - & $22.210,264$ & - & - & 440,170 \\
\hline 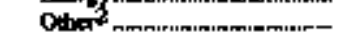 & - & - & - & - & - & - & - \\
\hline \multicolumn{8}{|l|}{ Mundispal } \\
\hline Prathes (Uonsend KWL) & 2,734 & - & - & 315,489 & - & - & sas \\
\hline Cod (dallin) & 1008,046 & - & - & 392041 & - & - & $1,387,147$ \\
\hline Dumpind Curge Ody I....... & - & $\rightarrow$ & - & - & - & - & - \\
\hline 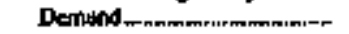 & - & - & - & - & - & - & - \\
\hline Enery & 108,046 & - & - & 392,041 & - & - & $1,387,19$ \\
\hline 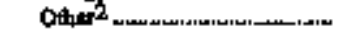 & - & - & - & - & - & - & - \\
\hline \multicolumn{8}{|l|}{ Copperite } \\
\hline 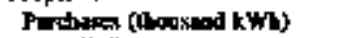 & 13,025 & - & - & 1,449 & - & 67 & $99,2+1$ \\
\hline 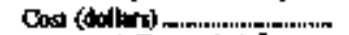 & $9(1) \cos 6$ & - & $=$ & $30,8,80$ & - & $6, \pi 3$ & 2,332,950 \\
\hline 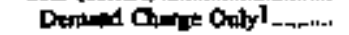 & - & - & - & - & - & - & - \\
\hline 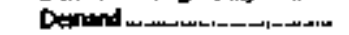 & - & - & - & - & - & - & - \\
\hline 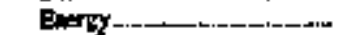 & 954,110 & - & - & 302580 & - & 6,733 & $2,332,950$ \\
\hline O中中 & f,946 & - & - & - & - & - & - \\
\hline \multicolumn{8}{|l|}{ Jبהם } \\
\hline Pordusea (thounind $\mathbf{L} W / \mathrm{h}$ ) & $1,284,437$ & $\rightarrow$ & - & -15.725 & - & 4,749 & $-12,986$ \\
\hline 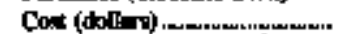 & $24,6 \mathrm{~F}_{1}, 13 \mathrm{~B}$ & $\rightarrow$ & - & $-525 \sqrt{125}$ & - & 20,745 & 808119 \\
\hline Denud Cavero Ody $J_{\text {nn--.- }}$ & - & - & - & - & - & - & - \\
\hline 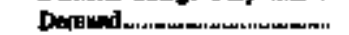 & - & - & - & - & - & - & - \\
\hline Energy & $22,508,779$ & - & - & $-525,10018$ & - & 20,343 & 91.1886 \\
\hline Olber ${ }^{2}$, mirmm & 591739 & $\rightarrow$ & - & - & - & - & 716,993 \\
\hline \multicolumn{8}{|l|}{ Tots } \\
\hline Dothanes (thootund KWh) & $4,205,279$ & - & 275,555 & $1,53[, 8 \times 2$ & - & 999,616 & 556,594 \\
\hline Cost (dotterst) & 116045,718 & - & $7,333,8000$ & 6066,196 & - & $46,171,856$ & $13,878,491$ \\
\hline Dewound Courge Ody 1 _t..... & - & - & - & - & - & - & - \\
\hline 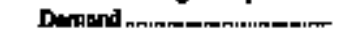 & - & $\rightarrow$ & - & 28.761 .790 & - & $24,034,413$ & - \\
\hline 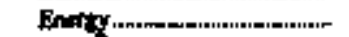 & $110,125,413$ & - & 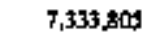 & $34,0004,418$ & - & 21,207,AO, & $13,161,558$ \\
\hline Odeg & $5,924,305$ & $ـ$ & - & - & - & I30,404 & T(6,93] \\
\hline
\end{tabular}

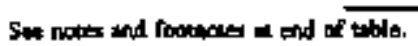


Table 19. Electricity Purchases by Investor-Owned Utilities, by State, 1994 (Conthued)

\begin{tabular}{|c|c|c|c|c|c|c|c|}
\hline 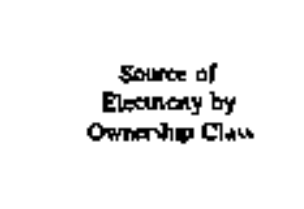 & 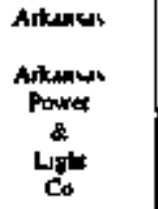 & 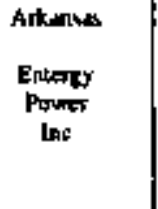 & $\begin{array}{l}\text { Calbevrtus } \\
\text { Paphe } \\
\text { Gin \& } \\
\text { Ekectre } \\
\text { Co }\end{array}$ & 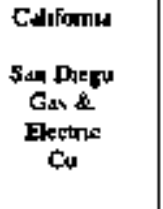 & 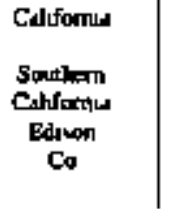 & $\begin{array}{l}\text { Colordido } \\
\text { Poblic } \\
\text { Samo: } \\
\text { Co of } \\
\text { Coloredo }\end{array}$ & 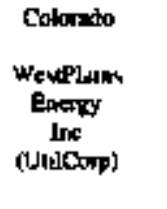 \\
\hline 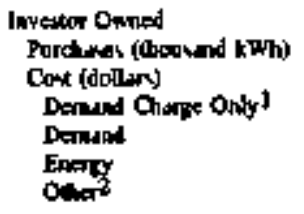 & $\begin{array}{r}3,563,932 \\
219,931,678 \\
- \\
197,117,703 \\
26,644,175 \\
-\end{array}$ & $\begin{array}{l}115,098 \\
1.944,255 \\
- \\
- \\
1.444,255 \\
-\end{array}$ & $\begin{array}{l}690,4013 \\
27,957,528 \\
- \\
2,992,406 \% \\
24,961,464 \\
-\end{array}$ & $\begin{array}{l}1,017,605 \\
11,, 694,582 \\
\overrightarrow{64,985,0801} \\
52,08,501 \\
-\end{array}$ & $\begin{array}{r}1,750,625 \\
n, 700,088 \\
16,919,500 \\
24,255,844 \\
12,517,255 \\
7,489\end{array}$ & $\begin{array}{r}1,557,952 \\
61.196,369 \\
- \\
33,397,515 \\
26,711,274 \\
657,244\end{array}$ & $\begin{array}{r}987,941 \\
35.311 .5100 \\
180 \\
18,593.200 \\
19.649,843 \\
-2.971,803\end{array}$ \\
\hline 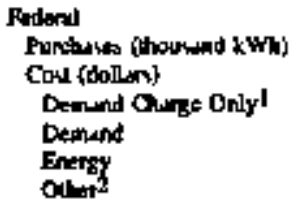 & 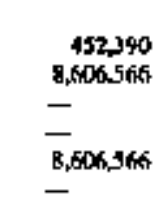 & $\begin{array}{l}- \\
\overline{-} \\
\overline{-} \\
-\end{array}$ & $\begin{array}{r}32,250 \\
10,295,517 \\
-\quad \\
10,29,276 \\
-\end{array}$ & $\begin{array}{l}\quad 385,071 \\
7,114,744 \\
- \\
- \\
7,104,744 \\
-\end{array}$ & $\begin{array}{r}1,127,737 \\
6,345.789 \\
- \\
13,528,919 \\
55,825,880 \\
-1189,010\end{array}$ & $\begin{array}{l}15,550 \\
-75.775 \\
- \\
-255,229 \\
=179,454\end{array}$ & $\begin{array}{l}z \\
z \\
= \\
=\end{array}$ \\
\hline 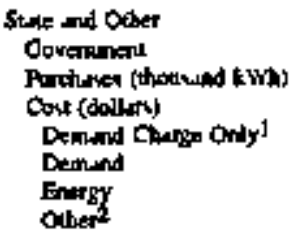 & $\begin{array}{l}\bar{z} \\
\overline{-} \\
\overline{-}\end{array}$ & $\begin{array}{l}- \\
\ddot{-} \\
\overline{-} \\
-\end{array}$ & $\begin{array}{l}1,045,196 \\
39,643,473 \\
- \\
676,598 \\
38,966,875 \\
-\end{array}$ & $\begin{array}{l}1,840,343 \\
42,135,511 \\
- \\
3,575.1109 \\
38,760,111 \\
-\end{array}$ & 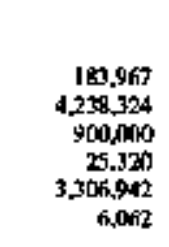 & $\begin{array}{l}1,146,727 \\
61,757,863 \\
- \\
42,821,0007 \\
18,929,461] \\
-\end{array}$ & $\begin{array}{l}= \\
= \\
= \\
=\end{array}$ \\
\hline 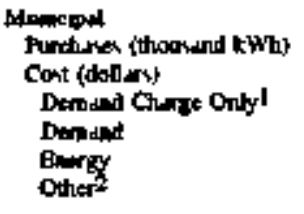 & $-_{-}^{-4,213}$ & $\begin{array}{l}- \\
- \\
- \\
-\end{array}$ & $\begin{array}{l}73,852 \\
5,66 !, 613 \\
- \\
1,464,012 \\
4,193,661 \\
=\end{array}$ & $\begin{array}{r}\$ 26,096 \\
12,411,7] 1 \\
- \\
3,721,516 \\
3,690,255 \\
-\end{array}$ & 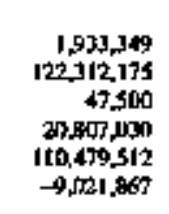 & $\begin{array}{r}5,874 \\
53,641 \\
-\quad \\
40,0199 \\
50563 \\
-12,971\end{array}$ & 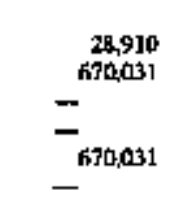 \\
\hline 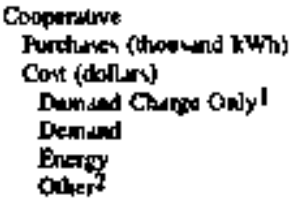 & $\begin{array}{l}2,170,949 \\
41,750,599 \\
- \\
41,750,599 \\
-\end{array}$ & $\begin{array}{l}- \\
\overline{-} \\
\overline{-} \\
-\end{array}$ & $\begin{array}{l}174,947 \\
4,0 \text { ơ } 1,979 \\
- \\
\overline{4,071,978} \\
-\end{array}$ & $\begin{array}{r}44,246 \\
9,113,160 \\
- \\
4,752,000 \\
5,061,160 \\
-\end{array}$ & $\begin{array}{r}10,550 \\
20 \%, 343 \\
-\quad \\
=\quad 190,543 \\
10,8101\end{array}$ & $\begin{array}{r}7,689,5,56 \\
146,426,263 \\
- \\
91,204,969 \\
19,369,916 \\
51,378\end{array}$ & $-_{-}^{121,200}$ \\
\hline 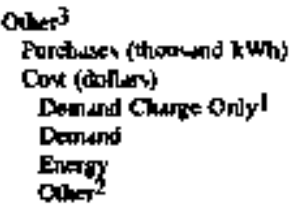 & $\begin{array}{l}3,0898,647 \\
58,054,018 \\
- \\
- \\
58,1054,18 \\
-\end{array}$ & $\begin{array}{l}\overline{-} \\
\bar{z} \\
\bar{z}\end{array}$ & $\begin{array}{r}22,350,057 \\
1,721,129,338 \\
5, \\
519,296,807 \\
1,201,832,731 \\
-\end{array}$ & $\begin{array}{l}3,319,797 \\
132,636,935 \\
- \\
68,188,364 \\
z+425,5+1 \\
-\end{array}$ & 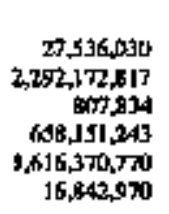 & $\begin{array}{r}2,116,148 \\
144,389,512 \\
- \\
109,018,412 \\
35,779,492 \\
608\end{array}$ & $\begin{array}{l}- \\
z \\
z \\
-\end{array}$ \\
\hline 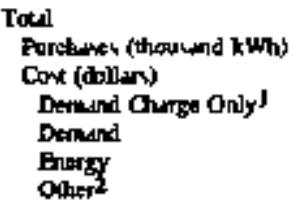 & $\begin{array}{l}9,212,580 \\
326,376,658 \\
- \\
193,317,703 \\
135, \sqrt{100,955} \\
-\end{array}$ & $\begin{array}{l}1,5,098 \\
1,944,255 \\
- \\
- \\
1,944,255\end{array}$ & $\begin{array}{r}25,958,309 \\
1,80 \%, 799,75 ? \\
- \\
524,438,757 \\
1,264,320,956 \\
-\end{array}$ & $\begin{array}{l}9,595,158 \\
341,75,303 \\
-\overline{145,001,961} \\
196,759,342 \\
-\end{array}$ & $\begin{array}{r}32,542,250 \\
2,561,978,536 \\
18,674,314 \\
316,368,356 \\
1,818,698,902 \\
7,734,444\end{array}$ & $\begin{array}{r}8,311,787 \\
414,099,422 \\
- \\
282,695,205 \\
130,697,391 \\
706,840\end{array}$ & $\begin{array}{r}1,021,525 \\
36,102,736 \\
180 \\
18,393,280 \\
20,491,6181 \\
-2,971.803\end{array}$ \\
\hline
\end{tabular}

Sot notes and frodrotes ix end of table 
Tabłe 19. Dectrictity Purchasea by Irvestor-Owned Utilities, by State, 1994 (Contlnned)

\begin{tabular}{|c|c|c|c|c|c|c|c|}
\hline $\begin{array}{c}\text { Somite of } \\
\text { Electrictsy by } \\
\text { Onmersity Cins }\end{array}$ & 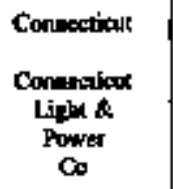 & 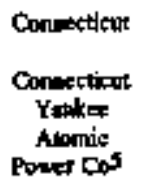 & 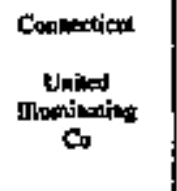 & 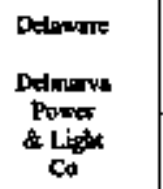 & 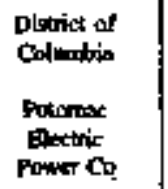 & 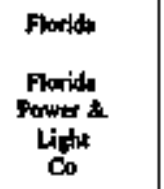 & 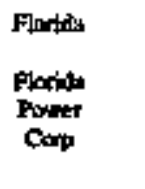 \\
\hline \multicolumn{8}{|l|}{ Inwerdor-Owined } \\
\hline Pontuess (thosind rtwh) & $3,9005,052$ & - & 44,050 & $2,725,360$ & 3,012,3]3 & $9 \times 172$ & 5,500 \\
\hline 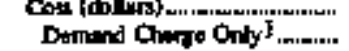 & $\begin{array}{l}185,582,735 \\
-\end{array}$ & - & $\begin{array}{l}20.700,074 \\
-\end{array}$ & $65,1,853,433$ & $17,7.10,957$ & - & - \\
\hline Demed d & - & - & $16,593,685$ & 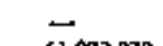 & 260s任 & - & $4,655,423$ \\
\hline 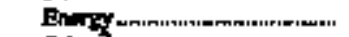 & $43,966,528$ & - & 4.J06, Ing & $6], 693,1033$ & 4272806 & $16.955 .70 \mathrm{~s}$ & $17,081,874$ \\
\hline 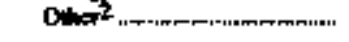 & $10,615,967$ & - & & - & $122,2 \times 5,591$ & - & 一 \\
\hline \multicolumn{8}{|l|}{ Fodall } \\
\hline Pultiven (thousund IWh) & - & - & - & - & - & - & 44,47 \\
\hline Cod (dollars) & - & - & - & - & - & - & $4 \$ 9,436$ \\
\hline Demined Chacga Only] & - & - & 一 & - & - & r & - \\
\hline 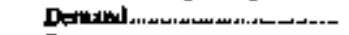 & - & - & - & - & - & - & - \\
\hline Bperty & - & - & - & - & - & - & 40,430 \\
\hline 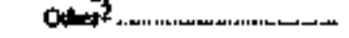 & - & - & - & - & $\leftarrow$ & - & - \\
\hline \multicolumn{8}{|l|}{ Sinte und Other } \\
\hline Punturses (thoumd twh) & 473,813 & - & בד, & - & - & $1,0 \$ 6,206$ & 一 \\
\hline Cod (dbllow) & $36,931,569$ & - & 63245 & - & - & $43,115,396$ & - \\
\hline Denend Chinge Orby' & - & - & - & - & - & - & - \\
\hline 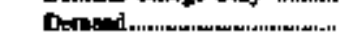 & $\rightarrow$ & - & - & - & - & 23,464060 & - \\
\hline 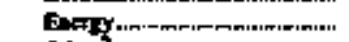 & $36,723,333$ & - & 67,745 & - & - & $19,709,316$ & - \\
\hline 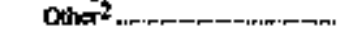 & $2 \pi, 726$ & - & - & - & 一 & - & - \\
\hline \multicolumn{8}{|l|}{ Metietpu } \\
\hline Ponthess (Unowsand KWh) & $20 \times 553$ & - & - & 41,797 & - & $1,490,450$ & $1,001,973$ \\
\hline 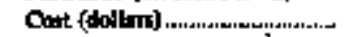 & $13,059,815$ & - & - & 1,898,750 & - & 134375.915 & +2516919 \\
\hline Derond Chere Only? & - & - & - & - & - & $=$ & $=$ \\
\hline 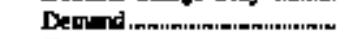 & - & - & - & - & - . & $81,54,1356$ & $17,491,208$ \\
\hline 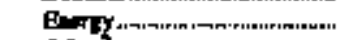 & $13,059,915$ & - & - & $1.896,6,1]$ & - & $32,550,899$ & $25,105,691$ \\
\hline 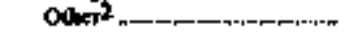 & - & - & 一 & 2,117 & - & 200,660 & - \\
\hline \multicolumn{8}{|l|}{ Coopertive } \\
\hline Panthen (thouand kWh) & - & - & - & - & - & 7n6s; & 41, 918 \\
\hline 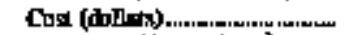 & - & - & - & - & 5 sax,9015 & $14,428,208$ & 7511900 \\
\hline Demand Chare Onfy] & - & - & - & - & - & - & 一 \\
\hline Defordind_us.--ー-_-ーーー & - & - & - & $\longrightarrow$ & 一 & - & - \\
\hline 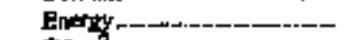 & - & - & - & - & - & $14,427,525$ & $7,511,090$ \\
\hline 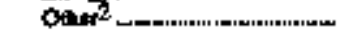 & 一 & - & - & - & $5,538,946$ & 기 & - \\
\hline \multicolumn{8}{|l|}{ ouker } \\
\hline 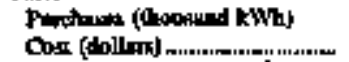 & $\begin{array}{r}10,321,248 \\
373,693,463\end{array}$ & - & $\begin{array}{r}1,222,533 \\
53,723,283\end{array}$ & $\begin{array}{r}999,402 \\
25,613,115\end{array}$ & 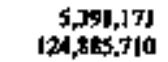 & $\begin{array}{r}9,710,451 \\
04,725,342\end{array}$ & 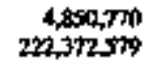 \\
\hline Dering Chare Dely' & - & - & - & - & - & - & - \\
\hline 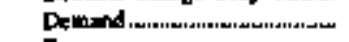 & - & - & $28,378,784$ & 314,545 & - & $299,237,128$ & II5,0gen,790 \\
\hline 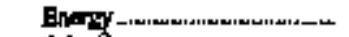 & $370,699,155$ & - & $25,54,500$ & $22,003,036$ & 110,540 ,fitil & 175, D68,201 & $101,251,763$ \\
\hline 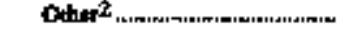 & 55,054 und & 十 & 一 & $7,765.491$ & 14,24050 & - & - \\
\hline \multicolumn{8}{|l|}{ Totnl } \\
\hline 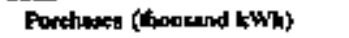 & $J 4,906,6,54$ & - & $1,657,683$ & 3.766 .1145 & $8,40,484$ & $15,90,2,191$ & $6,889,790$ \\
\hline Con (dodlen) & $571,2616,672$ & - & $74,490,706$ & $89,474,09$ & $301,216,572$ & $6+3,260,54$ & $294,650,123$ \\
\hline Demand Dhrtye Only], & - & - & 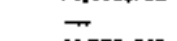 & - & - & - & - \\
\hline 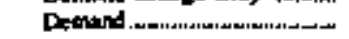 & - & - & $4,772,66$ & 314,548 & 262560 & $40,257,554$ & 137.JSt.41 \\
\hline 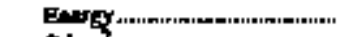 & $414,308,03\rfloor$ & - & $29,710,037$ & $05,792,742$ & $158,756,46$ & 2re,7yוfits & $137,500,642$ \\
\hline 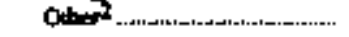 & IS6,8T,84] & - & - & $3.367,614$ & 142.16x<本 & 201.371 & - \\
\hline
\end{tabular}

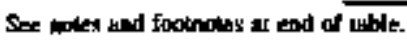


Table 19. Eaectricity Purchases by investor-Owned Utitties, by Sinte, 1994 (Continued)

\begin{tabular}{|c|c|c|c|c|c|c|c|}
\hline $\begin{array}{c}\text { Sounes of } \\
\text { Exethtity by } \\
\text { Omberstip Cliay }\end{array}$ & $\begin{array}{l}\text { Plotid } \\
\text { Gnip } \\
\text { Cow }\end{array}$ & $\begin{array}{l}\text { Fotide } \\
\text { Tumps } \\
\text { Eletic } \\
\text { Ca }\end{array}$ & $\begin{array}{l}\text { Domigin } \\
\text { Geargin } \\
\text { Pomer } \\
\text { Co }\end{array}$ & 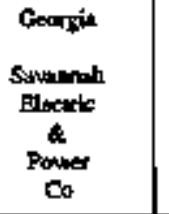 & $\begin{array}{l}\text { Himmil } \\
\text { Ctizizens } \\
\text { Utibities } \\
\mathrm{Co}^{4}\end{array}$ & 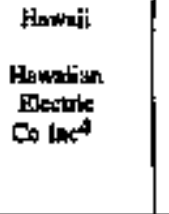 & 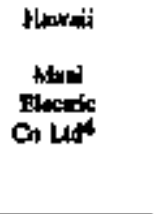 \\
\hline 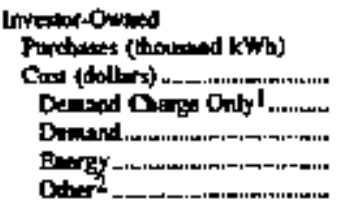 & $\begin{array}{l}88,767 \\
2,364,962 \\
- \\
- \\
2,364,962\end{array}$ & $\begin{array}{r}109,821 \\
17,688,539 \\
\overrightarrow{11,208,490} \\
4,41,144 \\
36,515\end{array}$ & $\begin{array}{l}2,72,627 \\
90,044,767 \\
- \\
- \\
-\end{array}$ & $\begin{array}{l}\quad 22,812 \\
-\quad 62,6 \$ 4 \\
- \\
-\end{array}$ & $\begin{array}{l}- \\
- \\
= \\
-\end{array}$ & $\begin{array}{l}- \\
- \\
- \\
-\end{array}$ & $\begin{array}{l}\bar{z} \\
\bar{z} \\
\bar{z}\end{array}$ \\
\hline 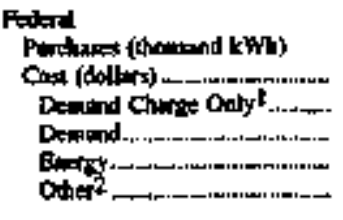 & $=^{-} \begin{array}{l}162,761 \\
168,413\end{array}$ & $\begin{array}{l}- \\
\overline{-} \\
\overline{-} \\
-\end{array}$ & 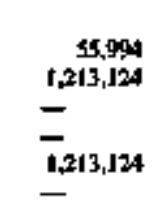 & $\begin{array}{l}2800 \\
- \\
-\end{array}$ & $\begin{array}{l}\bar{z} \\
\bar{z} \\
\bar{z}\end{array}$ & $\begin{array}{l}- \\
\overline{-} \\
\overline{-} \\
-\end{array}$ & $\begin{array}{l}z \\
z \\
z \\
-\end{array}$ \\
\hline 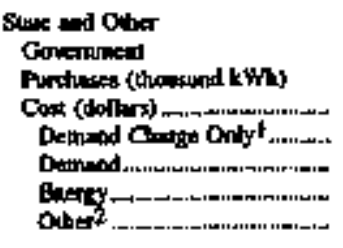 & $\begin{array}{r}\quad 281 \\
-\quad \begin{array}{r}6,320 \\
-\end{array}\end{array}$ & $\begin{array}{l}- \\
\overline{-} \\
\bar{z} \\
-\end{array}$ & $\begin{array}{l}1,531,906 \\
6,1+9,396 \\
- \\
119,4,35,670 \\
-23,246,274\end{array}$ & $\begin{array}{r}106 \\
-\quad \begin{array}{r}2397 \\
-\quad 2397\end{array}\end{array}$ & $\begin{array}{l}\bar{z} \\
= \\
\bar{z}\end{array}$ & $\begin{array}{l}- \\
- \\
- \\
-\end{array}$ & $\begin{array}{l}\bar{z} \\
\bar{z} \\
\bar{z}\end{array}$ \\
\hline 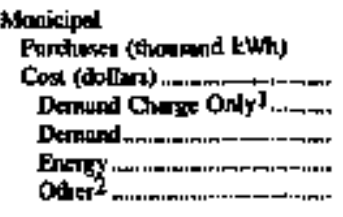 & $=^{-1.164}$ & 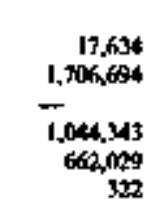 & 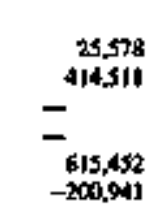 & $\begin{array}{r}139 \\
-\quad \begin{array}{r}5,412 \\
-\end{array} \\
-5,442\end{array}$ & $\begin{array}{l}- \\
\overline{-} \\
\overline{-} \\
-\end{array}$ & $\begin{array}{l}- \\
- \\
- \\
-\end{array}$ & $\begin{array}{l}- \\
\overline{-} \\
\overline{-} \\
-\end{array}$ \\
\hline 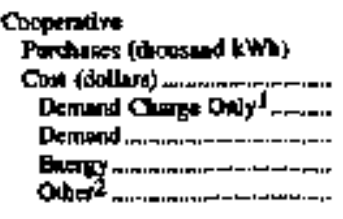 & $\begin{array}{l}12,119 \\
-\quad 1980900 \\
- \\
-\end{array}$ & $\begin{array}{r}7,876 \\
259,494 \\
-\quad 17,407 \\
238,965 \\
3,632\end{array}$ & $\begin{array}{l}2.04040 \\
75,912962 \\
= \\
75,912.962 \\
=\end{array}$ & ${ }^{-} \begin{array}{r}843 \\
-15,199\end{array}$ & $\begin{array}{l}- \\
- \\
- \\
-\end{array}$ & $\begin{array}{l}- \\
- \\
- \\
-\end{array}$ & $\begin{array}{l}- \\
= \\
= \\
-\end{array}$ \\
\hline 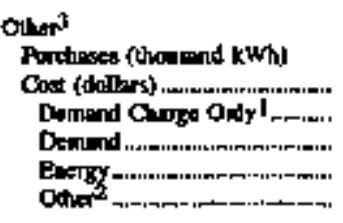 & $\begin{array}{l}1,277,329 \\
29,627,620 \\
= \\
6,111,912 \\
21,715,718 \\
=\end{array}$ & $\begin{array}{l}459.168 \\
50,782,479 \\
- \\
6,483,330 \\
7,299,149 \\
-\end{array}$ & 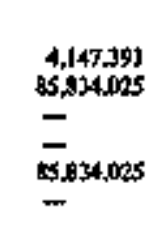 & $\begin{array}{l}2,491,578 \\
\$ 6,060,125 \\
\overline{2} \\
2,147,7719 \\
53,912,355 \\
-\end{array}$ & $\begin{array}{l}75,297 \\
5,794,504 \\
- \\
2,156,100 \\
3,638,594 \\
-\end{array}$ & $\begin{array}{l}2,977,412 \\
21,137,499 \\
- \\
136,125,334 \\
193,212,165\end{array}$ & $\begin{array}{l}102,002 \\
7,501,167 \\
- \\
2,101,558 \\
5,482,209 \\
-\end{array}$ \\
\hline 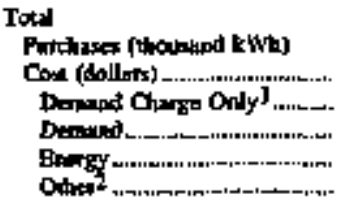 & $\begin{array}{l}1,4100,093 \\
02,579,693 \\
- \\
6,111,992 \\
26,457,791 \\
-\end{array}$ & $\begin{array}{r}594,499 \\
33,436,716 \\
- \\
24751,570 \\
12,640,687 \\
42,459\end{array}$ & $\begin{array}{l}11,169,500 \\
149,608,785 \\
- \\
- \\
-20,05,10,100 \\
-23,49,215\end{array}$ & $\begin{array}{l}2,962,798 \\
56,436,500 \\
- \\
2,20,7100 \\
54,688,730 \\
-\end{array}$ & $\begin{array}{r}75,290 \\
5,794,504 \\
- \\
2,156,1000 \\
3,658,504 \\
-\end{array}$ & $\begin{array}{l}2,977,412 \\
23,397,499 \\
= \\
-\overline{126,725,534} \\
107,212,165\end{array}$ & $\begin{array}{l}102,192 \\
7,584,167 \\
- \\
2,101,058 \\
5,412,20 \% \\
-\end{array}$ \\
\hline
\end{tabular}

Sue notes and fookonem th end of trble. 
Table 19. Electricity Purchases by Investor-Owaed Utilities, by State, 1994 (Continued)

\begin{tabular}{|c|c|c|c|c|c|c|c|}
\hline 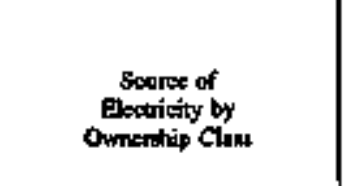 & 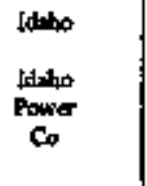 & 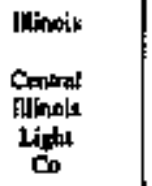 & 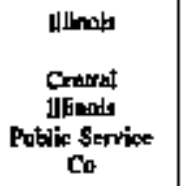 & 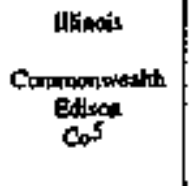 & $\begin{array}{l}\text { Dinoth } \\
\text { Eletric } \\
\text { Energy } \\
\text { Inc }\end{array}$ & $\begin{array}{l}\text { Minats } \\
\text { Illinels } \\
\text { Porwe } \\
\text { Co }\end{array}$ & $\begin{array}{l}\text { Ulinodr } \\
\text { Mt Cunnel } \\
\text { Prblic } \\
\text { Utiliny } \\
\text { Co }\end{array}$ \\
\hline 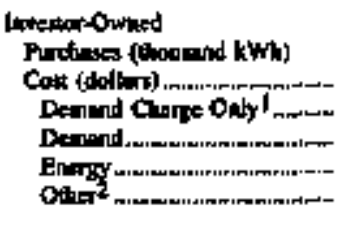 & $\begin{array}{l}1,177,087 \\
28,796,062 \\
\overline{-2,190, j 16} \\
28.176,378 \\
-\end{array}$ & $\begin{array}{l}32 t, 117 \\
6,807,110 \\
- \\
3,595,100 \\
4,912,010 \\
-\end{array}$ & $\begin{array}{l}3,059,097 \\
4,096,526 \\
- \\
6,121,244 \\
2,972,282 \\
-\end{array}$ & 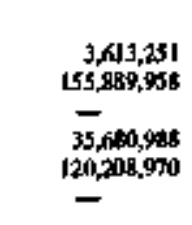 & $\begin{array}{l}\$, 022,169 \\
124,740,261 \\
- \\
- \\
124,740,261 \\
=\end{array}$ & $\begin{array}{l}2,677,057 \\
41,734,287 \\
- \\
5,126,477 \\
36,617,810 \\
-\end{array}$ & $\begin{array}{l}191,156 \\
6,710,617 \\
- \\
3,300,516 \\
3,410,301 \\
-\end{array}$ \\
\hline 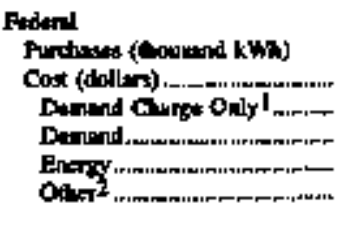 & 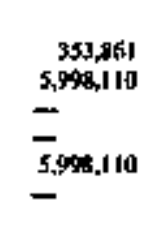 & $\begin{array}{l}\bar{z} \\
\overline{-} \\
\bar{z}\end{array}$ & $\begin{array}{l}1,17,969 \\
3,911,620 \\
- \\
100,0004 \\
3,811,608 \\
-\end{array}$ & $\begin{array}{l}= \\
= \\
= \\
=\end{array}$ & 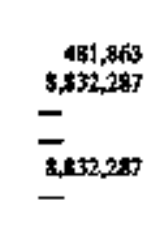 & $\begin{array}{r}239,16 \% \\
6,103,357 \\
m \\
102,000 \\
5,951,353 \\
-\end{array}$ & $\begin{array}{l}z \\
z \\
z \\
=\end{array}$ \\
\hline 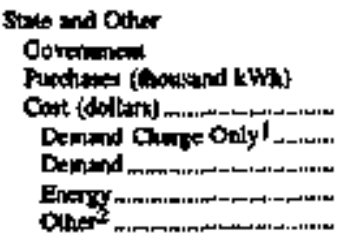 & 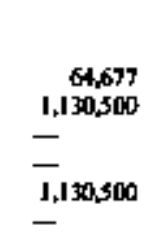 & $\begin{array}{l}\overline{-} \\
\overline{-} \\
\overline{-}\end{array}$ & $\begin{array}{l}- \\
- \\
- \\
=\end{array}$ & $\begin{array}{l}\overline{-} \\
\overline{-} \\
\overline{-} \\
-\end{array}$ & $\begin{array}{l}\overline{-} \\
\overline{-} \\
\overline{-}\end{array}$ & $\begin{array}{l}= \\
\bar{z} \\
\overline{-} \\
=\end{array}$ & $\begin{array}{l}z \\
z \\
- \\
-\end{array}$ \\
\hline 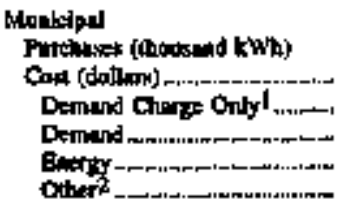 & $\begin{array}{l}\text { 47,660 } \\
-951.858 \\
- \\
-951.858\end{array}$ & 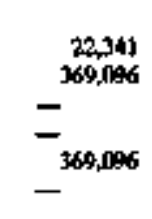 & $\begin{array}{r}16,096 \\
-292,460 \\
-\quad \begin{aligned} 14,400 \\
278,060\end{aligned}\end{array}$ & $=^{5,2,400}$ & $\begin{array}{l}- \\
z \\
z \\
-\end{array}$ & 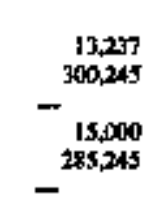 & $\begin{array}{l}- \\
\overline{-} \\
\overline{-} \\
-\end{array}$ \\
\hline 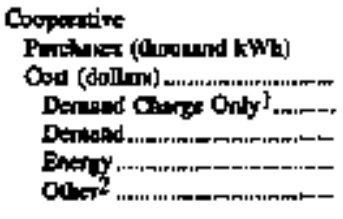 & 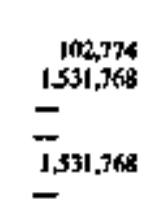 & $\begin{array}{l}z \\
\bar{z} \\
\bar{z}\end{array}$ & $\begin{array}{r}53,100 \\
1,013,117 \\
-\quad 10,000 \\
1,000,117 \\
-\end{array}$ & $\begin{array}{l}\bar{z} \\
\bar{z} \\
\bar{z}\end{array}$ & $\begin{array}{l}\bar{z} \\
\bar{z} \\
\overline{-}\end{array}$ & $\begin{array}{r}180,923 \\
1,924,419 \\
- \\
264,100 \\
3,600,709 \\
-\end{array}$ & $\begin{array}{l}- \\
z \\
z \\
-\end{array}$ \\
\hline 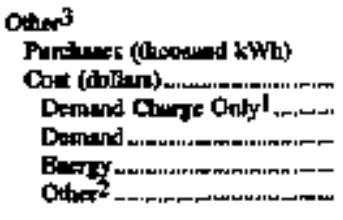 & $\begin{array}{l}5,3,395 \\
24,704,670 \\
- \\
2,194,341 \\
2,590,325 \\
-\end{array}$ & $\begin{array}{r}J 1,186 \\
272,43] \\
-\quad 85,804 \\
-\quad 190,6127\end{array}$ & $\begin{array}{r}36,597 \\
1,174,326 \\
-\quad \\
16,000 \\
1,194,326 \\
-\end{array}$ & 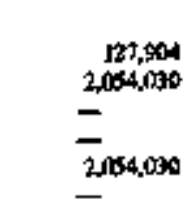 & $\begin{array}{l}-230,926 \\
- \\
= \\
250,926\end{array}$ & 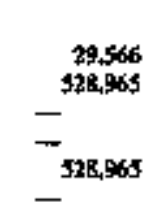 & $\begin{array}{l}\bar{z} \\
\overline{-} \\
\overline{-}\end{array}$ \\
\hline 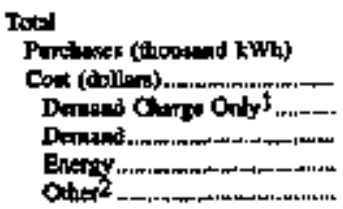 & $\begin{array}{l}2,278,454 \\
60,192,9,16 \\
- \\
-135,975 \\
60,178,943 \\
-\end{array}$ & $\begin{array}{l}75,4,146 \\
9,448,637 \\
- \\
3,976,904 \\
3,471,730 \\
-\end{array}$ & $\begin{array}{l}3,3,00,848 \\
55,490,066 \\
- \\
6,321,644 \\
49,168,42 \\
-\end{array}$ & $\begin{array}{l}3,743,675 \\
5,57.994,346 \\
\overline{35,600,948} \\
122,313,400 \\
-\end{array}$ & 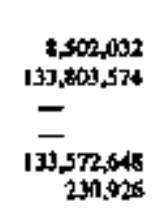 & 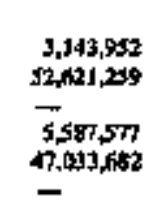 & $\begin{array}{l}191,156 \\
6,710,617 \\
- \\
3,300,316 \\
7,410,301 \\
-\end{array}$ \\
\hline
\end{tabular}

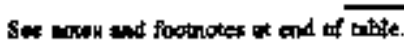


Table 19. Electricity Purchnses by Investor-Owned Utilities, by State, 1994 (Conlinued)

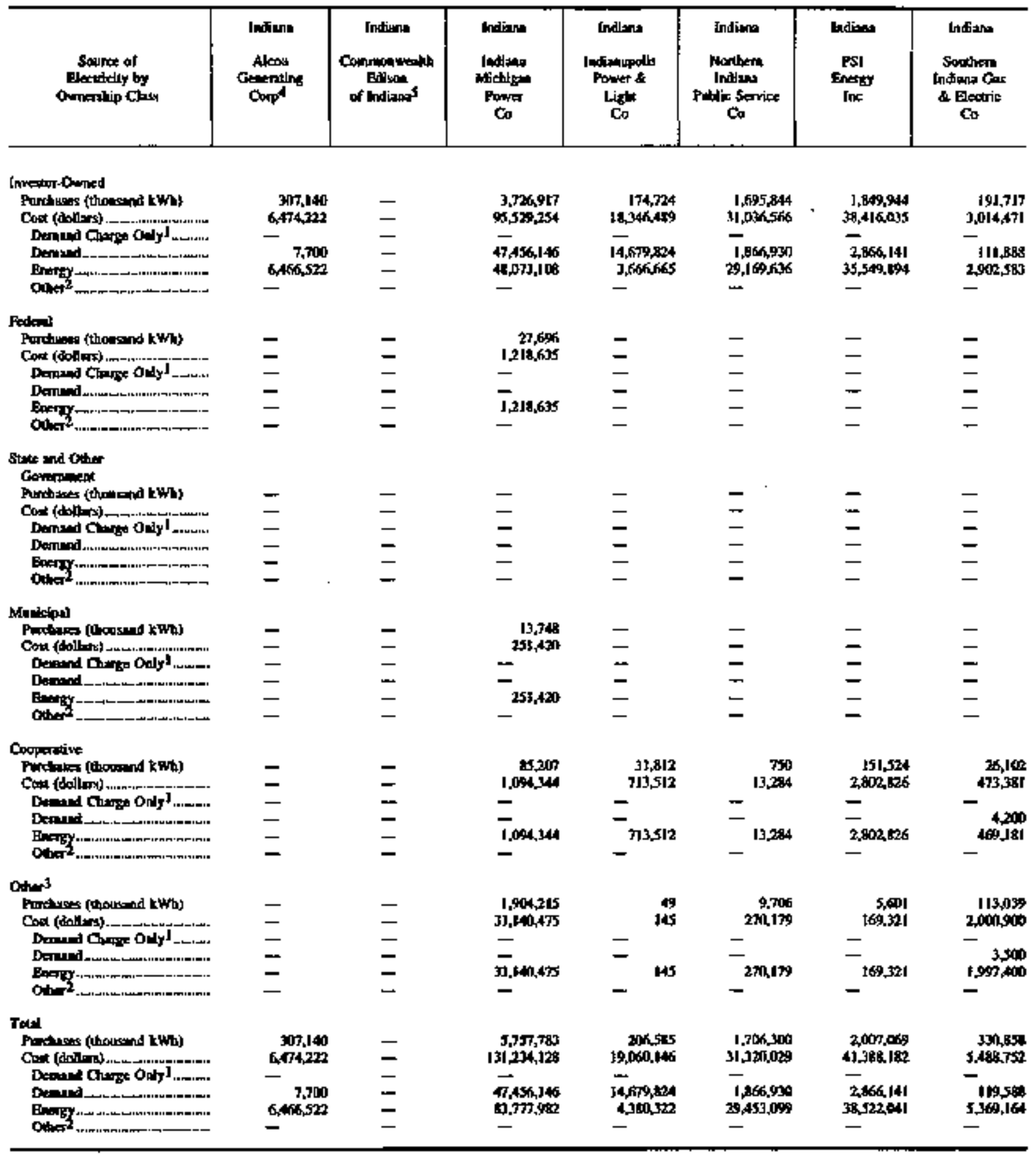

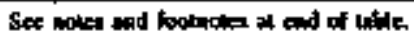


Table 19. Electricity Purchases by Investor-Omned Utilities, by State, 1994 (Confinued)

\begin{tabular}{|c|c|c|c|c|c|c|c|}
\hline 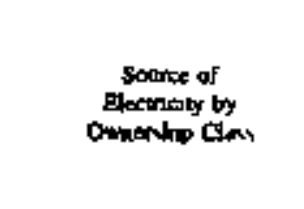 & 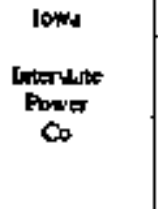 & $\begin{array}{c}\text { lowd } \\
\text { Tes } \\
\text { Utulues } \\
\text { the }\end{array}$ & 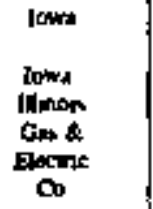 & 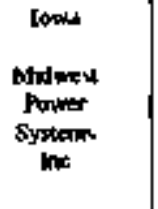 & 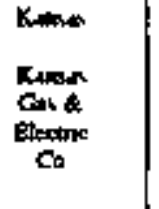 & 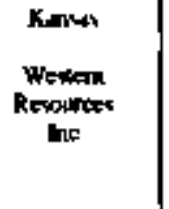 & 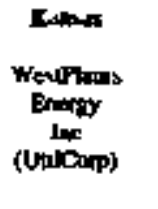 \\
\hline \multicolumn{8}{|l|}{ Ineter Durned } \\
\hline Pordhen (thomend kWh) & $1,837,204$ & $1,6,7,92]$ & 510,977 & $254,3,32$ & 245, ,ifit 6 & 82,362 & J15,46] \\
\hline 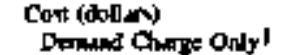 & $\begin{array}{l}50,263,1998 \\
-\end{array}$ & $\begin{array}{l}46,644.149 \\
-\end{array}$ & $\frac{9,279,665}{-}$ & $4,221.223$ & $4,296,419$ & $1.585,614$ & $2,304,085$ \\
\hline Demind & $24,567,000$ & 1E.241,3N & - & - & $=$ & $\vec{a}$ & 37,036 \\
\hline Energy & $24,675,167$ & $28,410,443$ & $97279,6 \mathrm{Kr}$ & $4,221,223$ & 4,296419 & $1.5885,4.14$ & $2259,70 \%$ \\
\hline Oaher 2 & $1,021,0 \mathrm{II}$ & - & - & - & - & $\rightarrow$ & $-2,247$ \\
\hline \multicolumn{8}{|l|}{ Federal } \\
\hline 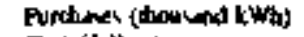 & 39.960 & If0,561 & - & 151.117 & - & - & - \\
\hline Cont (dollwr) & $65 h 442$ & $1,672,587$ & - & $2,609,089$ & - & - & - \\
\hline Dariand Orye Otyrl & - & - & - & - & - & - & - \\
\hline Demand & - & $=$ & - & $=$ & - & - & - \\
\hline Energy & 656,642 & $1,672,587$ & - & 2,609 , dos & - & - & - \\
\hline $0 a_{k} x^{2}$ & - & - & - & - & - & - & - \\
\hline \multicolumn{8}{|l|}{$\begin{array}{l}\text { Stut ant Other } \\
\text { Governmank }\end{array}$} \\
\hline Prrchas (thourand lWWh) & 4,947 & 95,271 & 20,292 & $1,534,429$ & 6,k45 & $2 \pi, 100$ & - \\
\hline 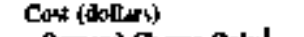 & 8B,295 & I,424,09\% & 388,006 & $119,228,906$ & 100,013 & $3,4[1,93]$ & - \\
\hline Demend Chege Only' & - & - & - & $1,380,000$ & - & - & - \\
\hline Dernand & & - & - & 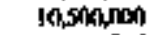 & - & - & - \\
\hline Energy & 89,298 & $1,428,096$ & 388,006 & $10,971,726$ & lino, 013 & $3,411,93]$ & - \\
\hline$O \mathrm{um}^{2}$ & & $\rightarrow$ & - & $90, \pi 7,1000$ & - & - & - \\
\hline \multicolumn{8}{|l|}{ Minnetsed } \\
\hline Furchiran (thoound kWh) & 3,232 & 65,980 & 11, 133 & 364,835 & - & 3,268 & - \\
\hline Cost (dollors) & 78,919 & $1,852,635$ & 221,584 & $3.312,768$ & - & $1,932,372$ & - \\
\hline Dembend Ghurge Odyl & $\$ 0,000$ & 1807,000 & - & h\$n, 000 & - & - & 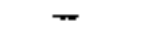 \\
\hline Demond & & 379.652 & - & 996,000 & - & $1,385,90 \pi$ & - \\
\hline Enerty & 48.919 & $1,314,346$ & 221,584 & $2,656,768$ & - & 346,455 & - \\
\hline anter & $\rightarrow$ & $-21,360$ & - & - & - & $\rightarrow$ & - \\
\hline \multicolumn{8}{|l|}{ Conparbye } \\
\hline 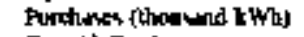 & $365,09 \%$ & 679.894 & 186,279 & 675,974 & I何.528 & 69 & 606,492 \\
\hline 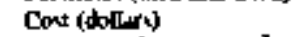 & $7,252.222$ & $32,325,474$ & 3004.286 & $11,2 h 5,157$ & 1,4 促, 49 & 1.420 & $10,105,008$ \\
\hline Demand Charge Oaly I & - & 233.678 & - & $10,1 \% 6$ & - & - & - \\
\hline Demand & $2,173,617$ & - & - & $1,3] 6,693$ & - & - & - \\
\hline Eneroy & $4,623, \mathrm{~mm}$ & $12,161,616$ & $3,0174,285$ & $10,059,268$ & $1,468,499$ & 1,420 & Jil. $105,0 \mathrm{ass}$ \\
\hline Othos ${ }^{2}$ & 455,528 & โชด & - & - & - & - & - \\
\hline \multicolumn{8}{|l|}{ Oture ${ }^{3}$} \\
\hline Porthanes (Itoctand KWh) & $m$ & 51,097 & 71,912 & 45,257 & 61,0m7 & 9,716 & - \\
\hline $\cos (\operatorname{dos} \theta \sin )$ & - & $4,671,493$ & 517,57 & $-2,344,2,0$ & 1.278 .678 & $2) 2,360$ & - \\
\hline 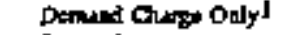 & - & - & - & 98,075 & - & - & - \\
\hline Derned & - & $3,982,860$ & - & 977,298 & - & - & - \\
\hline Eneryy & - & 090.613 & $517,57\}$ & 1,035048 & 1.278 .879 & $22+300$ & - \\
\hline 0 the & - & - & - & $-4,406,521$ & - & $\rightarrow$ & - \\
\hline \multicolumn{8}{|l|}{ Tand } \\
\hline Parthen (lboround \&Wh) & $2,250,442$ & $2,823,228$ & axpsy & $3,145,947$ & 391,316 & 372,015 & 721,943 \\
\hline Con (dollnorit) & $58,339,279$ & $64,794,434$ & 13 All, 113 & $34 B, 8,2,943$ & $7,343,809$ & 7.163 .757 & $12,469,153$ \\
\hline Denind Chety Orly' & 30,000 & 453,6775 & - & $2,1 \cap 8,271$ & - & - & $\rightarrow$ \\
\hline Deanind & $26,740,0,1]$ & $22, \mathrm{fMM}, 2 \mathrm{SB}$ & - & $13,189,991$ & $\rightarrow$ & $1,5 t 5,50 ?$ & 37,036 \\
\hline Enargy & $35,002,123$ & $45,757,701$ & ]$^{3}, 411,113$ & $34,343,022$ & $7.143,819$ & $5.377,810$ & $12,374,364$ \\
\hline Othent 2 & $1,476,519$ & $-25,183$ & - & $88,971,6,59$ & - & - & $-2,217$ \\
\hline
\end{tabular}

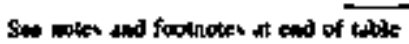


Table 19. Electricity Purchases by Investor-Omed Utilities, by State, 1994 (Continaed)

\begin{tabular}{|c|c|c|c|c|c|c|c|}
\hline 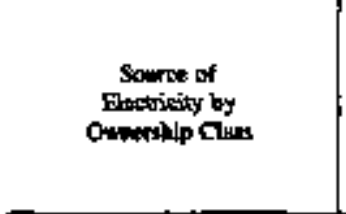 & $\begin{array}{l}\text { Kentecky } \\
\text { Kenuedy } \\
\text { Porrer } \\
\text { Co }\end{array}$ & 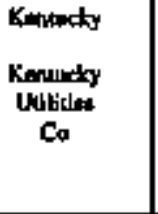 & 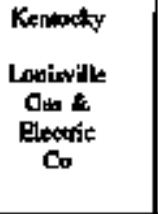 & 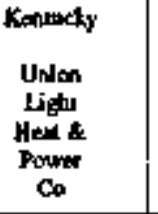 & $\begin{array}{l}\text { Loviximat } \\
\text { Condyat } \\
\text { Ola } \\
\text { Rinutios }\end{array}$ & 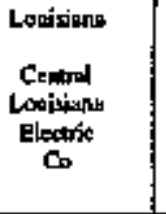 & $\begin{array}{l}\text { Louisima } \\
\text { Lonipimu } \\
\text { Powtr \& } \\
\text { Lijut } \\
\text { Co }\end{array}$ \\
\hline \multicolumn{8}{|l|}{ Ifrestor-Omed } \\
\hline Portheser (boosind kWh) & $2.743,413$ & 1,585,440 & 206,488 & 3,213,371 & - & 39,879 & $1,79.187$ \\
\hline 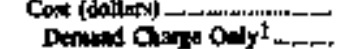 & $\frac{73,791,623}{-}$ & $39,854,968$ & $-3,64,127$ & $135.340,01018$ & $\bar{z}$ & $-^{644,467}$ & $\begin{array}{l}95,179,964 \\
-\end{array}$ \\
\hline Delled_-_C_. & $40,6 \mathrm{AB}, 90 \mathrm{NZ}$ & 21. 36,153 & 213 & $59,343,392$ & $=$ & 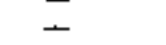 & $75,450,468$ \\
\hline 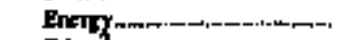 & $33,1+2,721$ & $18,474.502$ & $1,831,582$ & $89,162,984$ & - & $644,46 i 7$ & $19,729,496$ \\
\hline 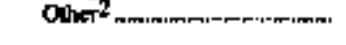 & - & II.673 & $1,817,332$ & $-13.186,368$ & - & - & - \\
\hline \multicolumn{8}{|l|}{ Fodenl } \\
\hline Potilines (1)ouvand kWh) & 10,247 & 28.445 & 113,044 & 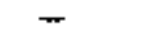 & - & - & 519,158 \\
\hline Cad & 450,530 & $1,110,320$ & 2866,708 & - & - & - & $11,782,8 \leq 0$ \\
\hline 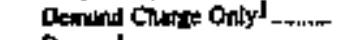 & - & - & - & - & - & - & - \\
\hline Demend & - & - & 2,066 & - & - & - & - \\
\hline 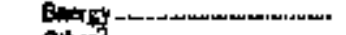 & 450,330 & $1,110,320$ & $2 \sqrt{5}+1,512$ & - & - & - & $11.782,650$ \\
\hline Oiher & - & - & - & - & - & - & - \\
\hline \multicolumn{8}{|l|}{ Sute and Othe } \\
\hline Purchioses (thooand kwth) & - & - & 160 & - & - & 73,640 & 73,627 \\
\hline 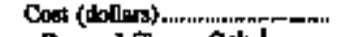 & - & - & 11,290 & - & - & J,B59,8:4 & $1,584,344$ \\
\hline 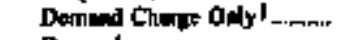 & - & - & - & - & - & - & - \\
\hline 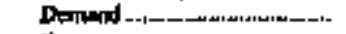 & - & - & - & $=$ & - & - & - \\
\hline Exeny & - & - & 11,200 & $m$ & - & $1,859,684$ & $1,584,344$ \\
\hline Other' & - & - & - & - & - & - & - \\
\hline \multicolumn{8}{|l|}{ Muripipd } \\
\hline 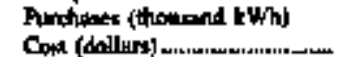 & 5,169 & $1,451,391$ & - & - & - & S.210 & \\
\hline Gopd (dollars) & 92,236 & $20,447,294$ & - & - & - & 202917 & $-111,968$ \\
\hline 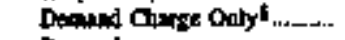 & - & - & - & - & - & $=$ & - \\
\hline 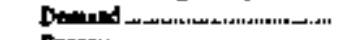 & - & $4,281,014$ & - & - & - & - & - \\
\hline Bnargy & $92,2 \mathrm{~B} 6$ & $16,164,192$ & - & - & - & 202,917 & $-10,968$ \\
\hline O1- & 一 & - & - & - & - & - & - \\
\hline \multicolumn{8}{|l|}{ Coopernatro } \\
\hline Punthares (thorind kith) & 9,587 & 1,621 & 220,200 & - & - & 165,73 & 369,324 \\
\hline 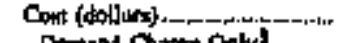 & 206,589 & 29,788 & $3,227,073$ & $m$ & - & $3.167,185$ & $30,522,182$ \\
\hline 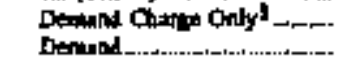 & - & - & - & - & - & - & - \\
\hline 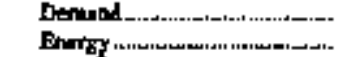 & - & - & 101.230 & - & 二 & T, & 10. \\
\hline 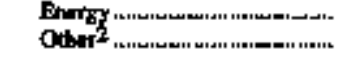 & 206,589 & 29.789 & $3,0,1,833$ & - & - & $3.167,005$ & 10.522 .182 \\
\hline \multirow{2}{*}{\multicolumn{8}{|c|}{$\theta_{h} \mathbf{a}^{3}$}} \\
\hline & & & & & & & \\
\hline & $1,109,680$ & - & - & - & - & 547,474 & 9,419,521 \\
\hline 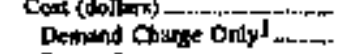 & $20,024,028$ & 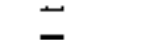 & 二 & - & - & $12,255,847$ & $24 T, 598.764$ \\
\hline 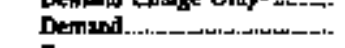 & - & $\overline{-}$ & $\overline{-}$ & $\overline{-}$ & $\bar{z}$ & $\overline{-}$ & 二 \\
\hline 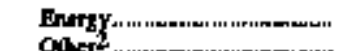 & $20,024,028$ & - & - & - & - & $\overline{11,379,2] 4}$ & 271] \\
\hline $\mathrm{Ol}^{2} \mathrm{~S}^{2} \ldots$ & - & - & - & - & - & 876,613 & $13,034,063$ \\
\hline \multicolumn{8}{|l|}{ Tat:l } \\
\hline Ponchusess (thoorind $\mathbf{t}$ Why & $3,869,396$ & 3,0669917 & 382942 & $3,21,931$ & - & 830,481 & 12334,826 \\
\hline & 94565.056 & $\frac{61,442,272}{-}$ & $9.754,1108$ & $135,740,008$ & - & $18,130,260$ & $366,364,136$ \\
\hline 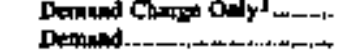 & $\overline{0, A B, 902}$ & $\overline{25} \times a+9.167$ & $-_{204509}$ & $\overline{59,743,392}$ & $\overline{-}$ & $\bar{z}$ & $\overline{35.450,46 t}$ \\
\hline & $53,016,154$ & $3 s_{1} 781,232$ & $1,352,227$ & $89,188,984$ & - & $\overline{17,253,627}$ & $20,139,415$ \\
\hline Oater & - & 11,875 & $1,817,312$ & $-35,186,768$ & - & BTh, 613 & $13,904,0 \times 3)$ \\
\hline
\end{tabular}

Set noter and lowlockes is and of tobles 
Table 19. Electricity Purchases by Investor-Owned Utllittes, by State, 1994 (Conthned)

\begin{tabular}{|c|c|c|c|c|c|c|c|}
\hline $\begin{array}{l}\text { Source of } \\
\text { Electrisicy by } \\
\text { Owerhip cless }\end{array}$ & $\begin{array}{l}\text { Lonielme } \\
\text { New Orieany } \\
\text { Putlic } \\
\text { Sarvice } \\
\text { Inc }\end{array}$ & $\begin{array}{l}\text { Locicisis } \\
\text { Somthwettem } \\
\text { Eloctric } \\
\text { Pownt } \\
\text { Co }\end{array}$ & 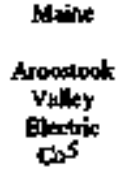 & 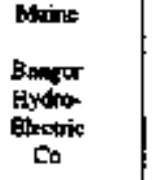 & 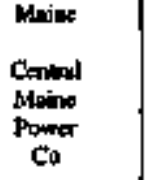 & 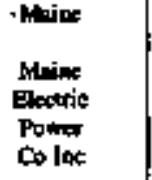 & $\begin{array}{l}\text { Mrin } \\
\text { Mrice } \\
\text { Public } \\
\text { Sorvice } \\
\text { Co }\end{array}$ \\
\hline \multicolumn{8}{|l|}{ Itprearomoned } \\
\hline 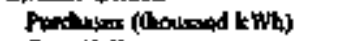 & $1,6 \neq 0,342$ & 72,975 & - & $1,152,897$ & $3,950,031$ & - & 39,814 \\
\hline 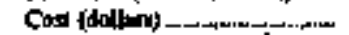 & J193,78s,776 & $1,891,664$ & - & $20,253,109$ & $118,3,32,237$ & - & $9,500,205$ \\
\hline Dempend Conge Onbl......... & - & - & - & - & - & - & - \\
\hline 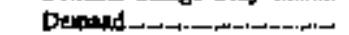 & $95,0 / 5,496$ & - & - & $11,201,678$ & - & - & - \\
\hline 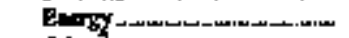 & $10,313,280$ & $1,847,259$ & - & $17,055,431$ & $36,461,500$ & - & $9,99,206$ \\
\hline 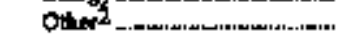 & - & 41.365 & - & - & $81,870,620$ & $\rightarrow$ & - \\
\hline \multicolumn{8}{|l|}{ Fedorl } \\
\hline 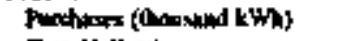 & 122,697 & - & - & - & - & - & - \\
\hline Call (Gollons) & $2,7,74,976$ & - & - & - & - & - & - \\
\hline 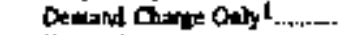 & - & - & - & - & - & - & - \\
\hline Dentand & - & - & - & - & - & - & - \\
\hline Enetry & $2,334,916$ & - & - & - & - & - & - \\
\hline 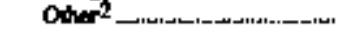 & - & - & - & - & - & - & - \\
\hline \multirow{2}{*}{\multicolumn{8}{|c|}{$\begin{array}{l}\text { Stule and Oatert } \\
\text { Gowemanent }\end{array}$}} \\
\hline & & & & & & & \\
\hline Purchrses (bround kWh) & - & 5.6508 & - & - & - & - & - \\
\hline 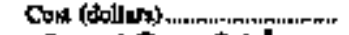 & - & 97,490 & - & $\rightarrow$ & - & - & - \\
\hline Demed Oherge Onlyl......... & - & - & - & - & $\rightarrow$ & - & - \\
\hline 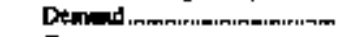 & - & - & - & - & - & - & - \\
\hline Enorly & - & 97.40 & - & - & - & - & - \\
\hline Dther & - & - & - & - & - & - & - \\
\hline \multicolumn{8}{|l|}{ Municetpul } \\
\hline Purdines (thoosind kMh) & 2 & 58,694 & - & - & 4754 & - & - \\
\hline 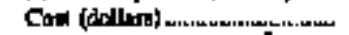 & $-2,1734$ & 909.825 & - & - & $\$ 95,174$ & - & - \\
\hline Decand Oharge Only' & - & - & $\leftarrow$ & - & - & - & - \\
\hline 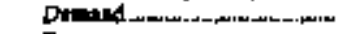 & - & - & - & - & - & - & - \\
\hline Endy & $-2,17 d$ & 301,425 & - & - & $\$ 9179$ & - & - \\
\hline Other ${ }^{2}$ & - & - & - & - & - & - & - \\
\hline \multicolumn{8}{|l|}{ Cooprative } \\
\hline 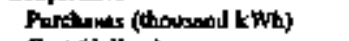 & 111,772 & $1, \mathbf{1 2 3 , 0 9 8}$ & - & - & - & - & - \\
\hline 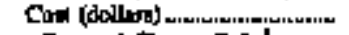 & $2,\{187,863$ & $3,142,586$ & 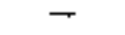 & - & - & - & - \\
\hline Drinand Chorge Onlyl .......... & - & $\rightarrow$ & - & - & - & - & - \\
\hline Derand & - & $=$ & - & - & - & - & - \\
\hline 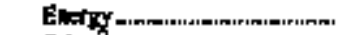 & $2,007.853$ & $8.742,586$ & - & - & $\rightarrow$ & - & - \\
\hline Otho' & - & - & - & - & $\neg$ & - & - \\
\hline \multicolumn{8}{|l|}{ Oiler } \\
\hline Purchusex (thomsaded kWh) & $1,729,346$ & s.7.796 & - & 54,189 & $4,850,856$ & $1,054,102$ & 367,326 \\
\hline 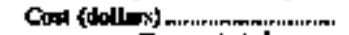 & $37,725,025$ & $9,207,992$ & - & $65,461,454$ & $386,698,1808$ & $22,044,694$ & $59,57,063$ \\
\hline Denrand Gure Onty l........... & - & - & - & - & - & - & - \\
\hline Deorund ................................ & - & - & - & - & - & - & - \\
\hline ENG & $36,72,387$ & $7,506,396$ & - & $65,481,454$ & $390,827,7 B 5$ & $22,016,497$ & $19,576,0613$ \\
\hline Ohand ${ }^{2}$ & $1,0,4,4]$ & $1,201,656$ & - & - & $-4,725.977$ & 30,197 & - \\
\hline \multicolumn{8}{|l|}{$T 00$} \\
\hline Punchesses (tromand kWh) & $0,577.159$ & $2,148,96.1$ & - & $1,691,093$ & 8,83B,541 & J,fi:4, InR & 785,140 \\
\hline Code & $145,935,206$ & 20.241 .557 & - & $93,734,563$ & $505,436,324$ & $22,046,694$ & $\Rightarrow, 56,2618$ \\
\hline Denand Chope Oolyl......... & - & - & $=$ & - & - & - & - \\
\hline Dentand inm & $93,075,496$ & $=$ & - & $11,201,678$ & - & - & - \\
\hline Exary & $51,056,272$ & $18,897,536$ & z & $\{2,552,1885$ & 427,681,472 & $22,016,497$ & $29,506,258$ \\
\hline 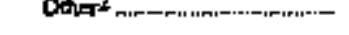 & $1,103,438$ & $1,340,121$ & - & - & $7,744,652$ & 30,197 & - \\
\hline
\end{tabular}

Ses enter and foctoroces at and of tribles. 
Table 19. Electricity Purchuses by Investor-Owned Utilities, by State, 1994 (Contianed)

\begin{tabular}{|c|c|c|c|c|c|c|c|}
\hline 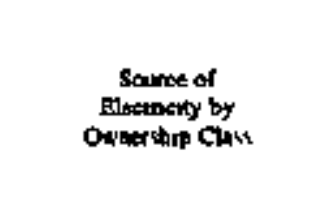 & 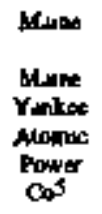 & 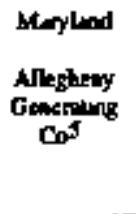 & 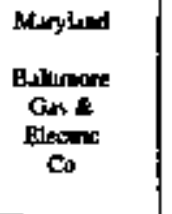 & $\begin{array}{l}\text { Maryland } \\
\text { Fodamex } \\
\text { Edrom } \\
\text { Co }\end{array}$ & 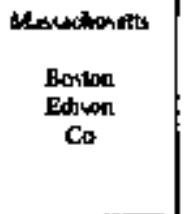 & $\begin{array}{l}\text { Alsurthests } \\
\text { Cumbridge } \\
\text { Plocene } \\
\text { Liglu } \\
\text { Co }\end{array}$ & 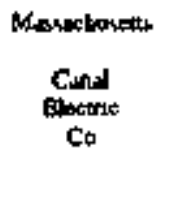 \\
\hline \multicolumn{8}{|l|}{ Invartor Owned } \\
\hline Purthuse (thoosund $\mathbf{k}$ why & - & - & 4397, , & 3.798.624 & $2451.17 \sqrt{4}$ & 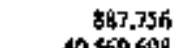 & 31,520 \\
\hline Cosk (dolnw) & - & - & $144,391,67$ & $216,601,88]$ & 100 , sol 941 & $40,69 ., 988$ & $14,697,913$ \\
\hline Denand Charte Oplyl & - & - & - & - & $-430,000$ & 436,142 & 2322202 \\
\hline Datonad & - & - & 51,905519 & $49,736,312$ & $62,930,730$ & 29,97257 & $8,418,597$ \\
\hline Energy & - & - & $64,399,393$ & $02,241,1 \mathrm{m3}$ & $37,561,211$ & J0,660,979 & $3,353,164$ \\
\hline Other & - & - & $20,056,755$ & 74, fid, NAS & - & - & - \\
\hline \multicolumn{8}{|l|}{ Fonderal } \\
\hline Burchone (how wond kwh) & - & - & - & - & 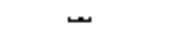 & - & - \\
\hline Cons (dolior) & - & - & - & - & - & - & - \\
\hline Demind Dime Onty I & - & - & - & - & - & - & - \\
\hline Demend & - & - & - & - & - & - & - \\
\hline Eosry & - & $=$ & - & - & - & - & - \\
\hline Other ${ }^{2}$ & - & - & - & - & - & $\rightarrow$ & - \\
\hline \multirow{2}{*}{\multicolumn{8}{|c|}{$\begin{array}{l}\text { Shen and Oher } \\
\text { Coyernment }\end{array}$}} \\
\hline & & & & & & & \\
\hline Purthuser (Oacosed kWL) & 一 & - & - & - & $\begin{array}{r}13,915 \\
30,573\end{array}$ & $\overline{-}$ & - \\
\hline Cors (dollan) & - & - & - & - & $-^{306,573}$ & - & $=$ \\
\hline Dentind Churge Onhly' & - & - & - & - & $-_{41,8 n s}$ & - & - \\
\hline Denand & - & - & - & - & 41,8015 & - & - \\
\hline Emedy & - & - & - & - & 264,763 & - & - \\
\hline Other ${ }^{2}$ & - & - & - & - & - & - & - \\
\hline \multicolumn{8}{|l|}{ Matucipal } \\
\hline Purcheses (thooted kWh) & - & - & - & 246 & $16,09 \mid$ & - & - \\
\hline Cou (dodlow) & - & - & - & 4,029 & 306,462 & - & - \\
\hline Cemand Charge Onlyl & - & - & - & - & - & - & - \\
\hline Demand & - & - & - & hs & 27,321 & - & - \\
\hline Emargy & - & - & - & 1.964 & 308,841 & - & - \\
\hline Other ${ }^{2}$ & - & - & - & - & - & - & - \\
\hline \multicolumn{8}{|l|}{ Cuopatativet } \\
\hline Ponshdest (thoutund kth) & - & - & 156,133 & - & - & $=$ & - \\
\hline $\operatorname{Cos}(\mathrm{dell} \ln \mathrm{x})$ & - & - & $2,548,736$ & - & - & - & - \\
\hline Deriond Ginge Only" & - & - & - & $m$ & - & - & - \\
\hline Dernumd & - & - & - & - & - & - & - \\
\hline Enerdy & - & - & $2,548,285$ & - & - & - & - \\
\hline Oh- $\pi^{2}$ & - & - & - & - & - & - & - \\
\hline \multicolumn{8}{|l|}{ Gaher ${ }^{3}$} \\
\hline Purchlones (boovand $k$ will) & - & - & $1,719,869$ & 112,693 & 4.974.1595 & 44i3.289 & 135,924 \\
\hline Cost (dollun) & - & - & $59,991,107$ & 342,679 & $24:, 119,49)$ & $32,168,515$ & $11,5 \$ 1,70 \mathrm{~g}$ \\
\hline Demend Cherge Only' & - & - & - & - & $-3,510,380$ & $\therefore$ & - \\
\hline Derendud & - & - & 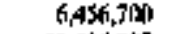 & 13,098 & $40,920,854$ & 18.852 .800 & $10,2,2,3,34$ \\
\hline Energy & - & - & $53,536,787$ & 723,390 & $203,719,023$ & $11,357,730$ & $1,369,374$ \\
\hline Olbet ${ }^{2}$ & - & - & - & - & - & $1,823,925$ & - \\
\hline \multicolumn{8}{|l|}{ Youl } \\
\hline Puntures (0roowand kWh) & - & - & $6,269,650$ & $5,911,365$ & $7, A S 5,400$ & $1,551,045$ & 477444 \\
\hline $\operatorname{Cov}$ (dolleri) & - & - & $21 \times, 939,050$ & $217,48,549$ & $341,784,173$ & $72,609,2,13$ & $23,675,691$ \\
\hline Demend Chuse Only1 & - & - & - & - & $-3,540,150$ & 436,142 & $2,322.212$ \\
\hline Dermund & - & - & $96,362,229$ & $49.752,116$ & $103,920,310$ & $48,355,397$ & I8,630,93] \\
\hline enty & - & - & $120,484,0106$ & $92,472,337$ & $241,783.843$ & $2,018,749$ & 4.722 .539 \\
\hline Oher $2^{2}$ & - & - & $29,066.755$ & $34,\{24,096$ & - & $1,327,925$ & - \\
\hline
\end{tabular}

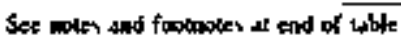


Table 19. Mectricity Purchases by Investor-Owned Utilities, by State, 1994 (Continned)

\begin{tabular}{|c|c|c|c|c|c|c|c|}
\hline $\begin{array}{c}\text { Source of } \\
\text { Elatidely by } \\
\text { Owerthip Class }\end{array}$ & 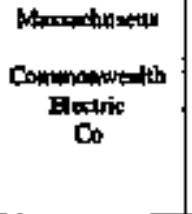 & 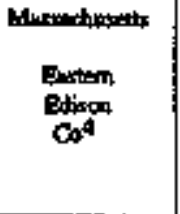 & 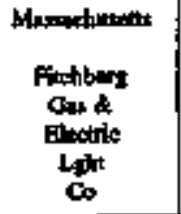 & 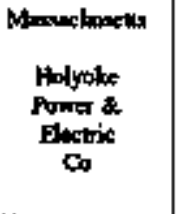 & 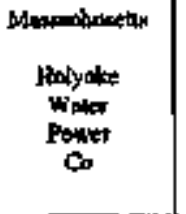 & 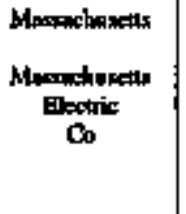 & 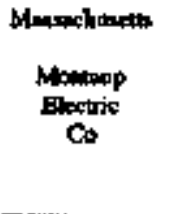 \\
\hline 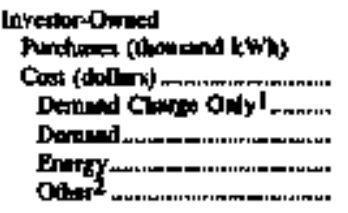 & $\begin{array}{r}1,928,844 \\
32636,041 \\
3,90,01,400 \\
94,316,699 \\
28,683,186 \\
35,756\end{array}$ & 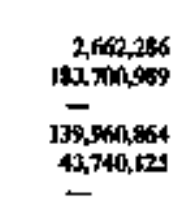 & $\begin{array}{l}110,604 \\
4,372,352 \\
- \\
3,099,730 \\
1,272,619 \\
-\end{array}$ & $\begin{array}{l}917,123 \\
32,196,134 \\
- \\
- \\
17,361,396 \\
14,435,448\end{array}$ & $\begin{array}{l}84,262 \\
29,80,851 \\
- \\
\overline{15,414,403} \\
14,45,448\end{array}$ & $\begin{array}{r}16,457,414 \\
1,074,325,6149 \\
- \\
552,152,218 \\
058,327,649 \\
161,845,692\end{array}$ & $\begin{array}{l}2364,421 \\
535,094,606 \\
- \\
304,50,375 \\
30,594,027 \\
-\end{array}$ \\
\hline 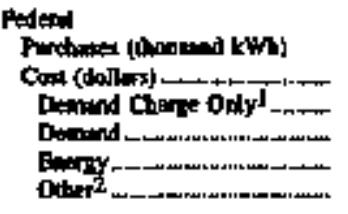 & $\begin{array}{l}\bar{z} \\
\overline{-} \\
\bar{z}\end{array}$ & $\begin{array}{l}\bar{z} \\
\overline{-} \\
\overline{-}\end{array}$ & $\begin{array}{l}\bar{z} \\
\bar{z} \\
\bar{z}\end{array}$ & $\begin{array}{l}\overline{-} \\
\overline{-} \\
\overline{-}\end{array}$ & $\begin{array}{l}\overline{-} \\
\overline{-} \\
-\end{array}$ & $\begin{array}{l}z \\
z \\
z\end{array}$ & $\begin{array}{l}\bar{z} \\
\bar{z} \\
\overline{-}\end{array}$ \\
\hline 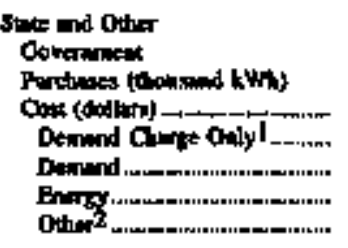 & $\begin{array}{l}- \\
z \\
- \\
-\end{array}$ & $\begin{array}{l}z \\
z \\
z \\
-\end{array}$ & $\begin{array}{r}16,946 \\
-\quad 16 \% 565 \\
-\quad\end{array}$ & $\begin{array}{l}\bar{z} \\
\overline{-} \\
\overline{-}\end{array}$ & $\begin{array}{l}- \\
\overline{-} \\
\overline{-}\end{array}$ & $\begin{array}{l}\bar{z} \\
\bar{z} \\
-\end{array}$ & $\begin{array}{r}3,042 \\
784,504 \\
-\quad 033,520 \\
-\end{array}$ \\
\hline 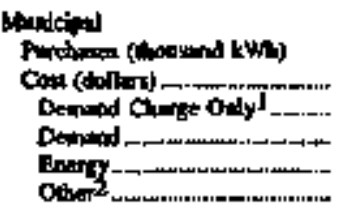 & $\begin{array}{l}\bar{z} \\
\bar{z} \\
\overline{-}\end{array}$ & $\begin{array}{r}236 \\
22,917 \\
-\quad 19,200 \\
-\quad 1,627\end{array}$ & $\begin{array}{l}\bar{z} \\
\bar{z}\end{array}$ & $\begin{array}{l}\overline{-} \\
\bar{z} \\
\overline{-}\end{array}$ & $\begin{array}{l}- \\
z \\
z\end{array}$ & $=\frac{323}{32.319}$ & $\begin{array}{r}57,651 \\
5,574,155 \\
\overline{3,920,030} \\
1,655,322 \\
-\end{array}$ \\
\hline 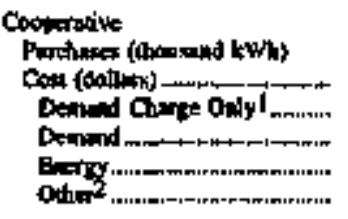 & $\begin{array}{l}- \\
\overline{-} \\
- \\
-\end{array}$ & $\begin{array}{l}= \\
z \\
z\end{array}$ & $\begin{array}{l}\bar{z} \\
\overline{-} \\
\overline{-}\end{array}$ & $\begin{array}{l}\overline{-} \\
\bar{z} \\
\overline{-}\end{array}$ & $\begin{array}{l}\bar{z} \\
\overline{-} \\
\overline{-}\end{array}$ & $\begin{array}{l}\bar{z} \\
\bar{z} \\
-\end{array}$ & $\begin{array}{l}\bar{z} \\
\bar{z} \\
\bar{E}\end{array}$ \\
\hline 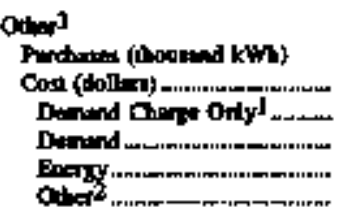 & $\begin{array}{r}2,19,970 \\
160959,195 \\
251,759 \\
78,740,419 \\
96,572,654 \\
-14,605,237\end{array}$ & $\begin{array}{l}129 \\
9,179,552 \\
= \\
-\quad 2,200 \\
9.176,952\end{array}$ & $\begin{array}{l}269,791 \\
15,22, \pi 75 \\
- \\
10,37,737 \\
4,64,134 \\
-\end{array}$ & $\begin{array}{l}\overline{-} \\
\overline{-} \\
\overline{-}\end{array}$ & $\begin{array}{l}30415 \\
-\quad 36477 \\
- \\
-\end{array}$ & $=^{37,564}$ & $\begin{array}{r}32,847 \\
27,591,540 \\
475,470 \\
13,313,613 \\
14,102,459 \\
-\end{array}$ \\
\hline 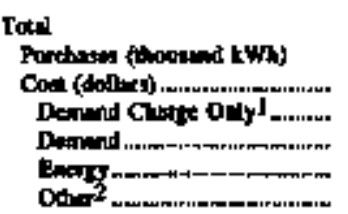 & $\begin{array}{r}4,748,462 \\
28,895,636 \\
4,152,159 \\
173,197,111 \\
125,255,840 \\
-24,559,411\end{array}$ & $\begin{array}{r}2,662,651 \\
192,900,648 \\
- \\
139,994,144 \\
13,745,952 \\
9,176,552\end{array}$ & $\begin{array}{r}381,761 \\
19,764,172 \\
- \\
13,461,45 \\
4,285,704 \\
-\end{array}$ & $\begin{array}{l}917,023 \\
32,396,804 \\
- \\
- \\
17,761,3 \% 6 \\
14,135,410\end{array}$ & $\begin{array}{l}916,697 \\
30,2,4,4 \times 8 \\
- \\
\overline{15.779,140} \\
14,45.440\end{array}$ & $\begin{array}{r}16,459,135 \\
1,074,411,552 \\
- \\
552,152,218 \\
158,493,512 \\
163,845,102\end{array}$ & $\begin{array}{r}3,347,064 \\
169.349,104 \\
475,970 \\
122,390,02 \\
46,482,790 \\
-\end{array}$ \\
\hline
\end{tabular}

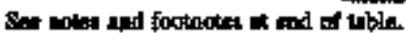


Table 19. Mtectricify Purchases by Investor-Owned Utilities, by State, 1994 (Continued)

\begin{tabular}{|c|c|c|c|c|c|c|c|}
\hline 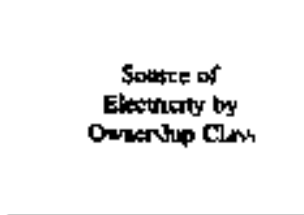 & 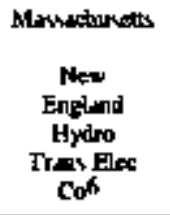 & $\begin{array}{l}\text { Mrivehorets } \\
\text { New } \\
\text { Earlend } \\
\text { Power } \\
\text { Co }\end{array}$ & 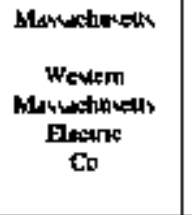 & 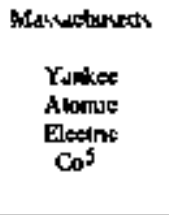 & $\begin{array}{l}\text { Mustugen } \\
\text { Conwumar } \\
\text { Ponler } \\
\text { Co }\end{array}$ & $\begin{array}{l}\text { Muchygas } \\
\text { Detrat } \\
\text { Eflson } \\
\text { Co }\end{array}$ & 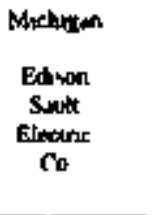 \\
\hline \multicolumn{8}{|l|}{ Iwevent Oimed } \\
\hline 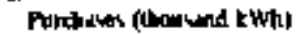 & - & $3,456,207$ & (15.17\% & - & $1,970,207$ & $1,904,55 \mathrm{~d}$ & 420,7 放 \\
\hline Cons (dallon) & - & $23,931,406$ & 79,822010 & - & 40 EXA 412 & $4 k_{1}, 125,100$ & $16 \$ 54,776$ \\
\hline beseud Oure Onby" & - & - & - & - & - & - & - \\
\hline Demind & - & $52,1100,407$ & - & - & $5,722,527$ & $21,310,497$ & $6,028,528$ \\
\hline Energy & - & $80,045,909$ & $\{1,784,042$ & - & $14,921,763$ & 14813,616 & $9,822,828$ \\
\hline Othents & - & $\mathrm{JOH}, 791,090$ & 28,410178 & - & $-987 \mathrm{~h}$ & - & 30,48 \\
\hline \multicolumn{8}{|l|}{ Rederil } \\
\hline Pwothea (thon und kWb) & - & - & - & - & - & - & 18 \&ill-3 \\
\hline Cod $\{$ doltent & - & - & - & - & - & - & 1.724223 \\
\hline Durnand Churge Only] & - & - & - & - & - & - & - \\
\hline Demond & - & - & - & - & - & - & - \\
\hline Bangy & - & - & - & - & - & - & 1,724223 \\
\hline Dhest & - & - & - & - & - & - & - \\
\hline \multicolumn{8}{|l|}{ Saste and Oba } \\
\hline 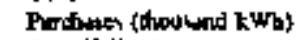 & - & 17ד. & 126 & - & 7.924 & 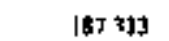 & - \\
\hline Cost (dollers) & - & 6.710 & 1,931 & - & 97, I I I I l l l & $8,823,770$ & - \\
\hline Dopind Oing Otyly & - & - & - & - & - & - & - \\
\hline Denend & - & - & - & - & - & - & - \\
\hline Eneray & - & 4,3 रा & 3.931 & - & 97,II I & $2,852,5 \mathrm{~m} 7$ & - \\
\hline $00 x^{2}$ & - & - & - & - & - & $5,971,263$ & - \\
\hline \multicolumn{8}{|l|}{ Mankeppd } \\
\hline Puntos (thoouthd kWt) & - & - & 152 & - & $498,40]$ & 5,5976 & - \\
\hline Cont (doldans) & - & - & 2,197 & - & 15.706, I, H & Sx635 & - \\
\hline Deniend Curge Only I & - & - & - & - & - & - & - \\
\hline Depland & - & - & $=$ & - & $24,768,291$ & - & - \\
\hline Energy & - & - & 2,177 & - & $10,5 \mathrm{M}, 24]$ & 65,6535 & - \\
\hline Olater $x^{2}$ & - & - & - & - & -26400 & - & - \\
\hline \multicolumn{8}{|l|}{ Opoperitivi } \\
\hline Pathines (thonamd $k W / a$ ) & - & $1.4 \%$ & - & - & $39,49 t$ & - & 46 \\
\hline Cont $(d-1)+r y)$ & - & 29.116 & - & - & $1,129,978$ & - & $2 A 12$ \\
\hline Demisand Churge Ondy' & - & $=$ & - & - & - & - & - \\
\hline Dement & - & - & - & - & 311,364 & - & - \\
\hline Energy & - & 29.J IEN & - & - & 818,614 & - & 2,412 \\
\hline Outrot & - & - & - & - & - & - & - \\
\hline \multicolumn{8}{|l|}{ orlens } \\
\hline Punctuses (thoundnd 1 w/h) & - & $5,576,102$ & $1,236,66-18$ & - & $11,929,929$ & $4,503,595$ & - \\
\hline Cout (doldint) & - & $279,610,222$ & $44,758,895$ & - & 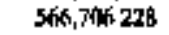 & $103,577.281$ & - \\
\hline Dewend Clige Ody' & - & - & - & - & - & - & - \\
\hline Defpand & - & - & - & - & $356,148,705$ & $30,929,141$ & - \\
\hline Enerty & - & $231,0+10,413$ & $44,758,885$ & - & 231,214753 & $95,299,333$ & $=$ \\
\hline Other ${ }^{2}$ & - & $46,399.812$ & - & - & $-\$ 57,2 \% 0$ & $1,148,007$ & $m$ \\
\hline \multicolumn{8}{|l|}{ Tod } \\
\hline Purchmex (hoon:und kWh) & - & $11.375,519$ & $2,252,325$ & - & 14,458, 104 & $6,601,038$ & 559,087 \\
\hline Cons (dolliwas) & - & $55,583,158$ & $34,565,373$ & - & $64), 873767$ & $158,992,789$ & $18,281,416$ \\
\hline Deniend Cing Only' & - & $=$ & - & - & - & - & - \\
\hline Dernund & - & $52,100,4 m$ & - & - & $386,950,8877$ & $22,240,628$ & $6,025,528$ \\
\hline Enengy & - & $311,091,829$ & $36,147,195$ & - & $25,616,384$ & 129,432,091 & $11,549,4618$ \\
\hline$\theta(1)=a^{2}$ & - & $1512,200,962$ & $2 B, 478, I \pi B$ & - & $-693,500$ & $7,325,0 \mathrm{~m}$ & 303,420 \\
\hline
\end{tabular}

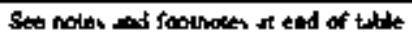


Table 19. Electricity Purchases by Investor-Owned Utilties, by State, 1994 (Continuod)

\begin{tabular}{|c|c|c|c|c|c|c|c|}
\hline 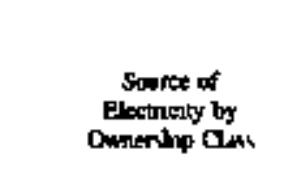 & 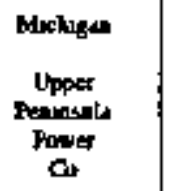 & 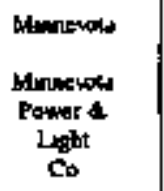 & 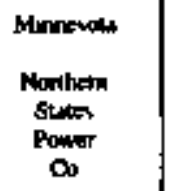 & $\begin{array}{l}\text { Mmewtat } \\
\text { Ouda } \\
\text { Tadt } \\
\text { Power } \\
\text { Co }\end{array}$ & $\begin{array}{l}\text { Dhsuruppi } \\
\text { Murusappi } \\
\text { Power \& } \\
\text { Lught } \\
\text { Co }\end{array}$ & 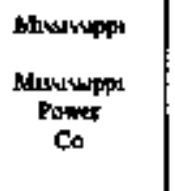 & 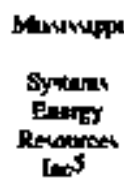 \\
\hline 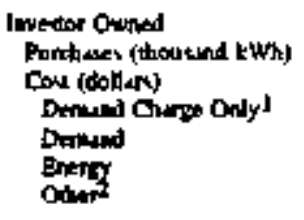 & $\begin{array}{r}594,459 \\
16,284,123 \\
- \\
5,641,104 \\
10,4648,126 \\
-5,109\end{array}$ & 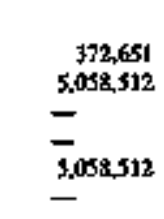 & $\begin{array}{r}1,330,3119 \\
68,711,815 \\
\overline{2} \\
2,881,446 \\
18,855,540 \\
47,614,629\end{array}$ & 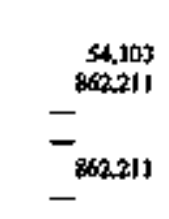 & $\begin{array}{r}1,158,160 \\
200,250,155 \\
- \\
13,992,978 \\
21,269,17 \\
-\end{array}$ & 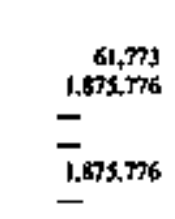 & $\begin{array}{l}= \\
= \\
=\end{array}$ \\
\hline 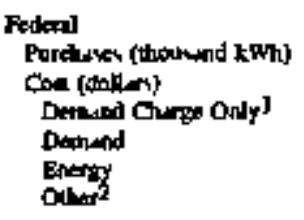 & $\begin{array}{l}\overline{-} \\
\bar{z} \\
\bar{z}\end{array}$ & 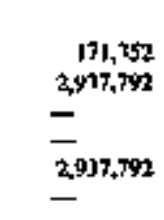 & $\begin{array}{l}268,100 \\
4,725,1048 \\
- \\
-7,725,058 \\
-\end{array}$ & $\begin{array}{l}- \\
- \\
- \\
-\end{array}$ & $\begin{array}{l}261,321 \\
4,910,365] \\
- \\
4,970,359 \\
-\end{array}$ & 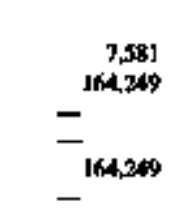 & $\begin{array}{l}- \\
= \\
= \\
-\end{array}$ \\
\hline 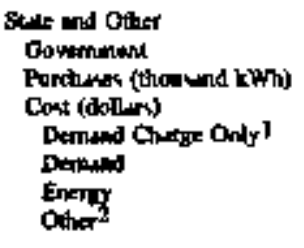 & $\begin{array}{l}- \\
- \\
- \\
-\end{array}$ & $\begin{array}{l}495,243 \\
7.148,768 \\
- \\
= \\
7.148,768 \\
=\end{array}$ & $\begin{array}{l}155,5301 \\
2816,418 \\
= \\
= \\
2,816,418 \\
-\end{array}$ & $\begin{array}{l}524,359 \\
7,073,748 \\
- \\
- \\
7,074,748 \\
-\end{array}$ & $\begin{array}{l}z \\
- \\
- \\
-\end{array}$ & $\begin{array}{r}28 B \\
-\quad 6,41 \\
-\quad 6491\end{array}$ & $\begin{array}{l}z \\
z \\
z \\
-\end{array}$ \\
\hline 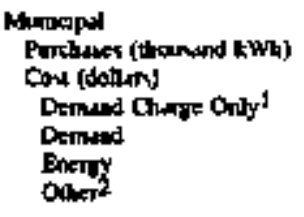 & $\begin{array}{l}= \\
\overline{-} \\
=\end{array}$ & 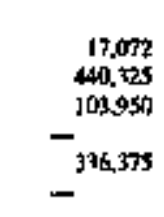 & $\begin{array}{r}72,441 \\
2346,067 \\
- \\
630,0010 \\
1,716,067 \\
=\end{array}$ & $\begin{array}{l}1,34,934 \\
2,04,545 \\
= \\
2,624541 \\
-\end{array}$ & $-^{\quad 11402}$ & 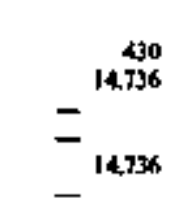 & $\begin{array}{l}\bar{z} \\
\bar{z} \\
\overline{-}\end{array}$ \\
\hline 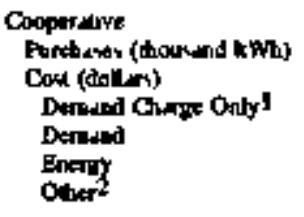 & $\begin{array}{l}- \\
= \\
= \\
-\end{array}$ & $\begin{array}{l}2,521,495 \\
58,473,708 \\
- \\
38,481.978 \\
19,751.778 \\
-\end{array}$ & $\begin{array}{r}753,989 \\
24,922,519 \\
- \\
16,07,207 \\
8,849,312 \\
-\end{array}$ & $\begin{array}{l}353,046 \\
6,955,521 \\
+ \\
1,311,414 \\
5,644,101 \\
-\end{array}$ & $\begin{aligned} & 218,093 \\
& 4,447,440 \\
&- \\
& \overline{4,447,445} \\
&-\end{aligned}$ & $\begin{array}{l}-^{4,318} \\
- \\
-126.509 \\
\end{array}$ & $\begin{array}{l}\bar{z} \\
\bar{z} \\
=\end{array}$ \\
\hline 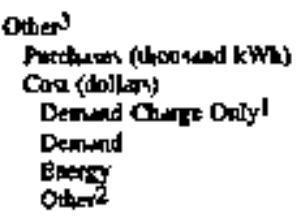 & $\begin{array}{l}= \\
= \\
=\end{array}$ & $\begin{array}{r}302,399 \\
10,762,003 \\
- \\
3,06,393 \\
10,235,3700 \\
-7\end{array}$ & $\begin{array}{r}6,997,107 \\
193,939,934 \\
- \\
86,407,103 \\
107,531,831 \\
-\end{array}$ & $\begin{array}{r}683,699 \\
11,801,188 \\
- \\
1,467,612 \\
10,353,556 \\
-\end{array}$ & $\begin{array}{l}J, 17,474 \\
25,296,235 \\
- \\
- \\
25,296,238 \\
-\end{array}$ & $\begin{array}{r}3,673,860 \\
69,065,136 \\
-\quad 627,591 \\
68,426,792 \\
11,843\end{array}$ & $\begin{array}{l}\bar{z} \\
\bar{z} \\
\overline{-}\end{array}$ \\
\hline 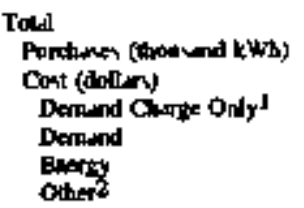 & $\begin{array}{r}594,459 \\
16,284,123 \\
- \\
5,641,104 \\
10,548,126 \\
-5,107\end{array}$ & $\begin{array}{r}4,320,212 \\
67,781,138 \\
100,950 \\
42,108,263 \\
45,568,925 \\
-\end{array}$ & 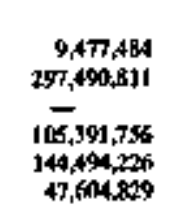 & $\begin{array}{l}1,749,901 \\
28,717,209 \\
\overline{2,799,046} \\
25,958.163 \\
-\end{array}$ & $\begin{array}{l}4,029,764 \\
23,012,510 \\
- \\
178,992,978 \\
56,025,632 \\
-\end{array}$ & 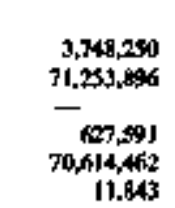 & $\begin{array}{l}- \\
- \\
- \\
-\end{array}$ \\
\hline
\end{tabular}

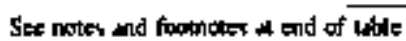


Tahte 19. Electricity Purchases by Investor-Owned Ut:Hites, by State, 1994 (Continoed)

\begin{tabular}{|c|c|c|c|c|c|c|c|}
\hline 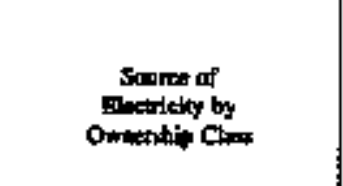 & 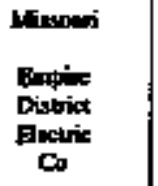 & 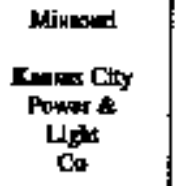 & 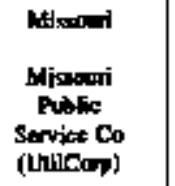 & 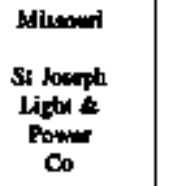 & 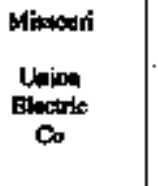 & 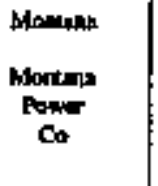 & 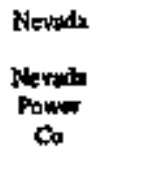 \\
\hline 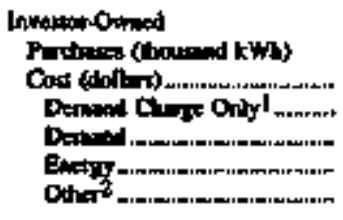 & 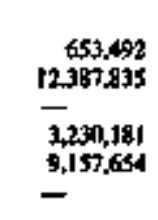 & $\begin{array}{r}201,856 \\
11.23,541 \\
-\quad \\
34,451 \\
9,296,994 \\
2,490,0001\end{array}$ & 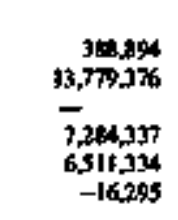 & $\begin{array}{l}214,340 \\
7,112,740 \\
\overline{1,679,500} \\
3,433,140 \\
-\end{array}$ & $\begin{array}{l}7,423,463 \\
191,463,625 \\
\overline{36,511,705} \\
103,961,920 \\
-\end{array}$ & $\begin{array}{r}704,660 \\
15.440,629 \\
55,350 \\
-\quad 15,899,266 \\
-4,967\end{array}$ & 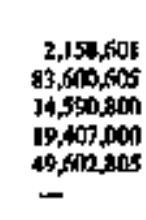 \\
\hline 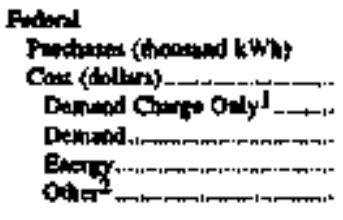 & $\begin{array}{l}- \\
\overline{-} \\
\overline{-} \\
-\end{array}$ & $\begin{array}{l}- \\
\overline{-} \\
= \\
=\end{array}$ & $\begin{array}{l}- \\
- \\
z \\
-\end{array}$ & $\begin{array}{l}- \\
\overline{-} \\
\overline{-} \\
\overline{-}\end{array}$ & $\begin{array}{r}4 \pi, 729 \\
10,626,217 \\
-\quad \\
10,119,21\} \\
-\end{array}$ & $\begin{array}{r}627,196 \\
32,530,752 \\
500,000 \\
-\quad \\
31,551,374 \\
72,478\end{array}$ & $\begin{array}{l}129,255 \\
2,952,170 \\
- \\
= \\
2,502,150 \\
390,200\end{array}$ \\
\hline 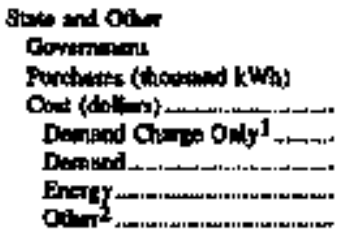 & $\begin{array}{l}129,996 \\
2,270,653 \\
- \\
3,58,6140 \\
1,931,965 \\
-\end{array}$ & $\begin{array}{l}110,903 \\
1,40,344 \\
- \\
\overline{1.44,544} \\
-\end{array}$ & $\begin{array}{l}= \\
= \\
=\end{array}$ & $\begin{array}{l}201,292 \\
298,723 \\
- \\
- \\
2966,721\end{array}$ & $\begin{array}{l}z \\
\overline{-} \\
=\end{array}$ & $\begin{array}{l}-m \\
- \\
-\end{array}$ & $\begin{array}{r}1,207,200 \\
26,775,291 \\
- \\
5,384,706 \\
29,629,539 \\
96,046\end{array}$ \\
\hline 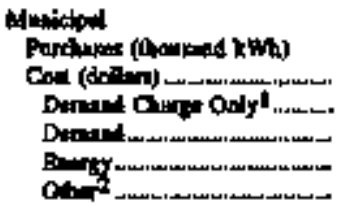 & $\begin{array}{r}34,743 \\
1,012,569 \\
- \\
146,176 \\
-84,303\end{array}$ & $\begin{array}{l}1, \pi 4,951 \\
- \\
- \\
1,907,151 \\
1,97,800\end{array}$ & $\overline{-}^{5,627}$ & 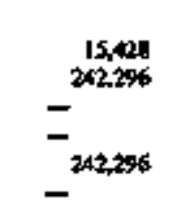 & $\begin{array}{l}\bar{z} \\
\bar{z} \\
\overline{-}\end{array}$ & $\begin{array}{r}312,799 \\
1.952 .609 \\
-45.154 \\
5.906 .755 \\
-\end{array}$ & 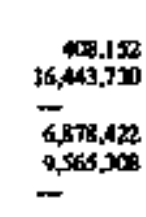 \\
\hline 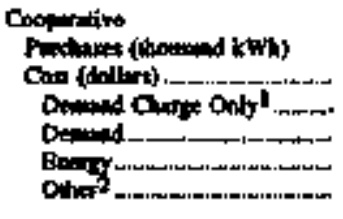 & 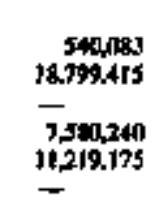 & $\begin{array}{l}331,8222 \\
14, \pi 32,276 \\
- \\
\overline{7,776,024} \\
9,256,250\end{array}$ & 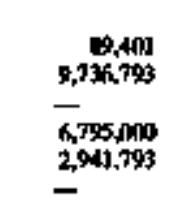 & 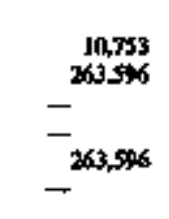 & 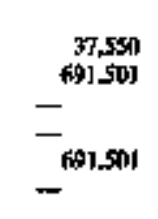 & $\begin{array}{r}1,102,909 \\
19.710,41 \\
72,450 \\
-\quad \\
19,63 \%, 019 \\
-\end{array}$ & $-^{2,025}$ \\
\hline 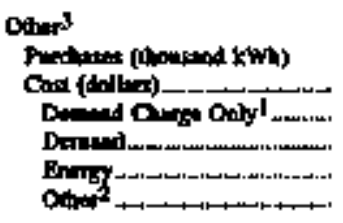 & 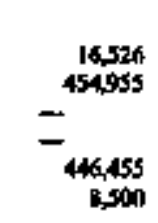 & $\begin{array}{l}1,9,476 \\
-890,6022 \\
- \\
1,890,692 \\
-\end{array}$ & $\begin{array}{l}- \\
\overline{-} \\
\overline{-} \\
-\end{array}$ & $\begin{array}{l}-^{109} \\
- \\
52,645 \\
52,685\end{array}$ & 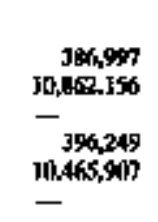 & $\begin{array}{r}365,440 \\
19,234,060 \\
- \\
3,1674,138 \\
15,414,331 \\
-56,78]\end{array}$ & $\begin{array}{l}2,291,3915 \\
127,342,340 \\
- \\
71,131,617 \\
56,210,731 \\
-\end{array}$ \\
\hline 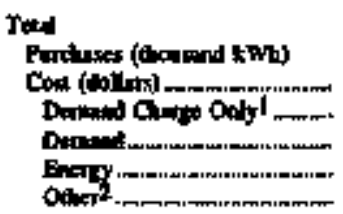 & $\begin{array}{r}1,394,600 \\
34,925,427 \\
- \\
11,25,2855 \\
23,6+9,642 \\
1,500\end{array}$ & $\begin{array}{r}972,46 \\
3,905,504 \\
- \\
36,151 \\
20,64,602 \\
13,234,050\end{array}$ & $\begin{array}{r}485,917 \\
20,64,109 \\
- \\
14,079,337 \\
9,57,967 \\
-16,295\end{array}$ & $\begin{array}{r}5,2,382 \\
10,658,0104 \\
- \\
1,579,600 \\
8,925,755 \\
52,685\end{array}$ & $\begin{array}{l}8,318,739 \\
162,643,499 \\
- \\
39,404,954 \\
129,208,545 \\
-\end{array}$ & $\begin{array}{r}3,013,004 \\
89,376,922 \\
1,127,600 \\
1,921,972 \\
64,432,040 \\
11,110\end{array}$ & $\begin{array}{r}6,193,140 \\
25,157,169 \\
1,590,010 \\
102,801,745 \\
139,473,258 \\
1,291,266\end{array}$ \\
\hline
\end{tabular}

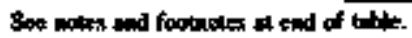


Table 19. Electricly Porchoses by Investor-Owned Utilities, by State, 1944 (Contimued)

\begin{tabular}{|c|c|c|c|c|c|c|c|}
\hline $\begin{array}{c}\text { Sourde of } \\
\text { Fintelely by } \\
\text { Omershlp Chink }\end{array}$ & 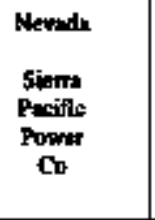 & $\begin{array}{l}\text { New Hinpthin } \\
\text { Conersd } \\
\text { Filetic } \\
\text { Cot }\end{array}$ & 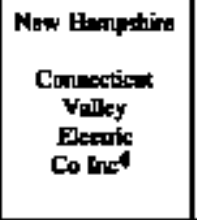 & 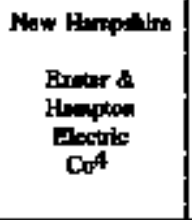 & $\begin{array}{c}\text { New Hympdire } \\
\text { Comile } \\
\text { Stute } \\
\text { Flectic } \\
\text { Cot }\end{array}$ & $\begin{array}{l}\text { New Humputine } \\
\text { Con:t } \\
\text { Buy } \\
\text { Power } \\
\text { Corp }\end{array}$ & New Hineplitime \\
\hline 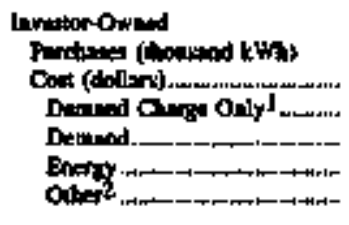 & $\begin{array}{l}1,943,236 \\
63,586,708 \\
- \\
26,73,500 \\
15,351.903 \\
1,461,265\end{array}$ & $\begin{array}{r}488,154 \\
34,26,7,742 \\
\overrightarrow{16,073,007} \\
9,287,186 \\
1,501,599\end{array}$ & $\begin{array}{r}13,348 \\
9,466,2 \% 6 \\
- \\
7,27 \%, 724 \\
1,914,974 \\
216,594\end{array}$ & $\begin{array}{r}511,392 \\
34,092,226 \\
+ \\
16,948,710 \\
9,728,900 \\
9,324,546\end{array}$ & $\begin{array}{l}719,363 \\
45,167,679 \\
7 \\
21,997,400 \\
16,144,384 \\
7,129,865\end{array}$ & $\begin{array}{l}= \\
\overline{-} \\
\overline{-} \\
-\end{array}$ & $\begin{array}{l}\bar{z} \\
\overline{-} \\
\overline{-}\end{array}$ \\
\hline 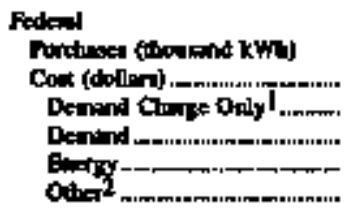 & 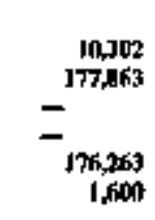 & $\begin{array}{l}= \\
= \\
z \\
=\end{array}$ & $\begin{array}{l}\bar{z} \\
\bar{z} \\
=\end{array}$ & $\begin{array}{l}= \\
= \\
= \\
=\end{array}$ & $\begin{array}{l}\bar{z} \\
\bar{z} \\
\bar{z}\end{array}$ & $\begin{array}{l}z \\
z \\
=\end{array}$ & $\begin{array}{l}\bar{z} \\
\overline{-} \\
\overline{-}\end{array}$ \\
\hline 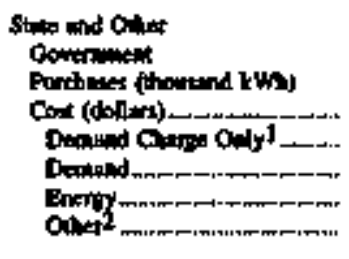 & $\begin{array}{r}62,905 \\
1905,698 \\
- \\
205,000 \\
1.705,538 \\
-\end{array}$ & $\begin{array}{l}\overline{-} \\
\overline{-} \\
\overline{-}\end{array}$ & $\begin{array}{rr}\quad 65 \\
-\quad 1,655 \\
-\quad 1,653\end{array}$ & $\begin{array}{l}- \\
- \\
- \\
-\end{array}$ & $\begin{array}{l}- \\
\overline{-} \\
\overline{-} \\
\overline{-}\end{array}$ & $\begin{array}{l}- \\
- \\
- \\
-\end{array}$ & $\begin{array}{l}- \\
\overline{-} \\
\overline{-} \\
-\end{array}$ \\
\hline 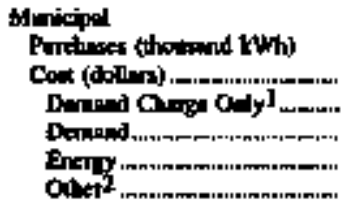 & $\begin{array}{l}18,061 \\
6,414,468 \\
- \\
\overline{6,256,968} \\
15,504\end{array}$ & $\begin{array}{l}z \\
\overline{-} \\
\bar{z}\end{array}$ & $\begin{array}{r}1,872 \\
145,041 \\
-\quad 28,786 \\
-116,245\end{array}$ & $\begin{array}{l}\bar{z} \\
\bar{z} \\
=\end{array}$ & $\begin{array}{l}= \\
= \\
=\end{array}$ & $\begin{array}{l}\bar{z} \\
\bar{z} \\
=\end{array}$ & $\begin{array}{l}\bar{z} \\
\overline{-} \\
\overline{-}\end{array}$ \\
\hline 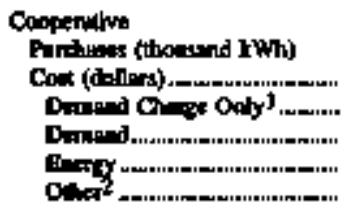 & $\begin{array}{l}484,395 \\
10,068,118 \\
- \\
3,124,000 \\
6,948,118 \\
-\end{array}$ & $\begin{array}{l}- \\
- \\
- \\
- \\
-\end{array}$ & $\begin{array}{l}z \\
z \\
z \\
=\end{array}$ & $\begin{array}{l}= \\
\ddot{-} \\
= \\
=\end{array}$ & $\begin{array}{l}\bar{z} \\
\bar{z} \\
\bar{z}\end{array}$ & $\begin{array}{l}\bar{z} \\
\bar{z} \\
\bar{z}\end{array}$ & $\begin{array}{l}\bar{z} \\
\bar{z} \\
=\end{array}$ \\
\hline 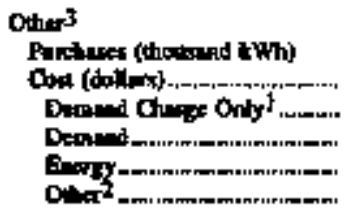 & $\begin{array}{r}802,963 \\
40,50,317 \\
+ \\
2,407,714 \\
40,4416,448 \\
-287,46\end{array}$ & $\begin{array}{l}- \\
z \\
- \\
z\end{array}$ & 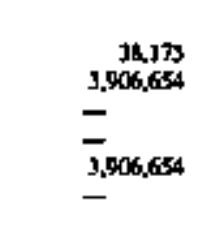 & $\begin{array}{l}\bar{z} \\
\bar{z} \\
=\end{array}$ & 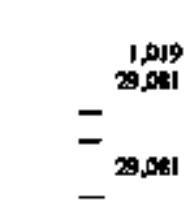 & $\begin{array}{r}8,159 \\
-\quad 82 \\
-\quad 8152\end{array}$ & $\begin{array}{l}\bar{z} \\
\overline{-} \\
\overline{-}\end{array}$ \\
\hline 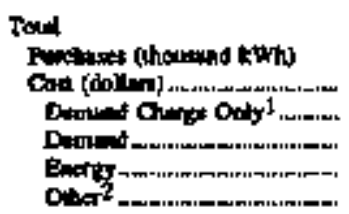 & 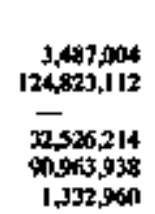 & $\begin{array}{r}48,114 \\
34,263,742 \\
- \\
16,075,042 \\
9,287,186 \\
8,901,509\end{array}$ & $\begin{array}{r}172,460 \\
13,519.636 \\
- \\
3,259,54 \\
5,961,524 \\
258,594\end{array}$ & $\begin{array}{r}511,392 \\
36,042,22 x \\
- \\
{[6,444,7101} \\
9,724,9501 \\
9,204,56\end{array}$ & 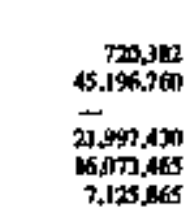 & $\begin{array}{r}\text { 4.99 } \\
-\quad 852 \\
-\quad 8.152 \\
-\quad 8\end{array}$ & $\begin{array}{l}\overline{-} \\
\overline{-} \\
= \\
=\end{array}$ \\
\hline
\end{tabular}

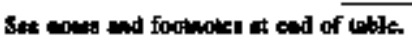


Table 19. Electricity Purchases by Investor-Owned Utitities, by State, 1994 (Continued)

\begin{tabular}{|c|c|c|c|c|c|c|c|}
\hline 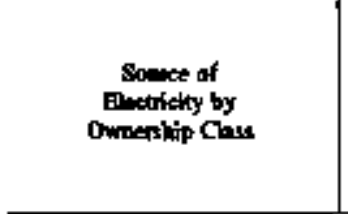 & $\begin{array}{l}\text { New Humparition } \\
\text { New } \\
\text { Englond } \\
\text { Hytro-Tintu } \\
\text { Cosp }\end{array}$ & 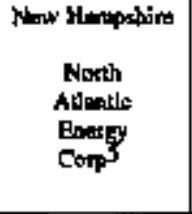 & $\begin{array}{c}\text { Now Hompnimite } \\
\text { Puble } \\
\text { Serriet } \\
\text { Co of NH }\end{array}$ & $\begin{array}{l}\text { Now Harpachite } \\
\text { UNuTUL } \\
\text { Powst } \\
\text { Corp }\end{array}$ & 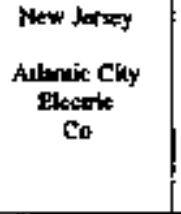 & 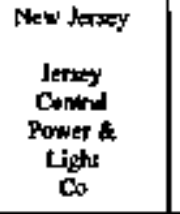 & 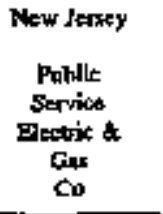 \\
\hline \multicolumn{8}{|l|}{ bovetor Ound } \\
\hline Parchne (llopand kWh) & - & - & $3,527,180$ & 574382 & $2,801.277$ & $1,452,541$ & 4.906, dns \\
\hline Cost (dollox)-C- - & - & - & $169,091,846$ & $36,961,992$ & $108,724,662$ & $247,050,823]$ & $119,966,945$ \\
\hline 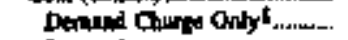 & - & - & - & - & - & $2,891,927$ & - \\
\hline 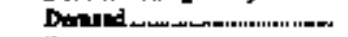 & - & - & - & $27,122,155$ & - & 158.997 .132 & - \\
\hline 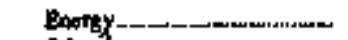 & - & م- & $24,771,499$ & $9,609,824$ & $54,8,0,422$ & $85,9,96,440$ & $159,946,945$ \\
\hline Ot-3 & - & - & $16,320,347$ & - & $50.064 .24 \mathrm{P}$ & $-274,5 T 2$ & - \\
\hline \multicolumn{8}{|l|}{ Fedaral } \\
\hline 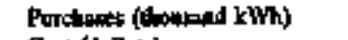 & $=$ & - & - & - & - & - & - \\
\hline 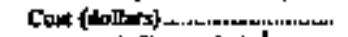 & - & - & - & - & - & - & - \\
\hline Deniang Oimte Onlyl. & - & - & - & - & - & - & - \\
\hline Depund__-................_- & - & - & - & - & - & - & - \\
\hline Entroty & - & - & - & - & - & - & - \\
\hline 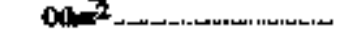 & - & - & - & - & - & - & - \\
\hline \multicolumn{8}{|l|}{ Shave und Oula } \\
\hline Poneluese (thoosund twh) & - & - & 221 & 2891 & - & - & $+01,79 \%$ \\
\hline 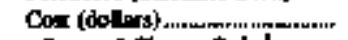 & - & - & 3.385 & 66,481 & - & - & $2[39,0081$ \\
\hline Denued Cherge dedy I & - & - & - & - & - & - & - \\
\hline 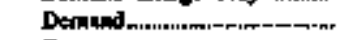 & - & - & - & - & - & - & - \\
\hline Endry & - & - & 3,308 & $64,48]$ & - & - & $2,139,081$ \\
\hline Oblar & - & - & - & - & - & - & - \\
\hline \multicolumn{8}{|l|}{ Mancicipal } \\
\hline 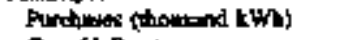 & - & - & 8,402 & - & 39.787 & 1.529 .816 & - \\
\hline $\cos (\mathrm{do}(\operatorname{ser})$ & - & - & 312,369 & - & $1,42,580$ & $111.126,349$ & - \\
\hline Deriand Oarge Onlyl_....-. & - & - & - & - & - & - & - \\
\hline 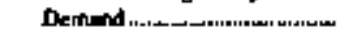 & - & - & - & - & - & 1,522 & -- \\
\hline Entry & $\rightarrow$ & سـ & 712,369 & ع. & $1,41,6143$ & $111,124,827$ & - \\
\hline Daber & - & - & - & - & \$57 & - & $\rightarrow$ \\
\hline \multicolumn{8}{|l|}{ Conpentive } \\
\hline Aindwess (thound lWh) & - & - & [34,85] & - & - & 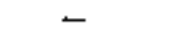 & $-r$ \\
\hline Cont (dollins)........ & - & - & $10,073,074$ & - & - & - & $-r$ \\
\hline Denend Gane Only 1 & - & - & - & - & - & - & - \\
\hline 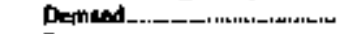 & - & - & - & - & - & - & - \\
\hline Enereygy & - & - & 450,368 & - & - & - & - \\
\hline 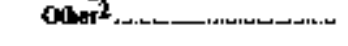 & - & - & $9,62,7,6$ & - & - & - & 一 \\
\hline \multicolumn{8}{|l|}{ G:Jat } \\
\hline Purcheses (thowand $k$ Wha) & - & - & 2605,966 & 411,260 & $2,219,978$ & $4,605,0 \mathrm{~d} 2$ & $7,936,05$ \\
\hline 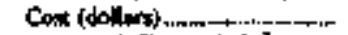 & - & - & $124,680,961$ & $26,393,868$ & 149,3BG,S55 & 240,421,981 & $395,545,432$ \\
\hline Denind Chate Oelyly & - & - & - & $1,154,724$ & - & - & $=$ \\
\hline Densund ....-............-- & - & - & - & $15,10,0,537$ & - & $3,417,216$ & $46,362,774$ \\
\hline 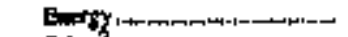 & - & - & {$[24,680,96]$} & $10,078,607$ & $72,309,0057$ & $232,749,941$ & $299,397,971$ \\
\hline 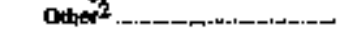 & - & - & - & - & 77,077518 & $4,24,744$ & $.164,313$ \\
\hline \multicolumn{8}{|l|}{ Tatd } \\
\hline 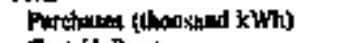 & - & - & $6,34,620$ & 990,593 & $5,090,042$ & $11,887,369$ & $12,974,662$ \\
\hline 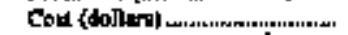 & - & - & $324,561,678$ & $61,362,331$ & 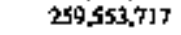 & $998,609.1 \mathrm{~s}$ & $457,651,458$ \\
\hline Drontad Ohrote Only' & - & - & - & $1,154,724$ & - & $2,801,927$ & $\overrightarrow{.}$ \\
\hline 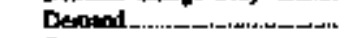 & - & - & - & 42,422695 & $\overline{-}$ & $162,005,670$ & 46398,774 \\
\hline 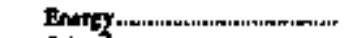 & - & - & $150,618,56 \leq 5$ & 19.784912 & $128,591,102$ & $429,311,298$ & $411,503,997$ \\
\hline Ohat & - & - & $173,943,505$ & $=$ & $130,962,615$ & 3.990, 172 & $-161,317$ \\
\hline
\end{tabular}

Sef roves and foomoces at end of toble. 
Table 19. Electriclity Purchases by Inpestor-Owned Utilities, by State, 1994 (Continued)

\begin{tabular}{|c|c|c|c|c|c|c|c|}
\hline $\begin{array}{c}\text { Soute of } \\
\text { Electricity by } \\
\text { Oranensip Oals }\end{array}$ & $\begin{array}{l}\text { New lendey } \\
\text { Rockland } \\
\text { Eloctis } \\
\text { Cof }\end{array}$ & 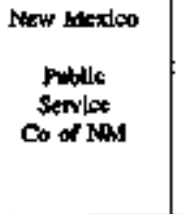 & 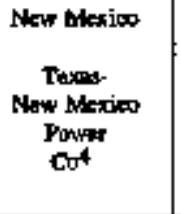 & 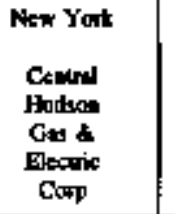 & $\begin{array}{l}\text { New Yort } \\
\text { Consolidered } \\
\text { Edicon } \\
\text { Co-NY } \\
\text { ImF }\end{array}$ & $\begin{array}{l}\text { New Yok } \\
\text { Long } \\
\text { Lcland } \\
\text { Ligitina } \\
\text { Co }\end{array}$ & $\begin{array}{l}\text { New York } \\
\text { Long } \\
\text { Sudil } \\
\text { Inef } 6\end{array}$ \\
\hline \multicolumn{8}{|l|}{ Inwascr-onened } \\
\hline & $1,339,412$ & 367,790 & $1,675,598$ & $1.769,960$ & $7,396,901$ & $1,135,694$ & - \\
\hline 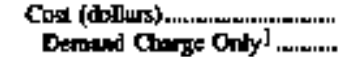 & 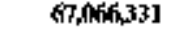 & $14,750,0 \% 3$ & 51.454 .897 & $26,872,971$ & $158,422,072$ & $25,340,483$ & - \\
\hline & - & - & $\rightarrow$ & - & - & - & - \\
\hline 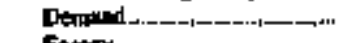 & $26,785,992$ & $3,0000,000$ & $18,477,768$ & $\bar{\pi}$ & - & $\bar{\pi}$ & - \\
\hline Entaty & $43,487,98 \mathrm{~J}$ & $7.445,376$ & 7,398,565 & $34,872,971$ & $55 R .422,072$ & $25,395,452$ & - \\
\hline Ofknt & $6,793,258$ & $264,7] 7$ & $2,618,544$ & - & - & 3,001 & - \\
\hline \multicolumn{8}{|l|}{ Fodersl } \\
\hline 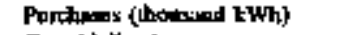 & - & - & - & - & - & - & - \\
\hline 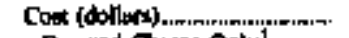 & - & 11,135 & - & - & - & - & - \\
\hline Dapinad Ohige Onty & - & - & - & - & - & - & - \\
\hline 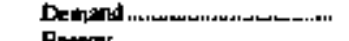 & - & - & $\rightarrow$ & - & - & - & - \\
\hline Bnergy & - & - & - & - & - & - & - \\
\hline 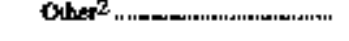 & - & $11.1,35$ & $\rightarrow$ & - & - & - & - \\
\hline \multirow{2}{*}{\multicolumn{8}{|c|}{$\begin{array}{l}\text { Stwe and Other } \\
\text { Combenoent }\end{array}$}} \\
\hline & & & & & & & \\
\hline Purchays (0nomirand KWB) & 二 & 793,062 & - & $\operatorname{los} x(x)$ & I, 18,288 & 2724001 & - \\
\hline 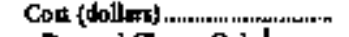 & $\overline{-}$ & 605,991 & - & $3,847,079$ & $\pi, 32,612$ & $24,246,640$ & - \\
\hline De:nand Churge Oaly I....... & - & - & - & $1,352,400$ & - & - & - \\
\hline 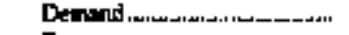 & - & $5,741,553$ & - & 431.975 & $37,841,585$ & $51,95], 200$ & - \\
\hline 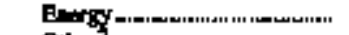 & - & $14,555,525$ & $\rightarrow$ & $2,02,01$ & 41531,427 & $62,343,4,4$ & - \\
\hline 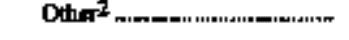 & 一 & $-19,691,1685$ & - & - & - & - & - \\
\hline \multicolumn{8}{|l|}{ Mumiripal } \\
\hline Purtusyes (boolond kWD) & - & 2,404 & I30 & - & - & 95 & $\rightarrow$ \\
\hline Cont (dbalurs) & - & 56,993 & $2,86+1$ & - & - & 4,921 & - \\
\hline Dowind Churge Only I ur...... & - & - & - & - & - & - & - \\
\hline 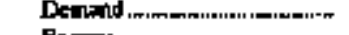 & - & - & - & $=$ & - & - & - \\
\hline 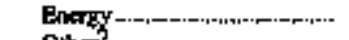 & - & 56,963 & - & - & - & 4.921 & - \\
\hline 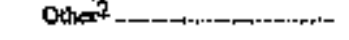 & - & $2 B$ & 2,80 & - & - & - & - \\
\hline \multicolumn{8}{|l|}{ Copprative } \\
\hline 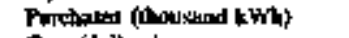 & - & 240 & 一 & - & - & - & - \\
\hline 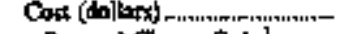 & - & ]I, 133 & - & - & - & - & - \\
\hline 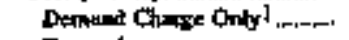 & - & - & - & - & - & - & - \\
\hline 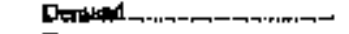 & - & - & 一 & - & - & - & - \\
\hline Enrth & - & 4.995 & - & $\rightarrow$ & - & - & - \\
\hline Olwer & - & 6.138 & - & - & - & - & - \\
\hline \multicolumn{8}{|l|}{ Other 3} \\
\hline Purkave (housued (KWh) & $\bar{z}$ & $-93,752$ & $1,2,2,974$ & 261,464 & $16,928,200$ & $\begin{array}{r}4,259,689 \\
67,467064\end{array}$ & - \\
\hline 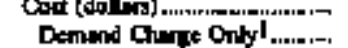 & $\overline{-}$ & $\frac{1,158,564}{-}$ & 61,674,946 & $10,462,056$ & - & $\begin{array}{l}167,402,9646 \\
-\end{array}$ & $\overline{-}$ \\
\hline 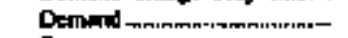 & - & - & $36,259,713$ & 71,292 & $1 \mathrm{Bg}, 0,10,088$ & $\overline{-}$ & - \\
\hline 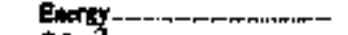 & - & $33, \mathbf{1 8 7}$ & $1,676,389$ & $10,391,164$ & $318,415,396$ & 167,462 米化 & - \\
\hline 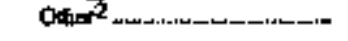 & - & $1,125,377$ & $27,138,842$ & - & $36,4,34,578$ & - & - \\
\hline \multicolumn{8}{|l|}{ Tow } \\
\hline Putcluser (Monkund kWL) & $1,219,412$ & $1,075,764$ & $2,918,682$ & $1,634,025$ & $21,143,392$ & $7,6+64,103$ & - \\
\hline Cost (dollast................ & - & $56,53,009$ & 113.137 .703 & $41,2,0 x$ & $781,754,749$ & $267,100,000$ & 二 \\
\hline 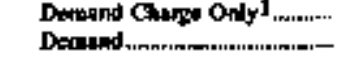 & $21.785,1928$ & $\overline{12,761,551}$ & $53,297,483$ & $\begin{array}{r}1,352,400 \\
503,270\end{array}$ & $\overline{236,751,267}$ & $\overline{11,951,2000}$ & $\overline{-}$ \\
\hline 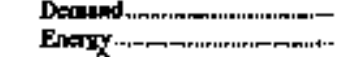 & $0,480,951$ & $\begin{array}{l}12,761,351 \\
22,696,048\end{array}$ & $\begin{array}{r}35,297,493 \\
9,074,954\end{array}$ & $39,360,2736$ & $\begin{array}{l}230,731,207 \\
5,38,369,395\end{array}$ & $\begin{array}{l}11,901,2010 \\
25,146, \pi 99\end{array}$ & - \\
\hline Other ${ }^{2}$..,-- & 1,795258 & $-18280,690$ & $48,760,266$ & - & $36,534,578$ & 5,031 & - \\
\hline
\end{tabular}

Soc novet and foctneter un and of tolle. 
Table 19. Electricity Purchoses by Inwestor-Owned Utllittes, by Stote, 1994 (Continued)

\begin{tabular}{|c|c|c|c|c|c|c|c|}
\hline 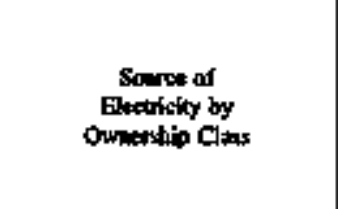 & 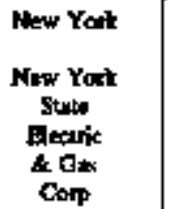 & $\begin{array}{l}\text { Now Yoik } \\
\text { Ningen } \\
\text { Molumek } \\
\text { Power } \\
\text { Corp }\end{array}$ & 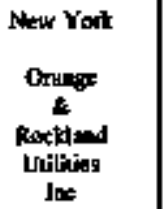 & $\begin{array}{l}\text { New Yort } \\
\text { Rochester } \\
\text { Gur \& } \\
\text { Elecorte } \\
\text { Corp }\end{array}$ & $\begin{array}{l}\text { Nonh Compling } \\
\text { Curolito } \\
\text { Powir \& } \\
\text { Ligh } \\
\text { Co }\end{array}$ & $\begin{array}{c}\text { Nonh C-politp } \\
\text { Duted } \\
\text { Power } \\
\text { Co }\end{array}$ & 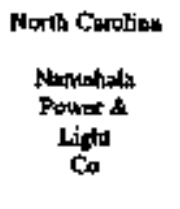 \\
\hline 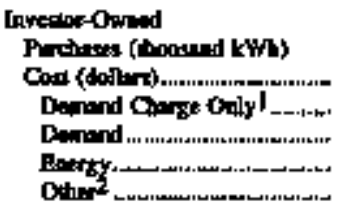 & $\begin{array}{r}1,087,010 \\
10,654,393 \\
- \\
4,417,343 \\
5,499,859 \\
377,334\end{array}$ & $\begin{array}{r}312,721 \\
4,193,617 \\
6,090 \\
265,0277 \\
8,918,992 \\
2,676\end{array}$ & $\begin{array}{r}756,533 \\
20,458,796 \\
4,251,307 \\
300,013 \\
13,907,248 \\
2\end{array}$ & $\begin{array}{r}349,715 \\
7,497,246, \\
- \\
7,76,6913 \\
6,735,593 \\
-\end{array}$ & $\begin{array}{r}2,922,749 \\
132,737,394 \\
\overline{76,5132,3404} \\
56,104,9014 \\
532\end{array}$ & $\begin{array}{r}74,575 \\
14,040,083 \\
- \\
11,645,520 \\
2,376,112 \\
18,451\end{array}$ & $\begin{array}{r}518,221 \\
24,401,377 \\
- \\
13,546,565 \\
11,754,812 \\
-\end{array}$ \\
\hline 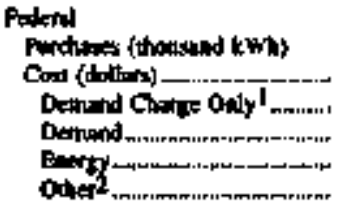 & $\begin{array}{l}\bar{z} \\
\bar{z} \\
\bar{z}\end{array}$ & $\begin{array}{l}- \\
\overline{-} \\
\overline{-} \\
-\end{array}$ & $\begin{array}{l}\overrightarrow{-} \\
\overline{-} \\
= \\
-\end{array}$ & $\begin{array}{l}- \\
\overline{-} \\
\overline{-} \\
-\end{array}$ & $\begin{array}{l}101,208 \\
2,421,834 \\
- \\
- \\
2,421,834 \\
-\end{array}$ & $\begin{array}{l}- \\
\overline{-} \\
\overline{-} \\
-\end{array}$ & $\begin{aligned} 2,617 \\
3,521,548 \\
= \\
7, \times 96,400 \\
725,148\end{aligned}$ \\
\hline 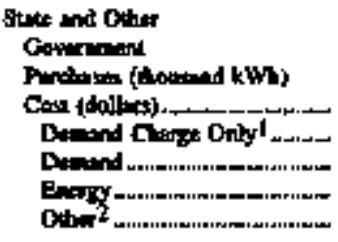 & $\begin{array}{r}656,754 \\
6412,744 \\
- \\
t, 116,0100 \\
3 \$ 45,944 \\
1,450,610\end{array}$ & $\begin{array}{r}8,073,501 \\
125,069,573 \\
7,457,000 \\
25,213,919 \\
92,43,694 \\
-\end{array}$ & $\begin{array}{l}319,63 \\
7,967,509 \\
-\quad \\
690,000 \\
7,277,849 \\
-\end{array}$ & $\begin{array}{l}1.375,620 \\
22,515,451 \\
- \\
8,003,017 \\
14,622,414 \\
-\end{array}$ & $\begin{array}{l}\quad 80,210 \\
3,726,159 \\
= \\
3,726,159 \\
-\end{array}$ & 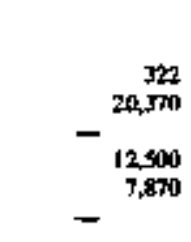 & $\begin{array}{l}- \\
\overline{-} \\
\overline{-} \\
-\end{array}$ \\
\hline 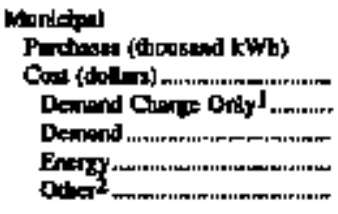 & 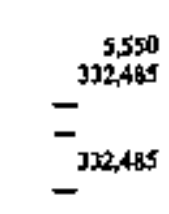 & $\begin{array}{l}7 \times 1,099 \\
49, J 46,967 \\
- \\
\overline{45.196,967} \\
-\end{array}$ & $\begin{array}{l}\bar{z} \\
\bar{z} \\
\bar{z}\end{array}$ & $\begin{array}{l}- \\
- \\
z \\
-\end{array}$ & $\begin{array}{r}67,419 \\
64,461,697 \\
- \\
49,652,914 \\
9,210,212 \\
1,518,491\end{array}$ & $\begin{array}{r}6,236,044 \\
429,611,093 \\
-186,490 \\
361,395,401 \\
65,302,789 \\
3,100,399\end{array}$ & $\begin{array}{l}= \\
= \\
= \\
=\end{array}$ \\
\hline 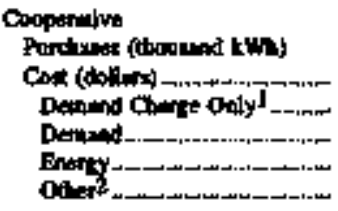 & $-^{22,457}$ & $\begin{array}{l}- \\
- \\
- \\
- \\
-\end{array}$ & $\begin{array}{l}- \\
\overline{-} \\
\overline{-} \\
-\end{array}$ & $\begin{array}{l}- \\
- \\
- \\
-\end{array}$ & $\begin{array}{l}- \\
= \\
- \\
-\end{array}$ & 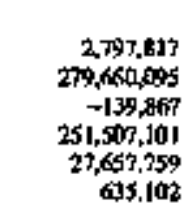 & $\begin{array}{l}- \\
-- \\
= \\
-\end{array}$ \\
\hline 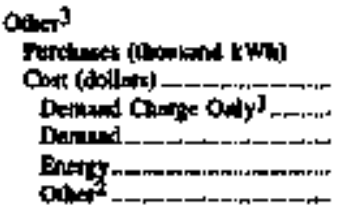 & 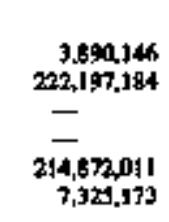 & $\begin{array}{r}16,253,953 \\
031,616,492 \\
-\quad 719,997 \\
924,212,250 \\
6,683,855\end{array}$ & $\begin{array}{r}528,7 m 7 \\
24,204,605 \\
-\quad 390,419 \\
19,009,694 \\
724,582\end{array}$ & $\begin{array}{r}210,832 \\
4,401,616 \\
- \\
446,77 y \\
3,94,831\end{array}$ & $\begin{array}{r}9,289,622 \\
210,146,227 \\
- \\
37,462,711 \\
172,94,722 \\
-11,22,\end{array}$ & $\begin{array}{l}704,129 \\
29,904,636 \\
- \\
29,934,016 \\
-\end{array}$ & $\begin{array}{l}\overline{2}, 195,521 \\
- \\
- \\
2,195,524\end{array}$ \\
\hline 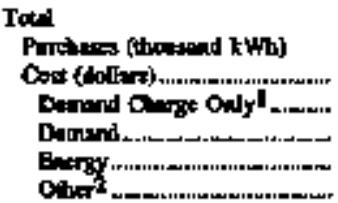 & $\begin{array}{r}5,639,505 \\
229,619,401 \\
- \\
5,533,443 \\
224,532,756 \\
9553,704\end{array}$ & $\begin{array}{r}25,432,464 \\
1,1+1,066,249 \\
3,458,690 \\
26,198,943 \\
1,930,721,443 \\
6,486,739\end{array}$ & $\begin{array}{r}1,6724,878 \\
4,630,844 \\
4,251,029 \\
1,720,432 \\
2,274,791 \\
724,582\end{array}$ & $\begin{array}{l}1,936,167 \\
34,414,327 \\
- \\
9,304,499 \\
26,112,838 \\
-\end{array}$ & $\begin{array}{r}7,031,257 \\
413,243,723 \\
7 \\
169,740,145 \\
244,407,853 \\
3,547,837\end{array}$ & $\begin{array}{r}9,833,787 \\
753,265,677 \\
-326,357 \\
624,550,522 \\
125,276,560 \\
3,762,952\end{array}$ & $\begin{array}{r}540,1544 \\
35,118,449 \\
- \\
21,42,465 \\
11,490,960 \\
2,195,524\end{array}$ \\
\hline
\end{tabular}

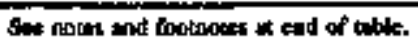


Table 19. Electricity Purchnses by Investor-Owned Utilitles, by State, 1994 (Continued)

\begin{tabular}{|c|c|c|c|c|c|c|c|}
\hline $\begin{array}{c}\text { Sowree of } \\
\text { Dectricity by } \\
\text { armenhlp Cless }\end{array}$ & 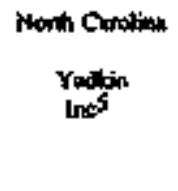 & 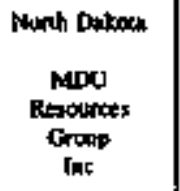 & $\begin{array}{l}\text { Oltio } \\
\text { Oener } \\
\text { Cos }\end{array}$ & $\begin{array}{c}\text { Obio } \\
\text { Clacineuti } \\
\text { Gus \& } \\
\text { Elestric } \\
\text { Co }\end{array}$ & 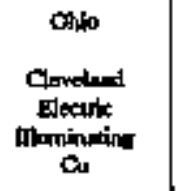 & $\begin{array}{c}\text { Onip } \\
\text { Colmmbur } \\
\text { Southeth } \\
\text { Pown Co }\end{array}$ & $\begin{array}{l}\text { Otio } \\
\text { Doytom } \\
\text { Powter \& } \\
\text { Llyit } \\
\text { Ca }\end{array}$ \\
\hline \multicolumn{8}{|l|}{ Inestoroushod } \\
\hline 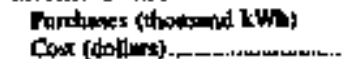 & - & 227,691 & - & 870,600 & $2,093,573$ & 388,175 & 791,213 \\
\hline 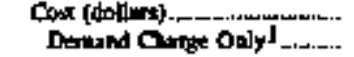 & $\bar{z}$ & $\frac{6,816,027}{-}$ & $\bar{z}$ & $\frac{15,949,925}{-}$ & $\begin{array}{l}132,390,465 \\
-\end{array}$ & $\begin{array}{l}11,564,651 \\
-\end{array}$ & $\begin{array}{l}18,390,394 \\
-\end{array}$ \\
\hline Denand.m-r.r., & - & $2,659,869$ & - & 131,800 & $4,8 n, 575$ & $1 \$ 2,264$ & $2.911,355$ \\
\hline Enctit & - & 4.156 .158 & س & $32,690,433$ & 128551526 & $11,4: 2,197$ & $15,268,491$ \\
\hline OQn ${ }^{2} \ldots$ & - & 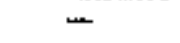 & - & $J, 030,685$ & $-538 \leqslant 56$ & - & 21554 \\
\hline \multicolumn{8}{|l|}{ Fedonal } \\
\hline 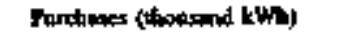 & - & 25,139 & - & - & - & 25,187 & - \\
\hline 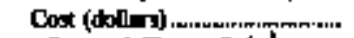 & - & 172,529 & - & - & - & $1,109,371$ & - \\
\hline 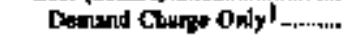 & - & ב & - & - & - & - & - \\
\hline 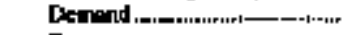 & - & - & $=$ & - & - & - & - \\
\hline Emprgy & - & 390.587 & - & - & - & $1, \sqrt{07}, 171$ & - \\
\hline Othe $a^{2}$ mimmann & 一 & $-166,058$ & $=$ & - & - & - & - \\
\hline \multirow{2}{*}{\multicolumn{8}{|c|}{ Sutte and Other }} \\
\hline & & & & & & & \\
\hline 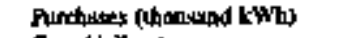 & $=$ & 3,975 & $=$ & - & - & - & - \\
\hline $\operatorname{Cost}(\operatorname{lol})$ & - & 49.97 & $=$ & - & - & - & - \\
\hline 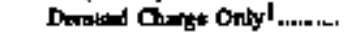 & - & - & - & - & - & - & - \\
\hline - & - & - & - & - & - & - & - \\
\hline 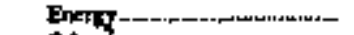 & $\rightarrow$ & $49,9 n$ & - & - & - & - & - \\
\hline Oalef & - & - & - & - & - & - & - \\
\hline \multicolumn{8}{|l|}{ Mlmicipd } \\
\hline 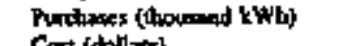 & - & 1, EN0 & - & - & 152 & $\mathbf{1 0 , 9 6 1}$ & \\
\hline 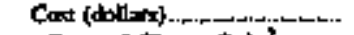 & - & $29,3) 2$ & $=$ & - & 3:5 & 247,455 & 6,446 \\
\hline Demund & - & - & - & - & - & - & - \\
\hline 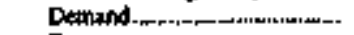 & - & - & - & - & 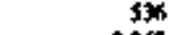 & - & - \\
\hline 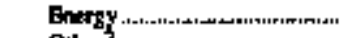 & - & 25,312 & - & - & 3,362 & 247.495 & 6,466 \\
\hline Other ${ }^{3}$ & - & - & - & - & - & - & - \\
\hline \multicolumn{8}{|l|}{ Cooperative } \\
\hline 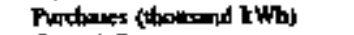 & - & 61,335 & - & $248,9] 3$ & - & 25,095 & מב \\
\hline 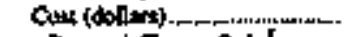 & - & $12,636,323$ & - & $5,0 \mathrm{~J} 5,614$ & - & $530,2+2$ & - \\
\hline 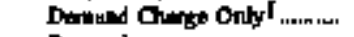 & - & - & - & - & - & - & - \\
\hline Dentudi & - & 8,674719 & - & 210000 & - & - & - \\
\hline Enarby & - & $3,563.630$ & $=$ & $4,5] 2,605$ & - & 510,242 & - \\
\hline 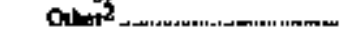 & - & $-2,2,14$ & - & 299.009 & $\neg$ & - & - \\
\hline \multicolumn{8}{|l|}{ Othen ${ }^{3}$} \\
\hline Poncluses (hroonsad kWh) & - & - & - & - & 6], 159 & $3,184,081$ & In5,697 \\
\hline 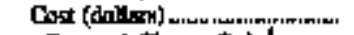 & - & - & - & - & $1,843,379$ & $121 . J 05,85$ & 2,888,915 \\
\hline 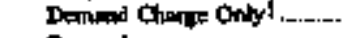 & - & - & - & - & - & - & - \\
\hline 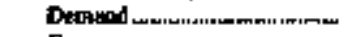 & - & - & - & - & - & - & 155,558 \\
\hline Endify & - & - & - & - & $\mathbf{3}, \mathbf{B A 3}, \mathrm{API}$ & $621,109,253$ & 2729,357 \\
\hline O10 & - & - & - & - & - & - & - \\
\hline \multicolumn{8}{|l|}{ Totad } \\
\hline Ponthot (thooushed kWh) & - & 699.740 & m & $1,159,353$ & $2,54,8384$ & $3,693,702$ & tox,sent \\
\hline 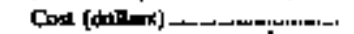 & - & $19,704,160$ & - & $28,461,539$ & $134,738,052$ & $134,509,612$ & $21,286,773$ \\
\hline 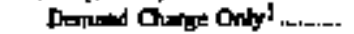 & - & - & - & - & - & - & - \\
\hline 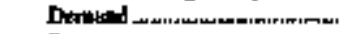 & - & 11.334 .564 & - & 321,8013 & $4,878,1] 1$ & 152,264 & 3,146,913 \\
\hline Emerty- & - & $8,537,872$ & - & I7,210, & $1374,098,597$ & $134,357,348$ & $18,000,3,34$ \\
\hline Other & - & $- 1 6 8 \longdiv { 2 9 2 }$ & - & $3,329,694$ & -578.456 & - & 215.548 \\
\hline
\end{tabular}

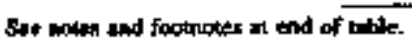


Table 19. Electricity Purchases by Investor-Owned Utilltes, by Stale, 1994 (Continued)

\begin{tabular}{|c|c|c|c|c|c|c|c|}
\hline $\begin{array}{c}\text { Sounte of } \\
\text { Electioniny by } \\
\text { Ownendip Clas }\end{array}$ & $\begin{array}{l}\text { Otho } \\
\text { Indinut } \\
\text { Lentucky } \\
\text { Electrte } \\
\text { Conp5 }\end{array}$ & $\begin{array}{l}\text { Otio } \\
\text { Otio } \\
\text { Bdiken } \\
\text { Cu }\end{array}$ & $\begin{array}{c}\text { Olio } \\
\text { Obin } \\
\text { Powr } \\
\text { Cu }\end{array}$ & $\begin{array}{l}\text { Chlo } \\
\text { Otio } \\
\text { Valley } \\
\text { elociric } \\
\text { Corp }\end{array}$ & $\begin{array}{c}\text { Otio } \\
\text { Toledo } \\
\text { Edikon } \\
\text { Co }\end{array}$ & 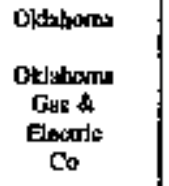 & 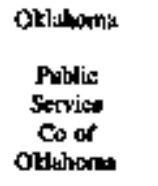 \\
\hline \multicolumn{8}{|l|}{ Invelas Ohined } \\
\hline Phisuss (thouned kWh) & - & $1,493,168$ & $1,315, \sqrt{126}$ & $9,354,871$ & 616,378 & 314, 158 & 104,5tin \\
\hline Cond (dollow) & - & $37,043,628$ & $36,775,845$ & $136,339,896$ & $36,399,654$ & $51,773,204$ & $2,591,673$ \\
\hline Dremend OMrge Onfy & - & - & - & - & - & - & - \\
\hline 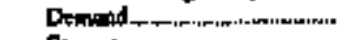 & - & $8,279,482$ & $26 \mathrm{D}, 141$ & - & $2,60,4,382$ & - & - \\
\hline 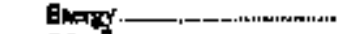 & - & $28,600,200$ & $35,915,704$ & - & {$[3,4,4,3,3 \mid$} & $30,405,519$ & $2,594,673$ \\
\hline 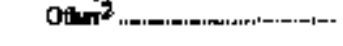 & - & 73,747 & - & $136,399,806$ & 330,88 I & 1.167,685 & - \\
\hline \multicolumn{8}{|l|}{ Federal } \\
\hline Funchuset (thousund KWh) & - & - & 43,317 & - & - & - & 5,357 \\
\hline Cont $($ ablyn) & - & - & $1.849,909$ & - & - & - & J1057) \\
\hline 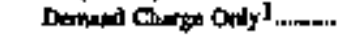 & - & - & - & - & - & - & - \\
\hline 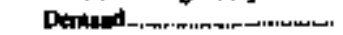 & - & - & - & - & - & - & - \\
\hline Eneryy & - & - & $1,899,0,090$ & - & - & - & 100,571 \\
\hline Onder & - & - & - & - & - & - & - \\
\hline \multicolumn{8}{|l|}{ Stant wod Ohth } \\
\hline 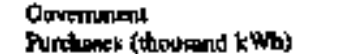 & & & & & & & \\
\hline 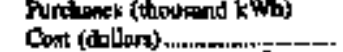 & $\bar{z}$ & $z$ & $=$ & $\bar{z}$ & $\overline{-}$ & $\overline{-}$ & 23,52 \\
\hline 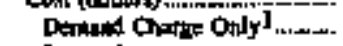 & - & $\overrightarrow{-}$ & $\overline{-}$ & - & - & - & - \\
\hline 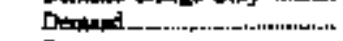 & - & - & - & - & - & - & - \\
\hline Bnory & - & - & - & - & - & - & 418,072 \\
\hline Othere & - & - & - & - & - & - & - \\
\hline \multicolumn{8}{|l|}{ Bdunkespal } \\
\hline 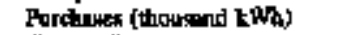 & - & 42 & 21,648 & $m$ & - & - & - \\
\hline Con (dollon) & - & 710 & 385,599 & - & - & - & - \\
\hline Deriund Charge Oaly ${ }^{1}$ & - & - & - & - & - & - & - \\
\hline 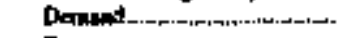 & - & - & - & - & ב & - & - \\
\hline 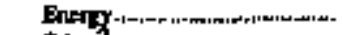 & - & $\pi 0$ & J85,539 & - & - & - & -- \\
\hline 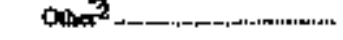 & - & - & - & - & - & - & - \\
\hline \multicolumn{8}{|l|}{ Coopertive } \\
\hline Punctules (thoosind kWh) & - & 578,276 & 43,251 & - & $=$ & 285.793 & 51,352 \\
\hline Cod (dallary) ........_. & - & $15,258,784$ & 657,506 & - & - & $5.270,496$ & $1,3] 5,225$ \\
\hline 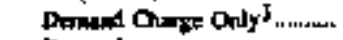 & - & - & - & - & - & - & - \\
\hline 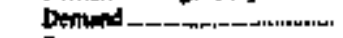 & - & $1,470,300$ & - & - & - & 455,986 & - \\
\hline 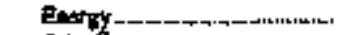 & - & $13,78,094$ & 657,508 & - & - & $4,814,510$ & $1.215,225$ \\
\hline 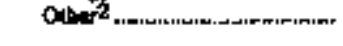 & - & - & - & - & $\rightarrow$ & - & -- \\
\hline \multicolumn{8}{|l|}{$0 \mathrm{an}^{3}{ }^{3}$} \\
\hline Posting (lhasad kWh) & $\rightarrow$ & -363 & $1,017,128$ & - & 113,505 & 3,186,937 & $1,202,11 \mathrm{Rz}$ \\
\hline Coan $\{$ doltans & - & $\rightarrow$ & 26.847 .106 & - & $3,845,454$ & $211,657,971$ & $30,298,205$ \\
\hline Demend Gimpte Onlyt....... & - & - & - & - & - & - & -- \\
\hline 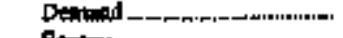 & - & - & - & - & 722,577 & - & - \\
\hline 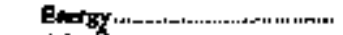 & - & - & $21,357.106$ & - & $3,163,475$ & $29,107,556$ & $216,089,999$ \\
\hline Oth & 一 & 一 & - & - & $-40,598$ & $182,549,815$ & $\mid(1,30,2,2,16$ \\
\hline \multicolumn{8}{|l|}{ Tatd } \\
\hline 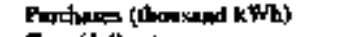 & - & $2,161, J 23$ & $2,540,299$ & $9,354,87 J$ & 739,183 & $4,386,883$ & $1,386,274$ \\
\hline 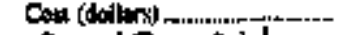 & - & $52,302,722$ & 59.955.907 & J35,339,8PX & $20,245,108$ & $22 \pm, 701,071$ & $34,833,746$ \\
\hline Deprand Onge Onby 1 & - & - & - & - & - & - & - \\
\hline Dearand nr... & - & $9.749,7 B 2$ & 260,141 & - & $3,326,959$ & 455,986 & - \\
\hline 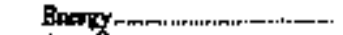 & $\rightarrow$ & $42,479,193$ & $59,690,766$ & - & ] $6,627,266$ & $44,527,585$ & $24,525,500$ \\
\hline $0 \operatorname{lon}^{3}$ & - & 73,747 & - & $136,339,806$ & $29,4,283$ & $193,717,500$ & $10,3 \cap \mathbb{R}, 206$ \\
\hline
\end{tabular}

Soe novea und foothotiog it end of toble 
Table 19. Electricity Purchases by Investor-Owned Utilifies, by State, 1994 (Continumed)

\begin{tabular}{|c|c|c|c|c|c|c|c|}
\hline $\begin{array}{l}\text { Socres of } \\
\text { Entricily by } \\
\text { Owmentip } 0 \text { ind }\end{array}$ & $\begin{array}{l}\text { Ongon } \\
\text { Facificonp }\end{array}$ & 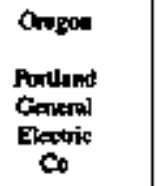 & 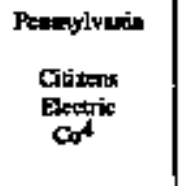 & 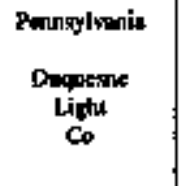 & 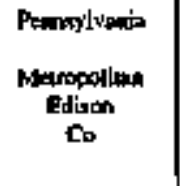 & 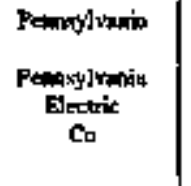 & 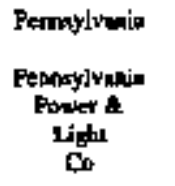 \\
\hline \multicolumn{8}{|l|}{ Invedor.0 Onnod } \\
\hline 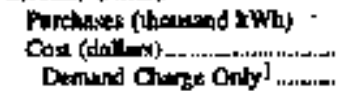 & $\begin{array}{r}2,363,146 \\
66,562,608 \\
-\end{array}$ & $\begin{array}{r}3,855,031 \\
98,300,390 \\
10,734,1000\end{array}$ & $\begin{array}{r}159,600 \\
6,499,944 \\
-\end{array}$ & $\begin{array}{r}520,200 \\
18,061,264 \\
-\end{array}$ & $\begin{array}{r}\text { K\$1,203 } \\
39,623,1571 \\
4,729,665\end{array}$ & $\begin{array}{r}431,027 \\
14,4,55,6144 \\
1,471,022\end{array}$ & $\begin{array}{l}1,788,198 \\
4.512 .654 \\
-\end{array}$ \\
\hline Demand & $14,583,600$ & 2,8010000 & 2,2007219 & $3,500,682$ & $14,010,843$ & $2,423,36, ?$ & $1,005,541$ \\
\hline Ematy-- & $51,103,843$ & $\begin{array}{r}34,943,950 \\
-142,556\end{array}$ & $\begin{array}{l}1,633,90 m \\
-38 \pm, 602\end{array}$ & 10560584 & $20,882,642$ & In,781,157 & $\begin{array}{l}73,492,920 \\
10,04,593\end{array}$ \\
\hline \multicolumn{8}{|l|}{ Fedend } \\
\hline Partusen (thousand (WWh) & 240,699 & 348,729 & - & - & - & - & - \\
\hline Cost (dollurs) & $81,270,032$ & $12,351,300$ & - & - & - & - & - \\
\hline Denurd Charge Only' 1 mirin. & $77,4,0,996$ & $5.832,140$ & - & - & - & - & - \\
\hline 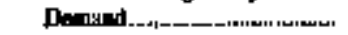 & - & - & - & - & - & - & - \\
\hline 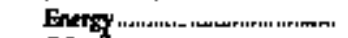 & $1,879,1136,6$ & $6,479,307$ & - & - & - & - & - \\
\hline Ober2 & - & $-1,073$ & - & - & - & - & - \\
\hline \multicolumn{8}{|l|}{ State and Ober } \\
\hline Govanminak & & & & & & & \\
\hline 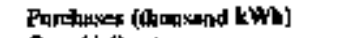 & 1,154,759 & 4,370,950 & - & - & $1 B ; 066$ & 25,463 & $49,466 \mathrm{i}$ \\
\hline 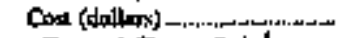 & $57,980,318$ & $87,829.360$ & - & - & $258, \mathrm{TMI}$ & 282,358 & 720,940 \\
\hline 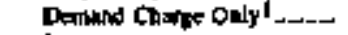 & - & - & - & - & - & - & - \\
\hline Bermend_....._.............. & 468,869 & - & - & - & 167,909 & J5X.487 & - \\
\hline 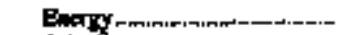 & $17,497,969$ & $75,102,422$ & - & - & 90,792 & 125,471 & 720,943 \\
\hline Othet & $40,073,383$ & $12,726.968$ & - & - & - & - & - \\
\hline \multicolumn{8}{|l|}{ Mhetedput } \\
\hline Punclasas (lhoolwad kWh) & 62,117 & $757,85 \%$ & - & 37,964 & 3,900 & 27 & $29,9 n$ \\
\hline Cost (dolburit) & 3],515,156h & $16,97,1897$ & - & $1,07,840$ & 297,918 & 10,612 & $1,799,260$ \\
\hline 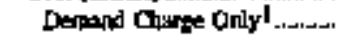 & - & 571,140 & - & - & - & - & - \\
\hline Dentad__........................... & $21,210,064$ & - & - & - & - & - & - \\
\hline 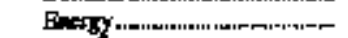 & $10,104,992$ & $16,278,490$ & - & $1,471,840$ & 297,918 & 10,612 & $1,798,260$ \\
\hline Other & - & $121.16 ?$ & - & - & - & - & - \\
\hline \multicolumn{8}{|l|}{ Cooperative } \\
\hline 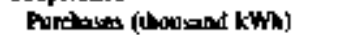 & $1,999, B M 7)$ & - & - & - & - & $1 \mathrm{~L}_{1}, 172$ & 25,525 \\
\hline 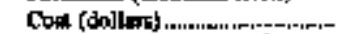 & $5 ., 127,151$ & - & - & - & - & 116,968 & 362,486 \\
\hline Deprand Chure Only' & - & - & - & $m$ & - & - & - \\
\hline Denond & $11.173 \operatorname{sing}$ & $=$ & - & - & - & - & - \\
\hline 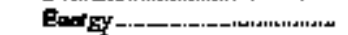 & $41,148,851$ & - & - & - & - & 116968 & 362,496 \\
\hline 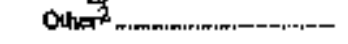 & - & - & - & - & - & - & - \\
\hline \multicolumn{8}{|l|}{ Dther } \\
\hline 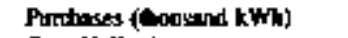 & $1,155,416$ & $1,665,661$ & - & 88.275 & 1,394,643 & $2,850,162$ & $4,169,904$ \\
\hline Cost (dolbrs) & $50,671.940$ & -4.8601044 & - & 20 吹.169 & $146,696,792$ & $143,154,170$ & $199,630,573$ \\
\hline 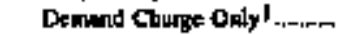 & - & - & - & - & 69,904 & - & - \\
\hline 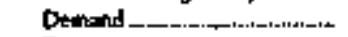 & $18,349,486$ & $7,450,092$ & - & - & $., 745,029$ & $3,174,7 \$ 11$ & 5.119 .260 \\
\hline $\mathrm{Ba}$. & 47, $, 3 \times 1,043$ & $3,1,469,916$ & - & 2069.169 & $142,953,628$ & $140,024,491$ & $194,191,954$ \\
\hline Other ${ }^{2}$ & $562,4] 1$ & $-45,980,116$ & - & - & $-71,764$ & $-45,101$ & 302,459 \\
\hline \multicolumn{8}{|l|}{ Jold } \\
\hline Puntuses (tooconat kWh) & $9,445,944$ & $10,998,429$ & ISP,tion & $\approx 26 f_{1} 3319$ & $4,086,3204$ & $3,317,061$ & 6,065,064 \\
\hline Con (dolbry) & $349,122.215$ & $2] 0,671,097$ & $6,499,944$ & $21,202,2+5$ & $186,876,561$ & $158,229,712$ & $287,017,92 x$ \\
\hline Demind Churge Only I........ & $T M, A M B S \%$ & $17,17,110$ & - & - & $4,799,569$ & 1,4100 & - \\
\hline Denowa & 65,7155519 & $10,2 \pm 0,1202$ & 2,207219 & $5,900,082$ & $17,923,774$ & $5,755,034$ & $6,124,800$ \\
\hline $\mathrm{Burgy}$ & $161,794,734$ & $216,519,535$ & $4,633,907$ & $17,201,393$ & $164,224,980$ & ]$\times 1 \times 0,978,699$ & $270,506,473$ \\
\hline Othex 2. & $41,210,956$ & $-33,275,710$ & $-381,162$ & - & $=71,764$ & 24,957 & $10,39 \pi, 5 \leq 2$ \\
\hline
\end{tabular}

Sece noles and foolnotes at wit of with. 
Table 19. Electricity Purchases by Investor-Owned Utilities, by State, 1994 (Continued)

\begin{tabular}{|c|c|c|c|c|c|c|c|}
\hline 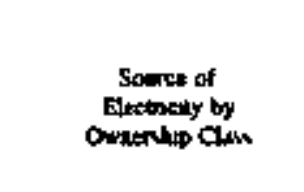 & 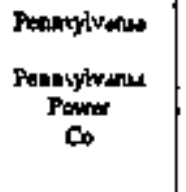 & 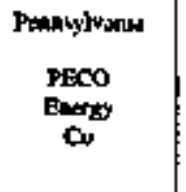 & 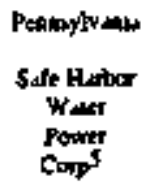 & 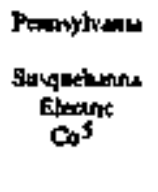 & 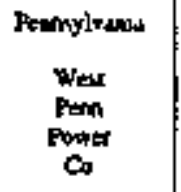 & 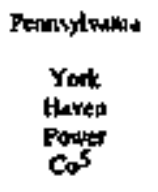 & 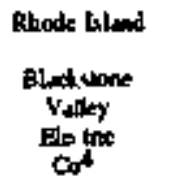 \\
\hline \multicolumn{8}{|l|}{ Invesupe Ouned } \\
\hline Puachenes (dhound kWh) & 656,473 & $2,991,686$ & - & - & 1,186,812 & - & $1,009,340$ \\
\hline $\operatorname{Cog}(d-1)(\alpha, n)$ & $12,570,174$ & $23,182,193$ & - & - & $155,223,472$ & - & $94,948, \sqrt{103}$ \\
\hline Dorband Charge 0dy1 & - & $3,135,025$ & - & - & - & - & $\rightarrow$ \\
\hline Derear & - & 642,630 & - & - & $57,704,1 \% 1$ & - & $71,100,319$ \\
\hline Enangy & $12,570,174$ & 615,794 & - & - & $59,731,292$ & - & $20,414,213$ \\
\hline 0 ats & - & $18,774,740$ & - & - & $34,788,040$ & - & $1,411,476$ \\
\hline \multicolumn{8}{|l|}{ Fedend } \\
\hline Protowe (fhoound kwh) & - & - & - & $m$ & - & - & - \\
\hline Cont (dordur) & - & - & - & - & - & - & - \\
\hline 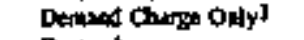 & $m$ & - & - & - & - & - & - \\
\hline Derinumet & - & - & - & - & - & - & - \\
\hline Enrरत्र & - & - & - & - & - & - & - \\
\hline Onber & - & - & - & - & - & - & - \\
\hline \multicolumn{8}{|l|}{ Sule and Othe } \\
\hline Punchuses (llowiond kWh) & - & 70,6033 & - & - & - & - &.- \\
\hline $\operatorname{Cos}(d 0 / \pi x)$ & - & 305,205 & - & - & - & - & - \\
\hline Denind Charge Onlyl & - & $\rightarrow$ & - & - & - & - & - \\
\hline Derwind & - & - & - & - & - & - & - \\
\hline Enersy & - & 20s,abs & - & - & - & - & $\rightarrow$ \\
\hline $04 \mathrm{des}^{2}$ & - & $\rightarrow$ & - & - & - & - & - \\
\hline \multicolumn{8}{|l|}{ Monapal } \\
\hline Purchens (thowend $\mathbf{k}$ Wh) & - & - & - & - & 3 39 & - & -- \\
\hline Cou (dollin) & - & - & - & - & 5,49 & - & -- \\
\hline Denind Drege Only I & - & ــ & - & - & - & - & - \\
\hline Dund & - & מـ & - & - & 89 & - & זـ \\
\hline epery & - & - & - & - & 5,400 & - & $\rightarrow$ \\
\hline Other ${ }^{2}$ & - & - & - & - & - & - & - \\
\hline \multicolumn{8}{|l|}{ Cooporive } \\
\hline Pupthes (thoound kWh) & - & - & - & - & - & - & $\rightarrow$ \\
\hline $\operatorname{Cos}(\mathrm{dollim})$ & - & - & - & - & - & - & $m$ \\
\hline Dorind Girs: Oalyl & - & - & - & - & 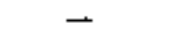 & $\leftarrow$ & - \\
\hline Depund & - & - & - & - & - & - & $\rightarrow$ \\
\hline Enorgy & - & - & - & - & - & - & $-r$ \\
\hline$O$ has $\sigma^{2}$ & - & - & - & - & - & - & -- \\
\hline \multicolumn{8}{|l|}{$\operatorname{coth}^{3}$} \\
\hline Ponduns (Ilamyend kWb) & BTo & $3,83,609$ & - & - & $2,24: 384$ & - & 3,332 \\
\hline Cot (dallin) & $13.5 \mathrm{~m}$ & 145,663,21.5.5 & - & - & $92,195,651$ & - & 25,800 \\
\hline Deand Dane Onkyl & - & - & - & - & - & - & - \\
\hline Dener & & 13,023,1808 & - & - & $52,106,662$ & - & $\rightarrow$ \\
\hline Bintry & 13,572 & $130,891,99]$ & - & - & $42,01000,967$ & - & $21+\$ 00$ \\
\hline onere & - & $1,347,412$ & - & - & - & - & - \\
\hline \multicolumn{8}{|l|}{ Total } \\
\hline Diratares (thoudend kWh) & 657,349 & $7,985,928$ & - & - & $6,135,533$ & - & I, \\
\hline Cos (dollent) & $12,513,746$ & $169,556,603$ & - & - & $247, A>A$, GIST & - & Ma, $969, \mathrm{Br}$ \\
\hline Denend Chuffe Onlyl & - & $3,13,025$ & 一 & - & - & - & - \\
\hline 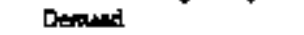 & $\rightarrow$ & $13,6 \% 6,510$ & - & - & $109,810,910$ & - & TI.1001319 \\
\hline Enerty & 12589.746 & $132.2129 \%$ & $=$ & - & 96,825669 & - & $20,496,013$ \\
\hline Otan 2 & - & 20.522 .152 & - & - & 34,788,040 & - & $1,433,476$ \\
\hline
\end{tabular}

Sec notes and foorrocen it red of toble 
Twble 19. Elextricily Purchases by Investor-Omed Utitities, by State, 1994 (Conthaed)

\begin{tabular}{|c|c|c|c|c|c|c|c|}
\hline 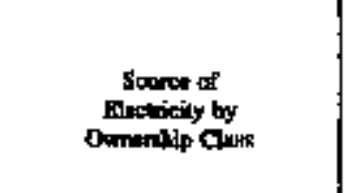 & 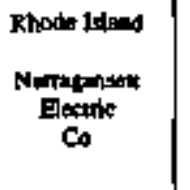 & $\begin{array}{l}\text { Rhote klund } \\
\text { Dewpor } \\
\text { Carpic }\end{array}$ & 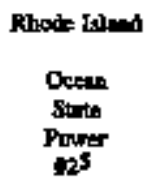 & 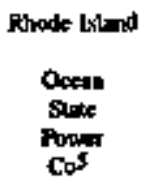 & $\begin{array}{l}\text { Sonifh Cursilan } \\
\text { Lochturt } \\
\text { Pon's } \\
\text { Co }\end{array}$ & 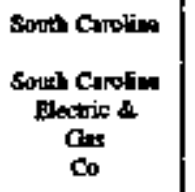 & 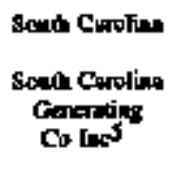 \\
\hline 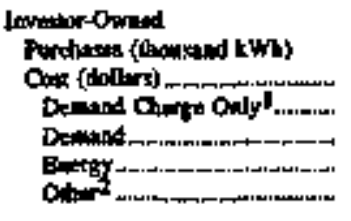 & 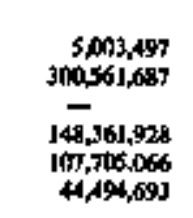 & $\begin{array}{r}542,825 \\
35,49,9401 \\
- \\
24,773,242 \\
7,555,294 \\
1,569,300\end{array}$ & $\begin{array}{l}- \\
- \\
- \\
-\end{array}$ & $\begin{array}{l}z \\
z \\
z \\
-\end{array}$ & $\begin{array}{r}27,, 563 \\
12,94,4,77 \\
7 \\
6,674,716 \\
7,516,923 \\
-1,244,144\end{array}$ & 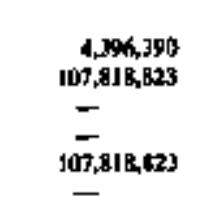 & $\begin{array}{l}z \\
= \\
z \\
-\end{array}$ \\
\hline 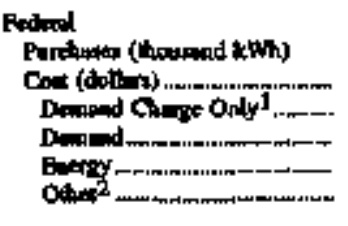 & $\begin{array}{l}\overline{-} \\
\overline{-} \\
\overline{-}\end{array}$ & $\begin{array}{l}- \\
\overline{-} \\
\overline{-}\end{array}$ & $\begin{array}{l}\overline{-} \\
\bar{z} \\
\overline{-}\end{array}$ & $\begin{array}{l}z \\
z \\
z \\
-\end{array}$ & $\begin{array}{l}- \\
- \\
- \\
-\end{array}$ & $\begin{array}{l}\bar{z} \\
\bar{z} \\
\bar{z}\end{array}$ & $\begin{array}{l}\overline{-} \\
\overline{-} \\
\overline{-}\end{array}$ \\
\hline 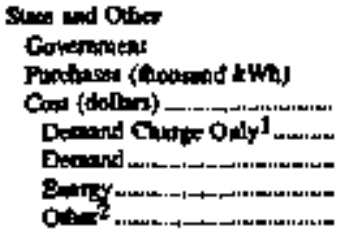 & $\begin{array}{l}\bar{z} \\
\bar{z} \\
\bar{z}\end{array}$ & $\begin{array}{l}= \\
= \\
=\end{array}$ & $\begin{array}{l}- \\
\overline{-} \\
\overline{-} \\
-\end{array}$ & $\begin{array}{l}\vec{z} \\
\vec{E} \\
\overline{-}\end{array}$ & $\begin{array}{l}\bar{z} \\
\bar{z} \\
\bar{z}\end{array}$ & 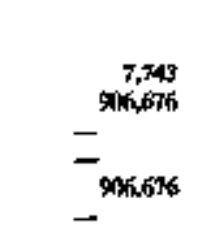 & $\begin{array}{l}\bar{z} \\
\bar{z} \\
\overline{-}\end{array}$ \\
\hline 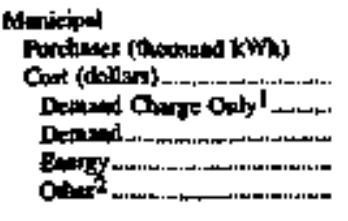 & $\begin{array}{l}= \\
= \\
z\end{array}$ & $\begin{array}{r}4,543 \\
1,268,567 \\
-\quad \\
1,034,669 \\
233,886 \\
-\end{array}$ & $\begin{array}{l}\text { Z} \\
\text { - } \\
\text { - } \\
-\end{array}$ & $\begin{array}{l}- \\
- \\
- \\
- \\
-\end{array}$ & $\begin{array}{l}\bar{z} \\
\bar{z} \\
\bar{z}\end{array}$ & $\begin{array}{l}- \\
\overrightarrow{-} \\
\vec{E} \\
-\end{array}$ & $\begin{array}{l}\overline{-} \\
\bar{z} \\
\overline{-}\end{array}$ \\
\hline 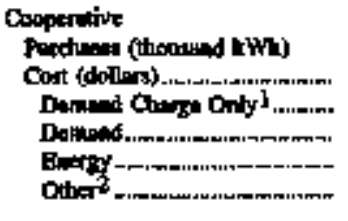 & $\begin{array}{l}z \\
\overline{-} \\
\overline{-}\end{array}$ & $\begin{array}{l}z \\
\overline{-} \\
\overline{-}\end{array}$ & $\begin{array}{l}\bar{z} \\
\bar{z} \\
\bar{z}\end{array}$ & $\begin{array}{l}\bar{z} \\
\bar{z} \\
\overline{-}\end{array}$ & $\begin{array}{l}z \\
z \\
\overline{-} \\
-\end{array}$ & 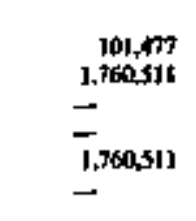 & $\begin{array}{l}\bar{z} \\
\bar{z} \\
- \\
-\end{array}$ \\
\hline 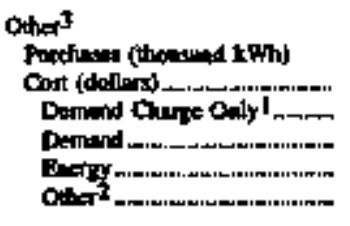 & $-^{11,255} \begin{array}{r}115.024 \\
- \\
-^{113.824}\end{array}$ & $\begin{array}{r}18,659 \\
1,22,131 \\
-\quad \\
531,621 \\
688,759 \\
1, \pi]\end{array}$ & $\begin{array}{l}\bar{z} \\
\bar{z} \\
\bar{z}\end{array}$ & $\begin{array}{l}\bar{z} \\
\bar{z} \\
=\end{array}$ & $\begin{array}{r}\begin{array}{r}2,939 \\
155,962\end{array} \\
-\quad \begin{array}{l}67, J 48 \\
8 B, 814\end{array}\end{array}$ & 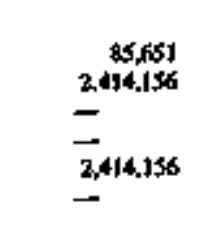 & $\begin{array}{l}z \\
\overline{-} \\
\overline{-} \\
-\end{array}$ \\
\hline 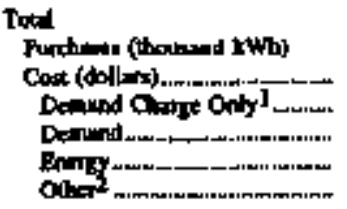 & 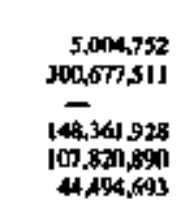 & $\begin{array}{r}566,027 \\
37,906,5398 \\
- \\
27,909,532 \\
8,77,985 \\
1,571,021\end{array}$ & $\begin{array}{l}\overline{-} \\
\overline{-} \\
\bar{z}\end{array}$ & $\begin{array}{l}\overline{-} \\
\bar{z} \\
\bar{z} \\
=\end{array}$ & $\begin{array}{r}375,502 \\
13,105,499 \\
- \\
6,674,718 \\
3,585,071 \\
-3,155,330\end{array}$ & 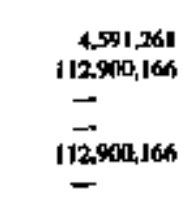 & $\begin{array}{l}z \\
\overline{-} \\
- \\
-\end{array}$ \\
\hline
\end{tabular}

Sec woon and footinglys an and of tablo. 
Table 19. Ełectricity Porcheses by Investor-Owned Utilities, by State, 1994 (Continued)

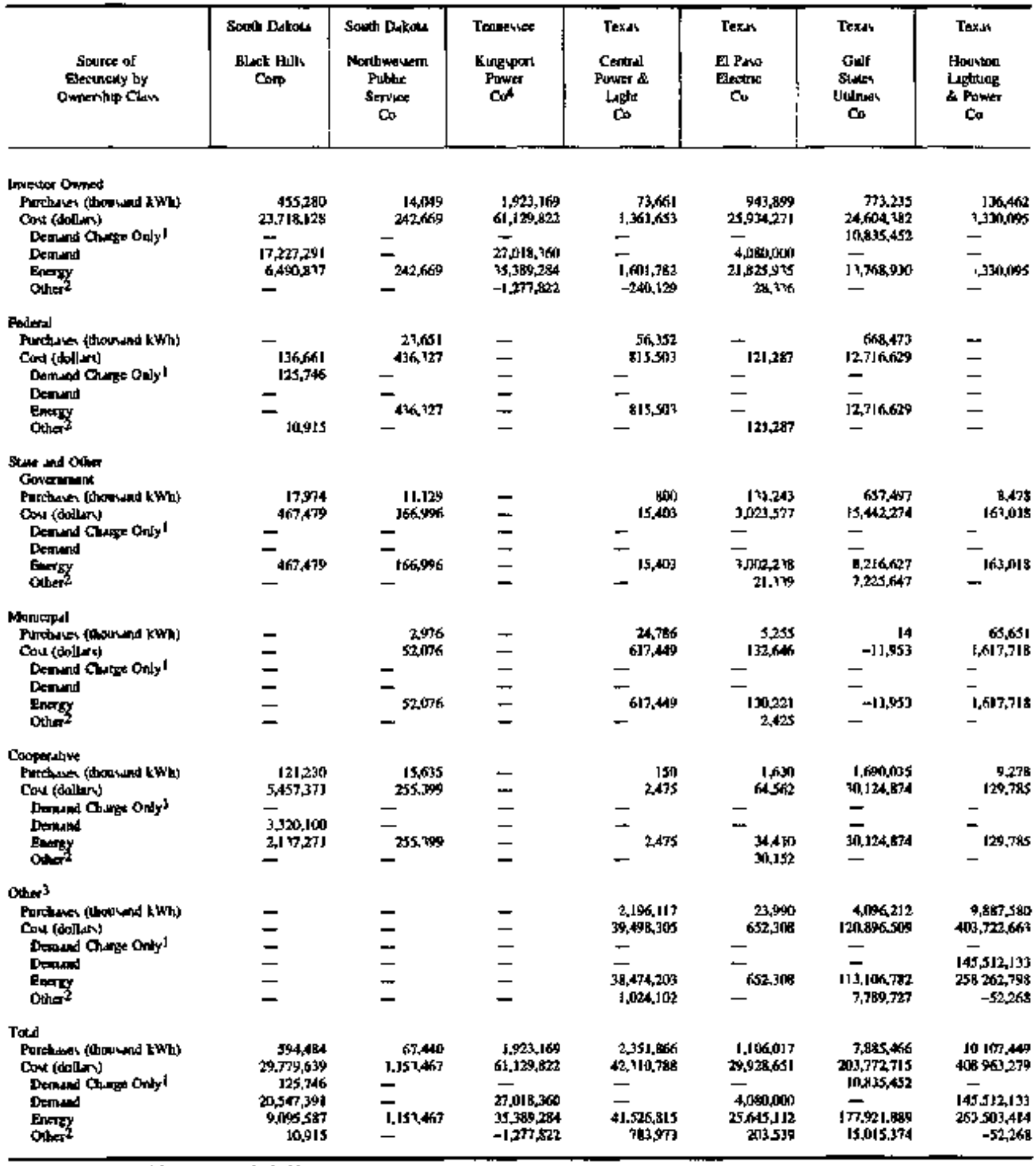

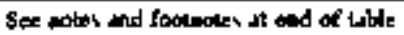


Table 19. Electricity Purchases by Inrestor.0wned Utilities, by State, 1994 (Continued)

\begin{tabular}{|c|c|c|c|c|c|c|}
\hline 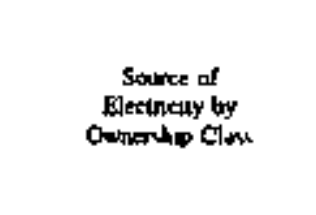 & 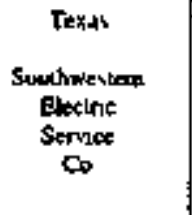 & 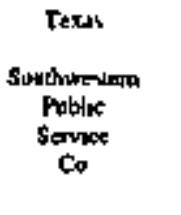 & $\begin{array}{l}\text { Toxds } \\
\text { Texus } \\
\text { Ualities } \\
\text { Eleterus } \\
\text { Co }\end{array}$ & 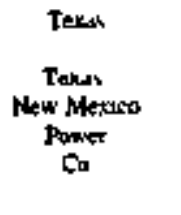 & $\begin{array}{l}\text { Texos } \\
\text { Wort } \\
\text { Texm } \\
\text { Uatum } \\
\text { Ca }\end{array}$ & 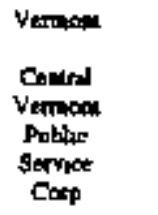 \\
\hline \multicolumn{7}{|l|}{ Invetor Oonned } \\
\hline Punden (froound kWh) & 45B, ,483 & 4,491 & fis,91tr & $1,5=0,970$ & 9,093 & $1,83,167$ \\
\hline $\begin{array}{l}\text { Cont (dollow) } \\
\text { Dewned Chare Only } 1\end{array}$ & $22,971,744$ & $-_{-}^{127.631}$ & $1,627,9615$ & $81,100,189$ & $-612,228$ & $\begin{array}{r}72,455,218 \\
1,928,930\end{array}$ \\
\hline Demand & $12,677,027$ & - & $=$ & $51,760,421$ & - & $\$ 2,196,469$ \\
\hline Energy & $8,58 J, 416$ & - & $1,452.921$ & $4,6 \mathrm{~T}, \mathrm{fil}$ & $586 \sqrt{62}$ & $13,42,414$ \\
\hline Ouler & $1,712,701$ & 127,601 & 145,044 & $24,656,155$ & $26 . J 76$ & 4.037,405 \\
\hline \multicolumn{7}{|l|}{ Feiderl } \\
\hline hircheres f(toosund (WW) & - & 40 & - & - & - & - \\
\hline $\cos (d o l i n)$ & - & 752 & - & - & - & - \\
\hline Denumd Chure Only' & - & - & - & - & - & $\pi$ \\
\hline Demend & - & - & - & - & - & - \\
\hline Enary & - & - & - & - & - & - \\
\hline OAnen 2 & - & $7 \$ 2$ & - & - & - & - \\
\hline \multirow{2}{*}{\multicolumn{7}{|c|}{ 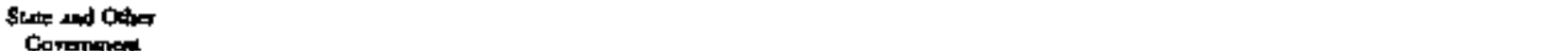 }} \\
\hline & & - & 7,417 & - & & \\
\hline 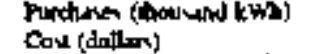 & $\begin{array}{r}692,604 \\
21,505,6100\end{array}$ & $\overline{-}$ & 14,998 & - & - 6.176 & $11,802,493$ \\
\hline Detwent Gunge Only ${ }^{1}$ & - & - & - & - & - & - \\
\hline Demand & 6.859 .798 & - & - & - & - & $4,553,434$ \\
\hline Em & $11,035,068$ & - & 193,428 & - & - & $7,042,918$ \\
\hline Othent & $1,610,734$ & - & 3,5617 & - & 6,176 & 93,728 \\
\hline \multicolumn{7}{|l|}{ Munecipal } \\
\hline 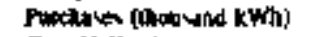 & - & 245 & 49.101 & - & 2,115 & 12,044 \\
\hline Cow (dollan) & - & 4.577 & $1,164,168$ & - & 64518 & 551,307 \\
\hline Demind Chatge Osay' & - & - & - & - & - & - \\
\hline Detand & - & - & - & - & - & 253,365 \\
\hline Entry & - & - & $1,089,15]$ & - & 57,2721 & 317,842 \\
\hline Other $x^{2}$ & - & 4,577 & 影 415 & - & 6,698 & - \\
\hline \multicolumn{7}{|l|}{ Copperatave } \\
\hline 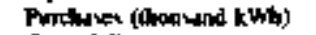 & - & 75 & 24,716 & - & - & 2893 \\
\hline Cou (dollex) & - & 76,857 & 284.18 I & - & $1, A 83$ & 140.298 \\
\hline Demind Curbe Oaly' & - & - & - & - & - & - \\
\hline Deannd & - & - & - & - & - & 54,598 \\
\hline Enery & - & - & $3 B J, 245$ & - & - & $85 \mathrm{x} n$ \\
\hline Othor & - & 36,857 & -97064 & - & 1,683 & - \\
\hline \multicolumn{7}{|l|}{ Other 3} \\
\hline 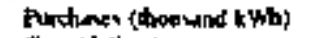 & -2.158 & $2 \$ 1.769$ & II, 509,56 i I & 1,195 & 267,448 & $1,255,8,85$ \\
\hline Cont (dolland & $-49,912$ & 4231.016 & $498,110,426$ & 24,558 & $4,459,853$ & $54,272,053$ \\
\hline Dermad ongefe ONy' & - & - & - & - & - & - \\
\hline Deriend & $-210,145$ & - & $231,0 \leqslant 0, \pi 78$ & - & - & $20,968,029$ \\
\hline Bneryy & $-231,5018$ & - & 767,029,6AB & 24,558 & $4,38,037$ & $32,952,531$ \\
\hline Quher & $-57,259$ & $4,231,018$ & - & - & 78,816 & $\mathbf{3 5 0 , 4 9 3}$ \\
\hline \multicolumn{7}{|l|}{ Tous } \\
\hline 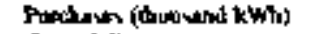 & $1,14 b, 489$ & 247,320 & $11,4 t 0,751$ & $1,645,165$ & 269,456 & $3,594,452$ \\
\hline $\cos$ (dollen) & $45,977,432$ & 4,401, EVS & $301,323,726$ & $81,127,349$ & $5.144,458$ & $139,108,856$ \\
\hline Deruend Ohinge Oaly' & - & - & - & - & - & I,928,900 \\
\hline Deondd & $19,327,241)$ & - & $231,0,0,0,778$ & $31,769, A 24$ & - & $78,006,895$ \\
\hline Enerty & $21,363,976$ & - & $270,110,595$ & $4.702,17]$ & $5,024,909$ & $53, \mathrm{BOI}, 4 \mathrm{AS}$ \\
\hline Othis & $5,266,216$ & $4,404,835$ & 102,353 & $24,556,155$ & 119,549 & $5,281,624$ \\
\hline
\end{tabular}


Table 19. Electricily Purchases by Investor-Owned Utilities, by State, 1994 (Continued)

\begin{tabular}{|c|c|c|c|c|c|c|}
\hline 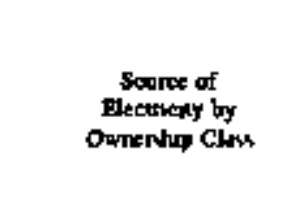 & 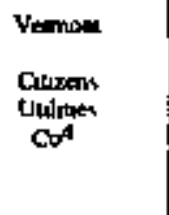 & $\begin{array}{l}\text { Vownot } \\
\text { Oran } \\
\text { Mountum } \\
\text { Powar } \\
\text { Conp }\end{array}$ & 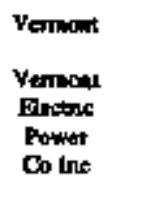 & $\begin{array}{l}\text { Vemont } \\
\text { Venoment } \\
\text { Electine } \\
\text { Tans } \\
\text { Cont }\end{array}$ & 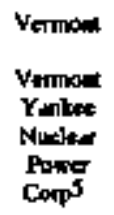 & $\begin{array}{c}\text { Yryanu } \\
\text { Populamer } \\
\text { Ca }\end{array}$ \\
\hline 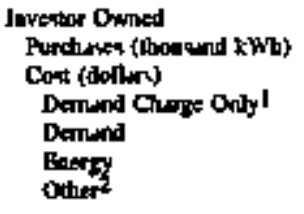 & $\begin{array}{r}42,670 \\
2,338,675 \\
- \\
1,332,779 \\
997,0 \% 6 \\
9,000\end{array}$ & $\begin{array}{r}843,194 \\
34,812,111 \\
\overrightarrow{25,062,972} \\
6,17,2,554 \\
1,646,585\end{array}$ & $\begin{array}{r}909,096 \\
30,943,972 \\
- \\
18,475,519 \\
11,605,972 \\
862,481\end{array}$ & $\begin{array}{l}= \\
z \\
= \\
=\end{array}$ & $\begin{array}{l}- \\
- \\
- \\
-\end{array}$ & $\begin{array}{l}1,135,628 \\
29,40,138 \\
- \\
3,309,602 \\
26,1) 9,536 \\
-\end{array}$ \\
\hline 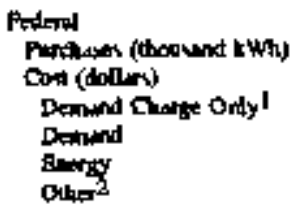 & $\begin{array}{l}- \\
- \\
- \\
-\end{array}$ & $\begin{array}{l}\tilde{-} \\
\overline{-} \\
-\end{array}$ & $\begin{array}{l}= \\
= \\
= \\
=\end{array}$ & $\begin{array}{l}z \\
= \\
= \\
=\end{array}$ & $\begin{array}{l}= \\
= \\
= \\
=\end{array}$ & $\begin{array}{l}53,259 \\
2,31,611 \\
- \\
= \\
-\end{array}$ \\
\hline 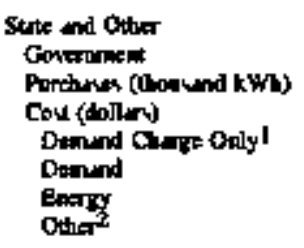 & $\begin{array}{l}- \\
- \\
- \\
-\end{array}$ & 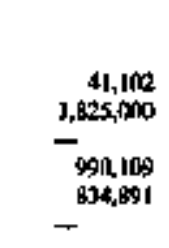 & $\begin{array}{l}z \\
z \\
z \\
-\end{array}$ & $\begin{array}{l}z \\
z \\
z \\
=\end{array}$ & $\begin{array}{l}- \\
- \\
- \\
-\end{array}$ & $\begin{array}{l}\overline{-} \\
\overline{-} \\
\overline{-}\end{array}$ \\
\hline 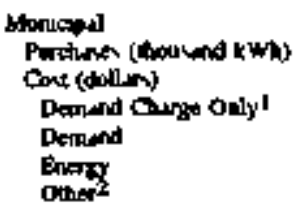 & ${ }_{-}{ }^{95,925}$ & $\begin{array}{r}34,48 \\
1,488,806 \\
-1,063 \\
949,474 \\
540,195 \\
-\end{array}$ & $\begin{array}{l}- \\
- \\
- \\
-\end{array}$ & $\begin{array}{l}- \\
\overline{-} \\
\overline{-} \\
\overline{-}\end{array}$ & $\begin{array}{l}z \\
z \\
z \\
-\end{array}$ & 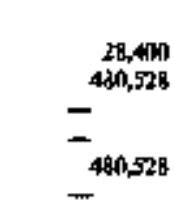 \\
\hline 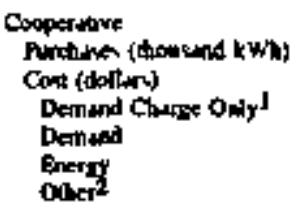 & $\begin{array}{l}\quad 89 \\
-\quad 899 \\
-\quad 899\end{array}$ & $\begin{array}{r}27,512 \\
629,430 \\
-\quad 259,360 \\
-370,008 \\
-\end{array}$ & $\begin{array}{l}= \\
= \\
= \\
=\end{array}$ & $\begin{array}{l}- \\
\overline{-} \\
\overline{-} \\
=\end{array}$ & $\begin{array}{l}- \\
- \\
z \\
-\end{array}$ & 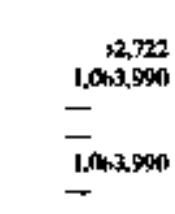 \\
\hline 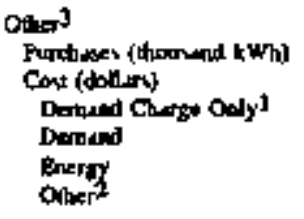 & $\begin{array}{l}219.158 \\
12,727,398 \\
- \\
6,237,715 \\
6,489683 \\
-\end{array}$ & $\begin{array}{r}1,063,503 \\
37,251,622 \\
-2,161 \\
5,45,851 \\
28, \pi 79,287 \\
34,615\end{array}$ & $\begin{array}{l}- \\
- \\
- \\
-\end{array}$ & $\begin{array}{l}- \\
\overline{-} \\
\bar{z} \\
\overline{-}\end{array}$ & $\begin{array}{l}\bar{z} \\
\bar{z} \\
\overline{-}\end{array}$ & $\begin{array}{l}10,414,895 \\
286,412,837 \\
- \\
- \\
286,412,817 \\
-\end{array}$ \\
\hline 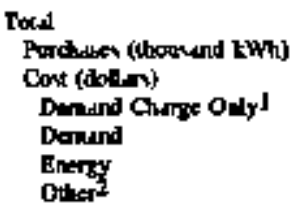 & $\begin{array}{r}264,179 \\
15.163,097 \\
- \\
7,570,494 \\
7,383,600 \\
9,1000\end{array}$ & $\begin{array}{r}2,029,059 \\
36,076,977 \\
-3,224 \\
35,207,796 \\
3,4,691,216 \\
3,681,200\end{array}$ & $\begin{array}{r}909,096 \\
30,943,972 \\
- \\
18,475,519 \\
11,605,972 \\
863,481\end{array}$ & $\begin{array}{l}- \\
\overline{-} \\
\overline{-} \\
\overline{-}\end{array}$ & $\begin{array}{l}- \\
- \\
= \\
-\end{array}$ & $\begin{array}{r}11,6,64,904 \\
119,7 \times 8,124 \\
- \\
3,309,602 \\
316,418,522 \\
-\end{array}$ \\
\hline
\end{tabular}

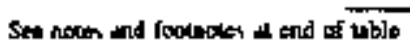


Table 19. Dectricity Purchases by Imvestor-Omned Utflities, by State, 1994 (Continued)

\begin{tabular}{|c|c|c|c|c|c|c|}
\hline $\begin{array}{c}\text { Sorrot of } \\
\text { Bectocky by } \\
\text { Owarilp Clm }\end{array}$ & 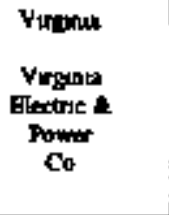 & 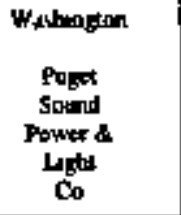 & 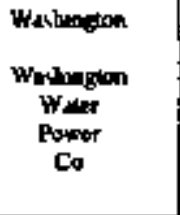 & 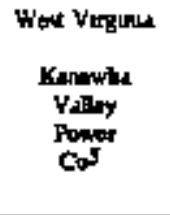 & 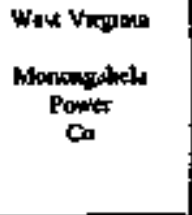 & 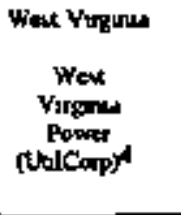 \\
\hline 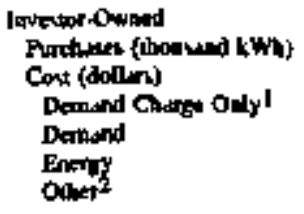 & $\begin{array}{r}3,80,200 \\
12,005,509 \\
5,521,000 \\
51,201,953 \\
62,294,546 \\
-\end{array}$ & $\begin{array}{r}2,512,73 \\
8,749,918 \\
201,4100 \\
42,724,928 \\
45,924,998 \\
-\end{array}$ & $\begin{array}{r}1,516,673 \\
38,808,249 \\
622.125 \\
- \\
38,266.124 \\
-\end{array}$ & $\begin{array}{l}- \\
- \\
= \\
- \\
-\end{array}$ & 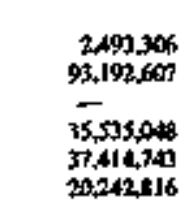 & $\begin{array}{r}374,010 \\
13,571,195 \\
- \\
6,767,248 \\
8,03,678 \\
-919,731\end{array}$ \\
\hline 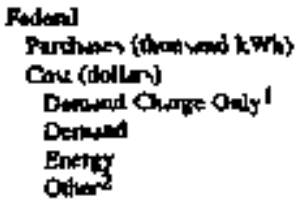 & $\begin{array}{l}= \\
\bar{z} \\
\bar{z}\end{array}$ & $\begin{array}{r}2,1052,164 \\
64,969,145 \\
- \\
82,1080 \\
61,962,145 \\
4,202,253\end{array}$ & $\begin{array}{r}660,413 \\
22,8,4,798 \\
3,16,2,952 \\
- \\
25,537,564 \\
150,264\end{array}$ & $\begin{array}{l}\overline{-} \\
\overline{-} \\
\overline{-} \\
=\end{array}$ & $\begin{array}{l}\bar{z} \\
\bar{z} \\
\bar{z}\end{array}$ & $\begin{array}{l}\overline{-} \\
\bar{z} \\
\bar{z}\end{array}$ \\
\hline 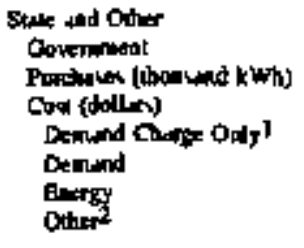 & 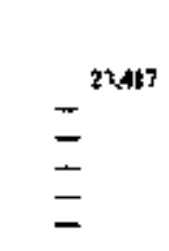 & $\begin{array}{l}6,418,946 \\
86,503,611 \\
- \\
1,077,010 \\
85,426,611 \\
-\end{array}$ & $\begin{array}{l}1,346,923 \\
11,725,161 \\
- \\
- \\
11,726,161 \\
-\end{array}$ & $\begin{array}{l}\overline{-} \\
\overline{-} \\
\overline{-}\end{array}$ & $\begin{array}{l}\bar{z} \\
\bar{z} \\
\bar{z}\end{array}$ & $\begin{array}{l}\bar{z} \\
\overline{-} \\
\bar{z}\end{array}$ \\
\hline 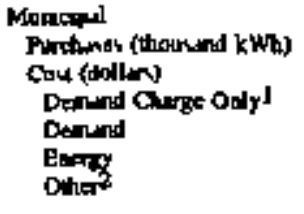 & $\begin{array}{r}717 \\
-\quad 43775 \\
-76,149 \\
-\quad 3676\end{array}$ & $\begin{array}{l}1,203,927 \\
35,195,944 \\
= \\
- \\
35,185,944 \\
-\end{array}$ & $\begin{array}{l}101,745 \\
2,343,900 \\
100,0100 \\
-7,243,500 \\
-\end{array}$ & $\begin{array}{l}\overline{-} \\
\overline{-} \\
\overline{-}\end{array}$ & $\begin{array}{r}184 \\
-\quad 2981 \\
-\quad 2,913\end{array}$ & $\begin{array}{l}\overline{-} \\
\overline{-} \\
\overline{-} \\
\overline{-}\end{array}$ \\
\hline 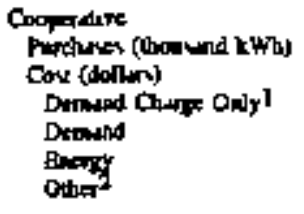 & $\begin{array}{l}2,533,510 \\
125,648,121 \\
- \\
51,774,477 \\
61,868,549 \\
-\end{array}$ & $\begin{array}{l}\bar{z} \\
\overline{-} \\
\overline{-}\end{array}$ & $\begin{array}{l}-2748 \\
-\quad 2748 \\
-\quad\end{array}$ & $\begin{array}{l}\overline{-} \\
\overline{-} \\
\overline{-} \\
-\end{array}$ & $\begin{array}{l}\bar{E} \\
\bar{E}\end{array}$ & $\begin{array}{l}- \\
\overline{-} \\
\overline{-} \\
-\end{array}$ \\
\hline 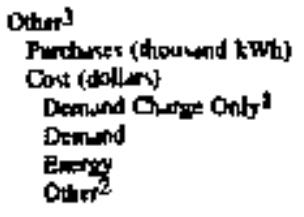 & $\begin{array}{l}9,525,1050 \\
713,716,060 \\
-728,900 \\
513,035,125 \\
25,019,840 \\
-\end{array}$ & $\begin{array}{r}4,100,232 \\
117,3 \% 6,184 \\
- \\
180,467 \\
151,986,0] 9 \\
-4,819,32\end{array}$ & $\begin{array}{l}696,683 \\
24,471 \% 99 \\
- \\
- \\
24,790,651 \\
41,348\end{array}$ & $\begin{array}{l}- \\
- \\
z \\
-\end{array}$ & 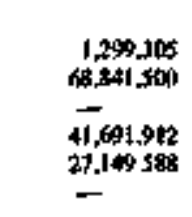 & $\begin{array}{l}\bar{z} \\
\bar{z} \\
\bar{z}\end{array}$ \\
\hline 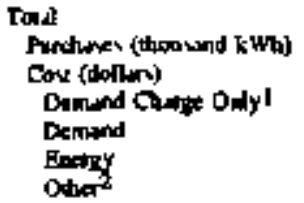 & $\begin{array}{r}15,859,916 \\
1,024,113,403 \\
4,781,104 \\
646,112,699 \\
381.219,604 \\
-\end{array}$ & $\begin{array}{r}16,2618,142 \\
39,794,800 \\
200,000 \\
44815,267 \\
410,385,617 \\
-40,60,0,079\end{array}$ & 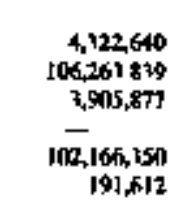 & $\begin{array}{l}- \\
\bar{E} \\
\bar{z}\end{array}$ & $\begin{array}{r}3,799,985 \\
162,077,098 \\
- \\
77,207,008 \\
64,167,264 \\
20,242,816\end{array}$ & 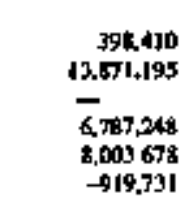 \\
\hline
\end{tabular}

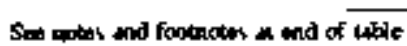


Table 19. Electricity Perchuses by Investor.Owned Utilities, by State, 1994 (Continned)

\begin{tabular}{|c|c|c|c|c|c|c|}
\hline 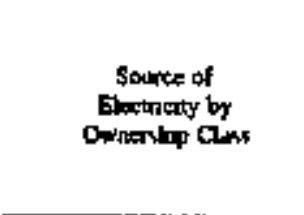 & $\begin{array}{l}\text { West Yirgond } \\
\text { Wheelang } \\
\text { Paver } \\
\text { Cod }\end{array}$ & 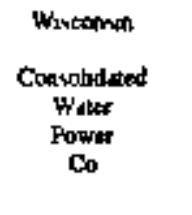 & 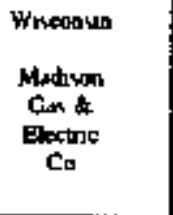 & 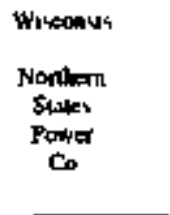 & $\begin{array}{l}\text { Wivoomm } \\
\text { Norditweiten } \\
\text { Wixeoniln } \\
\text { Elesine } \\
\text { Co }\end{array}$ & 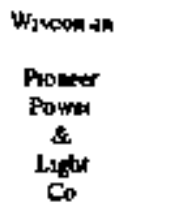 \\
\hline 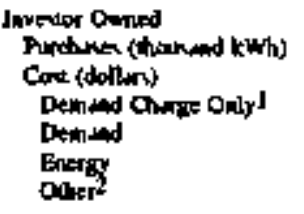 & 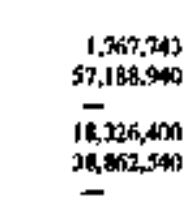 & $\begin{array}{l}3,067,694 \\
26,177,406 \\
- \\
6,56,5,994 \\
19,613,410 \\
-\end{array}$ & $\begin{array}{l}414,521 \\
9,280,121 \\
1,640,010 \\
1,331,666 \\
6,306,455 \\
-200,501\end{array}$ & 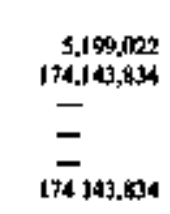 & 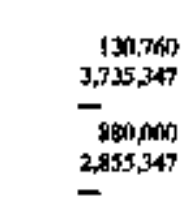 & $\begin{array}{r}24,131 \\
309,339 \\
- \\
364,260 \\
\text { J10,994 } \\
-61,915\end{array}$ \\
\hline 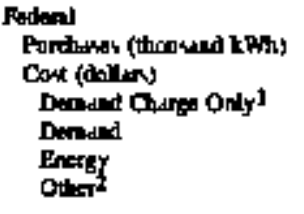 & $\begin{array}{l}- \\
- \\
- \\
- \\
=\end{array}$ & $\begin{array}{l}= \\
= \\
= \\
-\end{array}$ & $\begin{array}{l}- \\
\overline{-} \\
\overline{-} \\
-\end{array}$ & $\begin{array}{l}- \\
- \\
= \\
= \\
-\end{array}$ & $\begin{array}{l}- \\
\overline{-} \\
\overline{-} \\
\bar{z}\end{array}$ & $\begin{array}{l}- \\
\overline{-} \\
\overline{-} \\
-\end{array}$ \\
\hline 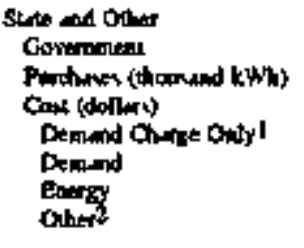 & $\begin{array}{l}= \\
= \\
= \\
=\end{array}$ & $\begin{array}{l}= \\
= \\
= \\
-\end{array}$ & $\begin{array}{l}\bar{z} \\
\overline{-} \\
-\end{array}$ & $\begin{array}{l}- \\
\overline{-} \\
- \\
-\end{array}$ & $\begin{array}{l}\bar{z} \\
\bar{z} \\
\bar{z}\end{array}$ & $\begin{array}{l}= \\
z \\
- \\
-\end{array}$ \\
\hline 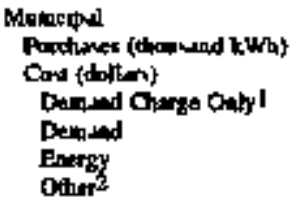 & $\begin{array}{l}\bar{z} \\
\overline{-} \\
\overline{-}\end{array}$ & $\begin{array}{l}- \\
\overline{-} \\
\overline{-}\end{array}$ & 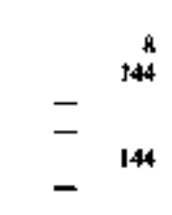 & $\begin{array}{l}z \\
= \\
= \\
=\end{array}$ & $\begin{array}{l}- \\
\overline{-} \\
- \\
-\end{array}$ & $\begin{array}{l}z \\
\overline{-} \\
- \\
-\end{array}$ \\
\hline 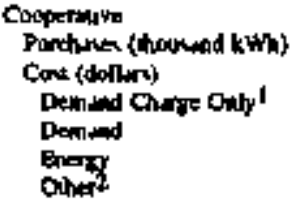 & $\begin{array}{l}= \\
= \\
= \\
=\end{array}$ & $\begin{array}{l}z \\
- \\
= \\
-\end{array}$ & $e^{\quad \begin{array}{r}5,581 \\
-115,457\end{array}}$ & $\begin{array}{l}- \\
z \\
= \\
-\end{array}$ & $\begin{array}{l}- \\
\overline{-} \\
\overline{-} \\
-\end{array}$ & $\begin{array}{l}\bar{F} \\
\bar{z} \\
=\end{array}$ \\
\hline 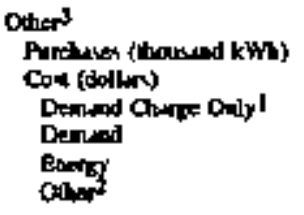 & $\begin{array}{r}6,198 \\
209,842 \\
-\quad \\
06,109 \\
107,743\end{array}$ & $\begin{array}{l}z \\
- \\
= \\
=\end{array}$ & $\begin{array}{r}20,713 \\
619,465 \\
-\quad \\
-\quad 619,163 \\
502\end{array}$ & $\begin{array}{l}z \\
z \\
z \\
-\end{array}$ & $\begin{array}{l}- \\
= \\
= \\
-\end{array}$ & $\begin{array}{l}- \\
z \\
z \\
-\end{array}$ \\
\hline 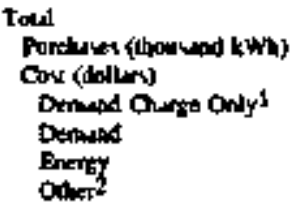 & $\begin{array}{r}1,773,941 \\
57,392,782 \\
- \\
10,326,400 \\
38,958,619 \\
107,743\end{array}$ & $\begin{array}{l}1,041,694 \\
26,177,466 \\
- \\
6563,994 \\
19,613,410 \\
-\end{array}$ & $\begin{array}{r}4011,828 \\
10,015,357 \\
3,6401,190 \\
1,3,31,6606 \\
7,24], 219 \\
-199,498\end{array}$ & $\begin{array}{l}5,199,022 \\
174,143,534 \\
= \\
= \\
= \\
174,147,8344\end{array}$ & $\begin{array}{l}130,760 \\
3,735,347 \\
- \\
\quad 890,000 \\
2,855,347 \\
-\end{array}$ & $\begin{array}{r}24,131 \\
809.339 \\
-\quad \\
364,260 \\
50,994 \\
-61,915\end{array}$ \\
\hline
\end{tabular}

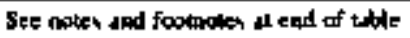


Table 19. Electriclty Purchases by Invesior-Owned Utilittes, by Stale, 1994 (Continued)

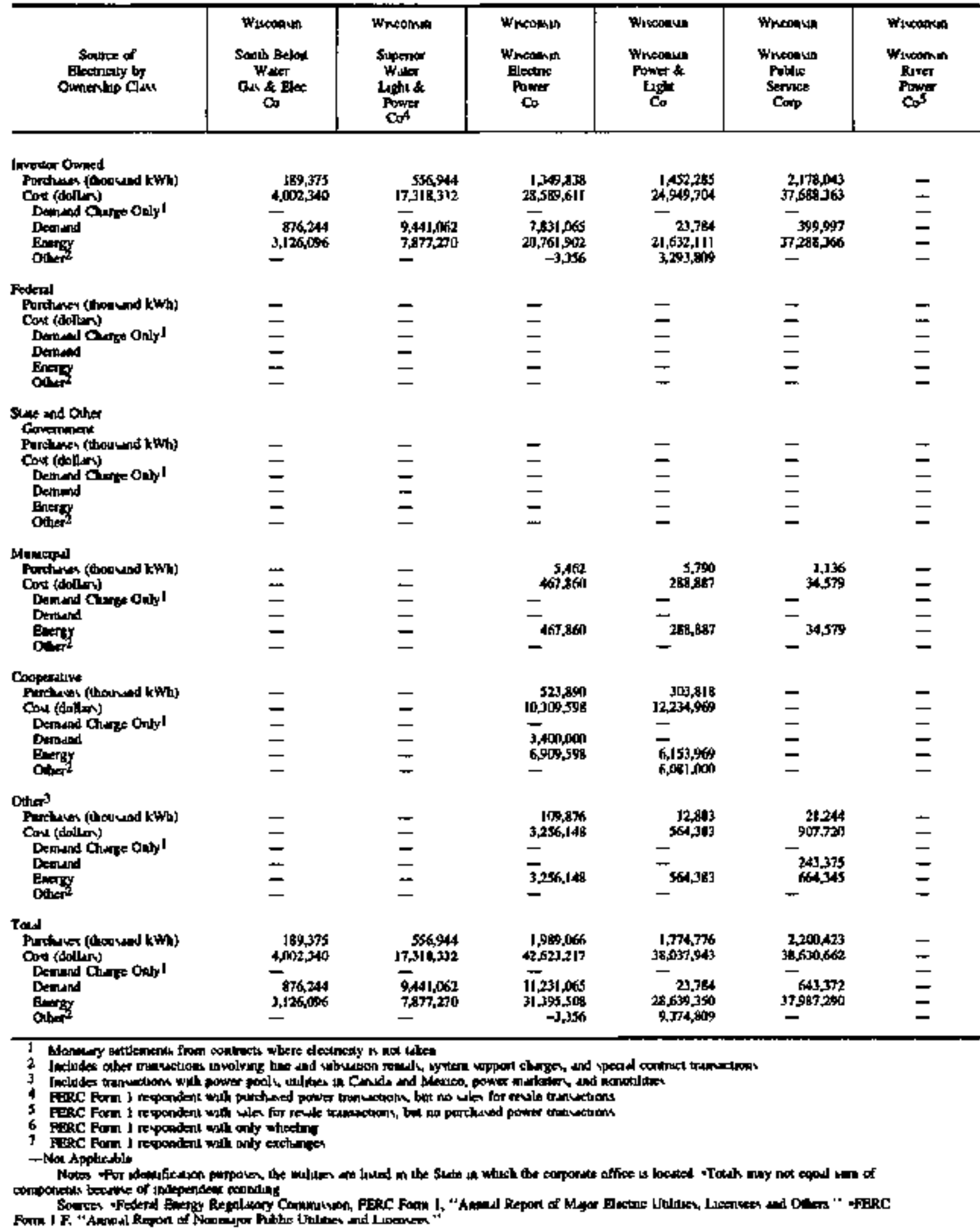


Table 20. Electricity Sales Ior Resale by Investor-Owned Utillles, by State, 1994

\begin{tabular}{|c|c|c|c|c|c|c|c|}
\hline 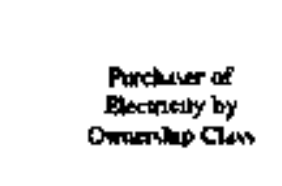 & $\begin{array}{c}\text { Alsbures } \\
\text { Alabwnd } \\
\text { Powner } \\
\text { Co }\end{array}$ & 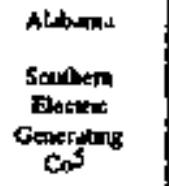 & 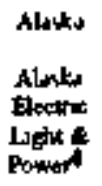 & $\begin{array}{l}\text { Anzons } \\
\text { Anzont } \\
\text { Poblic } \\
\text { Servicx } \\
\text { Co }\end{array}$ & $\begin{array}{l}\text { Arroons } \\
\text { Centriny } \\
\text { Ponter } \\
\text { Conps }\end{array}$ & $\begin{array}{c}\text { Anzoxa } \\
\text { Curzam } \\
\text { Unluke } \\
\infty\end{array}$ & $\begin{array}{l}\text { Aneon } \\
\text { Tecion } \\
\text { Eector } \\
\text { Pownit } \\
\text { Co }\end{array}$ \\
\hline 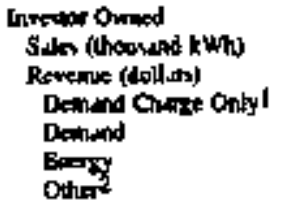 & $\begin{array}{l}2,896,901 \\
198,624,210 \\
- \\
- \\
198,424,210\end{array}$ & $\begin{array}{l}4.557 .521 \\
148,965,816 \\
= \\
- \\
148,965.816 \\
-\end{array}$ & $\begin{array}{l}- \\
- \\
- \\
-\end{array}$ & 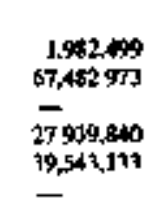 & $\begin{array}{r}1104 \\
-\quad 1704\end{array}$ & $\begin{array}{l}- \\
\overline{-} \\
\overline{-} \\
-\end{array}$ & $\begin{array}{l}1,560,6 \times 8 \\
4,481,072 \\
- \\
0,965,9010 \\
31,515,172 \\
-\end{array}$ \\
\hline 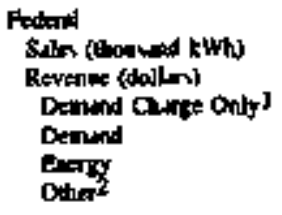 & 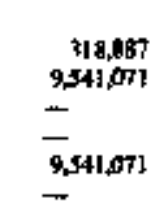 & $\begin{array}{l}- \\
\overline{-} \\
- \\
-\end{array}$ & $\begin{array}{l}- \\
\overline{-} \\
\overline{-} \\
-\end{array}$ & $\begin{array}{l}256,525 \\
11.591,226 \\
- \\
6,735.760 \\
5.245 .466 \\
-\end{array}$ & $\begin{array}{l}\bar{z} \\
\bar{z} \\
=\end{array}$ & $\begin{array}{l}z \\
\bar{z} \\
=\end{array}$ & $\begin{aligned} & 2,154 \\
& 1,320,194 \\
&= \\
&- \\
& 1,325,194 \\
&=\end{aligned}$ \\
\hline 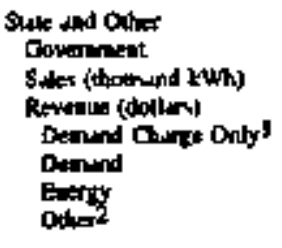 & 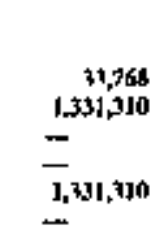 & $\begin{array}{l}- \\
- \\
\overline{-} \\
-\end{array}$ & $\begin{array}{l}z \\
= \\
= \\
=\end{array}$ & $\begin{array}{l}281,37 \\
10181,294 \\
- \\
5,147,400 \\
5,1169,824 \\
-\end{array}$ & $\begin{array}{l}\bar{z} \\
\overline{-} \\
\overline{-}\end{array}$ & $\begin{array}{l}- \\
\overline{-} \\
z \\
=\end{array}$ & $\begin{array}{r}995,958 \\
45,2 B 7, A B 7 \\
- \\
25,203,499 \\
21,008,989 \\
-\end{array}$ \\
\hline 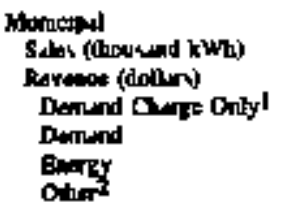 & $\begin{array}{r}3,132,122 \\
131,947,929 \\
- \\
50,121,399 \\
90,207,226 \\
-8,341,694\end{array}$ & $\begin{array}{l}= \\
= \\
=\end{array}$ & $\begin{array}{l}- \\
- \\
- \\
-\end{array}$ & $\begin{array}{r}190,0900 \\
4,2+4,664 \\
- \\
941,302 \\
3,261,342 \\
-\end{array}$ & $\begin{array}{l}\bar{z} \\
\bar{z} \\
=\end{array}$ & $\begin{array}{l}= \\
= \\
= \\
=\end{array}$ & $\begin{array}{r}129,634 \\
2.576 .186 \\
=\quad 105,000 \\
2,431.386 \\
=\end{array}$ \\
\hline 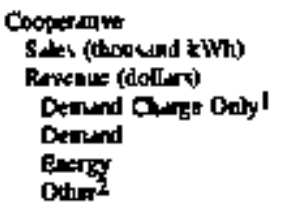 & $\begin{array}{r}791,172 \\
14,556,055 \\
- \\
6,727,691 \\
8,479,491 \\
-649,723\end{array}$ & $\begin{array}{l}z \\
- \\
\overline{-} \\
=\end{array}$ & $\begin{array}{l}\overline{-} \\
\overline{-} \\
\overline{-} \\
-\end{array}$ & 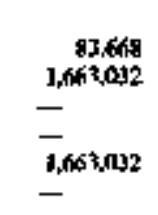 & 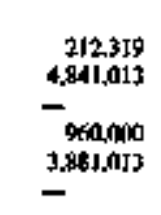 & $\begin{array}{l}- \\
= \\
= \\
=\end{array}$ & $\begin{array}{l}204,903 \\
4,18 \%, 314 \\
= \\
= \\
4,189,314 \\
=\end{array}$ \\
\hline 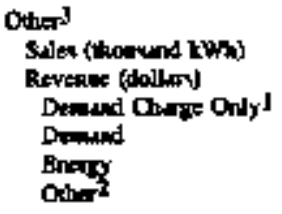 & $\begin{array}{r}8,432,769 \\
16,2,400,154 \\
- \\
- \\
158,441,4199 \\
4,936,445\end{array}$ & $\begin{array}{l}\bar{z} \\
\bar{z} \\
=\end{array}$ & $\begin{array}{l}- \\
\bar{z} \\
=\end{array}$ & 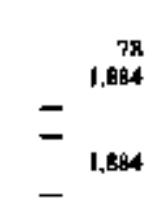 & 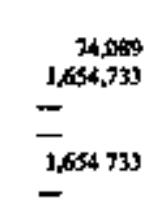 & $\begin{array}{r}1,393 \\
117,527 \\
-\quad 51,793 \\
37,638 \\
28,096\end{array}$ & 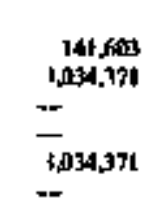 \\
\hline 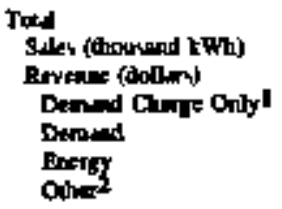 & $\begin{array}{r}15,207,7199 \\
519,521,529 \\
\overline{56,848,488} \\
46,6,72,017 \\
-4,151,976\end{array}$ & 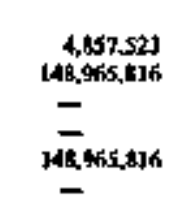 & $\begin{array}{l}\overline{-} \\
\bar{z} \\
\bar{z}\end{array}$ & $\begin{array}{l}2,764,210 \\
95,15,173 \\
- \\
401,400,172 \\
54,756, \pi 01 \\
-\end{array}$ & 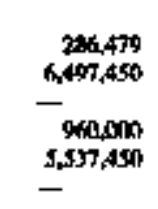 & $\begin{array}{r}1,353 \\
117327 \\
-\quad \\
51.793 \\
37,608 \\
28,096\end{array}$ & $\begin{array}{l}1,105,520 \\
0,1388,624 \\
- \\
34,349,399 \\
60,539,225 \\
-\end{array}$ \\
\hline
\end{tabular}

Sec mon and footioted at and of thile 
Table 20. Electricity Sales for Resale by Investor-Owned Uthities, by State, 1994 (Continued)

\begin{tabular}{|c|c|c|c|c|c|c|c|}
\hline 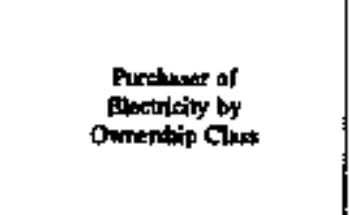 & 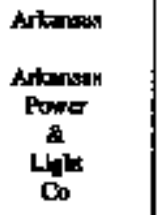 & 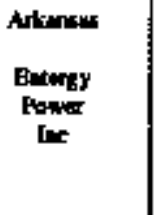 & 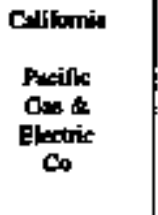 & $\begin{array}{c}\text { Colifonite } \\
\text { sin Dingo } \\
\text { Ond } \\
\text { Elentric } \\
\text { Co }\end{array}$ & 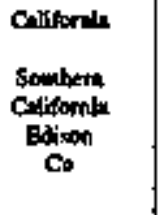 & $\begin{array}{l}\text { Codorodo } \\
\text { Publis } \\
\text { Sarrice } \\
\text { Co of } \\
\text { Colorodo }\end{array}$ & 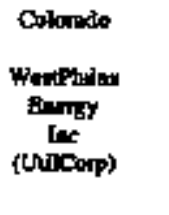 \\
\hline 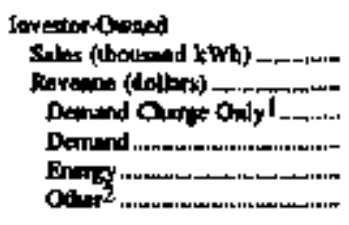 & $\begin{array}{l}1,463,812 \\
33,200,497 \\
- \\
69,690,146 \\
15,530,291 \\
-\end{array}$ & $\begin{array}{l}1,341,410 \\
25,345,327 \\
- \\
\overline{25,345,327} \\
-\end{array}$ & $\begin{array}{r}975,595 \\
44,705,474 \\
- \\
4,4055,507 \\
31,583,725 \\
46,242\end{array}$ & $\begin{array}{r}169,212 \\
4,612,302 \\
142,000 \\
14,625 \\
4,155,677 \\
-\end{array}$ & 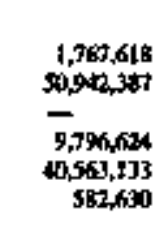 & $\begin{array}{r}1,176,026 \\
39,853,184 \\
\overline{19,138,760} \\
27,6,96,844 \\
-2,959,456\end{array}$ & $=\frac{92}{3,201}$ \\
\hline 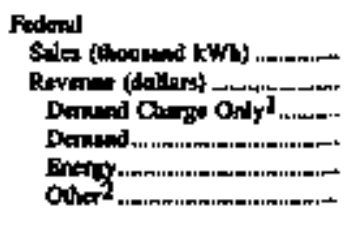 & $\begin{array}{l}\bar{z} \\
\bar{z} \\
\overline{-}\end{array}$ & $\begin{array}{l}- \\
265,000 \\
- \\
- \\
265,010\end{array}$ & 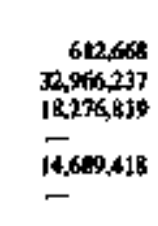 & $-^{-57,512}={ }^{676,601}$ & $\begin{array}{l}1,629,196 \\
37,390,057 \\
- \\
- \\
77,3 \times 0,057 \\
-\end{array}$ & 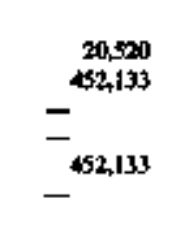 & $\begin{array}{l}\overline{-} \\
\overline{-} \\
-\end{array}$ \\
\hline 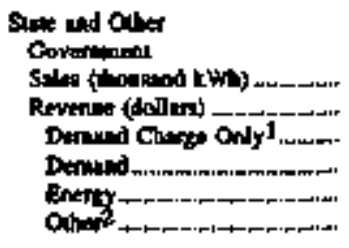 & $\begin{array}{l}- \\
\text { J90,960 } \\
190,960 \\
- \\
- \\
-\end{array}$ & $\begin{array}{l}- \\
\overline{-} \\
- \\
-\end{array}$ & 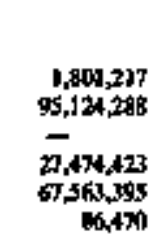 & $\begin{array}{r}346,698 \\
10,712,749 \\
- \\
2,418,645 \\
2,290,644 \\
2\end{array}$ & 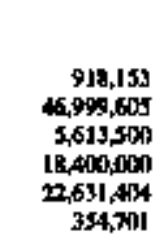 & 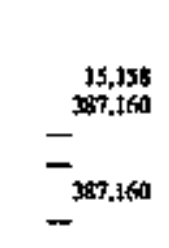 & $\begin{array}{l}\overline{-} \\
\overline{-} \\
\overline{-}\end{array}$ \\
\hline 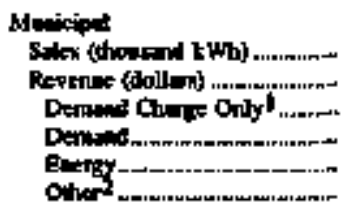 & $\begin{array}{l}1,713,311 \\
75,210,600 \\
111,072 \\
21,153,074 \\
5,615,814 \\
-\end{array}$ & $\begin{array}{l}- \\
\overline{-} \\
\overline{-} \\
-\end{array}$ & $\begin{array}{r}867,864 \\
4,529,866 \\
- \\
2,932,290 \\
25,137,066 \\
460,550\end{array}$ & $\begin{array}{l}112,052 \\
4,514,107 \\
- \\
1,188,000 \\
3,3] 6,007 \\
-\end{array}$ & $\begin{array}{r}1,812,138 \\
55,692,419 \\
- \\
18,565,619 \\
36,617,461 \\
509,129\end{array}$ & $\begin{array}{r}91,662 \\
3,042,642 \\
- \\
2,300,517 \\
-155,332\end{array}$ & $\begin{array}{r}1,242 \\
-\quad 4,457 \\
2,645 \\
29,575 \\
14,241\end{array}$ \\
\hline 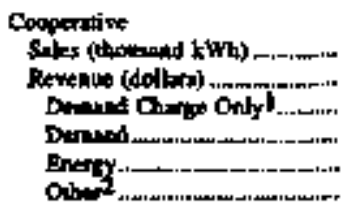 & $\begin{array}{l}1,487,104 \\
46,292,6919 \\
- \\
17,325,784 \\
28,964,9105 \\
-\end{array}$ & $\begin{array}{r}44,035 \\
12,520,455 \\
- \\
10,54,1,246 \\
9,134,769 \\
-770,550\end{array}$ & $\begin{array}{l}- \\
= \\
= \\
=\end{array}$ & $-^{38,715}$ & 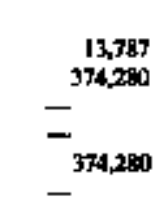 & $\begin{array}{l}7,775,025 \\
76,499,547 \\
- \\
48,279,650 \\
35,174,220 \\
-6,959, \pm 25\end{array}$ & $\begin{array}{l}= \\
= \\
= \\
-\end{array}$ \\
\hline 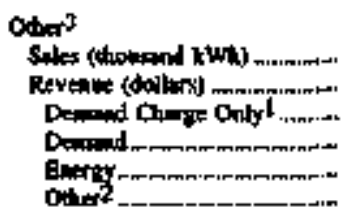 & $\begin{array}{l}10,837,593 \\
238,219,148 \\
- \\
-\overline{234,219,148} \\
-\end{array}$ & $\begin{array}{l}- \\
\bar{z} \\
\overline{-} \\
-\end{array}$ & $\begin{array}{r}18,400 \\
1,170,387 \\
-\quad 16,047 \\
40,099 \\
601,241\end{array}$ & $\begin{array}{r}98,197 \\
2,461,636 \\
-\quad 1,8100 \\
2,43,136 \\
-\end{array}$ & $\begin{array}{r}157,279 \\
4,924,779 \\
-\quad \\
770,000 \\
1,803,607 \\
351,172\end{array}$ & $\begin{array}{r}\begin{array}{r}140 \\
2,840\end{array} \\
-\quad 2,840\end{array}$ & $\begin{array}{l}- \\
\bar{z} \\
\overline{-}\end{array}$ \\
\hline 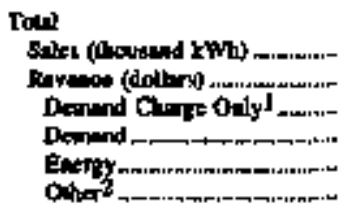 & $\begin{array}{r}15,56], 824 \\
39,233,794 \\
132,1921 \\
36,569,604 \\
34,332,158 \\
-\end{array}$ & $\begin{array}{r}1,795,445 \\
44,935,792 \\
-\overline{10,561,246} \\
34,880,0 \% \\
-306,550\end{array}$ & $\begin{array}{r}4,25,947 \\
20,496,250 \\
18,276,819 \\
34,524,221 \\
121,430,703 \\
1,255,503\end{array}$ & 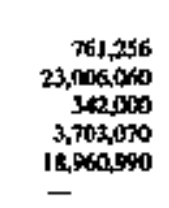 & $\begin{array}{r}6,768,171 \\
196,723,516 \\
1,613,500 \\
47,532,443 \\
14,379,942 \\
1,797,631\end{array}$ & 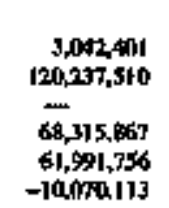 & $\begin{array}{r}1,334 \\
49,654 \\
-\quad 2,641 \\
32,774 \\
14,241\end{array}$ \\
\hline
\end{tabular}

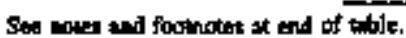


Table 20. Electricity Sales for Resale by Inveslor-Owned Utilities, by State, 1994 (Contimed)

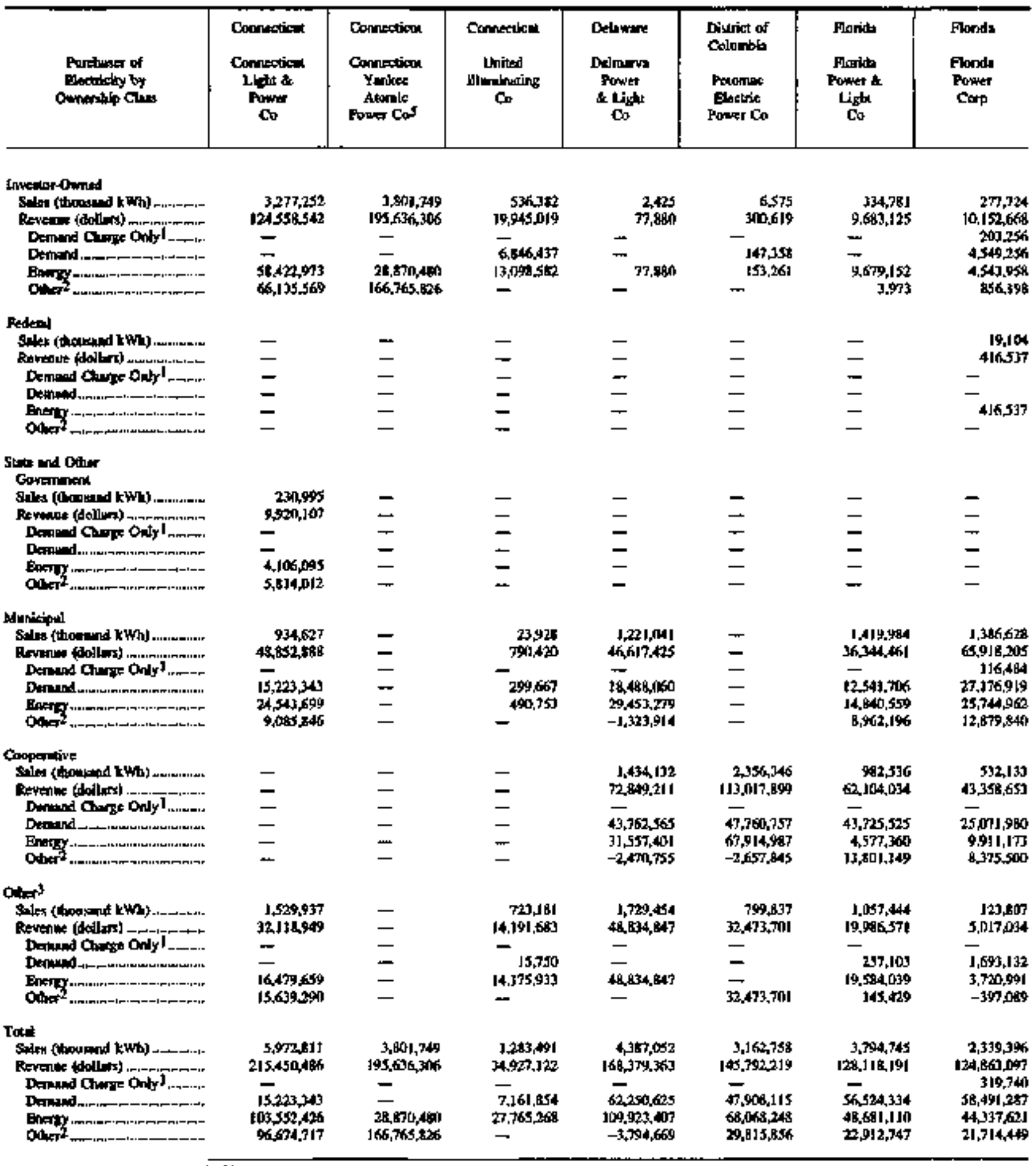

Sez notas and fooknower an ead of the 
Table 28. Electricity Sales for Resale by lnvestor-Owned Utilities, by State, 1994 (Continued)

\begin{tabular}{|c|c|c|c|c|c|c|c|}
\hline 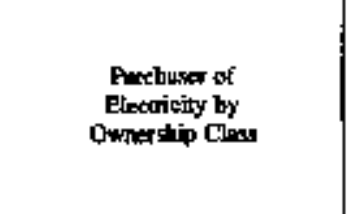 & $\begin{array}{c}\text { Ponidh } \\
\text { Culf } \\
\text { Power } \\
\text { Co }\end{array}$ & $\begin{array}{c}\text { Fotink } \\
\text { Tampi } \\
\text { Elocrie } \\
\text { Co }\end{array}$ & $\begin{array}{l}\text { Goontil } \\
\text { Georgia } \\
\text { Paver } \\
\text { Co }\end{array}$ & 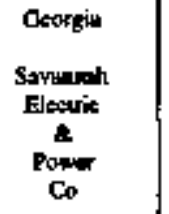 & 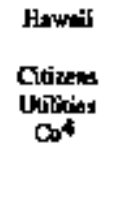 & 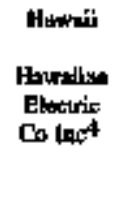 & 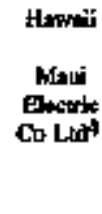 \\
\hline \multicolumn{8}{|l|}{ Inverionotined } \\
\hline Silek (bomaged kWh) & $972 \times 30$ & $1,151,512$ & $5,554,062$ & 145,423 & - & - & - \\
\hline 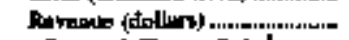 & $47,195,886$ & $13,122,628$ & $135,200,051$ & $3,413,796$ & - & - & - \\
\hline Fenowd Orroce only 1 ........ & - & - & $\rightarrow$ & - & - & - & - \\
\hline 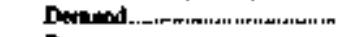 & $24,631,122$ & $30,873,004$ & $65,034,315$ & 445,497 & - & - & - \\
\hline 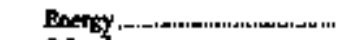 & $24,935,241$ & $24,245,220$ & 20.168 .736 & $2,968,359$ & - & - & - \\
\hline 0 & $-2,570,497$ & 4,404 & - & - & - & - & - \\
\hline \multicolumn{8}{|l|}{ Federal } \\
\hline 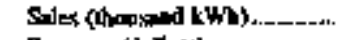 & 71,722 & - & 402,053 & 24,102 & - & $m$ & - \\
\hline 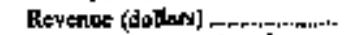 & $2,251.225$ & - & $14,423,310$ & $2,1,137$ & - & - & - \\
\hline Deneand Orage Ooly' & - & - & - & - & $\rightarrow$ & - & $\rightarrow$ \\
\hline 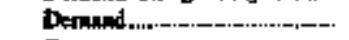 & - & - & $\rightarrow$ & - & - & - & - \\
\hline Entrog & 2011,275 & - & $14,423,310$ & $\mathbf{2 1} \mathbf{1} 13 t$ & - & - & - \\
\hline Qher & - & - & - & - & - & - & - \\
\hline \multicolumn{8}{|l|}{ 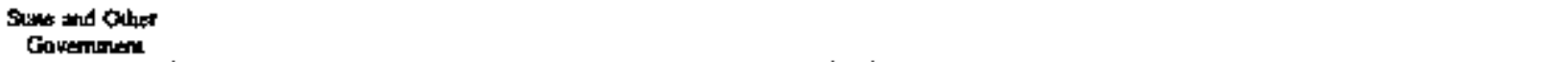 } \\
\hline 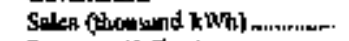 & 67739 & - & $+639,020$ & 2,557 & - & - & - \\
\hline Perenue (dorlers) & 265,250 & - & $-8,911,3,2$ & IDOSTi24 & - & - & - \\
\hline Demond Onde Only I n....... & - & - & $\rightarrow$ & - & - & - & - \\
\hline Das & - & - & - & - & - & - & - \\
\hline Energy, & 265,290 & - & I[190],403 & 100,624 & - & - & - \\
\hline 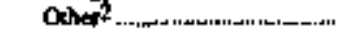 & - & - & -19812713 & - & - & - & - \\
\hline \multicolumn{8}{|l|}{ Moricipal } \\
\hline Sples (Gromband kWh & 348,404 & B31,787 & 1.461 .259 & $2 n, 781$ & - & - & - \\
\hline Revant (dolldxs) & $15,914,570$ & $21,790,3$; & $99,765.266$ & $996, \pi 92$ & - & - & - \\
\hline Denued Ghroe Only' & - & - & - & - & - & - & - \\
\hline 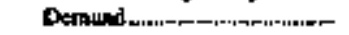 & $7,865,900$ & $6,792,439$ & $25,995,050$ & 2,510 & - & - & - \\
\hline 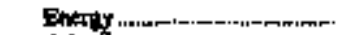 & $5,745.185$ & 14,947,935 & $28.5 \times 8913$ & 394,282 & - & - & - \\
\hline 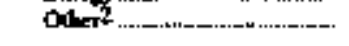 & -296575 & - & -388.59 & - & - & - & - \\
\hline \multicolumn{8}{|l|}{ Cooperive } \\
\hline 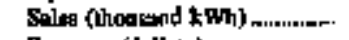 & 2965 & $11 \%, 602$ & $2,597 \leq 15$ & 236 & - & - & - \\
\hline Revenwe (dolltri) . . & 55, J65 & $3,530,031$ & $194.153,567$ & $3,15 \%$ & $=$ & - & - \\
\hline Denuad Conare Onby I....,.,. & - & - & - & - & $=$ & - & - \\
\hline 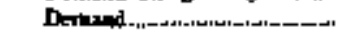 & - & 1,359,636 & $97,750,000$ & - & - & - & - \\
\hline 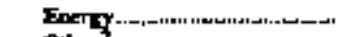 & $8 s_{+} 1 x_{6}$ & $2,1 \div 0,375$ & $60,405,347$ & 3,257 & - & - & - \\
\hline Other ${ }^{2}$ & - & - & - & - & $\rightarrow$ & - & - \\
\hline \multicolumn{8}{|l|}{ Othan' } \\
\hline 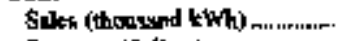 & tonsst? & - & $3.166,566$ & $9 \%, 62 ?$ & - & - & - \\
\hline 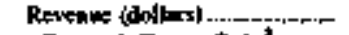 & $17,791,197$ & - & 6,1550,793 & 6990,622 & - & - & - \\
\hline 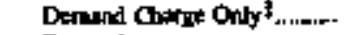 & - & - & - & - & - & - & - \\
\hline 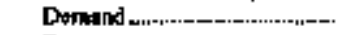 & $2 n, 70 n$ & - & - & 4,071,654 & - & - & - \\
\hline 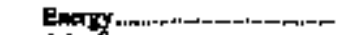 & 17518,490 & - & $67,853,793$ & $2,121,968$ & - & 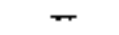 & - \\
\hline OHhot & - & - & - & - & - & - & - \\
\hline \multicolumn{8}{|l|}{ Tond } \\
\hline 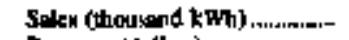 & $2,293,0027$ & $2,101.99$ & 11004535 & 294,716 & - & - & - \\
\hline 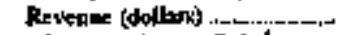 & $80,463,884$ & $70,433,023$ & $412,480,653$ & $11,231,728$ & - & - & - \\
\hline Derpan Charge Only 1 & - & - & - & - & - & - & - \\
\hline 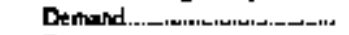 & 32.769 .789 & $29,0105,069$ & $184,779,365$ & $4,919,65]$ & - & - & - \\
\hline 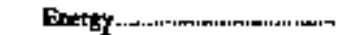 & $53,369,265$ & $41,42,350$ & $248,311,602$ & $6, \$ 12$ 1577 & - & - & - \\
\hline 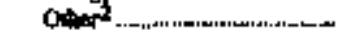 & $-2,667,072$ & 4,404 & $-20, f_{i 01}, 312$ & - & - & - & - \\
\hline
\end{tabular}

See ane and foornotre on and of trible 
Table 20. Electricity Sales for Resale by Investor-Omined Utilities, by State, 1994 (Conthuted)

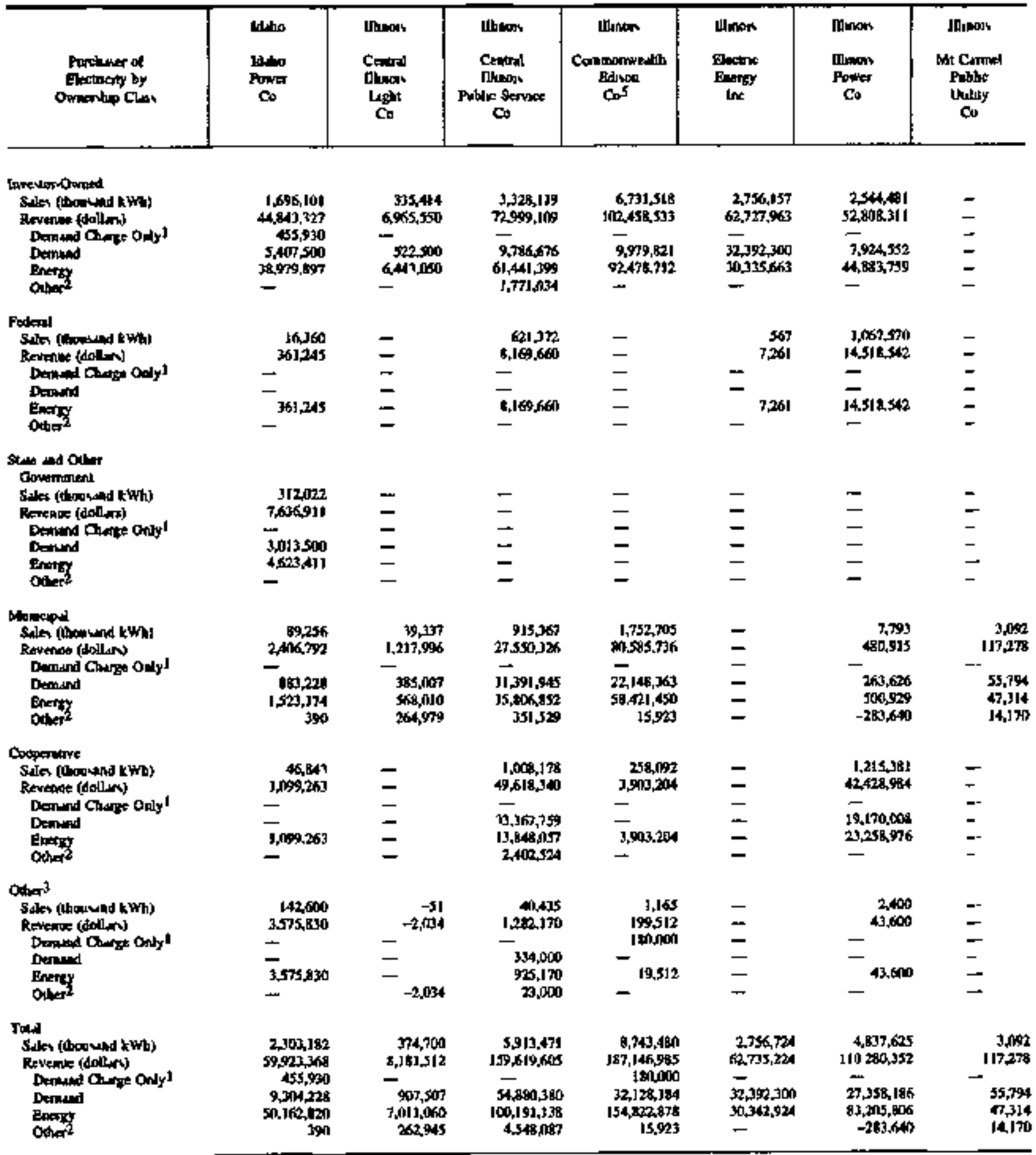

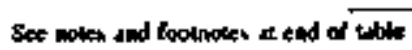


Table 20. Electrichy Sales for Resale by Investor-Owned Utilities, by State, 1994 (Continned)

\begin{tabular}{|c|c|c|c|c|c|c|c|}
\hline $\begin{array}{l}\text { Purcbuter of } \\
\text { Bectricity by } \\
\text { Owerthid Class }\end{array}$ & $\begin{array}{l}\text { Intions } \\
\text { Alese } \\
\text { Goneating } \\
\text { Conf4 }\end{array}$ & $\begin{array}{l}\text { Indians } \\
\text { Componementh } \\
\text { Edbon } \\
\text { of Indingls }\end{array}$ & $\begin{array}{l}\text { Inding } \\
\text { Indienta } \\
\text { Michipan } \\
\text { Paurer } \\
\text { Co }\end{array}$ & 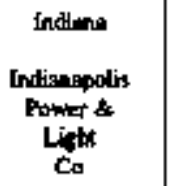 & 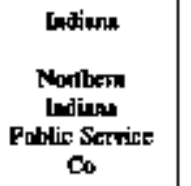 & $\begin{array}{l}\text { Indiann } \\
\text { PSI } \\
\text { Rempy } \\
\text { Inx }\end{array}$ & $\begin{array}{l}\text { Indipon } \\
\text { Somitem } \\
\text { Indlonu Gus } \\
\text { \& Electris } \\
\infty\end{array}$ \\
\hline \multicolumn{8}{|l|}{ Invrotor-Owined } \\
\hline 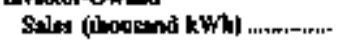 & 14,1358 & 1.672 .835 & $2,779,179$ & 256,119 & 252,752 & $3,419, \pi \mathbf{s}$ & 325,309 \\
\hline Revenoe (dollurs) -.r..-- & 269,463 & $98,871,261$ & 89,593,9019 & 4,SPS,9ts? & $5,240,5,2$ & $62,432,093$ & $6,842,964$ \\
\hline Danund Charge ONy $1_{\text {minn- }}$ & - & - & - & - & - & - & - \\
\hline Dasind d & - & $25,72+290$ & 4), 660,033 & 268,121 & 253.142 & $3,285,031$ & 7,200 \\
\hline Energy & 241,463 & $73,142,575$ & $4,924,876$ & $4,14], 266$ & $4,907,400$ & se,68]+062 & $6,139,066$ \\
\hline OAd & - & - & - & - & - & - & 65,295 \\
\hline \multicolumn{8}{|l|}{ Fadkal } \\
\hline Salen (lloovond KWh) ... & - & - & 9,948 & - & - & - & - \\
\hline 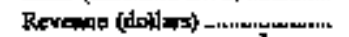 & - & - & 27,443 & - & - & - & 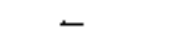 \\
\hline 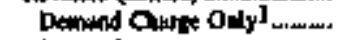 & - & - & - & - & - & - & - \\
\hline 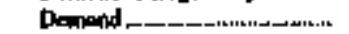 & - & - & - & - & - & - & - \\
\hline Bnorgy & - & - & 277443 & - & - & - & - \\
\hline Olket2 & - & - & - & - & - & - & - \\
\hline \multirow{2}{*}{\multicolumn{8}{|c|}{ Strte and Other }} \\
\hline & & & & & & & \\
\hline 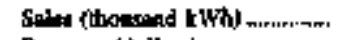 & سـ & - & 113,923 & - & - & $1,300,330$ & - \\
\hline 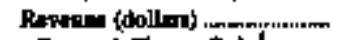 & - & - & $6,741,702$ & - & $\mathbf{3 6 , 8 1 4}$ & $39,992,9014$ & - \\
\hline Denued Chorge Onlyl ....... & - & - & - & مع & - & - & - \\
\hline 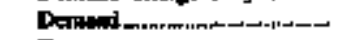 & - & - & $5,2 \pi, 000$ & - & - & IB, & - \\
\hline 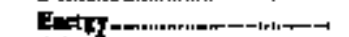 & - & - & $1,48], 108$ & - & $36,8] \mathbf{4}$ & $22,954,064$ & - \\
\hline Other ${ }^{2}$ & - & - & $11,6 \notin 0$ & - & - & $-1,348,251$ & - \\
\hline \multicolumn{8}{|l|}{ Monicipal } \\
\hline Sules (thound kWh) ..-__ & - & - & $2,201,131$ & - & 218,108 & $\operatorname{sen} 218$ & 500,155 \\
\hline Berenoe (dolders) n n & $m$ & - & 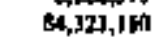 & - & $10,674,143$ & $16,5648,889$ & $22,621, \pi 77$ \\
\hline Demsind Gurge Ody $1_{\text {,rinin. }}$ & - & - & - & - & 1,844002 & - & - \\
\hline 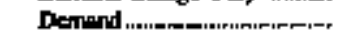 & ـ & - & $50,075,571$ & - & 2722937 & $2,321,031$ & $10,069,092$ \\
\hline 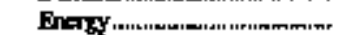 & - & - & $30,236,773$ & - & $8,214,736$ & $9,221,913$ & $11,526,495$ \\
\hline 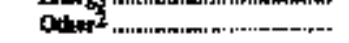 & - & - & $3,013,166$ & - & $-2,107,357$ & $-47,055$ & $1,325,990$ \\
\hline \multicolumn{8}{|l|}{ cooptrative } \\
\hline 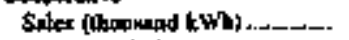 & - & - & $6 \pm 2.446$ & 205,683 & 73,233 & $1,987,956$ & 3n; \\
\hline Reverven (dorlars) & $m$ & - & $21.201,435$ & $4,268,296$ & $5,569,966$ & $46,006,577$ & $1,896,378$ \\
\hline 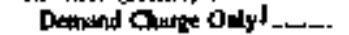 & - & - & - & - & - & - & 7,560 \\
\hline Demand & - & - & $12,468,566$ & 739,872 & $4,184,750$ & $23,0 \div 5.224$ & $\sin 5600$ \\
\hline 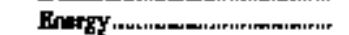 & - & - & $B, 508,678$ & $0,6] n, 2399$ & $2.384,265$ & $24,410,640$ & 906,578 \\
\hline Obteres & - & - & 224,184 & $-84,775$ & - & -538.851 & - \\
\hline \multicolumn{8}{|l|}{ ounery } \\
\hline sile (lhougd kWh) .......... & - & - & $5.383,123$ & - & 83 & 163,833 & 371,278 \\
\hline Ravenet (dotlurs) _.................. & - & - & $150,791,216$ & - & 1,154 & $2,640,623$ & 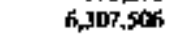 \\
\hline 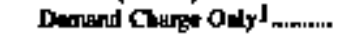 & سـ & - & 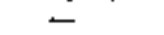 & - & - & - & - \\
\hline Dentand u...u & - & - & $3,54,5213$ & - & - & 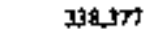 & 69,875 \\
\hline Energy. & عـ & - & $147,298,696$ & - & 1.154 & 2,Fin, 860 & $6,2+7,631$ \\
\hline 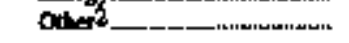 & - & - & - & - & - & -105.614 & - \\
\hline \multicolumn{8}{|l|}{ rotul } \\
\hline Snles (aknowpd kWb)__-_- & 14,138 & $1,672,835$ & $11,146,992$ & 481,897 & 564,166 & $6,941.389$ & 1,234,8in4 \\
\hline Revenoe (dollons).....-- - & 248,463 & $96,671,269$ & 352.898.888 & $8, m 8,283$ & $22,521,609$ & $169,781,016$ & $37,668.622$ \\
\hline Demend O Oure Ody I & - & - & - & - & $5,044,077$ & - & 7,5018 \\
\hline Darond_- & - & $25,728,868$ & ]15,972,290 & $1,607.953$ & 3.1601809 & 52366.774 & $11.126,667$ \\
\hline Binergy & 248,467 & $73,142,375$ & 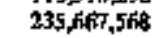 & 7,752105 & $15,624,310$ & $118,381,039$ & $2 x, 61 \mathrm{~J}, 1,0$ \\
\hline 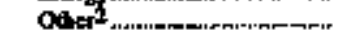 & - & - & $(, 25), 030$ & mB1, vos & $-2,107,55 ?$ & $-2,166,799$ & $1,921,285$ \\
\hline
\end{tabular}

Ses notes and foolncta a end of the 
Table 20. Electricity Sales for Resale by Investor-0wned Utalities, by State, 1994 (Continued)

\begin{tabular}{|c|c|c|c|c|c|c|c|}
\hline $\begin{array}{l}\text { Purcheser of } \\
\text { Electinsty by } \\
\text { Ounerbly Clas }\end{array}$ & $\begin{array}{l}\text { Iowy } \\
\text { Intervane } \\
\text { Power } \\
\text { Co }\end{array}$ & $\begin{array}{c}\text { low. } \\
\text { IES } \\
\text { Uinumen } \\
\text { Inc }\end{array}$ & 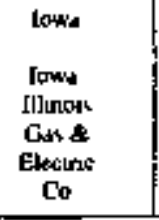 & 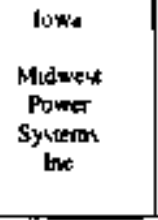 & $\begin{array}{c}\text { Kamn } \\
\text { Kand } \\
\text { Gan } \\
\text { Eledine } \\
\text { Co }\end{array}$ & 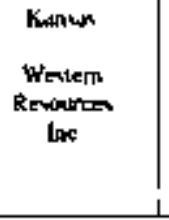 & 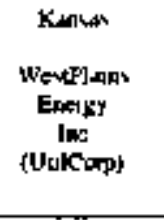 \\
\hline 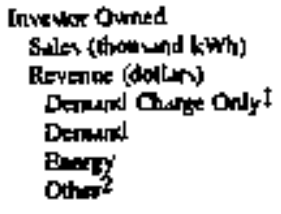 & $\begin{array}{r}221,997 \\
4,207,9511 \\
5,0100 \\
917,740 \\
2,209,210 \\
-\quad\end{array}$ & $\begin{array}{r}474,057 \\
9,945,429 \\
- \\
1,749,826 \\
7,269,398 \\
966,215\end{array}$ & $\begin{array}{l}1,2010,751 \\
22,595,599 \\
- \\
7,569,459 \\
19,1029,1111 \\
-\end{array}$ & $\begin{array}{l}1,11+, 492 \\
13,1026,465 \\
- \\
18,859,600 \\
14,166,655 \\
-\end{array}$ & $\begin{array}{r}705,645 \\
14,665,646 \\
- \\
2,745,036 \\
12,293,874 \\
26,776\end{array}$ & $\begin{array}{r}94,7201 \\
2,269,913 \\
-\quad 1,940 \\
2,258,877 \\
-\end{array}$ & $\begin{aligned} & 53,014 \\
& 1,184,155 \\
&- \\
&- \\
& 1,184,150 \\
&-\end{aligned}$ \\
\hline 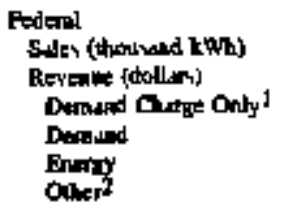 & 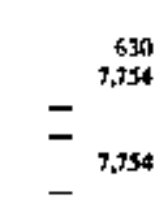 & 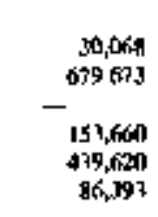 & $\begin{array}{l}- \\
z \\
- \\
-\end{array}$ & $\begin{array}{l}\quad 52,455 \\
-\quad 760,2867 \\
- \\
-760,1867\end{array}$ & $\begin{array}{l}z \\
z \\
= \\
-\end{array}$ & $\begin{array}{l}\bar{z} \\
\bar{z} \\
\overline{-}\end{array}$ & $\begin{array}{l}- \\
- \\
- \\
\overline{-}\end{array}$ \\
\hline 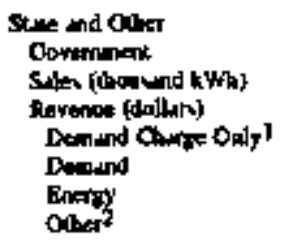 & 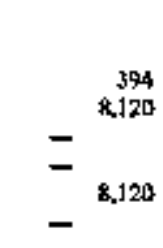 & $\begin{array}{l}\text { 18,016 } \\
-\quad \\
- \\
=\end{array}$ & $\begin{array}{l}11,6055 \\
- \\
- \\
{ }^{196,314}\end{array}$ & $-^{52,454} \begin{array}{r}52,35 \\
52,355\end{array}$ & $\begin{array}{r}25,528 \\
960,241 \\
-\quad 709,000 \\
-551,241 \\
-\quad\end{array}$ & $\begin{array}{r}242,642 \\
4,074,123 \\
- \\
1,654,3 \pm 0 \\
1,279,743 \\
-\end{array}$ & $\begin{array}{l}- \\
- \\
- \\
- \\
-\end{array}$ \\
\hline 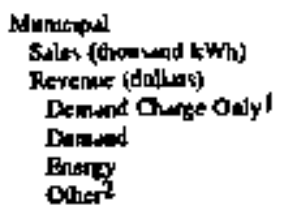 & $\begin{array}{l}247.745 \\
8.751,51205 \\
- \\
3,14,157 \\
5,746,867 \\
-\end{array}$ & $\begin{array}{r}\$ 8,426 \\
15,828,527 \\
- \\
6,199,299 \\
5,415,553 \\
4,191,465\end{array}$ & $\begin{array}{r}42,823 \\
1,287,815 \\
- \\
626,493 \\
674,820 \\
26,502\end{array}$ & $\begin{array}{r}712,056 \\
19,464,111 \\
- \\
9,070591 \\
10,298,5201 \\
-\end{array}$ & $\begin{array}{r}746,251 \\
38,732,9 \text {, } \\
- \\
2,482,645 \\
16,078,782 \\
170,712\end{array}$ & $\begin{array}{r}972,442 \\
27,431,654 \\
- \\
10,489,290 \\
16, \pi 78,545 \\
162,854\end{array}$ & $\begin{array}{r}219,522 \\
6899,1551 \\
- \\
2,560,043 \\
4534,0100 \\
-735,007\end{array}$ \\
\hline 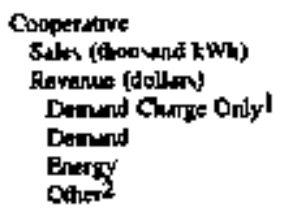 & $\underbrace{}_{-} \begin{array}{r}7.188 \\
- \\
-\end{array}$ & $\begin{array}{r}684,778 \\
30,248,431 \\
- \\
151,647 \\
9,343,597 \\
203,287\end{array}$ & $\begin{array}{r}2,418 \\
527,244 \\
-\quad 10,18010 \\
-\quad 392,244\end{array}$ & $\begin{array}{r}157,692 \\
5,123,331 \\
12,540 \\
-\quad 110,611 \\
-\end{array}$ & $\begin{array}{r}34675 \\
2,974,249 \\
-\quad 375,1999 \\
595.159 \\
-\end{array}$ & $\begin{array}{r}999,499 \\
32,858,486 \\
- \\
14,356,557 \\
18,494,3066 \\
2,697\end{array}$ & $\begin{array}{r}203.816 \\
6137,347 \\
- \\
2176,945 \\
4057,367 \\
-96,965\end{array}$ \\
\hline 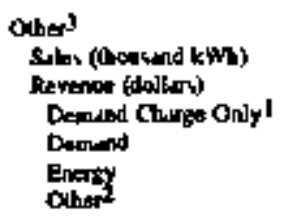 & $\begin{array}{l}\bar{z} \\
\overline{-} \\
\overline{-} \\
-\end{array}$ & $\begin{array}{r}19,595 \\
165,586 \\
-\quad 80,0006 \\
-\quad 135586\end{array}$ & $\begin{array}{l}\quad 30,654 \\
-\quad 322,416 \\
-\quad 322,434\end{array}$ & $\begin{array}{r}55,326 \\
995,753 \\
-\quad 290,656 \\
785,197 \\
-\quad 297\end{array}$ & 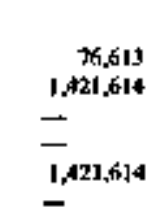 & $\begin{array}{l}z \\
z \\
\bar{z} \\
=\end{array}$ & $\begin{array}{l}\bar{z} \\
\bar{z} \\
=\end{array}$ \\
\hline 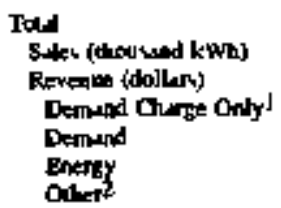 & $\begin{array}{r}477,954 \\
13,569,245 \\
5,000 \\
3,927,893 \\
9,235,352 \\
-\end{array}$ & $\begin{array}{l}1,704,940 \\
17,271,45,3 \\
- \\
8,444,392 \\
23,375,311 \\
5,419350\end{array}$ & $\begin{array}{r}6,311,247 \\
24,932,764 \\
- \\
4,3,4,951 \\
20,574,913 \\
26,502\end{array}$ & $\begin{array}{r}2,297,845 \\
59.327,403 \\
12,6405 \\
28,220,849 \\
11,090.914 \\
-\end{array}$ & $\begin{array}{r}1,559,974 \\
78,749,939 \\
- \\
7,511,621 \\
31,040,670 \\
197,448\end{array}$ & $\begin{array}{l}2,309, \text { ind } \\
67,493,181 \\
- \\
26,511,367 \\
40,816,457 \\
165,477\end{array}$ & $\begin{array}{r}476,252 \\
14,180,557 \\
- \\
4736,989 \\
975,372 \\
-332,097\end{array}$ \\
\hline
\end{tabular}

Set noth and flonomoner all end of table 
Table 20. Electricity Sales for Resale by Investor-Owaed Utilities, by State, 1994 (Continued)

\begin{tabular}{|c|c|c|c|c|c|c|c|}
\hline 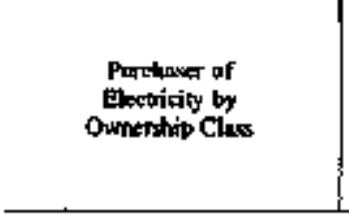 & $\begin{array}{l}\text { Kenocky } \\
\text { Kontucky } \\
\text { Pawr } \\
\text { Co }\end{array}$ & $\begin{array}{l}\text { Kenuecty } \\
\text { Kenuecky } \\
\text { Utilities } \\
\text { Co }\end{array}$ & 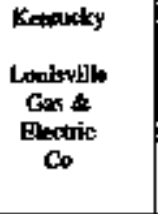 & 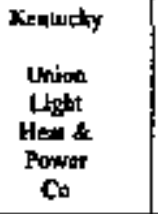 & 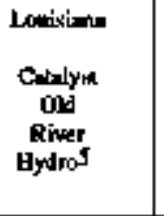 & $\begin{array}{l}\text { Lovinipax } \\
\text { Centril } \\
\text { Looivinus } \\
\text { Electrir } \\
\text { Co }\end{array}$ & $\begin{array}{c}\text { Loulviana } \\
\text { Lodisimen } \\
\text { Power \& } \\
\text { Lighr } \\
\text { Co }\end{array}$ \\
\hline \multicolumn{8}{|l|}{ Inrestor-OMned } \\
\hline Salke (thoodand kHAl ........... & 299,180 & $2,188,6949$ & 1,0901475 & - & 886,494 & 82 & - \\
\hline Revenoe (dollurs) & $12,795,517$ & $42,579,0015$ & $20,343,981$ & - & $53,421,495$ & $2.1 \%$ & - \\
\hline Denupd Chesge Only')......... & 453,086 & - & - & س & - & - & - \\
\hline 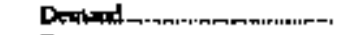 & $2,656,843$ & $7,813,317$ & $1,271,985$ & - & - & - & - \\
\hline 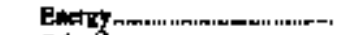 & $9,695,889$ & $35,57,626$ & $19,271,9 \%$ & - & $55,424,495$ & - & - \\
\hline Odun & - & $-817,858$ & - & - & - & 2,196 & - \\
\hline \multicolumn{8}{|l|}{ Pesieral } \\
\hline 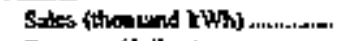 & 3,647 & $115, \sqrt{1} \times \mathbf{4}$ & 587,609 & $m$ & - & - & - \\
\hline Revende fdallarist & 101,658 & $2,170,676$ & J自, & - & - & - & - \\
\hline 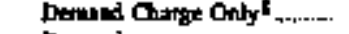 & - & - & - & - & - & - & - \\
\hline 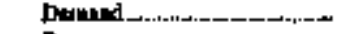 & - & - & - & - & - & - & $=$ \\
\hline 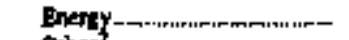 & 101,650 & $2,171,676$ & $10,410,42)$ & 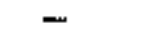 & - & - & - \\
\hline 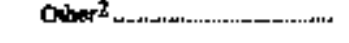 & - & - & - & - & - & - & - \\
\hline \multirow{2}{*}{\multicolumn{8}{|c|}{ Sunte and Otier }} \\
\hline & & & & & & & \\
\hline Reviay (dollars) & - & $\bar{m}$ & $2,946,781$ & $\overline{-}$ & $\overline{-}$ & 87,986 & 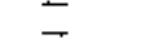 \\
\hline 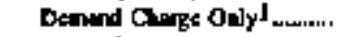 & - & - & - & - & - & - & - \\
\hline 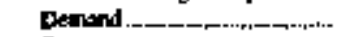 & - & - & 462,672 & - & - & - & $\rightarrow$ \\
\hline 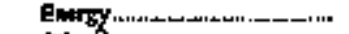 & - & - & $2,484,115$ & - & - & - & - \\
\hline 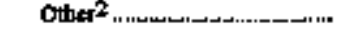 & - & - & - & - & - & 87,986 & - \\
\hline \multicolumn{8}{|l|}{ Maniagut } \\
\hline Soles (Lrousand KW/W) & I I89,352 & $1,467,759$ & 61,022 & T,464 & 36,586 & Jumins & - \\
\hline 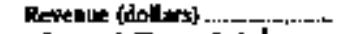 & $55 \$ 6.884$ & $44,7 k 1.935$ & $1,042,163$ & $2,167,929$ & $3,597,810$ & $8,067,321$ & - \\
\hline Dentind Cbugr Ody J & - & - & - & - & - & - & - \\
\hline 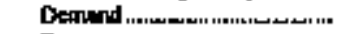 & 2353.132 & $21,336,734$ & - & 665_398 & - & 575,873 & $=$ \\
\hline 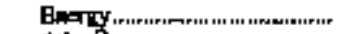 & $3,223,192$ & $34,26,3,090$ & 1.042367 & $1.409,274$ & 1.537816 & 428922 & - \\
\hline Others & $35: 560$ & $-10,813,989$ & - & $-17,793$ & $\rightarrow$ & $2,042,526$ & - \\
\hline \multicolumn{8}{|l|}{ Cooperative } \\
\hline 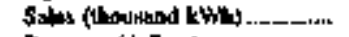 & 176 & $\$, 690$ & 315,923 & - & - & 2,328 & - \\
\hline Revenue (dallars] $-1-$ & 9,048 & 139,240 & $3,645,906$ & 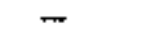 & - & 85,974 & - \\
\hline Demend Corgo Ody 1 _........ & - & - & - & - & - & - & - \\
\hline Denpand ........... & & 23,2000 & $1,344,051$ & - & - & 29,640 & - \\
\hline 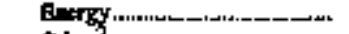 & 9.2d5 & 108,548 & $4,311,855$ & $=$ & - & - & - \\
\hline 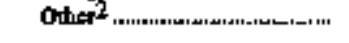 & - & - & - & - & - & 36,334 & - \\
\hline \multicolumn{8}{|l|}{ Otherl } \\
\hline 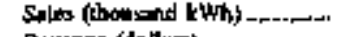 & 2820,574 & - & 214.45 & $B$ & - & $61,5) 7$ & 786,443 \\
\hline 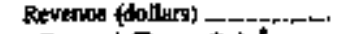 & $75,285,751$ & $m$ & $2,130,515$ & 950 & - & $2,090,498$ & $35,406,359$ \\
\hline 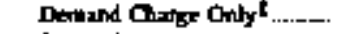 & - & - & - & - & $\rightarrow$ & - & - \\
\hline 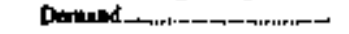 & 1298,566 & - & 66,105 & - & - & 332,420 & - \\
\hline 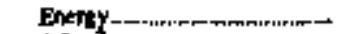 & $33,983.215$ & - & 2.064513 & - & - & 930 & $35,406,359$ \\
\hline ollentra & - & - & - & 990 & - & $1,757,548$ & - \\
\hline \multicolumn{8}{|l|}{ Tots } \\
\hline 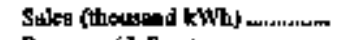 & $3,004,225$ & $3,776,991$ & 2015,313 & 47,472 & $943, \pi 80$ & $394,5,36$ & 786,443 \\
\hline Revelos ( & $59,7 \pm 5,468$ & $89,665,444$ & $42,720,026$ & $2,058,319$ & $58,972,3 / 15$ & $10,314,359$ & $35,406,358$ \\
\hline Danind Cture Only 1 ......... & 453,1286 & - & - & - & - & & - \\
\hline 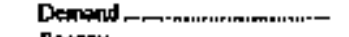 & (3) & $29,175,251$ & $3,141,331$ & 646,388 & - & 937.933 & $\overrightarrow{3 c}$ \\
\hline 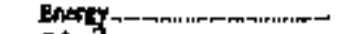 & $47,003,30]$ & 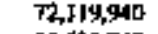 & $39,579,293$ & $1,409,274$ & $38,962,315$ & 429,952 & $35,406,359$ \\
\hline OAha" & 16,564 & $-11,629,747$ & - & $-[2,34]$ & - & $8.946,590$ & - \\
\hline
\end{tabular}

Sod wort end footentes at end of whlk. 
Table 20. Electriclty Sales for Resile by Investor-Owned Utilities, by State, 1994 (Continued)

\begin{tabular}{|c|c|c|c|c|c|c|c|}
\hline 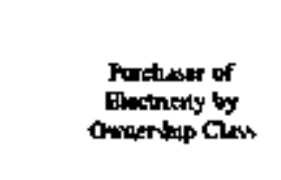 & 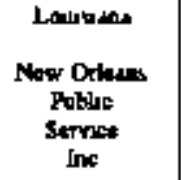 & 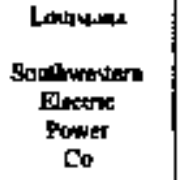 & 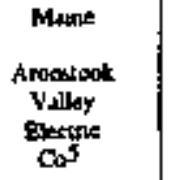 & $\begin{array}{l}\text { Hrine } \\
\text { Bungor } \\
\text { Hytho } \\
\text { filectore } \\
\text { Co }\end{array}$ & $\begin{array}{l}\text { Mare } \\
\text { Central } \\
\text { Munno } \\
\text { Powrer } \\
\text { Co }\end{array}$ & $\begin{array}{l}\text { Malue } \\
\text { Mane } \\
\text { Elactine } \\
\text { Powet } \\
\text { Co Ins }\end{array}$ & $\begin{array}{l}\text { Maune } \\
\text { Mation } \\
\text { Public } \\
\text { Serive } \\
\text { Ca }\end{array}$ \\
\hline \multicolumn{8}{|l|}{ [netor Onned } \\
\hline & 一 & 6],814 & 42,095 & $-_{12137}$ & $\begin{array}{r}54,545 \\
1,4,405\end{array}$ & $1,017,078$ & 45,408 \\
\hline 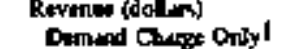 & 二 & $\underline{-}_{-}^{1,323,279}$ & $\frac{1,570,7356}{-}$ & $\begin{array}{l}12.137 \\
12.157\end{array}$ & $1,201.410$ & $21,1001,398$ & ${ }^{990,427}$ \\
\hline Dennd & - & - & - & - & - & - & g,त्राW \\
\hline Emergy & - & $1,325.279$ & $1,570, \mathrm{~ms}$ & - & $1,247,408$ & $20,103,398$ & 942,427 \\
\hline Other & - & - & - & - & - & - & - \\
\hline \multicolumn{8}{|l|}{ Feterd } \\
\hline 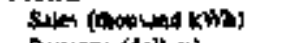 & - & - & $\rightarrow$ & $\rightarrow$ & - & - & - \\
\hline Revenoe (dollers) & - & $=$ & - & - & - & - & - \\
\hline Duned Ohes Oably & - & - & - & - & - & - & - \\
\hline Demsad & - & - & - & - & - & 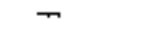 & - \\
\hline Enerty & - & $=$ & - & - & - & - & $=$ \\
\hline $00 x^{2}$ & - & - & - & - & - & - & $T$ \\
\hline \multicolumn{8}{|l|}{ Stwe and Olbst } \\
\hline Shaes (thowed 2Wh) & - & I5 & - & - & 1,230 & - & - \\
\hline Revestang (Jallan) & - & 29,273 & - & - & 102,824 & - & - \\
\hline Demud Q Q Ody? & - & - & 一 & - & - & - & - \\
\hline Domind & - & - & - & - & $m$ & - & - \\
\hline Eargy & - & 29,279 & - & - & Im, Bzd & - & $=$ \\
\hline Dthe ? $^{2}$ & - & - & - & 一 & - & - & $m$ \\
\hline \multicolumn{8}{|l|}{ Mrlocppl } \\
\hline 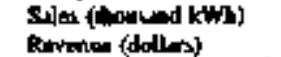 & - & 429,632 & - & Ifig & 105,055 & - & 78,672 \\
\hline 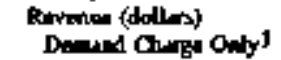 & - & $12,819,807$ & - & 13.545 & $6,120,949$ & - & $5,431,255$ \\
\hline 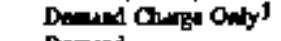 & - & - & - & - & - & - & $\bar{t}$ \\
\hline Derewed & عـ & $4,65 \times 421$ & - & 4,657 & $2,724.173$ & - & $2.590,158$ \\
\hline Enoroy & - & $8,667,386$ & - & 8,908 & $3,372,211$ & - & \$4, II J \\
\hline Othen & - & - & - & - & 2As6t & - & $2,147,366$ \\
\hline \multicolumn{8}{|l|}{ Cooperyture } \\
\hline suler (thoonend kwh) & - & $2,647,542$ & - & 11,536 & 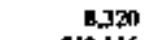 & - & $\operatorname{ll}, \sin 2$ \\
\hline Ravemos (de!lma) & - & $49,652.547$ & - & 923,024 & 519.116 & - & Mnsm \\
\hline Drinind Ouge Only? & - & - & - & $\pi$ & - & - & - \\
\hline Dearend & - & $18,483,265$ & - & $\tan , 469$ & 2596401 & - & 390,455 \\
\hline Enery & - & 31.074 .158 & - & 653,555 & 244,764 & - & 101,829 \\
\hline other 2 & - & 125.120 & - & ع & 14,712 & - & 317,286 \\
\hline \multicolumn{8}{|l|}{ oth ${ }^{3}$} \\
\hline Subs (thournad kWh) & 294,302 & $2,053,250$ & - & 112801 & J,401,554 & 66,724 & $\begin{array}{r}48,549 \\
9040\end{array}$ \\
\hline 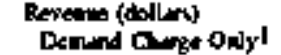 & $9,572,520$ & $38,866,121$ & - & $12 \pi 7, \infty 93$ & $2 \pi .997 .447$ & $20 \mathrm{H} \cdot 29 \%$ & 850.479 \\
\hline Demind ange Ongl & - & - & - & - & - & - & $-\ldots$ \\
\hline $\begin{array}{l}\text { Demband } \\
\text { Enoryy }\end{array}$ & - & - & - & - & - & - & $\begin{aligned} 30,336 \\
4\end{aligned}$ \\
\hline $\begin{array}{l}\text { Enory } \\
\text { Dother }\end{array}$ & 9,572520 & $36,349,003$ & - & $12,737,897$ & $27.997,447$ & 2013,099 & 648,437 \\
\hline Diter & - & 317,618 & 一 & - & - & 30,197 & 151,706 \\
\hline \multicolumn{8}{|l|}{ Tord } \\
\hline 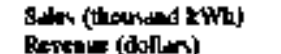 & 294,302 & 5,18B,949 & 40,09 & 124,506 & $1,571.604$ & $1,084,102$ & $\begin{array}{r}384,27] \\
894,731\end{array}$ \\
\hline 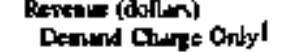 & $9,572,520$ & ${ }_{-}^{\ln 2,73005}$ & $1,570,716$ & $\begin{array}{r}13,686,683 \\
12,15 ?\end{array}$ & $\frac{13997.740}{-}$ & $22,046,694$ & $8,01.73]$ \\
\hline Demend & - & $23,139,6 \pm 6$ & - & 314,106 & $2,989,813$ & - & $9047, \operatorname{mat}$ \\
\hline Enerey & 9.572520 & 79.141 , INI & 1 sxa.xis & $13,460,360$ & $72,964,654$ & $22,016,497$ & 1,37s,sol \\
\hline $00 \mathbf{r}^{2}$ & - & 42,218 & - & - & 39,273 & 30,197 & $2,616,318$ \\
\hline
\end{tabular}

See nower and forthowiol al and af ible 
Table 29. Bectricity Sales for Resale by Investor-Owned Utilifies, by State, 1994 (Contimued)

\begin{tabular}{|c|c|c|c|c|c|c|c|}
\hline $\begin{array}{l}\text { Purchans of } \\
\text { Elecitidyy by } \\
\text { Ownorinio Clean }\end{array}$ & $\begin{array}{l}\text { Maine } \\
\text { Maine } \\
\text { Yamker } \\
\text { Alorpic } \\
\text { Power } \\
\text { Cos }\end{array}$ & 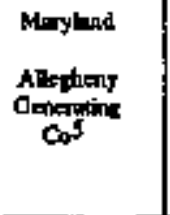 & 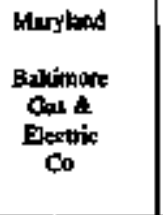 & $\begin{array}{l}\text { Mryilend } \\
\text { Powones } \\
\text { Edson } \\
\text { Co }\end{array}$ & $\begin{array}{l}\text { Mrosechnetus } \\
\text { Bonten } \\
\text { Edisom } \\
\text { Co }\end{array}$ & 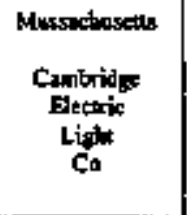 & 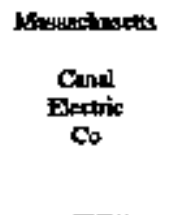 \\
\hline \multicolumn{8}{|l|}{ Ipqeator Dwned } \\
\hline 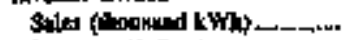 & 6.205061 & - & J,205,958 & 3.053 .274 & $1,251,497$ & - & 4.335 .140 \\
\hline Revenine (doflarst & 162,930320 & 44,51,000 & $25,785,112$ & Indsog,004 & $92,519,56$ & - & $190,213,051$ \\
\hline 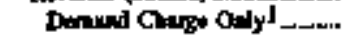 & - & - & - & - & 750284 & - & 65,766 \\
\hline 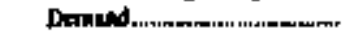 & $133,323,435$ & - & - & $57,154,5168$ & $7,6,6,622$ & - & I(H, 413,454 \\
\hline 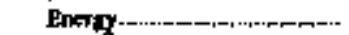 & 20.107245 & - & $25.785,112$ & $47,190,468$ & $14,172,657$ & - & $86,341,659$ \\
\hline Ole ${ }^{2}$ & - & $84,652,000$ & - & $1 \times 2,678$ & - & $-m$ & - 800 , \\
\hline \multicolumn{8}{|l|}{ Fodant } \\
\hline 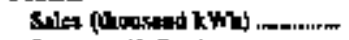 & ב. & 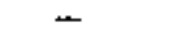 & - & - & - & - & - \\
\hline Reverese (dolprs) & - & - & - & - & - & - & - \\
\hline Demund Gurge 0dy & - & - & - & - & - & - & - \\
\hline Depnend...rnnm - .... ur..... & - & - & - & - & - & - & - \\
\hline Eperyy, & س & - & - & - & - & - & - \\
\hline 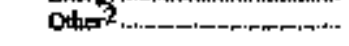 & - & - & - & - & - & - & - \\
\hline \multirow{2}{*}{\multicolumn{8}{|c|}{ SLre and Other }} \\
\hline & & & & & & & \\
\hline 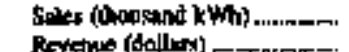 & - & - & - & - & - & - & - \\
\hline 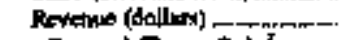 & - & - & - & - & - & - & - \\
\hline Demend Charpe Only' I....... & - & - & - & - & - & $=$ & - \\
\hline 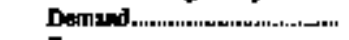 & - & - & - & - & - & - & - \\
\hline Entrgy & - & - & - & - & - & - & - \\
\hline 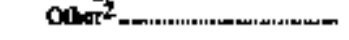 & - & - & - & - & - & - & - \\
\hline \multicolumn{8}{|l|}{ Mluminipd } \\
\hline 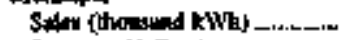 & $\sin , 007$ & - & - & 574,586 & 693,59 & 98,857 & $2,5 \mathrm{mn}$ \\
\hline 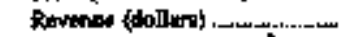 & $9,111,139$ & - & - & $22,004, \pi 3$ & 4,1200024 & $5,459,122$ & NS, 8885 \\
\hline Deround Chorge Ontyl & - & - & - & - & JNB, 437 & - & - \\
\hline 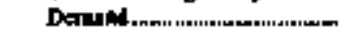 & $7,493,443$ & - & - & $10,424,025$ & $32,682, \sqrt{2} 23$ & 4087,564 & 12.429 \\
\hline Exenty--_,-.._- & $1,57,696$ & - & - & $10,21,034$ & $11,232,664$ & $1,557,851$ & 36,456 \\
\hline $0 \mathbf{l n}^{2}-1$ & - & - & - & $1,359,113$ & - & $-192,393$ & - \\
\hline \multicolumn{8}{|l|}{ Copperitive } \\
\hline 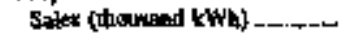 & 69,535 & - & - & 35,245 & - & - & \\
\hline 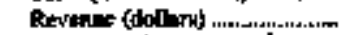 & 1,815,238 & - & - & $1,997,429$ & - & - & לד? \\
\hline Denud Gurge Onlyi ....... & - & - & - & - & - & - & - \\
\hline 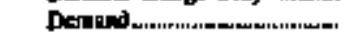 & $1,490,968$ & - & - & 7נ0,9J/ & - & - & 355 \\
\hline Entroy & 324,290 & - & مـ & 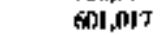 & - & - & $7 \times 2$ \\
\hline onet & - & - & - & $\mathrm{BS}, \mathbf{4 6 2}$ & - & - & - \\
\hline \multicolumn{8}{|l|}{$\mathrm{OOhag}^{3}$} \\
\hline Soles (thotosued tWh) & $=$ & - & $1,477,826$ & 165.639 & $1,141,678$ & 204,757 & - \\
\hline 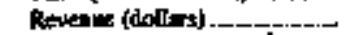 & - & - & $92,2+2,25$ & $4,1629+7$ & $5,133,493$ & $1,676 \mathrm{J3} 3$ & - \\
\hline Detind Chirg Oaly I...... & - & - & - & - & - & $=$ & - \\
\hline 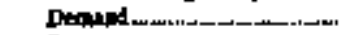 & - & - & - & 521,276 & $9,791,156$ & - & - \\
\hline 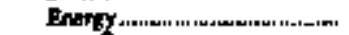 & - & - & 92.242 .275 & T, $(40], 611$ & 27342337 & $2,767,561$ & - \\
\hline ריוגיה & - & - & - & - & - & 918,72 & - \\
\hline \multicolumn{8}{|l|}{ Total } \\
\hline Sules (thoukned LWh) & $6,621,509$ & - & $5,(403,794$ & $0,226,744$ & $3,093,028$ & $30,3,024$ & 4037.646 \\
\hline 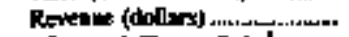 & $173,056,797$ & $84,853,1000$ & $108,027,3] 7$ & $1,12,073,38$. & $173,693,9890$ & $9,129,255$ & $190,253,073$ \\
\hline 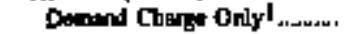 & - & & - & - & $855, n_{21}$ & - & 6,766 \\
\hline Der & $142,797,426$ & - & - & {$[\{7,8] 0,900$} & $120,090,40 \mid$ & 4,0897,664 & $104,426,338$ \\
\hline Bnagy & $3 F, 059,33 \mathrm{~J}$ & - & (18,027,397 & $61,654,410$ & $\$ 2,747,858$ & $4,025,412$ & $86,598,897$ \\
\hline Oxtor ${ }^{2}$ & - & BA,BS3,MFA & - & $1,607.973$ & - & 216.179 & $-807,82 \mathrm{~B}$ \\
\hline
\end{tabular}

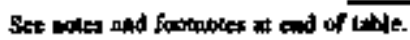


Tabte 20. Electricity Sales for Resale by Investor -Owned Utilities, by State, 1994 (Contimued)

\begin{tabular}{|c|c|c|c|c|c|c|c|}
\hline 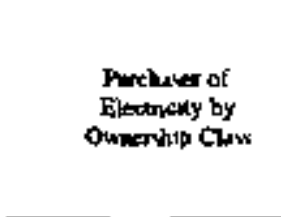 & 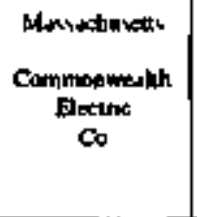 & 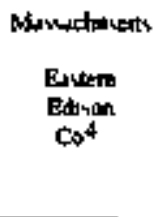 & 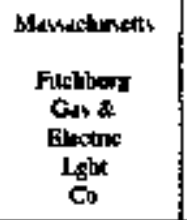 & 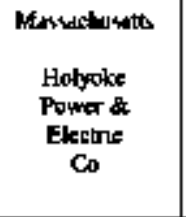 & $\begin{array}{l}\text { Mtmuthinems } \\
\text { Holycke } \\
\text { Wuter } \\
\text { Power } \\
\text { Co }\end{array}$ & 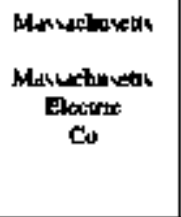 & 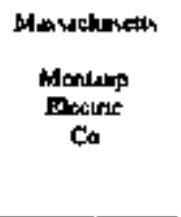 \\
\hline 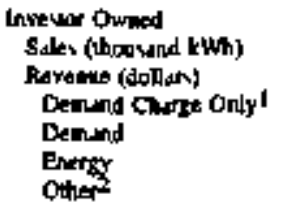 & $\begin{array}{r}r 4,167 \\
1,637,0 \$ 4 \\
-\quad \\
273,930 \\
1,37,270 \\
-9,156\end{array}$ & $\begin{array}{l}- \\
- \\
- \\
-\end{array}$ & $\begin{array}{r}541 \\
-59.250 \\
24,669 \\
-4589\end{array}$ & $\begin{array}{l}896,262 \\
29,849,927 \\
- \\
= \\
15,414,479 \\
14,435,448\end{array}$ & $\begin{array}{l}917,023 \\
32,196,833 \\
= \\
= \\
13,761,985 \\
14,435448\end{array}$ & $\begin{array}{r}3,279 \\
265,699 \\
-\quad \\
48,872 \\
128,869 \\
87,964\end{array}$ & $\begin{array}{r}4480,482 \\
319091,600 \\
- \\
236317,178 \\
68994,694 \\
14179,728\end{array}$ \\
\hline 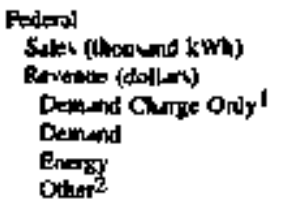 & $\begin{array}{l}\bar{z} \\
= \\
\overline{-}\end{array}$ & $\begin{array}{l}\bar{z} \\
\bar{z} \\
=\end{array}$ & $\begin{array}{l}\overline{-} \\
\overline{-} \\
\overline{-}\end{array}$ & $\begin{array}{l}\bar{z} \\
\overline{-} \\
\bar{z}\end{array}$ & $\begin{array}{l}= \\
= \\
= \\
=\end{array}$ & $\begin{array}{l}- \\
\overline{-} \\
\overline{-} \\
\overline{-}\end{array}$ & $\begin{array}{l}- \\
- \\
- \\
- \\
-\end{array}$ \\
\hline 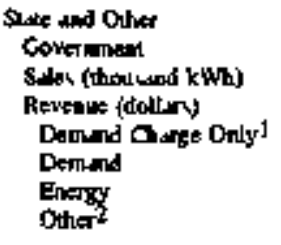 & $\begin{array}{l}\overline{-} \\
\overline{-} \\
\overline{-}\end{array}$ & $\begin{array}{l}z \\
- \\
\overline{-} \\
-\end{array}$ & $\begin{array}{r}35 \\
-\quad 8,009 \\
\quad 0,250 \\
-\quad 1,785\end{array}$ & $\begin{array}{l}- \\
\overline{-} \\
\overline{-} \\
-\end{array}$ & $\begin{array}{l}= \\
= \\
= \\
-\end{array}$ & $\begin{array}{l}z \\
- \\
- \\
-\end{array}$ & $\begin{array}{l}132,900 \\
4941,542 \\
- \\
2159,413 \\
2782,129 \\
=\end{array}$ \\
\hline 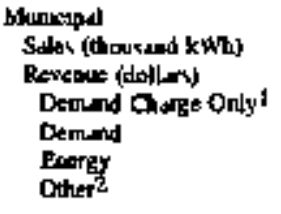 & $\begin{array}{l}\bar{z} \\
- \\
\overline{-} \\
-\end{array}$ & $\begin{array}{l}- \\
\overline{-} \\
- \\
- \\
-\end{array}$ & $\begin{array}{l}\overline{-} \\
\overline{-} \\
\bar{z}\end{array}$ & $\begin{array}{r}30,761 \\
2,345,09 \\
- \\
1,51,5206 \\
789,067 \\
16,502\end{array}$ & $\begin{array}{r}2,366 \\
-\begin{array}{r}40,769 \\
15,477\end{array} \\
-5,367\end{array}$ & 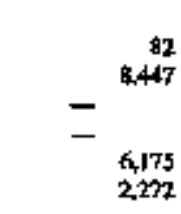 & $\begin{array}{l}24,694 \\
9315,272 \\
= \\
5424,028 \\
3891,246 \\
-\end{array}$ \\
\hline 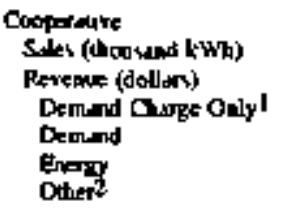 & $\begin{array}{l}= \\
\overline{-} \\
= \\
=\end{array}$ & $\begin{array}{l}\bar{z} \\
\bar{z} \\
\bar{z}\end{array}$ & $\begin{array}{l}- \\
\overline{-} \\
\overline{-} \\
-\end{array}$ & $\begin{array}{l}- \\
\overline{-} \\
\overline{-} \\
=\end{array}$ & $\begin{array}{l}= \\
= \\
=\end{array}$ & $\begin{array}{l}- \\
- \\
= \\
-\end{array}$ & $\begin{array}{l}- \\
\overline{-} \\
= \\
=\end{array}$ \\
\hline 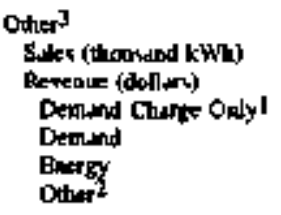 & $\begin{array}{r}1,005,182 \\
22,106,752 \\
- \\
144,677 \\
19,440,464 \\
1,911,603\end{array}$ & $\begin{array}{l}- \\
- \\
- \\
- \\
-\end{array}$ & $\begin{array}{l}\quad 7,769 \\
-490,106 \\
- \\
-\end{array}$ & $\begin{array}{l}\overline{-} \\
= \\
= \\
-\end{array}$ & $\begin{array}{l}981,062 \\
25,311,231 \\
- \\
- \\
13,518,621 \\
12,592,612\end{array}$ & $\begin{array}{r}6,228 \\
674,216 \\
-\quad \\
166,61 k) \\
331,125 \\
136,491\end{array}$ & $\begin{array}{r}826,174 \\
16093.973 \\
-\quad 530,900 \\
15323,079 \\
-\end{array}$ \\
\hline 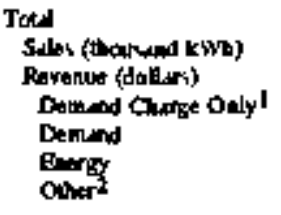 & $\begin{array}{r}1,179,349 \\
20,773,816 \\
- \\
1,058,507 \\
20,812,896 \\
1,902,573\end{array}$ & $\begin{array}{l}- \\
\overline{-} \\
\overline{-} \\
-\end{array}$ & $\begin{array}{r}8,345 \\
557,791 \\
-\quad 34,917 \\
526,474 \\
-\end{array}$ & $\begin{array}{r}917,623 \\
32.193,7966 \\
- \\
1,507,920 \\
16,273,436 \\
14,492,350\end{array}$ & $\begin{array}{r}1,900,451 \\
51,548,855 \\
-\quad \\
35,422 \\
31,860,006 \\
26,630,423\end{array}$ & $\begin{array}{r}9,5,35 \\
94 \mathrm{~d}, 362 \\
-\quad \\
215,472 \\
466,163 \\
2 x 6,723\end{array}$ & $\begin{array}{r}3586,250 \\
349442,397 \\
- \\
244471,317 \\
90791,142 \\
14179,728\end{array}$ \\
\hline
\end{tabular}

Ste mater foodnotes at aod of whe 
Table 24. Electricily Sales for Resale by Investor-Owned Utilities, by State, 1994 (Continued)

\begin{tabular}{|c|c|c|c|c|c|c|c|}
\hline $\begin{array}{l}\text { Puncluner of } \\
\text { Electingily by } \\
\text { Ownathip Class }\end{array}$ & 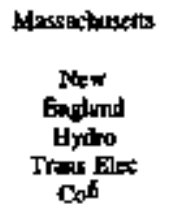 & 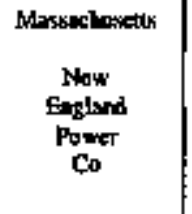 & 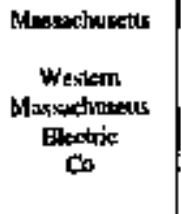 & $\begin{array}{l}\text { Messuxtursetts } \\
\text { Yunlate } \\
\text { Alumic } \\
\text { Elestric } \\
\text { Cos }\end{array}$ & $\begin{array}{c}\text { Mintigen } \\
\text { Consumets } \\
\text { Powe } \\
C_{0}\end{array}$ & $\begin{array}{l}\text { Mletien } \\
\text { Deanow } \\
\text { Elisom } \\
\text { Co }\end{array}$ & $\begin{array}{l}\text { Michipon } \\
\text { Edisom } \\
\text { swill } \\
\text { Eetric } \\
\text { Co }\end{array}$ \\
\hline \multicolumn{8}{|l|}{ Invextor-O Onened } \\
\hline Sskex (Lhound tWhi) & - & $22,8 n .131$ & $1 \$ 0,896$ & - & $1,486,1155$ & $1.573,616$ & - \\
\hline Reventule (dolbry) - - - & - & $1,448,819,673$ & $3,590,857$ & $\$ 3,694,321$ & $44,652,699$ & 32.549 .727 & - \\
\hline Devend Charge Only ${ }^{1}$ & 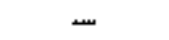 & - & - & - & - & - & - \\
\hline 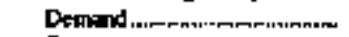 & - & $220,664,831$ & 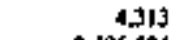 & - & $13,470,960$ & $2,706,6,83$ & - \\
\hline 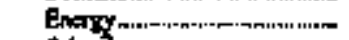 & - & 192132103 & 3406534 & $55,684,52]$ & $39,155,871$ & $29,843,044$ & - \\
\hline 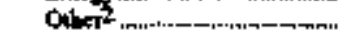 & 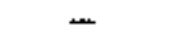 & 234,022759 & 180,010 & - & -7.124 .192 & - & - \\
\hline \multicolumn{8}{|l|}{ Fedetal } \\
\hline 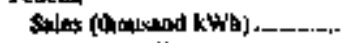 & - & - & - & - & - & - & - \\
\hline 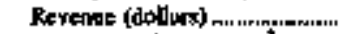 & - & - & - & - & - & - & - \\
\hline 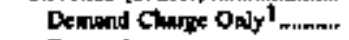 & - & - & - & - & - & ש- & - \\
\hline Detuend ..... & - & - & - & - & $-m$ & - & - \\
\hline 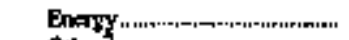 & - & - & - & عـ & - & - & - \\
\hline Othor & 一 & - & - & - & - & - & - \\
\hline \multicolumn{8}{|l|}{ Stent and Dher } \\
\hline 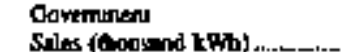 & & 41,020 & 125,50 & - & 160.585 & [23,86] & \\
\hline 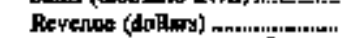 & $\bar{z}$ & $2,747,618$ & 347399 & - & $\$ \$ \$ 27,900$ & $2,456,397$ & $\overline{-}$ \\
\hline Denaed Chere Onlys & - & - & - & - & - & - & - \\
\hline 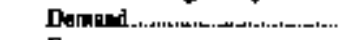 & - & $3,485,310$ & - & - & 752,000 & 83,760 & - \\
\hline 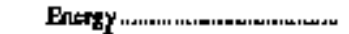 & - & 857,1256 & 313,609 & - & $3,071,000$ & $2,352,8866$ & - \\
\hline 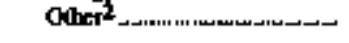 & - & 401,252 & 35,74ה & - & - & {$[9,64]$} & - \\
\hline \multicolumn{8}{|l|}{ Mnoicipd } \\
\hline Sald (thoorand kWh) ............. & - & 611.712 & 28.769 & - & $3 \pi, 441$ & 499,670 & - \\
\hline Beyeroe (dolknky) & - & 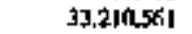 & $576,7 \%$ & - & $12,711,201$ & $16,87 \pi, 59]$ & - \\
\hline 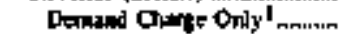 & - & - & - & - & - & - & - \\
\hline Danud & - & $16,228,384$ & - & - & $3,391,844$ & 5.901 .106 & - \\
\hline Eneryy & - & $11,388,9] 2$ & 532,317 & - & $11,6 \pi, 732$ & $12,633,1866$ & - \\
\hline Oaket 2 - & - & $1,393,195$ & 44,479 & - & $-2,318,283$ & $-1,6 \leqslant 7,401$ & - \\
\hline \multicolumn{8}{|l|}{ Coopenulve: } \\
\hline Sale (thourad KWh) .............. & - & 6,781 & - & - & 53,60 & B:04,837 & 143,558 \\
\hline Revalpe (doltuts) , & - & 433,631 & - & - & $1,824,981$ & 526 GST,DKA & $0,984,412$ \\
\hline Dantund Chrofe Onlyl & - & - & - & - & $\rightarrow$ & - & - \\
\hline Denaudad n, & - & $(8) .351$ & - & - & $116, \beta, 1$ & $16,100,692$ & 409.850 \\
\hline 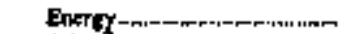 & - & [45.708 & - & - & {$[.6] 4,216$} & $18,311,824$ & $3,289.190$ \\
\hline Olker ${ }^{2}$ - & - & 101,812 & - & - & -504int & $-1,747,452$ & 265,372 \\
\hline \multicolumn{8}{|l|}{ Onber ${ }^{3}$} \\
\hline Sales (thoongend l'Wh) & & 129,2615 & $1,142,631$ & - & $452,6 \in 8$ & $-21,029$ & - \\
\hline Reverno (dolinns) ..._........... & - & $3,026.876$ & $31,858,980$ & - & $11,332,750$ & $-941,470$ & - \\
\hline 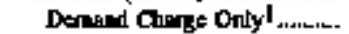 & - & - & - & - & - & - & - \\
\hline Desinaind & - & - & - & $m$ & $2,261,0,60$ & 3,891 & - \\
\hline Energy & - & $2,562,300$ & $-10,902,997$ & - & $7,445,000$ & $-176,614$ & - \\
\hline 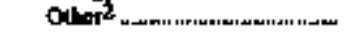 & - & 464,576 & $42,76], 977$ & - & $3,4,16,750$ & $-76 \mathrm{~A}, 707$ & - \\
\hline \multicolumn{8}{|l|}{ Toetl } \\
\hline 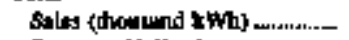 & 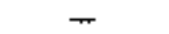 & $23,665,959$ & $1,117,846$ & - & $2,529,550$ & $2,920,065$ & 149,554 \\
\hline ReYerioe (do]lntr).................. & - & $1,488,234,599$ & 36.27, 1022 & $55,684,521$ & 74324,680 & 53.606i,499 & $3,934,452$ \\
\hline Denowd Change Onlyl........ & - & - & & - & - & - & - \\
\hline 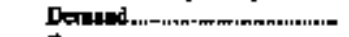 & - & 2401.599 .9 .16 & 4313 & - & 201540,645 & $24,7,5,0 \%$ & 4098 \\
\hline Enagy & - & 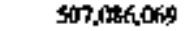 & T, & $55,640,521$ & $62,164,819$ & $62,964,326$ & $3,289.190$ \\
\hline 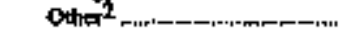 & - & 240,5bs,5y4 & $43,022,246$ & - & $-8,382,801$ & $-4,151,919$ & 265,372 \\
\hline
\end{tabular}

She woter and foothonet at and of tride. 
Table 20. Elextricity Sales Ior Resale by Investor Owned Utilities, by State, 1994 (Continued)

\begin{tabular}{|c|c|c|c|c|c|c|c|}
\hline $\begin{array}{l}\text { Purchurex of } \\
\text { Elestnesty by } \\
\text { Ownerathp CLis, }\end{array}$ & 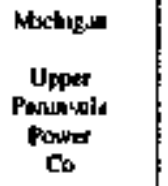 & 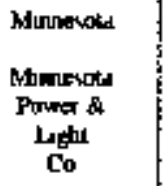 & $\begin{array}{l}\text { Monnevos } \\
\text { Morthern } \\
\text { States } \\
\text { Powes } \\
\text { Co }\end{array}$ & $\begin{array}{c}\text { Mmesoda } \\
\text { Ouga } \\
\text { Talt } \\
\text { Puwar } \\
\text { Co }\end{array}$ & $\begin{array}{l}\text { Mistrupippt } \\
\text { Misusupp } \\
\text { Powrer \& } \\
\text { Esthe } \\
\text { Co }\end{array}$ & $\begin{array}{l}\text { Misuluppy } \\
\text { Mrsusuppt } \\
\text { Powar } \\
\text { Cu }\end{array}$ & 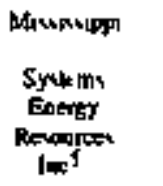 \\
\hline \multicolumn{8}{|l|}{ Invenor Onmed } \\
\hline 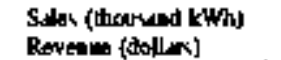 & $\begin{array}{r}17,406 \\
7 d, 521\end{array}$ & $\begin{array}{r}3,592,497 \\
35,058,528\end{array}$ & $\begin{array}{r}9,573,894 \\
247,128,5200\end{array}$ & $\begin{array}{r}809, \text { 혀 } 0 \\
17,725,005\end{array}$ & - & $\begin{array}{r}413,981 \\
10,116,261\end{array}$ & $\begin{array}{r}86,57.218 \\
472.540 .442\end{array}$ \\
\hline Dernund Cunge Only 1 & - & - & - & - & - & - & - \\
\hline Demund & 309,652 & $16,548,278$ & $3,506,856$ & AED, SXK & - & $1,895,598$ & $\pi$ \\
\hline Eartsy & 47,857 & I9,992 & $99,347,001$ & $37,245,005$ & - & $B, 220,761$ & $472,547,4+2$ \\
\hline Qtuct & -5.948 & $-3,4 x_{2}, 21$ & $134,215,461$ & - & - & $m$ & - \\
\hline \multicolumn{8}{|l|}{ Fodalal } \\
\hline SAle thouland kWhy & - & $4245 ?$ & 76,5010i & - & - & 65,268 & - \\
\hline 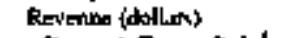 & - & $4-19,524$ & $454, \mathrm{kn}$ & - & - & $1,052,821$ & - \\
\hline Deluatid Coutye Onhyl & - & - & - & - & - & - & - \\
\hline Demand & - & - & - & - & - & $\rightarrow$ & - \\
\hline Bmaty & 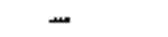 & 64t52d & 454,307 & - & - & $1,952,161$ & - \\
\hline Ch=t & - & - & - & - & - & - & - \\
\hline \multicolumn{8}{|l|}{$\begin{array}{l}\text { State and Oiker } \\
\text { Gougmruent }\end{array}$} \\
\hline Sules (thontand kWh) & - & 5.557 & 89.533 & 88,855 & - & 6,912 & - \\
\hline ReYenoe (doNhaT) & - & 118? & $1,678,743$ & $1,400,8008$ & $m$ & 272,486 & - \\
\hline Denend Chupe Only' & - & $\rightarrow$ & - & - & - & - & - \\
\hline Daraped & 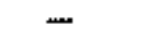 & - & - & - & - & - & - \\
\hline Eutsty & - & 118,710 & $1,879,743$ & 1408,808 & - & 272,496 & - \\
\hline Cther & - & - & - & - & 一 & - & - \\
\hline \multicolumn{8}{|l|}{ Mtancepy } \\
\hline Sdles (thoukat kWh) & $9: 277$ & 552 恻 & $1,661,094$ & 124,268 & - & 86297 & - \\
\hline Revenux (do|lers) & $3,383_{N}, 355$ & 17 的了 称 & 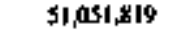 & $2,140,380$ & - & $2,386,584$ & - \\
\hline Danand Charpe Ondyl & $=$ & - & - & - & - & - & - \\
\hline Danund & $1.351 \$ 12$ & $9, \cos 5115$ & 17640,045 & 109,816 & - & 69,017 & - \\
\hline Enनly & $2,064,368$ & 9,10054128 & 32827,856 & $2010,32]$ & - & $1,742,732$ & - \\
\hline ouker? & -32325 & $-1,1863839$ & 563,918 & $-10,163$ & - & 554,839 & - \\
\hline \multicolumn{8}{|l|}{ Coopermut } \\
\hline Siles (thontand $k W h$ ) & 37,365 & 13,281 & 70,7II & 54,712 & - & $1,937,913$ & - \\
\hline Rovenpe (dollwa) & $1 \mathrm{901}, 537$ & 467284 & 1,270787 & 99246 & - & $74,015,888$ & - \\
\hline Danend Charge Only] & - & - & - & - & - & - & - \\
\hline Denond & ולה, ולא, & I26, LIYA & - & - & - & 40,000 & - \\
\hline Enatg & 954,167 & 31,284 & $1,270,787$ & 991,496 & - & $48,636,967$ & - \\
\hline Othot & -E],557 & - & - & - & - & $35,178,92 \mathrm{t}$ & - \\
\hline \multicolumn{8}{|l|}{ Other ${ }^{3}$} \\
\hline S.les (thoulaod kWh) & 一 & $10, \infty 20$ & 61,349 & 60,422 & 1,590,659 & 189,576 & - \\
\hline Reverese (dollwn) & - & 119,590 & $1,316,162$ & 799.062 & $5,474,724$ & $9,938,547$ & - \\
\hline Derumd Charge Onlyt & - & - & - & - & - & - & - \\
\hline Demund & - & ع & שس & - & - & f., 161,332 & - \\
\hline Bnogy & - & 115,590 & $1,316,162$ & 799,062 & $54,474,724$ & $3.777,215$ & - \\
\hline Other? & - & - & - & - & 一 & 一 & 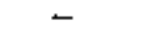 \\
\hline \multicolumn{8}{|l|}{ Tould } \\
\hline 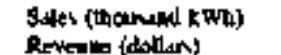 & J164104 & $\begin{array}{r}1,831,113 \\
57,41,376\end{array}$ & $\begin{array}{r}11,493,487 \\
03,100378\end{array}$ & $1,145,667$ & $\begin{array}{l}1,590,653 \\
54,474,724\end{array}$ & $\begin{array}{r}2,735,347 \\
9692697\end{array}$ & $\begin{array}{r}8,653,218 \\
472,543,442\end{array}$ \\
\hline Demerd Darge Drby' & - & - & - & 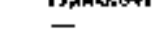 & - & - & 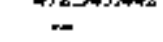 \\
\hline Derpud & $2,328,395$ & $25,759,401$ & 21, If64,1]1 & 539,516 & - & $8,525,943$ & - \\
\hline Enower & $\begin{array}{r}3,456,188 \\
-56,370\end{array}$ & $\begin{array}{l}31,228,457 \\
-2,546554\end{array}$ & 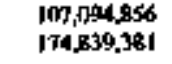 & $\begin{array}{r}\mid[,-486,18 B \\
-10,163\end{array}$ & $54,474.324$ & $\begin{array}{l}54,472,984 \\
35,733,760\end{array}$ & $\begin{array}{l}472,543,442 \\
-\end{array}$ \\
\hline
\end{tabular}

Set noter ind foctinuter a and of ato 
Table 20. Electrichy Sales for Ressle by Investor-Owned Utilties, by State, 1994 (Continued)

\begin{tabular}{|c|c|c|c|c|c|c|c|}
\hline 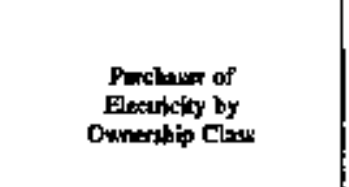 & 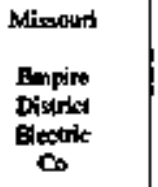 & 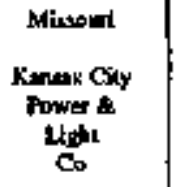 & 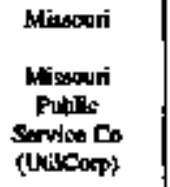 & 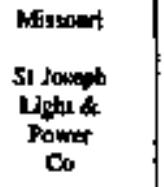 & $\begin{array}{c}\text { Missouri } \\
\text { Unicon } \\
\text { Elextric } \\
0\end{array}$ & $\begin{array}{c}\text { Montwa } \\
\text { Montear } \\
\text { Power } \\
\text { Co }\end{array}$ & $\begin{array}{l}\text { Nonde } \\
\text { Neneda } \\
\text { Poner } \\
\text { Ca }\end{array}$ \\
\hline \multicolumn{8}{|l|}{ Invetion-Owad } \\
\hline Siles (thoostind $\mathbf{x} \mathbf{W h}$ ) .........-in & 30,035 & $2,494,117$ & 伆,72x & 23,199 & $5.106,010)$ & $2.1008,861$ & 1,538 \\
\hline 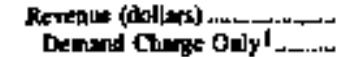 & $5,304,410$ & $37,599,869$ & $1,385,526$ & $-401,406$ & 121.151 .319 & $\frac{62,462,912}{-}$ & $-90,362$ \\
\hline Demind & $1,991,872$ & 63,196 & - & - & $26,914,334$ & $22,012,920$ & - \\
\hline 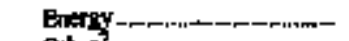 & $3,296,313$ & $16,9,0,245$ & $1,585,526$ & 401,406 & $99,239,985$ & $40,389,992$ & $\infty 0,362$ \\
\hline 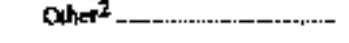 & $2 \times, 2016$ & 514,4300 & - & - & - & - & - \\
\hline \multicolumn{8}{|l|}{ Festem } \\
\hline 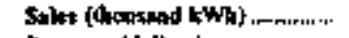 & - & - & - & - & 194,434 & $\mathbf{J , 0 2 5 , 0 1 3}$ & - \\
\hline 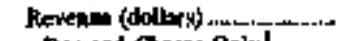 & - & - & - & - & 3,116 , 伤 & $23,036,816$ & - \\
\hline Detrand Charge Oaly' & - & - & - & - & - & - & - \\
\hline 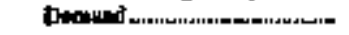 & - & - & - & - & - & - & - \\
\hline 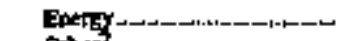 & - & $\rightarrow$ & - & - & $3,116,067$ & $23,036,816$ & - \\
\hline 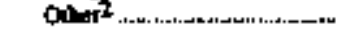 & - & - & $\rightarrow$ & - & - & - & - \\
\hline \multicolumn{8}{|l|}{ Surte and Olk } \\
\hline 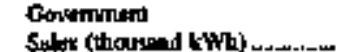 & 4.518 & $76,4 \% 7$ & 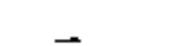 & Rex 2 & - & 9069 & t55.178 \\
\hline Reve:1e (dollers) & 57,027 & $1,624,853$ & - & 200,144 & - & 219,452 & $4,877,571$ \\
\hline Oennead Chore Onlyl....... & - & - & س & - & - & - & - \\
\hline 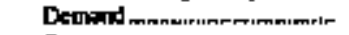 & - & - & - & - & - & - & 305,347 \\
\hline Energy & 87,027 & $1,6064,053$ & - & $21 k, 144$ & - & 219,452 & 4,505,959 \\
\hline 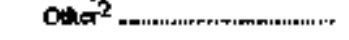 & 一 & - & - & - & - & - & 26,265 \\
\hline \multicolumn{8}{|l|}{ Murictpul } \\
\hline 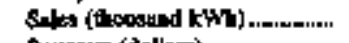 & 220,978 & 939, if6 & 175,953 & $\mathrm{I}, 600$ & $1,21,03$ & [222,J11 & 64,101 \\
\hline 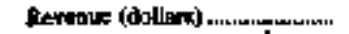 & $7,674,709$ & $21,055,054$ & $6,4=1,560$ & $35, n 54$ & $43,761,569$ & $43,230,071$ & $2,025,030$ \\
\hline Dunind Chenge Only 1 ,........ & - & - & - & - & - & - & - \\
\hline 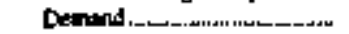 & $2,672,893$ & $1, \infty, 6,6 \pi$ & $2,143,682$ & - & $2,000,604$ & $22,163,890$ & 986,322 \\
\hline Enary & $5.1201, B] 6$ & $13,009,560$ & $5,346,525$ & $35,0 \mathbf{B S} \mathbf{4}$ & $41,760,960$ & $2 J, 106,191$ & $|, A| 6, \mid 7]$ \\
\hline 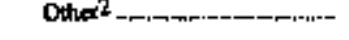 & - & $5,684,01$ & -1 ,10yoge? & 一 & - & - & $-77,446$ \\
\hline \multicolumn{8}{|l|}{ Cosportitize } \\
\hline Soleg (thousund KWh) . & 13.J5] & $1,288,826$ & 91,238 & 189,204 & 746,920 & 445,949 & 199,792 \\
\hline 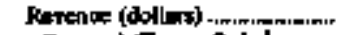 & 497,059 & $18,655,116$ & $1,355,344$ & 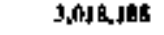 & $25,92 x, 139$ & {$[5,065,06]$} & $4,518,601$ \\
\hline Deniand There Onlyl & - & - & - & - & - & - & - \\
\hline 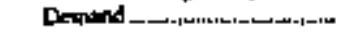 & 279.121 & 689,394 & - & - & $\rightarrow$ & $4,18,401$ & 636,413 \\
\hline 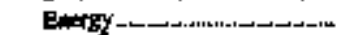 & 218,738 & $18,166,722$ & $1,355,344$ & $3,0 \mathrm{JB}, \mathrm{INt}$ & $25,96,190$ & $1,030,600$ & $3,8+2,20$ \\
\hline QWhert2 & - & - & - & - & - & - & 4.570 \\
\hline \multicolumn{8}{|l|}{ Other 3} \\
\hline Sales (Lonsund kWh) & - & 32,194 & - & - & 2,060,569 & 22,734 & -3, EOI \\
\hline 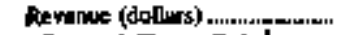 & - & 550,040 & - & - & $35,849,539$ & 292,523 & $-82,972$ \\
\hline 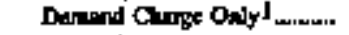 & - & - & - & - & - & - & - \\
\hline 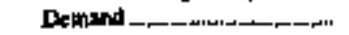 & - & 一 & - & - & $\rightarrow$ & - & - \\
\hline 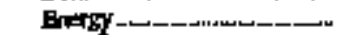 & - & 5St,049 & - & - & $35,049,334$ & 375013 & 11,025 \\
\hline Olher2 & - & - & $\rightarrow$ & - & $\rightarrow$ & -82490 & -93.999 \\
\hline \multicolumn{8}{|l|}{ Tot:l } \\
\hline Sales (4honanad ktwh) .......... & 511,73\% & $4,821,285$ & $327 A 27$ & 2m, 185 & 9336,190 & 4,03,575 & J58 \\
\hline 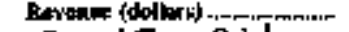 & 13560,005 & $79,618,941$ & $9,221,380$ & $3,660,792$ & $229,006,439$ & 144,596,855 & $11,608,615$ \\
\hline 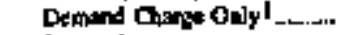 & - & - & $\rightarrow$ & - & - & $m$ & - \\
\hline Demind .............................. & $4,943,846$ & $2,261,258$ & $2,143,682$ & - & $2 B, 914,938$ & $48,561,211$ & $1.928,0096$ \\
\hline 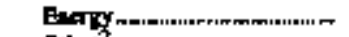 & 8593,954 & $71,211,431$ & $8,197,395$ & $3,660,79$ & $200,891,495$ & $9 \times 558,144$ & 94900.743 \\
\hline 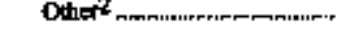 & $26,2 n s$ & $6,199,252$ & - & - & - & -82040 & $-140,296$ \\
\hline
\end{tabular}

Str noter and foodnotes an end of thble. 
Table 20. Electricity Sales for Resale by Invesior-Owned Utilities, by State, 1994 (Continued)

\begin{tabular}{|c|c|c|c|c|c|c|c|}
\hline 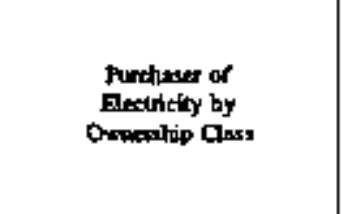 & 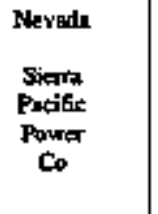 & $\begin{array}{c}\text { Nex Harpuhdre } \\
\text { Confard } \\
\text { Elentric } \\
\text { Cof }\end{array}$ & 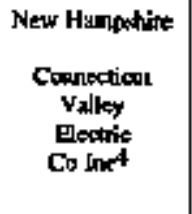 & 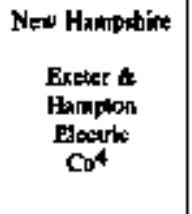 & $\begin{array}{c}\text { New Bisuphine } \\
\text { Grante } \\
\text { Strone } \\
\text { Bdeswic } \\
\text { Cot }\end{array}$ & 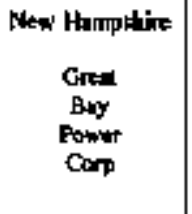 & $\begin{array}{c}\text { New Homphtie } \\
\text { New } \\
\text { Buglend } \\
\text { Trans } \\
\text { Eles } \\
\text { Con }\end{array}$ \\
\hline \multicolumn{8}{|l|}{ Inmentor Ouned } \\
\hline 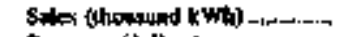 & $37, \Omega / 2$ & - & - & - & - & $6 \mathrm{~s} 2,098$ & - \\
\hline 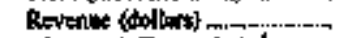 & 1.00K.986 & - & - & - & - & $15,6 \kappa R 22]$ & - \\
\hline 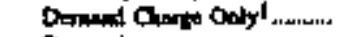 & - & - & - & - & - & - & - \\
\hline 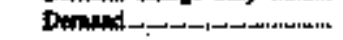 & 181.867 & - & - & - & - & $12,71\{, 048$ & -. \\
\hline 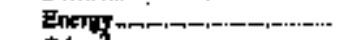 & BNo.103 & - & - & - & - & $4,034,179$ & - \\
\hline 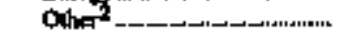 & 13,016 & - & - & - & - & $-1,082,0100$ & $m$ \\
\hline \multicolumn{8}{|l|}{ Fuden } \\
\hline 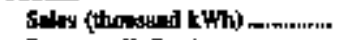 & 22811 & - & - & - & - & - & - \\
\hline 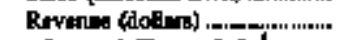 & $1,704.239$ & - & - & - & - & - & - \\
\hline Dend Cuge Only 1 -..... & - & - & - & - & - & - & - \\
\hline Deandand & 914,682 & - & - & - & - & - & - \\
\hline Extry & 857568 & - & - & - & - & - & -- \\
\hline Other & $-68,011$ & - & - & - & - & - & - \\
\hline \multicolumn{8}{|l|}{ Star othor } \\
\hline Salos (thoterad KWh) & 106,740 & - & - & - & - & - & - \\
\hline 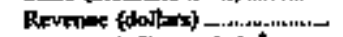 & $5,869,750$ & - & $m$ & - & $-m$ & - & - \\
\hline 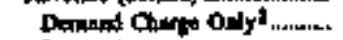 & - & - & $m$ & - & - & - & - \\
\hline 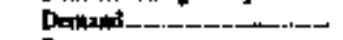 & $1,868,112$ & - & - & - & - & - & -- \\
\hline 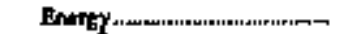 & $4,101,128$ & - & - & - & - & - & -- \\
\hline 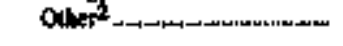 & $-99,490$ & - & - & - & - & - & - \\
\hline \multicolumn{8}{|l|}{ Mrexipal } \\
\hline 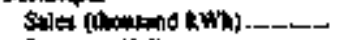 & 55574 & - & - & - & - & 37,453 & $m$ \\
\hline Revenoe fdolbrof & $3,112,216$ & - & - & - & - & sdefts: & - \\
\hline 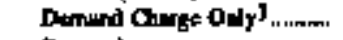 & - & - & - & - & - & - & - \\
\hline 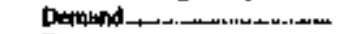 & 911,048 & - & - & - & - & $6 \$ 1.093$ & - \\
\hline Entryy.............................. & $2,49,9,578$ & - & - & - & - & 234,519 & -- \\
\hline Oater & -349.410 & - & - & - & - & 526 & - \\
\hline \multicolumn{8}{|l|}{ Coopernutwe } \\
\hline sales (lousend TWh) & 63 & - & - & - & - & - & - \\
\hline 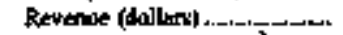 & 1,677 & - & - & - & - & - & -- \\
\hline Demend Ohege ady' & - & - & - & - & - & - & - \\
\hline Dment & & - & - & - & - & - & - \\
\hline Bntry & 1,677 & - & - & - & - & - & - \\
\hline 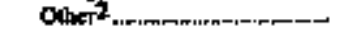 & - & - & - & - & - & - & -- \\
\hline \multicolumn{8}{|l|}{0} \\
\hline 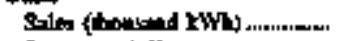 & - & - & - & - & - & 32,813 & - \\
\hline Revenies fdoll:ry ..................... & - & - & - & - & - & 301,283 & - \\
\hline Deviund Chorge 0 dy ${ }^{1}$ & - & - & - & - & - & - & - \\
\hline 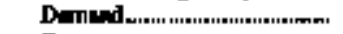 & سה & - & - & - & - & 503,834 & - \\
\hline Ferty................ & - & - & - & - & - & I7t,898 & - \\
\hline Olk- & - & - & - & - & - & 33,501 & - \\
\hline \multicolumn{8}{|l|}{ Toted } \\
\hline Solka (thorangad KWhi) _...-_. & 222230 & - & - & - & - & 759.124 & - \\
\hline 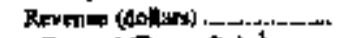 & $11,694,868$ & - & - & - . & - & $17,661,648$ & - \\
\hline Denend On-gige Only 1 & - & $=$ & - & - & - & - & $=$ \\
\hline Dernimd & $3,935,209$ & - & - & - & - & $13,671,003$ & - \\
\hline 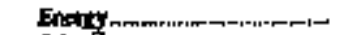 & $\$ 259,054$ & - & - & - & 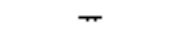 & $4,440,5 \%$ & - \\
\hline 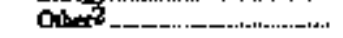 & $-499,895$ & - & - & - & - & $-1,049,973$ & - \\
\hline
\end{tabular}

Soc woles and forvtnoter he end of toble. 
Table 20. Electricity Sales for Resale by Invesior-Owned Utilitles, by State, 1994 (Continued)

\begin{tabular}{|c|c|c|c|c|c|c|c|}
\hline 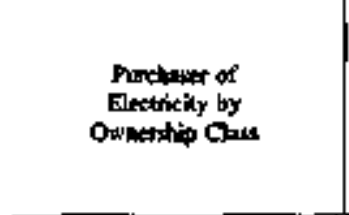 & 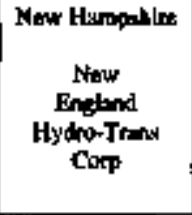 & $\begin{array}{l}\text { New Herophine } \\
\text { North } \\
\text { Atlogtic } \\
\text { Energy } \\
\text { Corp }\end{array}$ & $\begin{array}{c}\text { New Hortphire } \\
\text { Poostre } \\
\text { Service } \\
\text { Co of No }\end{array}$ & $\begin{array}{l}\text { Nex Hanpedite } \\
\text { UNITL } \\
\text { Powner } \\
\text { Corp }\end{array}$ & $\begin{array}{c}\text { Now keray } \\
\text { Allatic Ciy } \\
\text { Bectic } \\
\text { Co }\end{array}$ & 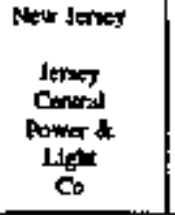 & $\begin{array}{l}\text { Nom Jofoly } \\
\text { Pontlic } \\
\text { Sorvice } \\
\text { Eloctice \& } \\
\text { Gey } \\
\text { Co }\end{array}$ \\
\hline \multicolumn{8}{|l|}{ Invesposoned } \\
\hline Solks (thenthe kWh) & - & $2,229,370$ & $1,528,945$ & 909,456 & हो.8ज़ & 33.756 & 207,403 \\
\hline Revenied (dollors) & - & 143.043 .170 & $45,2=30$ & $69.923,08$ & $1,535,800$ & $1,[4], 297$ & $11,713,919$ \\
\hline Deand Chatye Only' & - & - & - & - & - & - & 一 \\
\hline 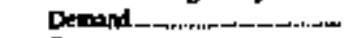 & - & - & 66,391 & $33,063,+3)$ & - & 104,540 & 324,553 \\
\hline Energy & - & $3,142,835$ & $27,19:, 360$ & $19,016,116$ & $1,555,890$ & $1,038,757$ & $4,210,009$ \\
\hline Qther ${ }^{2}$............................. & - & 115.900.285 & $18,558,958$ & $17,843,605$ & - & - & $7,3199,357$ \\
\hline \multicolumn{8}{|l|}{ Fodent } \\
\hline Sales (thookend twh & - & - & - & - & - & - & - \\
\hline 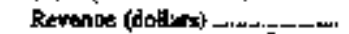 & - & - & - & - & - & - & - \\
\hline Demend Gevget Ody & - & - & - & ـ & - & - & - \\
\hline 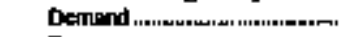 & - & - & - & - & - & - & - \\
\hline Enerfy & - & - & - & - & - & - & - \\
\hline OAter & - & - & - & 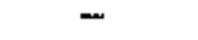 & - & - & - \\
\hline \multicolumn{8}{|l|}{ Strte and Other } \\
\hline Dowernsatin & & & & & & & \\
\hline 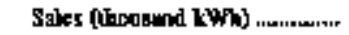 & - & - & 54,334 & - & - & - & - \\
\hline 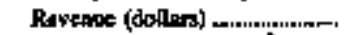 & - & $=$ & 1,2659965 & $\neg$ & - & - & - \\
\hline Denond Carge Orbly:....... & - & - & - & - & - & - & - \\
\hline Densand .......................... & - & - & - & - & - & - & - \\
\hline Entroy & - & - & $1,121.768$ & - & $m$ & - & - \\
\hline 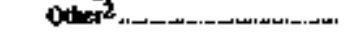 & - & - & 92,138 & - & - & 一 & - \\
\hline \multicolumn{8}{|l|}{ Mmiction } \\
\hline 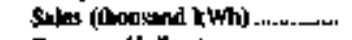 & - & - & 119.223 & - & 29nsts & - & $133+1027$ \\
\hline 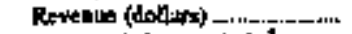 & - & - & $7,563,6 m$ & - & $10,262,057$ & $-23,6] 6$ & $7,365,107$ \\
\hline Deankind Chawe only $1 . . . . . .$. & - & - & - & - & - & - & - \\
\hline 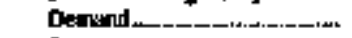 & - & - & $1.954,875$ & - & - & - & $1,200,4,0$ \\
\hline 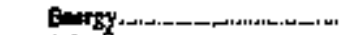 & - & - & $5,109,047$ & - & $6,49,176$ & - & $4, m, 610$ \\
\hline 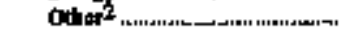 & - & - & 498,455 & - & $4,267,911$ & -21616 & $1.29 \mathrm{~d}, 167$ \\
\hline \multicolumn{8}{|l|}{ Cooperolitye } \\
\hline 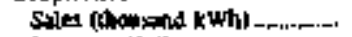 & - & - & 559,258 & - & - & - & - \\
\hline Revervoe (dallersy) . & - & - & 47.161 .277 & - & - & - & - \\
\hline 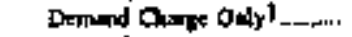 & 一 & 一 & - & - & - & - & - \\
\hline 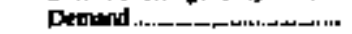 & - & - & $16,848,617$ & - & - & - & - \\
\hline EAtry & - & - & $20,451,281$ & - & - & $=$ & - \\
\hline Other & - & - & $1,464,379$ & - & - & - & - \\
\hline \multicolumn{8}{|l|}{ Other } \\
\hline 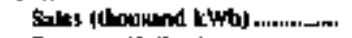 & - & - & $2,323,905$ & c.75s & $1,521,2008$ & 787,750 & $1,118,953$ \\
\hline 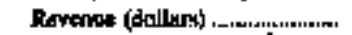 & - & - & $45,930,327$ & 479,502 & 201052522 & $17,643,6077$ & 23.525.725 \\
\hline Demund Chrof Only' ......... & - & - & - & - & - & - & 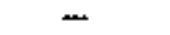 \\
\hline 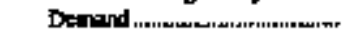 & - & - & - & - & - & $9,778, \pi 06$ & - \\
\hline 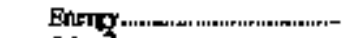 & - & - & $45,787,687$ & 970,512 & $41,219,105$ & $13.864,931$ & 19,97B, \\
\hline Oher ${ }^{2}$ & - & - & 142,631 & - & $3,3,417$ & - & $3,647,097$ \\
\hline \multicolumn{8}{|l|}{ Tot:d } \\
\hline Sales (thpusand kWh) , & - & $2,219,370$ & \$, 585 56 & {$[, 001,211$} & $1.849,221$ & 821,396 & $1,459,415$ \\
\hline 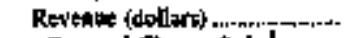 & - & $143,043,170$ & 148.346 .581 & m,902990 & $54,3 \times 0,4] 1$ & 18,763,318 & $42,704,631$ \\
\hline Demand Gepe odyl & - & - & - & - & - & - & - \\
\hline 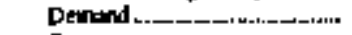 & - & - & $18,869,575$ & $33,063,737$ & - & 1887.246 & 1514,963 \\
\hline Energy-----....---_-_en & - & $0.142,885$ & II8,, 19.94 & $19,995,61$ \& & $4,269,083$ & $14,500,668$ & $29,059,267$ \\
\hline ollogot & - & $135.900,285$ & $20,757,060$ & $17,843,635$ & K161328 & $-23,616$ & $12,130,581$ \\
\hline
\end{tabular}

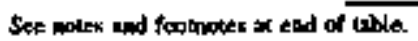


Table 20. Electrictity Sales for Resale by Investoc-Owned Uttitles, by State, 1994 (Conthued)

\begin{tabular}{|c|c|c|c|c|c|c|c|}
\hline 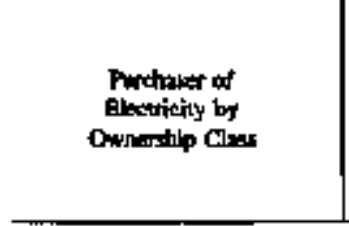 & 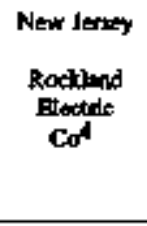 & $\begin{array}{c}\text { New Mexica } \\
\text { Pablle } \\
\text { Senite } \\
\text { Co of NMA }\end{array}$ & $\begin{array}{l}\text { New Merico } \\
\text { Teras- } \\
\text { New Mexico } \\
\text { Power } \\
\text { Có }\end{array}$ & 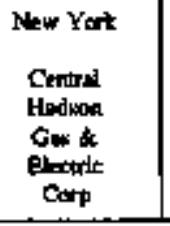 & $\begin{array}{l}\text { Now Yort } \\
\text { Consoliduted } \\
\text { Edingn } \\
\text { Co-NY } \\
\text { bet }\end{array}$ & 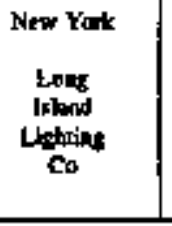 & $\begin{array}{c}\text { New Yod } \\
\text { Inomg } \\
\text { Inthe }\end{array}$ \\
\hline 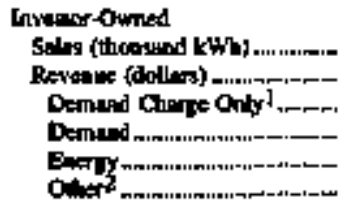 & $\begin{array}{l}- \\
z \\
z \\
-\end{array}$ & $\begin{array}{l}2,119,8391 \\
72,158,574 \\
\overline{31,999,313} \\
36,410,204 \\
908,997\end{array}$ & $\begin{array}{l}z \\
z \\
z \\
-\end{array}$ & $\begin{array}{r}73,140 \\
2,374,624 \\
- \\
335,120 \\
2,039,104 \\
-\end{array}$ & $\begin{array}{l}1,004,351 \\
23,174,076 \\
- \\
= \\
- \\
23,174,075\end{array}$ & $\begin{array}{r}1,600 \\
-\quad 4,600 \\
- \\
-\end{array}$ & $\begin{array}{l}- \\
- \\
- \\
-\end{array}$ \\
\hline 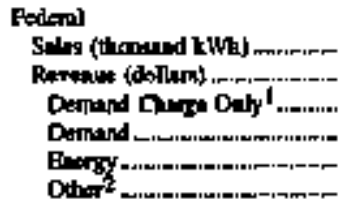 & $\begin{array}{l}\bar{z} \\
\bar{z} \\
=\end{array}$ & $\begin{array}{l}17 \pi, 241 \\
1,36 R, 297 \\
- \\
-1,768,297 \\
-\end{array}$ & $\begin{array}{l}\bar{z} \\
\bar{z} \\
\bar{z}\end{array}$ & $\begin{array}{l}- \\
- \\
\overline{-} \\
-\end{array}$ & $\begin{array}{l}- \\
- \\
- \\
-\end{array}$ & $\begin{array}{l}- \\
- \\
- \\
-\end{array}$ & $\begin{array}{l}- \\
- \\
- \\
-\end{array}$ \\
\hline 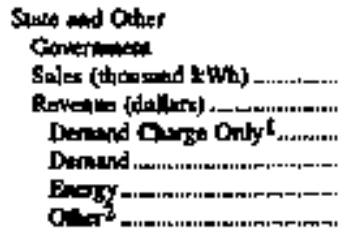 & $\begin{array}{l}= \\
= \\
= \\
=\end{array}$ & $\begin{array}{r}274,885 \\
12,324,718 \\
\overline{7567,010} \\
4,757,765 \\
119\end{array}$ & $\begin{array}{l}- \\
= \\
z \\
=\end{array}$ & $\begin{array}{l}-\begin{array}{r}4,386 \\
4,386\end{array} \\
- \\
-\end{array}$ & $\begin{array}{l}2,098,370 \\
- \\
= \\
2,098,370\end{array}$ & $\begin{array}{l}= \\
= \\
- \\
-\end{array}$ & $\begin{array}{l}\bar{z} \\
\bar{z} \\
\overline{-}\end{array}$ \\
\hline 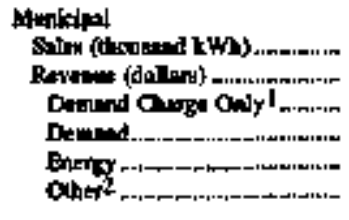 & $\begin{array}{l}\bar{z} \\
\overline{-} \\
\overline{-}\end{array}$ & $\begin{array}{r}99.143 \\
2.180 .174 \\
- \\
560,1001 \\
1,521,593 \\
96,511\end{array}$ & $\begin{array}{l}z \\
\bar{z} \\
-\end{array}$ & $\begin{array}{l}- \\
z \\
z \\
-\end{array}$ & $\begin{array}{l}- \\
z \\
- \\
-\end{array}$ & $\begin{array}{l}- \\
- \\
- \\
-\end{array}$ & $\begin{array}{l}- \\
\overline{-} \\
- \\
-\end{array}$ \\
\hline 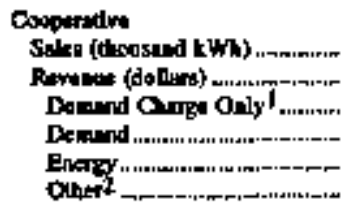 & $\begin{array}{l}\bar{z} \\
\bar{z} \\
\bar{z}\end{array}$ & $\begin{array}{r}192,435 \\
7,178,411 \\
\overline{3,319,9001} \\
3,696,625 \\
161,886\end{array}$ & $\begin{array}{l}\bar{z} \\
\bar{z} \\
\bar{z}\end{array}$ & $\begin{array}{l}- \\
- \\
- \\
-\end{array}$ & $\begin{array}{l}- \\
z \\
z \\
-\end{array}$ & $\begin{array}{l}\bar{z} \\
\bar{z} \\
\overline{-}\end{array}$ & $\begin{array}{l}- \\
\overline{-} \\
= \\
=\end{array}$ \\
\hline 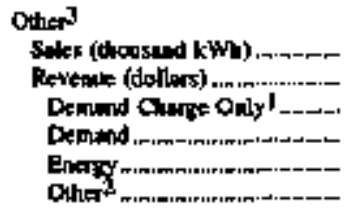 & $\begin{array}{l}= \\
= \\
=\end{array}$ & $\begin{array}{r}508,759 \\
-789,648 \\
-7,111,643 \\
14,111,511 \\
9,134,511 \\
-2,035,792\end{array}$ & $\begin{array}{l}= \\
= \\
=\end{array}$ & $\begin{array}{l}399,700 \\
10,699,961 \\
- \\
- \\
19,659,961 \\
-\end{array}$ & $\begin{array}{l}\text { 7e0,072 } \\
2,965.415 \\
= \\
= \\
= \\
20.925,415\end{array}$ & $\begin{array}{l}79,493 \\
14,84,716 \\
= \\
\overline{14,84,710} \\
=\end{array}$ & $\begin{array}{l}- \\
= \\
= \\
=\end{array}$ \\
\hline 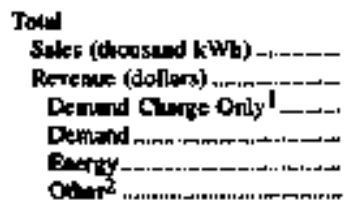 & $\begin{array}{l}- \\
- \\
- \\
-\end{array}$ & $\begin{array}{r}3,361,933 \\
96,820,516 \\
- \\
60,397,896 \\
59,290,615 \\
-22,861,975\end{array}$ & $\begin{array}{l}= \\
\overline{-} \\
\bar{z}\end{array}$ & 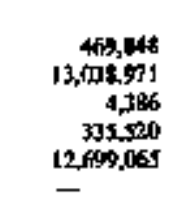 & $\begin{array}{l}1.784,773 \\
4,057,851 \\
- \\
- \\
- \\
49,057,851\end{array}$ & $\begin{array}{l}372,48] \\
14, B 24518 \\
- \\
\overline{14,894518} \\
-\end{array}$ & $\begin{array}{l}\bar{z} \\
\bar{z} \\
\bar{z}\end{array}$ \\
\hline
\end{tabular}

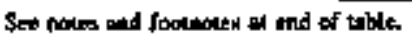


Table 26. Deedricily Sales for Resale by Intestor-Owaed Utiltites, by State, 1994 (Continued)

\begin{tabular}{|c|c|c|c|c|c|c|c|}
\hline 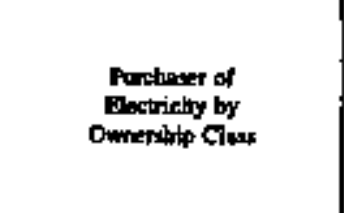 & 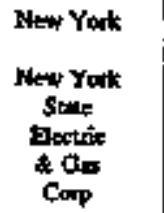 & 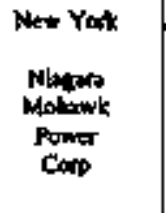 & $\begin{array}{l}\text { Ner Yoth } \\
\text { Oratite } \\
\text { Roddind } \\
\text { Inc }\end{array}$ & $\begin{array}{l}\text { Neq Yok } \\
\text { Rochenter } \\
\text { Con k } \\
\text { Eloctis } \\
\text { Cap }\end{array}$ & 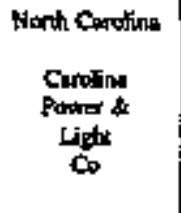 & 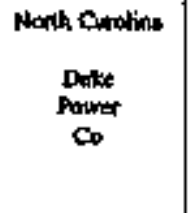 & 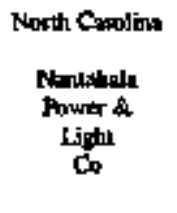 \\
\hline Invation-Ow & & & & & & & \\
\hline 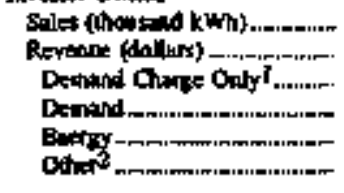 & $\begin{array}{r}3,52,940 \\
70,986,184 \\
-\quad 2,521 \\
70,95 \times, 663 \\
-\end{array}$ & $\begin{array}{l}2,8 \times 92,952 \\
65,857,180 \\
+ \\
2,978,453 \\
62,918,655 \\
=\end{array}$ & $\begin{array}{r}1,413,260 \\
68,494,040 \\
\overrightarrow{22,558,377} \\
16,055,769 \\
52,929\end{array}$ & $\begin{array}{r}69,276 \\
1,497,044 \\
- \\
- \\
-\end{array}$ & $\begin{array}{r}469,513 \\
19,132,635 \\
- \\
5,689,205 \\
15,449,430 \\
-\end{array}$ & $\begin{array}{l}2,73,0,01 \\
129,227,180 \\
- \\
60836,265 \\
69,104,927 \\
=714,012\end{array}$ & $\begin{array}{r}\quad 2,617 \\
12, \sqrt{31,255} \\
\overrightarrow{1}+, 405,520 \\
-725,735\end{array}$ \\
\hline EMandend & & & & & & & \\
\hline 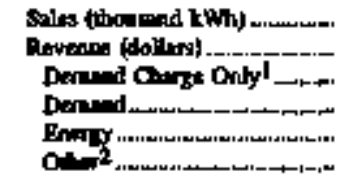 & $\begin{array}{l}- \\
- \\
- \\
-\end{array}$ & $\begin{array}{l}- \\
- \\
- \\
-\end{array}$ & $\begin{array}{l}- \\
- \\
- \\
-\end{array}$ & $\begin{array}{l}- \\
- \\
- \\
-\end{array}$ & $\begin{array}{r}90,136 \\
1204,984 \\
100,000 \\
-1,14,984 \\
-\end{array}$ & $\begin{array}{l}- \\
- \\
- \\
-\end{array}$ & 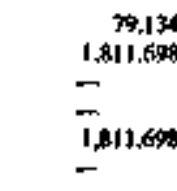 \\
\hline 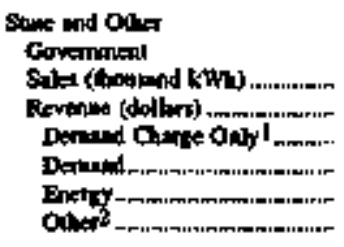 & $\begin{array}{l}574,986 \\
10,546,715 \\
- \\
- \\
10,546,115 \\
-\end{array}$ & $\begin{array}{r}21,9,922 \\
4,163,444 \\
- \\
138,134 \\
4,12910 \\
-\end{array}$ & $\begin{array}{r}55 \% \\
1,800 \\
-\quad 1,800 \\
-\quad\end{array}$ & $\begin{array}{l}133,006 \\
2,478,306 \\
- \\
- \\
-\end{array}$ & $\begin{array}{r}40,930 \\
-\quad 996,145 \\
- \\
-\quad 096,145\end{array}$ & $\begin{array}{r}52,557 \\
1,130,343 \\
-\quad 90,000 \\
1,037,343 \\
-\end{array}$ & $\begin{array}{l}- \\
- \\
- \\
-\end{array}$ \\
\hline 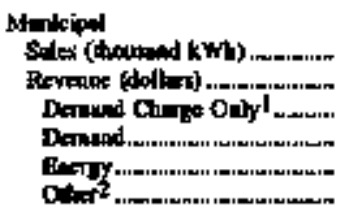 & $\begin{array}{r}17,238 \\
596,382 \\
- \\
-59,382\end{array}$ & $\begin{array}{r}13,036 \\
375,729 \\
-\quad 136,941 \\
238,948\end{array}$ & $\begin{array}{l}- \\
- \\
- \\
-\end{array}$ & $\begin{array}{l}- \\
- \\
- \\
-\end{array}$ & $\begin{array}{r}4,651,087 \\
203,341,644 \\
- \\
57,356,748 \\
145,672,392 \\
2,312,644\end{array}$ & $\begin{array}{r}4,276,65] \\
218,298,822 \\
- \\
130,329,192 \\
92,936,110 \\
-4,946,490\end{array}$ & $\begin{array}{r}30,855 \\
046,960 \\
-\quad \\
640,489 \\
290,274 \\
16,0 \% 9\end{array}$ \\
\hline 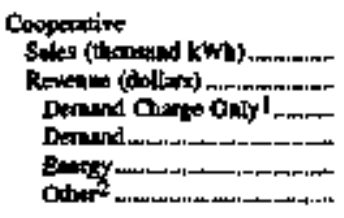 & $\begin{array}{l}-100,0000 \\
- \\
- \\
-\end{array}$ & $\begin{array}{l}- \\
- \\
- \\
-\end{array}$ & $\begin{array}{l}- \\
- \\
- \\
-\end{array}$ & $\begin{array}{l}- \\
- \\
- \\
-\end{array}$ & $\begin{array}{l}5,257,179 \\
26,426,566 \\
- \\
- \\
295,225,591 \\
-2,35,525\end{array}$ & $\begin{array}{l}2,584,786 \\
160,594,792 \\
- \\
128,364,673 \\
3,24,119 \\
=\end{array}$ & $\begin{array}{r}19,065 \\
601,439 \\
-\quad \\
114,704 \\
17,786 \\
13,149\end{array}$ \\
\hline 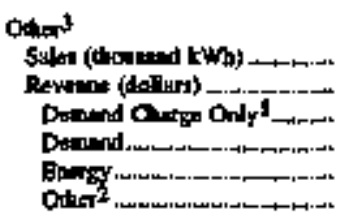 & $\begin{array}{l}2,716,492 \\
59,672537 \\
- \\
59,672,537 \\
-\end{array}$ & $\begin{array}{l}4,415,601 \\
87,399,211 \\
- \\
2,103,140 \\
-\end{array}$ & $\begin{array}{l}225,736 \\
5,927,648 \\
- \\
\overline{5018,834} \\
100,814\end{array}$ & 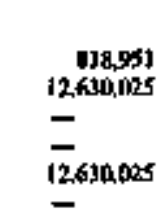 & $\begin{array}{l}-47,732 \\
-10,282,527 \\
- \\
- \\
-5,266,260 \\
-1,014,267\end{array}$ & $\begin{array}{l}309,561 \\
5,925,152 \\
- \\
\overrightarrow{11,227,090} \\
-5,401,939\end{array}$ & $\begin{array}{r}24,513 \\
789511 \\
- \\
525,214 \\
21,069 \\
2127\end{array}$ \\
\hline 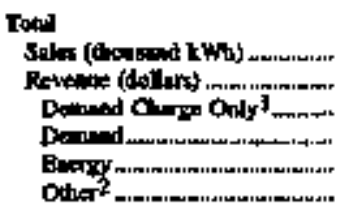 & $\begin{array}{r}6,827,656 \\
141,901,518 \\
- \\
299,527 \\
141,572,297 \\
-\end{array}$ & $\begin{array}{l}7,441,511 \\
157,875,544 \\
- \\
1,256,596 \\
352,548,098 \\
-\end{array}$ & $\begin{array}{r}1,642,592 \\
74,62,517 \\
\overrightarrow{20} \\
2,514,317 \\
51,876,403 \\
161,797\end{array}$ & $\begin{array}{l}1,021,733 \\
16,006,375 \\
- \\
\overline{16,601,375} \\
-\end{array}$ & $\begin{array}{r}10,442,113 \\
500,559,587 \\
902,000 \\
61,009,955 \\
448,922,188 \\
-11,502,548\end{array}$ & $\begin{array}{r}9,955,558 \\
515,172,499 \\
= \\
319,025,130 \\
206,629,789 \\
-11,082,430\end{array}$ & $\begin{array}{r}136,554 \\
16,280,764 \\
- \\
12,906,927 \\
3,235362 \\
56,475\end{array}$ \\
\hline
\end{tabular}

Sed wout and fostanci an end of trible 
Table 20. Electricity Sales for Resale by Investor Owned Utlitties, by State, 1994 (Continued)

\begin{tabular}{|c|c|c|c|c|c|c|c|}
\hline 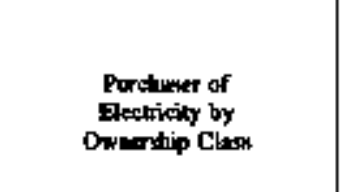 & $\begin{array}{l}\text { Noth Curotian } \\
\text { Youkin } \\
\text { Inos }\end{array}$ & 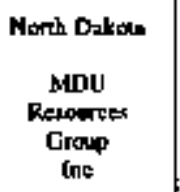 & $\begin{array}{c}\text { Otid } \\
\text { AEs } \\
\text { Cengating } \\
\text { Cos }\end{array}$ & 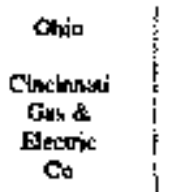 & 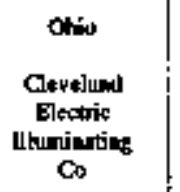 & $\begin{array}{l}\text { Ohid } \\
\text { Cohornbos } \\
\text { Sondhem } \\
\text { Power Co }\end{array}$ & $\begin{array}{l}\text { Otion } \\
\text { Durson } \\
\text { Power } 4 \\
\text { Llelu } \\
\text { Co }\end{array}$ \\
\hline \multicolumn{8}{|l|}{ 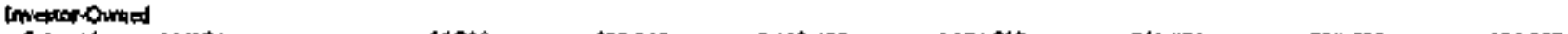 } \\
\hline Soles (thensond tWh) ...........rin & 55.790 & 221.340 & $9.119,496$ & $4,174,718$ & 36] I I I I I & 734,555 & 296,087 \\
\hline 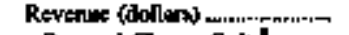 & $1,0 \$ 5,7645$ & $3,973,809$ & $235,973,742$ & $158,907,450)$ & $4,157.307$ & $31,742,506$ & $7,100,872$ \\
\hline 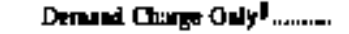 & - & - & - & - & - & $1,305,676$ & -- \\
\hline 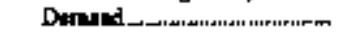 & - & - & $47,414,398$ & $69,29,872$ & $163,, 102$ & $6,754,047$ & B41,659 \\
\hline Finrsgy & $1,055.765$ & $3,971.800$ & $180,559,404$ & $109,761,672$ & $6,137 . \Omega$ an & 23.883 .389 & $(0,261,0] 1$ \\
\hline Oher & $m$ & - & - & $-8.151,090$ & $-2,143,165$ & - & \\
\hline \multicolumn{8}{|l|}{ Peideral } \\
\hline Sules (thothod KWh) & - & 119,855 & - & - & - & B,205 & - \\
\hline 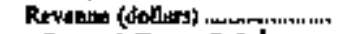 & - & $1,619,334$ & - & - & - & 248,604 & - \\
\hline Demapd Chage Onlyl......... & - & - & - & - & - & - & -- \\
\hline Detend & - & - & - & - & - & - & - \\
\hline 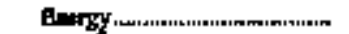 & - & $1,615.374$ & 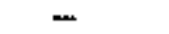 & - & - & 249,604 & - \\
\hline Other & - & - & - & - & - & 一 & - \\
\hline \multicolumn{8}{|l|}{ 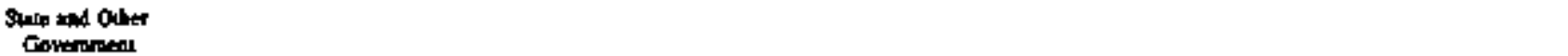 } \\
\hline 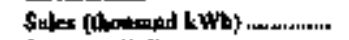 & - & 24,2 & - & - & - & - & - \\
\hline Revene (dol|rs) & - & 43,675 & - & - & - & - & - \\
\hline Demisod Corge 0dy 1 ........ & - & - & - & - & - & - & -- \\
\hline 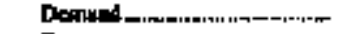 & - & - & - & - & - & - & -- \\
\hline Energy. & - & $42,3,675$ & - & - & - & - & -- \\
\hline Dators & - & - & - & - & - & - & $r$ \\
\hline \multicolumn{8}{|l|}{ Mariciod } \\
\hline 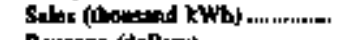 & - & 55,373 & - & 417,424 & 112416 & (19B,BA9 & $6 \times 8,757$ \\
\hline 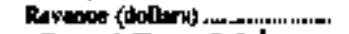 & - & B77, I60 & - & 12,657, 1913 & $4,449,6,35$ & $22,041.476$ & $17,170,733$ \\
\hline Denroed Choge Dolyl ......riner & - & - & - & - & - & - & - \\
\hline Detrond....nm & - & - & - & $3,6,61,554$ & $621,0.84$ & $6,905,992$ & $6,318,422$ \\
\hline 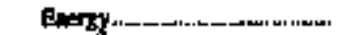 & - & B7T, I60 & - & \$, 996,243 & $.1 .916,0187$ & ] $5,[35,484$ & $12,300,554$ \\
\hline Other & - & - & - & Snits & $-5,516$ & - & -1.457 .643 \\
\hline \multicolumn{8}{|l|}{ Coophrative } \\
\hline 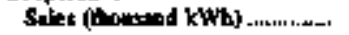 & - & 25,032 & - & 90.176 & - & $46 \%$ & - \\
\hline 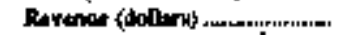 & - & 280.251 & - & $1,564,1960$ & س & 20.879 & -- \\
\hline Denuyed Ohroge Onlyl .......... & - & - & - & - & - & - & - \\
\hline 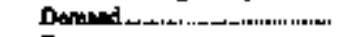 & - & - & - & - & - & - & - \\
\hline 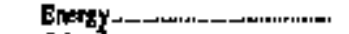 & - & 190,251 & - & $1,198,6376$ & - & 23,879 & -- \\
\hline 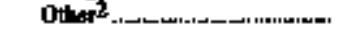 & 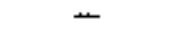 & - & - & 365,554 & - & - & - \\
\hline \multicolumn{8}{|l|}{ other ${ }^{3}$} \\
\hline 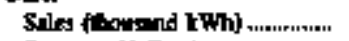 & - & مـ & - & -39 & 999.460 & I.139.39d & -- \\
\hline 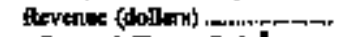 & - & - & - & $-4,692$ & $11,291,750$ & $24,361,455$ & -- \\
\hline Danud Charpe Oaly" & - & - & - & - & - & - & $-r$ \\
\hline Donund-, & - & - & - & - & - & $3,18], 852$ & -- \\
\hline 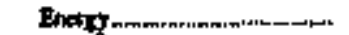 & - & - & - & - & {$[1,3355,016$} & $21,581,603$ & - \\
\hline Other & - & - & - & $-4,692$ & $-44,266$ & - & - \\
\hline \multicolumn{8}{|l|}{ Tot } \\
\hline 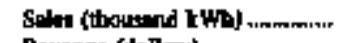 & 55.790 & 44,492 & 9.119 .496 & 4,fita, 25y & $1,672,947$ & $2,779,111$ & 164.844 \\
\hline 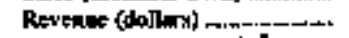 & $1,1165,766$ & $7,270,229$ & $205,973,742$ & $173,124,761$ & $19,997,692$ & $78,839,920$ & $24,273,2,69$ \\
\hline Demand Charte Oaly' __- & - & - & - & - & - & $1,100,0770$ & - \\
\hline Denand & & & $47,414,338$ & $6 x, 698,436$ & 784476 & ] $6,861, \mathrm{BSI}$ & $\tau, 160.081$ \\
\hline 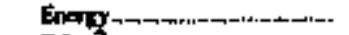 & 1,105,765 & $7,270,29$ & 186,559,4149 & $113,966,511$ & $21,358,183$ & 611,872953 & $1 \mathrm{H}=5 \mathrm{~T}, 565$ \\
\hline Ding $\mathbf{G}^{2}$ & - & - & - & $\rightarrow 7,790,176$ & -2274967 & - & $=1,457,461$ \\
\hline
\end{tabular}

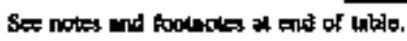


Table 20. Electricity Sales for Resale by Invesior-Owned Utilitles, by State, 1994 (Comlinwed)

\begin{tabular}{|c|c|c|c|c|c|c|c|}
\hline $\begin{array}{l}\text { Purchner of } \\
\text { Eloctricity by } \\
\text { Ownership Cluss }\end{array}$ & $\begin{array}{l}\text { Otio } \\
\text { folliping } \\
\text { Kentendy } \\
\text { Eloctini } \\
\text { Conps }\end{array}$ & $\begin{array}{c}\text { Ohio } \\
\text { Ohlo } \\
\text { Eursom } \\
\text { Co }\end{array}$ & $\begin{array}{c}\text { Otio } \\
\text { Oado } \\
\text { Porwer } \\
\text { Co }\end{array}$ & $\begin{array}{l}\text { Ohío } \\
\text { Otio } \\
\text { Vylley } \\
\text { Elatio } \\
\text { Con }\end{array}$ & $\begin{array}{c}\text { OAlib } \\
\text { Toleso } \\
\text { Editon } \\
\text { Co }\end{array}$ & 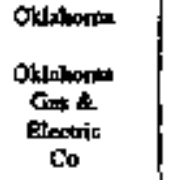 & 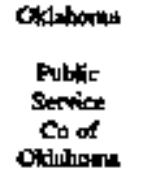 \\
\hline \multicolumn{8}{|l|}{ Imesust-Owined } \\
\hline 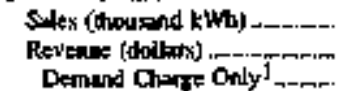 & $\begin{array}{l}9,945,739 \\
131,1111,221 \\
-\end{array}$ & $\begin{array}{r}5,653,442 \\
196,969,468 \\
-\end{array}$ & $\begin{array}{r}3,604,390 \\
125,739,164 \\
1,898,167\end{array}$ & $\begin{array}{l}1,732,354 \\
23,167,677 \\
-\end{array}$ & $\begin{array}{r}1,74,299 \\
115,811,029 \\
-\end{array}$ & $\begin{array}{r}\$ 28,702 \\
8,517,805 \\
-\end{array}$ & $\begin{array}{r}\text { 130,54010 } \\
4,729,616 \\
-\end{array}$ \\
\hline 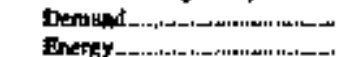 & $\begin{array}{l}31,830,915 \\
91,279,306\end{array}$ & $\begin{array}{l}96,394,114 \\
97,999,633\end{array}$ & $\begin{array}{l}30,694,249 \\
92,055,652\end{array}$ & $\begin{array}{r}3,370,604 \\
19.797,063\end{array}$ & $\begin{array}{r}227,363 \\
115,372,578\end{array}$ & ssitgens & $\begin{array}{l}1,117,417 \\
3,520,038\end{array}$ \\
\hline 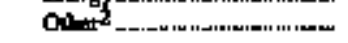 & - & $2,6 \mathrm{AS}, \mathrm{nI}$ & - & - & It 0888 & - & 92,161 \\
\hline \multicolumn{8}{|l|}{ Federpl } \\
\hline Soles (thoouand kWh) & - & - & 15,230 & - & - & 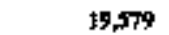 & 56,044 \\
\hline 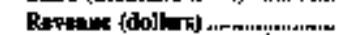 & - & - & $\$ 3.620$ & - & - & 597,120 & 2770893 \\
\hline 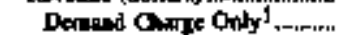 & - & - & - & - & - & - & - \\
\hline Denuad.......ririm & - & - & - & - & - & 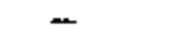 & $1,117.747$ \\
\hline Bow & $\longrightarrow$ & - & 424,623 & - & - & 284,337 & $15 x, 443$ \\
\hline 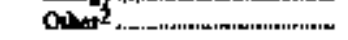 & - & - & - & - & - & -197217 & 127,700 \\
\hline \multicolumn{8}{|l|}{ Stake and OUher } \\
\hline 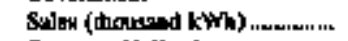 & - & - & - & - & - & 296,284 & 50,052 \\
\hline Revenos (dollans) ...,............... & - & - & - & - & - & $9,414,9,5$ & $5,326,192$ \\
\hline 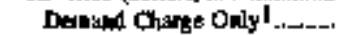 & - & - & - & - & - & - & - \\
\hline 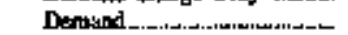 & - & - & $=$ & - & - & $3,045,363$ & 390,0010 \\
\hline Enetry & - & $\pi$ & $\rightarrow$ & - & $m$ & 2004,763 & $1,123,257$ \\
\hline 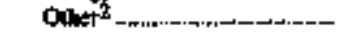 & - & - & - & - & - & $-2, \sin , 241$ & $3,804,825$ \\
\hline \multicolumn{8}{|l|}{ Manicipul } \\
\hline Sales f(thonand kWh\} & - & $1 \mathbf{M}, \mathbf{1 3 9}$ & $1,199,868$ & - & 652,142 & 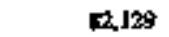 & 35,250 \\
\hline Revenne (dolltrts) .................. & - & $6,406,454$ & $17,37,520$ & - & $20,229,274$ & 2,SER,9II & $1,143.875$ \\
\hline 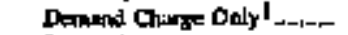 & - & - & - & $m$ & - & $\rightarrow$ & - \\
\hline 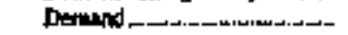 & - & $2,611,370$ & $\{3,213,460 \mathrm{~J}$ & - & $12,705,280$ & $1,292,22 \mathrm{~J}$ & 78,249 \\
\hline Eurgy & - & $3,+416,748$ & $24,150,857$ & - & 16,512,407 & $2,463,102$ & $1,007,626$ \\
\hline Other & - & 146,376 & $\rightarrow$ & - & 17,497 & $-17,412$ & - \\
\hline \multicolumn{8}{|l|}{ Coopentuive } \\
\hline Salex (thourned kW) & - & - & 615,035 & - & 5,050 & 690,603 & 1,629 \\
\hline 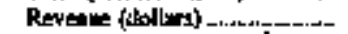 & - & - & $12,147, \mathrm{Bg}$ & - & 207,876 & $2 \sqrt{2}, 794,864$ & 6. 726 \\
\hline 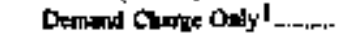 & - & - & $\rightarrow$ & - & 一 & - & - \\
\hline Dem=md & - & - & - & - & $\mathbf{I O F , 4 9 I}$ & $8,787,523$ & 0,137 \\
\hline E & - & - & $12,147,899$ & - & 100,785 & IB,BI7,339 & torits \\
\hline 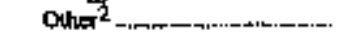 & - & - & - & - & - & $-6, \sin 9,598$ & - \\
\hline \multicolumn{8}{|l|}{ Other ${ }^{3}$} \\
\hline Soles (thonkapd kWh) & - & 92.586 & $8,059,971$ & - & 176,731 & 33,700 & $1,225,361$ \\
\hline Rereave (dothrs) & - & 194.7\% & $276,457,272$ & - & 3017256 & 005,262 & $21,425,587$ \\
\hline Dermend Churge Only lat-_-_ & - & - & - & - & - & - & - \\
\hline 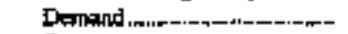 & - & 21,1519 & $5,434,894$ & - & 410,767 & - & - \\
\hline 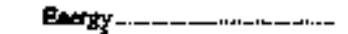 & - & 473,497 & $271,022,37 \mathrm{~g}$ & - & $2,661,076$ & $805,2 \times 2$ & $2 J, 295,321$ \\
\hline Other & - & - & - & - & -54597 & - & 130266 \\
\hline \multicolumn{8}{|l|}{ T매제 } \\
\hline Svlex (thonaugd kWh) & $9,045.7,79$ & $5,870,207$ & $13,484,712$ & $1,352,354$ & $2,540,220$ & $1,489,997$ & 1,508,908 \\
\hline 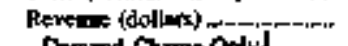 & J 1,110221 & $203, \mathrm{Bg}$, 712 & $452.146,478$ & $23,167,677$ & $142,261,439$ & $43,000,947$ & $35,457,849$ \\
\hline Demmend Crope Oalyl, & - & - & $1.849,167$ & & & & \\
\hline Dentand & $37,830,915$ & 99,156:1,13? & $\Rightarrow 5+6,902$ & $3,370,614$ & $13,450,9019$ & $13,125,107$ & 2,712550 \\
\hline 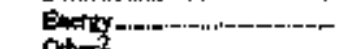 & $93,279,306$ & $102,001,818$ & $400,710,409$ & $19,797,060]$ & $128,53,5,5360$ & $39,961,608$ & $20,590,134$ \\
\hline 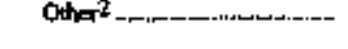 & & $2,792,067$ & & - & -264002 & $-10,008,768$ & \\
\hline
\end{tabular}

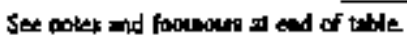


Table 20. Electrictify Sales for Resale by Investor-0wned Utilities, by State, 1994 (Conlimued)

\begin{tabular}{|c|c|c|c|c|c|c|c|}
\hline 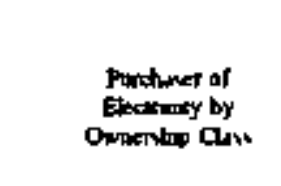 & $\begin{array}{l}\text { Oneon } \\
\text { Punficort }\end{array}$ & 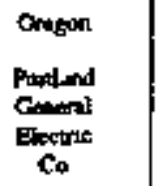 & 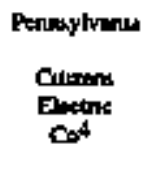 & 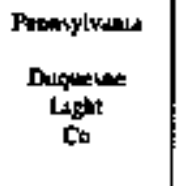 & 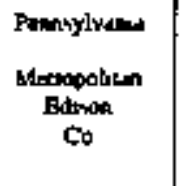 & 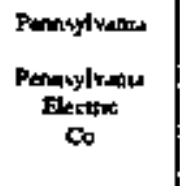 & 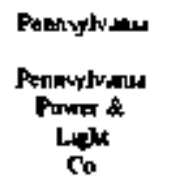 \\
\hline 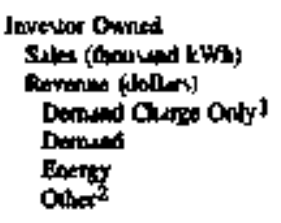 & $\begin{array}{r}10,675,127 \\
362,177,066 \\
75 \\
153,571,434 \\
206,951,49 \\
1,655,183\end{array}$ & $\begin{array}{r}468,338 \\
26,767,289 \\
- \\
16,901,0019 \\
10,233,5419 \\
-36,6,231\end{array}$ & $\begin{array}{l}- \\
= \\
= \\
=\end{array}$ & $\begin{array}{l}3,211,962 \\
56,281,505 \\
- \\
15,616,901 \\
42,634,6014 \\
-\end{array}$ & $\begin{array}{r}5,342 \\
572,613 \\
-\quad 0,274 \\
521,494 \\
1,849\end{array}$ & $\begin{array}{r}132,363 \\
5,796,064 \\
- \\
2,144,684 \\
2,8919,585 \\
841,799\end{array}$ & $\begin{array}{r}6,663,805 \\
323,268,006 \\
- \\
2,110,100 \\
91, B 54,529 \\
229,310,135\end{array}$ \\
\hline 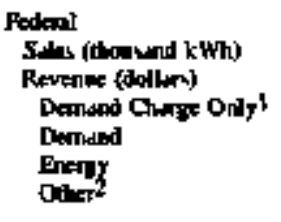 & $\begin{array}{l}2,85 \%, 154 \\
87,872,527 \\
= \\
- \\
=\end{array}$ & 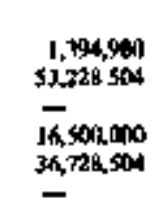 & $\begin{array}{l}z \\
- \\
z \\
=\end{array}$ & $\begin{array}{l}\overline{-} \\
\overline{-} \\
\overline{-}\end{array}$ & $\begin{array}{l}z \\
= \\
= \\
=\end{array}$ & $\begin{array}{l}\overline{-} \\
= \\
= \\
=\end{array}$ & $\begin{array}{l}\bar{z} \\
\bar{z} \\
\bar{z}\end{array}$ \\
\hline 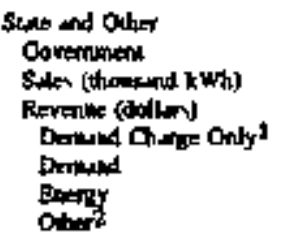 & $\begin{array}{r}1.115,6166 \\
35,709,662 \\
7,240 \\
13,043,159 \\
18,500,391 \\
1501,866\end{array}$ & $\begin{array}{r}321,608 \\
11,772,724 \\
- \\
11,6109, \pi 00 \\
-\end{array}$ & $\begin{array}{l}- \\
z \\
z \\
-\end{array}$ & $\begin{array}{l}- \\
- \\
- \\
-\end{array}$ & $\begin{array}{l}- \\
- \\
- \\
-\end{array}$ & $\begin{array}{l}- \\
\overline{-} \\
\bar{z} \\
-\end{array}$ & $\begin{array}{l}124161 \\
2816206 \\
- \\
- \\
2,816206 \\
=\end{array}$ \\
\hline 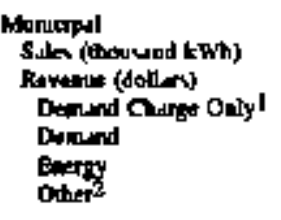 & $\begin{array}{r}361,316 \\
38,620,977 \\
305,700 \\
22,694,454 \\
16,539,011 \\
-717,766\end{array}$ & $\begin{array}{r}99,975 \\
2,129,998 \\
- \\
5,7210,000 \\
2,09,998 \\
-\end{array}$ & $\begin{array}{l}\overline{-} \\
\bar{z} \\
=\end{array}$ & $\begin{array}{r}12.356 \\
846,501 \\
-\quad \\
454,009 \\
372,680 \\
19,799\end{array}$ & $\begin{array}{r}94,404 \\
141,551 \\
- \\
127,476 \\
1,073,269 \\
45,514\end{array}$ & $\begin{array}{r}349,688 \\
12018.305 \\
- \\
5,049662 \\
6,312,499 \\
256.203\end{array}$ & $\begin{array}{r}744613 \\
15,3890626 \\
- \\
12,351,954 \\
22,726,672 \\
-\end{array}$ \\
\hline 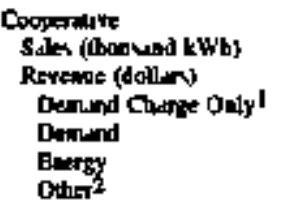 & $\begin{array}{r}56,336 \\
1,493,199 \\
320 \\
36,952 \\
1,455,927 \\
-\quad\end{array}$ & $\begin{array}{l}- \\
- \\
- \\
-\end{array}$ & $\begin{array}{l}\bar{z} \\
\bar{z} \\
\bar{z}\end{array}$ & $\begin{array}{l}\bar{z} \\
\bar{z} \\
\bar{z}\end{array}$ & $\begin{array}{l}= \\
= \\
= \\
=\end{array}$ & 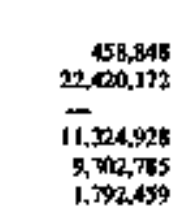 & $\begin{array}{r}19,539 \\
935,215 \\
- \\
333,21 k \\
592,439 \\
-\end{array}$ \\
\hline 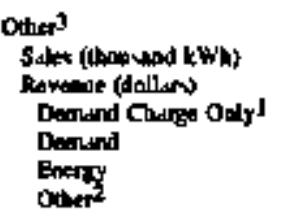 & $\begin{array}{r}159,984 \\
6,579,932 \\
850 \\
3,430,000 \\
3,099,002 \\
-\end{array}$ & $\begin{array}{r}17,779 \\
414,571 \\
-\quad 146,504 \\
267,585 \\
+\quad\end{array}$ & $\begin{array}{l}\bar{z} \\
\bar{z} \\
\bar{z}\end{array}$ & $\begin{array}{r}\quad 148 \\
-\quad 1403 \\
-\quad 14003\end{array}$ & $\begin{array}{r}395,671 \\
11.374744 \\
- \\
211.791 \\
11,162,942 \\
-\end{array}$ & $\begin{array}{l}1,485,347 \\
75,712,391 \\
- \\
2,414,493 \\
26,997,900 \\
-\end{array}$ & 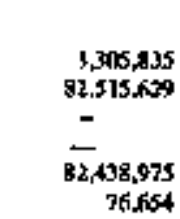 \\
\hline 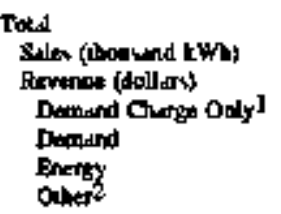 & $\begin{array}{r}15,624,573 \\
532,727,358 \\
313,070 \\
196,832,999 \\
314,499,988 \\
1,089,281\end{array}$ & $\begin{array}{l}2, \pi 0,670 \\
105,911,685 \\
- \\
39,676,688 \\
67,000,651 \\
-7,6,251\end{array}$ & $\begin{array}{l}z \\
= \\
\bar{z}\end{array}$ & $\begin{array}{r}1,224,466 \\
19,1401,016 \\
- \\
15,101,9,90 \\
4,019.297 \\
19,799\end{array}$ & 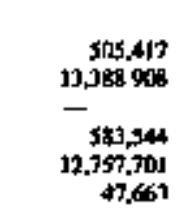 & $\begin{array}{l}2,25,946 \\
75,596,935 \\
- \\
27,2,27,755 \\
45,62,719 \\
2,690,461\end{array}$ & 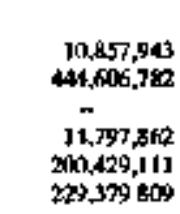 \\
\hline
\end{tabular}

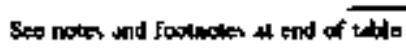


Tahle 20. Electricity Sales for Resale by Inveslor-Owned Utilities, by State, 1994 (Contimaed)

\begin{tabular}{|c|c|c|c|c|c|c|c|}
\hline 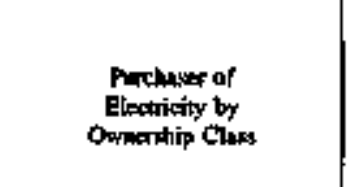 & 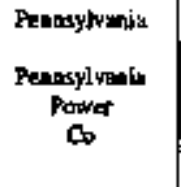 & $\begin{array}{c}\text { Pentisyonia } \\
\text { PECo } \\
\text { Bagky } \\
\text { Co }\end{array}$ & 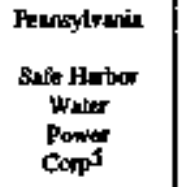 & 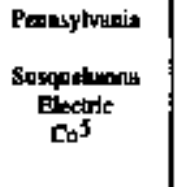 & $\begin{array}{c}\text { Penreylyaris } \\
\text { Wey } \\
\text { Pese } \\
\text { Power } \\
\text { Ca }\end{array}$ & $\begin{array}{c}\text { Penurgylyenix } \\
\text { Yoit } \\
\text { Hruan } \\
\text { Power } \\
\text { Cos }\end{array}$ & 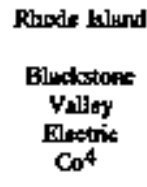 \\
\hline \multicolumn{8}{|l|}{ Invedophomind } \\
\hline Stlex (boulend kWh)........ & 934,698 & $10,50,1,419$ & 1.291800 & $1,954,107$ & $4,990,067$ & 137,566 & - \\
\hline Revenwa (dodlond) & $31,173, \mathrm{BS9}$ & $288,345,229$ & 25.8702800 & Rotions & $161,203,462$ & $6,49,688$ & - \\
\hline Deraind Ghwjo Onlys & - & - & - & - & - & - & - \\
\hline 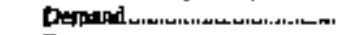 & $15,538,594$ & $35,557,526$ & - & $6,677,579$ & $7,337,044$ & - & - \\
\hline Evergy................................... & IS, 63.35 .304 & $254,431,559$ & - & $2,417,013$ & $n, 325,315$ & $5,349,698$ & - \\
\hline 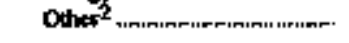 & - & $-1,649,856$ & $2 B, 870,23 n$ & $-321 \mathrm{~d} 182$ & $8,340,703$ & - & - \\
\hline \multicolumn{8}{|l|}{ Feckent } \\
\hline salex (dwousind KWh) & - & - & - & - & - & - & - \\
\hline Revenec (dolbext .................. & - & ـ & - & - & - & - & - \\
\hline 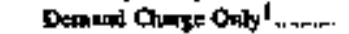 & - & - & - & - & - & - & - \\
\hline 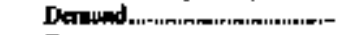 & - & - & - & - & $\rightarrow$ & - & - \\
\hline Eneroy & - & - & - & - & - & - & - \\
\hline 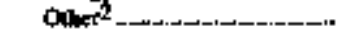 & - & - & - & - & $\rightarrow$ & - & $m$ \\
\hline \\
\hline $\begin{array}{l}\text { Cohmmens } \\
\text { Sales (lhoorind kWh) }\end{array}$ & - & 23,595 & - & - & - & - & - \\
\hline 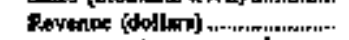 & - & 486,278 & - & - & - & - & - \\
\hline Denand Charg Onlyl....... & - & - & - & - & - & - & - \\
\hline Derond & - & - & - & - & - & - & - \\
\hline 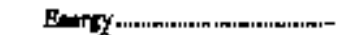 & - & 486,278 & - & - & - & - & - \\
\hline 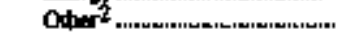 & - & - & - & - & - & - & - \\
\hline \multicolumn{8}{|l|}{ Mnicipol } \\
\hline SHek (thousind $k$ W) & 141,872 & $\rightarrow$ & - & - & 302500 & - & - \\
\hline Revesous (dollurs) & 7ABS,990 & - & - & - & $10,510,300$ & - & - \\
\hline Derpand Oante Ody 1 & - & $=$ & - & - & - & - & - \\
\hline Dersand & $2,929.796$ & - & - & - & $\$, 521$, fils & - & - \\
\hline Eum & $4,556,094$ & - & - & - & 5315,151 & - & - \\
\hline OAthot & - & - & - & - & $\sin 534$ & - & - \\
\hline \multicolumn{8}{|l|}{ Coppentive } \\
\hline Salen (hbounend kWh) ........... & - & 179.453 & - & - & $24], 029$ & - & - \\
\hline Reventu (doll & - & $3,438,007$ & - & - & $9,499,418$ & - & - \\
\hline Danumi Comse Only' & - & - & - & - & - & - & - \\
\hline 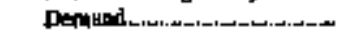 & - & - & - & - & $4,340,119$ & - & - \\
\hline 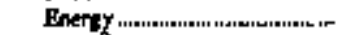 & - & J.43A, 160 & - & - & $1,212,986$ & - & - \\
\hline 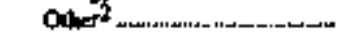 & - & - & - & - & $\$ 46,313$ & - & - \\
\hline \multicolumn{8}{|l|}{ Other ${ }^{3}$} \\
\hline Saks (thoud kWh) & - & 781,781 & - & - & 282,307 & - & - \\
\hline Revelü (dollars) & - & $23,750,816$ & - & $16,51,212$ & 6.767.75 & - & - \\
\hline Damand Cherpe Only l......... & - & - & - & - & - & - & - \\
\hline 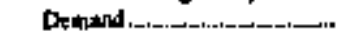 & - & - & - & - & 1061267 & - & - \\
\hline 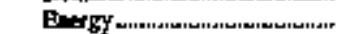 & سـ & $23,750,816$ & - & - & $5,706,448$ & - & - \\
\hline Other ${ }^{2}$......................... & - & - & - & $16,571,212$ & - & - & - \\
\hline \multicolumn{8}{|l|}{ Tad } \\
\hline Suks (thoonund kWh) .............. & 1,076,57M & $11,674,248$ & $1,291,800$ & $1,954,107$ & $5,815,843$ & 137,596 & - \\
\hline Remediw (dolkrs) & $39,659,729$ & $3+6,020,330$ & $23,870,280$ & $25.374,947$ & IBS, 6800,895 & $6,349,698$ & - \\
\hline Dentand Ctome Only 1 ......... & - & - & - & - & $=$ & - & - \\
\hline Othotnd.minn & $28,468,390$ & $35,553,526$ & - & $6,67,670$ & 87360,143 & - & - \\
\hline Everty & 20.191 .338 & 202106,600 & - & $2,467,047$ & $92,9+10,010$ & $6,349,698$ & - \\
\hline $0, x_{1}$ & $=$ & $-t, 643, B 56$ & $28,87,2 \times 0$ & $16,250,030$ & 9.460 .550 & - & - \\
\hline
\end{tabular}

Ste nolts and foothotes it end of lible. 
Table 20. Electricity Sales for Resale by Investor-Owned Utillties, by State, 1994 (Continued)

\begin{tabular}{|c|c|c|c|c|c|c|c|}
\hline 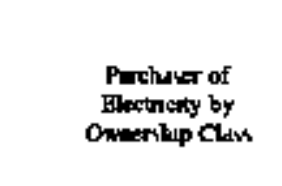 & 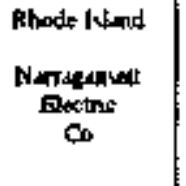 & $\begin{array}{l}\text { Racude lulend } \\
\text { Nespont } \\
\text { Elodtre } \\
\text { Corp }\end{array}$ & 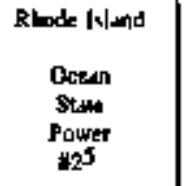 & $\begin{array}{c}\text { Rhouk [1]und } \\
\text { Orem } \\
\text { Sult } \\
\text { Puower } \\
D_{0}^{3}\end{array}$ & 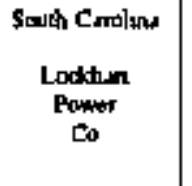 & 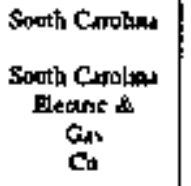 & 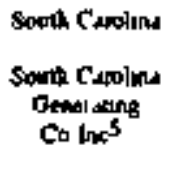 \\
\hline \multicolumn{8}{|l|}{ Inverter Oumed } \\
\hline 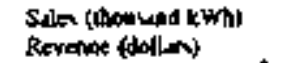 & $\begin{array}{r}591 \\
69831\end{array}$ & $\bar{z}$ & $\begin{array}{r}2,063,409 \\
1[1], 565,4566\end{array}$ & $\begin{array}{r}2,043, \pi n 9 \\
91,800,141\end{array}$ & $\overline{-}$ & $\begin{array}{r}84,534 \\
2,47,9083\end{array}$ & $\begin{aligned} \pi 27,033 \\
9, \pi 9,192\end{aligned}$ \\
\hline 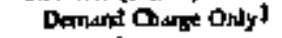 & - & - & - & - & - & - & $=$ \\
\hline Demand & - & - & $7 月, 011, B S B$ & 60,692008 & - & - & 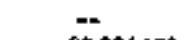 \\
\hline Enetsy & 62, Hing & - & $20,20 \%, 0080$ & $19,112,478$ & - & $0,467,908$ & 92,796192 \\
\hline$a d x=t^{2}$ & 6,522 & - & $1.147,7 n g$ & 4005.454 & - & - & $\neg \pi$ \\
\hline \multicolumn{8}{|l|}{ Fetiers } \\
\hline Silen (thowsand $\mathbf{k W h}$ ) & - & - & - & - & - & - & -- \\
\hline Revenu: (dollurs) & - & - & - & - & - & - & - \\
\hline Demond Oarge Odyl & - & ـ & - & - & س & - & -- \\
\hline Deand & - & - & - & - & - & - & - \\
\hline Buryy & - & - & - & - & - & - & -- \\
\hline Othe ${ }^{2}$ & - & - & - & - & - & - & -- \\
\hline \multicolumn{8}{|l|}{ Sust dad Other } \\
\hline Sukb (loooud kWh) & - & - & - & - & - & 89,641 & - \\
\hline Ruveade (dollerv) & - & - & - & - & - & $3,624, \mathrm{BSB}$ & -- \\
\hline Deanind Curge Only I & - & - & - & - & - & - & - \\
\hline Dentand & - & - & - & - & - & $1,79,487$ & - \\
\hline Enary & - & - & - & - & - & $1,852,903$ & -- \\
\hline Oband 2 & - & $m$ & - & - & - & $-18,5] 2$ & - \\
\hline \multicolumn{8}{|l|}{ Mhangopal } \\
\hline Suls (Lhow wad kwh) & - & - & - & - & 129476 & 837.672 & - \\
\hline Revanum (dollon) & - & - & - & - & $6.3 \mathrm{JB}, 3] 4$ & $32,325,406$ & - \\
\hline Denind arge Conly! & - & - & - & - & - & - & سـ \\
\hline Derrind & - & - & - & - & $3.764,127$ & 36,241, J65 & - \\
\hline E-m & - & - & - & - & $2,393,935$ & $36,275,972$ & - \\
\hline Ooture ${ }^{2}$ & - & - & - & - & $1 f 0,252$ & $-192,331$ & - \\
\hline \multicolumn{8}{|l|}{ Coopdane } \\
\hline Silst (thousind KWh) & - & - & - & - & - & 562,322 & - \\
\hline Revenua (dollen) & - & - & - & - & - & $6,756,599$ & - \\
\hline Dened andere Onby' & - & - & - & - & - & - & - \\
\hline Derand & - & - & - & - & - & $3.627 .39 \mathrm{~d}$ & - \\
\hline Energy & - & - & - & - & - & $3,161,164$ & - \\
\hline Onter & - & - & - & - & - & $-31,997$ & - \\
\hline \multicolumn{8}{|l|}{$040^{3}$} \\
\hline Sten (thourand tith) & - & 12.5300 & - & - & - & $21,25]$ & $=$ \\
\hline Reverne (dodlary) & - & $6 * 4,900$ & - & ـ & - & 528.857 & - \\
\hline 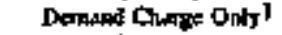 & - & - & - & - & - & - & - \\
\hline Denusnd & - & - & - & - & - & - & - \\
\hline Enersty & - & 6090 & - & - & - & 528,867 & - \\
\hline$a 0 n^{3}$ & - & - & - & - & - & - & - \\
\hline \multicolumn{8}{|l|}{ Troxd } \\
\hline 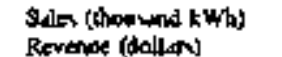 & $\begin{array}{r}591 \\
68,8 \div 1\end{array}$ & $\begin{array}{r}12530 \\
680,500\end{array}$ & $\begin{array}{r}2,067,409 \\
101,565,656\end{array}$ & $\begin{array}{r}2,043,779 \\
91,830,340\end{array}$ & $\begin{array}{r}129,476 \\
6,318314\end{array}$ & $\begin{array}{r}1,195,422 \\
46,743,620\end{array}$ & $\begin{array}{r}1,727,1330 \\
97,796,192\end{array}$ \\
\hline Dembund Cherge Only1 & - & - & 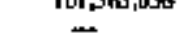 & - & - & - & $=$ \\
\hline Danand & $-\infty 100$ & - & $78,011,808$ & $68,692,208$ & $0,764,127$ & $21,659,586$ & - \\
\hline Boengy & $\begin{array}{r}62,309 \\
6,522\end{array}$ & $-^{680,500}$ & $\begin{array}{r}20.206,1889) \\
3,347,7 n g\end{array}$ & $\begin{array}{r}[9,1] 2,4] 8 \\
4,075,454\end{array}$ & $\begin{array}{r}2,743.975 \\
161258\end{array}$ & $\begin{array}{r}25.286,814 \\
-242.760\end{array}$ & $\begin{array}{l}97.796,192 \\
--\end{array}$ \\
\hline
\end{tabular}

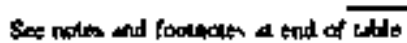


Table 20. Electricity Sales for Resale by Investor-Owned Utilltes, by State, 1994 (Continued)

\begin{tabular}{|c|c|c|c|c|c|c|c|}
\hline 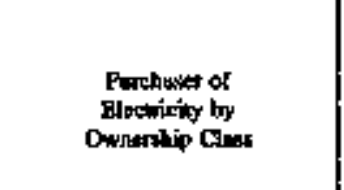 & 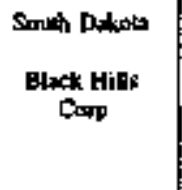 & 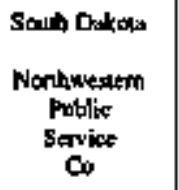 & 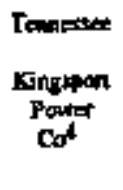 & 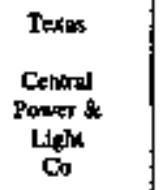 & $\begin{array}{c}\text { Telas } \\
\text { El Fas } \\
\text { Electric } \\
\text { Co }\end{array}$ & 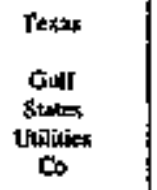 & $\begin{array}{l}\text { Texus } \\
\text { Hodston } \\
\text { lipining } \\
\text { \& Power } \\
\text { Co }\end{array}$ \\
\hline \multicolumn{8}{|l|}{ Lavedir-Owaned } \\
\hline Satis (bonond kwh) & 1000 & 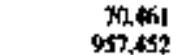 & $\frac{-}{-}$ & 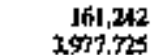 & $\begin{array}{r}521,161 \\
14524,559\end{array}$ & - & $\begin{array}{r}297,9019 \\
28,290009\end{array}$ \\
\hline 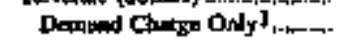 & - & - & - & - & - & - & - \\
\hline Defrond & - & - & - & عـ & 5,400100 & - & $20,907,129$ \\
\hline ENerg, & - & 957,452 & - & $3,006,170$ & $5,728,962$ & $=$ & $3,382,0100$ \\
\hline Other. & 一 & - & - & 97,555 & $3.395,597$ & - & - \\
\hline \multicolumn{8}{|l|}{ Ptederil } \\
\hline Silas (llonetind k'Wh) & - & $28,9,01$ & 一 & - & - & - & 一 \\
\hline Revenow (dollars) & - & 172,262 & - & - & $m$ & - & - \\
\hline Dempend Ongy Only' & - & - & - & - & - & - & - \\
\hline 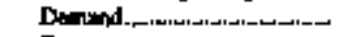 & - & 一 & $\longrightarrow$ & - & - & - & 一 \\
\hline 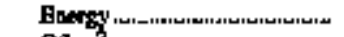 & - & 172,252 & - & - & - & - & 一 \\
\hline Oth: & 一 & - & - & - & 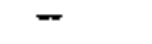 & - & - \\
\hline \multicolumn{8}{|l|}{ 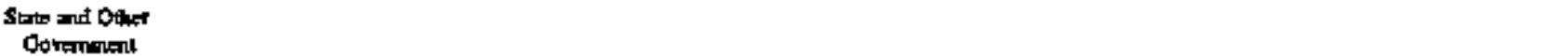 } \\
\hline 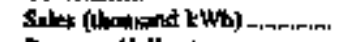 & - & 12999 & - & - & 755,173 & 71,035 & 16,525 \\
\hline 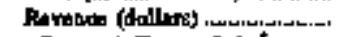 & - & 171,715 & - & 7,276 & $49,964,938$ & $7,595,364$ & 399.489 \\
\hline Dempend Chrge Orly't__... & - & - & 一 & - & - & - & - \\
\hline 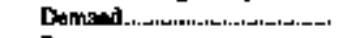 & - & - & - & - & $35,40,0,10$ & $5,505,575$ & - \\
\hline 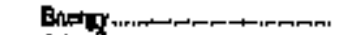 & - & 17t.71s & - & - & $3,000,266$ & $2,153,10015$ & 399,489 \\
\hline 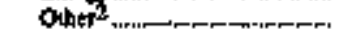 & - & - & - & 7.276 & $11,426,672$ & $-3,616$ & 一 \\
\hline \multicolumn{8}{|l|}{ Maricipel } \\
\hline Suls (lboanond twh) ............. & 166579 & 9,955 & - & $146,00 ?$ & 8sto & 168,293 & 130,689 \\
\hline Reverue (dedllus) & $7,425,149$ & 399.735 & - & $5,5 \mathbf{5 0 1} 39$ & 10,857 & $7,310,314$ & 3,694,0日7 \\
\hline Dempend Charge Doly I........ & - & - & - & - & - & - & - \\
\hline 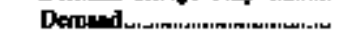 & $3,199,815$ & 187.226 & 一 & $3,346,404$ & - & $3,2,92,609$ & - \\
\hline 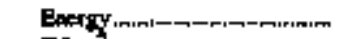 & $\$, 699,061$ & 112,766 & 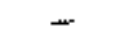 & $3,414,000$ & $10,7 \mathrm{t}\}$ & $1,017.705$ & $3,188,007$ \\
\hline 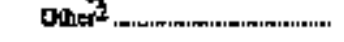 & $32 \pi, 273$ & 9,803 & - & BIk,945 & 30 & - & - \\
\hline \multicolumn{8}{|l|}{ Cooperiliv } \\
\hline 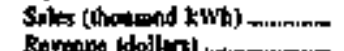 & - & 26,601 & - & 296760 & 28,760 & 396,011 & $38 B, 661$ \\
\hline 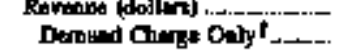 & - & $-^{342,461}$ & - & $\begin{array}{l}34,047,415 \\
-\end{array}$ & $\begin{array}{l}1,363,780 \\
-\end{array}$ & $15,4 B B, 459$ & 1906.185 \\
\hline 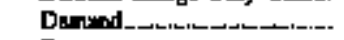 & - & - & - & $17,055,016$ & - & $7,159,988$ & - \\
\hline Eocrgy & - & 342,461 & - & $16,691,900$ & 938,323 & $8,328,919$ & 6,906, It:5 \\
\hline olk ${ }^{2}$ & - & - & 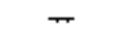 & $30,0,419$ & 428,457 & -448 & - \\
\hline \multicolumn{8}{|l|}{ Qlber ${ }^{3}$} \\
\hline Sukk (llomiand kWht & - & 33,122 & - & $2 \tan 648$ & 999,81: & $2,876,278$ & - \\
\hline Beythute (dalless) & - & 477.797 & - & $7,161,044$ & $44.790,197$ & $67.744,614$ & 一 \\
\hline Datuned Chege Only1 & - & 一 & - & - & - & - & 一 \\
\hline 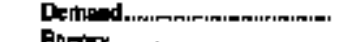 & 一 & 14,060 & - & - & $27.572,146$ & - & 一 \\
\hline 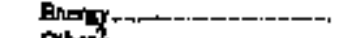 & - & 462.925 & - & $5,804,4] 3$ & $2,500.118$ & $67,744,614$ & 一 \\
\hline 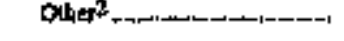 & - & 792 & - & $1.364,567$ & $13,117,903$ & - & - \\
\hline \multicolumn{8}{|l|}{ Tokin } \\
\hline 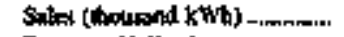 & 167,479 & 182,092 & - & $1,255,465$ & $2,245,694$ & $3,511,557$ & 873,855 \\
\hline 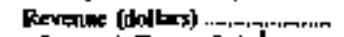 & $7.425,149$ & $2,43 r, 422$ & - & sin.tis & 110,656,331 & $98,228,751$ & $39,399,769$ \\
\hline 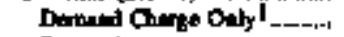 & - & - & - & - & - & - & - \\
\hline 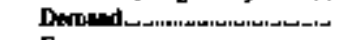 & $3,199,815$ & 2APr,306 & - & IB, 403,420 & $69,002,146$ & $15,988,572$ & 20 然, 129 \\
\hline 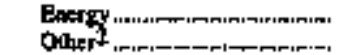 & $\begin{array}{l}3,608,061 \\
527,273\end{array}$ & $\begin{array}{r}2,419,521 \\
19,595\end{array}$ & - & $\begin{array}{r}2 k, 916,62 ? \\
3,466,262\end{array}$ & $12,258,381$ & $\begin{array}{r}32,34,263 \\
-1,064\end{array}$ & $\begin{array}{l}\text { I8, } 652,640 \\
-\end{array}$ \\
\hline
\end{tabular}

Ses notes and fostrutes a cond of toble. 
Table 20. Electricity Sales for Resale by Investor-Owaed Uthities, by State, 1994 (Continued)

\begin{tabular}{|c|c|c|c|c|c|c|}
\hline $\begin{array}{l}\text { Puncture of } \\
\text { Electecily by } \\
\text { Owrenip Clus }\end{array}$ & $\begin{array}{l}\text { Takes } \\
\text { Souktherken } \\
\text { Elestric } \\
\text { Serwite } \\
\text { Co }\end{array}$ & $\begin{array}{l}\text { Texth } \\
\text { Sowhweutm } \\
\text { Fubhe } \\
\text { Sernes } \\
\text { Co }\end{array}$ & 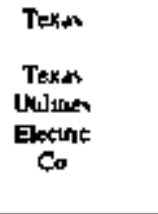 & $\begin{array}{c}\text { Texas } \\
\text { Tekas } \\
\text { New Moxios } \\
\text { Power } \\
0\end{array}$ & 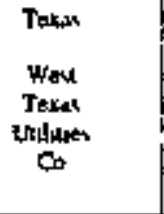 & 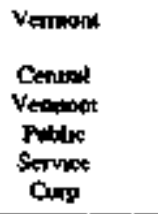 \\
\hline 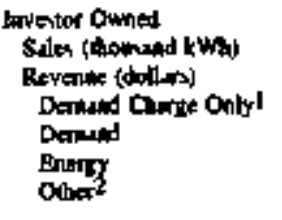 & $\begin{array}{r}2,303 \\
110,068 \\
-\quad \\
40,842 \\
62,909 \\
6,317\end{array}$ & 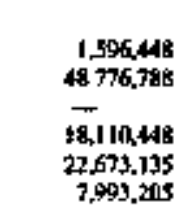 & 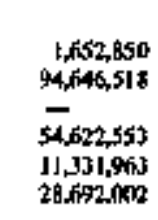 & $\begin{array}{l}\vec{z} \\
\overrightarrow{-} \\
\vec{E}\end{array}$ & $\begin{array}{r}124,442 \\
1,609,503 \\
- \\
1,698,241 \\
2,699,921 \\
3,342\end{array}$ & 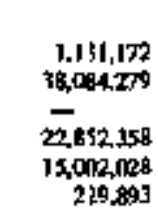 \\
\hline 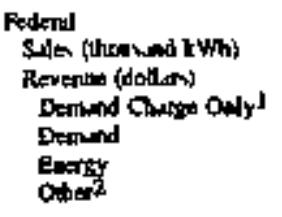 & $\begin{array}{l}- \\
- \\
- \\
-\end{array}$ & $\begin{array}{l}r^{6,917} \\
- \\
- \\
-\end{array}$ & $\begin{array}{l}= \\
= \\
= \\
=\end{array}$ & $\begin{array}{l}\overline{-} \\
\bar{z} \\
\bar{I}\end{array}$ & $\begin{array}{l}\overline{-} \\
\bar{z} \\
\overline{-}\end{array}$ & $\begin{array}{l}- \\
\overline{-} \\
\overline{-} \\
-\end{array}$ \\
\hline 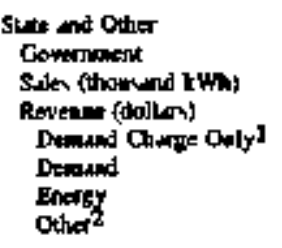 & $\begin{array}{l}\bar{z} \\
\bar{z} \\
\bar{m}\end{array}$ & $=^{76,975}$ & $\begin{array}{l}\$ 6,741 \\
1,233,863 \\
- \\
- \\
1,233,865 \\
-\end{array}$ & $\begin{array}{l}\bar{z} \\
= \\
= \\
-\end{array}$ & $\begin{array}{ll}- & 4010 \\
- & \\
- & \\
& 40\end{array}$ & 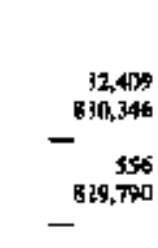 \\
\hline 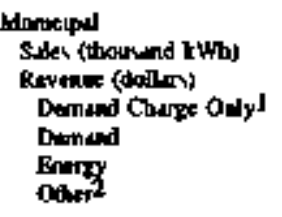 & $\begin{array}{l}\overline{-} \\
\overline{-} \\
\overline{-} \\
-\end{array}$ & $\begin{array}{l}62,171 \\
17,989,465 \\
- \\
4,112,640 \\
5,354,679 \\
8,521,146\end{array}$ & $\begin{array}{r}168,151 \\
18,558,898 \\
- \\
340,825 \\
17,977,759 \\
271,374\end{array}$ & $\begin{array}{l}\vec{E} \\
\vec{E} \\
\overrightarrow{-}\end{array}$ & $\begin{array}{r}94,961 \\
3230,792 \\
-\quad \\
954,216 \\
2,201,477 \\
72,899\end{array}$ & 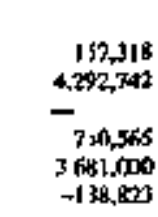 \\
\hline 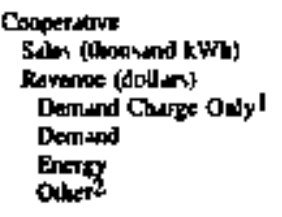 & $\begin{array}{r}97,951 \\
4,396,327 \\
- \\
1,592,237 \\
2,674,740 \\
129,310\end{array}$ & $\begin{array}{r}4,251,295 \\
149,446,675 \\
- \\
51,359,643 \\
12,291,323 \\
83,879,709\end{array}$ & $\begin{array}{l}1,925,897 \\
120,284,100 \\
- \\
70,515,0] 1 \\
11,217,106 \\
38,480,983\end{array}$ & $\begin{array}{r}3,130 \\
251.137 \\
-\quad \\
101,315 \\
108,272 \\
19,480\end{array}$ & $\begin{array}{r}1,401,591 \\
48,408,819 \\
- \\
16,556,547 \\
31,02,427 \\
20,845\end{array}$ & 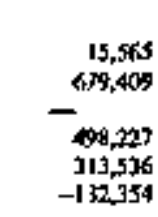 \\
\hline 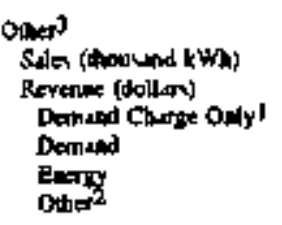 & $\begin{array}{r}1,000 \\
5,195 \\
-\quad 23,817 \\
27,312 \\
5,066\end{array}$ & $\begin{array}{l}194,756 \\
2,372,489 \\
- \\
- \\
1,296,764 \\
-924,284\end{array}$ & $\begin{array}{l}\bar{z} \\
\overline{-} \\
\overline{-} \\
\bar{z}\end{array}$ & $\begin{array}{l}\overline{-} \\
\overline{-} \\
\bar{z} \\
\overline{-}\end{array}$ & $\begin{array}{r}421,963 \\
10,832,825 \\
-\quad 12,640 \\
8,371,706 \\
2,48,39 \%\end{array}$ & $\begin{array}{r}200,459 \\
17,614,416 \\
- \\
9,906,300 \\
7,658,116 \\
-\end{array}$ \\
\hline 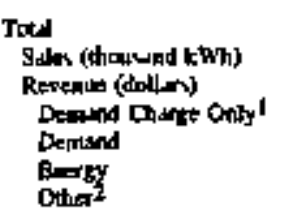 & $\begin{array}{r}1061,254 \\
4,562,590 \\
- \\
1,656,996 \\
2,764,941 \\
140,693\end{array}$ & $\begin{array}{r}6,665,027 \\
218,772,535 \\
- \\
75,592,731 \\
43,721,024 \\
99,464,776\end{array}$ & $\begin{array}{r}4,51,539 \\
21,72,379 \\
-\overline{125,551,389} \\
41,761,991 \\
67,474,299\end{array}$ & $\begin{array}{r}3,330 \\
251,137 \\
-\quad 103,385 \\
108,272 \\
59,480\end{array}$ & $\begin{array}{r}2,050,959 \\
67,076,379 \\
- \\
19,221,0613 \\
49,300,794 \\
2,551,927\end{array}$ & $\begin{array}{r}1,636,923 \\
61,521,192 \\
- \\
34,048,106 \\
27,44,470 \\
-41,284\end{array}$ \\
\hline
\end{tabular}

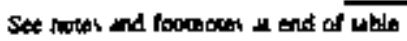


Table 20. Electrieliy Sales for Resale by Investor-Owned Utilithes, by State, 1994 (Continned)

\begin{tabular}{|c|c|c|c|c|c|c|}
\hline 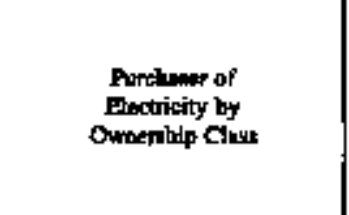 & 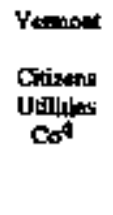 & $\begin{array}{l}\text { Vermont } \\
\text { Orean } \\
\text { Mounin } \\
\text { Power } \\
\text { Corp }\end{array}$ & 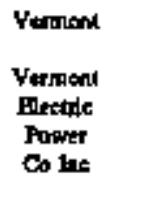 & $\begin{array}{l}\text { Veminat } \\
\text { Venomot } \\
\text { Eleculc } \\
\text { Thing } \\
\text { Cor }\end{array}$ & 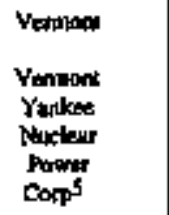 & $\begin{array}{l}\text { Viroipix } \\
\text { Appolseltan } \\
\text { Ponver } \\
\text { Co }\end{array}$ \\
\hline \multicolumn{7}{|l|}{ Inventothingod } \\
\hline Sther (Utalesed kWh) & $\rightarrow$ & 317,69t & 179.496 & - & $4,315,547$ & 4,758.614 \\
\hline Devepup (doplins) & - & $1 \mathrm{t}, 0 \mathrm{~J} 6,82 \mathrm{t}$ & $15,410,466$ & - & $162,777,189$ & $164, \pm 50,415$ \\
\hline 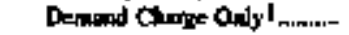 & - & - & - & - & - & $2,368,400$ \\
\hline 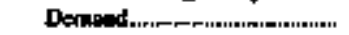 & - & $4999,29 n$ & $3,617,978$ & - & - & $55,67, \mathrm{Jn}$ \\
\hline Energy & - & 6.061026 & $7,990,629$ & - & - & $106,6[4,643$ \\
\hline $\operatorname{OAph}^{2}$ & - & $-42,995$ & $1, \$ 29$ & - & $152,257,189$ & - \\
\hline \multicolumn{7}{|l|}{ Fesiendil } \\
\hline Saln (thourand kWh) & - & - & - & - & - & 19,120 \\
\hline Reverve $(d a l i n x)$. & - & - & - & - & - & $520+4$ \\
\hline Daluned Chrye Ontyr'......... & - & - & - & - & - & - \\
\hline Oarimpond & - & - & - & - & - & - \\
\hline 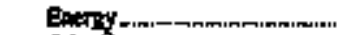 & - & - & - & - & - & 529,842 \\
\hline Whert & - & - & - & - & - & - \\
\hline \multicolumn{7}{|l|}{ Stove und OWh } \\
\hline Salay (chousond kWh) ........... & - & 264 & - & - & - & $=$ \\
\hline 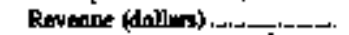 & - & 2,805 & - & - & - & - \\
\hline Deneund Gurge Onbly......... & - & - & - & - & - & - \\
\hline Dentad & - & $\rightarrow$ & - & - & - & - \\
\hline Euergy & - & no, ons & - & - & - & - \\
\hline Other & - & - & - & - & - & - \\
\hline \multicolumn{7}{|l|}{ Minicipal } \\
\hline Siles (0oded kWh) & - & 33,1662 & 339,778 & - & - & 240.301 \\
\hline 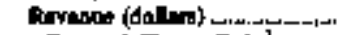 & - & $1,085,281$ & $12,267,427$ & - & - & $\mathbf{5 1 , 8 9 0 , 0 3 3 2}$ \\
\hline Denomed Cherge Onlyl ...uc.... & - & - & 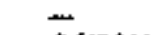 & - & - & $=$ \\
\hline Denomd.......................... & - & 642043 & $3,647,904$ & - & - & $35,511,200$ \\
\hline 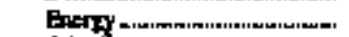 & - & $1.126,288$ & 2997,824 & - & - & $46,368,8390$ \\
\hline 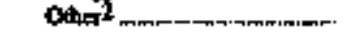 & - & $-83,530$ & G61.699 & - & - & - \\
\hline \multicolumn{7}{|l|}{ Cooperilive } \\
\hline 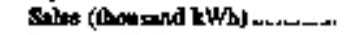 & - & 4,911 & 89,822 & - & - & 184,801 \\
\hline Revemine (daillen) & - & 345,954 & $3,286,109$ & - & - & $7,173,623$ \\
\hline Deaund Gugze Onlyl 1 ....t-." & - & $244 n$ & - & - & - & - \\
\hline Derind & - & $174,90,0$ & 2,40957 & - & - & $3,635,957$ \\
\hline Bntry & - & 180,477 & 67,519 & - & - & 1,531,\$6 \\
\hline Q6her & - & $=$ A,, $\mathbb{B}$ & 199013 & - & - & - \\
\hline \multicolumn{7}{|l|}{$0 t^{3}{ }^{3}$} \\
\hline 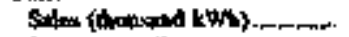 & - & 36,436 & - & - & ـ & $1,930,494$ \\
\hline Rovane fdoturs & - & $20 A_{1} 10 \mathrm{~A}$ & - & - & - & $36,977,724$ \\
\hline 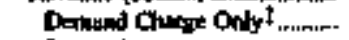 & - & - & - & - & - & - \\
\hline 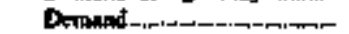 & - & - & - & - & - & $3,751,447$ \\
\hline 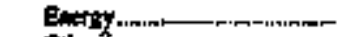 & - & 264,101 & - & - & - & $33,626,277$ \\
\hline Ding & - & - & - & - & - & - \\
\hline \multicolumn{7}{|l|}{ Iot } \\
\hline Soldm (Bow & - & 367,426 & 902,096 & - & 4,315547 & $9,378,520$ \\
\hline 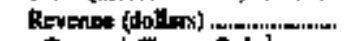 & - & $13,520,939$ & $30,949,972$ & - & $162,757.199$ & $291,411,236$ \\
\hline 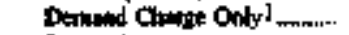 & - & $2, A 40$ & - & - & - & $2,376,410$ \\
\hline 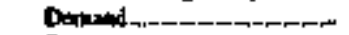 & - & 6 (1012240 & $18,475,539$ & - & - & $90,365,978$ \\
\hline Entroy -m, & - & $7,640,567$ & $11,616,972$ & - & - & $190,67.458$ \\
\hline alver ${ }^{2}$ & $=$ & $-135,918$ & B62,AB! & - & $162,757,189$ & - \\
\hline
\end{tabular}

Ses noles and frowotes ot end of uble 
Table 20. Electricity Sales for Resale by Investor-0wned Utilities, by State, 1994 (Continned)

\begin{tabular}{|c|c|c|c|c|c|c|}
\hline 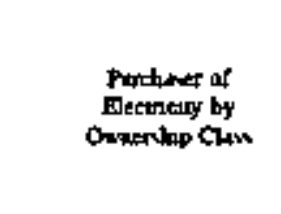 & 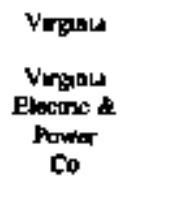 & 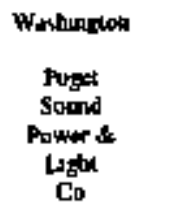 & 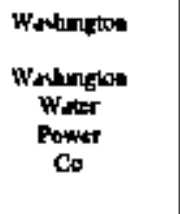 & 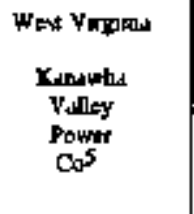 & 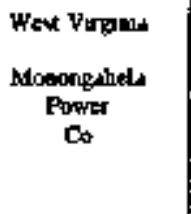 & 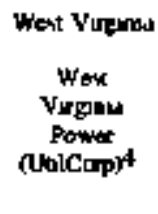 \\
\hline \multicolumn{7}{|l|}{ Snvestor Onned } \\
\hline SAln (hoonded kWh) & 175.682 & 1,792, a2d & 2212,804 & 269,384 & $4,006,995$ & - \\
\hline freverioe tdollen) & $9.9101,130$ & $36,759,212$ & $72 \sin , \pi 2$ & $3,940,559$ & $145,511,982$ & - \\
\hline Denand Orape Oobl & - & 50,0000 & $J$ IR, T24, ONA & - & - & - \\
\hline Denuas & $6,473,136$ & - & $9,319,167$ & $2,966,440$ & $42,6310,823$ & - \\
\hline Enery & $3,456,934$ & $36,281,344$ & S245LEOS & 994,119 & $64,599,977$ & - \\
\hline ourex & - & 27,364 & - & - & $38,341,792$ & - \\
\hline \multicolumn{7}{|l|}{ Fodornl } \\
\hline Sthe (thoseund k'th) & - & B17,812 & 90,159 & - & - & - \\
\hline Reveniwe (dollen) & - & $19,314,054$ & $1,91 \leqslant, 247$ & - & - & - \\
\hline Dernad Gonge Only' & - & - & - & - & - & - \\
\hline Demand & - & 400,0100 & - & - & - & - \\
\hline Enatgy & - & IB,9/4,054 & $1,916,247$ & - & - & - \\
\hline Ouber & - & - & - & - & - & - \\
\hline \multicolumn{7}{|l|}{ Stut mad b4tar } \\
\hline Salen (therowd KWh) & - & 66.164 & 469,806 & - & - & - \\
\hline Reveriwo (dollar) & - & $1,391,158$ & $13,548,238$ & - & - & - \\
\hline Denand ondre ontyl & - & - & 305,132 & - & - & - \\
\hline Denedwi & - & - & $4,8 ? 1,500$ & - & - & ـ \\
\hline Entray & - & $1,391,159$ & $0,371,39 \%$ & - & - & - \\
\hline athent & - & - & - & - & - & - \\
\hline \multicolumn{7}{|l|}{ Mtarnopplal } \\
\hline Sils (thoulnd twh) & $1,026,519$ & $241.56 \mathrm{~d}$ & $4\{$ 1554 & - & $\$ 5 ., 056$ & - \\
\hline Revenus (dollas) & $45.393,392$ & $6,475,619$ & $1.900,280$ & - & $2,889,669$ & - \\
\hline Denand Chwrge Onlyl & - & - & 751,998 & - & - & - \\
\hline Derand & $25,216,962$ & I, $1,3,47 \mid$ & 87,416 & - & $1,259.102$ & - \\
\hline Bnogy & $21,848,188$ & $5,042,910$ & 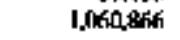 & - & $1,819.264$ & - \\
\hline $0 h=2$ & $-1,711,750$ & 119.276 & - & - & $-188,677$ & - \\
\hline \multicolumn{7}{|l|}{ Copparative } \\
\hline 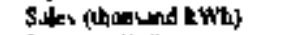 & $4,931,736$ & - & - & - & 49,346 & - \\
\hline Retrelue (danter) & 195,354,01d & - & - & - & $1,926,438$ & - \\
\hline Dendand Chuge Ondy I & - & - & - & - & - & - \\
\hline Dewand & 95,38:,429 & - & - & - & 1.019 .118 & - \\
\hline Entry & J04,537,795 & - & - & - & $1,136,592$ & 一 \\
\hline Duket 2 & $-4,364210$ & - & - & - & $-215,262$ & - \\
\hline \multicolumn{7}{|l|}{ Obet } \\
\hline Sales (thousend $k$ Wh) & 900556 & 46.174 & 672013 & - & 141,835 & - \\
\hline Reverum (dollun) & $26,339.623$ & $1,018,025$ & $1,513.49$ & - & $3,566,910$ & - \\
\hline Domonad Conge Ody & 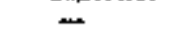 & - & 340,000 & - & - & - \\
\hline Dephanul & $2,737,815$ & 20,459 & 316,793 & - & 290,258 & - \\
\hline Eoergy & $21, \mathrm{fOH}, 308$ & 996,785 & $1,508,172$ & - & $1,176,552$ & - \\
\hline Ouber 2 & - & 5I & $-651,070$ & - & - & - \\
\hline \multicolumn{7}{|l|}{ الנMT } \\
\hline Sallos (bootand KWh) & $7,133,984$ & 2.962 .741 & 2886,886 & $20 \% 384$ & $4,283,232$ & - \\
\hline Retenoe (dothirs) & $277,176,659$ & $64,553,1616$ & $91,386,006$ & $3,940,599$ & $151,904,999$ & - \\
\hline Denund Codag Onlyl & - & so, (010 & $12,131,000$ & - & - & - \\
\hline Denind & $129,8018,362$ & $1,370,090$ & 14534,480 & $2,986,40$ & $45,295,281$ & - \\
\hline Amergy & $153,444,265$ & $62,626,751$ & $65,711,286$ & 954,119 & $70,671,775$ & - \\
\hline Other2 & $-6 \mathrm{~ms} 5 \mathrm{sin}$ & 147,727 & -650 & - & $37,937,841$ & - \\
\hline
\end{tabular}

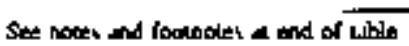


Table 20. Electriclty Sales for Resale by Investor-Owned Utilities, by State, 1994 (Continned)

\begin{tabular}{|c|c|c|c|c|c|c|}
\hline 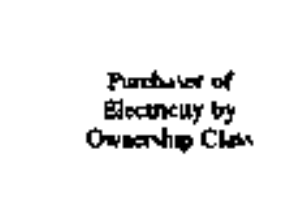 & 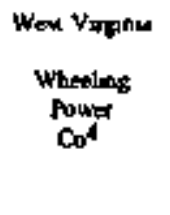 & 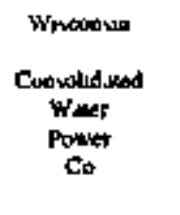 & 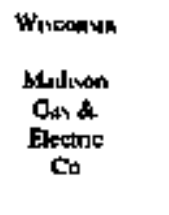 & $\begin{array}{c}\text { Wromus } \\
\text { Nonthem } \\
\text { Sures } \\
\text { Pour } \\
\text { Co }\end{array}$ & 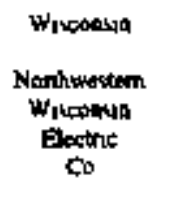 & 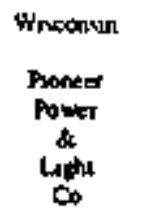 \\
\hline \multicolumn{7}{|l|}{ Inivedor Opmed } \\
\hline 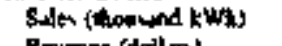 & $=$ & $\mathbf{4 6 1}$ & 26,340 & 469,029 & - & 10,784 \\
\hline 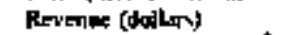 & - & 2,897 & $1,753,50515$ & $47,504,829$ & - & 427,494 \\
\hline Donend Ohrye Onlyl & - & - & - & - & - & - \\
\hline Demped & $=$ & - & $1,002 m 0$ & - & - & 129. fino \\
\hline 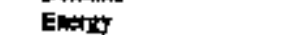 & - & 2096 & 356.505 & - & - & $\$ 35,590$ \\
\hline Ohen 2 & - & - & - & 47,60M.829 & - & $-41,49$ \\
\hline \multicolumn{7}{|l|}{ Fedard } \\
\hline S.An (thou wond Is Wh) & - & - & - & - & - & - \\
\hline Reverier fdollond & - & - & - & - & - & - \\
\hline Detound Cherge Only! & - & - & - & - & - & - \\
\hline Dernati & - & - & - & - & - & - \\
\hline Enasy & - & - & 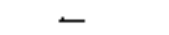 & - & - & - \\
\hline Detes & - & - & - & - & - & - \\
\hline \multirow{2}{*}{\multicolumn{7}{|c|}{ Sute and Oover }} \\
\hline & & & & & & \\
\hline Shiks (hoorand kWh) & - & - & - & - & - & - \\
\hline Revente (dollun) & - & - & - & - & - & - \\
\hline Demind Oruse Only 1 & - & - & - & - & - & - \\
\hline Deondrd & - & - & - & - & - & - \\
\hline Enary & - & - & - & - & - & - \\
\hline Other & - & - & - & - & - & - \\
\hline \multicolumn{7}{|l|}{ Mancipal } \\
\hline Sales (thosend KWh) & - & - & 8,250 & 436,978 & 4,638 & س \\
\hline Revenue (dollenv & - & - & 225,162 & $17,361.1106$ & 212,369 & - \\
\hline 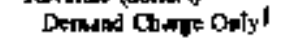 & - & - & - & - & - & - \\
\hline Danand & - & - & - & $6,54], 364$ & 58,300 & - \\
\hline ENרזy & - & - & 225,162 & $10,482.785$ & 152,237 & - \\
\hline $0 \mathrm{a}=\mathrm{C}^{2}$ & - & - & - & 136,757 & 1,323 & - \\
\hline \multicolumn{7}{|l|}{ Couparate } \\
\hline Sules (thoonnd leWh) & - & - & - & - & - & - \\
\hline Revenue (deflor) & - & - & - & - & - & - \\
\hline Demund Durge Orly! & - & - & - & - & - & - \\
\hline Demued & - & מـ & - & $=$ & - & - \\
\hline Entargy & - & - & - & - & - & - \\
\hline Dhent & - & - & - & - & - & 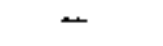 \\
\hline \multicolumn{7}{|l|}{ Olert } \\
\hline Sules (thoovend kWh) & - & - & - & 1,292 & - & - \\
\hline Revenue (aoller) & 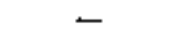 & - & - & gotal & - & - \\
\hline Denend Sodrge Only I & - & - & - & - & - & - \\
\hline Deofund & - & - & - & - & - & - \\
\hline Energy & - & - & r & - & - & - \\
\hline$a_{1} a^{2}$ & - & - & - & SA,64I & - & - \\
\hline \multicolumn{7}{|l|}{ Totsl } \\
\hline Slev (lhoound HWb) & - & 461 & 34,990 & 841,249 & 4,838 & 10,394 \\
\hline Rexame (dollur's) & - & 2,897 & $1,58366 ?$ & 65,018,576 & 21270 & 423,494 \\
\hline Demenil Oendrge Only 1 & - & - & - & - & - & - \\
\hline Demind & - & - & ], Onz, anN & $6,543,564$ & 58,809 & 129,6 \\
\hline Emergy & - & 2857 & $5 B \mid, f(i, 7\}$ & $10,682,785$ & 132,313 & 135,300 \\
\hline Other? & - & - & - & $4,792,237$ & 1.12 & $-\$ 1.496$ \\
\hline
\end{tabular}

See notes and foodecents and of table 
Table 20. Electricity Sales for Resale by Investor-Owned Utilities, by Stait, 1994 (Coutimued)

\begin{tabular}{|c|c|c|c|c|c|c|}
\hline 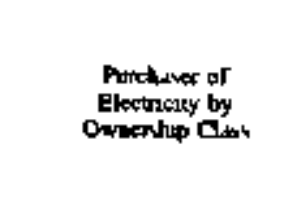 & $\begin{array}{l}\text { Wixconin } \\
\text { Sourh Belogt } \\
\text { Witter } \\
\text { Cat \& Elec } \\
\text { Co }\end{array}$ & 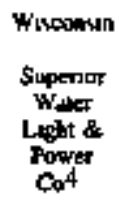 & $\begin{array}{l}\text { Worooush } \\
\text { Wpromith } \\
\text { Eketing } \\
\text { Powrer } \\
\text { Co }\end{array}$ & 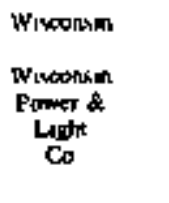 & 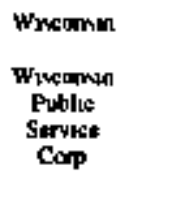 & $\begin{array}{l}\text { Whitcouve } \\
\text { Wicomen } \\
\text { Rumer } \\
\text { Power } \\
\text { Cas }\end{array}$ \\
\hline 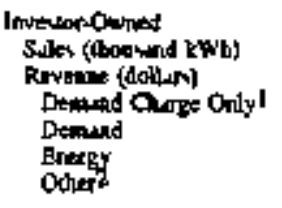 & $\begin{array}{r}5,180 \\
125,974 \\
-\quad 27,784 \\
-\quad 102,190\end{array}$ & $\begin{array}{l}\bar{z} \\
\bar{z} \\
\bar{z}\end{array}$ & 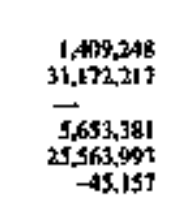 & $\begin{array}{r}40,356 \\
10,407,370 \\
\\
1,240,504 \\
7,574899 \\
1,59], 967\end{array}$ & 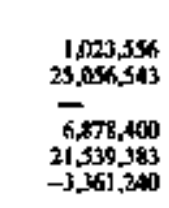 & $\begin{array}{l}105,744 \\
4,953,252 \\
- \\
4,953,252 \\
-\end{array}$ \\
\hline 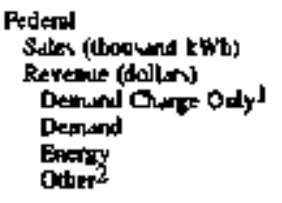 & $\begin{array}{l}\overline{-} \\
\bar{z} \\
\overline{-}\end{array}$ & $\begin{array}{l}\bar{z} \\
\bar{z} \\
\bar{z}\end{array}$ & $\begin{array}{l}\overline{-} \\
\overline{-} \\
\overline{-}\end{array}$ & $\begin{array}{l}z \\
z \\
z \\
z\end{array}$ & $\begin{array}{l}\bar{z} \\
\bar{z} \\
\bar{z}\end{array}$ & $\begin{array}{l}\bar{z} \\
\bar{z} \\
\bar{z}\end{array}$ \\
\hline 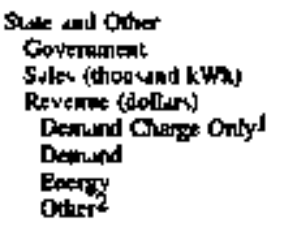 & $\begin{array}{l}z \\
\bar{z} \\
\bar{z}\end{array}$ & $\begin{array}{l}= \\
\bar{z} \\
=\end{array}$ & $\begin{array}{l}\bar{z} \\
\bar{z} \\
\bar{z}\end{array}$ & $\begin{array}{l}\bar{z} \\
\bar{z} \\
\bar{z}\end{array}$ & $\begin{array}{l}\bar{z} \\
\bar{z} \\
\bar{z}\end{array}$ & $\begin{array}{l}\bar{z} \\
\bar{z}\end{array}$ \\
\hline 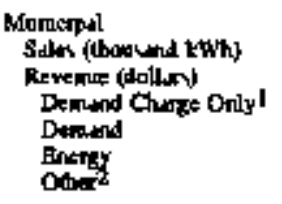 & $\begin{array}{l}\bar{z} \\
\bar{z} \\
\bar{z}\end{array}$ & $\begin{array}{l}\bar{z} \\
\bar{z} \\
\bar{z}\end{array}$ & $\begin{array}{l}1,173,447 \\
45,123,188 \\
-\overline{19,124,097} \\
27,444,474 \\
-1,345,378\end{array}$ & $\begin{array}{l}1,999,160 \\
04,774,900 \\
-\overline{16,875,372} \\
41,303,919 \\
-3,404,390\end{array}$ & $\begin{array}{l}1,054,252 \\
30,511,704 \\
\overline{13,591,157} \\
25,115,1571 \\
-1,289,234\end{array}$ & $\begin{array}{l}\bar{z} \\
\bar{z} \\
\bar{z}\end{array}$ \\
\hline 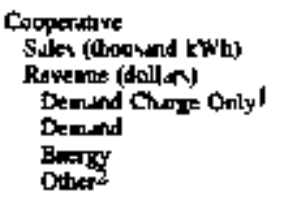 & $\begin{array}{l}\bar{z} \\
\bar{z} \\
\bar{z}\end{array}$ & $\begin{array}{l}\bar{z} \\
\bar{z} \\
\bar{z}\end{array}$ & $\begin{array}{r}298,390 \\
10,508,396 \\
\overline{4,274,304} \\
6,572,112 \\
-3,38,120\end{array}$ & $\begin{array}{r}474,104 \\
15,820,575 \\
- \\
7,400,740 \\
9,858,270 \\
-1,088,435\end{array}$ & $\begin{array}{r}10,314 \\
393,621 \\
- \\
190,375 \\
261.904 \\
-610,658\end{array}$ & $\begin{array}{l}= \\
\bar{z} \\
\bar{z}\end{array}$ \\
\hline 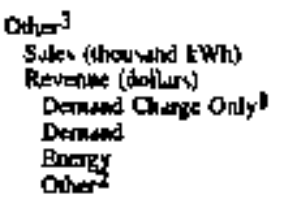 & $\begin{array}{l}= \\
= \\
=\end{array}$ & $\begin{array}{l}\bar{z} \\
\bar{z} \\
\bar{z}\end{array}$ & $\begin{array}{l}= \\
\bar{z} \\
=\end{array}$ & $\begin{array}{l}-20936 \\
-\sin , 160 \\
- \\
- \\
- \\
-\sin .160\end{array}$ & $\begin{array}{l}= \\
\bar{z} \\
=\end{array}$ & $\begin{array}{l}= \\
= \\
=\end{array}$ \\
\hline 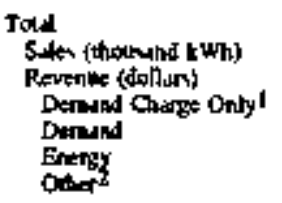 & $\begin{array}{r}6,180 \\
125,974 \\
-\quad 21,784 \\
-192,190\end{array}$ & $\begin{array}{l}= \\
= \\
= \\
=\end{array}$ & $\begin{array}{l}2,881,685 \\
1,80,3,311 \\
- \\
28,951,777 \\
59,589,579 \\
-1,728,655\end{array}$ & $\begin{array}{l}2,760,654 \\
90,402,685 \\
- \\
35,516,616 \\
58,737,0097 \\
-1,851,018\end{array}$ & $\begin{array}{r}2,0181,122 \\
55,969,958 \\
= \\
20,759.9,12 \\
46,919.158 \\
-11,711,132\end{array}$ & $\begin{array}{l}195,744 \\
4,9 \times 3,262 \\
= \\
- \\
4.952,252 \\
-\end{array}$ \\
\hline
\end{tabular}

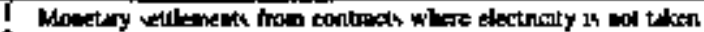

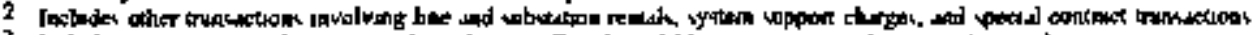

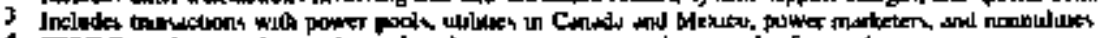

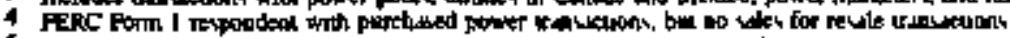

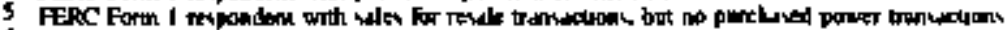

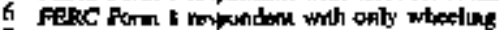

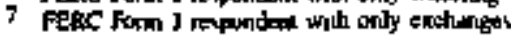

$\rightarrow$ Slot Apolicabie

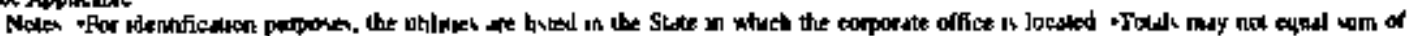
corponenti bectube of adependert rooriling

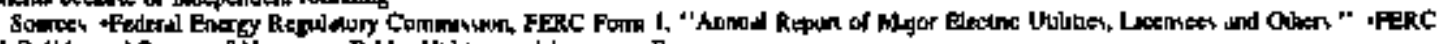

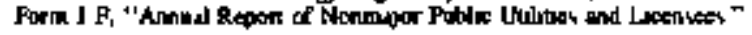


Table 21. Electriclty Exchanges by Investor-Owned Utilities, by State, 199

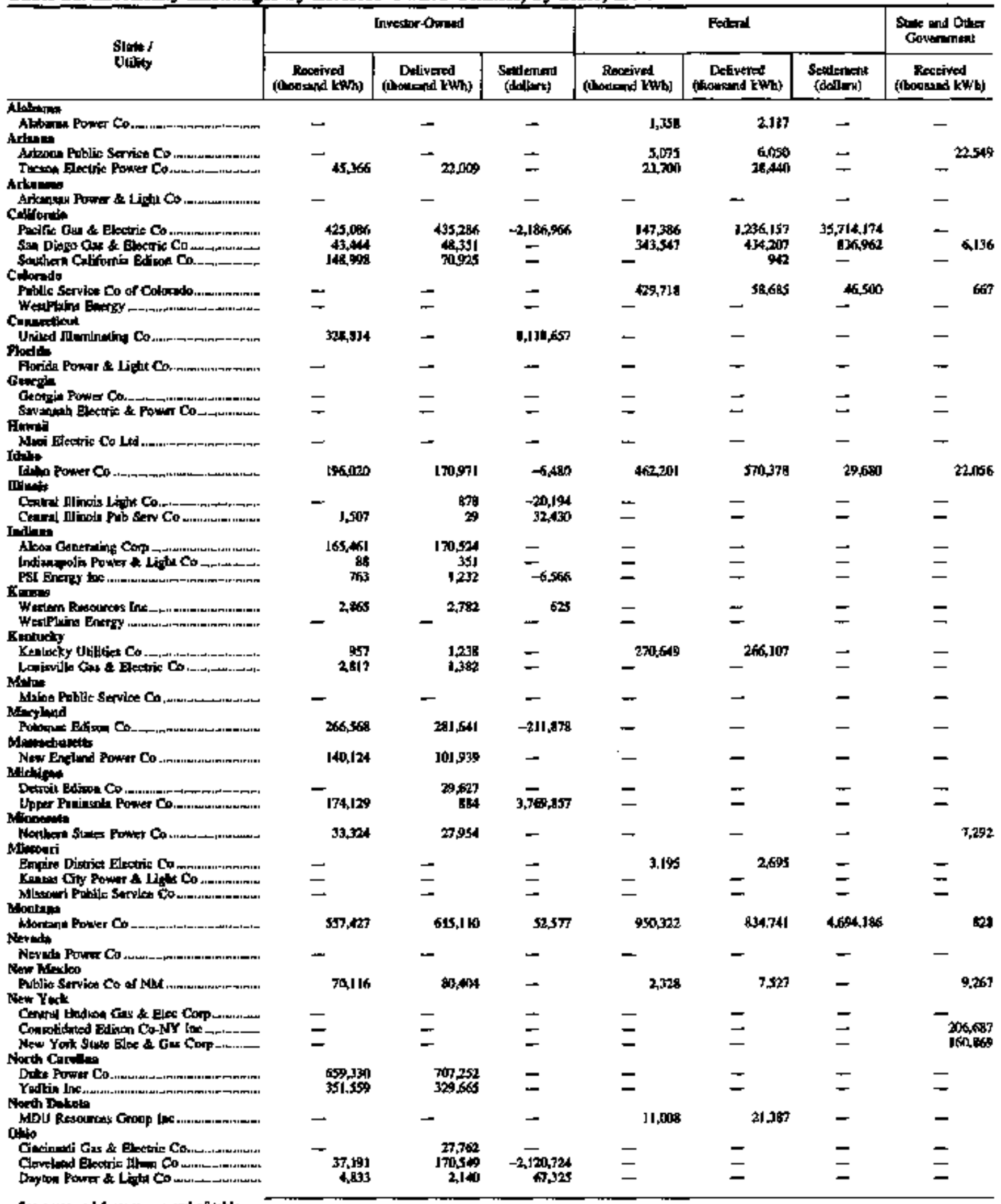


Table 21. Electricity Erchanges by Irvestor-O wned Utililies, by State, 1994 (Conlinued)

\begin{tabular}{|c|c|c|c|c|c|c|c|}
\hline \multirow{2}{*}{$\begin{array}{l}\text { Strite } t \\
\text { Uutyly }\end{array}$} & \multicolumn{2}{|c|}{$\begin{array}{l}\text { Stuxe and Oulet } \\
\text { Gowemnont }\end{array}$} & \multicolumn{3}{|c|}{ Municepul } & \multicolumn{2}{|c|}{ Coopersume } \\
\hline & 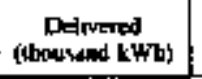 & $\begin{array}{l}\text { Sealumem } \\
\text { (dollow) }\end{array}$ & $\begin{array}{l}\text { Recelved } \\
\text { (Ohovind kWh) }\end{array}$ & $\begin{array}{l}\text { Dellwered } \\
\text { (illowitind } k W h)\end{array}$ & 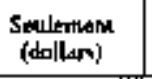 & 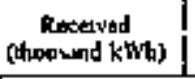 & 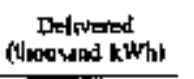 \\
\hline 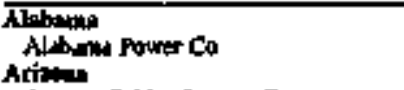 & - & - & - & - & - & - & - \\
\hline 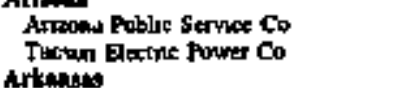 & -3 & 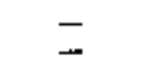 & - & $\overline{-}$ & $\overline{-}$ & $-{ }_{12,099}$ & $-{ }_{12, n \in \mathbb{1}}$ \\
\hline 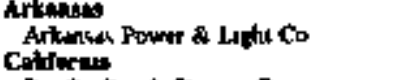 & - & - & - & - & - & - & - \\
\hline 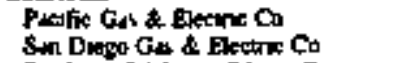 & -5.917 & $\underline{-}$ & $-^{41951}$ & $-\quad$ & 1,752 & $\overline{-}$ & $\vec{\sigma}$ \\
\hline 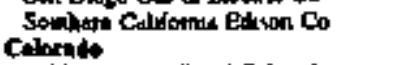 & - & - & 16,175 & 16,175 & - & - & - \\
\hline 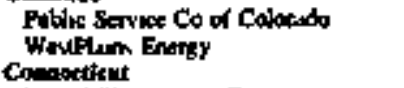 & -12.130 & $\overline{-}$ & - & $-\quad 1$ เ 23 & $I^{115,236}$ & - & $\overline{-}$ \\
\hline $\begin{array}{l}\text { Louted lionomang Co } \\
\text { Dorida }\end{array}$ & - & - & 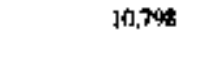 & 14 Bri & - & - & - \\
\hline $\begin{array}{l}\text { Fonde Power \& Light Ca } \\
\text { Gengel }\end{array}$ & - & - & - & - & - & 3622 & 1.71ः \\
\hline 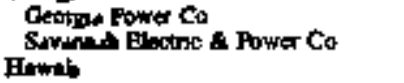 & $\overline{-}$ & $\overline{-}$ & $\bar{z}$ & $\bar{z}$ & 二 & $\overline{-}$ & $\overline{-}$ \\
\hline Idobo Elotinc Ca La & - & - & 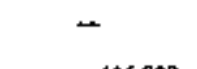 & - & - & - & - \\
\hline Idolu Power Co & 12920 & - & 126,000 & 126 Non & - & - & - \\
\hline 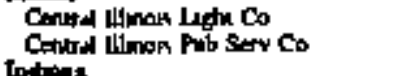 & $\overline{-}$ & $\overline{-}$ & $\begin{array}{l}1.656 \\
1.185\end{array}$ & $1,4,44$ & $\begin{array}{r}4,558 \\
211.14010\end{array}$ & $\overline{-}$ & $\overline{-}$ \\
\hline & $z$ & $\bar{z}$ & $\overline{-}$ & $\overline{-}$ & z & $\overline{-}$ & - J \\
\hline & - & - & - & $\overline{-}$ & - & 2,418 & 21,132 \\
\hline 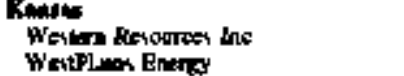 & - & - & - & - & - & - & - \\
\hline 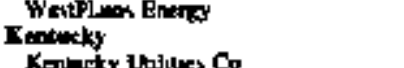 & - & - & - & - & - & - & - \\
\hline 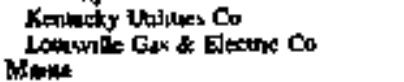 & $\overline{-}$ & $\overline{-}$ & $\overline{-}$ & $=$ & $\overline{-}$ & $\begin{aligned} 121,036 \\
849\end{aligned}$ & $\begin{array}{r}12,711 \\
338\end{array}$ \\
\hline 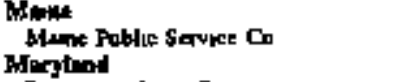 & - & - & - & - & - & - & - \\
\hline 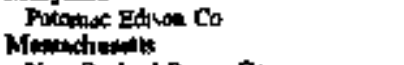 & - & - & - & - & - & - & - \\
\hline 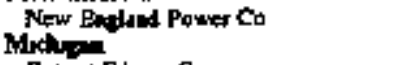 & - & - & - & - & - & - & - \\
\hline $\begin{array}{l}\text { Detrout Bdisa Ca } \\
\text { Uppet Pretinauld Power Co }\end{array}$ & $\overline{-}$ & $\ddot{z}$ & $T_{5,5017}$ & -1522 & $-\overline{-120,0011}$ & $\overline{-}$ & $\overline{-}$ \\
\hline $\begin{array}{l}\text { Mriniots } \\
\text { Northare Sustes Powa Co } \\
\text { Mrsmeri }\end{array}$ & - & - & - & - & - & - & - \\
\hline 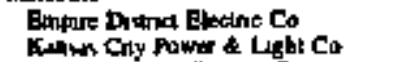 & $\overline{-}$ & $\overline{-}$ & $\bar{z}$ & $\overline{-}$ & 二 & $-9,334$ & $-, 0,315$ \\
\hline $\begin{array}{l}\text { Mhowir Poble Servate Co } \\
\text { Monlate }\end{array}$ & - & - & - & - & - & 6.395 & fin 789 \\
\hline 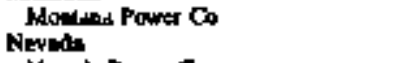 & - & 9,108 & - & - & - & - & - \\
\hline $\begin{array}{l}\text { Nerade Ponter Co } \\
\text { New Bentes }\end{array}$ & - & - & - & - & - & - & - \\
\hline $\begin{array}{l}\text { Pobles Servme Co of Nb } \\
\text { New Yalk }\end{array}$ & 9,051 & - & 5.713 & 10,261 & - & 74780 & $16,05 B$ \\
\hline 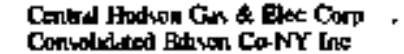 & $\overline{3}_{312612}$ & $5 \bar{T} 700000$ & 二 & $\overline{-}$ & $\overline{-}$ & $\bar{z}$ & $=$ \\
\hline 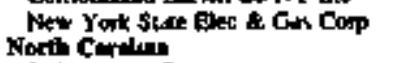 & 243,875 & 2732400 & - & - & - & - & $\overline{-}$ \\
\hline 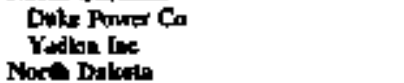 & $\overline{-}$ & $\overline{-}$ & $1,267,261$ & $\begin{array}{l}1,067,401 \\
-\end{array}$ & $1,753,238$ & $\frac{1,913,764}{-}$ & $-^{1,000,8655}$ \\
\hline 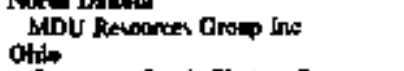 & - & $\pi$ & - & - & - & - & - \\
\hline 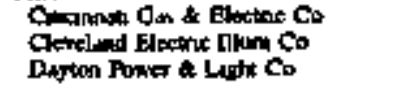 & $\frac{-}{m}$ & $\overline{\overline{-}}$ & $\begin{array}{l}135 \\
-\end{array}$ & $\overline{-}^{12,190}$ & $\begin{array}{l}7 \text { Th, } \\
-\end{array}$ & $\bar{z}$ & ${ }_{-}$ \\
\hline
\end{tabular}


Table 21. Electricity Exchanges by Investor-Owaed Utilltites, by State, 1994 (Conilnued)

\begin{tabular}{|c|c|c|c|c|c|c|c|}
\hline \multirow{2}{*}{ State } & \multirow{2}{*}{ 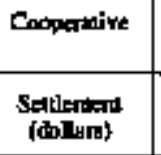 } & \multicolumn{3}{|c|}{ Ound } & \multicolumn{3}{|c|}{ Total } \\
\hline & & $\begin{array}{c}\text { Recrived } \\
\text { fhoosind kWh) }\end{array}$ & $\begin{array}{c}\text { Delingted } \\
\text { (thogsind } \mathrm{kWh} \text { ) }\end{array}$ & 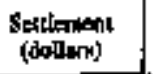 & (fhosingend kith) & (tholinkend $k$ Wh) & $\begin{array}{l}\text { Sellkment } \\
\text { (dolles) }\end{array}$ \\
\hline 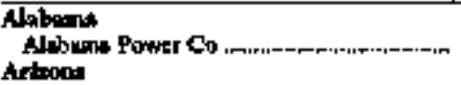 & $\rightarrow$ & - & - & - & 1,358 & 2,117 & - \\
\hline 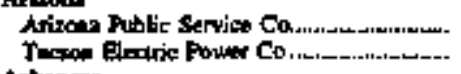 & $\overline{-}$ & $\overline{-}$ & $\overline{-}$ & - & $\begin{array}{l}27,644 \\
83,165\end{array}$ & $\underset{62,145}{409}$ & $\overline{-}$ \\
\hline 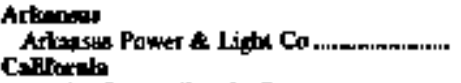 & 一 & 268,457 & $220 ; 210$ & - & 268,457 & 221,200 & - \\
\hline 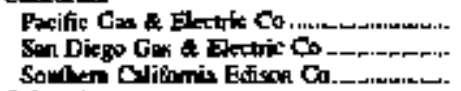 & $\bar{z}$ & $z^{\text {Innons }}$ & $\begin{array}{l}-39,343 \\
-\end{array}$ & $\begin{array}{l}2,00 \%, 386 \\
-\end{array}$ & $\begin{array}{l}714,500 \\
393,127 \\
165.173\end{array}$ & $\begin{array}{r}1,70,906 \\
489,475 \\
309042\end{array}$ & $\begin{array}{l}36,713,346 \\
836969 \\
-\end{array}$ \\
\hline 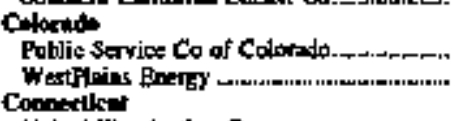 & $\overline{-}$ & - נונ & -236 & $-3+9$ & 430,437 & $\begin{array}{r}72,658 \\
2.66\end{array}$ & $\begin{array}{r}-62,786 \\
-130\end{array}$ \\
\hline 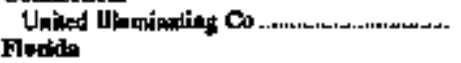 & - & 7,245 & - & 422,300 & 346,857 & 14,456 & 8,54356 \\
\hline 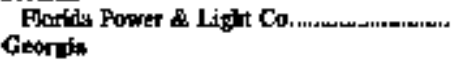 & - & - & - & - & 3.622 & 3.718 & - \\
\hline 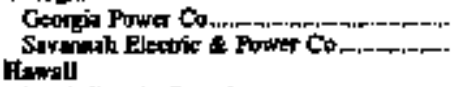 & $\overline{-}$ & $\begin{array}{l}311,268 \\
176,333\end{array}$ & $\begin{array}{r}63,969 \\
372,043\end{array}$ & $\overline{-}$ & $3: 1,268$ & $\begin{array}{r}63,543 \\
372,043\end{array}$ & - \\
\hline 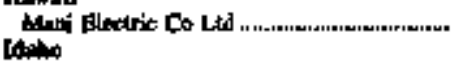 & - & $2, f i 09$ & 6.415 & $-171,862$ & 2,609 & 6,415 & $-171,862$ \\
\hline 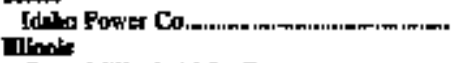 & - & - & - & - & $B A K, 2 D 7$ & 840,269 & 23,200 \\
\hline 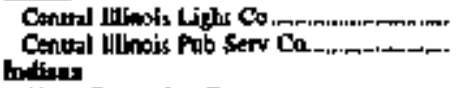 & $\overrightarrow{-}$ & 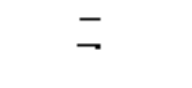 & 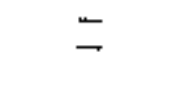 & $\overline{-}$ & $\begin{array}{l}1, \pi 56 \\
2,592\end{array}$ & 2322 & $\begin{array}{r}-15,636 \\
52,620\end{array}$ \\
\hline 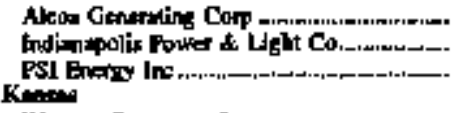 & $\bar{T}_{18,604}$ & $\begin{array}{r}21 \\
76,429\end{array}$ & $\overline{-}_{\mathbf{7 5 , 7 4 4}}$ & $\bar{z}$ & $\begin{array}{l}165,160 \\
109 \\
90,610\end{array}$ & $\begin{array}{r}170524 \\
515 \\
96,10 \mathrm{~s}\end{array}$ & $\overline{\bar{z}}$ \\
\hline 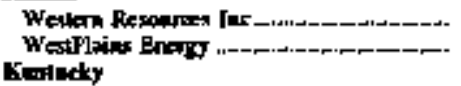 & $\bar{z}$ & - & 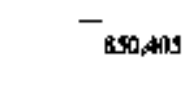 & $\overline{-}$ & $\begin{array}{r}2,865 \\
.51,365\end{array}$ & $2,30,403$ & - \\
\hline 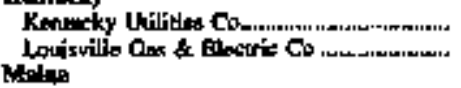 & 二 & 32 & $\overline{-}$ & $\overline{-}$ & 192, 104 & $\begin{array}{r}90,056 \\
1,720\end{array}$ & 二 \\
\hline Mine Pabtle Servien Co....................... & - & 658 & - & - & 650 & $\rightarrow$ & - \\
\hline 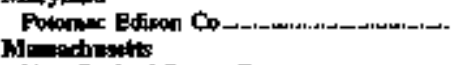 & - & - & - & - & 266,568 & 281,641 & $-211,878$ \\
\hline 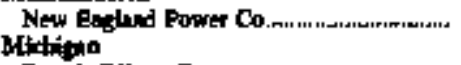 & - & - & - & - & 140,124 & Jin],939 & - \\
\hline 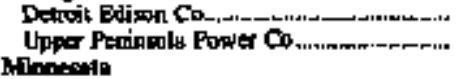 & $\overline{-}$ & 278 & 923 & - 4098 & $\overline{I P Y, 914}$ & $\begin{array}{l}29,420 \\
17,029\end{array}$ & 3,654844 \\
\hline 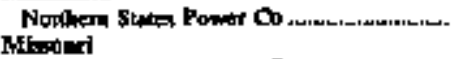 & - & - & 102,532 & - & 10,616 & 130,486 & - \\
\hline 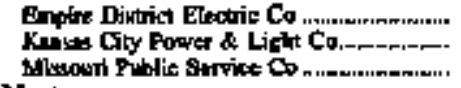 & 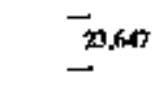 & $\bar{z}$ & $\bar{z}$ & $\bar{z}$ & $\begin{array}{r}3,195 \\
9,734 \\
61,395\end{array}$ & $\begin{array}{r}2,695 \\
7,515 \\
0,389\end{array}$ & $\bar{z}^{-}$ \\
\hline 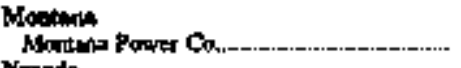 & - & 42 & - & - & 1500,619 & $1,449.851$ & $4,755,871$ \\
\hline 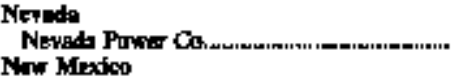 & مـ & sminn & 81000 & - & YA, & 8], 100 & - \\
\hline 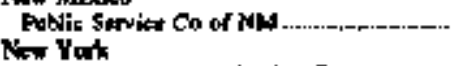 & - & 9,009 & 2,086 & - & 165,213 & 125,367 & - \\
\hline 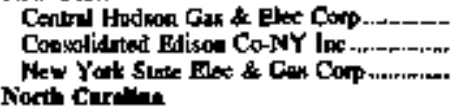 & $\bar{z}$ & $z^{69,991}$ & $\overline{-}^{5,968}$ & $\bar{z}$ & 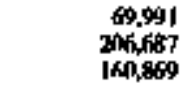 & $\begin{array}{r}3,968 \\
313,142 \\
240,875\end{array}$ & $\sin _{2,732,4 \times 0}$ \\
\hline 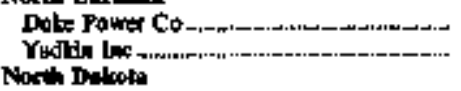 & $2,37), 305$ & $-56,009$ & $-\quad 50,265$ & $\overline{-}$ & $\begin{array}{r}3,876,394 \\
351,559\end{array}$ & 3,405,780 & $4,124,543$ \\
\hline 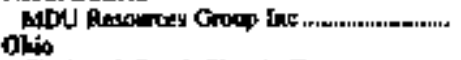 & - & - & - & - & 11,008 & 21,387 & - \\
\hline 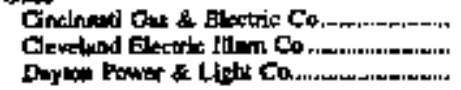 & $\bar{z}$ & $-^{62,1033}$ & ${ }^{-}$ & $\begin{array}{l}- \\
z\end{array}$ & $\begin{array}{r}62,1668 \\
37,191 \\
4,853\end{array}$ & $\begin{array}{r}191,459 \\
170,549 \\
2,140\end{array}$ & $\begin{array}{r}71,109 \\
-2,120,724 \\
6,7,325\end{array}$ \\
\hline
\end{tabular}


Table 21. Electricity Exchanges by Investor-Onned Utilities, by State, 1994 (Cantinued)

\begin{tabular}{|c|c|c|c|c|c|c|c|}
\hline \multirow{2}{*}{ Stated } & \multicolumn{3}{|c|}{ Invosien-Owned } & \multicolumn{3}{|c|}{ Fodornd } & \multirow{2}{*}{ 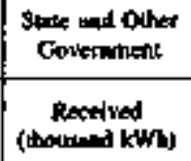 } \\
\hline & 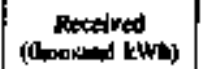 & 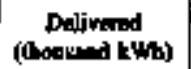 & $\begin{array}{l}\text { Suthent } \\
\text { (dellont) }\end{array}$ & $\begin{array}{c}\text { Pesedved } \\
\left.\text { (thaosind } \mathbf{k}^{\mathbf{W}} / \mathrm{b}\right)\end{array}$ & 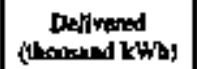 & $\begin{array}{l}\text { Settlonimat } \\
\text { (dollan) }\end{array}$ & \\
\hline \multicolumn{8}{|l|}{$\overline{\text { ans }}$} \\
\hline Gio Bdixom Co... & ID6, It5] & $16,1,37$ & 2237,916 & - & - & - & - \\
\hline 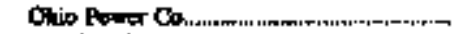 & 4,250 & 2,12 & - & - & - & - & - \\
\hline Tolizdo Edipo Co & 37,638 & 21,413 & $1,4+4,700$ & - & - & - & - \\
\hline Obluhine & & & & & & & \\
\hline 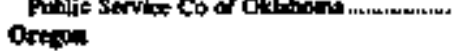 & 27,965 & 27,363 & - & $123,76]$ & $12 x, 146$ & T2Aנה & 760,199 \\
\hline 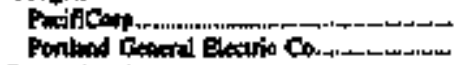 & $\begin{array}{l}30,304 \\
488,183\end{array}$ & $\begin{array}{l}619,359 \\
559,575\end{array}$ & 20,227 & $13,184,614$ & $\begin{array}{r}13,579,965 \\
463,074\end{array}$ & $\begin{aligned}-38,506,294 \\
84,376\end{aligned}$ & $\begin{aligned} 317,404 \\
56,393\end{aligned}$ \\
\hline$P=$ & & & & & & & \\
\hline 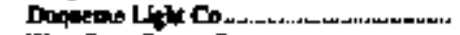 & 171,852 & $3 \$ 2,330$ & - & שמ & - & - & - \\
\hline 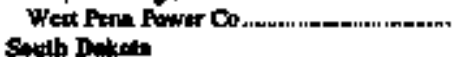 & 130,162 & 145,930 & -230544 & - & - & - & + \\
\hline 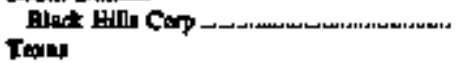 & 6,436 & 6,436 & - & 9.90 & $9, \mathrm{~mm}$ & - & - \\
\hline a foner \& Lifit Ca & 59,302 & 61,384 & $-5,568$ & - & $\leftarrow$ & - & $2], 968$ \\
\hline 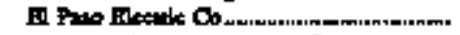 & 17,500 & 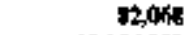 & - & - & - & - & 21.14 \\
\hline 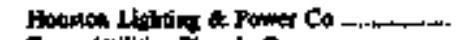 & $4,377,399$ & $12,966,989$ & - & - & - & - & 356,127 \\
\hline 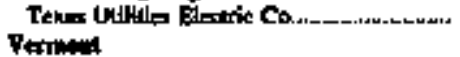 & $15,6 \pm 9.405$ & $7,907,559$ & - & - & - & - & $-200,90$ \\
\hline 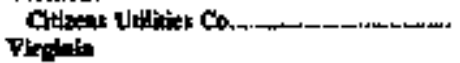 & 57,893 & 34,542 & 73,168 & - & - & - & - \\
\hline 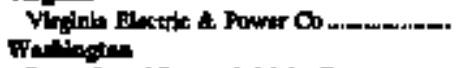 & 35,144 & 21,J植 & 一 & 12,01 & 12248 & - & 一 \\
\hline 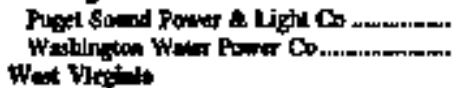 & $\begin{array}{l}415,1006 \\
770,873\end{array}$ & $\begin{array}{l}413,100 \\
653,2 \times 0\end{array}$ & $1,900,420$ & $\begin{array}{l}226,945 \\
300,526\end{array}$ & $\begin{array}{l}304,580 \\
470,455\end{array}$ & $\begin{aligned}-531,556 \\
-1,840,653\end{aligned}$ & $\begin{array}{l}7,104 \\
4,322\end{array}$ \\
\hline 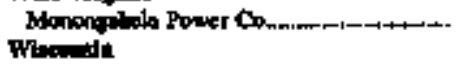 & T1.06 & 79,434 & $-196,078$ & - & - & - & - \\
\hline 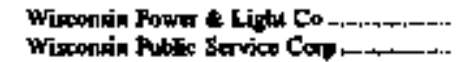 & $\begin{array}{l}11,655 \\
4105\end{array}$ & 2811 & $\bar{z}$ & $\overline{-}$ & $\overline{-}$ & $\bar{z}$ & $\bar{z}$ \\
\hline
\end{tabular}

see notes and footrones at and of thile 
Tabte 21. Electricity Exchanges by Investor-Owned Utillties, by State, 1994 (Continued)

\begin{tabular}{|c|c|c|c|c|c|c|c|}
\hline \multirow{2}{*}{. Dutity } & \multicolumn{2}{|c|}{$\begin{array}{l}\text { State and Other } \\
\text { Gavenumequ }\end{array}$} & \multicolumn{3}{|c|}{ Menicipal } & \multicolumn{2}{|c|}{ Coopentive } \\
\hline & $\begin{array}{c}\text { Dellowerod } \\
\text { (thousend }\end{array}$ & $\begin{array}{l}\text { Setulemeat } \\
\text { (dollars) }\end{array}$ & $\begin{array}{c}\text { Recolined } \\
\text { thoosend KWhy }\end{array}$ & 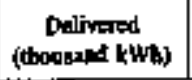 & $\begin{array}{l}\text { Setthement } \\
\text { (dolloming }\end{array}$ & 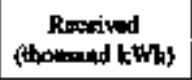 & 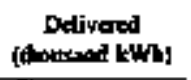 \\
\hline \multicolumn{8}{|l|}{$\overline{0 \%}$} \\
\hline CAdo Bdinon Conm & - & - & - & - & - & - & $\rightarrow$ \\
\hline 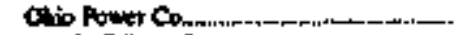 & - & - & 20,95 & 20,058 & - & - & - \\
\hline 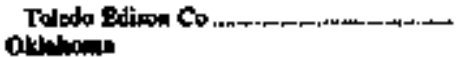 & - & - & - & - & - & - & - \\
\hline 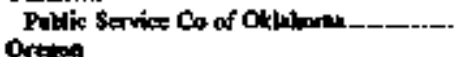 & 257,125 & - & - & - & - & - & - \\
\hline Puiffen & $\begin{array}{r}311,776 \\
64,932\end{array}$ & $-474,859$ & $\begin{array}{l}\mathbf{4 6 , 7 4 4} \\
52,746\end{array}$ & $\begin{array}{l}42,949 \\
41,468\end{array}$ & 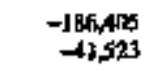 & $-^{125098}$ & -96.025 \\
\hline Peponjhald & & & & & & & \\
\hline 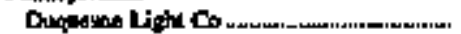 & - & - & - & - & - & - & - \\
\hline 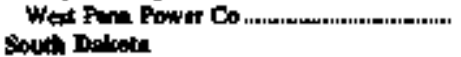 & - & - & - & - & - & - & - \\
\hline 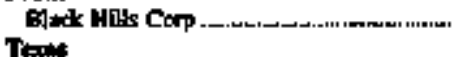 & - & - & - & - & - & - & - \\
\hline \multicolumn{8}{|l|}{ Then: } \\
\hline Condral Pown \& Light Co & 20,311 & - & - & - & - & 64.446 & 69267 \\
\hline 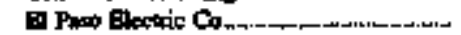 & 11,362 & - & - & - & - & - & - \\
\hline 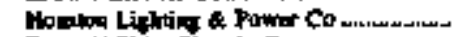 & 0,167 & - & $4,22,9,99$ & 7,133 & - & - & - \\
\hline Texps It & I,811,535 & - & $4,607,664$ & $10,231,59$ & - & - & - \\
\hline 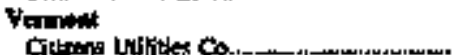 & & & & & & & \\
\hline 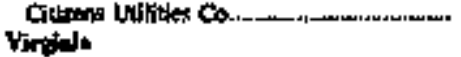 & - & - & - & - & - & - & - \\
\hline 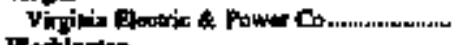 & - & - & - & - & - & - & - \\
\hline 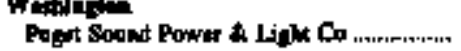 & 13,487 & $-57,793$ & 114,750 & I1d,945 & -500692 & - & - \\
\hline 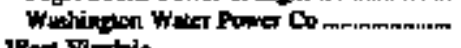 & I6.513 & $-\mathrm{J} 26 \mathrm{A12}$ & - & - & - & - & - \\
\hline 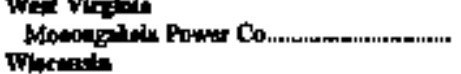 & - & - & - & - & - & - & - \\
\hline 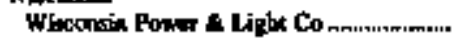 & س & - & - & - & - & - & - \\
\hline 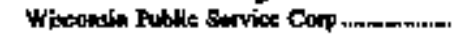 & - & - & - & - & - & - & - \\
\hline
\end{tabular}


Table 21. Electrictity Exchanges by Investor-Owned Utillites, by State, 1994 (Continued)

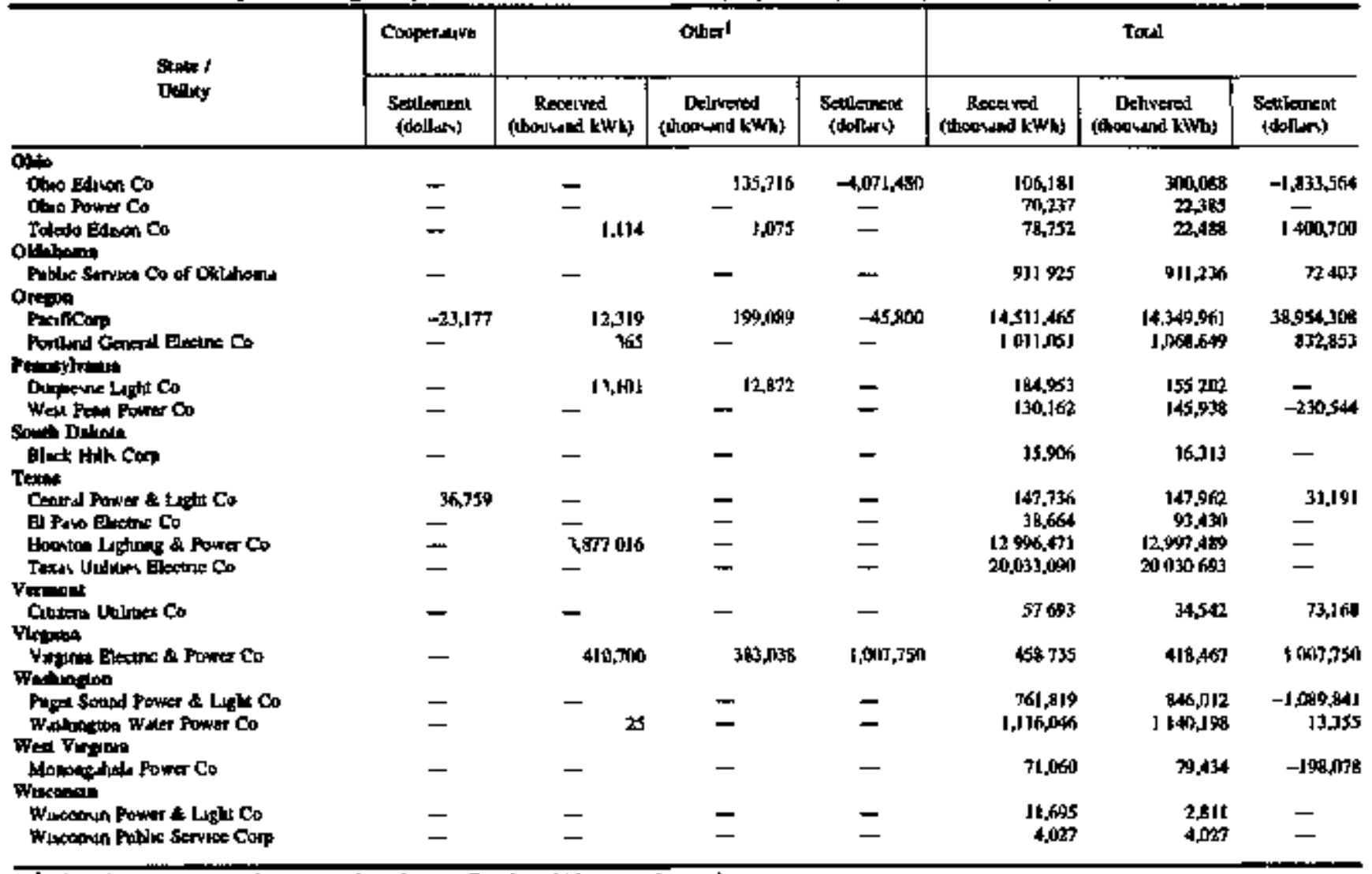

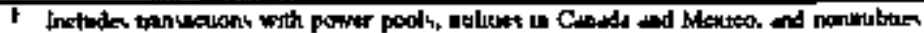

Nlot Aplueptit

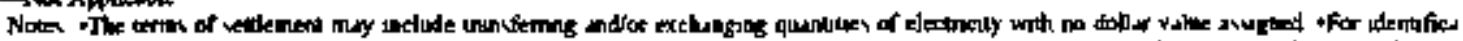

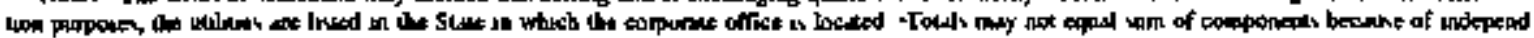
enc wounding

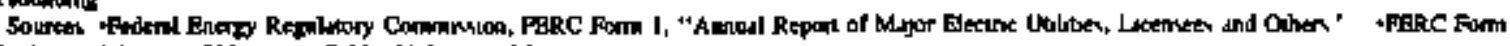

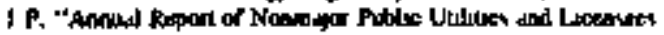


Tabte 22. Electricity Purchases by Investor-0med Uthities Not Reported to the Federal Eneroy Regulalory Commission, by State, 1994

\begin{tabular}{|c|c|c|c|c|c|c|c|}
\hline \multirow{2}{*}{$\begin{array}{l}\text { Stolt l } \\
\text { Unitity }\end{array}$} & \multicolumn{7}{|c|}{ 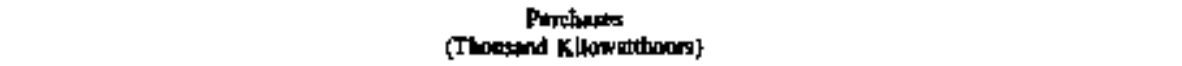 } \\
\hline & Intestin- & Foderi & $\begin{array}{l}\text { Stat and } \\
\text { Oaler } \\
\text { Odromment }\end{array}$ & Mtunielpal & Coppernitse & Gather & Total \\
\hline \multicolumn{8}{|l|}{ Aloo:he } \\
\hline 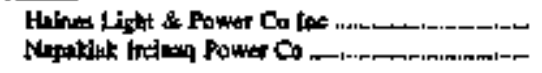 & -57 & - & - & - & - & 424 & 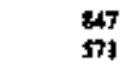 \\
\hline \multicolumn{8}{|l|}{ Adrocen } \\
\hline 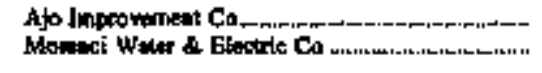 & - & $\longrightarrow$ & 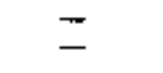 & $\overline{-}$ & $=27,033$ & $-9,150$ & $\begin{array}{r}9,450 \\
27,030\end{array}$ \\
\hline \multicolumn{8}{|l|}{ Rimuse } \\
\hline 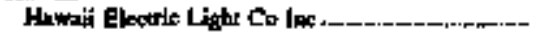 & 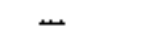 & - & - & - & - & $353,44 \mathbf{t}$ & 153,448 \\
\hline \multicolumn{8}{|l|}{ Mnnmin } \\
\hline Champlon Indenutional Cosp .......................... & 2,278 & - & - & - & - & - & 2,2808 \\
\hline 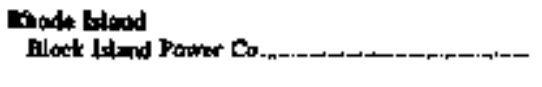 & - & - & - & - & - & 9 & 9 \\
\hline Chent & & & & & & & \\
\hline 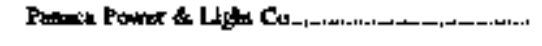 & - & - & 6,027 & - & - & - & (4, \\
\hline 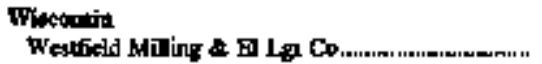 & Inj84 & - & - & - & - & - & 10,084 \\
\hline
\end{tabular}

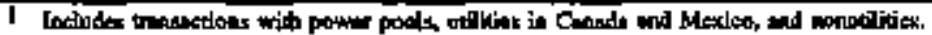

$\rightarrow$ Not Applicuilde

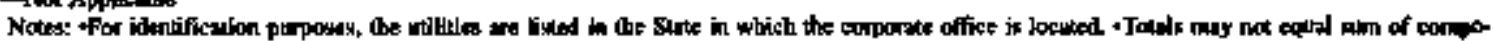
nows becurese of independert roouding.

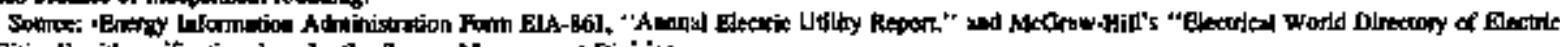

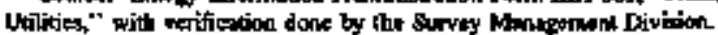


Table 23. Electricity Wheeling by Investor-Owned Utilties, by State, 1994

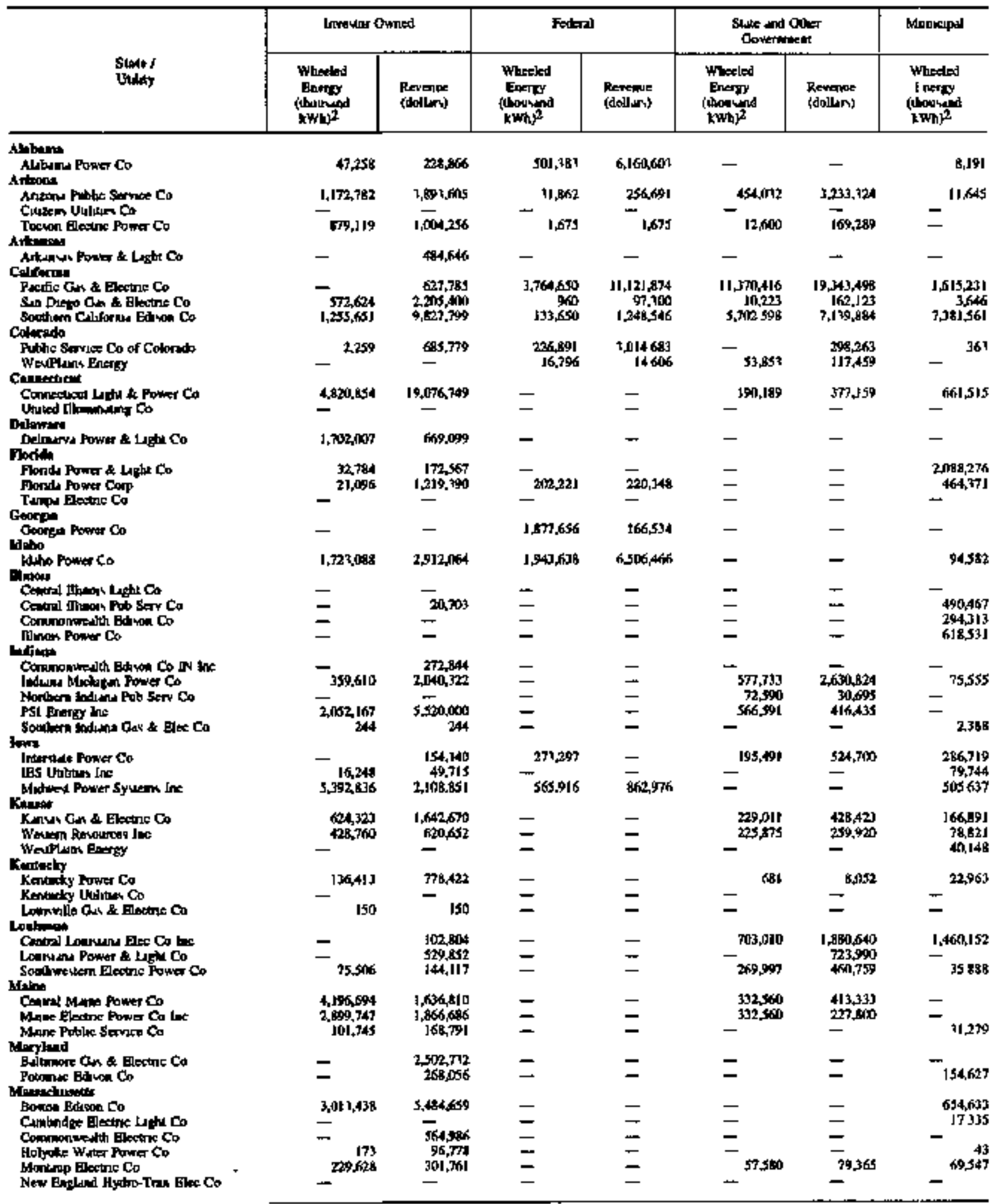


Table 23. Electrlcity Wheeliug by Investor-Owned Utilities, by State, 1994 (Continued)

\begin{tabular}{|c|c|c|c|c|c|c|c|}
\hline \multirow[b]{2}{*}{ Stimef } & \multirow{2}{*}{$\begin{array}{l}\text { Morucipal } \\
\text { Revenus } \\
\text { (dollew) }\end{array}$} & \multicolumn{2}{|c|}{ Cooperutre } & \multicolumn{2}{|l|}{ Oakrl } & \multicolumn{2}{|l|}{ Tous } \\
\hline & & 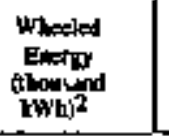 & $\begin{array}{l}\text { Reventue } \\
\text { (dollari) }\end{array}$ & 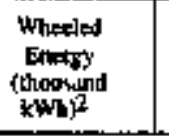 & $\begin{array}{l}\text { Revephe } \\
\text { (doldari) }\end{array}$ & 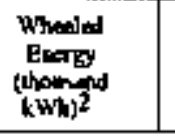 & $\begin{array}{l}\text { Revenue } \\
\text { (dodtmr) }\end{array}$ \\
\hline 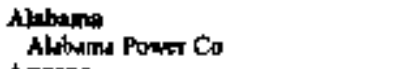 & 19.856 & 1,74809 & $7,501,505$ & - & - & 2304,921 & 13 9I0,800 \\
\hline 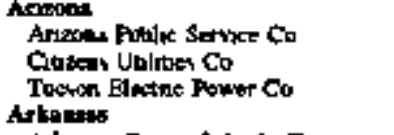 & $\begin{array}{l}143,656 \\
-\end{array}$ & $\begin{array}{c}184, B 51 \\
-\quad 96,1, K 38\end{array}$ & 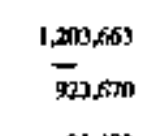 & $\begin{array}{r}272,436 \\
-\quad 651 \\
-\quad\end{array}$ & $\begin{array}{l}774,757 \\
-\end{array}$ & $\begin{array}{r}2127.6018 \\
651 \\
1,855,0052\end{array}$ & 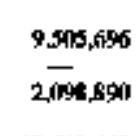 \\
\hline Ankan us Punver 4 Lindi Co & - & - & 91.483 & - & $17,04,1333$ & - & $17,619,462$ \\
\hline 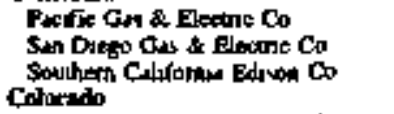 & $\begin{array}{r}14,556,040 \\
7,158 \\
16,901,187\end{array}$ & ${ }^{-}$ & $\begin{array}{l}\overrightarrow{\mathrm{Jj}_{7}, 724} \\
\end{array}$ & $-^{528,227}$ & 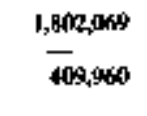 & $\begin{array}{r}17,078,524 \\
587,453 \\
14,569,651\end{array}$ & $\begin{array}{r}47,451,269 \\
2,471,981 \\
35,665,1061\end{array}$ \\
\hline $\begin{array}{l}\text { Fobluc Semoe Co of Colorats } \\
\text { Westrluns Energy } \\
\text { Conneclycut }\end{array}$ & $149,64\}$ & $\begin{array}{l}698,672 \\
697,093\end{array}$ & $\begin{array}{r}1,970,9 / 68 \\
753,34,3\end{array}$ & $r_{19896}$ & $\tilde{g}_{2, J 73}$ & $\begin{array}{r}1,138,185 \\
127,6988\end{array}$ & $\begin{array}{l}6,119,350 \\
\text { S97, } 1901\end{array}$ \\
\hline 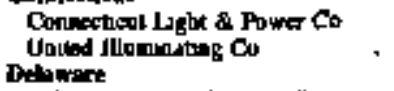 & 2961,909 & $\overline{-}$ & $\overline{-}$ & $\begin{aligned} 49+159 \\
31,0+2\end{aligned}$ & $\begin{array}{l}3.143,140 \\
3677,218\end{array}$ & $\begin{array}{l}6,6 \times 5,617 \\
51,042\end{array}$ & $\begin{array}{r}25,560,997 \\
367,218\end{array}$ \\
\hline 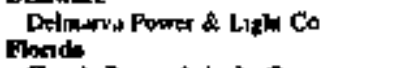 & - & - & - & - & 1,705 & 1.xosing & $6 m, 8015$ \\
\hline 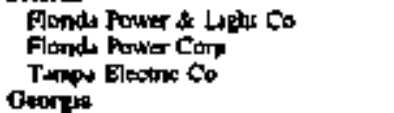 & $\begin{array}{l}7,200,397 \\
3,078,736 \\
-\end{array}$ & $\begin{array}{r}7,467,369 \\
-\quad 31,349 \\
-\end{array}$ & $\begin{array}{l}10,653,591 \\
8,696,530 \\
-\end{array}$ & $\begin{array}{l}\$ 14,427 \\
567.406 \\
703,495\end{array}$ & $\begin{array}{l}1,201,449 \\
2,277,3015 \\
2059,359\end{array}$ & $\begin{array}{l}3,902,846 \\
1,286,443 \\
703,495\end{array}$ & $\begin{array}{l}19.264 .914 \\
15.480 .645 \\
2 \operatorname{tog} 359\end{array}$ \\
\hline $\begin{array}{l}\text { Georgu Powe Ca } \\
\text { Lato }\end{array}$ & - & - & - & - & - & $1,877,696$ & 166,534 \\
\hline Idtho Power Co & 980,120 & - & - & 9,216 & 15,850 & 1.730 .504 & [n.41450 \\
\hline 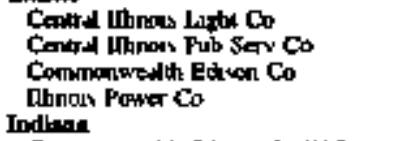 & $\begin{array}{l}-\overline{417,610} \\
1,559,308 \\
2,307.735\end{array}$ & $\begin{array}{l}29,855 \\
-\end{array}$ & $\begin{array}{l}56,154 \\
7,067,669 \\
= \\
-\end{array}$ & $\begin{array}{l}\overline{-} \\
\overline{-}\end{array}$ & $\begin{array}{l}\bar{z} \\
\bar{z}\end{array}$ & $\begin{array}{r}23,855 \\
1,434,383 \\
744,313 \\
618,591\end{array}$ & $\begin{array}{r}66,154 \\
13.905 .973 \\
1,559,308 \\
2,307,735\end{array}$ \\
\hline Comunantedth Rition Co tN Ine & - & - & - & - & - & - & 272,844 \\
\hline 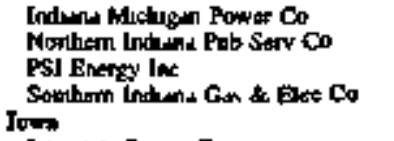 & $\begin{array}{l}486,951 \\
-\end{array}$ & $\begin{array}{r}542, \operatorname{sing} \\
982,230 \\
2,735,729 \\
14,876\end{array}$ & $\begin{array}{r}4,989,6001 \\
40,5+2 \\
4,198,949 \\
69,484\end{array}$ & $\overline{-}^{-30,332}$ & $\begin{array}{r}93.377 \\
4,505 \\
-\quad 13,314\end{array}$ & $\begin{array}{r}1,52,175 \\
1,0,1,820 \\
5,357,097 \\
47,418\end{array}$ & $\begin{array}{r}10,241,094 \\
437,742 \\
10,] 35,324 \\
84, \sqrt{704}\end{array}$ \\
\hline 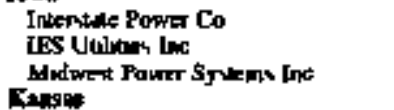 & $\begin{array}{r}1,171,896 \\
10,000 \\
1,039, \pi 21\end{array}$ & $\begin{array}{r}500,547 \\
85,092 \\
1,191,445\end{array}$ & $\begin{array}{l}1,263,922 \\
107,118 \\
474,787\end{array}$ & $\begin{array}{r}\mathbf{1 6}, 941 \\
40,904\end{array}$ & $\overline{\overline{100}}(000$ & 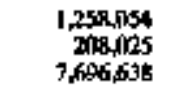 & $\begin{array}{r}3,1,17,9648 \\
37,6,633 \\
4,465,635\end{array}$ \\
\hline 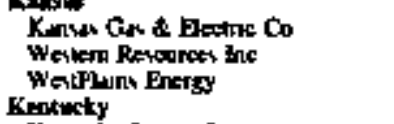 & $\begin{array}{l}110.341 \\
159.190 \\
778.504\end{array}$ & $\begin{array}{l}41,553 \\
921,042 \\
267,245\end{array}$ & $\begin{array}{l}2,845,796 \\
5,185,413 \\
1,003,475\end{array}$ & $\begin{array}{r}12,050 \\
-\quad 10000\end{array}$ & $\begin{array}{l}16,023 \\
16023 \\
-\end{array}$ & $\begin{array}{r}1,4,2,825 \\
1,46,5,549 \\
397,393\end{array}$ & $\begin{array}{l}5,341, \text {, } 533 \\
\times, 241, \text {,ns } \\
2,181,979\end{array}$ \\
\hline 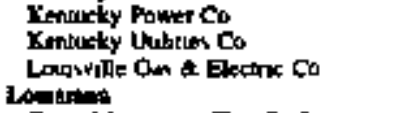 & $\begin{array}{l}121,622 \\
-\end{array}$ & $\begin{array}{l}61,1689 \\
921,046 \\
186,99\end{array}$ & $\begin{array}{r}191,262 \\
1,01,981 \\
18 \times, 795\end{array}$ & ${ }_{-}^{7,758}$ & $\begin{array}{l}54,653 \\
-\end{array}$ & $\begin{array}{l}231,123 \\
926,046 \\
165,945\end{array}$ & $\begin{array}{r}1,199,011 \\
1,400,941 \\
105,945\end{array}$ \\
\hline 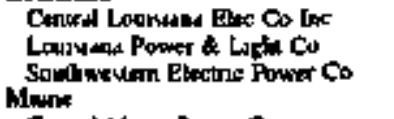 & $\begin{array}{l}3,765,600 \\
\overline{124,734}\end{array}$ & $\begin{array}{l}1,540,5835 \\
\frac{2}{2,1}, 0,504\end{array}$ & $\begin{array}{l}5,146,838 \\
1,910,285 \\
4,987, \$ 32\end{array}$ & $\overline{-}_{61,738}$ & $\frac{\bar{z}}{160,429}$ & $\frac{3,206,748}{2,57,3,953}$ & $\begin{array}{r}10,395,892 \\
5,164,123 \\
5,877,571\end{array}$ \\
\hline 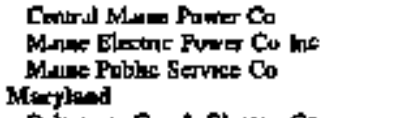 & $\begin{array}{l}\bar{z}_{13,931} \\
\end{array}$ & $\bar{z}$ & $\bar{z}$ & $\begin{array}{r}273,562 \\
64,724 \\
137,385\end{array}$ & $\begin{array}{r}1,51981 \\
48], 015 \\
1,709,068\end{array}$ & $\begin{array}{r}4,802,816 \\
3,298,031 \\
470,419\end{array}$ & $\begin{array}{l}3,274,124 \\
2,573,501 \\
1,930,7901\end{array}$ \\
\hline 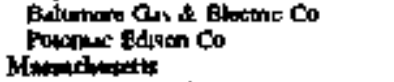 & $\overline{961, f i s 5}$ & $\bar{z}$ & $\overline{-}$ & $-\omega_{1062}$ & $\begin{array}{r}1,127,415 \\
0,332\end{array}$ & ${ }_{168.589}$ & $\begin{array}{l}3,670,147 \\
1,312,483\end{array}$ \\
\hline 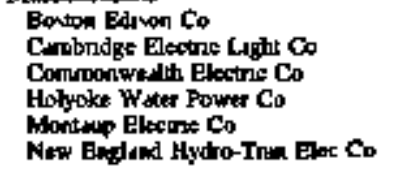 & $\begin{array}{l}1,921,715 \\
171,287 \\
248,320 \\
212,155 \\
-\end{array}$ & $\begin{array}{l}\bar{z} \\
\bar{z} \\
\bar{z}\end{array}$ & $\begin{array}{l}\bar{z} \\
\bar{z} \\
\bar{z}\end{array}$ & $\begin{array}{l}1,5,51,58 \\
24,163 \\
- \\
- \\
-\end{array}$ & $\begin{array}{r}4,1201,839 \\
351,727 \\
211,429 \\
2,190 \\
2,160,363 \\
49,693.131\end{array}$ & $\begin{array}{r}5.219 .329 \\
41.497 \\
-\quad 216 \\
1.150 .590\end{array}$ & $\begin{array}{r}11,527,213 \\
522,014 \\
1,024,729 \\
99,014 \\
2,780,644 \\
0,895,131\end{array}$ \\
\hline
\end{tabular}


Table 23. Bectricity Wheeling by Envestor-0waed Uthities, by State, 1994 (Continued)

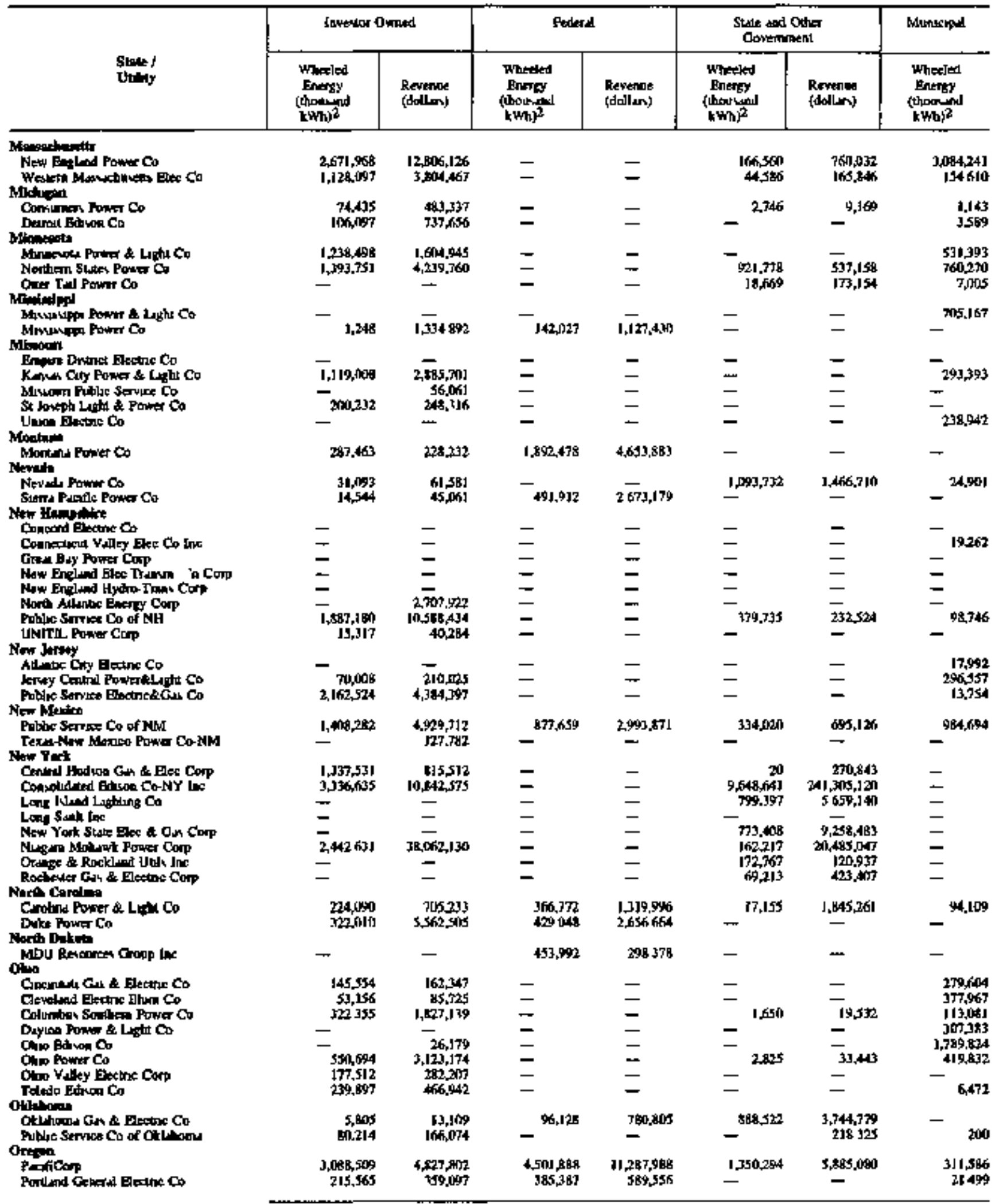


Table 23. Electricity Wheeling by Inrestor-Owned Utllthes, by State, 1994 (Continued)

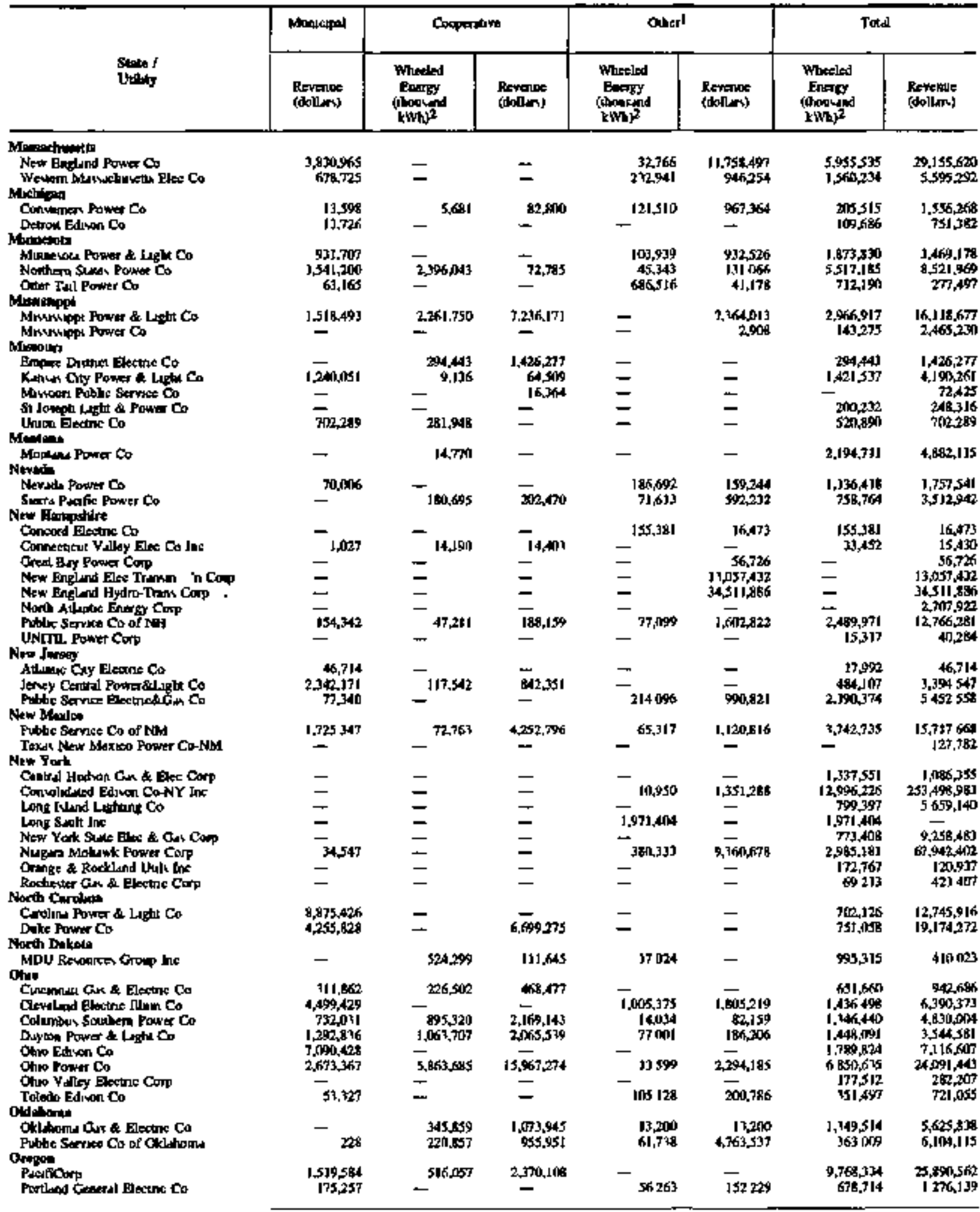


Table 23. Electricity Wheling by Investor-Owned Utillies, by State, 1994 (Continued)

\begin{tabular}{|c|c|c|c|c|c|c|c|}
\hline \multirow[b]{2}{*}{ 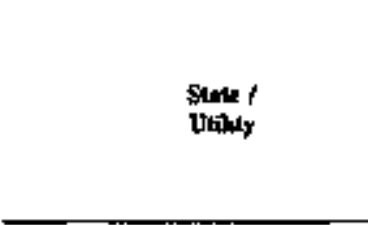 } & \multicolumn{2}{|c|}{ Enventor Onned } & \multicolumn{2}{|c|}{ Fededal } & \multicolumn{2}{|c|}{ 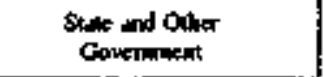 } & \multirow{2}{*}{ 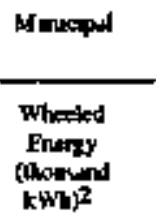 } \\
\hline & 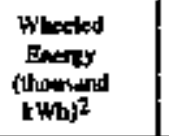 & $\begin{array}{l}\text { ReYenve } \\
\text { (doller) }\end{array}$ & 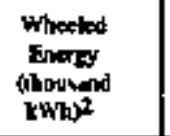 & $\begin{array}{l}\text { Acrentue } \\
\text { (ddilav }\end{array}$ & $\begin{array}{l}\text { Wheeled } \\
\text { Energy } \\
\text { (bound } \\
\text { KWh't }\end{array}$ & $\begin{array}{l}\text { Ravenos } \\
\text { (dellary) }\end{array}$ & \\
\hline \multicolumn{8}{|l|}{ 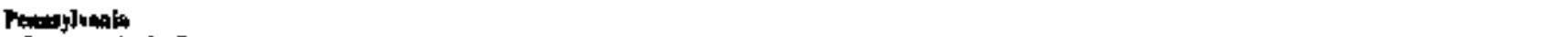 } \\
\hline 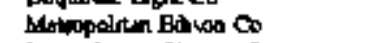 & $<2,201$ & $6,208,293$ & - & - & - & $\overline{-}$ & - 51,276 \\
\hline 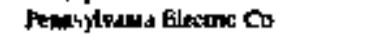 & 2462,649 & $9,353,165$ & 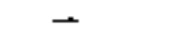 & - & سـ & - & 298,39 \\
\hline 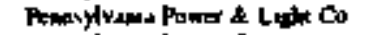 & 982.715 & $2,634.772$ & - & - & $=$ & - & 21,059 \\
\hline 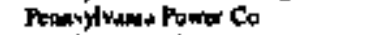 & - & $4,1 \$ 4$ & - & - & - & - & B.2Al \\
\hline PECó Exing to & - & - & - & - & - & - & - \\
\hline Was Pran Fower Ca & - & 65,070 & - & - & - & - & 200,544 \\
\hline \multicolumn{8}{|l|}{ 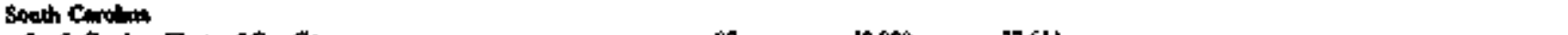 } \\
\hline 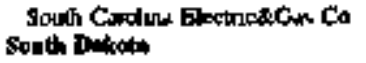 & - & 87 & 19,099 & 37 6IJ & - & - & - \\
\hline Slat malls Cont. & - & $m$ & - & - & B,96: & 55,764 & 29,440 \\
\hline Nondwentere Publoc Servece $\infty$ & - & - & 45,545 & 51.063 & - & - & 3,381 \\
\hline \multicolumn{8}{|l|}{ Tex: } \\
\hline 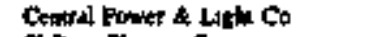 & - & 985,000 & - & - & - & 123,314 & 一 \\
\hline A Pareont Co & 171,423 & $325, B 18$ & - & - & $246,3,35$ & $1,236,496$ & - \\
\hline Golf States Itulukes Co & - & 719,059 & - & - & 699,978 & 3.169 .130 & 28,560 \\
\hline Howton Lephitupg \& Powne Co & $6,341,913$ & $8,810,04$ & - & - & 4,201 & (73) & 63,336 \\
\hline 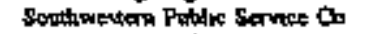 & $5,4 \mathbf{4 5}$ & $19, \mathbf{9 t}$ & - & - & - & - & 200 \\
\hline Tern Utinkes Electune Co & 6,254,659 & $11,09,150$ & - & - & $7,, 970$ & 118,933 & $3,297,421$ \\
\hline Texas Now Aloxiso Power Co & $2,631,136$ & S.tanding & - & - & - & - & - \\
\hline Wast Fexas Unulter Co & - & 818,338 & - & - & - & 601,220 & - \\
\hline \multicolumn{8}{|l|}{ Vermed } \\
\hline Cruted Vemont Pob Serv Corp & 0,815 & 15,568 & - & - & - & - & 153,443 \\
\hline Cousent Ubluten Co & 23,709 & 926,075 & - & - & - & - & 17,571 \\
\hline Gree: Mrounlan Poomer Cap & $10 . \% 1$ & {$[1, \pi]$} & - & - & - & - & $293, \sqrt{68}$ \\
\hline Yembat Eectinc Pover Co dn & $1,589,352$ & $0,209,263$ & - & - & - & - & 690,927 \\
\hline \multirow{2}{*}{\multicolumn{8}{|c|}{ Votpole }} \\
\hline & & & & & & & \\
\hline Applathen Powa Co & 687,602 & 3083300 & 9735 & $\$ 73,020$ & 3 1578 & 42,368 & $144 \leq 17$ \\
\hline 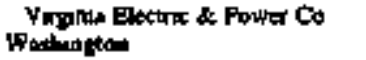 & - & - & $50 \mathrm{~N}+371$ & $2,302.265$ & - & - & 698,463 \\
\hline 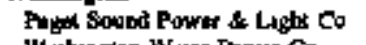 & 62,504 & 45,538 & 487326 & $1, \operatorname{sen} 35 t^{2}$ & $\$ 49.4 .5$ & 197,498 & 790\$13 \\
\hline 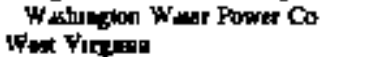 & \multicolumn{6}{|c|}{ Viet Virpard } & 641,188 \\
\hline Momong held Power co & - & 9,736423 & - & - & - & - & $t 13598$ \\
\hline \multirow{2}{*}{\multicolumn{8}{|c|}{ Wrocenting }} \\
\hline & & & & & & & \\
\hline Northwoveam Wheonim Elac Co & - & - & - & - & - & $=$ & - \\
\hline Sopanor Water Lugher.Ponver $\mathrm{Co}$ & 17 & 49.203 & - & - & - & - & - \\
\hline 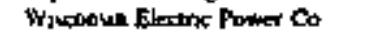 & 298 ] & 4068,862 & - & - & - & - & $1,172,407$ \\
\hline 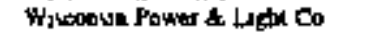 & 329,572 & 539,745 & - & - & - & - & 33007 \\
\hline Wuseonun Pable Servise Corp & 24ton & 8,400 & - & - & $m$ & - & 28,231 \\
\hline
\end{tabular}


Tabte 23. Electricity Wheeling by Investor-Owned Utilities, by State, 1994 (Continued)

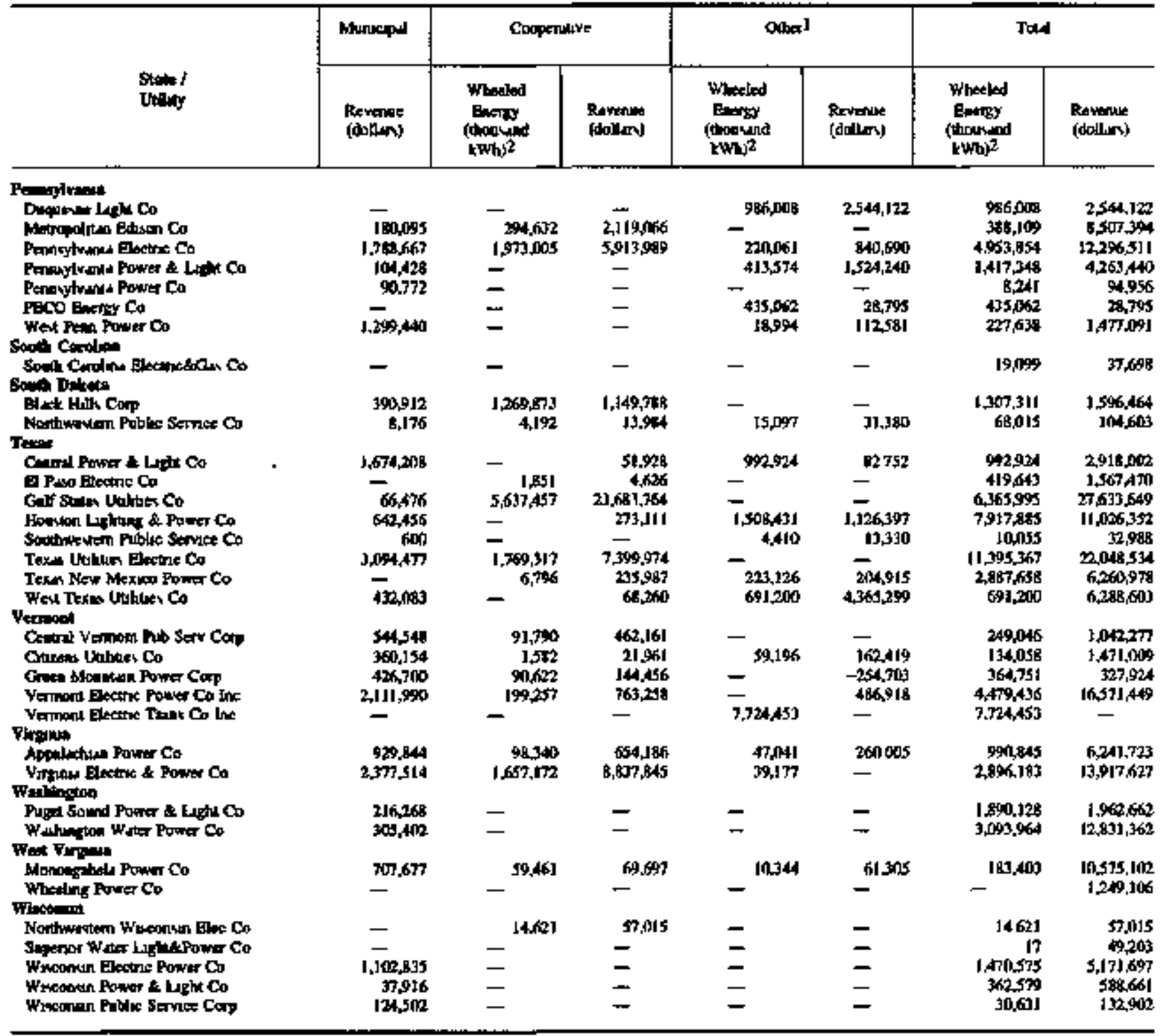

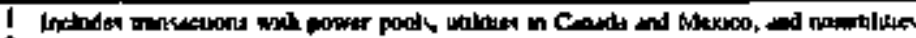

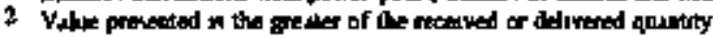

- Nor thomerhis

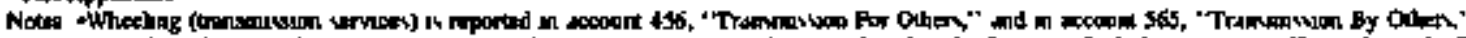

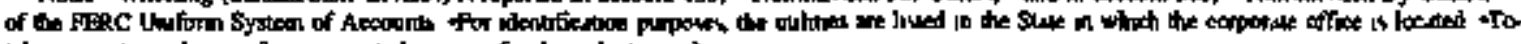

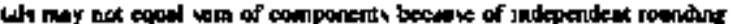

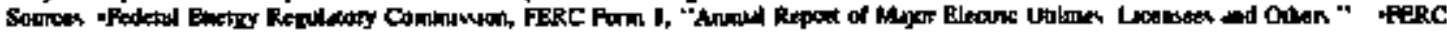

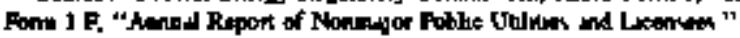


Table 24. Electricity Wheeling to Investor-Owned Utilities, by State, 1994

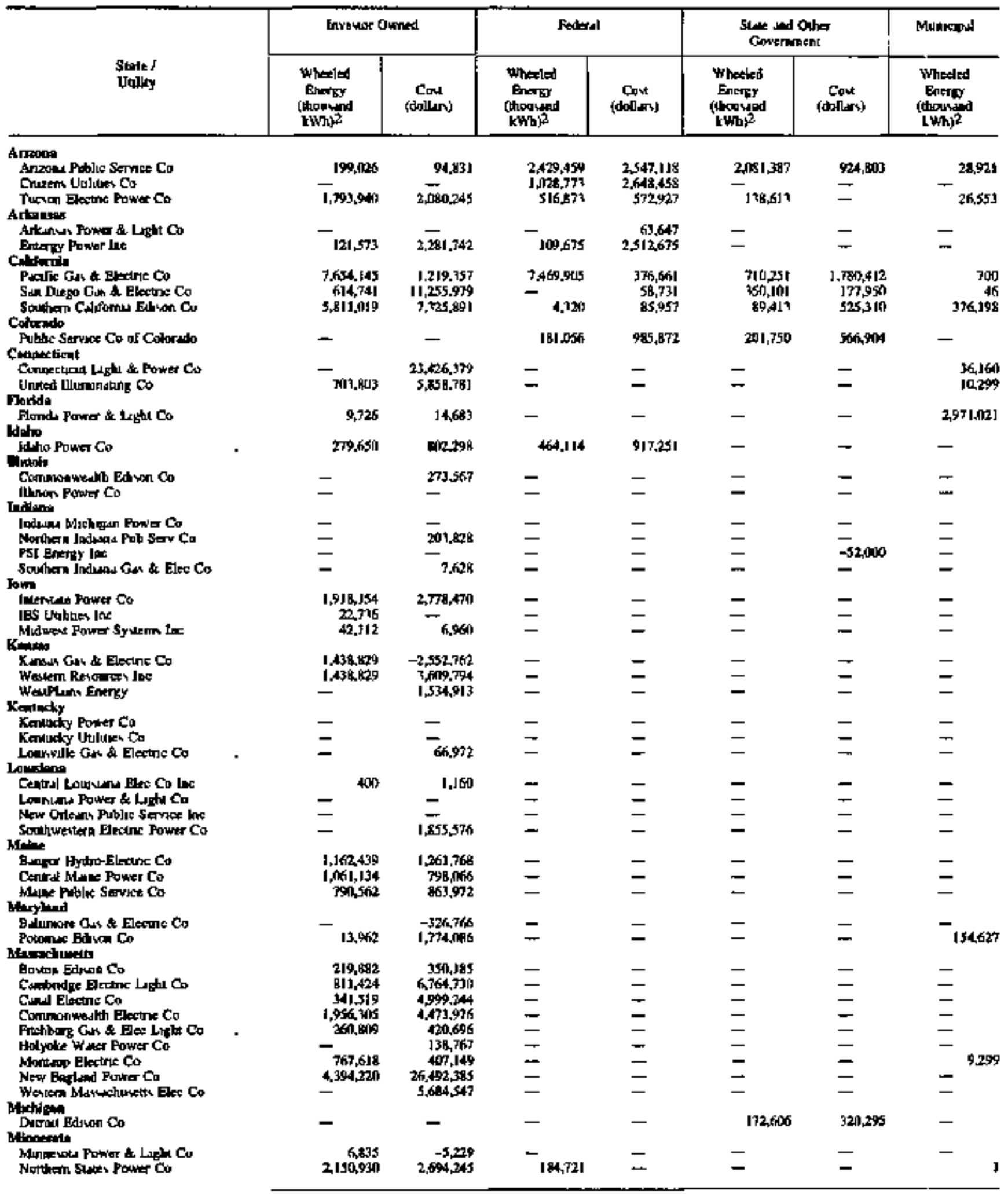

Set foun and footones and of wble 
Table 24. Electricity Wheeling to Investor-Owned Utilities, by State, 1994 (Continaed)

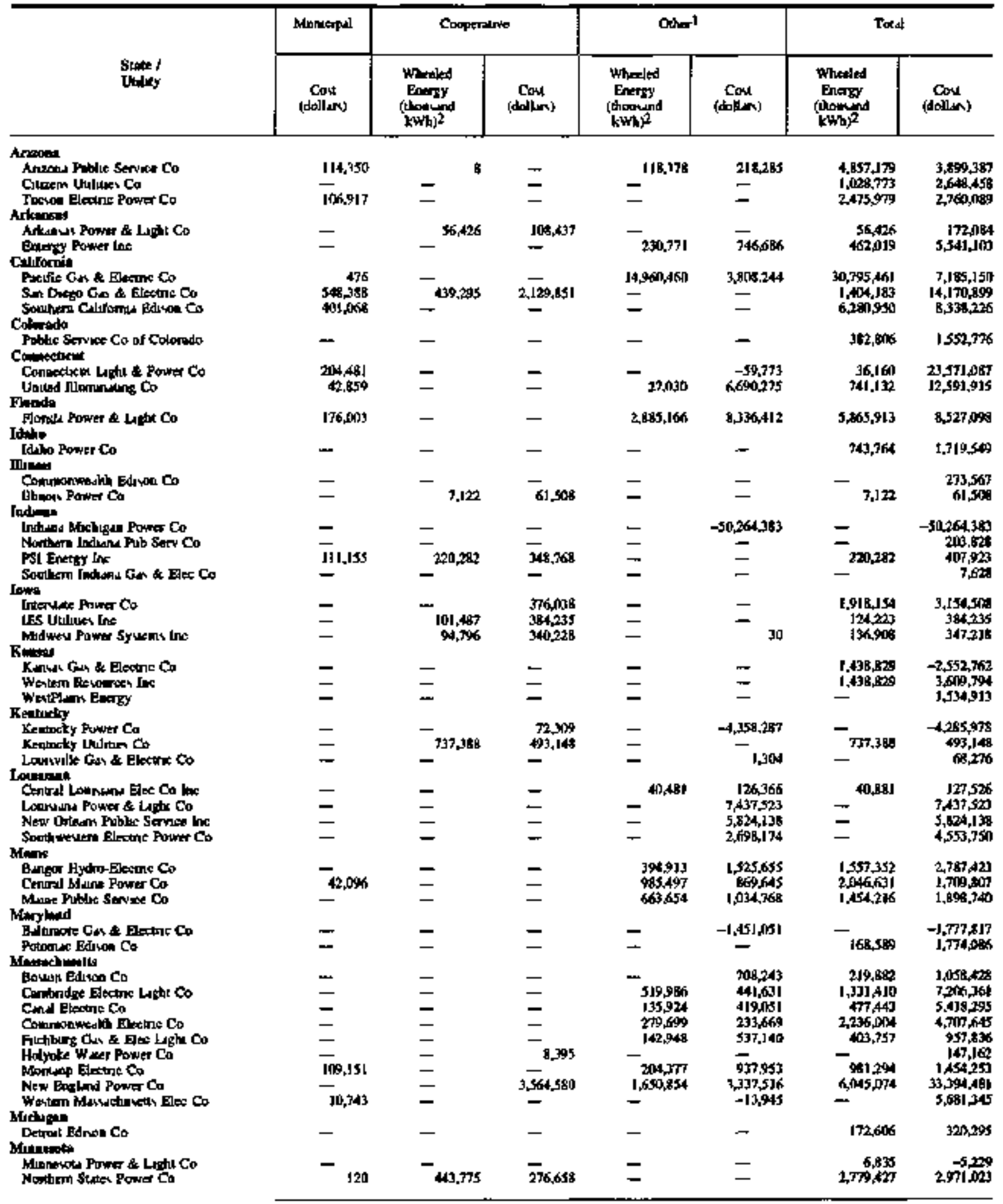


Table 24. Electricity Wheeling to Investor-Owned Utilities, by State, 1994 (Continued)

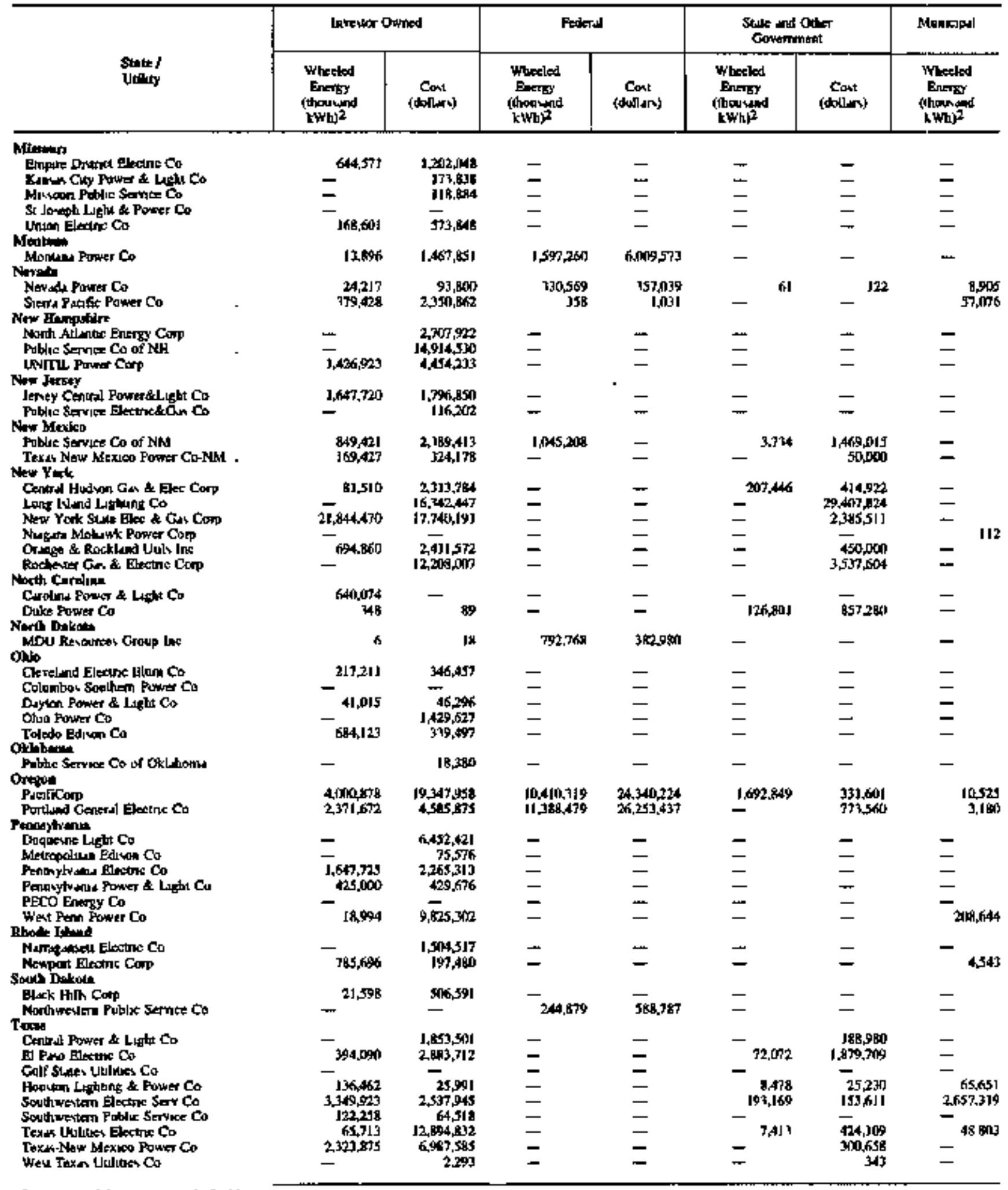


Table 24. Electricky Wheeling to Investor-Owned Utilities, by State, 1994 (Continaed)

\begin{tabular}{|c|c|c|c|c|c|c|c|}
\hline \multirow[b]{2}{*}{ 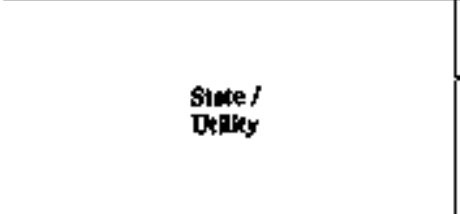 } & \multirow{2}{*}{ Moricilpul } & \multicolumn{2}{|c|}{ Cocperroive: } & \multicolumn{2}{|c|}{ Oubarl } & \multicolumn{2}{|c|}{ 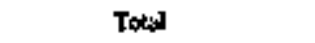 } \\
\hline & & 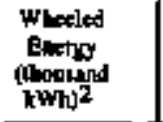 & $\underset{\text { (dollons) }}{\operatorname{Cos}}$ & 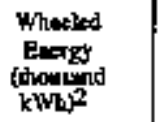 & $\begin{array}{c}\text { Conil } \\
\text { (da)lams) }\end{array}$ & 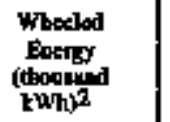 & $\begin{array}{c}\text { Cost } \\
\text { (dollarsi) }\end{array}$ \\
\hline \multicolumn{8}{|l|}{ Mistouxt } \\
\hline Errpis Distikt Eleatric Co & - & - & - & - & - & (4453) & $1,292,1,48$ \\
\hline 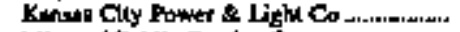 & 157,652 & - & - & - & ]4,BBDO & - & 346,290 \\
\hline 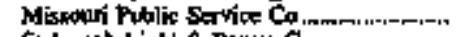 & - & - & 6.383 & $m$ & - & - & 125,267 \\
\hline 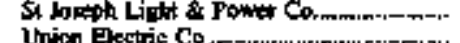 & - & 919 & 15,610 & 475 & - & 1094 & 15,600 \\
\hline 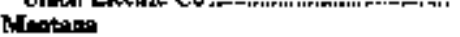 & & & & & & & \\
\hline Mopuraz Pawer Co & - & 3,507 & $99,286 \mathrm{~F}$ & - & - & $1,614,669$ & $7,4,6,3110$ \\
\hline Nevidy Poger Co ...rm & 280000 & - & - & - & - & 363,752 & $480,9 \% 1$ \\
\hline 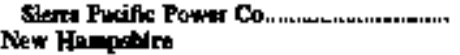 & 303,338 & - & - & 24,165 & - & Wilgh & 2.655 .231 \\
\hline 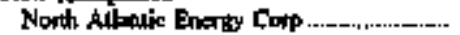 & - & - & - & - & - & - & $2,707,92$ \\
\hline Puthle Service Co of NH............ritimen. & - & - & 137,918 & - & 255,163 & - & $15,347,1 \pi 0$ \\
\hline 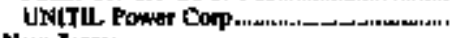 & - & - & - & $3 \% 4,874$ & G $6 \operatorname{2n} 459$ & $1,823.797$ & $5,1074,592$ \\
\hline \multicolumn{8}{|l|}{ New Jeriey } \\
\hline 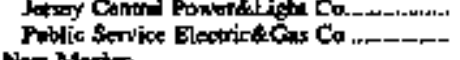 & $\overline{-}$ & $\overline{-}$ & $\overline{-}$ & $\overline{-}$ & - & $1,047,720$ & 116,210 \\
\hline 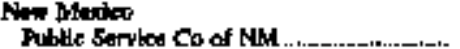 & - & $75 \sqrt{349}$ & 76,320 & - & - & $1,973,411$ & 3,934745 \\
\hline Teras.New Merico Powet Co-NM.-.-- & - & - & - & - & - & 169,427 & 374,178 \\
\hline 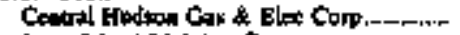 & - & - & - & - & 53,945 & 288.936 & $2782,6 \leq 1$ \\
\hline 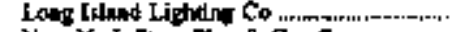 & - & - & - & $4,498,091$ & $1,128,479$ & $4,438,091$ & $46,078,750$ \\
\hline New Yort Stur Elec \& Gur Cor & - & - & - & - & Syls & $21,84,470$ & afh, $546,2+2$ \\
\hline 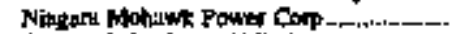 & 336 & - & - & 1,7IR0A9 & $1,851,782$ & $1,7,20 \mathrm{~J}$ & J,BS2, t18 \\
\hline 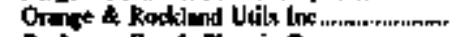 & - & - & - & - & $B 78,145$ & 89ASGN & $3,739.717$ \\
\hline Roxheswer Gue \& Eecric Cor __.............. & - & - & - & - & -265544 & - & 15.480,0,102? \\
\hline 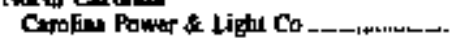 & - & - & - & - & - & $\{40,0\} 4$ & - \\
\hline Dnke Power Co .............................. & - & - & - & - & - & 127,649 & 897,369 \\
\hline 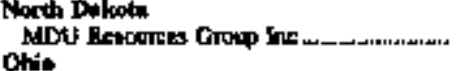 & - & 4,161 & 1,465 & 10,8015 & - & (n), 7 的) & 364,463 \\
\hline 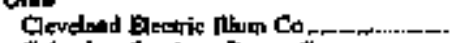 & - & - & - & - & - & 217,211 & 346,457 \\
\hline 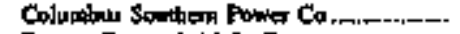 & - & - & - & - & $30,869,700$ & & $30,06,700$ \\
\hline 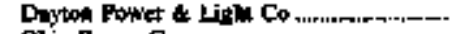 & - & - & - & - & - & 41,015 & 46,296 \\
\hline 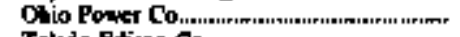 & - & - & - & - & $14324: 80 \%$ & & $15.754,433$ \\
\hline 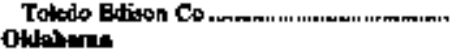 & - & - & - & 4,5] إ & 345,816 & 228,613 & 685.333 \\
\hline 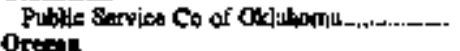 & - & - & - & - & $1,480,169$ & - & $1.498,549$ \\
\hline 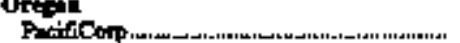 & 337,009 & $\mathbf{9 1 2 3 , 1 1 2}$ & 1,957996 & - & \multicolumn{3}{|c|}{ Oregan } \\
\hline 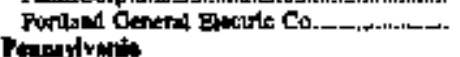 & & 758 & & $-18,996$ & $13,757,859$ & $32,945,499$ \\
\hline Dequeno Liebt Co & - & - & - & - & - & $=$ & $6,452,42 \mathrm{~J}$ \\
\hline Shinopolltan Bdl hat Co............................. & - & - & - & - & 299,762 & - & 375,738 \\
\hline 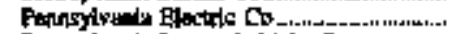 & - & - & - & - & - & $1,647.725$ & 2265,313 \\
\hline 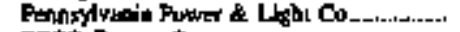 & - & - & - & - & - & $425, \mathrm{Bt}=\mathrm{n}$ & 429,676 \\
\hline PECó Enery Co & - & - & - & 475, , Nit2 & 24,795 & $435,0,5$ & 28,795 \\
\hline 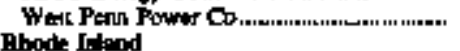 & \multicolumn{7}{|c|}{ Phode Iltand } \\
\hline Noristanted Electric Co. & - & - & - & - & _ & - & 1.504517 \\
\hline 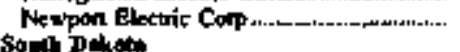 & $131 \sqrt{616}$ & - & - & 4,754 & $18 \mathrm{~d} 29 \mathrm{t}$ & Nos,sizs & 512,57 \\
\hline & - & - & - & $2 t=98$ & Shas \\
\hline Nonlwestm Poblie Swries Co .............. & - & 2,852 & 9,262 & - & $\neg$ & 247,311 & 398,049 \\
\hline \\
\hline 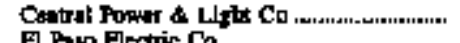 & 07,206 & - & $=$ & - & $4,606,195$ & ${ }_{160.162}$ & $6,715,110$ \\
\hline 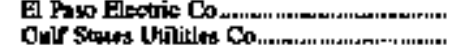 & $\bar{z}$ & $\bar{z}$ & $\bar{z}$ & $\bar{z}$ & $10,699,464$ & - & $10,699,484$ \\
\hline 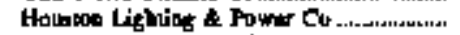 & 30,024 & 9.278 & 1,551 & - & - & 219.869 & 86,106 \\
\hline Soothweston Eledric sen Co.................. & 99,866 & $2,657,319$ & 43,549 & - & - & B.857,730 & 2830,971 \\
\hline Soulrweken Poblic Service Co & - & 5.171 & 3,070 & - & - & 127,429 & 67,588 \\
\hline Jexas Utiotios Flectric Co..---1-1 & $m 1, m$ & 21.7וח & $-258,381$ & - & $-12816,434$ & 140639 & $1,015,403$ \\
\hline 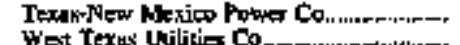 & $4+87.89$ & $\check{-}$ & $8,4 \times 1 \times 7$ & $\check{-}$ & $\begin{array}{l}1,625 \\
308064\end{array}$ & 2203.875 & $2,740,19$ \\
\hline Mititio Co & & & & & & & \\
\hline
\end{tabular}

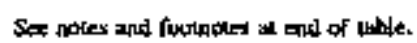


Table 24. Electricity Whecling to Investor-Owned Utilities, by State, 1994 (Continned)

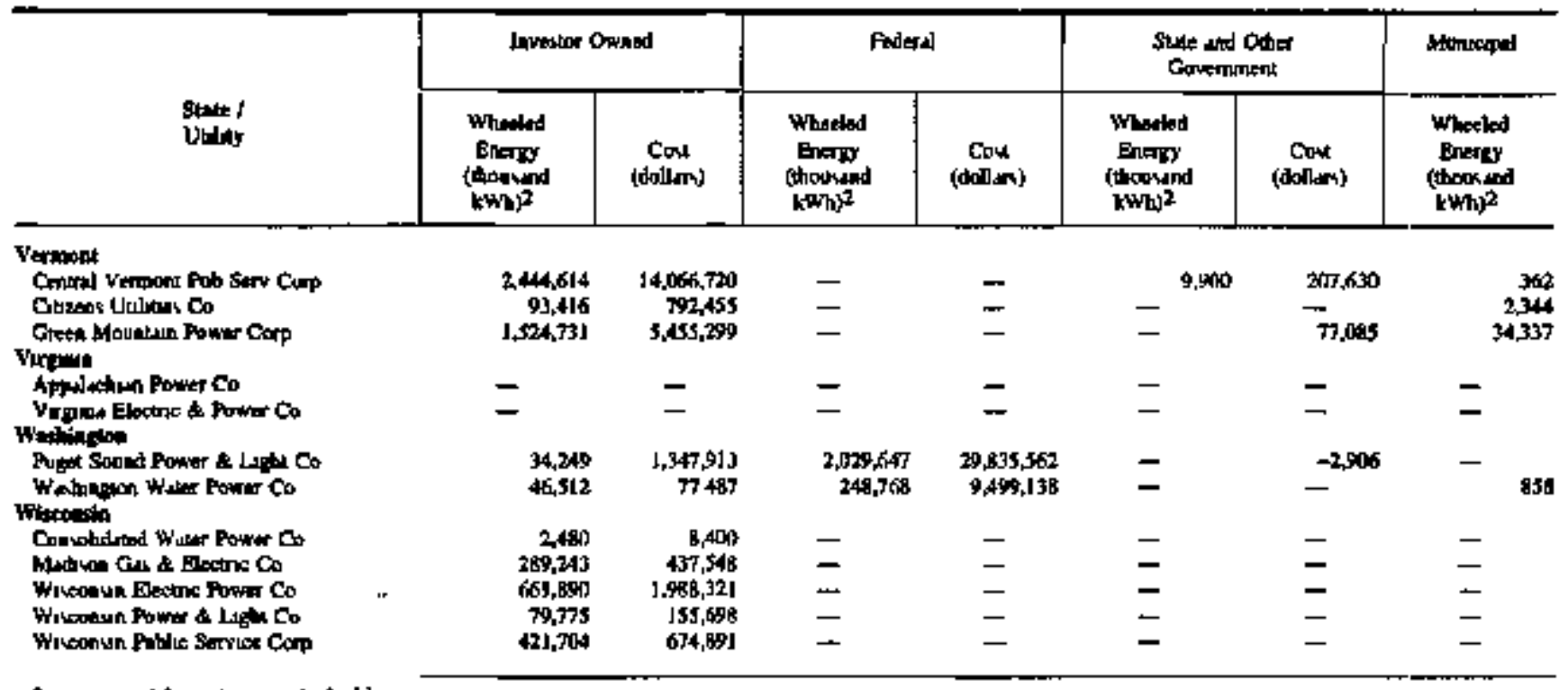

Table 24. Electrisily Wheeling to Investor-Owned Utilities, by State, 1994 (Conlinued)

\begin{tabular}{|c|c|c|c|c|c|c|c|}
\hline \multirow[b]{2}{*}{$\begin{array}{l}\text { Stan f } \\
\text { Dallog }\end{array}$} & \multirow{2}{*}{ Mumerpul } & \multicolumn{2}{|c|}{ Coxportive } & \multicolumn{2}{|c|}{ Olbe' } & \multicolumn{2}{|l|}{ Total } \\
\hline & & 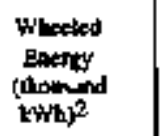 & $\begin{array}{c}\text { Cont } \\
\text { (didlur) }\end{array}$ & 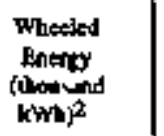 & $\begin{array}{c}\text { Cost } \\
\text { (dowlm, }\end{array}$ & 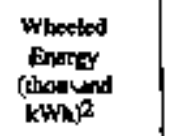 & $\begin{array}{l}\text { Cors } \\
\text { (iblun) }\end{array}$ \\
\hline \multicolumn{8}{|l|}{ Verned } \\
\hline 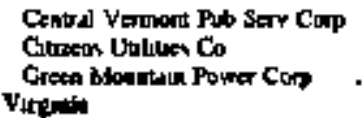 & $\begin{array}{r}127 \\
9,659 \\
99,626\end{array}$ & $\frac{-}{-}$ & $\underbrace{2,685}$ & $\overline{-}_{258,513}$ & $\begin{array}{l}-6,578 \\
3,929,365\end{array}$ & $\begin{array}{r}2,454,876 \\
95.760 \\
1,817,581\end{array}$ & $\begin{array}{r}14,277,162 \\
1,431,692 \\
9,551,575\end{array}$ \\
\hline 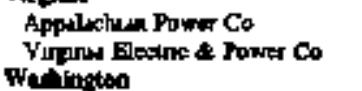 & $=$ & $\begin{array}{l}2,464 \\
1,062\end{array}$ & $\begin{array}{r}23,484 \\
9,016\end{array}$ & - & $\begin{array}{c}10,228,164 \\
-\end{array}$ & $\begin{array}{r}2,164 \\
4,052\end{array}$ & $\begin{array}{r}|0,75|, 648 \\
9,816\end{array}$ \\
\hline 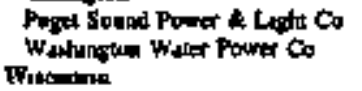 & $\begin{array}{r}-92,998 \\
1,665\end{array}$ & - & $\overline{24}^{-104}$ & - & - & $\begin{array}{r}2,060,956 \\
296,138\end{array}$ & $\begin{array}{l}31,097,57] \\
\text { ज्र,604,394 }\end{array}$ \\
\hline 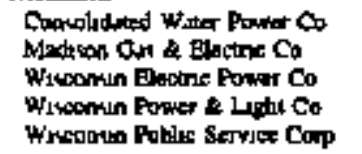 & $\begin{array}{l}- \\
= \\
= \\
=\end{array}$ & $\begin{array}{l}- \\
- \\
-\end{array}$ & $\begin{array}{l}- \\
- \\
-\end{array}$ & $\begin{array}{l}- \\
- \\
-\end{array}$ & $\begin{array}{l}- \\
- \\
-\end{array}$ & $\begin{array}{r}2,440 \\
289,243 \\
661,890 \\
79,775 \\
41,704\end{array}$ & $\begin{array}{r}3,40 \\
47,348 \\
1,988,321 \\
135,698 \\
674,691\end{array}$ \\
\hline 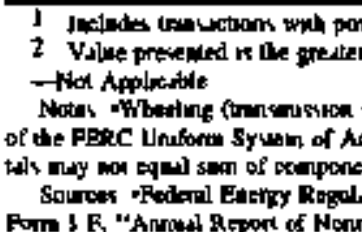 & 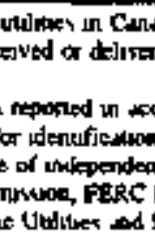 & 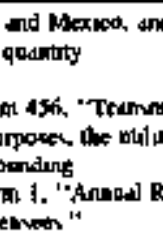 & th of 3100r & If in whidh & ess sind 0 & 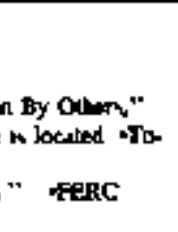 & \\
\hline
\end{tabular}




\section{Bulk Power Transactlons by Federal and Other Publicly Owned Utilities}

\section{Overview}

This chapter presents electricity trade data for pub. licly owned utilities that repor on Form EIA.4Iz, "Annual Repont of Public Electric Utilities." These data are separated into three types of public ownership: Federal. State and Other Government Utilities, and Municipalities. Summary totals by calendar year (except for Federal) cannat be provided because filings by utilities were based on different fiscal year's and ending months.

Figure 8. Electrleity Purchases and Costs by Federal Utilities, Fiscal Year 1994

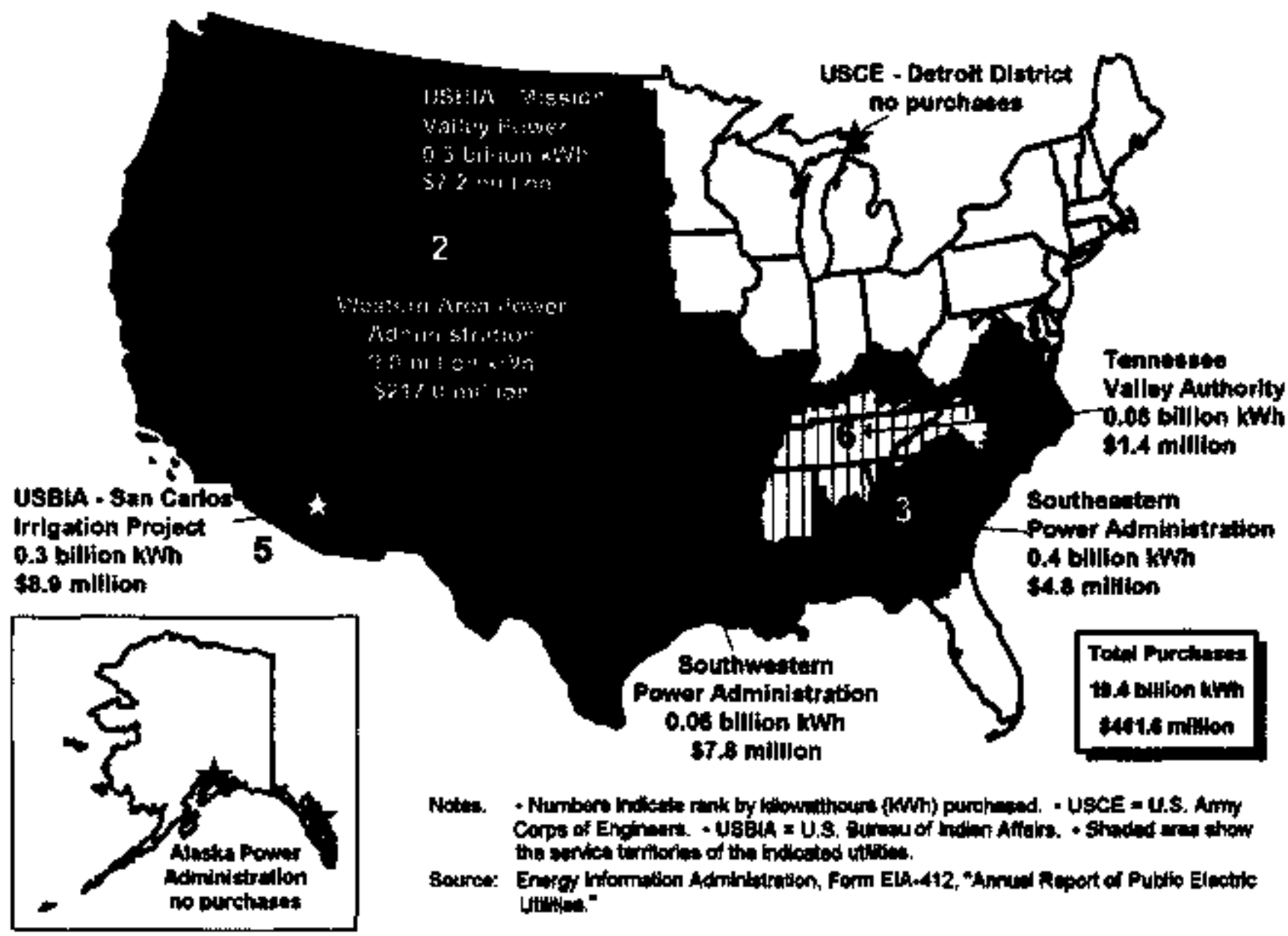

Individual transactions by publie utilities with other utilities by class of ownership are identified for purchased power and sales for resale data. Form ELA-412 collects only aggregate information (on a sample basis) on exchanges and wheeling to the "Energy Account Balance" schedule. Summixry daca from Form
EIA-B61, "Annual Electric Utility Report," are used to provide completo the coverage for the public atilities becanse it is collected from all electric utilities. However, Form EIA-86I does not identify individual transaction partners. 


\section{Tables}

This cbapter presents cost, reyenue, and quantity data on purchssed power and sales for resale for the following types of public utilities.

- Federal utilities (Tables 25 and 26)

- State and other government utilities (Tables 27 and 28)

- Municipalities (Tables 29 and 30)

- Electricity puschases by publicly owned utilities not listed elsewhere (Table 31 ).

Tables 25 through 30 provide information on the total cost and revenue associated with the total quantity of purchssed power and sales for resale. Other utitities which do not teport on the Form EJA-412 becatse of their small size are shown in Table 31 . Table 32 provides information on exchanges and wheeling for public electric utilities. In 1990 , Form ElA-4 I2 was updated to collect data on the different categories of power and energy and the associated value of electric utility trade. However, the value of the categories were not subdivided into Demand, Energy, and Other for revenues and costs.

The public utilities are listed by State according to the maitling addresses of their administrative offices. The fiscal-year ending date for each pubtic utility is also included in the tables. Federal utilities are jisted by major project, office, or administrative reporting level.

\section{Summary}

Federal power authorities dominare electric trade by publicly owned utilities. However, regional differences among the Fedejal authorities exist because of legal authority given and restrictions placed in the Federal laws that guide their activities. Many of these differences are due to Federal floond prevention and irrigation requirements at hydroeleciric projects or the limitations of corresponding transmission system operared by the power marketing authotitities.

FIgure 9. Electricity Sales for Resale and Revenue by Federal Utilitles, Fiscal Year 1994

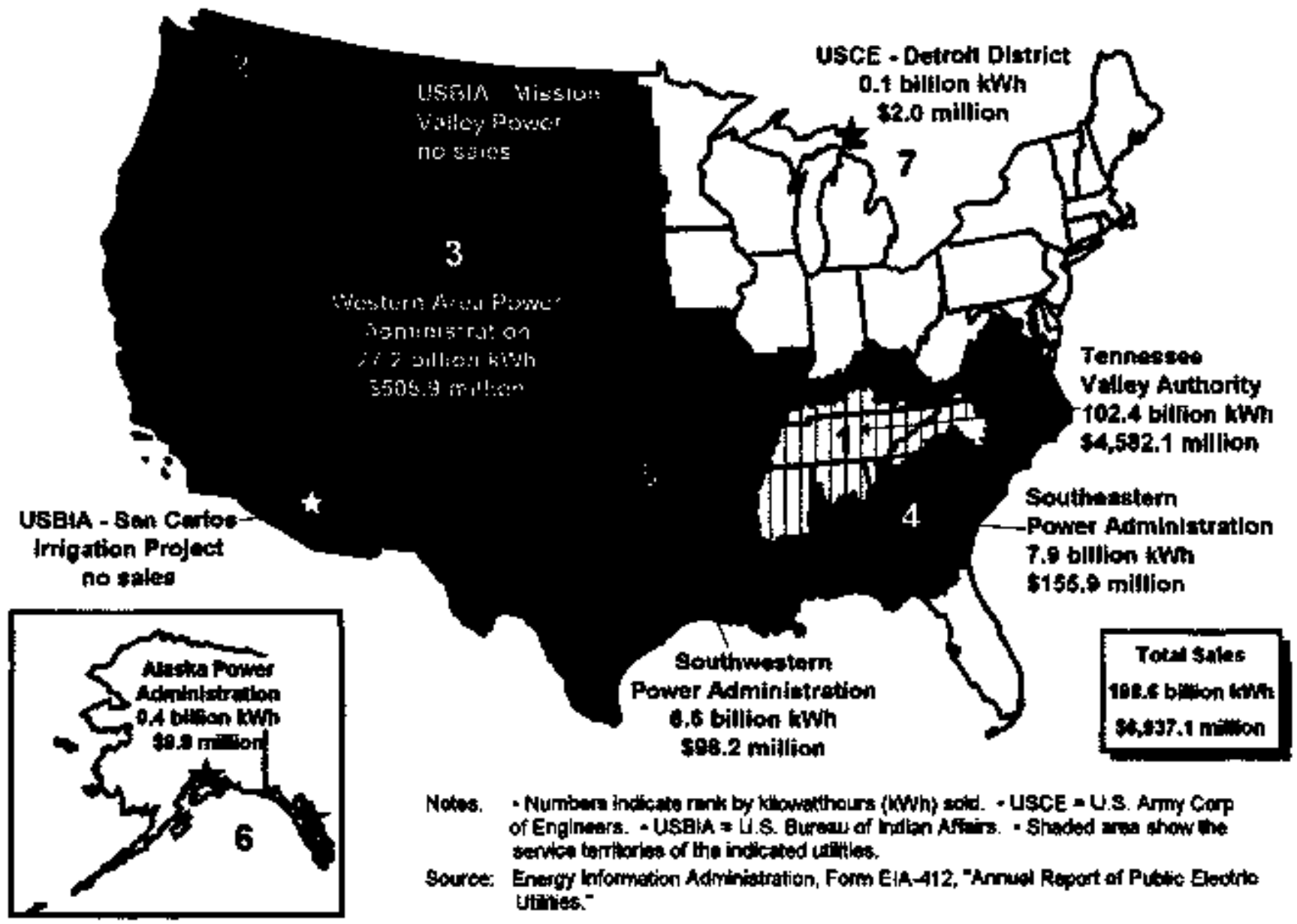

State power agencies and otber' public autborities purchase large amounts of electricity for resale to their wholesale customers. Some of these organizations have been sel up to acquire power for special in-State or regional projects, such as water transfer for irrigation and/or public consamption. In orther cases. 
States bave established power brokering agencies to purchase bulk power for redistribution to member movicipalities and cooperatives at tower costs.

Municipalities purchase far more power than they sell for resale. Their purchases are almost always redjs- tributed to the ultimate consumers of electricity. Those Inunicipal utilities involved in sales for resale usually have large generating capability, frequently including hydroelectric facilities and/or jointly owned thermal generators.

Figure 10. Electrielty Purchases and Costs by the Top Ten State and Other Government Utilities, Fiscal Year 1994

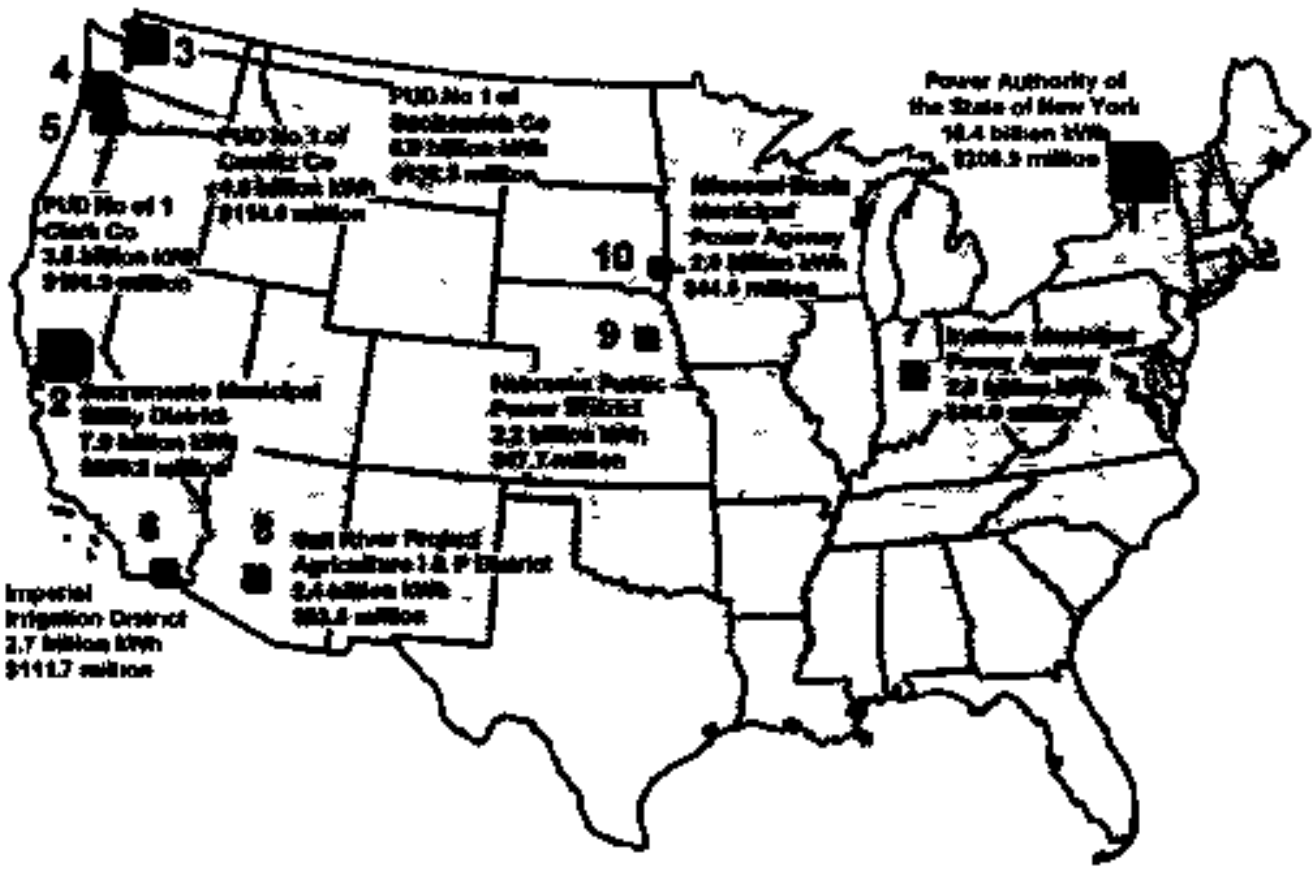

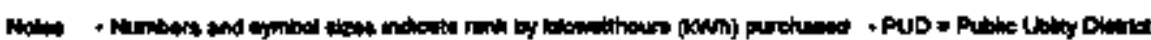

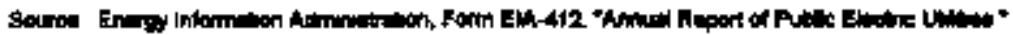


Figure 11. Electricity Sales for Resale and Revenue by the Top Ten State and Other Government Utilitles, Fiscal Year 1994

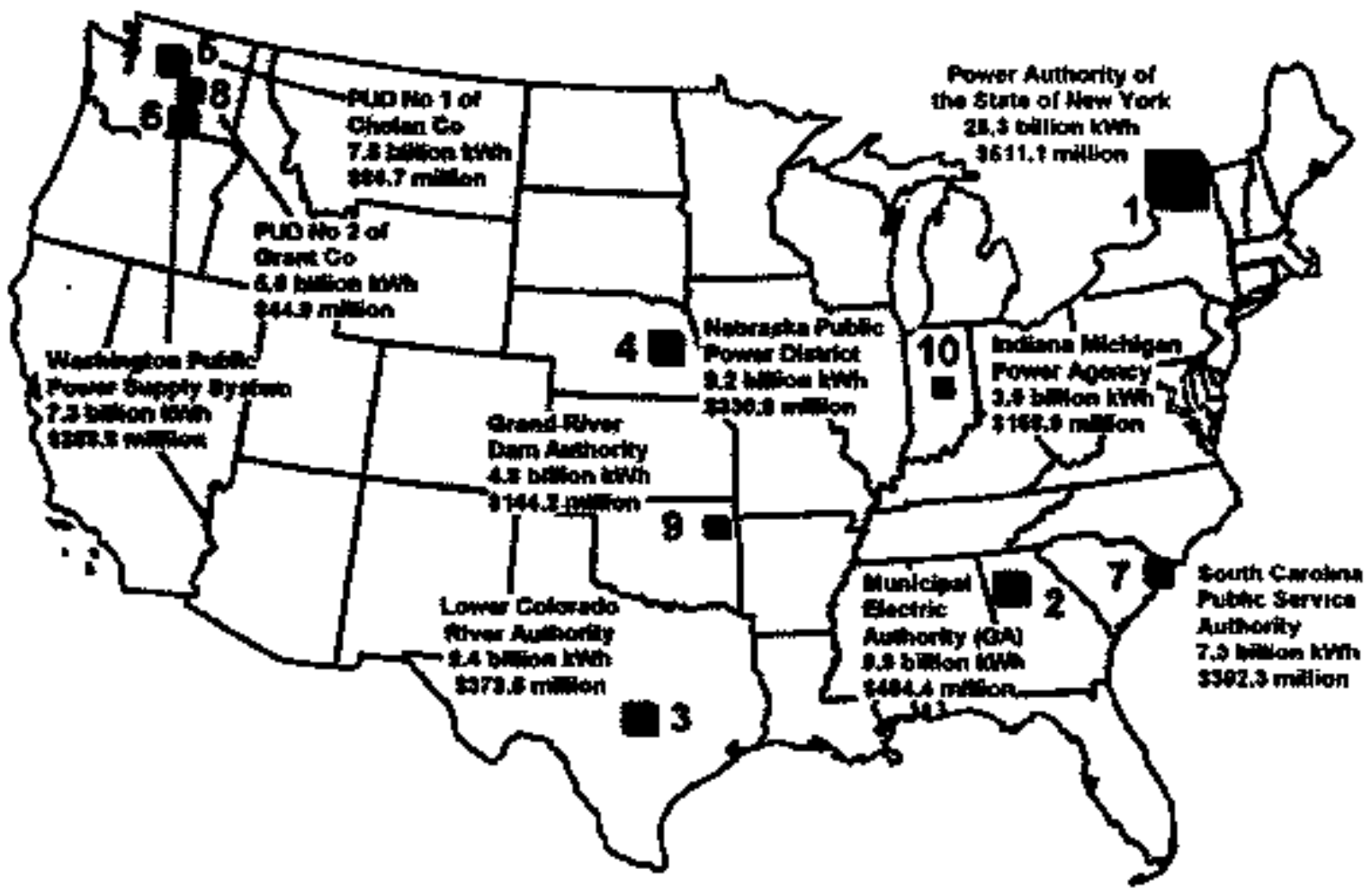

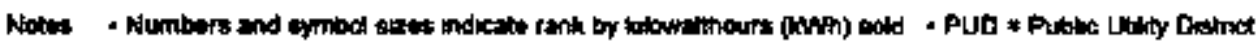

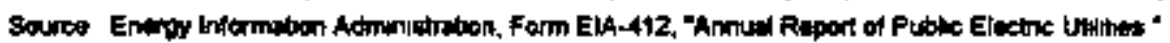


Figure 12. Electrleity Purchases and Costs by the Top Ten Munjclpal Utilities, Flscal Year 1994

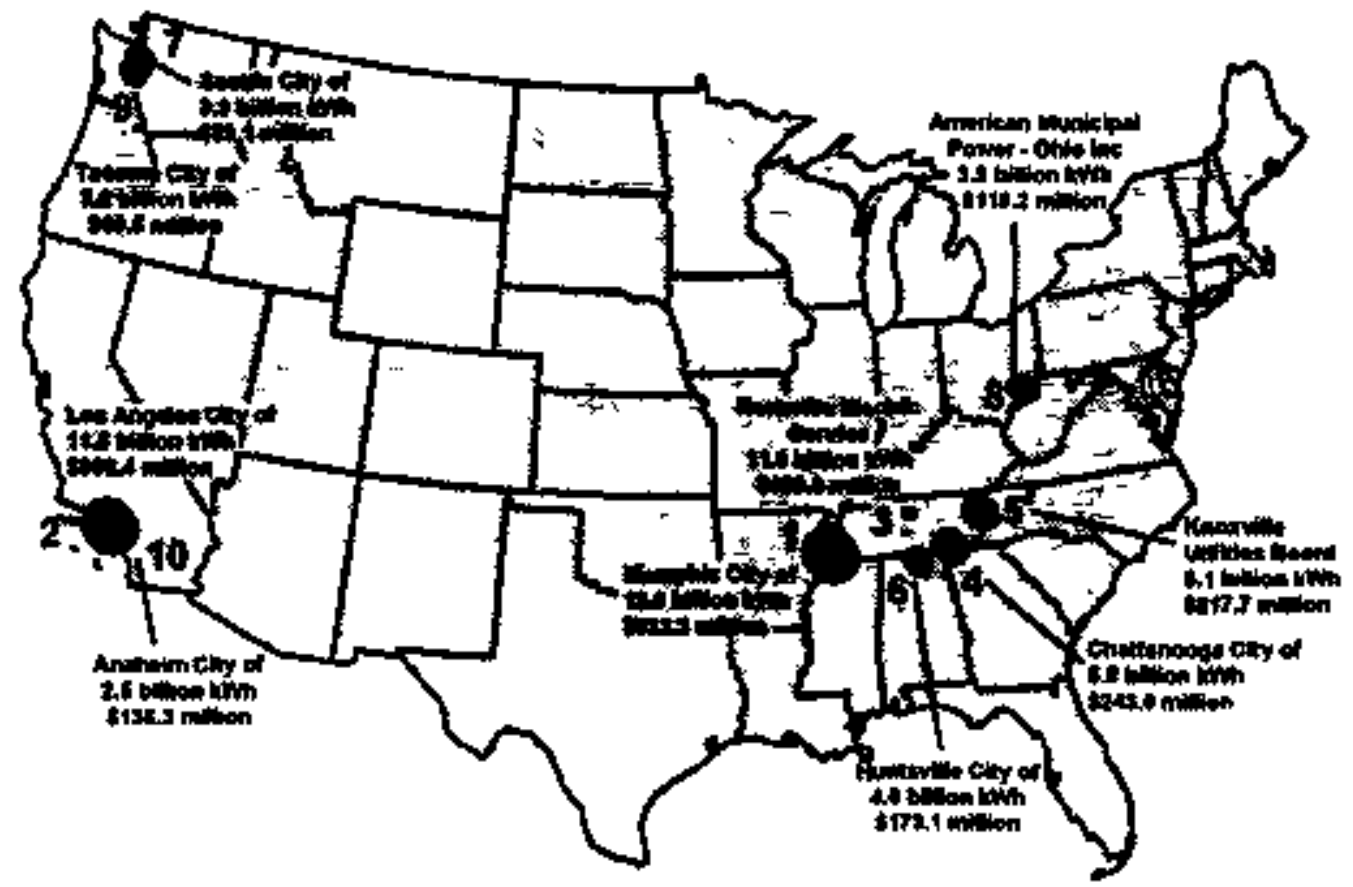

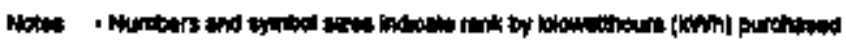

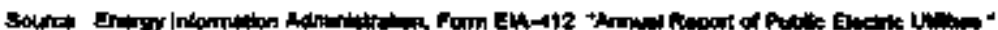


Figure 13. Electrlcity Sales for Resale and Revenue by the Top Ten Municlpal Utilities, Flseal Year 1994

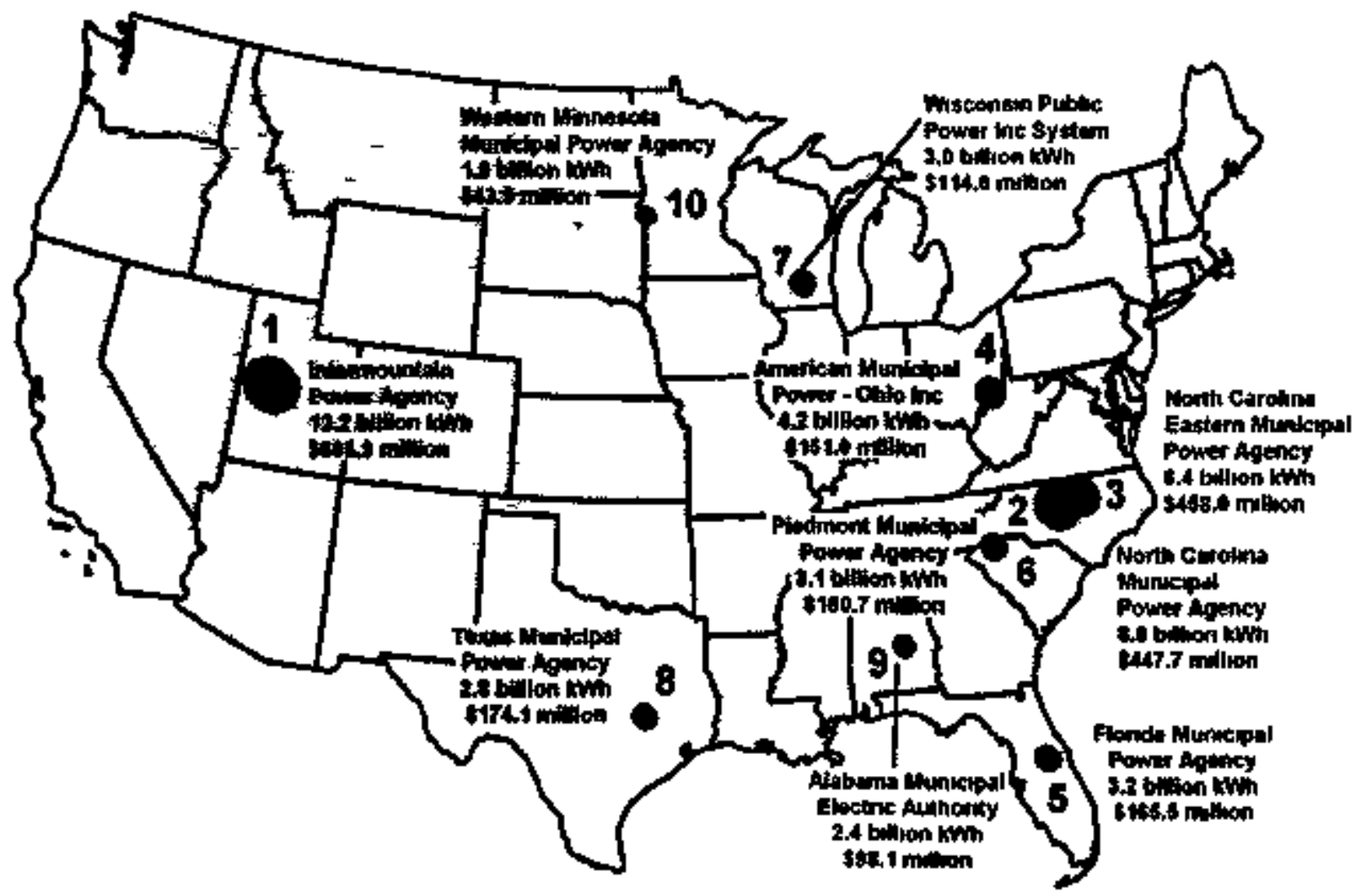

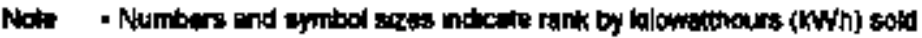

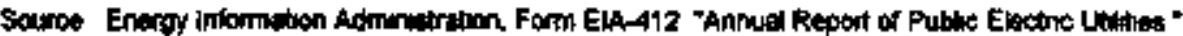


Table 25. Electricity Purchases by Federal U1ilities, Fiscal Year Ending September 30, 1994

\begin{tabular}{|c|c|c|c|c|c|c|c|c|c|c|}
\hline \multirow{3}{*}{$\begin{array}{l}\text { Strte of } \\
\text { Dutly }\end{array}$} & & \multicolumn{9}{|c|}{ Soproe of electinaty } \\
\hline & & \multicolumn{2}{|c|}{ Inwetor Onened } & \multicolumn{3}{|c|}{ Fectersl } & \multicolumn{2}{|c|}{ 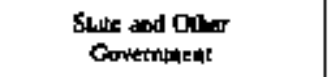 } & \multicolumn{2}{|c|}{ Muspapl } \\
\hline & & 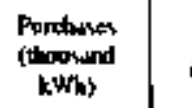 & $\underset{\text { dodtant }}{\operatorname{Cos}}$ & \multicolumn{2}{|c|}{ 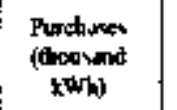 } & $\underset{\text { (dodtar) }}{\text { Cont }}$ & 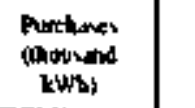 & $\operatorname{Cos}$ & $\begin{array}{l}\text { Plarbulat } \\
\text { (thothand } \\
\text { k'W/b) }\end{array}$ & $\operatorname{cost}_{(d \operatorname{doth})}$ \\
\hline \multicolumn{2}{|l|}{ 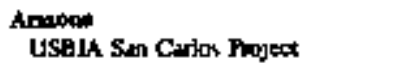 } & - & - & \multicolumn{2}{|l|}{-} & - & - & - & - & - \\
\hline \multicolumn{2}{|l|}{ Coloraft Ares Power Adnun } & $5.167,739$ & $124,492,959$ & \multicolumn{2}{|c|}{89,418} & 1.786271 & 473,632 & $8,67 T, 6344$ & 617,329 & $25,813,378$ \\
\hline $\begin{array}{l}\text { Georpa } \\
\text { Southentern Pown Admin }\end{array}$ & . & $19,58 \mathrm{x}$ & 400.663 & \multicolumn{2}{|l|}{-} & - & 179.574 & 2320.114 & - & - \\
\hline 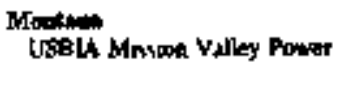 & . & 84582 & $1,259.917$ & \multicolumn{2}{|c|}{210.879} & $5, \mathrm{RT}, \mathrm{SAR2}$ & - & - & - & - \\
\hline \multicolumn{7}{|l|}{ Oldphons } & - & - & - & - \\
\hline 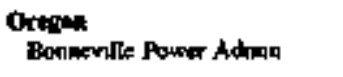 & & $4 . \mathrm{milom}$ & $115,296,762$ & & 2,264 & HA. $.79 \%$ & $3 \mid 5,000$ & $7.660,187$ & 178,253 & $4,016,645$ \\
\hline $\begin{array}{l}\text { Trannswes } \\
\text { Terneshed Valloy Avthonty }\end{array}$ & & - & - & - & & - & - & - & - & - \\
\hline US Toud & & 10008059 & $241,465.155$ & & S591 & $7.964,7,2$ & $968 ; 206$ & $18,557,935$ & 795,782 & $29,830,023$ \\
\hline & & & & & & Soprof of $\mathrm{E}$ & lestncity & & & \\
\hline & & $\mathrm{CoOp}$ & 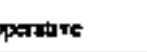 & & & othe & & & Fonel & \\
\hline & & $\begin{array}{l}\text { Porthres } \\
\text { (arooted kWth) }\end{array}$ & Cor & & & Primchuses & $\begin{array}{c}\text { Cont } \\
\text { (d) }\end{array}$ & $\begin{array}{l}\text { Eurchon } \\
\text { (thourind }\end{array}$ & k'th) & $\begin{array}{l}\text { Cour } \\
\text { (dellan) }\end{array}$ \\
\hline $\begin{array}{l}\text { Armoves } \\
\text { USBLA Sun Crolo, Propt }\end{array}$ & & - & - & - & & 273,130 & B, 152,465 & & 273.936 & $8,852,463$ \\
\hline 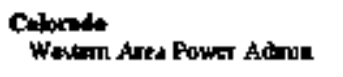 & & $2,217,399$ & & 394,981 & & 452.524 & $17,958,392$ & & $0 \notin 2,362$ & $217,043,615$ \\
\hline Geotget & & $16 J,(2005$ & & 064,2283 & & 一 & - & & 40.165 & $4,765,005$ \\
\hline $\begin{array}{l}\text { Meokent } \\
\text { USBIA Miriseon Yalley PoN }\end{array}$ & & - & - & - & & 1,041 & 25,487 & & $20 x \leq 02$ & $7,159,1666$ \\
\hline 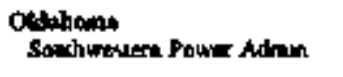 & & - & - & - & & - & $5 . \mathrm{BH} 6,7 \mathrm{II}$ & & 55,186 & $7,751,565$ \\
\hline $\begin{array}{l}\text { Orboge } \\
\text { Bounevilla Pawar Alarun }\end{array}$ & & $1,034,544$ & & 044,046 & & $7,049,794$ & $64,367,6897$ & & 299.859 & $214,694,322$ \\
\hline 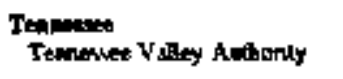 & & - & - & - & & 75,2013 & 1,356, & & 75.203 & $1,356,879$ \\
\hline Us, Tatol & & $3,412,939$ & & $* \mathbf{D}$,2ss & & $3,850,1852$ & 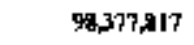 & & 403,007 & 461, , 22917 \\
\hline
\end{tabular}

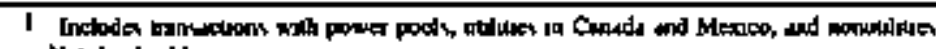

$\rightarrow$ Not Apheste

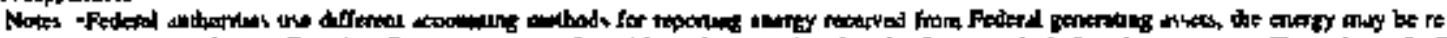

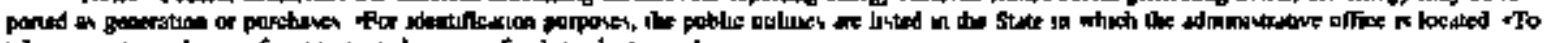

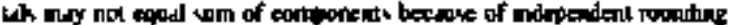

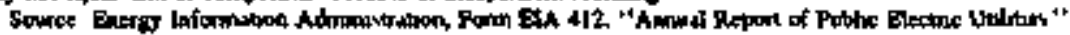


Table 26. Electricity Sales for Resale by Federal Utilities, Fiscal Yen Ending Septemiber 30, 1994

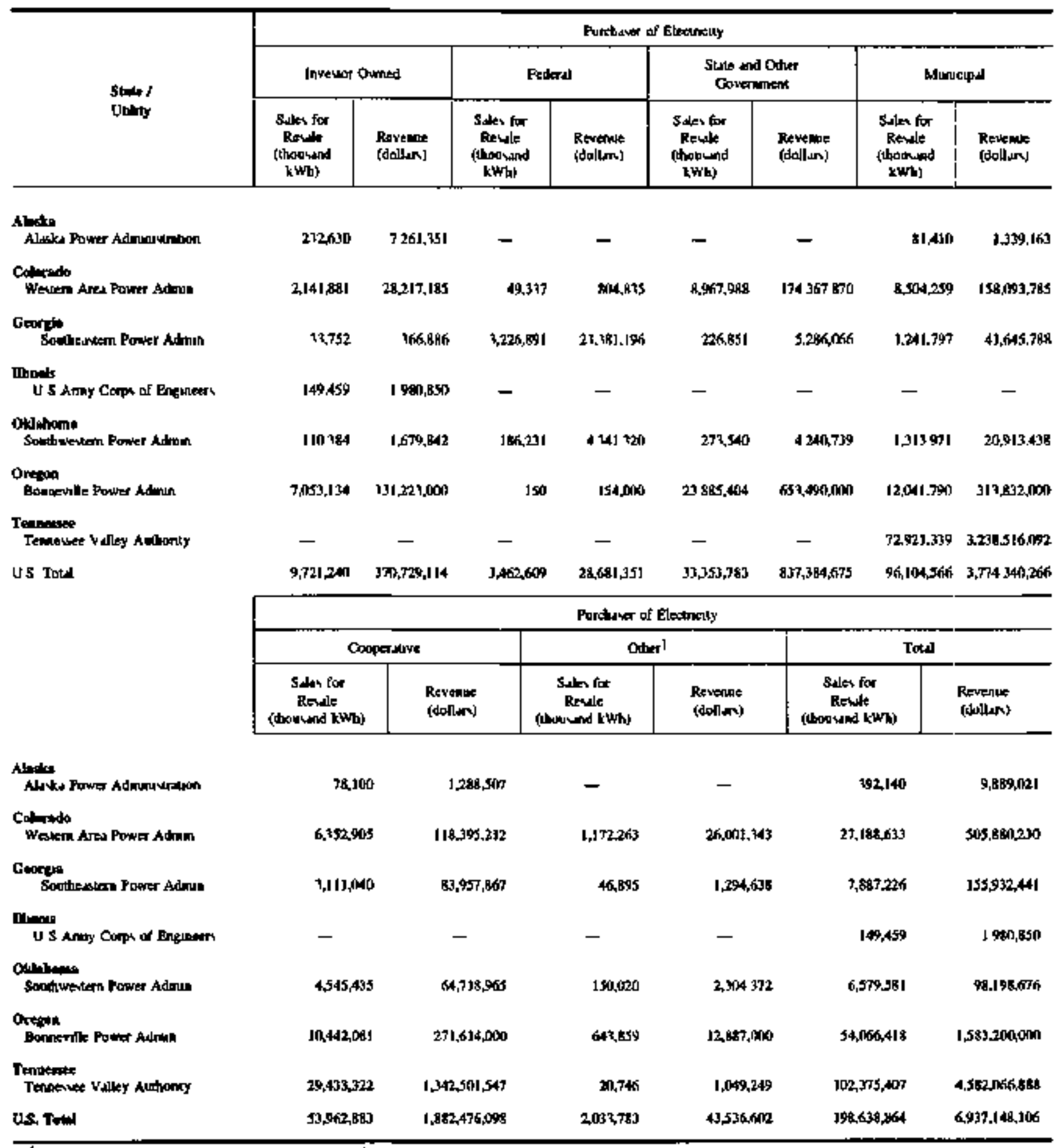

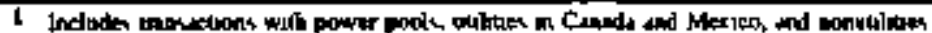

- Nhat Applesible

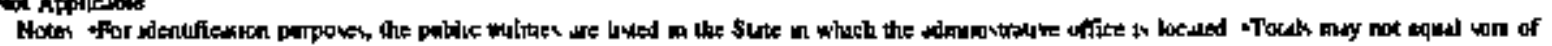
exnocmens bocioce of indeperdent roundang
}

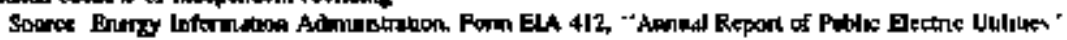


Tahle 27. Electricity Purchases by State and Otber Governunent Utilities, Flscal Year 1994

\begin{tabular}{|c|c|c|c|c|c|c|}
\hline \multirow{3}{*}{ 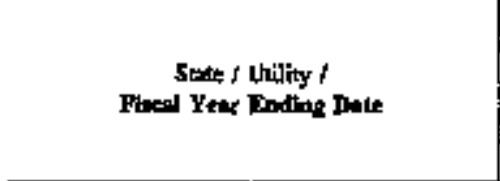 } & \multicolumn{6}{|c|}{ Somse of Electricity } \\
\hline & \multicolumn{2}{|c|}{ 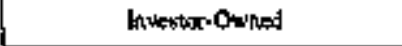 } & \multicolumn{2}{|c|}{ Federel } & \multicolumn{2}{|c|}{ Manicipal } \\
\hline & Popalasess & $\underset{(\cos 10 \sin )}{\cos }$ & 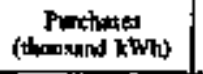 & $\begin{array}{c}\text { Cost } \\
\text { (ddilarrat) }\end{array}$ & 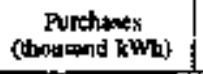 & $\begin{array}{c}\text { Cost } \\
\text { (twolloms) }\end{array}$ \\
\hline \multicolumn{7}{|l|}{ Artroan } \\
\hline Firn Poter & - & - & $\sin 012$ & $13,803,795$ & - & - \\
\hline 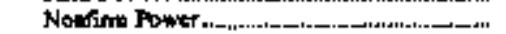 & - & 5,004 & - & - & - & - \\
\hline & - & $5, \sqrt{104}$ & 490,032 & $13,800,795$ & - & - \\
\hline 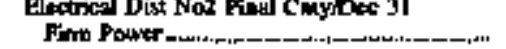 & - & - & - & - & - & - \\
\hline 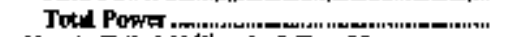 & - & - & - & - & - & - \\
\hline Nevejo Jirbal Uálicy AmhDec J] & & & & & & \\
\hline 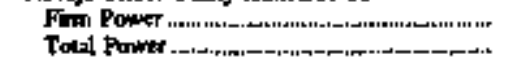 & $\begin{array}{l}4.56,140 \\
436, \sqrt{49}\end{array}$ & $\begin{array}{l}17,624,477 \\
17,624,477\end{array}$ & $\begin{array}{l}112,783 \\
112,783\end{array}$ & $\begin{array}{l}J, B \times 6,947 \\
J, B \times 6,947\end{array}$ & $\overline{-}$ & $\overline{-}$ \\
\hline Sall Riva Pod Af I \& P Diul/Ap 30 & & & & & & \\
\hline Firtat Powr & 334,535 & 24,941382 & 737,220 & $28,251,438$ & 一 & $\overline{1}$ \\
\hline 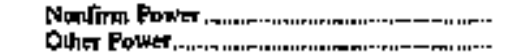 & $=17090$ & $4,196,850$ & $\begin{array}{l}131,490 \\
14786\end{array}$ & 2357,610 & $-59,055$ & $\frac{1, J 24,715}{-}$ \\
\hline Iotel Pow & 505.A7S & 20.158 .272 & $\operatorname{Ag} 20$ & 3.618.248 & 39 , 165 & $1.124,715$ \\
\hline Tchowo O Odhen Utilky An(WDes 3! & & & & & & \\
\hline Fren Powit . & 70,365 & $5,588,449$ & 13,536 & $159,57]$ & - & - \\
\hline 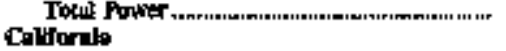 & 73,265 & $5.598,49$ & 11S24 & $159,57 \mid$ & 一 & 一 \\
\hline \multicolumn{7}{|l|}{ 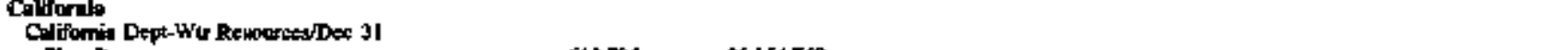 } \\
\hline 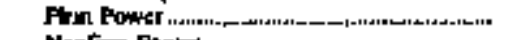 & 613,799 & $2 x, 154,769$ & - & - & - & - \\
\hline 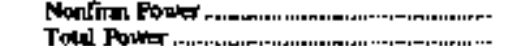 & $\begin{aligned} 401899 \\
644,644\end{aligned}$ & $\begin{aligned} 7926201 \\
25947389\end{aligned}$ & 21,455 & 383,060 & $\begin{array}{l}14 J, 180 \\
141180\end{array}$ & $\begin{array}{l}6,337,141 \\
6,337,141\end{array}$ \\
\hline \multicolumn{7}{|l|}{ 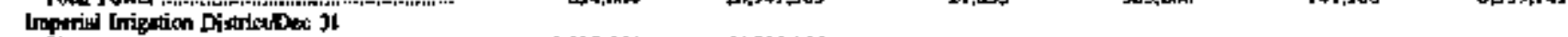 } \\
\hline Fith Fower & 1,497,466 & $64.0] 6,166$ & - & - & - & - \\
\hline 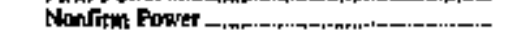 & 187,999 & 4,206,975 & 164,705 & $1,082,842$ & - & - \\
\hline Iodel Powter & $1.295,561$ & $68,223,141$ & 164,705 & $1,082,842$ & - & - \\
\hline \multicolumn{7}{|l|}{ Modene infrytlon Distried } \\
\hline Fon Pown & \$4, 155 & $6,175,3 * 1$ & 46,652 & $1 .-339,300$ & $6+8,061$ & $25,293,408$ \\
\hline 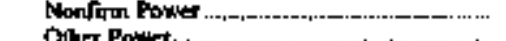 & $\begin{array}{r}170,816 \\
2,410\end{array}$ & $\frac{3.862813}{-}$ & $\begin{aligned} 126,1992 \\
-\end{aligned}$ & $2.181,8$ & 2,50 & $\underline{t}, 233,674$ \\
\hline 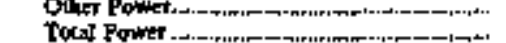 & $23,7,+10$ & $\overline{10,034,153}$ & 1,72744 & $\overline{3,62 i, 718}$ & 360.551 & $26,43,048$ \\
\hline \multicolumn{7}{|l|}{ 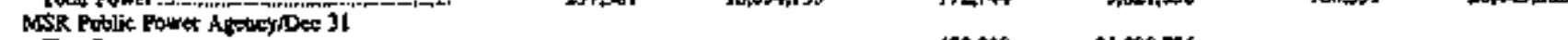 } \\
\hline Frm Power & - & - & 420,010 & $21.298,725$ & - & - \\
\hline Toxul Powng . & س & - & 422,010 & $21,292,725$ & - & - \\
\hline \multicolumn{7}{|l|}{ 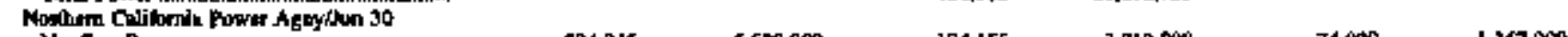 } \\
\hline 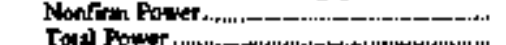 & 204045 & ${ }_{5}^{5,518,000}$ & 124,456 & J,019,0,000 & 74,009 & $1,967,000$ \\
\hline \multicolumn{7}{|l|}{ 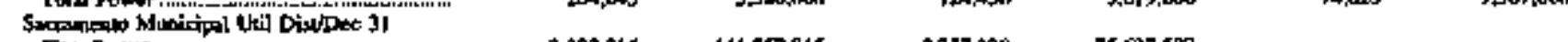 } \\
\hline 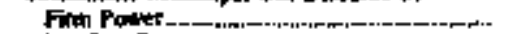 & $2,307,315$ & $141,057,815$ & $2,737,229$ & $75,697,587$ & - & - \\
\hline 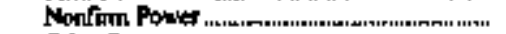 & $1,499,942$ & $33,446,595$ & 68,440 & $1,463,095$ & 103,682 & $2,176,357$ \\
\hline 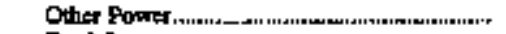 & & $\vec{a}$ & - & - & - & \\
\hline 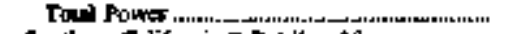 & $3,807,2,7$ & $175,704,410$ & $2,805,672$ & $77,300,682$ & 103,602 & $2,176,057$ \\
\hline Sorthem Doliformin P P AJIm 30 & & & & & & \\
\hline Nonfrem Power & - & - & 102,47 & $2.399,670$ & - & - \\
\hline 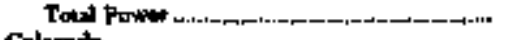 & - & - & La2,47 & $2,3919,6 \times 10$ & - & - \\
\hline \multicolumn{7}{|l|}{ 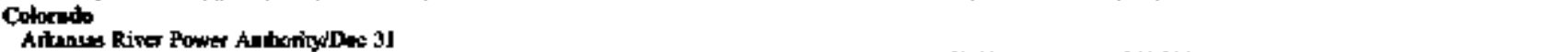 } \\
\hline 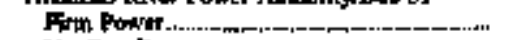 & - & - & 83,604 & 1,7610198 & - & $m$ \\
\hline 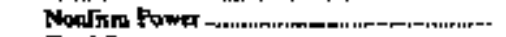 & - & - & $30, B+2$ & 545,993 & 134,601 & $4,247,265$ \\
\hline Todd Power rimmen........ & - & - & 134,536 & $2,394,331$ & 134,691 & $4,247,285$ \\
\hline \multicolumn{7}{|l|}{ Tohte River Power Awhorilyilenc 31} \\
\hline Frot Powir & - & $\rightarrow$ & 727,365 & 12,699,885 & - & - \\
\hline Monfinm Buwa & 692 & $20,16]$ & 1,443 & 34,928 & 375 & 0,165 \\
\hline \multirow{2}{*}{\multicolumn{7}{|c|}{ 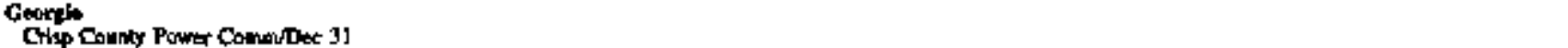 }} \\
\hline & & & & & & \\
\hline Naeflm Fowa - & 4aAt & $\{57,888\}$ & 30 nts & $1,146,6] J$ & - & - \\
\hline Tatd Power & 4,464 & 157,387 & 30,025 & $1.144,611$ & - & - \\
\hline \multicolumn{7}{|l|}{ Mapinipal Elowtris AntroritydDec 31} \\
\hline 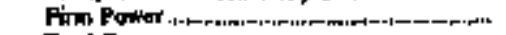 & ANB, 409 & 11,418000 & - & - & - & - \\
\hline Totod Pow & 440,09 & $11,418,000$ & - & - & - & - \\
\hline Indimitu & & & & & & \\
\hline 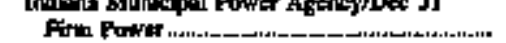 & $1,949,341$ & 58.991 .874 & - & - & 654,654 & $24,981,249$ \\
\hline Nondititil Power & - & 58,836 & - & - & - & $=$ \\
\hline Toxol Pow & $1,949,341$ & $99 ., 50,650$ & - & - & fSA,, 54 & $24,920,249$ \\
\hline
\end{tabular}

See notes and fortontes an wid of tuble. 
Table 27. Electricity Purchases by State and Other Government Utilities, Fiscal Year 1994 (Continued)

\begin{tabular}{|c|c|c|c|c|c|c|}
\hline \multirow{3}{*}{ 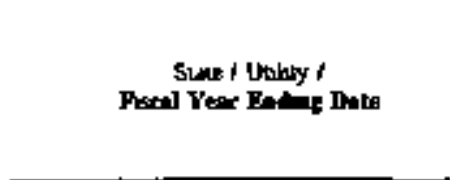 } & \multicolumn{6}{|c|}{ Schutin of Elecuntity } \\
\hline & \multicolumn{2}{|c|}{ Coophralve } & \multicolumn{2}{|c|}{ Other 1} & \multicolumn{2}{|c|}{ Totd } \\
\hline & $\begin{array}{l}\text { Pantunes } \\
\text { (thoovend twh) }\end{array}$ & $\underset{\text { (doflurs) }}{\text { Caty }}$ & $\begin{array}{l}\text { Purthave } \\
\text { (avound tiWh) }\end{array}$ & $\begin{array}{c}\text { Cont } \\
\text { (dollent }\end{array}$ & $\begin{array}{l}\text { Ponthues } \\
\text { (Croound twh) }\end{array}$ & $\underset{\text { Cout }}{\text { (dollmin) }}$ \\
\hline \multicolumn{7}{|l|}{ Amene } \\
\hline Fime Power & \multicolumn{2}{|c|}{ 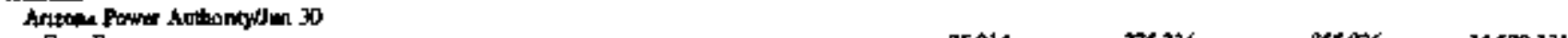 } & 25,914 & 775,936 & B5s,926 & 14,579, \\
\hline Nowim Fowed & - & - & - & - & - & \\
\hline Totd Rower & - & - & 25,914 & $775 . \pm 1+k$ & 855,926 & $14,584,135$ \\
\hline \multicolumn{7}{|l|}{ 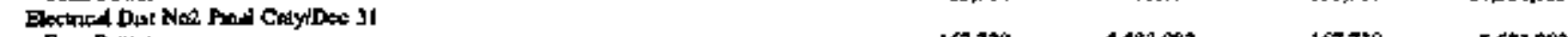 } \\
\hline Totel Power & - & - & 167.730 & $5,583,582$ & 167,750 & 550.98 \\
\hline \multicolumn{7}{|l|}{ 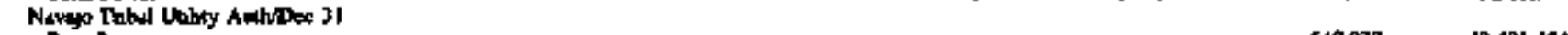 } \\
\hline Told Power & - & - & - & - & 548,932 & $19,49+, 424$ \\
\hline \multicolumn{7}{|l|}{ 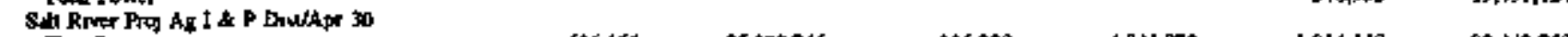 } \\
\hline $\begin{array}{l}\text { Nomfin Power } \\
\text { Olher Power }\end{array}$ & $-77,932$ & $1,736,1243$ & $\begin{array}{l}32,15 \mathrm{R} \\
15,255\end{array}$ & -606405 & $\begin{array}{r}411.167 \\
19,641\end{array}$ & $10.194 . T^{\prime} 13$ \\
\hline Toul hower & TH, 087 & $27,114,809$ & $2 \times 3,675$ & $5,46 \$ 2453$ & $2,414,948$ & $93,465,47 \mathrm{~J}$ \\
\hline \multicolumn{7}{|l|}{ 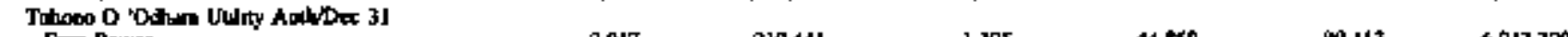 } \\
\hline $\begin{array}{l}\text { Fon Power } \\
\text { Tald Pariser }\end{array}$ & 2,017 & $\begin{array}{l}2] 10,441 \\
210,441\end{array}$ & ], 305 & 44.659 & 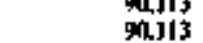 & 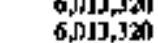 \\
\hline \multirow{2}{*}{\multicolumn{7}{|c|}{ 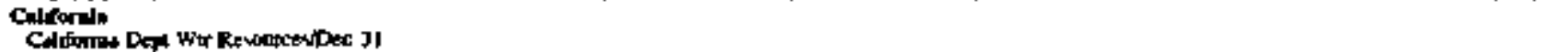 }} \\
\hline & & & & & & \\
\hline Fim Pawe & - & $\overline{-}$ & - & $\overline{7}$ & 613.795 & 24.154 .769 \\
\hline 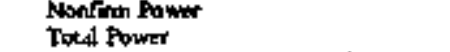 & 二 & $\bar{z}$ & $3 m, 014$ & (3, 336, 158 & $\begin{array}{r}510,938 \\
1,124,733\end{array}$ & $\begin{array}{l}\ln , 835,979 \\
17,005,748\end{array}$ \\
\hline \multicolumn{7}{|l|}{ 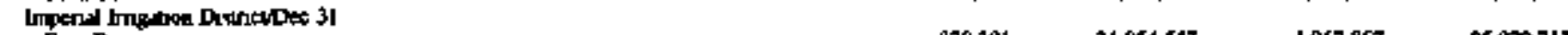 } \\
\hline Fum Power & - & - & 870,301 & $31,954,547$ & $1,867,867$ & $95,970,713$ \\
\hline Norim Prwa & - & - & 417,396 & $10,457,219$ & 770,2106 & $15,747,006$ \\
\hline Totd Power & - & $\rightarrow$ & $1,287,2007$ & $42,411,766$ & $2,738,073$ & $1[1,717,749$ \\
\hline \multicolumn{7}{|l|}{ 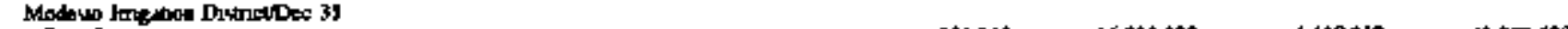 } \\
\hline Noptimat Paw & - & - & $\begin{array}{l}331359 \\
249,5 \times 0\end{array}$ & $\begin{array}{l}16,233,326 \\
21,462,555\end{array}$ & $\begin{array}{r}1,120,217 \\
608,915\end{array}$ & $\begin{array}{l}9, \pi 53538 \\
30,740,878\end{array}$ \\
\hline Otha Powe & - & $\rightarrow$ & - & - & 2413 & - \\
\hline Tots Pow & - & - & S8,9629 & $39,695, \mathrm{BS3}$ & $1,75], 605$ & $79,794,456$ \\
\hline \multicolumn{7}{|l|}{ MSR Poblic Power AsencitDer 31} \\
\hline Fim Pownor & - & - & - & - & 420,010 & $21,294,725$ \\
\hline \multirow{2}{*}{\multicolumn{6}{|c|}{ 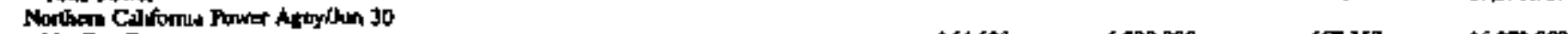 }} & $21,298.725$ \\
\hline & $m$ & $=$ & & $0,299,000$ & $6.7,358$ & $36,273,0 \times 0$ \\
\hline Totsl Ppore & - & - & 261,628 & $6,299,000$ & 667,159 & $36,273,000$ \\
\hline 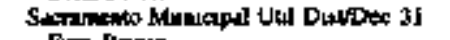 & & & & & & \\
\hline Fim Pown & - & 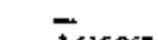 & - & & $5,004,544$ & $217, \$ 95,4182$ \\
\hline Nonfirm Pown & InI, fikh & 16]5,959 & 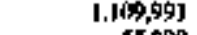 & \$1,415.6|6 & $2,883,724$ & 33.347 .622 \\
\hline Oing Pawn & & & $-55,990$ & & -55990 & - \\
\hline Tokd Power & 101,660 & $1,615,959$ & $1,054,001$ & 34,445,616 & 7,872276 & $290,343,024$ \\
\hline 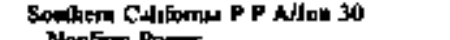 & & & & & & \\
\hline Nonfing Pomp & - & - & - & 32,237 & 102447 & $2,345,917$ \\
\hline $\begin{array}{l}\text { Totil Fown } \\
\text { Colocod? }\end{array}$ & - & - & - & $\mathbf{5 2 , 2 3 7}$ & 102447 & $2,341,907$ \\
\hline 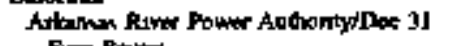 & & & & & & \\
\hline Fim Powe & - & - & - & 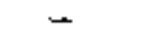 & 03.604 & $1,769,3,38$ \\
\hline Nonfim Power & 58,532 & $2,000,115$ & - & - & 224,065 & 6,B39, 193 \\
\hline Totd Fow & 58,532 & 2.946, I]S & - & - & 3(17.759 & B,EXK,,T3] \\
\hline 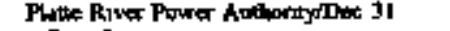 & & & & & & \\
\hline Fim Power & - & - & - & - & 727,365 & $32,639,895$ \\
\hline Nowim Power & 3,152 & If0,5s2 & - & -14.158 & 9.664 & 209, , $1 \mathrm{AB}$ \\
\hline Tot Pavier & 7,152 & If0552 & - & $-14,15 \pi$ & 737.109 & 12549 \\
\hline 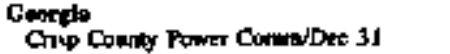 & & & & & & \\
\hline 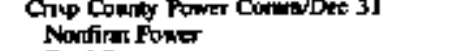 & - & $\rightarrow$ & 266,336 & 10,003, BAS & 825 & d1.373.J473 \\
\hline Tous Pouser & $=$ & $\vec{z}$ & 266,336 & in,039,845 & 30.829 & $1,398,343$ \\
\hline 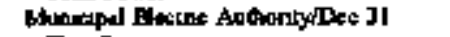 & & & & & & \\
\hline Fill Pown & - & - & 9,6903 & 462,000 & 458.102 & $11,880,000)$ \\
\hline Tohd Pown & - & - & 9,693 & 452.000 & 458,102 & 11,830000 \\
\hline 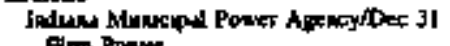 & & & & & & \\
\hline finm Power & - & - & - & - & $2,403,995$ & $83,973,073$ \\
\hline Namfin Power & - & - & - & - & - & 58,826 \\
\hline Tould Power & - & - & - & $\rightarrow$ & $2,603,985$ & 84031,899 \\
\hline
\end{tabular}

Ses noter and foolsowey at end of thble 
Table 27. Electricity Purchases by State and Other Governuent Utilties, Fiscal Year 1994 (Continned)

\begin{tabular}{|c|c|c|c|c|c|c|}
\hline \multirow{3}{*}{ 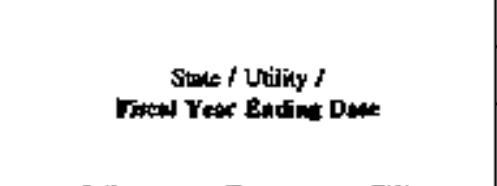 } & \multicolumn{6}{|c|}{ Sonets of Electrocity } \\
\hline & \multicolumn{2}{|c|}{ Invetha-Drned } & \multicolumn{2}{|c|}{ Fotend } & \multicolumn{2}{|c|}{ Menicafpel } \\
\hline & $\begin{array}{l}\text { Fonthuse } \\
\text { (Moorand kWh) }\end{array}$ & $\begin{array}{c}\text { Cod } \\
\text { (dollent }\end{array}$ & 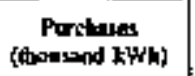 & $\underset{\text { (doTtart })}{\operatorname{Cost}}$ & $\begin{array}{l}\text { Puntased } \\
\text { (Thousand kWh) }\end{array}$ & $\underset{\text { (dollars) }}{\text { Cost }}$ \\
\hline \multicolumn{7}{|l|}{ Ranes } \\
\hline 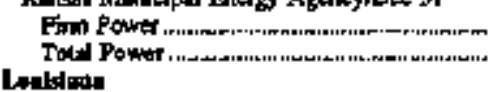 & $\begin{array}{l}218,341 \\
25.8,342\end{array}$ & $\begin{array}{l}7,569,010 \\
7,5,59,000\end{array}$ & $\begin{array}{l}32.5901 \\
32590\end{array}$ & $\begin{array}{l}491,100 \\
491,1000\end{array}$ & - & - \\
\hline \multicolumn{7}{|l|}{ 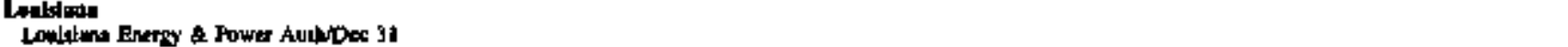 } \\
\hline EIm Rower & - & - & 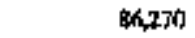 & $1,472,274$ & JB.5.I59 & $19,993,360$ \\
\hline 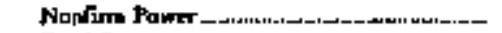 & 3.364 & 87,966 & - & - & 31,558 & $1,3 \pm 5,003$ \\
\hline \multicolumn{4}{|l|}{ 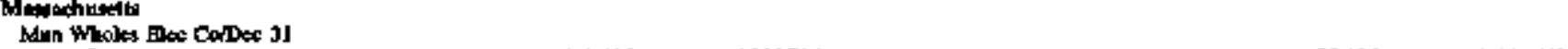 } & $1,472,274$ & 446,347 & $15,328,367$ \\
\hline 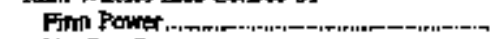 & 147,360 & 5.345 .724 & - & - & 7,805 & $3,461,540$ \\
\hline 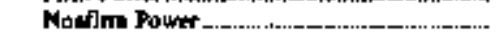 & 240 & 5,925 & $=$ & - & 16,161 & dongne \\
\hline 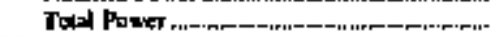 & 147,600 & 5.351 .649 & $=$ & - & 941046 & 3862742 \\
\hline \multicolumn{7}{|l|}{ 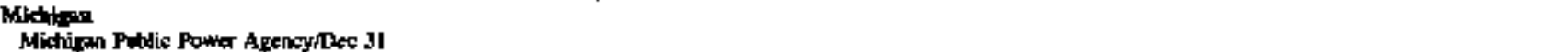 } \\
\hline 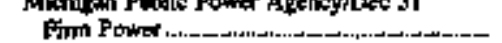 & 216,865 & 11,621, , ans & - & - & 455,055 & 13005842 \\
\hline 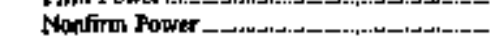 & 30 & 1,541 & - & - & 394 & 7205 \\
\hline 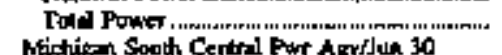 & 290,895 & IJ.仿立BAG & - & عـ & 455.469 & $13.013,047$ \\
\hline Pirm Powe & 139.J83 & $3,624,696$ & - & - & 7,977 & 557,588 \\
\hline 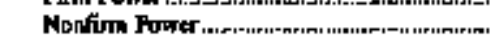 & 79,194 & $1,700,619$ & - & - & 21.216 & 46,722 \\
\hline 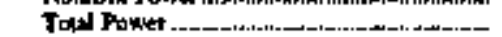 & 214,277 & $5,325,314$ & - & - & 29,193 & $1,004,310$ \\
\hline \multirow{2}{*}{\multicolumn{7}{|c|}{ Minoste }} \\
\hline \multicolumn{3}{|l|}{ Soothem Alinuesota Mtua P Aquyba 31} & & & & \\
\hline 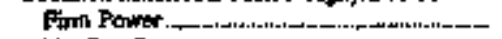 & 1,328 & 49,130 & - & - & 276,736 & $12,135,597$ \\
\hline Noufim Power & 13.257 & 295,784 & 8917 & 168,765 & 1,477 & $1,372,619$ \\
\hline 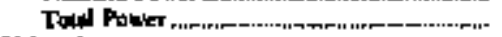 & 14585 & 344,918 & 8,917 & 168,765 & 279,213 & $13,508,216$ \\
\hline \multirow{2}{*}{\multicolumn{7}{|c|}{ 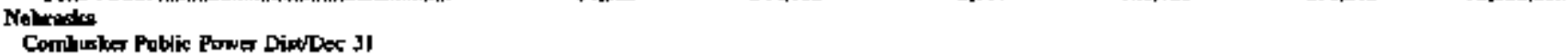 }} \\
\hline & & & & & & \\
\hline Fim Pownt & - & $\rightarrow$ & - & - & - & - \\
\hline 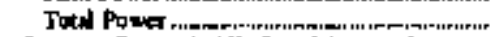 & - & - & - & - & - & - \\
\hline Duwwon Comery Rublic Rur bistroes 31 & & & & & & \\
\hline Fri Pown & - & - & - & - . & - & - \\
\hline Totnl Powr & - & - & - & - & - & - \\
\hline \multicolumn{7}{|l|}{ 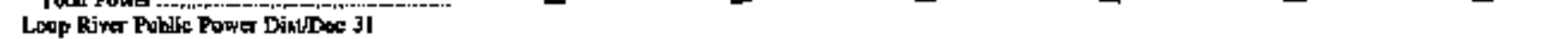 } \\
\hline Firtin Power & - & - & - & $=$ & - & - \\
\hline 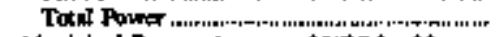 & - & - & - & - & - & - \\
\hline \multicolumn{7}{|l|}{ Meripd Bartgy Agancy of NEMter if } \\
\hline Fint Ponerer & 一 & - & $\mid 23,2]$ & $2,6,0,11,7$ & J65, & $6,111,879$ \\
\hline 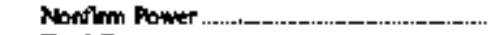 & 93 & 3,963 & - & $=$ & 14,200 & $277,0 \mathrm{n}$ \\
\hline 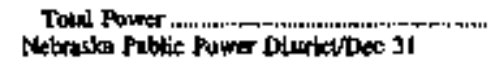 & 93 & 3,367 & 123,211 & $2,6,0,113$ & JaJ,Jk9 & 638981 \\
\hline Finn Powet .... & - & $\rightarrow$ & 739.561 & $17.184,396$ & 13,976 & $1,524,48$ \\
\hline Woaflm Pante & 239.498 & $1,747,598$ & 205,541 & $3,416,785$ & 132,143 & $1,719,359$ \\
\hline 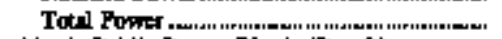 & 339,498 & $3,747,598$ & 944,102 & $29,601,58]$ & 145,118 & $3,243.33]$ \\
\hline Narris Public Power Disarituton 31 & & & & & & \\
\hline 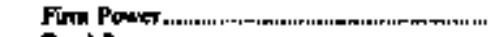 & - & - & - & - & - & - \\
\hline Tocal Power ._-_.., & - & - & - & - & - & - \\
\hline \multicolumn{7}{|l|}{ Onuhu Poblis Porwa Didrictods JI } \\
\hline Find Pouver & - & - & 378,417 & $5,090,090$ & - & 273,160 \\
\hline 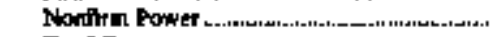 & - & $\rightarrow$ & - & - & - & - \\
\hline \multicolumn{7}{|l|}{ 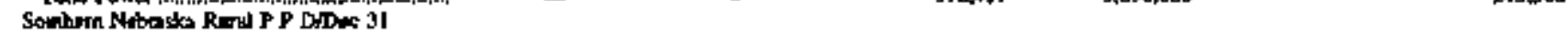 } \\
\hline 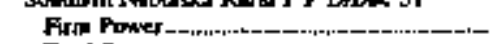 & $\longrightarrow$ & - & - & - & - & - \\
\hline 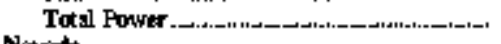 & 一 & - & - & - & - & - \\
\hline Nevide & & & & & & \\
\hline Fin Power & - & 306,689 & $1,544,6188$ & $24,834,578$ & - & - \\
\hline Nonfirm Pawer ........_-_..t......._................... & $\mathbf{1 4 2 , 7 1 3}$ & $4,785,763$ & - & - & - & - \\
\hline 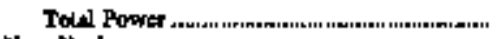 & 542,70? & 4.592 .452 & $1,544, \operatorname{Ban} 8$ & $24,9,14,578$ & - & $m$ \\
\hline 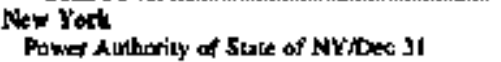 & & & & & & \\
\hline 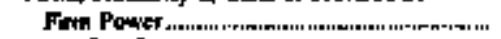 & 33,636 & |,A21,]低 & - & - & - & - \\
\hline 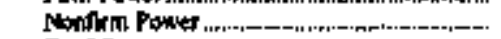 & 1,152767 & $24,291,436$ & - & - & - & - \\
\hline 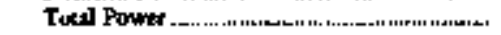 & $1.386,453$ & $25.714,677$ & - & 一 & $\rightarrow$ & - \\
\hline
\end{tabular}

See notes und forpokex ur ead of trble. 
Table 27. Electrictity Purchases by State and Other Government Uutlitles, Fiscal Year 1994 (Continued)

\begin{tabular}{|c|c|c|c|c|c|c|}
\hline \multirow{3}{*}{ 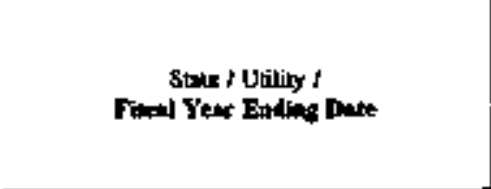 } & \multicolumn{6}{|c|}{ Suluet of Electricily } \\
\hline & \multicolumn{2}{|c|}{ Cuxpenulve } & \multicolumn{2}{|c|}{ Otha' } & \multicolumn{2}{|c|}{ Tot: } \\
\hline & 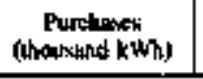 & 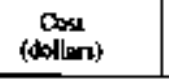 & $\begin{array}{c}\text { Purchevas } \\
\text { (thookund kWh) }\end{array}$ & $\begin{array}{c}\text { Coss } \\
\text { (dollins) }\end{array}$ & $\begin{array}{c}\text { Punchusex } \\
\text { (llookind } \mathbf{k} W \mathrm{Wh}\end{array}$ & $\underset{\text { (didinns) }}{\operatorname{Cos} x}$ \\
\hline \multicolumn{7}{|l|}{ 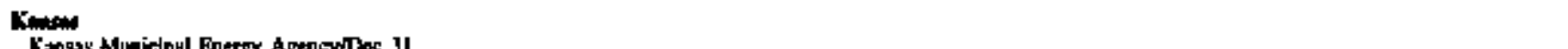 } \\
\hline 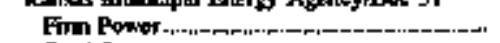 & 13,318 & 683,000 & - & - & 264,340 & 9.743,00, \\
\hline Totil Pount & 13,318 & EBg & - & - & 264,240 & $8,743,0001$ \\
\hline \multicolumn{7}{|l|}{ Lamidien } \\
\hline 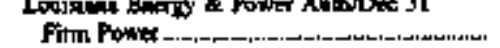 & - & - & $1,3,193$ & $4,077,995$ & 585,452 & $19,543,6129$ \\
\hline Honfinn Power...................... & 215 & 1596 & 294,426 & $3.092,674$ & 293,963 & 6517.251 \\
\hline Fotol Prwat & 215 & 0,590 & 347,619 & 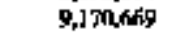 & $\$ 79.615$ & $26,060,982$ \\
\hline \multicolumn{7}{|l|}{ Mrandhunets } \\
\hline Fim Power ....n & - & - & s40120s & $26,799,331$ & 765,470 & $35,606,395$ \\
\hline 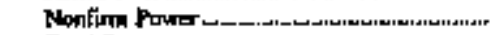 & - & - & BA,, 389 & $5,8 / 2,436$ & 96 & $0,249,163$ \\
\hline Totthl Fowst & - & - & 6anssid & $32,641,2 \times 3]$ & $86225 n$ & $4,, 655,758$ \\
\hline \multicolumn{7}{|l|}{ 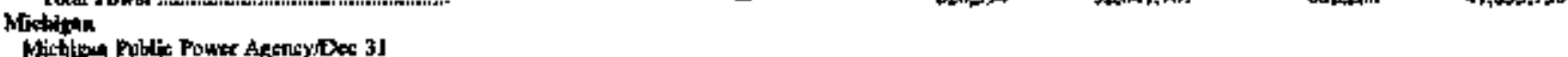 } \\
\hline Fim Powet........... & - & - & - & - & 664,920 & $24,627,147$ \\
\hline 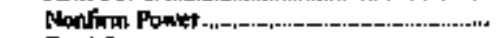 & - & - & - & - & & 8,746 \\
\hline 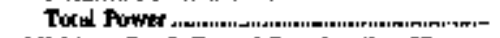 & - & - & - & - & 665344 & $24,635,893$ \\
\hline \multicolumn{7}{|l|}{ 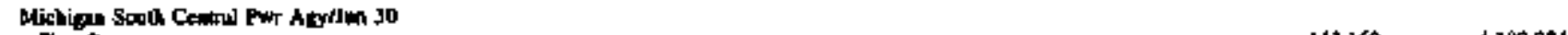 } \\
\hline 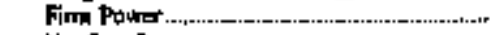 & - & - & - & - & 149,160 & $4,182,204$ \\
\hline 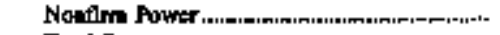 & - & $\longrightarrow$ & - & - & 100,310 & $2,17,340$ \\
\hline 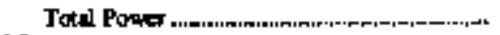 & - & - & - & - & 243,470 & $6,359,624$ \\
\hline \multicolumn{7}{|l|}{ 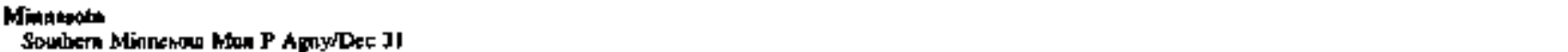 } \\
\hline Fim Pown & - & - & - & - & 27B-15i4 & $12,184,727$ \\
\hline Nonflm Power_.................... & 25,580 & 473, and & 36,085 & 408585 & 85.316 & 2718.764 \\
\hline \multirow{2}{*}{\multicolumn{7}{|c|}{ Nerito }} \\
\hline & & & & & & \\
\hline 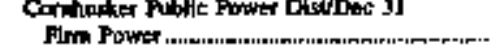 & 2 Ma,06s & $6,430,689$ & - & - & 200,083 & 6430600 \\
\hline Tolal Powe & 20,005 & $6,430,689$ & - & - & 2PMn, 155 & $0,430,109$ \\
\hline \multicolumn{7}{|l|}{ Dowron Conuy Poblic PwT DiedDes JI } \\
\hline Firn Powro, & 269,511 & $B, 698,902$ & - & - & 39,611 & B, $\mathrm{NAA}, 002$ \\
\hline Toct1 Power _." & 26511 & $8,69 \%$,9,12 & - & - & 269,611 & B. $6909.90 \%$ \\
\hline Loop River Patlie Power Dikt/Der 31 & & & & & & \\
\hline First Pow & - & - & 926,852 & $2 \pi, 2 \sqrt{3} 3,062$ & $\$ 26,852$ & $28,263,1182$ \\
\hline Toral Power & $=$ & - & 426,852 & $2 B, 263,0 B 2$ & 926,852 & $28,269,1002$ \\
\hline 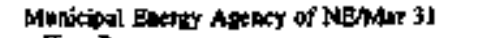 & & & & & & \\
\hline Fm Power & 59,789 & $1,4,82,040$ & 55,584 & $1,592,990$ & 407.553 & $11,46,993$ \\
\hline Nonfim Fower & 19,671 & 410,253 & $\$ 64,332$ & $6,298,290$ & 45B,316 & 6,968,948 \\
\hline Told Power & T9,4601 & I,BB2,3H & 459,916 & $7,871,263$ & 895, B, & $\mid 8,605,941$ \\
\hline 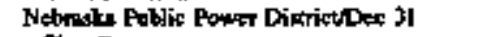 & & & & & & \\
\hline 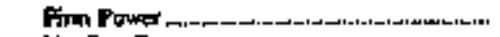 & - & - & 448,408 & $13, \infty 3,598$ & $1,201,945$ & $32,402,881$ \\
\hline 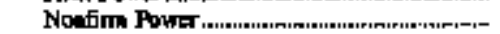 & 293,7013 & $4,681.124$ & 132946 & $1,7,2,78$ & $1,003,800$ & $15,137,635$ \\
\hline Tount Powor & 299. 203 & $4,681,124$ & 581,354 & $15,466,376$ & $2,294,775$ & $47,740,516$ \\
\hline Iorts Poblie Power DbtriadDex I & & & & & & \\
\hline Finm Power-, & - & ع & 41,034 & $13,400,349$ & 41,134 & $13,403,349$ \\
\hline Tocel Powner ...s...... & - & - & 41,134 & $13,410,349$ & 41,134 & $13,409,34$ \\
\hline 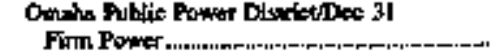 & $2 * 0$ & 338,640 & - & - & & \\
\hline & - & - & 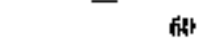 & 2.70n & $\begin{aligned} 378,657 \\
50\end{aligned}$ & $3,70,1800$ \\
\hline Toded Powner & 240 & 33:,640 & 6at & 2,700 & 378,717 & $5,764,780$ \\
\hline 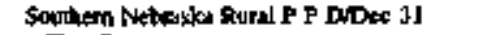 & & & & & & \\
\hline Firm Poswer & 435,547 & $14,896,360$ & - & - & 435,547 & $14,896,360$ \\
\hline 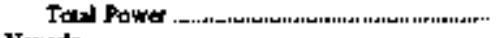 & 435,547 & J4,53,J6in & - & - & 435347 & $14,896,360$ \\
\hline Neyeda & & & & & & \\
\hline 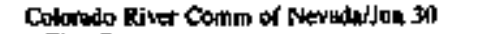 & & & & & & \\
\hline Fin Power & - & - & - & - & $1,544,968$ & $29,241,3 \times 7$ \\
\hline 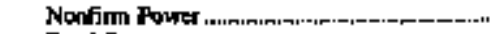 & - & - & 52,826 & $1,318,124$ & 195529 & $5,793,807$ \\
\hline Tots Pawer & - & - & 52,526 & $1,718,124$ & $1,740,397$ & $30,945,154$ \\
\hline 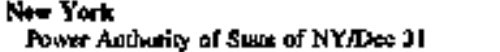 & & & & & & \\
\hline 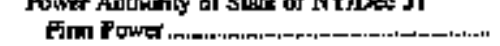 & - & - & 7,463, fi9] & $154,051,584$ & $7,497,179$ & $155,474,745$ \\
\hline Monitim Power & - & - & $1,73,284$ & 30.179 .782 & $2,926,051$ & $94,01,258$ \\
\hline Told Power & - & - & $0,236,977$ & $184,231.346$ & $10,423,470$ & $209.946,00 \mathrm{~J}$ \\
\hline
\end{tabular}

Soe nowa und looknowich ent ead of toble 
Table 27. Ehectricity Purchases by State and Other Gowetnment Ljthitfes, Fiscal Year 1994 (Continoed)

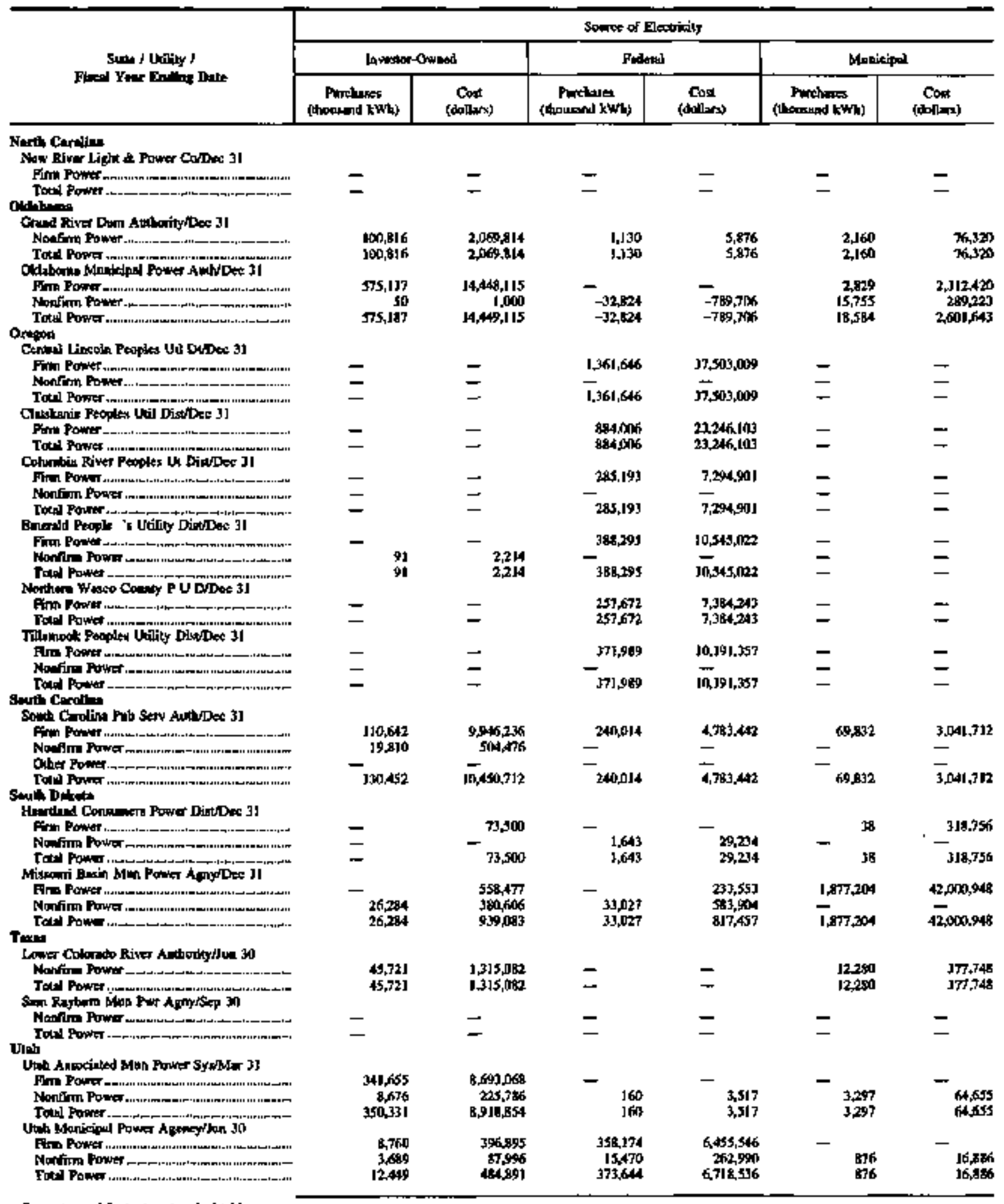

Sal notes and footacks and of table. 
Table 27. Electricity Purchases by State and Other Government Utnitfes, Flacal Year 1994 (Continued)

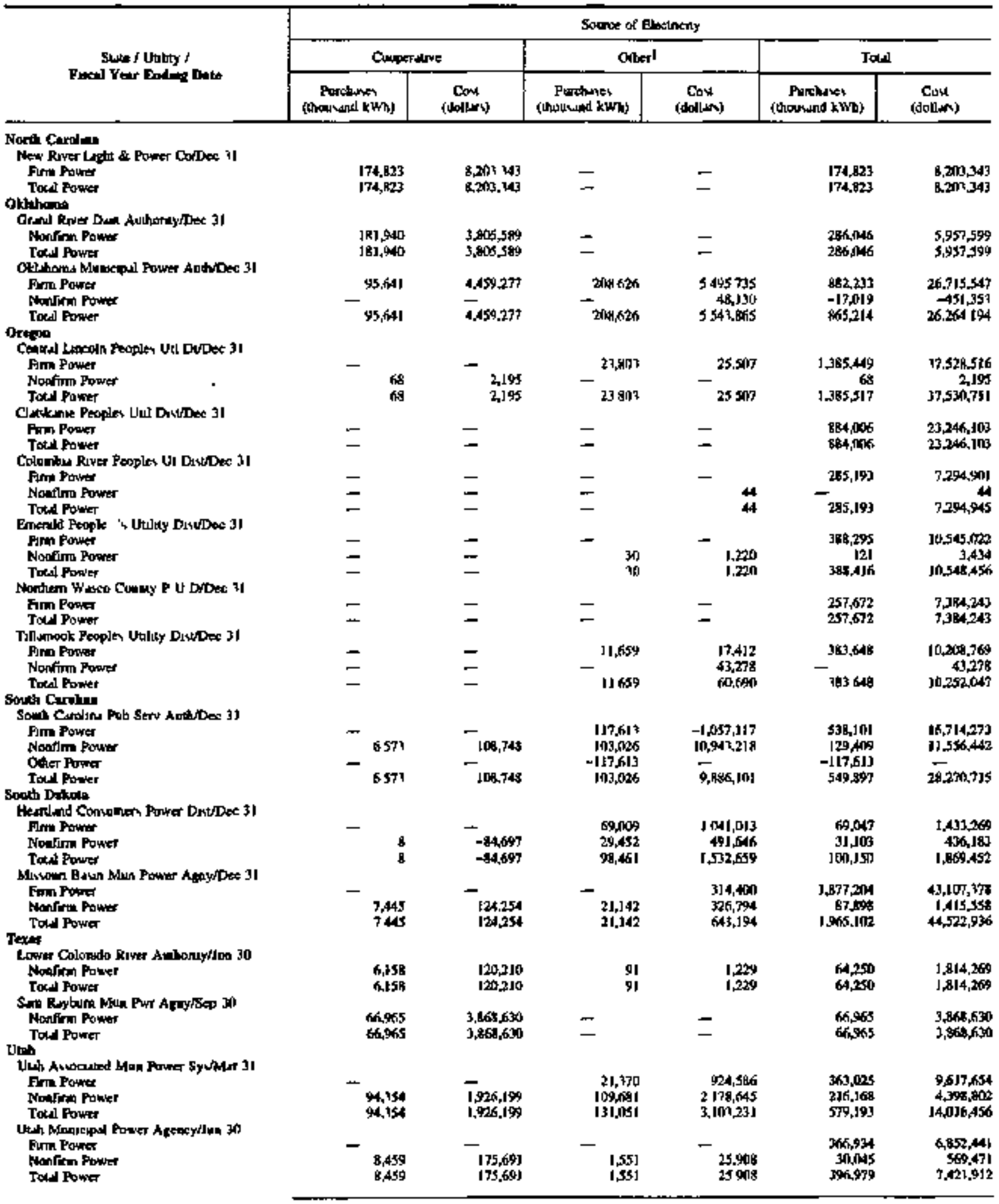


Table 27. Electricity Parchases by State and Other Goverament Utalities, Fiscal Year 1994 (Continued)

\begin{tabular}{|c|c|c|c|c|c|c|}
\hline \multirow{3}{*}{ 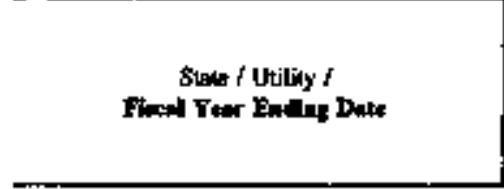 } & \multicolumn{6}{|c|}{ Soure of Electrixity } \\
\hline & \multicolumn{2}{|c|}{ Inreata-Oined } & \multicolumn{2}{|c|}{ Fodaral } & \multicolumn{2}{|c|}{ Meniciplal } \\
\hline & $\begin{array}{c}\text { Ponsturet } \\
\text { (lloosind trowh) }\end{array}$ & $\begin{array}{c}\text { Cost } \\
\text { [dertlorst) }\end{array}$ & 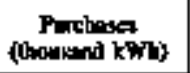 & Cod & $\begin{array}{l}\text { Porchnas: } \\
\text { (thourand kwh) }\end{array}$ & $\begin{array}{c}\text { Cos } \\
\text { (dollen) }\end{array}$ \\
\hline \multicolumn{7}{|l|}{ 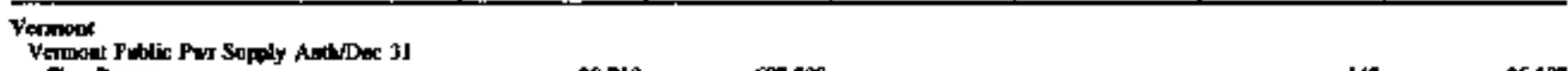 } \\
\hline Firm Power . & 20,710 & 695,500 & - & - & 142 & 26,187 \\
\hline 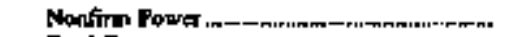 & $\$ 15$ & 5,112 & 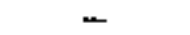 & - & - & - \\
\hline 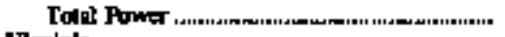 & 20,025 & 703,692 & - & 一 & 142 & 26,187 \\
\hline Vigingiria toch Elestric Savicethen 20 & & & & & & \\
\hline Fin Power & $\begin{array}{l}215,474 \\
215,474\end{array}$ & 7.674998 & - & - & - & - \\
\hline 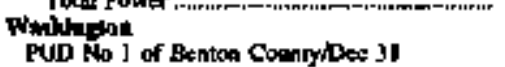 & & & & & & \\
\hline Fin Fowar & $\sigma$ & - & $1,431,178$ & $3 t, 965,566$ & 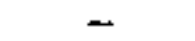 & - \\
\hline 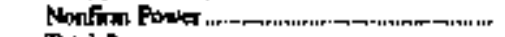 & - & - & - & - & - & - \\
\hline 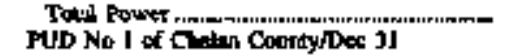 & - & 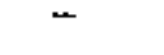 & 1,A31.178 & $38,965,366$ & 一 & - \\
\hline Himp Power & 13,579 & $1,233,941$ & - & - & - & - \\
\hline Nonfm Power & 161,0105 & 956,742 & 25,517 & 996, 793 & - & - \\
\hline 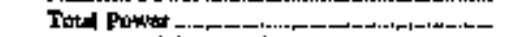 & 258,594 & $2,170,690$ & 25,917 & 596,793 & - & - \\
\hline PUD No I w Clalbith CoustydDex 31 & & & & & & \\
\hline 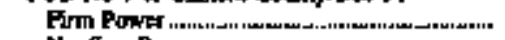 & - & - & भाazor & $1+1926,161$ & - & - \\
\hline 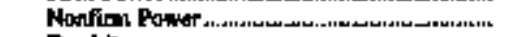 & - & - & - & - & - & - \\
\hline Told Pow & - & - & 500,2017 & $13,526,161$ & - & - \\
\hline 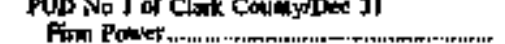 & - & - & $1,476,221$ & 97.714060 & $\leftarrow$ & $\rightarrow$ \\
\hline 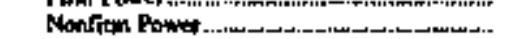 & 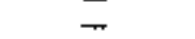 & - & - & - & - & - \\
\hline Total Pawn & - & $\rightarrow$ & $3,476,223$ & $97,714,040$ & - & - \\
\hline PUD Ho I of Conitix ConntylDec 31 & & & & & & \\
\hline 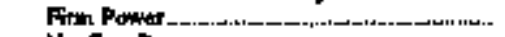 & - & - & 4,321,7iा & 112.411 .314 & $\rightarrow$ & - \\
\hline 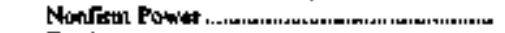 & - & - & - & - & 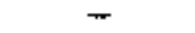 & 一 \\
\hline Toul Powar & - & - & $4,321,70\}$ & $112,411,714$ & - & 一 \\
\hline 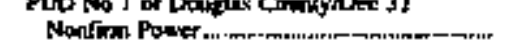 & - & $=$ & - & 一 & 一 & - \\
\hline Tokyl Pown .......... & - & - & - & - & - & - \\
\hline & & & & & & \\
\hline Prin Pow & 一 & 一 & 651,370 & $17,11 \leq 840$ & - & 一 \\
\hline & - & - & 一 & 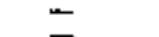 & 一 & 一 \\
\hline 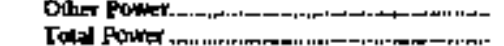 & $=$ & - & - & $\overline{17} 11590$ & - & - \\
\hline 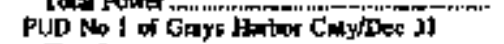 & - & - & 631,454 & $17,113,840$ & - & - \\
\hline Firme Powver & - & - & $7,0 \mathbf{1} 22,22$ & $29,305,787$ & - & - \\
\hline 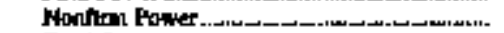 & - & - & - & - & - & - \\
\hline Toxill Power & - & - & $1,042,222$ & 29,325787 & - & - \\
\hline Pitd No I of Klickine Conmytout 31 & & & & & & \\
\hline 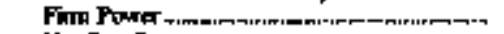 & 一 & 一 & 298,6897 & $7,617,667$ & - & - \\
\hline 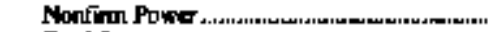 & 一 & - & 一 & - & - & - \\
\hline 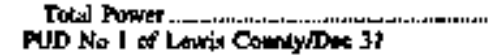 & 一 & - & 298,687 & $7,617,6$ fit? & - & - \\
\hline 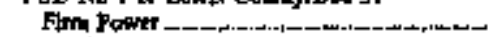 & $m$ & - & 707575 & $17,843,727$ & - & - \\
\hline 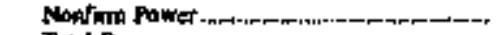 & - & 一 & - & - & - & - \\
\hline 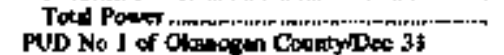 & - & - & 307,575 & $17,842,721$ & - & - \\
\hline 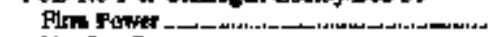 & - & ש & 355.46亿 & $9,5,790$ & - & - \\
\hline 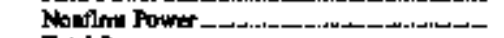 & - & 一 & - & + & - & - \\
\hline Told Pawer & - & - & 355,463 & $9,57), 193$ & - & - \\
\hline FUD Mo J of Pend Orejle Citydoec is & 7506 & $7945 \mathrm{man}$ & 10185 & $78+170$ & & \\
\hline 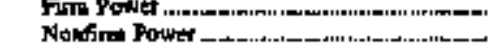 & 13,300 & 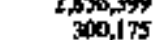 & $2164,4,18$ & 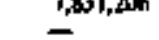 & 257.es & $\overline{102 \pi} 00 \mathrm{~s}$ \\
\hline 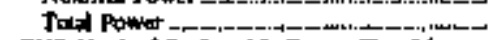 & 90,464 & $3, \sqrt{56,574}$ & 264,456 & $7,831,20 \%$ & 257,455 & $1,027,015$ \\
\hline 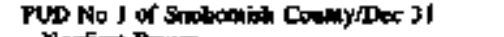 & & & & & & \\
\hline Nowfint Power & 14814 & $1,750,235$ & 4,948699 & $134,464,734$ & - & - \\
\hline 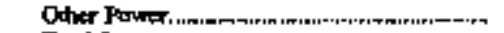 & - & & & $\Rightarrow$ & - & - \\
\hline 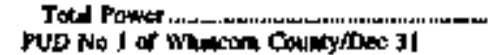 & 84.814 & $1,750,435$ & 4948698 & $134,464,73 d$ & - & - \\
\hline 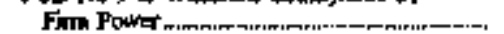 & - & - & 171,659 & $4,463,403$ & س & - \\
\hline Totid Pawer u... & - & - & 171,659 & $4,463,403$ & 一 & - \\
\hline 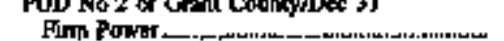 & - & - & $513,17]$ & $12, \sqrt{103}, 253$ & $\rightarrow$ & - \\
\hline 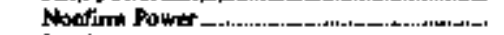 & - & 29,456 & 465 & 75.102 & - & - \\
\hline Tot Power & - & $20 A 56$ & $513,6,16$ & $12.15 \% 355$ & - & - \\
\hline
\end{tabular}

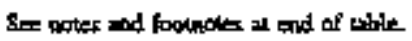


Table 27. Electricity Purchases by State and Other Government Utilities, Fiseal Xear 1994 (Contímued)

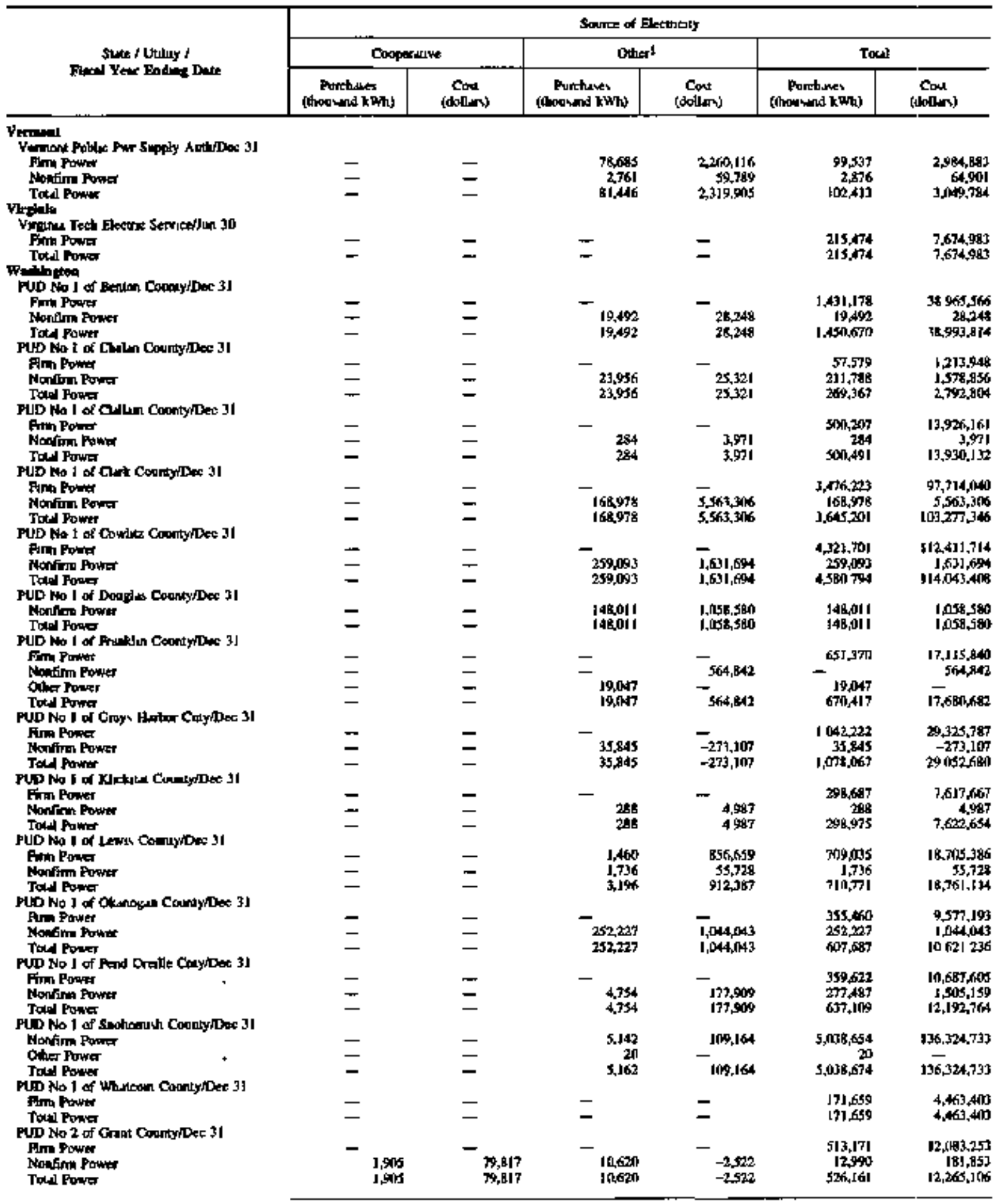

Sec mont and foothotes at end of thble 
Table 27. Electricity Purchases by State and Othex Governutent Utilities, Fiscal Year 1994 (Continned)

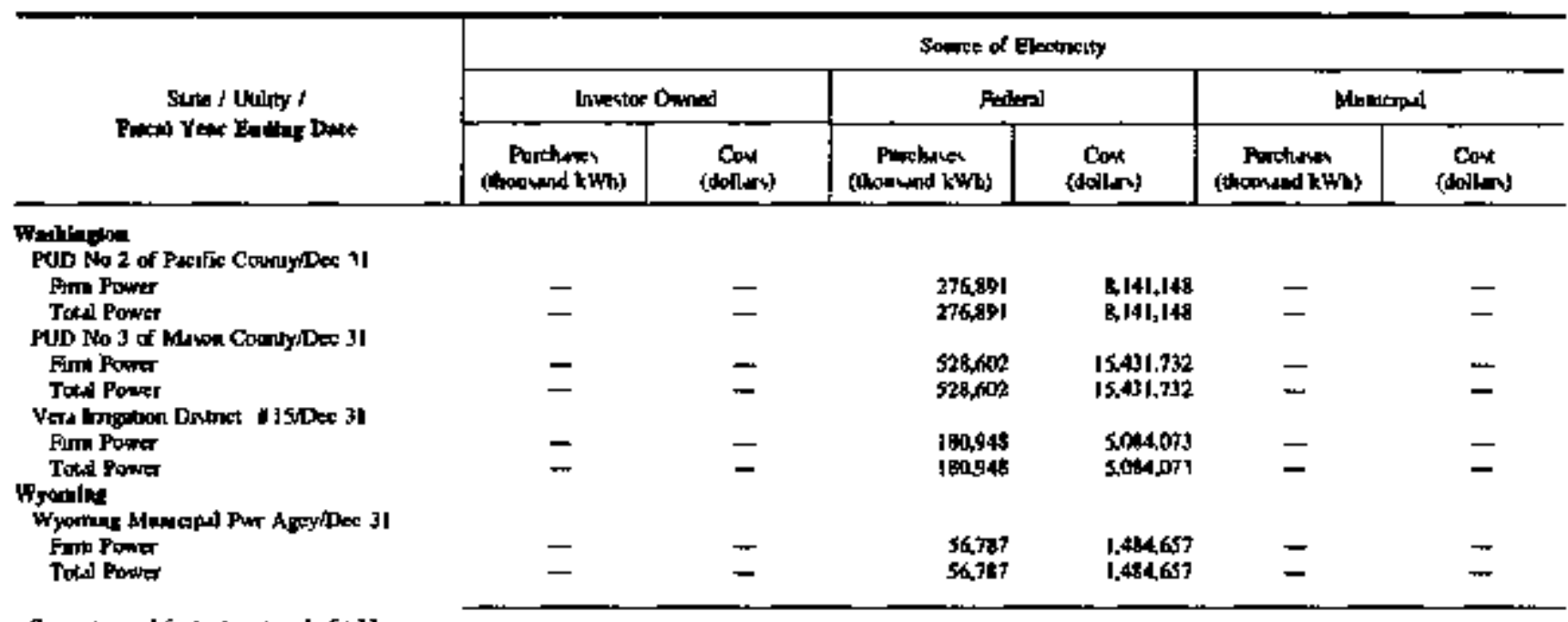

Table 27. Electritity Purchases by State and Oher Governmment Utilities, Fiscal Year 1994 (Contisnated)

\begin{tabular}{|c|c|c|c|c|c|c|}
\hline \multirow{3}{*}{ 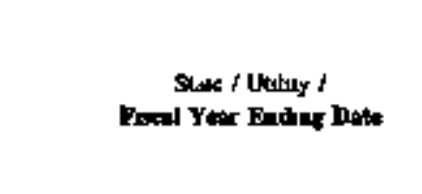 } & \multicolumn{6}{|c|}{ Soure of Eecureniy } \\
\hline & \multicolumn{2}{|c|}{ Coopstarve } & \multicolumn{2}{|c|}{ Other? } & \multicolumn{2}{|c|}{ Told } \\
\hline & 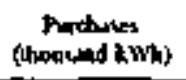 & $\underset{\text { Cont }}{(\text { dofland }}$ & $\begin{array}{l}\text { Puncheves } \\
\text { (thounent kw/h) }\end{array}$ & $\underset{\text { (donlas) }}{\text { Cond }}$ & 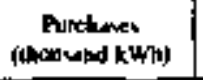 & $\begin{array}{c}\text { Con } \\
\text { (dilla) }\end{array}$ \\
\hline \multicolumn{7}{|l|}{ Thellagton } \\
\hline Fiास POW & - & - & - & - & 276,895 & $8,141,148$ \\
\hline Toud Pown & - & - & - & - & $2 \pi, 891$ & $8,141,140$ \\
\hline \multicolumn{7}{|l|}{ 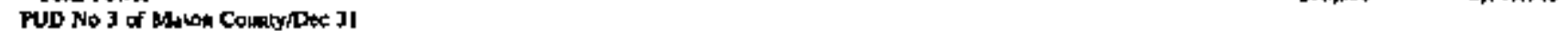 } \\
\hline Prom Ponver & - & - & - & - & s26,6n2 & $15,431,732$ \\
\hline Tocd Power & - & - & - & - & $\operatorname{ses} \sin 2$ & $15,491,732$ \\
\hline 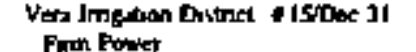 & - & - & - & - & 180,948 & 5.084073 \\
\hline Tacel Pount & - & - & - & - & 180,948 & sosutns \\
\hline \multicolumn{7}{|l|}{ Pyoming } \\
\hline Fin Poutr & - & - & $\rightarrow$ & - & 56,767 & $1,484,657$ \\
\hline Total Poutrer & - & - & - & $=$ & 36.787 & $1,484,657$ \\
\hline
\end{tabular}

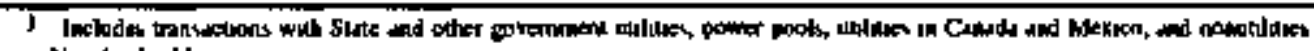
-Nor Applicdle

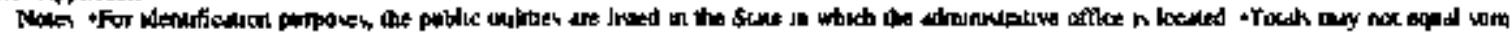
of compontints beculise of indapeadest fionding

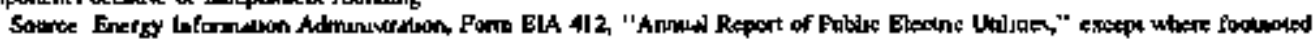


Table 28. Electricity Sales tor Resale by State and Other Government Utilities, Fiscal Year 1994

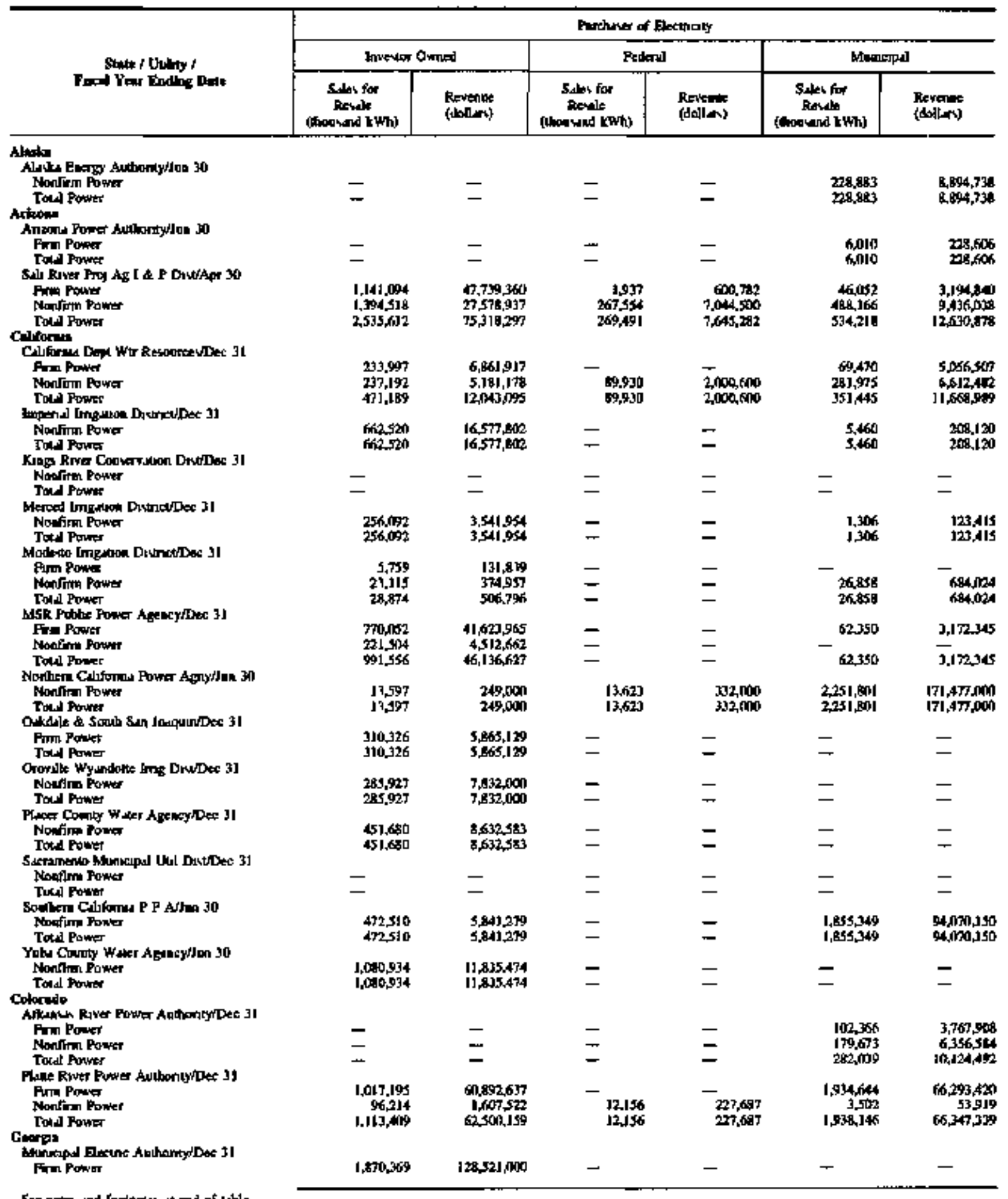


Table 28. Elecirtelity Saltes tor Resale by State and Other Government Utities, Fiscal Year 1994 (Continued)

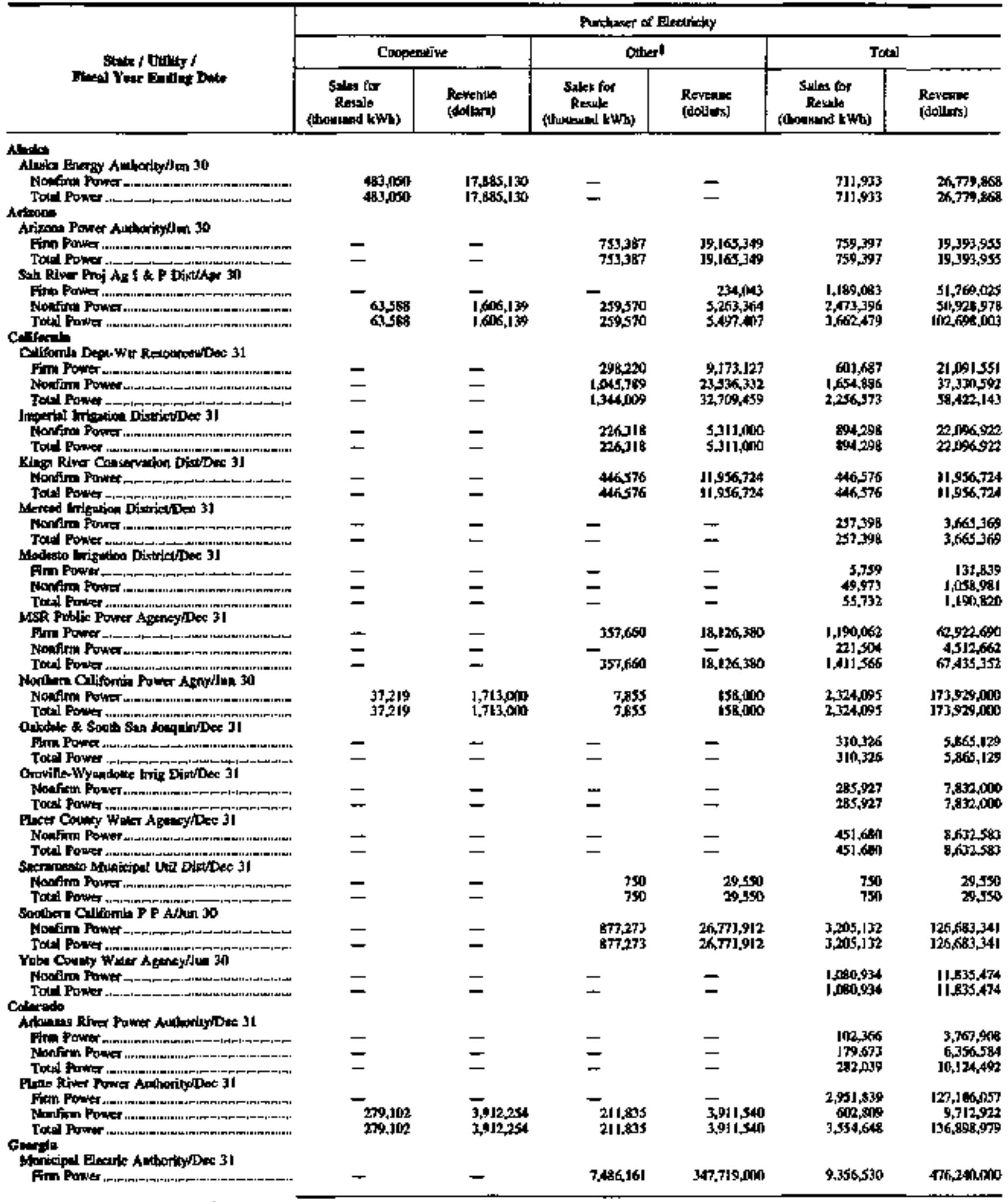

Sec noces and footrotis as end of toble 
Table 28. Electricity Sales for Resale by State and Other Government Utilities, Fiscal Year 1994 (Continned)

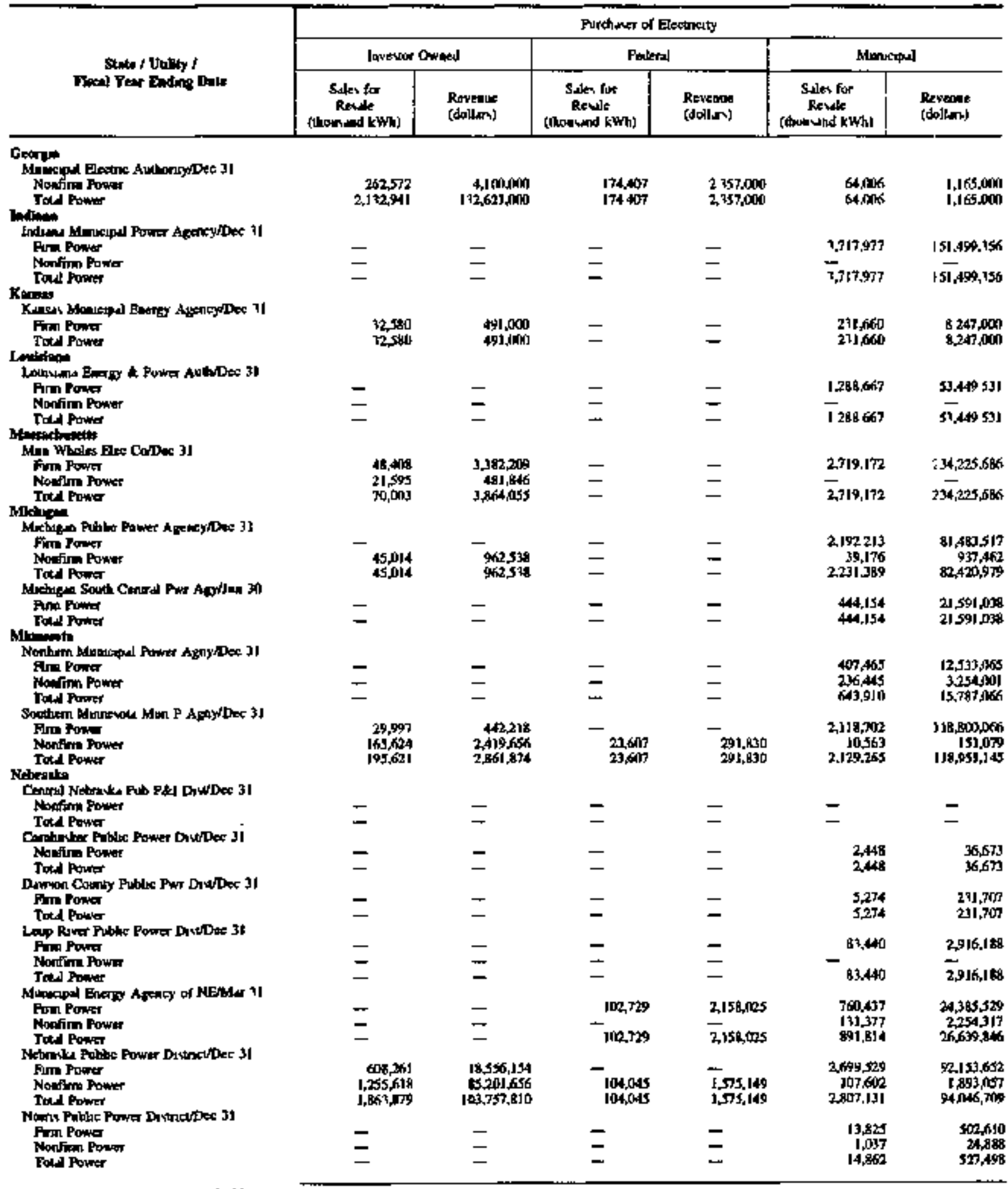

Set noter and footencen at and of wble 
Tabje 28. Electricity Sales for Resale by Siate and Obher Government Utllites, Fiscal Year 1994 (Continued)

\begin{tabular}{|c|c|c|c|c|c|c|}
\hline \multirow{3}{*}{ 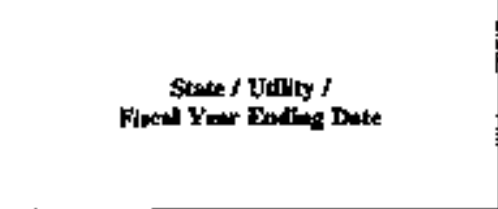 } & \multicolumn{6}{|c|}{ Purchoner of Elocrisity } \\
\hline & \multicolumn{2}{|c|}{ Cooperstive } & \multicolumn{2}{|c|}{ Outer 1} & \multicolumn{2}{|c|}{ Todn } \\
\hline & 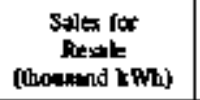 & 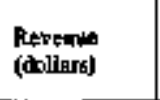 & 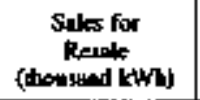 & $\begin{array}{l}\text { Reyenut } \\
\text { (dollatis) }\end{array}$ & 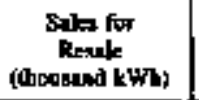 & $\begin{array}{l}\text { Rtweluat } \\
\text { (dohlurs) }\end{array}$ \\
\hline \multicolumn{7}{|l|}{ 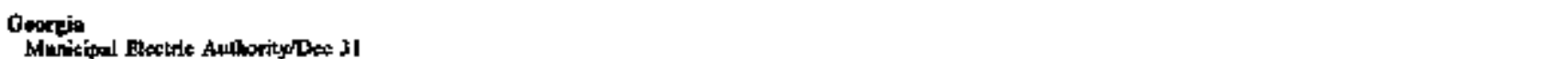 } \\
\hline 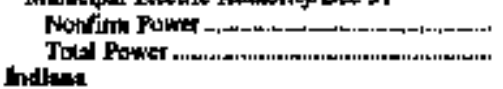 & - & $\overline{-}$ & $\begin{array}{r}31,948 \\
7,518,169\end{array}$ & 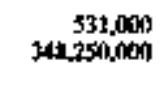 & $\begin{array}{r}592,933 \\
9,889,463\end{array}$ & $\begin{array}{r}8,153,010 \\
484,393010\end{array}$ \\
\hline \multicolumn{7}{|l|}{ 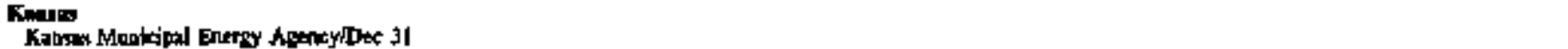 } \\
\hline Fim Pown & - & - & - & - & 254200 & $8,738,000$ \\
\hline \multicolumn{5}{|l|}{ 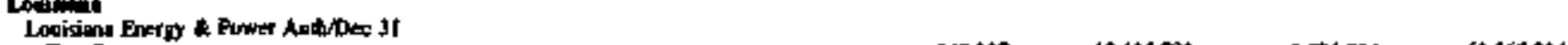 } & 261,240 & $8.738,0010$ \\
\hline Flm Pewter..... & 一 & - & 242,387 & $10,115,793$ & $1.5+1,054$ & 61565,724 \\
\hline 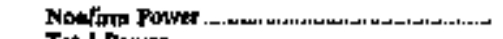 & - & - & $1, B 72$ & 75,738 & 1,872 & 75,738 \\
\hline \multicolumn{7}{|l|}{ Mann Wholes Dlec CoDDex 11} \\
\hline 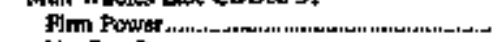 & 一 & - & 56,266 & $6,587,5,55$ & $2,023,846$ & $24,190,440$ \\
\hline 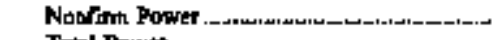 & 一 & - & - & -[]$, 086, x+40$ & 21,595 & $-12,604,994$ \\
\hline 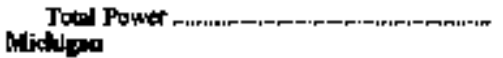 & - & - & 56,266 & $-6,504,295$ & $2,8<5,441$ & $251,585,446$ \\
\hline \multicolumn{7}{|l|}{ 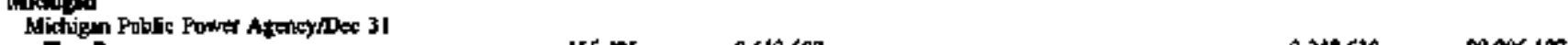 } \\
\hline Firm Power & 156,425 & $8,612,600$ & - & - & $2,3+0,698$ & 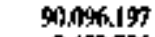 \\
\hline 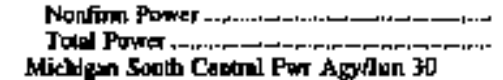 & - I56,4:5 & $8,612,600$ & $\begin{array}{l}20,256 \\
27,216\end{array}$ & $\begin{array}{l}52,726 \\
522,726\end{array}$ & $\begin{array}{r}107,406 \\
2,456,044\end{array}$ & $\begin{array}{r}2,422,726 \\
92,55,923\end{array}$ \\
\hline Fim Power & - & - & - & - & 44.154 & $21,591,038$ \\
\hline 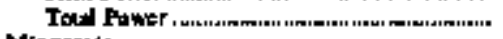 & - & - & - & $\rightarrow$ & 44,154 & $21,591,008$ \\
\hline \multicolumn{7}{|l|}{ 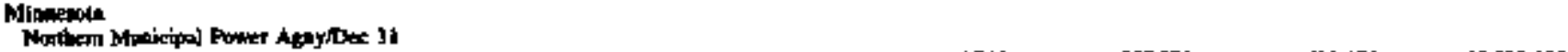 } \\
\hline Fim Pown & $m$ & عـ & $14, \mathbf{3} 13$ & 557,301 & 22.178 & $11,090,456$ \\
\hline 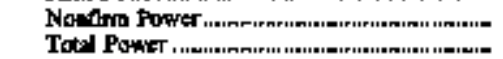 & $\begin{array}{l}54,883 \\
54,883\end{array}$ & $\begin{array}{l}2 J, 485,534 \\
2 J, 485,534\end{array}$ & $\begin{array}{l}48512 \\
63,295\end{array}$ & $\begin{array}{r}306,002 \\
1,36,593\end{array}$ & $\begin{array}{l}339,910 \\
762,104\end{array}$ & $\begin{array}{l}25,445,597 \\
18,531,943\end{array}$ \\
\hline Southern Minmesok Mom P AupryDec 3] & & & & & & \\
\hline 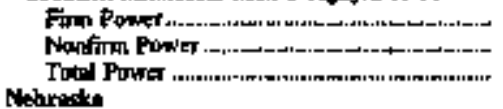 & $\begin{array}{r}1,157 \\
176,8800 \\
17 \$, 037 ?\end{array}$ & $\begin{array}{r}19,130 \\
2,665,248 \\
2,6,14376\end{array}$ & $\begin{array}{l}2,818 \\
34,2691 \\
37,1998\end{array}$ & $\begin{array}{r}45,767 \\
562,451 \\
608,218\end{array}$ & $\begin{array}{r}2,152,674 \\
110,064 \\
2,5,1,628\end{array}$ & $\begin{array}{r}19,307,18] \\
6 \sqrt{19}, 264 \\
125,387,446\end{array}$ \\
\hline \multicolumn{7}{|l|}{ Nehradk } \\
\hline 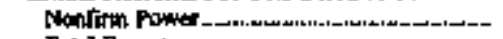 & - & - & 986,589 & In.295, 999 & 385,188 & Jn295,999 \\
\hline Totel Pown , & - & - & 386,189 & $10,295,999$ & 386,189 & $10,295,999$ \\
\hline Conduoder Pablic Pawer Diedthoc Il & & & & & & \\
\hline 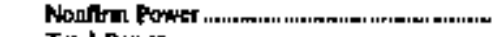 & 一 & - & 一 & 一 & 2,448 & 36,673 \\
\hline 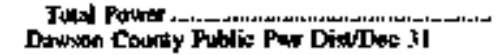 & - & - & - & $m$ & 2,448 & 36573 \\
\hline 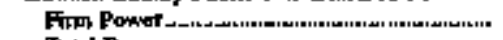 & - & - & - & - & 5,774 & $231,70 \%$ \\
\hline 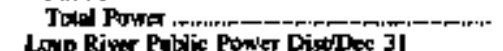 & - & - & - & - & 5,274 & 231,707 \\
\hline 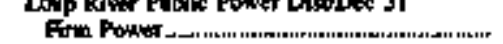 & $200,0,5$ & $6,335 ., 2010$ & - & $\rightarrow$ & $2: 3,0,5$ & $9,247,508$ \\
\hline 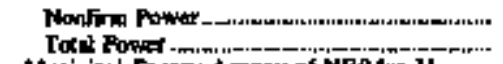 & - 200,003 & $\overline{6} 131,320$ & $\begin{array}{l}128,208 \\
128,205\end{array}$ & $\begin{array}{l}4,132,260 \\
4.137,360\end{array}$ & $\begin{array}{l}126,205 \\
411,730\end{array}$ & $\begin{array}{r}4,117,360 \\
13,384,864\end{array}$ \\
\hline Muninipul Everty Asency of NEAdkr II & & & & & & \\
\hline 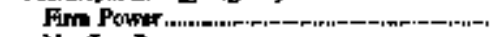 & 一 & - & - & - & 853,166 & 26,343554 \\
\hline 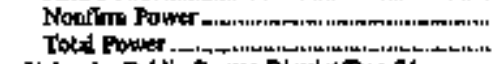 & $\begin{array}{l}3,618 \\
3,6058\end{array}$ & $\begin{array}{l}60,961 \\
63,963\end{array}$ & 727 & $\begin{array}{l}19,864 \\
10,864\end{array}$ & $\begin{array}{l}135,772 \\
995,979\end{array}$ & $\begin{array}{r}2,1338,144 \\
28,041,696\end{array}$ \\
\hline Nebpiks Public Powner DivertedDec 31 & & & & & & \\
\hline 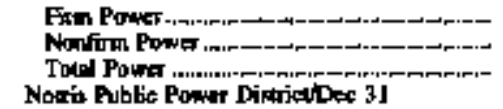 & $\begin{array}{r}91,716 \\
625,161 \\
716,57\end{array}$ & $\begin{array}{r}1,110,691 \\
9,069,985 \\
12,170,679\end{array}$ & $\begin{array}{r}3,724,816 \\
28,750 \\
0,753566\end{array}$ & 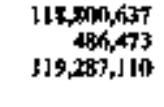 & $\begin{array}{l}7,124,723 \\
2,121,176 \\
9,245,498\end{array}$ & $\begin{array}{r}232,621,134 \\
98216,318 \\
320,837,452\end{array}$ \\
\hline Frm Power_ons & - & - & - & 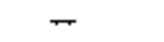 & 17,805 & 500610 \\
\hline 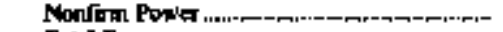 & 一 & - & 一 & 一 & $1, \mathbb{B B ?}$ & 24,B898 \\
\hline 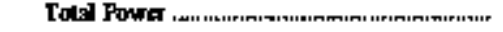 & - & 一 & - & 一 & 14,862 & 527,498 \\
\hline
\end{tabular}

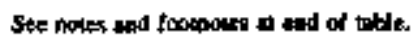


Table 28. Electricity Sales for Resale by State and Other Government Utilthes, Fiscal Year 1994 (Continued)

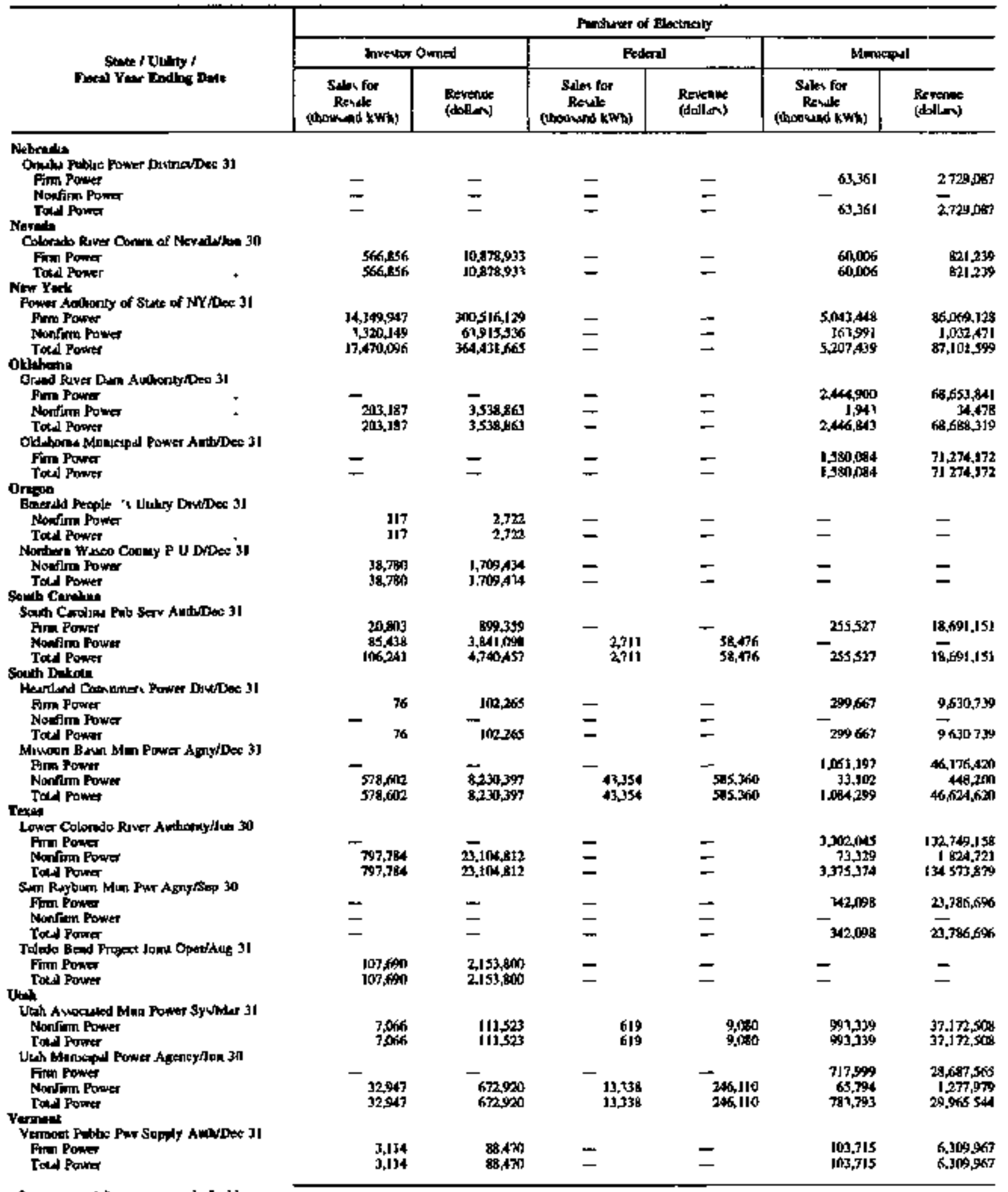

Ser noter ind footuoles at and of bele 
Tabłt 28. Electricity Sales for Resale by State and Other Government Utilities, Fiscal Year 1994 (Continoed)

\begin{tabular}{|c|c|c|c|c|c|c|}
\hline \multirow{3}{*}{ 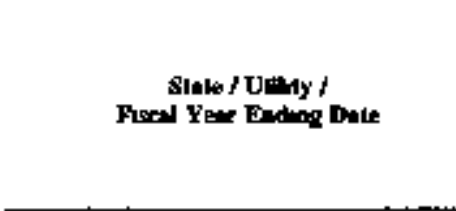 } & \multicolumn{6}{|c|}{ Ponture of Elostenty } \\
\hline & \multicolumn{2}{|c|}{ Coopenwive } & \multicolumn{2}{|c|}{ Otheri } & \multicolumn{2}{|c|}{ Trad } \\
\hline & 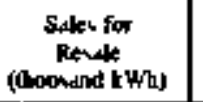 & $\begin{array}{l}\text { Reveride } \\
(\text { (WAR }\end{array}$ & 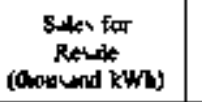 & $\begin{array}{l}\text { Rerenue } \\
\text { (dorlines) }\end{array}$ & $\begin{array}{c}\text { Suke for } \\
\text { Revde } \\
\text { (thoound kWh) }\end{array}$ & $\begin{array}{l}\text { Rewerue } \\
\text { (dollarivit }\end{array}$ \\
\hline \multicolumn{7}{|l|}{ Nebraken } \\
\hline Firm Power & - & - & - & - & 61,361 & $2,729,087$ \\
\hline Nowrim Power & - & - & 114,128 & $1,482,126$ & 114,128 & $1,482,124$, \\
\hline \multicolumn{7}{|l|}{$\begin{array}{l}\text { Nevedis } \\
\text { Colorodo Rive } 0\end{array}$} \\
\hline 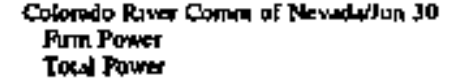 & $\begin{array}{l}152, \text { n10 } \\
152,010\end{array}$ & $\begin{array}{l}2,754,516 \\
2,794,516\end{array}$ & 186,280 & $\begin{array}{l}2,9 \mathrm{JB}, 389 \\
2,9 \mathrm{JB}, 789\end{array}$ & 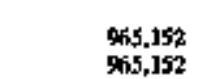 & $\begin{array}{l}17,393,077 \\
17,393,077\end{array}$ \\
\hline \multirow{2}{*}{\multicolumn{7}{|c|}{ 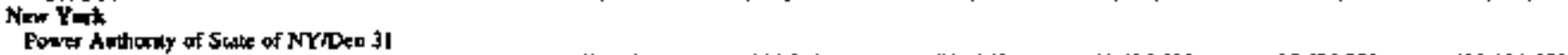 }} \\
\hline & & & & & 20.519303 & $408,104,656$ \\
\hline Tonfim Powa & - & $8,273,871$ & 202.539 & $\begin{array}{l}11,0,5,086 \\
37,562,531 \\
57.248,217\end{array}$ & 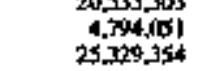 & $\begin{array}{l}102,950,696 \\
51,055,352\end{array}$ \\
\hline \multicolumn{7}{|l|}{ Ohliningina } \\
\hline 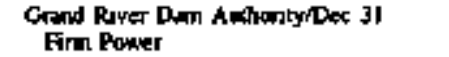 & 1827 A A & $x+46 x_{1}, 813$ & 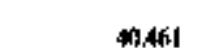 & 863,987 & $4,312,770$ & 133,984,64I \\
\hline 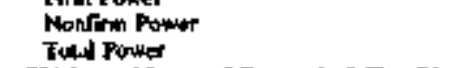 & $\begin{array}{l}71,0018 \\
1,899,217\end{array}$ & $\begin{array}{l}1,169,116 \\
6,635,129\end{array}$ & $\begin{array}{l}2100195 \\
250,497\end{array}$ & $\begin{array}{l}5,495,796 \\
6,359,723\end{array}$ & $\begin{array}{r}487,064 \\
4,799, R J 4\end{array}$ & $\begin{array}{l}10237393 \\
1442221034\end{array}$ \\
\hline 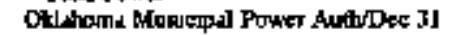 & & & & & & \\
\hline Furn Power & - & - & - & - & $1,530,0004$ & $71,274,172$ \\
\hline \multirow{2}{*}{\multicolumn{7}{|c|}{ 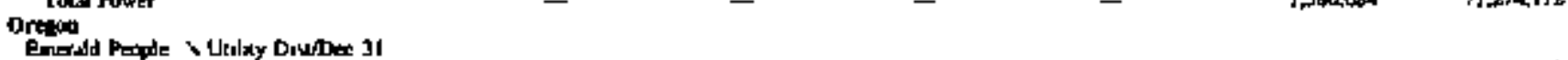 }} \\
\hline & & & & & & \\
\hline Nonsin Powet & $m$ & - & - & - & 117 & 2,72 \\
\hline Tocdi Power & - & - & - & - & II7 & 2,72 \\
\hline 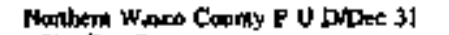 & & & & & & \\
\hline Nombind Pown & - & - & - & - & 38,380 & $1,709,434$ \\
\hline $\begin{array}{l}\text { Totdl Power } \\
\text { Souxh Caroties }\end{array}$ & - & - & - & - & 38,780 & $1.709,434$ \\
\hline \multicolumn{7}{|l|}{ 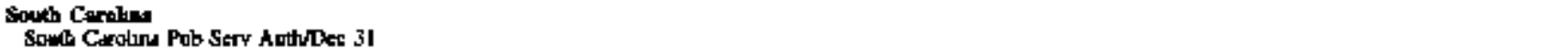 } \\
\hline 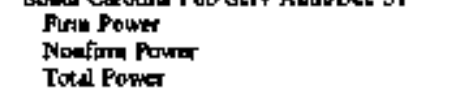 & $\begin{array}{r}6,907,249 \\
18,792 \\
6,922,1191\end{array}$ & $\begin{array}{l}277,520,906 \\
361,864 \\
27 t, 28,171\end{array}$ & $\begin{array}{r}\text { If, } 135 \\
1,213 \\
14548\end{array}$ & $\begin{array}{r}468,527 \\
94,360 \\
963,782\end{array}$ & $\begin{array}{l}7,1189,514 \\
111,144 \\
7,391,098\end{array}$ & $\begin{array}{r}297,979,797 \\
4,759,300 \\
302,399,037\end{array}$ \\
\hline \multirow{2}{*}{\multicolumn{7}{|c|}{ 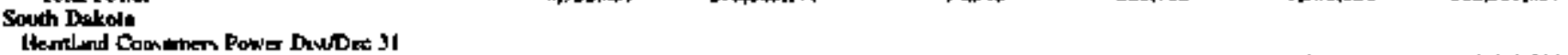 }} \\
\hline & & - & - & - & $299,74]$ & $4,533,0,0 \mathrm{~d}$ \\
\hline Nontom Power & - & - & 133,915 & $5,706,209$ & 137915 & $1,705,209$ \\
\hline Totd Apras & - & - & 173915 & $5,705,209$ & 439,658 & [1],4]B,213 \\
\hline 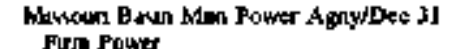 & & & & & & \\
\hline $\begin{array}{l}\text { Furn Power } \\
\text { Nopefont Pown }\end{array}$ & - & - & - & $-121,939$ & 1,051,J97 & Afingsedti \\
\hline $\begin{array}{l}\text { Noofinu Nown } \\
\text { Toul Pouter }\end{array}$ & $\begin{array}{l}14,116 \\
14,116\end{array}$ & $\begin{array}{l}18 x_{1}, 117 \\
\text { IB }, 117\end{array}$ & $\begin{array}{l}177,748 \\
177,738\end{array}$ & $\begin{array}{l}2,517.447 \\
2,395,504\end{array}$ & $\begin{array}{r}846,932 \\
3,898,129\end{array}$ & 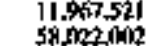 \\
\hline \multirow{2}{*}{\multicolumn{7}{|c|}{ Texwo }} \\
\hline 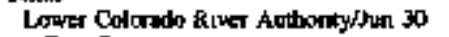 & & & & & & \\
\hline Firm Pauser & $5,0084,477$ & $2019,863,50 \%$ & - & - & R.387.012 & $342,612,667$ \\
\hline Nonforit hower & 559,9713 & $5,35,0,0,0$ & 26570 & f548.244 & $1,057,653$ & $30,928,737$ \\
\hline 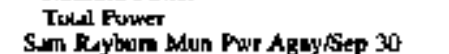 & $5,244,507$ & $215,214,499$ & 26,570 & $f+46,244$ & $9,444,065$ & $773,541,404$ \\
\hline 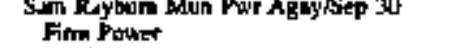 & - & - & - & - & $742.0 \%$ & $27,7 \pm 6,696$ \\
\hline Nonfim Prower & - & - & 282,177 & $4,547,978$ & 262,177 & 4,5*0,979 \\
\hline $\begin{array}{l}\text { Totil Porug } \\
\text { Toleto Band Projera lour Operiaug 3t }\end{array}$ & - & - & 282,137 & $4,540,978$ & 024,275 & 28327,674 \\
\hline $\begin{array}{l}\text { Fum Powa } \\
\text { Totdid Popter }\end{array}$ & - & $\bar{z}$ & $\begin{array}{l}107,600 \\
07,690\end{array}$ & $\underset{2,153,300}{2,150}$ & $\begin{array}{l}215,390 \\
215,380\end{array}$ & $\begin{array}{l}4,406,610 \\
\operatorname{lin}, 600\end{array}$ \\
\hline \multirow{2}{*}{\multicolumn{7}{|c|}{ 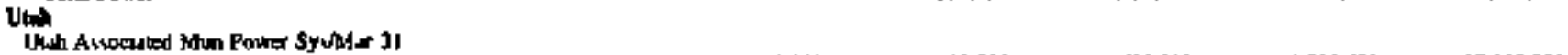 }} \\
\hline & & & & & & \\
\hline $\begin{array}{l}\text { Nonfim Power } \\
\text { Totel Poprer }\end{array}$ & $\begin{array}{ll}110 \\
110\end{array}$ & 2,243 & 10,553 & 61918 & $1,100,689$ & $\begin{array}{l}37,929.272 \\
37069.272\end{array}$ \\
\hline Tot Morier & & & 19,555 & 631,918 & $1,120,1889$ & $37.929,272$ \\
\hline Fimn Power & - & - & - & - & 717,599 & $28,687,565$ \\
\hline Nodfirma hrowes & $1,56,2$ & 27,413 & 1532 & 11,364 & 115.173 & $2,255,790$ \\
\hline \multirow{3}{*}{\multicolumn{7}{|c|}{ 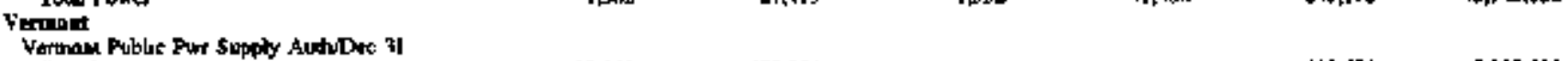 }} \\
\hline & & & & & & \\
\hline & & 689,364 & 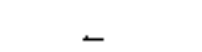 & - & 119, STI & $7,0057,8,0,1$ \\
\hline Towed Ppop & $12, \ldots+2$ & 689.364 & - & $ـ$ & II9 मसI & $7,057,801$ \\
\hline
\end{tabular}

See nowen and footnoted at end or whe 
Table 28. Electricity Sales for Ressle by State and Other Government Utilities, Fiscal Year 1994 (Conlinued)

\begin{tabular}{|c|c|c|c|c|c|c|}
\hline \multirow{3}{*}{ 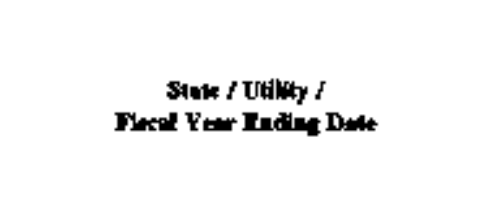 } & \multicolumn{6}{|c|}{ Aurchues of electrinthy } \\
\hline & \multicolumn{2}{|c|}{ Investor Orthed } & \multicolumn{2}{|c|}{ Fedor:l } & \multicolumn{2}{|c|}{ Monicipod } \\
\hline & $\begin{array}{c}\text { Sales for } \\
\text { kemle } \\
\text { (thourend } k \text { Wh) }\end{array}$ & $\begin{array}{l}\text { ReVemas } \\
\text { (dollers) }\end{array}$ & 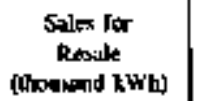 & $\begin{array}{l}\text { Rernowt } \\
\text { (dodlow) }\end{array}$ & 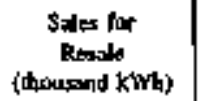 & $\begin{array}{l}\text { Retenute } \\
\text { (duTfra) }\end{array}$ \\
\hline \multicolumn{7}{|l|}{ WuD No I of Gelan Coantyidec 31} \\
\hline 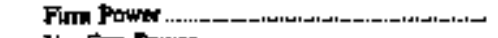 & 281,564 & $3,311,487$ & 985 & IZ,7@ & - & - \\
\hline 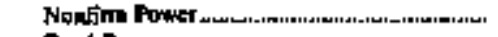 & $5,030,540$ & $6 \$, 597,846$ & 一 & 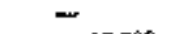 & $55,3,158$ & 6Din, 959 \\
\hline $\begin{array}{l}\text { Tot Power } \\
\text { PUD No I a Cuwfis Conuydot } 31\end{array}$ & $5,832,104$ & $71,719,333$ & 985 & 17,760 & 55.3468 & $6 \times 6,959$ \\
\hline Nonifm PowT & 16,943 & 208,414 & 一 & - & - & 一 \\
\hline 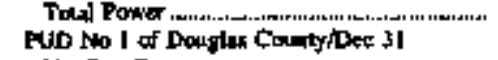 & $16, \$ 43$ & 269.414 & 一 & - & - & - \\
\hline 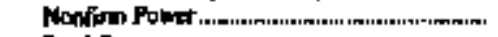 & $2,524,154$ & $19,929,8,51$ & - & - & 20.405 & 231,347 \\
\hline 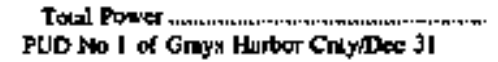 & $2,534,156$ & $19,929,851$ & $m$ & - & 17.05 & 231,847 \\
\hline 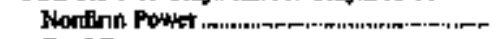 & 490,133 & $9,296,6665$ & - & - & - & - \\
\hline Tokni Fown, , & $100,15 s$ & $9,296, \pi K 5$ & - & - & 一 & - \\
\hline \multicolumn{7}{|l|}{ PUD No 1 of Pead Oredile Earythec 31} \\
\hline 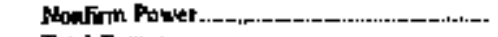 & 9094 & 397,736 & - & 一 & 134,966 & 1.24 .252 \\
\hline 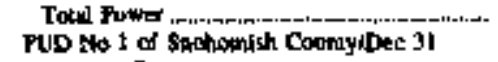 & 9,094 & \rfloor $\mathfrak{M}, 7 \mathbf{T} \mathbf{6}$ & - & - & 134,946 & $1,246.252$ \\
\hline Monfinm Potwer & 6,408 & $2,2018,759$ & - & 一 & $2 . J 65$ & 95.641 \\
\hline 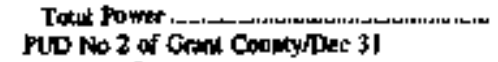 & 6,498 & $2,2018.7 \% 9$ & - & - & 2,763 & 96, 54.40 \\
\hline 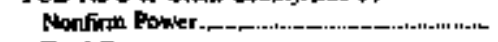 & $4,420,154$ & $36,8,6,2,268$ & 12,938 & 259,646 & 961399 & $6,404,069$ \\
\hline 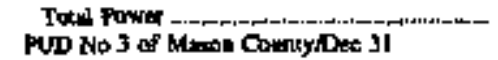 & $4,420,154$ & $36,862,208$ & 32,938 & 259.646 & 941315 & $6,404,062$ \\
\hline Ponfiph Poper & - & - & - & - & - & - \\
\hline 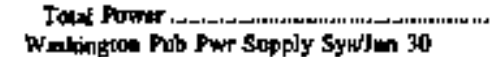 & - & - & - & - & - & - \\
\hline Find Power & - & 一 & - & 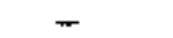 & - & - \\
\hline Nanfinn Poprer & - & - & $3,280,351$ & $356,183,000$ & - & - \\
\hline 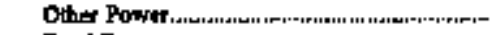 & - & 一 & 55,115 & - & - & - \\
\hline 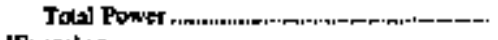 & - & - & $7,343,8666$ & $356,18,7,0000$ & 一 & - \\
\hline Wyomiti: & & & & & & \\
\hline Fim POMT & 一 & $\rightarrow$ & - & - & 151,155 & $5,29,2,060$ \\
\hline 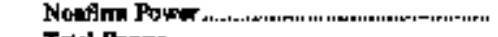 & - & - & 一 & - & ᄃ. & 57.888 \\
\hline 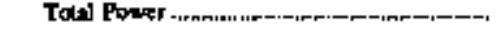 & $r$ & 一 & - & - & 192763 & 5.749 .948 \\
\hline
\end{tabular}

Sec noes and footackes at end of toble. 
Table 28. Electricity Sales tor Resale by State and Other Gopernment Utilifies, Fiscal Year 1994 (Continued)

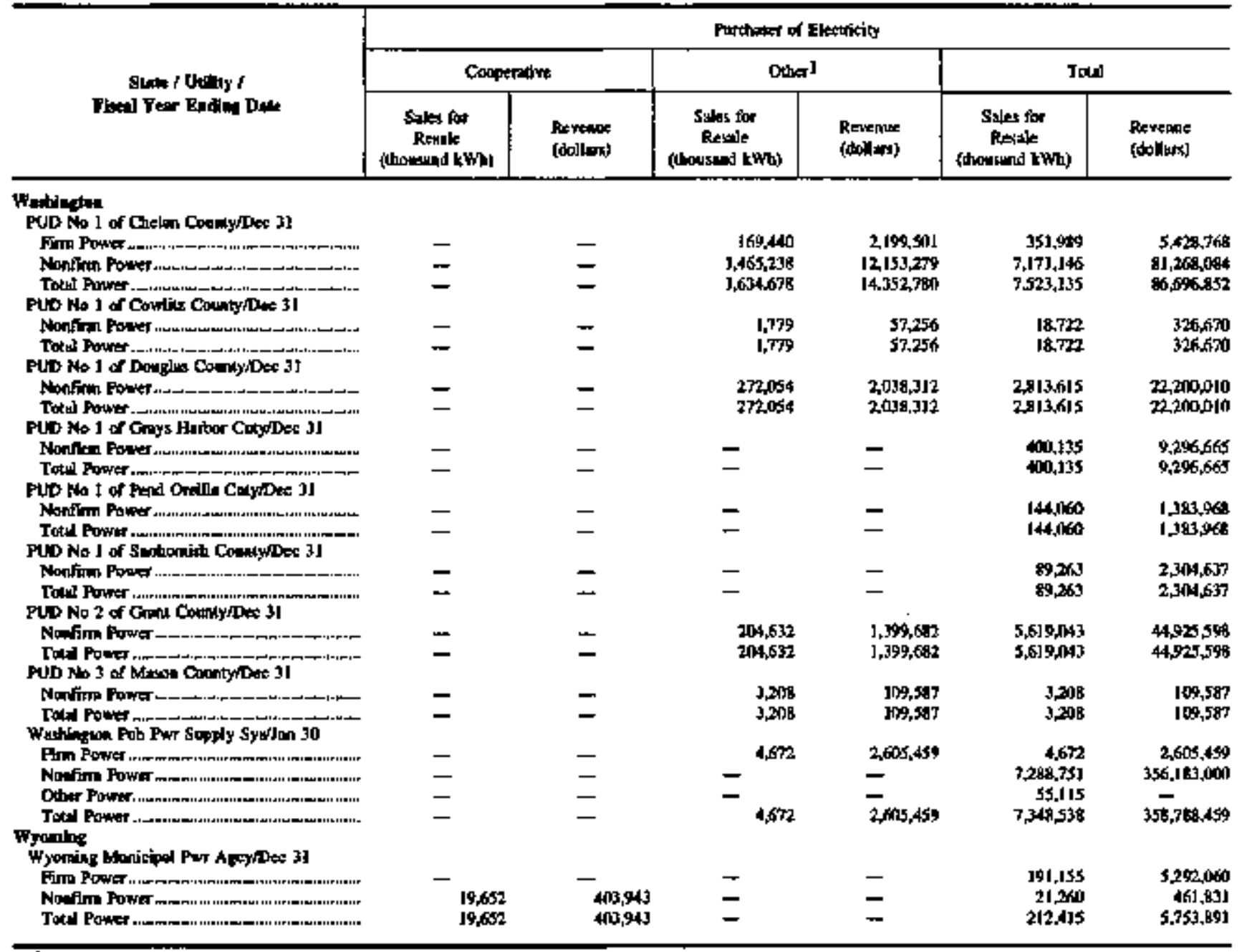

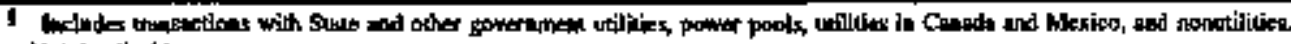

Tial Appition

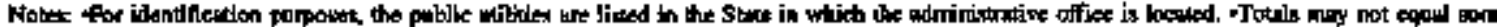

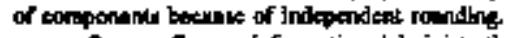

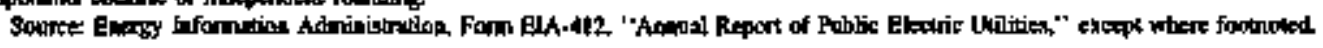


Table 29. Electricity Purchases by Municipal Utilites, Fiscal Year 1994

\begin{tabular}{|c|c|c|c|c|c|c|}
\hline \multirow{3}{*}{ 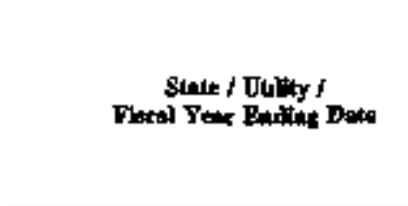 } & \multicolumn{6}{|c|}{ Sourse of Elotumy } \\
\hline & \multicolumn{2}{|c|}{ Invewor Owned } & \multicolumn{2}{|c|}{ Rederal } & \multicolumn{2}{|c|}{ 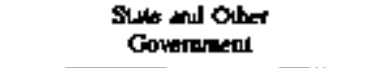 } \\
\hline & $\begin{array}{l}\text { Portluxas } \\
\text { (thoosend kWh) }\end{array}$ & $\begin{array}{c}C_{04} \\
\text { (dollam) }\end{array}$ & $\begin{array}{l}\text { Purchuser } \\
\text { (doodund twhy) }\end{array}$ & $\begin{array}{c}\text { Coit } \\
\text { (ddellurut }\end{array}$ & Punthes: & $\begin{array}{c}\text { Cont } \\
\text { fdollent }\end{array}$ \\
\hline \multicolumn{7}{|l|}{ Abobern: } \\
\hline \multicolumn{7}{|l|}{ 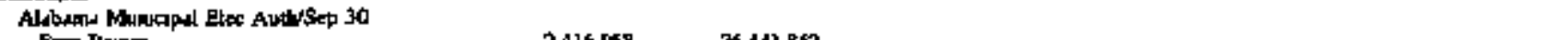 } \\
\hline Tokd Power & $2,416,088$ & $\begin{array}{ll}m, 411,8502 \\
76,411,862\end{array}$ & $\bar{z}$ & $\overline{-}$ & $\overline{-}$ & $\bar{z}$ \\
\hline \multicolumn{7}{|l|}{ 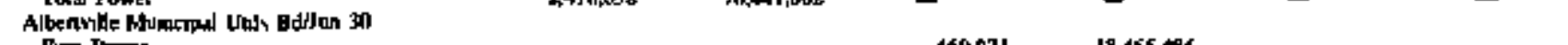 } \\
\hline $\begin{array}{l}\text { Fimi Pwort } \\
\text { Totd Power }\end{array}$ & - & - & 469,971 & $18,465,486$ & - & - \\
\hline \multicolumn{7}{|l|}{ Andalue Oly oftsep औ } \\
\hline Fim Power & - & - & - & - & - & - \\
\hline \multirow{2}{*}{\multicolumn{7}{|c|}{ Adues City abtoed 31}} \\
\hline & & & & & & \\
\hline Pum Power & - & - & 728.982 & $31,8+2,12]$ & - & - \\
\hline Totd Power & - & - & 728,982 & $31,822,127$ & - & - \\
\hline 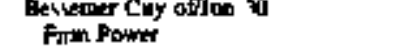 & - & - & 241,417 & $14,634,577$ & - & - \\
\hline Total Ponet & - & - & $4,3,1\}$ & $14,634,57 ?$ & - & - \\
\hline \multicolumn{7}{|l|}{ Collomin Poure Bowdrjon 30} \\
\hline Finn Power & - & - & 724,324 & ] $4,414,813$ & - & - \\
\hline Tald Pawet & - & - & 321,224 & $14,4,4,813$ & - & - \\
\hline Devertur Cisy offtan 50 & - & & & & - & \\
\hline Told Power & $\overline{-}$ & $\bar{z}$ & $1,461,320$ & $\sin , 904$ & $=$ & $z$ \\
\hline \multicolumn{7}{|l|}{ Dodum Chy offSep 30} \\
\hline Fim Porta & - & - & 77,575 & $3,543,435$ & - & - \\
\hline Tad Power & - & - & 73,575 & $1, \sqrt{43,405}$ & - & - \\
\hline \multicolumn{7}{|l|}{ Fitrente Cry offlum 30} \\
\hline Firm Powet & - & - & $1,6887,635$ & $48,125,501$ & عـ & 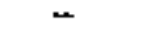 \\
\hline Trad Pawe & - & - & $1,0287.6355$ & $44,125.5 \mathrm{Br}$ & - & - \\
\hline Foley Crty of (Rivetra Utulvides 3J & & & & & & \\
\hline Fim Pown & - & - & 34,748 & $5,295,473$ & ع & - \\
\hline 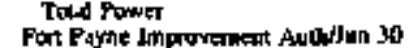 & - & - & 4,748 & $5.293,473$ & - & - \\
\hline Firm Power & - & - & 326,954 & 14, tos, 726 & - & - \\
\hline Tow Power & - & - & 320,954 & 14.395 .726 & - & - \\
\hline 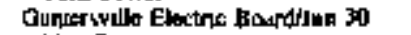 & & & & & & \\
\hline Fim Powr & - & - & 234,148 & Jn,3I.,8I I & - & - \\
\hline Tades Power & - & - & 234,148 & $10,31,81$ & - & - \\
\hline 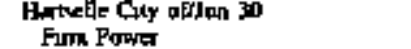 & $m$ & - & & 6.153) & - & \\
\hline Tard Pawter & - & $\bar{z}$ & $136,62 \pi$ & 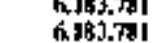 & $=$ & $\bar{z}$ \\
\hline Henuswdle Crty atssep 30 & & & & & & \\
\hline Fim Powe & 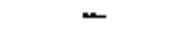 & - & $3,980,862$ & $373,000,649$ & - & - \\
\hline Toxal Porwar & - & 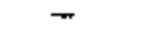 & 3980,802 & $173,064,649$ & - & - \\
\hline 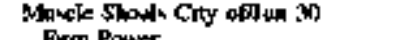 & & & & & & \\
\hline $\begin{array}{l}\text { Frm Powter } \\
\text { Totd Powert }\end{array}$ & $\bar{z}$ & - & 206,173 & $9.424,794$ & - & - \\
\hline $\begin{array}{l}\text { Totd Pown } \\
\text { Onelde Cuy opsen } 30\end{array}$ & - & - & $208,17 \%$ & $9,427,794$ & - & - \\
\hline $\begin{array}{l}\text { Opejpa Chy apsep } 30 \\
\text { Pim Power }\end{array}$ & & & & & & \\
\hline Pimm Power & 290,011 & $12.677,528$ & 28,138 & 1.254:016 & - & - \\
\hline Toul Power & 29a,all & 12.57528 & 28,138 & $1,254,016$ & - & - \\
\hline 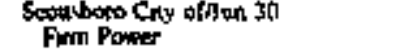 & w & - & 260000 & $1 d 060186$ & - & - \\
\hline Totdl Power & - & $\overline{-}$ & $\$ 66,002$ & $14 \times 2,38 \pi$ & $\bar{z}$ & $\bar{z}$ \\
\hline Sheficetd UnlutevJua 3n & & & & & & - \\
\hline Fm Power & - & - & 433,151 & $19,354,04 y$ & - & - \\
\hline Total Power & - & - & 433,151 & $19,354,049$ & - & - \\
\hline 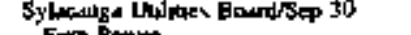 & & & & & & \\
\hline Fwin Pown & - & - & 24,154 & I,01 T,290, & $=$ & $=$ \\
\hline $\begin{array}{l}\text { Totad Pawer } \\
\text { Tnoy Chty of }\end{array}$ & - & - & 24,134 & $1,017,290$ & - & - \\
\hline Pirm Power & 2009,676 & 754.222 & 21. 308 & [6s,037 & - & - \\
\hline toul Power & 208,676 & $7,547,722$ & 21.305 & 655,037 & - & - \\
\hline 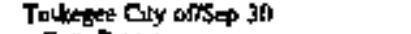 & & & & & & \\
\hline Frin Ponter & - & - & $\mid 7$, KI|B & 715,762 & - & - \\
\hline ICld Powe & - & - & $17,6.18$ & 715,762 & - & - \\
\hline Alasta & & & & & & \\
\hline 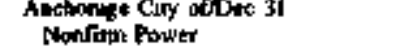 & - & - & 73,395 & $1.344,280$ & 98,45 & 3,605,206 \\
\hline Iotal Porwer & - & - & 73,395 & $1,344,28 n$ & $98,4 s$ & $3,600,256$ \\
\hline
\end{tabular}

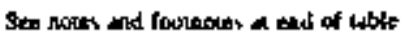


Table 29. Dectricily Purchases by Munteipal Utilites, Fiscal Year 1994 (Continued)

\begin{tabular}{|c|c|c|c|c|c|c|}
\hline \multirow{3}{*}{ Slave / Udity t } & \multicolumn{6}{|c|}{ Source of Elextriclyy } \\
\hline & \multicolumn{2}{|c|}{ Cocperainie } & \multicolumn{2}{|c|}{ OTh $\rightarrow$} & \multicolumn{2}{|c|}{ Tot: } \\
\hline & 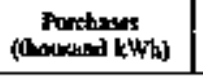 & Cod & (lionomid kwh & $\underset{\text { (dollors) }}{\cos }$ & (Alowingad kWh) & $\underset{\text { Conlurs) }}{\text { Cons }}$ \\
\hline \multicolumn{7}{|l|}{ 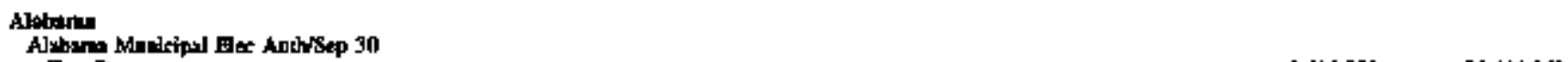 } \\
\hline Frm Fowner & - & - & - & - & $2,416,05 t$ & $26,4+1,800$ \\
\hline 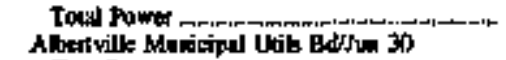 & - & - & - & - & $2,416,1758$ & $76,441,662$ \\
\hline Fm Power & - & - & - & 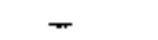 & 409971 & $18,465,186$ \\
\hline 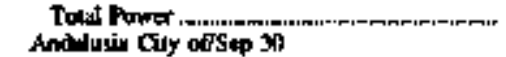 & - & - & - & - & 469,971 & $18,465,486$ \\
\hline Fim Power & 329,184 & $11,936,946$ & - & - & 329.184 & $11,936,966$ \\
\hline 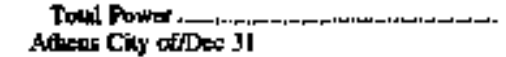 & 329,184 & $11,936,966$ & س & - & 209.184 & 119.16966 \\
\hline 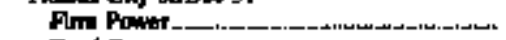 & - & - & - & - & 728,982 & $31,822,127$ \\
\hline 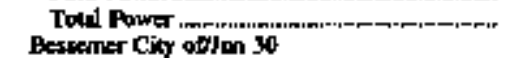 & - & - & - & - & 328,962 & $31,822,127$ \\
\hline Fim Povra & - & - & - & - & 3AJ,A63 & {$[4,634,577$} \\
\hline 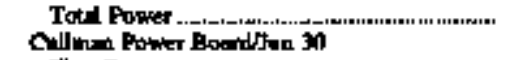 & - & - & - & - & 343,413 & $14,634,377$ \\
\hline 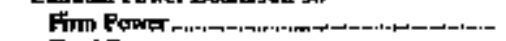 & - & - & - & - & $x_{24}, 724$ & $14,40 \mathrm{~d} B 13$ \\
\hline 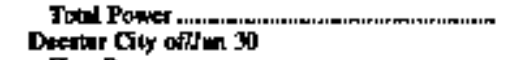 & - & - & - & - & 324,324 & $14,404,813$ \\
\hline 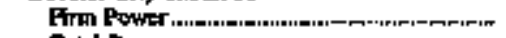 & سـ & - & - & - & $1,463,725$ & $56,225,934$ \\
\hline 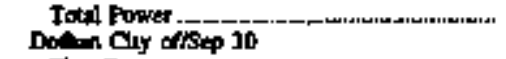 & - & - & - & - & $1,463,729$ & $36,228,934$ \\
\hline Finn Power-mer & - & - & 936,038 & $34,725,2 \times 19$ & $1,014,2) 3$ & $59,868,615$ \\
\hline 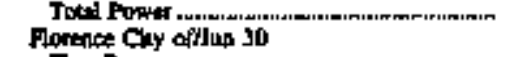 & - & - & $936,0,5 B$ & $36,725,291$ & $1,0 \mid 4,219$ & $\$ 9,868,695$ \\
\hline Firn Pourer & - & - & - & - & $1,097,635$ & $48,125,501$ \\
\hline 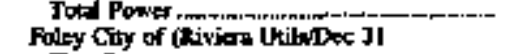 & - & - & - & $=$ & $1,000,693$ & $48,125,501$ \\
\hline 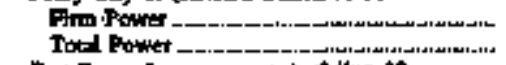 & $\overline{-}$ & $\overline{-}$ & $\begin{array}{l}992,863 \\
492,863\end{array}$ & $\begin{array}{l}22,146,648 \\
22,146,648\end{array}$ & $\begin{array}{l}527,6] 1 \\
527,611\end{array}$ & $\begin{array}{l}23,442,121 \\
23,442,121\end{array}$ \\
\hline 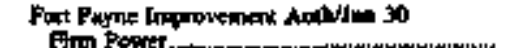 & & & & & & \\
\hline 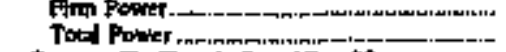 & $\overline{-}$ & $\bar{z}$ & $\bar{z}$ & $\bar{z}$ & $\begin{array}{l}324,954 \\
326,994\end{array}$ & $\begin{array}{l}14,310,726 \\
14,305,726\end{array}$ \\
\hline 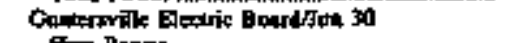 & & & & & & \\
\hline 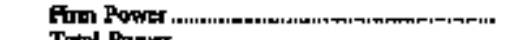 & - & - & - & - & 234,148 & $10,311,64)$ \\
\hline 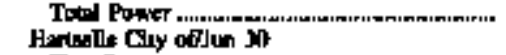 & - & - & - & - & 234,148 & 10.311 .551 \\
\hline Fin Powst & - & $m$ & - & - & 136,625 & $6,183,7 B \mid$ \\
\hline 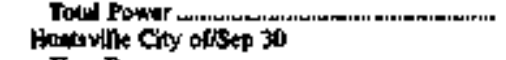 & - & - & - & - & 136,626 & $6,183,781$ \\
\hline Fim Pooner, & - & - & - & - & $3,944.012$ & $191,(10,0,640$ \\
\hline 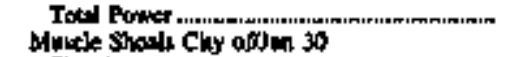 & - & - & - & - & $\$ .960,102$ & 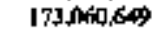 \\
\hline Eum Powar & - & - & - & - & 2018,170 & $9,427,794$ \\
\hline 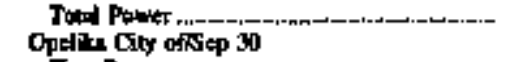 & - & - & - & - & 208,170 & $9.427,794$ \\
\hline Em Powes & - & - & - & - & 318,169 & $13,891,564$ \\
\hline 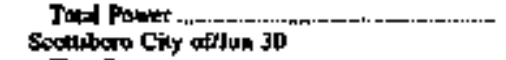 & - & - & - & - & 318,169 & $13,891,364$ \\
\hline Frn Poner & - & - & - & $\ldots$ & 364.892 & 14962.386 \\
\hline 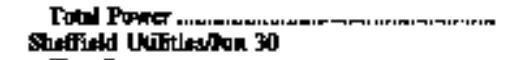 & - & - & - & - & 366,692 & 14962.386 \\
\hline 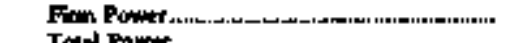 & - & - & - & - & 433,154 & $19,354,040$ \\
\hline 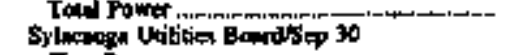 & 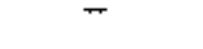 & - & $m$ & - & 433,154 & $19,354,048$ \\
\hline 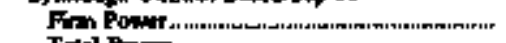 & - & - & 157,542 & $5,886,397$ & $381,8 \%$ & $6,903,687$ \\
\hline 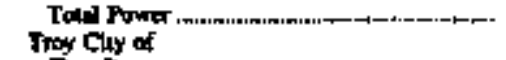 & - & - & 157542 & $5.685,397$ & $181,69 \%$ & 6,963,687 \\
\hline 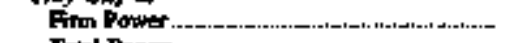 & - & - & - & - & 29,994 & 8.202 .759 \\
\hline 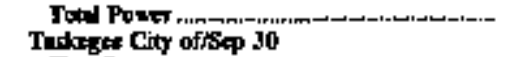 & - & - & - & - & 229,934 & $8,202,759$ \\
\hline 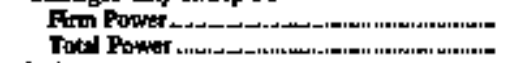 & $\bar{z}$ & $\overline{-}$ & $\begin{array}{l}139,098 \\
130,1998\end{array}$ & $\begin{array}{l}5,500,196 \\
5,501,196\end{array}$ & $\begin{array}{l}155,716 \\
15 \$, 316\end{array}$ & $\begin{array}{l}6,2,18,958 \\
6,2,18,958\end{array}$ \\
\hline Allite & & & & & & \\
\hline 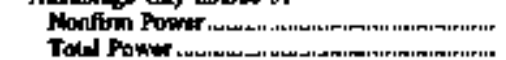 & 1,6800 & 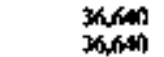 & $\bar{z}$ & $\overline{-}$ & $\begin{array}{l}173,570 \\
179,570\end{array}$ & $\begin{array}{l}4,966,136 \\
4,90,1 \% 6\end{array}$ \\
\hline
\end{tabular}

See noter and formates an and of teble. 
Table 29. Ejectricity Purchases by Municipal Utilitis, Fìscal Year 1994 (Continued)

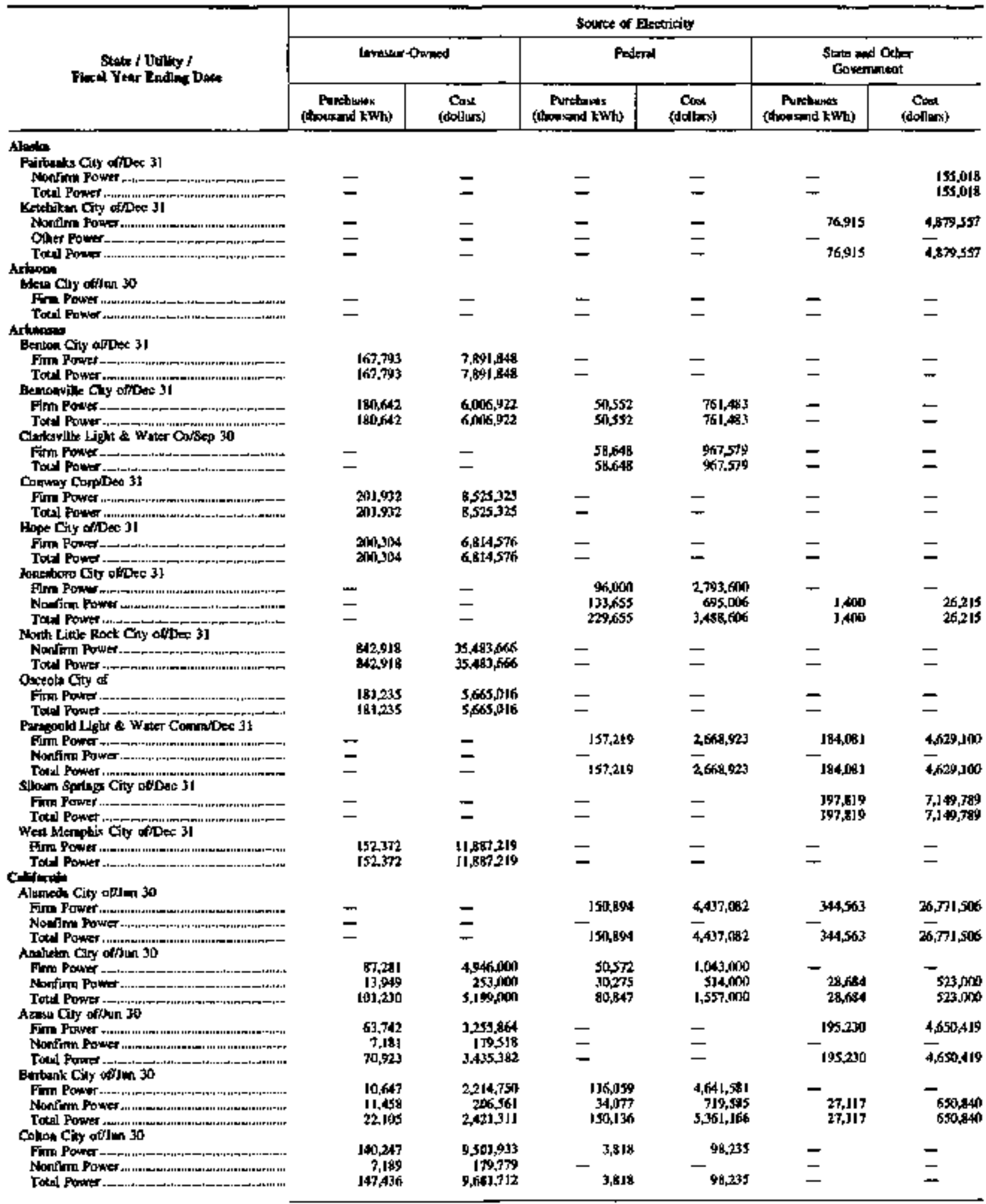

Sec noles and Epotwolex al and of the. 
Table 29. Electriclity Purchases by Munkipal Utililles, Fiscal Year 1994 (Continued)

\begin{tabular}{|c|c|c|c|c|c|c|}
\hline \multirow{3}{*}{ 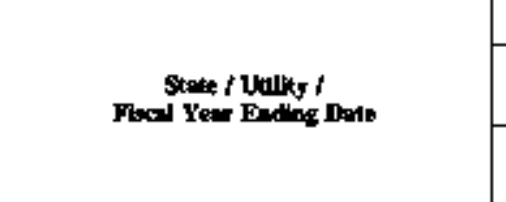 } & \multicolumn{6}{|c|}{ Solme of Elextrinity } \\
\hline & \multicolumn{2}{|c|}{ Cospanaive } & \multicolumn{2}{|c|}{ امبإO } & \multicolumn{2}{|c|}{ Total } \\
\hline & 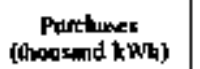 & $\begin{array}{c}\text { Cast } \\
\text { (dollors) }\end{array}$ & 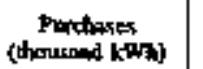 & Cost & $\begin{array}{l}\text { Porchoves } \\
\text { (thopenand kWh) }\end{array}$ & $\underset{(\operatorname{dollm} x)}{\operatorname{Cosd}}$ \\
\hline \multicolumn{7}{|l|}{ Alathes } \\
\hline \multicolumn{7}{|l|}{ Fribenly Chy offDec 31} \\
\hline Toxal Power & 15,630 & Sat,kTt & 19 jist3 & 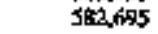 & 35,019 & $1,301,391$ \\
\hline \multicolumn{7}{|l|}{ 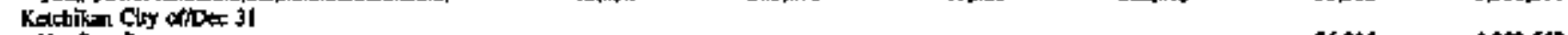 } \\
\hline Nonfim Rowa & - & ــ & - & - & 76,915 & $4,679,557$ \\
\hline Other Fowe-1, & - & - & 49 & - & & \\
\hline \multirow{2}{*}{\multicolumn{7}{|c|}{ Arewont }} \\
\hline & & & & & & \\
\hline Finn Powher & - & - & $3+2,080$ & 13,96,844 & 342,090 & $13,966,844$ \\
\hline 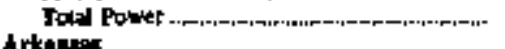 & - & - & $342, \pi 80$ & $13,966,344$ & 342060 & $13,946,844$ \\
\hline \multicolumn{7}{|l|}{ Arkanser } \\
\hline Gim Pown & - & - & $=$ & - & 167,793 & $7,891,848$ \\
\hline \multirow{2}{*}{\multicolumn{7}{|c|}{ Bentony lille City of Dee 31}} \\
\hline & & & & & & \\
\hline 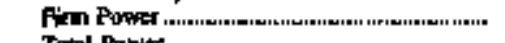 & - & - & - & - & 201,194 & 6,768,406 \\
\hline \multirow{2}{*}{\multicolumn{7}{|c|}{ 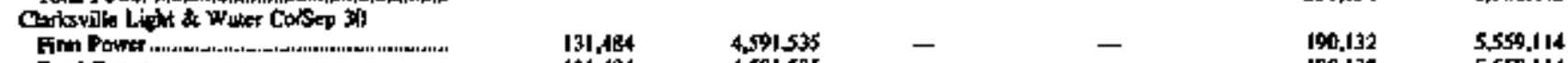 }} \\
\hline & |31,A34 & 4,591535 & - & - & 190,132 & $5,559,114$ \\
\hline & \multicolumn{5}{|c|}{ Conwsy Corpiow 3] } & $5,559,314$ \\
\hline 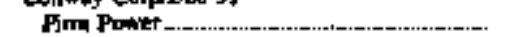 & - & $\longrightarrow$ & - & - & 2011,932 & $8,525,325$ \\
\hline \multirow{2}{*}{\multicolumn{7}{|c|}{ Hope Crty of IDec MI }} \\
\hline & & & & & & \\
\hline Fim Power & - & - & - & - & $2010,3 / 4$ & $6,814,576$ \\
\hline Toted Poner ..., & \multicolumn{6}{|c|}{ 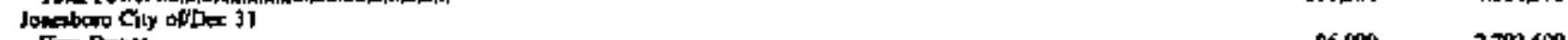 } \\
\hline 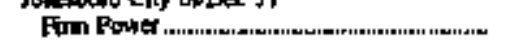 & - & - & - & - & 96,000 & $2,790,600$ \\
\hline Nonfint Power & - & - & $337,08 \mathrm{~J}$ & $5,864,243$ & 472,136 & $6,59,464$ \\
\hline Tatat Power :- & - & - & 337,081 & $5,868,243$ & 569,136 & $9,783,0644$ \\
\hline Nort Linte Roch Gity oflLes 31 & & & & & & \\
\hline 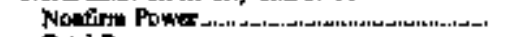 & - & - & $-134,6,20$ & $-6,645,577$ & $70 \% \times 290$ & $28,837,989$ \\
\hline Total Powar & - & - & $-1,34,620$ & $-6,645,8 \pi$ & 708,298 & $28,837,989$ \\
\hline 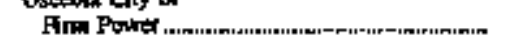 & - & - & $=$ & - & 181,235 & $5,6\} 5,016$ \\
\hline Totel Power & - & - & - & - & 181,235 & $5,645,016$ \\
\hline 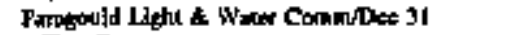 & & & & & & \\
\hline Fina Ponwer & - & - & - & - & 341,3006 & $7,299,093$ \\
\hline 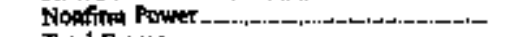 & sas & 23,450 & 3,3,305 & 637,926 & 34,nk & 66.536 \\
\hline Total Powter ... & 925 & 25,630 & I3, I301 & 637,926 & 775,526 & $7,959.579$ \\
\hline $\begin{array}{l}\text { SWoun Spring Bity of Dee } 31 \\
\text { Frm Powet. }\end{array}$ & - & - & $=$ & - & 197910 & 7100.789 \\
\hline Tothl Powrer & $\overline{-}$ & $\overline{-}$ & - & $\overline{-}$ & $\begin{array}{l}191,819 \\
1919\end{array}$ & $7,149,789$ \\
\hline Wed Memphax Cuy offoex 3] & & & & & & \\
\hline find Power & - & - & - & - & 152,372 & $11,897,219$ \\
\hline Total Power & - & - & - & - & 152,972 & $31,867,219$ \\
\hline Cosllorile & & & & & & \\
\hline 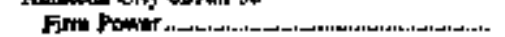 & - & - & - & 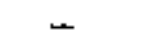 & 495,457 & $3 t, 200,58$ \\
\hline 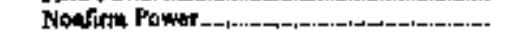 & - & - & - & $-1,418,758$ & - & $-[, 418,758$ \\
\hline Totd Fowro & - & - & - & $-1,418,758$ & $4 \% A S T$ & $29.7 \$ 9.830$ \\
\hline 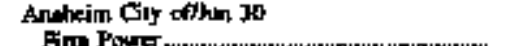 & & & & & 60104 & 260016000 \\
\hline $\begin{array}{l}\text { Fim Pown } \\
\text { No fin Pow }\end{array}$ & $\begin{array}{l}475,251 \\
213,827\end{array}$ & $\begin{array}{l}20,[2], 190 \\
2,95], 900\end{array}$ & $\overline{1} 559.671$ & $\overline{11 \times 6,06 a, 1000}$ & $\begin{array}{r}613,104 \\
1,846,406\end{array}$ & $\begin{array}{l}26,016,0,00 \\
190,307,00,5\end{array}$ \\
\hline Tota1 Fow & 689,1738 & $21,984,000$ & ],559,671 & IA 6,0 OSD, RAP & 2459510 & iJ6, $, 29,000$ \\
\hline Azuss Cly of then , in & & & & & & \\
\hline 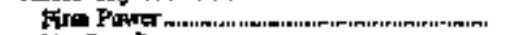 & - & - & 3,255 & $1,508,151$ & 269,227 & $9,414,434$ \\
\hline 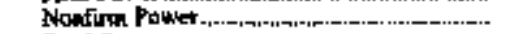 & - & - & 57597 & $1,233,536$ & 64,778 & 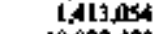 \\
\hline Totsd Power & - & - & 60,852 & $2.741,687$ & S2t, hos & $10,827,488$ \\
\hline Awthenk Cidy off $/ \mathrm{wn}$ 30 & & & & & & 49811.506 \\
\hline Firin Power & $\begin{array}{l}2 B_{3}, 30 \mathrm{~A} \\
32,958\end{array}$ & $\begin{array}{l}767,179 \\
64], 528\end{array}$ & $\frac{532,4 !}{2 n \leqslant 1}$ & 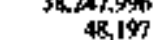 & 187,691 & 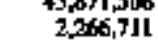 \\
\hline 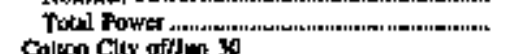 & 60,262 & $1,4188,707$ & $\$ 34,472$ & $38,296,190$ & 845,1992 & $48,138,217$ \\
\hline Co|em Clay offlum $\mathbf{x}$ & & & & & & \\
\hline Fime Panes & - & - & 145200 & $2,2,67,6$ f́d & 158,585 & $11,897,632$ \\
\hline Nominim Powst & $B, 424$ & $157,99 \%$ & 45,489 & 974,124 & $\begin{aligned} 61,102 \\
219,087\end{aligned}$ & $\begin{array}{r}1.344,899 \\
13,1007131\end{array}$ \\
\hline awar - & 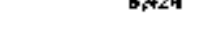 & & & & & $03, \sqrt{3},{ }_{0}$ \\
\hline
\end{tabular}

Srr notek and focknotes in end af thile 
Table 29. Electricity Purchases by Monicipal Utilities, Fiscal Year 1994 (Continued)

\begin{tabular}{|c|c|c|c|c|c|c|}
\hline \multirow{3}{*}{ 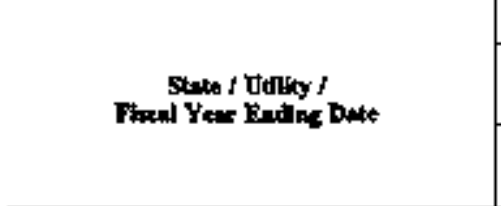 } & \multicolumn{6}{|c|}{ Source of Electrieity } \\
\hline & \multicolumn{2}{|c|}{ Investor-Oaned } & \multicolumn{2}{|c|}{ Pentral } & \multicolumn{2}{|c|}{ 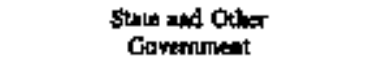 } \\
\hline & $\begin{array}{c}\text { Purclunes } \\
\text { (hookind kiwh) }\end{array}$ & $\underset{(\operatorname{dop} \text { Aors })}{\operatorname{Cos}}$ & $\begin{array}{l}\text { Porchick } \\
\text { (thows=nd twh) }\end{array}$ & $\underset{\text { (ddcilitst) }}{\operatorname{Cost}}$ & $\begin{array}{l}\text { Ponstures. } \\
\text { (thousud kiWh) }\end{array}$ & $\underset{(d \cos \mid \sin x)}{\operatorname{Cos}}$ \\
\hline \multicolumn{7}{|l|}{ Corllocian } \\
\hline \multicolumn{7}{|l|}{ Glendale Chy aftion 30} \\
\hline 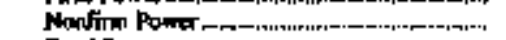 & 99,611 & $6,1 \% 6,231$ & 36,760 & 352,686 & 142,553 & $3,051,827$ \\
\hline \multirow{2}{*}{\multicolumn{7}{|c|}{ 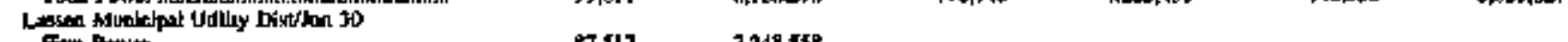 }} \\
\hline & 87517 & $7248,5 \times 8$ & - & - & - & - \\
\hline Pondin Pompror & 1,005 & 42,315 & 15,180 & 300,725 & 29,156 & 685,183 \\
\hline \multicolumn{7}{|l|}{ 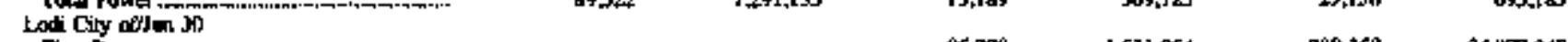 } \\
\hline Fin Pom & - & - & 85,0998 & {$[, 6] 1,0,54$} & 289.258 & $245 \pi 249$ \\
\hline 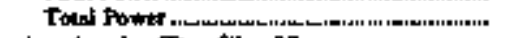 & - & - & 85,099 & $[, 6]], 064$ & $289.15 \mathrm{~B}$ & $24,979,249$ \\
\hline \multicolumn{7}{|l|}{ Le Angeles Cry of } \\
\hline Frim Pownt & $\begin{aligned} 841,5013 \\
83,923\end{aligned}$ & $45,054,599$ & $\begin{array}{l}651,966 \\
14,196\end{array}$ & $\begin{aligned} 13,301,589 \\
2,3 R 2,930\end{aligned}$ & $\begin{array}{l}749,287 \\
46,687\end{array}$ & $48,975,383$ \\
\hline Tonl Powet & 929,024 & 53.693 .551 & $+x_{1,162}$ & $16,084,555$ & 786,974 & $=0,74,612$ \\
\hline \multicolumn{7}{|l|}{ Pulo Alos City opyns 30} \\
\hline 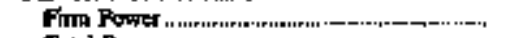 & - & - & 729,923 & 2A, 985,0000 & 350,938 & $10,135,000$ \\
\hline \multirow{2}{*}{\multicolumn{5}{|c|}{ Puswden Cuy ofthon 30}} & 358,993 & $10.135,000$ \\
\hline & & - & & 9,015,254 & 4,525 & \\
\hline Nonfritum Power & 18,597 & $1,239,7 \pi 3$ & 20,411 & ], 128,459 & 58.296 & $1,318,168$ \\
\hline 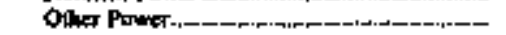 & & & 698 & & - & \\
\hline \multirow{2}{*}{\multicolumn{7}{|c|}{ 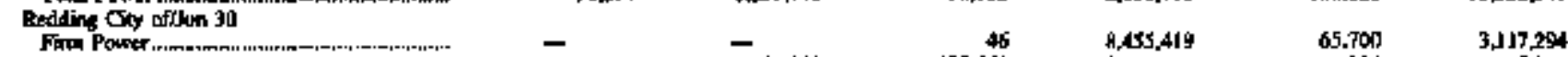 }} \\
\hline & - & - & 46 & $A, \Delta \leq 5,419$ & 65.70n & $3,117,294$ \\
\hline 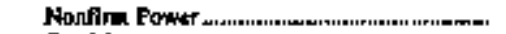 & 100,376 & 7,3001038 & 577.662 & 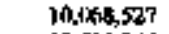 & 2nd & 38 \\
\hline Told Power & 100,376 & $7,309,038$ & $\$ 77,70 \mathrm{pg}$ & $1 B, 52,946$ & 65.904 & $3,124,6+2$ \\
\hline \multicolumn{7}{|l|}{ Riveride City othun 30} \\
\hline Flm Powt & 83,520 & $9,829,300$ & 37214 & $1.764,406$ & 64,4M & $3,246,890$ \\
\hline Nop:frm Powe & 27,330 & 637,301 & 16,903 & $291,7 \mathrm{~m}$ & 8,211 & $195,8,1]$ \\
\hline \multirow{2}{*}{\multicolumn{7}{|c|}{ Rontwle Ciy of tou 30}} \\
\hline & & & & & & \\
\hline 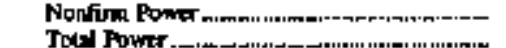 & - & $m$ & 376,761 & 19, 301,584 & 194,557 & $J 8,136,399$ \\
\hline 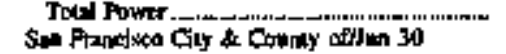 & - & - & 336,361 & $10,303,389$ & 199,957 & $18,136,590$ \\
\hline 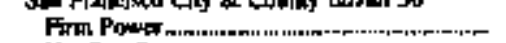 & 1,986 & 27963,880 & - & - & - & - \\
\hline 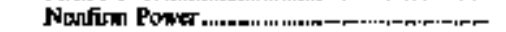 & 234,226 & $6,360,439$ & $2 n, 9 \mathrm{al}$ & 420.9016 & 275, .876 & $\overline{6}, 838,252$ \\
\hline 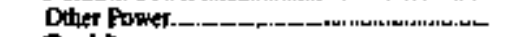 & 2,919 & - & - & - & - & \\
\hline 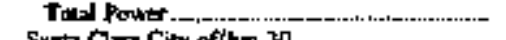 & $209,13 !$ & $29,324,316$ & 21501 & 420,3014 & 275,676 & $6,858.252$ \\
\hline 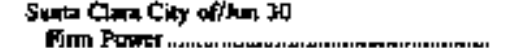 & & & & & & \\
\hline 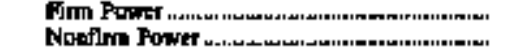 & $\begin{array}{l}315,01 y \\
9445\end{array}$ & $24,865,169$ & $\begin{array}{l}1,011,160 \\
65,163\end{array}$ & 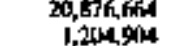 & - & - 1400 \\
\hline Tod Pow & 324,314 & $25,102,900$ & $3,036,123$ & $20,1,568$ & 69 & 1.40is \\
\hline 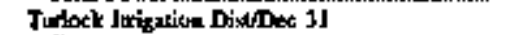 & & & & & & \\
\hline 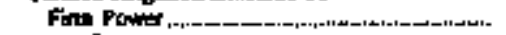 & 55,301 & $5,435,383$ & 18,994 & 591,443 & 249,373 & $21.226,862$ \\
\hline 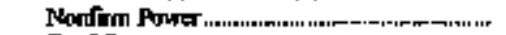 & d9,345 & $1.161 .8 \mathrm{dz}$ & 36, BOII & 562,341 & 193,145 & 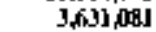 \\
\hline Totul Power & 105,146 & 6.597226 & 35,795 & $1,155,784$ & 42,518 & $24,857,943$ \\
\hline Vermon Gicy oflum an & & & & & & \\
\hline Find Powra & 747,219 & 16,233,935 & 82,520 & $1,356,763$ & 222.704 & 30.368 .448 \\
\hline 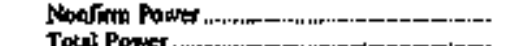 & 898 & 21,094 & B,794 & 32,869 & 294 & 14,236 \\
\hline 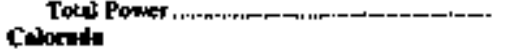 & 748,108 & $15,255,029$ & 91,356 & $1,969,4,32$ & 223.128 & $11,402,724$ \\
\hline Caloredo Sprlags Crty of Des 31 & & & & & & \\
\hline 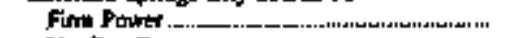 & - & - & 176,141 & $7,512,991$ & 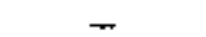 & - \\
\hline 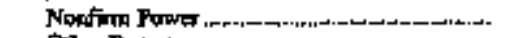 & 91,424 & 2,454.1046 & $2, \mathrm{ans}$ & $\mathrm{GMT}, \mathrm{Jat}$ & 36,443 & 1.191 .973 \\
\hline 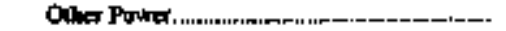 & & & & & $m$ & \\
\hline 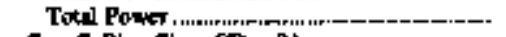 & 91,424 & $2,454,0 \% 6$ & $.183 .36 \mathrm{H}$ & 5. 1119,755 & Stid4s & 1.191 .973 \\
\hline Pun Cotilas Cly ofidene 31 & & & & & & \\
\hline 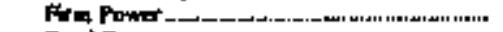 & - & - & - & - & 939,061 & $32,668,562$ \\
\hline 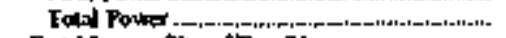 & - & - & - & - & 939, तNI & $32,668,562$ \\
\hline Font Morym Ciry astider 3I & & & & & & \\
\hline 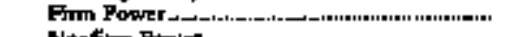 & - & - & 66,162 & $1,794,681$ & - & $\overline{2}, 0 \times 0$ \\
\hline 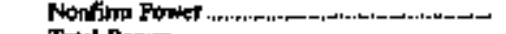 & - & - & $\longrightarrow$ & - & 101,454 & 2,207568 \\
\hline 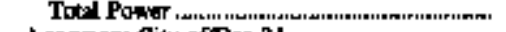 & 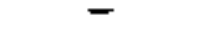 & - & $f(0,162$ & $1,75 \$, 681$ & ]0I,Æ4a & 20551518 \\
\hline Lougroons Ciuy ofidec 3I & & & & & & \\
\hline 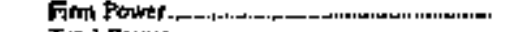 & - & - & - & - & 494,217 & I7.,199, ;01 \\
\hline 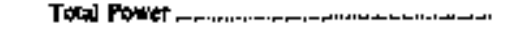 & 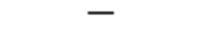 & - & & - & 494,217 & $17,079,301$ \\
\hline
\end{tabular}

Set motel and froenocs at end of wble. 
Tabłe 29. Electricity Purchases by Municfipal Utillites, Flscal Year 1994 (Contmued)

\begin{tabular}{|c|c|c|c|c|c|c|}
\hline \multirow{3}{*}{ 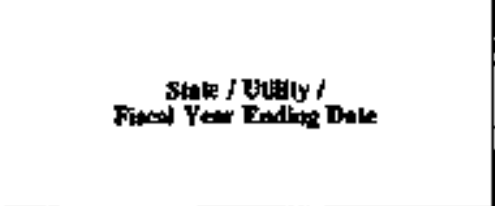 } & \multicolumn{6}{|c|}{ Sourea of Elocerletly } \\
\hline & \multicolumn{2}{|c|}{ Cosoperitive } & \multicolumn{2}{|c|}{ Otharl } & \multicolumn{2}{|c|}{ Tatal } \\
\hline & $\begin{array}{l}\text { Purchast: } \\
\text { olpowiond kthily }\end{array}$ & $\underset{\text { (dollaris) }}{\operatorname{Coth}}$ & Purdunes & $\begin{array}{c}\text { Cost } \\
\text { (dodins) }\end{array}$ & $\begin{array}{l}\text { Parchures } \\
\text { (houlind kiwh) }\end{array}$ & $\underset{\text { (don: }}{\operatorname{Con}}$ \\
\hline \multicolumn{7}{|l|}{ Callonle } \\
\hline 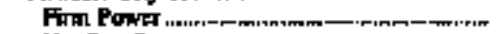 & - & - & - & - & 141,383 & $3,472,747$ \\
\hline 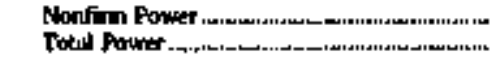 & $\begin{array}{l}1,188 \\
1,168\end{array}$ & $\frac{30,752}{30,752}$ & $\begin{array}{l}542,319 \\
542,319\end{array}$ & $\begin{array}{l}36,624,539 \\
26,624,539\end{array}$ & $\begin{array}{l}82,431 \\
963,8514\end{array}$ & $\begin{array}{l}36,646,074 \\
42,118,82.1\end{array}$ \\
\hline \multirow{2}{*}{\multicolumn{7}{|c|}{ 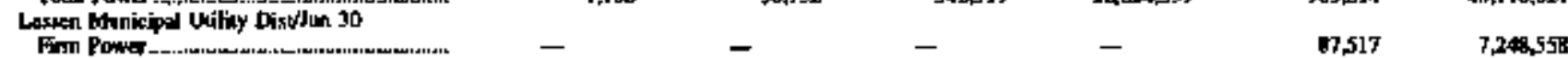 }} \\
\hline & - & - & - & - & 67,517 & $7,248,558$ \\
\hline 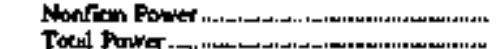 & $=$ & $\bar{z}$ & 4,140 & 748188 & 51),490 & $1,118,291$ \\
\hline \multicolumn{7}{|l|}{ Lodi Ciy ofJun 30} \\
\hline Fim Pown & - & - & - & عـ & $\$ 74256$ & $26,5 \times 0,13$ \\
\hline Totsl Power. & - & - & - & - & 374,254 & $2,590,313$ \\
\hline 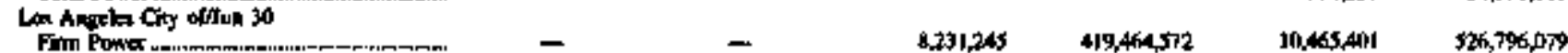 & & - & 8231245 & $419,464 \pi 2$ & 10,465 Anil & $324,796,0 \pi 9$ \\
\hline 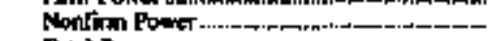 & 432.945 & 11329.511 & 920,000 & $\sin 590^{\circ}$ & $1,031.759$ & 81596.720 \\
\hline 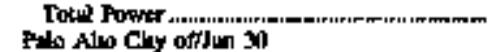 & 432,915 & $11,329,511$ & 8551,253 & 424,550351 & $11,49, J 60$ & $608,392,79$ \\
\hline Fin Powton & - & - & - & - & $1,078,8,55$ & $30,5201,0)$ \\
\hline 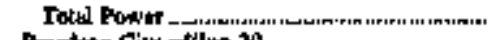 & - & - & - & - & 1.078 Ars & $30,530,0 x$ \\
\hline \multicolumn{7}{|l|}{ Parileng Ciky of Jun 38} \\
\hline 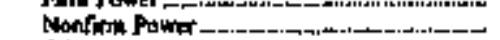 & I8.52\% & 397.774 & 9.390 & 2356,264 & 133,222 & $6,44,460$ \\
\hline 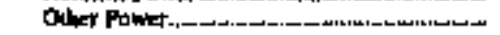 & - & - & & - & 68 & \\
\hline 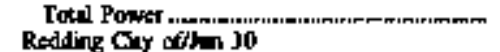 & 18,528 & 397,774 & 729.75s & $41,922,00$ & $93 ; 356$ & $55.430,4 m$ \\
\hline Frm Pown & - & - & - & - & 65,745 & $11,372,713$ \\
\hline 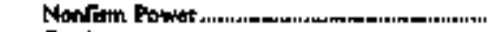 & - & - & 1,310 & 32,390 & 699552 & $17,418,0073$ \\
\hline 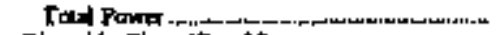 & - & - & 1,310 & 32,390 & 755,298 & $28,990,706$ \\
\hline \multicolumn{7}{|l|}{ Fiverside City of hat 30} \\
\hline Firm Poner & 230.745 & J2, ת6s.tm & 894,226 & $48,751,310$ & $1,364,112$ & $77,656,910$ \\
\hline 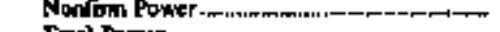 & 52906 & [2, 262,18] & 12.384 & 24,529 & 146,594 & $26,13,640$ \\
\hline \multirow{2}{*}{\multicolumn{7}{|c|}{ Rostrille Gay offm 30}} \\
\hline & & & & & & \\
\hline I & - & $\bar{z}$ & 二 & $\bar{z}$ & 37,318 & $28,439,982$ \\
\hline \multicolumn{7}{|l|}{ 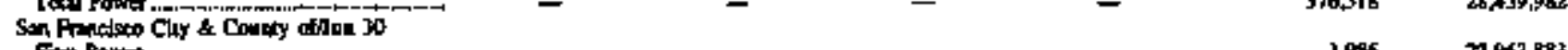 } \\
\hline Frtin Powd & - & - & - & - & 3,998 & $22,969,833$ \\
\hline 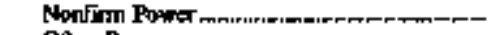 & - & - & (0).A65 & 0.014615 & 632,208 & $14,654,266$ \\
\hline 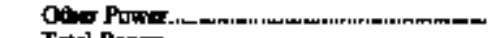 & - & - & & & 2,919 & \\
\hline 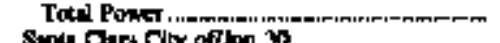 & - & - & $|a|, \mathbf{A b s}$ & 5, ill4675 & 697,693 & 37618.149 \\
\hline \multicolumn{7}{|l|}{ 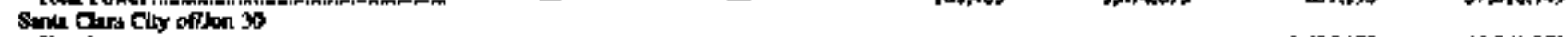 } \\
\hline 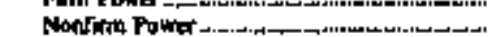 & $\overline{-}$ & $\bar{z}$ & - 26,978 & $\overline{71}=0 \times 00$ & $1,3260,179$ & $45,341,973$ \\
\hline Totul Power ....menn & - & - & 26,976 & $71,406,507$ & I אRS & $1190 n+9 x$ \\
\hline \multicolumn{7}{|l|}{ 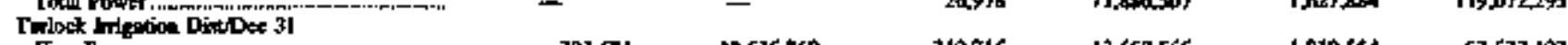 } \\
\hline Fim Power & I8J, I I & $22,615,640$ & 312715 & $13,667,5647$ & 1018554 & $53,5,7,129$ \\
\hline 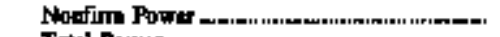 & & - & 3,763 & $4,317,531$ & JInNIS4 & $9,4 n_{1}, 3,36$ \\
\hline & 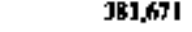 & $12,615,869$ & 343,478 & $17,725,137$ & 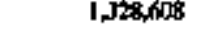 & $63,009,959$ \\
\hline Vawin Cily oflim $x$ & & & & & & \\
\hline 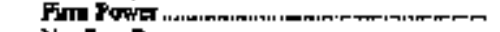 & - & - & - & - & $1,1092,443$ & $3,979,146$ \\
\hline Nonim Pantri & - & - & 738 & 1,65 & 10,847 & 49,934 \\
\hline Tots Power & - & - & 330 & 1.05 & 1,26290 & $37,029.000$ \\
\hline Colorido & & & & & & \\
\hline Colonudo Spring City ofidece 31 & & & & & & \\
\hline Fin Pow & - & - & - & - & 5766.141 & $7,502,591$ \\
\hline 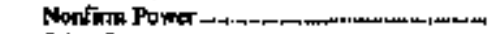 & 123,802 & $2,948,162$ & 154 & 4,004 & 279,700 & $7,208,349$ \\
\hline 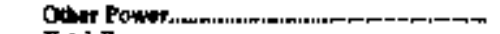 & & & -7.298 & & -729 & \\
\hline 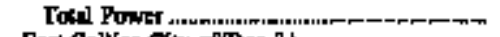 & 123.832 & $2,94.162$ & -3.14 & 4,004 & 644,447 & $14,797,9 \mathrm{~d} 0$ \\
\hline Fur Collins Chy ofrDec 3t & & & & & & \\
\hline Firn Pones & $\rightarrow$ & - & - & - & 939,061 & $32,6 f_{i}, 55$ \\
\hline 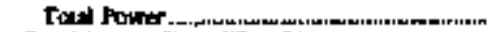 & - & - & - & - & 939,061 & $32,603,582$ \\
\hline Fort Mortate Ciny oflDet 3t & & & & & & \\
\hline Eim Paser-ר. & - & - & - & - & 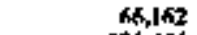 & $1,734,681$ \\
\hline 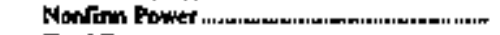 & - & - & سـ & - & 101,454 & $2,257,569$ \\
\hline Ifat Porw & 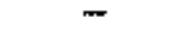 & - & - & - & 167,616 & $4,012,240$ \\
\hline Landmont City oftoce al & & & & & & \\
\hline 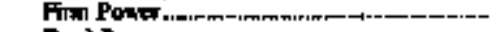 & - & - & - & $=$ & 494,217 & 17,079,301 \\
\hline 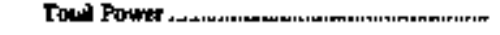 & - & - & - & - & 49,217 & $|7,074,30|$ \\
\hline
\end{tabular}

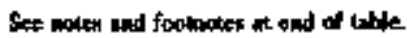


Tahł 29. Electricity Purchases by Municipal Utilities, Fiscal Year 1994 (Continued)

\begin{tabular}{|c|c|c|c|c|c|c|}
\hline \multirow{3}{*}{ 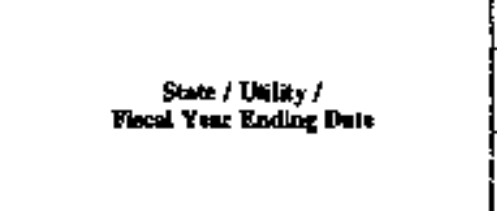 } & \multicolumn{6}{|c|}{ Source of Electricily } \\
\hline & \multicolumn{2}{|c|}{ Inqentor-OWacd } & \multicolumn{2}{|c|}{ Pederal } & \multicolumn{2}{|c|}{ State and Guler } \\
\hline & $\begin{array}{l}\text { Porthoneh } \\
\text { (thoopind twh }\end{array}$ & $\underset{\text { (dudtrx) }}{\operatorname{Cont}}$ & $\begin{array}{l}\text { Purchuses } \\
\text { (tboudsaw kath) }\end{array}$ & $\underset{\text { Cowt }}{\text { (dultstr) }}$ & $\begin{array}{l}\text { Parchuses } \\
\text { (1houxtisd L'Yh) }\end{array}$ & $\underset{\text { (dothers] }}{\operatorname{Can}}$ \\
\hline \multicolumn{7}{|l|}{ Caltorade } \\
\hline Fime Penwer & - & - & - & - & 406,852 & $13,426,247$ \\
\hline Total Powa ...................................................... & - & - & - & س- & $4 \times 6,852$ & $13,436,24 ?$ \\
\hline \multicolumn{7}{|l|}{ Comection } \\
\hline 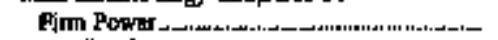 & 621,649 & $35,673,231$ & - & - & 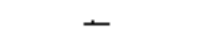 & - \\
\hline 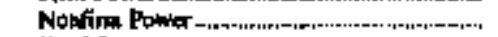 & - & - & - & - & 73,917 & 921,158 \\
\hline 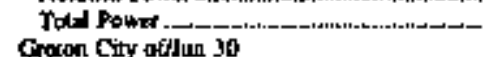 & 421,049 & $3,673,235$ & - & - & 73,917 & 921,159 \\
\hline 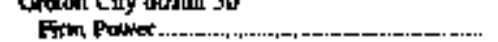 & - & $=$ & 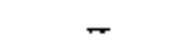 & - & - & - \\
\hline Tocel Power & - & - & - & - & - & - \\
\hline \multicolumn{7}{|l|}{ Norich Ghy offan 30} \\
\hline 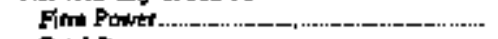 & - & - & - & - & - & - \\
\hline 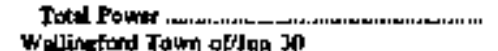 & - & - & - & - & - & - \\
\hline 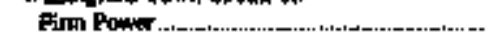 & 511.779 & Yh, IISA39 & - & - & - & - \\
\hline 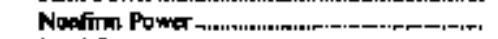 & 20,213 & 760,18 & - & - & - & - \\
\hline 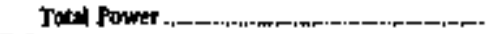 & 532,492 & $30,8 B 5,257$ & - & - & - & - \\
\hline \multicolumn{7}{|l|}{ 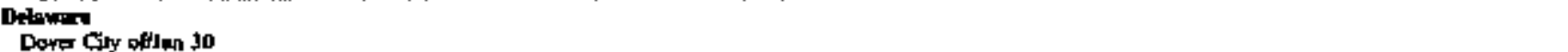 } \\
\hline Nonflu Power & $275,5,50$ & 7,0 a.,919 & ع & - & - & - \\
\hline 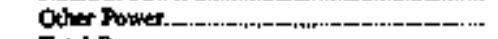 & $-43,022$ & - & - & - & - & - \\
\hline 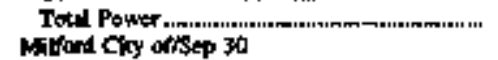 & 237.538 & $7,040,919$ & - & - & - & - \\
\hline 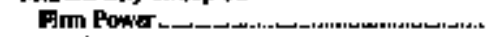 & 1482014 & 6,4979073 & - & - & - & $\longrightarrow$ \\
\hline 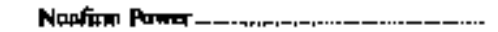 & - & - & - & - & - & - \\
\hline 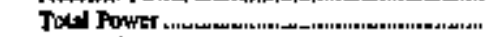 & 147,204 & K,497,07s & - & - & - & - \\
\hline Nowrek Sty offDes 31 & & & & & & \\
\hline Fin Power & 291,266 & $1,3,284,100$ & - & - & - & - \\
\hline 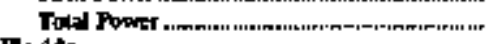 & 291266 & $13,234,100$ & - & - & - & - \\
\hline \multicolumn{7}{|l|}{ 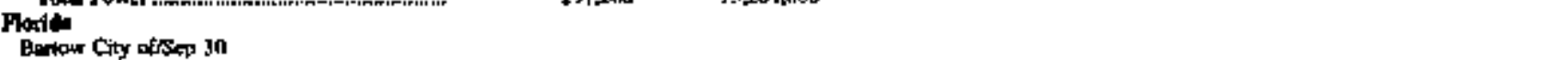 } \\
\hline 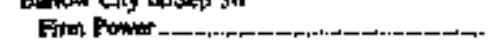 & 361,945 & $12,130,160$ & - & - & - & - \\
\hline Tot Popr & 261, yids & $12,130,166$ & - & - & - & - \\
\hline Forit Municipal Power AFecystep 30 & & & & & & \\
\hline 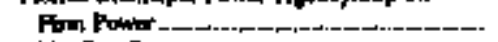 & 854,151 & $35,305,00 \mathrm{~PB}$ & - & - & - & - \\
\hline Ploafing Povra & $365,2,16$ & $2,55], \mathrm{DEO}$ & - & - & - & - \\
\hline Total Porw & $1,159,387$ & $38,162,000$ & - & - & - & - \\
\hline \multicolumn{7}{|l|}{ Fon Pieres Unditics Adstop 30} \\
\hline Nondition Powes & 64,377 & $1,935,426$ & - & - & - & - \\
\hline Totd Pownet & $\sqrt{4}, 3 \pi$ & $1,935,426$ & - & - & - & 一 \\
\hline \multicolumn{7}{|l|}{ Auinesrille Alegiond Utilitiensep in } \\
\hline 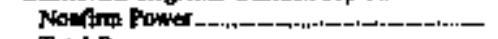 & 108,102 & 2,464,26010 & - & - & - & - \\
\hline Totd Poner & $108,110 \%$ & 2.464260 & - & - & - & - \\
\hline \multicolumn{7}{|l|}{ 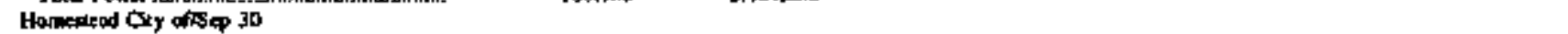 } \\
\hline 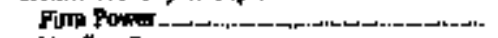 & 15.333 & $9 \ln 138$ & 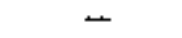 & - & - & - \\
\hline Nonfim Power ....................... & 35,689 & 919,200 & - & - & - & - \\
\hline 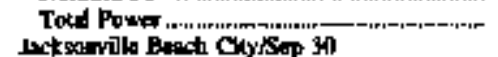 & stosen & $1,829,598$ & \multicolumn{2}{|c|}{ 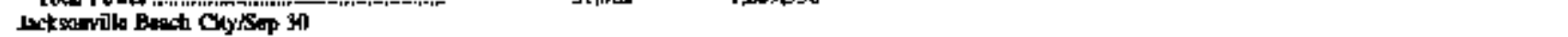 } & - & - \\
\hline 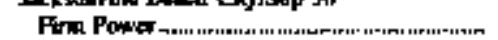 & - & - & - & - & - & - \\
\hline Nonflon Powte & 77,691 & $2,521,454$ & - & - & - & - \\
\hline 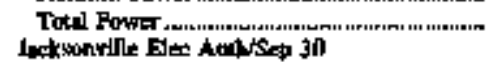 & J7, 491 & $2,521,454$ & - & - & - & - \\
\hline Firm Power & - & - & - & - & - & - \\
\hline 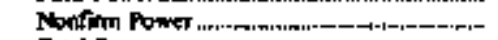 & $121,9 t 9$ & $2,272,105$ & - & - & 5,2740 & $109,9 \mathrm{ln}$ \\
\hline 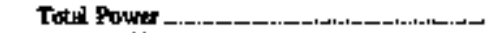 & 121.919 & 2772608 & - & - & 12tat & 109,40 \\
\hline Key West City arSp अn & & & & & & \\
\hline Fin Powrr & 4.38 .245 & $|4, y| 7,215$ & - & - & - & - \\
\hline Monfirm Powa & 6.49d & 176,489 & - & - & - & 一 \\
\hline Oher Powter....... & - & - & - & - & - & - \\
\hline 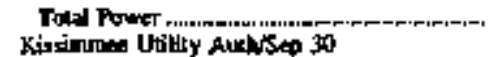 & 44,793 & $14,493,7013$ & - & - & - & - \\
\hline 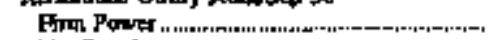 & $2(3,581$ & I1.,05B, I8I & - & - & - & - \\
\hline 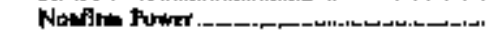 & $158,26 ?$ & $3,9 \$ 6,149$ & $\rightarrow$ & $\rightarrow$ & - & - \\
\hline Total Potulet .... & 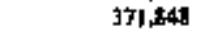 & $19,044,330$ & - & - & - & 一 \\
\hline
\end{tabular}

Ste notes and foodnoces an end of boble. 
Table 29. Electricity Purchases by Monicipal Utilties, Fiscal Year 1994 (Contínued)

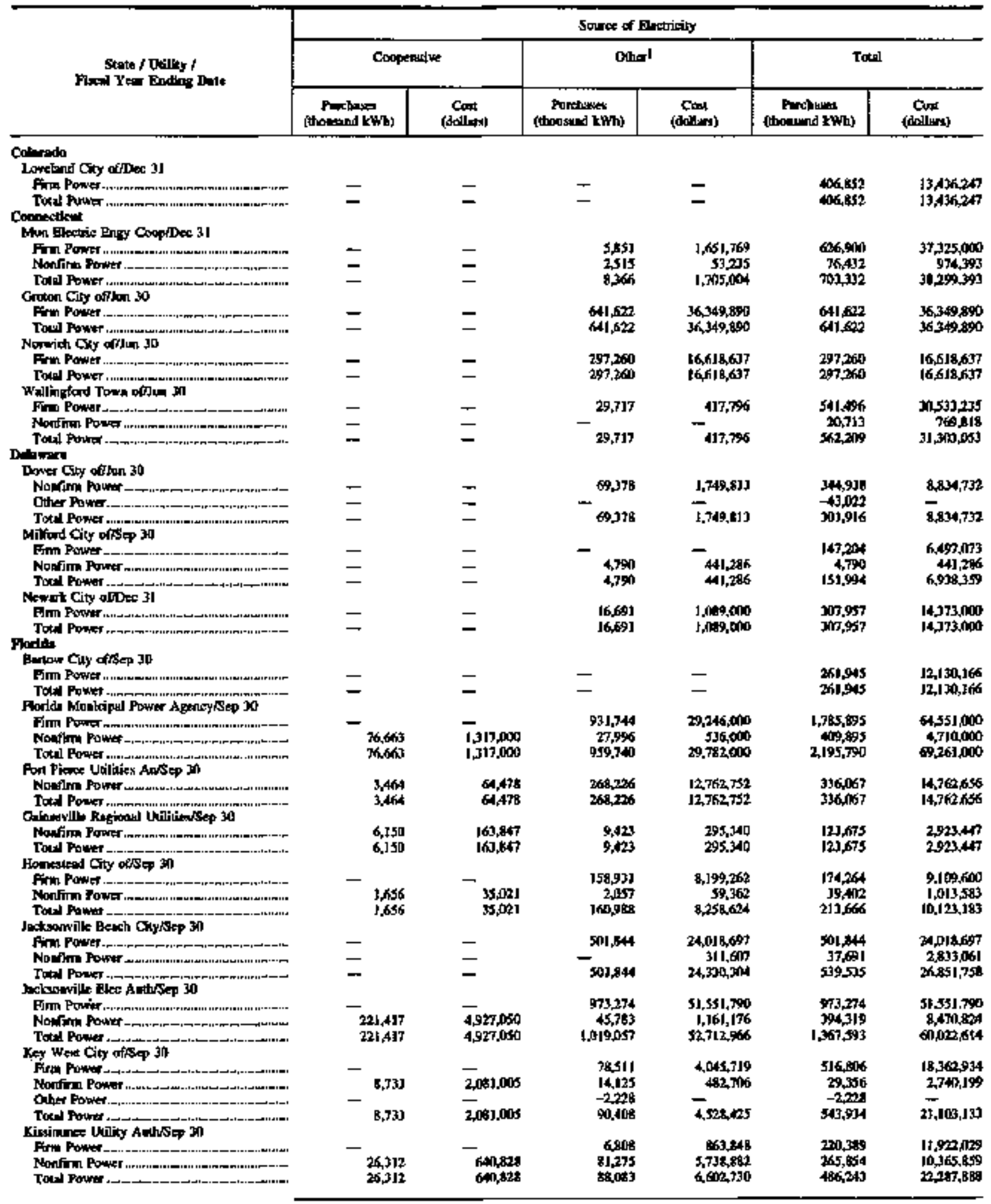

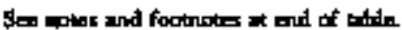


Tahte 29. Electricity Purchases by Municipol Utillites, Fiscal Year 1994 (Contimued)

\begin{tabular}{|c|c|c|c|c|c|c|}
\hline \multirow{3}{*}{ 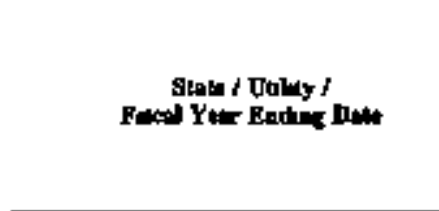 } & \multicolumn{6}{|c|}{ Soured of Elesencaty } \\
\hline & \multicolumn{2}{|c|}{ Invotor Owned } & \multicolumn{2}{|c|}{ Fvist } & \multicolumn{2}{|c|}{ Stule and Catser } \\
\hline & 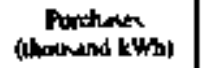 & Cont & Dinchant & $\underset{\text { (dall-r) }}{\text { Cint }}$ & (Whodhol & Contlat) \\
\hline \multicolumn{7}{|l|}{ Dooda } \\
\hline \multicolumn{7}{|l|}{$\begin{array}{l}\text { Lo Wart Coty tefse } 30 \\
\text { Monfin Power }\end{array}$} \\
\hline Toull Pow & 35049 & 95,688 & - & - & - & - \\
\hline Nonfinm Powa & 21,125 & 412,41 & - & - & - & - \\
\hline Tonst Fower & 21,135 & 412,44 & - & - & - & $=$ \\
\hline \multicolumn{7}{|l|}{ Lenburg Cry offSep $3 p$} \\
\hline Fin Punt & - & - & - & - & - & - \\
\hline \multicolumn{7}{|l|}{ 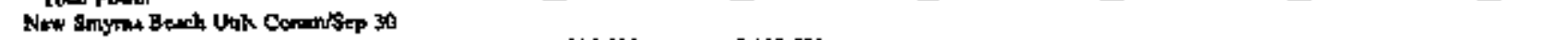 } \\
\hline Eun $F$ owat & 216,325 & $9,122,631$ & - & - & - & - \\
\hline Nonfim Powe & $4 A 60$ & 164,890 & - & - & - & - \\
\hline Totd Powter & 220,983 & 9,283511 & - & - & - & - \\
\hline \multicolumn{7}{|l|}{ Ocule Cty o.Sep 3A } \\
\hline Fim Promat & - & - & - & - & - & - \\
\hline \multicolumn{7}{|l|}{ Orande Uultues Commisep 30} \\
\hline Nonfin Paw & 400,749 & $7,682,699$ & - & - & - & - \\
\hline \multirow{2}{*}{\multicolumn{6}{|c|}{ st Gavd CAy ofsepp yp }} & - \\
\hline St Goud CAy of & & & & & & $\sigma_{-}$ \\
\hline Nofine Power & $1+110$ & \$1, 159,414 & $=$ & $=$ & Z & $\bar{z}$ \\
\hline Tout Power & 216,854 & $9,395,575$ & - & - & - & $\bar{z}$ \\
\hline \multicolumn{7}{|l|}{ Tullowhen Cuy ofsep op } \\
\hline Fin Bown & 32.,1108 & $\mathbf{6 3 3}, 0 \mathrm{KO}$ & - & - & - & - \\
\hline Moditu Powar & 25,393 & SThaxph & - & - & - & 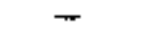 \\
\hline Other Popter & - & - & - & $m$ & 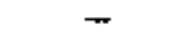 & - \\
\hline \multirow{2}{*}{\multicolumn{7}{|c|}{ Vero Besch Gty of $\rightarrow$, 30 }} \\
\hline & & & & & & \\
\hline Nanfim Powr & $3 \mathrm{~J}, 780$ & 349,267 & - & - & - & - \\
\hline Todd Pont & 31.790 & 349,267 & - & $m$ & - & - \\
\hline 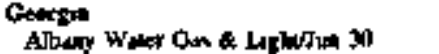 & & & & & & \\
\hline Prim Power & 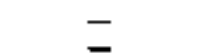 & $z$ & 76,622 & 2497,161 & $266,0 \%$ & $43,339,951$ \\
\hline Collow Culy of own 30 & & & & & & $43,351,0,031$ \\
\hline Fout Power & - & - & - & - & 326.102 & $15,6019,000$ \\
\hline Towd Power & - & - & - & - & 326,102 & If,609, ib0 \\
\hline Cakenvile Chry aftuma jo & & & & & & \\
\hline Fron Power & - & - & 25,707 & $\pi 3,715$ & 361,160 & $16,095,215$ \\
\hline Told Powete & - & - & 25,707 & 733,715 & 361,160 & $16,839,215$ \\
\hline Calloge Pak Cuy offluo 30 & & & & & & \\
\hline RuR Powt & - & - & 19,347 & $523 \sqrt{165}$ & $2+7,78$ & $14,329,398$ \\
\hline Toul Pawer & - & - & 19,347 & 927085 & 267285 & $14,929,399$ \\
\hline Corverton Cty offer 31 & & & & & & \\
\hline Fin Power & - & - & 19,009 & 599,886 & 213,197 & $10,330,947$ \\
\hline Tatol Ponter & - & - & 15,009 & $\$ 89,886$ & 213,197 & 10,339949 \\
\hline Dulion Cily offlow 90 & & & & & & \\
\hline Fint Poum & $421,74 z$ & $12,800,000$ & 68,257 & $1,80,000$ & - & ـ \\
\hline Tous Pourer & 423.742 & $12, \& B 000$ & 68,257 & $1,937,000$ & - & - \\
\hline Doorlu Cxy ufJln yn & & & & & & \\
\hline Fin Pown & - & - & 12549 & 42,099 & $2 \cos 203$ & $10,628,364$ \\
\hline To.s Pow & - & - & 12949 & 422009 & 307,283 & $10,628,369$ \\
\hline 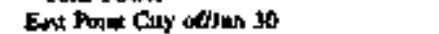 & & & & & & \\
\hline Fim Pown & - & - & 42,715 & $1,391,781$ & 291599 & $14,179,430$ \\
\hline Tosl Pown & - & - & 42,315 & $1,291,78$ & $29 ! 399$ & $14,179,430$ \\
\hline Pancerdd Wo Let \& Bndpec 31 & & & & & & \\
\hline Pum Powr & - & - & 15,670 & 400.27 & 165,749 & $B, 216,530$ \\
\hline Tox t hover & - & - & 15,670 & $409,2 \pi$ & 166,749 & $3,216,53$ ? \\
\hline Grifin Ory oftion 30 & & & & & & \\
\hline Pum Power & - & - & 61,546 & $7 \$ 9,019$ & 746.1P44 & $15,582,213$ \\
\hline Totd Fowrer & - & - & 67546 & 749,085 & 346,044 & $15, \$ 82.273$ \\
\hline L Groqfore Gty délum to & & & & & & \\
\hline Moofin Ponver & $\bar{z}$ & $=$ & $\overline{-}$ & $\overline{-}$ & 462001 & 24536,102 \\
\hline Tots Portar & & & & - & $462 \sqrt{201}$ & 21,536 I:2 \\
\hline
\end{tabular}

Sod noter and foctruteh at end of lable 
Tahle 29. Electricthy Porchases by Municipal Utilties, Fiscal Year 1994 (Continued)

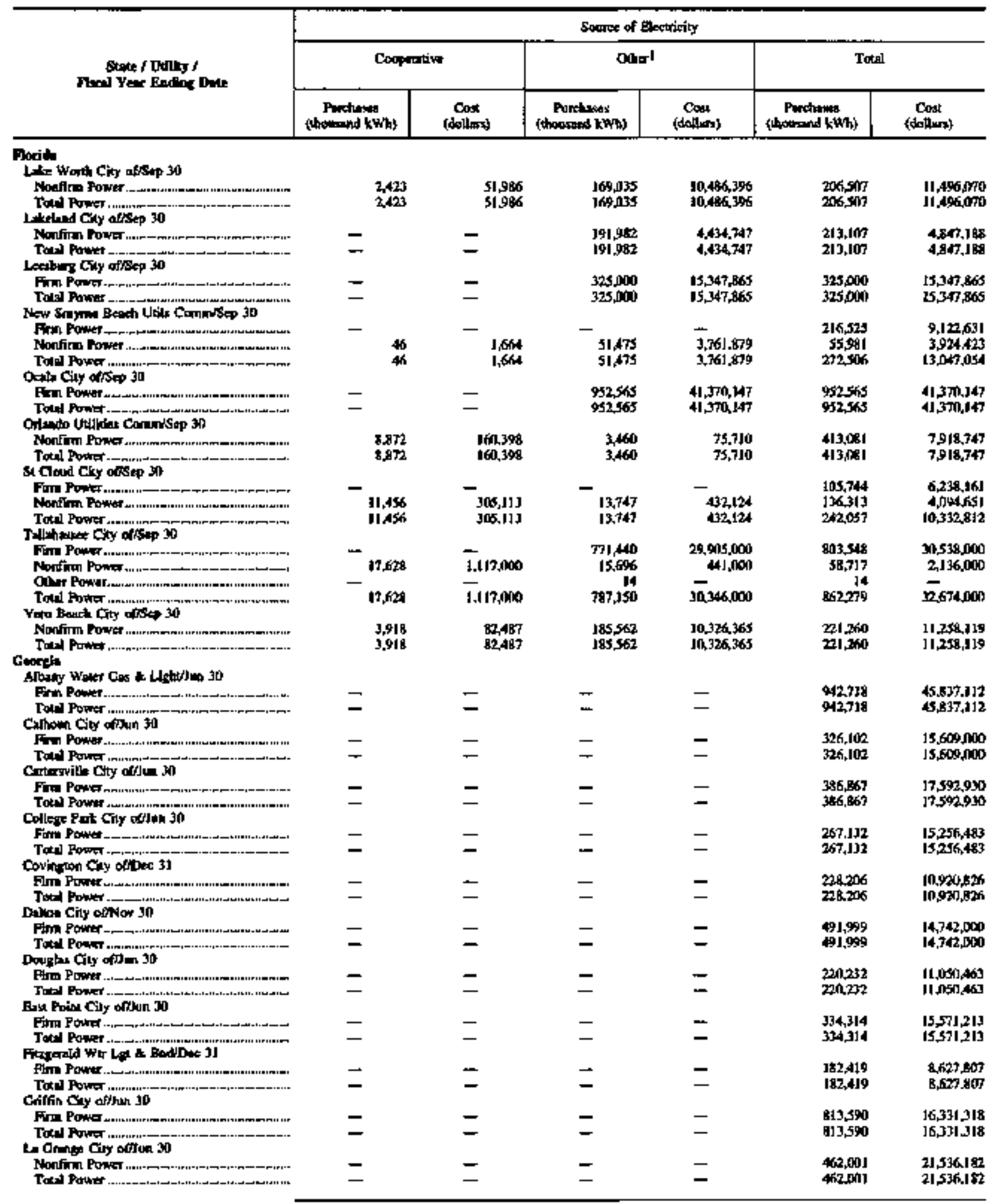

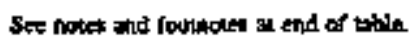


Table 29. Electrictty Purchases by Municjpal Utilities, Fiscal Year 1994 (Continued)

\begin{tabular}{|c|c|c|c|c|c|c|}
\hline \multirow{3}{*}{ 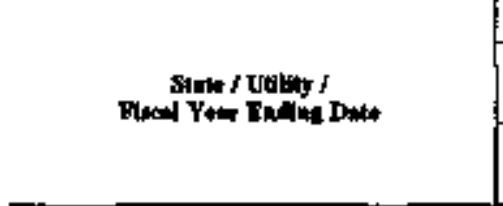 } & \multicolumn{6}{|c|}{ Source of electricity } \\
\hline & \multicolumn{2}{|c|}{ Inweraphowhed } & \multicolumn{2}{|c|}{ Federal } & \multicolumn{2}{|c|}{ Suive and Ootes } \\
\hline & 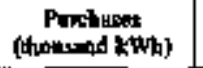 & $\begin{array}{c}\text { Cor } \\
\text { (dolnm) }\end{array}$ & $\begin{array}{c}\text { Portuses } \\
\text { (doopad kwh) }\end{array}$ & 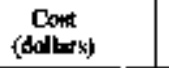 & 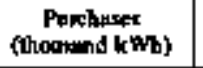 & $\begin{array}{c}\text { Dowat } \\
\text { (dollurst }\end{array}$ \\
\hline \multicolumn{7}{|l|}{ 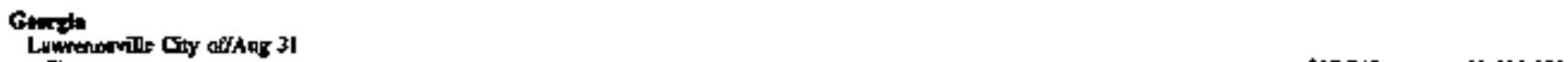 } \\
\hline Form Powar .... & - & - & - & - & 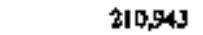 & $11,126,190$ \\
\hline 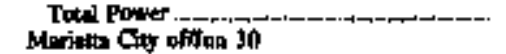 & - & - & - & - & 210,943 & $11,426,190$ \\
\hline Fin POTer & - & - & 45,603 & $1,778,572$ & 29.799 & $45.524,009$ \\
\hline 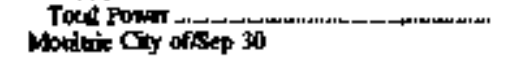 & - & - & 45,583 & $1,778,972$ & 29,799 & $45,524,609$ \\
\hline Fmu Powt & - & - & 25,227 & 664,618 & - & - \\
\hline 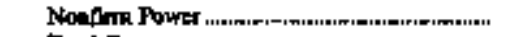 & - & - & - & - & 149,698 & $8,077,138$ \\
\hline 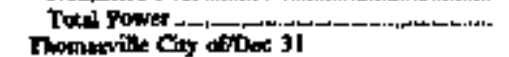 & - & - & 23,227 & 664,618 & $149,5 \% 8$ & $8,07,136$ \\
\hline Frin Power mm-and & - & - & 41,261 & $1,049,722$ & 371,057 & $19,528,398$ \\
\hline 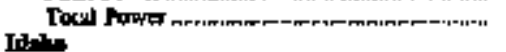 & - & - & $41,36]$ & $1,089,72-2$ & 371,957 & $19,925,398$ \\
\hline \multicolumn{7}{|l|}{ 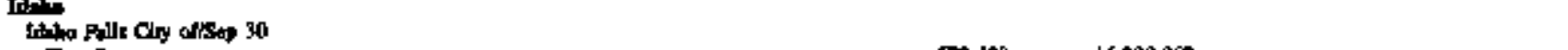 } \\
\hline Fim Pon & - & - & 572,458 & $16,300,062$ & - & - \\
\hline 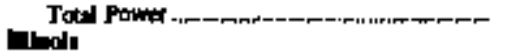 & - & - & 572.438 & $16,300,062$ & $\rightarrow$ & - \\
\hline \multicolumn{7}{|l|}{ Butuvin Chy ofdoec 3J } \\
\hline 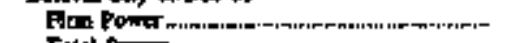 & $236,6,10$ & 10.5994 .41 & $\neg$ & - & - & - \\
\hline Jofd Pow & 234,630 & $911,589,414$ & - & - & - & - \\
\hline $\begin{array}{l}\text { Coness Chy ofithpr } 30 \\
\text { Erm Pown }\end{array}$ & 161,710 & $6,516,455$ & - & - & - & - \\
\hline Tast Pownt. & 161,710 & $6,116,455$ & - & - & - & - \\
\hline \multicolumn{7}{|l|}{ 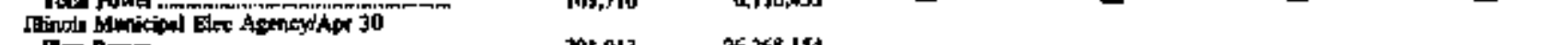 } \\
\hline 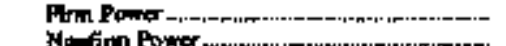 & $20 t, 0,3$ & $26,268,154$ & - & - & - & - \\
\hline 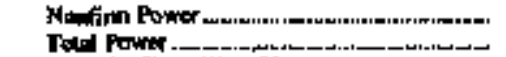 & $\begin{array}{r}63,743 \\
84,796\end{array}$ & $\begin{array}{l}1,737, \sin 59 \\
28,005,839\end{array}$ & $\bar{z}$ & $\bar{z}$ & $\bar{z}$ & $\vec{Z}$ \\
\hline \multicolumn{7}{|l|}{ Nuparpile Clyy offApr 30} \\
\hline 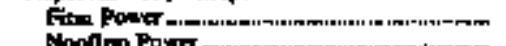 & K888896 & $13,901,860$ & - & - & - & - \\
\hline 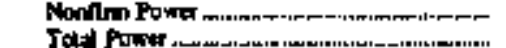 & - & Thon the & - & 一 & - & - \\
\hline \multicolumn{7}{|l|}{ 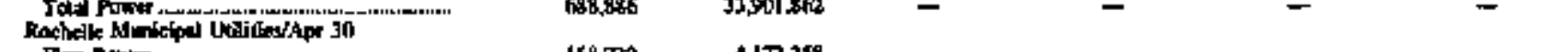 } \\
\hline 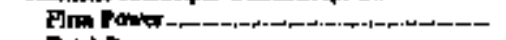 & 158,732 & $4,172,259$ & - & - & - & - \\
\hline \multicolumn{4}{|l|}{ Soriagfietd City ofisep 30} & & 一 & - \\
\hline 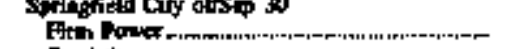 & 4],599 & $840,32 \mathrm{x}$ & - & - & - & $\longrightarrow$ \\
\hline Totd Power & 41,599 & $640,72 \pi$ & - & - & - & $\overrightarrow{-}$ \\
\hline $\begin{array}{l}\text { St Gurks Oity offAw } 30 \\
\text { Fin Pown }\end{array}$ & & & & & & \\
\hline & 369 . .75 & 17.128 .864 & - & 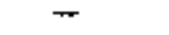 & - & - \\
\hline \multirow{2}{*}{\multicolumn{7}{|c|}{ 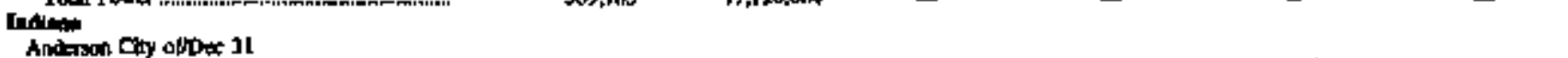 }} \\
\hline & & & & & & \\
\hline Fim Power & - & - & - & - & 660,57? & $28,215,57 \%$ \\
\hline 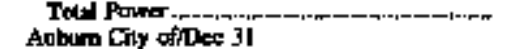 & - & - & - & - & $60,5 n$ & $28,215,076$ \\
\hline 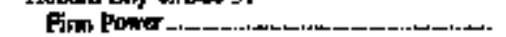 & 375,872 & $13,984.521$ & - & - & - & - \\
\hline \multirow{2}{*}{\multicolumn{7}{|c|}{ Blumpon City WDec 31}} \\
\hline & & & & & & \\
\hline 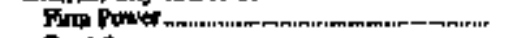 & 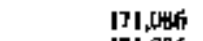 & $6,306,364$ & - & - & $\rightarrow$ & - \\
\hline Tad Powrer & 171,086 & 6.706, 764 & - & - & - & - \\
\hline \multicolumn{2}{|l|}{ 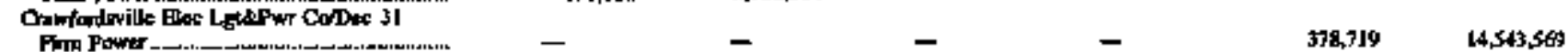 } & - & - & - & 378,719 & $14,543,569$ \\
\hline [onl Pownt & - & - & - & - & 378,719 & $14,549,569$ \\
\hline \multicolumn{7}{|l|}{ 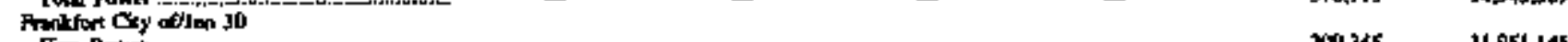 } \\
\hline 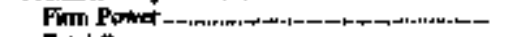 & - & - & - & - & 969,365 & $11,951,145$ \\
\hline 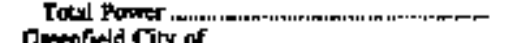 & \multicolumn{6}{|c|}{ Qmenfold City of } \\
\hline 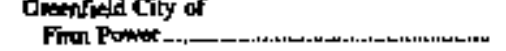 & - & - & - & - & 168876 & $6,810,731$ \\
\hline Tow Rowa & - & - & - & - & 968,876 & 6.810,7y] \\
\hline haper Gity ofDes 3) & & 9045.169 & & & & \\
\hline 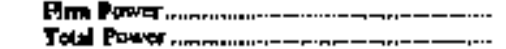 & 213,012 & $9, \sqrt{M 45,189}$ & $\overline{-}$ & $\overline{-}$ & $\overline{-}$ & $\bar{z}$ \\
\hline Lopenupor Ciy ofiDe= 31 & & & & & & \\
\hline 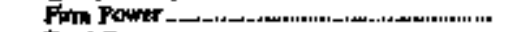 & 153,636 & $5,3,30,10$ & - & - & $\rightarrow$ & - \\
\hline 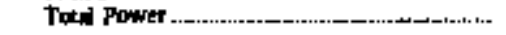 & 153,896 & $5,3,0,210$ & - & - & $=$ & - \\
\hline
\end{tabular}

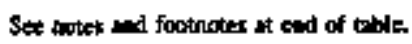


Table 29. Electricily Purchases by Munkclpal Utititits, Fiscal Year 1994 (Continued)

\begin{tabular}{|c|c|c|c|c|c|c|}
\hline \multirow{3}{*}{ State / Wellyy f } & \multicolumn{6}{|c|}{ Sprots of Dextricity } \\
\hline & \multicolumn{2}{|c|}{ Cosprative } & \multicolumn{2}{|c|}{ Cother 1} & \multicolumn{2}{|c|}{ Toxal } \\
\hline & 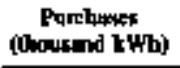 & $\begin{array}{c}\text { Cop } \\
\text { (d) llys) }\end{array}$ & 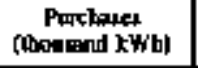 & $\begin{array}{c}\text { Dos } \\
\text { (dollers) }\end{array}$ & $\begin{array}{c}\text { Pumatape } \\
\text { (thousuitd kith) }\end{array}$ & $\underset{c}{C o m}$ \\
\hline \multicolumn{7}{|l|}{ Gtopta } \\
\hline 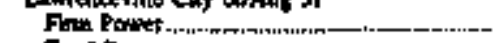 & - & - & - & - & 210,943 & |1,A26, I, \\
\hline 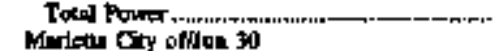 & - & - & - & - & 210,943 & $11,45.190$ \\
\hline Fim Power & - & - & - & - & 875,482 & $46,900,181$ \\
\hline 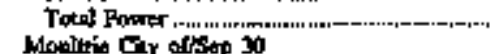 & - & - & - & - & 875,462 & $46,900.191$ \\
\hline 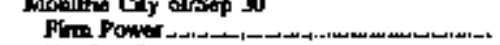 & - & - & - & - & 23,227 & KGA, \\
\hline 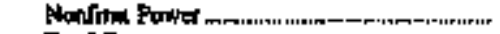 & - & - & - & - & 149,698 & $8,1797.198$ \\
\hline 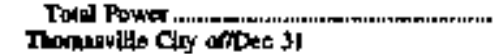 & - & - & - & 一 & 192,925 & $8,741.756$ \\
\hline Forth Pons & - & - & - & - & $413,2] 8$ & 201, 518,120 \\
\hline 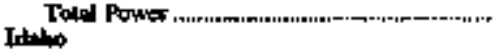 & - & - & - & - & 413,218 & $20,618,120$ \\
\hline \multicolumn{7}{|l|}{ [Who Fulh City ofisep 30} \\
\hline 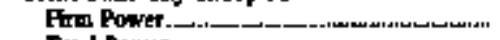 & - & - & - & - & $\sin 2,408$ & 16,30010062 \\
\hline Toed Powers & - & - & - & - & $\$ 72439$ & $16,30,012$ \\
\hline \multicolumn{7}{|l|}{ Difull: } \\
\hline Fim Power non ururim & - & - & - & - & 216,620 & $10,589,414$ \\
\hline 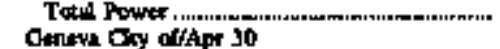 & ع & - & - & - & 246,640 & $10,549.414$ \\
\hline Firnt Power & - & - & $23,30 \%$ & 649,044 & J85,0J? & $6,765,499$ \\
\hline Told Penct, & - & - & 23,307 & 649,ni4 & 185,017 & $6,765,499$ \\
\hline \multicolumn{7}{|l|}{ Dhinow Maricipal Elec Agensy/Apr 30} \\
\hline 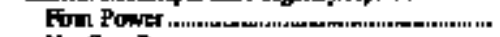 & - & - & 180,552 & 1.910 & 979,565 & $30,800,054$ \\
\hline 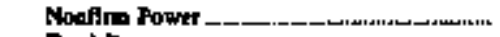 & - & - & - & & 6,743 & 1,737, G 65 \\
\hline Tould Pawket - & - & - & 188,552 & $4,531,919$ & $1,807, \mathrm{JnO}^{2}$ & $32,537,749$ \\
\hline Nepervilk Gity offap 30 & & & & & & \\
\hline Fin Power & - & - & - & - & 640,896 & $\$ 3,501.862$ \\
\hline Monflm Power & - & - & 5,825 & $257,71 B$ & ons & $237,7]$ \\
\hline \multirow{2}{*}{\multicolumn{7}{|c|}{ 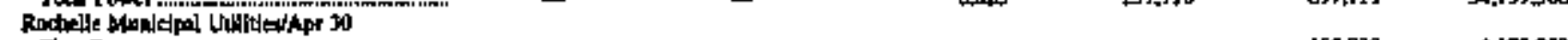 }} \\
\hline & & & & & & \\
\hline Fint Poun & - & - & - & - & 150,732 & $0,172,2918$ \\
\hline 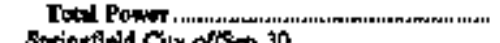 & - & سـ & 一 & 一 & 158,732 & 4.172258 \\
\hline \multicolumn{2}{|l|}{ Springfindd Gly oflso 30} & - & - & - & 41,599 & 840,326 \\
\hline 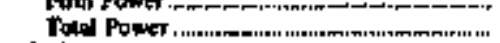 & - & $\overline{-}$ & $\overline{-}$ & $\overline{-}$ & 11,599 & 840,326 \\
\hline St Chare Gity offApr 30 & & & & & & \\
\hline 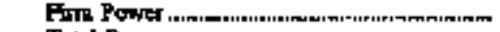 & - & - & $=$ & - & 369.765 & $17,128,064$ \\
\hline 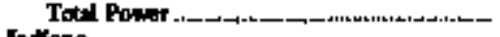 & - & - & - & - & 36,765 & $17, \sqrt{28,864}$ \\
\hline \multicolumn{7}{|l|}{ Anderpon Gity ofodec } \\
\hline 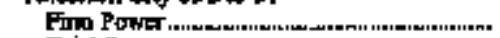 & - & - & - & - & 60.57 & $28,315,036$ \\
\hline Tits Pawer & - & - & - & - & 6ost7 & $28,215,076$ \\
\hline \multicolumn{7}{|l|}{ Aubam City of Des iJ } \\
\hline 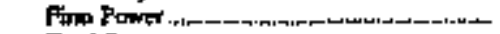 & - & - & - & - & 375,072 & [3,984,52] \\
\hline 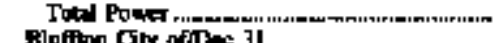 & 一 & - & - & - & 375,872 & $13,984,921$ \\
\hline \multicolumn{7}{|l|}{ Slofiton City ofiDe 31} \\
\hline Prow Power & - & - & - & - & I]10 & $6,70 n_{1}, 364$ \\
\hline \multicolumn{7}{|l|}{ 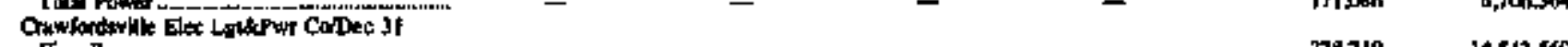 } \\
\hline Fith Powa & - & - & - & - & 378,719 & $14,543,569$ \\
\hline 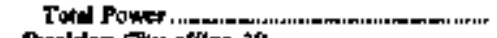 & - & - & - & - & 378,799 & $14,547,569$ \\
\hline \multicolumn{7}{|l|}{ Prydoxi Gily ofinn 30} \\
\hline 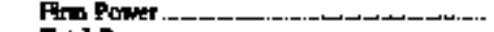 & - & - & - & - & 309,45 & $11, \$ 51,145$ \\
\hline 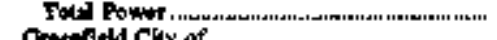 & - & - & - & - & 309045 & $11,951,145$ \\
\hline 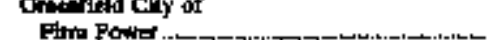 & & & & & & $6.810,731$ \\
\hline 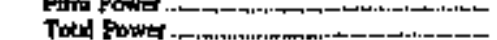 & - & $\bar{m}$ & - & 二 & 168876 & $5,810,731$ \\
\hline heper ons ofdes 31 & & & & & & \\
\hline 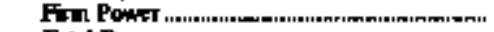 & - & - & - & - & 213,012 & $9, \sqrt{M} 5, \sqrt{169}$ \\
\hline 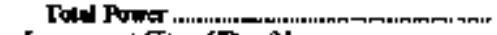 & - & - & - & - & 213,012 & $9,1 \mathrm{MS}, 169$ \\
\hline Logonsport Chy ofinec 3] & & & & & & \\
\hline 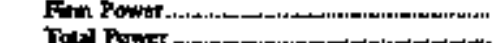 & 二 & - & $\bar{z}$ & - & 153.8.6 & $\$ 3,33,210$ \\
\hline 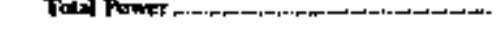 & & - & - & & 153.896 & $9,330,211$ \\
\hline
\end{tabular}

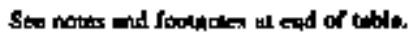


Talue 29. Electrieity Purchases by Mnniclpal Utlities, Flscal Year 1994 (Continued)

\begin{tabular}{|c|c|c|c|c|c|c|}
\hline \multirow{3}{*}{ 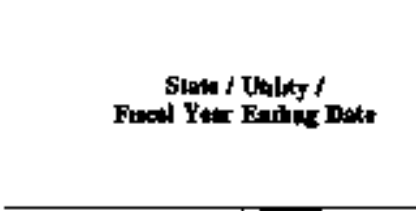 } & \multicolumn{6}{|c|}{ Sumtre of Pescreity } \\
\hline & \multicolumn{2}{|c|}{ investar Owned } & \multicolumn{2}{|c|}{ Fodetal } & \multicolumn{2}{|c|}{ State and Oekn } \\
\hline & (homing kwhy & $\underset{\text { collen }}{\text { Cout }}$ & 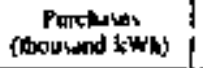 & (donts) & 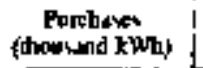 & $\begin{array}{c}\text { Con } \\
\text { (dollan) }\end{array}$ \\
\hline \multicolumn{7}{|l|}{ Frome } \\
\hline \multicolumn{7}{|l|}{ 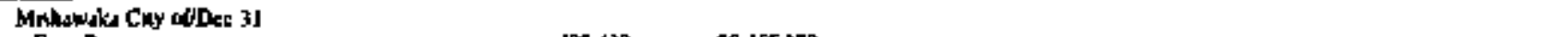 } \\
\hline Fim Powis & 482,459 & $20,465,973$ & - & - & - & - \\
\hline Totsl power & 42,499 & $20,465,130$ & - & - & - & - \\
\hline \multicolumn{7}{|l|}{ Pato City abdec 3 } \\
\hline $\begin{array}{l}\text { Find Pown } \\
\text { Tod Power }\end{array}$ & 二 & 二 & $\overline{-}$ & 二 & 206465 & $\begin{array}{l}5,729,786 \\
5,729756\end{array}$ \\
\hline \multicolumn{7}{|l|}{ 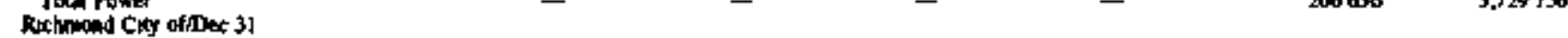 } \\
\hline Fim Power & - & - & - & - & $2 \mathrm{nh}, \mathrm{j04}$ & $33,427,901$ \\
\hline Total Pow:r & - & - & عد & - & $2 \mathrm{BO}, 104$ & 13,423, gin \\
\hline Whapgon ory of then & & & & & & \\
\hline $\begin{array}{l}\text { Fim Power } \\
\text { Totsl Power }\end{array}$ & 亡 & سـ & - & - & 162525 & 6,5729 \\
\hline \multirow{2}{*}{\multicolumn{7}{|c|}{ Core }} \\
\hline Allaev Cily ofthen 30 & & & & & & \\
\hline Nonfin Pond & 67,416 & 1. 1919.778 & - & - & - & - \\
\hline \multirow{2}{*}{\multicolumn{6}{|c|}{ 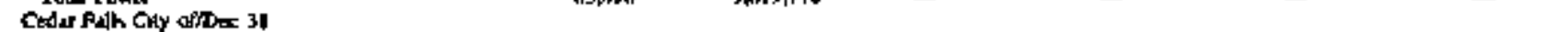 }} & - \\
\hline & & $1,350,897$ & - & - & - & - \\
\hline Othar Bower & $\rightarrow$ & $\frac{1}{0}$ & - & $=$ & $\bar{z}$ & $=$ \\
\hline Totd Power & $97,7 \mathrm{KI}$ & $1,350,897$ & - & - & - & - \\
\hline \multicolumn{7}{|l|}{ ול Musedien Ciky offDee } \\
\hline Noefinim Power & 9.270 & 160,42 & 1,625 & 65,475 & 24,735 & 328,035 \\
\hline \multirow{2}{*}{\multicolumn{7}{|c|}{ 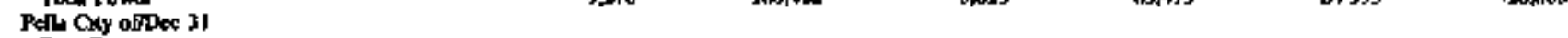 }} \\
\hline & & - & & & & \\
\hline Nosfim Fuxp & (1.053 & 463,369 & $\overline{-}$ & $\overline{-}$ & $\bar{z}$ & 二 \\
\hline Totd Pawer & 6,957 & 463,369 & - & - & - & $=$ \\
\hline \multirow{2}{*}{\multicolumn{7}{|c|}{ Kingist }} \\
\hline Onive Crty ofldoe 35 & & & & & & \\
\hline Fim Pow & - & - & 124 & 66 nils & - & - \\
\hline Nowimu Foner & 149,400 & 3548,865 & - & - & - & - \\
\hline $\begin{array}{l}\text { Totd Power } \\
\text { Cofferontle Oly of } \mathrm{Dec} 31\end{array}$ & $1 \$ 940$ & J543.846 & $\$, 346$ & 66,018 & 一 & - \\
\hline Nowfinte Power & 144,693 & $4 \pi 3_{3}, 9 \mathrm{~B}$ & 3,271 & J37,584 & - & - \\
\hline Total Pawer & 140,653 & $4,043,968$ & i & 137,484 & - & - \\
\hline Gailen City Cuty ofdrec I1 & & & & & & \\
\hline Fom Power & - & - & - & - & - & - \\
\hline Tots Power & - & - & $\sim$ & - & - & $=$ \\
\hline Maftutiven Cly offex il & & & & & & \\
\hline Form Power & 529.649 & $12,325,589$ & - & - & - & - \\
\hline Total Power & $\$ 29,559$ & $12,535,589$ & - & - & - & - \\
\hline Winfield Cly ofDer 31 & & & & & & \\
\hline $\begin{array}{l}\text { Noufmin Powa } \\
\text { Totsl Power }\end{array}$ & 91,649 & $2,616,335$ & 二 & $=$ & $84,53,19$ & $3,361,000$ \\
\hline Kolnaky & & $2,6] 6,375$ & & & & \\
\hline Bowleng Goen Chy afdec 31 & & & & & & \\
\hline Fure Power & - & - & 695sint & 捎, 131,894 & - & - \\
\hline Toxd Pown & - & 一 & 695 641 & $30,131,894$ & - & - \\
\hline Fnevkfot CIty ofIJan 30 & & & & & & \\
\hline Fim Power & 637,728 & $19,4] 4,2+4$ & - & - & - & - \\
\hline Toxd Powser & f33?,728 & 19,114245 & - & - & $=$ & - \\
\hline Frantla Chy offJum 30 & & & & & & \\
\hline Fin Power & - & - & 295,069 & $7,940,592$ & - & - \\
\hline Tode Pown & - & - & 395, 099 & $7,84 \mathrm{~B}, 592$ & سـ & - \\
\hline Gugaw Cuy of hon 3 . & & & & & & \\
\hline Mouffer Pomer & $\bar{z}$ & $\overline{-}$ & $\begin{array}{l}32,000 \\
32,000\end{array}$ & $11,479,057$ & - & - \\
\hline 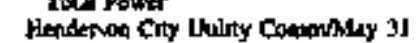 & & & 321,600 & $10,49,067$ & & - \\
\hline Fint Power & - & - & $\$ 6.530$ & 359.62 & - & - \\
\hline Nonfim Fower & 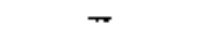 & - & - & - & - & - \\
\hline Told Powr & - & - & 16,530 & 3599,022 & - & - \\
\hline Hoplanville Cily offlon 30 & & & & & & \\
\hline Fim Power & - & - & 433,467 & $12,101,3]$ & - & - \\
\hline Toud Rowt & - & - & 433,467 & $|B| 01,,7 \geqslant \mid$ & - & - \\
\hline 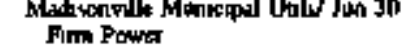 & 254535 & 8,746512 & - & - & $\rightarrow$ & - \\
\hline Tord powar & 284,555 & $8,746,512$ & - & - & - & - \\
\hline
\end{tabular}


Table 29. Electricity Purchases by Municipal Utilities, Fiscal Year 1994 (Continced)

\begin{tabular}{|c|c|c|c|c|c|c|}
\hline \multirow{3}{*}{ 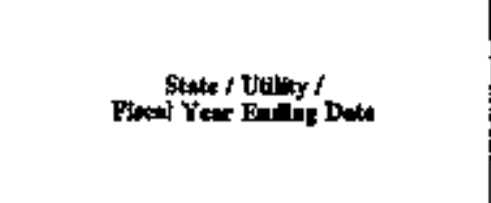 } & \multicolumn{6}{|c|}{ Somen of Eucticily } \\
\hline & \multicolumn{2}{|c|}{ Cuspotito } & \multicolumn{2}{|c|}{ 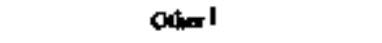 } & \multicolumn{2}{|c|}{ Totad } \\
\hline & 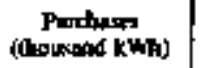 & $\underset{\text { Com }}{\text { Conflory) }}$ & 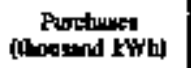 & $\underset{\text { (dollast }}{\text { Cont }}$ & $\begin{array}{l}\text { Porchang } \\
\text { (thoosed twh) }\end{array}$ & $\begin{array}{c}\text { Crmi } \\
\text { (dêllm) }\end{array}$ \\
\hline \multicolumn{7}{|l|}{ Ending: } \\
\hline \multicolumn{7}{|l|}{ Mishat tom Ciny offDec 31} \\
\hline \multicolumn{2}{|l|}{ forn Chy aboes 3 I } & - & - & - & 482,499 & $24,465,170$ \\
\hline Fitw Foros & - & - & - & - & $20,5,656$ & $3,729,756$ \\
\hline 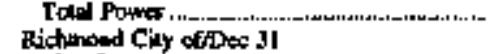 & - & - & - & - & 2015,666 & $5,729,756$ \\
\hline Finn Fower & - & - & - & - & 200,104 & $10,427,901$ \\
\hline 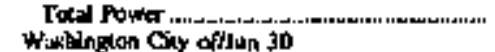 & \multicolumn{6}{|c|}{ Wistinglon Ciy oflum 30 } \\
\hline 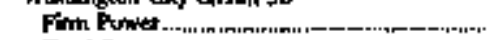 & - & - & - & - & $162, \pm 25$ & 6,572929 \\
\hline 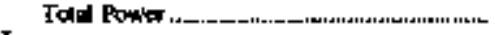 & - & - & - & - & 162,825 & 6,52928 \\
\hline \multicolumn{7}{|l|}{ Iown Chy ofion so } \\
\hline Nonfitin Pown & - & - & - & - & 60,416 & $1,019,37 t$ \\
\hline \multirow{2}{*}{\multicolumn{7}{|c|}{ 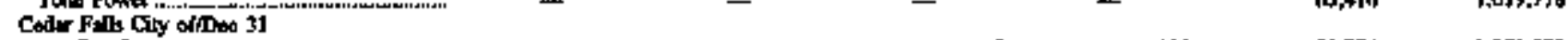 }} \\
\hline & & & & & & \\
\hline 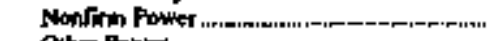 & - & - & , & 191 & 93,774 & 1,351,028 \\
\hline Olha Power & - & - & - & - & -4 & - \\
\hline Totul F9w口 & - & - & ! & 131 & 90.770 & $1,351,028$ \\
\hline \multicolumn{7}{|l|}{ Mosuline City offDes 31} \\
\hline 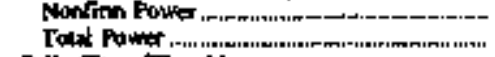 & $\begin{array}{l}10,950 \\
10,950\end{array}$ & $\begin{array}{l}178,967 \\
178,967\end{array}$ & $\begin{array}{l}285 \\
285\end{array}$ & $\begin{array}{l}4,767 \\
4,767\end{array}$ & $\begin{array}{l}48,465 \\
48,465\end{array}$ & $\begin{array}{l}737,6,66 \\
737,666\end{array}$ \\
\hline Pally Cry ofDet 31 & & & & & & \\
\hline 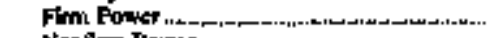 & - & - & - & 600,000 & - & 600,000 \\
\hline 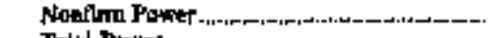 & - & - & 54,006 & 866,312 & 60,993 & 1.329 .681 \\
\hline 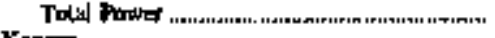 & - & - & 54.036 & $1,066,3 / 2$ & 64,993 & ],929,681 \\
\hline \multirow{2}{*}{\multicolumn{7}{|c|}{ Kavious Chty ofDec 31}} \\
\hline & & & & & & \\
\hline 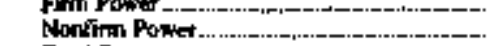 & - & 二 & $\overline{-}$ & 二 & 149.406 & 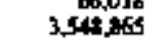 \\
\hline \multirow{2}{*}{\multicolumn{7}{|c|}{ 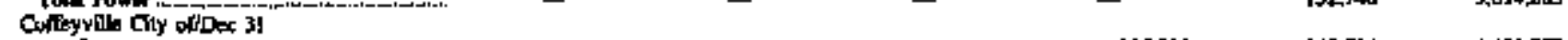 }} \\
\hline & & & & & & \\
\hline 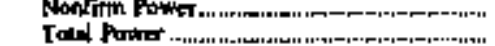 & $\bar{z}$ & $\bar{z}$ & $\overline{-}$ & 20,000 & 149,524 & $4,40,972$ \\
\hline Gurdes Chy City ofthet 31 & & & & & & \\
\hline Finn Ponver. & 178,114 & B,941,A17 & - & - & $178,[14$ & $8,941,417$ \\
\hline 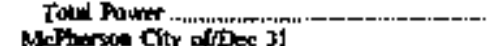 & IJ8,114 & $8,941,417$ & - & 一 & I74,I]4 & $8,94 \mid, A\}$ \\
\hline 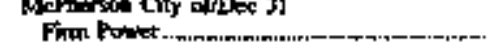 & - & - & - & - & 529,659 & $12,515,589$ \\
\hline Iod Power & - & - & - & $=$ & 529,699 & $12,535,569$ \\
\hline Winfiatd Ciky oftoec 31 & & & & & & \\
\hline 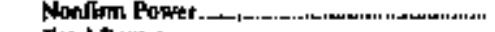 & - & - & - & - & 176,468 & $5,979,337$ \\
\hline 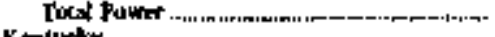 & - & - & - & - & I76,483 & $5,97,135$ \\
\hline Keively & & & & & & \\
\hline 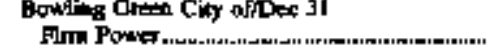 & - & - & - & $=$ & 65,641 & 32131.84 \\
\hline Totrl Power : & - & - & - & - & 699,641 & $30,131,891$ \\
\hline 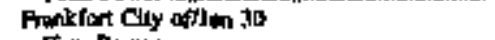 & & & & & & \\
\hline F的 Powt & - & - & - & - & 637,728 & $19.414,245$ \\
\hline 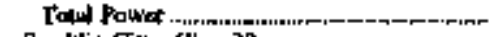 & - & - & - & - & 677,729 & $0.414,245$ \\
\hline Fraklin City wfllon 3A & & & & & & \\
\hline 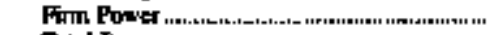 & - & - & - & - & 195809 & $7,845,592$ \\
\hline Tokul Pouter & - & - & - & - & 998,069 & $7,848,592$ \\
\hline Gkagow Chy of own TO & & & & & & \\
\hline 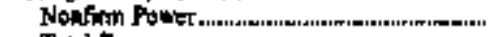 & - & - & - & - & 323,000 & $13,479,087$ \\
\hline Total bower & - & - & - & - & 327,010 & $13,479,087$ \\
\hline Hendesen Cily Utilicy Connulday 31 & & & & & & \\
\hline 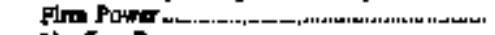 & - & ـ & - & - & $t 6,530$ & 359,602 \\
\hline 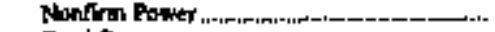 & 1,130 & 0,76 & - & - & 1.120 & 42,783 \\
\hline 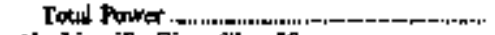 & 1,120 & Q,7?] & - & - & 17.,650 & 402,45 \\
\hline Hopkins rille Ciny afluou 30 & & & & & & \\
\hline 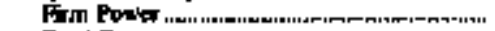 & $\longrightarrow$ & - & - & - & 431,467 & $18,101,771$ \\
\hline Totsl Fouver & - & - & - & - & 431,467 & IB.J11, 371 \\
\hline 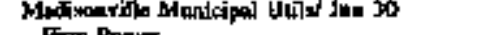 & & & & & & \\
\hline Fim, Pong & $=$ & $\bar{z}$ & $\bar{z}$ & - & 284,555 & $8,746,512$ \\
\hline Totuli Powner & - & - & - & - & 204035 & $8,746,012$ \\
\hline
\end{tabular}

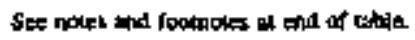


Tabie 29. Dlectricity Porchases by Municipal Uttillles, Fitscal Year 1994 (Continued)

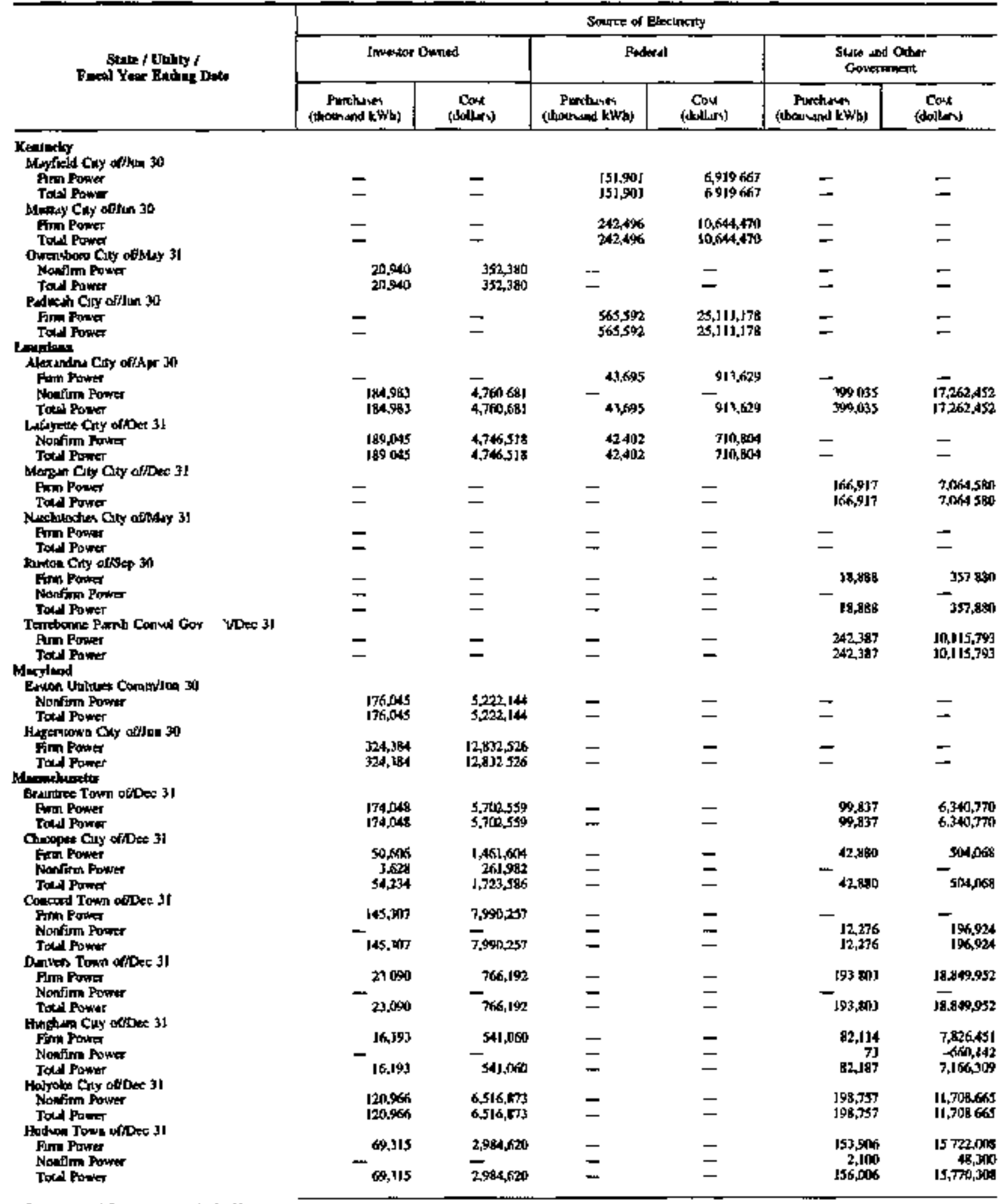

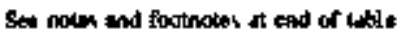


Table 29. Electricliy Purchases by Municipal Utilities, Fiscrl Year 1994 (Continued)

\begin{tabular}{|c|c|c|c|c|c|c|}
\hline \multirow{3}{*}{ 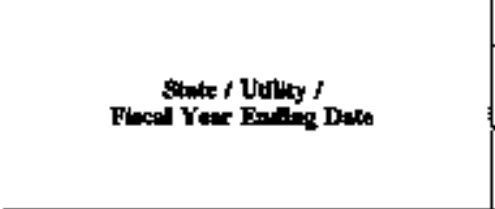 } & \multicolumn{6}{|c|}{ Sortce of Elextrithy } \\
\hline & \multicolumn{2}{|c|}{ Cocperative } & \multicolumn{2}{|c|}{ Oinerl } & \multicolumn{2}{|c|}{ Todal } \\
\hline & 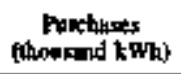 & $\underset{\text { (dollonk }}{\text { Cart }}$ & 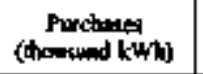 & $\begin{array}{c}\text { Cost } \\
\text { (dollax) }\end{array}$ & 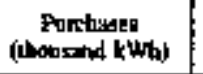 & $\begin{array}{c}\text { Coost } \\
\text { (dallaris) }\end{array}$ \\
\hline \multicolumn{7}{|l|}{ Ken:ally } \\
\hline Murfield Chy oftjon 3a & - & - & - & - & $|\leq|, \$ 01$ & $6,019,667$ \\
\hline Low Power & - & $\rightarrow$ & - & - & ISI, 的] & $6,919,667$ \\
\hline 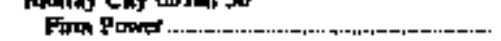 & - & - & $=$ & - & 242,406 & Mus44790 \\
\hline Tothl Ponetr -. & - & $\rightarrow$ & - & - & 242,496 & $50,644,470$ \\
\hline Owhondowa City of & - & - & - & - & 20,940 & 352,380 \\
\hline 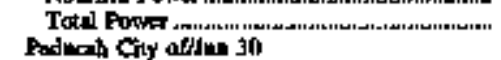 & - & - & - & - & 20,940 & 392,360 \\
\hline Fim Powef & 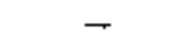 & - & - & - & $\mathbf{5 n d , 3 9 2}$ & $25,111,178$ \\
\hline 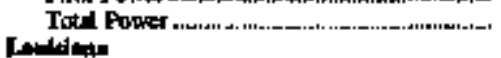 & - & - & - & - & 345,592 & $25,111,178$ \\
\hline \multicolumn{7}{|l|}{ INeximitia Chy off Apr 30} \\
\hline Fim Pow & - & - & - & - & 43,695 & $\$ 13,629$ \\
\hline Norim Powner & - & - & - & - & 584,018 & $22,829, \mathrm{J3}$ \\
\hline Totll Power & - & - & - & - & 62.713 & $22,936,762$ \\
\hline \multicolumn{7}{|l|}{ Lablete Cuy whot 3I } \\
\hline 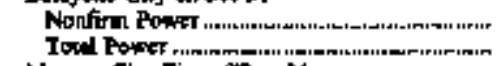 & $\overline{-}$ & $\underline{-}$ & $\begin{array}{l}1,474,468 \\
1,474,468\end{array}$ & $48,558,624$ & $\begin{array}{l}1,705,915 \\
1,705,915\end{array}$ & $\begin{array}{l}54,415,946 \\
54,415,946\end{array}$ \\
\hline \multicolumn{7}{|l|}{ Maras Chy Cing offos is } \\
\hline Fing Pourer & - & - & - & - & IS6,9I] & $30 \times 4,590$ \\
\hline \multicolumn{7}{|l|}{ 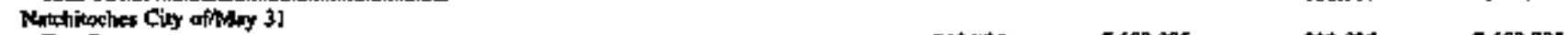 } \\
\hline Fin PQWer & - & - & $212 \mathrm{kits}$ & $7,609,8915$ & 212,000 & $7,609,300$ \\
\hline 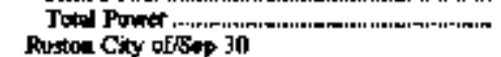 & - & - & 212005 & $7,609,806$ & 212,000 & $7,609,8005$ \\
\hline \multicolumn{7}{|l|}{ Rustoe CAy oESTp 30} \\
\hline Namitrim Power & - & - & -47011 & $\bar{j}, 095,001$ & $\begin{array}{l}18,988 \\
47,01 !\end{array}$ & $\begin{array}{r}357,8804 \\
1,095,670\end{array}$ \\
\hline \multirow{2}{*}{\multicolumn{7}{|c|}{ Temebann Puril Coused Gov vibes 31}} \\
\hline & & & & & & \\
\hline 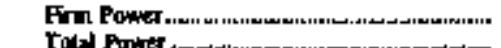 & - & - & - & - & 242,487 & $10,115,793$ \\
\hline \multirow{2}{*}{\multicolumn{7}{|c|}{ 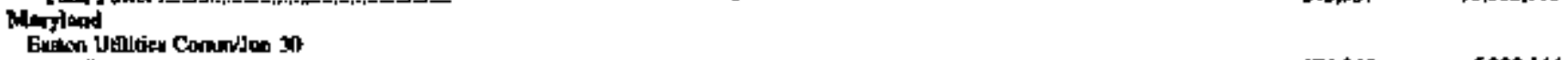 }} \\
\hline & & & & & & \\
\hline 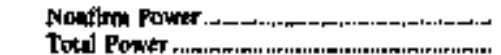 & - & - & - & - & $776, \mathrm{MAS}$ & $5,222, \sqrt{44}$ \\
\hline \multirow{2}{*}{\multicolumn{7}{|c|}{ Hagerdown Chy oflden 30}} \\
\hline & & & & & & \\
\hline 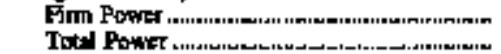 & - & - & - & - & 324,384 & $52,8,52524$ \\
\hline 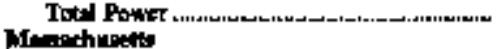 & س & - & - & - & 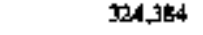 & $J 2,832,526$ \\
\hline Mraphupets & & & & & & \\
\hline 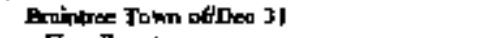 & & & & & & \\
\hline 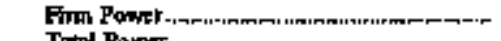 & $m$ & - & $6, \$ 90$ & 822,565 & $23 n, 779$ & $12,865,899$ \\
\hline 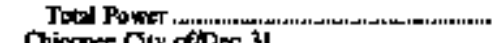 & - & $\rightarrow$ & 6.6901 & 822365 & 280.775 & $12,865,894$ \\
\hline Qhiepose Cuy of pec 31 & & & & & & \\
\hline 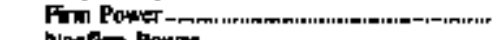 & $\bar{z}$ & $\Rightarrow$ & 166920 & $(0,220,97)$ & 260,406 & 12,186,6933 \\
\hline Nanfitm Rown & - & - & 22,260 & 1.065 .0 .897 & 25,858 & $1,327,069$ \\
\hline 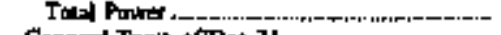 & - & - & 189,180 & $11.286,1658$ & 266,294 & 13513.712 \\
\hline Concond Town ofpec I & & & & & & \\
\hline Fim Popter & - & - & - & - & 145,300 & $7,997,257$ \\
\hline 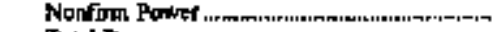 & - & - & - & - & 12,276 & I 9292 \\
\hline Tots Power & - & 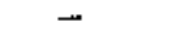 & 一 & - & 157.583 & $8,187,181$ \\
\hline Donvers Tom oftDec 31 & & & & & & \\
\hline Fink, Power _- & - & - & 80007 & 253.316 & 224,893 & $19,869,460$ \\
\hline 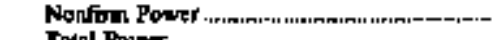 & - & - & 96,695 & $2,843,597$ & 96,695 & $2,404,588$ \\
\hline 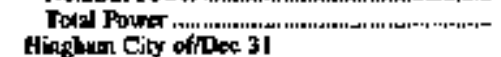 & - & - & IA4,615 & $3,096,904$ & 321,388 & 27.213040 \\
\hline Fon Pown & - & - . & 3.943 & 25,499 & 102,250 & B.793, 100 \\
\hline Nomfitin Powfif & - & $-73,1009$ & - & & +3 & $-733,111$ \\
\hline 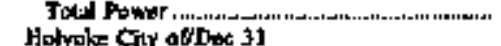 & & $-73,039$ & 3,943 & 125.489 & $\ln 2,323$ & $8,05 \% .819$ \\
\hline 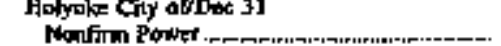 & - & - & 2,471 & 99.470 & 322,194 & 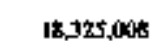 \\
\hline 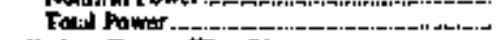 & - & - & 247 & 99.476 & 322.194 & 18925,0078 \\
\hline Hewon Town offDec Jl & & & & & & \\
\hline Pron Power ..... & - & - & 18,061 & $\begin{aligned} 765,800 \\
+1,000\end{aligned}$ & 241,282 & $19,472,129$ \\
\hline 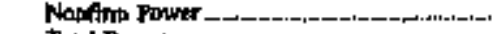 & 二 & $\bar{\Xi}$ & 7,539 & $2,243,923$ & 79.739 & $2,292,22]$ \\
\hline 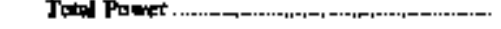 & & - & 95200 & 3.009 .72 & 320,521 & $21,764,651$ \\
\hline
\end{tabular}

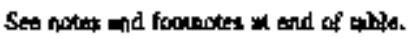


Talle 29. Electricity Furchases by Municipal Utilltes, Fiscal Year 1994 (Continned)

\begin{tabular}{|c|c|c|c|c|c|c|}
\hline \multirow{3}{*}{ 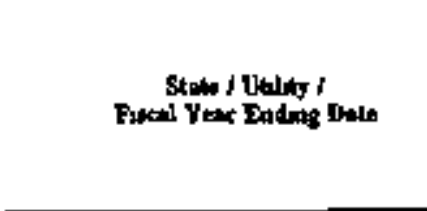 } & \multicolumn{6}{|c|}{ Souce of Elextncity } \\
\hline & \multicolumn{2}{|c|}{ Inwovet Ounned } & \multicolumn{2}{|c|}{ Rederal } & \multicolumn{2}{|c|}{$\begin{array}{l}\text { Stete and Other } \\
\text { Opuramumpix }\end{array}$} \\
\hline & $\begin{array}{l}\text { Purcheres } \\
\text { (thouldad twh) }\end{array}$ & $\begin{array}{c}\text { Cou } \\
\text { (doln) }\end{array}$ & Ploculuev & $\underset{\text { (dolln) }}{\operatorname{Cos} n}$ & 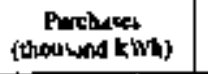 & Cost \\
\hline \multicolumn{7}{|l|}{ Manechuseth } \\
\hline \multicolumn{7}{|l|}{ Lutleno Tawn ofDec 31} \\
\hline Nonfind Powner & M,000 & 897,094 & - & - & 18,927 & $918, \$ 32$ \\
\hline Totd Ruwer & 54,007 & 897,094 & - & - & 10.927 & 958,532 \\
\hline \multicolumn{7}{|l|}{ Mesfield Iorm ofldec it } \\
\hline Fim Power & - & - & - & - & I32,A30 & $53,555,0 \mathrm{J6}$ \\
\hline \multirow{2}{*}{\multicolumn{7}{|c|}{ Mhddlebowalgt town ofroes $¥ 1$}} \\
\hline & & & & & & \\
\hline Nonitru Fower & 70,640 & $4,130,040$ & - & - & I02,0s & 7265,803 \\
\hline Total Pown & 20,640 & 4304046 & - & - & IM20N5 & $7,265,801$ \\
\hline \multicolumn{7}{|l|}{ Norh Aulktoroogh Town of $/ \mathrm{Dec}$ J] } \\
\hline Fim Pow口 & 21.360 & $1,000,103$ & - & - & 71 ,Band & $5.955,244$ \\
\hline Noprimg potwot & - & - & - & - & 1,127 & 15,990 \\
\hline Totd Pawer & 21,3601 & $1,0 \times 2.109$ & - & - & 71205 & $5,991,237$ \\
\hline \multicolumn{7}{|l|}{ 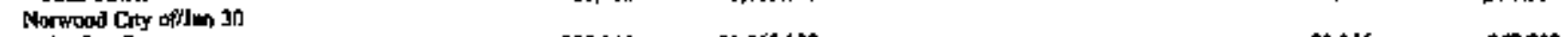 } \\
\hline Nonfine Power & 305,146 & $21,068,1,32$ & - & - & 21,346 & 25,790 \\
\hline ToLA Pिभाम & $\$ 05.146$ & $21,068,137$ & - & - & 21,346 & 257,790 \\
\hline \multicolumn{7}{|l|}{ Pesbody Cty offDes II } \\
\hline Fmn Powra & 46,958 & $2,051,3,8$ & - & - & 211,182 & $18,974,930$ \\
\hline Nonfirsm Power & 2,354 & 62391 & - & - & $\$ 544$ & 379,521 \\
\hline Tous Pown & 49442 & $2 \sqrt{13} 3,609$ & - & - & $215,20 x$ & 19.354 .360 \\
\hline 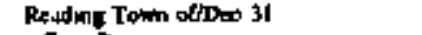 & & & & & & \\
\hline Firm Power & 259,759 & $16,640,531$ & - & - & 27,274 & $17,744,496$ \\
\hline Nonfim Pows & & 87.751 & - & - & - & [j0]1,ad] \\
\hline Tond Powror & 259,775 & $16,575,262$ & - & - & 227274 & $18,756,287$ \\
\hline Slwrewstury Ion'n affDec J। & & & & & & \\
\hline Frim Power & 95,569 & $4,0100,004$ & - & - & (5,961 & $4,41,839$ \\
\hline Nomfirm Pourer & 39,239 & 900,751 & - & - & - & - \\
\hline Iats Power & 125,800 & $5, \sqrt{4} \times 0,145$ & - & - & 80,991 & $4,41,439$ \\
\hline Trumton Cyy of Dec 3S & & & & & & \\
\hline Nonfinn Power & 107.678 & $2,735,463$ & - & - & 4225 & 643,563 \\
\hline Tanl Puwer & $149, \Omega B R$ & $2,735,961$ & - & - & 42,252 & 643,563 \\
\hline Wafield Iowm atodet II & & & & & & \\
\hline Firm Power & 2,725 & 3,201,173 & - & - & 142,301 & $10,287.667$ \\
\hline Nonfinm Power & & 276,252 & - & - & - & - \\
\hline Tot,l Ponver & 25.325 & $1,485,925$ & - & - & J42,301 & 10.287 , 每社 \\
\hline Wefledky Town afDec 31 & & & & & & \\
\hline Find Powtit & $185, y 00$ & In:266,8]? & - & - & 18,300 & 293,263 \\
\hline Nonifinom Power & - & & - & - & - & - \\
\hline Tocst Aower & I85,390 & In, $26 x, 817$ & - & - & 18,300 & 190.265 \\
\hline Wettleld Cry ofdec 31 & & & & & & \\
\hline Fitan Puner & 59,827 & $1,857,310$ & - & - & 20,857 & 17.974, 154 \\
\hline 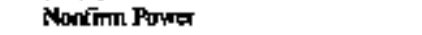 & 1.529 & 15,424 & - & - & - & - \\
\hline Tod止 Paw'it & +1,956 & 1,858,77,4 & - & - & 229.807 & $17,9,3,6,54$ \\
\hline Mlocingan & & & & & & \\
\hline Bay cuy Cly afthen 31 & & & & & & \\
\hline $\begin{array}{l}\text { Fith Power } \\
\text { Fold Power }\end{array}$ & $\begin{array}{l}2,55,5103 \\
295,500\end{array}$ & $\begin{array}{l}10,192,314 \\
10,192,114\end{array}$ & $\bar{z}$ & $\overline{-}$ & $\bar{z}$ & $\bar{z}$ \\
\hline 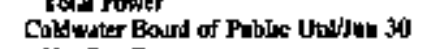 & & & - & & & 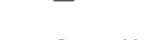 \\
\hline Nondinm Portra & $\rightarrow$ & - & - & - & 159,190 & 8218,491 \\
\hline Towl Fower & - & - & - & - & 199,190 & $8,218,49]$ \\
\hline Detrou Coy of then 30 & & & & & & \\
\hline Numfirsu Powar & 420,472 & $14,112,815$ & - & - & - & - \\
\hline Toksl Power & 429,472 & $14,112.895$ & - & - & - & - \\
\hline 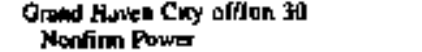 & - & - & - & - & 4514 & 121.746 \\
\hline Tad Power & - & - & - & - & 4,514 & 121.746 \\
\hline 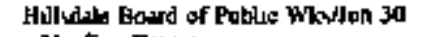 & & & & & & \\
\hline Nomitum Polvet & - & - & - & - & 1d2, & $6,365,216$ \\
\hline Torel Fuwar & - & - & - & - & 142,503 & $6,25,216$ \\
\hline Fothod Cly ofium id & & & & & & \\
\hline Frm Power & B, $16 \mathrm{G}$ & $2,897,30]$ & - & - & 311,120 & I1,671,5]6 \\
\hline Totel Power & 87,466 & $2,897,301$ & - & - & 311,128 & $11,671,516$ \\
\hline Linung Cuy ofitun 90 & & & & & & \\
\hline Nonikn Pover & - & - & $=$ & $\bar{z}$ & $\begin{array}{l}997,356 \\
097,356\end{array}$ & $37,816,537$ \\
\hline Tatd Power & - & - & - & - & $997,35 \%$ & $37,8[6,537$ \\
\hline
\end{tabular}

Sere moter and inothoter $a$ and of table 
Table 29. Electriclty Purchases by Mrenichal Utllities, Fiscal Year 1994 (Contlnued)

\begin{tabular}{|c|c|c|c|c|c|c|}
\hline \multirow{3}{*}{ 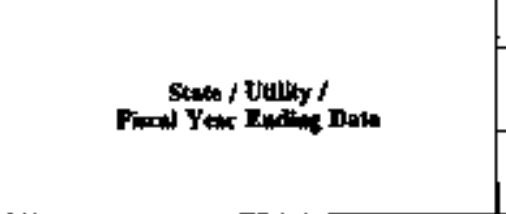 } & \multicolumn{6}{|c|}{ Sourte of Etcerdeily } \\
\hline & \multicolumn{2}{|c|}{ Coopentive } & \multicolumn{2}{|c|}{ Ollarl } & \multicolumn{2}{|c|}{ Tatad } \\
\hline & $\begin{array}{l}\text { Furctoses } \\
\text { (Ahoussond tWh) }\end{array}$ & $\begin{array}{c}\text { Coot } \\
\text { (dolnam) }\end{array}$ & $\begin{array}{c}\text { Ponchuses } \\
\text { (thersend kWh) }\end{array}$ & 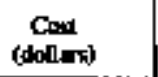 & 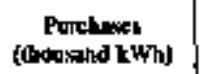 & $\underset{(\mathbf{d} k h \log s)}{\operatorname{Cosa}}$ \\
\hline \multicolumn{7}{|l|}{ Mequmbets } \\
\hline 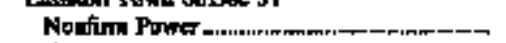 & 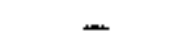 & - & $14 J, 46 t$ & $9,735,102$ & 174,421 & $11,591,1228$ \\
\hline 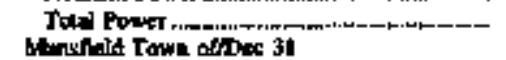 & - & - & $14 k, 4: ?$ & $9,715,102$ & 174,421 & 11.591 .028 \\
\hline 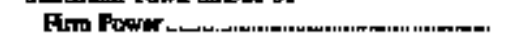 & - & - & 6,920 & 221.42 & 139,150 & $13, \pi 7,488$ \\
\hline Jald Power . & - & - & 6,920 & 221,42 & 139,350 & $19,776,486$ \\
\hline Nouflm Pown & - & - & - & - & 172,695 & $11,5 \%, 351$ \\
\hline Toted Power & - & - & - & - & 172,695 & $1 \mathrm{I}$ S95:A5I \\
\hline \multicolumn{7}{|l|}{ 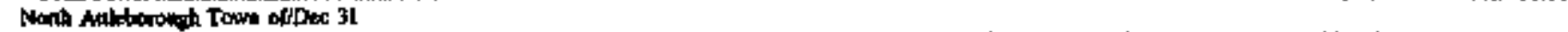 } \\
\hline 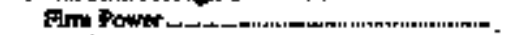 & - & - & 51,187 & $1,232,01$ & Ind.355 & $\$, 219,944$ \\
\hline Noxfun Pown - & - & - & - & - & $1, \notin 7$ & 35,990 \\
\hline \multirow{2}{*}{\multicolumn{7}{|c|}{ Norpood Cliy oftJun 39}} \\
\hline & & & & & & \\
\hline 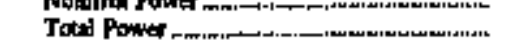 & - & - & - & - & 326,492 & $21,725,927$ \\
\hline \multicolumn{7}{|l|}{ Pewbody Oty ofDex 3! } \\
\hline Fitw Pown & - & - & - & - & 258,070 & $21,026,257$ \\
\hline 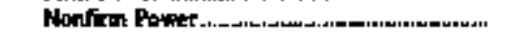 & - & - & 3,732 & 94.517 & 10930 & 536,629 \\
\hline 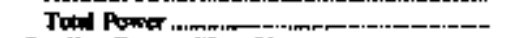 & - & - & 3.332 & 94517 & 260,000 & $21,362,886$ \\
\hline \multicolumn{7}{|l|}{ Retdieg Tow offoe 31} \\
\hline Fin Bown The & - & - & - & - & 487,003 & 3.234,937 \\
\hline Aonfin Pover._-_. & - & - & 93 & $-2,3,3,2$ & & 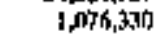 \\
\hline 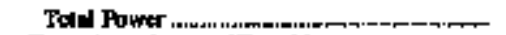 & - & - & 93 & $-23,302$ & $\$ 87.142$ & $35,311,267$ \\
\hline \multicolumn{7}{|l|}{ Stonewidury form ofldex it } \\
\hline 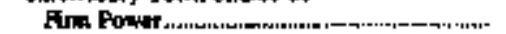 & ـ & - & - & - & 175,960 & $8,529,933$ \\
\hline 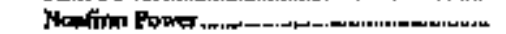 & - & 一 & - & - & 30,239 & 980,751 \\
\hline 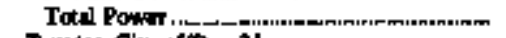 & - & - & - & - & 206,199 & $9.510,64$ \\
\hline \multicolumn{7}{|l|}{ Tunuke Cily offorer 31} \\
\hline 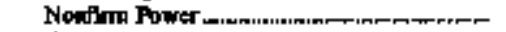 & - & - & $3,2 \pm 5$ & 474,627 & 149,215 & $3,854,151$ \\
\hline \multirow{2}{*}{\multicolumn{5}{|c|}{ Wurtefiedd Town ofthes 31}} & 149,215 & $3,854,151$ \\
\hline & - & - & - & - & 168,026 & $11,491,840$ \\
\hline 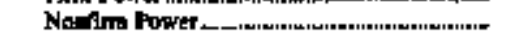 & - & - & 482 & 45.795 & 482 & $322 \sqrt{207}$ \\
\hline Totd Power & - & - & 182 & d5,79s & 168508 & $11,813,887$ \\
\hline \multicolumn{7}{|l|}{ Weflaley rawn offDec 31} \\
\hline Prm Powor .... & - & - & - & - & Phat, 690 & Insiffi, 1882 \\
\hline 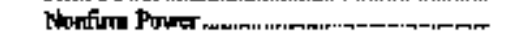 & - & - & ADB & 16.102 & 410 & 16.102 \\
\hline 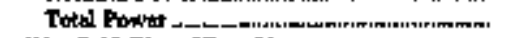 & - & - & 400 & if, ling & $24,10 \times 0$ & $10,576,134$ \\
\hline Wentrietd Cky ofDax II & & & & & & \\
\hline 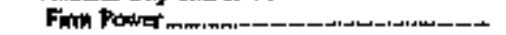 & - & - & 30,363 & 2,308,483 & 360,397 & $22,170,049$ \\
\hline Nonflm Povw & - & - & 4,136 & 454278 & 5ins & $487.7 \%$ \\
\hline Told Pows & $m$ & - & 35.199 & 2794,363 & 305,962 & $22,657,751$ \\
\hline Matipan & & & & & & \\
\hline Boy Chy Cly offron H & & & & & & \\
\hline 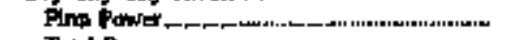 & - & - & - & - & $2955 \mathrm{sm}$ & $10,19,314$ \\
\hline Toted Powe & - & - & - & 一 & 203503 & I0,192314 \\
\hline 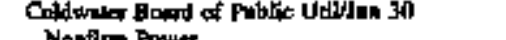 & & & & & & \\
\hline & - & - & - & - & 159,196 & $8,218,491$ \\
\hline Tots Powa & - & - & - & - & 159.190 & \&,2LB, A9I \\
\hline Dutroit Chy oflewn id & & & & & & \\
\hline Nontin Ponter & - & - & - & - & 429,472 & 14.J 12,815 \\
\hline 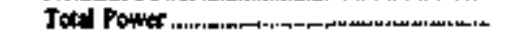 & - & - & - & - & 29,472 & 14] 12,5] 5$, \\
\hline Grad thues Ciny oflin 30 & & & & & & \\
\hline 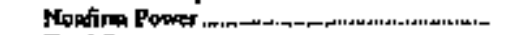 & - & - & - & - & $4 \$ 14$ & 121,746 \\
\hline 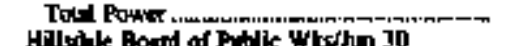 & - & - & 一 & 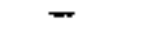 & 4514 & $121,74 \pi$ \\
\hline 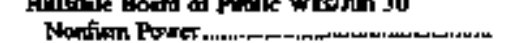 & - & - & - & - & $142 \mathrm{kP3}$ & 6.765 .216 \\
\hline Tovel Pourer & - & - & سـ & - & 142,063 & $6,365,216$ \\
\hline Hollond city ofiton 30 & & & & & & \\
\hline Finn Pown & - & - & - & - & 398,594 & $1 / 563,817$ \\
\hline 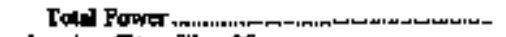 & - & $=$ & - & - & 398,594 & 14,569, a17 \\
\hline Lasing Cly ofluen 39 & & & & & & \\
\hline 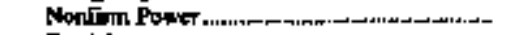 & - & - & - & - & 907,396 & $37,8 J 6,537$ \\
\hline 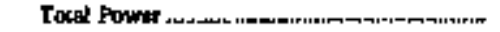 & - & - & - & - & 997,356 & $37,816,557$ \\
\hline
\end{tabular}

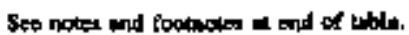


Table 29. Electricity Purchases by Monicipgl Utilities, Fiscal Year 1994 (Continued)

\begin{tabular}{|c|c|c|c|c|c|c|}
\hline \multirow{3}{*}{ 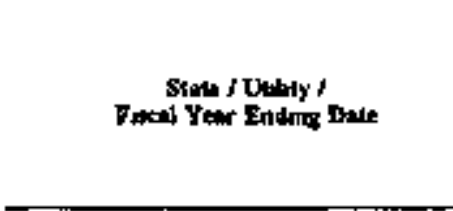 } & \multicolumn{6}{|c|}{ Soures of Blectnenly } \\
\hline & \multicolumn{2}{|c|}{ Inverlor Omined } & \multicolumn{2}{|c|}{ Pederal } & \multicolumn{2}{|c|}{ Sculf and other } \\
\hline & Purdines & $\begin{array}{c}\text { Cost } \\
\text { [dollors] }\end{array}$ & 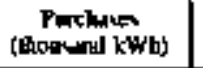 & $\underset{\text { (dollens) }}{\operatorname{Cos}}$ & 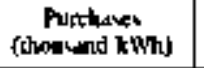 & $\begin{array}{l}\text { Cost } \\
\text { (d) }\end{array}$ \\
\hline \multicolumn{7}{|l|}{ Mrelupar } \\
\hline Noonfin Pow & $\$ 1,150$ & $m 9,23$ & $\longrightarrow$ & - & - & - \\
\hline Tokst Fower & 11,150 & $\pi 421$ & - & $\overline{-}$ & - & - \\
\hline \multicolumn{7}{|l|}{ sumpor Cry of $\$$ ep 30} \\
\hline Prim Prowa & 191,312 & $6,973,973$ & $\rightarrow$ & - & - & - \\
\hline Nonfinm Pown & & $\overline{600014}$ & $\bar{z}$ & $\bar{z}$ & $\overline{-}$ & - \\
\hline 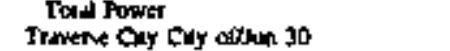 & 191,712 & $0,9 \pi, 0,11$ & & & - & - \\
\hline Fon Poner & - & - & 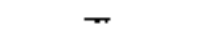 & - & 150946 & s,tw. I It \\
\hline Nomflesu Pover & - & - & $=$ & - & 59,0014 & $1,487.291$ \\
\hline Total power & - & - & - & - & 200,98 & $6,874,357$ \\
\hline $\begin{array}{l}\text { Wyandode Muncipd Sery Commsep } 30 \\
\text { Fim Power }\end{array}$ & & & & & - & \\
\hline $\begin{array}{l}\text { Fimm Poner } \\
\text { Tad Power }\end{array}$ & $\begin{array}{l}33,846 \\
75,846\end{array}$ & $\begin{array}{l}J, 108, d 2] \\
J, 198, \$ 13\end{array}$ & 二 & $\bar{z}$ & $\bar{z}$ & - \\
\hline Tealind Cty of Dec II & & & & & & \\
\hline Furn Power & - & - & - & - & $x 9584$ & $3,064,355$ \\
\hline Nonfinith Pother & - & - & - & - & 31,42 & $1,660,422$ \\
\hline \multirow{2}{*}{\multicolumn{7}{|c|}{ 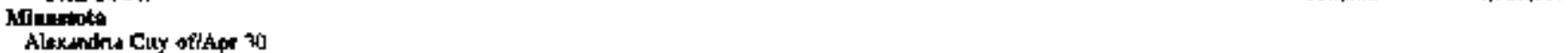 }} \\
\hline & & & & & & \\
\hline Fint Pow & - & - & 97,019 & 3198892 & 96.297 & $3,747,132$ \\
\hline Iods Powne & - & - & 97019 & $8,198,002$ & 96.297 & 3743,132 \\
\hline \multicolumn{7}{|l|}{ Andk Cuy of Dee 31} \\
\hline Frin Power & 218.704 & $8,894,813$ & $=$ & - & - & - \\
\hline \multicolumn{7}{|l|}{ Arum CuY wSep 30} \\
\hline Fint Power & - & - & - & - & $262 \leq 564$ & 14.537 .3611 \\
\hline Told Power & $m$ & - & - & - & 262556 & J4,537 $3 \mathrm{ki}$ \\
\hline \multicolumn{7}{|l|}{ Branem bry oftoec 31} \\
\hline 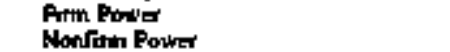 & -149.428 & $\frac{5,275.161}{-}$ & $\bar{z}$ & - & & - \\
\hline $\begin{array}{l}\text { Nonflin Pownt } \\
\text { Tatul Pawer }\end{array}$ & 149,428 & $\overline{5,275,161]}$ & $\overline{-}$ & $\bar{z}$ & $\bar{z}$ & $=$ \\
\hline \multicolumn{7}{|l|}{ Iasd Pawn } \\
\hline Frm Power & 167,816 & 6.734 .145 & - & - & - & - \\
\hline 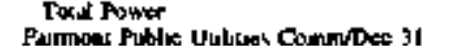 & 167:816 & 6.734 .145 & - & - & - & 一 \\
\hline Find Power & - & - & - & - & 146,474 & 8548850 \\
\hline Tad Power & - & - & - & - & 146.434 & 8548,950 \\
\hline \multicolumn{7}{|l|}{ Holotuniom Utaliber Comm/Dec } \\
\hline Fim Paurer & $25,09 y$ & 1,964,624 & - & $\overline{-}$ & - & - \\
\hline $\begin{array}{l}\text { Nonflim Power } \\
\text { Tous Power }\end{array}$ & $\begin{array}{l}\text { 104,910 } \\
\text { Jน1008 }\end{array}$ & ifial] & $\bar{z}$ & $\bar{z}$ & 425 & $\begin{array}{l}6,2 \pi \\
6,2 \pi\end{array}$ \\
\hline \multicolumn{7}{|l|}{ Murwh Gity ofsep 30} \\
\hline Putm Poure & - & - & 129,847 & J,551,569 & 273,722 & 6.759 .187 \\
\hline Namimm Power & - & - & - & - & - & \\
\hline Totd Pawer & - & - & 129,847 & $1,591,569$ & 233,722 & $6,759.187$ \\
\hline \multicolumn{7}{|l|}{ Moorterel Cily dider 31} \\
\hline $\begin{array}{l}\text { Pimm Powar } \\
\text { Totd Power }\end{array}$ & $\bar{z}$ & $\bar{z}$ & $\begin{array}{l}235,179 \\
235,179\end{array}$ & $\begin{array}{l}7,121,521 \\
7,121,521\end{array}$ & $\begin{array}{l}120,499 \\
120,449\end{array}$ & $4,762,257$ \\
\hline 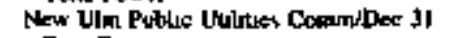 & & & & & & \\
\hline Pmm Powa & $133,6 \%$ & $3,100,34,7$ & - & - & - & - \\
\hline Tatd Power & 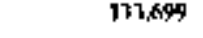 & $1,102,340$ & $=$ & - & - & - \\
\hline 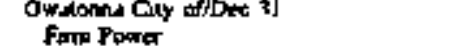 & - & - & - & - & 261,401 & 14.478 .513 \\
\hline Tulal Popdra & - & $=$ & $=$ & - & 264401 & $14,678,5] 3$ \\
\hline Rockeds Tubhe Uhbiliew Dee 31 & & & & & & \\
\hline Fimm Poung & - & - & - & - & $917, g_{1}$ & $49,594,285$ \\
\hline Nonflme Power & - & $\overline{-}$ & - & - & 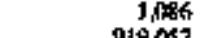 & 100,345 \\
\hline Tond Power & - & - & - & - & $9: 9,0\}$ & 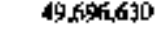 \\
\hline 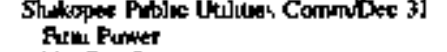 & 135.894 & $5,551,579$ & - & - & - & - \\
\hline Xonfin Power & - & & - & - & - & - \\
\hline Tas Power & 135,6344 & $5,553,579$ & $m$ & - & - & - \\
\hline 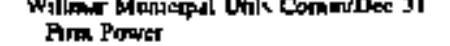 & 136,950 & $3,248,41]$ & 31,986 & 417,343 & - & - \\
\hline Nonfing Powe & 14,103 & 360,504 & $-_{31,486}$ & - & $\begin{array}{l}3,57 ? \\
\mathbf{3}, 577\end{array}$ & 55,948 \\
\hline Tond Power & 150,953 & $4,110,413$ & & & & $30,9,4$ \\
\hline
\end{tabular}

See woter and foctiouter at end of twbe 
Tabie 29. Eleciricily Purchases by Municipal Utaities, Fiscal Year 1994 (Continued)

\begin{tabular}{|c|c|c|c|c|c|c|}
\hline \multirow{3}{*}{ 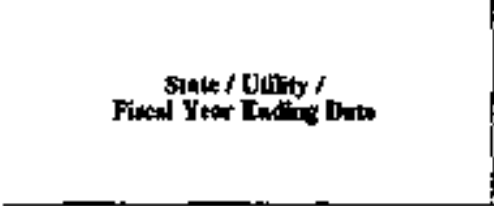 } & \multicolumn{6}{|c|}{ Source of Electricity } \\
\hline & \multicolumn{2}{|c|}{ Coopotitive } & \multicolumn{2}{|c|}{ other 1} & \multicolumn{2}{|c|}{ Total } \\
\hline & 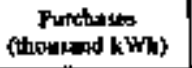 & Cond & $\begin{array}{c}\text { Porchatats } \\
\text { (thunosond } k \text { Wh) }\end{array}$ & $\begin{array}{c}\operatorname{Cosk} \\
(d a]: \sim r)\end{array}$ & 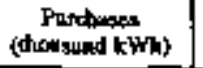 & 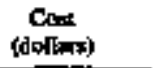 \\
\hline \multicolumn{7}{|l|}{ 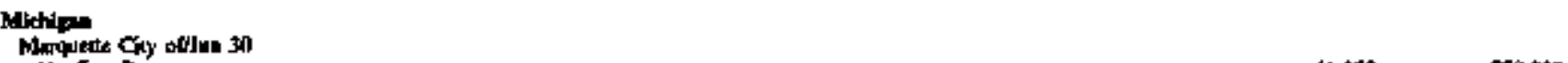 } \\
\hline Nonform Powner & - & - & - & - & 4],350 & 799,237 \\
\hline 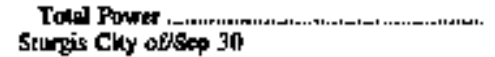 & - & - & - & - & 4,350 & 779,237 \\
\hline Fint Pren & - & - & - & - & 191,72 & $6,978,993$ \\
\hline 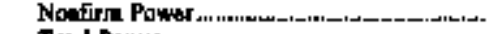 & - & - & 3,726 & $\mathrm{~d} \mathbf{I T , 0 0 8}$ & 3,726 & IIJ,03: \\
\hline Tous Paw & - & - & 0,726 & 117,038 & 195,498 & $7,005,971$ \\
\hline 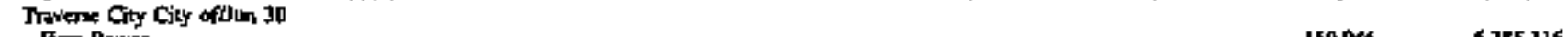 & - & - & - & - & 150,946 & $5,355, J] 6$ \\
\hline Nonfirm Power ..........-1_..................... & - & - & - & - & 39,034 & 140,281 \\
\hline Tokd Powre & - & - & - & - & $20 \% 980$ & $6,874,397$ \\
\hline \multicolumn{7}{|l|}{ Wyandowe Meniripal Serr CamnSep 30} \\
\hline 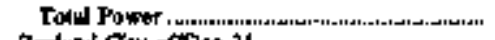 & - & - & - & $\overline{-}$ & 35,846 & $1,108,433$ \\
\hline & & & & & & \\
\hline 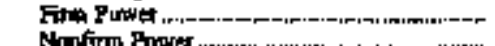 & - & - & - & - & 79,484 & $3,064,355$ \\
\hline 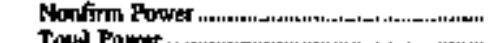 & - & - & - & - & N28 482 & $1,663.428$ \\
\hline & - & - & - & $-m$ & 150,066 & 4.727.m \\
\hline \multicolumn{7}{|l|}{ Dhineiglo } \\
\hline 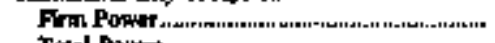 & - & - & - & - & $19 \$, 316$ & 4,941,934 \\
\hline \multicolumn{2}{|l|}{ Asookn City of Mer 31} & $\rightarrow$ & - & - & 193,316 & 4,91],934 \\
\hline 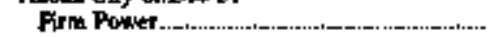 & - & - & - & - & 218,304 & $8.894,813$ \\
\hline \multirow{2}{*}{\multicolumn{4}{|c|}{ Autin Cidy of }} & - & 218,304 & $8,94,813$ \\
\hline & - & - & - & - & 262,556 & $14,5] 7,361$ \\
\hline & - & - & - & - & 262,556 & 14,597361 \\
\hline Grainend CHy ofd ec 31 & - & - & - & - & 199428 & $3,275,361$ \\
\hline 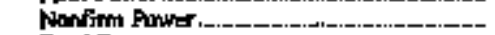 & - & - & - & -52994 & & -3299 \\
\hline 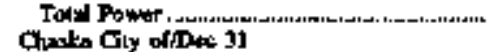 & \multirow{2}{*}{\multicolumn{6}{|c|}{ Charks Gly of Thec 31}} \\
\hline Proves Gly of & - & - & - & & & \\
\hline Jod Path & - & - & - & $=$ & 167,516 & $6,7+4,145$ \\
\hline \multicolumn{7}{|l|}{ 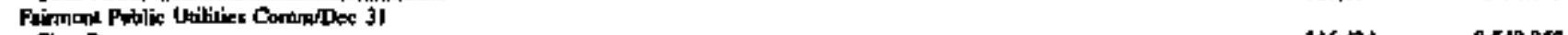 } \\
\hline 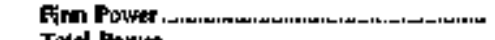 & - & - & - & - & 146,434 & $8,348, B 50$ \\
\hline \multicolumn{7}{|l|}{ 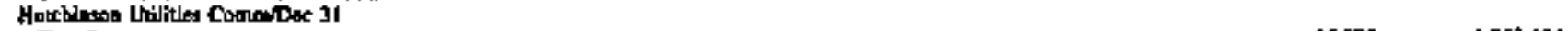 } \\
\hline 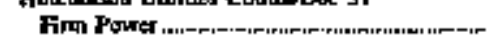 & $=$ & - & - & - & 25.096 & $3,96,3,624$ \\
\hline 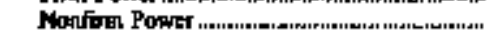 & 77,036 & $1,038,450$ & - & - & 182,289 & $2,745,343$ \\
\hline 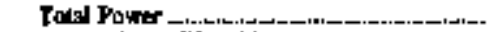 & 77,036 & $1, \square$, $8,4,4$ & - & - & $2 m .487$ & $4,769,367$ \\
\hline \multicolumn{7}{|l|}{ Mentod Ciny ofisap 30} \\
\hline 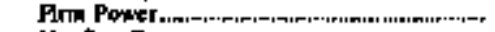 & - & - & - & - & 363,569 & $8 \mathrm{AID,756}$ \\
\hline 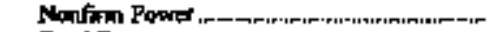 & - & - & 65t & 31,262 & & 31,852 \\
\hline 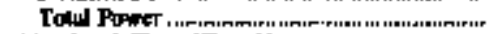 & - & - & GSI & 31,862 & 364,220 & 8,742616 \\
\hline Moortived Cty of Dec J1 & & & & & & \\
\hline 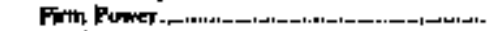 & - & - & - & - & 355.608 & $7,8 \pm 3,818$ \\
\hline 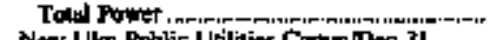 & - & $\rightarrow$ & - & - & 355,628 & 1.843818 \\
\hline New Ulm Poblic Utilaies Conm/Der 31 & & & & & & \\
\hline 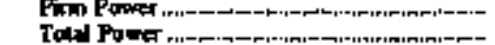 & $\bar{z}$ & $\overline{-}$ & $\bar{z}$ & $\bar{z}$ & 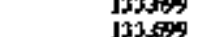 & $3,100,340$ \\
\hline Dwatonn City offoes 3$]$ & & & & & & \\
\hline Fard Power _.... & - & - & - & - & $\mathbf{2 6 4 , 4 0 1}$ & $4,478,513$ \\
\hline 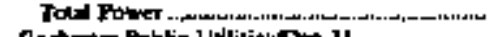 & - & - & - & - & $\mathbf{2 6 4 , 4 0 3}$ & $14,478,513$ \\
\hline 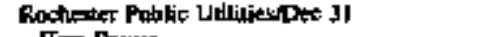 & & & & & & \\
\hline 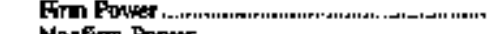 & - & - & - & - & 917,571 & 49594215 \\
\hline 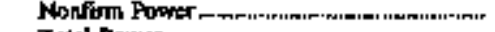 & $\ddot{z}$ & $\rightarrow$ & - & - & 1586 & 102,345 \\
\hline 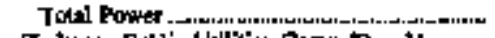 & - & - & - & - & 919,057 & $40,6 \times 6,6 \times 3)$ \\
\hline 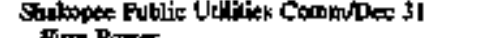 & & & & & & \\
\hline Fimn Power -...., & - $n$ & - & - & - & 175.894 & $3,533,579$ \\
\hline Nonfirn Powte & $9,0,03$ & 47,537 & - & - & 9,0 & $42.53 ?$ \\
\hline 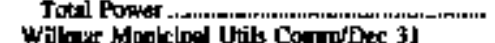 & 9,821 & $4 n \leq 9 t$ & - & - & J45. 的了 & $6,026,116$ \\
\hline 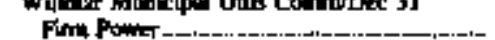 & - & - & - & - & $168,9] 6$ & $4.165,754$ \\
\hline 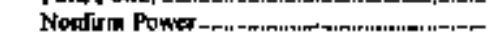 & - & - & - & $\pi$ & 17,600 & 418,452 \\
\hline | & - & - & - & - & 186,516 & 4384,206 \\
\hline
\end{tabular}

See noter und focotondex at and of luble. 
Table 29. Electricity Parchases by Mnnikipal Utilitles, Fiscal Year 1994 (Conthued)

\begin{tabular}{|c|c|c|c|c|c|c|}
\hline \multirow{3}{*}{ 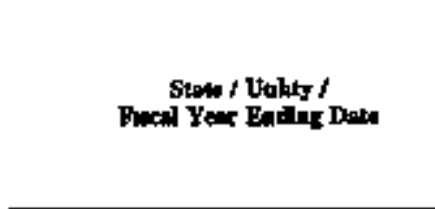 } & \multicolumn{6}{|c|}{ Senofs of Electintity } \\
\hline & \multicolumn{2}{|c|}{ Inwetor Owind } & \multicolumn{2}{|c|}{ Futaral } & \multicolumn{2}{|c|}{$\begin{array}{l}\text { stine and Orher } \\
\text { Govammenil }\end{array}$} \\
\hline & 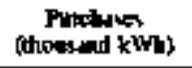 & $\underset{\text { Collent }}{\text { Cort }}$ & 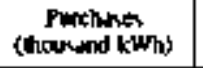 & Cold & 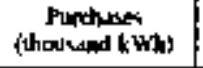 & $\begin{array}{c}\text { Cont } \\
\text { (dollaris) }\end{array}$ \\
\hline \multicolumn{7}{|l|}{ 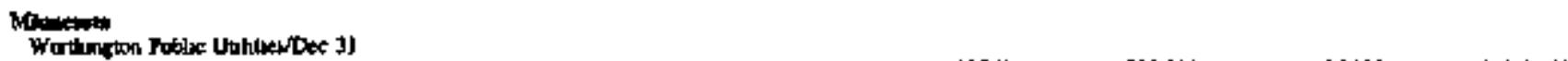 } \\
\hline 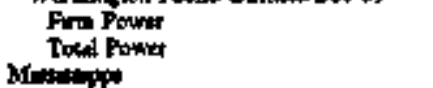 & 二 & $\overline{-}$ & $\begin{array}{l}56,758 \\
56,75 B\end{array}$ & $\begin{array}{l}71,221 \\
71,221\end{array}$ & $\begin{array}{l}86,902 \\
86,932\end{array}$ & $\begin{array}{l}3,689,393 \\
3,5893,383\end{array}$ \\
\hline \multicolumn{7}{|l|}{ 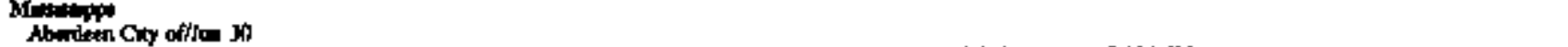 } \\
\hline Fim Power & $=$ & $=$ & 172.139 & $\begin{array}{l}3,204,593 \\
3,20+593\end{array}$ & - & $\approx$ \\
\hline Qhothdile Ciry offiep 30 & & - & $D_{4,1}$ & & & \\
\hline Fing Pount & - & - & - & - & - & - \\
\hline \multirow{2}{*}{\multicolumn{7}{|c|}{ 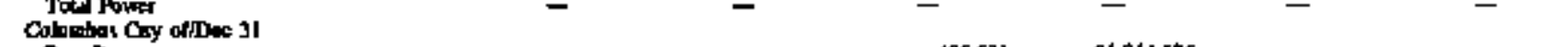 }} \\
\hline & - & - & $485,53\}$ & 21.244555 & - & \\
\hline Totel Pawы & - & - & $4 R 5 \times\}$ & 2124585 & $\overline{-}$ & - \\
\hline \multicolumn{7}{|l|}{ 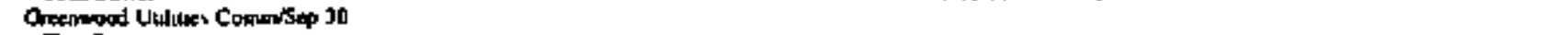 } \\
\hline Fin Powa & - & 一 & - & - & - & - \\
\hline \multicolumn{7}{|l|}{ Holly spand Chy ofjun 30} \\
\hline Nonfing Power & - & $\rightarrow$ & 223,162 & 9,758218 & - & - \\
\hline 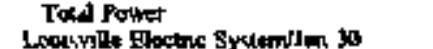 & - & - & 223,162 & 9758218 & - & - \\
\hline Frin Power & - & - & 165,221 & 3,059534 & سـ & - \\
\hline Tous Power & - & - & 165,221 & $3,069,534$ & - & - \\
\hline \multicolumn{7}{|l|}{ 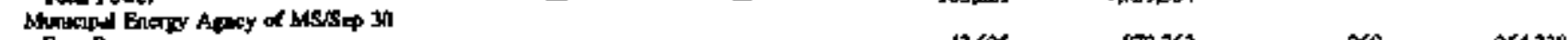 } \\
\hline $\begin{array}{l}\text { Prm Power } \\
\text { Nonfirm Powat }\end{array}$ & $\bar{z}$ & $\bar{z}$ & $-4 \sqrt{632}$ & ${ }^{872762}$ & 260 & $-254,318$ \\
\hline [oAls Power & 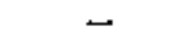 & 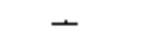 & 43,625 & 872,762 & 2 的 & 254338 \\
\hline \multicolumn{7}{|l|}{ Nes Abuy Cuy offine 30 } \\
\hline Prim Ponver & - & - & 230,531 & $11,390,567$ & - & - \\
\hline \multirow{2}{*}{\multicolumn{7}{|c|}{ OxGord City ofjon yb }} \\
\hline & & & & & & \\
\hline Totel Fowes & - & - & $\begin{array}{l}159,189 \\
158,185\end{array}$ & $2,11,+21$ & $=$ & $\overline{-}$ \\
\hline \multicolumn{7}{|l|}{ sutimle Cuy olunn 30} \\
\hline $\begin{array}{l}\text { Fim Powr } \\
\text { Todd Power }\end{array}$ & - & - & Mas, 169 & $14,511,449$ & - & - \\
\hline \multicolumn{7}{|l|}{ Topelo City offun 30} \\
\hline End Powar & - & - & 656,302 & $2 \neq, 630.984$ & - & - \\
\hline $\begin{array}{l}\text { Toul Powk } \\
\text { Wot Pont Cuy a fildm } 30\end{array}$ & - & - & 656,302 & $28,630,944$ & - & - \\
\hline \multicolumn{7}{|l|}{ West Point Cuy afinm 30} \\
\hline $\begin{array}{l}\text { Fin Poner } \\
\text { Tots Power }\end{array}$ & $\bar{z}$ & $\bar{z}$ & $\begin{array}{l}591,513 \\
191,515\end{array}$ & $8,300,942$ & $\bar{z}$ & $\bar{z}$ \\
\hline Mnoman & & & & & & \\
\hline 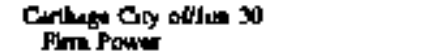 & & & & & & \\
\hline $\begin{array}{l}\text { Pim Paw } \\
\text { Noefirm Power }\end{array}$ & - & - & 22,636 & TS 3 B & 一 & - \\
\hline $\begin{array}{l}\text { Nooffrm lower } \\
\text { Toted Power }\end{array}$ & 二 & 二 & - 22,636 & -47878 & I,In] & 21,730 \\
\hline Cotempen Coy offing 30 & & & & & & \\
\hline Fim Porrer & 122045 & $12,615,1 / 2$ & - & - & - & - \\
\hline Nenfim Poner & & & - & - & - & - \\
\hline Totd Pawer & 22298 & $12,015,112$ & - & - & - & - \\
\hline Hernibul Cry oflon 30 & & & & & & \\
\hline Fim Pawer & 995,6] & $2,634,605$ & - & - & - & - \\
\hline Totd Power & 195.671 & $76,34,605$ & سـ & - & - & - \\
\hline 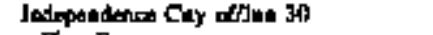 & & & & & & \\
\hline Fimp Pown & 744,351 & $18,687,570$ & - & - & - & - \\
\hline Nonfimm Mows & 34,252 & B34,564 & - & - & - & - \\
\hline Tatd Pown & $n 8,(n)$ & $19.521,134$ & - & - & - & - \\
\hline Kmont Cuy ofitun 30 & & & & & & \\
\hline Nontim Power & - & - & $29,24 !$ & $55,3,964$ & - & - \\
\hline Total Power & - & - & 29,244 & 553,964 & - & - \\
\hline Kitkmood Giy ofm- 31 & & & & & & \\
\hline Fim Power & 783,145 & $7,532.256$ & - & $\bar{z}$ & $\overline{-}$ & $\bar{z}$ \\
\hline 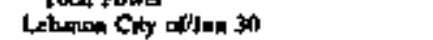 & & & & & & \\
\hline Fra Power & - & - & - & - & - & - \\
\hline Total & - & - & - & - & $=$ & - \\
\hline
\end{tabular}


Table 25. Electricity Purchases by Municipal Utitities, Fiscel Year 1994 (Continued)

\begin{tabular}{|c|c|c|c|c|c|c|}
\hline \multirow{3}{*}{ 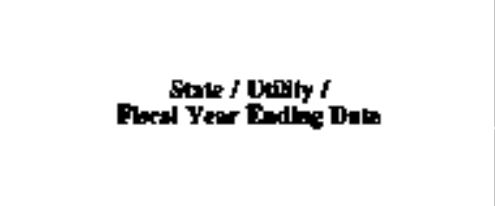 } & \multicolumn{6}{|c|}{ Somer Elativily } \\
\hline & \multicolumn{2}{|c|}{ Cooperntive } & \multicolumn{2}{|c|}{ onst } & \multicolumn{2}{|c|}{ Towil } \\
\hline & 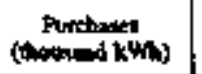 & $\underset{\text { (dotlens) }}{\text { Can }}$ & 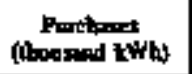 & Cod & (thosidand k'th) & $\underset{\text { (dollari })}{C}$ \\
\hline \multicolumn{7}{|l|}{ Milmenten } \\
\hline Fim Pothe & - & - & - & $一$ & 10,000 & $4,420,604$ \\
\hline Toat Wow & - & - & - & - & 140.690 & $4,420,604$ \\
\hline \multicolumn{7}{|l|}{ 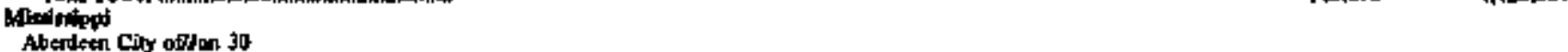 } \\
\hline Pm Power . & - & - & - & - & $172 \sqrt{135}$ & $7,204,593$ \\
\hline 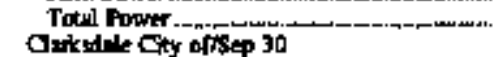 & - & - & - & - & 172,139 & $7,204,593$ \\
\hline Fint Ponef & - & - & 191,074 & $6,729,087$ & I91,007 & $6,329,887$ \\
\hline Total Purver & - & - & 191,074 & 6,329,087 & 191,074 & $6,329,887$ \\
\hline \multicolumn{7}{|l|}{ Colunber Cuy of Des 31} \\
\hline 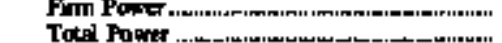 & 二 & $\vec{z}$ & 二 & $\bar{z}$ & $485,69 !$ & $\begin{array}{l}21,241,505 \\
21,244,5104\end{array}$ \\
\hline \multicolumn{7}{|l|}{ 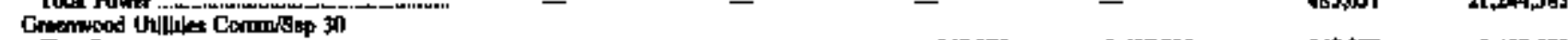 } \\
\hline Finn Power & - & - & 264,370 & $9,497,288$ & 268,370 & $9.497,288$ \\
\hline \multicolumn{4}{|l|}{ Holly Springs Cly offlus 30} & $9,47,288$ & 268,370 & 9,49728 \\
\hline 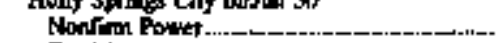 & - & - & - & - & 201,162 & 9758.218 \\
\hline 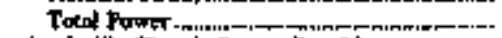 & - & - & - & - & 22,162 & $9.358,218$ \\
\hline 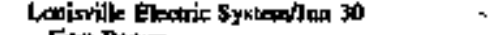 & & & & & & \\
\hline 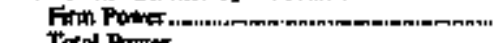 & - & - & - & - & 164.221 & $3, \sqrt{599}, 534$ \\
\hline \multicolumn{7}{|l|}{ Monleipel Energes Apery of Messep 30} \\
\hline 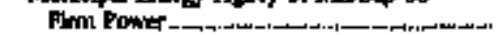 & 299,790 & $13,04,179$ & 120,787 & $4,9: 2,612$ & $\mathbf{4 6 4} \mathbf{4 6 2}$ & 19,149,Bg1 \\
\hline Nonfrith Power wam & & - & josis & $8,45,102$ & $J 2829$ & $\mathrm{~g}, 485, \mathrm{t}, \mathrm{W}$ \\
\hline 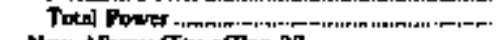 & 209.790 & $13 \Omega 00,179$ & 493.616 & 13.467 .714 & 137291 & $27,53,993$ \\
\hline \multirow{2}{*}{\multicolumn{7}{|c|}{ New Albeny City affor 30}} \\
\hline & - & - & - & - & $200,63)$ & II.79857 \\
\hline 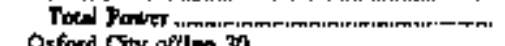 & \multicolumn{6}{|c|}{ Osford City oflJe 30} \\
\hline 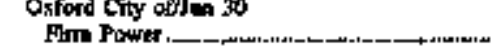 & - & - & - & - & 158. 185 & $7,111,42]$ \\
\hline 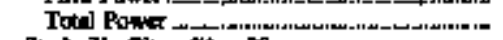 & 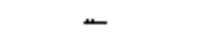 & - & - & - & ISB, 105 & $7,[1], 42 J$ \\
\hline \multicolumn{7}{|l|}{ Statwale City aplan 30} \\
\hline 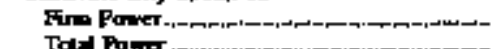 & - & - & - & - & $345, * 63$ & {$[0,5] 1,449$} \\
\hline \multicolumn{7}{|l|}{ Topedo City ofidoe 30} \\
\hline Fin Fork & - & - & - & - & 656,302 & $28,630,094$ \\
\hline Told Paw & - & - & - & - & 656,392 & $28,630,934$ \\
\hline \multicolumn{7}{|l|}{ 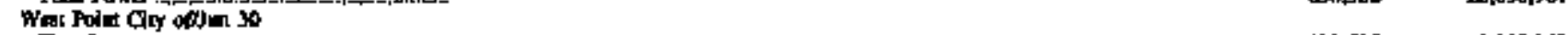 } \\
\hline 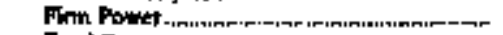 & - & - & - & - & 191 .515 & s,3in,, 42 \\
\hline \multirow{2}{*}{\multicolumn{7}{|c|}{ MEinut }} \\
\hline & & & & & & \\
\hline 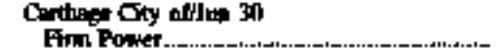 & - & - & & $6.199,330$ & ros.115 & 667118 \\
\hline Monfirm Powteт,_, & - 284: & - 6 재 & 257 & $-199,117$ & 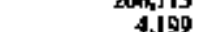 & $b_{-101,678}$ \\
\hline Towes pown & 2,841 & 67,700 & 189,736 & Gonk41? & 210,914 & $6,570,639$ \\
\hline 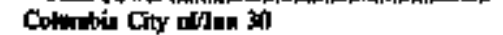 & & & & & & \\
\hline 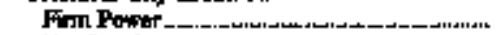 & - & - & 306,740 & $13,649,878$ & 732,725 & $25,6 G, 990$ \\
\hline 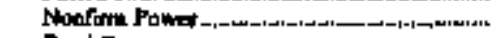 & 355 & 22,617 & 2,471 & 85,396 & 2,826 & $10 \$, 013$ \\
\hline 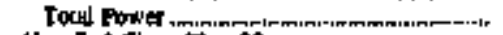 & 391 & 2017 & 312,211 & $13,731,274$ & T,15.551 & 24.7 , \\
\hline Hative City of Non 30 & & & & & & \\
\hline 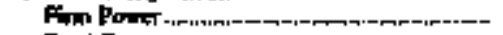 & - & 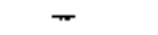 & - & - & 195.871 & $2,631,605$ \\
\hline Tolut Foper & - & - & - & - & |95,67] & $7,634,606$ \\
\hline Fin Pown & - & - & - & - & 744,251 & 18.687.078 \\
\hline Nooflatil Pawn & 23 & t,0) & - & - & 30,273 & 835,162 \\
\hline 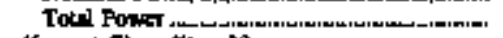 & 2] & $1,0 \% 8$ & 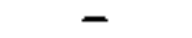 & - & $\pi 6,626$ & $19,522,532$ \\
\hline Komet Chy offJum 30 & & & & & & \\
\hline 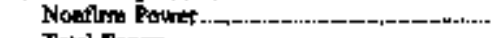 & - & $\rightarrow$ & 536,827 & $2,195,219$ & 156,0601 & $2,749,189$ \\
\hline 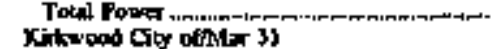 & $\pi$ & $=$ & 126,22 & $2,195,219$ & 136,063 & 2749,183 \\
\hline Fon Power & 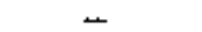 & ـ & - & - & 180,145 & 7.532256 \\
\hline Total Dow & - & - & - & - & 189.145 & $7,532.256$ \\
\hline 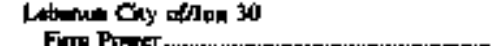 & $2 \times 3000$ & 6557899 & - & - & 181000 & 6.057 .800 \\
\hline 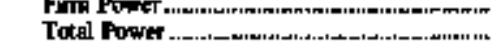 & 183010 & 6.857 .69 & - & - & 1890001 & $6,657,09$ \\
\hline
\end{tabular}

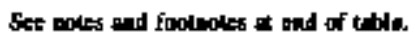


Table 29. Electricky Porchases by Monicipal Utilities, Fiscal Year 1994 (Continned)

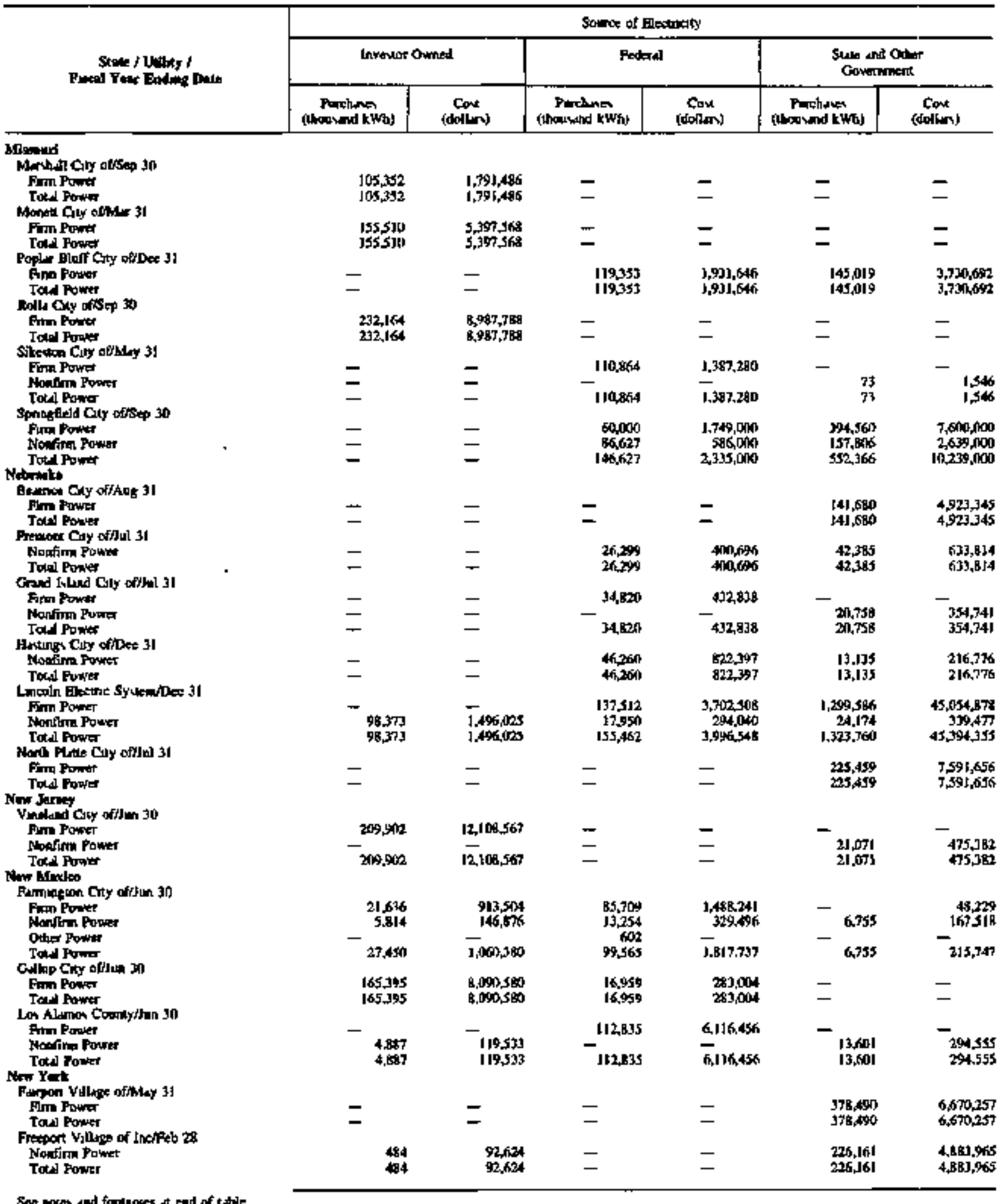


Table 29. Electricity Purchases by Manicipal Utilities, Fiscal Yenr 1994 (Continued)

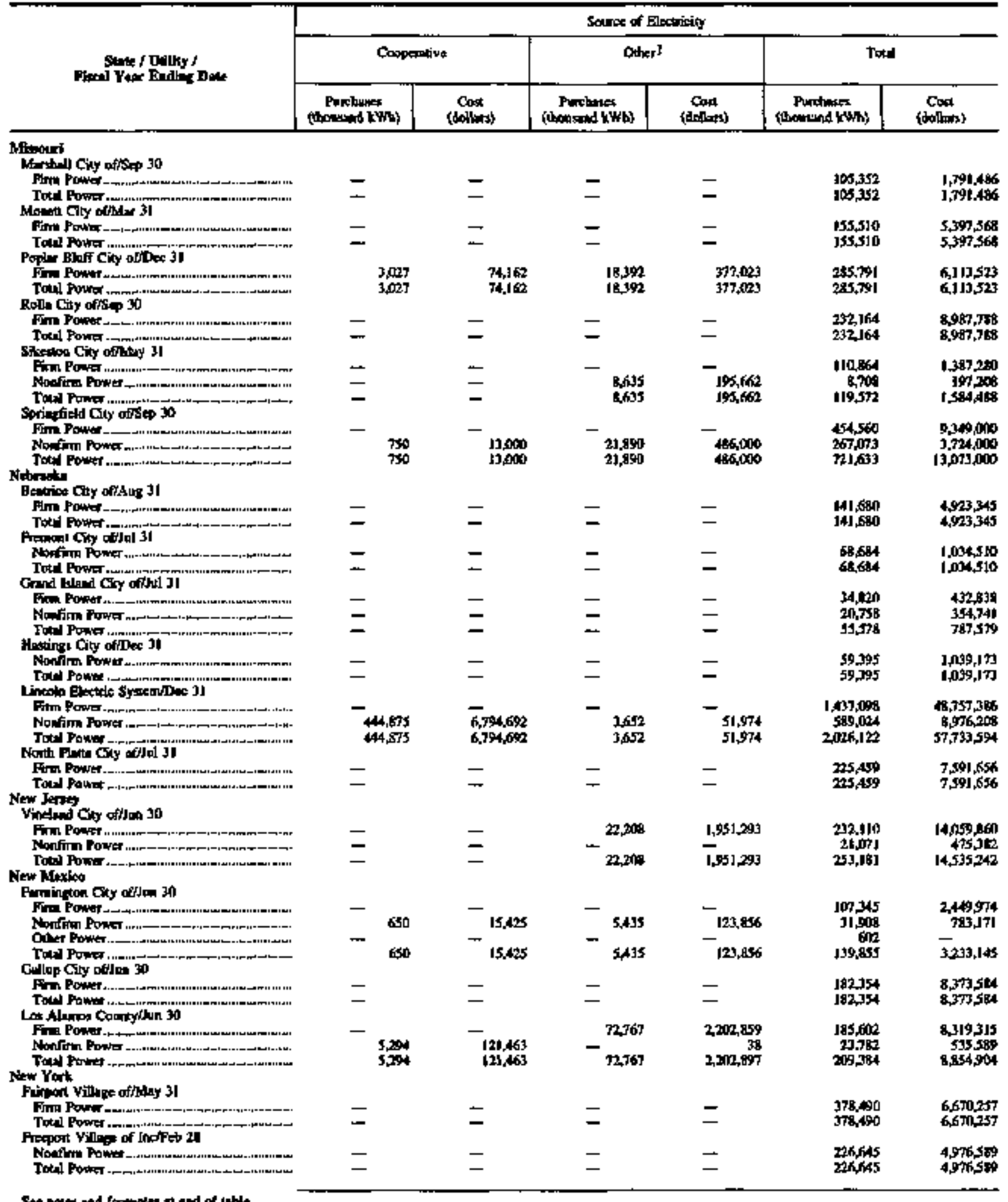

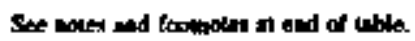


Table 29. Electricity Purchases by Municipal Utitities, Fiscal Year 1994 (Continued)

\begin{tabular}{|c|c|c|c|c|c|c|}
\hline \multirow{3}{*}{ 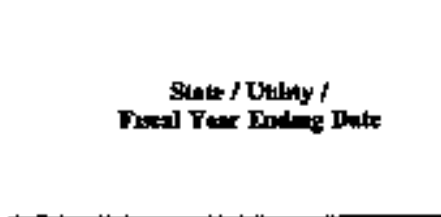 } & \multicolumn{6}{|c|}{ Soluce of Elowicaly } \\
\hline & \multicolumn{2}{|c|}{ Invertor Oromed } & \multicolumn{2}{|c|}{ Pederal } & \multicolumn{2}{|c|}{ Sitte ind Daher } \\
\hline & $\begin{array}{l}\text { Punthines } \\
\text { (lwoulund lWh) }\end{array}$ & (dalont & $\begin{array}{l}\text { Purtang } \\
\text { (thourind kith) }\end{array}$ & $\underset{\text { Cont }}{\operatorname{Con}}$ & 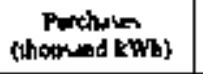 & (10) \\
\hline \multicolumn{7}{|l|}{ New Yat } \\
\hline Find Power & - & - & $m$ & - & $461,2,7$ & 5571,288 \\
\hline $\begin{array}{l}\text { Toul fower } \\
\text { Let Plect Viluge logthog } 30\end{array}$ & - & - & - & - & $46-1,767$ & $5,571,028$ \\
\hline Find Pown & - & - & - & - & 13,462 & $2,220,19]$ \\
\hline Total Power & - & - & - & - & $1,9,362$ & $2,226,197$ \\
\hline \multicolumn{7}{|l|}{ Dewend Town offDec 3 ) } \\
\hline Find Ponting Power & $-13,84 \pi$ & $\begin{array}{l}506,785 \\
152,796\end{array}$ & - & - & $-^{103,335}$ & $1,334,299$ \\
\hline Toul Powar & $11,5 \mathrm{~m}$ & $650 \leq 51$ & - & - & 1]93535 & 1,34299 \\
\hline \multicolumn{7}{|l|}{ 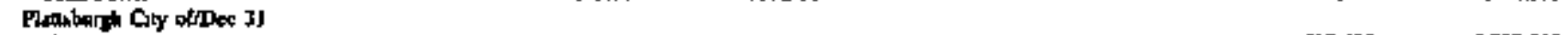 } \\
\hline Fint Fower & - & - & - & - & 5j9,4,999 & $5,737,2,20$ \\
\hline \multirow{2}{*}{\multicolumn{6}{|c|}{ Racknlle Centre Vildage offulay 31}} & 5.790 .020 \\
\hline Nonflion power & - & & & & & \\
\hline Todd Power & - & $\overline{-}$ & ב & $\overline{-}$ & 161,909 & $\begin{array}{l}3,740,730 \\
3,742,736\end{array}$ \\
\hline \multicolumn{7}{|l|}{ Soluy Villeze offldsy al } \\
\hline $\begin{array}{l}\text { Noiflin pown } \\
\text { Toty Powir }\end{array}$ & - & - & - & - & J51, 174 & $20,03 / 313$ \\
\hline 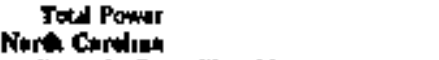 & - & - & - & - & $153, \sqrt{6} \mathrm{~T} 4$ & $2 \pi 03013$ \\
\hline \multicolumn{7}{|l|}{ Norit Cordich ofjon 30} \\
\hline Fim Potider & - & - & - & - & - & - \\
\hline Jad Pow & - & - & - & - & - & - \\
\hline 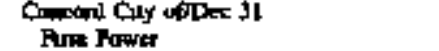 & $\$ 21.849$ & $19,000,691$ & 3,165 & 288.971 & - & - \\
\hline Tad Power & 421,899 & $19,000,691$ & 3,163 & $289,97]$ & ב. & - \\
\hline \multicolumn{7}{|l|}{ Euribeth Cry Coy offen 30} \\
\hline Fim Pown & - & - & 2,606 & 107,126 & - & - \\
\hline \multirow{2}{*}{\multicolumn{7}{|c|}{ Puyetorille Puble Wouk, Commilun 3 B }} \\
\hline & & & & & & \\
\hline Fim Poover & $1,431,127$ & $65,594,764$ & 4,06] & 236,797 & - & - \\
\hline Told Power & $1,433,027$ & $65,504,764$ & 4.061 & 236,797 & - & - \\
\hline \multicolumn{7}{|l|}{ Forn: Culy Town ofllat 30} \\
\hline Fim Power & 149,597 & $6,090,2 \$ 2)$ & 1,112 & 90,507 & - & - \\
\hline Totd Power & 149.697 & $6,(190,252$ & $3,1 / 2$ & 98527 & - & - \\
\hline \multicolumn{7}{|l|}{ Gevorde Cuy uefJun 30} \\
\hline Pim Porra & - & - & 6,129 & 509,927 & - & - \\
\hline Totd Pawer & - & - & 6,129 & 56.527 & - & - \\
\hline 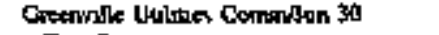 & & & & & & \\
\hline Find Powe & - & - & - & - & - & - \\
\hline Tot.l Pown & - & - & - & - & - & - \\
\hline 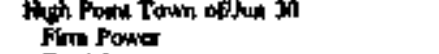 & - & - & - & - & - & - \\
\hline Total Powna & - & - & - & - & - & - \\
\hline Kmton Gry oflie 10 & & & & & & \\
\hline Fin Power & - & - & $9 \%$ & 60,933 & - & - \\
\hline Tot.1 Powtr & - & - & 996 & 63,933 & - & - \\
\hline Lexugtion Chy ofmon 30 & & & & & & \\
\hline Find Powner & - & - & ع & - & - & - \\
\hline Totsl Power & عـ & - & 一 & - & $=$ & - \\
\hline 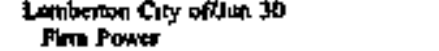 & - & - & 64to & $39,0 \mathrm{Ad}$ & - & - \\
\hline Tocst Pownt & - & - & 6hs & 39.104 & - & - \\
\hline Hoane Cily offlom 30 & & & & & & \\
\hline Find Poner & - & - & 2,953 & 314,307 & - & - \\
\hline Tord Power & - & - & 2953 & $3 \mathrm{~J} 4,307$ & - & - \\
\hline blotgman Ciry otjun 30 & & & & & & \\
\hline Find Power & - & - & 13,361 & 56,695 & - & - \\
\hline Toud Power & - & - & 13.361 & SQSAS & - & - \\
\hline 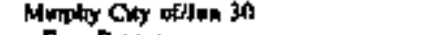 & & & & & & \\
\hline Fongen Poner & - & سـ & 139,887 & $5,584,479$ & - & - \\
\hline Tous Powr & - & - & 133,887 & $1,184,479$ & - & - \\
\hline 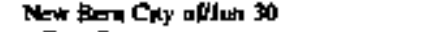 & & & & & & \\
\hline Furm Powur & - & $\overline{-}$ & 901 & 52,656 & $\overline{-}$ & $\overline{-}$ \\
\hline Jutil Pिw्न & - & - & 9्र1 & 52,656 & - & - \\
\hline
\end{tabular}

See colvh and foomoten st exd of thitp 
Table 29. Electricity Purchases by Muatcipal Utiltiss, Fiscal Year 1994 (Conthued)

\begin{tabular}{|c|c|c|c|c|c|c|}
\hline \multirow{3}{*}{ 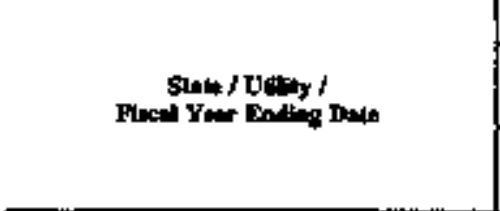 } & \multicolumn{6}{|c|}{ 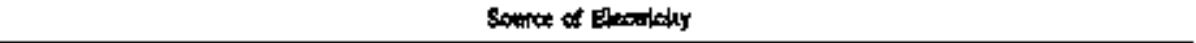 } \\
\hline & \multicolumn{2}{|c|}{ Cocperative } & \multicolumn{2}{|c|}{ Otha' } & \multicolumn{2}{|c|}{ Fold } \\
\hline & $\begin{array}{l}\text { Punsturex } \\
\text { (thoosond KWh) }\end{array}$ & $\underset{\text { (dot:lims) }}{\text { Cout }}$ & 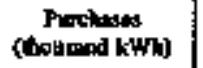 & 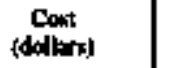 & $\begin{array}{l}\text { Purchosen } \\
\text { (uloorand kwhy }\end{array}$ & $\underset{\text { (dollors) }}{\text { Con }}$ \\
\hline \multicolumn{7}{|l|}{$\begin{array}{l}\text { New Yoct } \\
\text { Jerustown City ofites } 31\end{array}$} \\
\hline Fint Powst & - & - & _- & - & 461,367 & $5,571,028$ \\
\hline 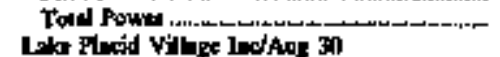 & - & - & - & - & 461,767 & $5,571,028$ \\
\hline Fim Pow & - & س & - & - & 199,362 & $2,228,197$ \\
\hline 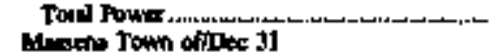 & - & - & - & - & 139,362 & $2,228,197$ \\
\hline frit Pouver & - & - & - & - & 151,942 & $1, \mathbb{1 d 1 , 0 8 4}$ \\
\hline 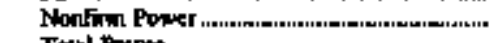 & - & - & 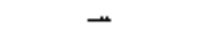 & - & - & $1527 \%$ \\
\hline 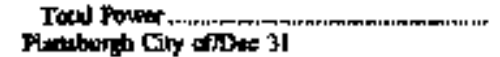 & - & - & - & - & 151,942 & $1,999,880$ \\
\hline 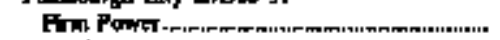 & - & - & - & - & 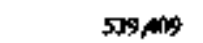 & S,7) \\
\hline 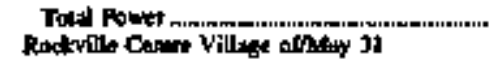 & - & - & - & - & 59940 & $5,737,800$ \\
\hline 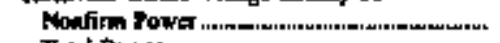 & - & - & - & - & J65,903 & $3,740,736$ \\
\hline & - & - & - & - & 163509 & $3,740,7,46$ \\
\hline 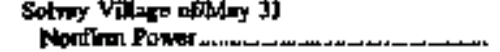 & - & - & - & - & $153, \sqrt{5} 44$ & 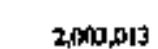 \\
\hline Toenl Power. & - & - & - & - & $15 j, 6,44$ & 2,00013 \\
\hline 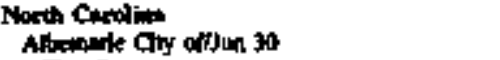 & & & & & & \\
\hline 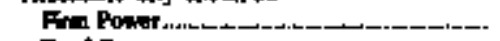 & - & - & $27 T, 113$ & $15,484,212$ & 237,113 & $15,46,212$ \\
\hline 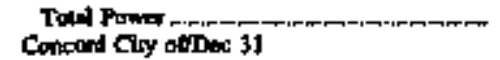 & - & - & 27,113 & $15,484,212$ & 207,113 & $15, A B 4212$ \\
\hline 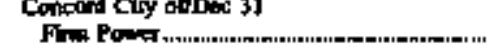 & - & - & - & - & 25,0602 & $19,292,662$ \\
\hline 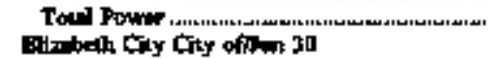 & - & - & - & - & 45,0062 & $19,792,562$ \\
\hline Plo Ponn & - & - & 251,352 & [8, RQ1,5,54 & 253,958 & $38,125,696$ \\
\hline 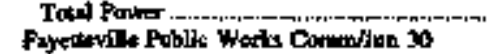 & - & - & 251,352 & $18,021,30$ & 253,968 & $18,128,596$ \\
\hline Fin Poth & - & - & - & - & 1,487,188 & 65,831, 61 \\
\hline 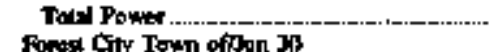 & - & - & - & - & $1 A J 7,188$ & $65,831,361$ \\
\hline 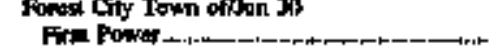 & - & $\pi$ & - & - & 150,609 & $0,1 E, T 79$ \\
\hline 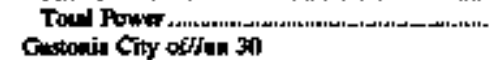 & - & - & - & - & $130,801,9$ & $6,189,7] 9$ \\
\hline Fine Powner___- & - & - & 624,427 & $30,67,815$ & $6 \times 0,556$ & $31,732.34$ \\
\hline 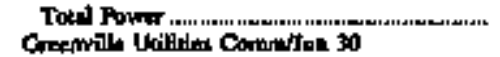 & $\rightarrow$ & - & 621427 & 30,47815 & 69,556 & $31,032,742$ \\
\hline Pmo Pown & - & - & $1.9 \pm 9.000$ & $n, m, 220$ & $1,159,000$ & $77,727,220$ \\
\hline Tord Porwer.-- & - & - & 1.189000 & $7,27,220$ & $1,139,000$ & $77,727.220$ \\
\hline 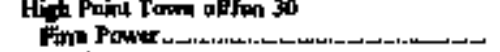 & - & - & g(64;3) & 47, X010, 倠: & 854,3] ] & $7 \pi, 308601$ \\
\hline Tow Powt & - & - & $\operatorname{si4313}$ & $4 \mathrm{~J}_{,}, \mathrm{JOA}, 60 \mathrm{~J}$ & 854,31] & 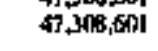 \\
\hline Einotol Chy oflun 30 & & & & & & \\
\hline 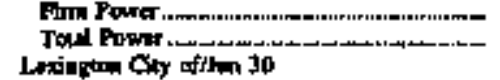 & $\bar{z}$ & $\bar{z}$ & 459,454 & $\begin{array}{l}30,505,027 \\
30,516,427\end{array}$ & 460,450 & $\begin{array}{l}30,569,355 \\
30,549,355\end{array}$ \\
\hline 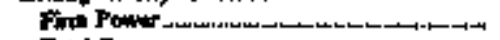 & - & - & 464,713 & $26,820,844$ & $46,7,7] 3$ & $20,890,844$ \\
\hline Thenl Poones. & - & - & 464,713 & $26,020,844$ & $46,7] 3$ & $26,832,144$ \\
\hline Finh pow & - & - & 2A3,365 & $19,4^{3} 4,252$ & 2014,034 & $19,473,306$ \\
\hline 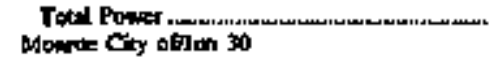 & - & - & 203,365 & 10,0425 & 284,014 & $19,473,306 \mathrm{~F}$ \\
\hline F & - & - & $\$ 27,156$ & $21,476,40$ & $4+0,09$ & $21,390,747$ \\
\hline 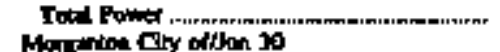 & - & - & 427,816 & $21,476,4 \times 0$ & 430, BNA & $25,390,747$ \\
\hline Monfonion Cly ofthan 10 & - & & & & & \\
\hline 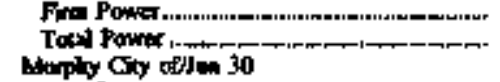 & - & - & 287,444 & 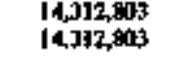 & $\begin{array}{l}300,809 \\
300,005\end{array}$ & $\begin{array}{l}14,7] 1,501 \\
14, \pi 1,501\end{array}$ \\
\hline 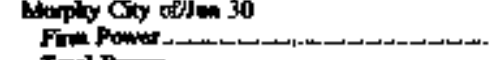 & - & - & - & - & $133, \$ 87$ & $5,584,479$ \\
\hline 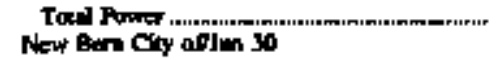 & - & - & - & - & $\mathbf{1 5 3 , 8 3 7}$ & $5,584,479$ \\
\hline 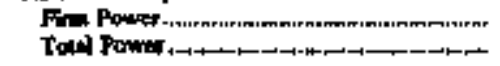 & $\dot{-}$ & $\overline{-}$ & $\begin{array}{l}381,192 \\
311,492\end{array}$ & $\begin{array}{l}27,192,329 \\
27,192,289\end{array}$ & $\begin{array}{l}382,393 \\
362,390\end{array}$ & $\begin{array}{l}27,244,885 \\
2724, \mathrm{BBS}\end{array}$ \\
\hline
\end{tabular}

Sal notes wod footuotes at end of thtle. 
Tanle 29. Electricizy Porchases by Monicipal Utilities, Ftecal Year 1994 (Continned)

\begin{tabular}{|c|c|c|c|c|c|c|}
\hline \multirow{3}{*}{ 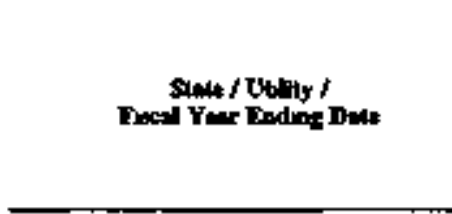 } & \multicolumn{6}{|c|}{ Sorpस्म of electricity } \\
\hline & \multicolumn{2}{|c|}{ Imestur Doned } & \multicolumn{2}{|c|}{ Foderd } & \multicolumn{2}{|c|}{$\begin{array}{l}\text { Stiv and othe } \\
\text { Govenmenk }\end{array}$} \\
\hline & $\begin{array}{l}\text { Prohdwh } \\
\text { (llousend } \mathrm{km} \text { ) }\end{array}$ & (douldory & 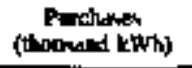 & $\begin{array}{c}\text { Con } \\
\text { (dollin) }\end{array}$ & $\begin{array}{l}\text { Portures } \\
\text { (lloocund kwh) }\end{array}$ & $\begin{array}{c}\text { Cost } \\
\text { (galles) }\end{array}$ \\
\hline \multicolumn{7}{|l|}{ North Cembang } \\
\hline Fin Pong Dar & $1,91,3,104$ & $107,480,000$ & - & - & 195.258 & $127+5000$ \\
\hline Nonfins Power & & 11.000 & - & - & - & - \\
\hline Tous fower & $1.971, \mathrm{MH}$ & $107,491,0001$ & - & - & 195,258 & $12,728,1500$ \\
\hline Fre Pow & $2,377,096$ & $117,922,000$ & - & - & - & $=$ \\
\hline $\begin{array}{l}\text { Tats Poocr } \\
\text { Racky Moow Cty atila } 30\end{array}$ & 2.377098 & i17,8=8000 & - & - & - & - \\
\hline Fin Power & - & $\rightarrow$ & - & 130,654 & - & - \\
\hline Noufinm Apwar & - & - & - & - & - & - \\
\hline 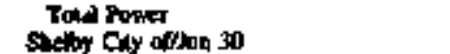 & - & - & - & 110,604 & - & - \\
\hline Fin Poowe & - & - & 2,748 & 206,561 & - & 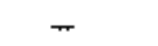 \\
\hline 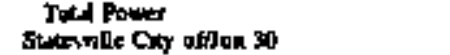 & - & - & 2,248 & 206,51 & - & - \\
\hline Fin Powar & 一 & - & 3.724 & 340,292 & - & - \\
\hline 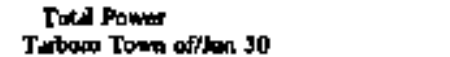 & - & - & 3,724 & 340,297 & $\rightarrow$ & - \\
\hline Fitu Pout & - & - & - & - & - & - \\
\hline 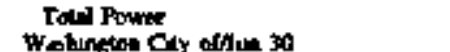 & - & - & - & - & - & - \\
\hline Fint pow & - & - & 2,023 & 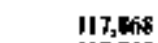 & - & - \\
\hline $\begin{array}{l}\text { Toul Powa } \\
\text { whon Cuy offun } 30\end{array}$ & - & - & 2022 & IIT,Eis & سـ & - \\
\hline Fin Pow & 一 & - & 2,206 & 128,608 & - & - \\
\hline Told how & 一 & - & 2006 & 128,696 & - & - \\
\hline \multicolumn{7}{|l|}{ Alo } \\
\hline Bum Power & $2,850,344$ & 98351,426 & - & - & 534,459 & $3,738,611$ \\
\hline Nothith Pook & $\overline{2} \cos 44$ & $1,779,129$ & $\overline{-}$ & $\overline{-}$ & - & $\overline{1}+0$ \\
\hline Bowleg Ond Cily ofdoct 31 & & & & & Non, & S, \\
\hline Fin Power & - & - & - & - & - & - \\
\hline Total Point & 一 & - & - & - & - & - \\
\hline $\begin{array}{l}\text { Exym Coty ofisop } 30 \\
\text { Nonfin Pow }\end{array}$ & 196 34] & $6,600,895$ & - & - & 5,399 & $9,0 \mathrm{~m}$ \\
\hline Total Pows & $196,4\}$ & $6,600,8 \% 6$ & - & - & 5399 & $\$, 0010$ \\
\hline Clap Cily aftoe 31 & & & & & & \\
\hline Fin Povitr & 149,189 & $5,5,0,0,484$ & - & - & - & - \\
\hline Clovelind Oty ofjen 30 & 149,1685 & $5,930,494$ & - & $=$ & & \\
\hline Nonfiren Power & - & - & - & - & $=$ & - \\
\hline Ioted Porrot & - & - & - & - & - & - \\
\hline 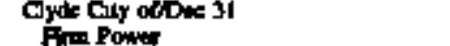 & 136050 & 4381施 & - & - & $=$ & - \\
\hline Totld Powrer & 136,059 & $4,131,227$ & 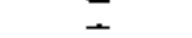 & $=$ & - & $\overline{-}$ \\
\hline Colponber Cay of Des 31 & & & & & & \\
\hline $\begin{array}{l}\text { Frith Power } \\
\text { Nonfion Power }\end{array}$ & 489 & $-_{75,128}$ & - & - & - & - \\
\hline $\begin{array}{l}\text { Nonfian Power } \\
\text { Pods Pown }\end{array}$ & 489 & 75,128 & $=$ & 二 & $=$ & $=$ \\
\hline Coystoon Pulk Cry old il & & & & - & & \\
\hline Fin power & 一 & - & - & 一 & - & - \\
\hline Total Ponver & - & - & 一 & 一 & $m$ & - \\
\hline 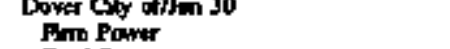 & 53,529 & $1,329,349$ & - & - & 9,327 & 430,719 \\
\hline Tatd Powa & 53,529 & 1279,949 & - & - & 9,727 & 430.719 \\
\hline 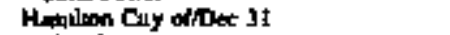 & & & & & & \\
\hline Fin Pown & 4, 1497 & 3.717 .426 & - & $\rightarrow$ & - & 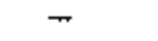 \\
\hline Nonfinm Pond & sing & 56,1900 & - & - & $=$ & $=$ \\
\hline Tasil Poner & $4 \mid, 643$ & $3,773,436$ & - & 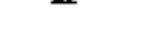 & - & - \\
\hline Nonfing Power & - & 一 & - & - & - & - \\
\hline To.d Pown & - & - & - & - & - & - \\
\hline Now Caty of & - & - & - & - & - & - \\
\hline To:d Power & - & - & - & - & - & - \\
\hline
\end{tabular}


Table 29. Elecirticity Purchases by Municipal Utilities, Fiseal Year 1994 (Contmmed)

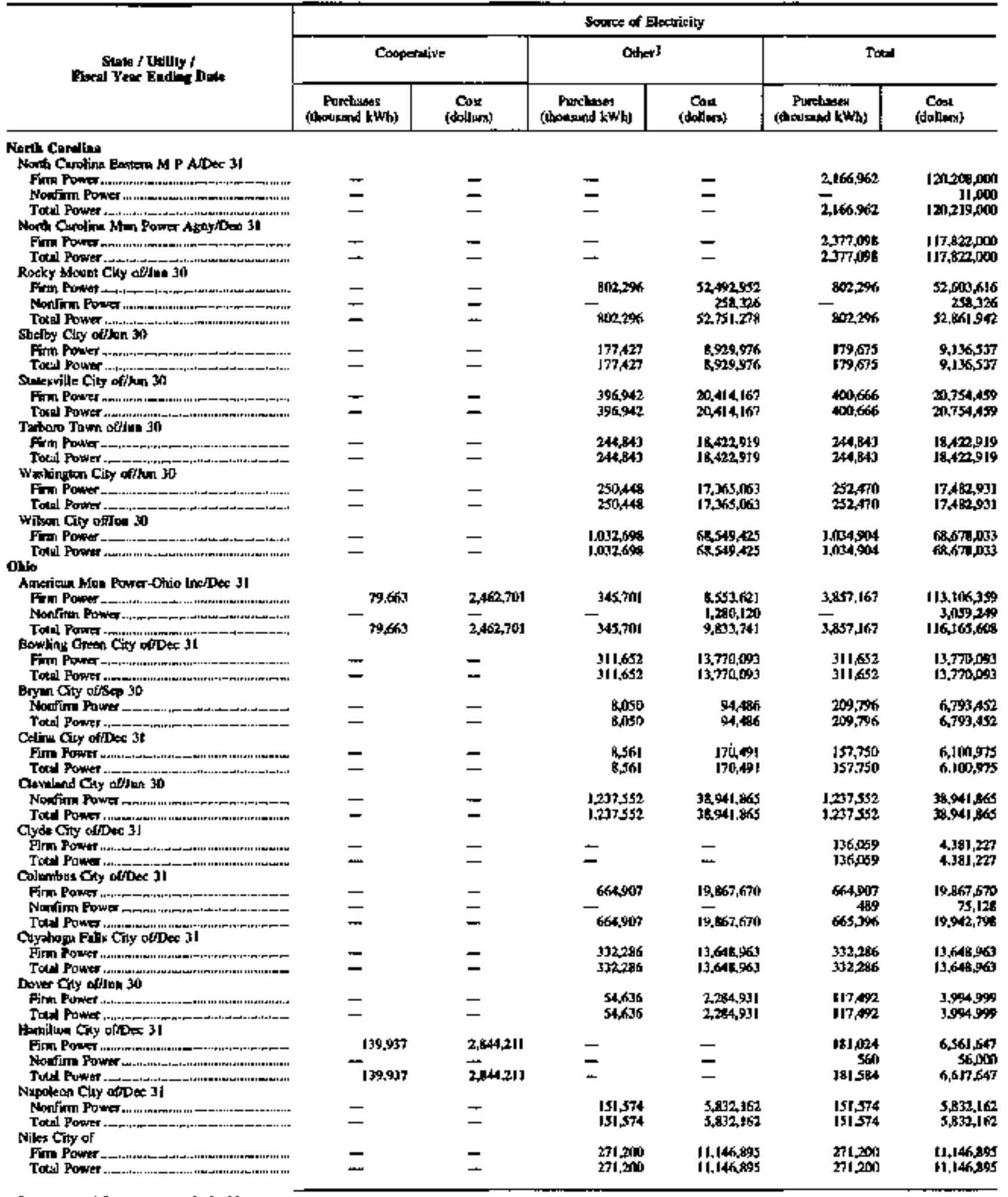

See wotex and foothotes at end of table 
Table 29. Elecirtitity Perchases by Monicipal Utitites, Fiseal Year 1994 (Continued)

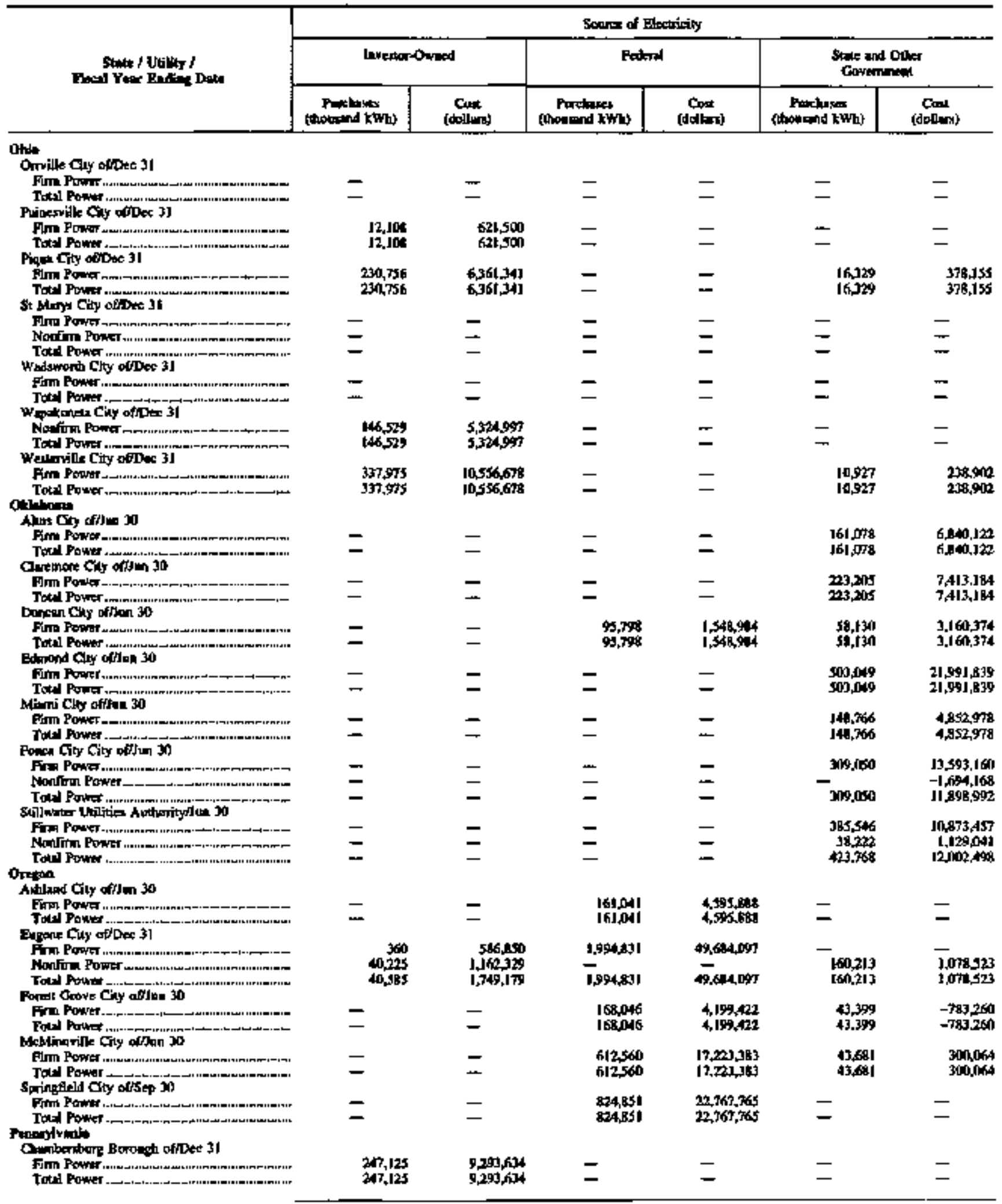

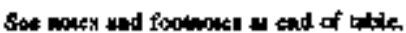


Table 29. Electricity Purchases by Munictpol Utllites, Fiscal Year 1994 (Contimed)

\begin{tabular}{|c|c|c|c|c|c|c|}
\hline \multirow{3}{*}{ Shote f Denlky t } & \multicolumn{6}{|c|}{ Soorse of Electricity } \\
\hline & \multicolumn{2}{|c|}{ Coopentive } & \multicolumn{2}{|c|}{ Oand } & \multicolumn{2}{|c|}{ Toded } \\
\hline & $\begin{array}{l}\text { Purchuwen } \\
\text { (thoosend } \mathbf{k} \text { Wh }\end{array}$ & $\underset{\text { (dolluns) }}{\text { Clost }}$ & $\begin{array}{l}\text { Prochesses } \\
\text { (thoestind kW'b) }\end{array}$ & $\underset{\text { (dollers) }}{\text { Con }}$ & $\begin{array}{l}\text { Porituos: } \\
\text { (Acosend kUth) }\end{array}$ & $\underset{\text { (dollext) }}{\text { Cont }}$ \\
\hline \multicolumn{7}{|l|}{ Oristime Cuy ofDer 31} \\
\hline PArm Pown & - & - & 35,125 & $1,668,400$ & 35, 125 & $1,566,490$ \\
\hline 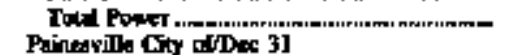 & - & $\rightarrow$ & 35,125 & $1,648,400$ & 35.125 & 1.668 .400 \\
\hline Futh Pown & - & - & 17,609 & 545,906 & 29,777 & $1,167,306$ \\
\hline 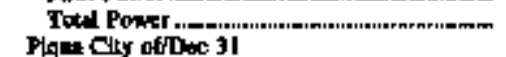 & - & - & 17,609 & 545,906 & $20 m$ & $1,167,306$ \\
\hline Fim Power.......... & - & - & - & - & 247,065 & $6,739,496$ \\
\hline 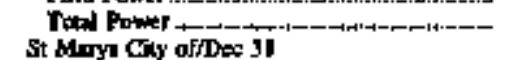 & - & - & - & - & 24985 & $6,739,496$ \\
\hline Fim Permer & - & - & 131,745 & $1,328,750$ & 131,745 & $3,320,750$ \\
\hline Nowim Rowet & - & - & 0,456 & 139,900 & 3AS6 & 131.900 \\
\hline 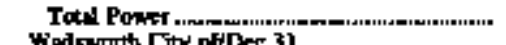 & - & 一 & 135,201 & $2,4614,600$ & 135,201 & $3,460,550$ \\
\hline $\begin{array}{l}\text { Wodrowth City ofDec 31 } \\
\text { Fin Powes }\end{array}$ & - & - & 221,904 & 8565,393 & 2000 & 6.56, 383 \\
\hline 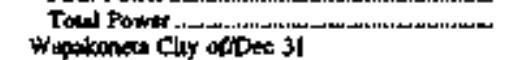 & - & - & 221,004 & $8,515,365$ & 201,500 & B,583.383 \\
\hline 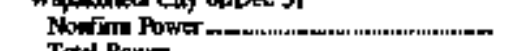 & - & 一 & 6,404 & 134,528 & $1 \leq 2933$ & $5.459,525$ \\
\hline 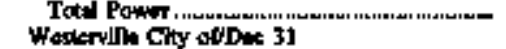 & - & - & 6,404 & $134,52 t$ & 152933 & 5.49525 \\
\hline Fin Prower - & $m$ & عـ & - & - & 349,902 & In,795,500 \\
\hline 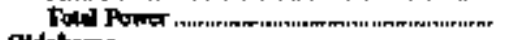 & - & - & - & - & $3 \mathrm{d8}, 502$ & $10,795,580$ \\
\hline \\
\hline 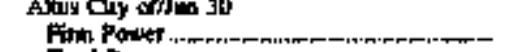 & - & - & - & - & 161.078 & $6.840,127$ \\
\hline Thas Mown & - & - & - & 二 & 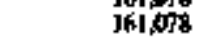 & $0,340.12$ \\
\hline \multicolumn{7}{|l|}{ 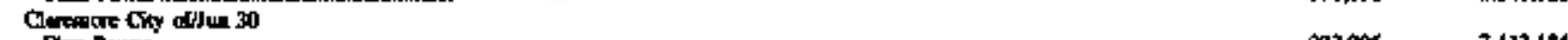 } \\
\hline Finn Powir & - & - & - & - & 2232015 & $7,433,184$ \\
\hline 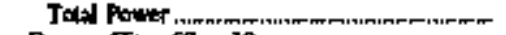 & - & - & - & - & 223,205 & $7,413,164$ \\
\hline \multicolumn{7}{|l|}{ Dapean Chy offar 30} \\
\hline 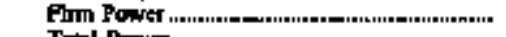 & - & - & - & - & 153,28 & 4,709, 058 \\
\hline 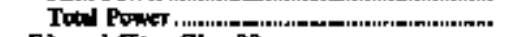 & - & - & - & - & 153,928 & $4,709,358$ \\
\hline \multicolumn{7}{|l|}{ Edimod Cly offon 30} \\
\hline 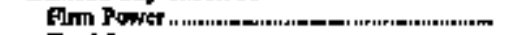 & - & - & - & - & 5no, 049 & 21),991,839 \\
\hline \multirow{2}{*}{\multicolumn{7}{|c|}{ Majul Chy oftion 30}} \\
\hline & & & & & & \\
\hline 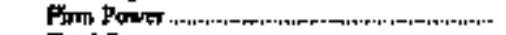 & - & - & - & - & 148,766 & $4,852,978$ \\
\hline 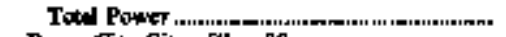 & - & - & - & - & 348.766 & $4,852,978$ \\
\hline \multicolumn{7}{|l|}{ Punce City City abjen 30} \\
\hline 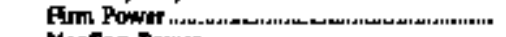 & - & - & - & - & 309,060 & $13593,161)$ \\
\hline 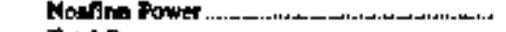 & - & - & - & - & - & $-1,104,168$ \\
\hline Jold Power & - & - & - & - & 309000 & $19,396,952$ \\
\hline \multicolumn{7}{|l|}{ Slillswler Uiliiles Amthrityllon 30} \\
\hline Fin Power .....-........-- & - & - & - & - & 385,546 & $10, B T 3, A S T$ \\
\hline 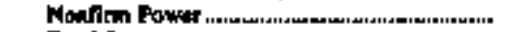 & - & - & - & - & 79222 & 1,129041 \\
\hline Iond Power & - & - & - & - & 423,768 & $12,012,488$ \\
\hline \multirow{2}{*}{\multicolumn{7}{|c|}{ Drequidend Chy ofJun 30}} \\
\hline & & & & & & \\
\hline 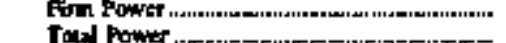 & - & - & - & $\rightarrow$ & [6],04] & $4,595,838$ \\
\hline 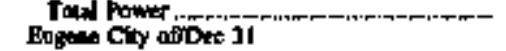 & 一 & - & - & - & F6INHI & $4,595, B B 89$ \\
\hline 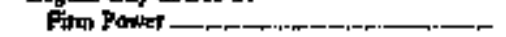 & - & - & - & - & $1,995, \sqrt{191}$ & $50,270,947$ \\
\hline Nawilm Fow & - & - & 197,656 & $1,2 \pi 3,066$ & $398, \sqrt{123}$ & $4,0,15,918$ \\
\hline 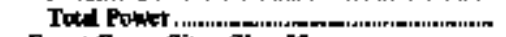 & - & - & 197,655 & $1,755,0,6$ & $2.3933,314$ & $34,276,365$ \\
\hline \multicolumn{7}{|l|}{ Forost Covere Ciny offlm 30} \\
\hline 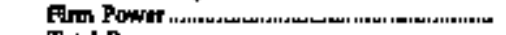 & - & سـ & 7,326 & $1,7 \pi 0,476$ & $2], \pi]$ & $5,126,5390$ \\
\hline 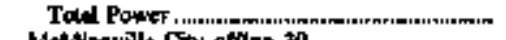 & - & - & $7,32 \pi$ & $1,230,476$ & 218,71 & $5,126,638$ \\
\hline 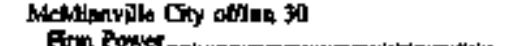 & & & & & & \\
\hline 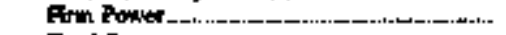 & $m$ & - & 9,835 & 24,65 & 666,076 & $17,548,1797$ \\
\hline 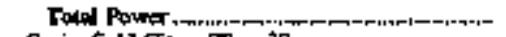 & - & - & 9,835 & 24,650 & 666,976 & $17,548,097$ \\
\hline Springfietd Cily arsep 30 & & & & & & \\
\hline Frm Powers & - & - & - & - & 20,05! & $22,76,765$ \\
\hline 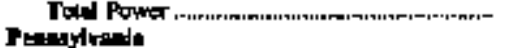 & - & $\pi$ & - & - & 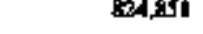 & $20,367,76$ \\
\hline 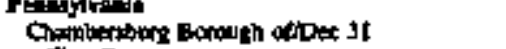 & & & & & & \\
\hline Firm $\mathrm{P}$ Oarar .......................... & - & - & - & - & 247,125 & $9,293,634$ \\
\hline 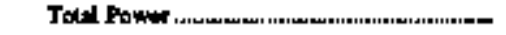 & - & - & - & $\rightarrow$ & 247.125 & $9,293,634$ \\
\hline
\end{tabular}


Toble 29. Electricity Purchases by Municipal Utilities, Fiscal Year 1994 (Continued)

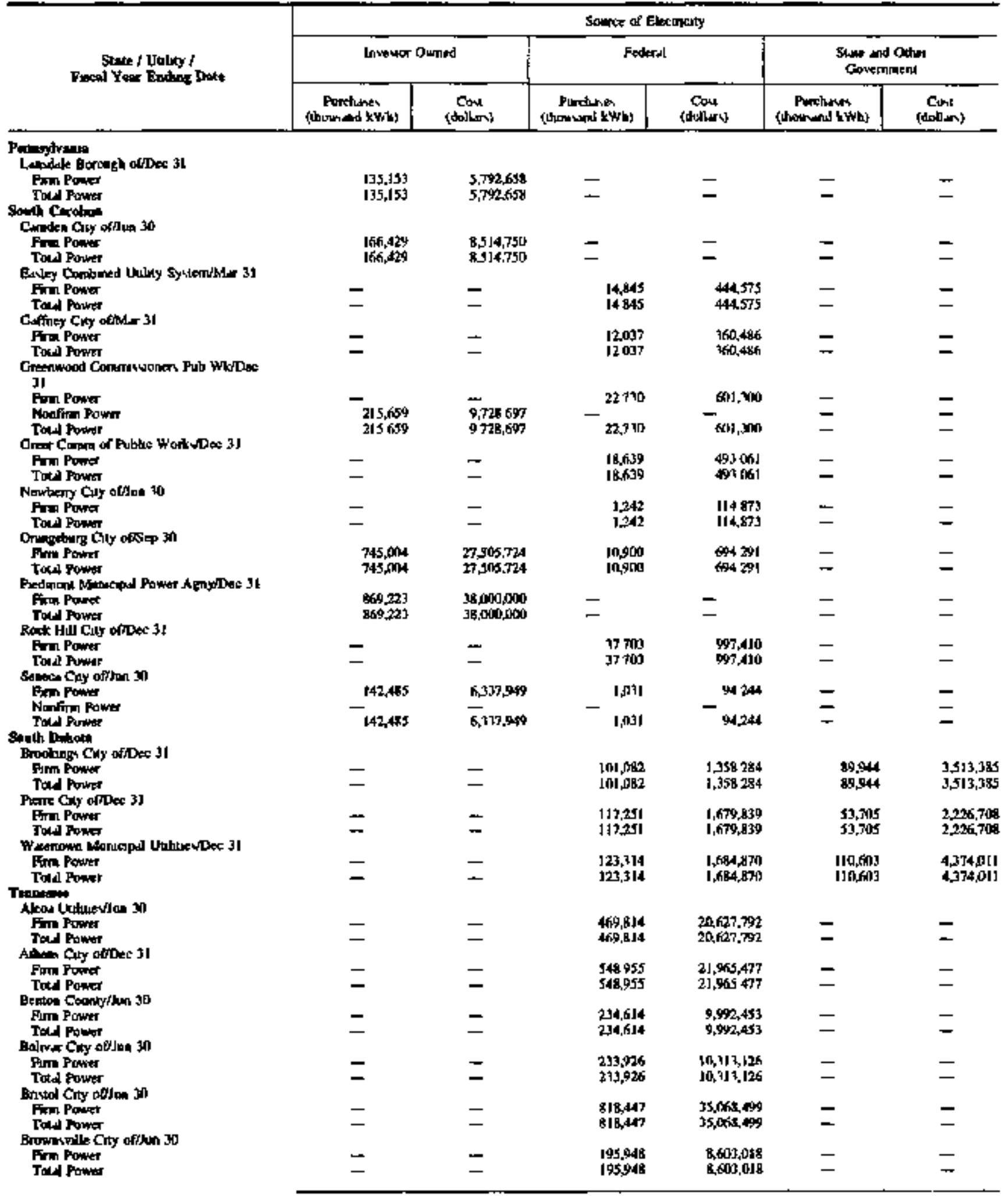

See notes and footentes it end of latite 
Table 29. Electricity Parchases by Manicipal Utilties, Fiscal Year 1994 (Continued)

\begin{tabular}{|c|c|c|c|c|c|c|}
\hline \multirow{3}{*}{ 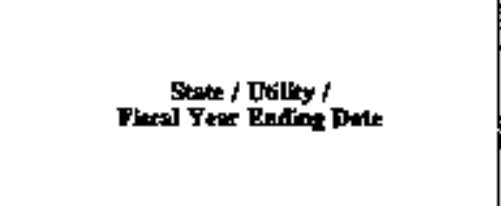 } & \multicolumn{6}{|c|}{ Soonse of Bectrility } \\
\hline & \multicolumn{2}{|c|}{ Coxpenutwe } & \multicolumn{2}{|c|}{ Othal } & \multicolumn{2}{|c|}{ rold } \\
\hline & 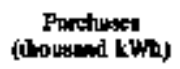 & Coin & 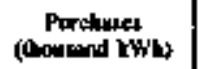 & $\underset{\text { (dollanit) }}{\text { Cod }}$ & $\begin{array}{l}\text { Phandiesers } \\
\text { (thausand } k W / h \text { ) }\end{array}$ & $\underset{\text { (don }}{\operatorname{Com}}$ \\
\hline \multicolumn{7}{|l|}{ Penmeyivento } \\
\hline Afim Powror & - & - & 8,020 & 154,781 & 143,973 & $5,947,439$ \\
\hline \\
\hline Sim Pouter & - & - & - & - & 166,429 & $8,514,750$ \\
\hline 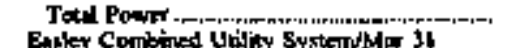 & - & - & - & - & 166,429 & $8,5 / 4,750$ \\
\hline 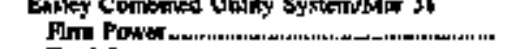 & - & $=$ & $212,346 \mathrm{i}$ & $10,640,4,56$ & 227.191 & 11,ass,051 \\
\hline 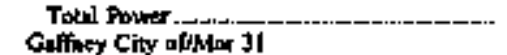 & - & - & 212346 & $10,642,456$ & 227,191 & $11,085,031$ \\
\hline 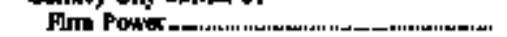 & - & 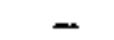 & 983,316 & $7,381,935$ & $195,15]$ & $7,70,421$ \\
\hline Toted Porvet & - & - & $183,2 t 6$ & $7,351,935$ & 195,153 & $7,702,421$ \\
\hline \multicolumn{7}{|l|}{ 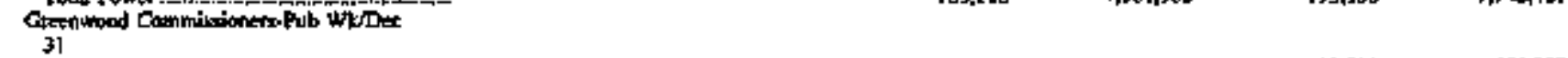 } \\
\hline Fim Powe & - & - & - & - & 22.730 & 601,306 \\
\hline Nodim PuWt & - & - & - & - & 255,6159 & 9.728 .69 \\
\hline Tall Power & . & - & m & - & 238389 & $10,929 \%$ \\
\hline \multicolumn{7}{|l|}{ 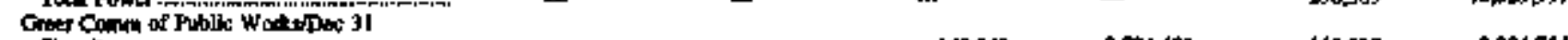 } \\
\hline Fint Pown & - & - & 149,949 & .731 .693 & 168558 & $8,224,734$ \\
\hline Totel Foner & - & - & 109.949 & $7,7 \mathrm{l}$ & 68,548 & 8224,734 \\
\hline \multicolumn{7}{|l|}{ Newteny Cky oflun 30} \\
\hline Pim Power & 二 & $\overline{-}$ & 146,732 & $7,560.121$ & 147,904 & $7,674,994$ \\
\hline \multicolumn{7}{|l|}{ 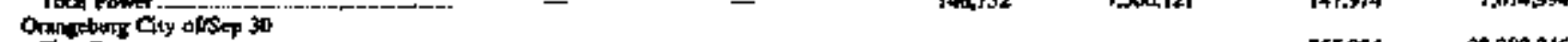 } \\
\hline Fm Pown & - & - & - & - & 795,904 & $2,200, B \mid 5$ \\
\hline 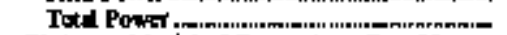 & - & - & $m$ & - & 795,900 & $28,20,0,315$ \\
\hline \multicolumn{7}{|l|}{ 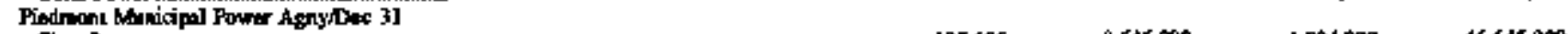 } \\
\hline 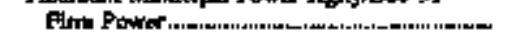 & - & - & I35,, 896 & $8,645,000$ & $1,004,009$ & $46,6,65,1000$ \\
\hline Joll Power & - & - & I35, RI: & $8,645,000$ & $1,004,909$ & $46,605,000$ \\
\hline \multicolumn{7}{|l|}{ Rack Hall Cily of Dee JI } \\
\hline Finm Power - _.......... & - & - & $67.1 \mathrm{~dB}$ & 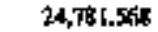 & 514,851 & 26.73897 \\
\hline Toter Fower, & - & - & A7?.t48 & $24,701,568$ & $514, B S 1$ & $25,778,978$ \\
\hline \multirow{2}{*}{\multicolumn{7}{|c|}{ 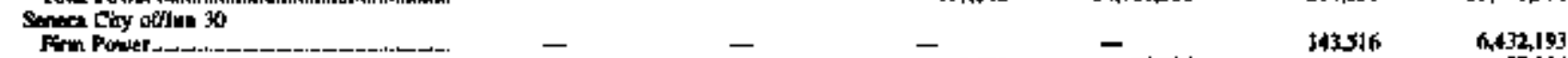 }} \\
\hline & - & - & - & - & 543516 & $0,432,193$ \\
\hline 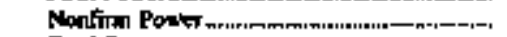 & - & - & 2,593 & $87,3 \mathrm{M}$ & 2,50 & 87,304 \\
\hline Toull Pow & - & - & 259 & 87,304 & 146,109 & 6.519A97 \\
\hline \multirow{2}{*}{\multicolumn{7}{|c|}{$\begin{array}{l}\text { Sount Dolrot: } \\
\text { Bnsokiage Cly of Dec } 31\end{array}$}} \\
\hline & & & & & & \\
\hline Pim Rompr & - & - & - & - & 191,006 & 4,87150 \\
\hline 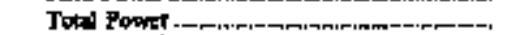 & - & - & - & - & 195, , 26 & $4,871,660$ \\
\hline Piere Giby of Dee JI & & & & & & \\
\hline & בع & - & - & - & 170.586 & 390,547 \\
\hline Tout Powt & - & 一 & - & - & J70,556 & 3.906547 \\
\hline Werengm Municiped UtilitiesDec 31 & & & & & & \\
\hline Fin Power & - & - & - & - & 239,917 & 6,050,88] \\
\hline 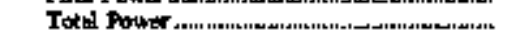 & - & - & - & - & 233,913 & fiNO58,BBI \\
\hline Thandiver & & & & & & \\
\hline Aleos Utintibevilum 30 & & & & & & \\
\hline 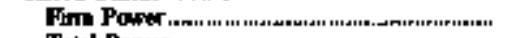 & - & - & - & - & 469,814 & $20,637,792$ \\
\hline 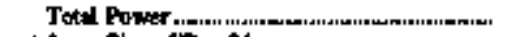 & - & - & - & - & $469, \mathrm{BI} 4$ & $20,107,792$ \\
\hline 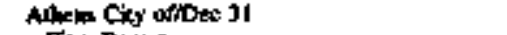 & & & & & & \\
\hline Fith Power & - & - & - & - & 545,955 & $21,965,477$ \\
\hline Toted Pown & - & - & - & - & 568,955 & $21,965,477$ \\
\hline Ecenton Coundythun 90 & & & & & & \\
\hline Firm Rower & - & - & - & - & 234,614 & $9,95,4,43$ \\
\hline 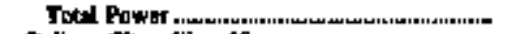 & - & - & - & - & 23,694 & $9,992.453$ \\
\hline Bolive City abjua 30 & & & & & & \\
\hline 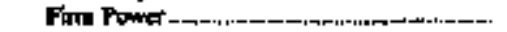 & - & - & - & - & 201924 & $10,313,126$ \\
\hline Total Power yen & - & - & - & - & $23,92 \pi$ & $10,319,126$ \\
\hline Brintad City oblim 30 & & & & & & 35068009 \\
\hline 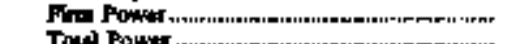 & $=$ & 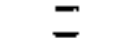 & $=$ & 二 & $\begin{array}{l}B 18,447 \\
B 18,46\end{array}$ & $35,068,496$ \\
\hline $\begin{array}{l}\text { Tow Powh ..... } \\
\text { Bnoweville City offon } 30\end{array}$ & & & & & $\mathbf{B} \mid \mathbf{d}, \mathbf{A F}$ & \\
\hline Fitu Poter & - & - & - & - & 195,948 & $8,607,015$ \\
\hline Toed Powwer & - & - & - & - & 195,948 & $8,604,018$ \\
\hline
\end{tabular}


Tabłe 29. Electricity Purchases by Manicipal Utillites, Fiecal Year 1994 (Contimued)

\begin{tabular}{|c|c|c|c|c|c|c|}
\hline \multirow{3}{*}{ 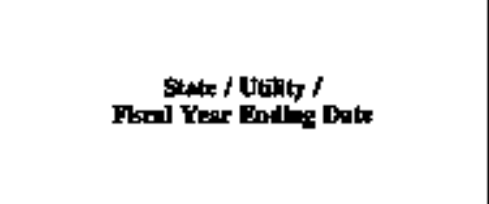 } & \multicolumn{6}{|c|}{ Somese of Electricity } \\
\hline & \multicolumn{2}{|c|}{ Invertor-Gunked } & \multicolumn{2}{|c|}{ Federall } & \multicolumn{2}{|c|}{$\begin{array}{l}\text { Sture ind Other } \\
\text { Govmmmen. }\end{array}$} \\
\hline & $\begin{array}{l}\text { Purthased } \\
\text { (thouked KW/W) }\end{array}$ & $\begin{array}{l}\text { Cont } \\
\text { (dollerin) }\end{array}$ & 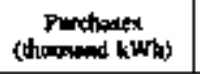 & (dollent) & 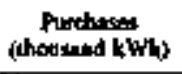 & (dillant) \\
\hline \multicolumn{7}{|l|}{ 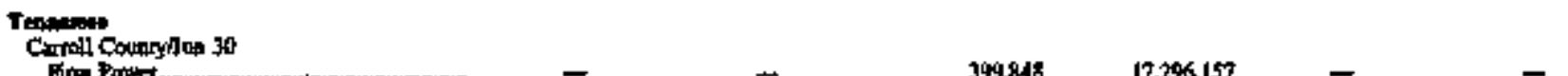 } \\
\hline 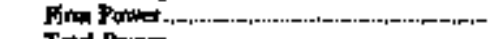 & - & - & 39984 형 & 17.294 .157 & - & - \\
\hline 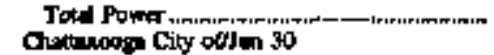 & - & - & 3998:18 & $17,296,157$ & - & - \\
\hline Fin Pown & - & - & $5,800,243$ & $243,038,957$ & - & - \\
\hline 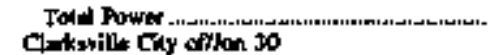 & - & - & $5,800,243$ & $243,038,957$ & - & - \\
\hline 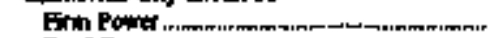 & - & - & 8\%227 & $9 x_{2}, 16,211$ & $m$ & 一 \\
\hline 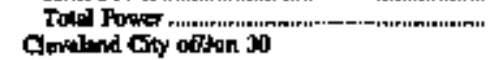 & 一 & - & 898.227 & $39.436,213$ & 一 & 一 \\
\hline 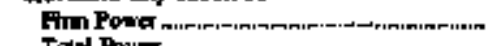 & $m$ & 一 & 94,640 & $40,104,472$ & - & 一 \\
\hline 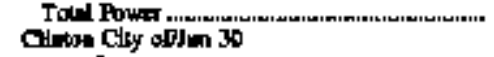 & 一 & 一 & 544,60 & $40,105,472$ & 一 & - \\
\hline Fin Fow & - & - & $65 t, 506$ & $28,390,945$ & - & - \\
\hline 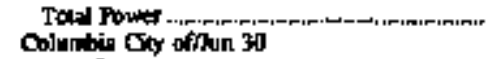 & $\rightarrow$ & - & 601,506 & $28,9 \times 0.945$ & - & - \\
\hline Fin Powlo & - & - & 540,173 & $20,962,538$ & - & - \\
\hline Totat Powes 1 & 一 & 一 & 540,173 & $23,962,138$ & - & - \\
\hline Flm Poping & - & - & 492,668 & $21,931,228$ & - & - \\
\hline 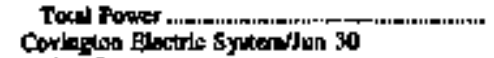 & 一 & - & 492,668 & $21,931,228$ & - & - \\
\hline Firill Powt _..._._._._. & - & - & 211,076 & 9,165, fat & - & - \\
\hline Total Poow & - & - & 211,076 & $9,165,606$ & - & - \\
\hline Donten City ofidum 30 & & & & & & \\
\hline 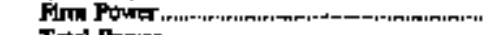 & - & - & 205 ath & 4.,149.191 & - & - \\
\hline 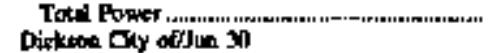 & 一 & - & $205 \Omega 36$ & S्र.149.19r & 一 & 一 \\
\hline Fing Ponller & - & - & Ka: 308 & $27,528,115$ & - & - \\
\hline 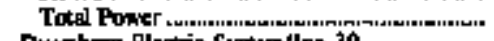 & 一 & - & 628,308 & $27,52,415$ & - & 一 \\
\hline Dyenberg Blectric SyrterestJon 30 & & & & & & \\
\hline 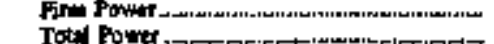 & - & - & 632,731 & $24,13,244$ & $\ddot{z}$ & 一 \\
\hline Eliegbalbon Cis of Jon Jin & & & n32, 131 & $24,4] 1, \Omega(14$ & - & - \\
\hline 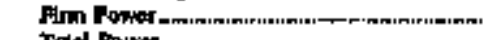 & - & - & 516218 & $22,611,156$ & - & - \\
\hline 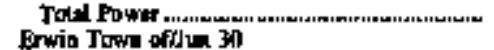 & 一 & - & ร18218 & $22,691,356$ & - & 一 \\
\hline Fin Power & - & - & 216.337 & $9,136,356$ & 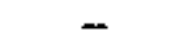 & - \\
\hline 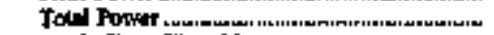 & - & - & 216,337 & $9,136,356$ & - & - \\
\hline Buswh Goy oftum 30 & & & & & & \\
\hline Fith Power & - & - & 153,607 & $6,254,839$ & - & - \\
\hline 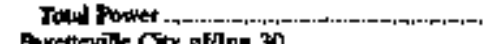 & - & - & $1 \$ 1,60 ?$ & $6,254,039$ & - & 一 \\
\hline 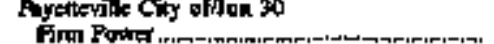 & - & - & torl,o4s & $16,745.56$ & - & - \\
\hline 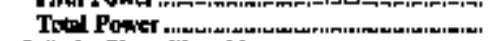 & - & - & 381,045 & $16,745,576$ & - & - \\
\hline Callatin Cry offlon 30 & & & & & & \\
\hline 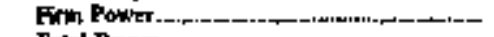 & 一 & - & 482,256 & $20,538,563$ & - & - \\
\hline 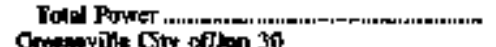 & 一 & $\rightarrow$ & $402 ; 56$ & 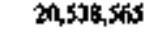 & $m$ & - \\
\hline Fint Pond & 一 & - & 951,754 & $99,460,991$ & - & - \\
\hline IDt Pawer & - & - & 952,754 & 39.461,99: & - & - \\
\hline Hadina Clay offlua 30 & & & & & & \\
\hline 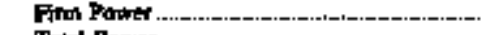 & 一 & - & 3y0,057 & $13,167,709$ & ع & 一 \\
\hline 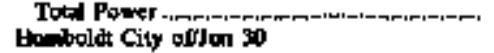 & - & 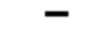 & 330,057 & $13,167,701$ & - & - \\
\hline 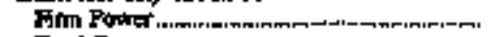 & - & - & 231,266 & $9,849,051$ & - & - \\
\hline 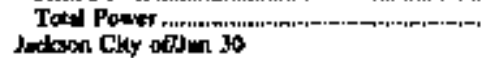 & - & ـ & 231,256 & $0,849,061$ & - & - \\
\hline Fin Paway & 一 & - & $1,254,746$ & $52,793,190$ & - & - \\
\hline 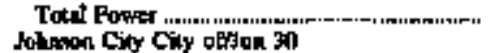 & - & 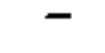 & $1,254,746$ & $52,793,190$ & 一 & $\longrightarrow$ \\
\hline Pind Power ..... & 一 & - & $1,643,723$ & $21,643,131$ & 一 & - \\
\hline Tothl Power & 一 & 一 & 1.643 .723 & $71,643,133$ & - & 一 \\
\hline 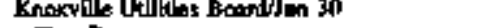 & & & & & & \\
\hline Fin Podher & 一 & - & $5,111,872$ & $217,335,744$ & 一 & 一 \\
\hline 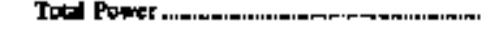 & 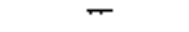 & 一 & $5,111,812$ & $217,3 \$ 1,744$ & - & - \\
\hline
\end{tabular}

Sec mowey and footnoter at end of unde. 
Table 29. Elettrictly Parcheses by Munitipal Utilities, Fiscal Year 1994 (Contimued)

\begin{tabular}{|c|c|c|c|c|c|c|}
\hline \multirow{3}{*}{ Siave f Udatty / } & \multicolumn{6}{|c|}{ Sownd of Elextrielly } \\
\hline & \multicolumn{2}{|c|}{ Coopentive } & \multicolumn{2}{|c|}{ Otherl } & \multicolumn{2}{|c|}{ Total } \\
\hline & Frackend & Con & 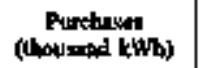 & 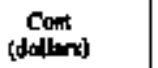 & 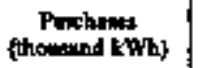 & $\underset{(\phi)|t| z)}{\operatorname{Cost}}$ \\
\hline \multicolumn{7}{|l|}{ Timangen } \\
\hline Fim Pown & - & - & - & - & 39,848 & $17,296,157$ \\
\hline 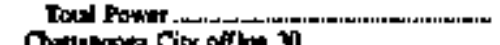 & - & - & - & - & 399,840 & $17,296,157$ \\
\hline 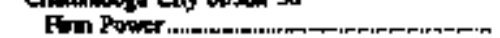 & ــ & - & - & - & $5,800,243$ & 243,0288997 \\
\hline Toted Power .... & - & - & - & - & $5,8200,243$ & $243,048,957$ \\
\hline 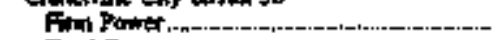 & - & - & - & 61,783 & Bgs,22] & $99,4 \pi, 904$ \\
\hline 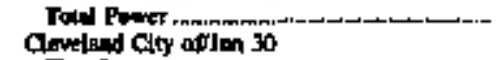 & - & - & - & 61,783 & 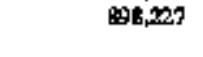 & $99 \mathrm{Am} .99 \mathrm{~A}$ \\
\hline Fim Poring & $=$ & - & - & - & 944,580 & $40,034,4 n$ \\
\hline 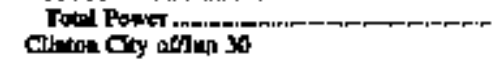 & - & - & - & - & 94,680 & $40,030,472$ \\
\hline frm Poomer & $\rightarrow$ & - & - & - & 651,506 & $28,390,945$ \\
\hline 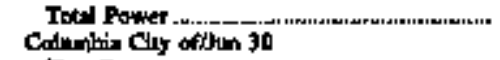 & - & - & - & - & 651596 & $28.390,045$ \\
\hline Fin Powr & - & - & - & - & $5 \$ 0,173$ & $20,962,138$ \\
\hline 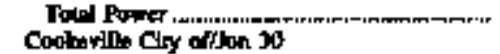 & - & - & - & - & $\$ \$ 0,133$ & 20.962 .138 \\
\hline Frm Powver & - & - & - & - & 42,6 的 & $21,931,228$ \\
\hline Topil Powrer & - & - & - & - & 492,568 & $21,931,228$ \\
\hline 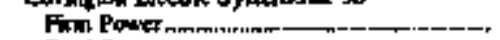 & - & - & - & $\neg$ & 211,096 & $9,165,606$ \\
\hline Tolud Pows & - & - & 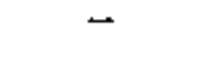 & - & 211,015 & $9, I 65,606$ \\
\hline 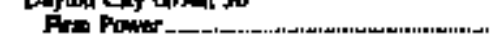 & - & - & - & - & 2ת5, & $9,149,19]$ \\
\hline 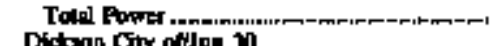 & - & - & - & - & 205,0856 & 9.14 .191 \\
\hline 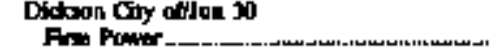 & - & - & - & 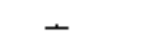 & 628,305 & $27,528,115$ \\
\hline 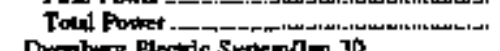 & - & - & - & - & 628,308 & 27528,115 \\
\hline 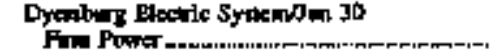 & - & - & - & - & $632 n 1$ & $24,411,204$ \\
\hline 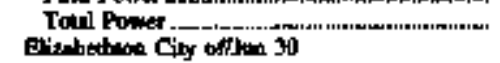 & - & - & - & - & 632.21 & $24,4,1,204$ \\
\hline Fimp Power & - & - & - & - & $51 \%, 218$ & $2,611,056$ \\
\hline 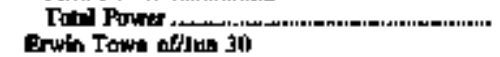 & - & - & - & - & 518,268 & 22611,256 \\
\hline 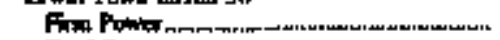 & - & - & - & - & 216,137 & $9,136,056$ \\
\hline 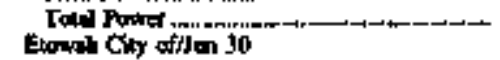 & - & - & - & - & 216,197 & $9,136,35 \%$ \\
\hline 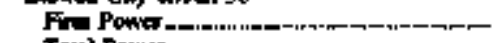 & - & - & - & - & $153,60 ?$ & 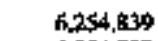 \\
\hline 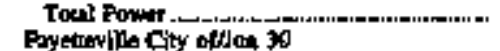 & - & - & - & - & 153,607 & $6,254,839$ \\
\hline Fin Pown & - & - & - & - & 381,045 & $16,745,376$ \\
\hline 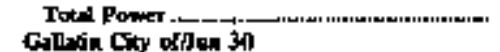 & - & - & - & - & $98 \mathrm{~J}$ (1045 & $16.745,376$ \\
\hline 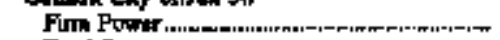 & - & - & - & - & 482,256 & $20,558,565$ \\
\hline 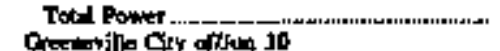 & - & - & - & - & 482.56 & 20573.546 \\
\hline 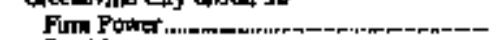 & - & - & - & - & 952,794 & $19,463,991$ \\
\hline 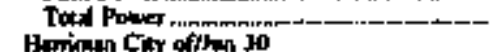 & - & 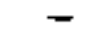 & - & - & 952,754 & $79.467,991$ \\
\hline 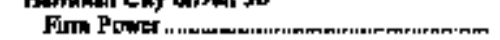 & - & - & - & - & 300,057 & $13,167,701$ \\
\hline Totel Power & 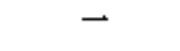 & - & - & - & 330,067 & 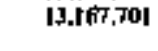 \\
\hline 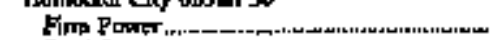 & - & - & - & - & 201,264 & $9,849,051$ \\
\hline Tored Power o...- & $\bar{n}$ & - & - & - & 231256 & 9649,051 \\
\hline 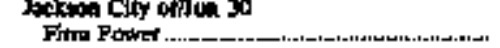 & - & - & - & 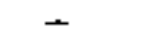 & $1,254,746$ & 51.793 .190 \\
\hline 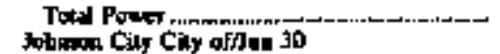 & 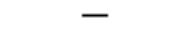 & - & - & 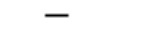 & $1,254,746$ & $52,793,190$ \\
\hline Fin Power & 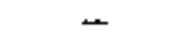 & - & - & - & $1.613,720$ & 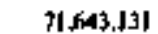 \\
\hline Total Powr & - & - & - & - & $1,643,723$ & Ti 643,191 \\
\hline 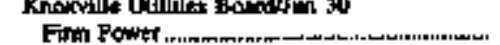 & - & - & 15,930. & 371,514 & 5,127,5!0 & 217657.251 \\
\hline 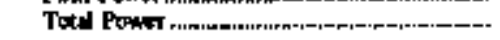 & - & - & 15,938 & 371,514 & $5,127, \mathbb{R I O}$ & $217,687,258$ \\
\hline
\end{tabular}

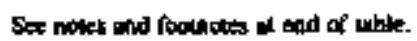


Trble 29. Electricity Purchasses by Manicipal Utllitios, Fiseal Year 1994 (Continued)

\begin{tabular}{|c|c|c|c|c|c|c|}
\hline \multirow{3}{*}{ 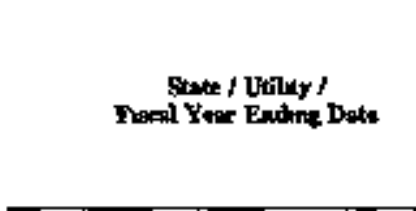 } & \multicolumn{6}{|c|}{ Sownd of Elecincity } \\
\hline & \multicolumn{2}{|c|}{ Intunerowod } & \multicolumn{2}{|c|}{ Fodiril } & \multicolumn{2}{|c|}{ 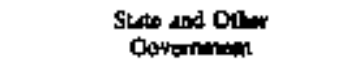 } \\
\hline & $\begin{array}{l}\text { Ponthass } \\
\text { (fiodund } 1 \text { Wh) }\end{array}$ & $\underset{\text { (doflus) }}{\text { Call }}$ & Punthases & 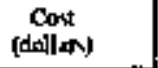 & 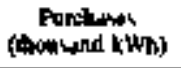 & $\underset{\text { (dollan) }}{\operatorname{Con}}$ \\
\hline \\
\hline 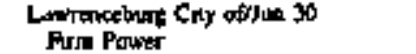 & - & - & 490286 & $20.3 \pi 7.3 B_{4}$ & & \\
\hline Tóal Potier & - & - & 490286 & $20.7 m, 734$ & $=$ & $\bar{z}$ \\
\hline Lefollete Cuy ofllom 30 & & & & & & \\
\hline $\begin{array}{l}\text { Fime Pourer } \\
\text { Totsl Power }\end{array}$ & $\rightarrow$ & - & 362.157 & $16,205,856$ & - & - \\
\hline \multicolumn{7}{|l|}{ Lobmon Clyy of Jen 30} \\
\hline Fur Pod Pawer & - & - & $362 \times 3 \times$ & $|5,864,87|$ & - & - \\
\hline Tow Foner & - & - & $262,21: 00$ & $(5,864,87)$ & - & - \\
\hline \multicolumn{7}{|l|}{ Lemer Chy Cay of the } \\
\hline $\begin{array}{l}\text { Fim Powir } \\
\text { Toxul Powtr }\end{array}$ & - & - & $1,204,0132$ & $45,684,413$ & - & - \\
\hline 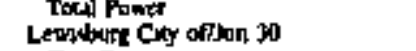 & - & - & $1,109,012$ & $45,680,413$ & - & - \\
\hline Fim Power & - & - & $289,14 t$ & $12,32,240$ & - & - \\
\hline Tots Power & - & - & 2894141 & $12,204,240$ & - & $\rightarrow$ \\
\hline \multicolumn{7}{|l|}{ Lexioum Cay ofjun 30} \\
\hline $\begin{array}{l}\text { Priw Prower } \\
\text { Totd Poyser }\end{array}$ & $\Rightarrow$ & $=$ & 415,525 & $18,257,131$ & - & - \\
\hline \multicolumn{7}{|l|}{ Lopdon City of oflum, } \\
\hline Firm Ponver & - & - & 290,931 & $11,499,838$ & - & - \\
\hline \multirow{2}{*}{\multicolumn{7}{|c|}{ Murgulle Uthituevian 30}} \\
\hline & & & & & & \\
\hline Toldel Poover & - & - & 365,476 & $23,368,460$ & - & $=$ \\
\hline \multicolumn{7}{|l|}{ 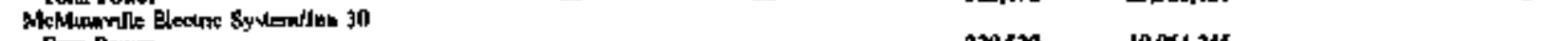 } \\
\hline $\begin{array}{l}\text { Pan Power } \\
\text { Tous Power }\end{array}$ & $\vec{F}$ & $\overline{-}$ & 230,530 & $10,0104,245$ & - & - \\
\hline \multicolumn{7}{|l|}{ Nemptos Cisy offlan 30} \\
\hline Prim Pown & - & - & $J 2,542,427$ & $53,3,220,5,521$ & - & - \\
\hline Tydu Powr & - & - & 12542420 & $533,220,521$ & - & - \\
\hline \multicolumn{7}{|l|}{ Mited $C x y$ offine 30} \\
\hline $\begin{array}{l}\text { Fint Power } \\
\text { Tous Pooket }\end{array}$ & - & 二 & 229.116 & $10,392,926$ & $\rightarrow$ & $\rightarrow$ \\
\hline \multicolumn{6}{|l|}{ Mominteren $6 x y$ oflan Jo } & 一 \\
\hline Frel Power & - & - & 694678 & $29,052,6 \pi]$ & - & - \\
\hline \multirow{2}{*}{\multicolumn{7}{|c|}{ Murfortesto Cny oflua 30 }} \\
\hline & & & & & & \\
\hline Prom Powner & - & - & 2ylinis & $39,340,817$ & - & - \\
\hline \multicolumn{7}{|l|}{ 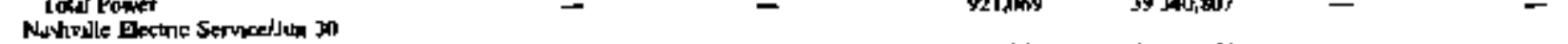 } \\
\hline Fim POWET & - & - & II, $000,1 / 2$ & 46B,982 555 & - & $\rightarrow$ \\
\hline Tokd Foner & - & - & $11,1000,362$ & $468,982,855$ & - & - \\
\hline Newport Gty offlutu 30 & & & & & & \\
\hline $\begin{array}{l}\text { Fotm Potres } \\
\text { Told Power }\end{array}$ & - & 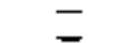 & 431,792 & $18,487,095$ & $\bar{z}$ & - \\
\hline OA Rudge Chy offlus HI & & & $81, \sqrt{192}$ & $16,487,093$ & & \\
\hline Fim Power & - & - & 498,655 & $21,143,+1202$ & - & - \\
\hline 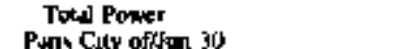 & - & $=$ & 498,605 & $21,347,622$ & - & - \\
\hline $\begin{array}{l}\text { Pund Clly offlan } 30 \\
\text { Ejtm Powet }\end{array}$ & - & - & 460,257 & $20,127,552$ & - & - \\
\hline Tots power & - & - & 46,257 & $20,12,352$ & - & $\check{-}$ \\
\hline Pulded Cny wfition 50 & & & & & & \\
\hline Frtm Fowet & - & - & 424.273 & $17,675,2511$ & $\boldsymbol{m}$ & - \\
\hline Toull Powet & - & - & 424273 & $17,676,250$ & - & - \\
\hline 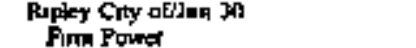 & - & - & 245 th5 & $30789-761$ & - & 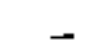 \\
\hline $\begin{array}{l}\text { Fim Pownd } \\
\text { Total Powet }\end{array}$ & - & 二 & 245,305 & $10,749,261$ & $\bar{z}$ & $\overline{-}$ \\
\hline Rodkwood Caly offJuen to & & & & & & \\
\hline Furd Ponter & - & - & 296,090 & $39,023,012$ & - & - \\
\hline Toul Powtr & - & - & 296090 & $19,027,012$ & - & - \\
\hline 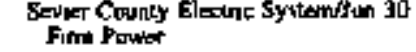 & - & - & 956965 & $42,67,8,803$ & - & - \\
\hline Total Powet & - & - & 956965 & $42,671,803$ & - & - \\
\hline 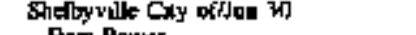 & & & & & & \\
\hline $\begin{array}{l}\text { From Power } \\
\text { Total Powar }\end{array}$ & $\overline{-}$ & $\bar{z}$ & $\begin{array}{l}335,768 \\
375,768\end{array}$ & $\begin{array}{l}34,898,754 \\
24,898,700\end{array}$ & 二 & $\bar{z}$ \\
\hline
\end{tabular}

Sete nothe and loodendes an and af uble 
Tahile 29. Eectricity Porchases by Municipal Utilities, Füscal Year 1994 (Continued)

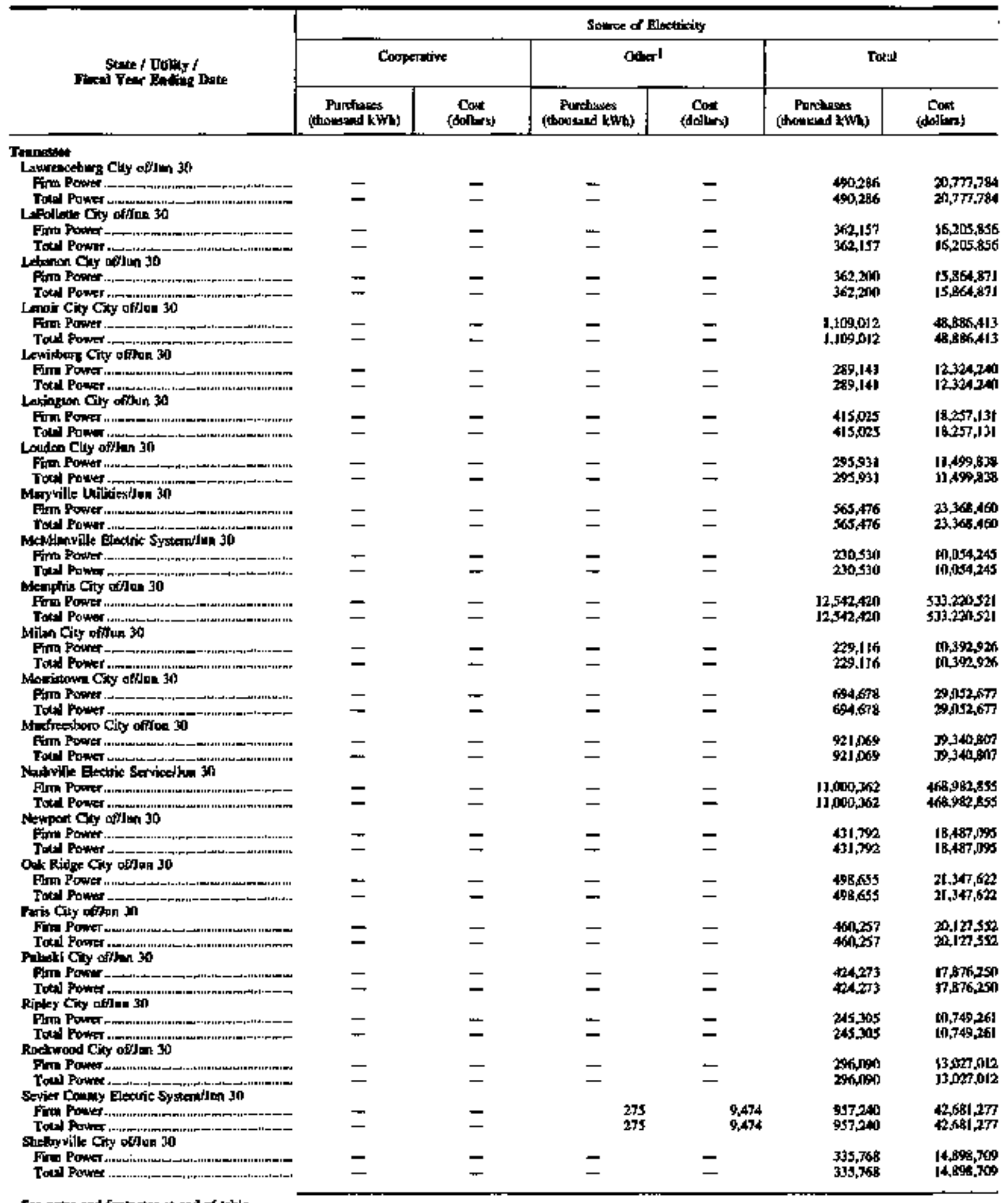

Sal notes and fooknoten a abd of tuble. 
Table 29. Electricity Purchases by Monicipal Utilifes, Fiscal Year 1994 (Continued)

\begin{tabular}{|c|c|c|c|c|c|c|}
\hline \multirow{3}{*}{ 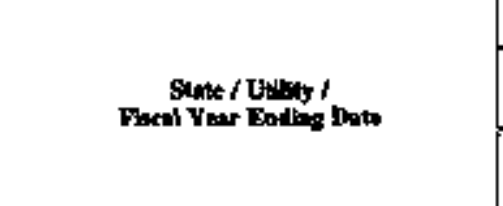 } & \multicolumn{6}{|c|}{ Sourtre of Elextricity } \\
\hline & \multicolumn{2}{|c|}{ Invextion OWhed } & \multicolumn{2}{|c|}{ Findetal } & \multicolumn{2}{|c|}{$\begin{array}{l}\text { State ind Other } \\
\text { Coyemnnem }\end{array}$} \\
\hline & 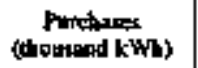 & $\underset{\text { (do]lest) }}{\text { Cost }}$ & Porclutat & $\begin{array}{c}\operatorname{Cos} x \\
\text { (destion) }\end{array}$ & 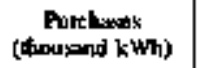 & $\begin{array}{c}\text { Conl } \\
\text { (donlw) }\end{array}$ \\
\hline \multicolumn{7}{|l|}{ 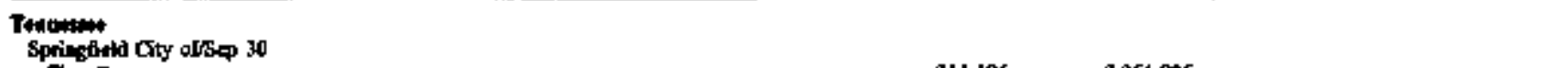 } \\
\hline 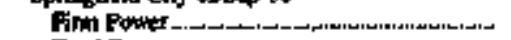 & - & - & 2H, Jak & $8,961,026$ & - & - \\
\hline $\begin{array}{l}\text { Todel Powed } \\
\text { swretwing Chy often } 30\end{array}$ & - & - & $211.11 \%$ & B.561.006 & - & - \\
\hline Fin Pover - & - & - & - & - & - & - \\
\hline Toxol Fower .... & - & - & - & - & - & - \\
\hline 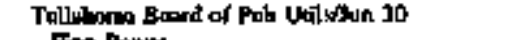 & & & & & & \\
\hline 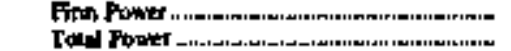 & $\overline{-}$ & $\overline{-}$ & $\begin{array}{l}2354,928 \\
2 \sin , 028\end{array}$ & $\begin{array}{l}12,469,134 \\
12,469,134\end{array}$ & $\bar{z}$ & \pm \\
\hline Union Ory Cty offrna \$n & & & & & & \\
\hline Fin Power, & - & - & 5S8,733 & $13.509,0 \% 9$ & - & - \\
\hline 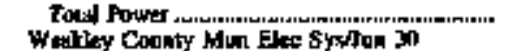 & - & - & 358.733 & $13,509,0898$ & - & - \\
\hline Firne Pouvef & - & - & $495,6 \mathbf{K}_{2}$ & $25,875,106$ & - & - \\
\hline 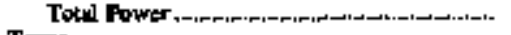 & - & - & 405,662 & 2f,875, 106 & - & - \\
\hline \multirow{2}{*}{\multicolumn{7}{|c|}{ Tavis City ofses 30}} \\
\hline & & & & & & \\
\hline 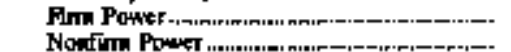 & -52800 & $\begin{array}{r}24,936 \\
167,392\end{array}$ & $\overline{-}$ & 二 & $-24 m$ & $\begin{array}{r}1,375 \\
5,37,599\end{array}$ \\
\hline Toted Pollter & 528,40 & $11,492,24 t$ & - & - & 24,025 & 533.914 \\
\hline \multicolumn{7}{|l|}{ Brophum Cay of Sap 30 } \\
\hline 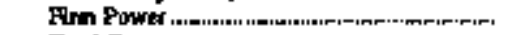 & - & - & - & - & 39,084 & 9990,012 \\
\hline Jot & - & - & - & - & 259.084 & $9,90000 \mathrm{me}$ \\
\hline \multicolumn{7}{|l|}{ 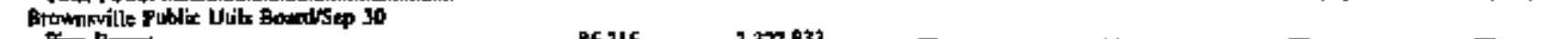 } \\
\hline 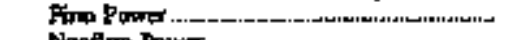 & B6,316 & $3,327,033$ & - & - & - & - \\
\hline 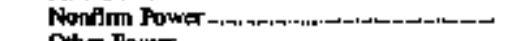 & $1 / 26,423$ & $2.175 A 13$ & - & 二 & 9,728 & $2.9 .1 \mathrm{~m}$ \\
\hline Othe Fowr & $-192 \pi 9$ & $\overline{5} 50.246$ & $\overline{-}$ & $\bar{z}$ & - 9,728 & -219.106 \\
\hline Brye Cly offses 30 & 196/ & 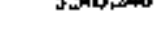 & & & $3, \sqrt{2}=8$ & \\
\hline form Punter & - & - & - & - & - & - \\
\hline Nonfitm Power & - & - & - & - & - & - \\
\hline 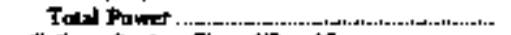 & - & $m$ & - & - & - & - \\
\hline \multicolumn{7}{|l|}{ 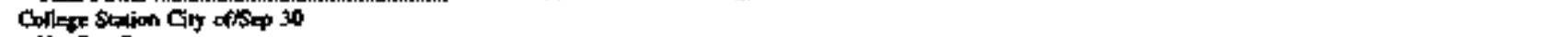 } \\
\hline 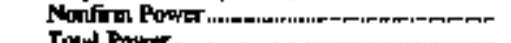 & - & - & - & - & - & - \\
\hline 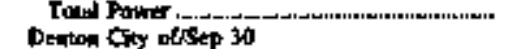 & - & - & - & - & 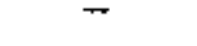 & - \\
\hline 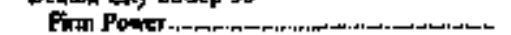 & - & - & - & - & - & - \\
\hline 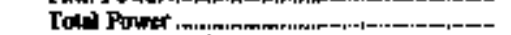 & - & - & - & - & - & - \\
\hline \multicolumn{7}{|l|}{ 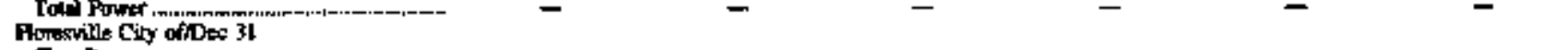 } \\
\hline Fin Potwer & - & - & - & - & - & - \\
\hline \multicolumn{7}{|l|}{ 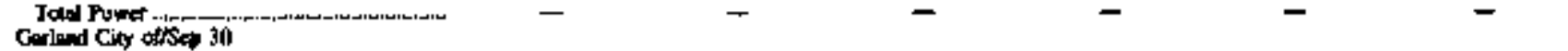 } \\
\hline Fm Poxtr. & - & - & - & - & - & - \\
\hline 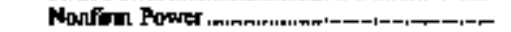 & - & - & - & - & - & - \\
\hline 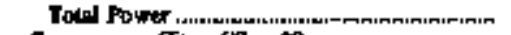 & - & - & - & - & - & - \\
\hline \multicolumn{7}{|l|}{ 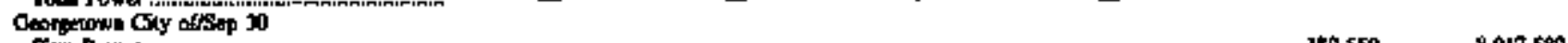 } \\
\hline Firn Poxer & - & - & - & - & 1t5.550 & $8,015,589$ \\
\hline 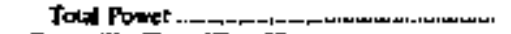 & - & - & - & - & 189,550 & 801758 \\
\hline \multicolumn{7}{|l|}{ Greenwille Gity offSo 30} \\
\hline Frm Panter & - & - & - & - & - & - \\
\hline 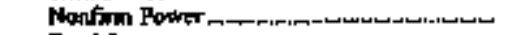 & - & - & - & - & - & - \\
\hline \multirow{2}{*}{\multicolumn{7}{|c|}{$\begin{array}{l}\text { Taus Pawer .... } \\
\text { Kervilik Publie Utility BoordSepp } 30\end{array}$}} \\
\hline & - & - & - & - & 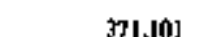 & $15.766 .6 \%$ \\
\hline 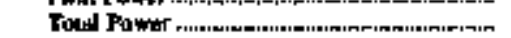 & - & - & - & - & 37,101 & $15,766, B 40$ \\
\hline \multicolumn{7}{|l|}{ Lebock Clyy of } \\
\hline Fith Ponter & 528,310 & $9,821,806$ & - & - & - & - \\
\hline 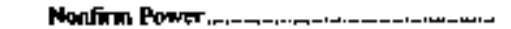 & 159.422 & $4,46 *, 7+27$ & - & - & - & - \\
\hline 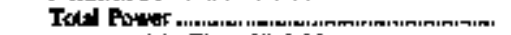 & $487,7,2$ & $14,290,083$ & - & - & - & - \\
\hline 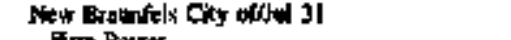 & & & & & & \\
\hline Finn Poures ...._- & $=$ & $=$ & $\bar{z}$ & $\bar{z}$ & 78.188 & soposon \\
\hline 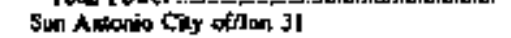 & & & & & & \\
\hline 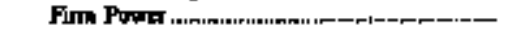 & - & 24,937 & - & - & - & \\
\hline Nowim Prunt & $\$ 99.192$ & $4,10,006$ & - & - & 12.16! & 289,522 \\
\hline 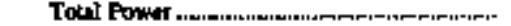 & 199,192 & $4,164,003$ & - & - & I2,08] & $269.6 n$ \\
\hline
\end{tabular}

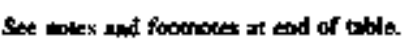


Table 29. Electricity Purchases by Muricţad Ut7lities, Fiseal Yeur 1994 (Continued)

\begin{tabular}{|c|c|c|c|c|c|c|}
\hline \multirow{3}{*}{ 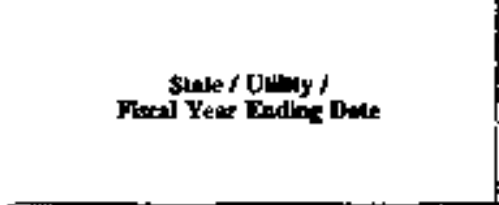 } & \multicolumn{6}{|c|}{ Somec of Elecerictry } \\
\hline & \multicolumn{2}{|c|}{ Coopentive } & \multicolumn{2}{|c|}{ Oty } & \multicolumn{2}{|c|}{ Tatl } \\
\hline & 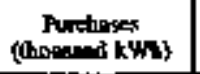 & (dollaris) & $\begin{array}{l}\text { Putstuser } \\
\text { (frosind XWh) }\end{array}$ & $\begin{array}{c}\text { Cout } \\
\text { (dollinit) }\end{array}$ & 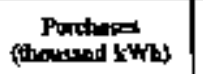 & $\begin{array}{c}\text { Cont } \\
\text { (dollens) }\end{array}$ \\
\hline \multicolumn{7}{|l|}{ Ttonisint: } \\
\hline 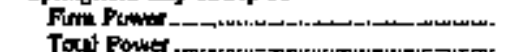 & - & - & - & - & 211,106 & $8,961,002$ \\
\hline 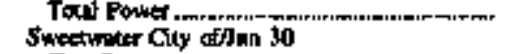 & - & - & - & - & 21).J06 & $8,561,025$ \\
\hline Fim Poowter & $\overline{-}$ & $\overline{-}$ & $\begin{array}{l}198,469 \\
796,469\end{array}$ & $\begin{array}{l}8,647,309 \\
6,647,409\end{array}$ & $\begin{array}{l}198,469 \\
198,469\end{array}$ & $\begin{array}{l}8,677,309 \\
8647,369\end{array}$ \\
\hline 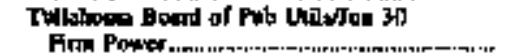 & & & & & & \\
\hline 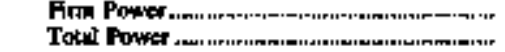 & - & - & - & - & 2300,926 & $12,469.134$ \\
\hline Undon Coy ouy ofilua 30 & - & - & - & - & 280,928 & $12,469, \sqrt{34}$ \\
\hline 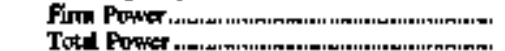 & - & - & - & - & 359,733 & 13,5090909 \\
\hline 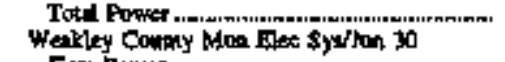 & - & - & - & - & 358,733 & 13,509 MBS \\
\hline 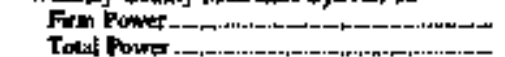 & - & - & - & - & 495,662 & $21.875,100$ \\
\hline \multirow{2}{*}{\multicolumn{7}{|c|}{ Tores }} \\
\hline & & & & & & \\
\hline 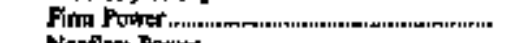 & - & - & - & - & - & 26311 \\
\hline 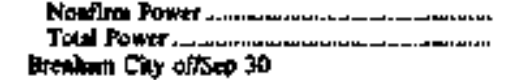 & $\begin{array}{l}62,439 \\
62,439\end{array}$ & $\begin{array}{l}1,112,5,33 \\
1, t 12,513\end{array}$ & $\begin{array}{l}20,5,45 \\
23,545\end{array}$ & $\begin{array}{l}1,938,308 \\
1+938,508\end{array}$ & $\begin{array}{l}616,457 \\
610,457\end{array}$ & 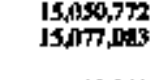 \\
\hline 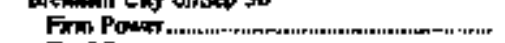 & - & - & - & - & $259,0,4$ & $9,990,010$ \\
\hline 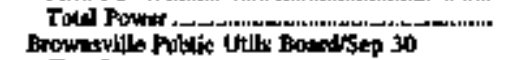 & - & - & 一 & - & 259,084 & 9990,002 \\
\hline 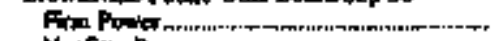 & - & - & 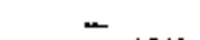 & - & 86,3t6 & 3.327833 \\
\hline 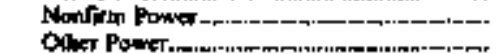 & $-\quad 3,193$ & $-^{139.960}$ & 1,746 & $-40,566$ & 23,096 & $2,569,035$ \\
\hline 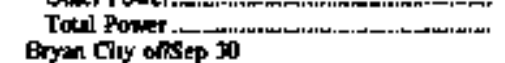 & 5,193 & 129,950 & 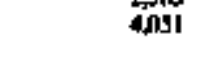 & $4 \$, 556$ & 211,711 & $5,896,368$ \\
\hline 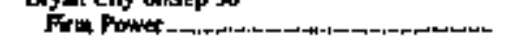 & - & - & - & $23,020,215$ & - & $20,824,215$ \\
\hline 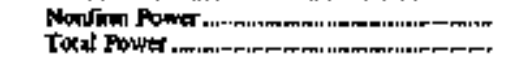 & $\bar{z}$ & $\bar{z}$ & $\begin{array}{l}655,962 \\
655,962\end{array}$ & $\begin{array}{l}9,27,446 \\
33,104,169\end{array}$ & $\begin{array}{l}656962 \\
656,962\end{array}$ & 9,27969 \\
\hline Collete Siwtion Cry of 'ses 30 & & & & & & \\
\hline 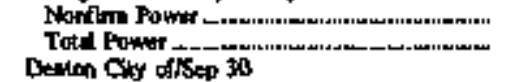 & $\overline{-}$ & $\overline{-}$ & 406,449 & $\begin{array}{l}22,693,021 \\
22,093,421\end{array}$ & 484,449 & $\begin{array}{l}22,693,021 \\
2,693, n 21\end{array}$ \\
\hline 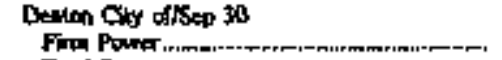 & - & - & $75 \$ 021$ & 35.150 .47 & $759 \mathrm{mi}$ & 31,1000 \\
\hline 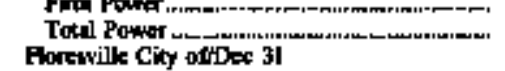 & $\rightarrow$ & - & 199,021 & $35,150,979$ & 159021 & $\begin{array}{l}35,150914 \\
35,150,979\end{array}$ \\
\hline 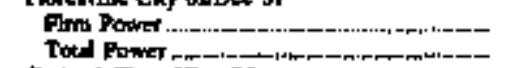 & $\overline{-}$ & $\overline{-}$ & $\begin{array}{l}157.657 \\
157,658\end{array}$ & $\begin{array}{l}6,266,26: \\
6,66,060\end{array}$ & $\begin{array}{l}1,97,657 \\
159,657\end{array}$ & $\begin{array}{l}6,266,268 \\
6,266,268\end{array}$ \\
\hline Ger and Cuy dfisep 70 & & & & & & \\
\hline 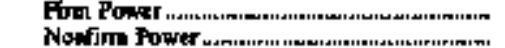 & 二 & $\overline{-}$ & $1,312,199$ & 64,847,686 & 1,312,869 & $68, B 47,696$ \\
\hline Tout Pawer & - & 二 & $1,417,132$ & $71,149,294$ & $1,417,132$ & $71,145,210$ \\
\hline Georgotown Chy offse 30 & & & & & & \\
\hline Fin Power & - & - & - & - & 189.500 & B:017,5e9 \\
\hline 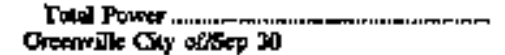 & - & - & - & - & 199550 & B,017,Sig \\
\hline 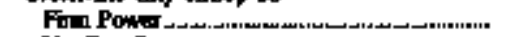 & - & - & $251,40]$ & $13.184, .328$ & 251,003 & $10,180,328$ \\
\hline 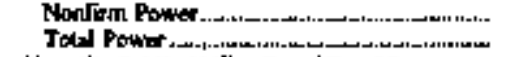 & $\begin{array}{l}13,130 \\
19,130\end{array}$ & $\begin{array}{l}300,200 \\
300,200\end{array}$ & $\begin{array}{l}166,657 \\
418,060\end{array}$ & $\begin{array}{r}3,844,446 \\
1,2,024,774\end{array}$ & $\begin{array}{l}179,787 \\
41,190\end{array}$ & $\begin{array}{r}4,1,44,546 \\
17,324,974\end{array}$ \\
\hline Karvilk Poblic Utilky Bowdsep 30 & & & & & & \\
\hline Ftron Power & - & - & - & - & $37, \sqrt{01}$ & $15,760,360$ \\
\hline 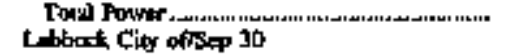 & 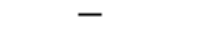 & - & - & - & $371,10 \mathrm{l}$ & $15,765, B 64$ \\
\hline 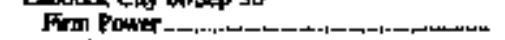 & - & - & - & - & 320,310 & $9,401,3 \times 6$ \\
\hline 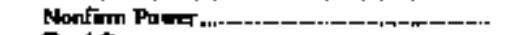 & - & - & - & - & 199,22 & $4,468,2 \mathrm{BR}$ \\
\hline 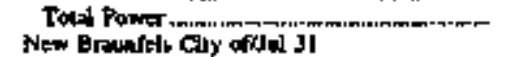 & - & - & - & - & 4.7 .732 & 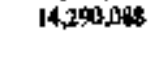 \\
\hline Fin Poner & - & - & - & - & 787,1888 & $30,009, \sqrt{144}$ \\
\hline Total Power & $=$ & - & - & - & 789,188 & $30,0190,044$ \\
\hline Fin Pawn & - & - & - & 788 & - & 25,875 \\
\hline 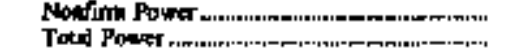 & 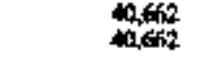 & $\begin{array}{l}557,9288 \\
557,978\end{array}$ & $\begin{array}{l}\$ 5,147 \\
\$ 5,147\end{array}$ & 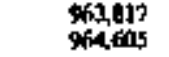 & 397,062 & $\begin{array}{l}5,054,3 \times 3 \\
5,900,2018\end{array}$ \\
\hline
\end{tabular}

See nowe and formotes at end of table 
Table 29. Bectricity Purchases by Municipal Utilities, Flseal Year 1994 (Continued)

\begin{tabular}{|c|c|c|c|c|c|c|}
\hline \multirow{3}{*}{ 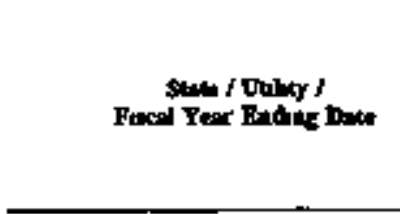 } & \multicolumn{6}{|c|}{ Sound of Electraliy } \\
\hline & \multicolumn{2}{|c|}{ Investor Owined } & \multicolumn{2}{|c|}{ Federol } & \multicolumn{2}{|c|}{ State end Coluer } \\
\hline & Ponchast & $\begin{array}{c}\text { Cost } \\
(\text { dollts) }\end{array}$ & $\begin{array}{c}\text { Porchires } \\
\text { (tbovsadd Kah) }\end{array}$ & $\underset{\text { (dallary }}{\operatorname{Cos} x}$ & 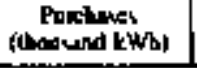 & Cost \\
\hline \multicolumn{7}{|l|}{ 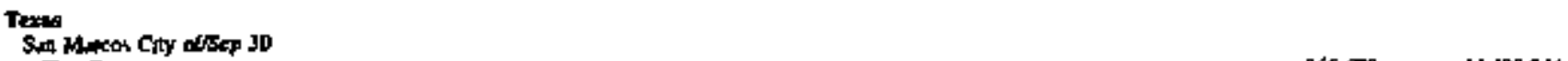 } \\
\hline Finm Porma & - & - & - & - & 366,670 & $14,493,244$ \\
\hline 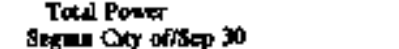 & - & - & - & - & 366,670 & $14,193,264$ \\
\hline Prom Ponver & - & - & - & - & 296,732 & $8,18,4,4]$ \\
\hline Nontirn Podta & - & - & - & - & - & \\
\hline Totel Pona & - & - & - & - & 205,732 & $8,181, A J 3$ \\
\hline 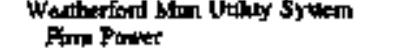 & - & - & - & - & - & - \\
\hline Tatel Power & $\overline{-}$ & - & - & - & - & $\overline{-}$ \\
\hline \multicolumn{7}{|l|}{ 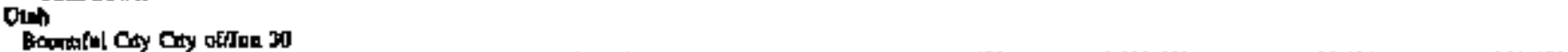 } \\
\hline $\begin{array}{l}\text { Pim Rotur } \\
\text { Told Powr }\end{array}$ & $\begin{array}{l}23,893 \\
\mathbf{2}, 893\end{array}$ & $\begin{array}{l}314,183 \\
714,183\end{array}$ & $\begin{array}{l}157,47\} \\
157,477\end{array}$ & $\begin{array}{l}3,021,462 \\
3,021,462\end{array}$ & 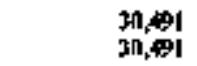 & $\begin{array}{l}931,480 \\
931,480\end{array}$ \\
\hline \multicolumn{7}{|l|}{ 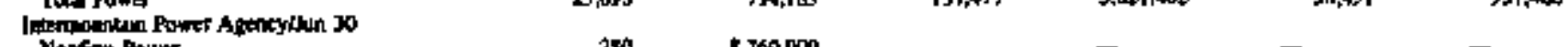 } \\
\hline Nowinn Power & 296 & 8.760,000 & - & - & - & 一 \\
\hline & 2 ton & 8,760,000 & - & - & - & - \\
\hline Fim Fonter & - & - & - & - & $127,6 \mathrm{fik}$ & $5,386,7) 1$ \\
\hline Nonfim Pawer & - & - & - & - & 40,345 & J (120,097 \\
\hline Told Power & - & - & - & - & 167.981 & 6406908 \\
\hline \multicolumn{7}{|l|}{ Murdy OWy aftion 30} \\
\hline 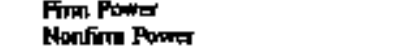 & $\overline{-}$ & 二 & - & $=$ & - 16.968 & 7040.740 \\
\hline $\begin{array}{l}\text { Nonfim Pond } \\
\text { Tots Pons }\end{array}$ & $\overline{-}$ & 二 & $\overline{-}$ & $\overline{-}$ & 165,948 & 7,049740 \\
\hline \multicolumn{7}{|l|}{ Prow Cily ConpJon 30} \\
\hline Fim Power & - & - & שـ & - & 369.617 & $2,718,956$ \\
\hline 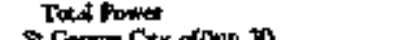 & - & - & - & - & 569.637 & $2718.9 \$ 6$ \\
\hline 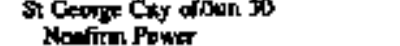 & & & & & & \\
\hline $\begin{array}{l}\text { Nomintin Power } \\
\text { Told Porat }\end{array}$ & $=$ & 二 & $\bar{z}$ & $=$ & $\begin{array}{l}244,575 \\
244575\end{array}$ & $10,177,634$ \\
\hline \multirow{2}{*}{\multicolumn{7}{|c|}{$\begin{array}{l}\text { Yomove } \\
\text { Burtayton Cry of }\end{array}$}} \\
\hline Burtagton Cixy of & & & & & & \\
\hline 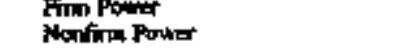 & $\begin{array}{l}127,618 \\
7,568\end{array}$ & $\begin{array}{l}0,57,913 \\
700,525\end{array}$ & 二 & $\bar{z}$ & $-21,023$ & $-258,421$ \\
\hline Tond Fower & 135,206 & 7, ons,d28 & - & - & 21,62 2 & 298421 \\
\hline \\
\hline $\begin{array}{l}\text { Bediund Chy offlim } 30 \\
\text { Eum Pawe }\end{array}$ & 200,858 & $7,57,597$ & B94 & 63,446 & - & - \\
\hline Totd Pows & 290,558 & $7,572,597$ & 894 & 63,446 & - & - \\
\hline \multicolumn{7}{|l|}{ Bnisto Undres Botridion Jo } \\
\hline Em Powar & - & - & 553,029 & $22,247,272$ & - & - \\
\hline \multirow{2}{*}{\multicolumn{7}{|c|}{ Dunvile Cuy of Jum so }} \\
\hline & & & & & & \\
\hline Fin Power & 670,398 & $90.165,746$ & 4,170 & $29 \%, 081$ & - & - \\
\hline Nobfint Powtr & - & $\overline{0}$ & - & - & - & - \\
\hline \multicolumn{7}{|l|}{ 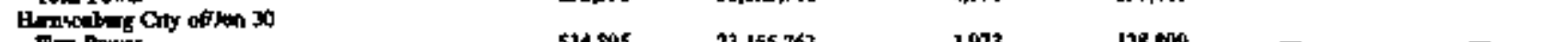 } \\
\hline Fim Powar & 54,895 & $22,155.763$ & 1.973 & 198,806 & - & - \\
\hline Tod Pows & 534.895 & $23.155,760$ & 1.973 & $198, \mathrm{BOS}$ & - & - \\
\hline \multicolumn{7}{|l|}{ Mankes City offlom 30} \\
\hline Fim Power & - & - & - & - & - & - \\
\hline \multirow{2}{*}{\multicolumn{7}{|c|}{ Matinswille Cug off Jom 36}} \\
\hline & & & & 84,595 & - & - \\
\hline $\begin{array}{l}\text { Fadl Power } \\
\text { Rutford Coty ofthen } 30\end{array}$ & 203.153 & $3,491, \pi 57$ & I.191 & 84,594 & - & $\overline{-}$ \\
\hline \multicolumn{7}{|l|}{ Rutiond Coy of the 30} \\
\hline $\begin{array}{l}\text { Nonfing Powr } \\
\text { Tow. Powe }\end{array}$ & 30,100 & $\begin{array}{l}30,572,175 \\
10572175\end{array}$ & $\bar{z}$ & $\bar{z}$ & 二 & - \\
\hline sitem on oflun 30 & & & - & - & 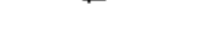 & - \\
\hline Fim poing & 148.852 & 125014,841 & 1.618 & 116,318 & - & - \\
\hline Topt Powat & 148,852 & 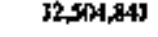 & 1.688 & $116,3] 8$ & - & - \\
\hline Walmetod & & & & & & \\
\hline Finu Paws & - & -7 & 172,826 & $5,215,286$ & - & - \\
\hline Toted Fonver & - & - & $172.62 x$ & 3219246 & - & - \\
\hline
\end{tabular}


Table 29. Electricky Purchases by Municipal Utilltis, Fiscal Year 1994 (Conlmued)

\begin{tabular}{|c|c|c|c|c|c|c|}
\hline \multirow{3}{*}{ 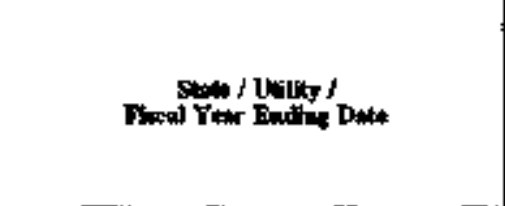 } & \multicolumn{6}{|c|}{ Soorte of Electrickty } \\
\hline & \multicolumn{2}{|c|}{ Cosperetyr } & \multicolumn{2}{|c|}{ Oatert 1} & \multicolumn{2}{|c|}{ Todd } \\
\hline & $\begin{array}{l}\text { Purchewk } \\
\text { (thookind kWh) }\end{array}$ & $\begin{array}{c}\text { Dons } \\
\text { (dolles) }\end{array}$ & 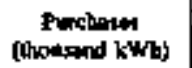 & $\underset{(\operatorname{dol} l \ln x)}{\cos }$ & $\begin{array}{l}\text { Ponchunes } \\
\text { (dinginand kwhy }\end{array}$ & $\underset{\text { (dollews) }}{\operatorname{Com}}$ \\
\hline \multicolumn{7}{|l|}{ Teney } \\
\hline Fim Pown - & - & - & - & - & 206,610 & $14,493,24$ \\
\hline 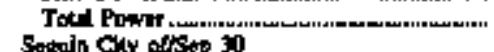 & تـ & - & - & - & 366,670 & $14,493,244$ \\
\hline Fim Porra & - & - & - & - & 200,732 & $8,18, \mathrm{Al}$ \\
\hline Monfilm Power & - & - & - & $-57,898$ & - & $-57,888$ \\
\hline Todd Powter - & - & - & $=$ & -57.888 & 205,732 & $8,123,525$ \\
\hline 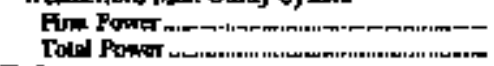 & $\begin{array}{l}47,413 \\
47,413\end{array}$ & $\begin{array}{l}2,390,176 \\
2,390,176\end{array}$ & $\begin{array}{l}179,150 \\
179,150 \mid\end{array}$ & 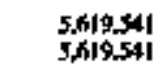 & $\begin{array}{l}22 \times 36393 \\
226363\end{array}$ & $2,000,717$ \\
\hline \multirow{2}{*}{\multicolumn{7}{|c|}{ Desil }} \\
\hline & & & & & & \\
\hline 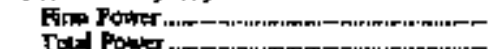 & - & - & - & - & 211,864 & 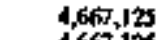 \\
\hline 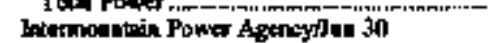 & & & & & & $0,66, \sqrt{2}$ \\
\hline Norefim Prow & - & - & - & - & 200 & $8,760,410$ \\
\hline Fot & - & - & - & - & 200 & s,7falone \\
\hline Laten Ciy oftum 30 & & & & & & \\
\hline 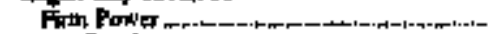 & - & - & 100.2856 & $2,110,110$ & 230,952 & $7,496,824$ \\
\hline 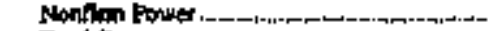 & - & - & - & & 40,315 & $1,020,197$ \\
\hline Told Ponet .... & 一 & - & 11732166 & 2.110 .116 & 27.26 & Asis.038 \\
\hline Momy Cily ofyman 30 & & & & & & \\
\hline 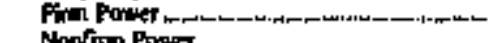 & - & $=$ & 131,199 & $2,342 \times 10$ & 131,199 & $2,3+2,500$ \\
\hline 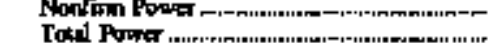 & $=$ & $\bar{z}$ & - & $\overline{2} 40 \mathrm{sen}$ & 165.968 & 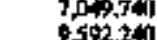 \\
\hline Prowe Chy Corpdon 3n & & & (18) & & & \\
\hline Fin Poner & - & - & - & $\rightarrow$ & $569 \curvearrowleft 7$ & $22,718,956$ \\
\hline 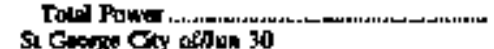 & - & - & - & - & 569,607 & $2,718,956$ \\
\hline 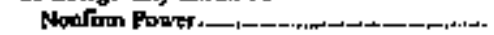 & $\longrightarrow$ & - & 100,726 & $1,957,961$ & 345301 & $12,146,65$ \\
\hline 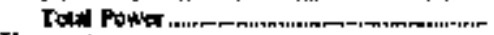 & - & - & I90,726 & 1.967.981 & 125. & $J 2.145,615$ \\
\hline \multirow{2}{*}{\multicolumn{7}{|c|}{$\begin{array}{l}\text { Vaimont } \\
\text { Boprlnaton City of }\end{array}$}} \\
\hline & & & & & & \\
\hline 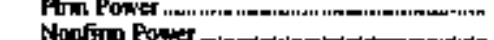 & $=$ & $\bar{z}$ & 100 & 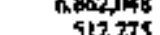 & 7,178 & $13,5 \times 1,172$ \\
\hline 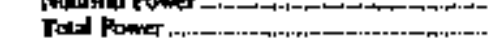 & $=$ & $=$ & 208,5 & 7374,393 & 30,806 & 1470,13 \\
\hline \multirow{2}{*}{\multicolumn{7}{|c|}{ Virelent }} \\
\hline & & & & & & \\
\hline Frm Pongr & - & - & - & - & 2011,752 & $7,636,043$ \\
\hline 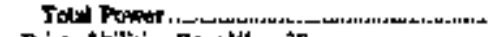 & - & - & - & - & 201,752 & $7,636,043$ \\
\hline \multicolumn{7}{|l|}{ 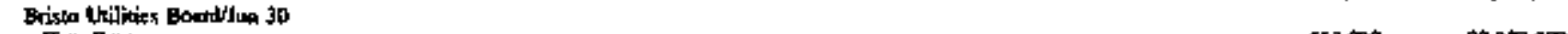 } \\
\hline 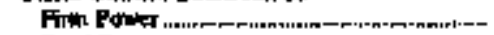 & - & - & - & - & 553,109 & $22,257,272$ \\
\hline Told Powar & - & - & - & $\rightarrow$ & 593,099 & $22,287,272$ \\
\hline \multicolumn{6}{|l|}{ Danvile Cly oflum 30} & \\
\hline Pron Pow & 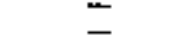 & $\bar{z}$ & $\bar{z}$ & $\rightarrow_{26,400}$ & -102000 & $36,+4,4,487$ \\
\hline twor ........-_-_..............--_-_... & - & - & - & 26,450 & 42,568 & $30,450,225$ \\
\hline \multicolumn{7}{|l|}{ Huminombery Cily offinn 70} \\
\hline 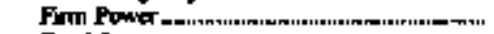 & - & - & - & ــ & 536,868 & $23,294,5 n$ \\
\hline 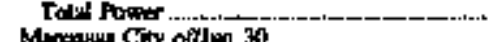 & - & $=$ & - & - & 536,868 & $20,294,572$ \\
\hline 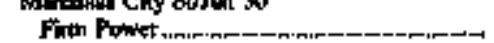 & - & - & $289,7 \$ 0$ & 11466484 & 287,700 & 11464 t4 \\
\hline 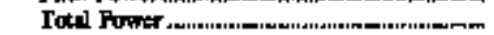 & - & - & 287,780 & $11,46,6,434$ & 287.74 & 11,40644 \\
\hline \multicolumn{7}{|l|}{ Msimuwllb Cxy ofr lon 30} \\
\hline From Power & - & - & - & - & 214,344 & $7,57,452$ \\
\hline Tochl follor & - & - & - & - & $2 I+344$ & $7,57,48$ \\
\hline \multicolumn{7}{|l|}{ Rudrod City afine 39} \\
\hline 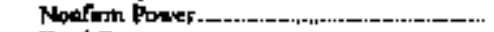 & - & - & - & - & 300,01 & $0,572,175$ \\
\hline 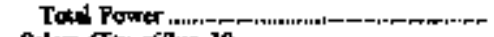 & - & - & - & - & 3000,101 & $10,572,115$ \\
\hline \multicolumn{7}{|l|}{ Siler Cy dijun 30} \\
\hline Fin Power & - & - & - & $\rightarrow$ & 350,490 & $32,627,939$ \\
\hline Toed Rown & - & - & - & - & 350,490 & $12,621,159$ \\
\hline \multicolumn{7}{|l|}{ 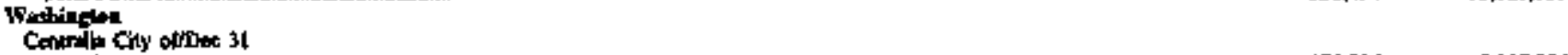 } \\
\hline Frim Pawer & - & - & - & - & 172,836 & $5,215,286$ \\
\hline 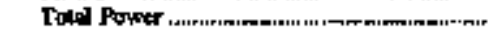 & - & - & - & - & 172826 & $5,215,216$ \\
\hline
\end{tabular}

Ses wotes ind footnoter st and of tobte 
Table 29. Electrictry Purchases by Municlpul Utilities, Fiscal Year 1994 (Continned)

\begin{tabular}{|c|c|c|c|c|c|c|}
\hline \multirow{3}{*}{ Stals / Undy I } & \multicolumn{6}{|c|}{ Sowne of Eloctincky } \\
\hline & \multicolumn{2}{|c|}{ Inrevto Opmed } & \multicolumn{2}{|c|}{ Foderal } & \multicolumn{2}{|c|}{ 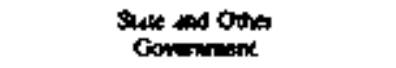 } \\
\hline & 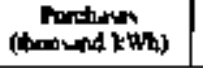 & 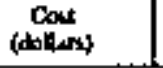 & 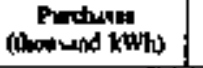 & (doditu & 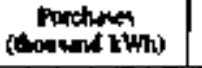 & $\begin{array}{c}\text { Con } \\
\text { (dofin) }\end{array}$ \\
\hline \multicolumn{7}{|l|}{ Wuataytom } \\
\hline \multicolumn{6}{|l|}{ 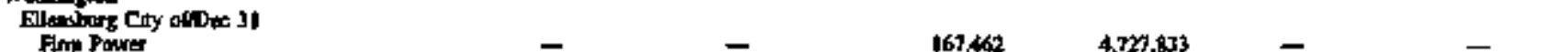 } & \\
\hline Totul Ponet & - & - & 367,462 & $4,727,803$ & - & - \\
\hline \multicolumn{7}{|l|}{ Puts Argules Coy aroec 31} \\
\hline Fim Power & - & - & 699,165 & $17,535,353$ & - & - \\
\hline \multicolumn{7}{|l|}{ Rrchlend Culy ofderc 30} \\
\hline Fim Pover & - & - & 62T,962 & $10,287,253$ & - & - \\
\hline Nomfim Powar & - & - & - & $=$ & - & - \\
\hline Tot Paw & \multicolumn{6}{|c|}{ Seink Cxy aftoes } \\
\hline 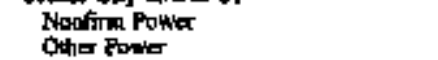 & $\begin{array}{l}237,1564 \\
28,655\end{array}$ & $4,123,526$ & $\begin{aligned} 2,44,736 \\
+8,025\end{aligned}$ & $52,901,932$ & $\begin{array}{l}595,078 \\
-2 \times 112\end{array}$ & 2,502007 \\
\hline Tot Power & 24,022 & $4,123,526$ & $2,522,761$ & $12,901,902$ & 568866 & 2,50200 \\
\hline Anser Poum & - & - & $2,2,7,72$ & $56,973,652$ & - & - \\
\hline $\begin{array}{l}\text { Noerim Pown } \\
\text { Tocat Power }\end{array}$ & $\begin{array}{l}\text { FI, } 565 \\
61,365\end{array}$ & $\begin{array}{l}1,177,316 \\
1,177,316\end{array}$ & $\begin{array}{r}297,706 \\
2518,488\end{array}$ & $\begin{array}{r}9,26,509 \\
65,165,461\end{array}$ & $\begin{array}{l}295,895 \\
791,895\end{array}$ & $\begin{array}{l}1,991,670 \\
1,931, \leqslant 70\end{array}$ \\
\hline \multicolumn{7}{|l|}{ Woctum } \\
\hline Fimm Power & - & - & - & - & - & - \\
\hline \multicolumn{7}{|l|}{ | I } \\
\hline Fim Pown & - & $\rightarrow$ & - & - & - & - \\
\hline Nonfirm Powet & - & - & - & - & - & - \\
\hline Tatsi Fowrer & - & - & - & - & - & - \\
\hline \multicolumn{7}{|l|}{ 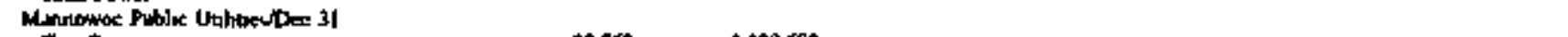 } \\
\hline Fimon Prow & $\begin{aligned} 92,759 \\
03502\end{aligned}$ & $3,129,512$ & - & $=$ & - & - \\
\hline $\begin{array}{l}\text { Nonfimn Power } \\
\text { Toted Power }\end{array}$ & 228,581 & $5,693,392$ & - & $\overline{-}$ & $\overline{-}$ & - \\
\hline \multicolumn{7}{|l|}{ 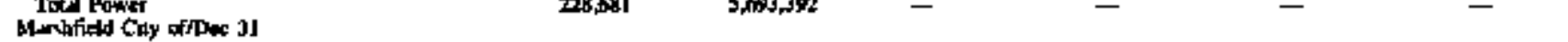 } \\
\hline Anm Power & 320,870 & $9,379,153$ & - & - & - & - \\
\hline 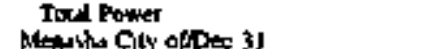 & 120,570 & $9,779,113$ & - & - & - & - \\
\hline Fim Power & - & - & - & - & - & - \\
\hline Toted Power & - & - & - & - & - & $\overline{-}$ \\
\hline \multicolumn{7}{|l|}{ New Loodar Cry ofldec 31} \\
\hline $\begin{array}{l}\text { Fm Pownes } \\
\text { Totd Power }\end{array}$ & $=$ & $\vec{F}$ & $E$ & - & - & - \\
\hline \multicolumn{7}{|l|}{$\begin{array}{l}\text { Totad Power } \\
\text { Oconornorroc Cuky offDec 3J }\end{array}$} \\
\hline Fim Powner & - & - & _. & - & - & - \\
\hline Totsl Power & - & - & - & - & - & - \\
\hline \multicolumn{7}{|l|}{ Phymoos Cuy offDex 31} \\
\hline Tots Power & 165,360 & 年 & - & $\bar{z}$ & $\bar{z}$ & $\overline{-}$ \\
\hline Reed buter Uthhy Cormmbec 30 & & & & & & \\
\hline Fum Pown & 3E4,B89 & $6,4 \pi, 924$ & - & - & - & - \\
\hline Tots Power & 564,8894 & $0,35,924$ & - & - & - & - \\
\hline 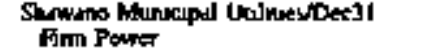 & - & - & - & - & & \\
\hline Totd Ponter & - & $\bar{z}$ & 二 & 二 & $\bar{z}$ & $\bar{z}$ \\
\hline 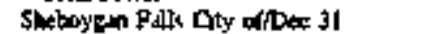 & & & & & & \\
\hline Frut Power & I9n.596 & $6,293,724$ & - & - & - & - \\
\hline Total Powar & 197596 & $6.299 .72 \%$ & - & - & - & - \\
\hline Surgean Bdy Combined Uak & & & & & & \\
\hline Fin Powar & 132,813 & $5,102,450$ & - & - & - & - \\
\hline Tokl Porter & 132,813 & $5,108,450$ & - & - & - & - \\
\hline Frm Power & - & - & - & - & - & - \\
\hline Fold Pourer & - & - & - & - & - & - \\
\hline 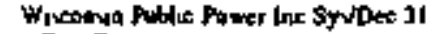 & & & & & & \\
\hline & $1,599,502$ & $0,0,234,095$ & - & - & - & - \\
\hline Noaftim Power & 757.18t & $12,000,734$ & 1,250 & $\operatorname{lin}_{1 \rightarrow 610}$ & 1,258 & I44 145 \\
\hline Wow power & $2,25 n, d 83$ & $n, 60,035$ & $\sqrt{23}$ & $1, \Delta, 0$ & & \\
\hline Fith Powet & 2007.902 & $6,840,105$ & - & - & - & - \\
\hline Toldal Poyer & 203.942 & $6,840,105$ & - & - & - & - \\
\hline
\end{tabular}

See nower ind foominger at the of wable 
Table 29. Flectriciky Porchases by Mmicipal Utilities, Fiscal Year 1994 (Continmed)

\begin{tabular}{|c|c|c|c|c|c|c|}
\hline \multirow{3}{*}{ 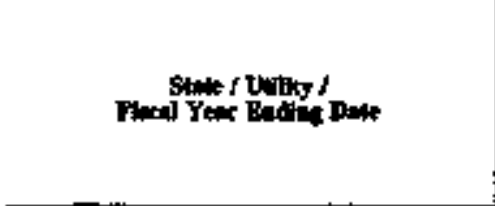 } & \multicolumn{6}{|c|}{ Somper of Eletrifilty } \\
\hline & \multicolumn{2}{|c|}{ Coopentix } & \multicolumn{2}{|c|}{ Othell } & \multicolumn{2}{|c|}{ Total } \\
\hline & $\begin{array}{l}\text { Paruluses } \\
\left.\text { (thootend } k W_{b}\right)\end{array}$ & 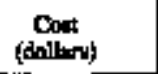 & 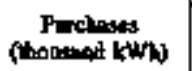 & $\begin{array}{c}\text { Con } \\
\text { (dollery) }\end{array}$ & $\begin{array}{l}\text { Puchnow } \\
\text { (thoosand kWhy }\end{array}$ & $\underset{\text { (dot } 12 x y)}{\cos x}$ \\
\hline \multicolumn{7}{|l|}{ Thellateon } \\
\hline Fin Pootr-_- & - & 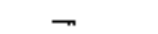 & - & - & 167,462 & $4,727,830$ \\
\hline 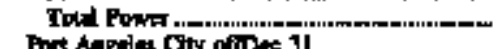 & - & - & - & - & 167,462 & $4,727,833$ \\
\hline 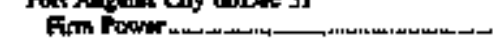 & - & - & $1,2,206$ & $17,9 \mid 3$ & St,05] & $17,53,266$ \\
\hline 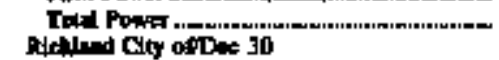 & - & - & 11,136 & 17,913 & 651,051 & $17,553,266$ \\
\hline Fin Power______._. & - & - & - & - & 602962 & $18,28,299$ \\
\hline Nolufing Bow & - & - & 19,778 & 20.06 & 19,378 & 20,405 \\
\hline Towd Pown . . & $\overline{-}$ & - & 19,375 & 20,406 & 647364 & $18,30,558$ \\
\hline 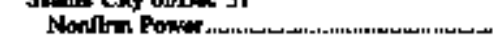 & - & - & 314235 & 2959,846 & $0,717,215$ & $89,126,031$ \\
\hline Other Pow & - & - & $-5,163$ & & 75,706 & \\
\hline 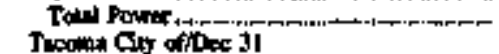 & - & - & 309012 & $29,598,546$ & $3,260,521$ & $89,226,331$ \\
\hline Fin Pown & - & - & - & - & $272, \pi 2$ & $56,778,952$ \\
\hline Noofim Pow & - & - & 524,247 & 736,460 & 971,353 & $10,3,955$ \\
\hline \multirow{2}{*}{\multicolumn{7}{|c|}{ 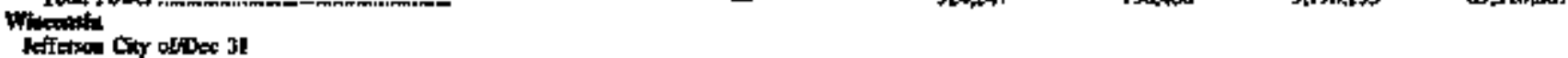 }} \\
\hline & & & & & & \\
\hline 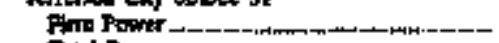 & - & 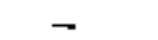 & 131818 & $1,132,597$ & 137,818 & $5,593,597$ \\
\hline 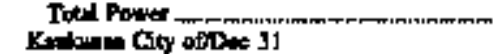 & - & 一 & 137,818 & 5.133 .597 & 137,818 & 5.113597 \\
\hline 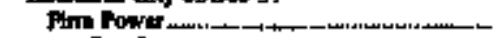 & - & - & SIR,geB & $\mathbf{3 7 , 6 5 6 , 1 2 7}$ & 518,983 & $17,026,127$ \\
\hline Nopofrom Pont & - & - & 2,634 & 26,567 & 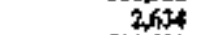 & 2656 \\
\hline TdA Powd , & 一 & - & 52162 & $17,660,694$ & setifti & $17,662,694$ \\
\hline \\
\hline 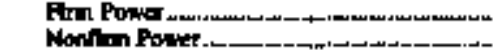 & 二 & $\bar{z}$ & $\bar{z}$ & 二 & $\begin{array}{r}92,759 \\
135,922\end{array}$ & $3,129,012$ \\
\hline Towl Pown & - & - & $=$ & - & 20881 & $5,690,392$ \\
\hline \multicolumn{7}{|l|}{ Mersoivd Cily ofdes 31} \\
\hline 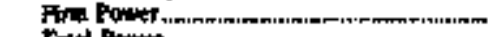 & - & - & - & - & 32080 & ד, \\
\hline \multicolumn{7}{|l|}{ Memax City of Dec JJ } \\
\hline Prm Poner & - & - & 54,900 & $18,645,899$ & S46,9:An & $18,643,899$ \\
\hline Tolal Powner & - & - & 346,900 & $12,645,899$ & 546900 & IB,645,899 \\
\hline \multicolumn{7}{|l|}{ Now Lomdon Criby of Dese 31} \\
\hline 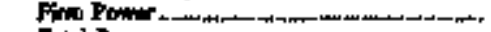 & - & - & 164,473 & $6,979,116$ & 166,473 & $6,379,1 \mathrm{tg}$ \\
\hline Totil Power & 一 & 一 & 166,473 & $4,379,116$ & 566,473 & $6,379,116$ \\
\hline \multicolumn{7}{|l|}{ 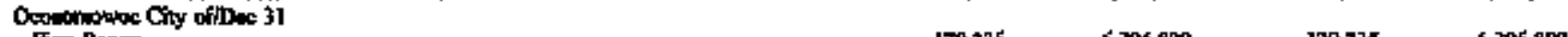 } \\
\hline 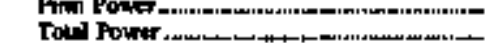 & $\bar{z}$ & 二 & 170,235 & 6.9690 & 170.235 & $6,394,891$ \\
\hline \multicolumn{7}{|l|}{ Plywanh Chy ofpec 34} \\
\hline Find Ponor & - & - & - & ـ & 355,360 & $5,593,164$ \\
\hline Toen Font & - & - & - & - & 165,760 & 5513,164 \\
\hline \multicolumn{7}{|l|}{ Readstorge Ut'Hly Commidese 30} \\
\hline 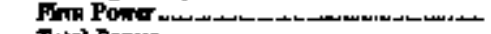 & - & - & - & - & IEA,BSE & $6,307,924$ \\
\hline \multicolumn{7}{|l|}{ 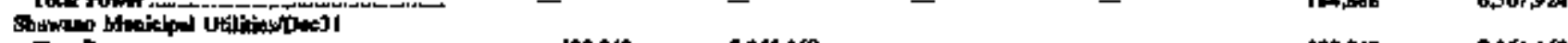 } \\
\hline 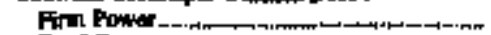 & 192,042 & $1,251,169$ & - & $=$ & 192012 & $7,251,169$ \\
\hline \multicolumn{7}{|l|}{ 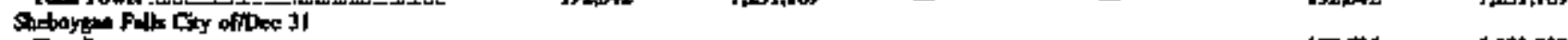 } \\
\hline Fith bowe no & - & - & - & - & 197,596 & $6.293_{T}, 728$ \\
\hline \multirow{2}{*}{\multicolumn{7}{|c|}{ sumgen Bay Coutired thits }} \\
\hline & & & & & & \\
\hline 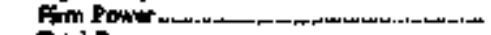 & - & - & - & - & 132,813 & $3,108,450$ \\
\hline Totol Power & - & - & - & - & J32,813 & $5,102,450$ \\
\hline Fith Pown, & - & - & 157,190 & 4015389 & 157.180 & 6,005330 \\
\hline Todal Power & 一 & - & $137+180$ & 6015,389 & I57.ID & 60016358 \\
\hline 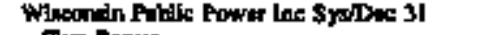 & & & & & & \\
\hline 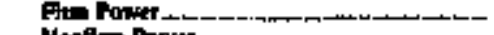 & - & 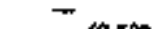 & $4,49]$ & 2936,073 & $1,543,993$ & $63,190,168$ \\
\hline Nanfim Power_-_, & $\begin{array}{l}3,115 \\
5,111\end{array}$ & $\begin{array}{l}68,352 \\
68532\end{array}$ & $\begin{array}{l}2,125 \\
6.16\end{array}$ & 2079,279 & $\begin{array}{r}767,129 \\
231+128\end{array}$ & $\begin{array}{l}12,14,5,519 \\
75,334,647\end{array}$ \\
\hline wiscondin Repids W WEL Comm & & & & & & \\
\hline 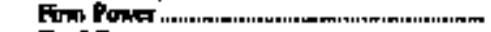 & - & - & - & - & 207,942 & $6.04,105$ \\
\hline 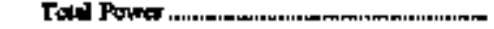 & - & - & - & - & 207,962 & $6,809,105$ \\
\hline
\end{tabular}


Table 29. Electricicy Purchases by Municipal Uitilities, Fiscal Year 1994 (Continued)

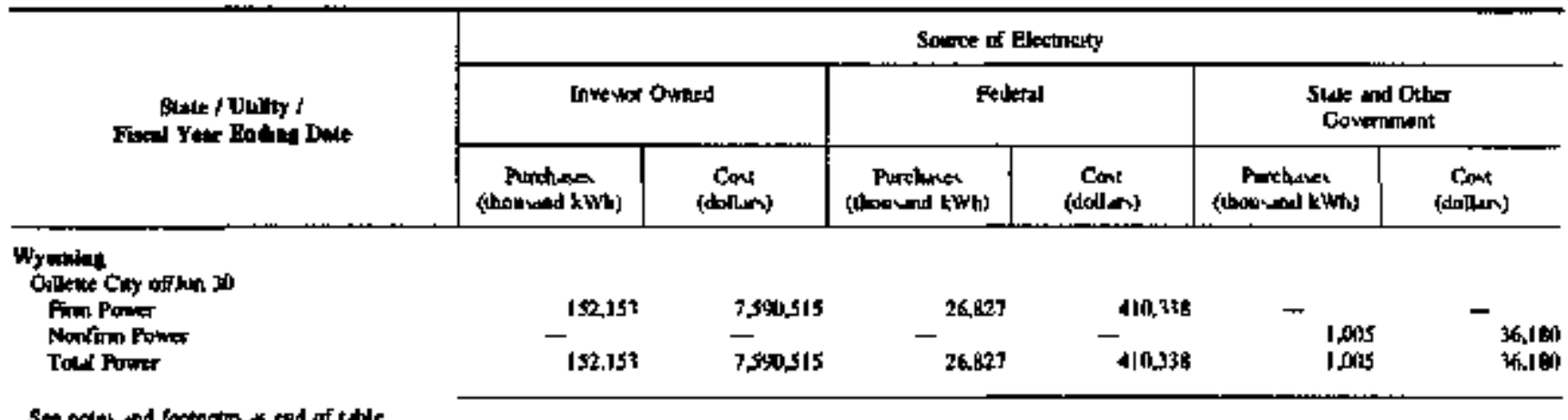

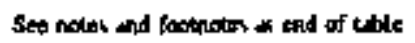

Table 29. Electricity Purchases by Municipal Utilities, Fisenl Year 1994 (Continued)

\begin{tabular}{|c|c|c|c|c|c|c|}
\hline \multirow{3}{*}{ 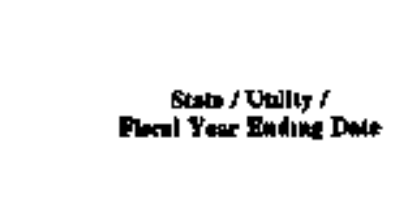 } & \multicolumn{6}{|c|}{ Somse of electinctity } \\
\hline & \multicolumn{2}{|c|}{ Conpyertius } & \multicolumn{2}{|c|}{ Othat } & \multicolumn{2}{|c|}{ Toks } \\
\hline & 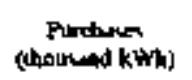 & 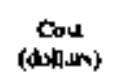 & 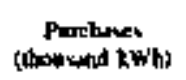 & $\underset{\text { (dolldent }}{C \infty}$ & 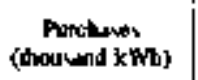 & 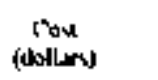 \\
\hline \multicolumn{7}{|l|}{ Fyroming } \\
\hline 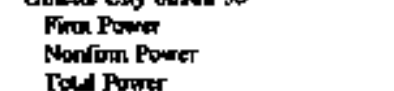 & $\frac{-}{-}$ & $=$ & $\frac{-}{-}$ & $\begin{array}{l}- \\
-\end{array}$ & $\begin{array}{r}178,080 \\
1,005 \\
179,983\end{array}$ & $\begin{array}{r}8,0001850 \\
76,180 \\
8037,003\end{array}$ \\
\hline
\end{tabular}

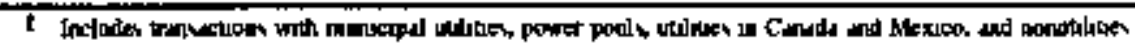

-Mon Appladible

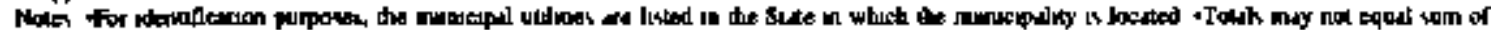

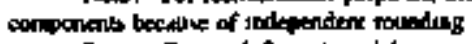

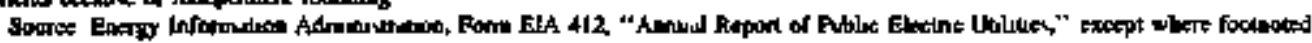


Table 30. Electricity Sales for Resale by Mnnicipal Utilities, Fiscal Year 1994

\begin{tabular}{|c|c|c|c|c|c|c|}
\hline \multirow{3}{*}{$\begin{array}{l}\text { Strie / Utility f } \\
\text { Plseal Year Fudies bowe }\end{array}$} & \multicolumn{6}{|c|}{ 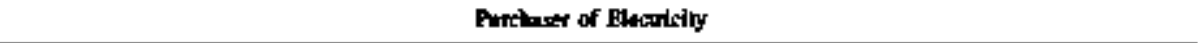 } \\
\hline & \multicolumn{2}{|c|}{ Innexpon Ourned } & \multicolumn{2}{|c|}{ Faderd } & \multicolumn{2}{|c|}{$\begin{array}{l}\text { Stive and Other } \\
\text { Gowernenl }\end{array}$} \\
\hline & 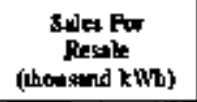 & $\begin{array}{l}\text { Revenwe } \\
\text { (dollers) }\end{array}$ & $\begin{array}{c}\text { Solven Ror } \\
\text { Retulk } \\
\text { (thousted EWh) }\end{array}$ & $\begin{array}{l}\text { Reverane } \\
\text { (dolden) }\end{array}$ & 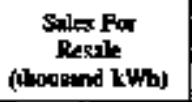 & $\begin{array}{l}\text { Revenue } \\
\text { (dollors) }\end{array}$ \\
\hline \multicolumn{7}{|l|}{ Alybant: } \\
\hline 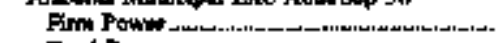 & - & - & - & - & - & - \\
\hline 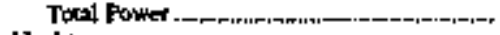 & - & - & - & - & - & - \\
\hline \multicolumn{7}{|l|}{ Alesk } \\
\hline 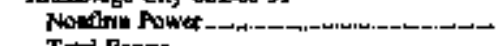 & - & - & - & - & - & $=$ \\
\hline \multicolumn{7}{|l|}{ Arthond } \\
\hline \multicolumn{7}{|l|}{ Beser Ciky off In 30} \\
\hline 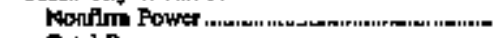 & - & $\rightarrow$ & - & - & - & - \\
\hline Total Power -_-_-_-_-_-_-_-_-_-_-_-_-_-_ & - & - & - & - & - & - \\
\hline \multicolumn{7}{|l|}{ Arkanses } \\
\hline Fimn Power ......................... & - & - & - & - & - & - \\
\hline 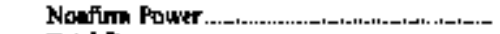 & - & - & - & - & - & - \\
\hline Jotal Power & - & - & - & - & - & - \\
\hline \multicolumn{7}{|l|}{ 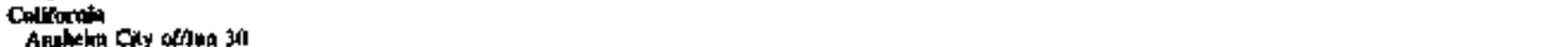 } \\
\hline 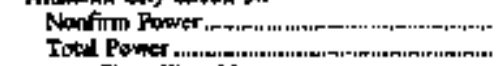 & $\begin{array}{l}713,937 \\
737.917\end{array}$ & $\begin{array}{l}13,856,19106 \\
13,836,0 \times 10\end{array}$ & 429 & IS, onon & 6,894 & $\begin{array}{l}147,000 \\
149,0100\end{array}$ \\
\hline Apase Cly offlon 30 & & & & & & \\
\hline Firm Powner & 120,130 & $2,944,834$ & - & - & - & - \\
\hline \multicolumn{7}{|l|}{ Burbonk City of Jin 30} \\
\hline Pim Powter & - & - & - & - & - & $m$ \\
\hline Nooflno Foner & 1,353 & 2906 & - & - & $\mathbf{2 , 2 3 2}$ & 4:, 316 \\
\hline Total Power & $1 \leqslant 5,3$ & 29,066 & - & - & $\mathbf{2 , 2 3 2}$ & $4+376$ \\
\hline \multicolumn{7}{|l|}{ 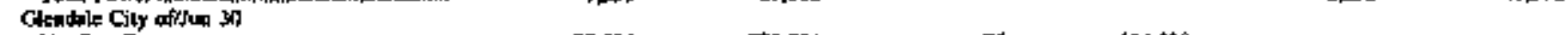 } \\
\hline Nanfim Foprer & 38,086 & 73,534 & 76 & 114,020 & - & - \\
\hline \multicolumn{7}{|l|}{ 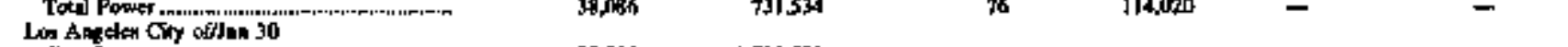 } \\
\hline 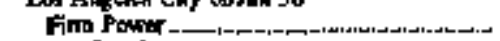 & $\pi, 888$ & $3,736,533$ & - & - & - & - \\
\hline Nodfon Prwer & 571250 & Q,407,605 & 130.931 & 2946,04 & 62,516 & $1,268,0 \mathrm{NB}$ \\
\hline \multicolumn{7}{|l|}{ Merepalluan Water Dlut/Jun 30} \\
\hline Noafing Porter & 136,213 & $6,789,694$ & - & - & 153,367 & 6,995,452 \\
\hline \multicolumn{6}{|l|}{ Pundera cuy ơdoun yo } & K.0.5.452 \\
\hline Fing Powtr-_. & - & - & - & - & - & - \\
\hline Nowim Rawer & 16,509 & 205,769 & - & - & $=$ & - \\
\hline Tod I Power & 16,509 & 298,768 & $\longrightarrow$ & - & - & - \\
\hline \multicolumn{7}{|l|}{ 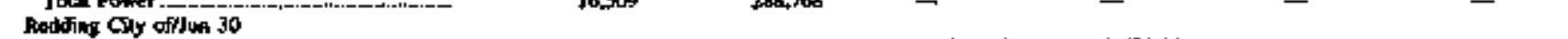 } \\
\hline Nonfim hower................. & - & - & 85,234 & $2,571,375$ & - & - \\
\hline 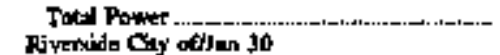 & - & 一 & 81,234 & $2,571,775$ & - & - \\
\hline & 198894 & $2,15 \$$, & - & - & - & - \\
\hline 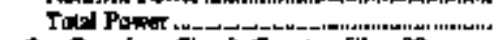 & 100,584 & $2,10,472$ & - & - & - & - \\
\hline 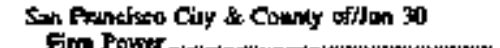 & & & & & & \\
\hline Fim Power & $\bar{z}$ & $\bar{z}$ & - & $\overline{3}$ & $32 x, 555$ & $19,006,858$ \\
\hline 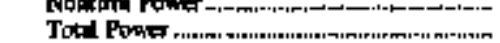 & $=$ & $\bar{z}$ & 120373 & $2,099.110$ & 7008,402 & $2,40,999$ \\
\hline 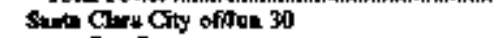 & & & & & & \\
\hline Nonfing Porwer__ & - & - & - & בـ & 30,815 & 851,659 \\
\hline Fod Paw & - & - & - & - & 30,815 & 851.659 \\
\hline 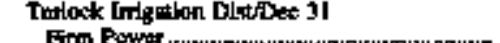 & - & & & 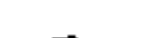 & & \\
\hline 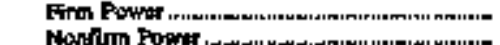 & $3 / 2$ & 1309 & - & $\overrightarrow{3318365}$ & 7 & $\begin{array}{r}68 \\
525,533\end{array}$ \\
\hline 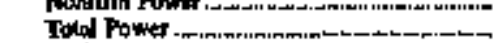 & $\$ 12$ & 158,852 & 122.987 & $3,318,65$ & $\begin{array}{l}13,595 \\
13,995\end{array}$ & 325,610 \\
\hline Coloredy & & & & & & \\
\hline 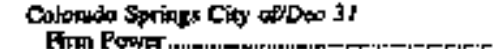 & & & & & & \\
\hline Fin Pow/ & 一 & - & - & - & - & - \\
\hline 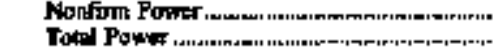 & $\begin{array}{l}43,174 \\
43,74\end{array}$ & 1,056048 & 整 & 15,000 & $=$ & $\bar{z}$ \\
\hline Congectkm & & & & & & \\
\hline Hon Extrte Enty Coophat II & & & & & & \\
\hline 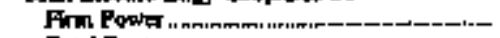 & - & - & - & - & - & - \\
\hline Jomol Paw口 & $m$ & - & - & - & - & - \\
\hline
\end{tabular}

ser war and foctinates at and of table. 
Tahle 30. Electricity Sales for Resale by Municipal Utilities, Fiseal Year 1994 (Continued)

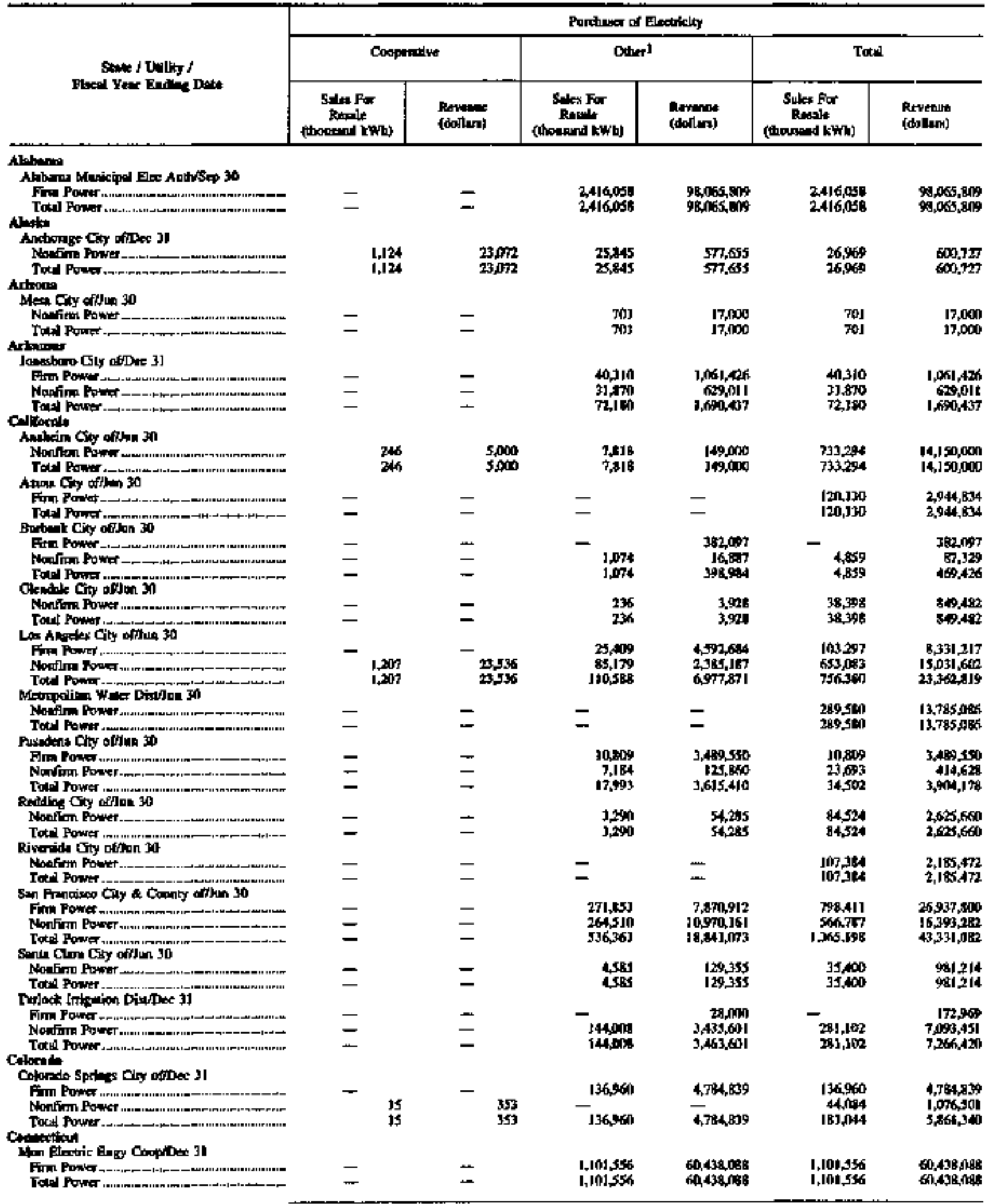

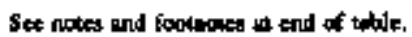


Tabłe 30. Dlectricity Sales for Resale by Municipal Utiltities, Fisen Year $19 \$ 4$ (Contimued)

\begin{tabular}{|c|c|c|c|c|c|c|}
\hline \multirow{3}{*}{ 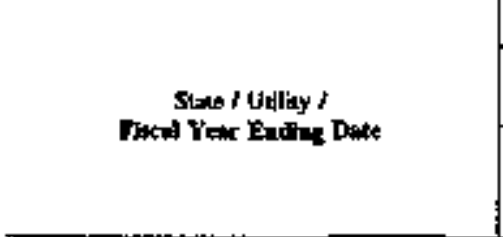 } & \multicolumn{6}{|c|}{ Partaner of Flectisky } \\
\hline & \multicolumn{2}{|c|}{ Invartor-Owaed } & \multicolumn{2}{|c|}{ 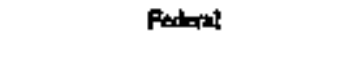 } & \multicolumn{2}{|c|}{ Stote and Other } \\
\hline & 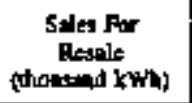 & $\begin{array}{l}\text { Rerenux } \\
\text { (dallisy) }\end{array}$ & 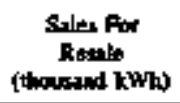 & $\begin{array}{l}\text { Reveose } \\
\text { (ddum) }\end{array}$ & 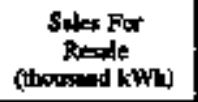 & $\begin{array}{l}\text { Reventus } \\
\text { (dollers) }\end{array}$ \\
\hline \multicolumn{7}{|l|}{$\begin{array}{l}\text { Connewetigat } \\
\text { Groton Cley of then } 30\end{array}$} \\
\hline 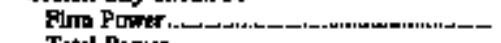 & 9.605 & 400,189 & - & - & - & - \\
\hline $\begin{array}{l}\text { Toted Power } \\
\text { Delnwere }\end{array}$ & 3.605 & $40 \mathrm{k}, 189$ & - & 一 & - & - \\
\hline Dover Chy of he औ & \multicolumn{2}{|c|}{ Delnwere } & & & & \\
\hline 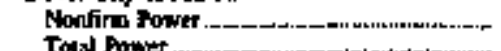 & - & - & - & - & - & - \\
\hline 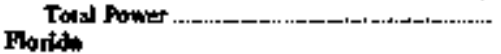 & - & - & - & - & - & - \\
\hline \multicolumn{7}{|l|}{ 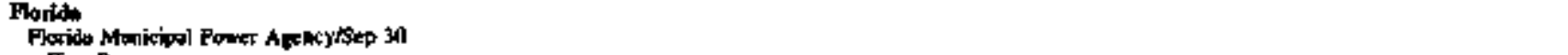 } \\
\hline 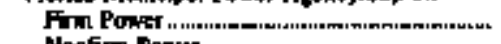 & - & - & - & - & - & - \\
\hline 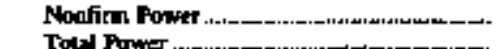 & 219 ग04 & 2538,000 & - & - & - & - \\
\hline \multicolumn{7}{|l|}{ 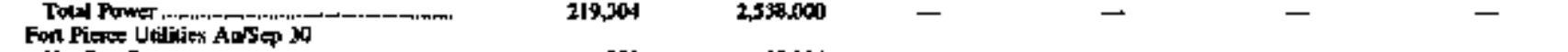 } \\
\hline 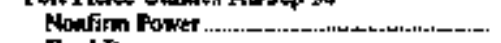 & 953 & 32,214 & - & - & عـ & - \\
\hline Tond Fower & 953 & 32,214 & - & - & - & ع. \\
\hline \multicolumn{7}{|l|}{ 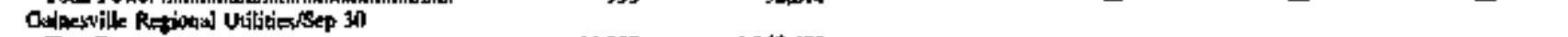 } \\
\hline 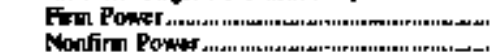 & 60,167 & $1,940,403$ & 一 & - & - & - \\
\hline 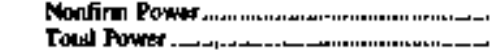 & $\begin{aligned} 69,8049 \\
135,976\end{aligned}$ & $\begin{array}{l}1,590,040 \\
3,591,403\end{array}$ & $\overline{-}$ & - & $\bar{z}$ & $\overline{-}$ \\
\hline \multirow{2}{*}{\multicolumn{7}{|c|}{ 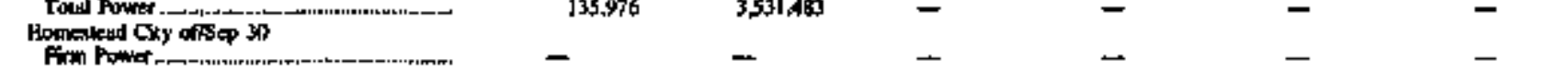 }} \\
\hline & - & - & - & ـ & - & - \\
\hline 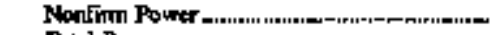 & 336 & J3,B7] & - & - & - & - \\
\hline \multicolumn{7}{|l|}{ 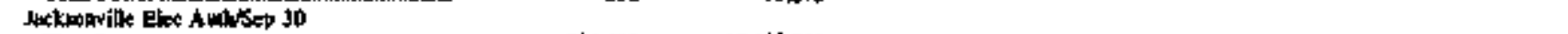 } \\
\hline 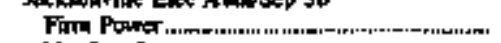 & 304,612 & $15,600,861$ & - & - & - & - \\
\hline Nonflow Power & 75.725 & $2,372,177$ & - & - & - & - \\
\hline \multicolumn{3}{|l|}{ Live Wont Cluy of 30} & & - & - & - \\
\hline Fim Pooner & - & - & - & - & - & - \\
\hline 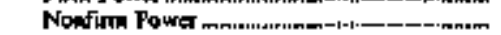 & 3,904 & 80,929 & $\overline{-}$ & $\overline{-}$ & $\bar{z}$ & $=$ \\
\hline Tats Rawer _-_-_-................. & 3.994 & 81,929 & - & - & - & - \\
\hline \multicolumn{7}{|l|}{ Likeland Chy offep 30} \\
\hline 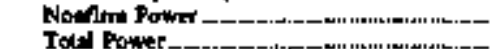 & 57 & $2,7 \rightarrow$ & $\bar{\pi}$ & $\bar{z}$ & $\bar{z}$ & $\bar{z}$ \\
\hline \multicolumn{7}{|l|}{ 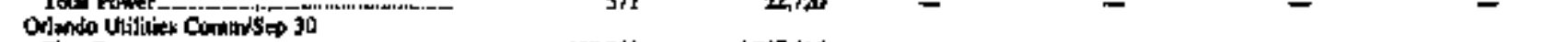 } \\
\hline 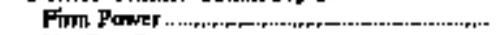 & 129,360 & $4,797,616$ & ـ & عـ & - & - \\
\hline Nonfinm Power ........... & 49,019 & $1,661,890$ & - & - & - & - \\
\hline 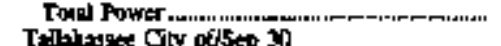 & 176,369 & t, 6 , & - & - & - & - \\
\hline Noarim Porer & 82,337 & 3,gm,noto & - & - & - & - \\
\hline 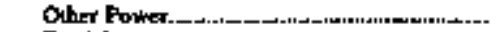 & - & & - & - & - & - \\
\hline Tolal Ponver - & 22,137 & $3,2827, n 006$ & - & - & - & - \\
\hline Yav Beach City of tsep 30 & & & & & & \\
\hline 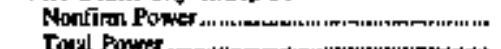 & $\begin{array}{l}1, \sin s \\
4,05\end{array}$ & 109.740 & $\bar{z}$ & - & - & - \\
\hline \multirow{2}{*}{\multicolumn{7}{|c|}{ Gearion Gixy opNor in }} \\
\hline & & & & & & \\
\hline 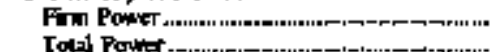 & $10, \pi]$ & 49,100 & - & - & - & - \\
\hline \multirow{2}{*}{\multicolumn{7}{|c|}{ 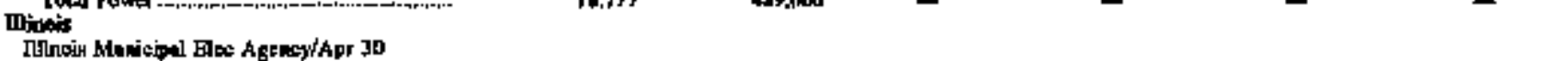 }} \\
\hline & & & & & & \\
\hline Fm Power & - & - & - & - & - & - \\
\hline 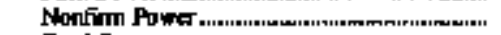 & - & - & - & - & 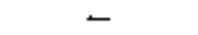 & - \\
\hline 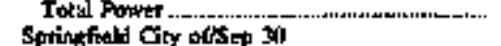 & 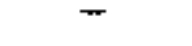 & $m$ & - & - & - & - \\
\hline 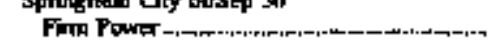 & - & - & - & - & - & - \\
\hline 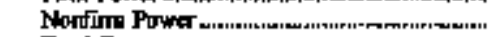 & J7.294 & 297,050 & 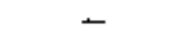 & ع & عـ & - \\
\hline 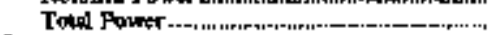 & 1.203 & 297,490 & - & - & - & عـ \\
\hline Indiene & & & & & & \\
\hline 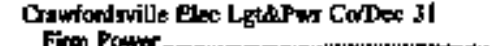 & & & & & & \\
\hline 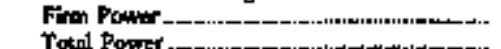 & - & - & - & - & $18, B_{306}$ & $2,040,823$ \\
\hline 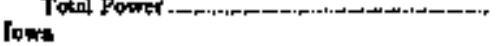 & & & - & 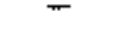 & 18,863 & \\
\hline Alnter coly oflum 30 & & & & & & \\
\hline 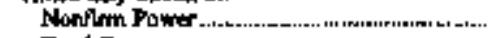 & $2 \sqrt[3]{36}$ & 20.368 & - & - & - & - \\
\hline 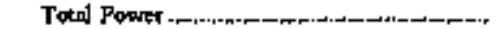 & $2 \mathrm{~m} 6$ & 20,768 & - & - & - & - \\
\hline
\end{tabular}

Ser noter and fbolecter at and of wble. 
Tabje 39. Electricity Sales tor Resale by Mumicipal Utillities, Fiscal Year 1994 (Continued)

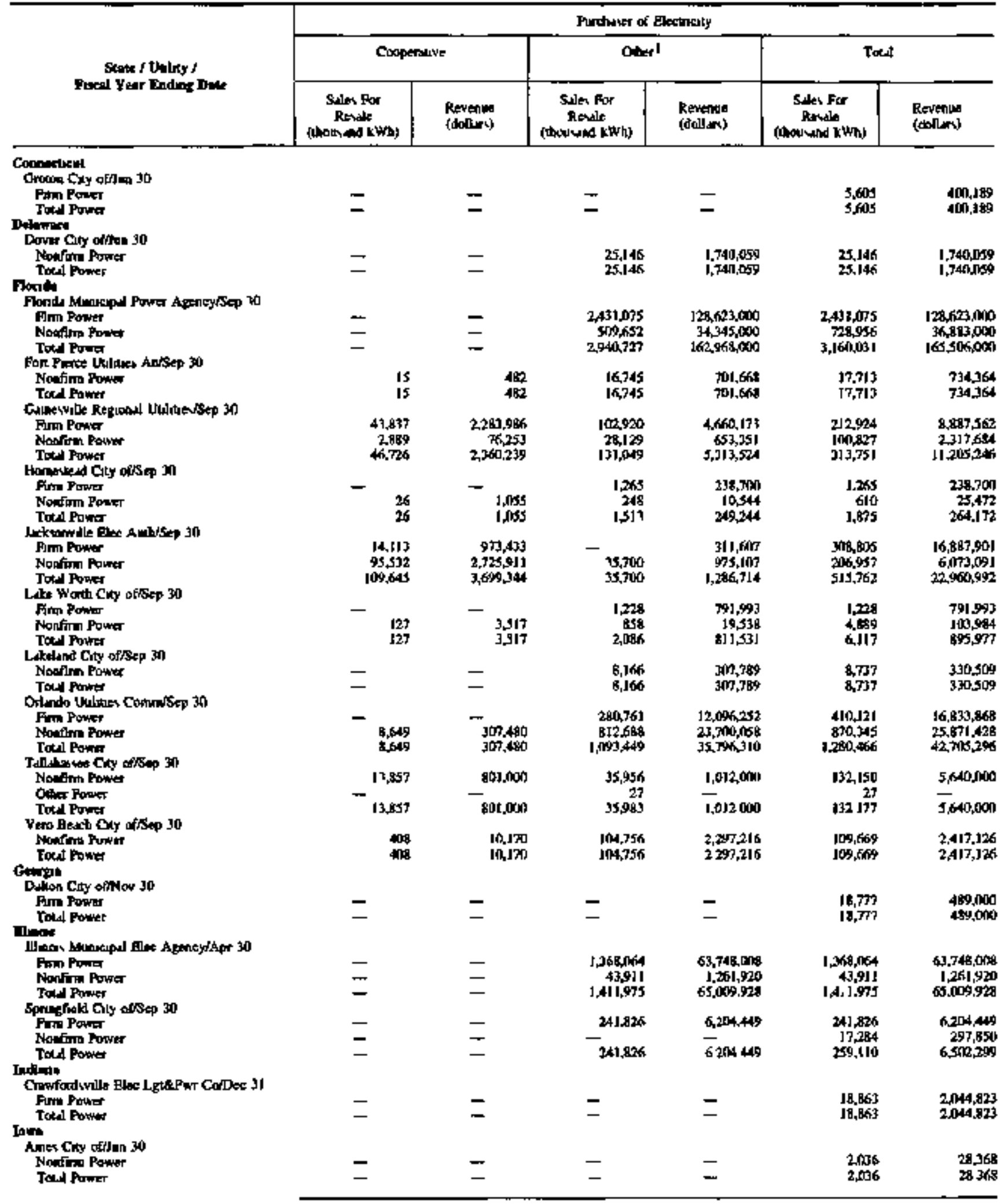

See aoter and feochones at and of uble 
Tabble 30. Elextricity Sales for Resale by Municipal Utilities, Fiscal Year 1994 (Continued)

\begin{tabular}{|c|c|c|c|c|c|c|}
\hline \multirow{3}{*}{ 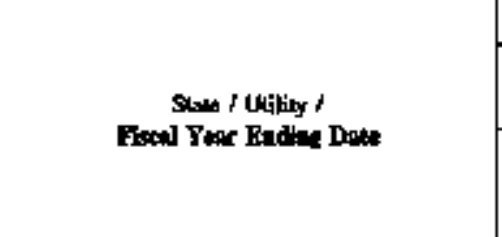 } & \multicolumn{6}{|c|}{ Putroter of Elettesily } \\
\hline & \multicolumn{2}{|c|}{ Inturarowad } & \multicolumn{2}{|c|}{ Foderll } & \multicolumn{2}{|c|}{$\begin{array}{l}\text { Sthte and Dolwer } \\
\text { Govtmument }\end{array}$} \\
\hline & $\begin{array}{c}\text { Sales Par } \\
\text { Restik } \\
\text { athoused kWl }\end{array}$ & $\begin{array}{l}\text { Rovarine } \\
\text { (dolleris) }\end{array}$ & 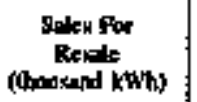 & 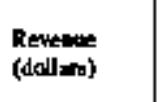 & $\begin{array}{c}\text { Selea For } \\
\text { Resale } \\
\text { (housind } k \text { Wh) }\end{array}$ & $\begin{array}{l}\text { Reveme } \\
\text { (dollons) }\end{array}$ \\
\hline \multicolumn{7}{|l|}{ 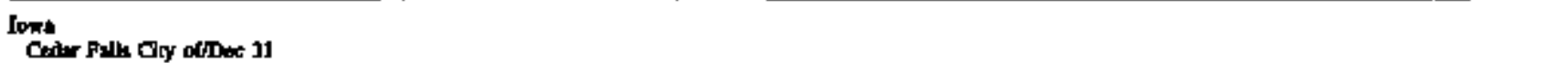 } \\
\hline Nonflen Pow & 12,028 & $2 \pi, 3 M$ & - & - & - & - \\
\hline 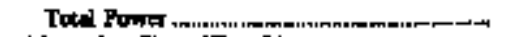 & 12,002 & $2 \pi n, 7 m$ & - & - & - & - \\
\hline Nusemine Cuy aldec 31 & & & & & & \\
\hline 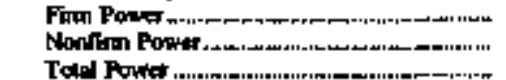 & $\begin{array}{l}- \\
260,8399 \\
260,899\end{array}$ & $\begin{array}{r}661,1531 \\
3,873,521 \\
4534.552\end{array}$ & $-\frac{1,295}{4235}$ & $\begin{array}{r}49,809 \\
49,899\end{array}$ & $-89,122$ & 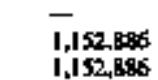 \\
\hline \multirow{2}{*}{\multicolumn{7}{|c|}{ 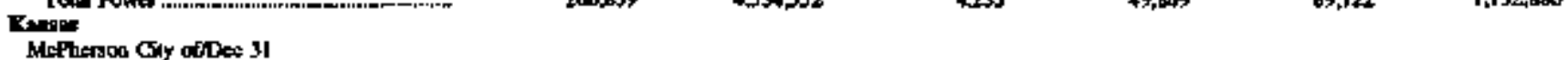 }} \\
\hline & & & & & & \\
\hline 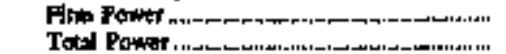 & $\begin{array}{l}3,567 \\
5,567\end{array}$ & $1,863,845$ & $\bar{z}$ & $\bar{z}$ & - & $\overline{-}$ \\
\hline Winfield Clyy offides 31 & & & & & & \\
\hline Fim Pown nom & - & - & - & - & - & - \\
\hline Totd Poner & - & - & - & - & - & - \\
\hline \multicolumn{7}{|l|}{ Bentudty } \\
\hline Nomfirm Prower & - & - & - & - & - & - \\
\hline Totd Powo & - & - & - & - & - & - \\
\hline 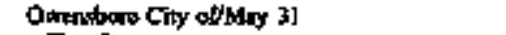 & & & & & & \\
\hline 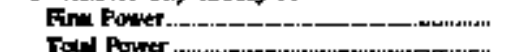 & $1,624,824$ & $21,72,650$ & - & - & - & - \\
\hline 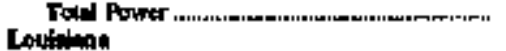 & $1,620,824$ & $21.722 .6 \%$ & - & - & - & - \\
\hline \\
\hline Fin Paser & - & - & - & - & 971921 & 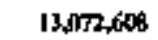 \\
\hline 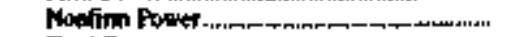 & IIH & 2.897 & - & - & - & - \\
\hline 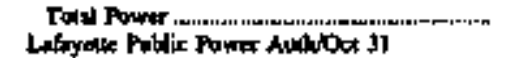 & IIH & 2,897 & - & - & 51,921 & $13,072,698$ \\
\hline 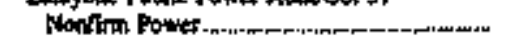 & - & - & - & - & - & - \\
\hline Told Poprer & - & - & - & - & - & - \\
\hline 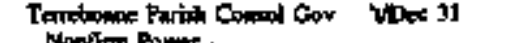 & & & & & & \\
\hline 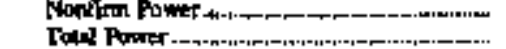 & - & $\overline{-}$ & $\overline{-}$ & $\bar{z}$ & 113,201 & $1,4 \times 8,804$ \\
\hline \multicolumn{7}{|l|}{ 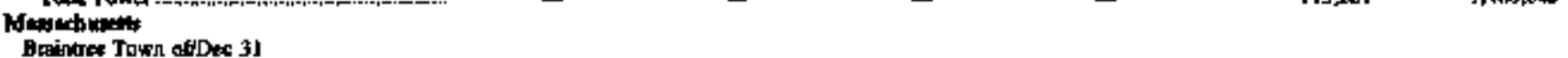 } \\
\hline finm $P(n k$ t & 38,030 & $1.02,807$ & - & - & - & - \\
\hline \multirow{2}{*}{\multicolumn{7}{|c|}{ Chicope Chy affore 31}} \\
\hline & & & & & & \\
\hline 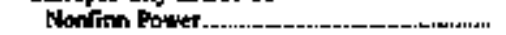 & 1,539 & 39.380 & - & - & - & - \\
\hline 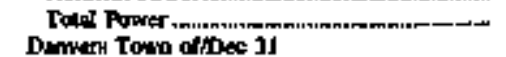 & 1,530 & 19,790 & - & - & - & - \\
\hline 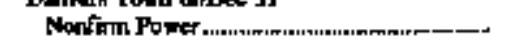 & - & - & - & - & 3,819 & 85,162 \\
\hline Tokel Pownef —_- & - & - & - & - & 3,819 & 86.160 \\
\hline \\
\hline 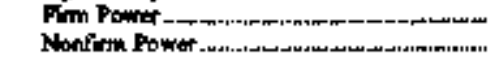 & - & - & $=$ & - & 155 & 41,714 \\
\hline 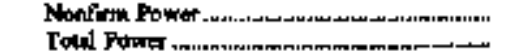 & $\begin{array}{l}1,500 \\
1,500\end{array}$ & $\begin{array}{r}\text { 46,583 } \\
\mathbf{4 6 . 5 8 3}\end{array}$ & $\bar{z}$ & $\overline{-}$ & - 155 & $-{ }_{41,714}$ \\
\hline Bodyobe Gity offDex 31 & & & & & & \\
\hline 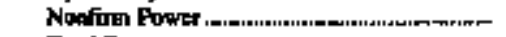 & - & - & - & - & $18, \leqslant 00$ & 440,100 \\
\hline \multirow{2}{*}{\multicolumn{7}{|c|}{ 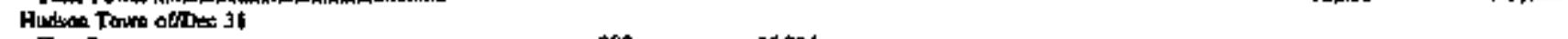 }} \\
\hline & & & & & & \\
\hline 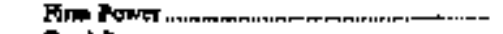 & 290 & 15294 & 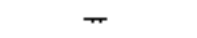 & - & - & - \\
\hline 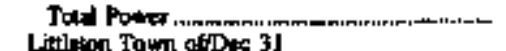 & \multicolumn{6}{|c|}{ Littleson Town ofDec $3 \mathrm{~J}$} \\
\hline 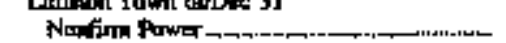 & 9,107 & 207,395 & - & - & 1,479 & 61,139 \\
\hline 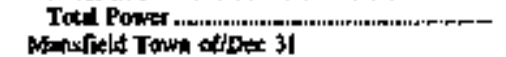 & 9.100 & 217,345 & - & - & 1,479 & fil, 139 \\
\hline Finn Pown- & - & - & - & 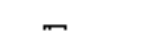 & sen & 901.912 \\
\hline 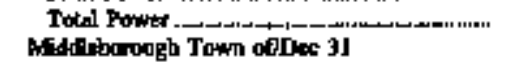 & - & 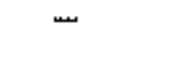 & - & - & 8611 & 90,912 \\
\hline 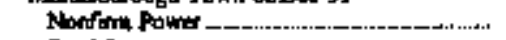 & - & - & - & - & 145 & 34.117 \\
\hline Totel Power are & - & - & - & - & 145 & 34.117 \\
\hline 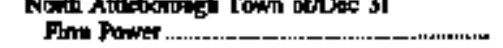 & - & - & - & - & 51 & 33,827 \\
\hline Told Proper & - & - & س & - & si & 33,827 \\
\hline
\end{tabular}

Sen motha bad foctnotes $x$ and of uble. 
Tabje 30. Electriclty Sales for Resale by Mumidipal titjlties, Fiscal Year 1994 (Contkmed)

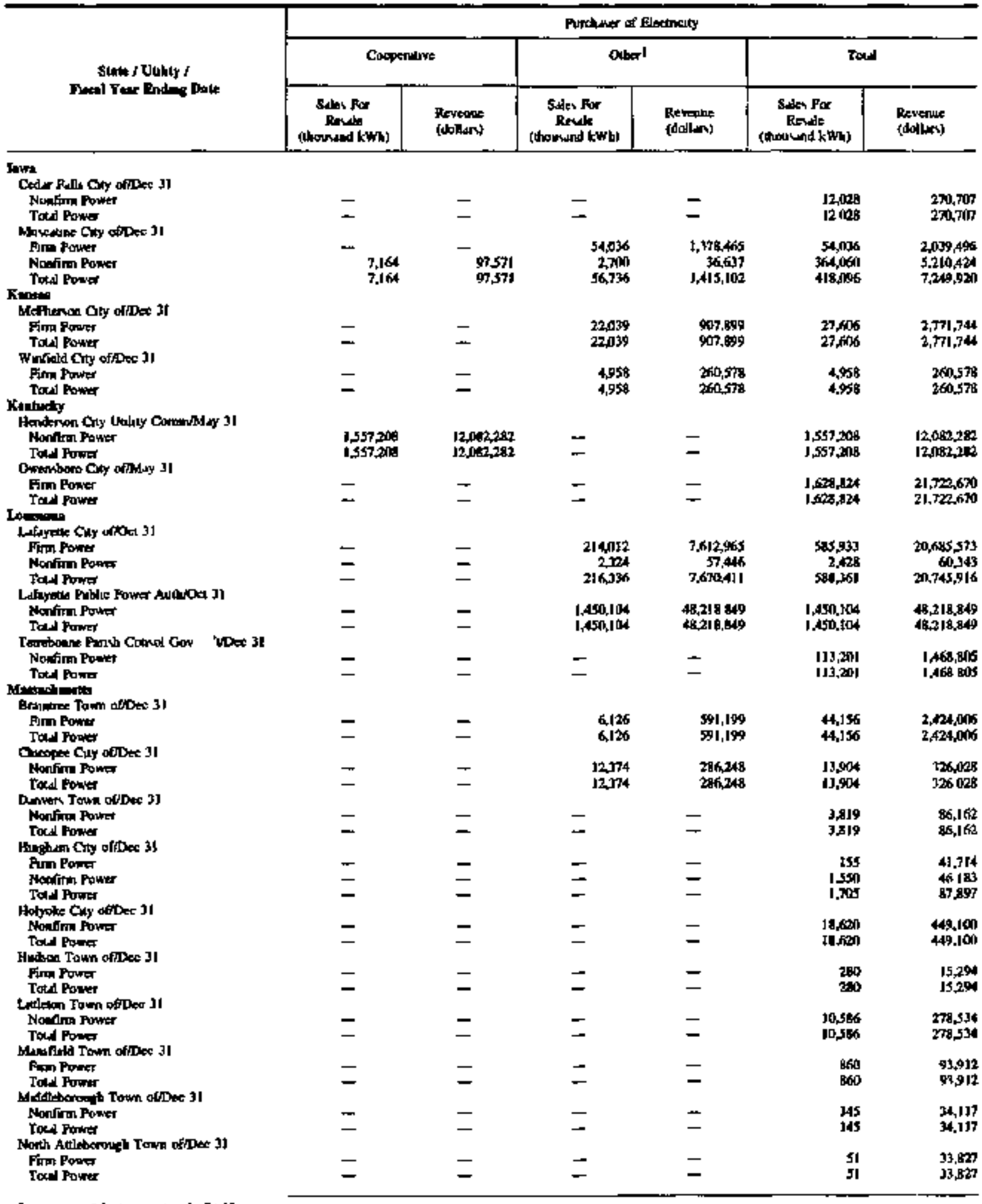


Table 30. Electricity Sales for Resale by Municlpal Utilities, Fiscal Year 1994 (Contimued)

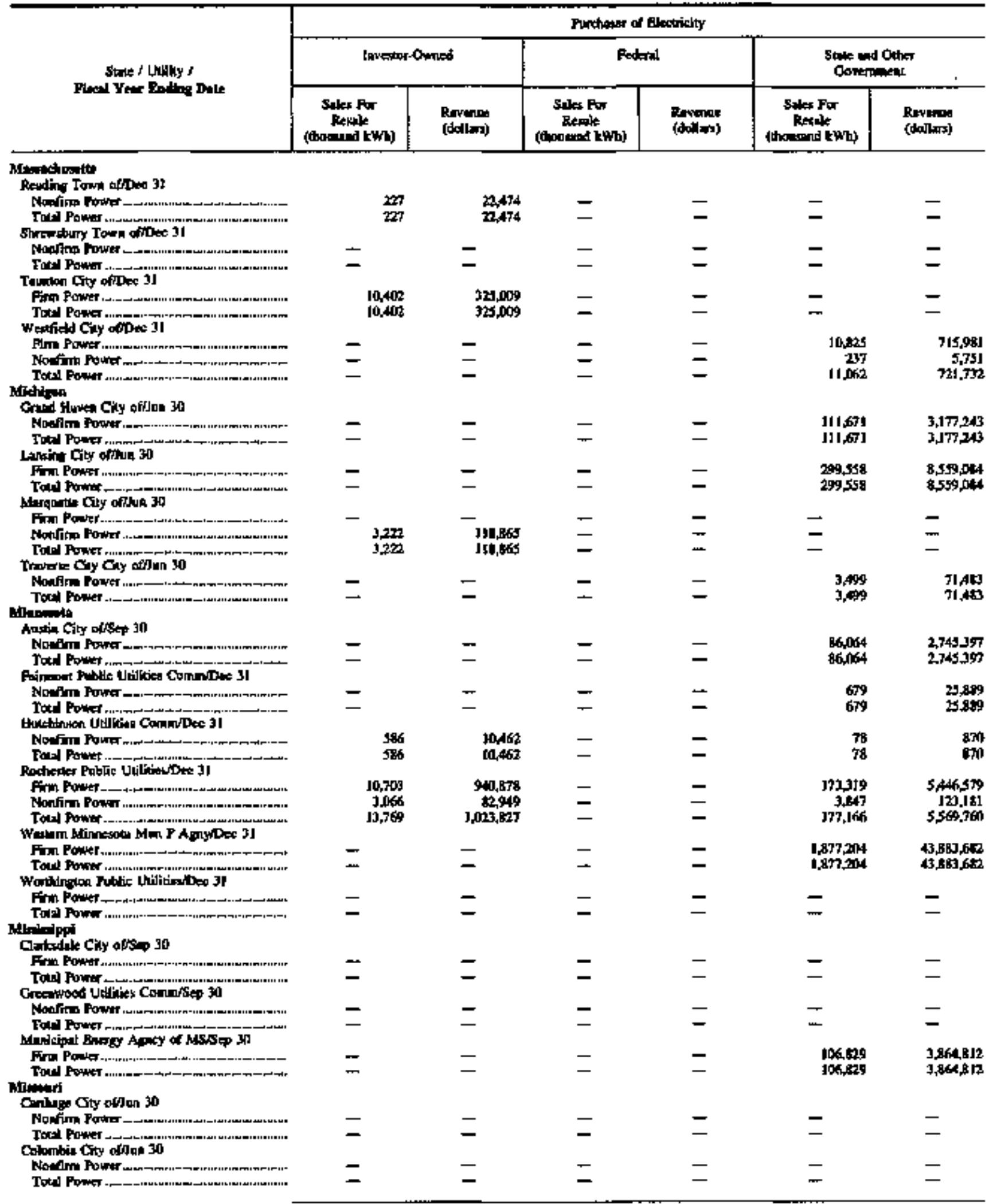

Sex noter and foothosests at and of whin. 
Table 30. Electricity Sales for Resale by Municipal Utilities, Fiscal Year 1994 (Continned)

\begin{tabular}{|c|c|c|c|c|c|c|}
\hline \multirow{3}{*}{ 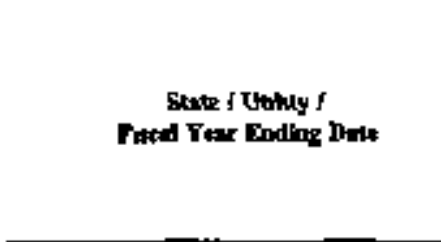 } & \multicolumn{6}{|c|}{ Ponthener of Electinaty } \\
\hline & \multicolumn{2}{|c|}{ Cospertate } & \multicolumn{2}{|c|}{ Ollat } & \multicolumn{2}{|c|}{ Towd } \\
\hline & 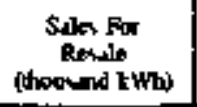 & $\begin{array}{l}\text { Revense } \\
\text { (dollary) }\end{array}$ & 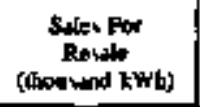 & $\begin{array}{l}\text { Revenwe } \\
\text { (dellan) }\end{array}$ & 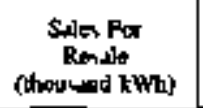 & $\begin{array}{l}\text { Brvenus } \\
\text { (Oodlers) }\end{array}$ \\
\hline \multicolumn{7}{|l|}{ Mavichnumit } \\
\hline Reding Jown dflDec JJ & - & - & 380 & 48,398 & $\omega$ & 30,872 \\
\hline $\begin{array}{l}\text { Toted Puwer } \\
\text { Slrewstury Torpm ofider } 31\end{array}$ & - & - & 380 & 4..59 & 60 & 70,872 \\
\hline 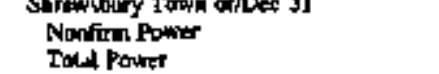 & 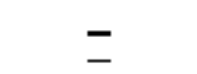 & $=$ & $\begin{array}{l}453 \\
453\end{array}$ & $\begin{array}{l}30,866 \\
30,85,65\end{array}$ & $\begin{array}{l}453 \\
453\end{array}$ & $\begin{array}{l}30,866 \\
30,366\end{array}$ \\
\hline \multicolumn{7}{|l|}{ Tamon Dty offoec } \\
\hline $\begin{array}{l}\text { Fum Power } \\
\text { Tox l Power }\end{array}$ & $\bar{z}$ & $\overline{-}$ & $\begin{array}{l}19,292 \\
19,292\end{array}$ & $\begin{array}{l}2,304,744 \\
2,304,744\end{array}$ & $\begin{array}{l}29,694 \\
29,604\end{array}$ & $\begin{array}{l}2,629,759 \\
2,629,753\end{array}$ \\
\hline \multicolumn{7}{|l|}{ Weatiledd Csty ofDe 31} \\
\hline Hem Fower & - & - & 9goj6 & 521,909 & I9,84] & $1.237,920$ \\
\hline Monfinm Pawt & - & - & - & - & 237 & 5.751 \\
\hline Tots Powne & - & - & 9,0נ6 & 521,939 & 20,078 & $1,240,67]$ \\
\hline \multicolumn{7}{|l|}{ 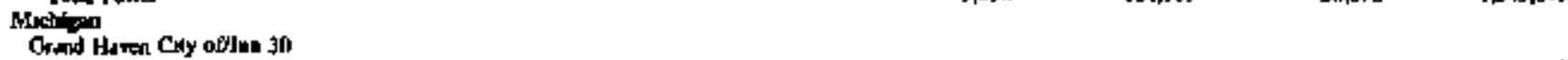 } \\
\hline Nonflisu Pawer & - & - & - & - & נונומונ & $3,17,24$ \\
\hline Totsl Powr & - & - & - & - & $111,6] \mathbf{3}$ & $3.177,24$ \\
\hline \multicolumn{7}{|l|}{ Larver Crty offlon 30} \\
\hline $\begin{array}{l}\text { Form Powts } \\
\text { Totil Power }\end{array}$ & 5,418 & 315,452, & - & $=$ & 304,976 & $8,874,536$ \\
\hline \multicolumn{7}{|l|}{ Minzilute Ocy offlon 30 } \\
\hline Finm Power & 11,164 & 634,066 & - & - & 11,164 & $674,0 \mathrm{M6}$ \\
\hline Nonfint Pawer & - & - & - & - & 3,222 & $1 / 8,8 \times 5$ \\
\hline Totdd Power & JI,IKA & 674.966 & - & - & 14,386 & 393,831 \\
\hline Travere Crty chy ailas 30 & & & & & & \\
\hline Wonflinn Popre & - & - & - & - & 3.499 & 71,483 \\
\hline Totd Power & - & - & - & - & J.499 & 71,483 \\
\hline \multirow{2}{*}{\multicolumn{7}{|c|}{ Minemil }} \\
\hline & & & & & & \\
\hline $\begin{array}{l}\text { Nooflim Pawer } \\
\text { Totil Powerer }\end{array}$ & 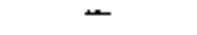 & - & - & - & $\$ 6,064$ & $2,745.197$ \\
\hline Total Power & - & - & - & - & Bh, 064 & $2,745,397$ \\
\hline \multicolumn{7}{|l|}{ Faurstont Polle Uulum Comminec 31} \\
\hline Nonfim Pownt & - & - & - & - & 679 & 27.889 \\
\hline Tousl power & - & - & - & س- & 679 & 25,889 \\
\hline 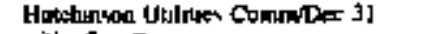 & & & & & & \\
\hline Nowfing Power & I & 30 & - & - & 669 & 11,052 \\
\hline Totd Powir & $\mathbf{I}$ & 90 & - & - & 605 & $1+.360$ \\
\hline 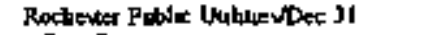 & & & & & & \\
\hline Pim Powa & - & - & - & - & 184,022 & $(6,357,4,57$ \\
\hline Nonfim Powts & 249 & 13.672 & 45 & 2,215 & 7,207 & 219,017 \\
\hline Tonal Pownt & 209 & $11,6 \% 2$ & 45 & 1,215 & 191,229 & $6,6,6,6,174$ \\
\hline 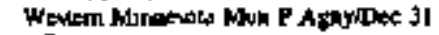 & & & & & & \\
\hline FitT Rower & $=$ & - & - & - & $1,877,294$ & $43,683,6 \% 1$ \\
\hline Tors Pomer & - & - & - & - & 1,877294 & 43.381 .682 \\
\hline 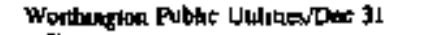 & & & & & & \\
\hline Fim Poung & - & - & 3.187 & 33,725 & 3,187 & 130,725 \\
\hline Tots Power & - & - & 3,189 & 130,725 & 3,187 & 120,725 \\
\hline Mtadshiji & & & & & & \\
\hline $\begin{array}{l}\text { Clatwide Cty of } \text { Sep } 30 \\
\text { Fum Pown }\end{array}$ & - & - & & & 100,553 & $3,1,5,519$ \\
\hline $\begin{array}{l}\text { Pum Power } \\
\text { Toted Pownter }\end{array}$ & - & - & $\begin{array}{l}10,553 \\
100,553\end{array}$ & $\begin{array}{l}3,07,319 \\
3,837,519\end{array}$ & $\begin{array}{l}100,333 \\
100,553\end{array}$ & $\begin{array}{l}3,0,5,519 \\
3,907,5] 9\end{array}$ \\
\hline Grearwood Uahug CommS $\Rightarrow$ in & & & & & & \\
\hline 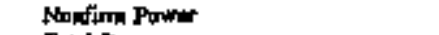 & - & - & 23,477 & $1,145,094$ & 20,477 & $1,145,04$ \\
\hline Tots Power & - & - & 20,477 & $1,145,0994$ & 20,477 & $1.145,094$ \\
\hline 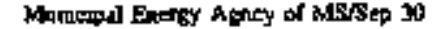 & & & & & & \\
\hline Fme Power & - & $\rightarrow$ & 790,460 & $26,991.151$ & B.7291 & $30,855,063$ \\
\hline Tots Power & - & - & 730,462 & 20,99I.ISI & 837291 & $70,895,963$ \\
\hline Musciend & & & & & & \\
\hline Curthuge Gry offlos 30 & & & & & & \\
\hline Noming Puwrer & ב & - & 35 & 1,8366 & 55 & 5,856 \\
\hline Tad Ponet & - & - & 53 & 1,856 & 55 & 7,856 \\
\hline Coluriben Crty offion 50 & & & & & & \\
\hline Nonflmo Power & - & - & 5 & $4,1,15$ & 57 & 4मI5 \\
\hline Toldal powtetr & - & - & 51 & 4,015 & $\$ 7$ & 4,015 \\
\hline
\end{tabular}

sec ate and foxpocesy and of thble 
Tabłe 30. Elecuricily Saks for Resale by Municipal Ulllties, Fiscal Year 1994 (Continoed)

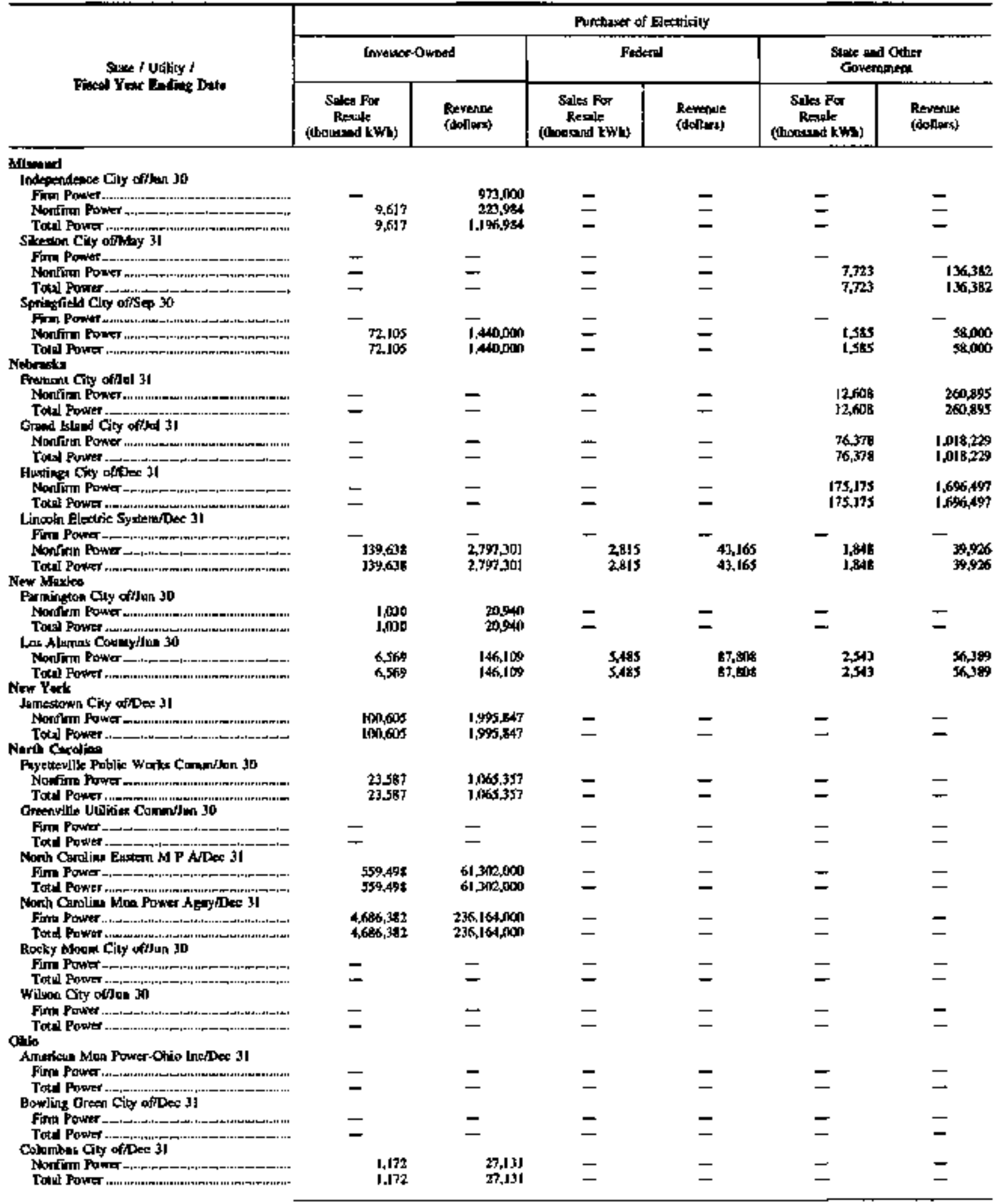

See noles and foolnuters at end of edale 
Table 34. Electricity Sales for Resale by Municipal Utilities, Fiscal Year 1994 (Continned)

\begin{tabular}{|c|c|c|c|c|c|c|}
\hline \multirow{3}{*}{ 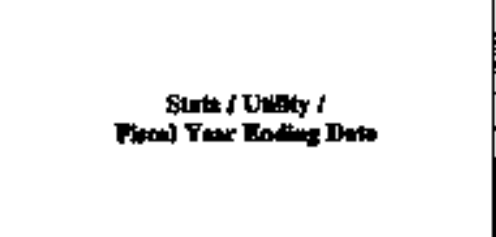 } & \multicolumn{6}{|c|}{ Perchane of Elactrictity } \\
\hline & \multicolumn{2}{|c|}{ Cospermine } & \multicolumn{2}{|c|}{ Qukerl } & \multicolumn{2}{|c|}{ Todd } \\
\hline & $\begin{array}{c}\text { Soles For } \\
\text { Reasle } \\
\text { (thowand (kw) }\end{array}$ & 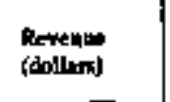 & 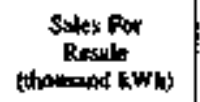 & $\begin{array}{l}\text { Reventet } \\
\text { (dolless) }\end{array}$ & 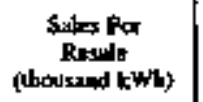 & 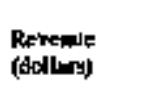 \\
\hline \multicolumn{7}{|l|}{$\begin{array}{l}\text { Misopint } \\
\text { Independence Clly ofilon } 30\end{array}$} \\
\hline Fim Pown & - & $=$ & - & - & - & 973,11000 \\
\hline Founfm Powrer & - & - & - & - & 9,617 & 223,984 \\
\hline Totel power & - & - & - & - & 9,517 & $1,196,99$ \\
\hline 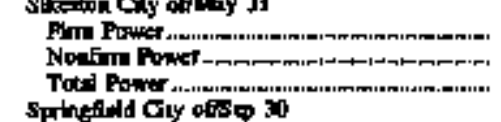 & $\begin{array}{l}-824 \\
824\end{array}$ & $-\underbrace{}_{\substack{1,0,25 \\
14,020}}$ & $\begin{array}{r}775,931 \\
603,867 \\
3,379,798\end{array}$ & $\begin{array}{l}29,946,660 \\
10,457,712 \\
40,404,372\end{array}$ & $\begin{array}{r}75,931 \\
612419 \\
1,98 B .345\end{array}$ & $\begin{array}{l}29,546, \pi 00 \\
10,606,422 \\
44,555,198\end{array}$ \\
\hline 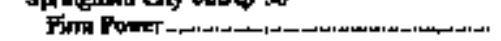 & - & - & 142981 & $3,002,0,00$ & 142,981 & $3,400,000$ \\
\hline 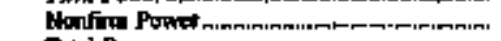 & 350 & 14,000 & 12.190 & 457.600 & 86.170 & 1.969000 \\
\hline 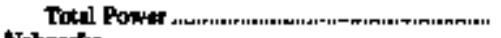 & 350 & 14,000 & 155.311 & 4,1759, tanta & 209.151 & 5.571 .00 \\
\hline \multicolumn{7}{|l|}{$\begin{array}{l}\text { Nebrovks } \\
\text { Prewout Chy aflut } 31\end{array}$} \\
\hline 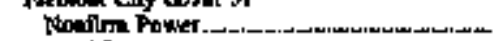 & - & - & - & - & 12,608 & 200,89 \\
\hline Total Powd & - & - & - & - & 12,698 & $264.8 \%$ \\
\hline 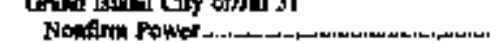 & - & - & - & - & $76,37 \mathrm{~B}$ & $5,018,225$ \\
\hline Told Power & - & - & - & - & $76,37 \mathrm{~B}$ & b. \\
\hline \multicolumn{7}{|l|}{ Hustingen Cry afdece II } \\
\hline Aowim Power & - & - & 17,309 & $1 \$ 3,092$ & 192.364 & $1,879,58$ \\
\hline Toted Power & - & - & 17.189 & 183,092 & 192,364 & $1,879,58$ \\
\hline Limoln Eectric Syytanther al & & & & & & \\
\hline 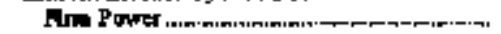 & $=$ & - & 72,903 & $2,132,356$ & 72.935 & $2.132,194$ \\
\hline 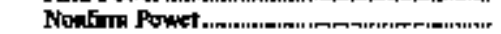 & 긱,500 & $10,283,475$ & $1,26 ?$ & 3942 & 858,116 & $13,199,285$ \\
\hline 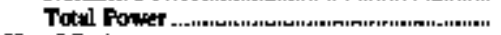 & 712550 & $10,282,475$ & $74,2,42$ & $2,162.778$ & $93 / .053$ & $15,525.14$ \\
\hline \multirow{2}{*}{\multicolumn{7}{|c|}{ Nem Manted }} \\
\hline & & & & & & \\
\hline Nomfirm Powet & - & - & 19,654 & $1,019, \mathbf{3} 3 \mathbf{2}$ & 201699 & $1.040,77$ \\
\hline Totul Powas & - & - & 19,639 & $1,019,032$ & 21,689 & $1,0,0,77$ \\
\hline 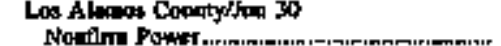 & 35,145 & 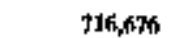 & 5,736 & 195,2016 & SB.498 & $1.292,18$ \\
\hline Tatal Power & 35,145 & $\$ 16,676$ & 8,756 & ins.206 & 58.498 & $1,200,18$ \\
\hline \multicolumn{7}{|l|}{ New Yact } \\
\hline Nonfim Powe & - & - & - & - & 200,405 & {$[, 945,84]$} \\
\hline Tous Ponver & - & - & - & - & Inopios & $1,995,84$ \\
\hline Nord Carolini & & & & & & \\
\hline \multicolumn{7}{|l|}{ 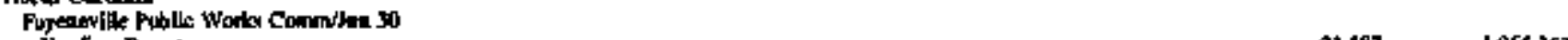 } \\
\hline & $=$ & - & $=$ & - & 21,587 & $1,065,357$ \\
\hline \multicolumn{7}{|l|}{ 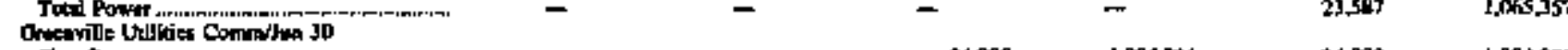 } \\
\hline Plm Ponver & - & - & $24 \sqrt{100}$ & $1,884,621$ & 24,000 & $1,884,\{2]$ \\
\hline Totell Fower & - & - & $24 \sqrt{3}(n)$ & $1,864,021$ & 24,010 & $1,534,02$ \\
\hline \multicolumn{7}{|l|}{ 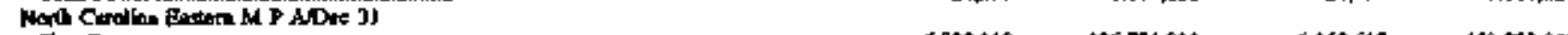 } \\
\hline 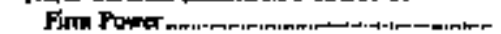 & - & - & $5,899,119$ & $396,721,0000$ & $6,568,617$ & 458,023,119x \\
\hline 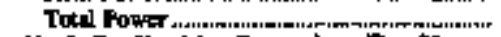 & - & - & 5.809,119 & $39,210 \mathrm{Dm}$ & $6,468,617$ & $458,023,190$ \\
\hline \multicolumn{7}{|l|}{ 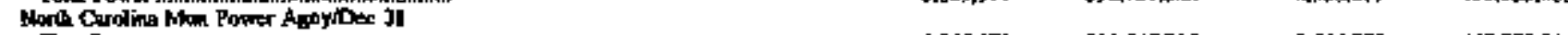 } \\
\hline 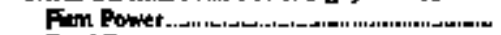 & - & - & $2,960,377$ & 211,545035 & $8,946,759$ & $447,709,93$ \\
\hline 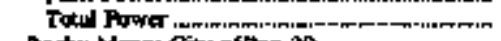 & - & - & 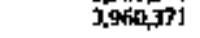 & $211,545,975$ & B.,646,753 & $44,208,93:$ \\
\hline \multicolumn{7}{|l|}{ Rocky Mooru City of ton 30} \\
\hline Flm Pown & - & - & 17,712 & $1.223,021$ & 17.712 & $1.223,42$ \\
\hline Toted Pown , , & - & - & 17,712 & $1,272,001$ & 17,712 & $1,225, n 2$ \\
\hline \multicolumn{7}{|l|}{ Wirom City ofpen 30} \\
\hline 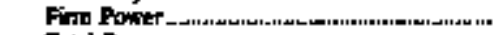 & - & - & 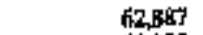 & $4,939,356$ & \$2.687 & 4.93 立. 35 \\
\hline Totel Powwer & - & - & 62,87 & $4,939,756$ & 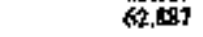 & $4,939,35$ \\
\hline Ohio & & & & & & \\
\hline 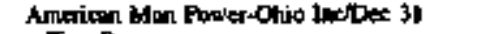 & & & & & & \\
\hline 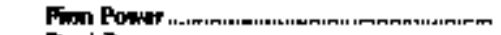 & - & - & $4,199,927$ & Isn,965,000 & $4,193,923$ & $150,965,0 \mathrm{hin}$ \\
\hline Trant Rawn & - & - & $4,193,927$ & $150,965,0003$ & $4,193.523$ & $150,965,000$ \\
\hline Bowling Gren Cry aflace 31 & & & & & & \\
\hline 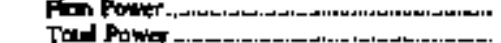 & $=$ & $=$ & 2818 & tof.79g & 2,018 & $\begin{array}{l}167.788 \\
165.78\end{array}$ \\
\hline Colwnibes City offDec JI & & & & & & \\
\hline 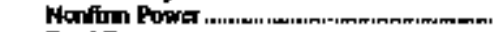 & - & - & $m$ & - & 1,172 & 27,13 \\
\hline 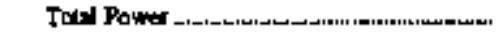 & - & - & - & - & 1.172 & 27,13 \\
\hline
\end{tabular}

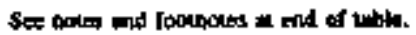


Table 30. Electriclity Sales tor Resele by Monicipal Utilities, Fiscal Year 1994 (Continued)

\begin{tabular}{|c|c|c|c|c|c|c|}
\hline \multirow{3}{*}{ 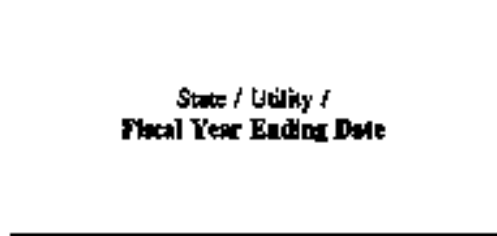 } & \multicolumn{6}{|c|}{ Puntuger of Electidity } \\
\hline & \multicolumn{2}{|c|}{ Invedtor-Oanned } & \multicolumn{2}{|c|}{ Fedmil } & \multicolumn{2}{|c|}{$\begin{array}{l}\text { Stove and Doher } \\
\text { Goneminum }\end{array}$} \\
\hline & $\begin{array}{c}\text { Sile For } \\
\text { Rexalk } \\
\text { (thookend KWh) }\end{array}$ & $\begin{array}{l}\text { Revendure } \\
\text { (dollens) }\end{array}$ & 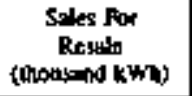 & $\begin{array}{l}\text { Reythue } \\
\text { (dotla) }\end{array}$ & 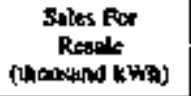 & $\begin{array}{l}\text { Revenute } \\
\text { (dorlory) }\end{array}$ \\
\hline \multicolumn{7}{|l|}{ Ohto } \\
\hline 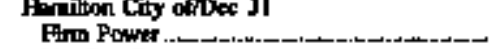 & - & 一 & 一 & - & - & - \\
\hline Now fim Power & $7.72 \pi$ & 72,634 & - & - & - & - \\
\hline 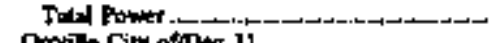 & 3,727 & 72634 & - & - & - & - \\
\hline $\begin{array}{l}\text { Orvile Ciry offace } 31 \\
\text { Fim Parer............ }\end{array}$ & & & & & & \\
\hline 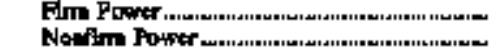 & 二 & $=$ & $=$ & - & $=$ & - \\
\hline 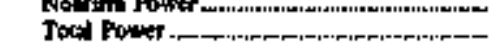 & $\overline{-}$ & $\overline{-}$ & $\overline{-}$ & $=$ & $=$ & $=$ \\
\hline Raga cily offece 31 & & & & & & \\
\hline 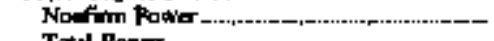 & $\pi$ & $6,46+1$ & - & - & - & - \\
\hline 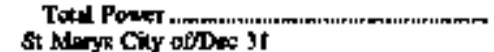 & $\boldsymbol{r}$ & 6,466 & - & - & - & - \\
\hline Fin Power & - & - & — & - & - & - \\
\hline Tokd Potur & 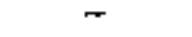 & - & - & - & - & - \\
\hline \multicolumn{7}{|l|}{ 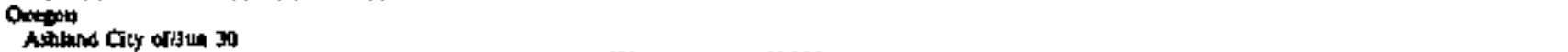 } \\
\hline 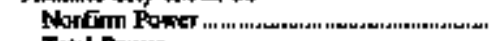 & 1,679 & 56,35s & - & - & - & - \\
\hline 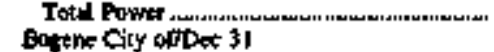 & 1,, Th9 & 56,785 & - & - & - & - \\
\hline 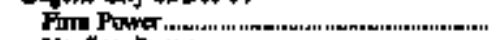 & - & - & - & - & 61,946 & 5.268 .706 \\
\hline 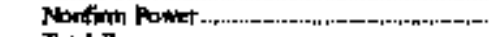 & 2ال1,159 & $5,069,859$ & d2,579 & $B 76, n 7$ & 40 & 1260 \\
\hline Toted Prower . & 21.159 & $5,069,259$ & 12.579 & 676,67 & 61,966 & 5.269 .966 \\
\hline \multirow{2}{*}{\multicolumn{7}{|c|}{ Souti Corrilnat }} \\
\hline & & & & & & \\
\hline Fim Pown & $1,562,075$ & $80,128,000$ & - & - & - & - \\
\hline 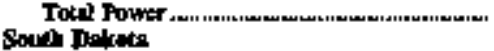 & $1,362,175$ & $80,128,000$ & - & - & - & - \\
\hline \multicolumn{7}{|l|}{$\begin{array}{l}\text { Souvi Dolcuta } \\
\text { Pieme Ciny offDet II }\end{array}$} \\
\hline Fin & مـ & & - & - & 14,617 & 291,086 \\
\hline 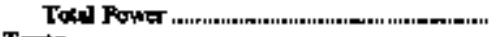 & - & - & - & - & 14,617 & 291,046 \\
\hline \multirow{2}{*}{\multicolumn{7}{|c|}{ 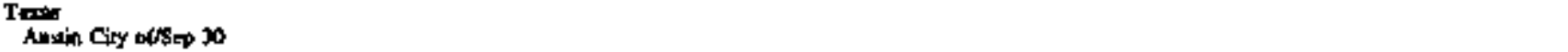 }} \\
\hline & & & & & & \\
\hline 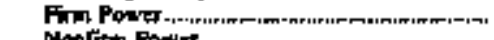 & - & - & - & - & - & - \\
\hline 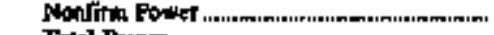 & 28511 & 49.469 & - & - & - & - \\
\hline 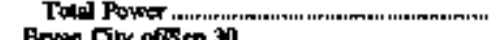 & 28,511 & $\$ 4,409$ & - & - & - & - \\
\hline \multicolumn{7}{|l|}{ Eryen Ciby ofsep 3n } \\
\hline 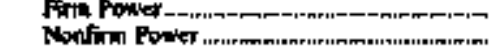 & $=$ & - & - & - & - & - \\
\hline 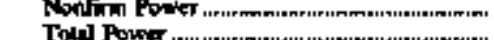 & $=$ & $\bar{Z}$ & - & - & - & - \\
\hline \multicolumn{7}{|l|}{ Deptom Chy of Sep 30} \\
\hline 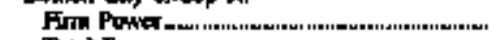 & - & - & - & - & - & - \\
\hline 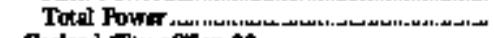 & - & - & - & - & - & - \\
\hline \multicolumn{7}{|l|}{ Gevlund Cly ofisep 30} \\
\hline 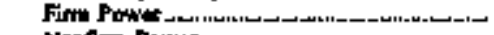 & - & - & - & - & - & - \\
\hline 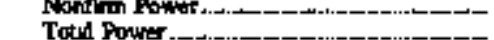 & $\bar{z}$ & 二 & 二 & 二 & - & 二 \\
\hline \multicolumn{7}{|l|}{ 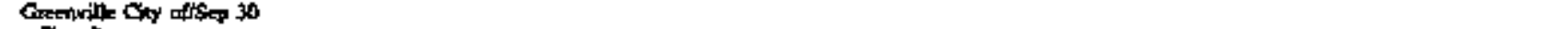 } \\
\hline 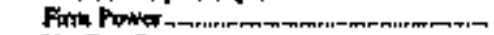 & - & - & - & - & - & - \\
\hline 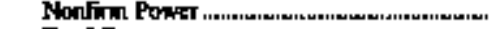 & - & - & - & - & - & - \\
\hline 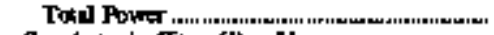 & - & - & - & - & - & - \\
\hline \multicolumn{7}{|l|}{ Sen Antoelo City of } \\
\hline 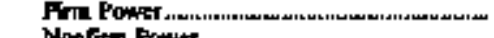 & - & - & - & - & - & - \\
\hline Nonfitin Fouter._-_- & 1] 6,969 & $3028,0 \%$ & - & - & 20,247 & 566,242 \\
\hline 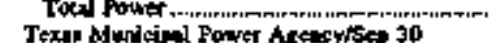 & 118569 & J020,04 & - & - & 20,267 & 506,242 \\
\hline 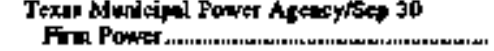 & & & & & & \\
\hline 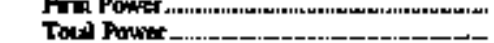 & - & - & - & - & - & - \\
\hline 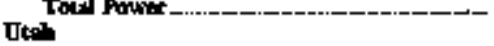 & - & - & - & - & - & - \\
\hline \multicolumn{7}{|l|}{ Utol } \\
\hline Pim Fown & 360,601 & $21,481, \sqrt{100}$ & - & - & - & - \\
\hline 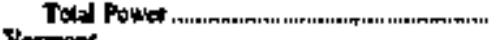 & 36t,68I & 3.,481,000 & - & - & - & - \\
\hline $\begin{array}{l}\text { Yermoes } \\
\text { Borlington Cly of }\end{array}$ & & & & & & \\
\hline Fith Powtron & 13,012 & 3,481,005 & - & - & 166 & $28,46 t$ \\
\hline 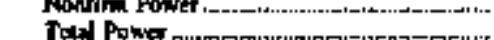 & 13,002 & 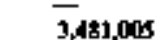 & $\vec{z}$ & - & 166 & $-28,464$ \\
\hline & & & & & & \\
\hline
\end{tabular}


Table 30. Electricity Sales for Resale by Mundcipal Utilities, Fiscal Year 1994 (Contimued)

\begin{tabular}{|c|c|c|c|c|c|c|}
\hline \multirow{3}{*}{$\begin{array}{l}\text { State / Uallky f } \\
\text { Fencl Year Lodine Date }\end{array}$} & \multicolumn{6}{|c|}{ Purcluare of Electicity } \\
\hline & \multicolumn{2}{|c|}{ Coopertive } & \multicolumn{2}{|c|}{ Other 1} & \multicolumn{2}{|c|}{ Totel } \\
\hline & $\begin{array}{c}\text { Sulex For } \\
\text { Retade } \\
\text { (lbogsand kWb) }\end{array}$ & $\begin{array}{l}\text { Revonute } \\
\text { (dedlurs) }\end{array}$ & $\begin{array}{l}\text { Soles For } \\
\text { Reple } \\
\text { (thotaland tWh) }\end{array}$ & $\begin{array}{l}\text { Rewenuad } \\
\text { (ddallares) }\end{array}$ & $\begin{array}{l}\text { Soles For } \\
\text { Rekuk } \\
\text { (Loogend kWhy }\end{array}$ & $\begin{array}{l}\text { Revaune } \\
\text { (dollariti }\end{array}$ \\
\hline 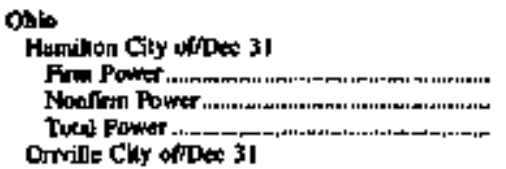 & $\underline{-}$ & $\overline{-}$ & $\begin{array}{l}135,955 \\
10,464 \\
146,419\end{array}$ & $\begin{array}{r}3,869,769 \\
89,644 \\
3,959,404\end{array}$ & $\begin{array}{r}135,959 \\
18,19 ! \\
154,146\end{array}$ & 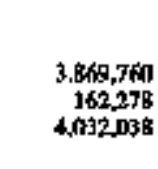 \\
\hline 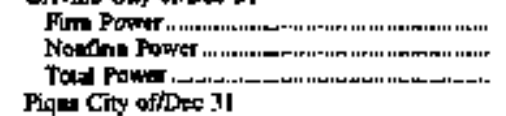 & $\begin{array}{l}- \\
-\end{array}$ & $\vec{z}$ & $\begin{array}{r}91,115,1 \\
6,257 \\
97,310\end{array}$ & $\begin{array}{l}1,607,563 \\
\text { MAM,848 } \\
1,912,41\end{array}$ & $\begin{array}{r}91,063 \\
6,257 \\
97,1010\end{array}$ & $\begin{array}{r}1,607,563 \\
304,848 \\
1,912,411\end{array}$ \\
\hline 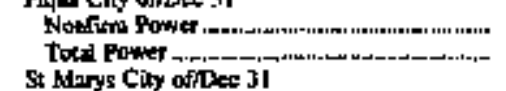 & $\overline{-}$ & 二 & $\overline{-}$ & $\overline{-}$ & $\frac{72}{72}$ & $\begin{array}{l}6,466 \\
6,466\end{array}$ \\
\hline 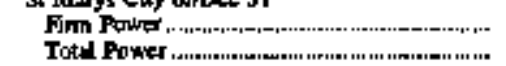 & $\overline{-}$ & $\overline{-}$ & $\begin{array}{l}5.159 \\
5.151\end{array}$ & $\begin{array}{l}163,9901 \\
16,9901\end{array}$ & $\begin{array}{l}5,159 \\
5,159\end{array}$ & $\begin{array}{l}163,990 \\
16,990\end{array}$ \\
\hline 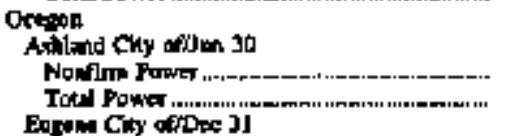 & $\overline{-}$ & $\overline{-}$ & $\overline{-}$ & - & $\begin{array}{l}1,679 \\
1,679\end{array}$ & $\begin{array}{l}56,785 \\
56,3,55\end{array}$ \\
\hline 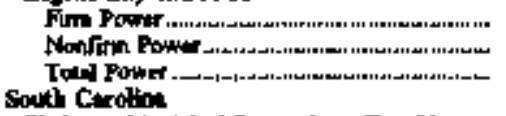 & $\underline{-}$ & $\underline{z}$ & $\begin{array}{r}31583 \\
31583\end{array}$ & $\begin{array}{r}-583,468 \\
589,468\end{array}$ & $\begin{array}{l}6,50,56 \\
285,365 \\
347,309\end{array}$ & $\begin{array}{r}3,268.765 \\
6,531265 \\
11,799,971\end{array}$ \\
\hline 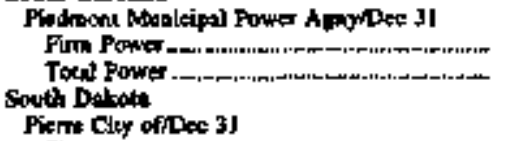 & $\overline{-}$ & $=$ & 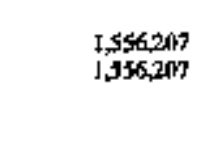 & $\begin{array}{l}80,549,000 \\
80,549,000\end{array}$ & $\begin{array}{l}3,119,282 \\
3,118,282\end{array}$ & $\begin{array}{l}160,677,000 \\
\text { Ifin, } 677 \text {, }\end{array}$ \\
\hline 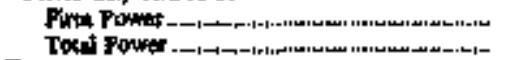 & $\overline{-}$ & $\bar{z}$ & $\overline{-}$ & $\overline{-}$ & $\begin{array}{l}14,617 \\
14,617\end{array}$ & $\begin{array}{l}291,506 \\
291,0166\end{array}$ \\
\hline 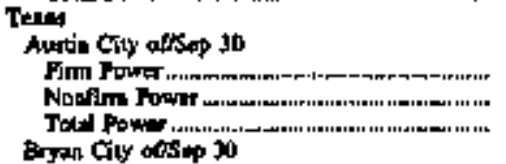 & 7, & $\begin{array}{l}\text { - } 139,096 \\
139,094\end{array}$ & $\begin{array}{l}179,033 \\
16,978 \\
19401401\end{array}$ & $\begin{array}{r}4,812,761 \\
15,165 \\
4,949,06 x\end{array}$ & $\begin{array}{l}179,0393 \\
17,930 \\
27,013\end{array}$ & $\begin{array}{r}4,8,2,781 \\
795,550 \\
5,606,331\end{array}$ \\
\hline 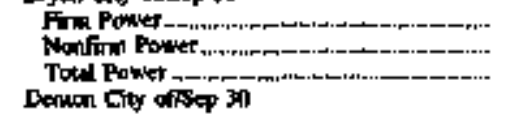 & $\bar{z}$ & $\vec{z}$ & $\begin{array}{c}606,067 \\
97,0678 \\
703,135\end{array}$ & $\begin{array}{r}26,439,062 \\
2,736,755 \\
25,769,357\end{array}$ & $\begin{array}{l}606,063 \\
97,068 \\
700,135\end{array}$ & $\begin{array}{r}26,493,0102 \\
2,39,355 \\
28,769,057\end{array}$ \\
\hline Fim Pown & $\overline{-}$ & $\vec{二}$ & $\begin{array}{l}175,250 \\
175,250\end{array}$ & $\begin{array}{l}7,097,478 \\
7,057,478\end{array}$ & $\begin{array}{l}175,250 \\
175,2510\end{array}$ & $\begin{array}{l}7,057,478 \\
7,057,478\end{array}$ \\
\hline 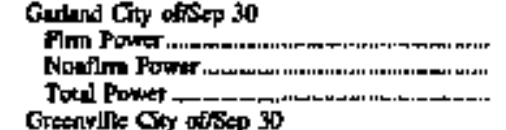 & $=$ & $\bar{z}$ & $\begin{array}{l}296.136 \\
543,630 \\
819.896\end{array}$ & $\begin{array}{l}11.737,470 \\
12,642,625 \\
24,416,099\end{array}$ & $\begin{array}{l}296,176 \\
54], 630 \\
899,816\end{array}$ & $\begin{array}{l}11,733 \mathrm{dTh} \\
12.642,625 \\
24,416.003\end{array}$ \\
\hline 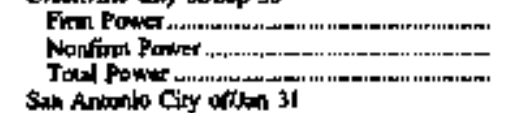 & $-\begin{array}{r}8,316 \\
8,316\end{array}$ & $\begin{array}{r}532,547 \\
-552,547\end{array}$ & $\begin{array}{r}37.27 \\
774 \\
38,151\end{array}$ & $\begin{array}{r}1,954,773 \\
5,692 \\
1,960,405\end{array}$ & $\begin{array}{r}45.593 \\
774 \\
46,367\end{array}$ & $\begin{array}{r}2,501,3201 \\
5,632 \\
2,512,952\end{array}$ \\
\hline 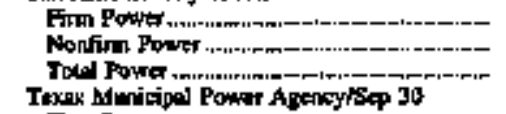 & $\begin{array}{r}\text { - } 31,673 \\
80,673\end{array}$ & $\begin{array}{r}6,093 \\
1,584,489 \\
1,592,5 \pm 2\end{array}$ & $\begin{array}{r}- \\
250,393 \\
250,353\end{array}$ & $\begin{aligned} J 1,513 \\
0,632,498 \\
0,650,451\end{aligned}$ & $\begin{array}{r}-42,864 \\
47,864\end{array}$ & $\begin{array}{r}24,606 \\
15,24,215 \\
15,23 \%, 821\end{array}$ \\
\hline Fon Power & $\overline{-}$ & $\bar{z}$ & $\begin{array}{l}2793,380 \\
2793,380\end{array}$ & $\begin{array}{l}174,146,0000 \\
174,146,000\end{array}$ & $\begin{array}{l}2,793,380 \\
2,793,3000\end{array}$ & $\begin{array}{l}374,146,1000 \\
374,146,100\end{array}$ \\
\hline 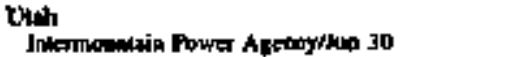 & & & & & & \\
\hline 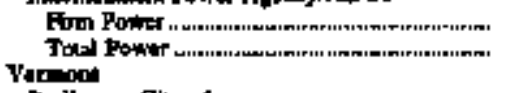 & $\bar{z}$ & $\overline{-}$ & $\begin{array}{l}11,824,513 \\
1,02,4,613\end{array}$ & $\begin{array}{l}670,428,000 \\
670,424000\end{array}$ & $\begin{array}{l}12,191,294 \\
12.191,294\end{array}$ & $\begin{array}{l}695,909,000 \\
695,909,000\end{array}$ \\
\hline 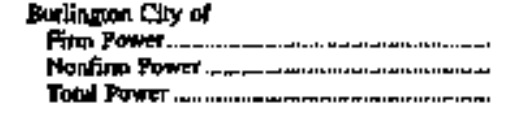 & $\bar{z}$ & $\vec{z}$ & 14,564 & $\begin{array}{l}- \\
849,182 \\
849,182\end{array}$ & $\begin{array}{l}13,378 \\
44,564 \\
57,342\end{array}$ & $\begin{array}{r}3,509.469 \\
869.182 \\
4,358.651\end{array}$ \\
\hline
\end{tabular}

Sep notank mat hookdous al add of teble. 
Table 30. Eleciricity Sales for Resale by Municipal Utilities, Fiseal Year 1994 (Continued)

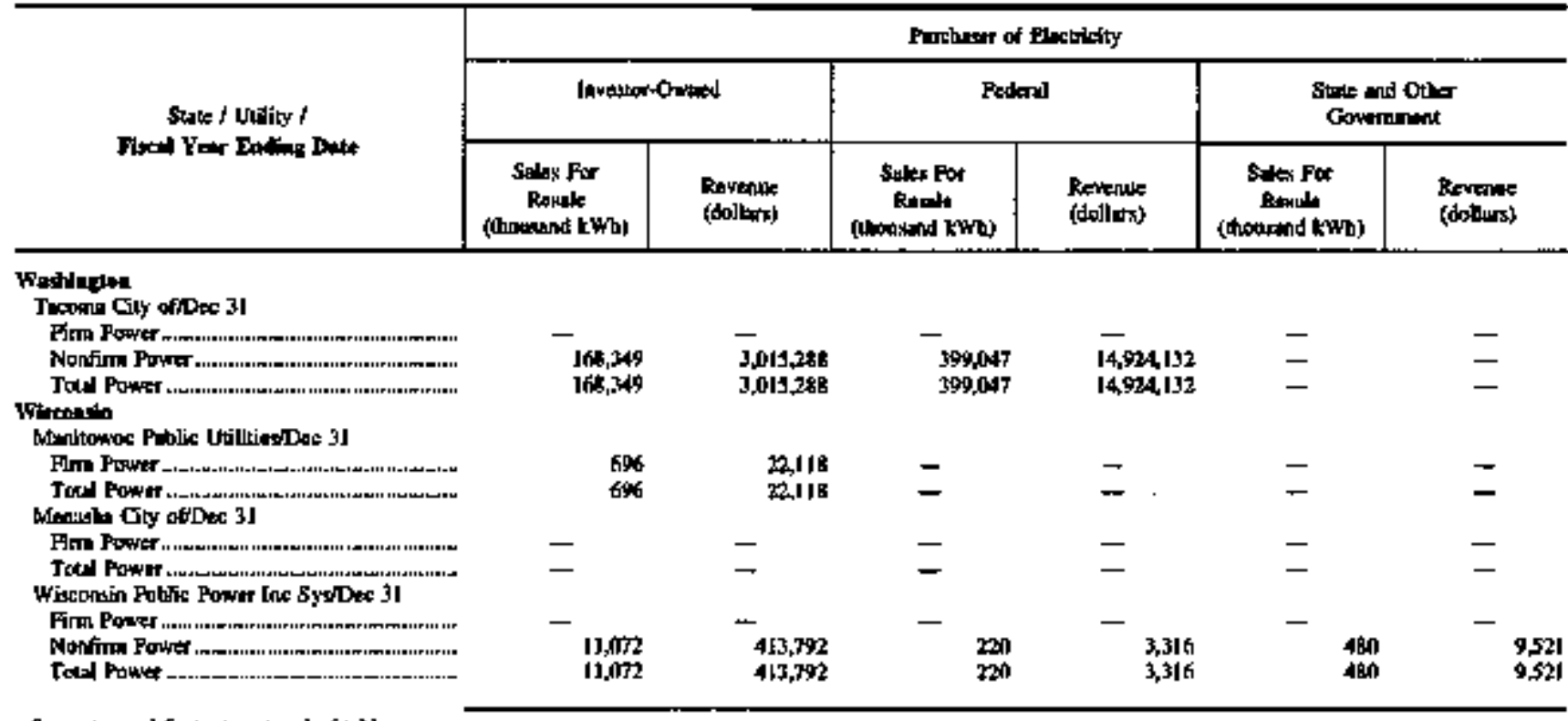

See notes and footected st end bo tuble.

Table 30. Electricity Sales for Resale by Municjpal Utilities, Fiscal Year 1994 (Contimued)

\begin{tabular}{|c|c|c|c|c|c|c|}
\hline \multirow{3}{*}{ 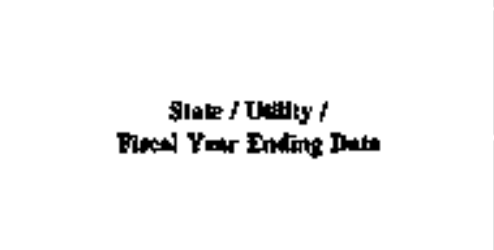 } & \multicolumn{6}{|c|}{ Prethater of Eherikity } \\
\hline & \multicolumn{2}{|c|}{ Cooperstive } & \multicolumn{2}{|c|}{ Ohanl } & \multicolumn{2}{|c|}{ Total } \\
\hline & $\begin{array}{c}\text { Suks Fur } \\
\text { Rexale } \\
\text { (atowkand kWh }\end{array}$ & $\begin{array}{l}\text { Revendit } \\
\text { (dollow })\end{array}$ & $\begin{array}{c}\text { Saken For } \\
\text { Benole } \\
\text { (booband twh) }\end{array}$ & $\begin{array}{l}\text { Revenu* } \\
\text { (doillias) }\end{array}$ & 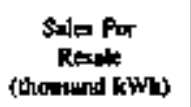 & $\begin{array}{l}\text { Revende } \\
\text { (dolluns) }\end{array}$ \\
\hline \multicolumn{7}{|l|}{ 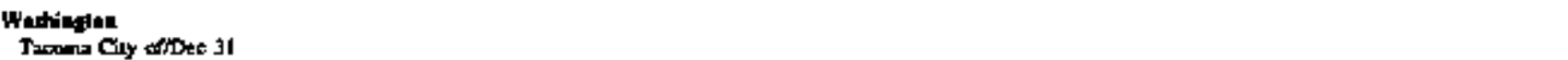 } \\
\hline Fim Patrer & - & - & 6,443 & 170,309 & 6,443 & IXn,30\% \\
\hline 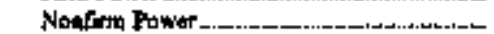 & - & $\neg$ & lofins & 180,693 & 578,079 & 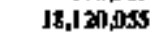 \\
\hline Tocal fower & - & $\neg$ & 17.115 & 350,944 & $\$ \$ 4,514$ & $18,290,364$ \\
\hline \multicolumn{7}{|l|}{ 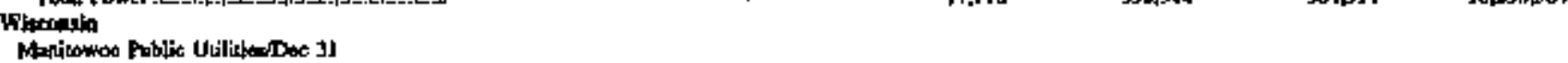 } \\
\hline Flon Pawer & - & $\neg$ & $=$ & - & (996 & 28,116 \\
\hline Total Poneer & - & - & - & - & 6 & 22,118 \\
\hline \multicolumn{7}{|l|}{ Monsulu Gity ofipar \$1 } \\
\hline Fim Powrer & - & - & 9,100 & $1,215,645$ & 3,100 & $1,215,565$ \\
\hline \multicolumn{7}{|l|}{ Whonde Puble Pouver Jac SywDec 31 } \\
\hline Fitm Power & - & - & $5,027,002$ & {$[13,603,14]$} & $3,527,002$ & 1]3,603,143 \\
\hline 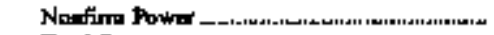 & 392 & 9.18] & 500 & 9,400 & 12,64 & 145,210 \\
\hline Tatd Pown & 392 & Y,IaJ & $3,027,502$ & {$[13.612 .54]$} & $3,3199,6,6$ & $114,046,357$ \\
\hline
\end{tabular}

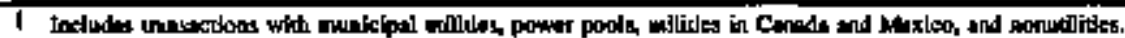

$\rightarrow$ Non Applicsble

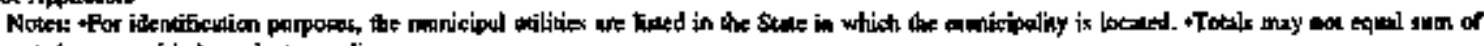
corpponentu bectuse of Indeperdert mounding.

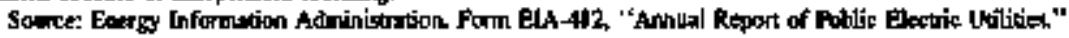


Table 31. Elextricity Purchases by Publicly Owned Utilities Not Reported on Form RaA-412, by State, 1994

\begin{tabular}{|c|c|c|c|c|c|c|c|}
\hline \multirow{2}{*}{ Sulte } & \multicolumn{7}{|c|}{ 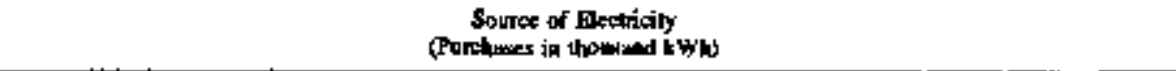 } \\
\hline & $\begin{array}{l}\text { Inwowor- } \\
\text { Orened }\end{array}$ & 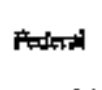 & 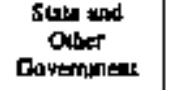 & honiejpal & Conperntive & Ohat' & Tots \\
\hline 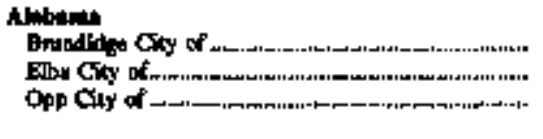 & $\bar{z}$ & $\overline{-}$ & $\overline{-}$ & $\bar{z}$ & $\begin{array}{l}28,675 \\
51,941 \\
73,868\end{array}$ & $\bar{z}$ & $\begin{array}{l}28,825 \\
\$ 1,941 \\
73,808\end{array}$ \\
\hline 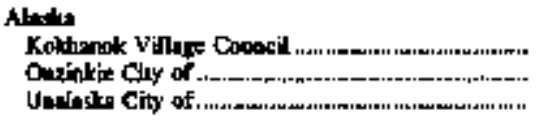 & $\overline{-}$ & $\overline{-}$ & $\begin{array}{l}- \\
-\end{array}$ & $\overline{-}$ & $\overline{-}$ & - 2,325 & $\begin{array}{r}131 \\
141 \\
2325\end{array}$ \\
\hline 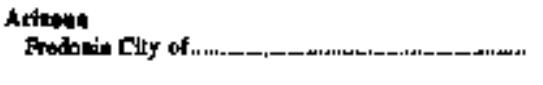 & 9,258 & - & - & $\omega$ & - & - & 9.58 \\
\hline 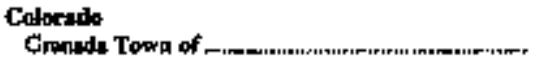 & - & - & - & 2,818 & - & - & 2818 \\
\hline 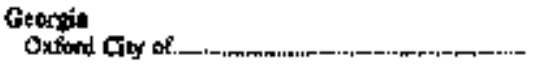 & - & - & $1,1 / 2$ & - & - & - & 11.142 \\
\hline 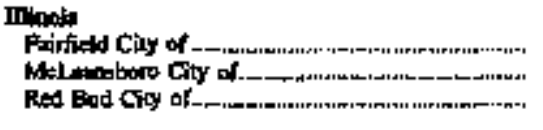 & $\overline{-}$ & $\overline{-}$ & $\overline{-}$ & $\bar{z}$ & $\begin{array}{l}71,513 \\
20,432 \\
34,185\end{array}$ & $\bar{z}$ & $\begin{array}{l}7,513 \\
20,432 \\
34,185\end{array}$ \\
\hline 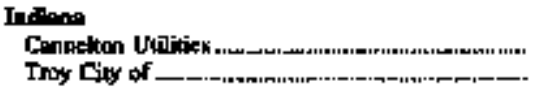 & $\overline{-}$ & - & $\overline{-}$ & $\begin{array}{r}58,590 \\
9.139\end{array}$ & $\overline{-}$ & $\bar{z}$ & $\begin{array}{r}18,590 \\
9,139\end{array}$ \\
\hline 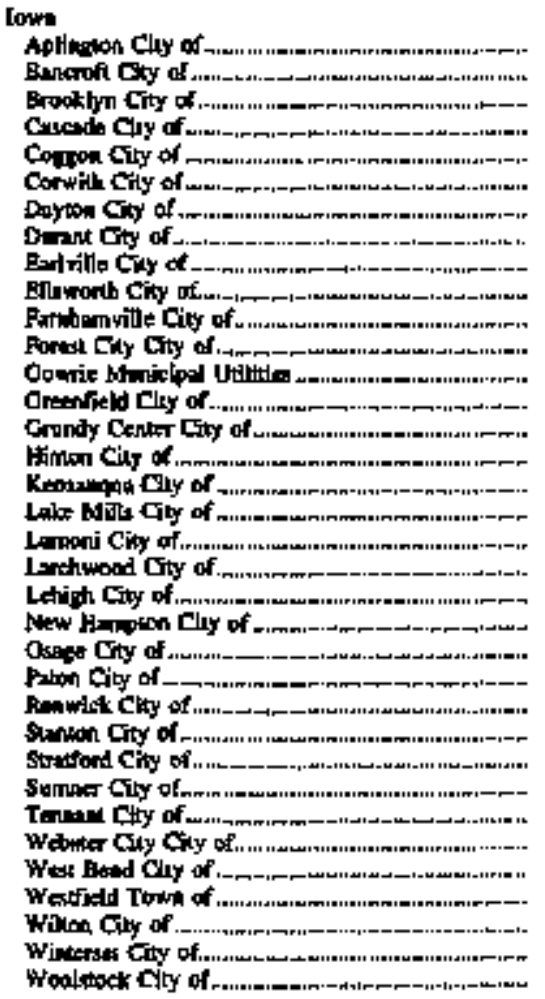 & $\begin{array}{l}= \\
= \\
= \\
= \\
= \\
= \\
= \\
= \\
= \\
= \\
= \\
= \\
= \\
= \\
= \\
= \\
= \\
= \\
=\end{array}$ & $\begin{array}{l}= \\
= \\
= \\
= \\
= \\
= \\
= \\
= \\
= \\
= \\
= \\
= \\
= \\
= \\
= \\
= \\
= \\
= \\
=\end{array}$ & $\begin{array}{l}= \\
= \\
= \\
= \\
= \\
= \\
= \\
= \\
= \\
= \\
= \\
= \\
= \\
= \\
= \\
= \\
= \\
= \\
=\end{array}$ & 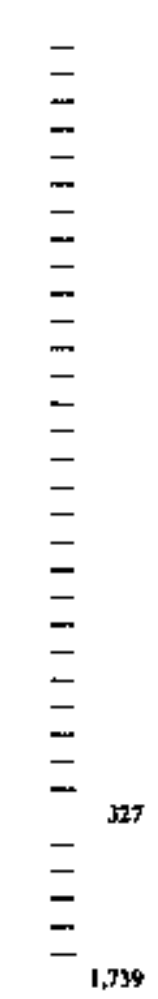 & 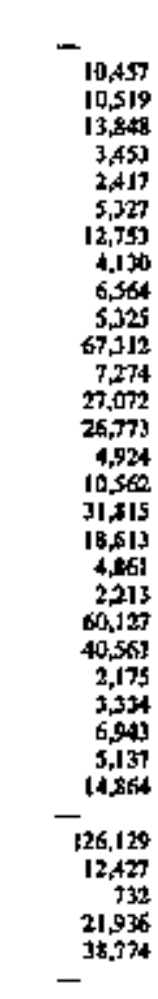 & $\begin{array}{l}= \\
= \\
= \\
= \\
= \\
= \\
= \\
= \\
= \\
= \\
= \\
= \\
= \\
= \\
= \\
=\end{array}$ & 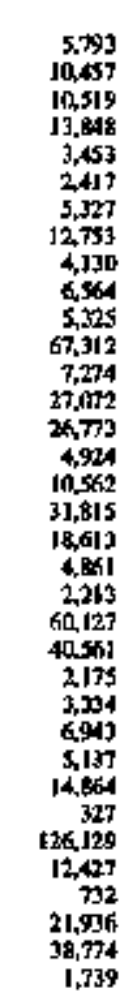 \\
\hline
\end{tabular}

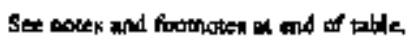


Table 31. Electriclty Purchases by Publichy Owned Utiltites Not Reported on Form ELA-412, by Stafie, 1994 (Continued)

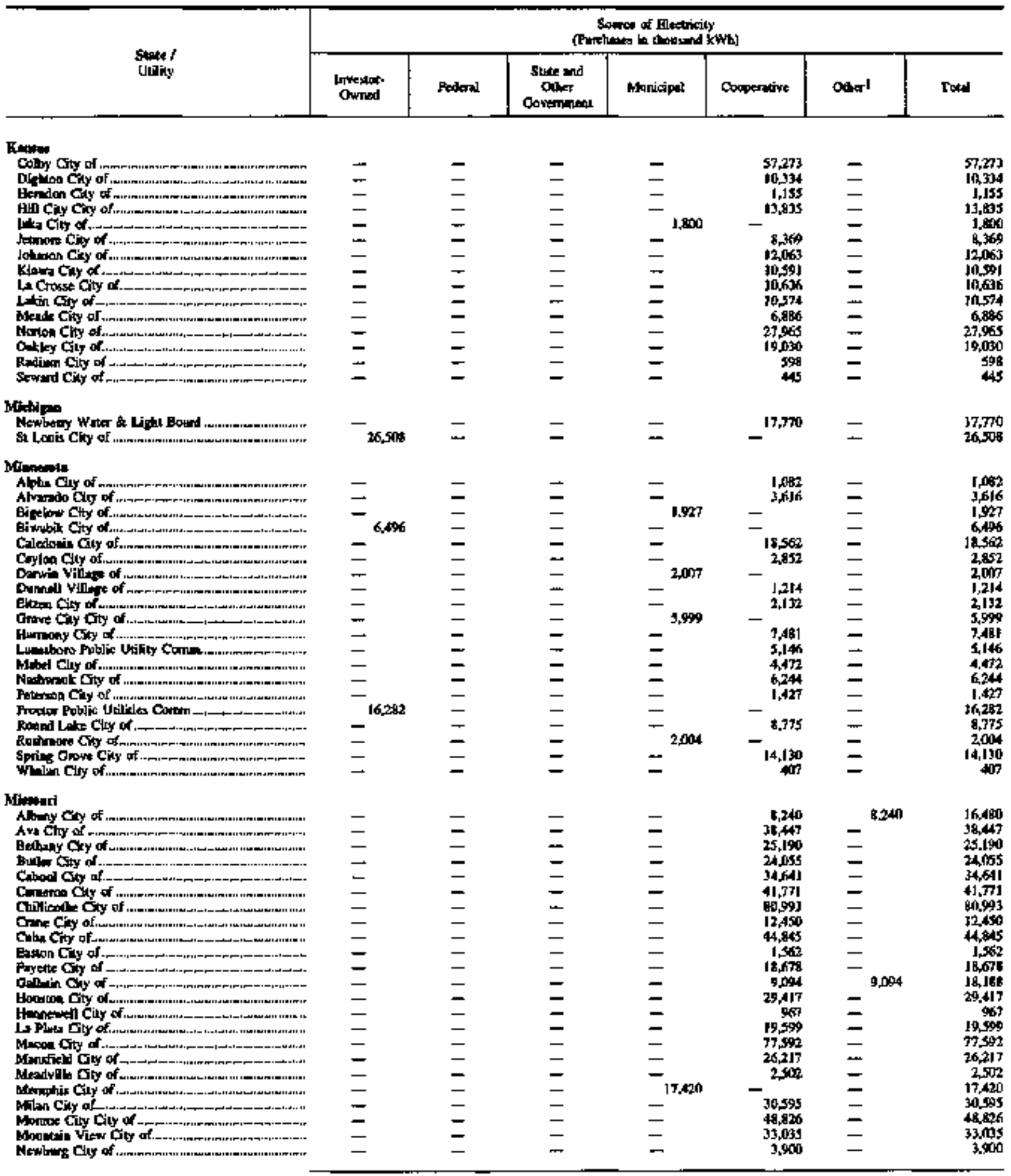

See noces ond footmoter at emd of tobde. 
Table 31. Electricity Parchases by Publicly Owned Uthitles Nat Reported on Form ELA-412, by State, 1994 (Contimued)

\begin{tabular}{|c|c|c|c|c|c|c|c|}
\hline \multirow{2}{*}{$\begin{array}{l}\text { Sure i } \\
\text { Uitily }\end{array}$} & \multicolumn{7}{|c|}{ 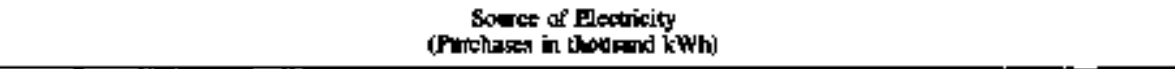 } \\
\hline & $\begin{array}{l}\text { Invertar- } \\
\text { Owrued }\end{array}$ & Fodentul & $\begin{array}{l}\text { State and } \\
\text { Othe } \\
\text { Governimax }\end{array}$ & Moticipal & Cocoperative & Dilend & Tots \\
\hline \multicolumn{8}{|l|}{ Minimin } \\
\hline 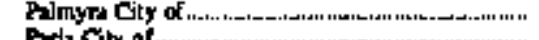 & - & - & - & - & 38,196 & - & 38.1996 \\
\hline 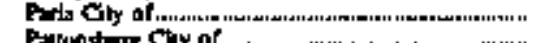 & - & - & - & - & 11,405 & - & 11,435 \\
\hline Prounders Chy of & - & - & - & - & 2,716 & - & 2,716 \\
\hline 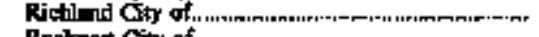 & - & - & - & - & 10,607 & - & 18,622 \\
\hline 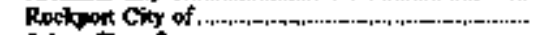 & - & - & - & - & 13,258 & - & 13,258 \\
\hline Solen Gity of & - & - & - & - & 51,500 & - & $51,3,3, P$ \\
\hline 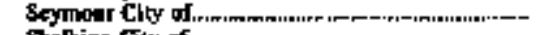 & - & - & - & - & 23,168 & - & 23,168 \\
\hline 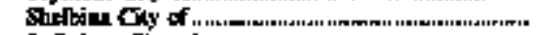 & - & - & - & - & 32,748 & $=$ & 32,748 \\
\hline 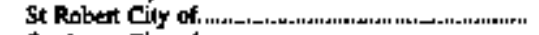 & - & - & - & - & 31.513 & - & 31.513 \\
\hline 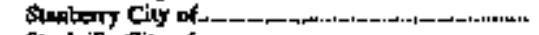 & $m$ & - & - & - & $9,8,33$ & - & 9,033 \\
\hline 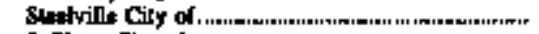 & - & - & - & - & 37,413 & $=$ & 17,413 \\
\hline 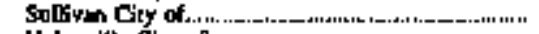 & - & - & - & - & 86,613 & - & 8 ghas \\
\hline 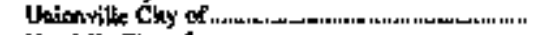 & - & - & - & - & - & 15,522 & 15,922 \\
\hline Vondia Cily of & - & - & - & - & 8,545 & 5,545 & $17,0 \% 0$ \\
\hline 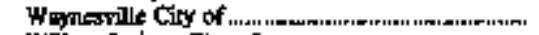 & - & - & - & - & $27,3 \mathbf{4}$ & - & 27,134 \\
\hline 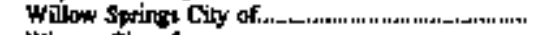 & - & - & - & - & 25,873 & - & 25,873 \\
\hline 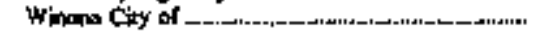 & - & - & - & - & 10,017 & - & In:a17 \\
\hline \multicolumn{8}{|l|}{ Nebcartal } \\
\hline 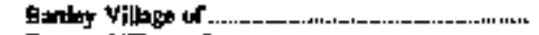 & - & - & 2,162 & - & $=$ & - & 2,162 \\
\hline 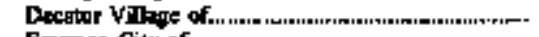 & - & - & 0.125 & - & - & 一 & 5,125 \\
\hline 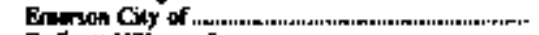 & בה & - & 5.840 & - & - & - & $5, B 40$ \\
\hline Endifoth Viliare of & - & - & - & GA7 & - & - & $\$ 47$ \\
\hline Nondwad Rond Pub Poyr Dixt & - & - & - & 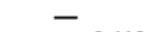 & 65,386 & - & 65,396 \\
\hline Paname Viltoge of & - & - & - & $\mathbf{1 , 4 4 1}$ & - & - & 1,441 \\
\hline 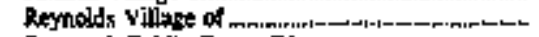 & - & - & - & 750 & - & - & 750 \\
\hline Rookevelt Public Power Diul_........._-_,............... & - & - & - & - & 45, הN4K & - & 45,896 \\
\hline Sa Pool Chty of ........................... & - & - & I7, 150 & - & - & - & 17,050 \\
\hline 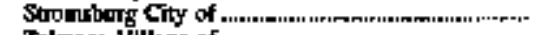 & - & - & 10, & - & - & - & J0, 1898 \\
\hline 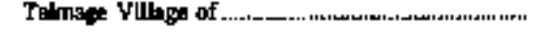 & - & - & - & $2,4 \$ 6$ & $=$ & - & 2446 \\
\hline \multicolumn{8}{|l|}{ Nevede } \\
\hline 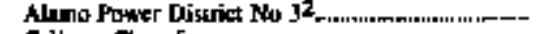 & - & - & In\&N7 & - & - & - & $10,24]$ \\
\hline 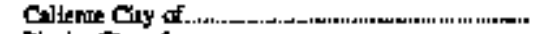 & - & - & 13.287 & - & - & - & 13,187 \\
\hline Piocter City of & 一 & - & & - & - & - & $\mathbf{6}, \mathbf{\Delta S I}$ \\
\hline \multicolumn{8}{|l|}{ Nm Madte } \\
\hline 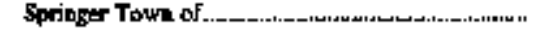 & 一 & - & - & 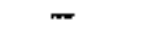 & 4,258 & - & 4,258 \\
\hline \multicolumn{8}{|l|}{ Noru Cardlan } \\
\hline Forntrin Town of & - & - & - & 7,331 & - & - & 7,331 \\
\hline 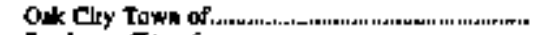 & - & - & - & - & 5,286 & - & 5,286 \\
\hline 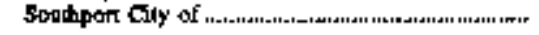 & 39,283 & - & - & - & - & - & 39,283 \\
\hline \multicolumn{8}{|l|}{ Ohis } \\
\hline 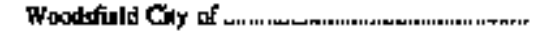 & - & - & - & - & $21,2 \pi$ & 一 & $21,2 \pi 0$ \\
\hline \multicolumn{8}{|l|}{ Oklahome } \\
\hline 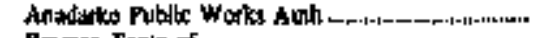 & - & - & - & - & 56,742 & - & 36,742 \\
\hline Bromun Jowin of & - & - & - & 1,024 & - & - & 1.64 \\
\hline Burlingen Ciuy of & - & - & - & - & 1经 & - & 1.270 \\
\hline Cordet̂ Clry of & - & - & - & - & 24,369 & - & 24,798 \\
\hline fon Swopty town of & - & - & - & - & 2,232 & $=$ & 2,232 \\
\hline Kow Chy City of & $1,06 \%$ & - & - & - & I, B69 & - & 2,138 \\
\hline Linflay Chy of & - & - & - & - & 24,788 & - & 24,789 \\
\hline Moordand Caly of & - & - & - & - & 8,146 & - & 8.146 \\
\hline \multicolumn{8}{|l|}{ Somedh Dakooka } \\
\hline ElR Point Chy of-rar............-, & - & - & - & - & 13,014 & - & 13,014 \\
\hline 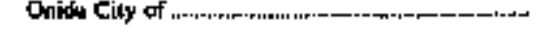 & - & $\theta$ & - & - & 6,916 & - & 6916 \\
\hline \multicolumn{8}{|l|}{ Texilu } \\
\hline Elean bity of & - & - & - & - & 23,839 & - & 23,879 \\
\hline Coniscon Gity of & - & - & - & - & 8,fIB & - & $8,61 \%$ \\
\hline Granbory Cily of.men. & - & - & - & - & 69,18 h & - & 69.180 \\
\hline Hesme City of & - & - & - & - & 42,526 & - & 12526 \\
\hline
\end{tabular}

Set notes and footenches an and of table 
Table 31. Elentricity Purchases by Publicly Owned Utiltities Not Reported on Form EIA-412, by State, 1994 (Continued)

\begin{tabular}{|c|c|c|c|c|c|c|c|}
\hline \multirow{2}{*}{$\begin{array}{l}\text { Stute f } \\
\text { Uintity }\end{array}$} & \multicolumn{7}{|c|}{ 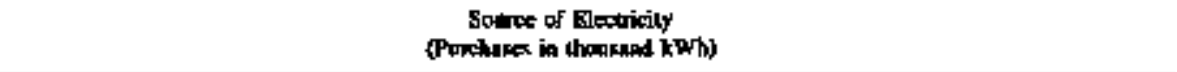 } \\
\hline & $\begin{array}{l}\text { Invaster- } \\
\text { Ormed }\end{array}$ & Fedfal & 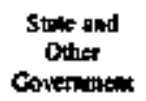 & Muscipil & Cooputativi & اיבים & Tod:d \\
\hline \multicolumn{8}{|l|}{ Trax } \\
\hline Hemphild Chy of, & - & - & 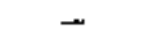 & - & 15,703 & - & 15.740 \\
\hline 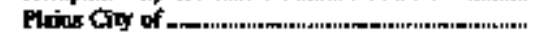 & - & - & - & - & 7,053 & 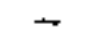 & 7,063 \\
\hline 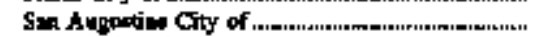 & - & - & - & - & 22939 & - & 2099 \\
\hline 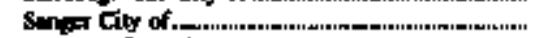 & - & - & - & - & 29824 & $\rightarrow$ & 29,824 \\
\hline 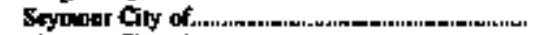 & 一 & - & - & - & 29.475 & $\rightarrow$ & 29,675 \\
\hline IEmping Cly of & - & - & 一 & - & 11,442 & - & 11,442 \\
\hline 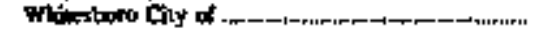 & - & - & - & - & 26,126 & - & 26,127 \\
\hline \multicolumn{8}{|l|}{ Placenten } \\
\hline Aredit City of & - & - & - & - & 60237 & $\longrightarrow$ & xastor \\
\hline 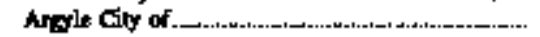 & - & - & - & - & 7838 & $\rightarrow$ & 7,838 \\
\hline Cublon Vileop of. & - & - & - & - & 6,579 & - & 6,579 \\
\hline Ganberlnd Ğty of & - & - & - & - & 90,632 & - & 30,432 \\
\hline 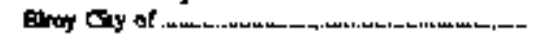 & - & - & - & $m$ & $|6| 2 \mid$, & $\rightarrow$ & 16.121 \\
\hline Fenifocre Cyty of & - & - & - & - & 29,710 & $\rightarrow$ & 29,310 \\
\hline 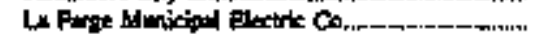 & - & - & - & 2,800 & 2,600 & $=$ & 5,600 \\
\hline 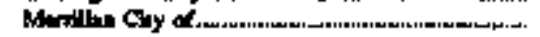 & - & - & - & - & $3,2,02$ & - & 1,254 \\
\hline 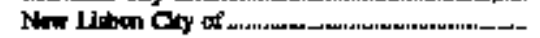 & - & - & - & - & 15,655 & - & 15,6ss \\
\hline Ywat Cy of & - & $m$ & $m$ & - & 4,238 & - & 4.218 \\
\hline \multicolumn{8}{|l|}{ Mration } \\
\hline Bupia Tonn of & - & - & - & - & 9,nst & - & 9.054 \\
\hline 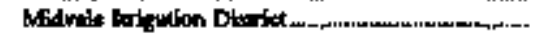 & - & - & - & - & 203 & $\longrightarrow$ & 233 \\
\hline
\end{tabular}

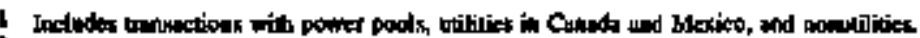

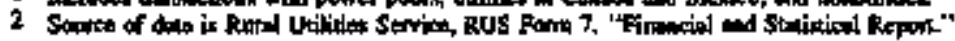

Not Applicalte

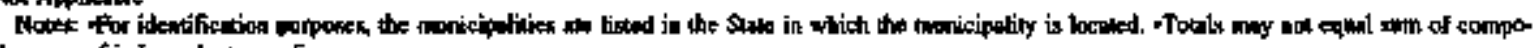
nents betowe of independent manding.

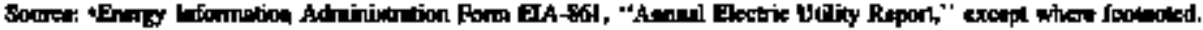


Table 32. Electricity Exchanges and Wheeling by Publicly Owned Utilities, 1994 (Thousand Kilowarthouss)

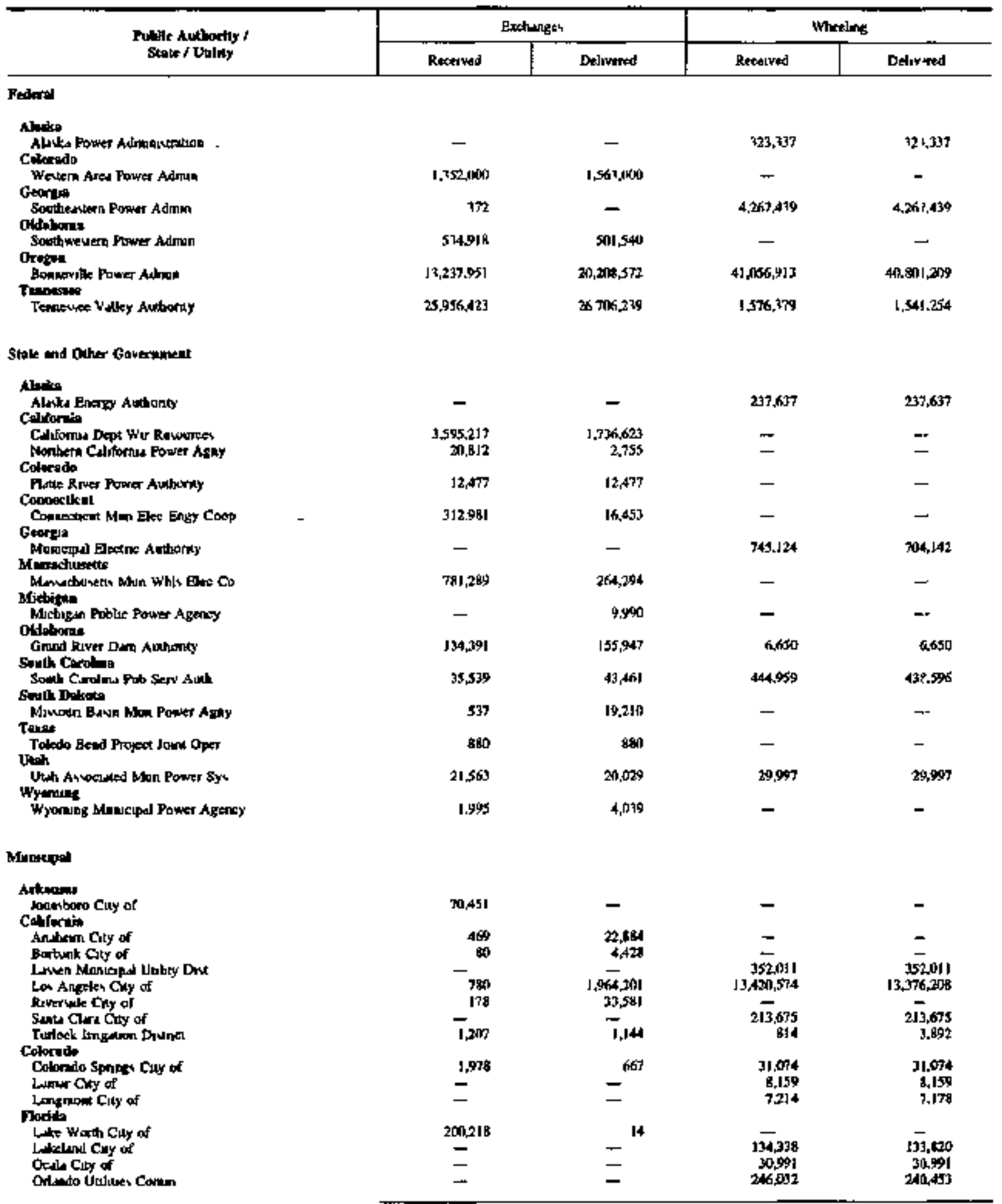

Sece notes ind foodncent at and of twble 
Table 32. Electricity Bxchanges and Wheeling by Poblicly Omned Utilities, 1994 (Thousand Kilowarthours) (Continued)

\begin{tabular}{|c|c|c|c|c|}
\hline \multirow{2}{*}{ 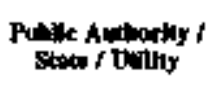 } & \multicolumn{2}{|c|}{ Exchenger } & \multicolumn{2}{|c|}{ Whating } \\
\hline & Recefved & Delfuents & Recolined & Dellyanes \\
\hline
\end{tabular}

\begin{tabular}{|c|c|c|c|c|}
\hline \multicolumn{5}{|l|}{ Mrridepel } \\
\hline \multicolumn{5}{|l|}{ low } \\
\hline 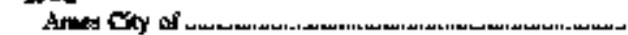 & 125 & 199 & - & - \\
\hline Heln Coyy of & 3,907 & J,EB & - & - \\
\hline Mngertine Cly of _..........t_-_...t__. & - & - & 6,279 & 28 \\
\hline 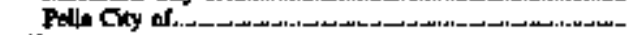 & 6,217 & 6.989 & - & - \\
\hline \multicolumn{5}{|l|}{ Kines } \\
\hline 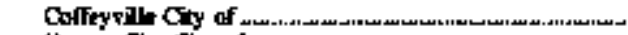 & 149,302 & 149,524 & - & - \\
\hline Kanps Giy Ciny of & - & 11,070 & $m$ & - \\
\hline 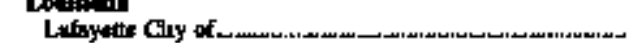 & & & & \\
\hline \multicolumn{5}{|l|}{ 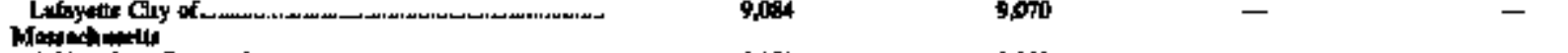 } \\
\hline 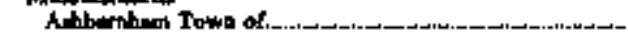 & s.t? & 3,12 & - & - \\
\hline Boybton Toph of & {$[\boldsymbol{n}, \boldsymbol{b}, \mathbf{J}$} & $2+\$ 36$ & - & - \\
\hline Brintree Towm of wir......... & $106,62]$ & 51,289 & - & - \\
\hline Chicopes Cly of & 1,089 & 2[12! & - & - \\
\hline Duever Town of & 125,918 & 29,246 & - & - \\
\hline Gengeoum Chy of & 12.162 & 2249 & - & - \\
\hline Grown Ciry of & J0,003 & 5907 & - & - \\
\hline Hin th= Chy of & 69.968 & 1468 & - & - \\
\hline Bolyole Cily of..... & 19,660 & 57,755 & - & - \\
\hline 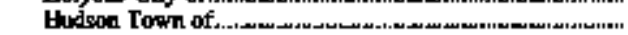 & $\mathrm{BI}, \mathrm{73 \textrm {K }}$ & 5,003 & - & - \\
\hline 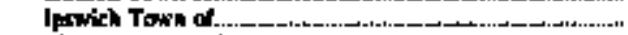 & 31.250 & 2490 & - & - \\
\hline 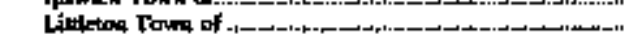 & 17j15 & 1030 & - & - \\
\hline Minufted Tows of & $71,05 \%$ & 10,569 & مـ & - \\
\hline Betklkesd Oty of & 24,942 & - & - & - \\
\hline Middoboroph To of of & 19,219 & 9,15 & - & - \\
\hline Nomb Atolboosongh Town of ..... & 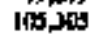 & 12,927 & - & - \\
\hline fuxtom town of & 6,80 & 2759 & - & - \\
\hline Neobody Clixy of & $182,0 m$ & 48055 & - & - \\
\hline hifestow Toure of & 3,068 & 502 & - & - \\
\hline Reving Tow/e of & 136,112 & $\$ 6,110$ & - & - \\
\hline Wyin Town of & 44,501 & 13006 & - & - \\
\hline Sowh Hedey Jown of & 4,35 & $3,8,3$ & - & - \\
\hline 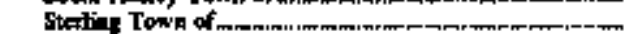 & 10514 & $3, \mathrm{~B} 2 \mathrm{~A}$ & - & - \\
\hline Twmen defy of & $3 J 6,391$ & 6060 & - & - \\
\hline 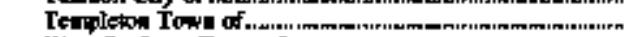 & 36,40 & $70 \%$ & $=$ & $\bar{m}$ \\
\hline Wert Boylume Tawn of & 37.930 & 6,074 & - & - \\
\hline Whotifild Ciny of & 66,150 & 26,983 & - & - \\
\hline \multicolumn{5}{|l|}{ 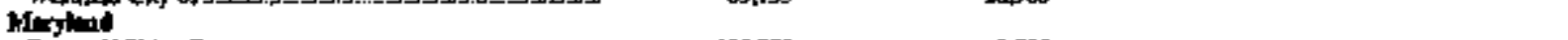 } \\
\hline \multirow{2}{*}{\multicolumn{5}{|c|}{ lilatilgen }} \\
\hline & & & & \\
\hline 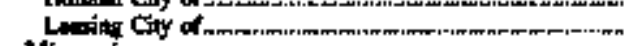 & 30 & 29 & - & - \\
\hline \multicolumn{4}{|l|}{ Misong } & \\
\hline & $-\overline{x+m}$ & $\overline{1}-1001$ & I2I & Juthent \\
\hline 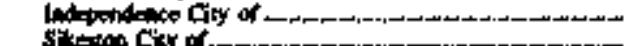 & $2007 x+4$ & 65007 & - & س \\
\hline & mili & 136075 & z & - \\
\hline & & & & \\
\hline Fyotitervile Poblle Worta Comm & 424,175 & 22,912 & - & - \\
\hline \multicolumn{4}{|l|}{ Neherds } & 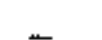 \\
\hline 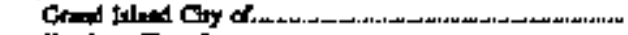 & 16 & - & $\bar{s} \mathbf{s u}$ & \\
\hline 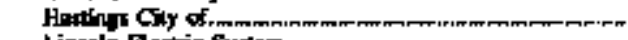 & - & $-\bar{t}_{\text {xat }}$ & 5,534 & 5,534 \\
\hline 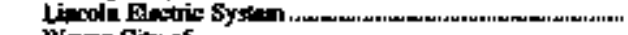 & - & $3,6,6$ rit $^{6}$ & 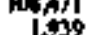 & 108,8713 \\
\hline \multirow{2}{*}{\multicolumn{5}{|c|}{ 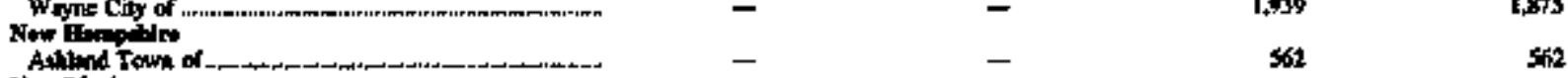 }} \\
\hline & - & - & 361 & 5 sit2 \\
\hline \multicolumn{5}{|l|}{ 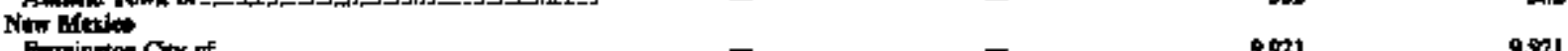 } \\
\hline & - & - & 9.921 & 9,821 \\
\hline Los Alim Coonty & \multicolumn{2}{|c|}{ Ohl } & - & \\
\hline 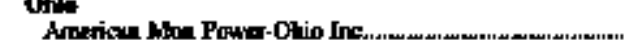 & - & - & 39.679 & 39,679 \\
\hline Getetand City of. & 二 & $\mathbf{2 , 2 0 2}$ & - & - \\
\hline Colwnows ofy of & - & - & 401,34 & 401,704 \\
\hline Horilug Gily of & $\underline{3,437}$ & 2016 & $\overline{1}, 550$ & $\overline{1}, 500$ \\
\hline A wille City of & $5 x$ & 606 & - & - \\
\hline
\end{tabular}

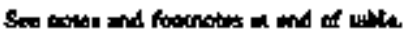


Table 32. Fibetricty Erchanges and Wroeling by Poblicty Owned Utilities, 1994 (Thousand Kilowathours) (Coatinued)

\begin{tabular}{|c|c|c|c|c|}
\hline \multirow{2}{*}{ 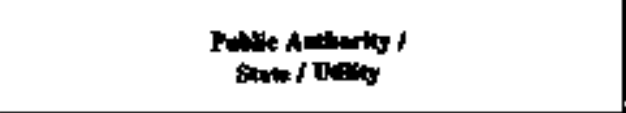 } & \multicolumn{2}{|c|}{ Exallants } & \multicolumn{2}{|c|}{ whetin: } \\
\hline & Rexived & Delinend & Received & Delinered \\
\hline \multicolumn{5}{|l|}{ 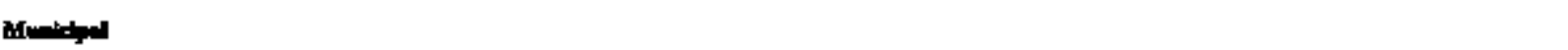 } \\
\hline \multicolumn{5}{|l|}{ ote } \\
\hline 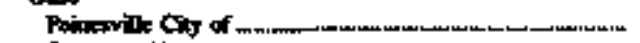 & 1,504 & $26 \mathbf{n}$ & - & $m$ \\
\hline St Mdres chy of . & 6,753 & 7.130 & - & - \\
\hline Onesper & & & & \\
\hline 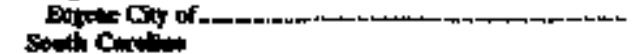 & $\mathbf{3 5 , 9 7}$ & 31644 & - & - \\
\hline 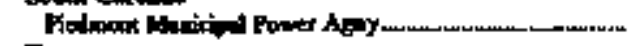 & 266,533 & 311,815 & - & - \\
\hline Teted & & & & \\
\hline Anitio Cily of & 64,05 & $\$ 9255$ & 20,606 & 23,796 \\
\hline 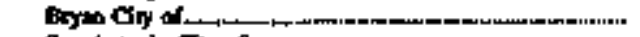 & nid & $2,00 \%$ & - & - \\
\hline s. & $1,397,427$ & $1,391,987$ & - & - \\
\hline 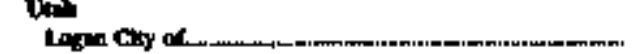 & - & - & 5,650 & 5,650 \\
\hline 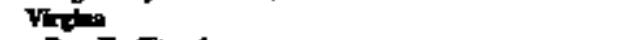 & & & & \\
\hline 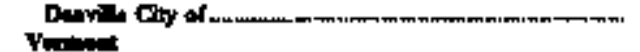 & - & - & 15,594 & 15,994 \\
\hline Beiniston City of & - & - & 1,949 & t,949 \\
\hline Bontsvith vilioge of & 4,786 & 2,557 & - & - \\
\hline Oflems Vinge of of & $\pi$ & $\pi$ & - & - \\
\hline 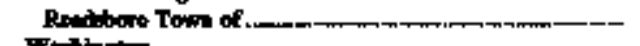 & 41 & 4 & - & - \\
\hline 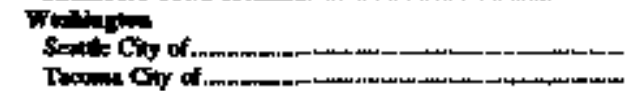 & $\begin{array}{r}5.978 .845 \\
1,197\end{array}$ & $\begin{array}{l}5.912917 \\
122992\end{array}$ & $\begin{array}{r}4,288 \\
1,206,419\end{array}$ & $\begin{array}{r}47,258 \\
1,286,417\end{array}$ \\
\hline
\end{tabular}

-Not Aptioble

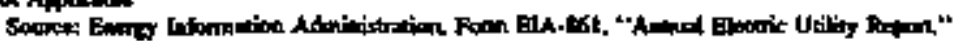




\section{Bulk Power Transactions by Cooperative Utilities}

\section{Overvlew}

This chapter presents electric trade data for coper. ative utilities, most of which are participants in the U. S. Department of Agriculture's Rural Utiljties Service (RUS) Cooperative Borrowers program. The data are separaled and presented for the Power Supply Botrowers and Distribution Borrowers. Power Supply Borrowers generate and purchase electricity for wholesale transactions with other electric utitities, while most Distribution Borrowers do not own generators and provide only distribution services to the final consumer. Cooperative borrowers are required to provide RUS with financial and operationsl data to support the RUS loan management progrand. The cooperative borrowers represent pearly all the cooperatives in the country.6

FIgure 14. Electrleity Purchases by Cooperative Utillties by State, 1994

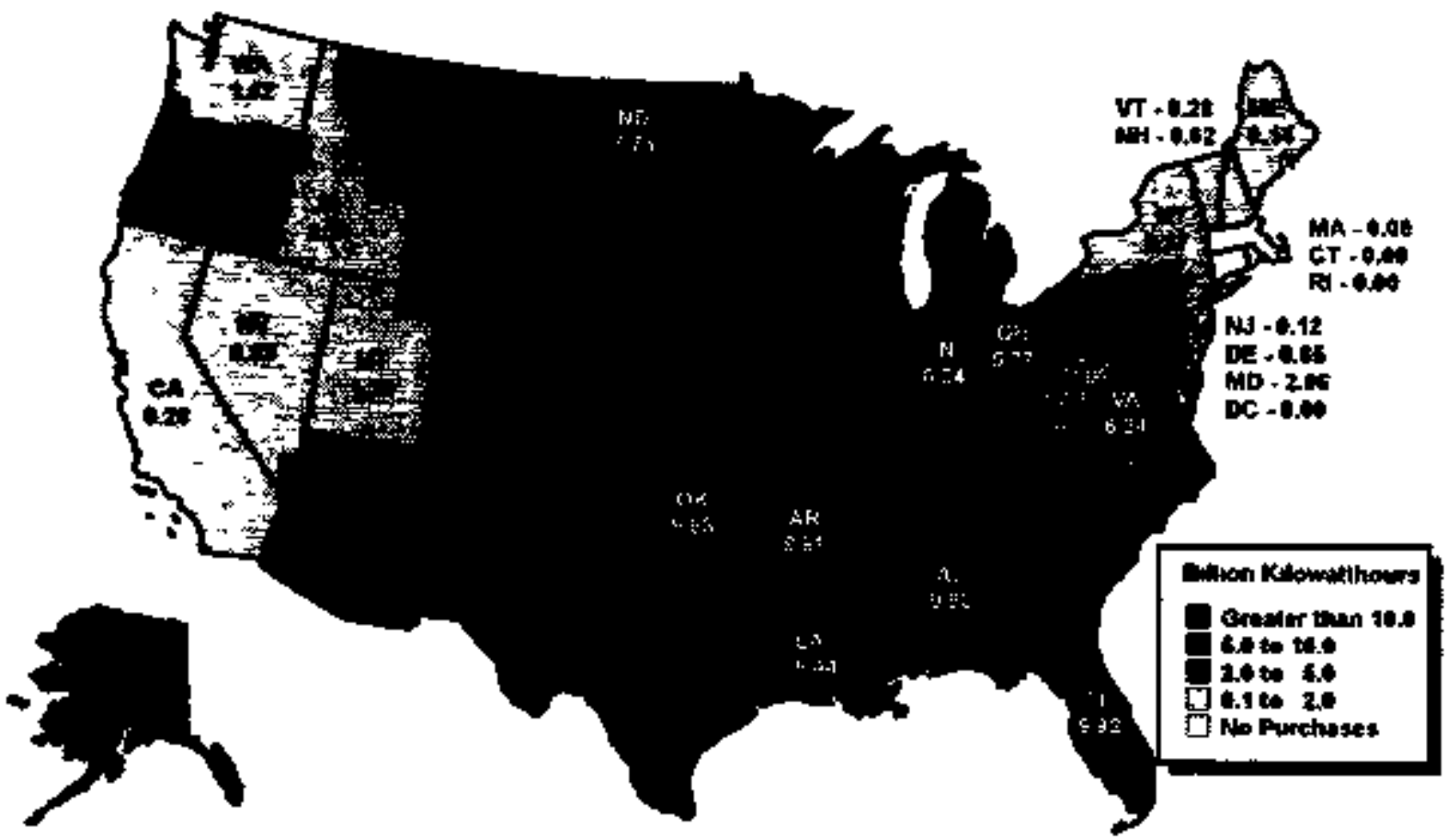

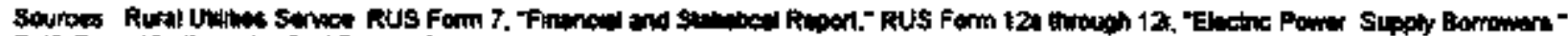

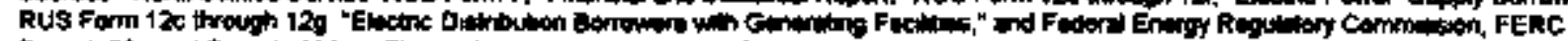

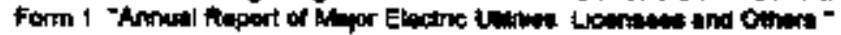

There are many types of cooperative assoctations, but five basic principles or features distinguish cooperatives from other types of private enterprise busjnesses.

- Ownersbip is held by membet-users.
- Control is on the basis of one vote per member, or on volume provided

- Operations have an at-cost (nomprofit) objective.

- Dividends on member cepital are límited.

6. There have beon tewer than 50 RUS bofrowers thal have pald ott theif Joans Those utllities are pat required to tile the RUS torms 
- Education is necessary for understanding and support. ${ }^{\text {? }}$

Cooperative entities are recognized by State statutes, which differ by State. Cooperatives can use a variety of generic labels depending on State statutes: rmutuals and cooperatives (Washington, Idaho, and Nevada); cooperatives, rural electric membership corporations (North Carolina); rural electric systems, electric power associations (Mississippi), etc. In addition, many cooperatives are incorporated. Federal legislation further defines cooperative activities. Cooperatives are organized for a variety of reasons including insurance, agricultural, telephone, water, and electrical services.

There were about 35 electruc cooperatives which predated the establishment of the Rural Electrification
Adtuinistration (REA), the prefecessor of RUS, in 1935. A number of these cooperatives were formed as mutual associations. Many of these eatly efforts, however, were not successful. It was the establishment of the REA and oher activicies stariting in 1935 that initiated the major development phase of cooperative electrification. This chapter focuses on pufchases by conperative borrowers because, at this time, RUS compurerizes only individual utility transactions with olher atilities for purchased power (Figure 14). Therefore, data on sales for resale are not presented in this chapter. Because RUS does not collect information on excbanges and wheeling, data from the Form ElA-861. "Annual Electric Utility Report," were used to provide aggregate data totals for the cooperative botrowers. Form EIA-861 is a mandatory form filed annually by all electne vtifities in the United States ob a calendar-year basis.

\section{Figure 15. Electricity Purchase Costs by Cooperative Utilitles by State, 1994}

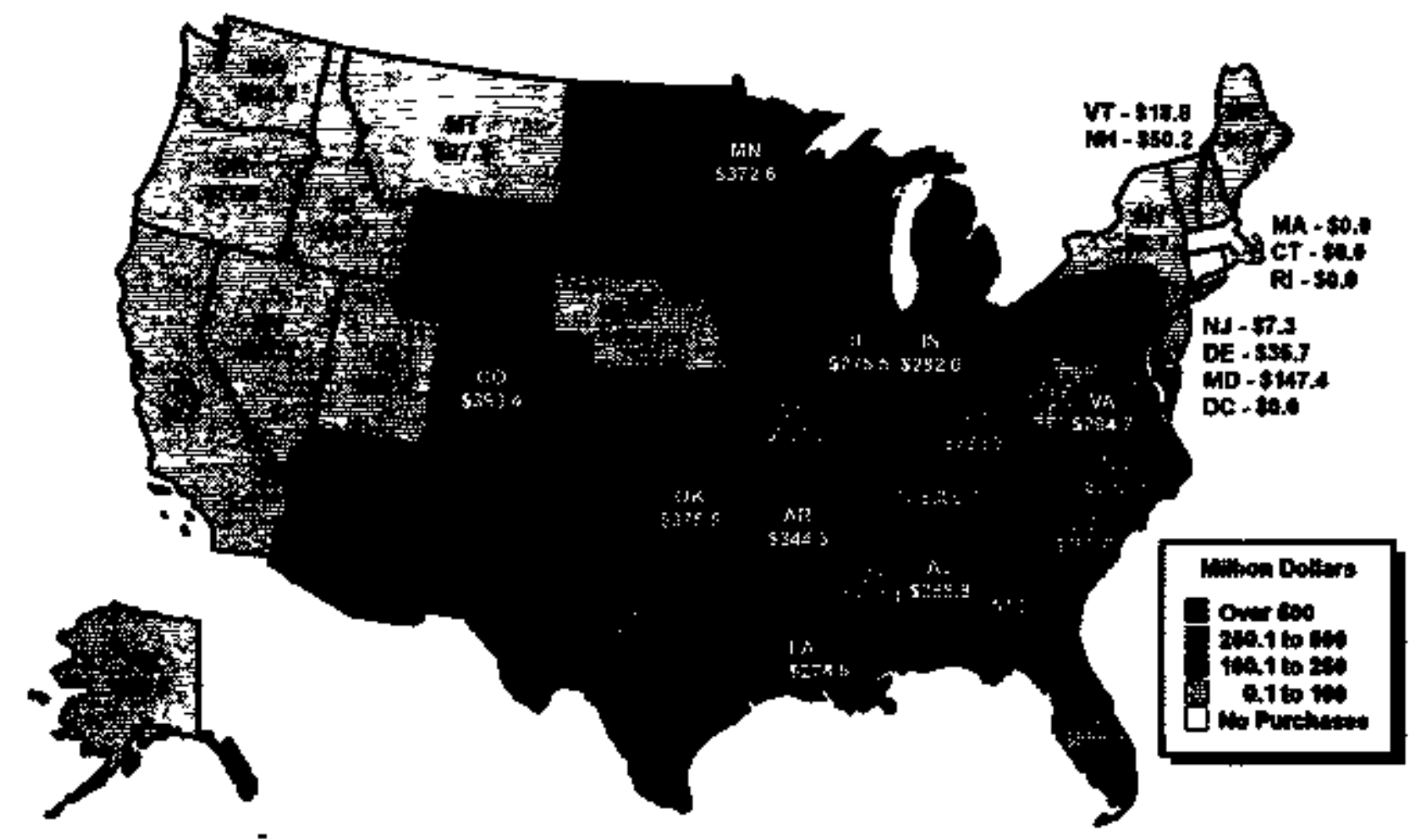

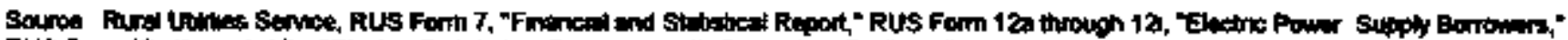

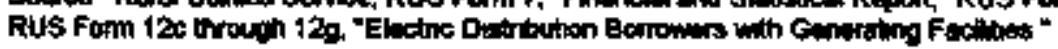

7 Intormatiop parapkrased and quoled erom Agricultural Cooperative Service, U $s$ Departmeal ot Agenculture, "Coopentive Pronciples and Legal Foudations," Cooperative Intarmalion Report I, Secliob I (Mareb 1982), p 3 


\section{Tables}

This chapter presents data on each of the cooperative utilities, most of which are RUS electric borrowers. These cooperatives purchased electricity from other cooperatives, investor-pwned, Federally owned, municipal utilities, and State authoritjes. Electricity purchases by power supply cooperatives are presented in Table 33 and by distribution cooperatives in Table 34. Cooperatives not listed elsewhere are presented in Table 35. Table 36 provides data on exchanges and transmission service transactions of the cooperative borrowers.

The tables present aggregate data on total quantity and value of each cooperative's electric utility trade (Figure 15). The reported quantities cannot be broken into îra and interruptible trade classifications. Nor is information available on demand, energy, and other revenues and costs. For ease of identification, the cooperative borrowers are listed by State according to the mailing address of the administrative office. This jisting does not necessarily reflect the electrical geographics of a cooperative's electrical system.

\section{Highlights}

While power supply cooperatives operate generation facilities to meet the needs of both bulk power requirenents of their membership and supply the endusing customer, no cooperatives were among the top 50 of all utitities for gerteration and count of end-vse customers. In part, this reflects the historical mission of cooperative ucilities to serve low-population artas. Yet, these cooperative utilities do provide service to large areas of the United States (Figure 17).

\section{FIgure 16. Eleotricity Purchases and Costs by the Top Ten Cooperbtlve Utilitles, 1994}

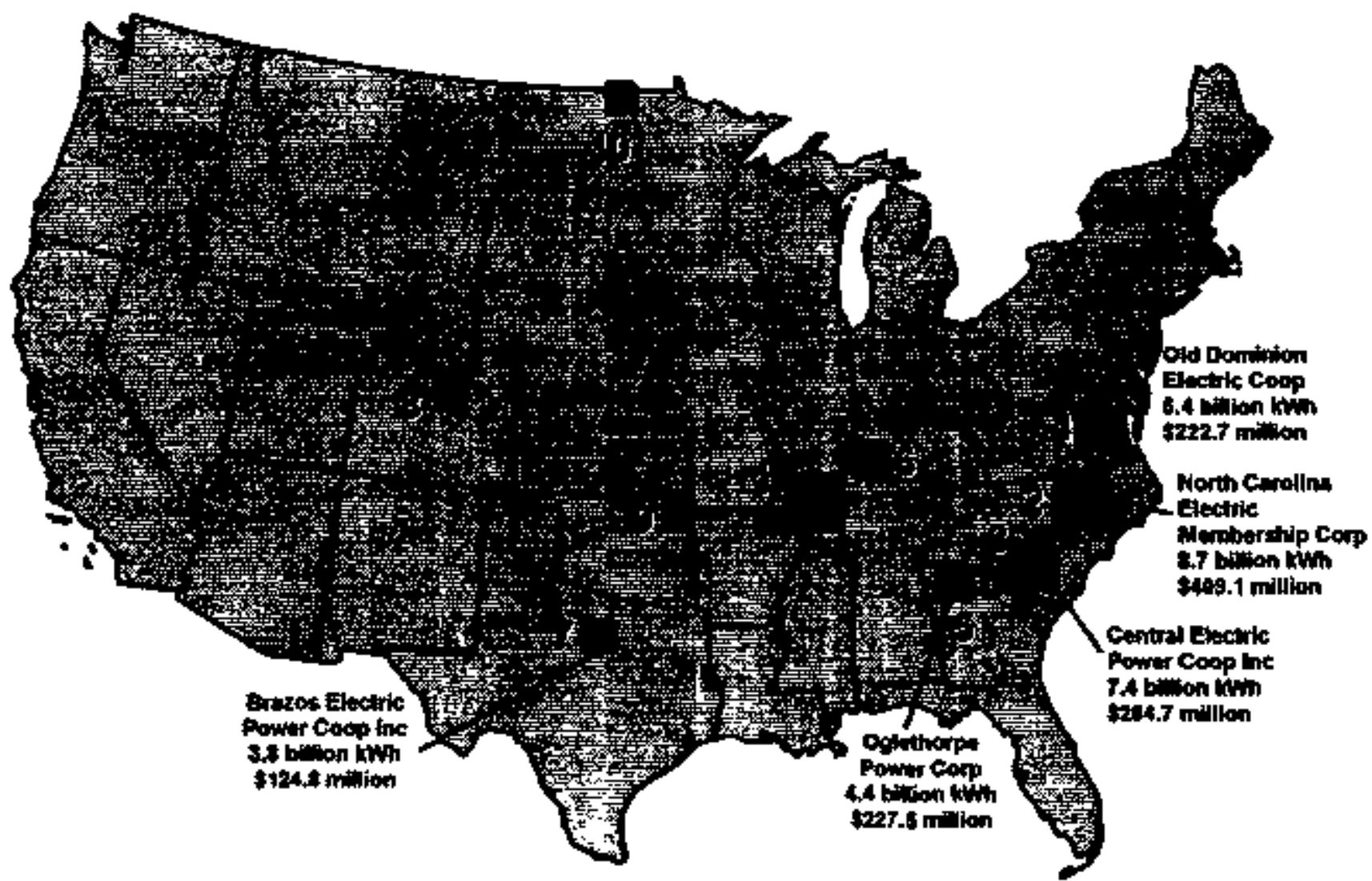

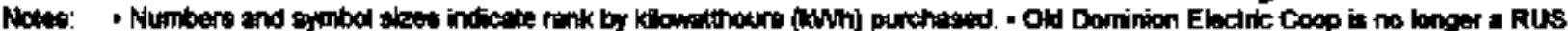
borrower and does not fie a form 12; worrce of this dita la FERC Form 1.

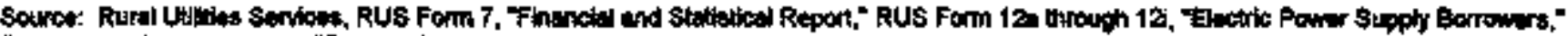

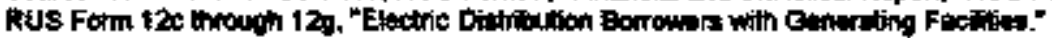




\section{Summary}

Power supply cooperatives, although small in total numbers, play an important role in the wholesale power resale market. Their large purchases and wideranging redistribution capability have put them in a highly visible position in the cooperative sector of the electric power jndustry. The amount of electricity a large power supply cooperative purchases is far greater than that of a typical distribution cooperative.

Many of these power suppliers are wholly owned by distribution cooperatives and supply only those coop. erarives as wholesale customers. Others are distribution cooperatives with excess generating capability. But as a group, power supply cooperatives continue to purchase more electric power from a wider range of sources.
Regional differences in establisbment of cooperatives primarily restulted from the histotical development of the clectric utility industry. Differences in the volume and type of trade can be telated to geographical concentration and the proximity of other utilities and transmission facilities. For instance, some large interconnected transmission systems cross cooperative service territories affording the cooperative multiple sources of power. In contrast, cooperatives that are geographically isolated may orly be able to bay from a single source.

Most distribution cooperaljyes sell power to the ultimate consumers who reside in their service territories. Cooperative service territories generally have a lower density of eustomers and many miles of distribution lines. Although distribution cooperarives do buy power from nearby investor-owned utiliıjes and government authorities, the large majority of distribution cooperatives purchase most of their supply from power supply cooperatives.

Figure 17. Electrlcity Purchases by Cooperative Utilities, 1994

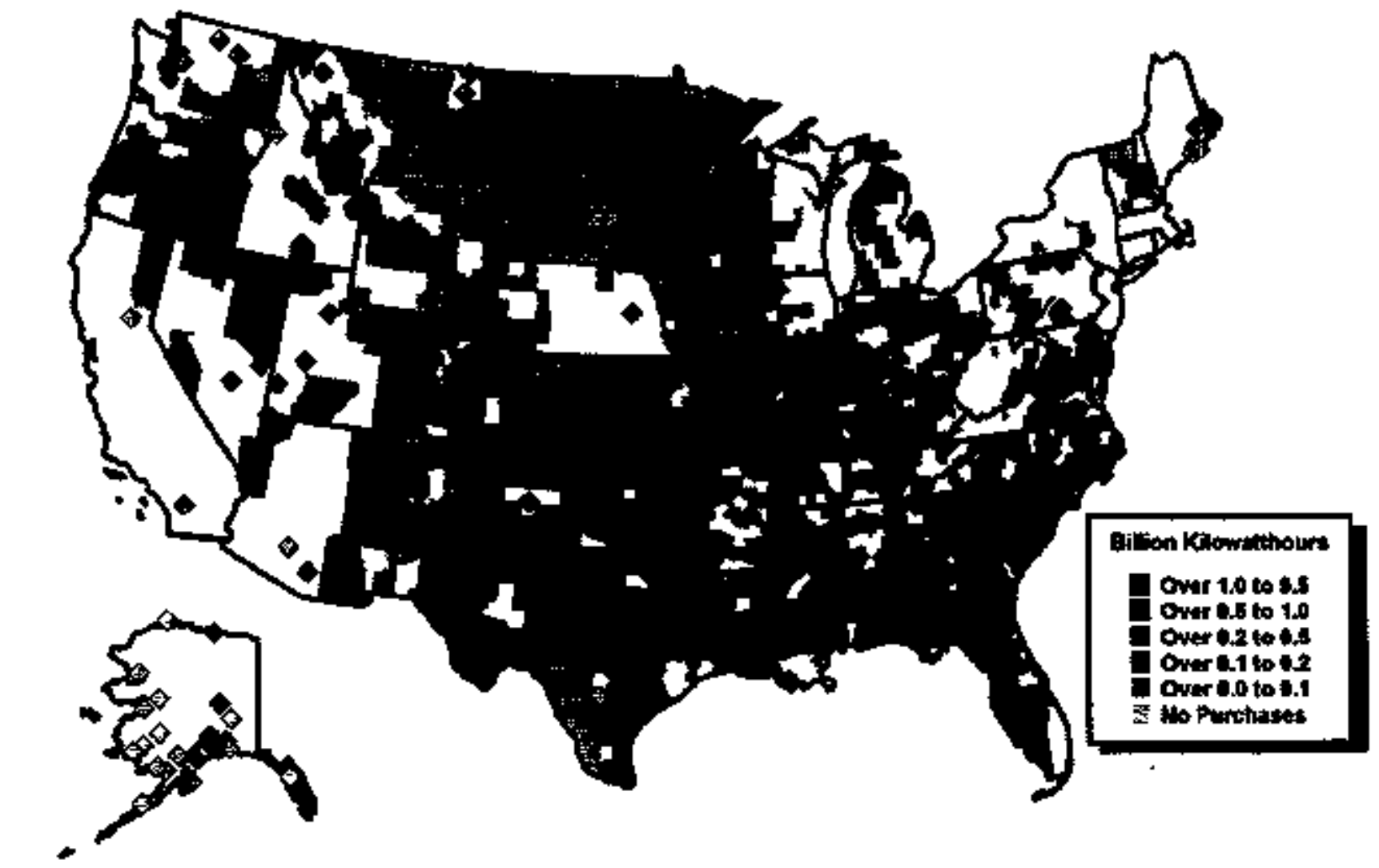

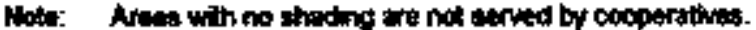

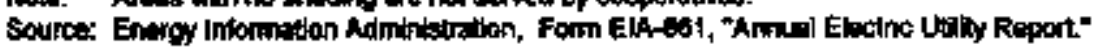


Tabie 33. Electricity Purchoses by the Power Supply Segnent of Cooperative Borrowers, by Stute, 1994

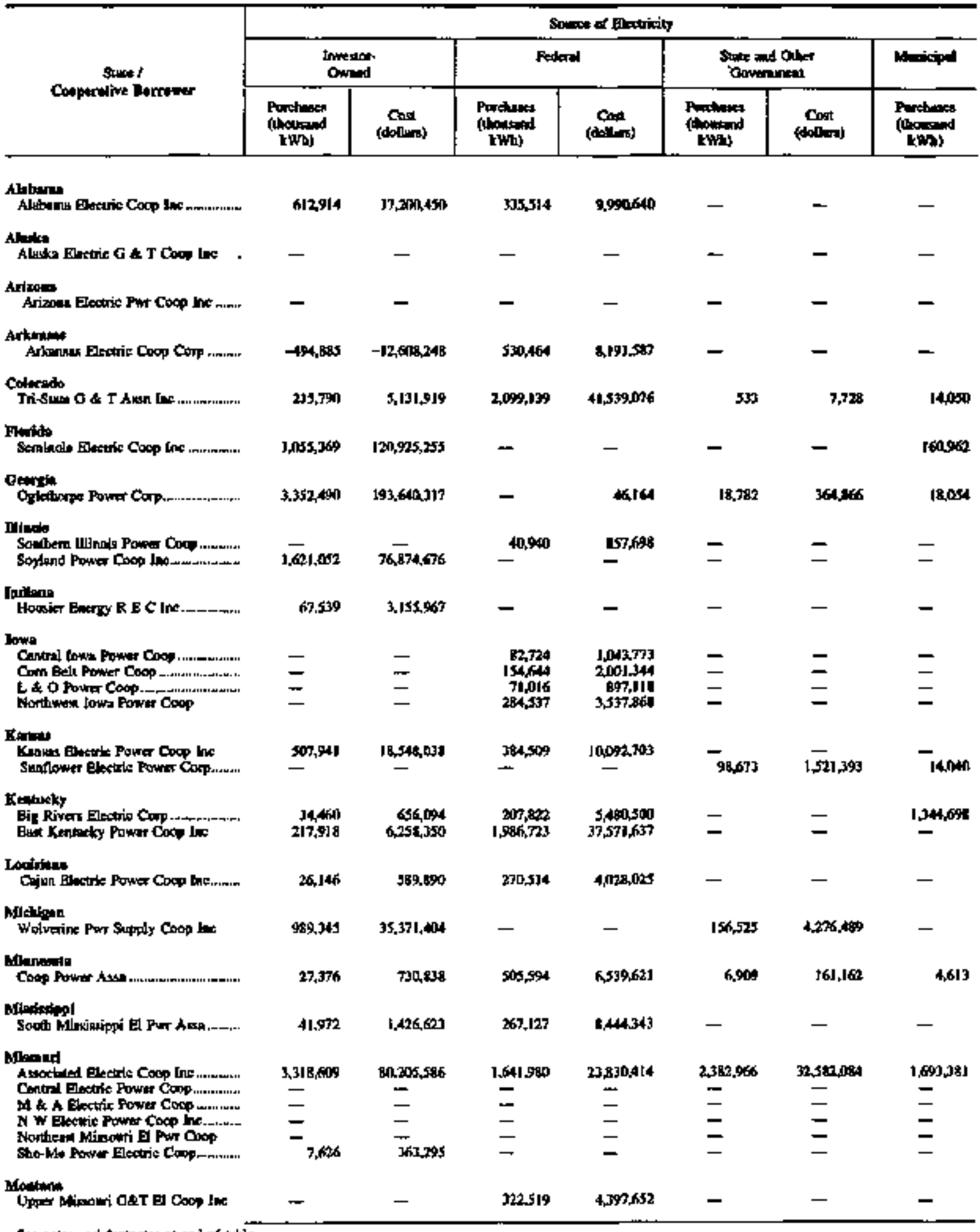

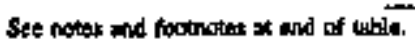


Table 33. Electridily Purchases by the Power Supply Segment of Cooperative Borrowers, by State, 1994 (Continued)

\begin{tabular}{|c|c|c|c|c|c|c|c|}
\hline \multirow{3}{*}{$\begin{array}{l}\text { Sinte / } \\
\text { Cooperative Dortome }\end{array}$} & \multicolumn{7}{|c|}{ Soures of Elequnaly } \\
\hline & \multirow{2}{*}{ Cont } & \multicolumn{2}{|c|}{ Cooperritiv: } & \multicolumn{2}{|c|}{ Otherl } & \multicolumn{2}{|c|}{ Totd } \\
\hline & & 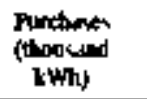 & $\underset{(\operatorname{dol})(x)]}{\operatorname{col}}$ & 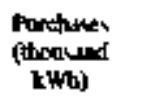 & 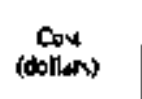 & 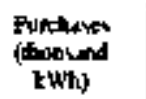 & $\underset{\text { (dolinin) }}{\text { Cont }}$ \\
\hline Alolume Detine Con Ins & - & I,395.106 & $30,975,837$ & - & - & $2,340,534$ & 48.166927 \\
\hline 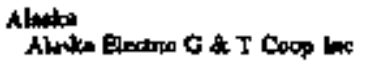 & - & 803,390 & $36,678,122$ & - & - & 860,390 & $26,678,127$ \\
\hline 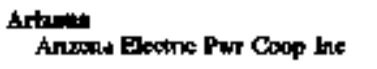 & - & - & - & 003.996 & $24.116,816$ & $902,9 \%$ & $29.116,8] 6$ \\
\hline 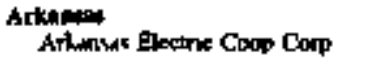 & - & $|\leq 64| 14 \mid$, & $26,691,316$ & - & - & $1,5 \% 9,420$ & $22,274,535$ \\
\hline 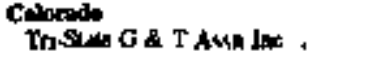 & 295,158 & $1,335,849$ & $51,136,199$ & 1,585 & 169,429 & $7,667,246$ & $90,279,509$ \\
\hline 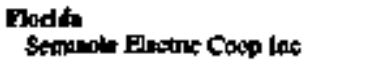 & $5,615,839$ & 185,589 & $3,493,122$ & 642 & 34,479 & $1,402,5 \leqslant 2$ & $J^{2} / 3,1604,609$ \\
\hline Oglempe Pow Cop . & 491,346 & 991, t61 & $32,934,0139$ & - & - & $4,381,087$ & $277,476,900$ \\
\hline 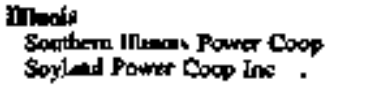 & $\bar{z}$ & $-{ }^{131}$ & $-^{8,930}$ & $\overline{-}$ & $\overline{-}$ & $\begin{array}{l}41.111 \\
1,621.0622\end{array}$ & $\begin{array}{r}666,628 \\
\pi, 17,6 \%\end{array}$ \\
\hline 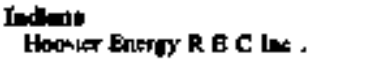 & - & 21.734 & $1,722,183$ & 22,535 & פ1].566 & 川,A|2 & $5,789.720$ \\
\hline 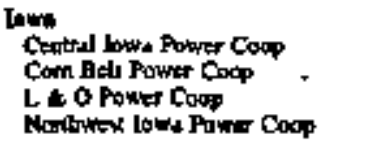 & $\begin{array}{l}= \\
\bar{z}\end{array}$ & 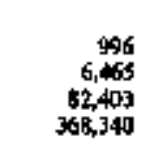 & $\begin{array}{r}33,420 \\
20,4,499 \\
3,397,918 \\
13,374,409\end{array}$ & $\begin{array}{l}219 \\
-\quad \\
\$ 19\end{array}$ & $\begin{array}{r}11,611 \\
16,440 \\
510.5 \%\end{array}$ & $\begin{array}{l}89.959 \\
161,109 \\
159,419 \\
453.6196\end{array}$ & $\begin{array}{r}1,108,809 \\
2,238,213 \\
4,205,036 \\
18,962,43\end{array}$ \\
\hline 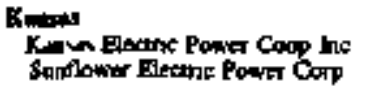 & $\overline{342,210}$ & $\underset{36}{27,757}$ & $\begin{aligned} 486,007 \\
004\end{aligned}$ & 二 & $\underset{2,531,574}{-}$ & $\begin{array}{l}975,70] \\
{[12,749}\end{array}$ & $\begin{array}{l}+1,588,3,282 \\
1,863,407\end{array}$ \\
\hline 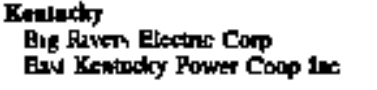 & $31,996,205$ & $\begin{array}{l}34,111 \\
39,475\end{array}$ & $\begin{aligned} 1,28,204 \\
1,207,529\end{aligned}$ & $\begin{array}{l}1,300 \\
3,848\end{array}$ & $\begin{array}{r}56,175 \\
147,155\end{array}$ & $\begin{array}{l}1,022,591 \\
2,247,064\end{array}$ & $\begin{array}{l}39,817,178 \mathrm{~B} \\
45, \mathrm{~J} 8,6,1]\end{array}$ \\
\hline Lowlenger & - & - & - & $\$ t, 186$ & 1.061 .753 & 347.946 & 5879672 \\
\hline 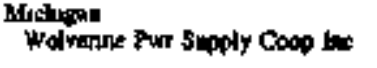 & - & - & - & - & 20 & 1.145870 & $39,607.922$ \\
\hline 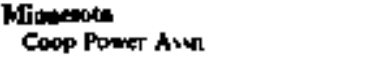 & 102.545 & 645.128 & $17,587,006$ & - & - & 1.189 .680 & $25,121,212$ \\
\hline 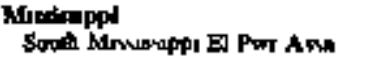 & - & $1,265,206$ & $31,318,819$ & 162,974 & $4,787,795$ & $1,737,959$ & $45,9 \pi, 580$ \\
\hline 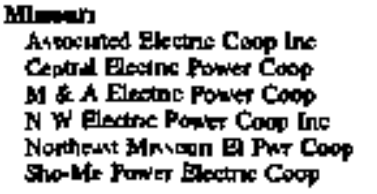 & $\begin{array}{l}36,604.156 \\
= \\
= \\
=\end{array}$ & $\begin{array}{r}443,599 \\
1,919,636 \\
1,085,003 \\
1,064,695 \\
1,093,419 \\
2,611,296\end{array}$ & $\begin{array}{l}11,957,205 \\
72,129,040 \\
40,802,076 \\
37,606,779 \\
31,880,879 \\
94,700,951\end{array}$ & $\begin{array}{l}z \\
\bar{z} \\
\bar{z}\end{array}$ & $\begin{array}{l}z \\
\bar{z} \\
z\end{array}$ & $\begin{array}{l}9,40,475 \\
1,919,6,46 \\
1,005,013 \\
1,064,496 \\
1,039,419 \\
2,618,862\end{array}$ & $\begin{array}{r}185,269,450 \\
22,129,040 \\
40,802,076 \\
37,606,799 \\
39,869,871 \\
95,063,646\end{array}$ \\
\hline 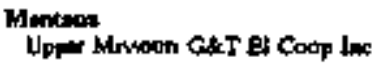 & - & 653,501 & $28,750,191$ & - & - & D7x.nal & $33.197,84\}$ \\
\hline
\end{tabular}

Set notes and footeocst and af thele 
Table 33. Electricity Purchases by the Power Supply Seginent of Cooperative Borrowers, by State, 1994 (Continumed)

\begin{tabular}{|c|c|c|c|c|c|c|c|}
\hline \multirow{3}{*}{ 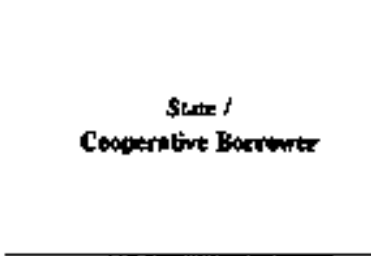 } & \multicolumn{7}{|c|}{ Sootete of gleninchy } \\
\hline & \multicolumn{2}{|c|}{ Investar. } & \multicolumn{2}{|c|}{ Fedaral } & \multicolumn{2}{|c|}{$\begin{array}{l}\text { Sinte and Olpa } \\
\text { Govennma }\end{array}$} & \multirow{2}{*}{ 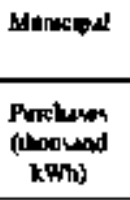 } \\
\hline & $\begin{array}{l}\text { Penclusen } \\
\text { (thousind } \\
\text { kWb) }\end{array}$ & Cont & $\begin{array}{l}\text { Fonchues } \\
\text { (thouniod } \\
\text { kWh }\end{array}$ & $\underset{\text { cont }}{\text { collens) }}$ & 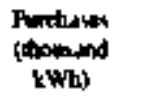 & $\underset{\text { Cont }}{\text { Contst }}$ & \\
\hline Nebealk & - & - & - & - & 2537.644 & 6878,260 & - \\
\hline 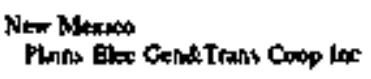 & 167,441 & 6,SIT,B36 & 357,556 & I39905,450 & 14,879 & 283,130 & 15 \\
\hline 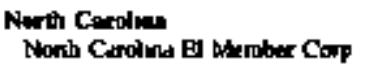 & $8,670,724$ & $409,112,374$ & - & - & - & - & - \\
\hline 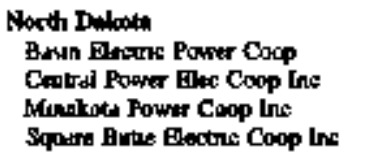 & $\begin{array}{l}-^{356,750} \\
-\end{array}$ & $\underbrace{43,739,472}_{-}$ & $\begin{aligned} \quad 0.551 \\
\$ 29,290 \\
700,6972\end{aligned}$ & $\begin{array}{l}5,485,1991 \\
4,145,039 \\
3,2] 8,942 \\
-\end{array}$ & $-^{2.139}$ & $\begin{array}{l}-30,407 \\
\frac{-}{1.135,246} \\
-\end{array}$ & $\bar{F}^{-}$ \\
\hline $\begin{array}{l}\text { Otwo } \\
\text { Bockeye Power Ins }\end{array}$ & 577,890 & 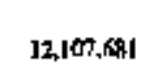 & 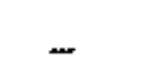 & - & - & - & - \\
\hline 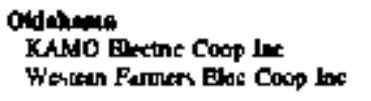 & $\begin{array}{r}39,515 \\
5,508\end{array}$ & $\begin{array}{r}1,399,306 \\
226,734\end{array}$ & $\overline{76 s, 920}$ & $\| \overline{A 11,59 \mid}$ & $\begin{aligned} 1.451 .709 \\
5.947\end{aligned}$ & $\begin{array}{r}\text { sn.724,sn } \\
128,015\end{array}$ & $\overline{-}$ \\
\hline 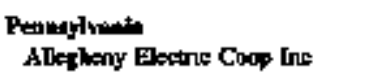 & 979,523 & 36578,319 & - & - & 267,359 & $1,73 \mathrm{~B}, 763$ & - \\
\hline 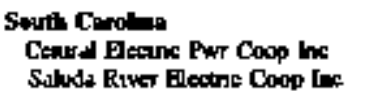 & $\begin{array}{r}\mathrm{BR}, 171 \\
1,271, \mathrm{BSS}\end{array}$ & $\begin{array}{r}4,754,145 \\
62,702,558\end{array}$ & 200504 & $\begin{array}{l}6,653,967 \\
-\end{array}$ & 20.57 .815 & $\begin{array}{c}231,483,492 \\
-\end{array}$ & $\overline{-}$ \\
\hline 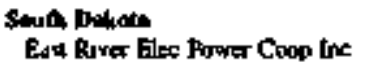 & - & $56,16 \%$ & 726,555 & $9,029,399$ & - & - & - \\
\hline 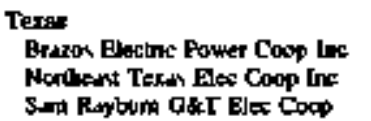 & $\begin{array}{r}1,447,553 \\
721,315\end{array}$ & $\begin{array}{l}50,0,12,745 \\
24,628,277\end{array}$ & $\begin{array}{l}128,854 \\
393,191\end{array}$ & $\begin{array}{l}3,179,177 \\
5,470,319\end{array}$ & $\begin{array}{l}193,900 \\
-\end{array}$ & $\begin{array}{l}7,67,392 \\
-\end{array}$ & $-^{66,17}$ \\
\hline 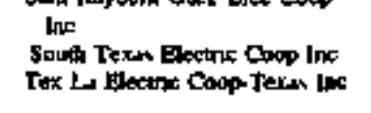 & $\begin{array}{l}527,716 \\
215,306 \\
61.176\end{array}$ & $\begin{array}{r}21,922,304 \\
1,485,600 \\
32,601,454\end{array}$ & $\overline{185.392}$ & $\begin{array}{r}-\overline{1,828,599} \\
30528\end{array}$ & $-1,075$ & $\begin{array}{l}\text { 219:0 } \\
-\end{array}$ & $\underbrace{}_{\substack{4009 \\
j 00,8089}}$ \\
\hline Jiwh & ו15.59 & 862,956 & 2480 & 57,648 & 1,878 & 4,897 & $\mathbf{J}, 140$ \\
\hline $\begin{array}{l}\text { Vernisn } \\
\text { Vernant Electne Get Coop Ine }\end{array}$ & 79,087 & $2,716,567$ & - & - & 17390 & 384,629 & - \\
\hline Wrocondind Poncer Coop & $15\}, 904$ & 2764858 & 43.861 & 812014 & 264,736 & $3,9+2.100$ & 11.139 \\
\hline
\end{tabular}

See noves and footnotos 44 end af table 
Table 33. Electricity Purchnses by the Power Supply Segment of Cooperative Borrowers, by State, 1994 (Continued)

\begin{tabular}{|c|c|c|c|c|c|c|c|}
\hline \multirow{3}{*}{$\begin{array}{c}\text { Siple I } \\
\text { Cosperntive Bortomer }\end{array}$} & \multicolumn{7}{|c|}{ Source of Elestnesty } \\
\hline & \multirow{2}{*}{$\begin{array}{c}\text { Munmpl } \\
\text { Cont } \\
\text { (dolliss) }\end{array}$} & \multicolumn{2}{|c|}{ Conperulve } & \multicolumn{2}{|c|}{ Other 1} & \multicolumn{2}{|c|}{ Tolal } \\
\hline & & $\begin{array}{l}\text { Purctures } \\
\text { (tromind } \\
\text { kWh) }\end{array}$ & $\underset{\text { (ADilon) }}{\operatorname{Cost}}$ & $\begin{array}{l}\text { Purchine } \\
\text { (thothund } \\
\text { kWla) }\end{array}$ & $\underset{\text { (Lond }}{\cos }$ & $\begin{array}{l}\text { Purclures } \\
\text { (thomidd } \\
\text { twh) }\end{array}$ & $\underset{\text { (dollonit) }}{\text { Cost }}$ \\
\hline $\begin{array}{l}\text { Nebesato } \\
\text { Noberke Flestre GeT Coop Int }\end{array}$ & - & - & - & - & - & 2397,644 & $82,878,261$ \\
\hline Nitw Nence & 352 & - & - & 982 & 23,714 & 940,573 & $24,330,54$ \\
\hline Nerd Candion & - & - & - & - & - & B.,67t, 324 & 499, Jn2,374 \\
\hline 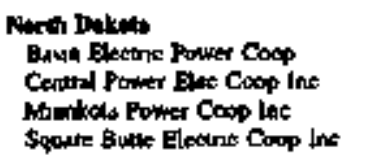 & $\begin{array}{l}\frac{-}{244,651} \\
-\end{array}$ & $\begin{array}{r}222,260 \\
685,044 \\
979,908 \\
12,124\end{array}$ & $\begin{array}{r}4,724,238 \\
25,299,0184 \\
2,954,368 \\
79.178\end{array}$ & $\begin{array}{l}-44,807 \\
\frac{1}{1088,722} \\
-\end{array}$ & $\begin{array}{l}56,0009 \\
- \\
16,215,879 \\
-\end{array}$ & $\begin{array}{r}281,327 \\
1,014,374 \\
3,194,541 \\
12,124\end{array}$ & $\begin{array}{r}10,889,068 \\
29,444,129 \\
73,668,718 \\
79,178\end{array}$ \\
\hline 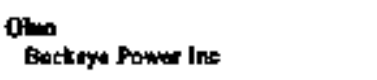 & - & - & - & - & - & $\operatorname{sng} 800$ & $12,107 / 81$ \\
\hline 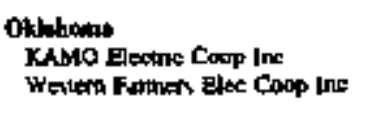 & $\overline{-}$ & $1,3+1,36\}$ & $65,100,784$ & $\overline{-}$ & $\overline{-}$ & $\begin{array}{r}3,231,601 \\
777,465\end{array}$ & $\begin{array}{r}117,204,613 \\
11,7 \pm 8,360\end{array}$ \\
\hline $\begin{array}{l}\text { Foonytuen: } \\
\text { Alligheny Electinc Coop he }\end{array}$ & - & - & - & - & - & $1,306,882$ & $98,317,0102$ \\
\hline 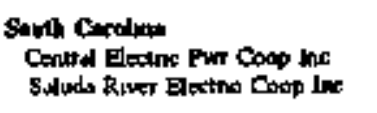 & $\overline{-}$ & $\overline{-}$ & $\overline{-}$ & $\overline{-}_{15,287}$ & $\overline{1,7363,326}$ & $\begin{array}{l}3,436,490 \\
1.257,172\end{array}$ & $\begin{array}{r}284,707, \mathrm{BP4} \\
\quad \mathrm{A4}, 085, \mathrm{BR4}\end{array}$ \\
\hline $\begin{array}{l}\text { South Diktate } \\
\text { Fint Ruser Elec Pown Copp the }\end{array}$ & - & 813,900 & $34,223,557$ & - & 5,587 & $1,540,458$ & $43,315,0018$ \\
\hline 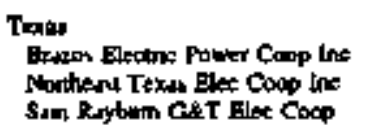 & $\begin{array}{l}119,421 \\
-\end{array}$ & $\begin{array}{l}1,260,313 \\
215,545\end{array}$ & $\begin{array}{r}46,612,239 \\
6,701212\end{array}$ & ${ }_{-}^{666,313}$ & $\frac{17,071,079}{-}$ & $\begin{array}{l}3,766,300 \\
1,270,054\end{array}$ & $\begin{array}{r}124,846,679 \\
36,799,800\end{array}$ \\
\hline 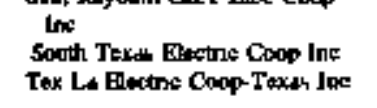 & $\begin{array}{r}-85,322 \\
2,225,702\end{array}$ & $\begin{array}{r}221,543 \\
1,174,910 \pi \\
195,752\end{array}$ & $\begin{array}{r}6,522,025 \\
20,577,253 \\
7,561,944\end{array}$ & $\begin{array}{r}9,8,340 \\
65,232 \\
28, R 14\end{array}$ & $\begin{array}{r}489,670 \\
21,075,154 \\
529,531\end{array}$ & $\begin{array}{r}746,139 \\
1,546,313 \\
1,100,497\end{array}$ & $\begin{array}{l}28,934,399 \\
48,473,366 \\
42,554,159\end{array}$ \\
\hline Uid & 21,660 & 14,613 & $185, \pm 40$ & 1,437 & 25, & $5 \sin 099$ & $1,289,686$ \\
\hline Ventood & - & - & - & - & $-24,1 \leqslant 0$ & $\%, 6 \pi$ & J,2ת,036 \\
\hline $\begin{array}{l}\text { Wutandin } \\
\text { Dearylind Power Coop }\end{array}$ & 179,175 & 519,202 & $8,131,3,50$ & 210 & 2.615 & 995,032 & $15,856,114$ \\
\hline
\end{tabular}

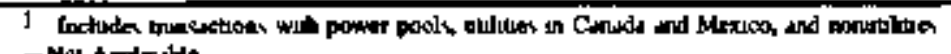
- Noh hadadite

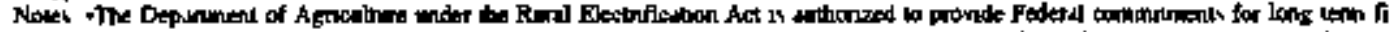

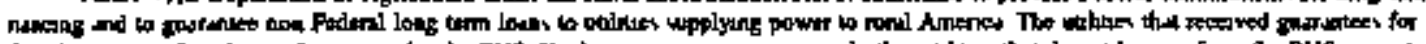

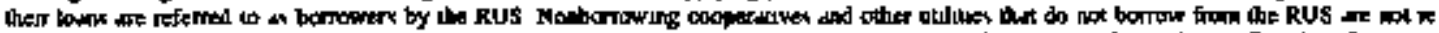

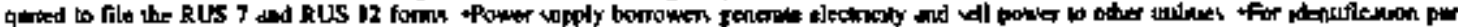

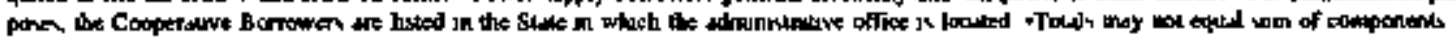
besure of undipandex mophint

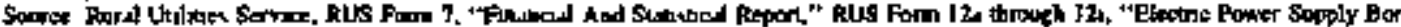

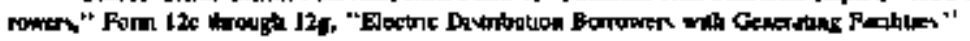


Table 34. Eleztricity Purchases by the Dtsiribution Segment of Cooperative Bormowers, by State, 1994

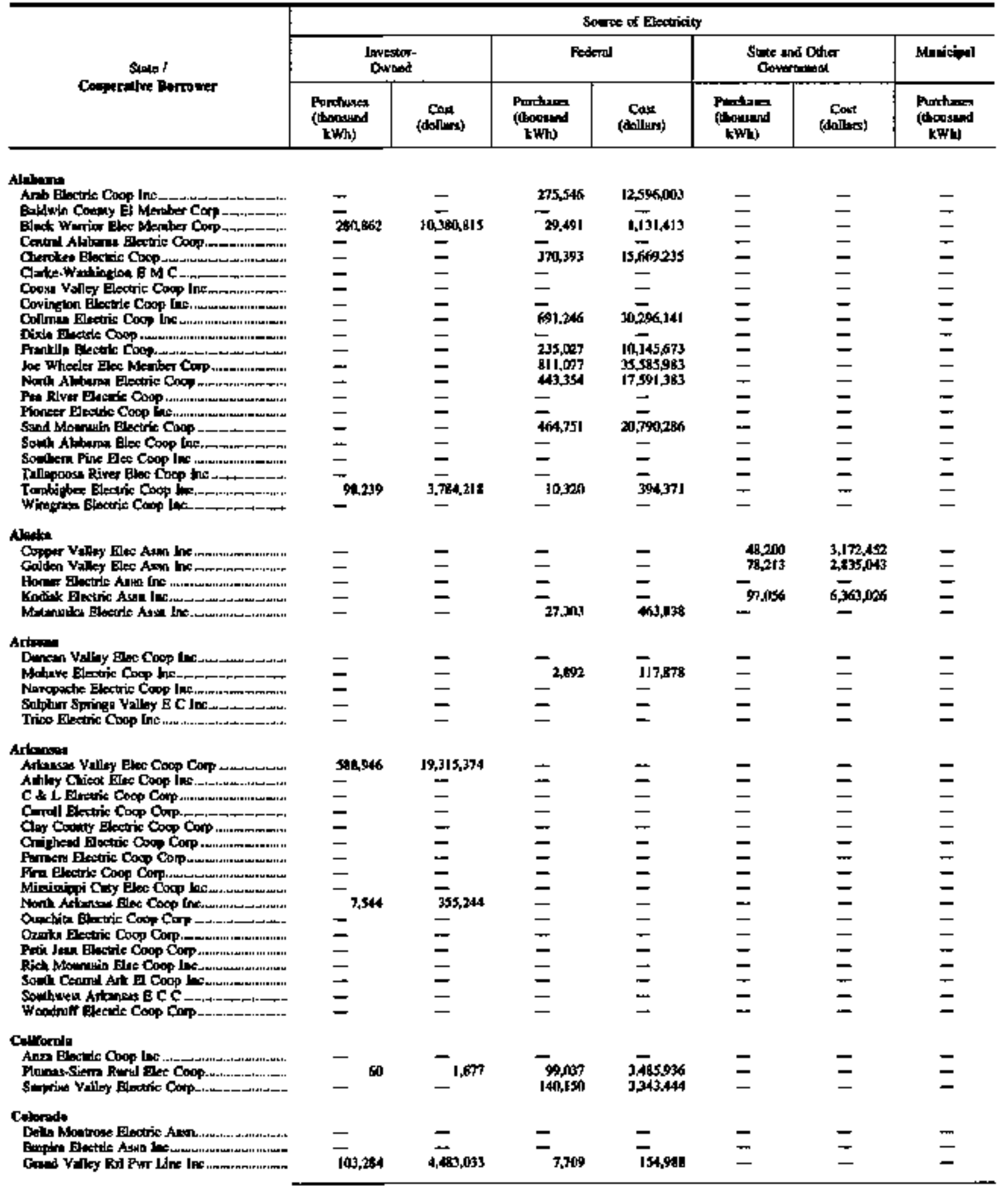

Ses noles and footnotes ut and of thble. 
Table 34. Electricity Purchases by the Distribution Segurent of Cooperative Borrowers, by Stale, 1994 (Continued)

\begin{tabular}{|c|c|c|c|c|c|c|c|}
\hline \multirow{3}{*}{ Cente of } & \multicolumn{7}{|c|}{ Sorece of Electincky } \\
\hline & \multirow{2}{*}{ 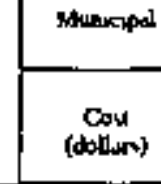 } & \multicolumn{2}{|c|}{ Coopetatut } & \multicolumn{2}{|c|}{ I I I } & \multicolumn{2}{|c|}{ Toxd } \\
\hline & & 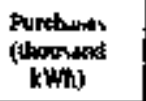 & (doll|cr) & 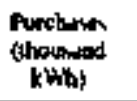 & $\underset{\text { (dorlas) }}{\operatorname{Cos}}$ & $\begin{array}{l}\text { Purchula } \\
\text { (1komind } \\
\text { kwh) }\end{array}$ & Dunc \\
\hline 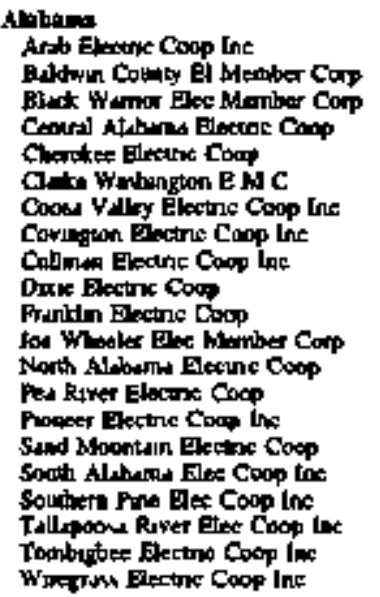 & $\begin{array}{l}= \\
= \\
= \\
= \\
= \\
= \\
= \\
= \\
= \\
= \\
= \\
=\end{array}$ & 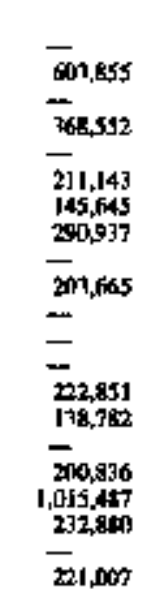 & 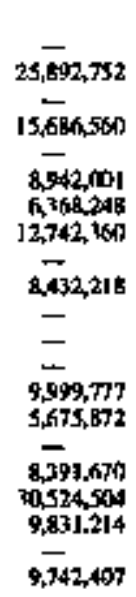 & $\begin{array}{l}- \\
- \\
- \\
- \\
- \\
- \\
- \\
- \\
- \\
- \\
- \\
- \\
- \\
- \\
-\end{array}$ & $\begin{array}{l}\overline{-} \\
- \\
- \\
= \\
= \\
= \\
= \\
= \\
= \\
- \\
= \\
= \\
-\end{array}$ & 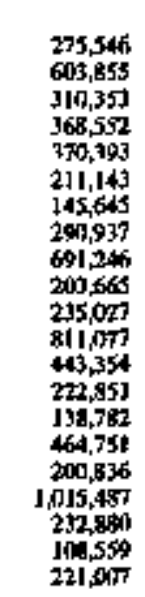 & 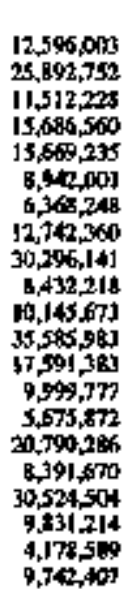 \\
\hline 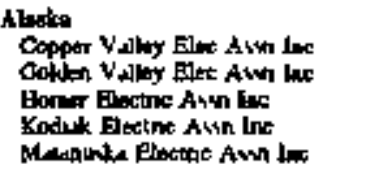 & $\begin{array}{l}- \\
- \\
-\end{array}$ & $\begin{array}{l}\overline{186,297} \\
429,942 \\
\overline{470,123}\end{array}$ & 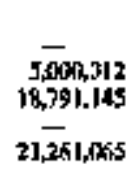 & $\begin{array}{r}232 \\
-\quad 461 \\
431\end{array}$ & $\begin{array}{r}5,195 \\
9,027 \\
2 \\
6,528 \\
19,227\end{array}$ & $\begin{array}{r}49,700 \\
264,742 \\
429,942 \\
91,219 \\
457,907\end{array}$ & $\begin{array}{r}3,177,847 \\
7,844,382 \\
18,791,147 \\
6,369,554 \\
21,74,136\end{array}$ \\
\hline 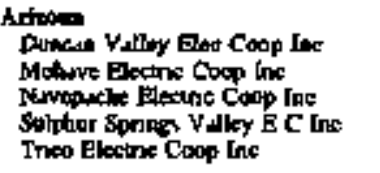 & $\begin{array}{l}- \\
- \\
-\end{array}$ & $\begin{array}{r}1,264,649 \\
41,135 \\
201,743 \\
410,153 \\
262, \pi 08\end{array}$ & $\begin{array}{l}51,3 m, 282 \\
22,856,806 \\
17,993,762 \\
21,692,865 \\
17,078,506\end{array}$ & $\begin{array}{l}- \\
- \\
-\end{array}$ & $\begin{array}{l}- \\
- \\
-\end{array}$ & $\begin{array}{r}1,261,645 \\
457,027 \\
21,742 \\
418,153 \\
262,708\end{array}$ & $\begin{array}{l}51,507,282 \\
22,974,716 \\
17,99,762 \\
21,692,866 \\
13,009,501\end{array}$ \\
\hline 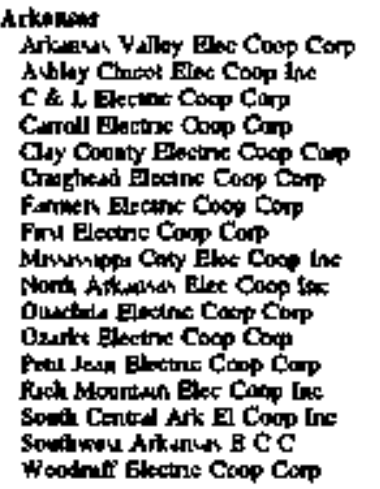 & $\begin{array}{l}- \\
- \\
- \\
- \\
= \\
= \\
= \\
- \\
- \\
-\end{array}$ & 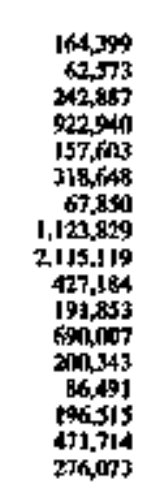 & 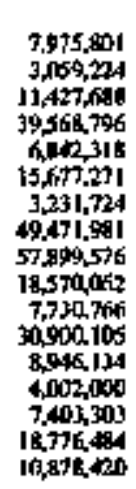 & $\begin{array}{l}= \\
= \\
= \\
= \\
= \\
= \\
= \\
= \\
= \\
-\end{array}$ & 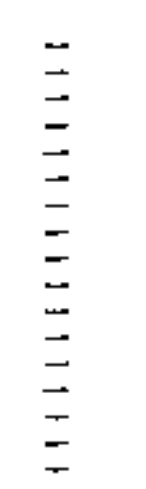 & 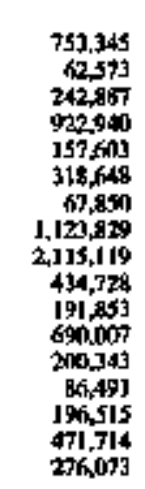 & 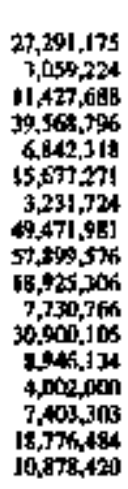 \\
\hline 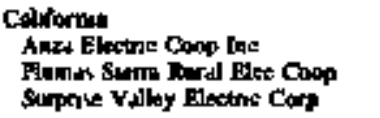 & $\frac{-}{-}$ & $\begin{array}{l}15,950 \\
-\end{array}$ & $\frac{1,681,201}{-}$ & - IBA & $-^{4,13}$ & $\begin{array}{r}35,958 \\
09,283 \\
140,150\end{array}$ & $\begin{array}{l}1,681,320 \\
3,491,787 \\
3,343,444\end{array}$ \\
\hline 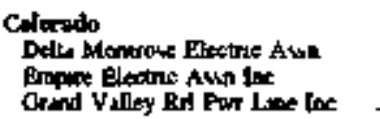 & $\overline{-}$ & $\begin{array}{l}4|3,84| \\
343,495 \\
-\end{array}$ & 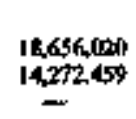 & $\bar{z}$ & $\overline{-}$ & $\begin{array}{l}413,841 \\
143,495 \\
110,993\end{array}$ & $\begin{array}{r}18,656,020 \\
14,272,499 \\
4,638,021\end{array}$ \\
\hline
\end{tabular}

Siec abter and foothotes a and af the 
Table 34. Electricity Purchases by the Distribation Segment of Cooperative Borrowers, by State, 1994 (Contuued)

\begin{tabular}{|c|c|c|c|c|c|c|c|}
\hline \multirow{3}{*}{$\begin{array}{c}\text { Sint i } \\
\text { Cocpercune Bocrower }\end{array}$} & \multicolumn{7}{|c|}{ 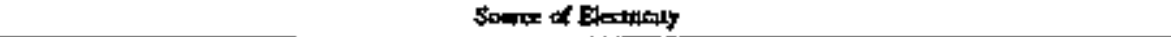 } \\
\hline & \multicolumn{2}{|c|}{$\begin{array}{c}\text { Imvestor } \\
\text { Owned }\end{array}$} & \multicolumn{2}{|c|}{ Pedatal } & \multicolumn{2}{|c|}{$\begin{array}{l}\text { Stath nd Otiver } \\
\text { Corvenumenl }\end{array}$} & \multirow{2}{*}{ 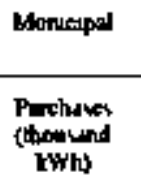 } \\
\hline & 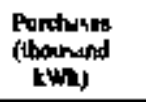 & $\begin{array}{c}\cosh \\
\text { (difltwis }\end{array}$ & 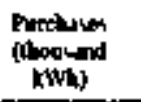 & $\underset{\text { (dollest) }}{\operatorname{cosi}}$ & $\begin{array}{l}\text { Purchuse: } \\
\text { (thowind } \\
\text { KWD) }\end{array}$ & $\begin{array}{c}\text { Cort } \\
\text { (dollon) }\end{array}$ & \\
\hline \multicolumn{8}{|l|}{ Colorndo } \\
\hline Genibe Coonty Rec Asn Inc & - & - & - & ـ & - & - & - \\
\hline 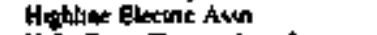 & - & - & 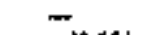 & - & - & - & - \\
\hline Holy Crou Bectire han tar & 459.914 & $25,017,945$ & 43.554 & 359,243 & 265,234 & $3,904,425$ & - \\
\hline 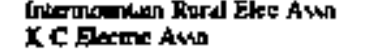 & $\pi 0,7 \times 0$ & $12,759,466$ & $13 J, 410$ & 2,755,257 & - & - & $=$ \\
\hline Lo PJas Eleopre Asm tre & $\overline{-}$ & $\overline{-}$ & $\overline{-}$ & $\overline{-}$ & 二 & $\ddot{-}$ & - \\
\hline Morgan Cowny Ruml Elec Awa & - & - & - & - & - & - & - \\
\hline 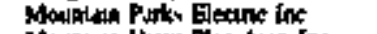 & - & - & - & - & - & - & - \\
\hline 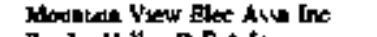 & - & - & - & - & - & - & - \\
\hline Ponde YAlley R E \& the & - & - & 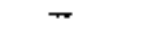 & - & - & - & - \\
\hline Sen I vod Electinc A ivi Ine & - & 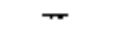 & - & - & - & - & - \\
\hline SA Lols Yalky R E C Inc & - & - & - & - & - & - & $=$ \\
\hline Su Mrgoel Pawn Asun Ex & - & - & - & $\rightarrow$ & - & - & - \\
\hline Sangro bo Choo Bloc Arn Ine & - & - & - & - & - & ـ & - \\
\hline 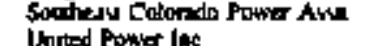 & - & - & - & - & - & - & $=$ \\
\hline Unrted Powing lact & 398 & 16,878 & - & - & - & - & - \\
\hline Whise Ruver Eletenc Awn inc & - & - & - & - & - & - & - \\
\hline 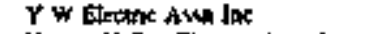 & & - & - & - & - & - & $=$ \\
\hline Yampa Valiey Electort AsW be & 372004 & IS,535,655 & 26,232 & 431,2600 & - & - & - \\
\hline \multicolumn{8}{|l|}{ Delowere } \\
\hline Delinwe Bedtec Coop lac & - & - & - & - & - & - & - \\
\hline \multicolumn{8}{|l|}{ Floadn } \\
\hline 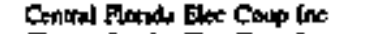 & - & - & 91,582 & 334031 & - & - & $m$ \\
\hline & $\bar{z}$ & 二 & - & - & س & - & 一 \\
\hline Day Bectos Coop lne & $\bar{z}$ & $\overline{-}$ & $=$ & $\bar{z}$ & 二 & 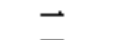 & 715 \\
\hline & 6015,39 & $23,446,150$ & 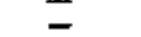 & $=$ & $\bar{z}$ & 二 & $\bar{z}$ \\
\hline 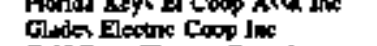 & - & - & - & - & - & - & $=$ \\
\hline Gutf Coult Dectre Cogp he & - & - & - & - & - & - & - \\
\hline Pested Row Elotene Coop lac & - & - & - & - & - & - & $\rightarrow$ \\
\hline Soloter Bloxthe Cusp Im & - & - & - & - & - & - & 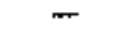 \\
\hline 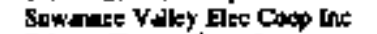 & - & - & 20,519 & 687.337 & - & - & \\
\hline Inlquan Electuse Coop Est & - & - & 68.135 & I,464.965 & - & - & 8,751 \\
\hline In County Electre Coop Ine & عـ & - & 25,747 & 748,313 & - & - & - \\
\hline Wey filnds ge coop Avil Inc & - & - & - & ع & - & - & - \\
\hline 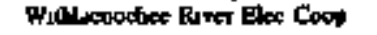 & - & - & - & - & - & - & - \\
\hline \multicolumn{8}{|l|}{ Content } \\
\hline 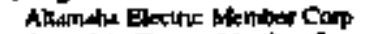 & - & - & 17,670 & 465,956 & - & - & - \\
\hline 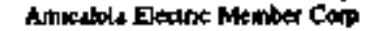 & - & - & 18,349 & $\$ 94,158$ & - & - & - \\
\hline Blus Fedge Boonten E M C & - & - & $342,6 \% 5$ & $4,78: 412$ & - & - & - \\
\hline Cunbuches Elestse Menber Corp & - & $=$ & E5, 948 & 396,523 & $=$ & - & - \\
\hline 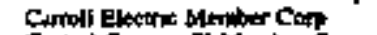 & 14 & 4,161 & 27,588 & 728510 & - & - & - \\
\hline 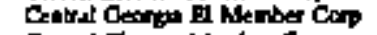 & - & - & 21,382 & 567,730 & - & - & - \\
\hline Candal Elestinc Member Corp & - & - & $4,0,81$ & 131,567 & - & - & - \\
\hline Cobs Electors Memberinup Conp & - & - & 61,656 & $1,605,536$ & - & - & - \\
\hline Colquit Eloctinc Mamban Conp & - & $=$ & 62,343 & $1,64,178$ & - & - & - \\
\hline Coweta Payetie El Menber Copp & - & - & $21.0 \times 4$ & 556,14 & - & - & - \\
\hline 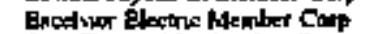 & - & - & 14,418 & 3890,126 & - & - & - \\
\hline fiac Beturc blenberilp Corp & $\rightarrow$ & - & 90.942 & $2,397,541$ & - & - & - \\
\hline 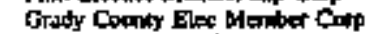 & - & - & 17,1032 & 449,547 & مـ & - & - \\
\hline Gonstion Powe Corp & - & - & 50,024 & $1,318, \mathbf{9 5 2}$ & - & - & - \\
\hline 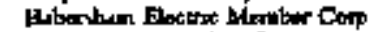 & - & ــ & 16,578 & 437069 & $\pi$ & - & - \\
\hline Hn Elotine Mentor Corp & - & - & 30,900 & 808,680 & - & - & - \\
\hline 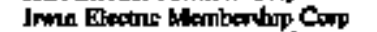 & - & - & 15,556 & 357,387 & מ- & - & - \\
\hline Jeken Electry Member Coup & - & - & 77,782 & $2,050,897$ & - & - & - \\
\hline 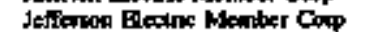 & - & - & 22,500 & 390,192 & - & - & - \\
\hline Lmar Evetre Menbernalp Colp & - & - & 10粕 & 289,122 & - & - & - \\
\hline 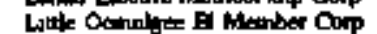 & - & - & 12.65 & 317,689 & - & - & - \\
\hline 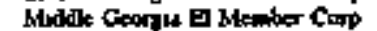 & - & - & $9, \mathbf{6 7}$ & 299 & - & - & - \\
\hline Norh George Elec Merrber Corp & - & - & $2,045,842$ & $87,528,442$ & - & - & - \\
\hline 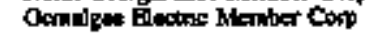 & - & - & 13,419 & 326,737 & - & - & - \\
\hline
\end{tabular}

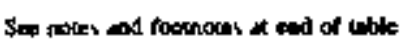


Table 34. Electricity Purchases by the Distrilention Segment of Cooperative Borrowers, by State, 1994 (Continued)

\begin{tabular}{|c|c|c|c|c|c|c|c|}
\hline \multirow{3}{*}{ 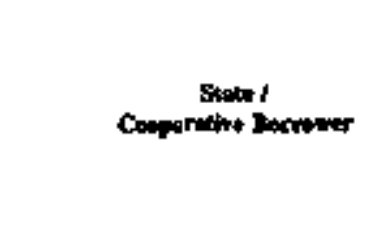 } & \multicolumn{7}{|c|}{ Socre of Electrocity } \\
\hline & \multirow{2}{*}{$\begin{array}{c}\text { Mencepil } \\
\text { Cost } \\
\text { (dollery }\end{array}$} & \multicolumn{2}{|c|}{ Cooparative } & \multicolumn{2}{|c|}{ ' } & \multicolumn{2}{|c|}{ Total } \\
\hline & & $\begin{array}{l}\text { Paplunes } \\
\text { (1)oound } \\
\text { kWh) }\end{array}$ & Cond & 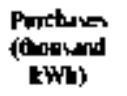 & $\begin{array}{c}\text { Cont } \\
\text { (dollm }\end{array}$ & $\begin{array}{c}\text { Pardudes } \\
\text { (thoubud } \\
\text { kWb) }\end{array}$ & $\begin{array}{c}\text { Cors } \\
\text { (dollers) }\end{array}$ \\
\hline 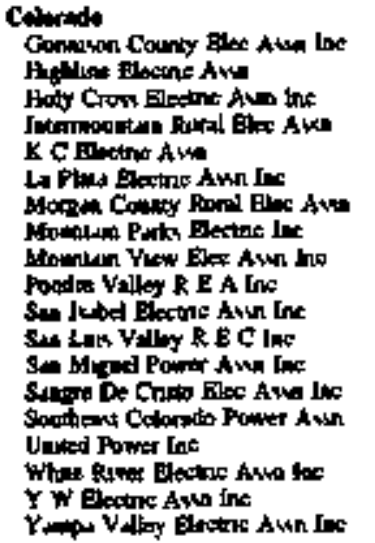 & $\begin{array}{l}- \\
= \\
= \\
= \\
= \\
= \\
= \\
= \\
= \\
= \\
=\end{array}$ & $\begin{array}{r}97,075 \\
327,392 \\
7,373 \\
- \\
371,203 \\
360,359 \\
198,072 \\
207,215 \\
15,0,011 \\
586,254 \\
392,983 \\
174,995 \\
123,251 \\
66,216 \\
168,625 \\
530,139 \\
96,769 \\
519,3155 \\
7\end{array}$ & 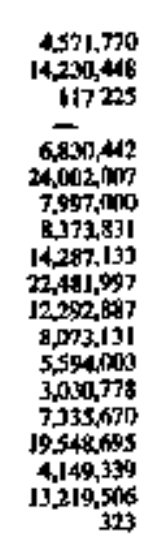 & $\begin{array}{l}- \\
- \\
- \\
= \\
- \\
- \\
= \\
- \\
- \\
- \\
-\end{array}$ & $\begin{array}{l}-1,276 \\
- \\
709.140 \\
= \\
- \\
- \\
- \\
- \\
- \\
- \\
- \\
- \\
-35,103 \\
-\end{array}$ & 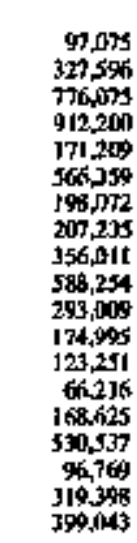 & 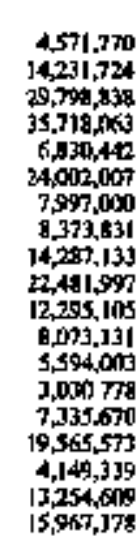 \\
\hline Delawart & 一 & 652,719 & 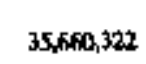 & - & 一 & 652.719 & $15,660,0,322$ \\
\hline 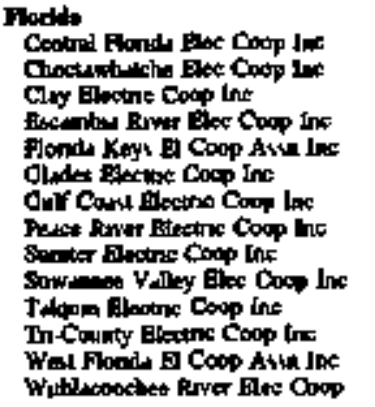 & $\begin{array}{l}- \\
51.156 \\
= \\
= \\
= \\
= \\
= \\
= \\
=\end{array}$ & 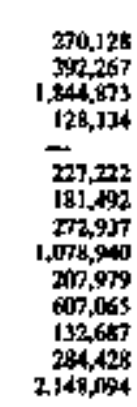 & 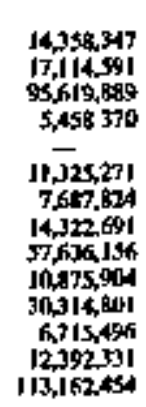 & $\begin{array}{l}- \\
- \\
- \\
- \\
- \\
- \\
- \\
- \\
-\end{array}$ & $\begin{array}{l}- \\
- \\
- \\
=0212 \\
- \\
- \\
- \\
= \\
- \\
- \\
-\end{array}$ & 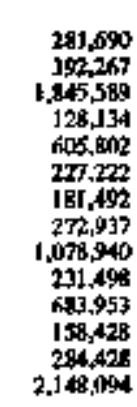 & 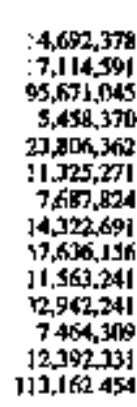 \\
\hline 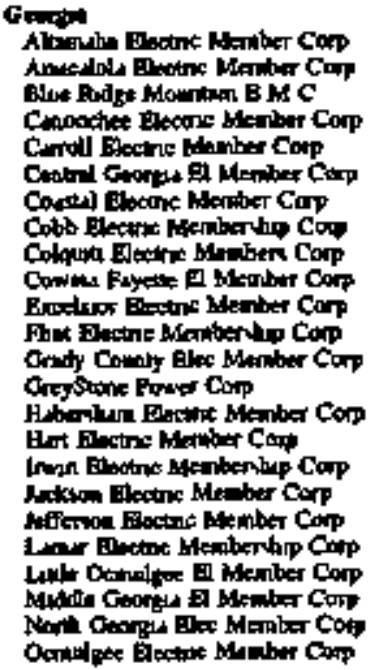 & $\begin{array}{l}= \\
= \\
= \\
= \\
= \\
= \\
= \\
= \\
= \\
= \\
= \\
= \\
=\end{array}$ & 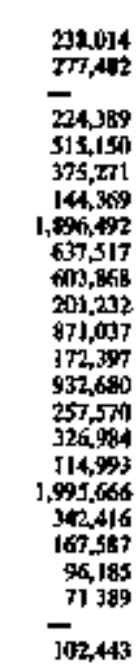 & 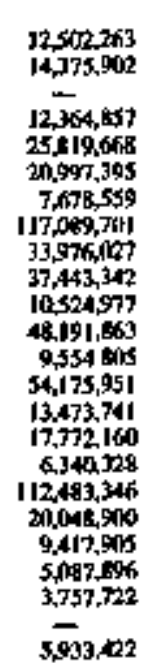 & $\begin{array}{l}- \\
- \\
- \\
- \\
- \\
- \\
- \\
- \\
- \\
- \\
- \\
- \\
- \\
- \\
-\end{array}$ & $\begin{array}{l}- \\
z \\
- \\
- \\
= \\
- \\
- \\
- \\
- \\
- \\
- \\
- \\
- \\
- \\
-\end{array}$ & 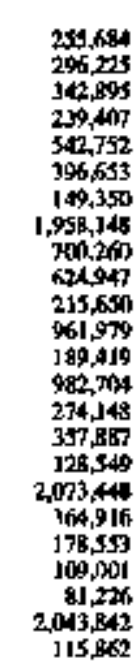 & 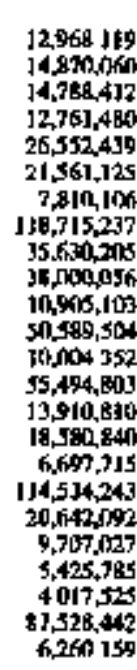 \\
\hline
\end{tabular}

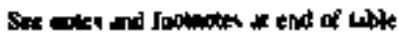


Table 34. Eectricity Purchases by the Distribution Segment of Cooperative Borrowers, by State, 1994 (Continmed)

\begin{tabular}{|c|c|c|c|c|c|c|c|}
\hline \multirow{3}{*}{$\begin{array}{c}\text { Sitre f } \\
\text { Cospermyra Bocrower }\end{array}$} & \multicolumn{7}{|c|}{ Soume of Eloctariry } \\
\hline & \multicolumn{2}{|c|}{$\begin{array}{l}\text { Innexpr } \\
\text { Owned }\end{array}$} & \multicolumn{2}{|c|}{ Fedarat } & \multicolumn{2}{|c|}{$\begin{array}{l}\text { Shate and Dolwer } \\
\text { Goxemmenl }\end{array}$} & \multirow{2}{*}{ 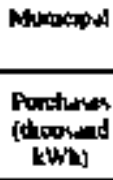 } \\
\hline & $\begin{array}{l}\text { Purtules } \\
\text { (thowind } \\
\text { kWh) }\end{array}$ & $\begin{array}{c}\text { Con } \\
\text { (dollery) }\end{array}$ & 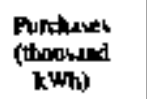 & $\begin{array}{c}\operatorname{Cou} \\
\text { (doluns) }\end{array}$ & $\begin{array}{l}\text { Pmichases } \\
\text { (thoptind } \\
\text { (1Wh) }\end{array}$ & $\underset{\text { (Sollownt }}{\text { Cont }}$ & \\
\hline \multicolumn{8}{|l|}{ Gatis } \\
\hline Oopmos Electiric Methet Cotp & $=$ & - & 129975 & 341,004 & - & - & - \\
\hline Otefendke Rural El Matber Copp & - & - & 15,379 & 406.154 & - & - & - \\
\hline Patauld Eectine Bestite Cop & - & - & 5,276 & 139,38 & - & - & - \\
\hline Plinum Eledine Member Corp & - & - & 16,725 & 41.714 & - & - & - \\
\hline Rayle Elecunc Menoberhip Corp & - & - & J6,943 & 46,697 & - & - & - \\
\hline Sotal Rorl Elec Menter Conp & - & - & 49,521 & 1.307 .870 & - & - & $=$ \\
\hline Sawnes Destre Bombern Cor & - & - & 31,159 & 827904 & - & - & - \\
\hline Sluh Pine Elec Moniter Corp & $\sigma$ & - & 7,816 & 206,153 & - & - & - \\
\hline Swinter Electinc Meruber conp & - & - & 18,372 & 485,200 & ــ & - & - \\
\hline Thret Notch Eles Memiter Cor & - & - & 19,244 & 318,547 & - & - & - \\
\hline In Gonpiy Flac Member Cocp & 136 & 12,807 & IN, J5S? & 260,593 & - & - & - \\
\hline Tr-Si= Bloxinc Merber Cup & - & - & 103,805 & $8,45 \mathrm{E}, 220$ & - & - & - \\
\hline Trwop Elestre deniben Conp & - & - & 17.815 & $504,46 \mathrm{~J}$ & - & - & - \\
\hline 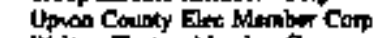 & - & - & $7,4 \mathrm{I}$ & 196.139 & - & - & - \\
\hline Wilten Foctue Mdember Copp & - & - & 48,721 & $1,294,502$ & - & - & - \\
\hline Wahulewon 림 Member Cop & - & - & 23,125 & 60,690 & - & - & - \\
\hline \multicolumn{8}{|l|}{ lom } \\
\hline Derw sar Power Co & - & - & 165,847 & $4,524,169$ & - & - & - \\
\hline Pall R1* Rutal Elac Coof Int & - & - & 174,308 & $4,428,963$ & - & - & - \\
\hline [dalo Onty LAP Goop Asid It: & - & - & 39.241 & $1,002,269$ & - & - & - \\
\hline Kootena Electro Coop ine & - & - & 250,742 & $6,734,514$ & - & - & - \\
\hline Lant Ruver Beseme Coop Inc & - & - & $79,0 \%]$ & $1,025,061$ & - & - & - \\
\hline Noriletin Lutins lace & - & - & 264,765 & $5,343,935$ & - & - & - \\
\hline Ruti Ruwe Rural Elec Copp inc & - & - & 214,267 & $5,070,728$ & - & - & - \\
\hline Sdroon Rryes Glecure Coop lac & - & - & $167,92 \%$ & 1,289,197 & - & - & - \\
\hline \multicolumn{8}{|l|}{$\ln (x)$} \\
\hline Adanis Elexpestat Coop & - & - & - & - & - & - & - \\
\hline Clay Eesine Coop Inc & - & - & - & - & - & - & - \\
\hline Clum Coanny bes Coop las & - & - & - & - & - & - & - \\
\hline Cuk Moulone Eleture Coop & - & - & - & - & - & - & $=$ \\
\hline Com Belt Electonc Coop lac & 17 & 1,358 & - & - & - & - & - \\
\hline Extern IJun Eleatnc Coop & - & - & - & - & - & - & - \\
\hline Egypten Eextre Coop Aiv & - & - & - & - & - & - & - \\
\hline Fankers Mohwl Eletixc Do & - & $=$ & - & - & - & - & - \\
\hline Dheons Rural Eleterse Co & - & - & - & - & - & - & - \\
\hline to Camll electine Coop Ins & - & - & - & - & - & - & $\rightarrow$ \\
\hline M I M Eketre Coep inc & - & - & - & - & - & - & - \\
\hline MaDenough Power Coop & - & - & - & - & - & - & - \\
\hline Menurd Electris Con & - & - & - & - & - & - & - \\
\hline Moruxe County Def Capplase & - & - & - & - & - & - & - \\
\hline Rord Bestnc Coeven Coop & - & سع & - & - & $=$ & - & - \\
\hline Sowbememen it Ele Cosp Inc & - & - & - & - & - & - & - \\
\hline Sopolherd Muran, $E$ lac Cocp, & - & - & - & - & - & - & - \\
\hline Sodbwewan Electnc Coop tac & - & - & - & - & - & - & - \\
\hline Spoon River Electnc Coop lns & - & - & - & - & - & - & - \\
\hline Tn Counry Blextre Coop fone & - & - & - & - & - & - & - \\
\hline 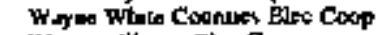 & - & - & - & - & - & - & - \\
\hline Wonter Hinol Elec Corp & - & - & - & - & - & - & - \\
\hline \multicolumn{8}{|l|}{ Indman } \\
\hline Boom Councy Ruml E MS & - & - & - & - & - & - & - \\
\hline Davwss Mrts County R B M C & - & - & - & - & - & - & - \\
\hline Decribr Courny Rutal E M C & - & - & - & - & - & - & - \\
\hline 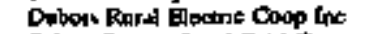 & - & - & - & - & - & - & - \\
\hline Folton Coonty Runl EMC & 54,197 & $2,481,518$ & - & - & - & - & - \\
\hline Haneock Counhy Rural E M C & - & - & - & - & - & - & - \\
\hline Hamban Coonty Rord B as C & - & - & - & - & - & - & - \\
\hline IA & 736,556 & $12,824,688$ & - & - & - & - & - \\
\hline 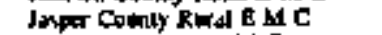 & - & - & - & - & - & - & - \\
\hline Idy County Real E A C & - & - & - & - & - & 一 & 一 \\
\hline Joliron Cownt Rural EMC & - & - & - & - & - & - & - \\
\hline Kentukee Valley Rand B BC C & - & - & - & - & - & $=$ & - \\
\hline
\end{tabular}

See nover and foctnoter at and of lible 
Table 34. Electricity Purchases by the Distribution Segment of Cooperative Borrowers, by State, 1994 (Conlinued)

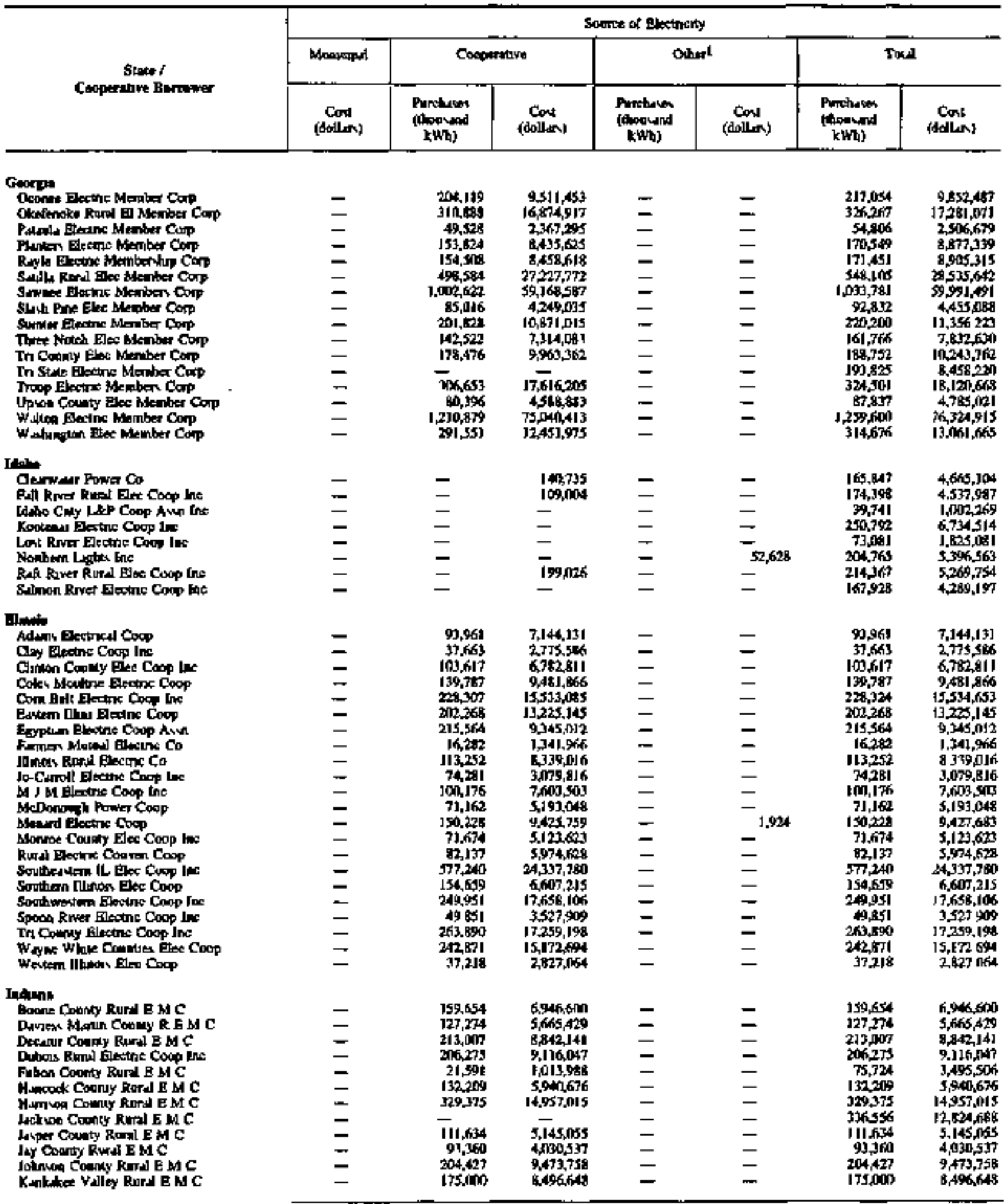

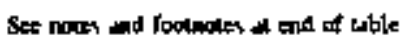


Table 34. Electricity Purchases by the Distribution Segment of Cocperative Barrowers, by State, 1994 (Contimued)

\begin{tabular}{|c|c|c|c|c|c|c|c|}
\hline \multirow{3}{*}{$\begin{array}{c}\text { State } \\
\text { Ceoperotare boctome }\end{array}$} & \multicolumn{7}{|c|}{ Scoune of electrety } \\
\hline & \multicolumn{2}{|c|}{$\begin{array}{l}\text { Inveriler } \\
\text { Owried }\end{array}$} & \multicolumn{2}{|c|}{ fulterd } & \multicolumn{2}{|c|}{ Stre and ond } & \multirow{2}{*}{ 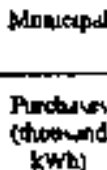 } \\
\hline & 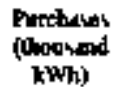 & $\begin{array}{c}\text { Coak } \\
\text { (dollust }\end{array}$ & 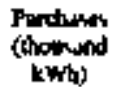 & $\underset{\text { (dollent }}{\text { Cond }}$ & 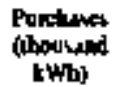 & $\begin{array}{c}\text { Cosu } \\
\text { (doblas) }\end{array}$ & \\
\hline
\end{tabular}

Indingt

Knox Obetry Real E M C

Koncula Couty Rerl EMC

Luprang Conty Roral E M C

MW की Connty Rorat BMC

Mami Car Conty Rm E M C

Hewlos Couns Rind E M C

Noble Conony Ruth EMC

Mothemion Red E M C

Orange Coenty Rutal B M C

Pate Coonty Rural E B C

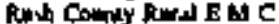

Shelloy Couney Rurd E MC

Sout Cemual thd Ana RBMC

Soubudver Inilum R B bI C

Southern Indian R E C bus

Stenken Conoty Roral E M C

Syllem toony Prol E MC

Tpromt Rord Eke Mambor Corp

Ifued Burl Bee Banber Cop

Julues Div Weotem IN REMAC

Wouth Coosty Rord E h C

Wrim Codny Rutd A M C

Wayec County Roral E M C

Whats Coundy Ruril $\mathrm{B}$ M C

tom

Adma County Coop Elestre Co Alladite Clyos El Coop Ine Benon Coopty Eloc Codp Aur

Buchum County Rrl Elec Corp

Bullex Conly REul Elec Coop

Calboun Coonty Eloe Crop Avin

Codat Valley Bltitie Coop

Chankar Valley Elec Coop lus

Clake Electre Cocp Ine

Entern low a Lughts.Powar Cosp

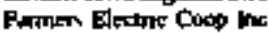

Arinkil Rniri Elextre Coop

Ghiddn Rutrd Electris Coop

Gindy County Raral Ber Coop

Guthe Courty Rurl E C A

Husodx Coonty Rord Elec Cosp

H-root Coiniy Rd Elec Con

Hawkey Tn Coundy Er Coop in:

Hrimbold Cowny R E C

IU Coority Rurd Flextise Coop

bowd Luter Dlacenc Conp

Lim Comk Rowal B C A

Lyon Rardl Electroc Cois

Msquates VAlley RA Elex Coop

Merthd County Bol Bles Coop

budlus Power Coop

Monera County Rurpl Ploc Coop

Whisubotod Yilley R E C

Nymin Eleste Coop In.

Oxcold Dlectine Coop In

Follk Coop Elac Ais

Plymewli Eletinc Coop Ava

Rides Eloxurs Coop int

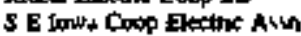

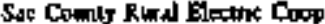

Slowr Electre Cop Aw

Soun Crawsord Rurd Plec Corp

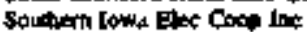

Set wore and footnotes at and of whe

$\begin{array}{ll}= & = \\ = & = \\ = & = \\ = & = \\ = & = \\ = & = \\ = & = \\ = & = \\ = & = \\ = & = \\ = & = \\ = & = \\ = & =\end{array}$

\begin{tabular}{|c|c|c|c|c|c|c|}
\hline \multirow{2}{*}{$=$} & - & - & - & - & - & - \\
\hline & - & - & - & - & - & - \\
\hline$=$ & - & - & - & - & - & - \\
\hline- & - & - & - & - & - & - \\
\hline- & - & - & - & - & - & - \\
\hline- & - & - & - & - & - & - \\
\hline- & - & - & - & - & - & - \\
\hline- & $\bar{I}$ & - & $T$ & - & - & - \\
\hline $\bar{z}$ & - & - & - & - & - & - \\
\hline$\overline{-}$ & - & - & - & $=$ & - & - \\
\hline- & - & - & - & - & $\overline{-}$ & $=$ \\
\hline- & - & $\pi$ & - & - & - & - \\
\hline- & - & - & - & - & - & - \\
\hline בـ & - & - & - & - & - & - \\
\hline- & - & - & - & - & - & - \\
\hline- & - & - & - & - & - & - \\
\hline- & - & - & - & $=$ & - & - \\
\hline- & - & - & - & - & - & - \\
\hline E & $\bar{z}$ & $\bar{z}$ & $\bar{z}$ & $=$ & $\bar{I}$ & Z \\
\hline- & - & - & - & - & - & - \\
\hline- & - & - & - & - & - & - \\
\hline- & - & - & - & - & - & - \\
\hline- & - & - & - & - & - & - \\
\hline- & - & - & - & - & - & - \\
\hline - & - & - & - & - & - & - \\
\hline - & - & - & - & - & - & - \\
\hline- & $\bar{Z}$ & $=$ & 二 & $=$ & 二 & $z$ \\
\hline- & - & - & $\sigma$ & - & - & - \\
\hline & - & - & - & $=$ & - & - \\
\hline - & - & - & - & - & - & - \\
\hline$m$ & - & - & - & - & - & 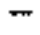 \\
\hline- & $\vec{a}$ & Z & $=$ & $=$ & $=$ & - \\
\hline & Z & - & - & - & $\bar{Z}$ & - \\
\hline- & - & - & - & - & - & - \\
\hline
\end{tabular}


Table 34. Electricity Purchases by the Distribution Seggment of Cooperative Borrowers, by State, 1994 (Continued)

\begin{tabular}{|c|c|c|c|c|c|c|c|c|c|c|}
\hline \multirow{3}{*}{ 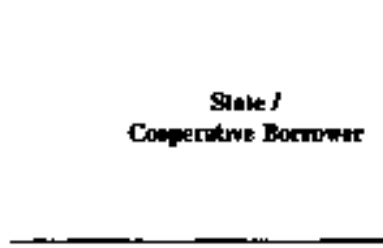 } & & \multicolumn{9}{|c|}{ Source of Electronty } \\
\hline & & \multirow{2}{*}{ 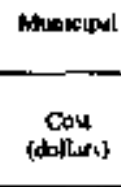 } & \multicolumn{2}{|c|}{ Cooperdive } & \multicolumn{4}{|c|}{0 ater } & \multicolumn{2}{|c|}{ Total } \\
\hline & & & $\begin{array}{l}\text { Purchures } \\
\text { (thowesdind } \\
\text { kWhy) }\end{array}$ & $\begin{array}{c}\operatorname{Cov} \\
(\boldsymbol{\alpha})\end{array}$ & $\begin{array}{l}\text { Ponchures } \\
\text { (thorkund } \\
\text { k'Wh) }\end{array}$ & & Cont & & $\begin{array}{l}\text { Purchuses } \\
\text { (thomind } \\
\text { XWh) }\end{array}$ & $\begin{array}{c}\text { Cont } \\
\text { (dollms) }\end{array}$ \\
\hline \multicolumn{11}{|l|}{ Indege } \\
\hline Knose county Rord E I M C & & - & 191931 & 8487,895 & - & & - & & $(93,92)$ & $8,487,895$ \\
\hline 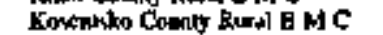 & & - & $20 \sin 9$ & 10,722714 & - & & - & & $2 \times 6,359$ & $10,722,14$ \\
\hline Lagrampt Cumby Roril E M C & & - & \$1, 862 & $1,557.898$ & - & & - & & ЯIB & $3,657,6 \%$ \\
\hline MNSt Comm Reral EMC & & - & 74,45 & 3,4Y2B89 & - & & - & & 74,415 & 3,4 20,889 \\
\hline Mhorm Com Coomy RUTA E M C & & - & R6, fien & $3.709,486$ & - & & - & & B5, fos & $3,719,486$ \\
\hline Nowton Conoly Ruml E \& C & & - & 24,490 & $1,145,816$ & - & & - & & 24,399 & $1,145,816$ \\
\hline Motke Cowrty Raral E MC & & - & 151,735 & $65 \$ 9,487$ & - & & - & & $151,7 * 3$ & $6,369,487$ \\
\hline Nopheamen Rurid E M C & & - & JMn,ns4 & $16,109.875$ & - & & - & & 370,034 & $16,309,875$ \\
\hline Onange Copne Rust E M C & & - & 81,293 & 3716375 & - & & - & & 81273 & $3,718,375$ \\
\hline Pate Cownty Rond B M C & & - & 162.10 & 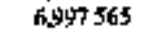 & - & & - & & 162,1197 & 6997,565 \\
\hline furt councy Rerl EMC & & - & 79,679 & $3,262,5145$ & - & & - & & 73,673 & $32,62,649$ \\
\hline Shelby Counly Roral E M C C & & - & 179,722 & 6,094.706 & - & & - & & 133,722 & $6,1094,306$ \\
\hline 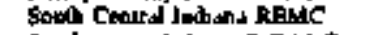 & & - & 779,846 & 17.4F, & 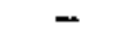 & & - & & 373,846 & $17,460,317$ \\
\hline Soolvestarn Initene R B B C & & - & 325.736 & 14846065 & - & & - & & 325,776 & 14,846065 \\
\hline Sowhem Inthand R E C Ine & & - & 121,068 & $5.519,414$ & - & & - & & 123,869 & $3,319,414$ \\
\hline Steuben Coundy Rord E MA C & & - & 95,195 & 4.25instis & - & & - & & 95,195 & 4.750605 \\
\hline SWhwn Coomy Rural E MC & & - & Ifs, 356 & $5,174,151$ & - & & - & & 115356 & 5,174751 \\
\hline Ipnowk Rarl Bles Menher Copp & & - & 266,373 & $12,003,1396$ & - & & - & & 266,370 & $12,093,836$ \\
\hline UnLed Rerl Bec Manber Cont & & - & $96,5,92$ & $16,297,060$ & - & & - & & $380,5,52$ & $16,237,060$ \\
\hline Lthues Das wettem IN RRaste & & - & 251,412 & $1,, 424,718$ & - & & - & & $251, A 12$ & $11,424,718$ \\
\hline Whith Ooumy Rurd E BA C & & - & 123,696 & $5,490,445$ & - & & $=$ & & 123,890 & $3,490,46$ \\
\hline 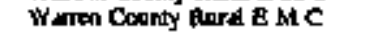 & . & - & 70,692 & $3,126,507$ & $=$ & & - & & 20.692 & 3,126, sक \\
\hline Whowe Cown'y Rurd B MC C & & - & $\sqrt{62,327}$ & $7,266,8688$ & - & & - & & $162 \pm 27$ & $7.266,868$ \\
\hline Whike Copny Rura E MC & & - & $J 001428$ & $4.799,55 \pi$ & - & & - & & INOA2R & $4.399,5,8$ \\
\hline \multicolumn{11}{|l|}{ lowe } \\
\hline Adsax Coliny Coop Elertme Co & & - & 27,363 & $1,750,997$ & - & & - & & 27,361 & $1,150,993$ \\
\hline Alwnakee Clayten ש Crop bre & & - & 122.196 & $5,739,897$ & - & & - & & 122,49 & $5.339,897$ \\
\hline Eenton Covary Elec Coop An, & & - & 62,123 & $3,078,599$ & - & & - & & 62,123 & $3,173,559$ \\
\hline Bochane Carnuy Rol Blac Coop & & - & B1, 45,56 & $4,204,07 ?$ & - & & - & & 83,456 & $1,204,6 \pi$ \\
\hline Batler Counny Rirnl Elex Coop & & - & IDT,5]2 & $4,029,99$ & & 6 & & $26 t$ & $30,5.58$ & $4,900,264$ \\
\hline Cllhour Conaty Elec Cod Axp & & - & 30,016 & $1,(2), 807$ & - & & - & & 30,916 & $1,427,809$ \\
\hline Cedr Yalley Bloctuc Coop & & - & 6il, 490 & $2.536,132$ & - & & - & & 61,940 & $2.536,132$ \\
\hline Chartoe Villey Elec Coop [oe & & - & 71,809 & 2834001 & - & & - & & 기요요 & $2.836,018]$ \\
\hline aks Blectrie Coop int & & - & 53,564 & 2770,484 & - & & - & & $\$ 3564$ & 2.720, $4 \mathrm{AH}$ \\
\hline Envem towa Lestidpower Coxp & & - & 327,300 & $16,534,760$ & - & & - & & 327318 & If, 574,760 \\
\hline Fumber Dectre Cocp he & & - & 109.435 & $4,742,216$ & - & & - & & 103,435 & $4,742,216$ \\
\hline 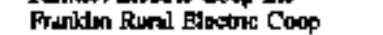 & & - & $\mathbf{3 k}, \mathrm{T}^{4} \mathbf{9}$ & 1856865 & - & & - & & 38.099 & $1,856,865$ \\
\hline OAsdon Rotal Electris Coop & & - & $17,5 \div 1$ & 1770,882 & $=$ & & - & & 37371 & $1,7 \pi 0,8992$ \\
\hline Ongady Cowiny Rursl Elax Coop & & - & 47,743 & $2,709,829$ & - & & - & & 47,743 & $2.309,829$ \\
\hline Golthe Condy RRTd EC A & & - & 59,712 & 2989746 & - & & - & & 99,712 & $2,984,746$ \\
\hline Hencock Cowny Rutal Elec Coop & & - & 4,54 & 2,133576 & - & & - & & 44,544 & $2,133,576$ \\
\hline Hemon Coonty An Btee Cocp & & $\rightarrow$ & $47, \$ 25$ & $1,862,191$ & - & & - & & 47,425 & $1,862 \quad 191$ \\
\hline HE In Coonty El Coop Ins & & - & JIR,755 & $5.131,656$ & & 2 & & 9) & 118,357 & $5,13], 746$ \\
\hline Hobolitr Copnty R EC & & - & 4P,BOA & $1,924,157$ & - & & - & & 40,504 & $1,924,157$ \\
\hline 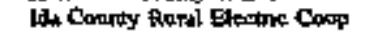 & & - & 35,529 & $1,368,012$ & - & & - & & 75.529 & $1,368, \mathrm{NH}_{2}$ \\
\hline [owfa I las Elextne Coop & & - & 20,393 & 10890,852 & - & & - & & 240,893 & $10,800,852$ \\
\hline owy Rord B C A & & - & 175,352 & 9502,391 & - & & - & & 175,352 & $9,502,391$ \\
\hline Lyan Rural flectax Coop & & - & 56.418 & $1,804,873$ & - & & $m$ & & 56,418 & $1, \sin , 871$ \\
\hline Nuquotala valley Rel piec Coop & & - & 223, rad & $11,834,804$ & - & & - & & 223,659 & $11,834,804$ \\
\hline 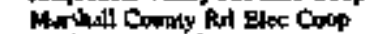 & & - & 70,740 & $3,637,135$ & - & & - & & 70,740 & $3,637,135$ \\
\hline af Power Coco & & - & $177,0 \%$ & $8,582,424$ & - & & - & & JTI, DE & B, 582,424 \\
\hline Mowoms Condy Rard gles Coop & & - & 36,0015 & $1,547,852$ & - & & - & & 3 WhAt & $1,547,851$ \\
\hline Nulhorbotins Valky R E C & & - & 70,432 & $2,742,951$ & - & & - & & 20,432 & $2,742,951$ \\
\hline Wym Dectac Coof Inc & & - & $2 I, 47 \mid$ & $1,057,797$ & - & & - & & $25,47 \mathrm{~J}$ & $1,097,797$ \\
\hline Oxcola Bactrie Coop la & & $\ldots$ & 25 .1\%7 & 799.900 & - & & - & & 25,087 & 799,903 \\
\hline pollu Coop Ber Avin & & عـ & $38,0 \leqslant 1$ & $1,964.194$ & - & & - & & $\mathbf{3 8 , 0 \times 1}$ & $1,964,19 \mathrm{~d}$ \\
\hline Plynopoth Electre Cog Axm & & $=$ & 68,924 & $2,701.297$ & & 2 & & 85 & $\$ 8,926$ & $2,701,378$ \\
\hline Rudet Electinc Copp bis & & - & $19,14 \pi$ & $t, 6012917$ & - & & - & & 33.146 & $1,600,947$ \\
\hline S B lowa Coop Electrac A hun & & - & 169,496 & $6,279,133$ & - & & - & & $10 \%, 46$ & $6.275,133$ \\
\hline 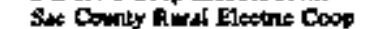 & & -_ & 21.154 & $1,061,076$ & - & & - & & 21,154 & I, DK[, nTh \\
\hline Shom Elecine Coop A4r & & - & 121,500 & $4,025,852$ & & 2 & & 64 & 121.582 & $4,325,9] 6$ \\
\hline 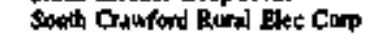 & & مـ & 70, KI & 2, inT,56] & - & & - & & 20,361 & 2,601, 363 \\
\hline phem Iow-1 Elec Cocp he & & - & 69,532 & $2,748,240$ & - & & - & & 69,500 & $2,748,340$ \\
\hline
\end{tabular}

Sen arter and foolnntex al end of whto 
Table 34. Electricity Purchases by the Distribution Segment of Cooperative Borrowers, by Stale, 1994 (Continued)

\begin{tabular}{|c|c|c|c|c|c|c|c|}
\hline \multirow{3}{*}{ 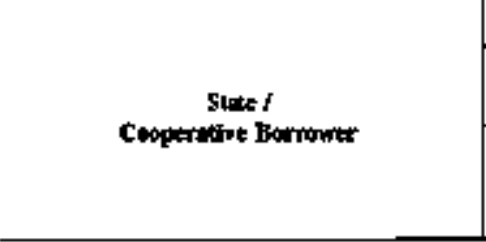 } & \multicolumn{7}{|c|}{ Solite of Elentrifity } \\
\hline & \multicolumn{2}{|c|}{$\begin{array}{l}\text { Invastur. } \\
\text { Ourned }\end{array}$} & \multicolumn{2}{|c|}{ Fedatal } & \multicolumn{2}{|c|}{ 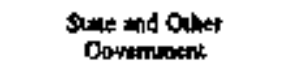 } & \multirow{2}{*}{ 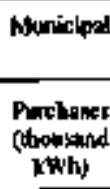 } \\
\hline & $\begin{array}{l}\text { Porctumea } \\
\text { (thowsulpd } \\
\text { twh] }\end{array}$ & (dod) & 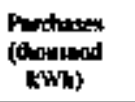 & $\begin{array}{c}\text { Cost } \\
\text { (doltart) }\end{array}$ & $\begin{array}{l}\text { Purchisser } \\
\text { (thopsund } \\
\text { kwh }\end{array}$ & $\underset{\text { (dollon) }}{\operatorname{con}}$ & \\
\hline \multicolumn{8}{|l|}{ Inewe } \\
\hline Ti P Rurar Blectic Coop & - & - & - & - & - & - & - \\
\hline Winduco Rurly Elec Coop Askrtr...-...... & - & - & - & ـ & - & - & - \\
\hline 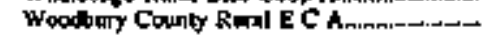 & - & - & - & ـ & - & - & - \\
\hline 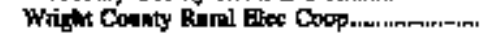 & - & - & ـ & - & - & - & - \\
\hline \multicolumn{8}{|l|}{ Kanum } \\
\hline Ark Valley Eloc Coop Aash luc.... & - & - & - & - & - & مـ & - \\
\hline 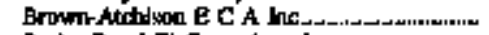 & - & - & - & - & - & - & - \\
\hline Bulker Rubd El Chop Axth lac & - & - & - & - & - & - & - \\
\hline C \& W Rord Elec Cop Asun [nc & - & - & - & - & - & - & - \\
\hline Cancy Valky E Cocp Aston bec & - & - & - & - & - & - & - \\
\hline Chys elecerte Coop lec & - & - & $m$ & - & - & - & - \\
\hline$D S \& O$ Rard $E C A$ Ine & - & - & - & - & - & - & - \\
\hline Dowiplon Eec Copp A=en Inc..., & 16,388 & 589,352 & - & - & - & - & - \\
\hline Fint Hills Rorl E C A Jnc......... & - & - & - & - & - & - & - \\
\hline 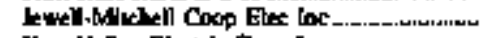 & - & - & - & - & - & - & - \\
\hline Kuw Valory Elexpic Coop Inc , , & 100,874 & $3,551,996$ & - & - & - & - & - \\
\hline 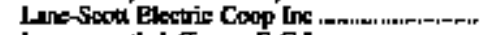 & - & $=$ & - & - & - & $m$ & - \\
\hline 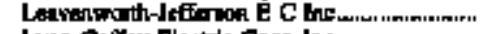 & - & - & - & - & - & - & - \\
\hline 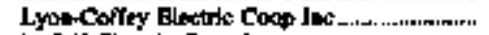 & $\rightarrow$ & - & - & - & - & - & - \\
\hline 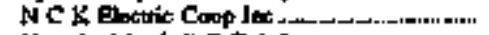 & - & - & - & - & - & - & - \\
\hline 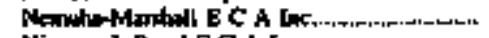 & $\mathbf{4 4 , 4 6 0}$ & $1,540,428$ & - & $m$ & - & - & - \\
\hline 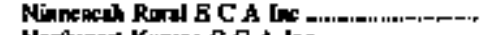 & - & - & - & - & - & - & - \\
\hline 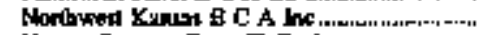 & - & - & - & - & - & - & $=$ \\
\hline Nonop-Desator Coop El Co lnc & - & - & - & - & - & - & - \\
\hline PR \& W Electrie Coop Asin Jac & - & - & - & - & - & - & - \\
\hline 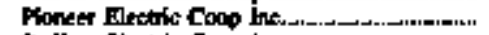 & - & - & - & - & - & - & - \\
\hline Redam eluobrie Coop lot & - & - & - & - & - & - & - \\
\hline 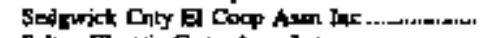 & - & - & - & - & - & - & - \\
\hline Sten Elatic Cogp Axn hesm & - & - & - & - & - & - & - \\
\hline 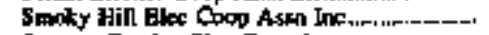 & - & - & - & - & - & - & - \\
\hline Sumost-Cowky Bles Coop Jwe & - & - & - & - & - & - & - \\
\hline 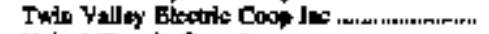 & - & - & $\rightarrow$ & ع. & - & - & - \\
\hline 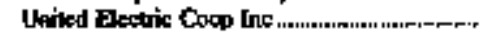 & - & - & - & - & - & - & - \\
\hline Viefory Elestric CoOp Anm lne & - & - & - & - & - & - & - \\
\hline 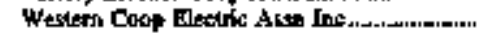 & - & - & - & - & - & - & - \\
\hline 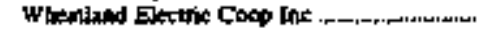 & - & - & - & - & - & - & - \\
\hline \multicolumn{8}{|l|}{ Keaducly } \\
\hline 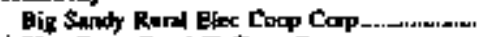 & - & - & - & - & - & - & - \\
\hline 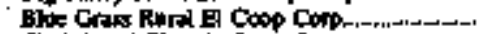 & - & - & - & - & - & - & - \\
\hline 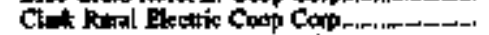 & - & - & - & - & - & - & - \\
\hline Ounberland Volley Rowil E C C........... & - & - & - & - & - & - & - \\
\hline Fumers Rurd Bloc Coop Copp & - & - & - & - & - & - & - \\
\hline 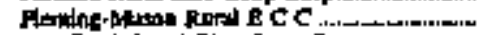 & - & - & - & - & - & - & - \\
\hline 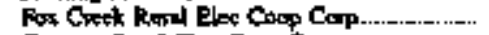 & - & - & - & - & - & - & - \\
\hline Gronen Ronl Elec Coop Copp & - & - & 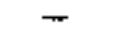 & - & - & - & - \\
\hline 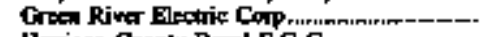 & - & - & - & - & - & - & - \\
\hline Hrrison Combly Raral E C C C & - & - & - & - & - & - & - \\
\hline funderico- Inion Rurt B C C C & - & - & - & - & - & - & - \\
\hline 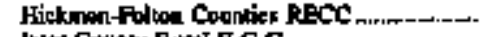 & - & - & 318,362 & $5,204,205$ & - & - & - \\
\hline Inds Covinty Rurat E C C & - & - & - & - & - & - & - \\
\hline 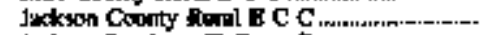 & - & - & - & - & - & - & - \\
\hline 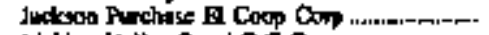 & - & - & - & - & - & - & - \\
\hline 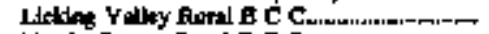 & - & - & - & - & - & - & - \\
\hline Mende Conpul Rand BCC & - & - & - & - & - & - & - \\
\hline 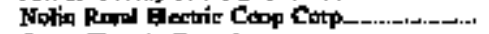 & - & - & - & - & - & - & - \\
\hline Oofer Blextric Coop lac & - & - & - & - & - & - & - \\
\hline 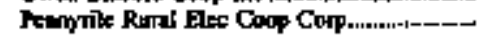 & $-m$ & - & a) 35.559 & 37.121 .890 & - & - & - \\
\hline Sul Rike Rurd E Coop Comp & - & - & - & - & - & - & - \\
\hline Sheloy Ruml Eles Conp Corp & - & - & - & - & - & - & - \\
\hline 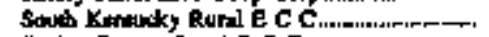 & $\rightarrow$ & - & - & - & - & - & - \\
\hline Taylor Coung Rural E C C C & - & - & - & - & - & - & - \\
\hline 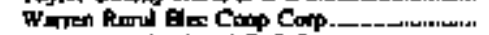 & - & - & $1,057,227$ & $4,745.199$ & - & - & - \\
\hline 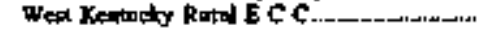 & - & - & 768,045 & $31,999,622$ & - & - & - \\
\hline
\end{tabular}

Sal woles and foctrotes at and of ibble. 
Table 34. Electricity Purchases by the Distrbution Segment of Cooperative Borrowers, by Stote, 1994 (Continued)

\begin{tabular}{|c|c|c|c|c|c|c|c|}
\hline \multirow{3}{*}{$\begin{array}{c}\text { Steide } \\
\text { Concernimp borrente }\end{array}$} & \multicolumn{7}{|c|}{ Sroure of Elestrinty } \\
\hline & \multirow{2}{*}{$\frac{\text { Mumicipul }}{\text { Cont }}$} & \multicolumn{2}{|c|}{ Cooporture } & \multicolumn{2}{|c|}{ Other 1} & \multicolumn{2}{|c|}{ Totod } \\
\hline & & 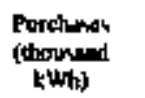 & (d) & $\begin{array}{l}\text { Porahues } \\
\text { (thoundid } \\
\text { l'wh) }\end{array}$ & Cons & $\begin{array}{c}\text { Parclesed } \\
\text { (dhoordid } \\
\text { kWb) }\end{array}$ & $\cos$ \\
\hline 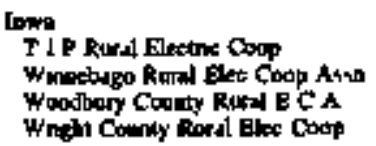 & $\begin{array}{l}- \\
- \\
-\end{array}$ & $\begin{array}{r}101,539 \\
37,784 \\
51,697 \\
97.272\end{array}$ & $\begin{array}{l}5,088,933 \\
1,580,789 \\
1,996,607 \\
3,132,334\end{array}$ & $\begin{array}{l}- \\
- \\
-\end{array}$ & $\frac{-}{-}$ & $\begin{array}{r}10,539 \\
37,784 \\
51,617 \\
87,212\end{array}$ & $\begin{array}{l}5, \sqrt{1}, 6,913 \\
1,500,349 \\
1,9 \%, 8, n \\
3,112,334\end{array}$ \\
\hline 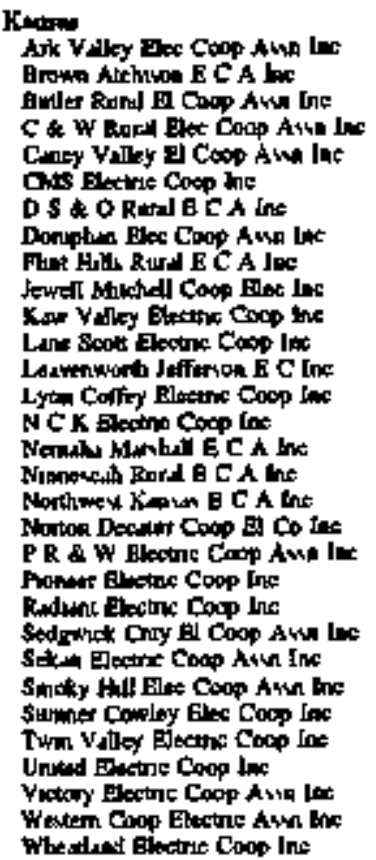 & 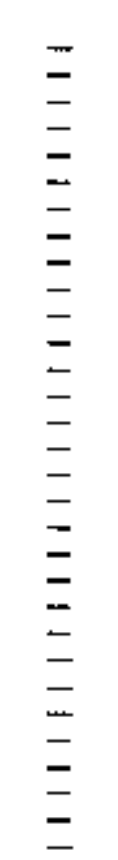 & 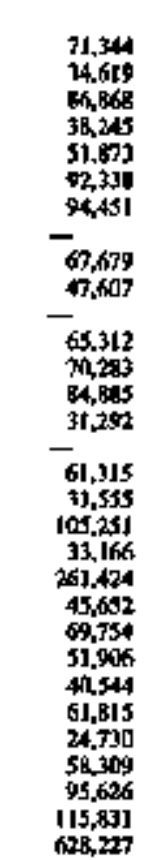 & 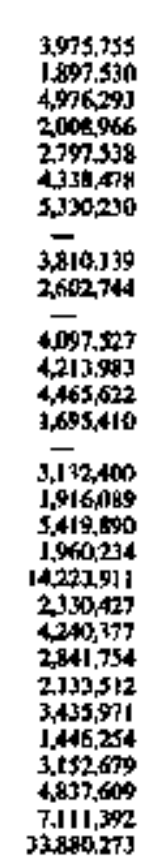 & 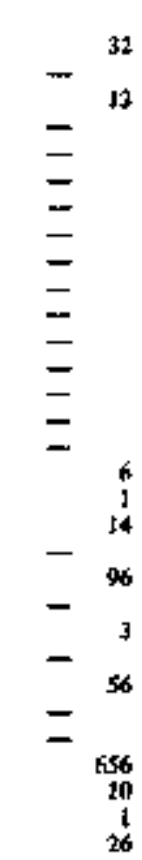 & 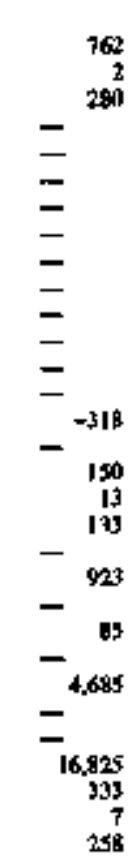 & 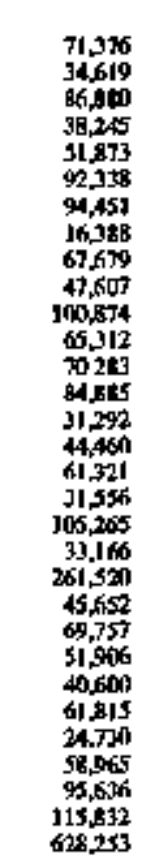 & 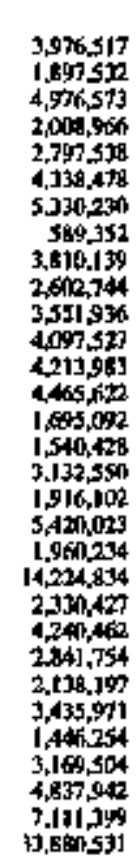 \\
\hline 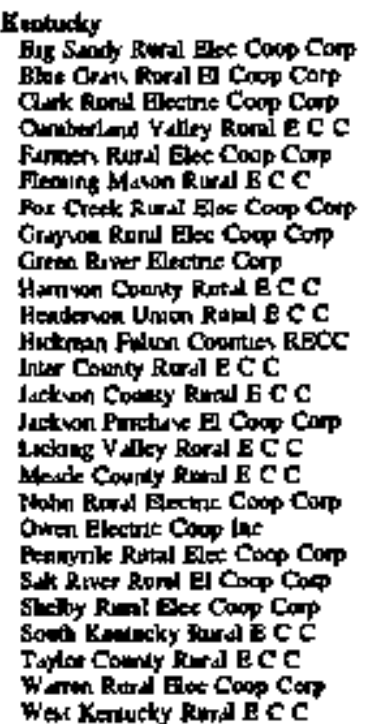 & 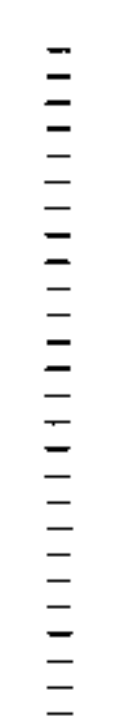 & 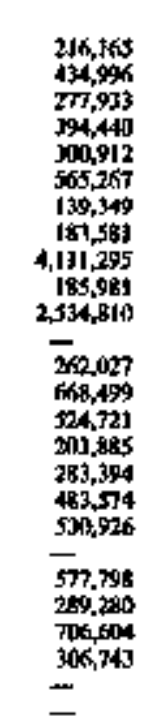 & 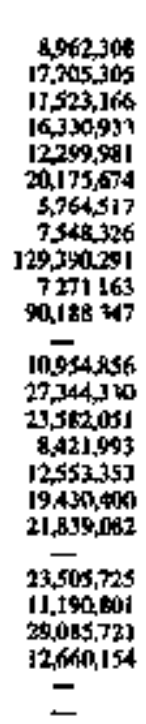 & $\begin{array}{l}- \\
- \\
= \\
= \\
- \\
- \\
-1.755 \\
- \\
- \\
- \\
- \\
- \\
-6 \\
- \\
- \\
- \\
- \\
-\end{array}$ & 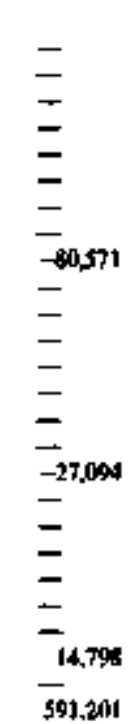 & 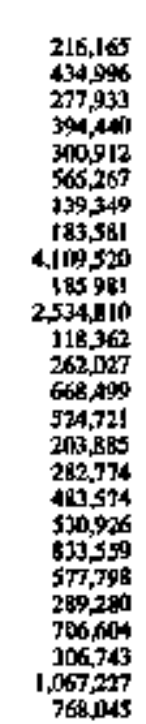 & 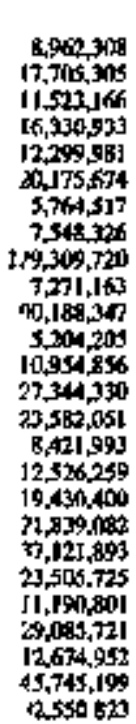 \\
\hline
\end{tabular}


Table 34. Electricity Purchases by the Dtstribation Segment of Cooptrative Borrowers, by State, 1994 (Continut)

\begin{tabular}{|c|c|c|c|c|c|c|c|}
\hline \multirow{3}{*}{$\begin{array}{c}\text { Sixef } \\
\text { Compenture Berrower }\end{array}$} & \multicolumn{7}{|c|}{ Solnte of Alectnery } \\
\hline & \multicolumn{2}{|c|}{$\underset{\text { Inverlor }}{\text { Owned }}$} & \multicolumn{2}{|c|}{ Federal } & \multicolumn{2}{|c|}{$\begin{array}{l}\text { Stale and Oler } \\
\text { Govammenl }\end{array}$} & \multirow{2}{*}{ 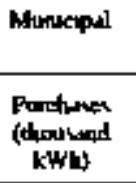 } \\
\hline & 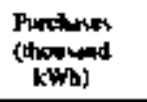 & $\underset{\text { Cont }}{\text { Colliar) }}$ & 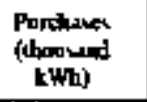 & $\begin{array}{c}\operatorname{Con} \\
(j \mathrm{al} / \mathrm{N})\end{array}$ & 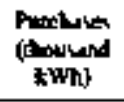 & $\underset{\text { Cons }}{\text { dathen) }}$ & \\
\hline 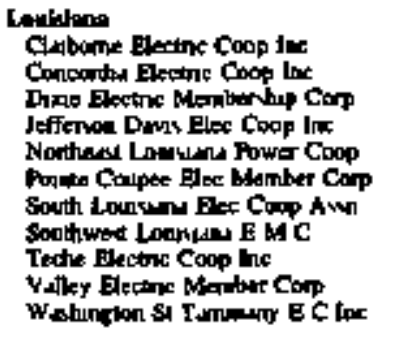 & $\begin{array}{l}\bar{z} \\
= \\
\bar{z} \\
\bar{z} \\
\bar{z} \\
\overline{-}\end{array}$ & $\begin{array}{l}\bar{z} \\
= \\
= \\
= \\
= \\
=\end{array}$ & $\begin{array}{l}= \\
= \\
= \\
= \\
= \\
= \\
=\end{array}$ & $\begin{array}{l}= \\
= \\
= \\
= \\
= \\
= \\
=\end{array}$ & $\begin{array}{l}= \\
= \\
= \\
= \\
= \\
= \\
=\end{array}$ & $\begin{array}{l}= \\
= \\
= \\
= \\
= \\
=\end{array}$ & $\begin{array}{l}= \\
= \\
= \\
= \\
= \\
=\end{array}$ \\
\hline 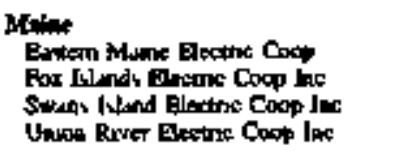 & $\begin{array}{r}32,729 \\
8,321 \\
2,063 \\
8,912\end{array}$ & $\begin{array}{r}1,54,225 \\
43,193 \\
162,154 \\
62,774\end{array}$ & $\begin{array}{l}\bar{z} \\
\bar{z}\end{array}$ & $\begin{array}{l}\overline{-} \\
\overline{-}\end{array}$ & $\begin{array}{l}- \\
- \\
-\end{array}$ & $\begin{array}{l}\bar{z} \\
\bar{z}\end{array}$ & $\begin{array}{l}\bar{z} \\
\overline{-}\end{array}$ \\
\hline 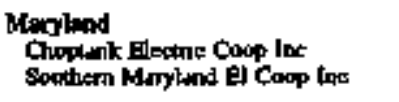 & $2 \sqrt{56,346}$ & $10, \overline{251.124}$ & $\overline{-}$ & $\overline{-}$ & - & - & - \\
\hline 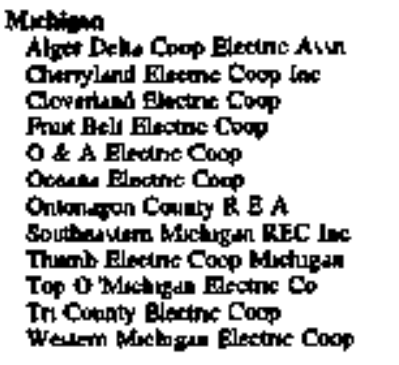 & $\begin{array}{l}4,806 \\
\overline{185,514} \\
= \\
= \\
22,053 \\
31,011 \\
115,826 \\
= \\
=\end{array}$ & $\begin{array}{l}1,843,002 \\
\overline{4,483,091} \\
\overline{-} \\
\overline{-} \\
935,572 \\
1,37,563 \\
4,609,405 \\
= \\
=\end{array}$ & $\begin{array}{l}\bar{z} \\
\bar{z} \\
\bar{z} \\
\bar{z} \\
\bar{z} \\
\overline{-}\end{array}$ & $\begin{array}{l}= \\
= \\
= \\
= \\
= \\
= \\
=\end{array}$ & $\begin{array}{l}= \\
= \\
= \\
= \\
\bar{z} \\
\bar{z}\end{array}$ & $\begin{array}{l}\bar{z} \\
\bar{z} \\
\bar{z} \\
\bar{z} \\
\bar{z} \\
\bar{z}\end{array}$ & $\begin{array}{l}{ }^{11,214} \\
= \\
= \\
= \\
= \\
= \\
=\end{array}$ \\
\hline 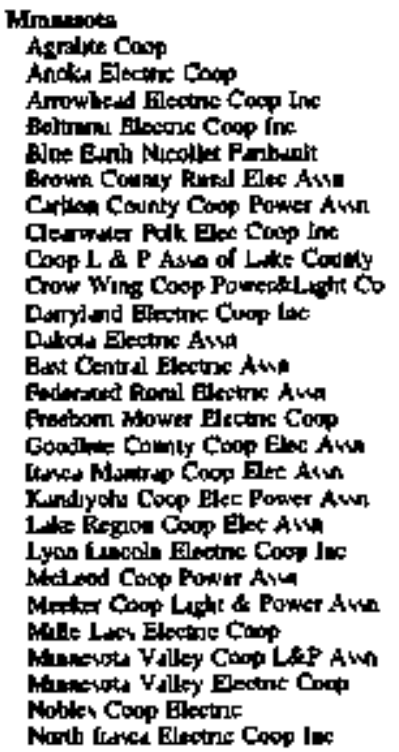 & 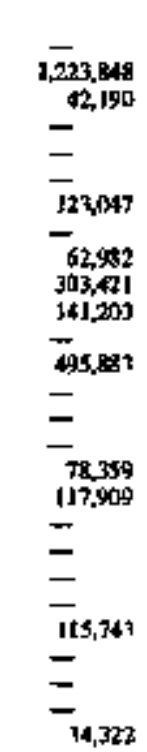 & 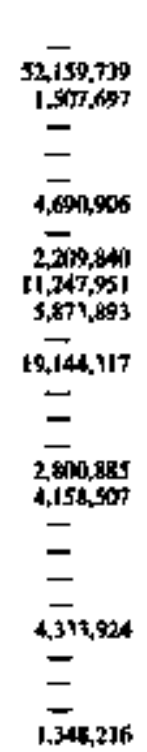 & $\begin{array}{l}= \\
= \\
= \\
= \\
= \\
= \\
= \\
= \\
= \\
= \\
= \\
23,916 \\
= \\
= \\
= \\
=5050 \\
= \\
=\end{array}$ & $\begin{array}{l}= \\
= \\
= \\
= \\
= \\
= \\
= \\
= \\
= \\
= \\
4 \\
= \\
= \\
= \\
= \\
730.150 \\
= \\
=\end{array}$ & $\begin{array}{l}\bar{z} \\
= \\
= \\
= \\
= \\
= \\
= \\
= \\
= \\
= \\
= \\
= \\
= \\
= \\
=\end{array}$ & $\begin{array}{l}= \\
= \\
= \\
= \\
= \\
= \\
= \\
= \\
= \\
= \\
= \\
= \\
= \\
=\end{array}$ & $\begin{array}{l}z \\
= \\
= \\
z \\
= \\
= \\
= \\
= \\
= \\
= \\
= \\
= \\
= \\
= \\
= \\
= \\
=\end{array}$ \\
\hline
\end{tabular}

See nowe and looknotev at ead of whll 
Table 34. Electricity Purchases by the Distribution Segment of Cooperative Borrowers, by State, 1994 (Continued)

\begin{tabular}{|c|c|c|c|c|c|c|c|}
\hline \multirow{3}{*}{$\begin{array}{l}\text { Stwt I } \\
\text { Cooperivivi Derromer }\end{array}$} & \multicolumn{7}{|c|}{ Somere of Eletroly } \\
\hline & \multirow{2}{*}{ 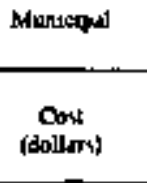 } & \multicolumn{2}{|c|}{ Cosperaye } & \multicolumn{2}{|c|}{0,1} & \multicolumn{2}{|c|}{ Tocel } \\
\hline & & 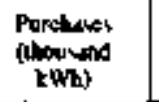 & $\begin{array}{c}\text { Cond } \\
\text { (dollew }\end{array}$ & 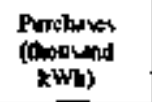 & $\begin{array}{c}\text { Cout } \\
\text { dollant }\end{array}$ & $\begin{array}{l}\text { Purctum } \\
\text { (thowind } \\
\text { kWh) }\end{array}$ & $\begin{array}{l}\text { Cont } \\
\text { (ddder) }\end{array}$ \\
\hline 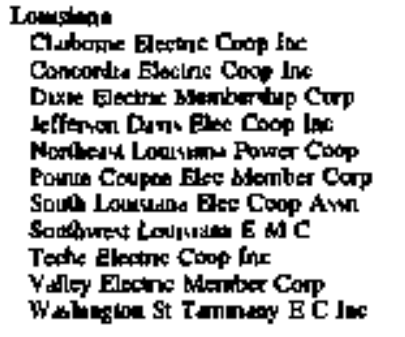 & $\begin{array}{l}\bar{z} \\
\bar{z} \\
\bar{z} \\
\bar{z} \\
\overline{-}\end{array}$ & 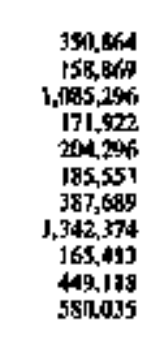 & 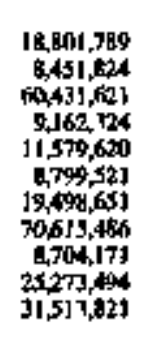 & $\begin{array}{l}= \\
\bar{z} \\
= \\
\bar{z} \\
= \\
\bar{z} \\
=\end{array}$ & $\begin{array}{l}= \\
= \\
= \\
= \\
= \\
= \\
=\end{array}$ & 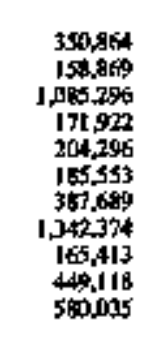 & 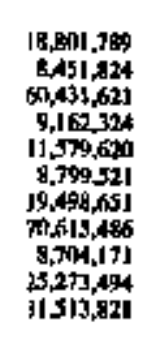 \\
\hline 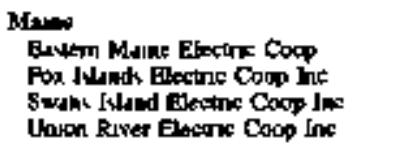 & $\begin{array}{l}\overline{-} \\
=\end{array}$ & $\begin{array}{l}\bar{z} \\
\overline{-}\end{array}$ & $\begin{array}{l}- \\
\overline{-}\end{array}$ & $\begin{array}{l}-87.455 \\
- \\
-\end{array}$ & $\begin{array}{l}4.108,072 \\
= \\
=\end{array}$ & $\begin{array}{r}120,214 \\
8,321 \\
20163 \\
8,012\end{array}$ & $\begin{array}{r}3,652,297 \\
44,193 \\
112,154 \\
627,774\end{array}$ \\
\hline 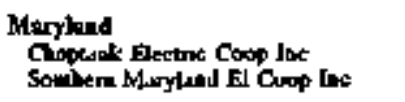 & - & $L^{618517}$ & $32,168,321$ & $=$ & $2, \sqrt{100,000}$ & $\begin{array}{r}606,517 \\
2,356,346\end{array}$ & $\begin{array}{r}12,168,321 \\
J 15.251,184\end{array}$ \\
\hline 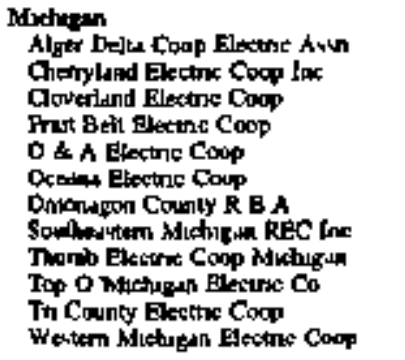 & $\begin{array}{l}162.286 \\
= \\
= \\
= \\
= \\
= \\
= \\
= \\
=\end{array}$ & $\begin{array}{l}\bar{z} \\
\overline{333,563} \\
\overline{-} \\
\overline{{ }_{11}, 466} \\
\overline{-} \\
= \\
=\end{array}$ & 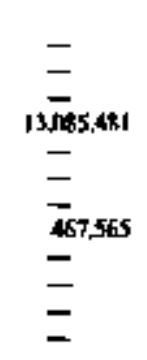 & 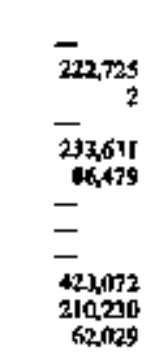 & 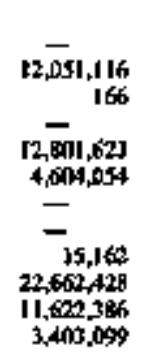 & 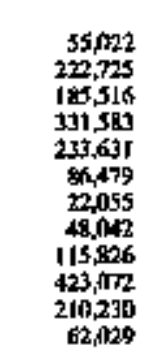 & 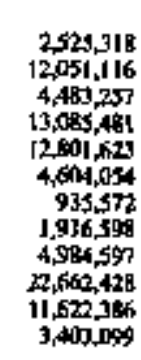 \\
\hline 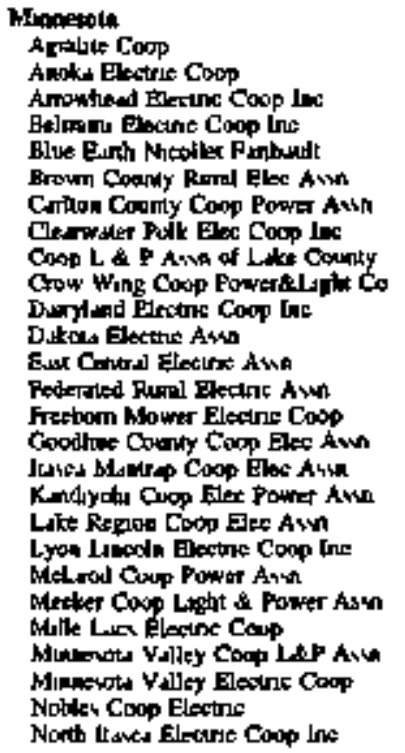 & $\begin{array}{l}= \\
= \\
= \\
= \\
= \\
= \\
= \\
= \\
= \\
= \\
= \\
= \\
= \\
=\end{array}$ & 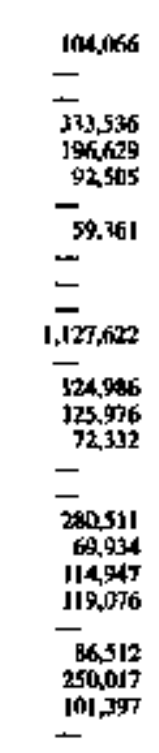 & 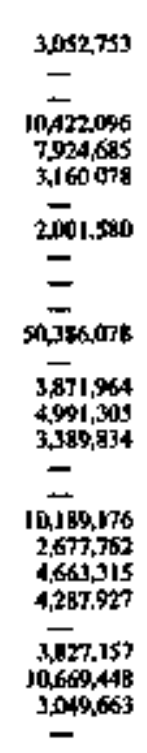 & 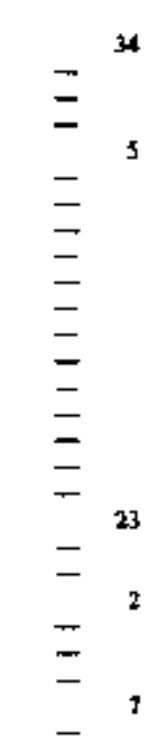 & 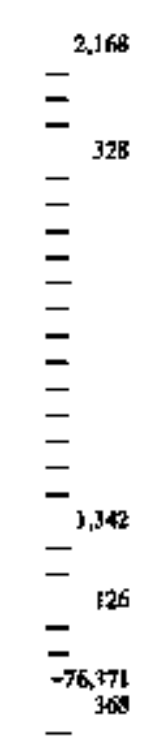 & 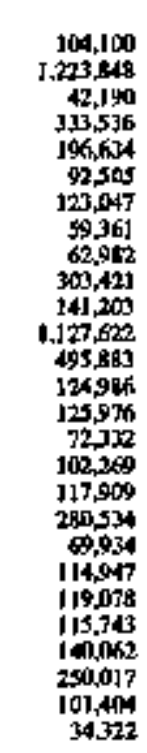 & 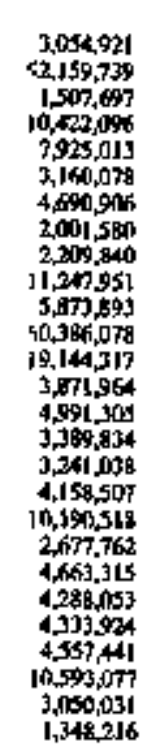 \\
\hline
\end{tabular}

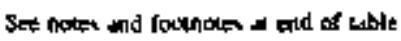


Table 34. Electricity Purchases by the Distrilhation Segment of Cooperative Borrowers, by State, 1994 (Conlinued)

\begin{tabular}{|c|c|c|c|c|c|c|c|}
\hline \multirow{3}{*}{$\begin{array}{c}\text { Stdet it } \\
\text { Coopwriter Docrower }\end{array}$} & \multicolumn{7}{|c|}{ Sonre of Elecunaty } \\
\hline & \multicolumn{2}{|c|}{$\begin{array}{l}\text { laveshor } \\
\text { Owned }\end{array}$} & \multicolumn{2}{|c|}{ Fexpent } & \multicolumn{2}{|c|}{$\begin{array}{l}\text { State and Othr } \\
\text { Orovernitemt }\end{array}$} & \multirow{2}{*}{ 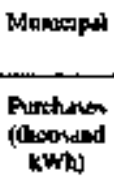 } \\
\hline & $\begin{array}{l}\text { Purchuset } \\
\text { (thoutund } \\
\text { kWh) }\end{array}$ & $\begin{array}{c}\text { Cout } \\
\text { (dollaty }\end{array}$ & 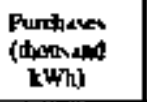 & $\underset{(d o D \text { Ins) }}{\operatorname{Cosu}}$ & 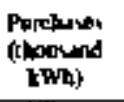 & $\begin{array}{c}\text { Cont } \\
\text { (dollon) }\end{array}$ & \\
\hline \multicolumn{8}{|l|}{ Minusatom } \\
\hline North Pre Blectre Coxp he & 09,052 & $3,391,441$ & - & - & - & - & - \\
\hline Nerou Ster Eletene Coop Int & & & - & - & - & - & - \\
\hline Worthem Eloctne Cosp Asse & $186,54 !$ & $7,268,509$ & - & - & - & - & - \\
\hline F X M Elenenc Coep hix & - & - & - & - & - & - & - \\
\hline People $、$ Coop Pondr A Am & - & - & - & - & $\rightarrow$ & - & - \\
\hline 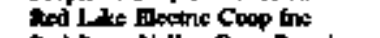 & - & - & - & - & - & - & - \\
\hline ked luver V dley Coop Por Awa & - & - & - & - & - & $\pi$ & - \\
\hline Redwood Derwis Cogo & - & - & - & - & $\rightarrow$ & - & - \\
\hline 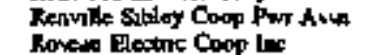 & $\bar{z}$ & 二 & - & 二 & $\rightarrow$ & - & - \\
\hline $\begin{array}{l}\text { Roxew Elotinc Coop las } \\
\text { Ronetione Floctine Alve }\end{array}$ & $\bar{z}$ & $\overline{-}$ & $\bar{z}$ & $\bar{z}$ & - & $\overline{-}$ & - \\
\hline 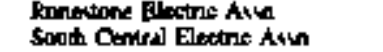 & 二 & - & - & $\overline{-}$ & $\bar{z}$ & $\bar{z}$ & 二 \\
\hline 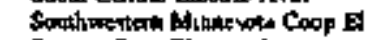 & - & - & - & - & - & - & - \\
\hline Strank Cosp Ekethre Axn & - & - & - & - & $\rightarrow$ & عـ & - \\
\hline Steele Wast Coov Elednse & - & - & - & - & $\rightarrow$ & - & - \\
\hline Todd Wuden Electonc Coop & - & - & - & - & - & $=$ & - \\
\hline Trwote elotint Cosp he & - & - & - & - & - & - & - \\
\hline In Connly forthe Coco & - & $=$ & - & - & $\rightarrow$ & - & - \\
\hline 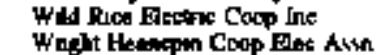 & 455,851 & $\operatorname{IE} \overline{0,12.647}$ & $\bar{z}$ & $\bar{c}$ & $\bar{F}+1+1+1+1$ & - & $\overline{-}$ \\
\hline \multirow{2}{*}{\multicolumn{8}{|c|}{ Brstimpp }} \\
\hline & & & & & & & \\
\hline 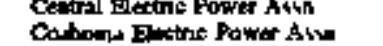 & - & 二 & (- ש- & - & $\Xi$ & $\bar{z}$ & $\bar{z}$ \\
\hline 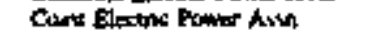 & - & - & - & - & - & - & $\overline{-}$ \\
\hline Delta Elecorc Pown A & - & - & - & - & - & - & - \\
\hline Draf Electnc Fower Aysh & - & - & - & 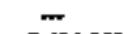 & $\rightarrow$ & - & - \\
\hline But Mrunuppi als Pwr Awe & 29997 & $11,246,222$ & 2007,363 & 8494859 & - & - & - \\
\hline 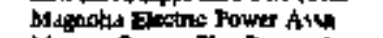 & - & - & & - & - & $=$ & - \\
\hline Mowo Cony Eles Pown Awu & - & - & 160,37 & 6,962593 & $\rightarrow$ & - & - \\
\hline 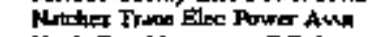 & - & - & 302,606 & $13,634,169$ & $\boldsymbol{-}$ & - & - \\
\hline 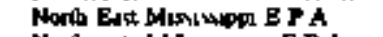 & $=$ & - & 337,006 & $14,217,776$ & - & - & - \\
\hline Norbocentrul Meruwpps E P A & - & - & 399.957 & $24,83075 \%$ & - & - & - \\
\hline Peerl Ravet Vuloy E Rwt kwn & - & - & - & - & - & - & - \\
\hline 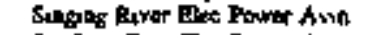 & - & - & - & - & - & - & - \\
\hline Sowbern Pune Dex Poper Abn & - & - & - & - & - & - & - \\
\hline Soellwed A4sushqpi E P A & - & - & - & - & - & - & - \\
\hline 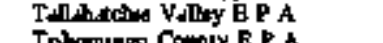 & - & - & $s s 1 . m 7$ & $24,176,116$ & - & - & 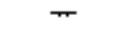 \\
\hline Irimonungor Comiy E P A & $z$ & - & 216,526 & $9,862,290$ & $\rightarrow$ & - & - \\
\hline Tombyghe Bectic Power Avn & $\bar{z}$ & $z$ & 814863 & 36472047 & $\rightarrow$ & - & - \\
\hline 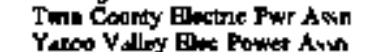 & $\overline{-}$ & $\overline{-}$ & $=$ & - & $\overline{-}$ & $\overline{-}$ & - \\
\hline 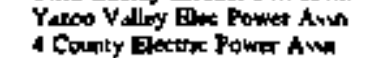 & - & $\overline{-}$ & $\overline{7}$ & $\overline{-}$ & $\bar{z}$ & $\overline{-}$ & - \\
\hline \multirow{2}{*}{\multicolumn{8}{|c|}{ Bus:ann }} \\
\hline & & & & & & & \\
\hline 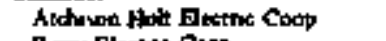 & - & - & - & - & - & - & - \\
\hline Buty Eloctuse Coop & 二 & $\overline{-}$ & - & - & - & - & - \\
\hline 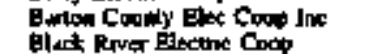 & 二 & $\bar{z}$ & $\overline{-}$ & $\bar{z}$ & $\bar{z}$ & $\bar{z}$ & $\ddot{z}$ \\
\hline 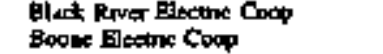 & - & $\mp$ & - & 二 & $\Rightarrow$ & - & - \\
\hline Caldway Pleconc Coop & - & - & - & - & $\rightarrow$ & - & - \\
\hline Canted Hiwow Elec Coop Jnc & - & - & - & - & $\rightarrow$ & - & - \\
\hline 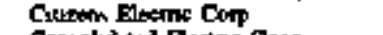 & 300.319 & 25113,393 & - & - & - & 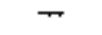 & - \\
\hline Consolichted Flestac Coos & - & - & - & - & - & - & - \\
\hline Gratond Eletns Coop in & - & - & - & - & 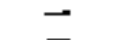 & - & - \\
\hline 0 แO Blostint Cosp int & $\overline{-}$ & 二 & 二 & בع & $\overline{-}$ & $=$ & - \\
\hline Fimer Glectinv Coop Ine & - & $\bar{z}$ & $\overline{-}$ & 二 & $=$ & $\bar{z}$ & $\bar{z}$ \\
\hline 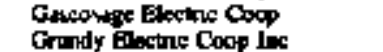 & $\bar{z}$ & $=$ & 二 & 二 & 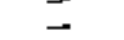 & $=$ & $\overline{-}$ \\
\hline Howand Elesenc Coop & - & - & - & - & - & - & - \\
\hline Bowath Orugun Elec Coop inc & - & - & - & - & - & - & - \\
\hline Imencosony Eloctic Cocp Asun & - & - & $=$ & - & - & - & - \\
\hline Laclede Eoctrite Coop late & $\overline{-}$ & 二 & $\bar{z}$ & $\overline{-}$ & $\overline{-}$ & 二 & 二 \\
\hline Lew Coomly Romd B CA A & - & - & - & - & - & - & - \\
\hline
\end{tabular}


Tabte 34. Electrtcity Purchases by the Distriluntion Segment of Cooperatlve Borrowers, by State, 1994 (Contlnued)

\begin{tabular}{|c|c|c|c|c|c|c|c|c|}
\hline \multirow{3}{*}{\multicolumn{2}{|c|}{ 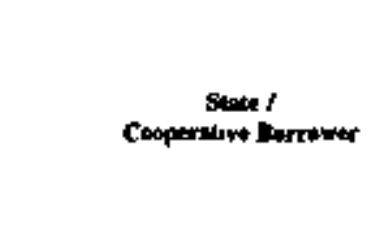 }} & \multicolumn{7}{|c|}{ Source of Eectintity } \\
\hline & & M/anneipat & \multicolumn{2}{|c|}{ Cooptrutu } & \multicolumn{2}{|c|}{ Oher } & \multicolumn{2}{|c|}{ Told } \\
\hline & & Cont & 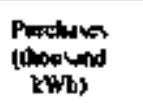 & $\begin{array}{c}\text { Cont } \\
\text { (dollan) }\end{array}$ & 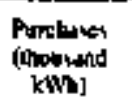 & Cont & 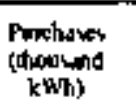 & $\begin{array}{c}\text { Coni } \\
\text { (dollnin }\end{array}$ \\
\hline 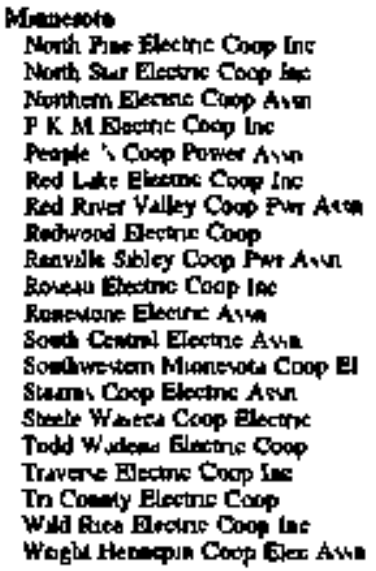 & . & $\begin{array}{l}- \\
= \\
- \\
- \\
= \\
= \\
= \\
= \\
= \\
- \\
- \\
= \\
-\end{array}$ & 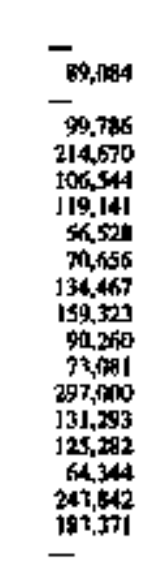 & 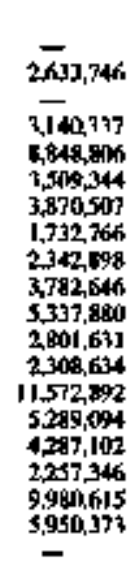 & $\begin{array}{ll}- & \\
- & \\
- & \\
- & \\
- & \\
- & 31 \\
- & 6 \\
- & \\
& 14 \\
- & 5 \\
- & 46 \\
- & \end{array}$ & 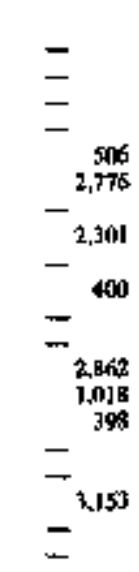 & 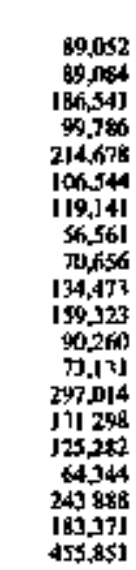 & 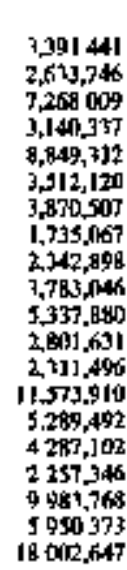 \\
\hline 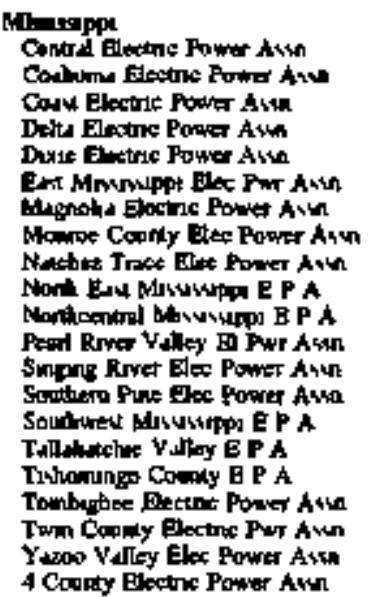 & $\dot{5}$ & $\begin{array}{l}- \\
= \\
- \\
= \\
= \\
- \\
- \\
= \\
- \\
- \\
- \\
= \\
-\end{array}$ & $\begin{array}{l}\overline{101,367} \\
922,189 \\
399,193 \\
505,375 \\
\overline{428,158} \\
- \\
- \\
\overline{-} \\
503,354 \\
911,1965 \\
1,359,475 \\
372,518 \\
- \\
- \\
239,376 \\
216,391 \\
-\end{array}$ & $\begin{array}{l}\overline{4}, 729,452 \\
41,683,291 \\
18,816,555 \\
23,849,426 \\
\overline{-} \\
19,45,115 \\
- \\
- \\
- \\
22,986,266 \\
11,544,091 \\
59,144,967 \\
16,726,905 \\
- \\
- \\
10,593,664 \\
7,899,604 \\
-\end{array}$ & $\begin{array}{l}- \\
- \\
- \\
- \\
= \\
- \\
- \\
- \\
- \\
- \\
- \\
- \\
- \\
- \\
-\end{array}$ & $\begin{array}{l}- \\
- \\
= \\
= \\
= \\
- \\
= \\
= \\
= \\
- \\
= \\
= \\
-\end{array}$ & 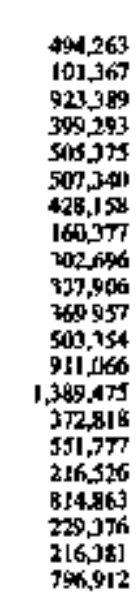 & 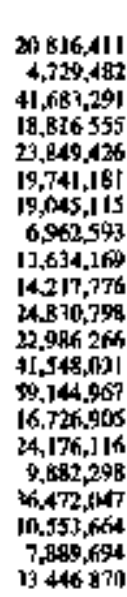 \\
\hline 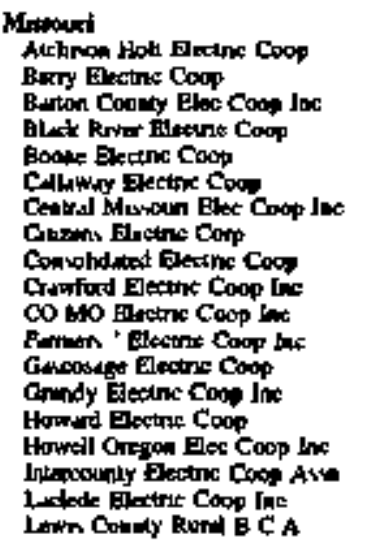 & & $\begin{array}{l}- \\
- \\
- \\
- \\
- \\
- \\
= \\
- \\
= \\
= \\
= \\
-\end{array}$ & 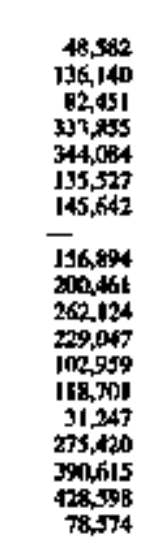 & 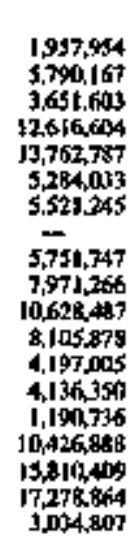 & $\begin{array}{l}- \\
- \\
- \\
- \\
- \\
- \\
- \\
- \\
- \\
- \\
- \\
- \\
-\end{array}$ & $\begin{array}{l}- \\
= \\
- \\
= \\
- \\
= \\
= \\
- \\
= \\
= \\
- \\
- \\
= \\
= \\
=\end{array}$ & 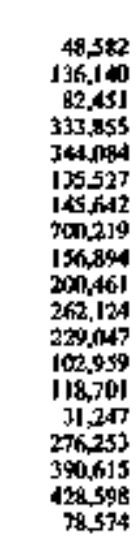 & 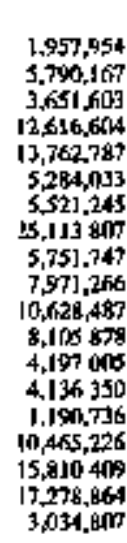 \\
\hline
\end{tabular}


Table 34. Elextricity Purchases by the Distribution Segnaent of Cooperative Borrowers, by State, 1994 (Continued)

\begin{tabular}{|c|c|c|c|c|c|c|c|}
\hline \multirow{3}{*}{$\begin{array}{c}\text { Stwe } I \\
\text { Cooperreme Barrower }\end{array}$} & \multicolumn{7}{|c|}{ Sowne of Elatncity } \\
\hline & \multicolumn{2}{|c|}{ Jowexter } & \multicolumn{2}{|c|}{ Federat } & \multicolumn{2}{|c|}{$\begin{array}{l}\text { Strote and Other } \\
\text { Gomemnimum }\end{array}$} & \multirow{2}{*}{ 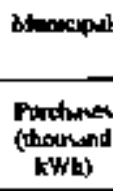 } \\
\hline & 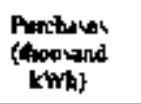 & $\underset{\text { Cont }}{\text { Collam }}$ & 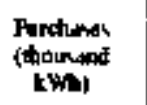 & 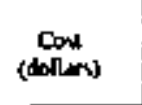 & 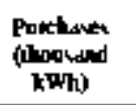 & Cons & \\
\hline 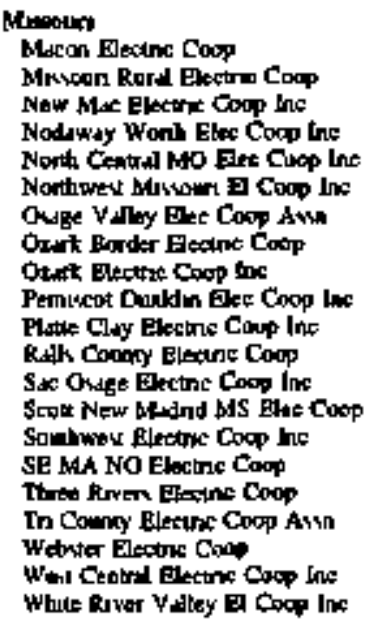 & $\begin{array}{l}= \\
= \\
= \\
= \\
= \\
= \\
= \\
= \\
= \\
= \\
= \\
= \\
=\end{array}$ & $\begin{array}{l}= \\
= \\
= \\
= \\
= \\
= \\
= \\
= \\
= \\
= \\
= \\
= \\
= \\
=\end{array}$ & $\begin{array}{l}\bar{z} \\
\bar{z} \\
\bar{z} \\
\bar{z} \\
\bar{z} \\
\bar{z} \\
\bar{z} \\
\bar{z} \\
\bar{z} \\
\bar{z}\end{array}$ & $\begin{array}{l}= \\
= \\
= \\
= \\
= \\
= \\
= \\
= \\
= \\
= \\
= \\
= \\
=\end{array}$ & $\begin{array}{l}= \\
= \\
= \\
= \\
= \\
= \\
= \\
= \\
= \\
= \\
= \\
= \\
=\end{array}$ & $\begin{array}{l}= \\
= \\
= \\
= \\
= \\
= \\
= \\
= \\
= \\
= \\
= \\
= \\
=\end{array}$ & $\begin{array}{l}= \\
= \\
= \\
= \\
= \\
= \\
= \\
= \\
= \\
= \\
= \\
= \\
=\end{array}$ \\
\hline 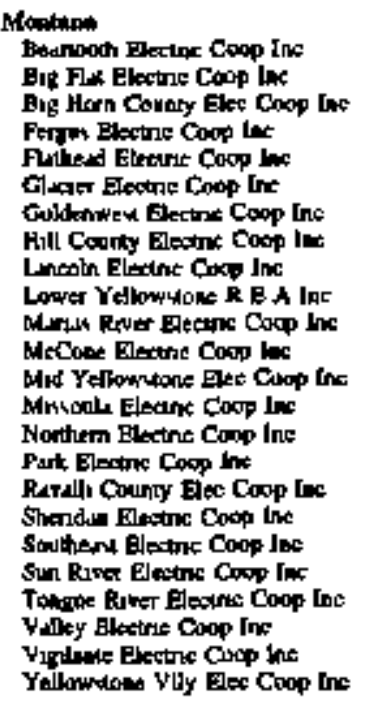 & $\begin{array}{l}= \\
= \\
= \\
= \\
= \\
= \\
= \\
= \\
= \\
= \\
= \\
= \\
=\end{array}$ & $\begin{array}{l}= \\
= \\
= \\
= \\
= \\
= \\
= \\
= \\
= \\
= \\
= \\
= \\
=\end{array}$ & 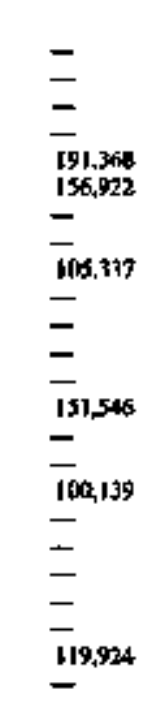 & 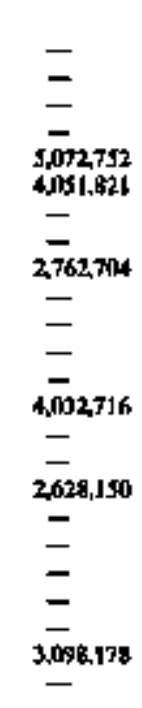 & $\begin{array}{l}= \\
= \\
= \\
= \\
= \\
= \\
= \\
= \\
= \\
= \\
= \\
= \\
= \\
=\end{array}$ & $\begin{array}{l}\bar{z} \\
= \\
= \\
= \\
= \\
= \\
= \\
= \\
= \\
= \\
= \\
= \\
= \\
=\end{array}$ & 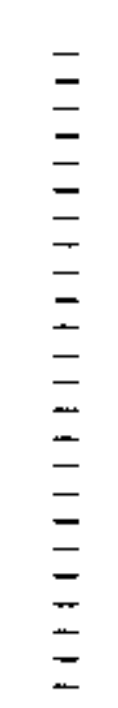 \\
\hline 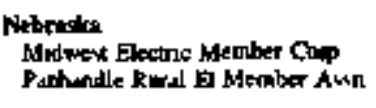 & $\overline{-}$ & $\overline{-}$ & $\overline{-}$ & $\overline{-}$ & $\overline{-}$ & - & $\overline{-}$ \\
\hline 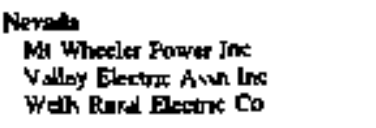 & $\overrightarrow{128,440}$ & $\begin{array}{r}90,519 \\
1,761,091 \\
14,298\end{array}$ & $\overline{\overline{4}}_{42,655}$ & $\frac{\overline{-}}{12,544,271}$ & $\overline{19 \%, 061}$ & $\underset{-}{J, 679,246}$ & $\bar{z}$ \\
\hline 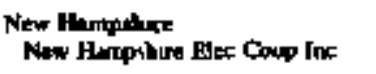 & 611.173 & $50,530,112$ & - & - & - & - & - \\
\hline $\begin{array}{l}\text { Now Jenty } \\
\text { Snyex Rud Eleant Coop In }\end{array}$ & - & - & - & - & - & - & - \\
\hline
\end{tabular}


Table 34. Plectriclty Parchases by the Distribution Segment of Cooperative Borrowers, by State, 1994 (Continued)

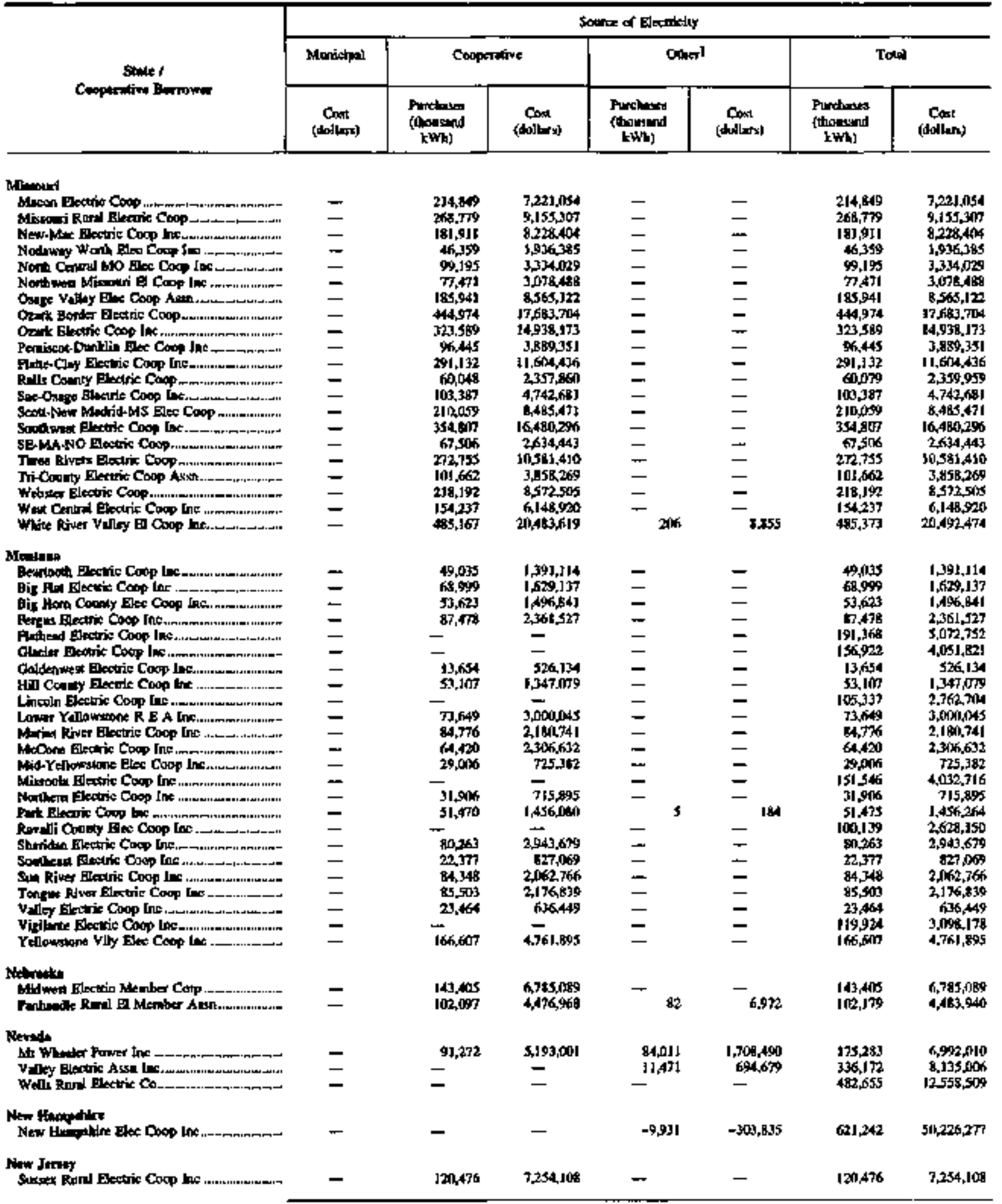

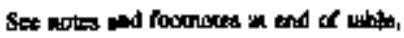


Table 34. Electricity Purchases by the Distribation Sezmunt of Conperative Borrowers, by State, 1994 (Continged)

\begin{tabular}{|c|c|c|c|c|c|c|c|}
\hline \multirow{3}{*}{ 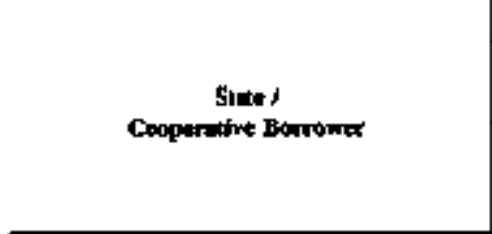 } & \multicolumn{7}{|c|}{ Sowne of Electicity } \\
\hline & \multicolumn{2}{|c|}{$\begin{array}{l}\text { Intexiot. } \\
\text { Onnet }\end{array}$} & \multicolumn{2}{|c|}{ Pedenal } & \multicolumn{2}{|c|}{ 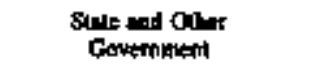 } & \multirow{2}{*}{ 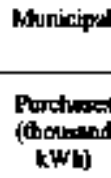 } \\
\hline & 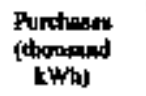 & $\underset{\text { (doflonis) }}{\operatorname{Cos}}$ & $\begin{array}{l}\text { Portakes } \\
\text { (thoutherd } \\
\text { kWh) }\end{array}$ & (1) & 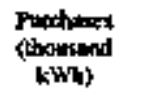 & Cont & \\
\hline \multirow{14}{*}{ 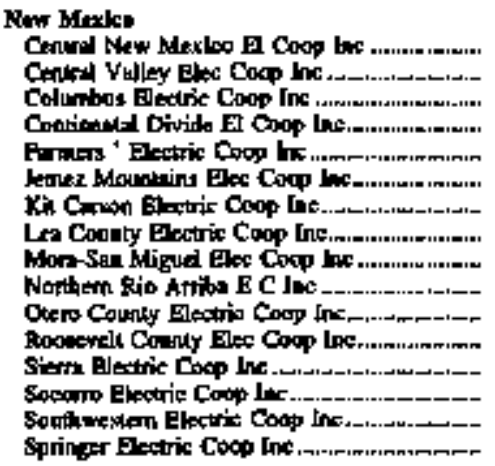 } & - & - & - & - & - & - & - \\
\hline & 346.166 & $11,530,14]$ & - & $=$ & $=$ & $=$ & - \\
\hline & - & - & - & - & - & - & - \\
\hline & $\overline{16}$ & $\overline{6009038}$ & $\overline{-}$ & $\bar{z}$ & 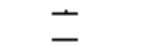 & 二 & - \\
\hline & 167.681 & $\underline{-}, 099,138$ & $\bar{z}$ & $\bar{z}$ & $\bar{z}$ & $\bar{z}$ & $\overline{-}$ \\
\hline & - & - & - & 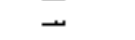 & - & - & - \\
\hline & 729,366 & $20,7 \times 4,902$ & - & - & - & - & - \\
\hline & - & - & - & - & - & - & $\pi$ \\
\hline & - & $=$ & - & - & - & - & - \\
\hline & $\overline{-}$ & $\overline{-}$ & - & - & - & - & - \\
\hline & 140.616 & $4,047,72)$ & - & - & - & - & - \\
\hline & $\overline{-}$ & - & $=$ & - & - & - & - \\
\hline & $\bar{m}$ & $\rightarrow$ & $\bar{z}$ & $\overline{-}$ & $\bar{z}$ & 二 & $\bar{z}$ \\
\hline & - & - & - & - & - & - & - \\
\hline \multirow{5}{*}{ 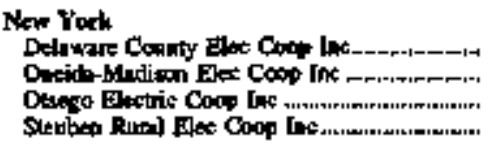 } & & & & & & & \\
\hline & - & - & - & - & 46,561 & 801,158 & - \\
\hline & - & - & - & - & $\mid[6,367$ & 349,976 & - \\
\hline & - & - & - & - & 4,700 & 859,976 & - \\
\hline & - & & & & & $1,050,360$ & - \\
\hline \multicolumn{8}{|l|}{ Nerth Carring } \\
\hline 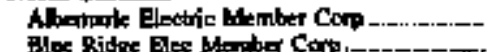 & - & - & 7.594 & 177,417 & - & - & - \\
\hline 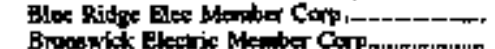 & - & - & 14,58 & 374,519 & - & - & - \\
\hline 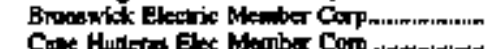 & - & - & 10,940 & 297,188 & - & - & - \\
\hline Cape Hullora Ele Menber Corp & - & - & - & - & - & - & - \\
\hline & - & - & B, 393 & $\begin{aligned} 165,533 \\
3655 ?\end{aligned}$ & $\bar{z}$ & $=$ & $=$ \\
\hline Central Batric Menber Cup & $=$ & $=$ & $\begin{array}{r}3,856 \\
j+300\end{array}$ & $\begin{array}{r}76,557 \\
857,478\end{array}$ & $\bar{z}$ & $\bar{z}$ & - \\
\hline 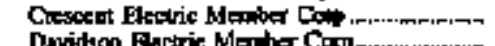 & - & $\bar{Z}$ & & & $=$ & - & - \\
\hline 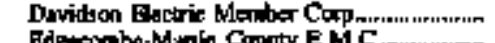 & $m$ & $\overline{-}$ & $T_{12,168}$ & $\overline{284,292}$ & $\overline{-}$ & - & - \\
\hline Edguombo-Manin Coonty E M C & - & 二 & 13,060 & & $\overline{-}$ & - & - \\
\hline 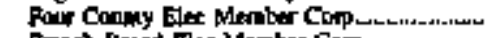 & $\overline{372.681}$ & $57, \overline{6} 18,848$ & 10105 & 250.390 & & $=$ & - \\
\hline 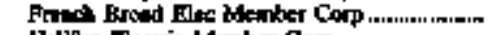 & & $37,616,845$ & $i_{i n g}$ & $\begin{array}{l}+26,162 \\
163,005\end{array}$ & $\bar{z}$ & - & - \\
\hline Halliss Eleteric Menber Conp......................... & $\bar{z}$ & $\overline{-}$ & 42 & & $\bar{z}$ & - & $\longrightarrow$ \\
\hline hintes thend b Menter Copp................... & - & - & & 2,402 & - & 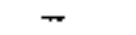 & - \\
\hline 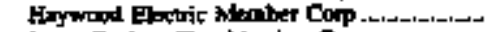 & - & - & 5,127 & If6,725 & - & - & - \\
\hline 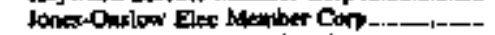 & - & - & 16,135 & $320 ; 313$ & - & - & - \\
\hline 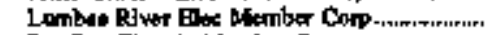 & - & - & 11.606 & 230,412 & - & - & - \\
\hline 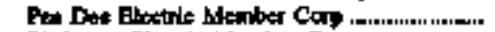 & - & - & In,01? & 203,982 & - & - & - \\
\hline 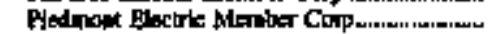 & - & - & 3,380 & $67.2 \mathrm{Q}$ & - & - & - \\
\hline 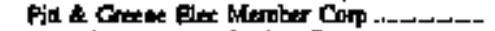 & - & - & 4,918 & 采, 627 & - & - & - \\
\hline Rundolph Electic Nember Corp.-.-- & - & $=$ & 11,229 & 222,935 & - & - & - \\
\hline 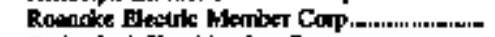 & - & - & 16.189 & 378,235 & - & - & - \\
\hline Roderfond Agec Mamber Cosp & - & - & 45,344 & $1.199,609$ & - & - & - \\
\hline Sopth River Elec Mernber Corp. & - & - & 19.045 & 378,087 & - & - & - \\
\hline 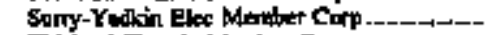 & - & $m$ & - & - & ـ & - & - \\
\hline 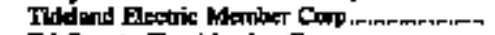 & - & - & 9.198 & 207,460 & - & - & - \\
\hline Thí County Eles Manber Coop & - & - & 9.6916 & $191,29 \%$ & - & - & - \\
\hline Uniom Eledric Rlemberinhtp Corp & - & - & 21,647 & 572,646 & - & - & - \\
\hline W-ike Electric Akphteribip Colp_.._-____- & - & - & 6,735 & $\$ 73,7,7$ & - & - & - \\
\hline \multicolumn{8}{|l|}{ Notet Dallow } \\
\hline 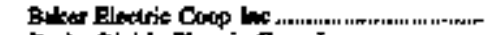 & - & - & - & - & - & - & - \\
\hline 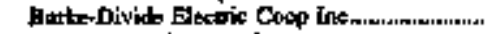 & - & - & - & - & - & - & - \\
\hline Chpind Electric Coop lac- & - & - & 12,705 & $\mid \mathbf{1 8 | \alpha |}$ & $=$ & - & $=$ \\
\hline 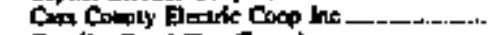 & - & - & - & - & - & $=$ & $=$ \\
\hline Evodier Raphl Elex Cocp Ins & - & - & - & - & - & - & - \\
\hline 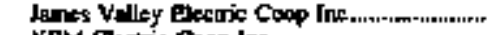 & 65 & 2,3166 & - & - & - & - & - \\
\hline KEM Eloctric Doop Ine & - & - & $31,9 t 8$ & 481,531 & - & - & - \\
\hline Nikentie Electric Coop foc.................. & - & - & - & - & - & - & - \\
\hline Halen Eefric Coop ing, & $=$ & - & - & 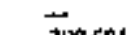 & - & - & - \\
\hline 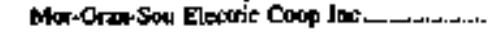 & - & - & 27,709 & 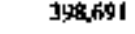 & - & 一 & - \\
\hline
\end{tabular}

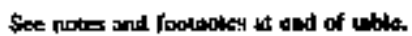


Table 34. Electricity Purchases by the Distribuntion Segment of Cooperative Borrowers, by \$tale, 1994 (Continned)

\begin{tabular}{|c|c|c|c|c|c|c|c|}
\hline \multirow{3}{*}{$\begin{array}{c}\text { Stule } \\
\text { Cepporidve Bermomer }\end{array}$} & \multicolumn{7}{|c|}{ Socrta of Eloctriflsy } \\
\hline & \multirow{2}{*}{$\begin{array}{c}\text { Muriciped } \\
\text { Coss } \\
\text { (dallex) }\end{array}$} & \multicolumn{2}{|c|}{ Cooperative } & \multicolumn{2}{|c|}{ Quer' } & \multicolumn{2}{|c|}{ Tokyl } \\
\hline & & $\begin{array}{l}\text { Purctabs } \\
\text { (0loound } \\
\text { kWh) }\end{array}$ & $\begin{array}{c}\text { Co:k } \\
\text { (dollent) }\end{array}$ & 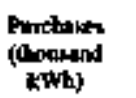 & 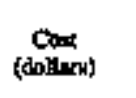 & $\begin{array}{c}\text { Punctures } \\
\text { (thoupind } \\
k \text { thl })\end{array}$ & $\begin{array}{c}\text { Cosit } \\
\text { (dedtur: }\end{array}$ \\
\hline 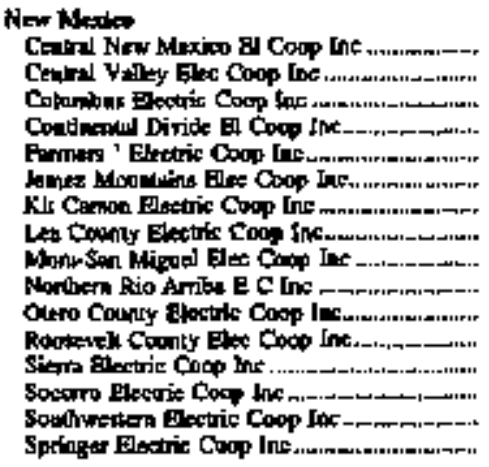 & $\begin{array}{l}- \\
\overline{-} \\
= \\
= \\
= \\
= \\
= \\
= \\
=\end{array}$ & 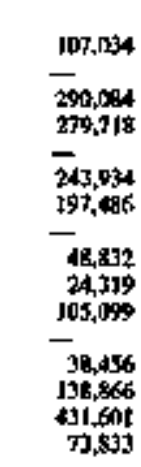 & 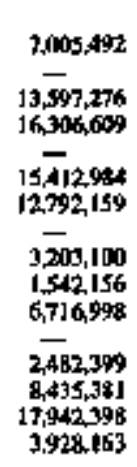 & $\begin{array}{l}- \\
- \\
- \\
z \\
- \\
- \\
- \\
- \\
- \\
- \\
-\end{array}$ & $\begin{array}{l}- \\
- \\
- \\
= \\
- \\
= \\
- \\
- \\
- \\
-\end{array}$ & 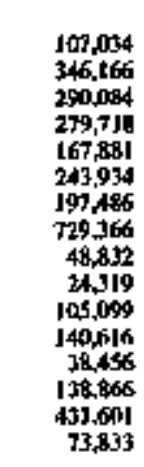 & $\begin{array}{r}7,005,492 \\
1,530,141 \\
13,597,276 \\
16,306,619 \\
6,199,135 \\
15,412,984 \\
12,792,159 \\
21,714,942 \\
3,201,100 \\
1,50,156 \\
6,316,998 \\
4,847,721 \\
2,492,399 \\
8,425,381 \\
17,942,391 \\
3,928,363\end{array}$ \\
\hline 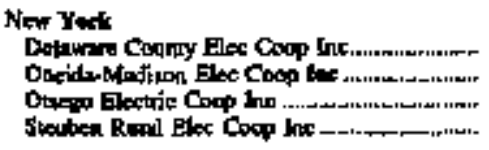 & $\begin{array}{l}- \\
- \\
-\end{array}$ & $\begin{array}{l}- \\
- \\
-\end{array}$ & $\begin{array}{l}- \\
- \\
-\end{array}$ & $\begin{array}{l}\bar{z} \\
\overline{-}\end{array}$ & $\begin{array}{l}-2,5800 \\
-\end{array}$ & $\begin{array}{l}46,561 \\
16,387 \\
44,700 \\
64,676\end{array}$ & $\begin{array}{r}801,052 \\
352,458 \\
809,576 \\
1,050,360\end{array}$ \\
\hline 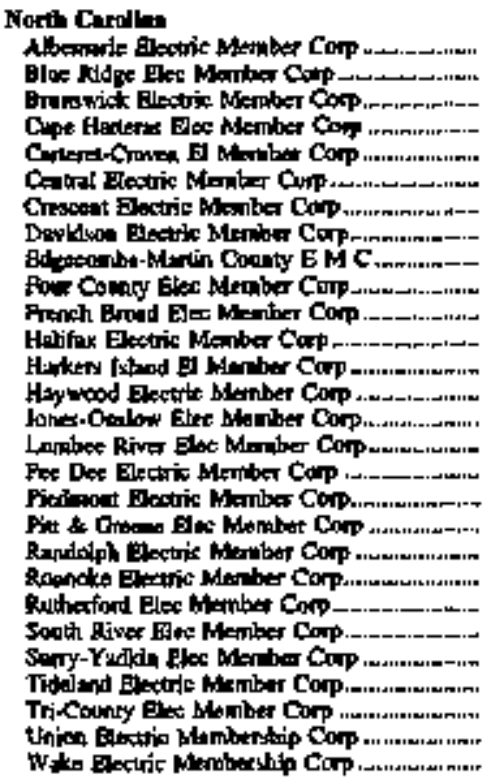 & $\begin{array}{l}- \\
= \\
- \\
= \\
= \\
- \\
= \\
- \\
- \\
- \\
- \\
- \\
- \\
- \\
- \\
-\end{array}$ & 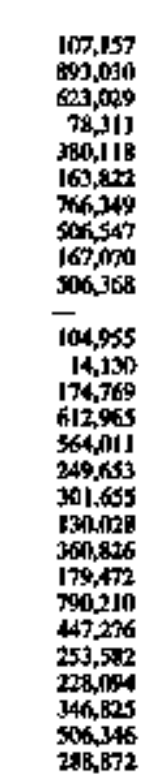 & 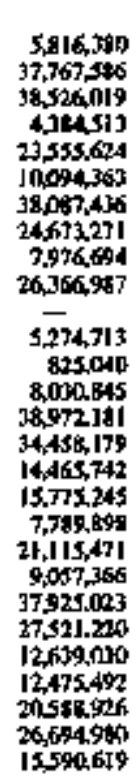 & $\begin{array}{l}-62 \\
- \\
= \\
- \\
= \\
= \\
- \\
= \\
= \\
- \\
- \\
= \\
- \\
- \\
- \\
- \\
= \\
- \\
-\end{array}$ & 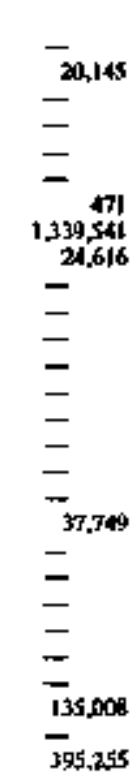 & 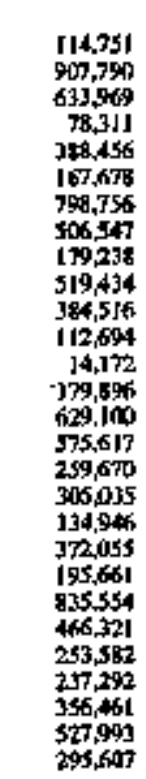 & 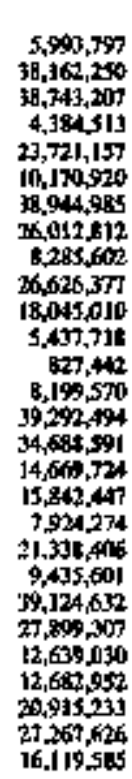 \\
\hline 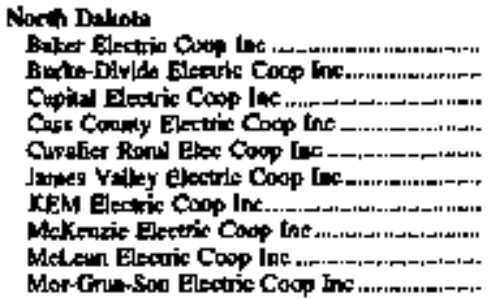 & $\begin{array}{l}E \\
- \\
- \\
- \\
- \\
-\end{array}$ & 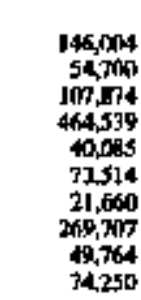 & $\begin{array}{r}5,91,129 \\
1,820,469 \\
4,478,964 \\
12,682,632 \\
1,430,667 \\
2,961,929 \\
993,543 \\
9,262,691 \\
1,916,673 \\
3,243,487\end{array}$ & $\begin{array}{l}- \\
= \\
= \\
= \\
= \\
-\quad 91 \\
\end{array}$ & $\begin{array}{l}- \\
= \\
= \\
-8,736 \\
= \\
\text { D,64465 }\end{array}$ & $\begin{array}{r}146,004 \\
54,760 \\
120,579 \\
464,599 \\
40,085 \\
73,579 \\
55,649 \\
269,707 \\
49,855 \\
101,571\end{array}$ & $\begin{array}{r}5,393,129 \\
1,820,869 \\
4,660,045 \\
12,682,632 \\
1,430,667 \\
2,944,235 \\
1,483,810 \\
9,762,691 \\
1,918,317 \\
3,644,413\end{array}$ \\
\hline
\end{tabular}

See ander and footencter at epd of table 
Table 34. Electridty Purchases by the Distribution Sejgent of Cooperative Borrowers, by State, 1994 (Continaed)

\begin{tabular}{|c|c|c|c|c|c|c|c|}
\hline \multirow{3}{*}{ 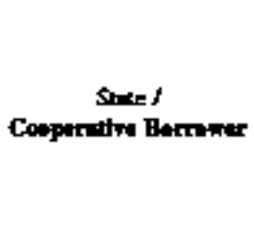 } & \multicolumn{7}{|c|}{ Sodtat of Electricity } \\
\hline & \multicolumn{2}{|c|}{$\begin{array}{l}\text { Investor } \\
\text { inated }\end{array}$} & \multicolumn{2}{|c|}{ Faderd } & \multicolumn{2}{|c|}{$\begin{array}{l}\text { Slote and Other } \\
\text { Conktatinem }\end{array}$} & \multirow{2}{*}{ 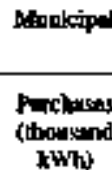 } \\
\hline & 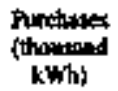 & (dollins) & $\begin{array}{c}\text { Ponduses } \\
\text { (thoogned } \\
\text { kthit) }\end{array}$ & Cod & 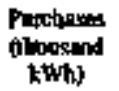 & $\begin{array}{c}\text { Coat } \\
\text { (dollans) }\end{array}$ & \\
\hline
\end{tabular}

Wheth Delloth

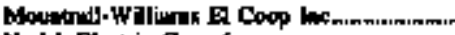
Nodrl Eloctric Cocop far

Noth Gontral alu Coop In

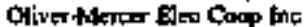

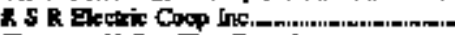

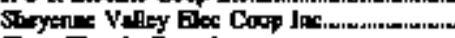

Slope Electric Coop Jne.

Th-Conaty Blectric Coos les.

Treneming Bloct

Wert Flán Bectic Coop tox

$$
\text { Oblo }
$$

Mdany Rerl Blectric Coop loc

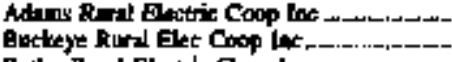

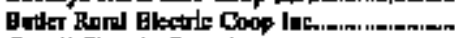

Comoll Bactris Coop lec

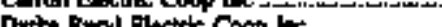

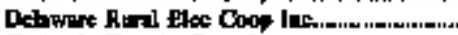

Fritmali Elocerde Coop hes.

Fromice Power Co

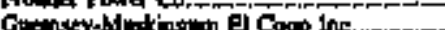

Hunoxk-Wood Electris Coop [n: ..............

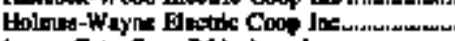

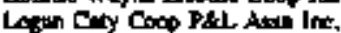

Lerindedina R E C tor

Hain

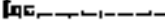

Midwed Elestric JPC

Momar Placaric Coop Inte.

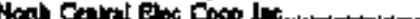

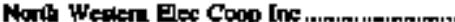

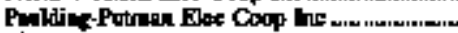

Piones Poral Eloc Cocp bec......................

Somb Cetaral Pontur Oo.

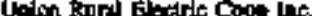

Lhited Bord Electic Coop the

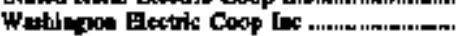

Oinluaps

Alfifo Elodric Cod Is

Coddo Eexctric Coop In

Condian Vally Elec Coos Jax.... nmmmm

Ceatral ford Electric Coop................-.....

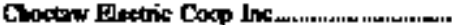

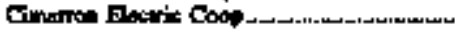

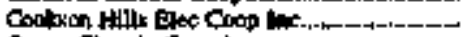

Cotin Elextric Conp Jac.

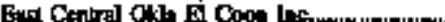

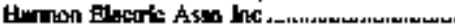

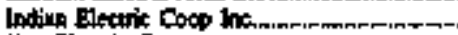

Kny electric Conp.

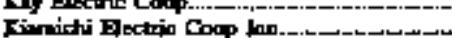

Gianh Bectric Coop Int.

Lits Ropdoe Elettrite Cocp In

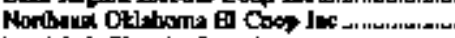

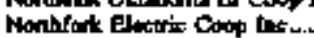

Nonhwerten Eectric Coos Ine

Coluhoms Eletric Coep bo

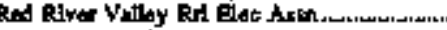

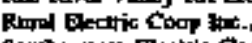

Sounknition Extrite Cocp the

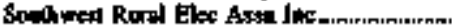

Tri-Cownty Eluctris Coop Ine.w-..................

Verdigrix Vulky Elec Coop Inc

Sw boled and footnoens at and of ublt.

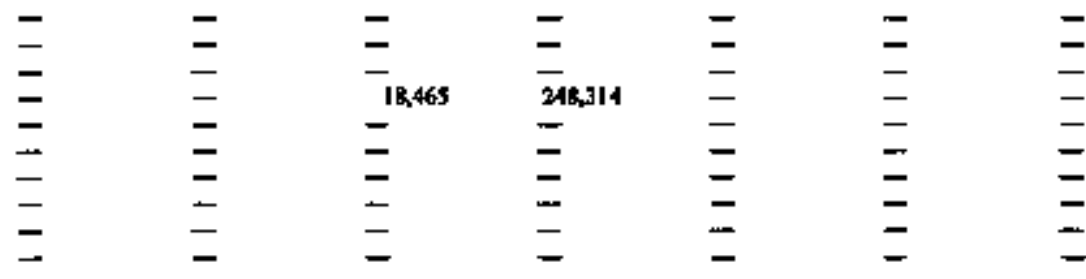

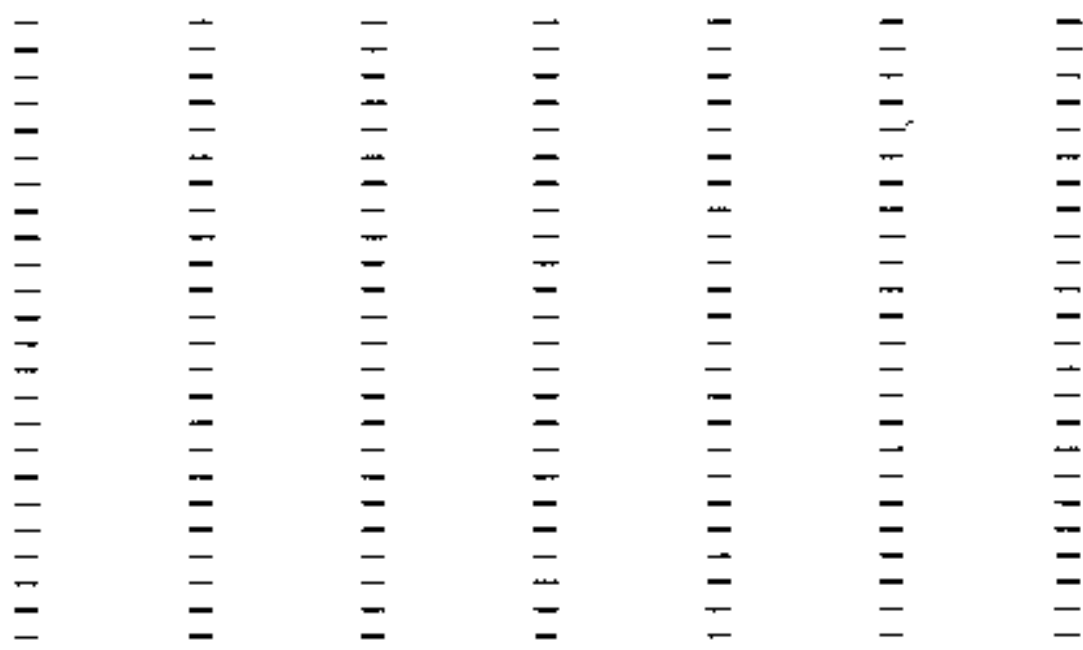

$=$
$=$
$=$
$=$
$=$
$=$
$=$
$=$
$=$
$=$
$=$

\begin{tabular}{|c|c|c|}
\hline- & - & \\
\hline & $\bar{z}$ & \\
\hline & - & \\
\hline$z$ & $z$ & \\
\hline- & - & \\
\hline $\bar{\tau}$ & $\bar{z}$ & \\
\hline$E$ & 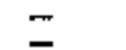 & \\
\hline- & - & \\
\hline- & $\bar{z}$ & \\
\hline$\overline{3 n, 6 \infty}$ & $13 \overline{6} \mathbf{6 s}, 198$ & \\
\hline$z$ & $=$ & \\
\hline & - & \\
\hline & - & \\
\hline & - & \\
\hline & - & \\
\hline & & \\
\hline
\end{tabular}

$=$
$=$
$=$
$=$ 
Table 34 Electricity Purchases by the Distribution Seggnent of Cooperative Borrowers, by State, 1994 (Continued)

\begin{tabular}{|c|c|c|c|c|c|c|c|}
\hline \multirow{3}{*}{ Stulk I } & \multicolumn{7}{|c|}{ Sowes of Flocwitisy } \\
\hline & \multirow{2}{*}{ 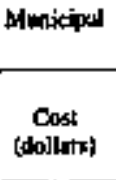 } & \multicolumn{2}{|c|}{ Cooperxive } & \multicolumn{2}{|c|}{ abn' } & \multicolumn{2}{|c|}{ Towal } \\
\hline & & 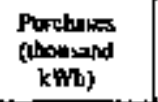 & $\begin{array}{c}\operatorname{Cos} x \\
\text { (dollosites }\end{array}$ & $\begin{array}{c}\text { Ponduces } \\
\text { (abonkund } \\
\text { KWh) }\end{array}$ & $\begin{array}{c}\text { Cos } \\
\text { (dolluss })\end{array}$ & $\begin{array}{c}\text { Porrhusel } \\
\text { (thomennd } \\
\text { kWhy }\end{array}$ & $\begin{array}{c}\operatorname{Cos} x \\
\text { [dolters }\end{array}$ \\
\hline 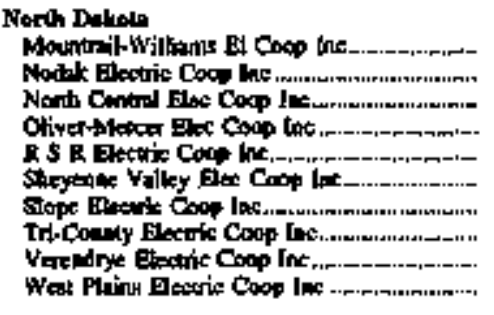 & $\begin{array}{l}- \\
= \\
= \\
= \\
= \\
=\end{array}$ & $\begin{array}{r}143,534 \\
524,457 \\
151,134 \\
160,194 \\
11,1782 \\
73,936 \\
53,965 \\
134,192 \\
291,536 \\
204,259\end{array}$ & $\begin{array}{r}4,628,399 \\
16,652,699 \\
5,622,249 \\
7,016,104 \\
4,511,690 \\
2,475,662 \\
2,1984,496 \\
4,576,706 \\
9,408,961 \\
2,792,918\end{array}$ & $\begin{array}{l}\bar{z} \\
= \\
\overline{-} \\
\overline{-}\end{array}$ & $\begin{array}{l}= \\
= \\
= \\
= \\
=\end{array}$ & $\begin{array}{r}141,594 \\
524,497 \\
151.134 \\
178,608 \\
317,483 \\
71,936 \\
53,464 \\
124.106 \\
281,596 \\
204,359\end{array}$ & $\begin{array}{r}4,628,799 \\
16,692,679 \\
5,622,29 \% \\
7,264,676 \\
\$, 511,690 \\
2,475,660 \\
2,094,496 \\
4,576,704 \\
9,408,961 \\
8,792,918\end{array}$ \\
\hline 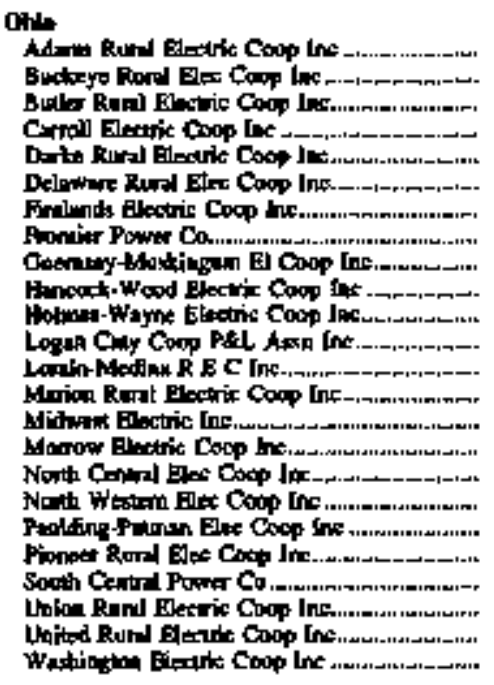 & $\begin{array}{l}= \\
= \\
= \\
= \\
= \\
= \\
= \\
= \\
= \\
= \\
= \\
= \\
= \\
=\end{array}$ & 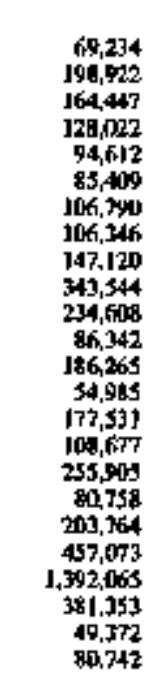 & 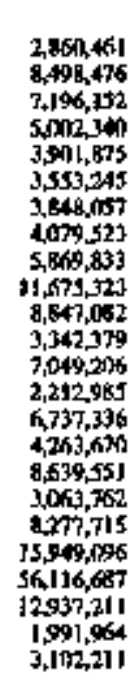 & $\begin{array}{l}\vec{z} \\
\vec{z} \\
= \\
= \\
= \\
= \\
= \\
= \\
= \\
= \\
= \\
=\end{array}$ & $\begin{array}{l}= \\
= \\
= \\
= \\
= \\
= \\
= \\
= \\
= \\
= \\
= \\
= \\
=\end{array}$ & 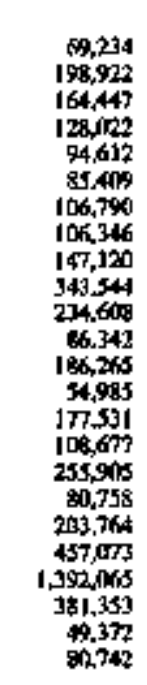 & 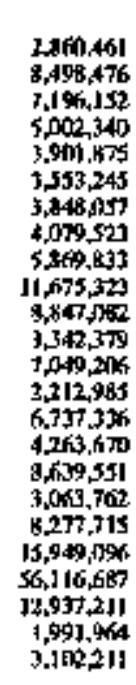 \\
\hline 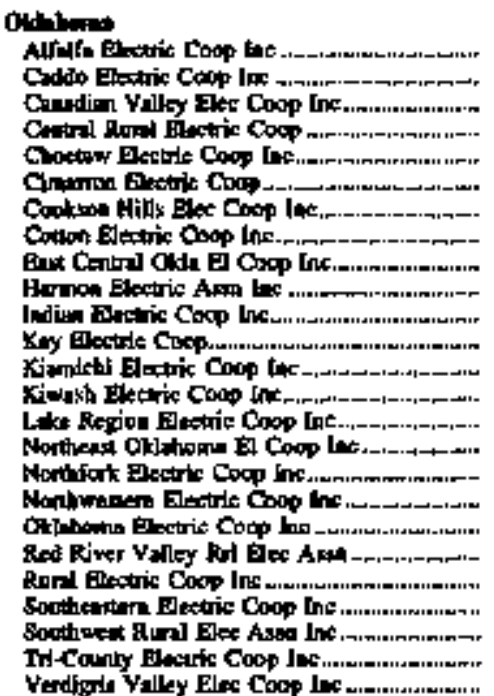 & $\begin{array}{l}= \\
= \\
= \\
= \\
= \\
= \\
= \\
= \\
= \\
= \\
= \\
= \\
=\end{array}$ & 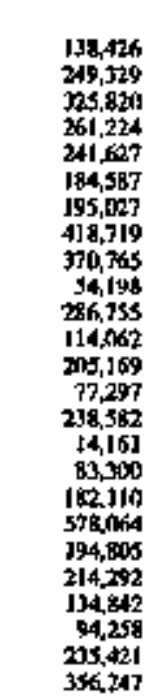 & 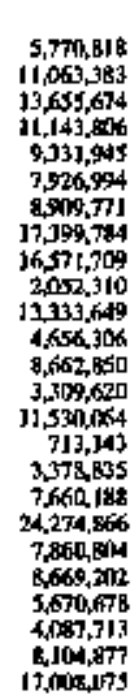 & $\begin{array}{l}\vec{z} \\
= \\
= \\
= \\
= \\
= \\
= \\
= \\
= \\
= \\
= \\
=\end{array}$ & $\begin{array}{l}= \\
= \\
= \\
= \\
= \\
= \\
= \\
= \\
= \\
= \\
= \\
= \\
= \\
= \\
= \\
=\end{array}$ & 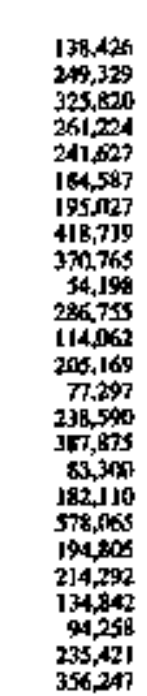 & 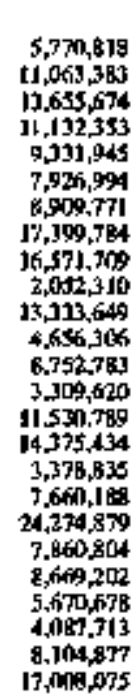 \\
\hline
\end{tabular}

See noter and rootioter at end of loble 
Table 34. Electricity Purctiases by the Distribution Sepment of Cooperative Borrowers, by State, 1994 (Continued)

\begin{tabular}{|c|c|c|c|c|c|c|c|}
\hline \multirow{3}{*}{$\begin{array}{c}\text { Sinde } t \\
\text { Coopero이 De Bocrower }\end{array}$} & \multicolumn{7}{|c|}{ Sounte of Electricity } \\
\hline & \multicolumn{2}{|c|}{$\begin{array}{l}\text { Inventor- } \\
\text { Owined }\end{array}$} & \multicolumn{2}{|c|}{ Foderal } & \multicolumn{2}{|c|}{ 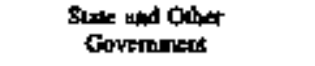 } & \multirow{2}{*}{ 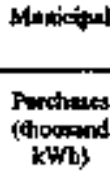 } \\
\hline & 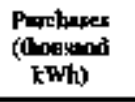 & Cont & 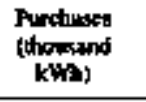 & $\underset{\text { (dolnents }}{\operatorname{Con}}$ & 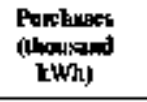 & $\underset{\text { (dollons) }}{\text { Cost }}$ & \\
\hline \multicolumn{8}{|l|}{ Drepose } \\
\hline 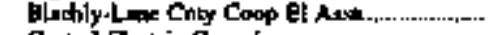 & - & - & 138,632 & $3,362,613$ & - & - & - \\
\hline Centril Destric Coop low & - & - & 432,658 & $11,964,559$ & - & - & - \\
\hline Colomba Bain Bec Coop lack...................... & - & - & $105,47]$ & $2,599,928$ & - & - & - \\
\hline 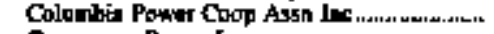 & - & - & - & - & 20258 & 322,036 & - \\
\hline Candunerd Powir Inc & 28 & $1,49 \%$ & 346,287 & $8,217,951$ & - & 96,645 & - \\
\hline Coar-Cuny Eloctric Coop Inf......................... & - & - & 290,698 & $7,684,972$ & - & - & - \\
\hline Dooghs Electric Coxp loc ............................ & - & - & 133,642 & $3,767,810$ & - & - & - \\
\hline Horley Electrte Coop Inx & - & - & 243,648 & $5,258,3,4$ & - & - & - \\
\hline 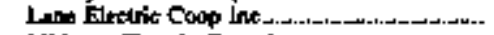 & - & - & 216,304 & $5,905,430$ & - & - & - \\
\hline 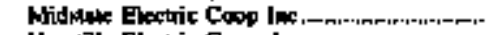 & - & - & 281,637 & $7,330,127$ & - & ع. & - \\
\hline Unetillo Electric Cong Ars & $\rightarrow$ & - & 660,711 & $(5,245,731$ & - & - & - \\
\hline 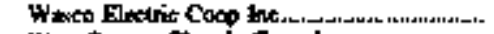 & - & - & 80,493 & $2,214,646$ & - & - & - \\
\hline 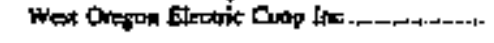 & - & - & K后, ㅁ19 & $1,3 \times 247$ & 一 & - & - \\
\hline \multicolumn{8}{|l|}{ Pennoykeld } \\
\hline Admins Dectic Cotp inc & - & - & - & - & - & - & - \\
\hline 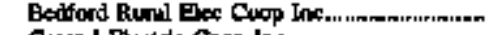 & - & - & - & - & - & - & - \\
\hline 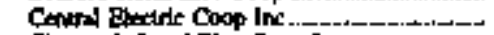 & - & - & - & - & - & - & - \\
\hline Cawerack Rond Dlec Cosp Inc................- & - & - & - & - & - & - & - \\
\hline 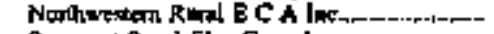 & - & - & - & - & - & - & - \\
\hline 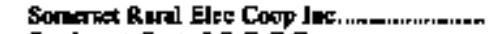 & - & $m$ & - & - & - & - & - \\
\hline Soophost Contril R E C Copp.................. & - & - & - & - & - & - & - \\
\hline Sullive Caunsy R E C Ins & - & - & - & - & - & - & $=$ \\
\hline 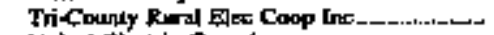 & - & - & - & - & - & - & - \\
\hline 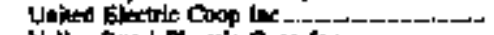 & - & - & - & - & - & - & - \\
\hline Volley Rural Bedtic Cosp Inx. & - & - & - & - & - & - & - \\
\hline 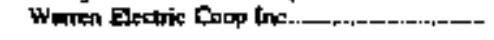 & - & - & - & - & - & - & - \\
\hline \multicolumn{8}{|l|}{ Soub Carvang } \\
\hline 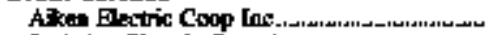 & - & - & - & - & - & - & - \\
\hline Bertetity Eloctic Coop Ine ........................ & - & - & - & - & - & - & - \\
\hline 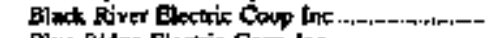 & - & - & - & - & - & - & - \\
\hline Blue Ridge Fletpic Coop lnc ................. & - & - & 35,396 & 91,420 & - & - & - \\
\hline 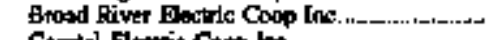 & - & - & In.221 & 2ThG & - & 一 & - \\
\hline 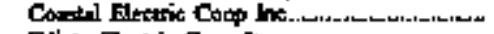 & - & - & - & - & - & - & - \\
\hline 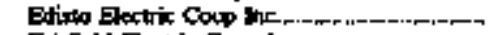 & - & - & - & - & - & - & - \\
\hline Firfiedd Exotic Cocp lnc & - & - & - & - & - & - & - \\
\hline Hory Fletric Cosp inc & - & - & - & $\bar{F}$ & - & - & - \\
\hline Lourtat Bertit Cocp lne & - & - & 26,600 & $\times 03.152$ & - & - & - \\
\hline 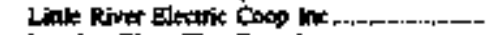 & - & - & 9,908 & 276,021 & - & - & - \\
\hline 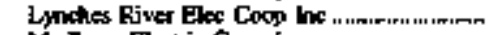 & - & - & - & - & - & - & 一 \\
\hline 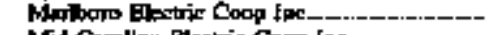 & - & - & - & - & - & - & - \\
\hline hid-Corolinu Biectric Coop ben & - & - & - & - & - & - & - \\
\hline 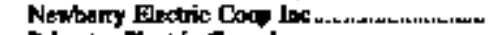 & - & - & - & - & - & - & - \\
\hline P. Inetes Electic Cocp he & - & - & - & $=$ & - & - & - \\
\hline 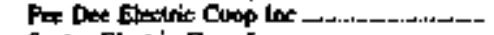 & - & - & - & - & - & - & - \\
\hline 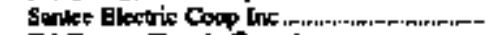 & - & - & - & - & - & - & - \\
\hline 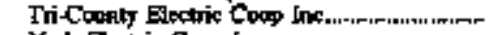 & - & - & - & - & - & - & - \\
\hline 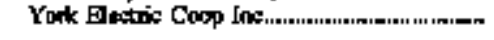 & - & - & 16,436 & 434,796 & - & - & 一 \\
\hline \multicolumn{8}{|l|}{ Sourth Ilencets. } \\
\hline Besilf Ploctrie Coop Ins.............................. & - & - & - & - & - & - & - \\
\hline 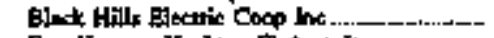 & - & س & - & - & - & - & - \\
\hline 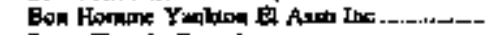 & - & - & - & - & - & - & - \\
\hline Bulte Electric CoOp Ins & - & - & - & - & - & - & - \\
\hline 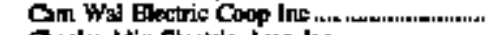 & - & - & - & - & - & - & - \\
\hline 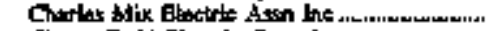 & - & - & - & - & - & - & - \\
\hline Ghary-Todd Flettic Coop lax & 455 & 17,147 & - & - & - & - & - \\
\hline 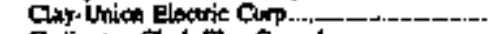 & $m$ & ש- & - & - & - & - & - \\
\hline 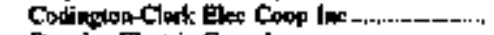 & - & - & - & - & - & - & - \\
\hline Dooglan Electric Coop Inc-ini-n & - & - & - & - & - & 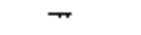 & - \\
\hline 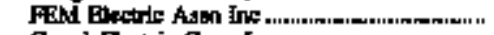 & - & - & - & $\bar{x}$ & - & - & - \\
\hline 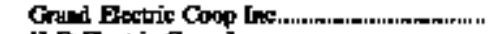 & - & - & 36,387 & $\sin 3357$ & - & - & $=$ \\
\hline 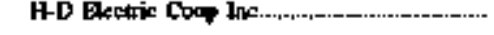 & - & - & - & - & - & - & - \\
\hline
\end{tabular}

Sed wont and forthoter at and or wbic. 
Table 34. Electrtity Purchases by the Disiribation Sepment of Cooperative Borrowers, by \$tate, 1994 (Contimued)

\begin{tabular}{|c|c|c|c|c|c|c|c|c|}
\hline & & \multicolumn{7}{|c|}{ Soure of Eletenety } \\
\hline & & \multirow{2}{*}{$\frac{\text { Monienpl }}{\text { Cont }}$} & \multicolumn{2}{|c|}{ Сexpadte } & \multicolumn{2}{|c|}{ Otherl } & \multicolumn{2}{|c|}{ Tots } \\
\hline$\pi$ & & & $\begin{array}{c}\text { Portules } \\
\text { (ltoweyd } \\
\text { kWyb) }\end{array}$ & $\begin{array}{c}\text { Cont } \\
\text { (dollex) }\end{array}$ & 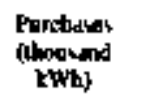 & $\begin{array}{c}\text { Cark } \\
\text { (doblary) }\end{array}$ & $\begin{array}{c}\text { Porthinet } \\
\text { (thoundind } \\
\text { lwht) }\end{array}$ & $\begin{array}{c}\text { Cost } \\
\text { (dollar) }\end{array}$ \\
\hline 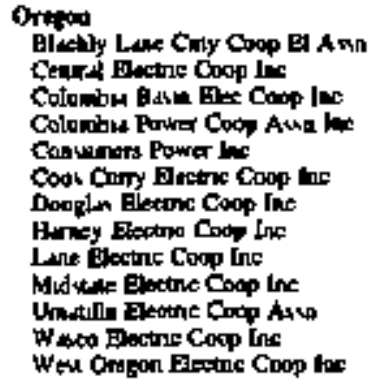 & & $\begin{array}{l}= \\
= \\
= \\
= \\
= \\
= \\
= \\
-\end{array}$ & $\begin{array}{l}z \\
- \\
z \\
z \\
- \\
z \\
- \\
-\end{array}$ & $\begin{array}{l}343,057 \\
238,550 \\
- \\
\overline{3} \\
305.286 \\
204,481 \\
- \\
- \\
\overline{-} \\
= \\
-\end{array}$ & $\begin{array}{l}- \\
- \\
= \\
= \\
- \\
-9,976 \\
- \\
=\end{array}$ & $\begin{array}{l}22,100 \\
- \\
= \\
- \\
= \\
- \\
= \\
= \\
=\end{array}$ & 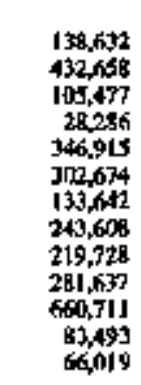 & 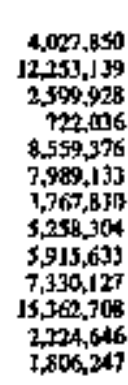 \\
\hline 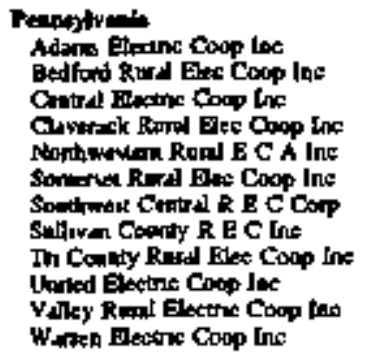 & & $\begin{array}{l}\overline{-} \\
- \\
= \\
= \\
= \\
= \\
-\end{array}$ & 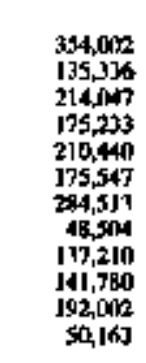 & $\begin{array}{r}21,019,275 \\
7,958,952 \\
12,210,589 \\
10,149,204 \\
11,523,522 \\
10,390,791 \\
17,092,145 \\
2,707,061 \\
8,294,157 \\
8,480,002 \\
11,234,267 \\
2,951,6159\end{array}$ & $\begin{array}{l}- \\
- \\
- \\
- \\
- \\
- \\
- \\
-\end{array}$ & $\begin{array}{l}- \\
- \\
- \\
- \\
- \\
- \\
20,5150 \\
- \\
- \\
-\end{array}$ & $\begin{array}{l}354,002 \\
135,336 \\
214,047 \\
175,203 \\
210,4010 \\
175,547 \\
244,513 \\
48,504 \\
137,210 \\
141,7801 \\
192,002 \\
50,163\end{array}$ & $\begin{array}{r}21,015,275 \\
7,958,952 \\
12,163,879 \\
10,149,280 \\
11,521,522 \\
10,390,793 \\
1,092,145 \\
2,727,596 \\
8,294,157 \\
8,480,002 \\
11,234,267 \\
2,95,605\end{array}$ \\
\hline 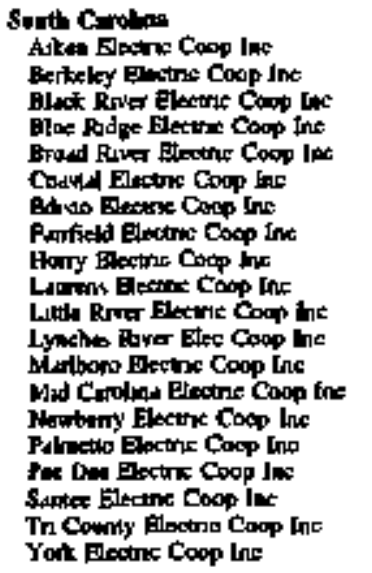 & i. & $\begin{array}{l}= \\
= \\
= \\
= \\
= \\
= \\
= \\
= \\
= \\
= \\
= \\
= \\
-\end{array}$ & 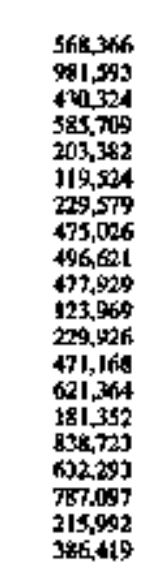 & 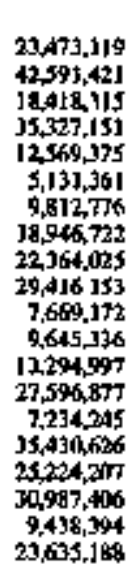 & $\begin{array}{l}- \\
- \\
- \\
- \\
- \\
- \\
- \\
- \\
- \\
- \\
- \\
- \\
- \\
-\end{array}$ & $\begin{array}{l}- \\
- \\
- \\
- \\
= \\
= \\
- \\
- \\
= \\
- \\
- \\
= \\
- \\
- \\
-4601\end{array}$ & 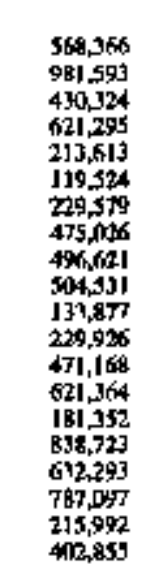 & 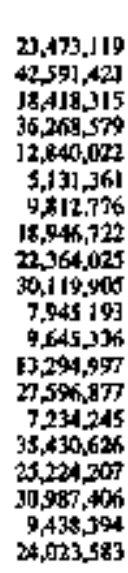 \\
\hline 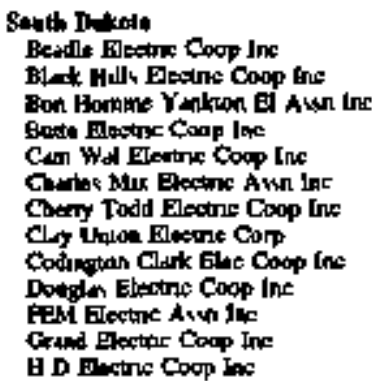 & $\cdot$ & $\begin{array}{l}= \\
= \\
= \\
= \\
= \\
= \\
= \\
=\end{array}$ & $\begin{array}{r}38,728 \\
76,171 \\
65,164 \\
59,797 \\
43,1289 \\
39,446 \\
80,786 \\
46,976 \\
67,759 \\
18,894 \\
45,223 \\
102,242 \\
35,938\end{array}$ & 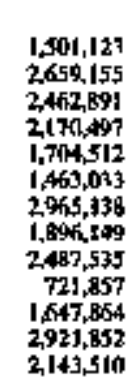 & $\begin{array}{l}- \\
- \\
- \\
- \\
= \\
= \\
- \\
- \\
-\end{array}$ & $\begin{array}{l}1,350 \\
- \\
- \\
= \\
= \\
= \\
-4,034 \\
= \\
-\end{array}$ & $\begin{array}{r}38,728 \\
76,173 \\
65,164 \\
59,797 \\
47,059 \\
39,486 \\
86,241 \\
50,956 \\
67,758 \\
18,894 \\
45,221 \\
139,629 \\
55918\end{array}$ & 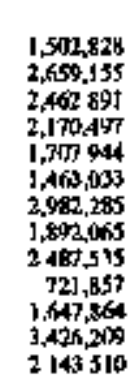 \\
\hline
\end{tabular}

Sta noceb and footnatos al and of table 
Table 34. Electricity Purchases by the Distribution Sepment of Cooperative Borrowers, by State, 1994 (Continued)

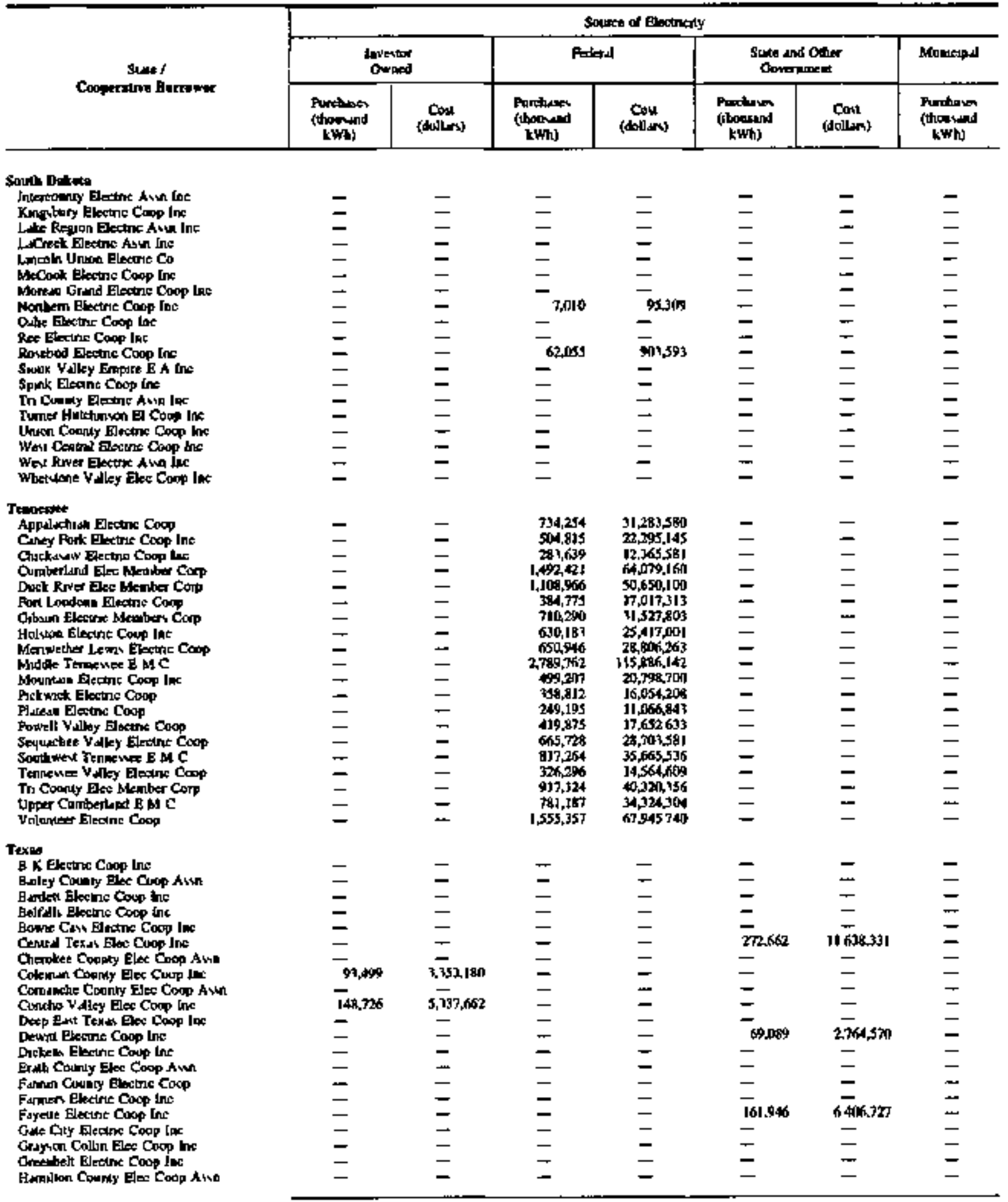

See woten and foosnotes st end of thide 
Table 34. Electricity Purcheses by the Distribution Segment of Cooperative Borrowers, by State, 1994 (Conthued)

\begin{tabular}{|c|c|c|c|c|c|c|c|}
\hline \multirow{3}{*}{ Sinet } & \multicolumn{7}{|c|}{ Soumes of Pletetnelly } \\
\hline & \multirow{2}{*}{ Mantuped } & \multicolumn{2}{|c|}{ Coopestuve } & \multicolumn{2}{|c|}{ Otherl } & \multicolumn{2}{|c|}{ Totel } \\
\hline & & 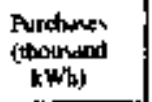 & $\underset{\text { (dollanin) }}{\operatorname{Cos}}$ & 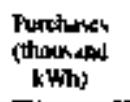 & $\underset{\text { (Don }}{\text { Cons) }}$ & $\begin{array}{l}\text { Forchness } \\
\text { (ditorndad } \\
\text { kWhy) }\end{array}$ & $\begin{array}{c}\text { Cont } \\
\text { (dollan) }\end{array}$ \\
\hline 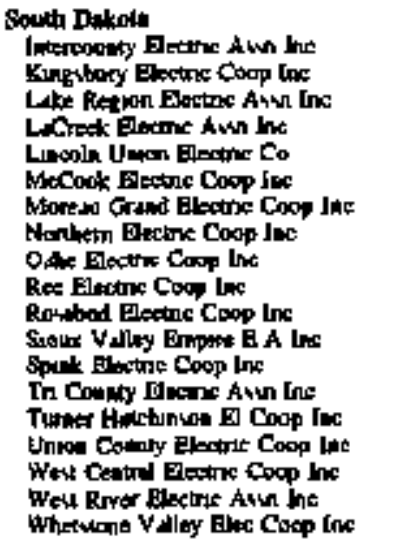 & $\begin{array}{l}= \\
= \\
= \\
= \\
= \\
= \\
= \\
= \\
= \\
=\end{array}$ & 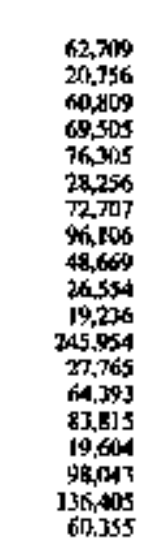 & 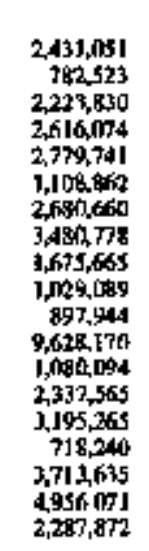 & $\begin{array}{l}\bar{z} \\
= \\
= \\
= \\
= \\
= \\
= \\
= \\
= \\
=\end{array}$ & $\begin{array}{l}= \\
= \\
= \\
= \\
= \\
= \\
= \\
\quad 118 \\
= \\
=\end{array}$ & 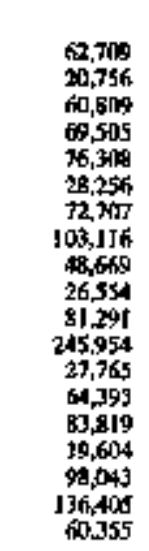 & 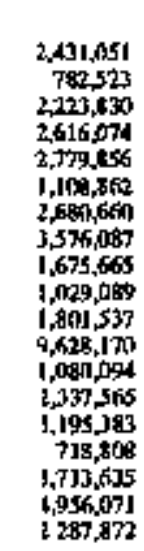 \\
\hline 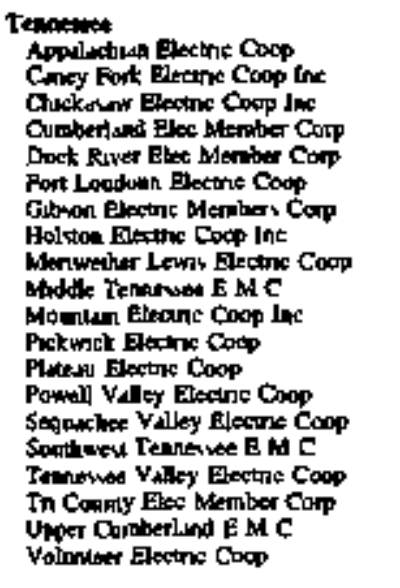 & $\begin{array}{l}= \\
= \\
= \\
= \\
= \\
= \\
= \\
= \\
= \\
= \\
= \\
=\end{array}$ & $\begin{array}{l}= \\
= \\
= \\
= \\
= \\
= \\
= \\
= \\
= \\
= \\
= \\
= \\
=\end{array}$ & $\begin{array}{l}= \\
= \\
= \\
= \\
= \\
= \\
= \\
= \\
= \\
= \\
= \\
=\end{array}$ & $\begin{array}{l}= \\
= \\
= \\
= \\
= \\
= \\
= \\
= \\
= \\
= \\
= \\
= \\
=\end{array}$ & $\begin{array}{l}= \\
= \\
= \\
= \\
= \\
= \\
= \\
= \\
= \\
= \\
= \\
= \\
-\end{array}$ & 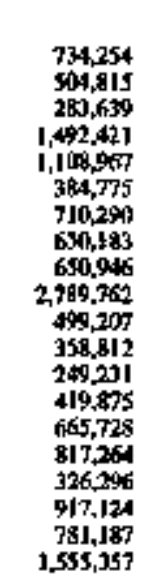 & 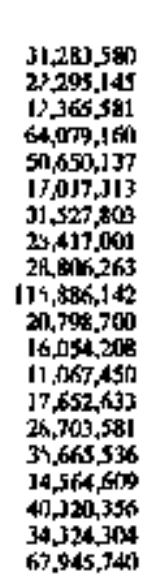 \\
\hline 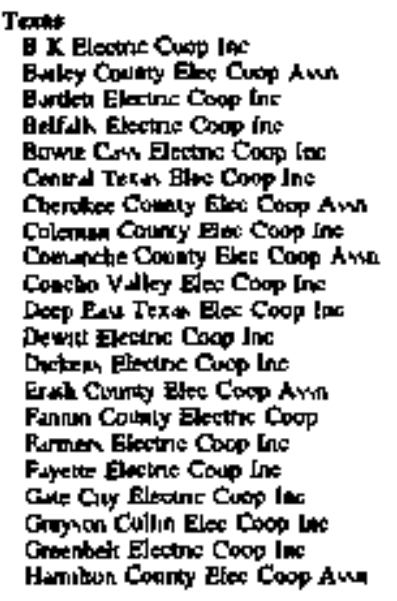 & $\begin{array}{l}= \\
= \\
= \\
= \\
= \\
= \\
= \\
= \\
= \\
= \\
=\end{array}$ & 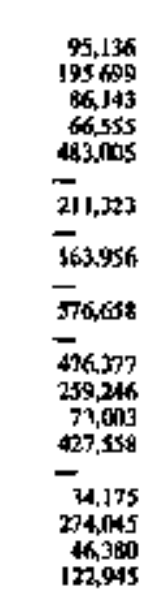 & 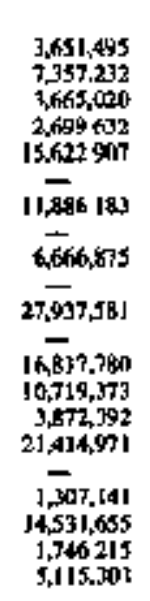 & $\begin{array}{l}= \\
= \\
= \\
= \\
= \\
= \\
= \\
= \\
= \\
= \\
= \\
= \\
=\end{array}$ & $\begin{array}{l}z \\
= \\
= \\
= \\
= \\
= \\
= \\
= \\
= \\
= \\
= \\
= \\
-\end{array}$ & 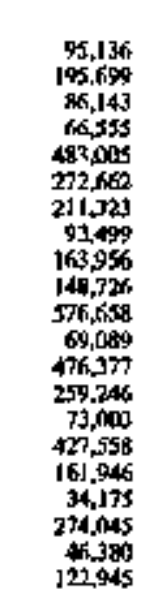 & 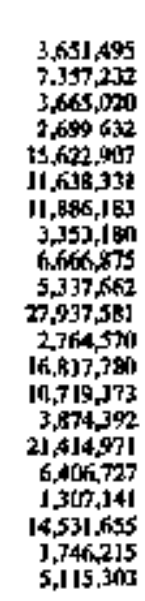 \\
\hline
\end{tabular}


Table 34. Electrielty Purchases by the Distribution Segment of Cooperative Borrowers, by Stute, 1994 (Continued)

\begin{tabular}{|c|c|c|c|c|c|c|c|}
\hline \multirow{3}{*}{ 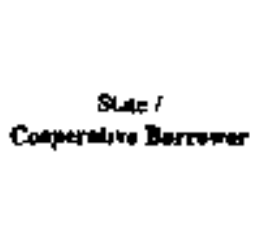 } & \multicolumn{7}{|c|}{ somes of blectupty } \\
\hline & \multicolumn{2}{|c|}{$\begin{array}{l}\text { Inwerton } \\
\text { Owrined }\end{array}$} & \multicolumn{2}{|c|}{ Federnl } & \multicolumn{2}{|c|}{$\begin{array}{l}\text { Stale and Collit } \\
\text { Goverument }\end{array}$} & \multirow{2}{*}{ 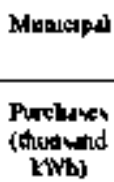 } \\
\hline & $\begin{array}{l}\text { Forthing } \\
\text { (thouland } \\
\text { kWha) }\end{array}$ & Cost & $\begin{array}{l}\text { Pording } \\
\text { (chowind } \\
\text { kwh) }\end{array}$ & $\begin{array}{l}\text { Cont } \\
\text { [dollam) }\end{array}$ & 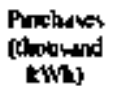 & $\begin{array}{c}\text { Cont } \\
\text { (dolum })\end{array}$ & \\
\hline
\end{tabular}

Thin

Hal Condy Deathe Coop

Hoision Conity Elec Coop lec

J A Electric Coop Inc

Jakion Eluzac Coop lin

Impar Newton Elec Coop in

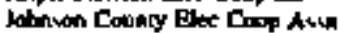

Kimes Electne Coop ine

Xofina Couts Dese Coop Int

Kimble Fectinc Cocp inc

luter Coung Da Coop Ain

1-4lob Country Eloctris Coop ine

Lughthows Binge Coop inc

Lyneger Electinc Coop Ins

Mutch Valey Blecunc Coop Ine

MoCulloch Euctic Coop Int

Malcandar Cospry bee Coop In

Nedin Eletine Coop In

Middweut Electere Coop Jar

Naverro Connly Elec Coop Ine

Ndvised Yalley Pitec Coop lic

Naw En Elestnc Coop Inc

Nowh Pluns Electro Coop Inc

Nunat Exetre Coop bit

Proold Haman Ele Cosp Iac

Roo Orande Dectric Coco ine

Rul Blates Elactint Coop Inc

Ruk County Elocanc Cocp linc

Sen Pumew Electinc Coop Int

South Plolar Elecane Coop Ir.

Scuibusat Taxes Bue Coop Inc

Stumblov Bettic Cocp lnc

Swant berinc Coos la

Tylor Eletric Coop Jae

Upher Burd Bla box Cop

$\checkmark$ retorld Electre Coop Inc

Whanon Condy Elec Coop It:

Wine Dectinc Coup Jnc

Wood Clowny Gientric Cow Inc

Uin)

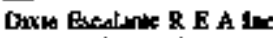

Fowredl Electiot Asun Inc

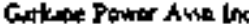

Mom Lite Electrix A'sn Ine

Vermod

Vorting Eloting Goop lat

Walungton Eectroc Coop Ine

Vurpaim

A \& N Elestens Copp

BARC Elequic Coop Ine

Central Virgunu Evecunts Dop

Oommundy Elscirjt Cocp

Grags Botridert Electint Coop

Hectleaburg Electoc Coop ins

Nothan Nect Elec Coxop Int

Nothom Virginte Aluc Coop

Prove Deante Eloctuc Coop

Rupphenanck Electinc Coop

Shenandont Valley Elac Coop

Sowbrath Elosinc Coop be

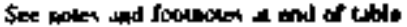

\begin{tabular}{|c|c|c|c|c|c|c|}
\hline- & - & - & - & - & - & - \\
\hline - & 一 & - & - & - & - & 一 \\
\hline - & - & - & - & - & - & - \\
\hline - & - & - & - & - & - & - \\
\hline- & - & - & - & - & - & - \\
\hline 一 & 一 & - & - & - & - & 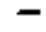 \\
\hline - & - & - & - & - & - & - \\
\hline- & $\overline{7}$ & - & - & - & - & - \\
\hline 14,201 & 312,417 & - & - & 11,492 & $1.261,876$ & - \\
\hline - & - & - & - & - & - & - \\
\hline- & - & - & - & ع. & - & - \\
\hline $\mathbf{1 3 , 2 1 8}$ & $54 H_{1}, 292$ & - & - & - & - & - \\
\hline $\operatorname{son}, 281$ & $19,464,392$ & - & - & - & - & - \\
\hline $5 \$ 2405$ & $25 \times 37,044$ & - & - & - & - & - \\
\hline- & - & - & - & 69452 & $2,671,497$ & - \\
\hline- & - & - & - & - & - & 一 \\
\hline 68,736 & $3.187,114$ & 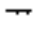 & - & - & - & $=$ \\
\hline 95,268 & 4.138.1063 & - & - & - & - & - \\
\hline - & - & - & - & - & - & 一 \\
\hline - & - & $\pi$ & - & - & - & $m$ \\
\hline - & - & - & - & $m$ & - & - \\
\hline 一 & - & - & - & - & - & - \\
\hline 一 & - & - & - & - & - & $m$ \\
\hline- & - & - & - & - & - & - \\
\hline 111,716 & $3,157,4 n$ & - & - & - & - & - \\
\hline- & - & - & - & - & - & - \\
\hline $283,56 \mathrm{~s}$ & 9,608,875 & - & - & - & - & - \\
\hline- & - & - & - & - & - & - \\
\hline$\overline{100,372}$ & $6 \overline{2} 18,1$ & - & - & $=$ & $=$ & $\bar{z}$ \\
\hline $\begin{array}{r}09,474 \\
69,42\end{array}$ & $2,544,137$ & $\overline{-}$ & $=$ & $\overline{-}$ & - & - \\
\hline- & - & - & - & - & - & - \\
\hline 188,167 & $7,190,2 \times 6$ & - & - & - & - & 一 \\
\hline - & - & - & - & - & - & - \\
\hline$=$ & $=$ & 二 & $\bar{z}$ & $\bar{z}$ & $=$ & - \\
\hline - & - & - & - & - & - & \\
\hline- & - & - & - & - & - & - \\
\hline
\end{tabular}

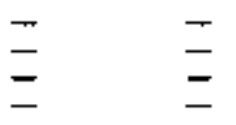

1276

$25, \sqrt{21}$

62 [3]

$1,019,846$

$\begin{array}{lc}\overline{-} & - \\ 35,699 & 18,067,191 \\ - & - \\ 37,09 & 2304,348 \\ - & - \\ - & - \\ - & - \\ - & - \\ - & -\end{array}$

Itis2

225,282

4.25s 693

${ }^{205}$

$1.102,45$

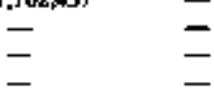

$-$

-

8,661

I45,2fi4

2.150

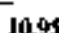

10,953

23,299

5,143

33.221

11.510

9,575

7.409

65,678

39.104

42,683

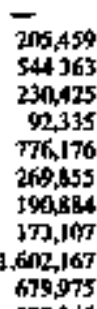

2015,450

544163

231425

92,335
776,176

269.55

I94.8.

17,167

679,975

99714 
Table 34. Electricity Purchases by the Distributhon Segnent of Cooperative Borrowers, by Stote, 1994 (Continued)

\begin{tabular}{|c|c|c|c|c|c|c|c|}
\hline \multirow{3}{*}{$\begin{array}{c}\text { Sapka f } \\
\text { Cooparallye Barmwer }\end{array}$} & \multicolumn{7}{|c|}{ Sutures of Electuneily } \\
\hline & \multirow{2}{*}{ Munacep } & \multicolumn{2}{|c|}{ Cucperstuve } & \multicolumn{2}{|c|}{ Other 1} & \multicolumn{2}{|c|}{ Tot } \\
\hline & & $\begin{array}{l}\text { Purchers } \\
\text { (droutund } \\
\text { (kWt) }\end{array}$ & $\begin{array}{c}\text { Cont } \\
\text { (do]lax })\end{array}$ & 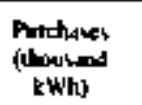 & $\underset{(t i o n d r)}{\text { Ond }}$ & 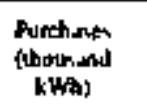 & $\begin{array}{c}\text { Cont } \\
\text { (doildr) }\end{array}$ \\
\hline \multicolumn{8}{|l|}{ Texp: } \\
\hline Hdl County Blecene Cusop & - & 218,944 & $9,11,394$ & - & - & 218,94 & $9,71,744$ \\
\hline Howito Conty Eles Coop lne & - & 218,199 & $11,451.202$ & - & - & 218,799 & $1,451,272$ \\
\hline I A C Elosthe Coog Ins & - & RR, 466 & $1,514,552$ & $\overline{-}$ & $=$ & 結, 4 G & 3514,552 \\
\hline 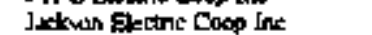 & - & 246.112 & $7,028,260$ & - & - & $146, \sqrt{12}$ & $7,628,268$ \\
\hline Japar Newton Bes Coop luc & - & 116,016 & 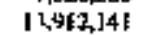 & - & - & ?]గ, 966 & $|x, y| 2,14 \mid$ \\
\hline blanion Counk Eec Coos Aiva & - & $569,0 \mathrm{A2}$ & $24,419,794$ & - & - & 160002 & $24,419,384$ \\
\hline Kimen Ejetnc Coop ine & - & 199.177 & $7,994,477$ & - & - & 159,177 & $7,904,473$ \\
\hline Kuimen Cowy Flex Cosp loc & - & 159,157 & [B, 122,15] & - & - & 159,053 & $[8] 22,15]$, \\
\hline Kmble Elextre Corp lis & - & - & - & - & - & $\$ \$ 1,692$ & $1.974,293$ \\
\hline 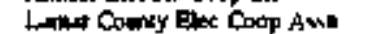 & $=$ & 109,25 & $5,826,996$ & - & $=$ & 109,297 & 5,R26,996 \\
\hline 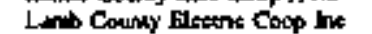 & - & 261,791 & 10,114, snos & - & - & 24,291 & $10,173,005$ \\
\hline Loghthoure Electinc Coop Jac & - & 217412 & $5 \leq 0 \geq 0050$ & - & - & 230,640 & 9,1149002 \\
\hline 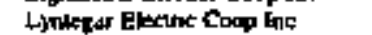 & - & - & - & - & - & $507,28]$ & $19,464,592$ \\
\hline 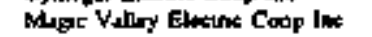 & - & - & - & - & - & 582,4115 & $28,393,044$ \\
\hline MoOulloct Electine Coop Inc & - & - & - & - & - & 69,452 & $2,671,497$ \\
\hline Aklewen Cawnty fler Coop lat: & - & 159.299 & Q.75it,521 & - & - & 159.289 & h,764,521 \\
\hline Melw Ekootnc Goop Inc & - & 20 & I0,045,112 & - & - & 3A8,B]6 & $17,232,426$ \\
\hline 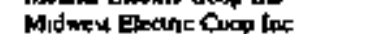 & - & 51,859 & $1,805,571$ & - & - & ₹49, In & $5.947,6306$ \\
\hline Nan-to Comar Ele Cusp loc & - & $203,7 \mathrm{Rg}$ & $8,446,650$ & - & - & 2117,360 & $8,446,6 S D$ \\
\hline Now troud V+hley Bles Coop bis & - & 269,819 & $10,769,247$ & - & - & $269,4 \%$ & In,769,243 \\
\hline New Exs Exotuc Coop fne & - & 27,455 & $13,648,796$ & - & - & 273,655 & $17,848,796$ \\
\hline Nonh Plans Blectoe Coup In & - & 374,79 & $4,74,541$ & s & jon & 14,384 & 4,734 , nd \\
\hline Noen lowerc Corp low & - & 145.617 & $7,7+9,797$ & - & - & 145,677 & $7,319,793$ \\
\hline 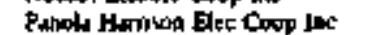 & - & 239.106 & $7, \% 1,007$ & - & - & 299,106 & $7,961,002$ \\
\hline Ros Grade Electine Coop Inc & - & 21.347 & 1,041562 & - & - & 193,469 & $0,201,039$ \\
\hline ful Bluas Elestow Coop Inc & $=$ & 140,110 & $4,995,867$ & - & - & 140,110 & $4,995,8$ त \\
\hline 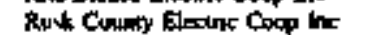 & - & - & - & - & - & 283,568 & $9.6010,875$ \\
\hline San Pathero Flatse Coop Inc & - & 376651 & $6,781,120$ & - & - & 136,fis 1 & $6,781,90$ \\
\hline Sooth Flums Eloetne Cosp Ins & - & 506517 & 192887,298 & - & $m$ & $50 \times 5 \pi$ & $19,287,298$ \\
\hline Sonthwex Texsis Ber Coop In & - & - & - & - & - & 190,572 & $6,218,891$ \\
\hline 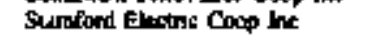 & - & - & - & - & - & 64,074 & 2540,077 \\
\hline Sorther Hastenc Coop Inc & - & 2noults: & $8,251,58$ & - & - & 296,439 & $825158 \mathrm{~J}$ \\
\hline Taylor Electes Coop In & - & - & - & - & - & $198 \% 169$ & $7,790,266$ \\
\hline Upther Rural Eloc Coop Cosp & - & 36elind & $19,700,540$ & - & - & 564,674 & 17,709540 \\
\hline 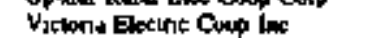 & - & 216531 & 12,467,210 & - & - & 276,531 & $12,467,230$ \\
\hline 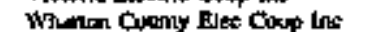 & - & J29,1601 & $6,482,760$ & - & - & 129,160 & 6,482760 \\
\hline Wive Eletinc Coop Ine & - & 193.612 & E.15T.119 & 18 & 479 & 19\}, fist & $8,197,018$ \\
\hline Wood Covwy Electinc Coop in & - & 400216 & $14,978.758$ & - & - & 400,716 & $14,978,758$ \\
\hline \multicolumn{8}{|l|}{ Utah } \\
\hline 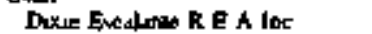 & - & 26,879 & $3,51,3,51\}$ & 95,876 & $1.716,22$ & 13,298 & $4,372,592$ \\
\hline Fowed erang Asm Inc & - & II.794 & 690,425 & & & 22516 & and \\
\hline 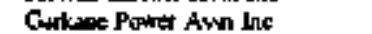 & - & 16807 & 603,379 & 84,766 & $2,290,168$ & 101,173 & 2890,497 \\
\hline 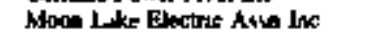 & - & 62,48 & $35,297,149$ & 13,456 & 410,12 & 925,086 & 4.964,164 \\
\hline \multicolumn{8}{|l|}{ Verponil } \\
\hline Verwont Electinc Coop Ins: & - & 1004,45 & $11,185.162$ & 8,113 & 813,613 & 125,825 & $12,820.926$ \\
\hline Wohungion Elosere Coup ine & 391,329 & 6.163 & 215.775 & 17,329 & B64,823 & 50,6126 & 2,6571197 \\
\hline \multicolumn{8}{|l|}{ Vuction } \\
\hline$A \& N$ Eketroc Coop & - & 105,847 & $8,|| 1,4,45 \mid$ & - & - & 175.847 & $8,1111,451$ \\
\hline BARC Getroc Coop ine & $=$ & 132,609 & $6,045,891$ & - & $-10,418$ & 141,622 & $6,240,923$ \\
\hline Centrod Yirpanit Electre Copop & - & - & - & - & - & 418,938 & $16,511,554$ \\
\hline 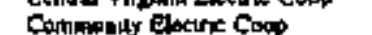 & - & 122,131 & 6,172014 & ـ & - & 14,519 & $6,600,459$ \\
\hline Galp Bodewert Blestre Cos, & - & - & - & - & $-31,970$ & 6].N00 & $2,364,719$ \\
\hline Mectlabers eloctrox coop be & - & 344,769 & $16,746,9 \times 6$ & - & - & $373.99 \mathrm{AB}$ & $17522+72$ \\
\hline Nonhyst Nect Elee Coop inc & $\rightarrow$ & JW9, D?9 & $9,123,527$ & - & - & $1900,5 \% 9$ & $4,397,3 \mathrm{kZ}$ \\
\hline Nothan Yopamo elec coop & - & $1,756,768$ & $91, \sqrt{1837,184}$ & - & - & $1,766,458$ & $91,8 \pi, 068$ \\
\hline Puece Ceorge Blectine Coop & - & 152,100 & B., 3,460 & - & - & 159,499 & $8,180,567$ \\
\hline Ropolantrock Elestine Coop & - & $1,7 y 3,723$ & $72,055,617$ & - & - & $1,801,401$ & $74,557,780$ \\
\hline 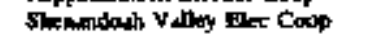 & - & 519,955 & $24,426,914$ & - & - & SG, 659 & $25,106,889$ \\
\hline Sconthude Elestex Coop Ins & - & 511.534 & $25,157,074$ & - & - & 554.217 & 26.154 .320 \\
\hline
\end{tabular}

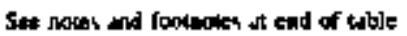


Table 34. Electricity Purchises by the Distribotion Sezment of Cooperntive Borrowers, by State, 1994 (Continued)

\begin{tabular}{|c|c|c|c|c|c|c|c|}
\hline \multirow{3}{*}{$\begin{array}{c}\text { Sute It } \\
\text { Cosperafive Bormonwer }\end{array}$} & \multicolumn{7}{|c|}{ Surote of electionty } \\
\hline & \multicolumn{2}{|c|}{ Oavents } & \multicolumn{2}{|c|}{ Fodort } & \multicolumn{2}{|c|}{$\begin{array}{l}\text { Strie and Other } \\
\text { Oomedruent }\end{array}$} & \multirow{2}{*}{ 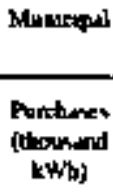 } \\
\hline & 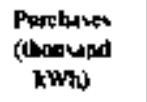 & $\begin{array}{c}\text { Con } \\
(\text { da! } ! n n)\end{array}$ & 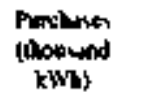 & Con & 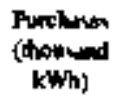 & Cont & \\
\hline \multicolumn{8}{|l|}{ Wabington } \\
\hline 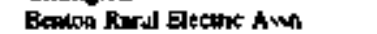 & - & - & $36+449$ & 6.979 .387 & - & - & - \\
\hline 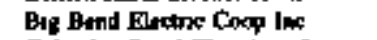 & - & - & 417,816 & $9,5 \leqslant 0,290$ & - & - & - \\
\hline Coturnbsu Rerd Eloc $A$ inn Inc & - & - & $229, \$ 09$ & $4,970,336$ & $=$ & - & - \\
\hline Lmoola Flestnc Coop hic & - & - & IOS, K21 & 268ting & - & - & - \\
\hline Neppetem VAlley Bec Coop Inc & - & - & 39,312 & $1,075,063$ & - & - & - \\
\hline Otdrogen Coomiy Elec Coop lnc & - & - & $38,17]$ & 1.916.348 & - & - & - \\
\hline Ores powet A Ligh Co & - & - & 150,484 & $4 \operatorname{lng}, 490$ & - & - & - \\
\hline Thetr Elocine Curop & $\rightarrow$ & - & 4,97 & $1,212,915$ & - & - & - \\
\hline \multicolumn{8}{|l|}{ Wen Vrovels } \\
\hline EAmson Rurd Elec Axm Inc & 49.515 & 1,940246 & - & - & - & - & - \\
\hline \multicolumn{8}{|l|}{ Wuscment } \\
\hline Addms Colomba Deane Coop & 320,837 & $10,808,172$ & - & - & - & - & - \\
\hline Emon Electinc Coop & - & - & - & - & - & - & - \\
\hline 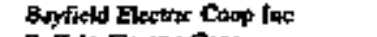 & - & - & - & - & - & - & - \\
\hline BoIlulo Electne Coop & $-7 \operatorname{ses}$ & Tanse & - & 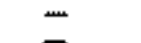 & - & - & - \\
\hline Chnord Wiveonun Eitc Coop & 75,545 & $2,494,213$ & - & - & - & - & - \\
\hline Oppous Valky Electine Coop & - & - & - & 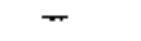 & - & - & - \\
\hline 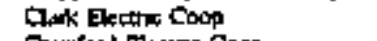 & - & - & - & - & - & - & - \\
\hline Crowifoed Elecenc Coop & - & - & $=$ & - & - & - & - \\
\hline Donn Councy Eloctro Coop & - & - & - & - & - & $m$ & - \\
\hline Es Cane Electnc Conp & - & - & - & - & - & - & - \\
\hline Gram Lafuyets Electre Coop & - & - & - & - & - & - & - \\
\hline Hed of teiken Elestne Coop & 40,194 & $1,354,769$ & - & - & - & - & - \\
\hline beckon Deecone Cocp lne & - & - & - & - & - & - & - \\
\hline Jump Riner Eleosing Coop Inc & - & - & - & - & - & - & - \\
\hline Dukidue Glectuc Coop & - & - & - & - & - & $=$ & - \\
\hline Demo Emenc Coop & 0,158 & 3.197 .138 & - & - & - & - & - \\
\hline Pienct Pepu Elextenc Coop & - & - & - & - & - & - & - \\
\hline Palk Gutitul Blextre Coop & - & - & - & - & - & - & - \\
\hline Prose Aleane Coop luc & - & - & - & - & - & - & - \\
\hline Ruchund Flactore Coop & - & - & - & - & - & - & - \\
\hline Rock Coomy Electre Coop Anu & $6.7,989$ & 2258.499 & - & - & - & - & - \\
\hline St Grax Elestinc Coop & - & - & - & - & - & - & - \\
\hline Tyylor Electse Coop & - & - & - & - & - & - & - \\
\hline Trempedient Electric Coop & - & - & - & - & - & - & ב- \\
\hline Vemion Electre Cocp & - & - & - & - & - & - & - \\
\hline 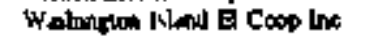 & 2.104 & 293399 & - & - & - & 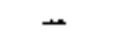 & - \\
\hline \multicolumn{8}{|l|}{$W_{\text {gnang }}$} \\
\hline Bis Horn Rerd Ekeanc Co & - & - & - & - & - & ع & - \\
\hline Brodpe Valky lasc Awn tor & - & - & $39,76 \pi$ & 727215 & - & - & - \\
\hline Cribou Power a loth line & - & - & - & - & - & - & - \\
\hline Gerlind Leth \& Rrive Co & - & - & - & - & - & - & $=$ \\
\hline Hot Sprngig Rarl Es Asun lac & - & - & - & - & - & - & - \\
\hline Lower Valloy Powner \& Ldu Ine & - & - & 454699 & $12,293,276$ & - & - & - \\
\hline 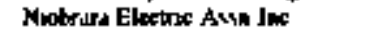 & - & - & - & - & - & - & $\pi$ \\
\hline Rewertion Valkg Flec AMn InT & - & - & - & - & - & - & - \\
\hline Rord Electne Do & - & - & - & - & - & - & - \\
\hline Shendan Jowman Rif Eles Asu & - & - & - & - & - & - & - \\
\hline Tn County Electhe Awh I I & - & - & - & - & - & - & - \\
\hline Whesland Rord Eec Atu It & $=$ & - & - & - & - & - & - \\
\hline Wynobs Co & - & - & - & - & - & - & - \\
\hline
\end{tabular}


Tabłe 34. Electricity Purchases by the Distrilontion Segment of Cooperative Borrowers, by State, 1994 (Continued)

\begin{tabular}{|c|c|c|c|c|c|c|c|}
\hline \multirow{3}{*}{$\begin{array}{c}\text { Etale } t \\
\text { Cosparnowa Bocroner }\end{array}$} & \multicolumn{7}{|c|}{ Sowree of Elecancely } \\
\hline & \multirow{2}{*}{ 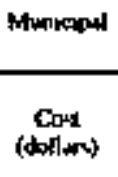 } & \multicolumn{2}{|c|}{ Coopative } & \multicolumn{2}{|c|}{ Ox=1 } & \multicolumn{2}{|c|}{ Thtal } \\
\hline & & 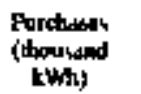 & Corl & 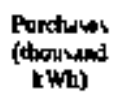 & $\begin{array}{c}\text { Caul } \\
\text { (dorldur) }\end{array}$ & 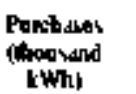 & $\underset{\text { Coxd }}{\text { (dollex) }}$ \\
\hline 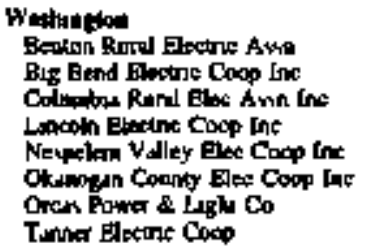 & $\begin{array}{l}z \\
z \\
z \\
z \\
-\end{array}$ & $\begin{array}{l}z \\
z \\
z \\
z \\
-\end{array}$ & $\begin{array}{l}272,154 \\
- \\
15 D_{1} / 83 \\
- \\
= \\
-\end{array}$ & $\begin{array}{l}- \\
- \\
\begin{array}{l}2,102 \\
1,187\end{array} \\
= \\
-\end{array}$ & 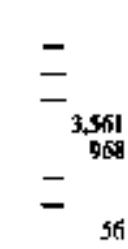 & $\begin{array}{r}36,449 \\
477,3] 6 \\
229,833 \\
111,923 \\
40,519 \\
35,177 \\
150,484 \\
44977\end{array}$ & $\begin{array}{l}9,243,541 \\
9,650,790 \\
4,974,336, \\
2,343,1925 \\
1,076,031 \\
1,006,388 \\
4,1990,490 \\
1,212,071\end{array}$ \\
\hline 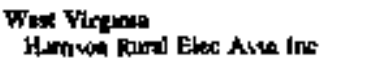 & - & 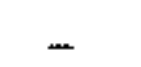 & - & - & - & 49.519 & 1940,246 \\
\hline 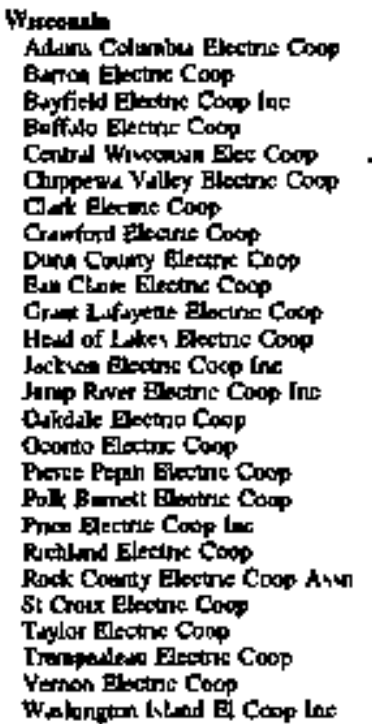 & 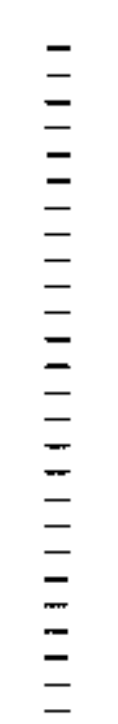 & 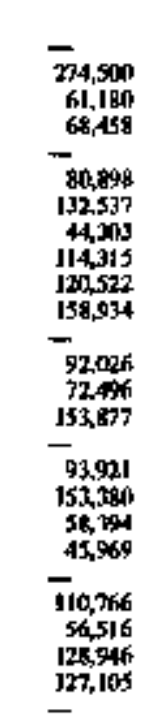 & 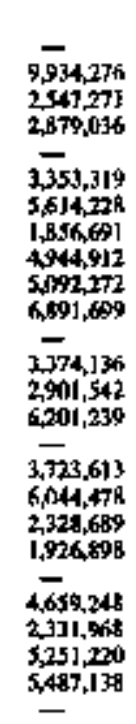 & $\begin{array}{l}\text { II' } \\
= \\
- \\
= \\
= \\
- \\
- \\
= \\
= \\
= \\
- \\
- \\
- \\
= \\
= \\
- \\
- \\
-\end{array}$ & $\begin{array}{l}3.959 \\
= \\
= \\
= \\
- \\
- \\
- \\
= \\
= \\
= \\
- \\
- \\
- \\
- \\
= \\
- \\
- \\
-\end{array}$ & 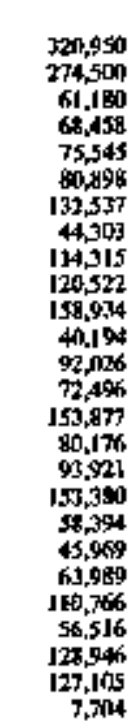 & 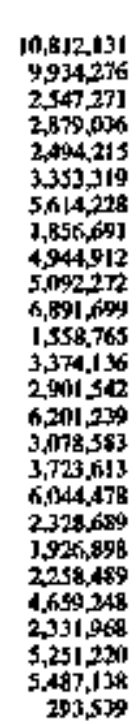 \\
\hline 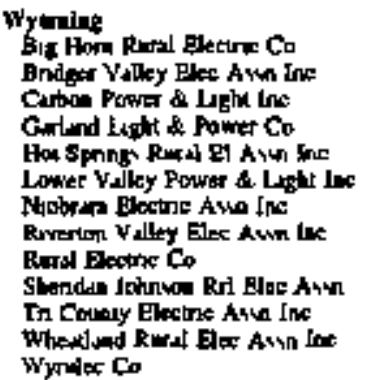 & $\begin{array}{l}z \\
= \\
= \\
= \\
= \\
= \\
=\end{array}$ & $\begin{array}{r}68,190 \\
44,549 \\
32,999 \\
17,400 \\
251,966 \\
- \\
53,550 \\
173,790 \\
187,234 \\
38,994 \\
1,161,800 \\
90,490 \\
138,450\end{array}$ & $\begin{array}{r}2,527,737 \\
2,460,958 \\
3,300,090 \\
734,944 \\
9,139,697 \\
399,272 \\
2,019,502 \\
6,624,845 \\
7,624,648 \\
2,406,549 \\
4,781,951 \\
1,566,362 \\
5,085,210\end{array}$ & $\begin{array}{l}{ }^{68} \\
= \\
- \\
18.999 \\
= \\
= \\
= \\
-\quad 1\end{array}$ & $\begin{array}{l}{ }^{4.417} \\
= \\
= \\
452.354 \\
= \\
= \\
= \\
=\quad 9\end{array}$ & $\begin{array}{r}68,259 \\
84,255 \\
82,599 \\
17,400 \\
251,986 \\
73,097 \\
53,530 \\
173,799 \\
183,234 \\
28,394 \\
1,161,800 \\
99,498 \\
138,451\end{array}$ & 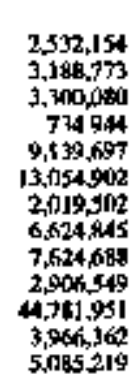 \\
\hline
\end{tabular}

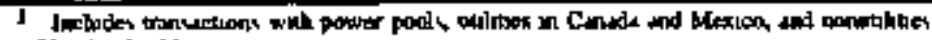

Nox Appliculie

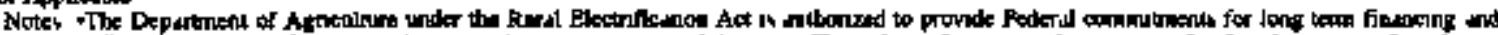

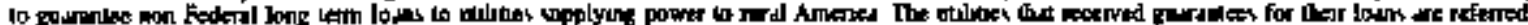

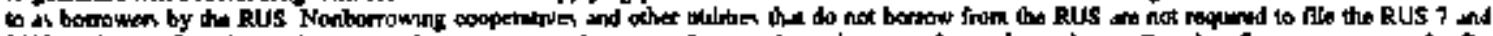

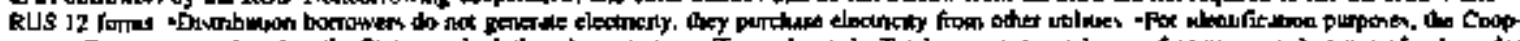

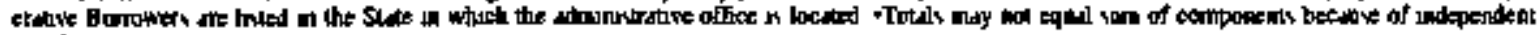
roouding

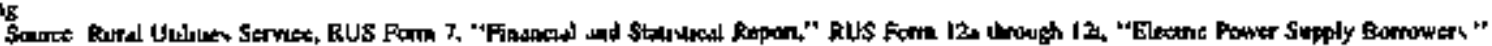

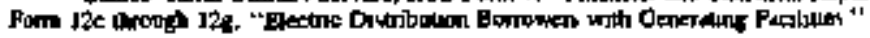


Table 35. Electricity Purchases by Cooperative Utilithes Not Reported to the Rurnl Utilithes Service, by State, 1994

\begin{tabular}{|c|c|c|c|c|c|c|c|}
\hline \multirow{2}{*}{$\begin{array}{l}\text { State } \\
\text { Ualdy }\end{array}$} & \multicolumn{7}{|c|}{ 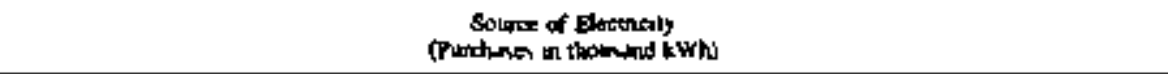 } \\
\hline & Iowertor & Fedaral & $\begin{array}{l}\text { Stur and } \\
\text { Other } \\
\text { Gowerwant }\end{array}$ & Mabiofil & Cosperstave & Other I & Tocat \\
\hline 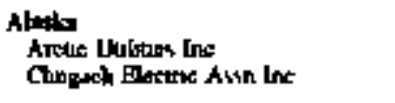 & $\overline{-}$ & $-5,84$ & $\overline{-}$ & $-5,144$ & $\overline{13}+214$ & 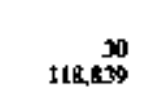 & 357.064 \\
\hline 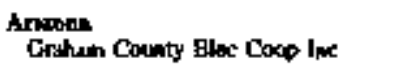 & - & - & - & - & 101033 & - & Int \\
\hline $\begin{array}{l}\text { Fland } \\
\text { Le Coonty Electine Coop Inc }\end{array}$ & - & - & - & - & 2213,69 & - & $2,2013,54 \mathrm{~B}$ \\
\hline 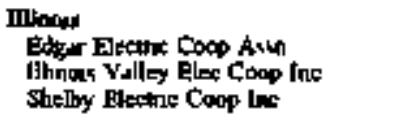 & $\bar{z}$ & $\bar{z}$ & $\bar{z}$ & $\bar{z}$ & $\begin{array}{l}54,554 \\
75,135 \\
195,734\end{array}$ & $\bar{z}$ & $\begin{array}{r}69,554 \\
75,135 \\
195,734\end{array}$ \\
\hline 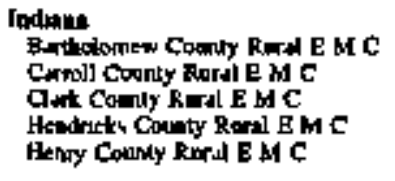 & $\begin{array}{l}\bar{z} \\
\bar{z} \\
-\end{array}$ & $\begin{array}{l}\bar{z} \\
\bar{z}\end{array}$ & $\begin{array}{l}\bar{z} \\
= \\
\overline{-}\end{array}$ & $\begin{array}{l}\bar{z} \\
\bar{z}\end{array}$ & $\begin{array}{l}149,59 \\
129,373 \\
223,267 \\
271,826 \\
143,110\end{array}$ & $\begin{array}{l}\bar{z} \\
\bar{z}\end{array}$ & $\begin{array}{l}199,543 \\
129,73 \\
209,267 \\
271,806 \\
143,100\end{array}$ \\
\hline 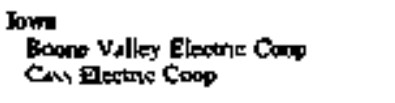 & $\overline{-}$ & $\overline{-}$ & $\bar{z}$ & $\overline{-}$ & $5,8 \pi 7$ & $\bar{z}$ & 5,873 \\
\hline 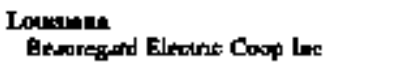 & - & - & - & - & 500,243 & - & 507,247 \\
\hline 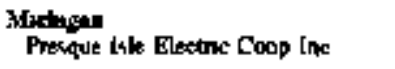 & - & - & - & $=$ & $195,4 \pi$ & - & 195470 \\
\hline 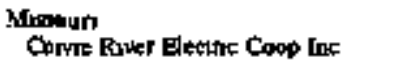 & - & - & - & - & 57275 & - & $572,27 s$ \\
\hline 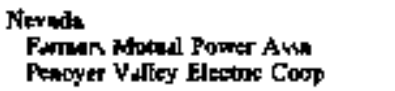 & $\overline{-}$ & $\bar{z}$ & - & $\overline{-}$ & $--^{1,260}$ & $=$ & 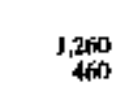 \\
\hline $\begin{array}{l}\text { Ote. } \\
\text { Lutong Kwal Edecture Ine } \\
\text { TnCounty Rorl Elec Coop Ine }\end{array}$ & $\underline{-}$ & $\bar{z}$ & $=$ & $\overline{-}$ & $\begin{array}{c}258,235 \\
52,510\end{array}$ & $=$ & $\begin{array}{r}258,235 \\
92,5017\end{array}$ \\
\hline 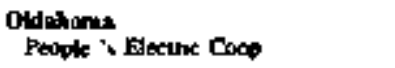 & $=$ & - & - & - & 256507 & - & 25697 \\
\hline $\begin{array}{l}\text { Pa:anylvoun } \\
\text { Nem Emitpme R B C Inc }\end{array}$ & - & - & - & - & 12,599 & - & 42.539 \\
\hline 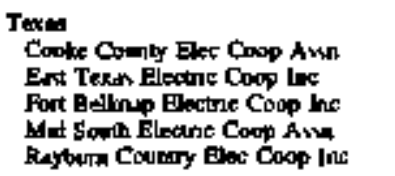 & $\begin{array}{l}\overline{456541} \\
\overline{-} \\
1,299,892\end{array}$ & $\begin{array}{l}\bar{z} \\
\bar{z} \\
2901031\end{array}$ & $\begin{array}{l}- \\
= \\
=\end{array}$ & $\begin{array}{l}\bar{z} \\
\bar{z}\end{array}$ & 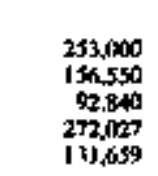 & $\begin{array}{l}\overline{201503} \\
= \\
=\end{array}$ & 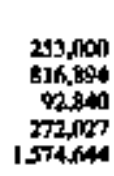 \\
\hline 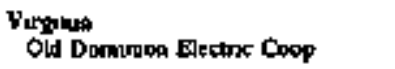 & $5,3701,4 \$ 2$ & - & - & Jis & - & 1,069 & $3,51, E R$ \\
\hline
\end{tabular}

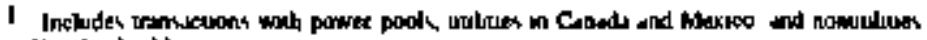

-Noi Apolicible

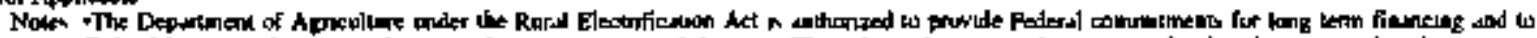

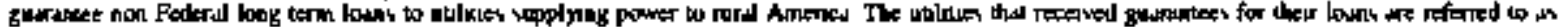

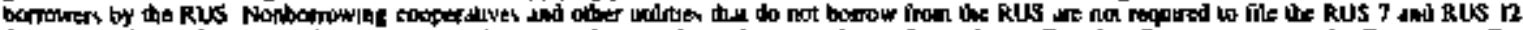

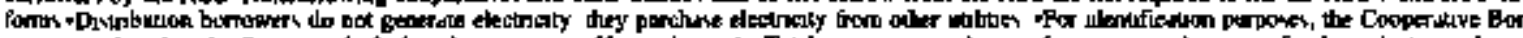

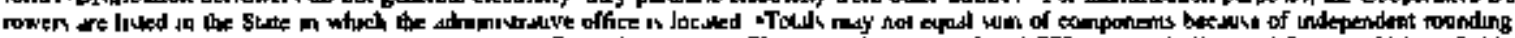

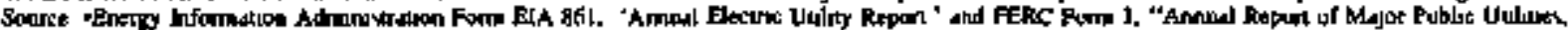

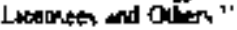


Table 36. Electricity Exchanges and Wheeling by Cooperattve Utillities, by State, 1994 (Thousand Kilowathours)

\begin{tabular}{|c|c|c|c|c|}
\hline \multirow{2}{*}{ State I } & \multicolumn{2}{|c|}{ Exchatyon } & \multicolumn{2}{|c|}{ Wheting } \\
\hline & 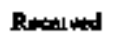 & 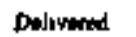 & Rexwived & Delareriod \\
\hline Alabara & $m$ & $=$ & 94,698 & 94,433 \\
\hline 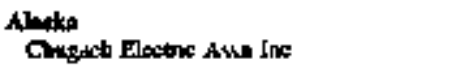 & 73,094 & 79,210 & $133,8 \pi$ & 123877 \\
\hline 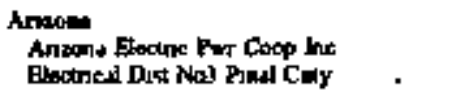 & $\begin{aligned} 49,090 \\
4.700\end{aligned}$ & $\begin{array}{r}33,096 \\
4,914\end{array}$ & $\frac{60,030}{-}$ & $\frac{9.67]}{-}$ \\
\hline 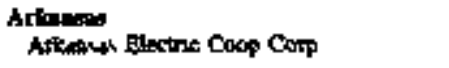 & $20,0,012$ & $2 R, 932$ & - & - \\
\hline 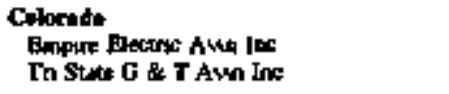 & $1, \overline{7,764,465}$ & $1 . \overline{65.2155}$ & 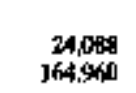 & $\begin{array}{r}23,519 \\
157.591\end{array}$ \\
\hline 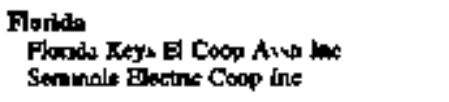 & $\overline{\mid 4,95]}$ & $\overline{18,9190}$ & $\begin{array}{r}538,072 \\
4,599\end{array}$ & 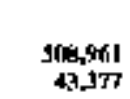 \\
\hline Idaln Dord Eleterc Co & - & - & $1,97\}$ & 1331 \\
\hline 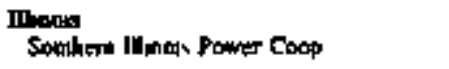 & 302015 & 257517 & - & - \\
\hline 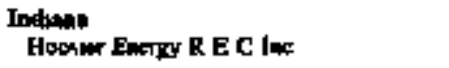 & 65,773 & 83,938 & $23 n_{1}, 282$ & 209,884 \\
\hline 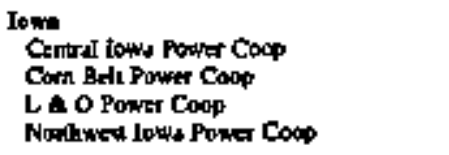 & $\begin{array}{c}1,679,424 \\
41,427 \\
151,217\end{array}$ & $\begin{array}{c}1,582,719 \\
10,616 \\
\frac{-}{146,349}\end{array}$ & $\frac{-}{99,913}$ & $\begin{array}{l}- \\
\overline{96,534} \\
318,144\end{array}$ \\
\hline 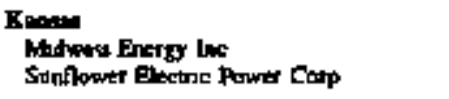 & -5 & 1.183 & $\overline{m i n}_{2 \pi 2}$ & $\overline{\pi j 2} 262$ \\
\hline 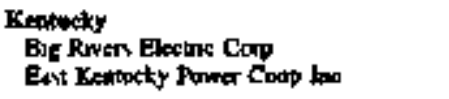 & $\begin{array}{r}17,005 \\
183,192\end{array}$ & $\begin{array}{l}48,946 \\
187,746\end{array}$ & $\begin{array}{l}1,664,231 \\
1,640,746\end{array}$ & $\begin{array}{l}1,669,231 \\
1.643 .746\end{array}$ \\
\hline $\begin{array}{l}\text { Mrineselat } \\
\text { Unded Pown Assn }\end{array}$ & 6,878 & 56,45: & 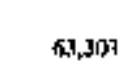 & 51.347 \\
\hline 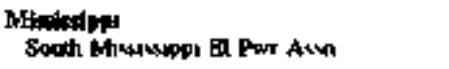 & 15,111 & 22,619 & 597,090 & $579, \pi / 83$ \\
\hline 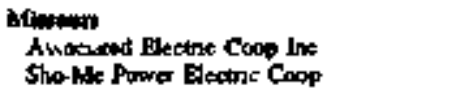 & 176,105 & 74,675 & $\begin{array}{l}701,645 \\
112,692\end{array}$ & $\begin{array}{l}648,010 \\
112582\end{array}$ \\
\hline Son Rewer Electre Coop lne & - & - & $3, \sqrt{19}$ & 3,110 \\
\hline 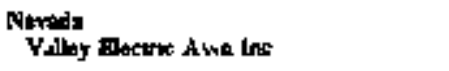 & 20,0103 & 17.253 & - & - \\
\hline $\begin{array}{l}\text { New Mrextes } \\
\text { Pland Ble: GenkTram Coop Inc }\end{array}$ & $19.7 m 2$ & 76,536 & - & - \\
\hline 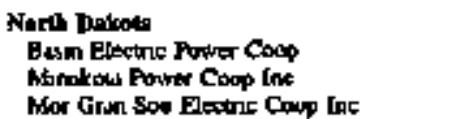 & $\begin{array}{l}\text { gtofis } \\
86,67\end{array}$ & $\overline{167549}$ & 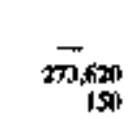 & $\overline{255.720}$ \\
\hline
\end{tabular}

Sot moth ad faxmotes at ced of thile 
Table 36. Ejectricity Erchanges and Wheeling by Cooperative Utilities, by State, 1994 (Thoustand Kilowanthours) (Continaten)

\begin{tabular}{|c|c|c|c|c|}
\hline \multirow{2}{*}{ 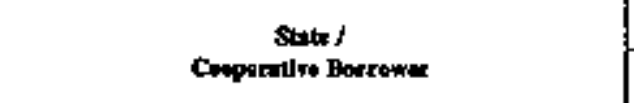 } & \multicolumn{2}{|c|}{ Exchanges } & \multicolumn{2}{|c|}{ Wheding } \\
\hline & Rexcived & Detivened & Received & Delisomad \\
\hline \multicolumn{5}{|l|}{ 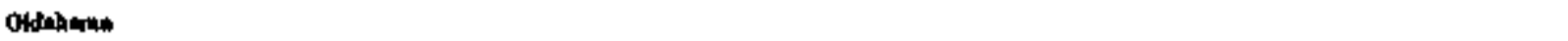 } \\
\hline 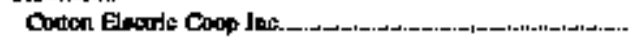 & $m$ & - & 6,110 & 6,103 \\
\hline 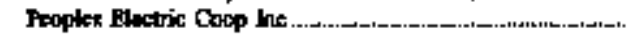 & - & - & 44,509 & 44,509 \\
\hline 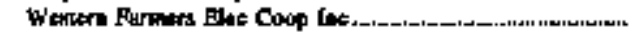 & 34,969 & 35,469 & - & - \\
\hline \multicolumn{5}{|l|}{ South Doiknt: } \\
\hline 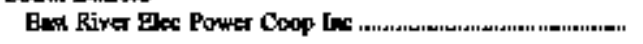 & - & - & $9+2634$ & 320.941 \\
\hline \multicolumn{5}{|l|}{$T=0$} \\
\hline 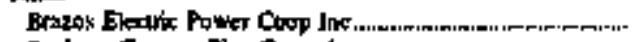 & $20,455,218$ & 20.430 .146 & 180,66 & 176,195 \\
\hline Rrybume Couny Ekx Cocp. the & 159,945 & I9R,491 & - & - \\
\hline 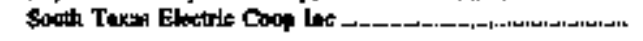 & - & 2810079 & - & 3,356 \\
\hline \multicolumn{5}{|l|}{ Uidh } \\
\hline Deveret Cenerotion te Tren Coop & 12,754 & 34,842 & \llcorner & - \\
\hline 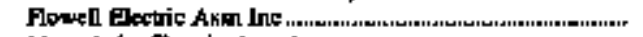 & 3,398 & 3,895 & - & - \\
\hline Broog Liłe Elentric Aan Ine ............................................ & 879 & $8 \times 9$ & - & $\rightarrow$ \\
\hline \multicolumn{5}{|l|}{ Yirdale } \\
\hline 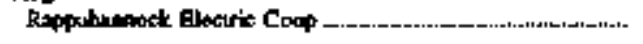 & - & - & $3.9: 7$ & 3.944 \\
\hline \multicolumn{5}{|l|}{ Wyouin: } \\
\hline 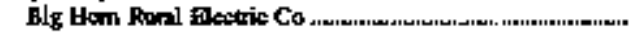 & - & - & $4 \mathbf{4 6}$ & 9.0052 \\
\hline
\end{tabular}

-Not Applicabla

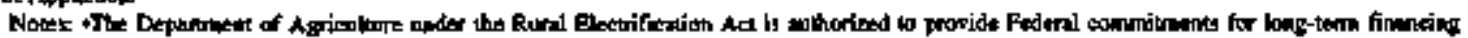

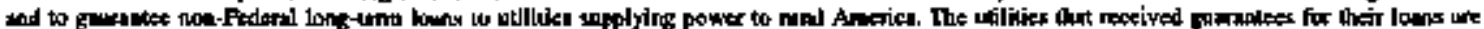

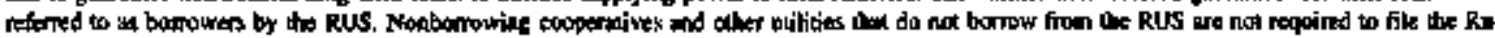

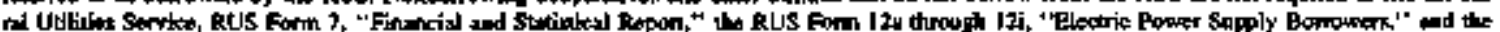

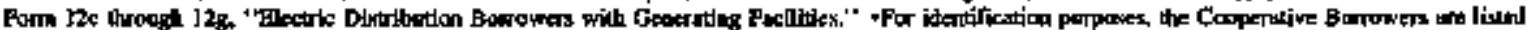

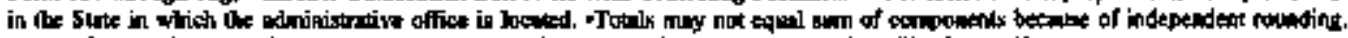

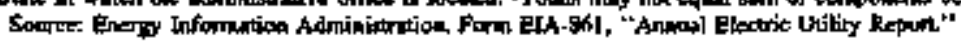




\section{Selected Information on the Physical Transmission System}

\section{Overvlew}

This chapter presents data on selected portions of the physical electrical system that investor- and publicly owned clectric utilities report on FERC Fom I, "Annual Report of Major Electric Udilitjes, Licensets and Others," and Form EJA-412, "Annual Report of Public Electric Utilities." Data on cooperative utilities cannot be provided because the filings submitted to the Retral Electification Adrinistration are not inclu. sive of the sare information rectuested from the other ownership classes. Summary totals (in NERC regions) of these collected dats are presented for the first rime. Figure 18 highlights the high voltage transmission system in the United States.

\section{Tables}

This chapter presents summary (by NERC Region) and individual statistics on voltage of transmissjop lines 138 kilovolts and above for both alternating and djrect cutrent lines. ${ }^{8}$ The data are shown in the categories of overhead and underground/submarine lines for both structural and circuit miles for the following types of utilities.

- Investot-owned electric utitities (Tables 37 and 38)

- Federal power authorities (Tables 39 and 40 )

- State and other government utilities (Tables 39 and 40)

- Municipalities (Tables 39 and 40).

Transmission lines added and watthour/line transformer information are not collected from public utilities. Fiscal-year ending dates for eacle public utility differ and are not jacluded in the tables. Transmission line data are, therefore, not necessarily for the same time period.

Tables 41 and 42 provide summary and detailed information on the transinission tines added by investor- owned electric utilities in 1994. Information is presented for lines 138 kilovolis and above for alteruating eurrent No direct current lines were added to 1994. In addition, tine cost information is presented and broken into the categories of land, structural, con. ductors and supporting equipment, and the total. Table 4I aggregates the line type by mileage ranges associated with the consuruetion andior removal of the transmission lines. Public urilities are not required to file this information on the Form EIA-412.

Negative nambers in the rables represed the removal of transmission mileage from the physical electrical system. Some negative numbers in the cost information represent removal of the cost of those lines and equipment from the ratebase. Upgrades to conductors and other equipment for transmission lines currently in operation in the transmission system may be shows without an associated line tength. Summary and delaj]ed information on line transiormer and watthout meter totals are provided by investor-owned electric utilities (Tables 43 and 44). This information is broken into the following categories: number of line transformers present at the beginning of the year; additions during the year; reductions during the year; total in use by customers; totel in use by the electric company; and summary information on what is kept in stack, locked at the customer's site, and in an inactive status. The line transformer capacity in megavoltanperes is also presented by the same categories.

\section{Summary}

Many other types of electrical equipment used in support for operating the electrical grid are not singled out for collection nor are the relationships of megayoltampere, reactive power, and megawatts highlighted. The information shown in this chapter does not deseribe the operation of the physical electrical system nor the costing of its services. Borh cost allocation and how engineering operations are linked to the contract trade of electricity are issues that are in the forefront for the changing electric power industry. 
Figure 18. Selected High-Voltage Transmission LInes in the Contlguous United States

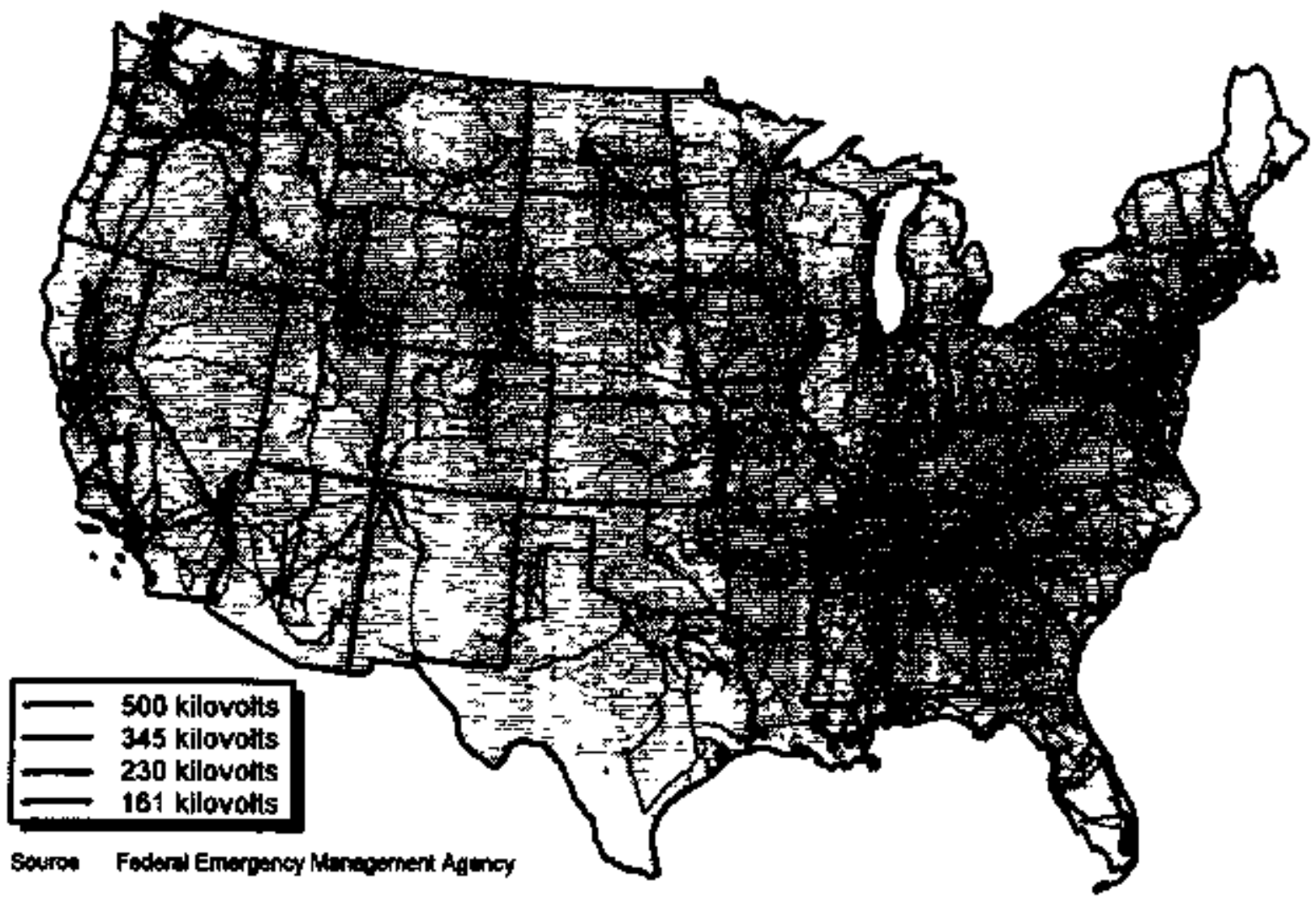


Table 37. Transmission Lime Statistics of Inveslar-Owned UtHities, by NERC Region, 1994

\begin{tabular}{|c|c|c|c|c|}
\hline \multirow{2}{*}{$\begin{array}{l}\text { Voltege of } \\
\text { Temoriftion } \\
\text { Line }\end{array}$} & \multicolumn{2}{|c|}{ Orefibead } & \multicolumn{2}{|c|}{$\begin{array}{l}\text { Uniergound } \\
\text { Submerine }\end{array}$} \\
\hline & $\begin{array}{l}\text { Strocturl } \\
\text { Miliss }\end{array}$ & $\begin{array}{l}\text { Circait } \\
\text { Mitum }\end{array}$ & $\begin{array}{c}\text { Streand } \\
\text { Millen }\end{array}$ & $\begin{array}{l}\text { Cirseit } \\
\text { Miflest }\end{array}$ \\
\hline \multirow{3}{*}{ 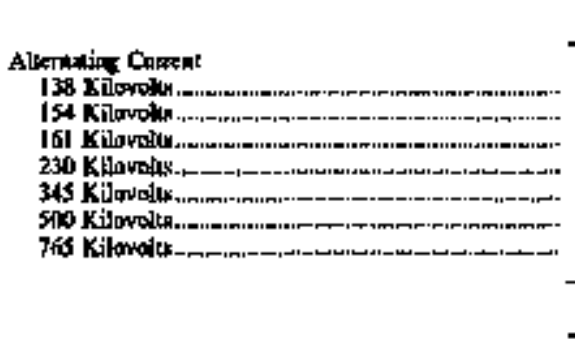 } & \multicolumn{4}{|c|}{ Invertor-Owped Vtritiks in ECAR } \\
\hline & $\begin{array}{r}10,840 \\
564 \\
819 \\
7,910 \\
1,011 \\
2,100\end{array}$ & $\begin{array}{r}24,315 \\
264 \\
564 \\
1,060 \\
12,903 \\
1,011 \\
2,020\end{array}$ & $\begin{array}{l}\quad 69 \\
- \\
- \\
-\end{array}$ & $\begin{array}{l}\text { 66 } \\
- \\
- \\
-\end{array}$ \\
\hline & \multicolumn{4}{|c|}{ 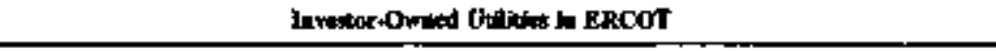 } \\
\hline \multirow[t]{2}{*}{ 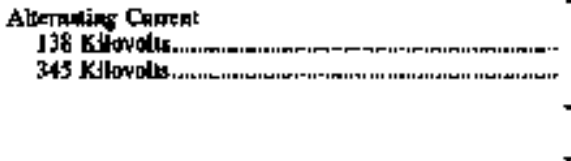 } & $\begin{array}{r}11,082 \\
4,190\end{array}$ & $\begin{array}{c}13,129 \\
7,817\end{array}$ & $\begin{array}{l}34 \\
15\end{array}$ & $\begin{array}{l}67 \\
15\end{array}$ \\
\hline & \multicolumn{4}{|c|}{ 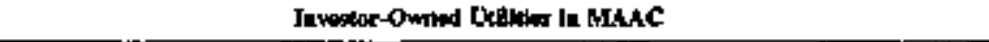 } \\
\hline \multirow[t]{2}{*}{ 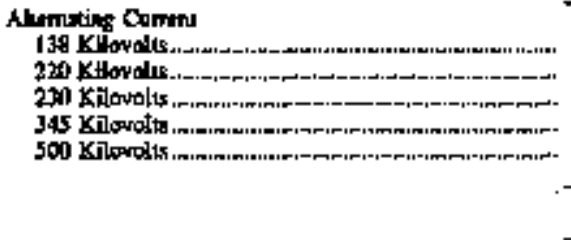 } & $\begin{array}{r}846 \\
472 \\
3,411 \\
153 \\
1,669\end{array}$ & $\begin{array}{r}1,6790 \\
570 \\
4512 \\
159 \\
1,689\end{array}$ & $\begin{array}{r}180 \\
6 \\
152 \\
-\quad 17\end{array}$ & $\begin{array}{r}208 \\
6 \\
204 \\
-\quad 17\end{array}$ \\
\hline & \multicolumn{4}{|c|}{ Inweslox-0wned Denldes in MALIN } \\
\hline \multirow[t]{2}{*}{ 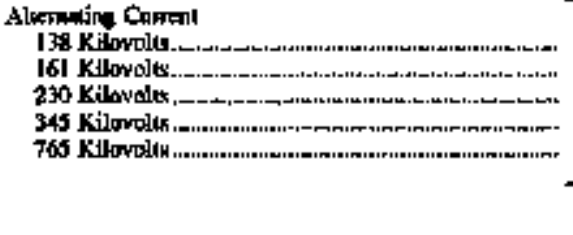 } & $\begin{array}{r}4122 \\
827 \\
190 \\
4,121 \\
91\end{array}$ & $\begin{array}{r}10,524 \\
833 \\
261 \\
541 \\
901\end{array}$ & $\begin{array}{l}-284 \\
- \\
-\end{array}$ & $\begin{array}{l}-295 \\
- \\
-\end{array}$ \\
\hline & \multicolumn{4}{|c|}{ 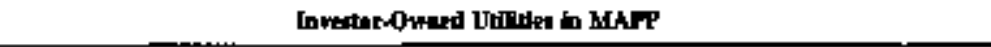 } \\
\hline \multirow[t]{2}{*}{ 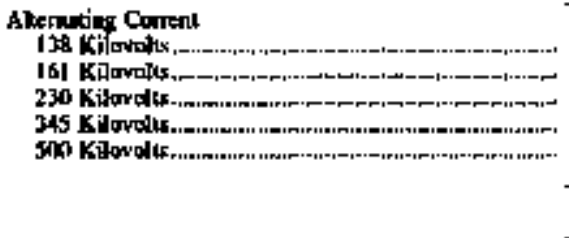 } & $\begin{array}{r}6 \\
3,042 \\
1,531 \\
2,318 \\
273\end{array}$ & $\begin{array}{r}6 \\
3,265 \\
1,555 \\
2,487 \\
273\end{array}$ & $\begin{array}{l}- \\
- \\
-\end{array}$ & $\begin{array}{l}- \\
- \\
-\end{array}$ \\
\hline & \multicolumn{4}{|c|}{ Invedor-0mmed Utilits Im NaCC } \\
\hline \multirow[t]{2}{*}{ 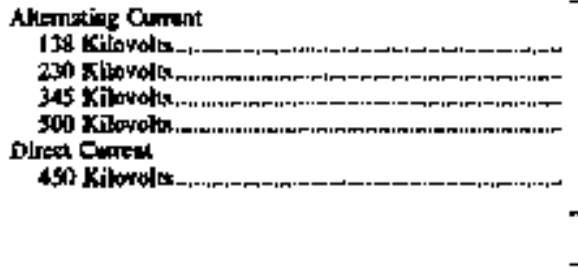 } & $\begin{array}{r}314 \\
9.362 \\
3.289 \\
10 \\
434\end{array}$ & $\begin{array}{r}548 \\
5,018 \\
5906 \\
50 \\
434\end{array}$ & $\begin{array}{r}\begin{array}{r}304 \\
6 \\
121\end{array} \\
-\quad\end{array}$ & $\begin{array}{r}47 \\
-\quad \begin{array}{r}62 \\
-\end{array}\end{array}$ \\
\hline & \multicolumn{4}{|c|}{ 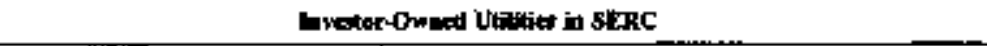 } \\
\hline \multirow[t]{2}{*}{ 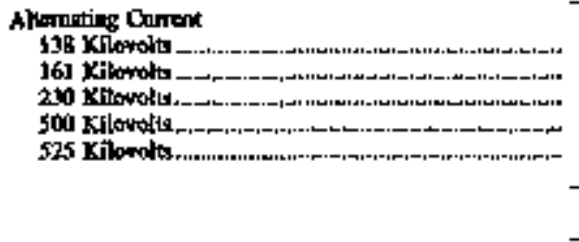 } & $\begin{array}{r}2,974 \\
390 \\
14,596 \\
0,061 \\
535\end{array}$ & $\begin{array}{r}2,476 \\
427 \\
18,357 \\
1062 \\
379\end{array}$ & $\begin{array}{l}19 \\
-\quad 61 \\
-\end{array}$ & 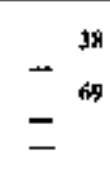 \\
\hline & \multicolumn{4}{|c|}{ 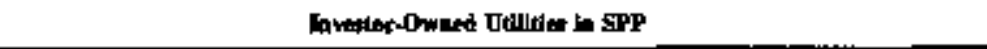 } \\
\hline 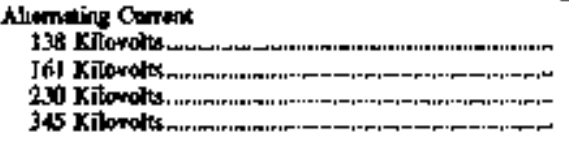 & $\begin{array}{l}7,453 \\
3.777 \\
4,216 \\
3.798\end{array}$ & $\begin{array}{l}3,394 \\
3,364 \\
4,707 \\
4,035\end{array}$ & $\begin{array}{r}43 \\
10 \\
-\quad 48 \\
\end{array}$ & $\begin{array}{r}47 \\
10 \\
-\quad 48\end{array}$ \\
\hline
\end{tabular}

See poles and foctorotes wa and af lable. 
Table 37. Transmission Line Statistics of Investor-Owned Utilities, by NERC Region, 1994 (Continued)

\begin{tabular}{|c|c|c|c|c|}
\hline \multirow{2}{*}{ 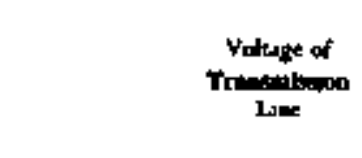 } & \multicolumn{2}{|c|}{ Onoliend } & \multicolumn{2}{|c|}{ 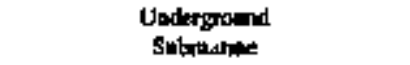 } \\
\hline & $\begin{array}{l}\text { Stroeaural } \\
\text { Milex }\end{array}$ & $\begin{array}{l}\text { Cutwal } \\
\text { Aflats }\end{array}$ & $\begin{array}{c}\text { Suncenrd } \\
\text { Meles }\end{array}$ & $\begin{array}{l}\text { Graurt } \\
\text { Maln }\end{array}$ \\
\hline & \multicolumn{4}{|c|}{ 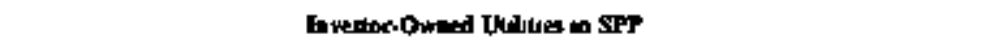 } \\
\hline \multirow[t]{2}{*}{ 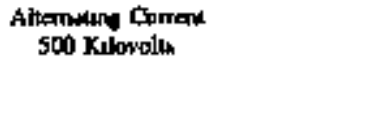 } & 2,076 & 2331 & - & - \\
\hline & \multicolumn{4}{|c|}{ 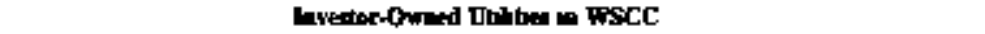 } \\
\hline 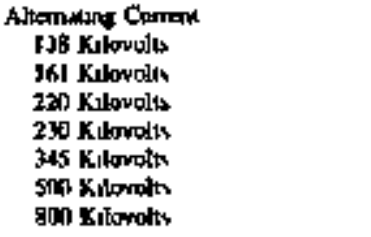 & $\begin{array}{r}3,800 \\
1,700 \\
2,707 \\
13275 \\
7,207 \\
6,1 \times 0 \\
313\end{array}$ & $\begin{array}{r}1,100 \\
1,039 \\
1,481 \\
16,9192 \\
1290 \\
7549 \\
113\end{array}$ & $\begin{array}{l}- \\
= \\
= \\
=\end{array}$ & $\begin{array}{l}= \\
= \\
= \\
=\end{array}$ \\
\hline gon Xitomolts & \multicolumn{4}{|c|}{ Coolpgoos US. Tut } \\
\hline 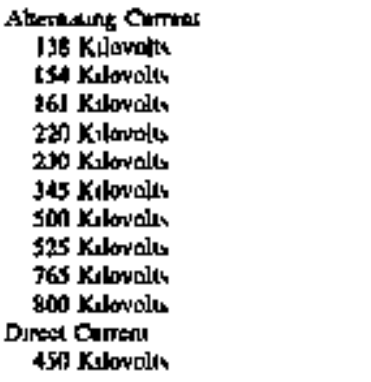 & $\begin{array}{r}52640 \\
66 \\
10,307 \\
3,179 \\
42,815 \\
2,678 \\
15,169 \\
575 \\
2.167 \\
.113 \\
414\end{array}$ & $\begin{array}{r}65,567 \\
264 \\
10 \mathrm{T72} \\
4053 \\
52365 \\
4858 \\
16,825 \\
575 \\
2,110 \\
113 \\
14\end{array}$ & $\begin{array}{r}957 \\
-\quad 19 \\
67 \\
3164 \\
= \\
= \\
-\end{array}$ & $\begin{array}{l}1,149 \\
-\quad 11 \\
\\
387 \\
- \\
235 \\
- \\
- \\
-\end{array}$ \\
\hline
\end{tabular}

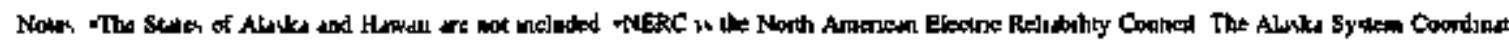

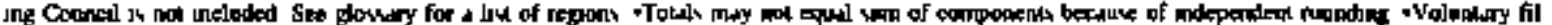

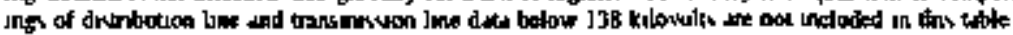

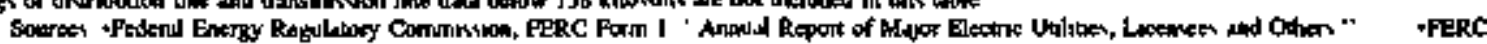

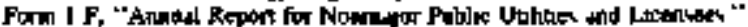


Table 38. Transmission Lime Statsilics of Investor-Owned Utilities, 1994

\begin{tabular}{|c|c|c|c|c|c|}
\hline \multirow{2}{*}{ 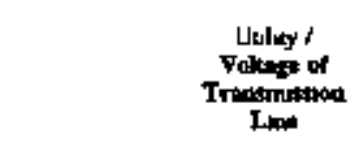 } & \multicolumn{2}{|c|}{ Orertaced } & \multicolumn{3}{|c|}{ 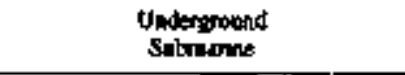 } \\
\hline & $\begin{array}{l}\text { Suroctint } \\
\text { Mila }\end{array}$ & $\begin{array}{l}\text { Cyreal } \\
\text { Blite }\end{array}$ & $\begin{array}{l}\text { Structurpl } \\
\text { Mhlon }\end{array}$ & Erewal & \\
\hline \multicolumn{6}{|l|}{$\begin{array}{l}\text { Aldbeme Power Compeny } \\
\text { Alkero, bing Coment }\end{array}$} \\
\hline IGI Kilovdlu & 325 & $72 T$ & - & - & \\
\hline 270 Xdowols & 1,342 & 1,347 & - & - & \\
\hline $300 \mathrm{~K}$ dovolis & 315 & 315 & - & - & \\
\hline \multicolumn{6}{|l|}{ 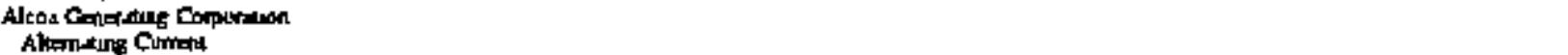 } \\
\hline I34 Kurvolits & 2 & 2 & - & - & \\
\hline \multirow{2}{*}{\multicolumn{6}{|c|}{ 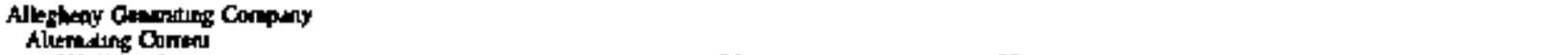 }} \\
\hline & & & & & \\
\hline \multirow{2}{*}{\multicolumn{6}{|c|}{ 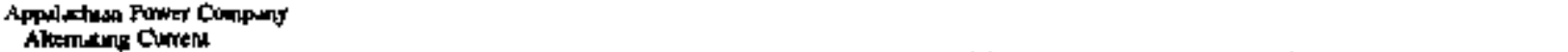 }} \\
\hline & & & & & \\
\hline 138 Kilovolu & 2045 & 3,192 & $\mathbf{I}$ & & ! \\
\hline 230 KNowdes & 16 & 91 & - & - & \\
\hline 345 Kulondith & 285 & 581 & - & - & \\
\hline SPD Kallords & & 95 & - & - & \\
\hline $7 \% 5$ Kinoudis & 64I & 64) & - & - & \\
\hline \multirow{2}{*}{\multicolumn{6}{|c|}{$\begin{array}{l}\text { Anucond Pobluc Service Cooupdiny } \\
\text { Alkinuting Comenl }\end{array}$}} \\
\hline & & & & & 14 \\
\hline 345 Kilunelis & 566 & 1,156 & - & - & \\
\hline 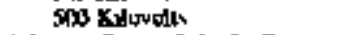 & 572 & 151 & - & - & \\
\hline \multirow{2}{*}{\multicolumn{6}{|c|}{ 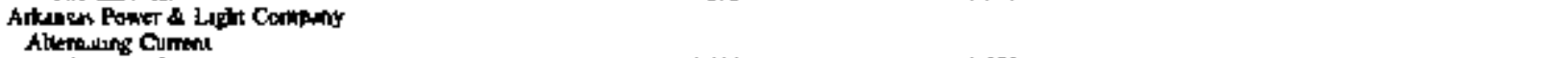 }} \\
\hline & & & & & \\
\hline 161 Kikodis & 1.411 & 1,459 & - & - & \\
\hline $230 \mathrm{~K} u$ ordis & IŞ9 & 258 & - & - & \\
\hline its Kulovdik & 44 & 44 & - & - & \\
\hline $\operatorname{son} \mathrm{K} L \mathrm{kavdl}$ : & 制 & 893 & - & - & \\
\hline \multirow{2}{*}{\multicolumn{6}{|c|}{$\begin{array}{l}\text { Alvux Cuy Eletne Company } \\
\text { Aluemeng Curment }\end{array}$}} \\
\hline & & & & & \\
\hline I 1 Xodoults & $a n$ & 301 & - & - & \\
\hline $230 \mathrm{XI}$ onolts & 127 & 133 & - & - & \\
\hline S00 Kulavolts & 24 & 24 & $\boldsymbol{\sigma}$ & - & \\
\hline \multirow{2}{*}{\multicolumn{6}{|c|}{ Balumore Gas \& Eleteine Co }} \\
\hline & & & & & \\
\hline 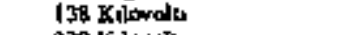 & 14 & 14 & $=$ & - & \\
\hline 230 Xulovoth & 179 & 305 & if & & 32 \\
\hline 500 Kulowdis & 205 & as & - & - & \\
\hline \multirow{2}{*}{\multicolumn{6}{|c|}{ Buts Hilk Corperathen }} \\
\hline 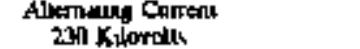 & & & & & \\
\hline \multirow{2}{*}{\multicolumn{6}{|c|}{ 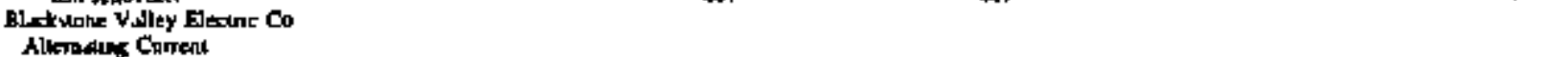 }} \\
\hline & & & & & \\
\hline 345 Kilowdic & 21 & 21 & - & - & \\
\hline 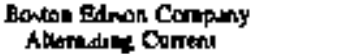 & & & & & \\
\hline 230 Kitonds & ן & 36 & - & - & \\
\hline 3.45 Xulovolh & IP6 & 115 & 21 & & 2] \\
\hline Coralin Powar \& Ludut Cornowny & & & & & \\
\hline 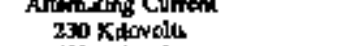 & 2.764 & 2910 & - & - & \\
\hline stop Kitavelis & 292 & $2 \$ 2$ & - & - & \\
\hline Ceatral Hwon Gas \& Elet Crop & & & & & \\
\hline 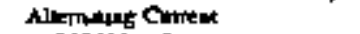 & & & & & \\
\hline 345 Xolaruts & 76 & 76 & - & - & \\
\hline Contral Illows Laths Compuny & & & & & \\
\hline Alwhong Comen & & & & & \\
\hline 138 Xilovolin & 226 & $\$ 77$ & - & - & \\
\hline 3dS Xolovolus & 47 & 83 & - & - & \\
\hline Cedtell Mlaver Pub Sery Co & & & & & \\
\hline Allatinthe Crimest & & & & & \\
\hline IJB Kulavoln & 1,472 & 0.474 & $=$ & - & \\
\hline IfI KDlorots & 5t & $5 B$ & - & - & \\
\hline 230 Kulowotts & 4 & 48 & - & - & \\
\hline 3ts Kalloyehts & 290 & 290 & - & - & \\
\hline 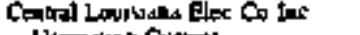 & & & & & \\
\hline Altemalus Cumpri & & & & & \\
\hline I38 KLbsadik & 622 & 674 & - & - & \\
\hline 230 Kitlondels & 442 & 458 & - & - & \\
\hline Sho Kulovalu & 63 & 67 & - & - & \\
\hline
\end{tabular}

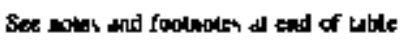


Table 38. Transatission Line Statistics of Investor-Owned Utlities, 1994 (Continued)

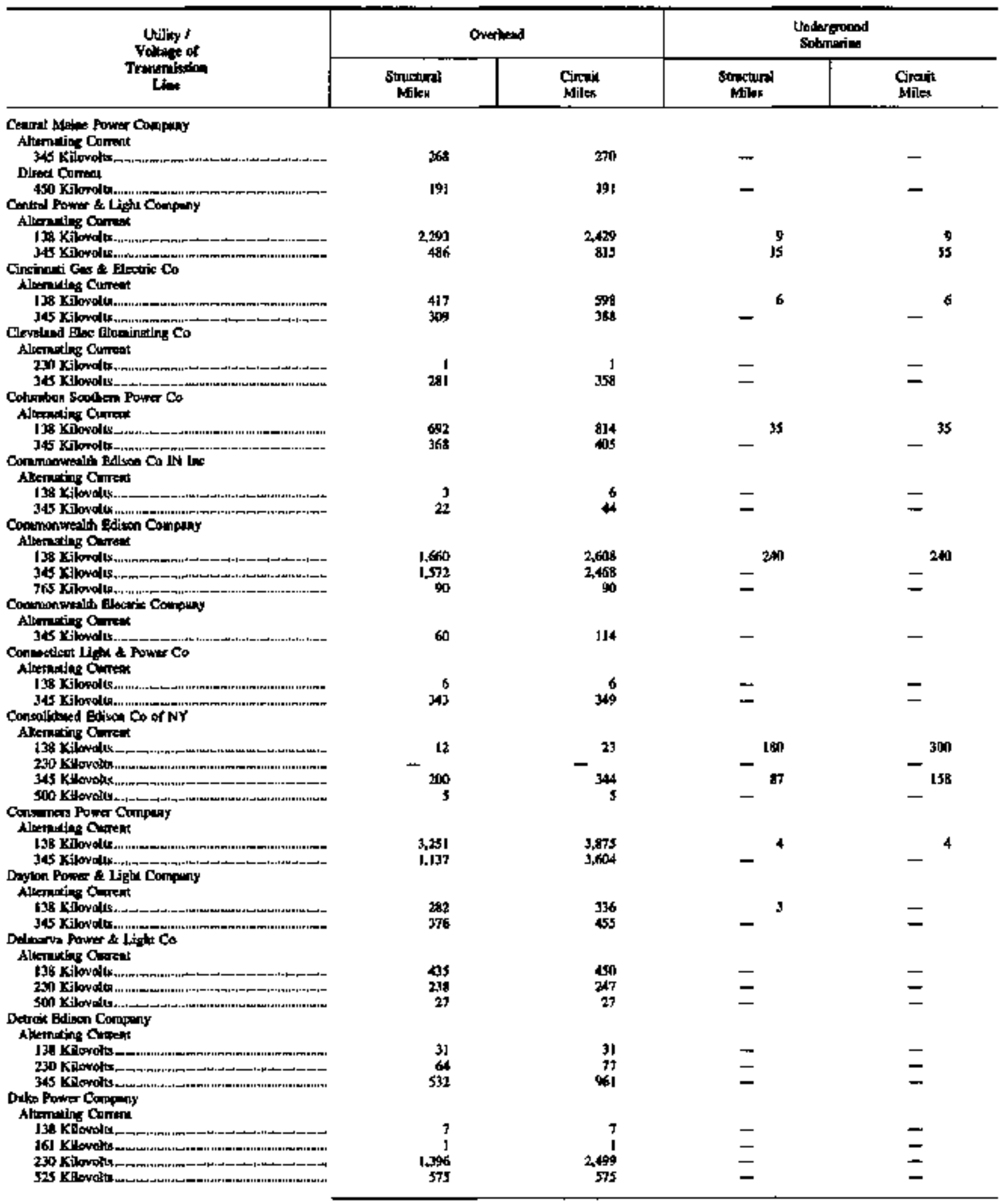

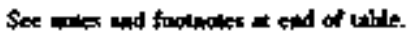


Table 38. Transinission Line Stotislies of Investor.Owned Ulilities, 1994 (Continned)

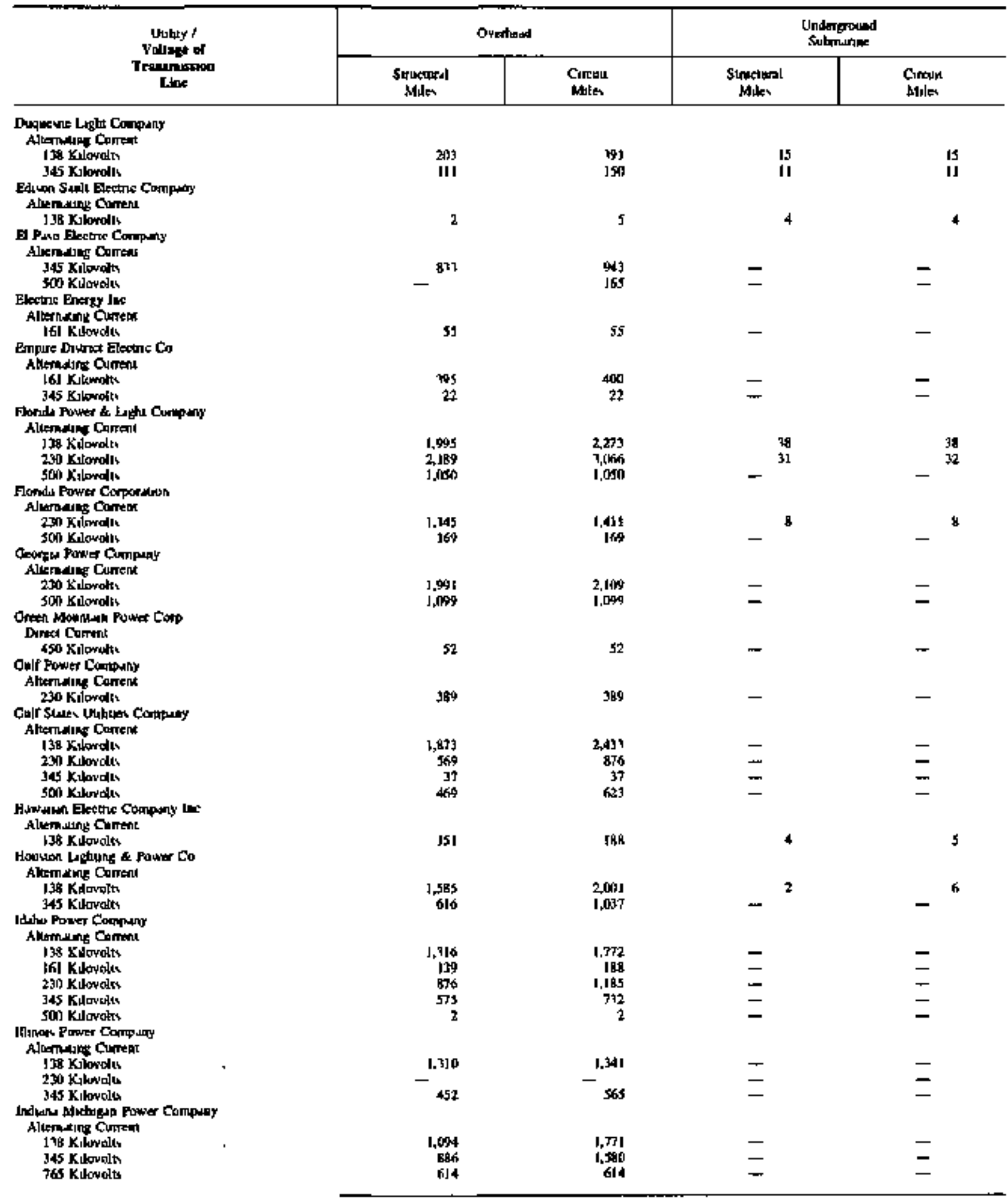

Sect notes ind foonders an end of twite 
Table 38. Transmission Line Statistios of Investor-Owned Utilities, 1994 (Continued)

\begin{tabular}{|c|c|c|c|c|}
\hline \multirow{2}{*}{ 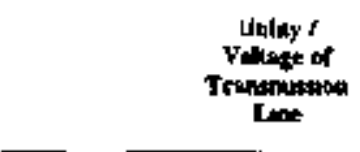 } & \multicolumn{2}{|c|}{ Dverilesd } & \multicolumn{2}{|c|}{$\begin{array}{l}\text { Underprousd } \\
\text { Selmentive }\end{array}$} \\
\hline & 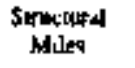 & $\begin{array}{l}\text { Cintan } \\
\text { Mives }\end{array}$ & $\begin{array}{l}\text { Sirtidural } \\
\text { Nitun }\end{array}$ & $\begin{array}{c}\text { Curcul } \\
\text { Mrles }\end{array}$ \\
\hline \multicolumn{5}{|l|}{ Indinnd Kentucky Eearse Corp } \\
\hline $\begin{array}{l}138 \text { Kalowdis } \\
245 \text { Kilondis }\end{array}$ & 3 & 2 & - & - \\
\hline \multirow{2}{*}{\multicolumn{5}{|c|}{ 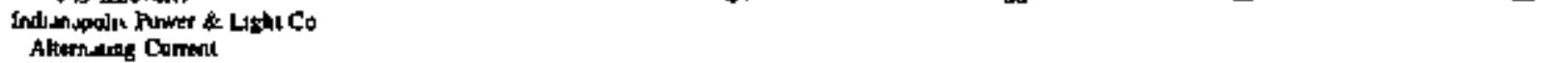 }} \\
\hline & & & & \\
\hline IHA Kidarolt & jis & 399 & - & - \\
\hline J45 Kilavolts & 440 & 475 & - & - \\
\hline Iatertutic Power Company & & & & \\
\hline $\begin{array}{l}\text { Allendunger Cumept } \\
\text { Ifi Kilowdis }\end{array}$ & 317 & तI & - & - \\
\hline $\begin{array}{l}\text { Ifil Kilowds } \\
345 \text { Kilowals }\end{array}$ & 135 & j1s & - & $\bar{z}$ \\
\hline \multirow{2}{*}{\multicolumn{5}{|c|}{$\begin{array}{l}\text { bowe blunos On \& Elex Co } \\
\text { Alinadues Cument }\end{array}$}} \\
\hline & & & & \\
\hline I6] Kuloweds & 34 & 181 & - & - \\
\hline 145 Kelovolus & 309 & $3 \times 5$ & - & - \\
\hline \\
\hline $\begin{array}{l}\text { Alsmang Conent } \\
161 \text { Kofovolts }\end{array}$ & 6318 & m & - & - \\
\hline 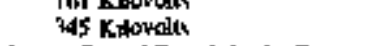 & 241 & 201 & - & $=$ \\
\hline \multirow{2}{*}{\multicolumn{5}{|c|}{ 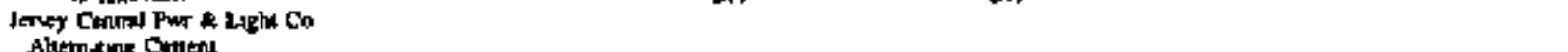 }} \\
\hline Aletmengs Domens & & & & \\
\hline 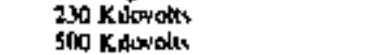 & 401 & ث10 & - & - \\
\hline \multirow{2}{*}{\multicolumn{5}{|c|}{ Kansas Cuy Pouser \& Lught Co }} \\
\hline & & & & \\
\hline I6I Kworolts & 832 & BA4 & in & \\
\hline J45 Kavolk & 294 & 297 & - & - \\
\hline \multirow{2}{*}{\multicolumn{5}{|c|}{$\begin{array}{l}\text { Kensan Oas \& Electinc Compuny } \\
\text { Alkematug Otwent }\end{array}$}} \\
\hline & & & & \\
\hline 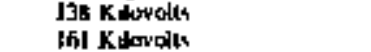 & $\begin{array}{l}462 \\
177\end{array}$ & $\begin{array}{l}511 \\
178\end{array}$ & $\overline{-}$ & $\overline{-}$ \\
\hline Jas Kutowalk & 6⿻一𠃋十 & 612 & - & - \\
\hline \multirow{2}{*}{\multicolumn{5}{|c|}{ Kenkinky Power Coenping }} \\
\hline & & & & \\
\hline 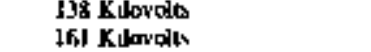 & 4 & 278 & - & - \\
\hline & 8 & 80 & $\overline{-}$ & $=$ \\
\hline 765 Kalonodh & 258 & 258 & $\bar{z}$ & $\bar{z}$ \\
\hline \multirow{2}{*}{\multicolumn{5}{|c|}{ Kenoocky Undeite Conpany }} \\
\hline Alkendung Cuntem & & & & \\
\hline 138 silowolu & 861 & 861 & - & - \\
\hline 16! Xilprodts & 518 & sth & - & - \\
\hline 345 Kularolks & 394 & 46 & - & - \\
\hline Solo Kelordts & 57 & 57 & - & - \\
\hline \multicolumn{5}{|l|}{$\begin{array}{l}\text { Xingypor Powes Compdny } \\
\text { Alkriading Corrent }\end{array}$} \\
\hline $\begin{array}{l}\text { Allorading Curreat } \\
158 \mathrm{~K} \text { lowolls }\end{array}$ & 22 & 43 & - & - \\
\hline \multirow{2}{*}{\multicolumn{5}{|c|}{ 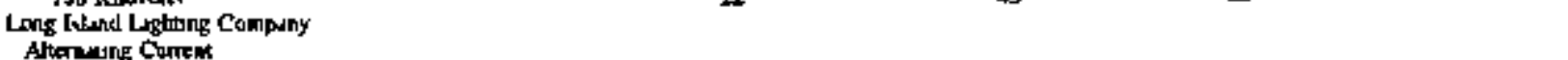 }} \\
\hline Alteraing Curew & & & & \\
\hline 138 Klomols & $2: 5$ & 223 & 121 & 12 \\
\hline 345 Kilonds & - & - & a & \\
\hline \multicolumn{5}{|l|}{ Loolused Power \& lugk Co } \\
\hline $\begin{array}{l}\text { AHeremag Oimem } \\
\text { I38 Kilomolk }\end{array}$ & 15 & I5 & - & - \\
\hline $\begin{array}{l}\text { I38 Kilomolk } \\
230 \text { Kulorolk }\end{array}$ & 487 & $\$ 2$ & 48 & -4 \\
\hline 345 Keforots & 17 & 17 & - & - \\
\hline 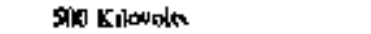 & 242 & 213 & - & - \\
\hline \multirow{2}{*}{\multicolumn{5}{|c|}{ 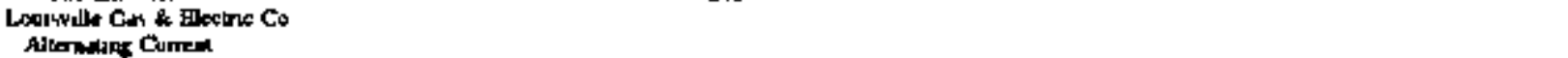 }} \\
\hline & & & & \\
\hline 138 Kilomols & 248 & 365 & I & \\
\hline 15s X1/ardits & 66 & 264 & - & - \\
\hline 34S Kilowolts & 102 & 197 & - & - \\
\hline 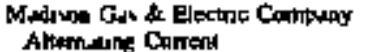 & & & & \\
\hline $\begin{array}{l}\text { Altomsing Conment } \\
\text { IIs Kilownitis }\end{array}$ & ו & 82 & 7 & \\
\hline 345 Kolorokts & 116 & 124 & - & - \\
\hline
\end{tabular}

Soe nown and footucles an end of table 
Table 38. Transmission Line Statistics of Investor-Owned Utilities, 1994 (Continued)

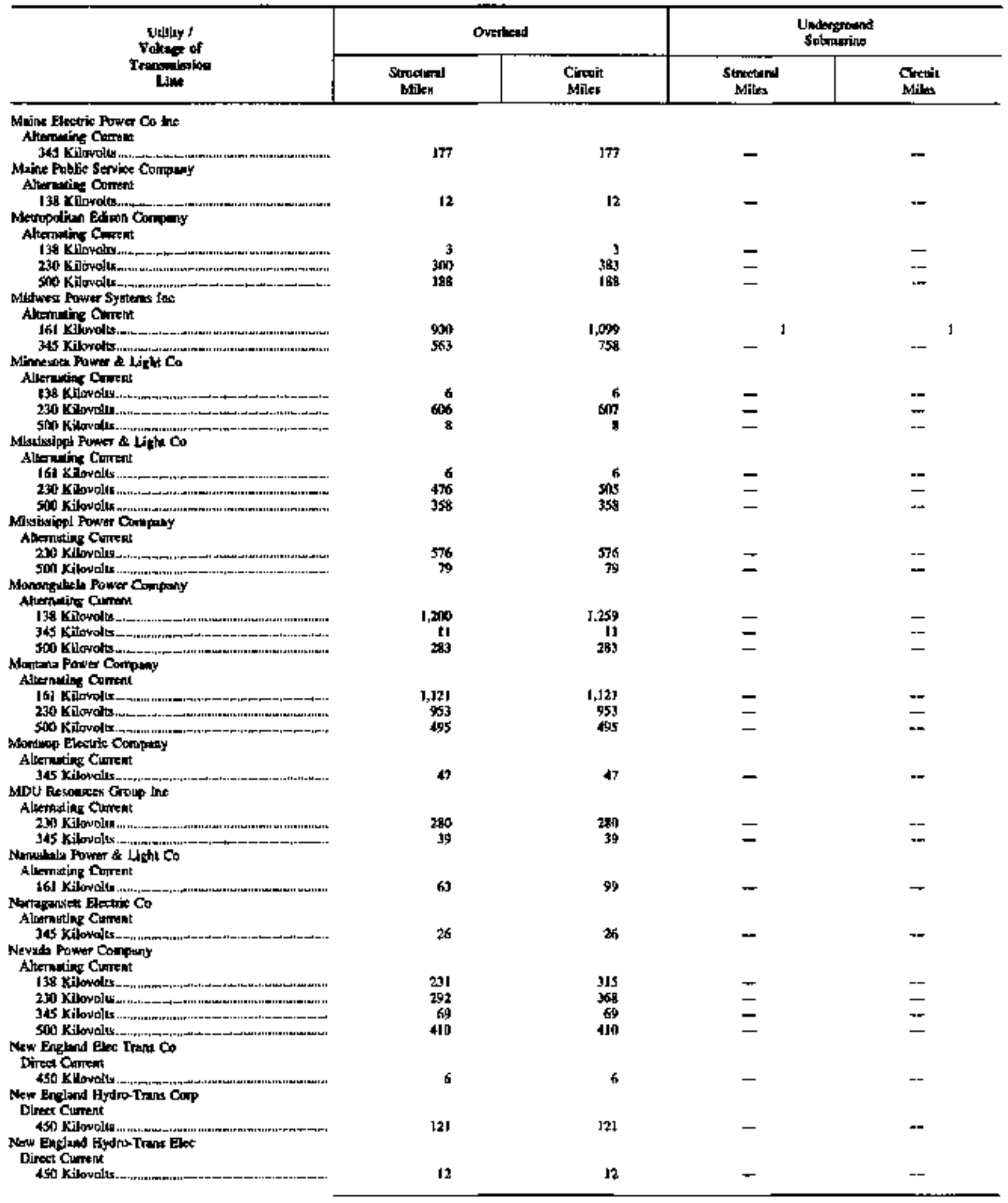

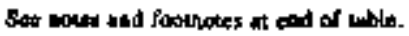


Table 38. Transmisston Line Statistics of Investor-Omed Utilities, 1944 (Conthmed)

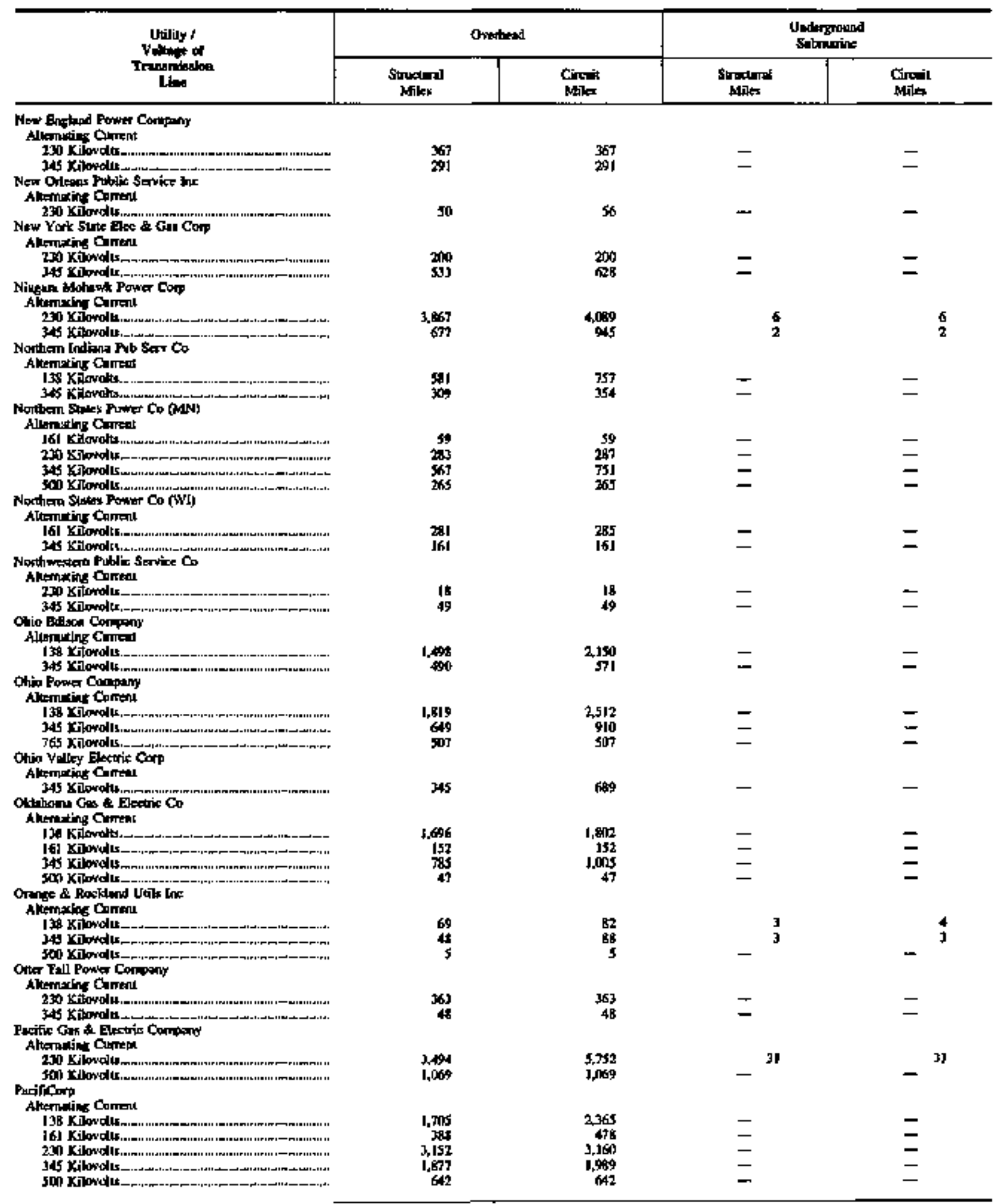

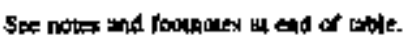


Table 38. Transontisslon Line \$tatistics of Investor-Owned Utllties, 1994 (Continuned)

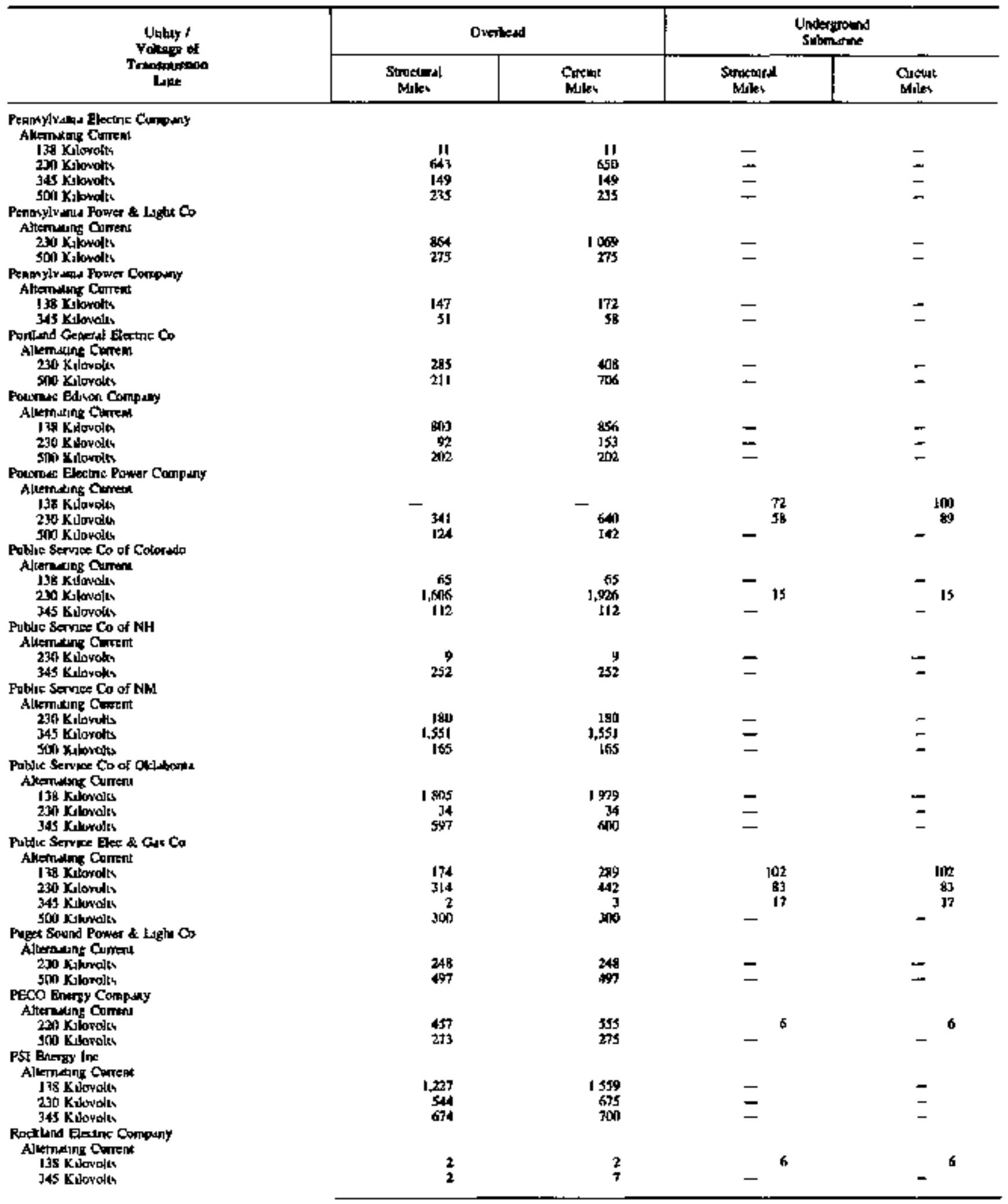

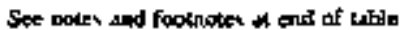


Table 38. Transmisstion Line Stalistics of Investor-Owned Utilities, 1994 (Continued)

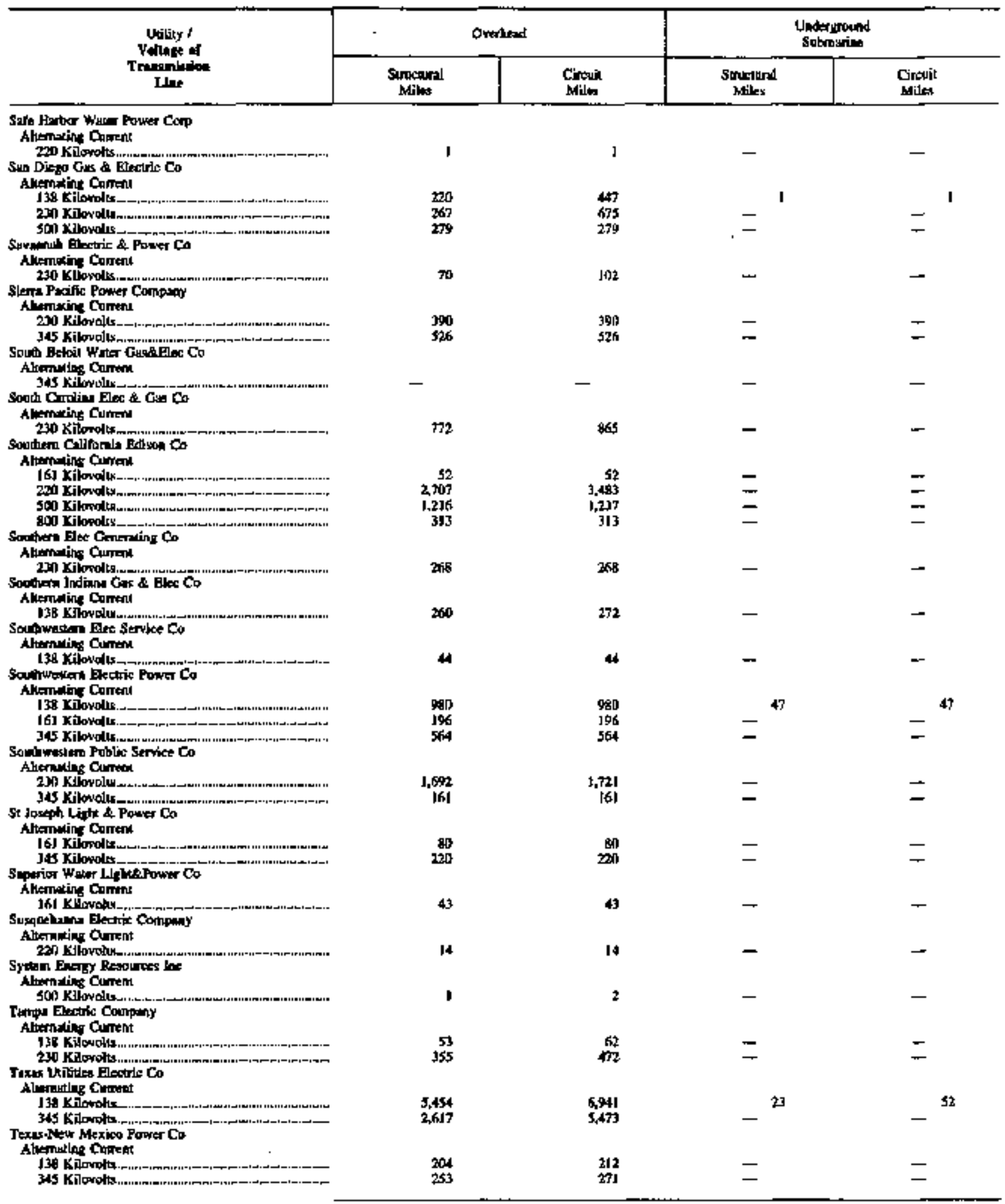

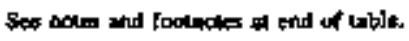


Table 38. Transmission Line Statistics of Iavestor-Owned Utilities, 1994 (Conlinued)

\begin{tabular}{|c|c|c|c|c|c|}
\hline \multirow{2}{*}{ 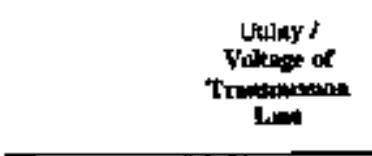 } & \multicolumn{2}{|c|}{ Antidid } & \multicolumn{3}{|c|}{$\begin{array}{l}\text { Uedutgroand } \\
\text { Subremente }\end{array}$} \\
\hline & $\begin{array}{c}\text { Strecturs } \\
\text { Malts }\end{array}$ & $\begin{array}{l}\text { Crewert } \\
\text { Malev }\end{array}$ & $\begin{array}{c}\text { Stroctural } \\
\text { Abtos }\end{array}$ & $\begin{array}{l}\text { CApप्या } \\
\text { Mtiles }\end{array}$ & \\
\hline \multicolumn{6}{|l|}{ 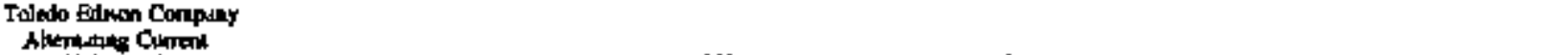 } \\
\hline $13 t$ Kiforolim & 589 & sis & $\sigma$ & - & \\
\hline $\begin{array}{l}345 \text { Kilorolth } \\
\text { Tuewon Elextinc Pawer Compuny }\end{array}$ & 134 & $15 \%$ & - & - & \\
\hline \multirow{2}{*}{\multicolumn{6}{|c|}{ Tuewon Flextinc Pawer Compiny }} \\
\hline & & ul & & & \\
\hline $\begin{array}{l}\text { 138 Killovalis } \\
345 \text { Ktowedis }\end{array}$ & ind & 34] & - & - & \\
\hline 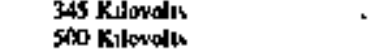 & 1,098 & $1,12.2$ & - & - & \\
\hline \multirow{2}{*}{\multicolumn{6}{|c|}{ 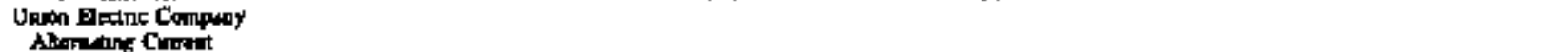 }} \\
\hline & & & & & \\
\hline 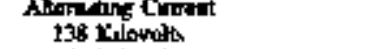 & 990 & 1,110 & - & - & \\
\hline $161 \mathrm{Kiforda}$ & 313 & 310 & $\bar{z}$ & 二 & \\
\hline 230 Kulowolts & 9n & 90 & - & - & \\
\hline 365 Kilowolts & 727 & 898 & - & - & \\
\hline \multirow{2}{*}{\multicolumn{6}{|c|}{ 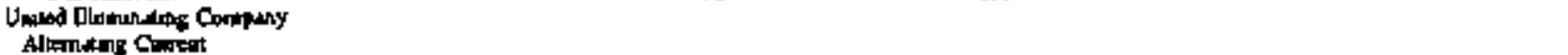 }} \\
\hline & & & & & \\
\hline \multirow{2}{*}{\multicolumn{6}{|c|}{ 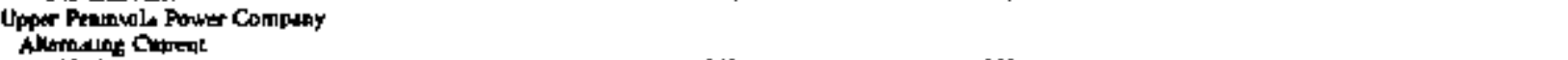 }} \\
\hline & & & & & \\
\hline 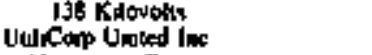 & 348 & 354 & - & - & \\
\hline 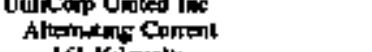 & & & & & \\
\hline Ifil Kilamols & 576 & 4010 & - & - & \\
\hline 345 Kowolts & 58 & 58 & - & - & \\
\hline \multicolumn{6}{|l|}{ Yatbod Electpe Poner Compiny } \\
\hline \multicolumn{6}{|l|}{ 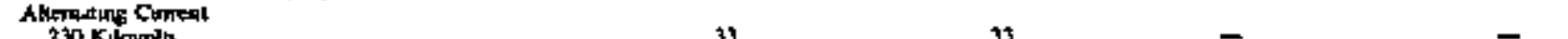 } \\
\hline $\begin{array}{l}230 \text { Kikgundk } \\
345 \text { Kulovolh }\end{array}$ & 92 & 52 & $=$ & $=$ & \\
\hline \multirow{2}{*}{\multicolumn{5}{|c|}{ 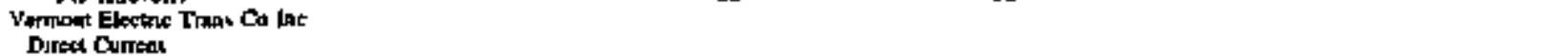 }} & \\
\hline & & & & & \\
\hline 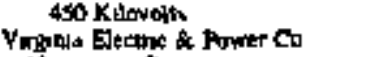 & 92 & 51 & - & - & \\
\hline \multicolumn{6}{|l|}{ 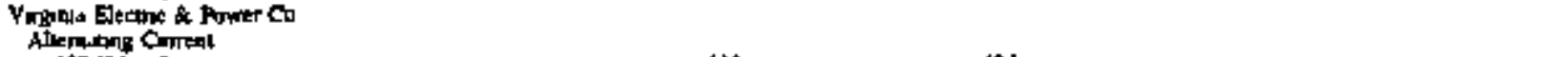 } \\
\hline IJB Kulovolts & 119 & 194 & - & - & \\
\hline 23 [l] Kullovols & $1,6 \mathrm{nN?}$ & 2,611 & 32 & & $2 \%$ \\
\hline \multirow{3}{*}{\multicolumn{6}{|c|}{ 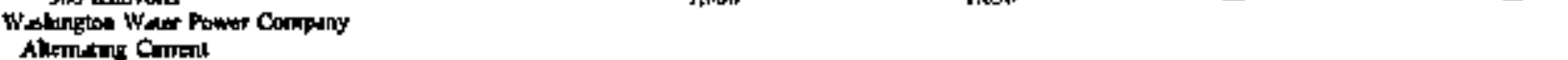 }} \\
\hline & & & & & \\
\hline & & & & & \\
\hline 230 Kilorols & 538 & SA5 & - & - & \\
\hline \multicolumn{6}{|l|}{ Weal Pim Prope Compaly } \\
\hline IJ kutorolu. & 1,108 & 1,346 & - & - & \\
\hline 200 Bilonolis & 72 & $\eta$ & - & - & \\
\hline 345 Kilonolis & 6 & h & - & - & \\
\hline S600 Kollomolss & 273 & 273 & - & - & \\
\hline \multirow{2}{*}{\multicolumn{6}{|c|}{ 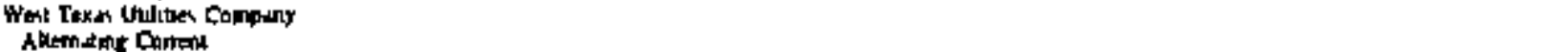 }} \\
\hline & & & & & \\
\hline IX8 Kilonolis & $1, \leqslant / 1 / 2$ & 1.502 & - & - & \\
\hline 340 Kulowols & 221 & 21 & - & - & \\
\hline 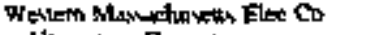 & & & & & \\
\hline Alleplang Cाrent & & & & & \\
\hline 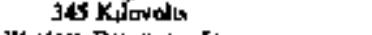 & Jos & 105 & - & - & \\
\hline Watern Revolutes Ine & & & & & \\
\hline Aloerndugf Cumens & & & & & \\
\hline $161 \mathrm{Kuluresh}$ & 152 & 152 & - & - & \\
\hline 230 Kilorath & 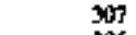 & M? & - & - & \\
\hline 345 Kikovolin & 336 & आ8 & $\leftarrow$ & - & \\
\hline Wheclung Power Condpathy & & & & & \\
\hline Altemanding Curreat & & & & & \\
\hline I38 Killorolts & 99 & jos & - & - & \\
\hline 345 Kdiwodk & 9 & 16 & - & $=$ & \\
\hline 500 Kilovols & 16 & 16 & - & - & \\
\hline Wjoponvin Eloctinc Pown Cu & & & & & \\
\hline Alomining oumeat & & & & & \\
\hline 139 KNovolk & 1,103 & 1,350 & 4 & & 52 \\
\hline 230 KNorolk & 52 & 123 & - & - & \\
\hline 345 Kslluyolts & 575 & 639 & - & - & \\
\hline
\end{tabular}

Set norel and foolnots a end of trible 
Table 38. Transmission Line Stutistics of Investor-Ormed Utllities, 1994 (Continued)

\begin{tabular}{|c|c|c|c|c|}
\hline \multirow{2}{*}{ 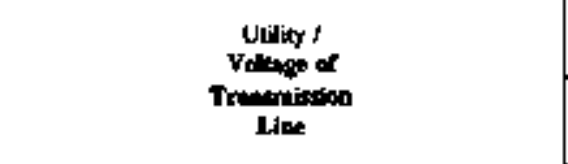 } & \multicolumn{2}{|c|}{ Onertiend } & \multicolumn{2}{|c|}{$\begin{array}{l}\text { Undepgoded } \\
\text { Subminitur }\end{array}$} \\
\hline & $\begin{array}{l}\text { Stroeumat } \\
\text { Mlles }\end{array}$ & 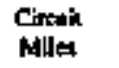 & $\begin{array}{c}\text { Sincwirll } \\
\text { Mikek }\end{array}$ & Cinedit \\
\hline \multicolumn{5}{|l|}{ 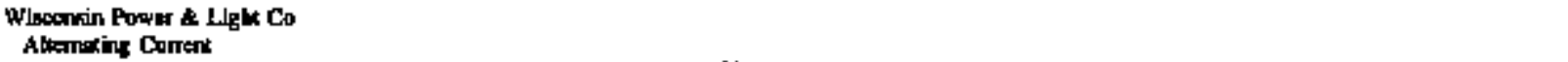 } \\
\hline I & $6 \mathbf{6}$ & 917 & - & $\sim$ \\
\hline 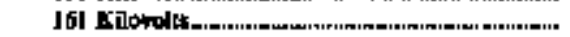 & I & 2 & 一 & - \\
\hline 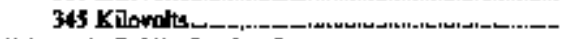 & 100 & 907 & - & - \\
\hline \multicolumn{5}{|l|}{ 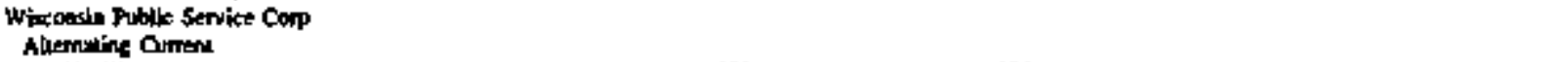 } \\
\hline 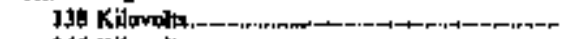 & 288 & 306 & - & - \\
\hline 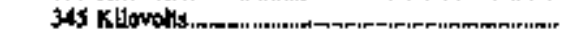 & 124 & 124 & - & $\rightarrow$ \\
\hline
\end{tabular}

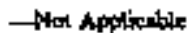

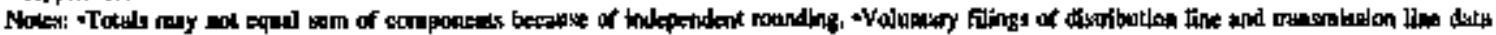

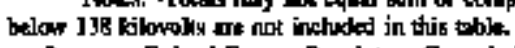

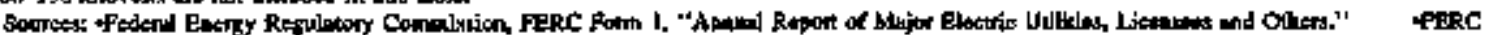

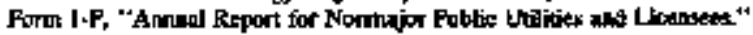


Table 39. Transmission Line Statistics of Public Utblities, by NERC Region, Fiscal Year 1994

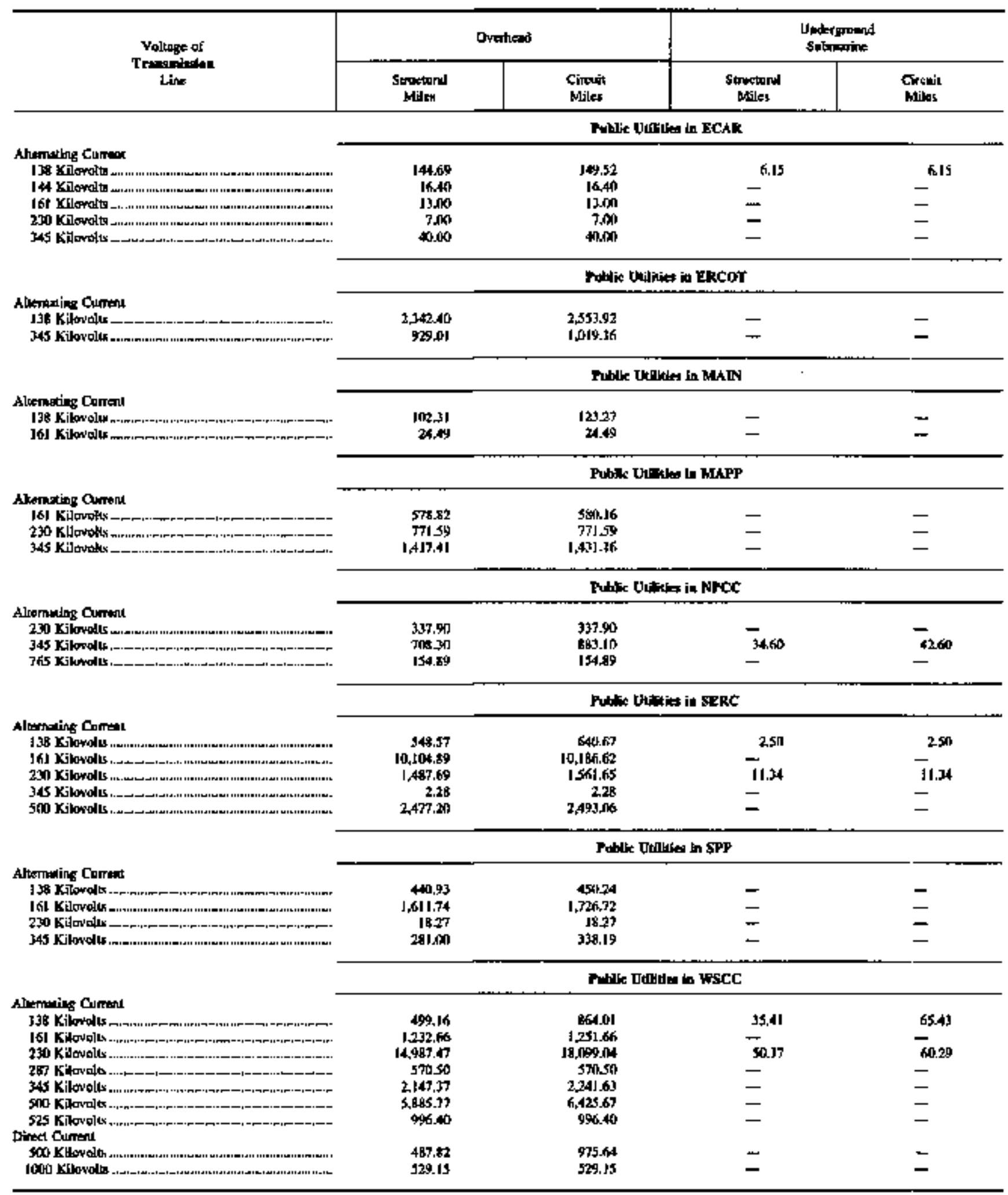

See woler and footholes at and of lokle. 
Table 39. Transunission Line Stattsties of Public Utiltites, by NERC Reglon, Fiscal Year 1994 (Conthued)

\begin{tabular}{|c|c|c|c|c|}
\hline \multirow{2}{*}{$\begin{array}{l}\text { Yoltuge of } \\
\text { Trangmition } \\
\text { ture }\end{array}$} & \multicolumn{2}{|c|}{ Overiead } & \multicolumn{2}{|c|}{$\begin{array}{l}\text { Undagoond } \\
\text { Sobmante }\end{array}$} \\
\hline & $\begin{array}{l}\text { Sorocenral } \\
\text { Males }\end{array}$ & $\begin{array}{l}\text { Cukewi } \\
\text { Malos }\end{array}$ & $\begin{array}{l}\text { Structurl } \\
\text { Ballo }\end{array}$ & $\begin{array}{l}\text { Cinswt } \\
\text { Mhles }\end{array}$ \\
\hline & \multicolumn{4}{|c|}{ 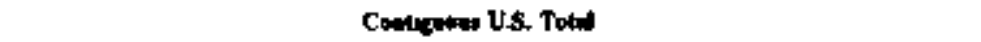 } \\
\hline \multicolumn{5}{|l|}{ Atherning Corrent } \\
\hline IJ8 Kulowolle & $4,11 \%$ D6 & 4,78163 & 4406 & $74 \mathrm{ng}$ \\
\hline 144 Kulow ollh & 160 & 1641 & - & - \\
\hline I61 Xeloralla & 13565600 & $13,782,65$ & - & - \\
\hline 230 Kelotiolls & I7ting 92 & 24,79545 & 6151 & $7+63$ \\
\hline 287 thlowalk & $\sin 50$ & 5 Tू 50 & - & - \\
\hline J45 Kulowilto & 3,52537 & 5,95552 & 3460 & 4260 \\
\hline 500 Kalondtas & $8,362,97$ & 891573 & 一 & - \\
\hline \$25 Elonoliv & 99640 & 996 to & - & - \\
\hline Th5 Kildroits & 154 & 15489 & 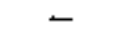 & - \\
\hline \multicolumn{5}{|l|}{ Drrest Dutent } \\
\hline SnO Kuldrotus & 4 in & gns 64 & 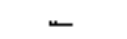 & - \\
\hline J0rה Xilonots & 52915 & 52915 & - & - \\
\hline
\end{tabular}

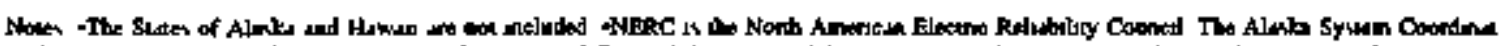

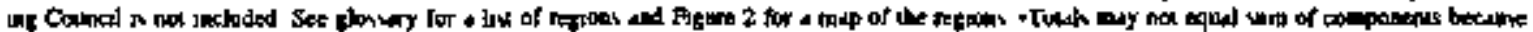

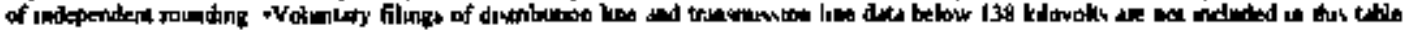

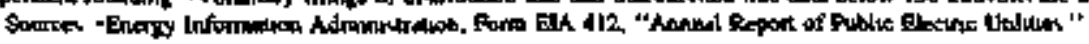


Table 4. Transmission Line Statestics of Poblic Utilities, Fiscal Year 1994

\begin{tabular}{|c|c|c|c|c|}
\hline \multirow{2}{*}{ 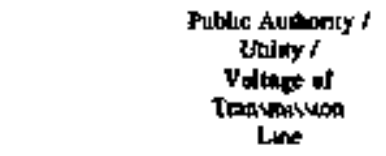 } & \multicolumn{2}{|c|}{ Orertew } & \multicolumn{2}{|c|}{$\begin{array}{l}\text { Wathrgound } \\
\text { Sobmature }\end{array}$} \\
\hline & $\begin{array}{l}\text { Stroclural } \\
\text { Mule }\end{array}$ & $\begin{array}{l}\text { Curcers } \\
\text { Mules }\end{array}$ & $\begin{array}{l}\text { Stroctual } \\
\text { Milex }\end{array}$ & $\begin{array}{c}\text { Cremer } \\
\text { Miley }\end{array}$ \\
\hline \multicolumn{5}{|l|}{ Puderol } \\
\hline \multicolumn{5}{|l|}{ Alnka Powe Adrumunumon } \\
\hline \multicolumn{5}{|l|}{ Bonnevillh Prower Adqun } \\
\hline 134 K dordis & 9230 & 92 आ & - & - \\
\hline 161 KLowdis & 46.57 & 4650 & - & - \\
\hline 230 Krtowolu & d,725 64 & 4.82614 & - & - \\
\hline 265 Kildods & 22740 & 2374 & - & - \\
\hline 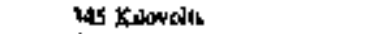 & 34360 & 42360 & - & - \\
\hline 500 Kavols & $4,328.90$ & 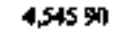 & - & - \\
\hline Drect curten & & & 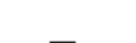 & \\
\hline \multirow{2}{*}{\multicolumn{5}{|c|}{ 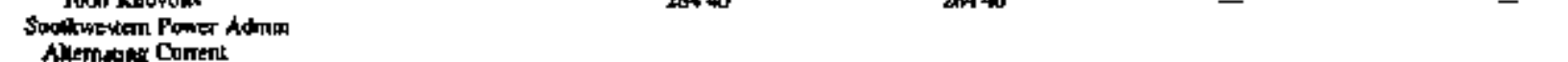 }} \\
\hline & & & & \\
\hline 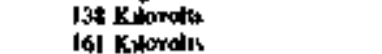 & I6A 12 & 16412 & - & $\overline{-}$ \\
\hline \multirow{2}{*}{\multicolumn{5}{|c|}{ Teansive Vulley Aublonly }} \\
\hline & & & & \\
\hline 161 Kisonels & 10,102 的 & $10,1 \pm 42$ & - & - \\
\hline 230 Kylowolth & 3007 & ชบ0 & - & - \\
\hline 345 Kiddeds & 228 & 228 & - & - \\
\hline \multirow{3}{*}{\multicolumn{5}{|c|}{ 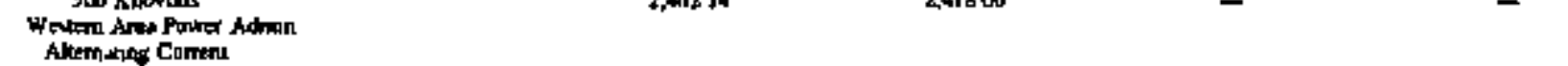 }} \\
\hline & & & & \\
\hline & & & & \\
\hline I3: Kitowols & 32742 & 65484 & - & - \\
\hline 16| K가어언ㄴ & 36925 & 86 & - & - \\
\hline 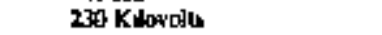 & 7,08623 & 9.12097 & - & $=$ \\
\hline 345 KAtordals & $i, 598$ & 1,61306 & - & - \\
\hline Strit Kovdu & 45034 & 45034 & - & - \\
\hline \multicolumn{5}{|l|}{ Steth and Other Coverrines } \\
\hline \multicolumn{5}{|l|}{ Alask Enoly Awhonsy } \\
\hline Alkemading Chrrenk & $\cos m \mathrm{~m}$ & 20600 & 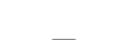 & \\
\hline \multirow{2}{*}{\multicolumn{5}{|c|}{ Callforma Dopt of Wuer Rer }} \\
\hline Atkenulang Coment & & & & \\
\hline \multirow{2}{*}{\multicolumn{5}{|c|}{ ormal Ruver Din Anthanty }} \\
\hline & & & & \\
\hline 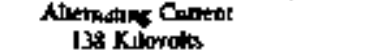 & 6171 & 6171 & - & \\
\hline 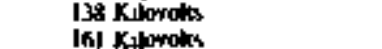 & 261 & 33374 & $\bar{z}$ & - \\
\hline $\begin{array}{l}\text { I6I Kiprotas } \\
345 \text { Kilprotes }\end{array}$ & 1396 & 1370 & - & - \\
\hline \multirow{2}{*}{\multicolumn{5}{|c|}{ Hearitud Cownerers Fowes DY }} \\
\hline & & & & \\
\hline 230 Kaloroles & 400 & 响 & - & - \\
\hline 345 Kilomolti & 1624 & 1624 & - & - \\
\hline \multirow{2}{*}{\multicolumn{5}{|c|}{ 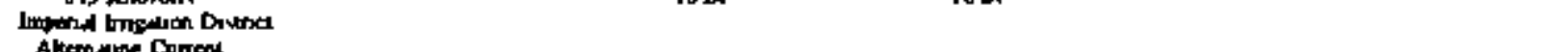 }} \\
\hline & & & & \\
\hline I6! Kalonolh & 30 & 32110 & - & - \\
\hline 200 Kitondis & 14470 & 14470 & - & - \\
\hline \multirow{3}{*}{\multicolumn{5}{|c|}{$\begin{array}{l}\text { Lower Colonato Rmer Authonty } \\
\text { Altming Comenl }\end{array}$}} \\
\hline & & & & \\
\hline & & & & \\
\hline I38 Kalorolts & 1.28922 & 1 , w9734 & - & - \\
\hline \multirow{2}{*}{\multicolumn{5}{|c|}{ Bhibegn Somth Centrol Fon AOY }} \\
\hline & & & & \\
\hline $\begin{array}{l}\text { Altomilups Ormen } \\
\text { J38 Kilovolus }\end{array}$ & 274 & 348 & - & - \\
\hline 345 Kallonolus & dón & 4000 & - & - \\
\hline \multirow{2}{*}{\multicolumn{5}{|c|}{ Nodevo Iminuor Dintra }} \\
\hline & & & & \\
\hline 230 Kutovalts & 6564 & 6564 & - & - \\
\hline
\end{tabular}

Set noten and fooknowe of end of bable 
Table 40. Transmission Line Statisties of Poblic Utilities, Fasal Year 1994 (Continued)

\begin{tabular}{|c|c|c|c|c|}
\hline \multirow{2}{*}{ 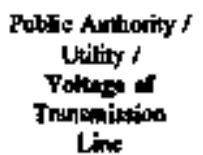 } & \multicolumn{2}{|c|}{ Drathend } & \multicolumn{2}{|c|}{ Underforind } \\
\hline & $\begin{array}{l}\text { Sractord } \\
\text { Miles }\end{array}$ & $\begin{array}{l}\text { Cimpoit } \\
\text { Mfles }\end{array}$ & $\begin{array}{c}\text { Stroctinal } \\
\text { MTit }\end{array}$ & $\begin{array}{l}\text { Chrenit } \\
\text { Miles }\end{array}$ \\
\hline
\end{tabular}

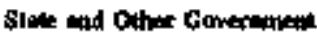

\begin{tabular}{|c|c|c|c|c|}
\hline \multicolumn{5}{|l|}{ 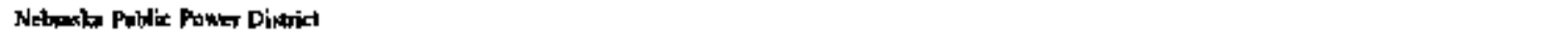 } \\
\hline \multicolumn{5}{|l|}{ Allernating Cument } \\
\hline I6] Kilondilo............. & 0.25 & 0.26 & $\rightarrow$ & - \\
\hline 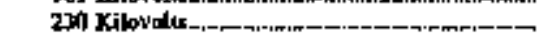 & 67.10 & 677.10 & - & - \\
\hline 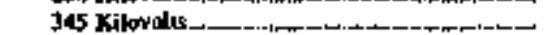 & 796,41 & $796, \boldsymbol{B J}$ & $\leftarrow$ & - \\
\hline \multicolumn{5}{|l|}{ 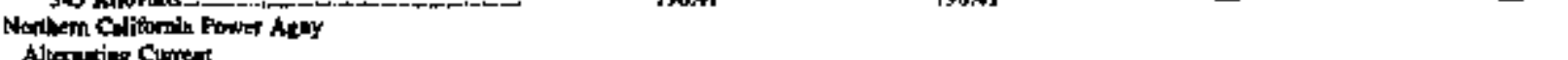 } \\
\hline 2 WP Kilovolls & 4l. In & 82,40 & - & - \\
\hline \multirow{2}{*}{\multicolumn{5}{|c|}{ 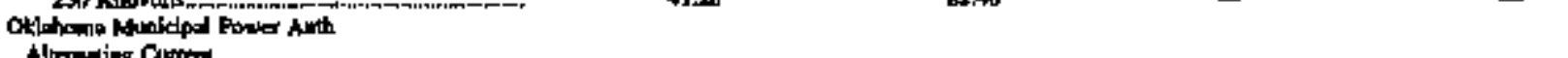 }} \\
\hline & & & & \\
\hline \multirow{2}{*}{\multicolumn{5}{|c|}{ 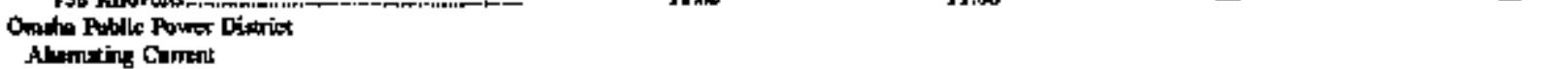 }} \\
\hline & & & & \\
\hline J6I Kollorolts. & $360 n$ & 360.7 & - & - \\
\hline 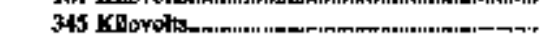 & 324.61 & 324,61 & - & - \\
\hline \multirow{2}{*}{\multicolumn{5}{|c|}{ 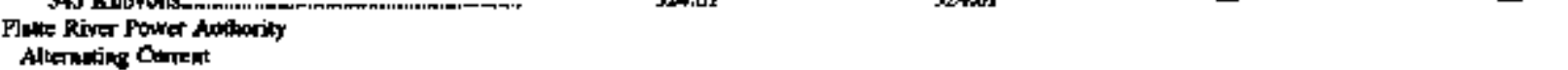 }} \\
\hline & & & & \\
\hline 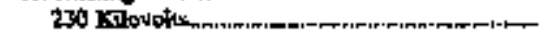 & 5,5330 & 163.30 & - & - \\
\hline 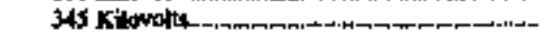 & 75.72 & 75.72 & - & - \\
\hline \multirow{2}{*}{\multicolumn{5}{|c|}{ 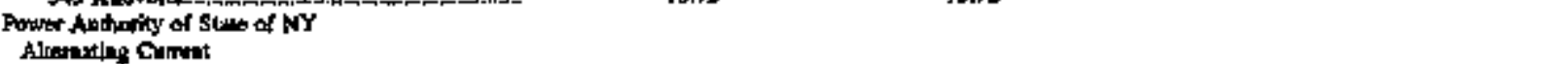 }} \\
\hline & & & & \\
\hline 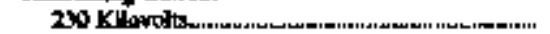 & 337,906 & 39790 & - & - \\
\hline 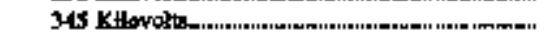 & 可边 39 & 183.10 & 346n & 1260 \\
\hline 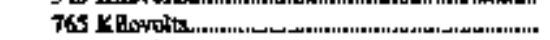 & 1346 & 154.89 & - & - \\
\hline \multicolumn{5}{|l|}{ PUL No I of Gela Coonty } \\
\hline Alenuting Cintert & & & & \\
\hline \multirow{2}{*}{\multicolumn{5}{|c|}{ PUD No I of Cowite Canity }} \\
\hline Alenting Curres & & & & \\
\hline 230 KIkmoth & 355 & 3.83 & - & - \\
\hline \multirow{2}{*}{\multicolumn{5}{|c|}{ PUD Ho I of Doods: Coowty }} \\
\hline Akemning Coment & & & & \\
\hline 230 CDotollsw & 82.00 & 8200 & - & - \\
\hline \multicolumn{5}{|l|}{ PUD No I of Lawle Counly } \\
\hline Alemrting Chents & & & & \\
\hline \multirow{2}{*}{\multicolumn{5}{|c|}{ PUD No 2 of Opas Cowaty }} \\
\hline Akemoting Comen: & & & & \\
\hline 230 Kilomol tr, & 9631 & ונה & - & - \\
\hline \multirow{2}{*}{\multicolumn{5}{|c|}{ 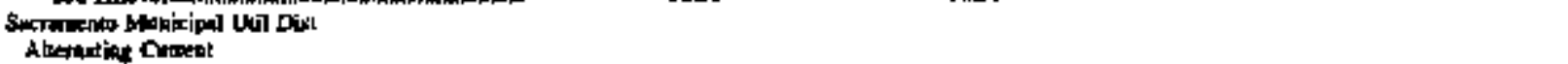 }} \\
\hline 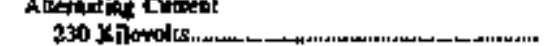 & & & & \\
\hline 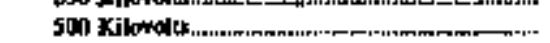 & 339.90 & 339.00 & - & - \\
\hline \multicolumn{5}{|l|}{ Solt River Prod Ag Implepwr } \\
\hline \multicolumn{5}{|l|}{ Alnomiting Cuman } \\
\hline 230 Xilionolve & 509 An & 60820 & - & - \\
\hline S25 tritomol ts. & 996,40 & 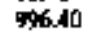 & - & $\pi$ \\
\hline \multicolumn{5}{|l|}{ South Copoliful toub Sor Anth } \\
\hline \multirow{3}{*}{\multicolumn{5}{|c|}{ 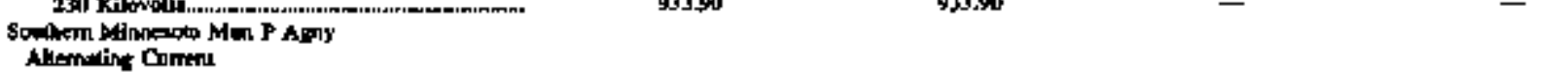 }} \\
\hline & & & & \\
\hline & & & & \\
\hline 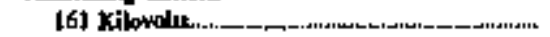 & 122.69 & 12269 & - & - \\
\hline 20 Kikwolus & 1730 & $\mathbf{J}, 30$ & - & - \\
\hline \multirow{2}{*}{\multicolumn{5}{|c|}{ 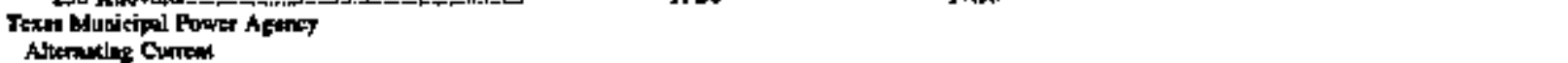 }} \\
\hline & & & & \\
\hline I]B Kilovalls:-_, & 15102 & 100,33 & - & - \\
\hline 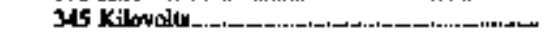 & Inew & 159.59 & - & - \\
\hline \multicolumn{5}{|l|}{ Thowo o othem Utility Areth } \\
\hline 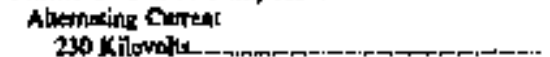 & Iflo0 & [0,10] & - & - \\
\hline
\end{tabular}

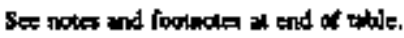


Table 40. Transmission Line Statistics of PubNic Utilities, Fiscal Year 1994 (Conlinwed)

\begin{tabular}{|c|c|c|c|c|}
\hline \multirow{2}{*}{ 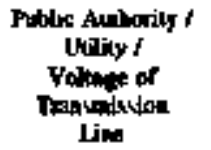 } & \multicolumn{2}{|c|}{ Drethend } & \multicolumn{2}{|c|}{$\begin{array}{l}\text { Uadergound } \\
\text { Sulvereint }\end{array}$} \\
\hline & $\begin{array}{c}\text { sumctival } \\
\text { Nille }\end{array}$ & $\begin{array}{l}\text { Giknu } \\
\text { Mulles }\end{array}$ & $\begin{array}{l}\text { Sthathisal } \\
\text { Naties }\end{array}$ & $\begin{array}{l}\text { Cirtuit } \\
\text { ulliser }\end{array}$ \\
\hline
\end{tabular}

State and Ober Gewecuned

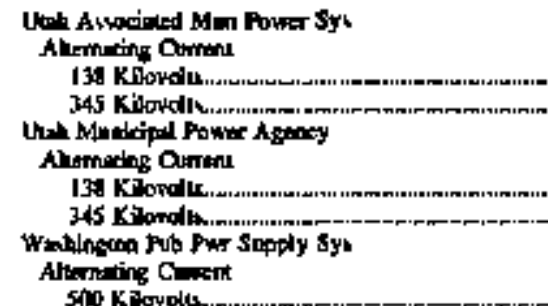

\begin{tabular}{|c|c|c|}
\hline $\begin{array}{l}21 / 00 \\
12 / 00\end{array}$ & $\begin{array}{l}21.00 \\
28.00\end{array}$ & - \\
\hline $\begin{array}{r}490 \\
106 \%\end{array}$ & $\begin{array}{r}4,90 \\
1069\end{array}$ & . \\
\hline 23.85 & 285 & - \\
\hline
\end{tabular}

Montival

Alextithu Gly or

Altenting Crmour

its Kikowolts

Anotin Clay of

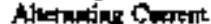

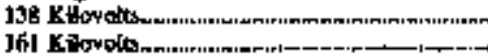

MS Kllovolts -

Rindy Pown Mortetip Anth

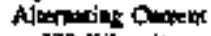

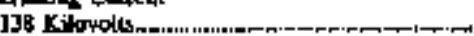

Brym Gily of

Altenitive Oarrest

198 K Klonolts.

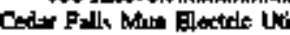

Afternting Corrent

Ifil Kilonoliti.

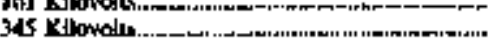

Cleveland Gity of

Afternties Currest

J]18 Xilonolu.

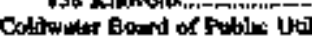

Alventutikg Crent

I3t Kionolts

Costiges Stration Ciny of

Allowhting Coment

13t Kiloride

Colorede Springs Ciay of

Aluerulaty Coment

$20 \mathrm{KLl}$ orod to

Colurbiu Gry of Water A Lith

Akerinting Cumet

161 Kllow dithe..................

(1.2)

16.21

241.24

4.73

179.85

241.24

179.69

25

2अ네

34.67

34.87

4.201

142.45

420

15,16

35.J6

6.15

Grarfondsvifle Eler I retpur Co

Altrencing Coment

138 Kllovolt

Dustos Cily of

Alterenting Corent

no Kliovole

Denvill City of Electric Div

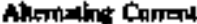

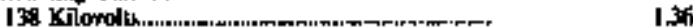

203

293

15.10

II

2738

35. B8

24,4

24.49

$12 \$$

1250

6008

6008

Daner City of

Atompins Commen

Is ricondus.

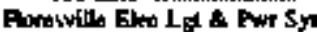

Akenoting Conrem

IX X] Konolu

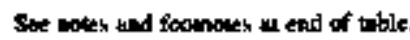


Table 40. Transmission Line Statistics of Public Utilities, Fiscal Year 194 (Conthoued)

\begin{tabular}{|c|c|c|c|c|}
\hline \multirow{2}{*}{ 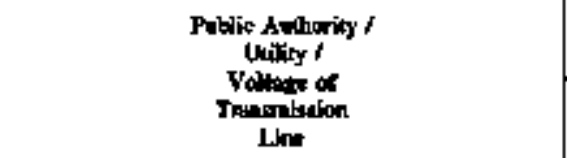 } & \multicolumn{2}{|c|}{ Oratiod } & \multicolumn{2}{|c|}{ 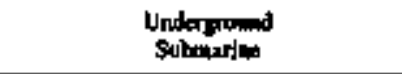 } \\
\hline & $\begin{array}{l}\text { Stroctar } \\
\text { alles }\end{array}$ & $\begin{array}{l}\text { Cirsoit } \\
\text { SHer }\end{array}$ & $\begin{array}{l}\text { Stroctoral } \\
\text { Bfles }\end{array}$ & $\begin{array}{l}\text { Cireoit } \\
\text { Btles }\end{array}$ \\
\hline \multicolumn{5}{|l|}{ 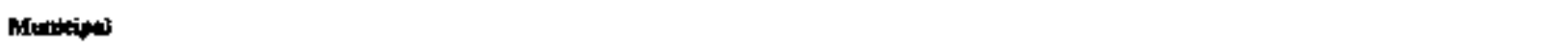 } \\
\hline 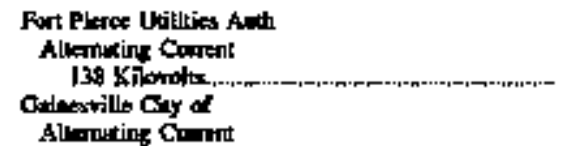 & Ton & 300 & $=$ & - \\
\hline 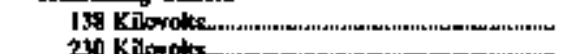 & 12031 & 210.1t & - & - \\
\hline 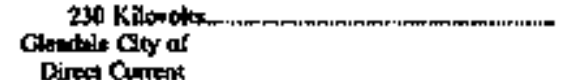 & | & 2.51 & - & - \\
\hline 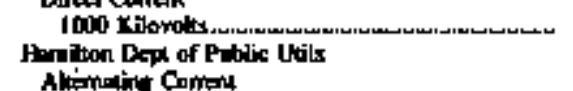 & 32.54 & 32.54 & - & - \\
\hline 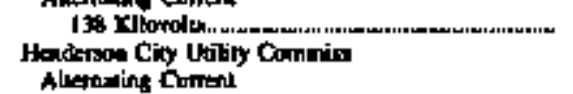 & 23.25 & 2025 & - & - \\
\hline 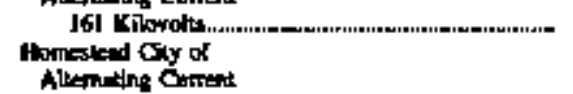 & 1,000 & 1000 & - & - \\
\hline 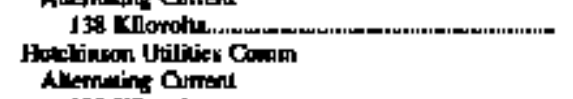 & 3.50 & 3st & - & - \\
\hline 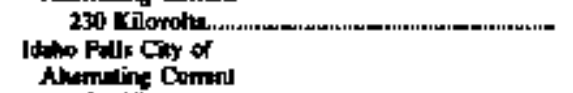 & 56.30 & 3830 & - & - \\
\hline 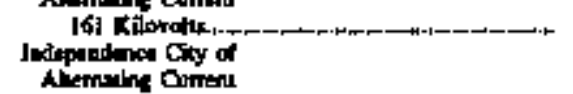 & 94.80 & 14.80 & - & - \\
\hline 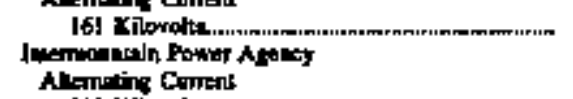 & 22.50 & 22.60 & - & - \\
\hline 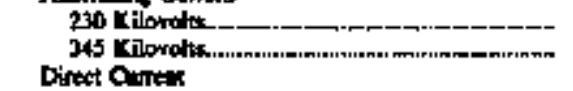 & $\begin{array}{l}\text { IAk:AJ } \\
\text { 100,56 }\end{array}$ & $\begin{array}{l}|14|, 4 \mid \\
\text { Inons6 }\end{array}$ & $\overline{-}$ & $\overline{-}$ \\
\hline 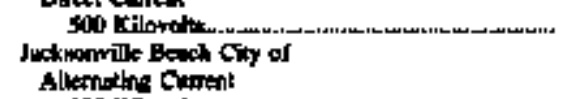 & 487.82 & प्रTS.jid & - & - \\
\hline 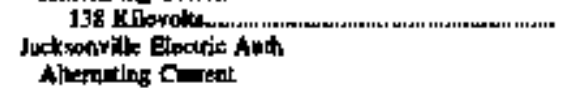 & 52,00 & 3200 & - & - \\
\hline 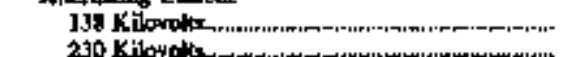 & 200.94 & $\begin{array}{l}297.17 \\
21746\end{array}$ & 2.50 & 2.50 \\
\hline 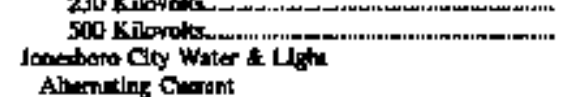 & 75,06 & 7506 & - & - \\
\hline 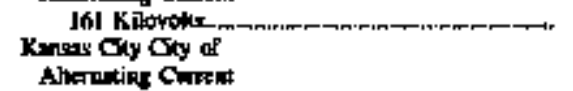 & 33,90 & 30.90 & - & - \\
\hline 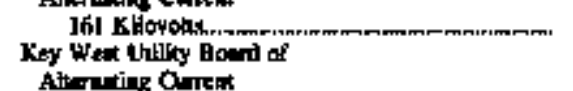 & 55.61 & f2.16 & - & - \\
\hline 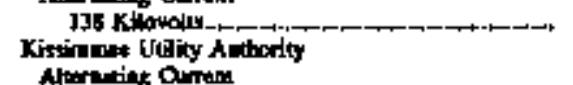 & 139.34 & 13938 & - & - \\
\hline 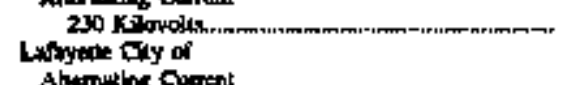 & 676 & 6768 & - & - \\
\hline 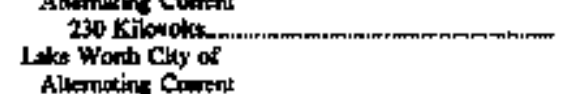 & 1400 & 14,00 & - & - \\
\hline 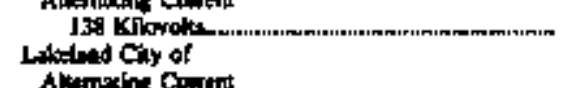 & 530 & 5.30 & - & - \\
\hline 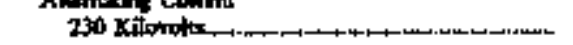 & 17.66 & 17.66 & - & - \\
\hline
\end{tabular}

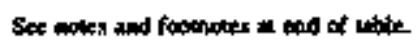


Table 40. Transsnission Line Statistics of Public Utilities, Fiseal Year 1994 (Conthued)

\begin{tabular}{|c|c|c|c|c|}
\hline \multirow{2}{*}{ 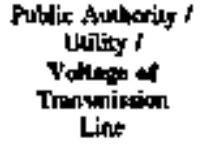 } & \multicolumn{2}{|c|}{ Ontitest } & \multicolumn{2}{|c|}{$\begin{array}{l}\text { Underpond } \\
\text { Sulpotaine }\end{array}$} \\
\hline & $\begin{array}{c}\text { Sancibond } \\
\text { Milen }\end{array}$ & $\begin{array}{l}\text { Clicuin } \\
\text { Milen }\end{array}$ & $\begin{array}{l}\text { Sunctond } \\
\text { Milks }\end{array}$ & $\begin{array}{l}\text { Cinenth } \\
\text { Mikn }\end{array}$ \\
\hline
\end{tabular}

Manizipal

Lapint Bood of Wrer d Ligh

Alteming Clment

$13 \mathrm{~B}$ Kilomolt

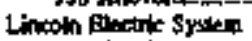

Altmilin Cmant

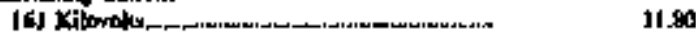

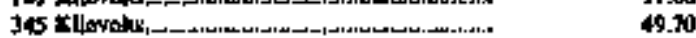

Lozanpon Bhricipal Wrilitare

Alteming ormal

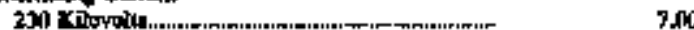

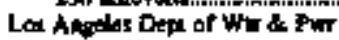

Alutetins Comont

130. Kilototes. 5136

20 Kllowolis

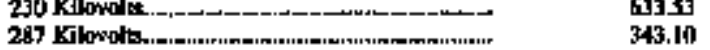

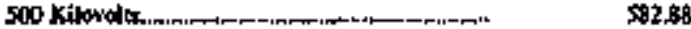

Dirten Cherent

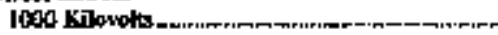

Loulsoille Hertiric Symen

Alingintin Coment

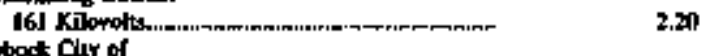

Labock Cly of

Alromenting Crment

isto Kilotolth

Mritull Clyy of

Atherentind Corpent

16J Kilopolth.

Mecaln Elntic \& Water Ut

Alatatien Cuntopt

135 Yluwales.

Bucropoliten Withe Ditrict

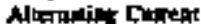$$
\text { 200 Xitorolth. }
$$

Murmy Ciby Puwe Deperiment

Alreating Craces

I3B Ki Kumblts

hase ane Pow and worer

Altan-ins: Cumbent

161) XFlevolus.

Neperryle City of

Alamelly Cureax

1385 sibro's:

Nathigathe Cly of

Altemiting Gement

$13 \mathbf{s}$ Klowals

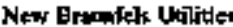

Aluratist Thipent

$138 \times 170$

Oeil Gty of

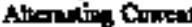

$230 \mathrm{Kil}$ Hods

Odunds Utifies Coramikion

Alteraving Comrä

2n X'Towalts

Divile Chity of

Aheminting Omeat

138 thtovelts

Omeneboro Maricipd Utiltie.

Akenioging Coment

144 Kilowolus.

Prow Chy Conpartion

Alemping Omept

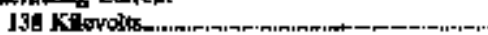

95

10

7.10

52.36

272.2]

4.27

I7, 16)

15. 19

305.60

I. $m$

453)

18.5

\$n

34,42

1295

117.16

B.80

16.40

4n
4669

I1.80

49.71

7,00

83.01

67811

24).10

244.15

21221

2.30

4.27

17.0. -

15.07

345.65

156

46.67

18.58

50

57.82

12.95

131.06

7.69

7.69

80

16.40

A)

$\begin{array}{ll}35,41 & \$ 3.40 \\ - & =\end{array}$

d21?

-

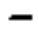

$m$

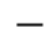

ㄴ.

-
Stet ortes and foodwols ax end of tenble. 
Table 40. Transmission Line Statistics of Public Utilities, Fiseal Year 1994 (Continued)

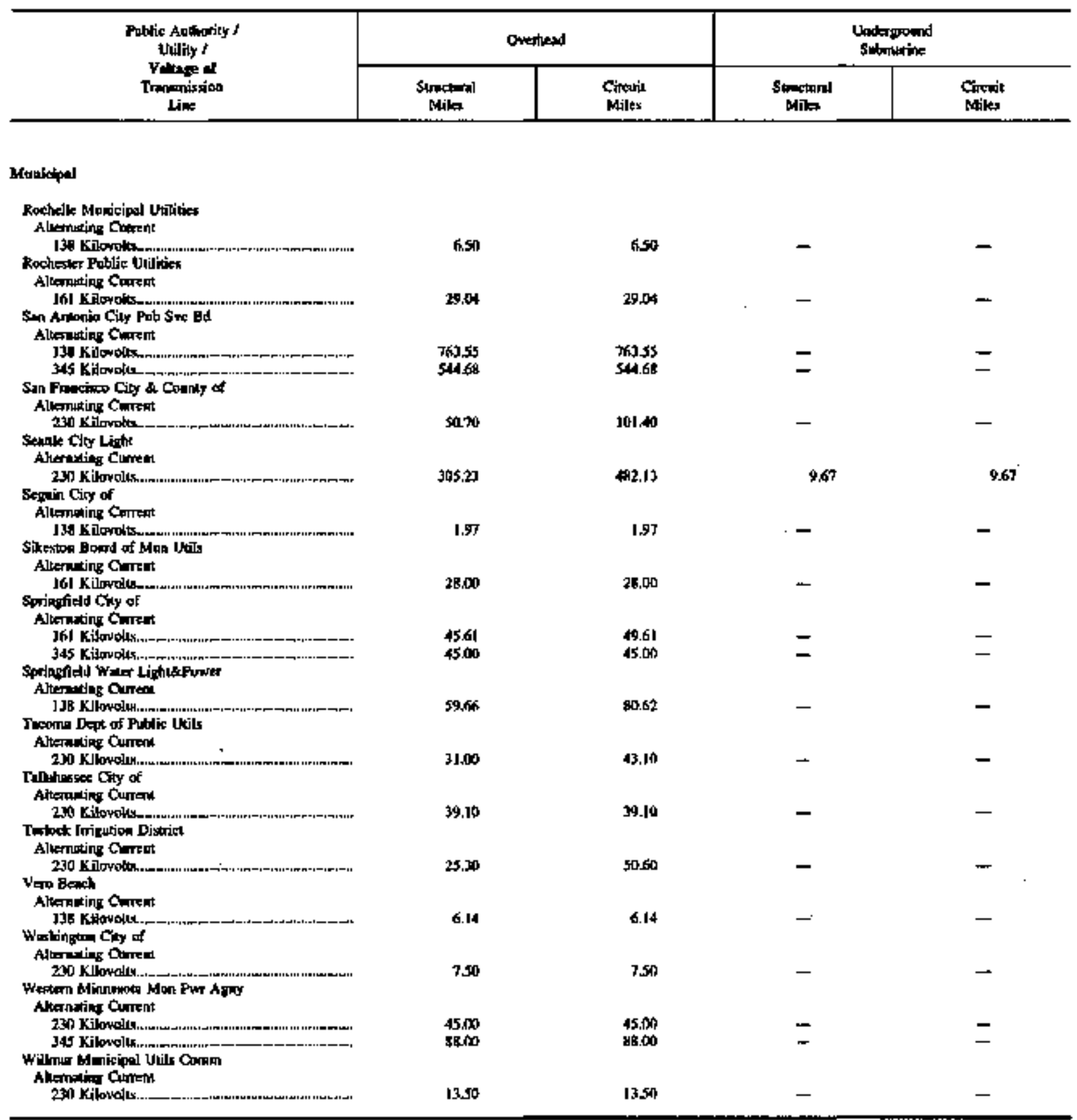

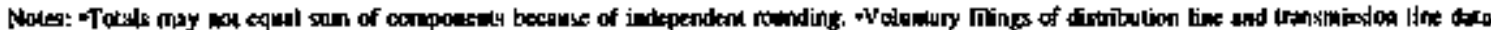

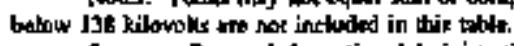

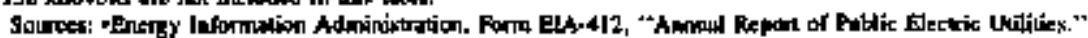


Table 41. Transmission Lines Added by Investor-Owned Utilities, by NRRC Region, 1994

\begin{tabular}{|c|c|c|c|c|c|}
\hline \multirow{2}{*}{ 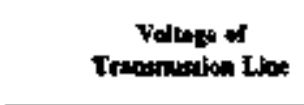 } & \multirow{2}{*}{$\begin{array}{l}\text { tune } \\
\text { Length } \\
\text { (netses) }\end{array}$} & \multicolumn{4}{|c|}{ Lae Coss (dollax) } \\
\hline & & 1 nald & Sinecinrs & Conitacens & Toul \\
\hline & \multicolumn{5}{|c|}{ 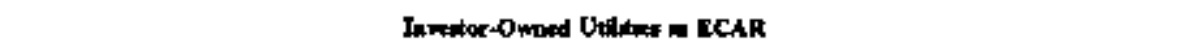 } \\
\hline \multirow[t]{2}{*}{$\begin{array}{l}128 \mathrm{kVAC} \\
2 \mathrm{kV} A C\end{array}$} & $\begin{array}{l}1042 \\
15 \text { \& }\end{array}$ & $\begin{array}{r}1,830,776 \\
12,720,0 \times 99\end{array}$ & $\begin{array}{l}2,414,722 \\
2537,027\end{array}$ & $\begin{array}{l}1,362,869 \\
1,445,666\end{array}$ & $\begin{array}{l}3,800,367 \\
16,700,748\end{array}$ \\
\hline & \multicolumn{5}{|c|}{ 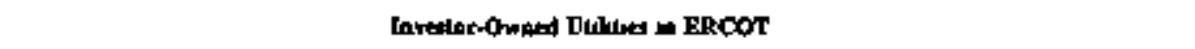 } \\
\hline \multirow[t]{2}{*}{ 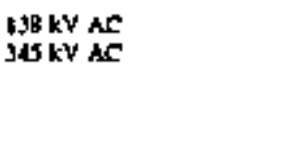 } & $\begin{array}{r}3935 \\
12351\end{array}$ & $3 . \overline{332861}$ & $\begin{array}{l}1,141,97) \\
36,29,946\end{array}$ & $\bar{z}$ & $\begin{array}{r}1,141,973 \\
42,123,457\end{array}$ \\
\hline & \multicolumn{5}{|c|}{ Invo:toc-O waod Dhltuse in MANC } \\
\hline \multirow[t]{2}{*}{$\begin{array}{l}138 \mathrm{kV} A C \\
200 \mathrm{kY} A C \\
745 \mathrm{kV} A C \\
5 \mathrm{kY} A C\end{array}$} & $\begin{array}{r}-370 \\
-\quad 411\end{array}$ & $\begin{array}{c}-\overline{800342} \\
-\overline{17,293,046}\end{array}$ & $\begin{array}{c}891,255 \\
7,479,614 \\
- \\
4758.8 .98\end{array}$ & $\begin{array}{r}2,979,843 \\
3,734,250 \\
32,137 \\
22,914,579\end{array}$ & $\begin{array}{r}3,781,090 \\
11,420,046 \\
32,100 \\
9,716,467\end{array}$ \\
\hline & \multicolumn{5}{|c|}{ 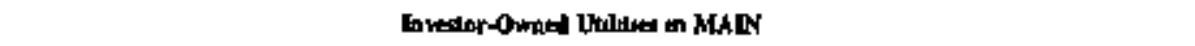 } \\
\hline \multirow[t]{2}{*}{$\begin{array}{l}13 \mathrm{kV} A C \\
345 \mathrm{AV} A \mathrm{C}\end{array}$} & $\begin{array}{l}5894 \\
4158\end{array}$ & $\begin{array}{l}2,100,680 \\
2,513,745\end{array}$ & $\begin{array}{l}9,737,531 \\
6,653,416\end{array}$ & $\begin{array}{l}8,914,776 \\
4,627,434\end{array}$ & $\begin{array}{l}20,459,987 \\
13,400,995\end{array}$ \\
\hline & \multicolumn{5}{|c|}{ Invertar-0wand Doltece u SERC } \\
\hline \multirow[t]{2}{*}{$\begin{array}{l}130 \mathrm{kY} A C \\
230 \mathrm{kV} A C \\
345 \mathrm{kY} A C \\
500 \mathrm{~kW} A C\end{array}$} & $\begin{array}{r}2016 \\
9316 \\
65 \\
6529\end{array}$ & 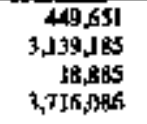 & $\begin{array}{r}4,912,6,34 \\
14,488,976 \\
55,110 \\
17,026,244\end{array}$ & $\begin{array}{r}2,62,256 \\
13,323,296 \\
55,600 \\
12,16,155\end{array}$ & $\begin{array}{r}7,604,541 \\
29,951,457 \\
128,885 \\
52,958,535\end{array}$ \\
\hline & \multicolumn{5}{|c|}{ Investoe-0 waed Uallies in SPP } \\
\hline \multirow[t]{2}{*}{$\begin{array}{l}138 \mathrm{kV} A C \\
3.15 \mathrm{KV} A C\end{array}$} & -421 & $\begin{array}{l}10+571 \\
250\end{array}$ & $\begin{array}{r}2.273,4010 \\
748,159\end{array}$ & $\begin{array}{l}T \$ 1,250 \\
95,6 \$ 1\end{array}$ & 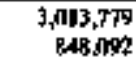 \\
\hline & \multicolumn{5}{|c|}{ 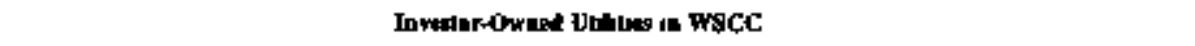 } \\
\hline \multirow[t]{2}{*}{$\begin{array}{l}138 \mathrm{kY} A C \\
230 \mathrm{kY} \text { AC }\end{array}$} & $\begin{array}{l}1168 \\
1445\end{array}$ & $\begin{array}{l}32,179 \\
2,005\end{array}$ & $\begin{array}{r}1,313,352 \\
3,976\end{array}$ & $\begin{array}{l}70,138 \\
737,659\end{array}$ & $\begin{array}{r}4,054,566 \\
76,052\end{array}$ \\
\hline & \multicolumn{5}{|c|}{ Condrgoos U.S. Totel } \\
\hline 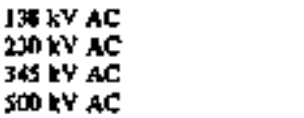 & $\begin{array}{l}14476 \\
127 \text { 57 } \\
165 \text { की } \\
111 \text { 4ी }\end{array}$ & $\begin{array}{r}4431,190 \\
6,743,104 \\
6,265.741 \\
20,969,132\end{array}$ & $\begin{array}{l}24,594,867 \\
24,509,569 \\
45,361,171 \\
64,575,192\end{array}$ & $\begin{array}{r}17, k 81,132 \\
\mid 18,241,263 \\
4,61 d, 234 \\
35,130,734\end{array}$ & $\begin{array}{r}46,184,411 \\
59.139,299 \\
56,841.136 \\
\mid 201,674,99 \%\end{array}$ \\
\hline
\end{tabular}

$\rightarrow$ Nox Applacibs:

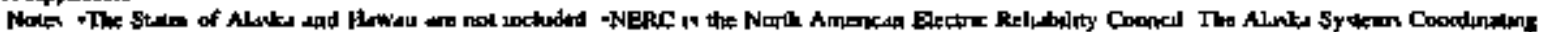

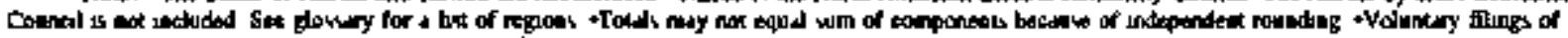

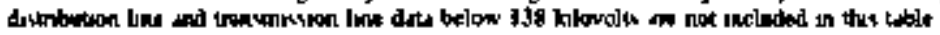

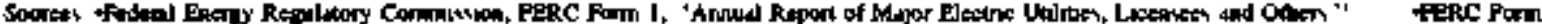

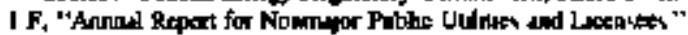


Table 42. Transnisslon Lines Added by Investor-Owned Utillites, 1994

\begin{tabular}{|c|c|c|c|c|c|}
\hline \multirow{2}{*}{$\begin{array}{c}\text { Dethy I } \\
\text { volthege of Lint }\end{array}$} & \multirow{2}{*}{$\underset{\text { Lergat }}{\operatorname{Lin}}$} & \multicolumn{4}{|c|}{ Lne Coxt (dnelles) } \\
\hline & & Lnd & Studcture & Conductorn & Total \\
\hline \multicolumn{6}{|l|}{ 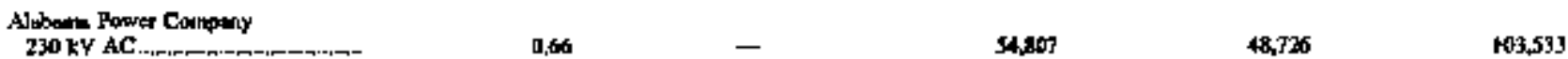 } \\
\hline \multicolumn{6}{|l|}{ Appoturebiun Fows Company } \\
\hline Jjo kY AC. & 286 & 159,453 & 629,253 & 751,694 & 1, 591 , 810 \\
\hline \multicolumn{6}{|l|}{ Bsuldume Gas a Electric Co } \\
\hline \multicolumn{2}{|l|}{ 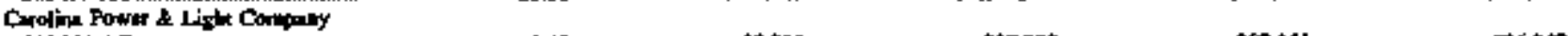 } & $1,468,47$ & 20.870 .968 & $\mid 2,874,9] \mid$ & $35,214,326$ \\
\hline $200 \mathrm{~V}$ AC & 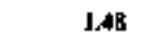 & 32,998 & 207,208 & 287,151 & 536,757 \\
\hline \multicolumn{6}{|l|}{ Centrol Dlinoit Fub \&erw Ca } \\
\hline 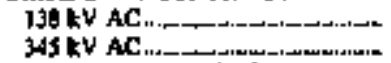 & $\begin{array}{l}4.74 \\
33.10\end{array}$ & $\begin{array}{r}988,594 \\
2,513,745\end{array}$ & $\begin{array}{l}5,391,252 \\
6,119,947\end{array}$ & $\begin{array}{l}5,919,418 \\
4,079,965\end{array}$ & $\begin{array}{l}12,219,214 \\
12,719,157\end{array}$ \\
\hline \multicolumn{6}{|l|}{ Cenrel Ponta \& Liqts Compeny } \\
\hline IJ8 kV AC. & 1.20 & - & $1,14,97$ & - & $1,141,59$ \\
\hline 36 kV AC & 11625 & $3,752.6 .1$ & $38,290,596$ & - & $42.083,457$ \\
\hline \multicolumn{6}{|l|}{ Cincinnuti Cins \& Destic Co } \\
\hline \multicolumn{6}{|l|}{ 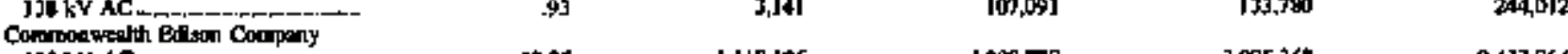 } \\
\hline $138 \mathrm{kV} A C_{-m n}$ & 33.05 & $1,1] 9,126$ & $4,238, \pi 0$ & $3,075,360$ & $8,433.264$ \\
\hline \multicolumn{6}{|l|}{ Dalke Putur Cochpady } \\
\hline \multicolumn{6}{|l|}{ 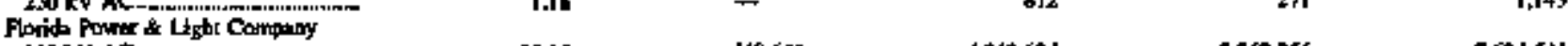 } \\
\hline $138 \mathrm{kV}$ AC- & 28.16 & 49,651 & $4,912,634$ & $2,262,256$ & $7,624,541$ \\
\hline $\begin{array}{l}230 \mathrm{kV} \text { AC } \\
500 \mathrm{kV} \text { AC }\end{array}$ & $11, \mathbf{6 3}$ & 194,720 & & 2416,596 & $3,6,69,814$ \\
\hline & 6529 & $3,7 \$ 6,0 \% 6$ & 17.9263294 & I2,216,ل55 & $32,958,35$ \\
\hline \multicolumn{6}{|l|}{ Elapidu Power Conpartion } \\
\hline 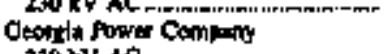 & 19.33 & $615,90]$ & $3,906,406$ & $3,006,406$ & 69628,713 \\
\hline 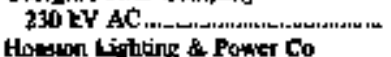 & B.63 & 30,562 & $1,22,596$ & - & $1,257,158$ \\
\hline \multicolumn{6}{|l|}{ Howon lighing \& Power Co } \\
\hline $138 \mathrm{kY} \mathbf{A C}_{3}$ & 645 & - & - & - & - \\
\hline 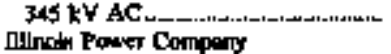 & 7.30 & - & - & - & - \\
\hline \multicolumn{6}{|l|}{ nelindel Pouer Compery } \\
\hline Indinsipotix Power \& Light Co & SAS & 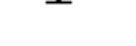 & 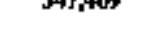 & 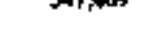 & 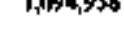 \\
\hline $178 \mathrm{kVAC}$ & 6,63 & - & - & - & - \\
\hline K-monx Gas of Electif Company & & & & & \\
\hline 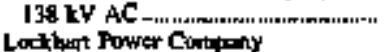 & 30 & - & 367, DNA & 323,264 & 696,305 \\
\hline 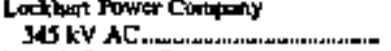 & . & 18.855 & 5550000 & 95,000 & 128,835 \\
\hline Netrot Powres Company & & & & & ت S \\
\hline $19 \mathrm{kV} A \mathrm{C}_{2}$ & ספר & - & 414,011 & J16,674 & 590,685 \\
\hline Ohio Edison Campany & & & & & \\
\hline $\begin{array}{l}13 \mathrm{~B} \text { kV AC } \\
\text { OAdohong Gut \& Eloxric Co }\end{array}$ & - & t.667.7\$2 & $1,647,378$ & 67,391 & $4,092,555$ \\
\hline س. & 3.91 & In,871 & $1,906,359$. & 427.965 & \\
\hline $345 \mathrm{kV} A C_{\ldots} \ldots$ & - & 250 & 748,159 & 99,683 & $2,34,496$ \\
\hline Pwificon & & & & & \\
\hline IS KV AC AC & 1.08 & 32,175 & 2007,764 & 3900008 & 2.457 .948 \\
\hline 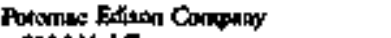 & & & & & \\
\hline 2) WV AC & 15.61 & $12,720,099$ & 2537,063 & $1,446,666$ & $16,7027,258$ \\
\hline Potemas Electibe Power Compary & & & & & \\
\hline 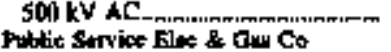 & 19.80 & $15, \pi 4,599$ & $26,394,032$ & 10129,840 & $5,20(n, n)$ \\
\hline I3B kV AC-_...... & - & - & 801,255 & 2990,07 & $3,781,098$ \\
\hline 2MA KV AC A & 3.X4 & $860,8+2$ & $7,479,614$ & $3,734,250$ & $11,720,046$ \\
\hline $345 \mathrm{kV} A \mathrm{C}_{-}$ & - & - & - & 32,103 & $32.1 \mathrm{~m}$ \\
\hline $\operatorname{sot} \mathrm{kV}$ AC & - & - & 283,8318 & 9,028 & 290,656 \\
\hline Sen Diego G* \& Bkectric Co & & & & & \\
\hline $230 \mathrm{YV} A C$ & 6.80 & 22,5 in & $2,19 \mathrm{~J}$. & 796,409 & 761.169 \\
\hline 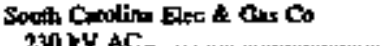 & & & & & \\
\hline 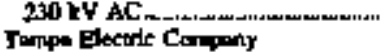 & 2250 & 1.204283 & $3, \mathbf{A G A}, \mathbf{d t a 2}$ & $5,00,147$ & $9,712,839$ \\
\hline $239 \mathrm{x} \forall \mathrm{AC}$ & 12.19 & - & - & - & - \\
\hline Texan Unilition Elactrit Co & & & & & \\
\hline $138 \mathrm{kV}$ AC & 31.70 & - & - & - & - \\
\hline Tochon Elentric Poner Clompony & & & & & \\
\hline 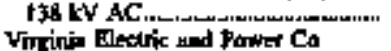 & 3.70 & - & mIsn & 194,456 & 1060.003 \\
\hline 2010 KV AC............................ & IS.9] & $1,0 \times 6,323$ & $5,527,167$ & $1,471,999$ & $8,151,509$ \\
\hline
\end{tabular}

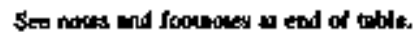


Table 42. Transmission Lines Added by Investar-Owned Utilities, 1994 (Continoed)

\begin{tabular}{|c|c|c|c|c|c|}
\hline \multirow{2}{*}{ 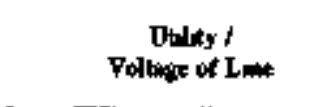 } & \multirow{2}{*}{$\begin{array}{c}\text { Lune } \\
\text { Lengl } \\
\text { (mules) }\end{array}$} & \multicolumn{4}{|c|}{ Line Corsi ilodlas } \\
\hline & & Lind & Stroçturte & Condurtuts & Futed \\
\hline Wulungiton Waler Popar & & & & & \\
\hline 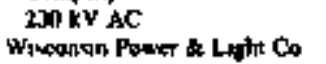 & 365 & 476 & 1,785 & 642 & 2,860 \\
\hline I3A LV AC & 紅 & - & 107,509 & - & Im, sols \\
\hline Wreomsin Pollic Servoce Cap & & & & & \\
\hline$J 38 \mathrm{kV} A C$ & 35 & - & - & - & - \\
\hline
\end{tabular}

- Slou Applesile

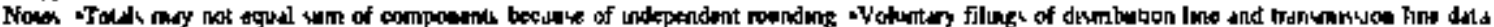

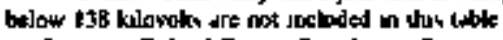

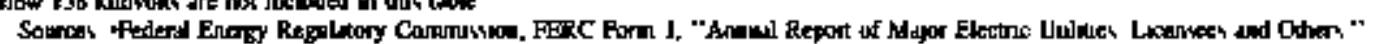

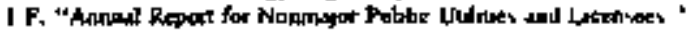

HERC Fom 
Table 43. Wathour Meter and Line Transtormer Statistics of Investor-Orned Utilities, by NERC Region, 1994

(Count and Megavoltampere)

\begin{tabular}{|c|c|c|c|c|c|c|c|}
\hline 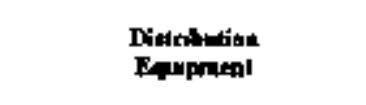 & $\begin{array}{l}\text { Nomber in } \\
\text { Beginning } \\
\text { of Yedr }\end{array}$ & 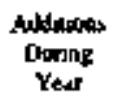 & $\begin{array}{l}\text { Reductuans } \\
\text { Danne } \\
\text { Yedr }\end{array}$ & $\begin{array}{l}\text { Numbet } 4 \\
\text { End of } \\
\text { Ytcd }\end{array}$ & $\begin{array}{l}\text { Is Stuck } \\
\text { Lodked, } \\
\text { and lintawe I }\end{array}$ & $\begin{array}{l}\text { In } \\
\text { Coutomert } \\
\text { Une }\end{array}$ & $\begin{array}{l}\text { Ua } \\
\text { Compiny }\end{array}$ \\
\hline & \multicolumn{7}{|c|}{ 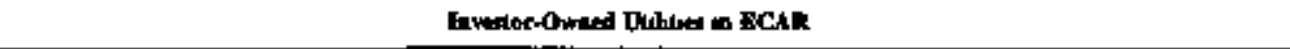 } \\
\hline \multirow[t]{2}{*}{ 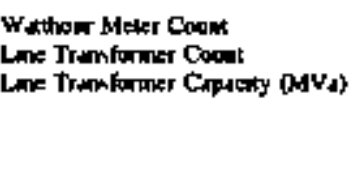 } & $\begin{array}{r}15,163,412 \\
4,124,662 \\
406,062\end{array}$ & $\begin{array}{r}484,500 \\
166,574 \\
17,1128\end{array}$ & $\begin{array}{r}40,045 \\
73,569 \\
3,9409\end{array}$ & $\begin{array}{r}15,247,947 \\
4,217,967 \\
419.1 \% 4\end{array}$ & $\begin{array}{l}597,017 \\
149,4,4] \\
46,060\end{array}$ & $\begin{array}{r}13,915,270 \\
3,753,964 \\
759,2614\end{array}$ & $\begin{array}{r}739,660 \\
314,573 \\
13,846\end{array}$ \\
\hline & \multicolumn{7}{|c|}{ haveder.10wned Uthles in ERCOT } \\
\hline \multirow[t]{2}{*}{ 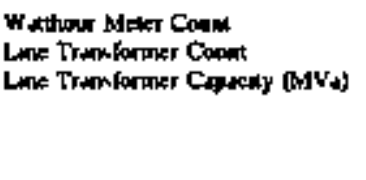 } & $\begin{array}{r}5,010,098 \\
1,514,663 \\
7 \pm, 859\end{array}$ & $\begin{array}{r}118,060 \\
42,59 \\
2,619\end{array}$ & $\begin{array}{r}6,2,52 \\
29,175 \\
1,475\end{array}$ & $\begin{array}{r}5,067,829 \\
1,59,745 \\
80,052\end{array}$ & $\begin{array}{r}17 f, f i T h \\
4,6,642 \\
4,169\end{array}$ & $\begin{array}{r}2,106,897 \\
41,7,979 \\
14,292\end{array}$ & $\begin{array}{r}2,284,295 \\
1, \sqrt{ } 69,966 \\
61,971\end{array}$ \\
\hline & \multicolumn{7}{|c|}{ 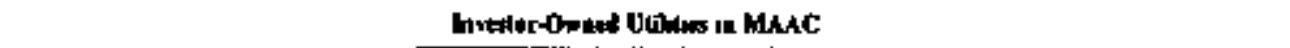 } \\
\hline \multirow[t]{2}{*}{ 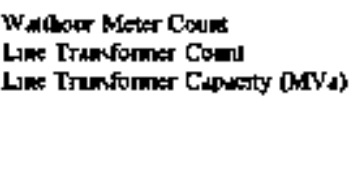 } & $\begin{array}{r}9,869,975 \\
1,904,796 \\
104,126\end{array}$ & $\begin{array}{r}333,62 \% \\
55,065 \\
3,017\end{array}$ & $\begin{array}{r}224,476 \\
26,4,16 \\
1,154\end{array}$ & $\begin{array}{r}9,579,128 \\
1,563,404 \\
105,7601\end{array}$ & $\begin{array}{r}603,734 \\
68,814 \\
6.516\end{array}$ & $\begin{array}{r}9,372,785 \\
1,711,9+7 \\
\text { BT,गJ। }\end{array}$ & $\begin{array}{r}2,609 \\
182,6100 \\
11,574\end{array}$ \\
\hline & \multicolumn{7}{|c|}{ Lrentor-0mad Duhles m MAFN } \\
\hline \multirow[t]{2}{*}{ 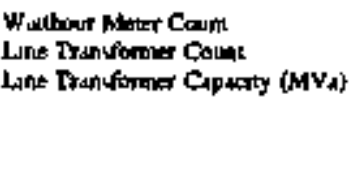 } & $\begin{array}{l}0,141,454 \\
1,71,1394 \\
1,4,4,12\end{array}$ & $\begin{array}{r}196,344 \\
13,661 \\
1,301\end{array}$ & $\begin{array}{r}187,333 \\
31,409 \\
2,259\end{array}$ & $\begin{array}{r}8,252,465 \\
1,735,856 \\
199,856\end{array}$ & $\begin{array}{r}194,877 \\
96,015 \\
8,786\end{array}$ & $\begin{array}{r}\text { g, } 065,597 \\
1,637,679 \\
190,919\end{array}$ & $\begin{array}{r}2,051 \\
2,162 \\
151\end{array}$ \\
\hline & \multicolumn{7}{|c|}{ Frestordamed Dhlites in MAPP } \\
\hline \multirow[t]{2}{*}{ 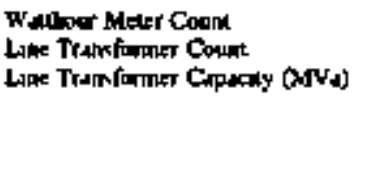 } & $\begin{array}{l}3,318,135 \\
759,164 \\
121,264\end{array}$ & $\begin{array}{r}115,354 \\
24,260 \\
4,974\end{array}$ & $\begin{array}{r}141,427 \\
12,276 \\
1,79 \mid\end{array}$ & $\begin{array}{r}7,213062 \\
7\} 1,183 \\
124,455\end{array}$ & $\begin{array}{r}171,919 \\
31,712 \\
12,306\end{array}$ & $\begin{array}{r}3,157,347 \\
596,993 \\
305,841\end{array}$ & $\begin{array}{r}2.776 \\
142,876 \\
6,306\end{array}$ \\
\hline & \multicolumn{7}{|c|}{ 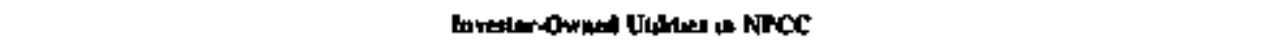 } \\
\hline \multirow[t]{2}{*}{ 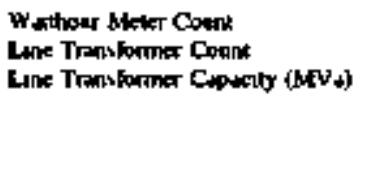 } & $\begin{array}{r}13,855,520 \\
2786,736 \\
748879\end{array}$ & $\begin{array}{l}475,1625 \\
111,441 \\
16,169\end{array}$ & $\begin{array}{r}373,026 \\
31,162 \\
3,972\end{array}$ & $\begin{array}{r}13,957,6: 5 \\
2,467,018 \\
361,036\end{array}$ & $\begin{array}{r}865,583 \\
205.158 \\
42,102\end{array}$ & $\begin{array}{r}10,848,227 \\
1,677,687 \\
271,955\end{array}$ & $\begin{array}{r}2,241,831 \\
1,164,173 \\
45,023\end{array}$ \\
\hline & \multicolumn{7}{|c|}{ 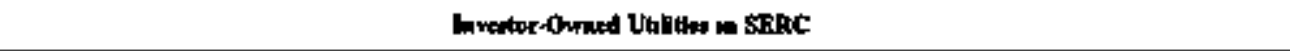 } \\
\hline \multirow[t]{2}{*}{ 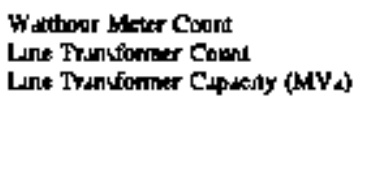 } & $\begin{array}{r}14,587,397 \\
4,214,813 \\
198,649\end{array}$ & $\begin{array}{r}55 B, 899 \\
157,112 \\
8,683\end{array}$ & $\begin{array}{r}335,648 \\
96,576 \\
4,734\end{array}$ & $\begin{array}{r}14,646,559 \\
4,265,09 \\
202998\end{array}$ & $\begin{array}{r}855,871 \\
101,252 \\
11,712\end{array}$ & $\begin{array}{r}13,943,895 \\
3,858,844 \\
177,354\end{array}$ & $\begin{array}{r}5,837 \\
305,313 \\
13,042\end{array}$ \\
\hline & \multicolumn{7}{|c|}{ Imotilomomed durese in SPP } \\
\hline 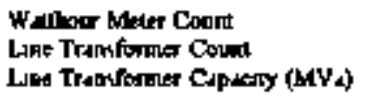 & $\begin{array}{r}6,411,712 \\
2,241,977 \\
94,496\end{array}$ & $\begin{array}{r}196,224 \\
74,0 \text {, } \\
3,620\end{array}$ & $\begin{array}{r}128,211 \\
76,7710 \\
1,378\end{array}$ & $\begin{array}{r}6,479,725 \\
2,279,274 \\
96,747\end{array}$ & $\begin{array}{r}271,335 \\
71,732 \\
6,329\end{array}$ & $\begin{array}{r}6,123,403 \\
2,193,099 \\
69,78]\end{array}$ & $\begin{array}{r}84,987 \\
11,446 \\
695\end{array}$ \\
\hline Lod Tratuforter Cspierry (MVL) & \multicolumn{7}{|c|}{ 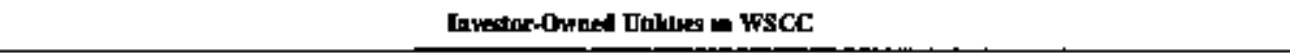 } \\
\hline \multirow[t]{2}{*}{ 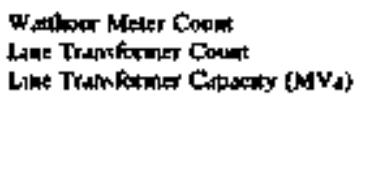 } & $\begin{array}{r}17,617, \pi 12 \\
3,702,162 \\
138,626\end{array}$ & $\begin{array}{r}626,115 \\
124,036 \\
6,811\end{array}$ & $\begin{array}{r}340,899 \\
70,877 \\
2,561\end{array}$ & $\begin{array}{r}17,903,638 \\
3,748,121 \\
143,146\end{array}$ & $\begin{array}{r}77,641 \\
17,059 \\
9,304\end{array}$ & $\begin{array}{r}17.125,164 \\
3,340,319 \\
121.268\end{array}$ & $\begin{array}{r}3,833 \\
231,743 \\
12,374\end{array}$ \\
\hline & \multicolumn{7}{|c|}{ Conteguns US. Told } \\
\hline 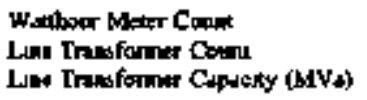 & $\begin{array}{r}93,973,115 \\
22,583,595 \\
1,685,677\end{array}$ & $\begin{array}{r}7,204,924 \\
219,49] \\
70,643\end{array}$ & $\begin{array}{r}2,532,007 \\
415,073 \\
21,024\end{array}$ & $\begin{array}{r}95,026,032 \\
22,976,613 \\
1,713,267\end{array}$ & $\begin{array}{r}4,517,594 \\
948,774 \\
147,250\end{array}$ & $\begin{array}{r}35,148,525 \\
15,54,4,519 \\
1,420,415\end{array}$ & $\begin{array}{r}5,369,9+3 \\
3,44,73,11 \\
165,734\end{array}$ \\
\hline
\end{tabular}

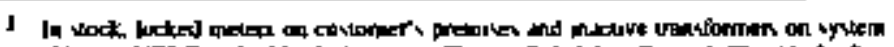

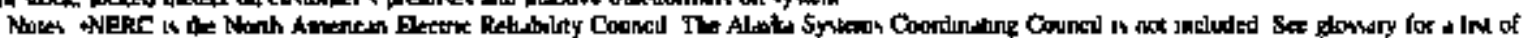

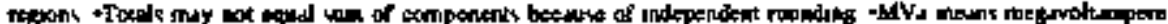

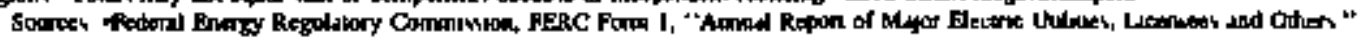


Table 44. Watthour Meter and Line Transformer Statistics of Investor-Owned Utillies, 1994 (Count and Megavoltampere)

\begin{tabular}{|c|c|c|c|c|c|c|c|}
\hline Dustribotan & $\begin{array}{l}\text { Nomber at } \\
\text { Dofanant } \\
\text { of Year }\end{array}$ & $\begin{array}{l}\text { Adinon } \\
\text { During } \\
\text { Yax }\end{array}$ & $\begin{array}{l}\text { Redacloas } \\
\text { Durunk } \\
\text { Yar }\end{array}$ & $\begin{array}{l}\text { Noniber a } \\
\text { End of } \\
\text { Yer }\end{array}$ & $\begin{array}{l}\text { In Slock, } \\
\text { Locked, } \\
\text { and Inetuve I }\end{array}$ & $\log _{\text {Und }}^{\text {In }}$ & 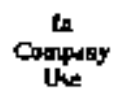 \\
\hline \multicolumn{8}{|l|}{ Alabane Power Conipury } \\
\hline 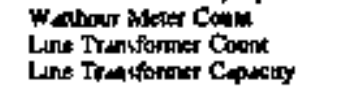 & $\begin{array}{l}1,240,450 \\
552,522\end{array}$ & $\begin{array}{l}\gamma_{1,015}, 015 \\
28,390\end{array}$ & $\begin{array}{l}37,757 \\
16.255\end{array}$ & $\begin{array}{r}1,27,5,688 \\
564,666\end{array}$ & $\begin{array}{l}75,787 \\
12.557\end{array}$ & $\begin{array}{r}1,194,397 \\
554,109\end{array}$ & $-{ }^{484}$ \\
\hline 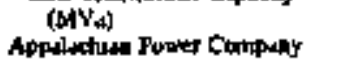 & 17978 & 1,068 & 483 & 18 sos & ass & 11,696 & - \\
\hline 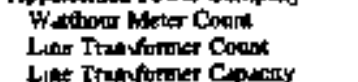 & $\begin{array}{l}89,9,95 \\
345,197\end{array}$ & $\begin{array}{l}21200 \\
17,604\end{array}$ & $\begin{array}{r}22.6599 \\
9,461\end{array}$ & $\begin{array}{l}\text { 8945799 } \\
353,196\end{array}$ & 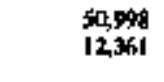 & $\begin{array}{l}315,715 \\
340,714\end{array}$ & 133 \\
\hline 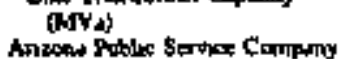 & II,120 & 734 & 25S & $115 \$ 9$ & Q1 & 10,660 & 18 \\
\hline 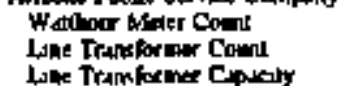 & $\begin{array}{l}70,5,9 \% 6 \\
193,944\end{array}$ & $\begin{array}{r}54,217 \\
6,8380\end{array}$ & $\begin{array}{r}1,90 \% \\
1,60 \%\end{array}$ & $\begin{array}{l}72,793 \\
199,144\end{array}$ & $\begin{array}{l}73.790 \\
16,075\end{array}$ & $\begin{array}{l}713,711 \\
183,469\end{array}$ & $-^{272}$ \\
\hline 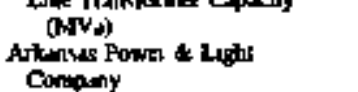 & 12,019 & 372 & 83 & $\sqrt{2}, 1008$ & 1.185 & 11,123 & - \\
\hline 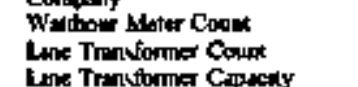 & $\begin{array}{l}50,563,3 \\
292,239\end{array}$ & 20,040 & 12,189 & $\begin{array}{l}677,662 \\
298,328\end{array}$ & $\begin{array}{l}46,4 \$ 8 \\
14,000\end{array}$ & $\begin{array}{l}\text { 62:.749 } \\
283.299\end{array}$ & $\begin{array}{l}3,425 \\
1,029\end{array}$ \\
\hline 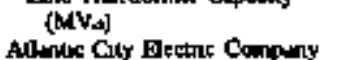 & 10,427 & 499 & 136 & 10,730 & 962 & 9.678 & 90 \\
\hline 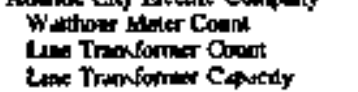 & $\begin{array}{l}41,081 \\
\mid 29, \text { Th? }\end{array}$ & $\begin{array}{r}20,455 \\
2,800\end{array}$ & 1,526 & $\begin{array}{l}483,160 \\
131,061\end{array}$ & $\begin{array}{r}16,479 \\
497 .\end{array}$ & $\begin{array}{l}\text { 476, } 614 \\
\text { I24, }\end{array}$ & $\begin{array}{l}137 \\
\text { 2n }\end{array}$ \\
\hline 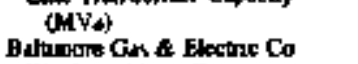 & 4,916 & 127 & 63 & 4.980 & 408 & 4,570 & 2 \\
\hline 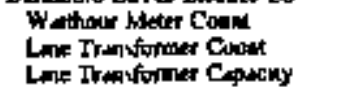 & $\begin{array}{r}\text { 1,J43.15n } \\
\text { J82,9n3 }\end{array}$ & $\begin{array}{r}67,643 \\
5,642\end{array}$ & $\begin{array}{r}32,497 \\
2,750\end{array}$ & $\begin{array}{l}\text { J.17B,296 } \\
\text { t85,795 }\end{array}$ & $\begin{array}{r}58,606 \\
3,736\end{array}$ & $\frac{1,138,124}{-}$ & 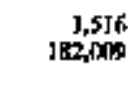 \\
\hline 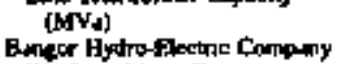 & 11,, ep? & 377 & IBY & $115 y$ & 473 & - & 11,524 \\
\hline 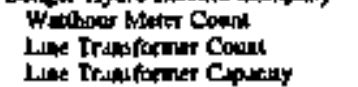 & $\begin{array}{r}108,556 \\
38,729\end{array}$ & $\begin{array}{l}2,656 \\
1,400\end{array}$ & $\begin{array}{l}1 \text { sit. } \\
447\end{array}$ & $\begin{array}{r}109,704 \\
39, \pi 92\end{array}$ & - & $\begin{array}{r}109,617 \\
39,461\end{array}$ & -97 \\
\hline 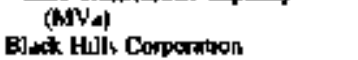 & 9:01] & 33 & 7 & 927 & - & 127 & - \\
\hline 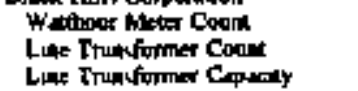 & $\begin{array}{l}56,646 \\
17.065\end{array}$ & IST1 & $\begin{array}{l}301 \\
\$ 91\end{array}$ & $\begin{array}{l}59,676 \\
18,168\end{array}$ & $\begin{array}{l}2,3718 \\
1,333\end{array}$ & $\$ 7,028$ & - \\
\hline B]eckide Yaltey Eleone Ca & 723 & 12 & 14 & BAI & 10 & 699 & - \\
\hline 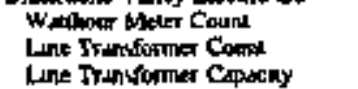 & $\begin{array}{l}50,980 \\
12,499\end{array}$ & $\begin{array}{r}1,958 \\
204\end{array}$ & $\begin{array}{r}3,663 \\
393\end{array}$ & $\begin{array}{l}91,213 \\
12,310\end{array}$ & $\begin{array}{l}4,267 \\
4.072\end{array}$ & $\begin{array}{l}86,093 \\
11,272\end{array}$ & $\begin{array}{l}15 \\
16\end{array}$ \\
\hline $\begin{array}{l}\text { (bavi) } \\
\text { Bontin Edron Comprisy }\end{array}$ & 737 & 13 & 25 & 725 & [56 & 575 & 」 \\
\hline 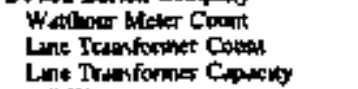 & $\begin{array}{r}754,227 \\
59,131\end{array}$ & $\begin{array}{r}45,376 \\
1,116\end{array}$ & $\begin{array}{r}19,2167 \\
264\end{array}$ & $\begin{array}{r}779,896 \\
\text { fal,675 }\end{array}$ & $\begin{array}{r}83,041 \\
6,477\end{array}$ & 690.799 & $\begin{array}{l}2,046 \\
54.201\end{array}$ \\
\hline 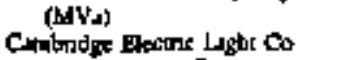 & 4934 & 132 & 28 & 4,538 & 627 & II & 4,733 \\
\hline 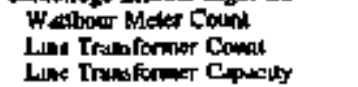 & $\begin{array}{r}1,5,944 \\
2,344\end{array}$ & $\begin{array}{r}645 \\
27\end{array}$ & $\begin{array}{r}57 \\
54\end{array}$ & $\begin{array}{r}4.512 \\
2.317\end{array}$ & $\begin{array}{l}2,079 \\
461\end{array}$ & $\begin{array}{r}45,904 \\
1,950\end{array}$ & 29 \\
\hline 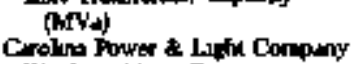 & 165 & $\$$ & 2 & 146 & $\mathbf{u}$ & 131 & I \\
\hline 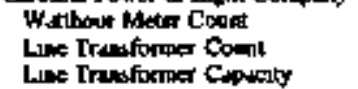 & $\begin{array}{r}3,053,763 \\
3 \pm 3,314\end{array}$ & $\begin{array}{l}\text { T6,604 } \\
\text { II,475 }\end{array}$ & $\begin{array}{l}9,930 \\
3,315\end{array}$ & $\begin{array}{r}1,130,401 \\
39 t, 434\end{array}$ & $\begin{array}{r}91,896 \\
8,814\end{array}$ & $\begin{array}{r}3,(135,063 \\
382,660\end{array}$ & $\begin{array}{r}532 \\
-\quad\end{array}$ \\
\hline 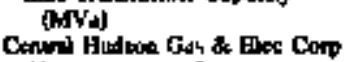 & 15,265 & GR & 120 & 15,744 & 68 & 15,062 & - \\
\hline 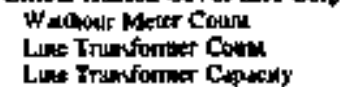 & $\begin{array}{r}274,412 \\
84,617\end{array}$ & $\begin{array}{l}8,233 \\
2,298\end{array}$ & $\begin{array}{l}6,498 \\
1,739\end{array}$ & $\begin{array}{r}276,316 \\
85,136\end{array}$ & 21,315 & 255,201 & $\overline{81.870}$ \\
\hline $\begin{array}{l}\text { (WYs) } \\
\text { Centrillinars degh Compiny }\end{array}$ & $2+300$ & 98 & 5 & 2400 & $1 \$ 7$ & - & 2043 \\
\hline 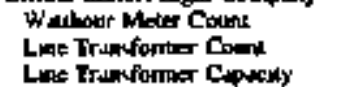 & $\begin{array}{r}2013,2,27 \\
63,157\end{array}$ & $\begin{array}{l}5,710 \\
2,105\end{array}$ & $\begin{array}{l}5,737 \\
1,2900\end{array}$ & $\begin{array}{r}203.249 \\
63,972\end{array}$ & $\begin{array}{l}6,752 \\
2,455\end{array}$ & $\begin{array}{l}\text { I94.457 } \\
61.467\end{array}$ & - \\
\hline ) & 2943 & I4⿻木口) & $\pi$ & 3이 & $1 * 4$ & 2827 & 一 \\
\hline
\end{tabular}

Sea wotes ind footnotes at end of uble 
Table 44. Watthour Meter and Line Transtormer Statistics of Investor-Owned Utilities, 1994 (Count and Megavoltrmuere) (Conkinued)

\begin{tabular}{|c|c|c|c|c|c|c|c|}
\hline Ditrílian & $\begin{array}{l}\text { Number a } \\
\text { Bofining } \\
\text { of Yeur }\end{array}$ & $\begin{array}{l}\text { Addinion: } \\
\text { Dring } \\
\text { Yerr }\end{array}$ & $\begin{array}{l}\text { Redectionn } \\
\text { Denin: } \\
\text { Yent }\end{array}$ & $\underset{\substack{\text { Nander at } \\
\text { Yedr }}}{\text { of }}$ & $\begin{array}{l}\text { In Stonk } \\
\text { Locked, } \\
\text { and Jnetive I }\end{array}$ & $\underset{\substack{\text { In } \\
\text { Unem }}}{\operatorname{lng}}$ & $\operatorname{Cimphy}_{\text {Une }}^{\ln }$ \\
\hline \multicolumn{8}{|l|}{ Ceneral ullnols Pub Serv Co } \\
\hline 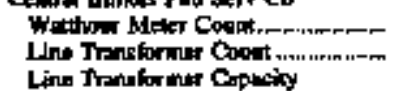 & $\begin{array}{l}341,279 \\
\text { Ing, } 716\end{array}$ & $\begin{array}{r}15,316 \\
2,357\end{array}$ & $\frac{10,732}{2,647}$ & $\begin{array}{l}30,663 \\
108,360\end{array}$ & $\begin{array}{r}|6,67| \\
1,187\end{array}$ & $\begin{array}{l}323,644 \\
\text { III.55 }\end{array}$ & 34: \\
\hline 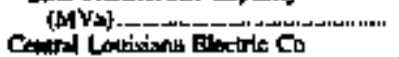 & 4,324 & 86 & 120 & 4,290 & 335 & 3,950 & 5 \\
\hline 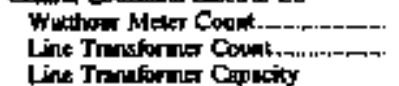 & $\begin{array}{l}240,254 \\
108,146\end{array}$ & $\begin{array}{l}3,502 \\
4,392\end{array}$ & 2.57 & $\begin{array}{l}2 \mathrm{d5}, 119 \\
109,585\end{array}$ & 6,224 & $\begin{array}{l}238,053 \\
105,635\end{array}$ & - \\
\hline 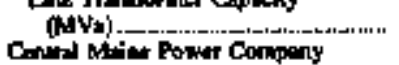 & 3,277 & 375 & 139 & 3317 & 25स) & 3,067 & - \\
\hline 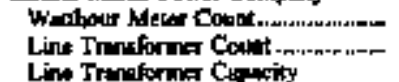 & $\begin{array}{l}34,979 \\
201,919\end{array}$ & $\begin{array}{r}17,121 \\
5,274\end{array}$ & $\begin{array}{r}15,319 \\
2,349\end{array}$ & $\begin{array}{l}545.977 \\
204944\end{array}$ & $\begin{array}{r}23,067 \\
1,000\end{array}$ & $=$ & $\begin{array}{l}522,120 \\
201,944\end{array}$ \\
\hline Ceris). & 4,600 & $J 30$ & 105 & 4,445 & $2 \pi t$ & - & 4,768: \\
\hline 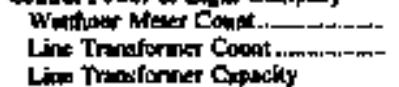 & $\begin{array}{l}618,980 \\
221500\end{array}$ & $\begin{array}{r}9,035 \\
61,145\end{array}$ & $\begin{array}{r}28,657 \\
3,374\end{array}$ & $\begin{array}{l}619,6165 \\
209,276\end{array}$ & $\begin{array}{l}34,352 \\
11,395\end{array}$ & $\begin{array}{l}586,669 \\
217,519\end{array}$ & $\begin{array}{l}645 \\
362\end{array}$ \\
\hline 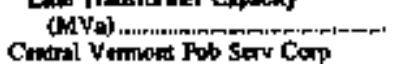 & 8255 & $\$ 75$ & 104 & 8,766 & 769 & 7,959 & 38 \\
\hline 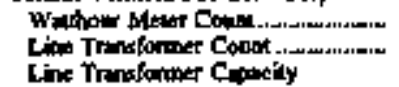 & $\begin{array}{r}183,115 \\
66,041\end{array}$ & $\begin{array}{l}7,991 \\
1,965\end{array}$ & $\begin{array}{l}6,464 \\
1,4] 4\end{array}$ & $\begin{array}{l}\mid 24.610 \\
66.591\end{array}$ & $\begin{array}{r}19,510 \\
1.517\end{array}$ & $\begin{array}{r}16-566 \% \\
64,912\end{array}$ & $\begin{array}{l}142 \\
162\end{array}$ \\
\hline 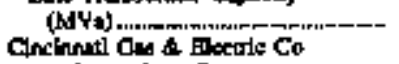 & 1.224 & 36 & 25 & 1,205 & 4 & 1,188 & 3 \\
\hline 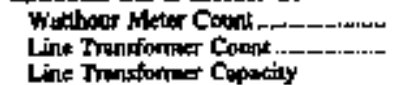 & $\begin{array}{l}675,210 \\
154,910\end{array}$ & $\begin{array}{r}23,567 \\
9,528\end{array}$ & $\begin{array}{l}17,499 \\
4,767\end{array}$ & $\begin{array}{l}6 \$ 1,785 \\
139,67 \mid\end{array}$ & $\begin{array}{l}56 r_{1}, 850 \\
18,879\end{array}$ & $\begin{array}{l}60,534 \\
140,792\end{array}$ & $\longrightarrow$ \\
\hline Cilivent Uillies Conpusy & 9,813 & 520 & 237 & 10,096 & 1.700 & 8,396 & - \\
\hline 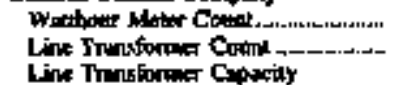 & $\begin{array}{r}306,366 \\
41,464\end{array}$ & $\begin{array}{l}5,732 \\
1,681\end{array}$ & $\begin{array}{r}1,533 \\
4,8\end{array}$ & $\begin{array}{r}110,465 \\
41,674\end{array}$ & $\begin{array}{l}32,911 \\
11,431\end{array}$ & $\begin{array}{l}65,31] \\
25,408\end{array}$ & $\begin{array}{r}12,4,43 \\
5,775\end{array}$ \\
\hline 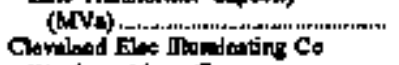 & 1,225 & 65 & 8 & 1,282 & 470 & 651 & 161 \\
\hline 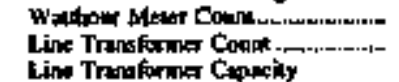 & $\begin{array}{l}803,499 \\
\mid 41 \leq 58\end{array}$ & $\begin{array}{r}11,506 \\
2,147\end{array}$ & $-^{2,081}$ & $\begin{array}{l}84,920 \\
143,705\end{array}$ & $\begin{array}{l}4,1899 \\
2,475\end{array}$ & $\begin{array}{l}840,791 \\
|4|, 230\end{array}$ & - \\
\hline $\begin{array}{l}\text { (hAVh) } \\
\text { Columbos Souldem Powa Co }\end{array}$ & 7.923 & 208 & - & 8,000 & 990 & 7.640 & - \\
\hline 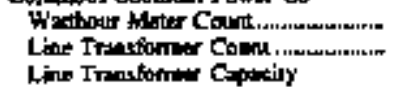 & $\begin{array}{l}628,597 \\
147,141\end{array}$ & $\begin{array}{r}39,605 \\
7,244\end{array}$ & $\begin{aligned} 26569 \\
2565\end{aligned}$ & $\begin{array}{l}\text { fi11929 } \\
151529\end{array}$ & $\begin{array}{l}32045 \\
5127\end{array}$ & 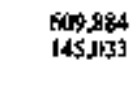 & - 374 \\
\hline 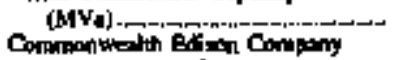 & 6,315 & 318 & 88 & $5, B] 5$ & 458 & 6,328 & 19 \\
\hline 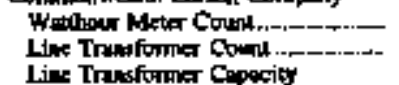 & $\begin{array}{r}3.749,615 \\
52+\$ 8 ?\end{array}$ & $\begin{array}{r}\text { I2t, } 0111 \\
13,644\end{array}$ & $\begin{array}{r}92,951 \\
3,393\end{array}$ & $\begin{array}{r}3,783,675 \\
538,118\end{array}$ & $\begin{array}{r}53,091 \\
7,256\end{array}$ & $\begin{array}{r}3,330,584 \\
530,95\end{array}$ & - \\
\hline 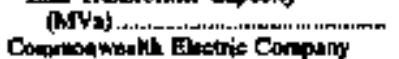 & 35.212 & 913 & 174 & $35, J 61$ & 1,239 & 34,522 & $\rightarrow$ \\
\hline 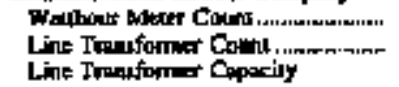 & $\begin{array}{r}394,792 \\
51,2188\end{array}$ & $\begin{array}{l}9,1591 \\
1,749\end{array}$ & $\begin{array}{r}7,249 \\
659\end{array}$ & $\begin{array}{r}316,897 \\
58,904\end{array}$ & $\begin{array}{l}7,646 \\
5,529\end{array}$ & $\begin{array}{r}79, \sqrt{301} \\
53,701\end{array}$ & $\begin{array}{r}196 \\
74\end{array}$ \\
\hline 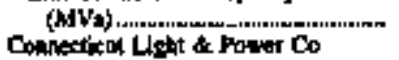 & 2,246 & 39 & 21 & 2.300 & 318 & 5.982 & 4 \\
\hline 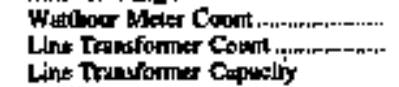 & $\begin{array}{r}1.520,459 \\
224,042\end{array}$ & $\begin{array}{r}95.737 \\
6,289\end{array}$ & 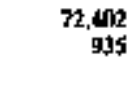 & $\begin{array}{r}1,141,794 \\
229,996\end{array}$ & $\begin{array}{r}52,413 \\
6,624\end{array}$ & $\begin{array}{r}1,091,269 \\
220,362\end{array}$ & $\begin{array}{l}112 \\
410\end{array}$ \\
\hline $\begin{array}{l}\text { (NY) } \\
\text { Connolidgred Bdison Co of NY }\end{array}$ & $t 11,136$ & 319 & 27 & 10,428 & 716 & 9,670 & \&2 \\
\hline 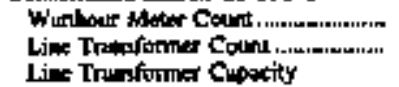 & $\begin{array}{r}3,190,615 \\
79,528\end{array}$ & $\begin{array}{r}74,251 \\
2,664\end{array}$ & $\begin{aligned} 64,997 \\
2,134\end{aligned}$ & 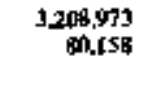 & $\begin{array}{r}211,532 \\
4,7,55\end{array}$ & $\begin{array}{r}2,096,438 \\
75,433\end{array}$ & $\begin{array}{r}90 \\
-\end{array}$ \\
\hline 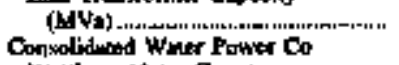 & 23.55 & 5.211 & dSt & 20,557 & 1,122 & 22,455 & - \\
\hline 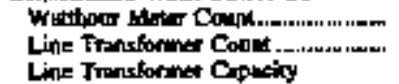 & $\begin{array}{l}1,227 \\
511\end{array}$ & $\begin{array}{r}214 \\
57\end{array}$ & $\begin{array}{r}9 \\
14\end{array}$ & $\begin{array}{r}1,432 \\
5,54\end{array}$ & $\begin{array}{l}305 \\
126\end{array}$ & - & $\begin{array}{r}1,127 \\
428\end{array}$ \\
\hline (HVis) & 10 & 3 & 一 & I3 & $\mathbf{5}$ & - & 8 \\
\hline
\end{tabular}

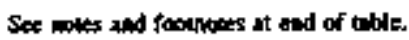


Table 44. Watthour Meter and Line Transformer Statistics of Inpestor-Owned Utilities, 1994 (Coumt and Megavoltampere) (Continued)

\begin{tabular}{|c|c|c|c|c|c|c|c|}
\hline $\begin{array}{l}\text { Instribulloa } \\
\text { Equpanent }\end{array}$ & $\begin{array}{l}\text { Nomber at } \\
\text { Bepinnint } \\
\text { of Your }\end{array}$ & $\begin{array}{c}\text { Adphowy } \\
\text { Derja: } \\
\text { Yeur }\end{array}$ & $\begin{array}{l}\text { Redoctrons } \\
\text { Darinst } \\
\text { Yedr }\end{array}$ & $\begin{array}{l}\text { Npmber } \Delta x \\
\text { End of } \\
\text { Yet }\end{array}$ & $\begin{array}{l}\text { In Sack } \\
\text { Locted, } \\
\text { and Inuctive ] }\end{array}$ & Ongments & In \\
\hline 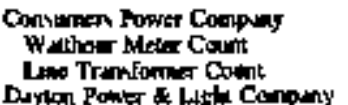 & $\begin{array}{r}1,57,, 3,36 \\
485,816\end{array}$ & $\begin{array}{l}48,251 \\
20,5146\end{array}$ & 34,911 & $\begin{array}{r}1,584,476 \\
490,262\end{array}$ & $\begin{array}{l}30,373 \\
32,814\end{array}$ & 1,545, 4m: & 696 \\
\hline 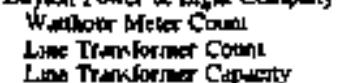 & $\begin{array}{l}\text { Tा16,31? } \\
140,353\end{array}$ & $\begin{array}{r}12,933 \\
4,989\end{array}$ & $\begin{array}{r}28,3,92 \\
1,2017\end{array}$ & $\begin{array}{l}490,918 \\
144,055\end{array}$ & $\begin{array}{r}21.696 \\
2,319\end{array}$ & $\begin{array}{l}468.970 \\
141.736\end{array}$ & 92 \\
\hline 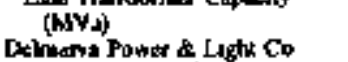 & 6.612 & 258 & 47 & 5,813 & 110 & $6, \pi, 3$ & - \\
\hline $\begin{array}{l}\text { Wuthow beter Coint } \\
\text { line Trantformer Count }\end{array}$ & $\begin{array}{l}412,651 \\
104,306\end{array}$ & $\begin{array}{r}24,189 \\
3,146\end{array}$ & $\begin{array}{r}11,501 \\
1,3,25\end{array}$ & $\begin{array}{l}425,219 \\
106,027\end{array}$ & $\begin{array}{r}25,582 \\
6,564\end{array}$ & $\begin{array}{r}399,450 \\
99,282\end{array}$ & 287 \\
\hline AMV & 4,857 & 142 & 47 & 4,952 & 446 & 4,503 & 3 \\
\hline 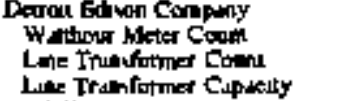 & $\begin{array}{r}7.158,902 \\
365.791\end{array}$ & 67,348 & $\begin{array}{l}5, \mathrm{ABR} \\
\text { S,Bftg }\end{array}$ & $\begin{array}{r}2,419,362 \\
386,707\end{array}$ & $\begin{array}{r}71,049 \\
\text { Fin, } 40,9\end{array}$ & $\begin{array}{r}2,346,551 \\
37,5241\end{array}$ & $\begin{array}{l}1,752 \\
2349\end{array}$ \\
\hline $\begin{array}{l}\text { (MVV) } \\
\text { Dele Power Compuny }\end{array}$ & 20,834 & 1,757 & 200 & 22.352 & 741 & 21,547 & 94 \\
\hline 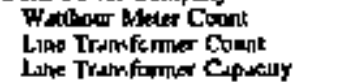 & $\begin{array}{l}1.846,049 \\
604,513\end{array}$ & $\begin{array}{l}64,082 \\
21,0808\end{array}$ & 55,1115 & $\begin{array}{r}1,859.116 \\
6190051\end{array}$ & $\begin{array}{l}70,363 \\
17,803\end{array}$ & $\begin{array}{l}1.786,096 \\
600,6202\end{array}$ & $\begin{array}{r}2,657 \\
375\end{array}$ \\
\hline (N1Ya) & 34,420 & 1.121 & 261 & 15,2807 & 1,379 & 31,882 & 19 \\
\hline 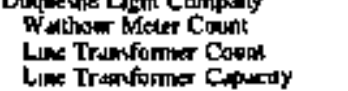 & $\begin{array}{l}621,304 \\
100,481\end{array}$ & $\begin{array}{r}16,1239 \\
1.965\end{array}$ & 14.401 & $\begin{array}{l}6,72,75 \\
101,445\end{array}$ & $\begin{array}{l}7,120 \\
2,210\end{array}$ & $\overline{-}$ & $\begin{aligned} 615,618 \\
99203\end{aligned}$ \\
\hline 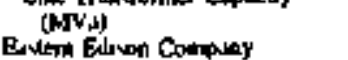 & 3,163 & 120 & 48 & 5,215 & 81 & - & 5,134 \\
\hline 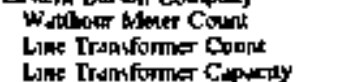 & X1, & $1,2,201$ & 3,596 & $2 \operatorname{lng} 107$ & $\begin{array}{l}3,0015 \\
3,437\end{array}$ & $\begin{aligned} 20,29 \\
29,589\end{aligned}$ & $\overline{-}$ \\
\hline $\begin{array}{l}\text { (WVA) } \\
\text { Bdison Sadt Electic Company }\end{array}$ & 1,596 & $m$ & 32 & 1,614 & 146 & 1,438 & - \\
\hline 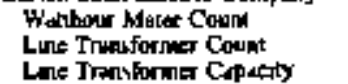 & $\begin{array}{l}21, \operatorname{tgn} \\
8, \ln 4\end{array}$ & $\begin{array}{l}6798 \\
287\end{array}$ & $\begin{array}{l}15010 \\
36\end{array}$ & $\begin{array}{r}21,682 \\
8,359\end{array}$ & $\begin{array}{l}393 \\
715\end{array}$ & $\begin{array}{r}21,255 \\
7,609\end{array}$ & 31 \\
\hline $\begin{array}{l}\text { (MVA) } \\
\text { E Pusa Blectrus Colmpuny }\end{array}$ & 259,370 & 9,887 & $1,6 \mathrm{~T}$ & 267.579 & 36,617 & 230,054 & BBS \\
\hline Wulpout Meter Coun & $\begin{array}{r}299,439 \\
64,454\end{array}$ & $\begin{array}{l}9,218 \\
3,975\end{array}$ & 2,481 & $\begin{array}{l}305,276 \\
66,412\end{array}$ & $\begin{array}{l}6,211 \\
1,476\end{array}$ & $\begin{array}{r}298,965 \\
64,80 x\end{array}$ & $\begin{array}{l}1+50 \\
130\end{array}$ \\
\hline 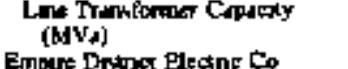 & 26535 & 99 & $\Gamma$ & 2,754 & J21 & 2,632 & I \\
\hline 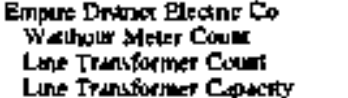 & $\begin{array}{r}137,766 \\
65,457\end{array}$ & $\begin{array}{l}8,791 \\
3,262\end{array}$ & $\begin{array}{r}2,215 \\
690\end{array}$ & $\begin{array}{r}141,282 \\
6 \%, 029\end{array}$ & $\begin{array}{l}17,311 \\
3,514\end{array}$ & $\begin{array}{r}126,97,3,3 \\
64,455\end{array}$ & -130 \\
\hline 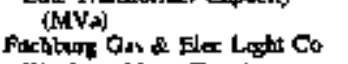 & 2,128 & 199 & 17 & 2,310 & 1,12 & 2,200 & II \\
\hline 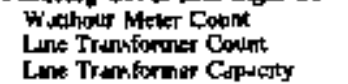 & $\begin{array}{r}26,563 \\
5,6575\end{array}$ & $\begin{array}{l}636 \\
121\end{array}$ & 806 & $\begin{array}{l}26,391 \\
5,666\end{array}$ & $\begin{array}{r}5,673 \\
319\end{array}$ & 24,712 & 5.39 \\
\hline $\begin{array}{l}\text { (MVA) } \\
\text { Flond Puntar \& Ligut Company }\end{array}$ & J64 & 1 & 4 & 167 & $\mathbf{2 0}$ & - & 147 \\
\hline 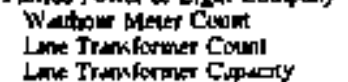 & $\begin{array}{r}3,516,847 \\
672,644\end{array}$ & $\begin{array}{r}146,059 \\
25.942\end{array}$ & $\begin{array}{r}106,6036 \\
17.524\end{array}$ & $\begin{array}{r}3,656,269 \\
68,1,060\end{array}$ & 209, & $\begin{array}{l}1,4 \% 6,098 \\
672,202\end{array}$ & $\begin{array}{r}367 \\
1,498\end{array}$ \\
\hline $\begin{array}{l}\text { (HVa) } \\
\text { Flond Power Corporston }\end{array}$ & 36,631 & 1,600 & 9 & $3\} .262$ & 846 & 36,300 & 115 \\
\hline 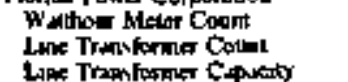 & 1.369,774 & $\begin{array}{l}34,657 \\
13,275\end{array}$ & 29,411 & $\begin{array}{l}1,368,527,7 \\
314,959\end{array}$ & $\begin{array}{l}\text { S.,ATR } \\
4.61 ? \text { ? }\end{array}$ & $1,284,590$ & 3012 IT2 \\
\hline (MVa) & 13,725 & 325 & 576 & 13,894 & 390 & 一 & 13,4 ts \\
\hline 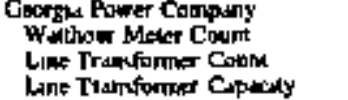 & $\begin{array}{l}1.793,128 \\
582,750\end{array}$ & $\begin{array}{l}45,012 \\
58,10 \times,\end{array}$ & $\begin{array}{l}35, \sqrt{155} \\
6,65]\end{array}$ & $\begin{array}{r}1,903,0 \$ 55 \\
594,012\end{array}$ & $\begin{array}{l}95,001 \\
25,897\end{array}$ & 1.708.104 & $\overline{-}$ \\
\hline 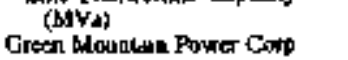 & 28,fi64 & 1,494 & 165 & 29,793 & 2,027 & 23,166 & - \\
\hline 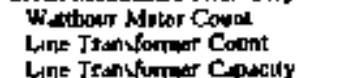 & $\begin{array}{l}84,426 \\
10,152\end{array}$ & $\begin{array}{r}1.860 \\
6,36\end{array}$ & $\begin{array}{r}1,167 \\
988\end{array}$ & $\begin{array}{l}85,119 \\
0,690\end{array}$ & $\begin{array}{l}7.463 \\
1.249\end{array}$ & $\begin{array}{ll}1 \\
21,541\end{array}$ & IIs \\
\hline$\left(M V_{d}\right)$ & 967 & 35 & a & b. 0072 & fis & 995 & 2 \\
\hline
\end{tabular}

Soe polet and foctrokes at ead of able 
Table 44. Watthonr Melex and Line Transformer Stotistics of Investor-Owned Utlities, 1994 (Coumk and Megavoltumpere) (Continued)

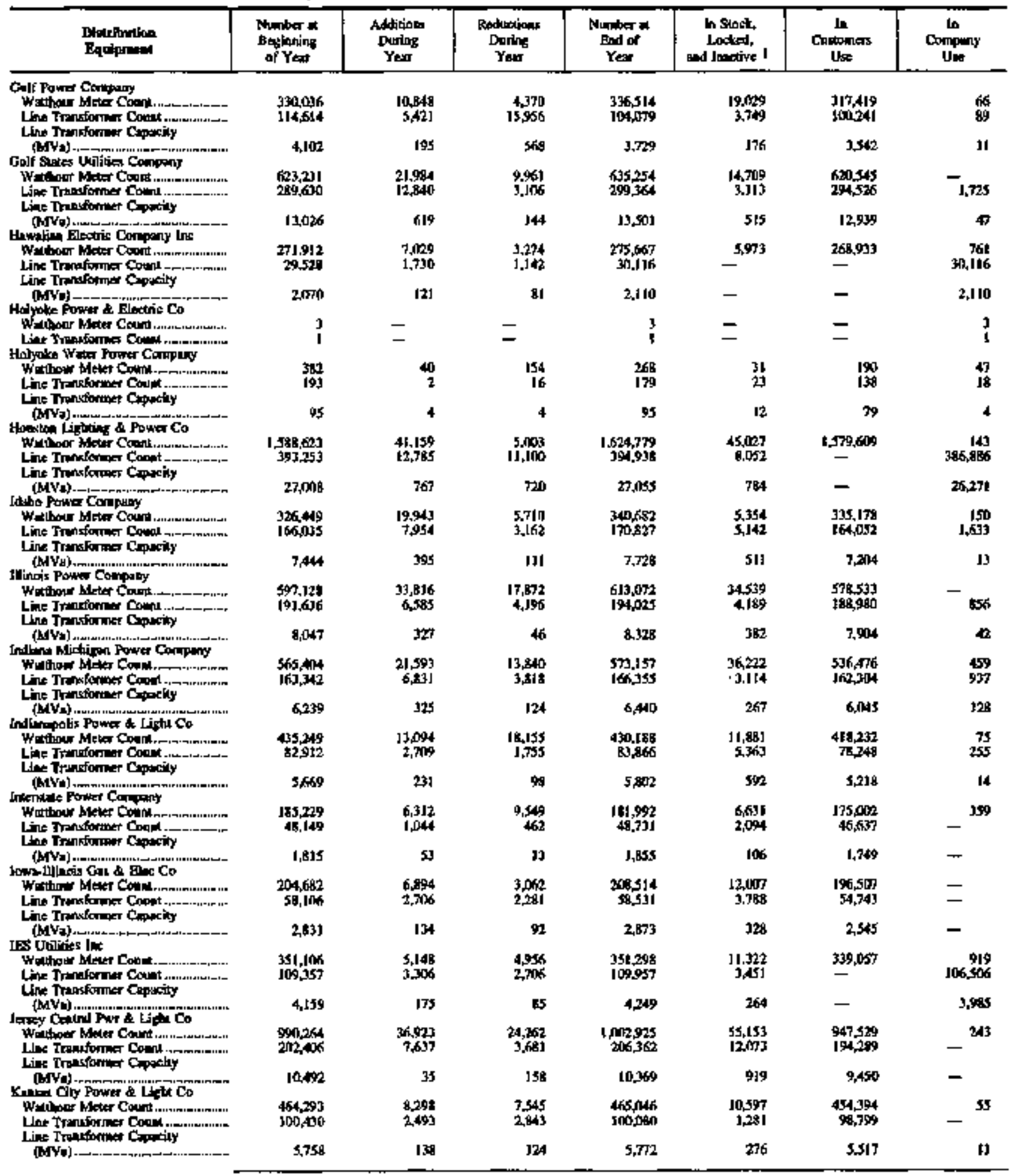

See nolss ind formotes at and of bojte. 
Table 44. Watthour Meter and Line Transformer Stalistics of Investor-Owned Utilities, 1994 (Count and Megavoltampere) (Contunued)

\begin{tabular}{|c|c|c|c|c|c|c|c|}
\hline 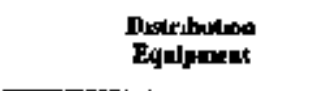 & $\begin{array}{l}\text { Nambar an } \\
\text { Bqumuni } \\
\text { of Ytut }\end{array}$ & $\begin{array}{l}\text { Adkintrosh } \\
\text { Dhank } \\
\text { Yeut }\end{array}$ & 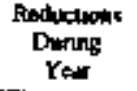 & 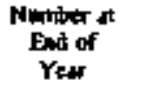 & 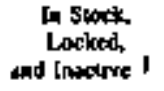 & 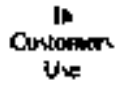 & $\begin{array}{l}\text { In } \\
\text { Cotmpany } \\
\text { Use }\end{array}$ \\
\hline \multicolumn{8}{|l|}{ Kands Gd4 \& Elestint Co } \\
\hline 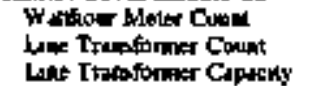 & $\begin{array}{l}275,206 \\
\text { ys.012 }\end{array}$ & $\begin{array}{l}6,72 B \\
2,774\end{array}$ & $\begin{array}{l}1,46] \\
1,494\end{array}$ & $\begin{array}{r}29,073 \\
99,302\end{array}$ & $\frac{3,801}{2,7018}$ & $\begin{array}{r}270,272 \\
94,424\end{array}$ & -170 \\
\hline $\begin{array}{l}\text { (WVa) } \\
\text { Kenlucty Pont Compeng }\end{array}$ & 4,501 & 159 & 47 & 4,611 & 269 & 4,322 & 24 \\
\hline 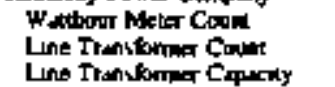 & $\begin{array}{l}130,4561 \\
81,671\end{array}$ & $\begin{array}{l}6,284 \\
4,088\end{array}$ & $\begin{array}{l}4.219 \\
1.566\end{array}$ & $\begin{array}{r}172521 \\
\text { BA201? }\end{array}$ & $\begin{array}{l}2,431 \\
2,525\end{array}$ & $\begin{array}{r}164037 \\
\text { BIS13 }\end{array}$ & 53 \\
\hline $\begin{array}{l}\text { (MVA) } \\
\text { Keameley Uulition Cumpeny }\end{array}$ & 2,497 & 152 & $46 \mathrm{~F}$ & 2, & [5] & 2,450 & 2 \\
\hline 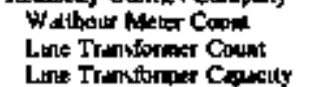 & $\begin{array}{l}000,492 \\
182,424\end{array}$ & $\begin{array}{r}14,104 \\
6,549\end{array}$ & 12.579 & $\begin{array}{l}\sin 217 \\
189,968\end{array}$ & $\begin{array}{r}41,175 \\
6,211\end{array}$ & $\begin{array}{l}460,254 \\
-\end{array}$ & $\begin{array}{r}788 \\
382,737\end{array}$ \\
\hline $\begin{array}{l}\text { (BYYd) } \\
\text { Xhowpon Powt Condpuny }\end{array}$ & 6,152 & 283 & 1 & 6,672 & 722 & - & 5,910 \\
\hline 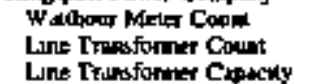 & $\begin{array}{l}42,137 \\
13.413\end{array}$ & $\begin{array}{l}714 \\
512\end{array}$ & $\begin{array}{l}182 \\
281\end{array}$ & $\begin{array}{l}\text { 47.56ig } \\
13, \mathrm{fiR}_{2}\end{array}$ & $\begin{array}{r}1,394 \\
436\end{array}$ & $\begin{array}{l}49,9+9 \\
13,238\end{array}$ & $\begin{array}{r}16 \\
8\end{array}$ \\
\hline $\begin{array}{l}\text { (bys) } \\
\text { Lockhn Powar Compeny }\end{array}$ & 372 & 28 & 11 & 589 & จ1 & 557 & I \\
\hline 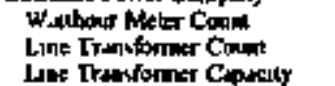 & $\begin{array}{l}5.793 \\
2.927\end{array}$ & 96 & -56 & 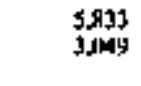 & $\begin{array}{l}204 \\
156\end{array}$ & $\begin{array}{l}5,594 \\
2,893\end{array}$ & $-\quad 35$ \\
\hline (MVL) & 84 & 8 & - & 92 & 6 & 36 & - \\
\hline 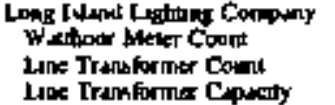 & $\begin{array}{l}1.156,704 \\
103,095\end{array}$ & $\begin{array}{r}43,521 \\
4,594\end{array}$ & $\begin{array}{r}33,947 \\
2,625\end{array}$ & $\begin{array}{l}1.166,578 \\
185,064\end{array}$ & $\begin{array}{l}49,566 \\
29.797\end{array}$ & $1,006,381$ & $\begin{array}{l}110.431 \\
155.327\end{array}$ \\
\hline 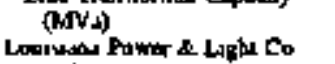 & 30,065 & Joj & 163 & If, 200 & $2, \operatorname{man}$ & - & 7,897 \\
\hline 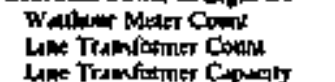 & $\begin{array}{l}657.685 \\
187.162\end{array}$ & $\begin{array}{r}16,183 \\
5,875\end{array}$ & $\begin{array}{r}13,175 \\
4,404\end{array}$ & $\begin{array}{l}646,693 \\
198553\end{array}$ & $\begin{array}{l}56,285 \\
12,594\end{array}$ & $\begin{array}{l}120,378 \\
174,398\end{array}$ & $\begin{array}{r}30 \\
1.681\end{array}$ \\
\hline $\begin{array}{l}\text { (MV) } \\
\text { Loossplle Gat \& Eloctinc Co }\end{array}$ & $\$, 2159$ & 136 & 209 & 9,416 & 1.201 & 7.979 & 513 \\
\hline 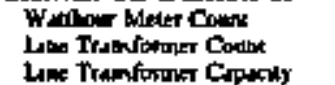 & $\begin{array}{r}361,873 \\
78,264\end{array}$ & $\begin{array}{l}1,156 \\
1,528\end{array}$ & $\begin{array}{l}9.019 \\
3.714\end{array}$ & $\begin{array}{r}354,0389 \\
76,178\end{array}$ & $\begin{array}{r}20,2882 \\
1,352\end{array}$ & $\begin{array}{r}333,542 \\
74,826\end{array}$ & -114 \\
\hline 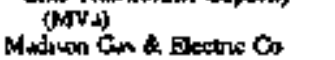 & 4,516 & $\mathbf{t 1 8}$ & LA2 & 4,242 & $x$ & 4,216 & - \\
\hline 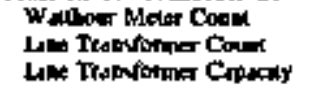 & $\begin{array}{r}119,530 \\
18,85 ?\end{array}$ & $\begin{array}{r}2.738 \\
146\end{array}$ & $\begin{array}{l}\text { 856 } \\
\text { t9l }\end{array}$ & $\begin{array}{r}121,192 \\
19,112\end{array}$ & $\begin{array}{l}3,5 \text { स: } \\
1,482\end{array}$ & $\begin{array}{r}117,973 \\
17,968\end{array}$ & $\begin{array}{l}78 \\
62\end{array}$ \\
\hline $\begin{array}{l}\text { (MV) } \\
\text { Mune Publese Sering Company }\end{array}$ & קובוב & 44 & 7 & 1,247 & 113 & 1.129 & $\mathbf{I}$ \\
\hline $\begin{array}{l}\text { Walloor Mut Cown } \\
\text { Lne Trunformer Count }\end{array}$ & $\begin{array}{l}35,656 \\
19.716\end{array}$ & 1,977 & 2,150 & $\begin{array}{l}35,483 \\
11,882\end{array}$ & $\begin{array}{l}1,441 \\
\sin \end{array}$ & $\begin{array}{l}33,658 \\
11,173\end{array}$ & $-\quad 152$ \\
\hline 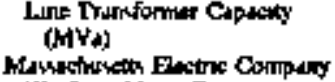 & 232,860 & $B, 231$ & 2341 & 238.710 & $26,5 \mathrm{BH}$ & 21t,809 & - \\
\hline 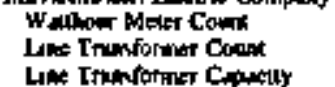 & $\begin{array}{r}1,012,065 \\
\text { J47,090 }\end{array}$ & $\begin{array}{r}24.5501 \\
1.931\end{array}$ & $\begin{array}{r}3,7,746 \\
2,003\end{array}$ & $\begin{array}{l}1,192, \sin 0 \\
146,598\end{array}$ & $\begin{array}{l}34,5212 \\
10,204\end{array}$ & $\begin{array}{l}967,895 \\
137 B>1\end{array}$ & 251 \\
\hline $\begin{array}{l}\text { (MV) } \\
\text { Man Eletinc Cornpiny lumited }\end{array}$ & 1.4t9 & 139 & $T \%$ & $3,54 \mathrm{t}$ & 14 & 6.409 & 198 \\
\hline 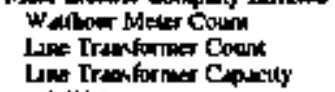 & $\begin{array}{l}55,797 \\
9,557\end{array}$ & $\begin{array}{r}1.875 \\
612\end{array}$ & 393 & $\begin{array}{r}5,239 \\
9,926\end{array}$ & $\begin{array}{r}57,239 \\
9,526\end{array}$ & - & - \\
\hline 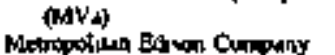 & 197 & 40 & 9 & $\mathbf{s} 8$ & 528 & - & - \\
\hline 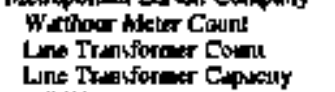 & $\begin{array}{l}497,059 \\
150,697\end{array}$ & $\begin{array}{l}9.911 \\
5531\end{array}$ & $\begin{array}{r}14,002 \\
1,658\end{array}$ & $\begin{array}{l}49,098 \\
154,610\end{array}$ & 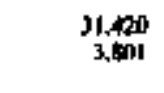 & $\begin{array}{l}4,0,660 \\
150,799\end{array}$ & -18 \\
\hline 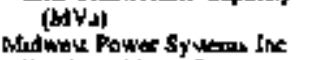 & 1,980 & 235 & 101 & 6,115 & 370 & 5,756 & 11 \\
\hline 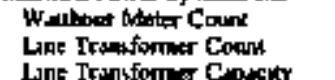 & $\begin{array}{l}450,730 \\
115,504\end{array}$ & $\begin{array}{r}20,787 \\
7.153\end{array}$ & $\begin{array}{l}9.752 \\
1.1+5\end{array}$ & $\begin{array}{l}461,765 \\
141,579\end{array}$ & $\begin{array}{r}17.787 \\
3.167\end{array}$ & $\begin{array}{l}44978 \\
139.412\end{array}$ & - \\
\hline $\begin{array}{l}\text { Dint } \\
\text { (MYAl) }\end{array}$ & 6,6885 & 242 & 47 & $6,9 \% 0$ & 271 & $6.7 \mathrm{~m}$ & - \\
\hline
\end{tabular}

See nodes abd froponolev al end af thble 
Table 44. Watthour Metor and Line Transformer Statistics of Investor-Owned Utilities, 1994 (Connt and Megavoltampere) (Contunued)

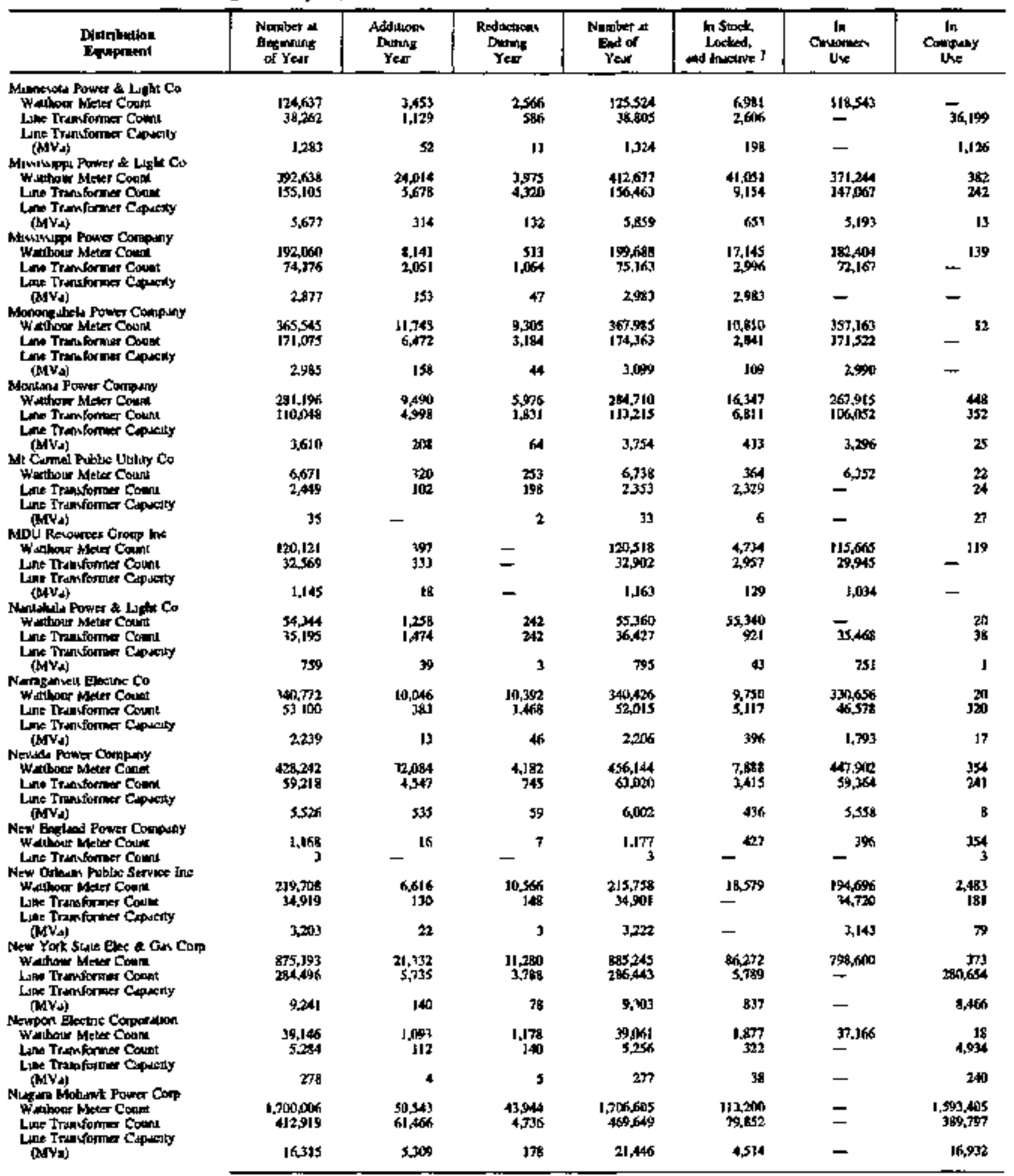

Seo notas and footendes at and of tite 
Talble 44. Watthour Meter and Line Transformer Statktics of Investor-Owned Uijlties, 1994 (Count and Megavolampere) (Continued)

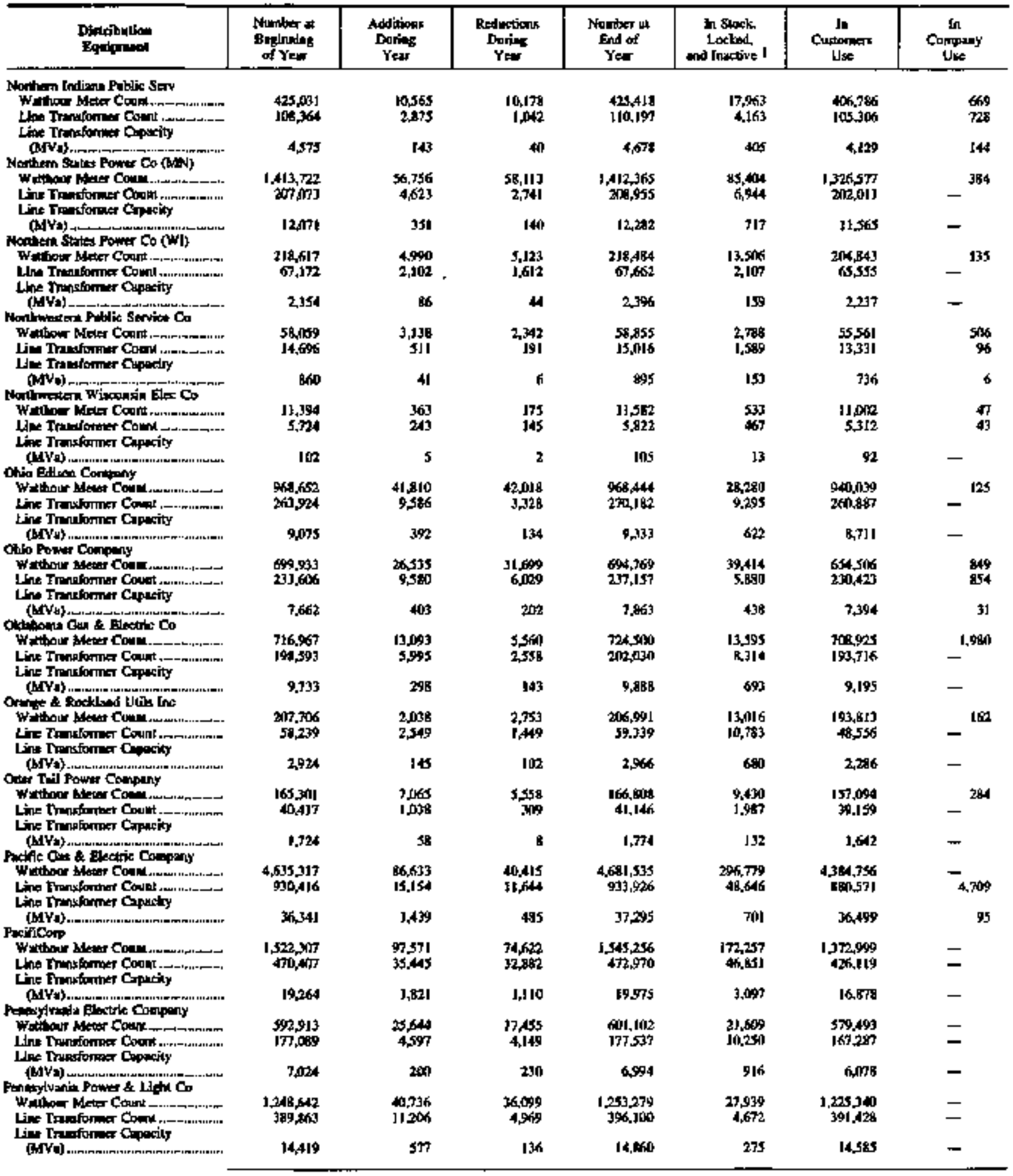

Ser arex and footonoces at ond of wble. 
Talole 44. Watthonr Meter and Line Transformer Statistics of lnvestor-Owned U1titifes, 1994 (Count and Megavodtampers) (Continned)

\begin{tabular}{|c|c|c|c|c|c|c|c|}
\hline $\begin{array}{l}\text { Diturifurtion } \\
\text { Equlpineat }\end{array}$ & $\begin{array}{l}\text { Nwabe at } \\
\text { Bepinimg } \\
\text { of Yin }\end{array}$ & $\begin{array}{l}\text { Adticions } \\
\text { Dering } \\
\text { Yturt }\end{array}$ & $\begin{array}{l}\text { Redoctions } \\
\text { During } \\
\text { Yey }\end{array}$ & $\begin{array}{l}\text { Nuniber } * \\
\text { End oll } \\
\text { Year }\end{array}$ & $\begin{array}{l}\text { In Stock } \\
\text { Loched, } \\
\text { whd Inuotine । }\end{array}$ & $\underset{\substack{\text { In } \\
\text { tones }}}{\cos }$ & $\underset{\substack{\text { In } \\
\text { Uarming }}}{ }$ \\
\hline \multicolumn{8}{|l|}{ Ptenylwasla Pown Coroping } \\
\hline 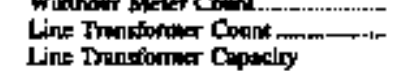 & 54,365 & $\begin{array}{l}3,68,4 \\
1,597\end{array}$ & $\begin{array}{l}3.491 \\
1,412\end{array}$ & 54,550 & 1,820. & 52,72 & - \\
\hline 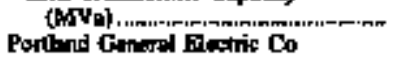 & 1.489 & 64 & 34 & 1523 & 919 & 1,425 & - \\
\hline 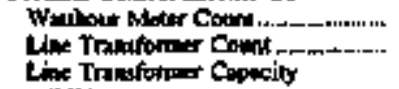 & $\begin{array}{l}656,3399 \\
363,499\end{array}$ & 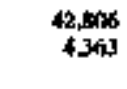 & $\operatorname{mA2I}$ & $\begin{array}{l}661.723 \\
165,225\end{array}$ & $\begin{array}{r}14,355 \\
6,605\end{array}$ & $\begin{array}{l}647,245 \\
{[58,390}\end{array}$ & 119 \\
\hline 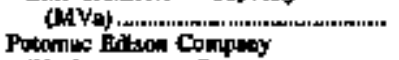 & 7,285 & 20 & 89 & 7,476 & $\$ 52$ & 2,004 & $m$ \\
\hline 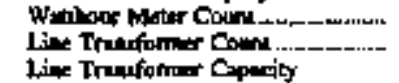 & $\begin{array}{l}373,152 \\
159,570\end{array}$ & $\frac{26,396}{5678}$ & $\begin{array}{l}13,9] 2 \\
3011\end{array}$ & $\begin{array}{l}3155376 \\
162,207\end{array}$ & $\begin{array}{l}16,0044 \\
5,15 \div 0\end{array}$ & 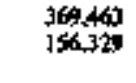 & $\begin{array}{l}33 \\
29\end{array}$ \\
\hline Potomet Electric Powar Compiny & 4,898 & 230 & 39 & 5,041 & 323 & 4,712 & 6 \\
\hline 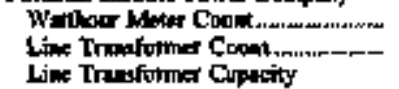 & $\begin{array}{l}710,178 \\
10,515\end{array}$ & $\begin{array}{r}21,022 \\
1519\end{array}$ & $\begin{array}{r}J 1.231 \\
563\end{array}$ & $\begin{array}{l}719099 \\
304,471\end{array}$ & $\begin{array}{l}99,359 \\
\text { InTht: }\end{array}$ & $\begin{array}{r}6.0,610 \\
9.6 .469\end{array}$ & $\overrightarrow{-}$ \\
\hline 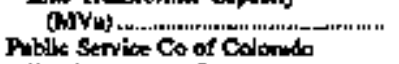 & 12,193 & 200 & 62 & 12,391 & 1,315 & 11,016 & - \\
\hline 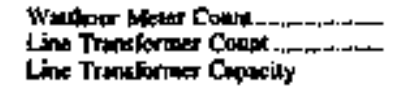 & $\begin{array}{l}1,003,207 \\
1,1,748\end{array}$ & 64,14h & 27,095 & $\begin{array}{r}1,0,52,332 \\
1735866\end{array}$ & $\begin{array}{r}4,9633 \\
7,016\end{array}$ & $1,0001,369$ & $\overline{16 \text { if } 3700}$ \\
\hline Pablic Servec Co of Nti & 9,010 & 324 & 145 & 9.789 & 162 & - & 9.227 \\
\hline 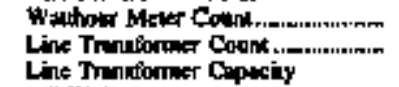 & $\begin{array}{l}505,367 \\
124,344\end{array}$ & $\begin{array}{r}10,203 \\
2,033\end{array}$ & $\begin{array}{l}7,304 \\
1,445\end{array}$ & $\begin{array}{l}508,466 \\
126,252\end{array}$ & $\begin{array}{r}26,596 \\
3,015\end{array}$ & $\begin{array}{l}48], 578 \\
123,237\end{array}$ & -2 \\
\hline 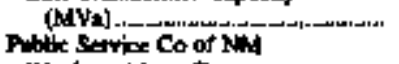 & $4,3: 2$ & 125 & 51 & 4,456 & $2 \% 9$ & 4,167 & - \\
\hline 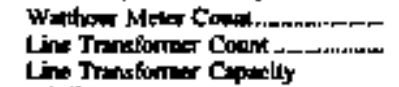 & $\begin{array}{l}30,217 \\
69,083\end{array}$ & $\begin{array}{r}840 \\
4215\end{array}$ & $\begin{array}{r}6,467 \\
147\end{array}$ & $\begin{array}{c}34,650 \\
12,951\end{array}$ & $\begin{array}{l}3,361 \\
2,0115\end{array}$ & $\begin{array}{r}32,, 285 \\
20,780\end{array}$ & $\begin{array}{r}86 \\
156\end{array}$ \\
\hline 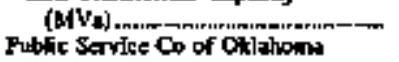 & 2,916 & ]和 & 16 & 3,060 & 128 & $2 ; 9$ & $\boldsymbol{2}$ \\
\hline 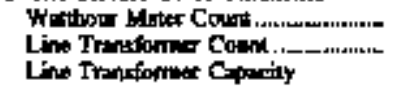 & $\begin{array}{l}493,717 \\
142,869\end{array}$ & $\begin{array}{l}6,723 \\
1,947\end{array}$ & $\begin{array}{l}17.732 \\
1.201\end{array}$ & $\begin{array}{l}46,705 \\
143,615\end{array}$ & $\bar{m}$ & $\begin{array}{l}482,798 \\
14,615\end{array}$ & - \\
\hline $\begin{array}{l}\text { (MYa) r. } \\
\text { Foblic Sarvice Elec \& Ges Co }\end{array}$ & $x, 394$ & 99 & 58 & 6,435 & - & 6,435 & - \\
\hline 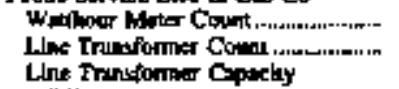 & $\begin{array}{r}1,966,2137 \\
304,757\end{array}$ & $\begin{array}{r}50,342 \\
6,577\end{array}$ & $\begin{array}{r}34,842 \\
4,524\end{array}$ & $\begin{array}{r}1,982,933 \\
\text { jof, } 110\end{array}$ & $\begin{array}{r}74,106 \\
3,382\end{array}$ & $\begin{array}{r}1,500,798 \\
300,728\end{array}$ & $\rightarrow^{218}$ \\
\hline 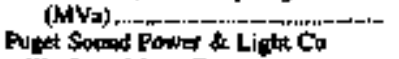 & 19,966 & $6 \%$ & 54 & 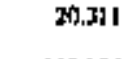 & 478 & I9.813 & - \\
\hline 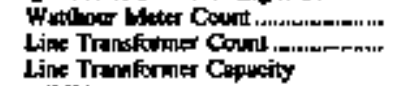 & $\begin{array}{l}802, \text { (40,0) } \\
251, \text { (1000 }\end{array}$ & $\begin{array}{r}34,248 \\
6,235\end{array}$ & $\begin{array}{l}8,902 \\
2, \operatorname{Bg} 29\end{array}$ & $\begin{array}{l}907,976 \\
254,446\end{array}$ & 48,760 & $\begin{array}{l}689,171 \\
249,156\end{array}$ & $\begin{array}{l}145 \\
116\end{array}$ \\
\hline 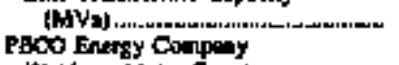 & 10,684 & 285 & 5 & 10,676 & 120 & 10,516 & 15 \\
\hline 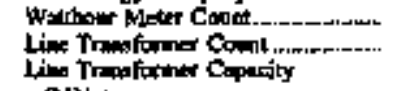 & $\begin{array}{l}\text { 1.758,M6t } \\
166,9641\end{array}$ & $\begin{array}{r}36,5,578 \\
5,54,8\end{array}$ & M.AT0 & $\begin{array}{r}1,762,900 \\
171,637\end{array}$ & 189.573 & $\begin{array}{l}157,168 \\
\text { I64, }\end{array}$ & $\begin{array}{l}168 \\
3 \pi 0\end{array}$ \\
\hline PGI Butg) & 11.442 & 441 & 49 & 11,830 & 719 & $1], 04$ & 34 \\
\hline 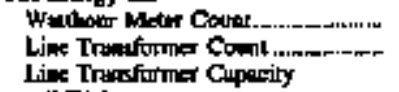 & $\begin{array}{l}667,597 \\
137,949\end{array}$ & $\begin{array}{r}25,791 \\
4, \pi \times n\end{array}$ & $\begin{array}{r}19,6537 \\
3,7 / 77\end{array}$ & $\begin{array}{l}673.531 \\
158.348\end{array}$ & $\begin{array}{r}10,558 \\
4,76\end{array}$ & $\begin{array}{l}622,276 \\
187,794\end{array}$ & 637 \\
\hline 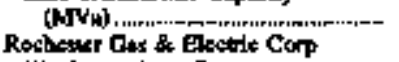 & 8,100 & 255 & 140 & 8,297 & 431 & 7.844 & $\boldsymbol{n}$ \\
\hline 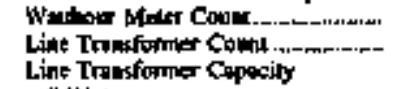 & $\begin{array}{r}358,529 \\
73,985\end{array}$ & $\begin{array}{l}679 \\
1,979\end{array}$ & - sil & $\begin{array}{r}359.306 \\
75.459\end{array}$ & $\begin{array}{r}11,516 \\
\text { A.160 }\end{array}$ & $\begin{array}{r}361.534 \\
67.299\end{array}$ & -26 \\
\hline 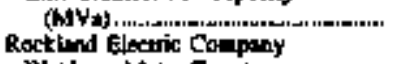 & 2,899 & 67 & 12 & 2,974 & 505 & 2,471 & - \\
\hline 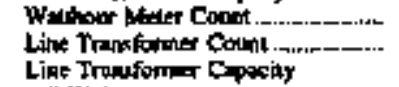 & $\begin{array}{l}68,438 \\
20,593\end{array}$ & $\begin{array}{l}347 \\
522\end{array}$ & $\begin{array}{l}\text { Bal } \\
\text { tint }\end{array}$ & $\begin{array}{l}67,944 \\
20,715\end{array}$ & $\begin{array}{l}3,928 \\
2,7 \% 5\end{array}$ & $\begin{array}{l}64.016 \\
13.920\end{array}$ & It \\
\hline 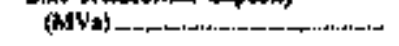 & 1,034 & $\mathbf{3 0}$ & $\mathbf{2 1}$ & $\mathbf{I}, 049$ & 169 & $\mathbf{8 7 4}$ & 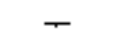 \\
\hline
\end{tabular}

Ser noies and fournater at end of bable, 
Table 44. Wattbons Meter and Line Transformer Statistics of Investor-Owned Utitities, 1994 (Count and Megavoltampere) (Continued)

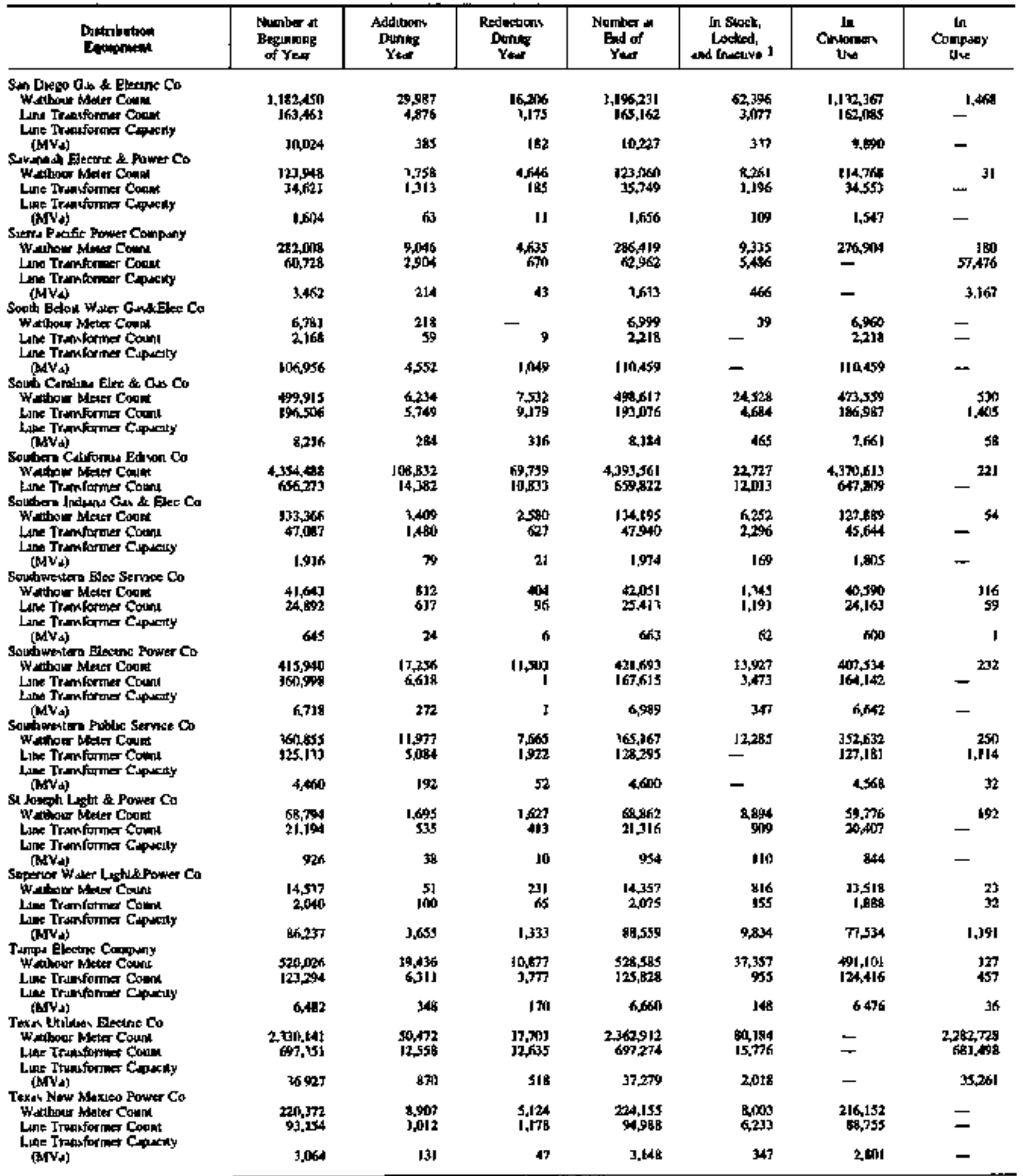

See noles and foolnotes st ead of thbt 
Table 44. Watthoor Meter and Line Transformer Stattsties of Investor-Owned Utllities, 1994 (Count and Megavoltampere) (Continued)

\begin{tabular}{|c|c|c|c|c|c|c|c|}
\hline 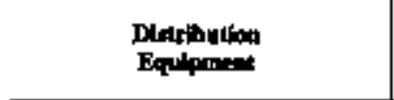 & $\begin{array}{l}\text { Nuriberit } \\
\text { Beglesing } \\
\text { of Year }\end{array}$ & $\begin{array}{l}\text { Additions } \\
\text { Durings } \\
\text { Yent }\end{array}$ & $\begin{array}{c}\text { Redections } \\
\text { Dupins } \\
\text { Year }\end{array}$ & $\begin{array}{l}\text { Nunber at } \\
\text { Fod of } \\
\text { Yed }\end{array}$ & $\begin{array}{l}\text { In Stoosk } \\
\text { Lasted, } \\
\text { andive I }\end{array}$ & 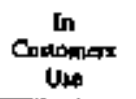 & $\begin{array}{c}\text { Io } \\
\text { Compony } \\
\text { Ulye }\end{array}$ \\
\hline 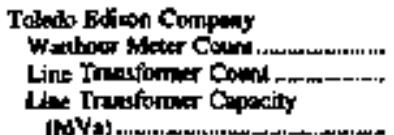 & $\begin{array}{l}304,960 \\
74,655\end{array}$ & $\begin{array}{l}6 \sin 0 \\
2,356 \\
149\end{array}$ & 23,603 & $\begin{aligned} 287,657 \\
n .191\end{aligned}$ & $\begin{array}{l}8,413 \\
2,441\end{array}$ & $\begin{array}{r}279,444 \\
74,750\end{array}$ & $\underline{-}$ \\
\hline 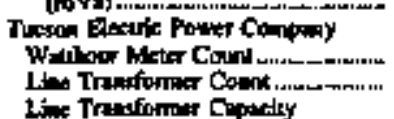 & $\begin{array}{r}306,538 \\
61,940\end{array}$ & $\begin{array}{l}11,535 \\
1,8,14\end{array}$ & $\begin{array}{l}4,142 \\
496\end{array}$ & $\begin{array}{l}31 \mathrm{~J}, 952 \\
02,768\end{array}$ & $\begin{array}{l}2.534 \\
2,469\end{array}$ & $\begin{array}{l}311,394 \\
60,390\end{array}$ & $=$ \\
\hline 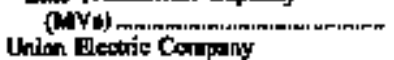 & 3,759 & 12) & \$4 & 3,849 & 234 & 3,619 & - \\
\hline 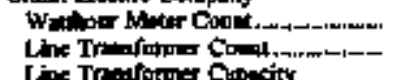 & $\begin{array}{r}1,293,591 \\
276, \pi 22\end{array}$ & $\begin{array}{r}41,456 \\
3,993\end{array}$ & $\begin{array}{r}17,018 \\
3,119\end{array}$ & $\begin{array}{r}1,317,989 \\
279,496\end{array}$ & 14,756 & $\frac{1,301,225}{243,257}$ & - \\
\hline 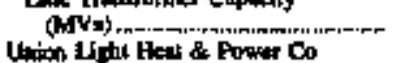 & 15,231 & 46 & 24 & 15,433 & 3,562 & 11,830 & 3 \\
\hline 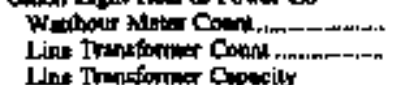 & 27.203 & 5,715 & $\begin{array}{r}5,697 \\
9801\end{array}$ & $\begin{array}{r}124,741 \\
n 7,602\end{array}$ & $\begin{array}{l}7,539 \\
1,412\end{array}$ & $\overline{-}$ & $\frac{112,152}{26,160}$ \\
\hline (ANP) & 1,482 & 9 & 44 & 1,337 & 10: & - & 1A29 \\
\hline 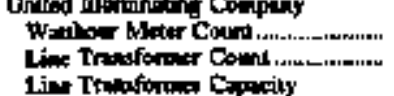 & $\begin{array}{l}326,645 \\
57,304\end{array}$ & $\begin{array}{r}16,547 \\
1,970\end{array}$ & $\begin{array}{l}16,146 \\
1,918\end{array}$ & $\begin{array}{l}327.146 \\
57,560\end{array}$ & $\begin{array}{l}24.790 \\
2511\end{array}$ & $\begin{aligned} 302,089 \\
34,476\end{aligned}$ & $\begin{array}{l}354 \\
179\end{array}$ \\
\hline 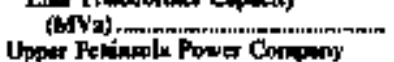 & 3,532 & $\operatorname{la}$ & 80 & 3,154 & 317 & 0,226 & I) \\
\hline 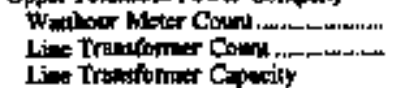 & $\begin{array}{l}62737 \\
19799\end{array}$ & $\begin{array}{l}3015 \\
301\end{array}$ & 1,127 & $\begin{array}{l}62.415 \\
19,772\end{array}$ & $\begin{array}{r}2543 \\
511\end{array}$ & $\begin{array}{l}59,765 \\
18,489\end{array}$ & $\stackrel{107}{72}$ \\
\hline 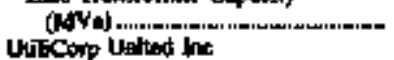 & 450 & 12 & 3 & 459 & 39 & 118 & 2 \\
\hline 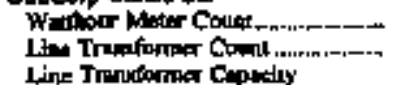 & $\begin{array}{l}347.716 \\
15 \%, 099\end{array}$ & 10,899 & $\begin{array}{l}5,558 \\
2,060\end{array}$ & $\begin{array}{l}353,127 \\
120,063\end{array}$ & $\begin{array}{l}10,69] \\
5,748\end{array}$ & $\begin{array}{l}266,616 \\
112,219\end{array}$ & $\begin{aligned} 7 x .878 t \\
2906\end{aligned}$ \\
\hline 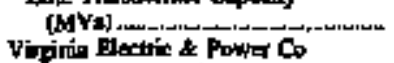 & 4,354 & 167 & 78 & $4.42 \mathrm{~J}$ & 4 & 3,803 & 187 \\
\hline 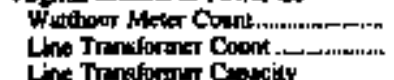 & $\begin{array}{r}1,932,754 \\
929,001\end{array}$ & 67,548 & $\begin{aligned} 33,550 \\
6, \mathrm{Ans}\end{aligned}$ & $\begin{array}{r}1,966,752 \\
53 \times, 774\end{array}$ & $\begin{array}{l}64,582 \\
11,245\end{array}$ & $\begin{array}{l}1,001,782 \\
324,31 !\end{array}$ & $\begin{aligned} 3,280 \\
1,278\end{aligned}$ \\
\hline 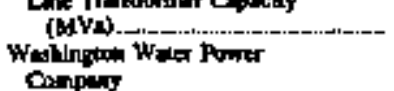 & 21,042 & 1,040 & 142 & 28,413 & 104 & 23,183 & 217 \\
\hline 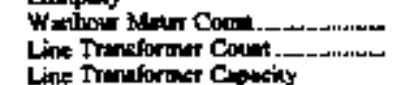 & 288,234 & $\begin{array}{l}31,523 \\
2,878\end{array}$ & 3,205 & $\begin{aligned} 29,6,282 \\
93,517\end{aligned}$ & $\frac{15,442}{2,626}$ & $\begin{array}{r}280,440 \\
90,891\end{array}$ & $\overline{-}$ \\
\hline 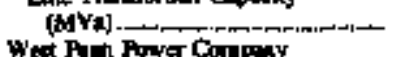 & 1504 & 108 & 33 & 3,579 & 17 & $1,4,172$ & - \\
\hline 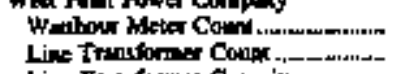 & $\begin{array}{l}69,720 \\
297,347\end{array}$ & $\begin{array}{r}21,077 \\
B, 182\end{array}$ & 21,394 & $\begin{array}{l}698,903 \\
301,353\end{array}$ & $\begin{array}{r}15,606 \\
4,741\end{array}$ & $\begin{array}{l}683,250 \\
296,612\end{array}$ & -4 \\
\hline 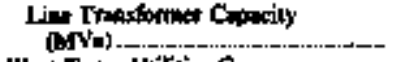 & 6,593 & $\mathbf{2 6}$ & 225 & 6.622 & 26] & I.36) & - \\
\hline 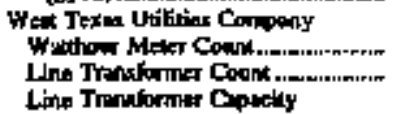 & 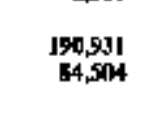 & $\begin{array}{l}7,398 \\
2,785\end{array}$ & $\frac{1,860}{792}$ & $\begin{aligned} 154,266 \\
8,497\end{aligned}$ & $\begin{array}{r}11,726 \\
2,950\end{array}$ & $\begin{aligned} {[\mathrm{IB1}, 877} \\
\mathrm{B}, \mathrm{S42}\end{aligned}$ & - \\
\hline 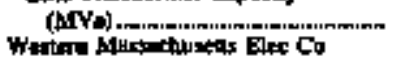 & 2,920 & 259 & 30 & 3, IdI & 209 & 2,932 & - \\
\hline 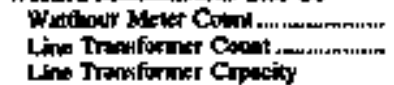 & $\begin{array}{l}216,306 \\
36,903\end{array}$ & 4,760 & $-^{6,683}$ & $\underset{\$ 2,706}{224.343}$ & 15,006 & $\begin{array}{r}208,307 \\
39,600\end{array}$ & In \\
\hline 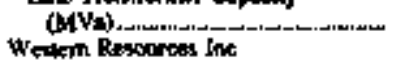 & 1,773 & 40 & - & $1,8]]$, & 201 & 1.709 & 3 \\
\hline 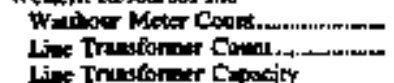 & $\begin{array}{l}327,739 \\
142,005\end{array}$ & $\begin{array}{l}12,47 \\
2,190\end{array}$ & $\begin{array}{l}8.42 \\
1,396\end{array}$ & 3,32044 & $\begin{aligned} 11,888 \\
5,916\end{aligned}$ & $\begin{array}{l}320,56 \\
1,52532\end{array}$ & $-{ }_{2,367}$ \\
\hline 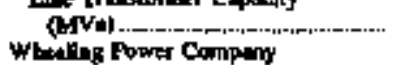 & 4,649 & 153 & 89 & 4,713 & 369 & 4,254 & 68 \\
\hline 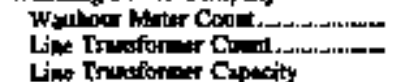 & 44,849 & $\begin{array}{l}793 \\
\$ 15\end{array}$ & 1.422 & 40.900 & 2,860 & $\begin{array}{l}41,319 \\
13,391\end{array}$ & $\begin{array}{l}2 f \\
49\end{array}$ \\
\hline (MYa) - & $\mathbf{1 5 4}$ & 20 & 12 & 402 & 33 & 423 & 6 \\
\hline
\end{tabular}

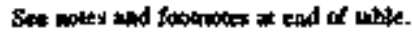


Table 44. Watthour Meter and Line Transformer Statistics of Investor-Owned Utilities, 1994 (Count and Megavoltampere) (Contjpued)

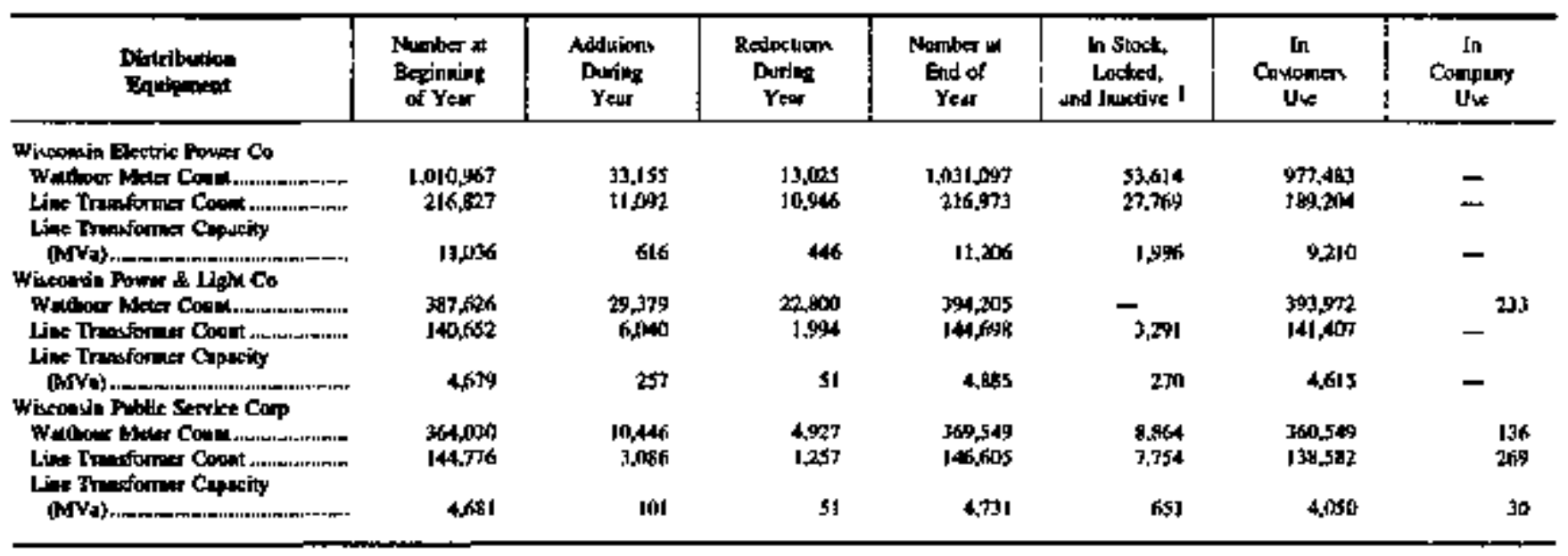

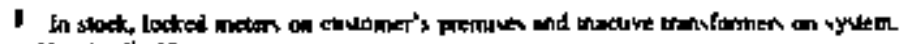

Not Aplicable

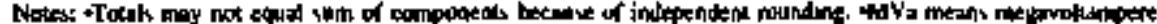

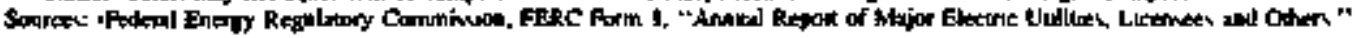




\section{Appendix A}

Federal

Energy

Regulatory

Commission

Regulation of

the Electric

Utility

industry 


\section{Federal Energy Regulatory Commission Regulation of the Electric Utility Industry}

The responsibility for regulating most interstate wholesale electric trade rests with the Federal Gop. ermment, and not with State or local govermments. Therefore, data on wholesale wade are collected within a framework of Federal laws and tegularions. In wholesale trade between electrically connected utilities, if even one utility is electrically connected to a utility that operates in a different Skate, then crade among any of these utilities is considered interstate and jutisulietional for the Federal Energy Regulacory Commission (FERC) togulatory putposes. Hence virtually all wholesale trade except that in Alaska, Hawaii, and part of Texas is considered interstate.

FERC is responsible for goveming interstate trade of wholesale electricity for jnvestor-owned utilities. In order to participate in wholesale electricity trade, uti]ities must file interconnection agreements (contracts) with the FERC. FERC exercises its regulatory athority difectly by approving interconnections between utilities, coordinating agreerneats to exchange power, and approving wholesale power rates. Authority for bulk power transactions is typjcally prearranged under interconnection contracts. These agreements define an indjvidual clectric utifjty's overall relationship to other utilities. In addition, they define how each will maintain its part of the electrical system operations. Qther contracts are often negotiated to bandle specific needs. In fact, one of the most common is for support of full or partial requirements power. Requirements contracts cover electric utilitites that have ether insuffictent or no generating capabilily to satisfy their customer load. They represent long-terus, firm power contractual obligations in which the terms and conditions obligate the selling electric utility to provide the buying electric utiliry a level of service equivalent to the seller's requirement to support its own retajl customers. These contracts may be with more than ane electric utility.

The FERC established a Uniform System of Accounts for the financial reporting of electric trade Iransactions. The tansactions reported are complex and involye specifics of contracts, simultaneous energy transactions, the uniotended receipt and delivery of energy, and the handling of energy losses. These accounts can be found in the FERC's "Accounting and Reporting Requirements For Public Utilities and Licensees." FERC requires only investor-owned utilities to fite financial information on electric trade. The Rural Electrification Administration (REA) requires its borrowers (usually cooperatives) to file similar information, while EIA requires similar information from publjc utjlities. Both the RUS and the EIA request the reporting of information based on the Uniform Sysken of Accounts.

Data on wholesale electricity trade are collected on Federal survey data forms that ace accounting-system based. They were designed for regulatory purposes to meet individual electric utility administrative and rate case ueeds as opposed to overall aggregate industry statistical and analytical purposes.

The FERC Forms 1 and 1-F are the primary financial reporting forms. Wholesale data are collected on 4 of the approximately 75 strvey schedules comprising the FERC Form 1. The wholestle electricity trade schedules are categorized as sales for resale, purchased power, snmmary of interchange, and transmission of electricity for or by others. These schedules correspond to one or more of the following accovnts from the Uniform Systen of Accounts as follows:

- Sales for Resale (447)

- A. This account shalt inclede the net billing for electricity supplied to other clectric utilities or to public authorities for resale purposes.

- B. Records sball be maintained so as to show the quantity of electricity sold and the revenue received from each customer.

- NOTE: Revenues from electricity supplted to other public utilities for nse by them and not for distribution shall be included in account 443. Commerial and lndustrial Sales, unless supptied under the same contract as and not readily separable from Jevenues includable in this accounc

- Purchased Power (555)

- A. This account shall include the cost at point of recoipt by the utility of electricity purchased for resale. It shall jnclude, also, net settlements for exchange of electricity or power, such as economy energy, off-peak energy for on-peak energy, spinning rescrve capacity, etc. In addition, the account shall include the net selt]eroenis for transactions under pooling of interconnection agrements wherein there is a balancing of debits and 
credits for energy, capacity, etc. Distinct purchases and sales shall not be recorded as exchanges and net amounts are only recorded merely because debit and credit amounts are combined in the voucher settlement.

- B. The records supporting this account shall show, by months, the demands and demand charges, kilowatthours and prices thereof under each purchase contract and the charges and credits under each exchange or power pooling contract.

- Other Electric Revenues (456)

- 5. Revenues from transmission of ejectricity of others over transmission facilities of the utility.

\section{- Transmission of Electricity By Orhers (565)}

- This account shall include amounts payable to others for the transmission of the utility's electricity over transmission facilities owned by others.

\section{Addition of Statistical Classiflcation Codes to the FERC Form 1}

The Federal Energy Regulatory Commission modified the FERC Form I, "Annual Report of Major Electrie Utilities, Licenses and Others" for the 1990 collection year. The Statistical Classification Codes are based on the orightal contractual terms and condirions of the service. The following codes were added to FERC Form 1:

- RQ - for requirements service. Requirements service is service which the supplier plans to provide on an ongoing basis (i.e., the supplier includes projected load for this service in its system resource planning). In addition, the reliability of requirements service must be the same as, or second only to, the supplier's service to its own ultimate consumers.

- LF - for tong-term service. "Long-term" means 5 years or longer and "firm" means that service cannot be interrupted for economic reasons and is intended to remajn reliable even under adverse conditions (e.g., the supplier must attempt to buy emergency energy from third parties to maintain deliveries of LF service). This category should not be used for long-term firm service which meets the definition of $\mathbf{R Q}$ service. For all transactions identified as $\mathrm{LF}_{y}$ provide in a footnore the termination date of the contract defined as the earliest date that either buyer or seller can unilaterally get out of the contract.

- $\mathrm{IF}$ - for intermediate-term firm service. The same as LF service except that "intermediate-term" means longer than 1 year but less than 5 years.

- SF - for short-terro firm service. Use this category for all firm services where the duration of each period of commitment for service is l year or less.

- LU - for long-term service from a designated generating unit. "Long-term" means 5 years or' longer. The availability and reliability of service, aside from transmission copstraints, must match the availability and reliability of the designated unit.

- IU - for intermediate-term service from a desig. naled generatiog unit. The same as LU service except that "intormedjate-term" means longer than 1 year but less than 5 years.

- EX - for exchange of electricity. Use this category for transactions involving a balancing of debits and credits for energy, capacity, etc. and any settlements for imbalanced exchanges.

- OS - for other service. Use this category only for those strvices which cannot be placed in the above-defined categories, such as all nonfirm service regardless of the length of the contract and service from designated units of less than 1 year. Describe the nature of the service in a foornote.

- AD - for out-of-period adjustments. Use this code for any accounting adjustments or "nune-ups" for service provided in prior reporting years. Provide an explanauton in a foosnote for exch adjustrnent.

\section{FEAC Accounting Release Number $A R-14$}

The Office of the Chief Accountant released an interpretstion of Account 555 in Accounting Relesse Number AR-14 effecrive January I, 199!. The format was question and answer. AR-14 is as follows:

Question: The text of Account 555, Purchased Power, states in part: "This acconnt shalj include the cost at point of receipt by the utility of electricity purchased for resale. It shall include, also, net settlements for exchange of electricity or power, such as economy entrgy, off-peak energy for on-peak energy, spinning reserve capacity, etc. In addition, the account shall include the net settlements for transactions under pooling or interconnection agreements wherein there is a balancing of debits and credits for energy. capacity, etc." Does this mean that Account 555 is to include all setclement amounts arising from power pool transactions regardless of how compensation for energy delivered to the power pool or capacity made avajiable to the powes pool is decerained and all settlement amounts for energy provided under nonpool contractual agreements in which the agreements contain provisions pertaining to both the receipt and delivery of energy?

Answer: No. The above requirement must be read in conjunction with the sentence is the accounting text that immediately follows the above quoce which stares: "Distinct purchases and sales shall not be recorded as exchanges and net amounts only recorded metely because debit and eredit amounts are conbined in the voucher settiement "It is the intent of the text of Account 555 to linit the use of the accourt to the cost of energy and capacity porchased and net sertlements arising from barter transactions. Gross amounts for compensation for energy delivered or eapacily made available to a power pool or delivered pursuant to other agreements where settlements are 
determined through application of specific rate schedules, "split-the-saving" calcutations, simulations, or other non-barter means must be recorded in the appro- priate operating revenue account and not netted with purchased power expenses properly recorded in Account 555. 
Appendix B

State, NERC

Region, and

Data Sources

for Electric

Utilities 


\section{State, NERC Region, and Data Sources for Electric Utilities}

Table B1. Inrestor-Owned Utilities, 1994

\begin{tabular}{|c|c|c|c|}
\hline Gtily & Satul & NRRC Rotiont & suctis Form \\
\hline 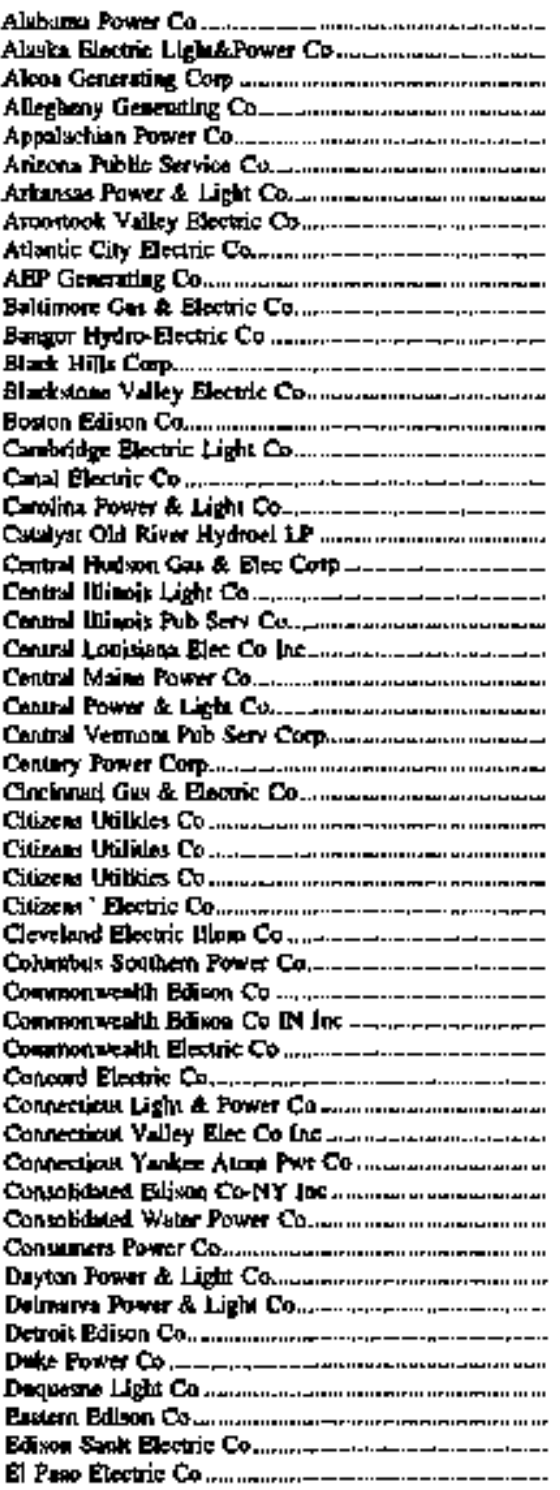 & 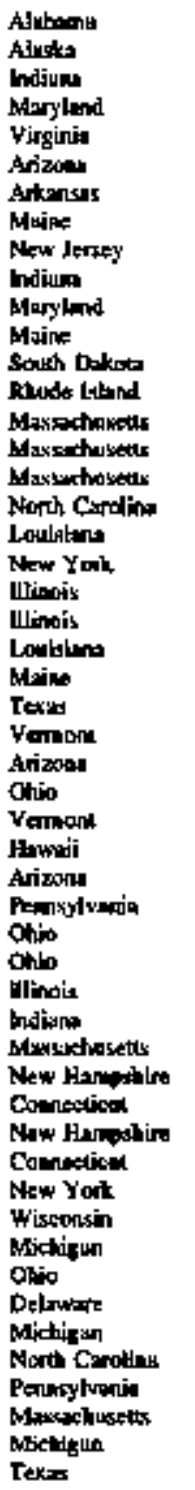 & 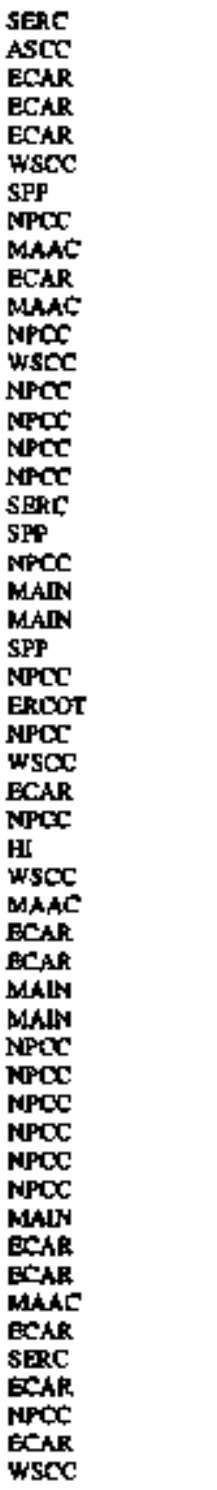 & 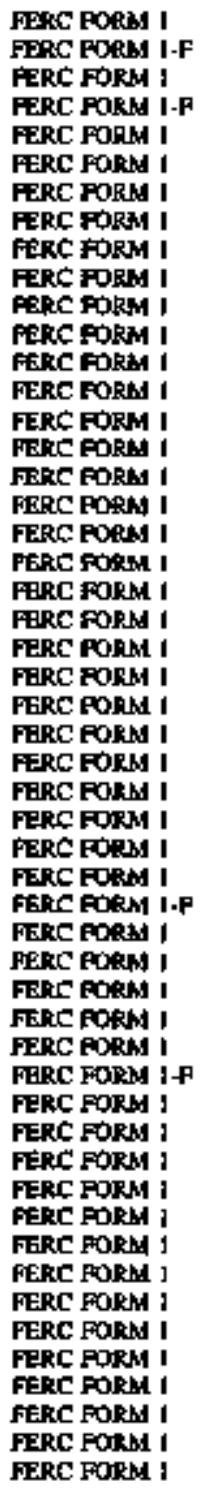 \\
\hline
\end{tabular}

Sot foofinotes a end of tylk. 
Table B1. Investorowned Utilities, 1994 (Continued)

\begin{tabular}{|c|c|c|c|}
\hline Untry & Stente1 & NIPRC Respen? & Sitroty Pans? \\
\hline 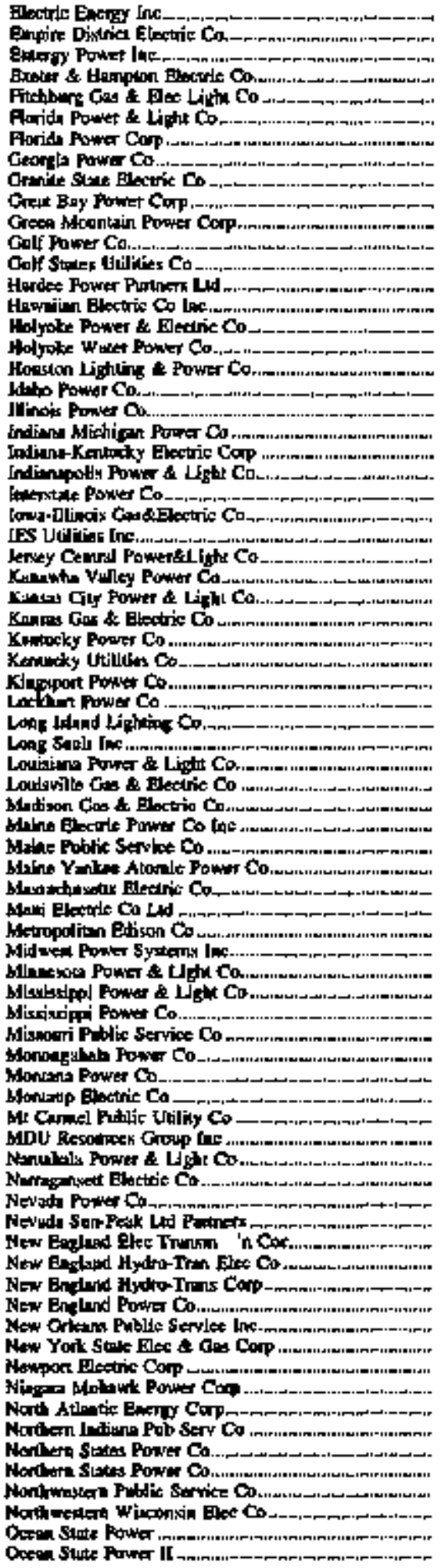 & 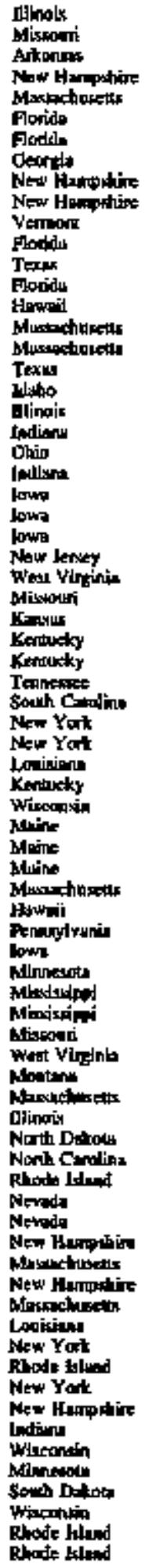 & 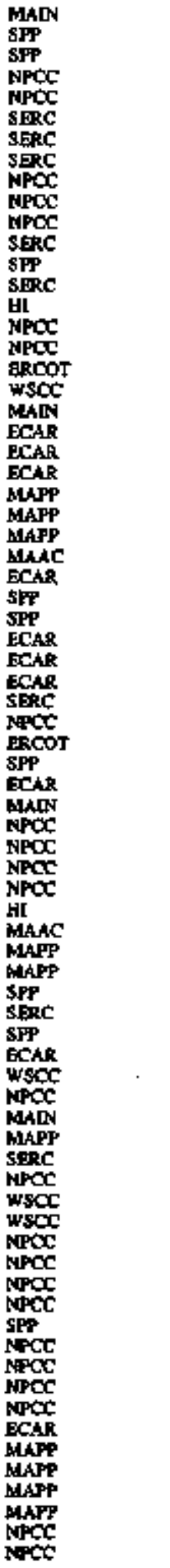 & 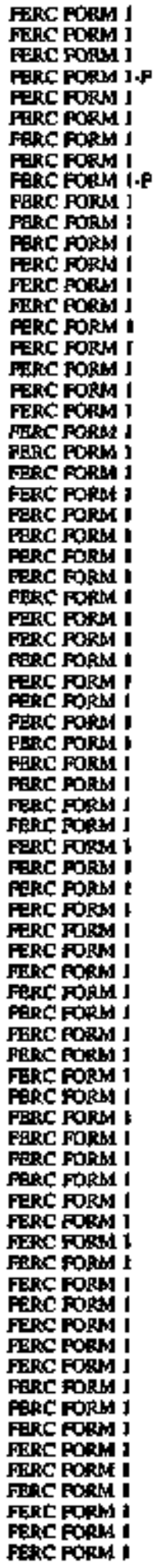 \\
\hline
\end{tabular}

Sed foolmolek ty thid of toble, 
Table B1. Investor-Omned Utilities, 1994 (Continued)

\begin{tabular}{|c|c|c|c|}
\hline Coring & Shat I & MARC Rejoot & Sumeg Parmo \\
\hline 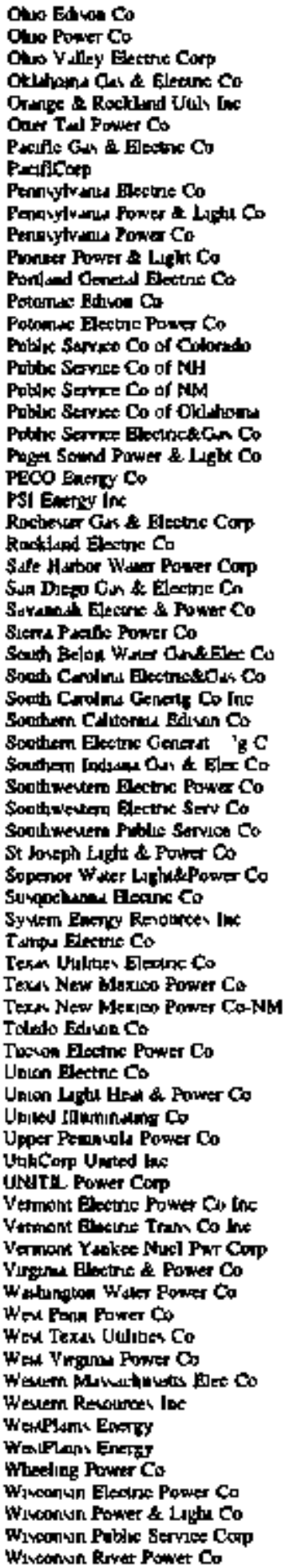 & 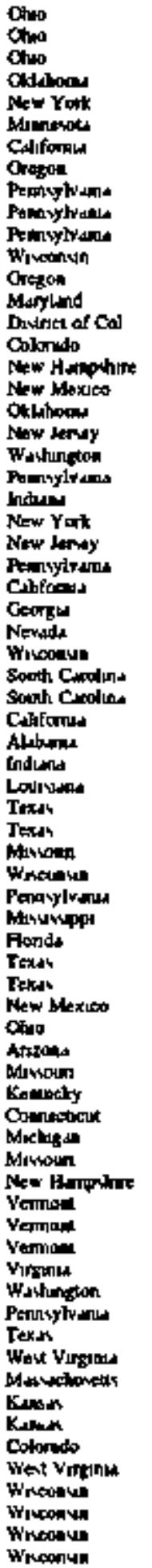 & 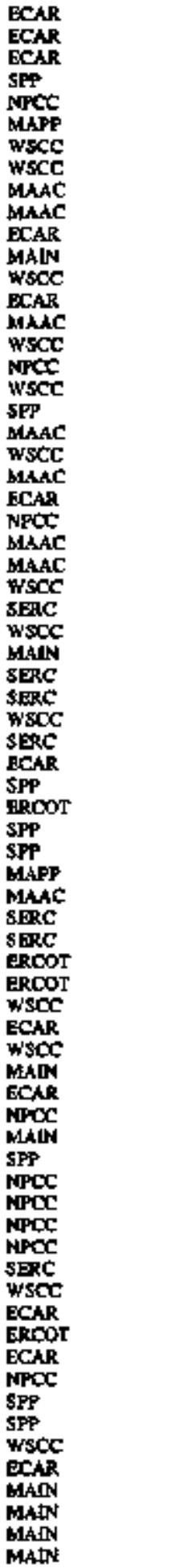 & 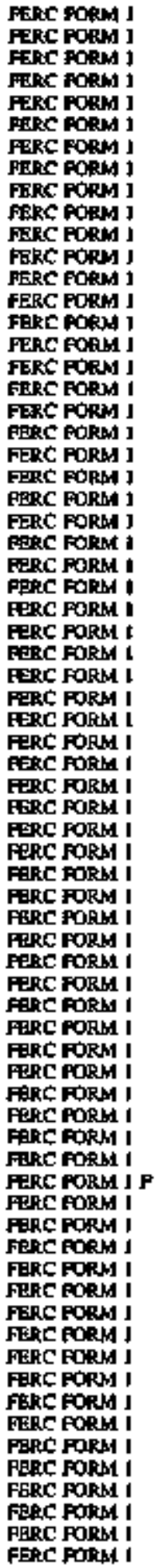 \\
\hline
\end{tabular}

Sec footwot at end of table 
Table B1. Investor-Owned Utilities, 1994 (Contimued)

\begin{tabular}{|c|c|c|c|}
\hline 1e:lik & St & NDRC Resione? & suxuly Fem? \\
\hline 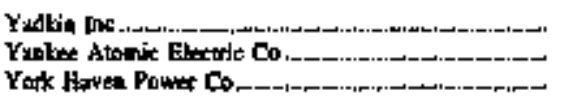 & 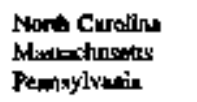 & $\begin{array}{l}\text { SRRC } \\
\text { NPCC } \\
\text { MAAC }\end{array}$ & $\begin{array}{l}\text { PERC FORN I } \\
\text { FERC FORM I } \\
\text { FERC FOEN I }\end{array}$ \\
\hline
\end{tabular}

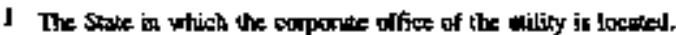

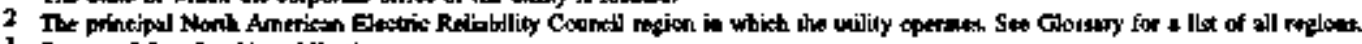

3. Sousce of dur for thes pablicution

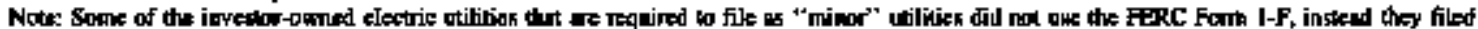

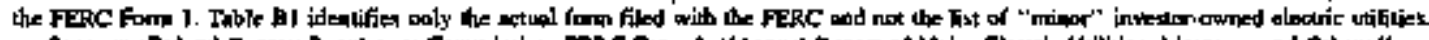

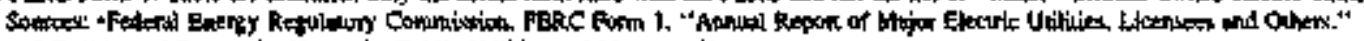
fexp

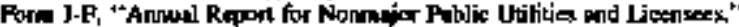


Table B2. Federal Utilities, 1994

\begin{tabular}{|c|c|c|c|}
\hline Vtinly & ston! & NREC Rrgon² & Smrvey Pare' \\
\hline 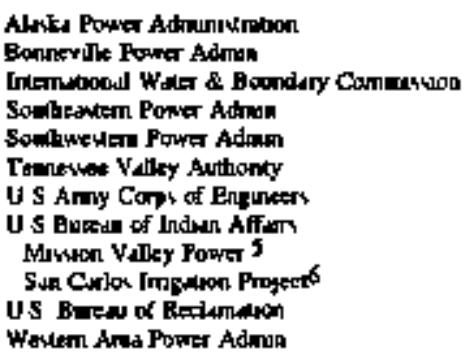 & 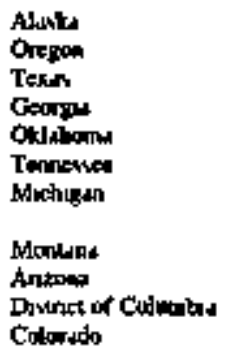 & $\begin{array}{l}\text { ASCC } \\
\text { WSCC } \\
\text { BRCOT } \\
\text { SERC } \\
\text { SPP } \\
\text { SERC } \\
\text { ECAC } \\
\text { WSCC } \\
\text { WSCC } \\
\text { WSC } \\
\text { WSCC }\end{array}$ & 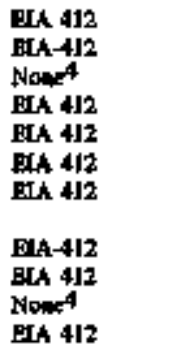 \\
\hline
\end{tabular}

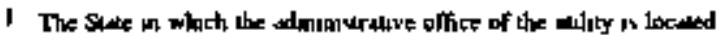

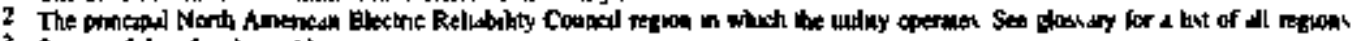

3 Sowre of dote for lins pubhemom

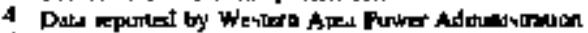

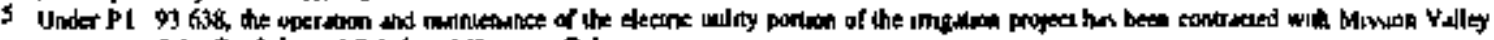

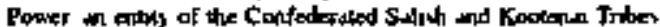

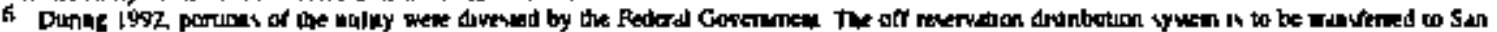

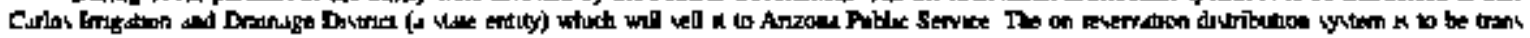

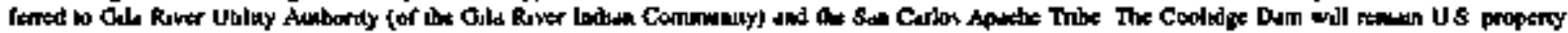

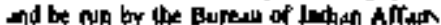

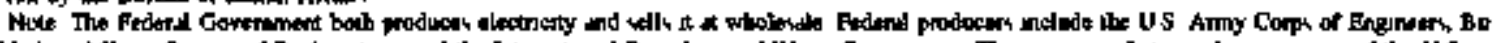

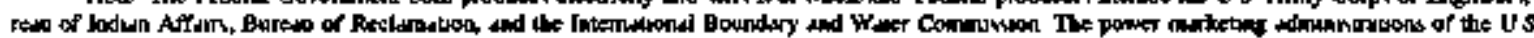

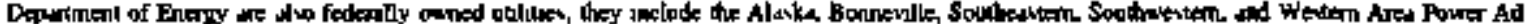

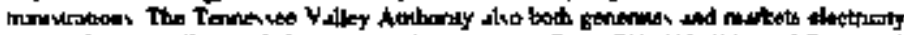

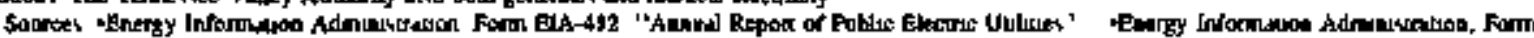

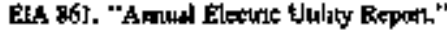


Table B3. State and Other Government Utiltties, 1994

\begin{tabular}{|c|c|c|c|}
\hline Unpiy & Sunte1 & NIDC Rejo-2 & Servey Focm 3 \\
\hline 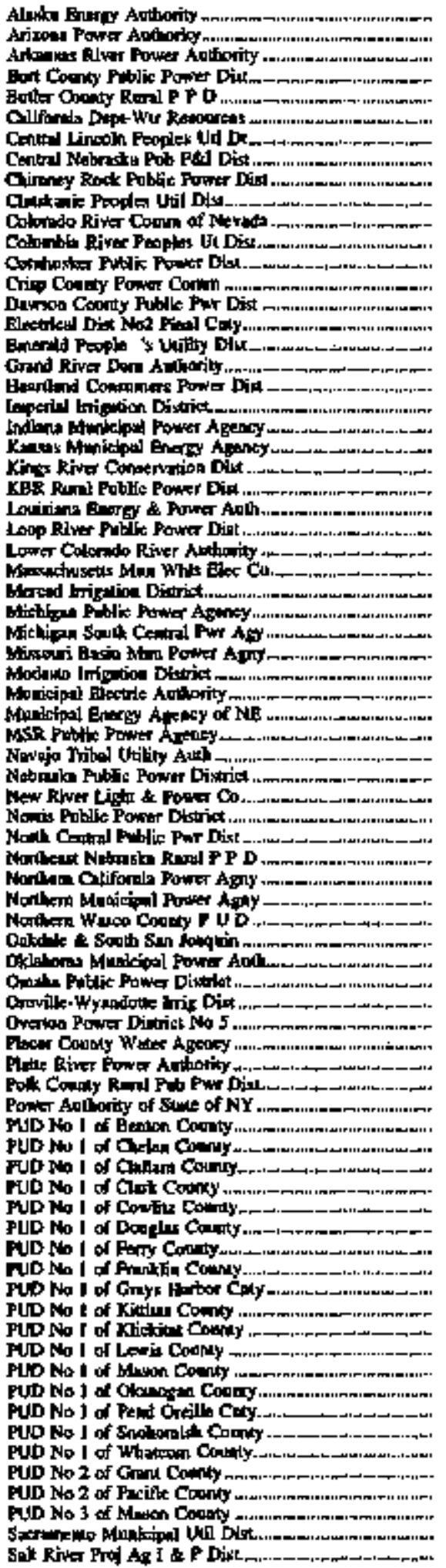 & 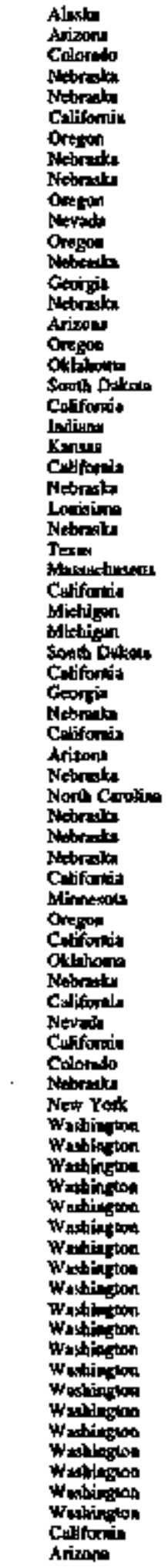 & 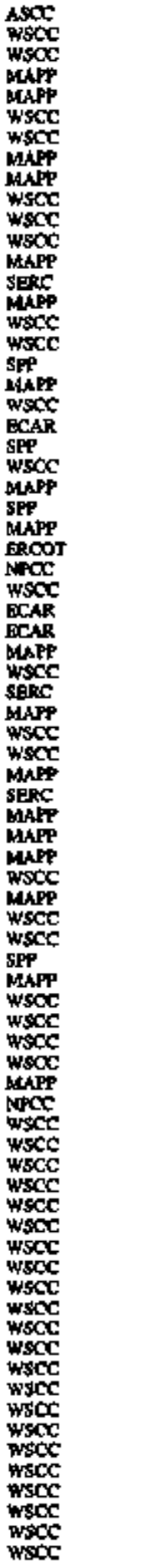 & 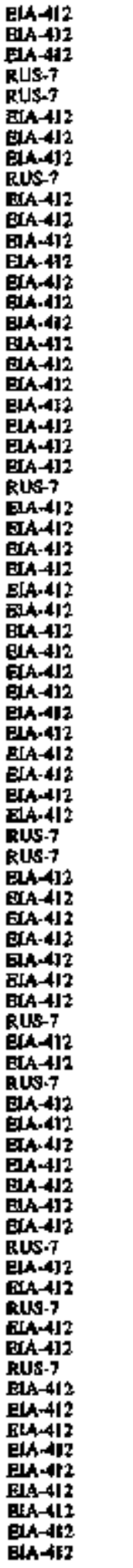 \\
\hline
\end{tabular}

See footuois in end of table. 
Table B3. State and Other Governenent Utilities, 1994 (Continued)

\begin{tabular}{|c|c|c|c|}
\hline Utiny & Sintel & NERC Beptar & Survpy Form ${ }^{3}$ \\
\hline 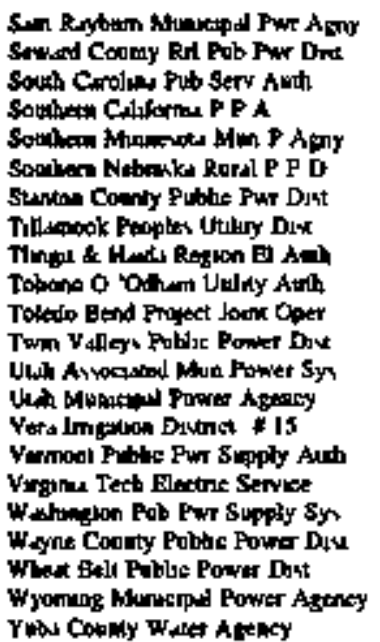 & 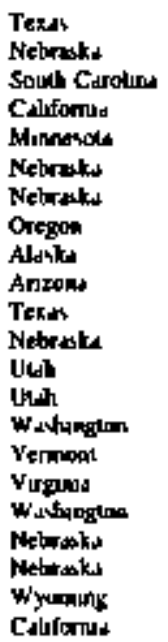 & $\begin{array}{l}\text { SPP } \\
\text { MAPP } \\
\text { SERCC } \\
\text { WSCY } \\
\text { MAPP } \\
\text { MLPP } \\
\text { MAPP } \\
\text { WSCC } \\
\text { ASCC } \\
\text { WSCC } \\
\text { SEP } \\
\text { MAPP } \\
\text { WSCC } \\
\text { WSCC } \\
\text { WSOC } \\
\text { MFOC } \\
\text { ECAR } \\
\text { WSCC } \\
\text { MAPP } \\
\text { WSCC } \\
\text { WSCC } \\
\text { WSCC }\end{array}$ & $\begin{array}{l}\text { EIA } 412 \\
\text { RUS ? } \\
\text { ELA } 112 \\
\text { EIA } 412 \\
\text { EIA } 412 \\
\text { ELA } 412 \\
\text { RUS ? } \\
\text { ELA-412 } \\
\text { RUS T } \\
\text { ELA } 412 \\
\text { ELA } 412 \\
\text { RLS ? } \\
\text { ELA } \$ 12 \\
\text { ELA } \$ 12 \\
\text { ELA } 412 \\
\text { ELA } 412 \\
\text { ELA } 412 \\
\text { ELA } 412 \\
\text { RUS T } \\
\text { RUS } \tau \\
\text { ELA } 412 \\
\text { ELA-412 }\end{array}$ \\
\hline
\end{tabular}

I T. Ste in which the whosity is Jocend

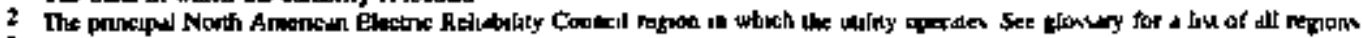

3 Sorrec of date for thes woblicanoe

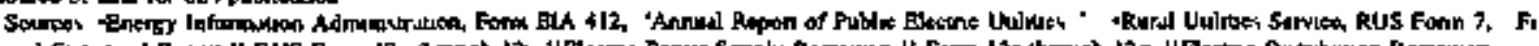

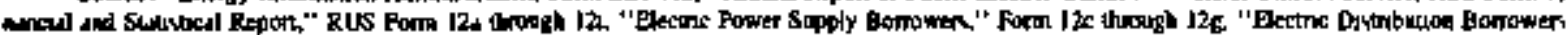

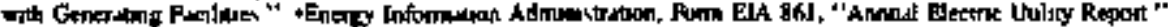


Table B4. Manicipal Uullities, 1994

\begin{tabular}{|c|c|c|c|}
\hline & & NARC Depont & Survey Forin"3 \\
\hline 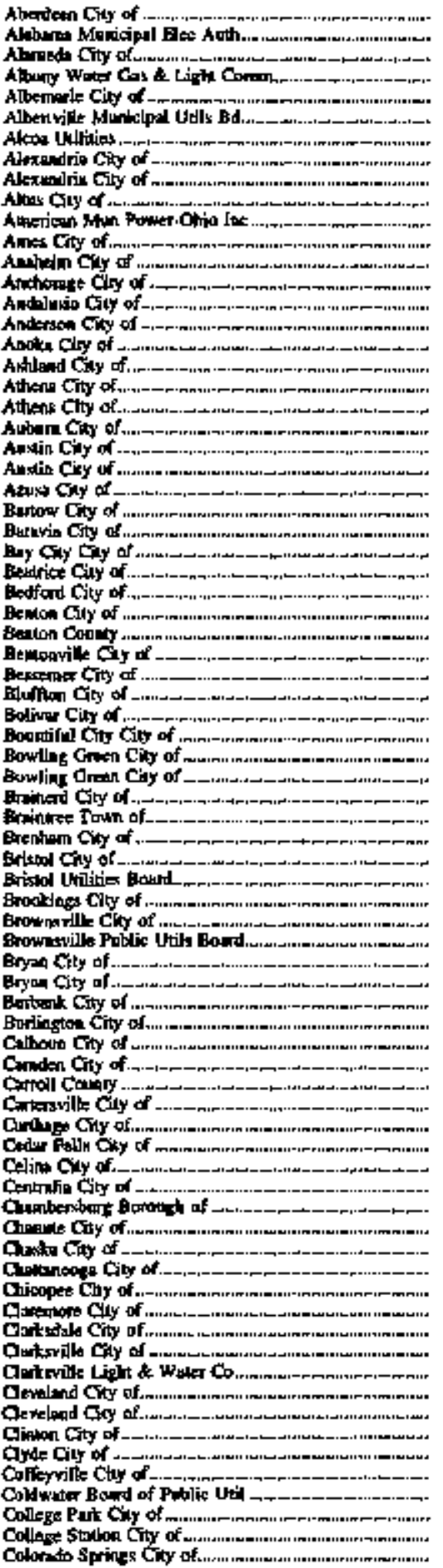 & 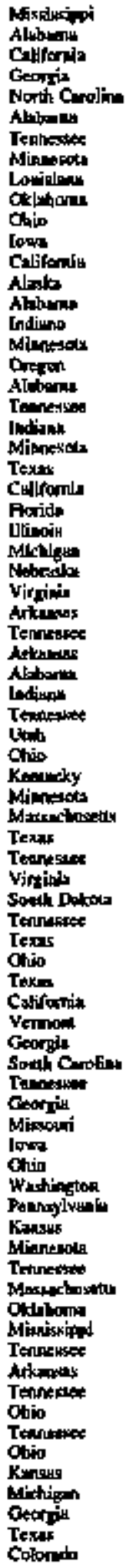 & 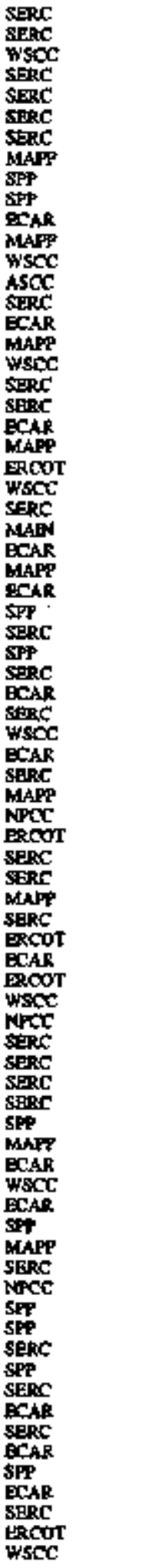 & 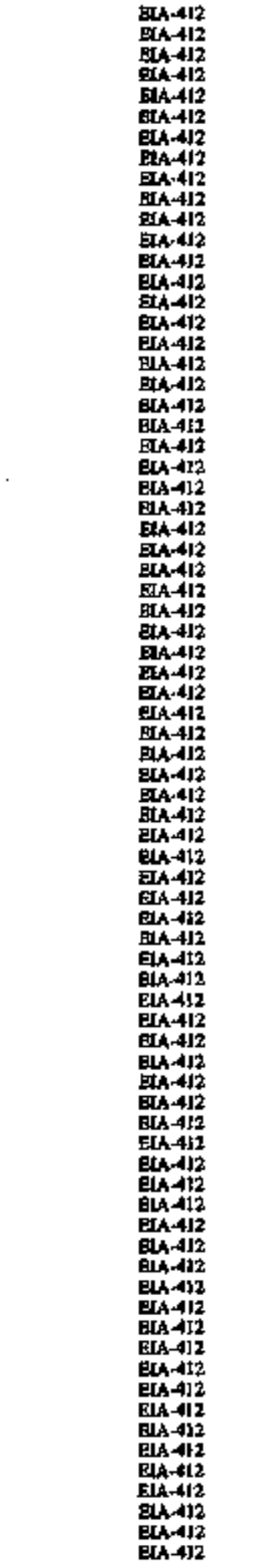 \\
\hline
\end{tabular}

See formolen et end of luble 
Table B4. Municipal Utilities, 1994 (Conthued)

\begin{tabular}{|c|c|c|c|}
\hline tleitity & Statl & NELC Reglof? & Sorvey Fonnt \\
\hline 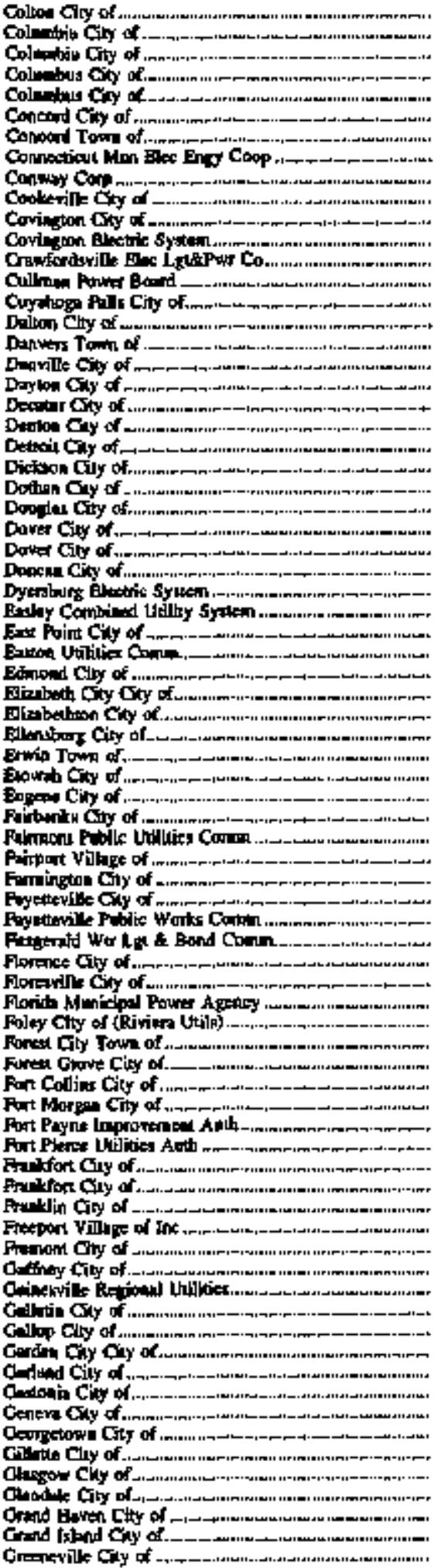 & 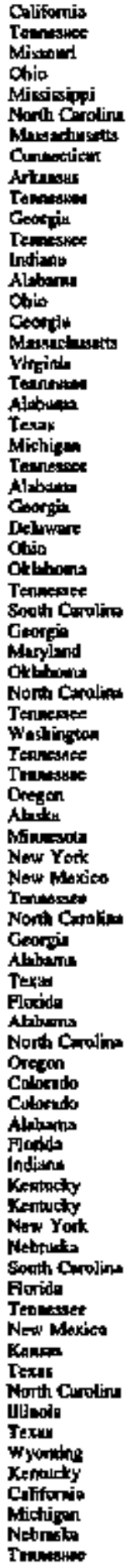 & 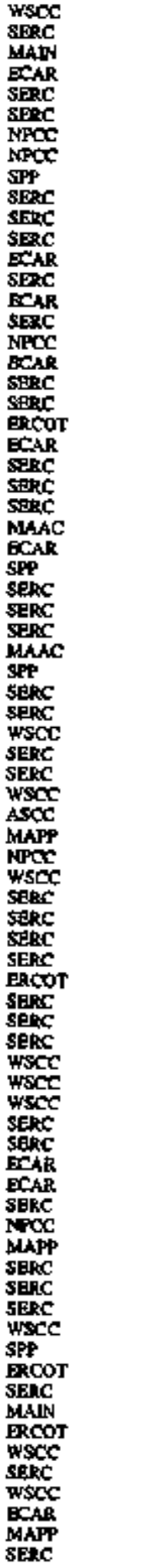 & 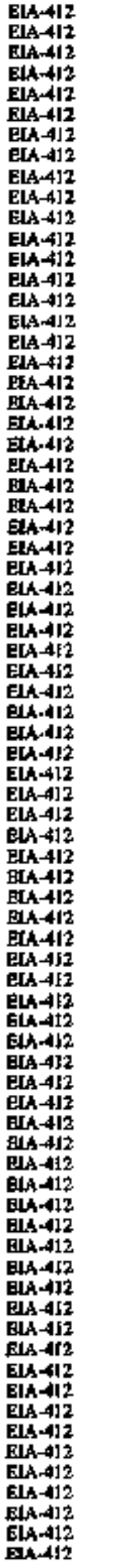 \\
\hline
\end{tabular}

Sex footnotes at end of whil 
Table B4. Municipal Utałtles, 1994 (Continued)

\begin{tabular}{|c|c|c|c|}
\hline Ut:llits & Stotal & NTRRC R-jing? & Survey Formo \\
\hline 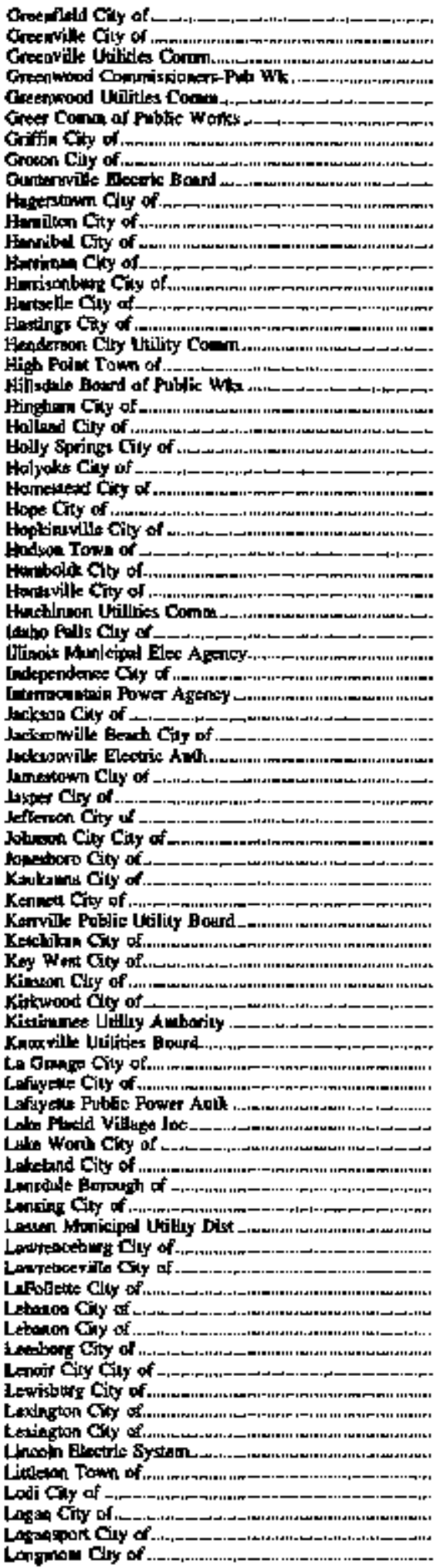 & 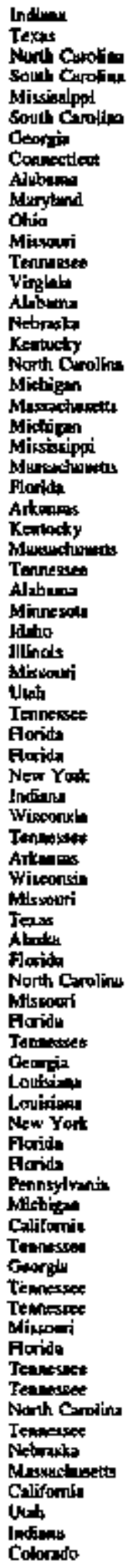 & 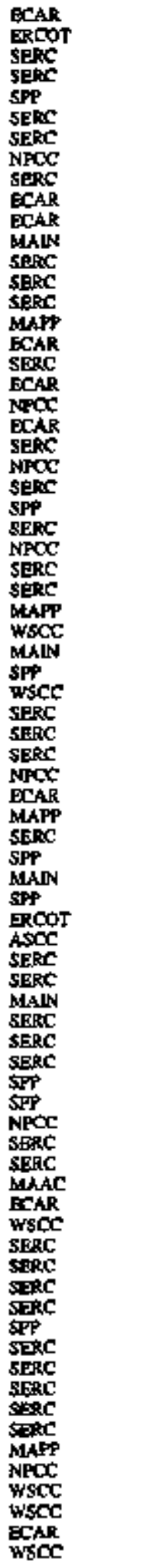 & 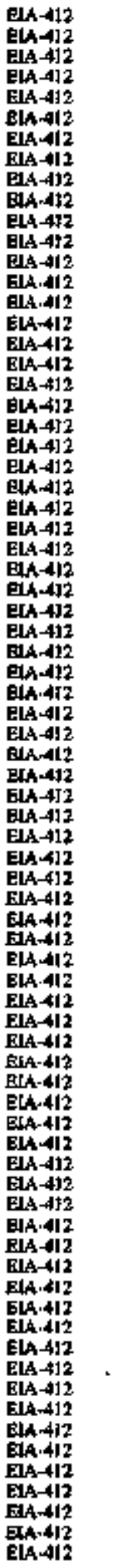 \\
\hline
\end{tabular}

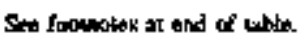


Tabke B4. Manicipal Utiaties, 1994 (Contimed)

\begin{tabular}{|c|c|c|c|}
\hline Uadiky & Stril & Nenc Repion & Survey Pocma \\
\hline 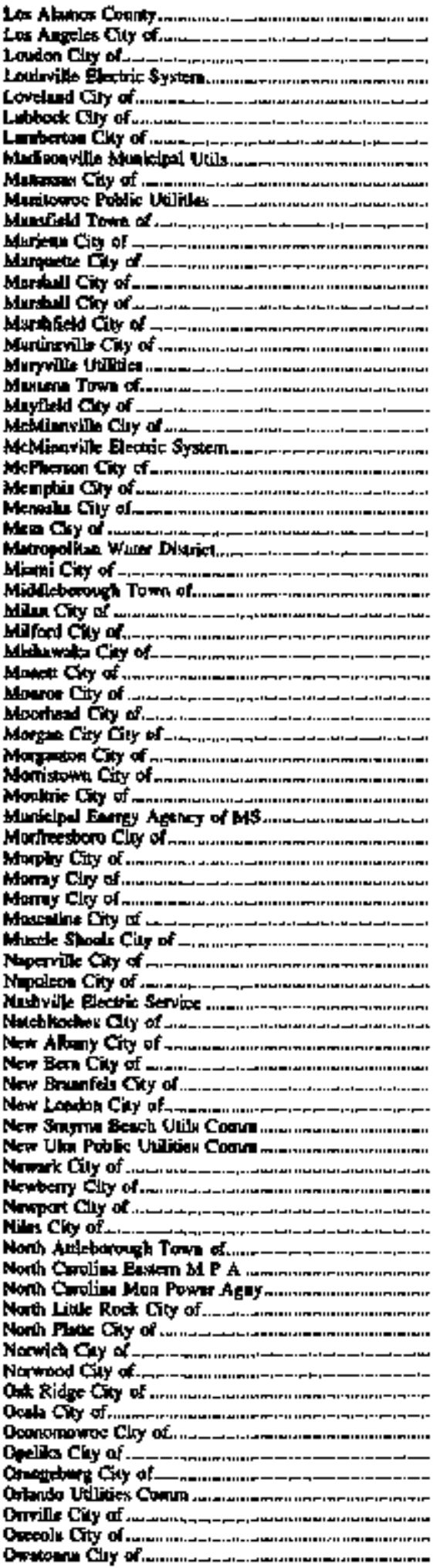 & 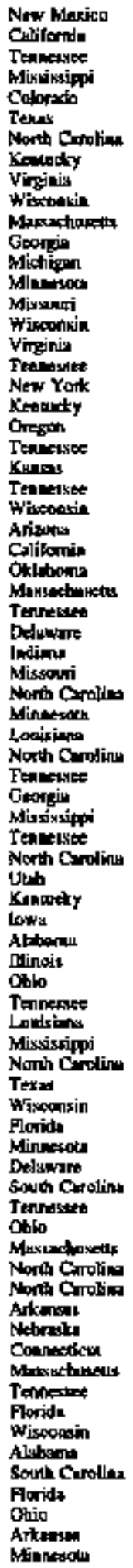 & 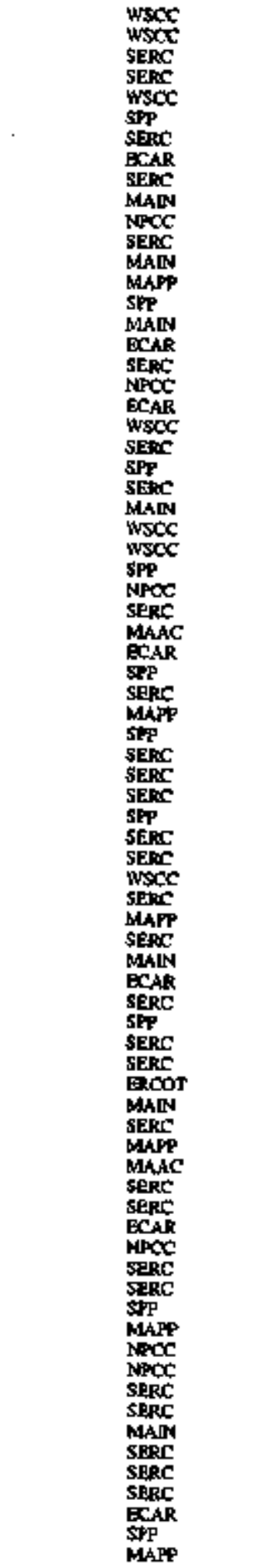 & 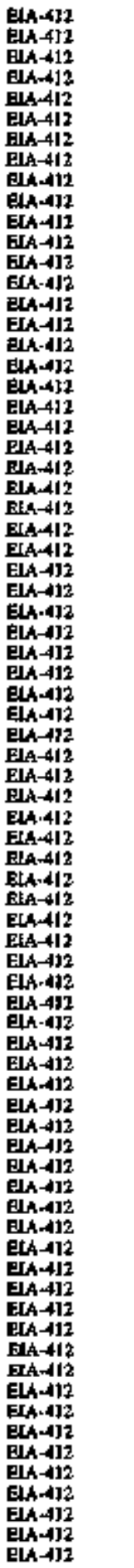 \\
\hline
\end{tabular}

Sex footnotes at end of uble. 
Table B4. Municipal Utilities, 1994 (Confinued)

\begin{tabular}{|c|c|c|c|}
\hline Uolity & Statal & NDEC Reglan 2 & Sevey Pan ${ }^{3}$ \\
\hline 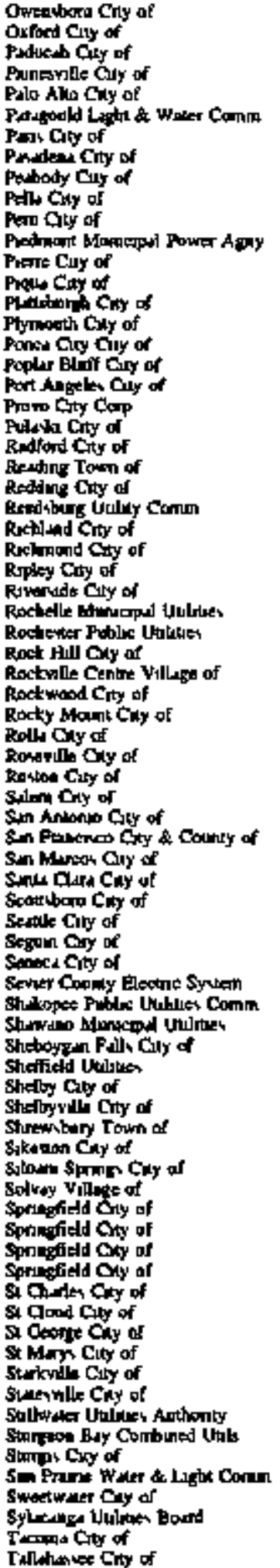 & 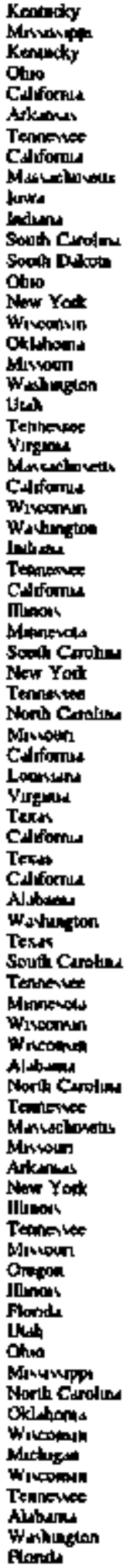 & 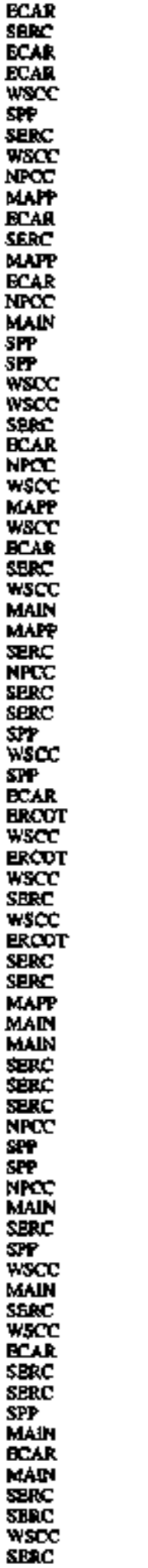 & 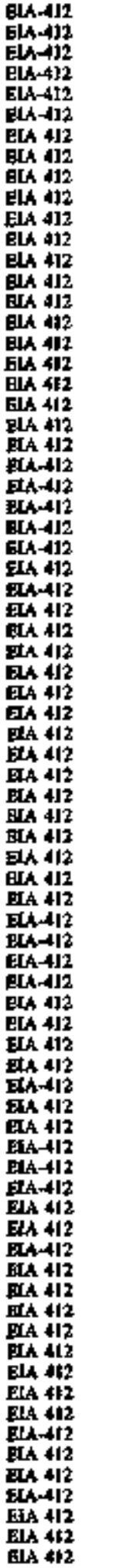 \\
\hline
\end{tabular}

See footnotus th and of uble 
Table B4. Municipal Utilities, 1994 (Continued)

\begin{tabular}{|c|c|c|c|}
\hline E) & Stat & NARC Ramant & Survty Tarmo \\
\hline 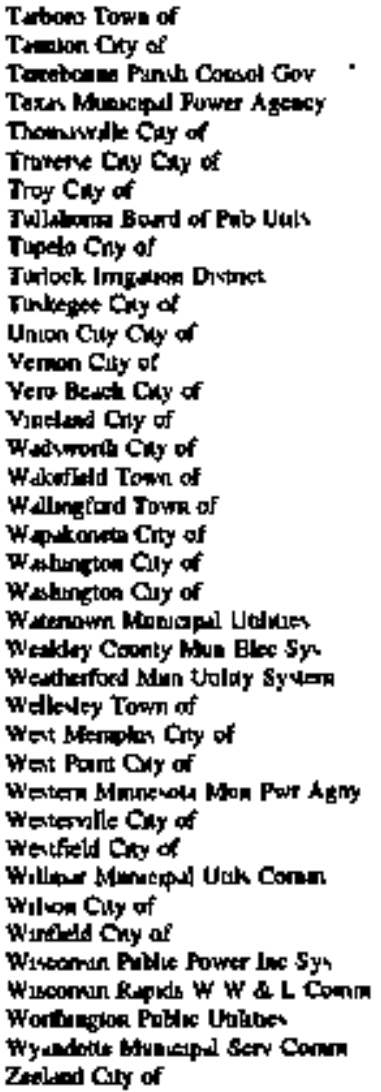 & 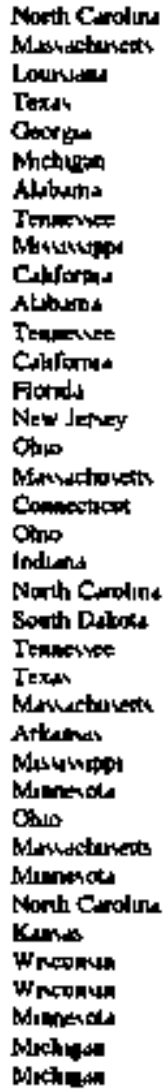 & $\begin{array}{l}\text { SERC } \\
\text { NPCC } \\
\text { SPP } \\
\text { ERCOT } \\
\text { SERC } \\
\text { SEAR } \\
\text { SERC } \\
\text { SERC } \\
\text { SERC } \\
\text { WSCC } \\
\text { SERC } \\
\text { SERC } \\
\text { MSCC } \\
\text { SPRC } \\
\text { MAAC } \\
\text { ECAR } \\
\text { NPCC } \\
\text { NPCC } \\
\text { ECAR } \\
\text { ECAR } \\
\text { SERC } \\
\text { MSAPF } \\
\text { SERC } \\
\text { ERCOI } \\
\text { NPCC } \\
\text { SPP } \\
\text { SERC } \\
\text { MAPP } \\
\text { ECAR } \\
\text { MPCC } \\
\text { MAPP } \\
\text { SERC } \\
\text { SPP } \\
\text { MAIN } \\
\text { MAPF } \\
\text { MAPS } \\
\text { ECAR } \\
\text { ECAR }\end{array}$ & 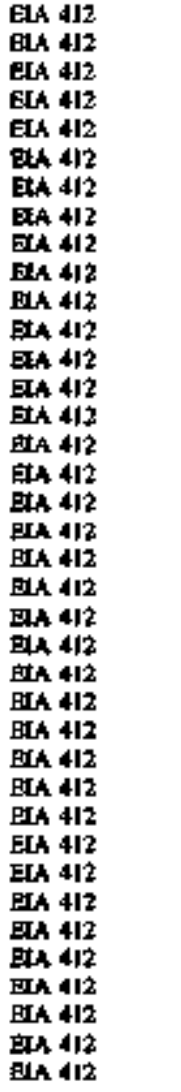 \\
\hline
\end{tabular}

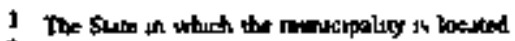

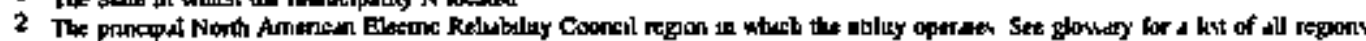

3 Sonter of date for los poblucatione

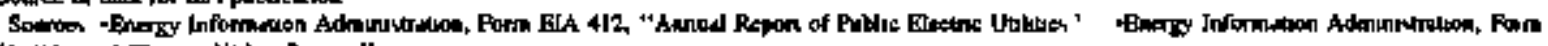
ELA 8fl, "Atrow Election Utiliry Bepon" " 


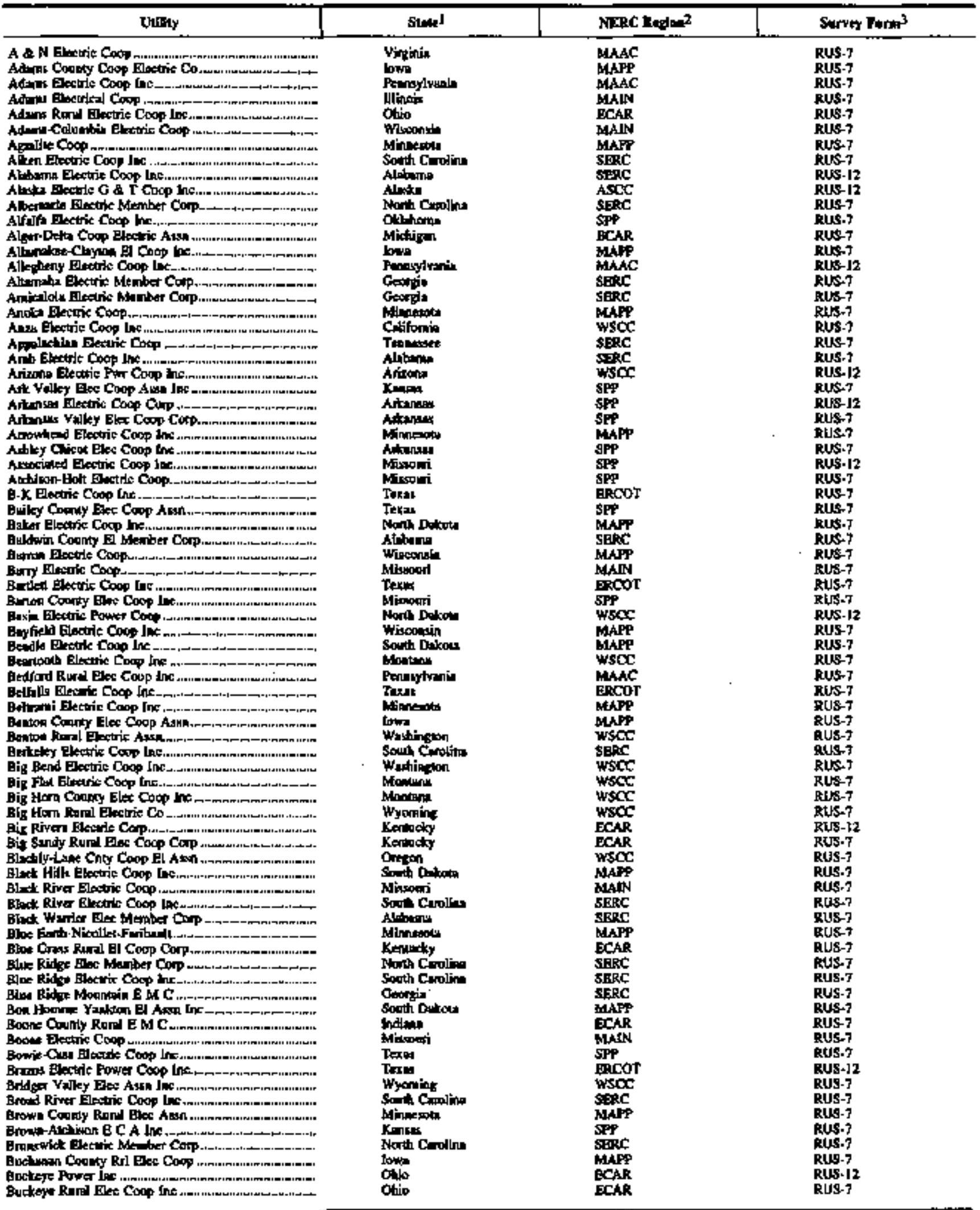

Ses toounotit al and of trible, 
Table B5. Cooperative Borrowers, 1994 (Continued)

\begin{tabular}{|c|c|c|c|}
\hline Uhily & Stented & ADPC Regha' & Suxwey Forme' \\
\hline 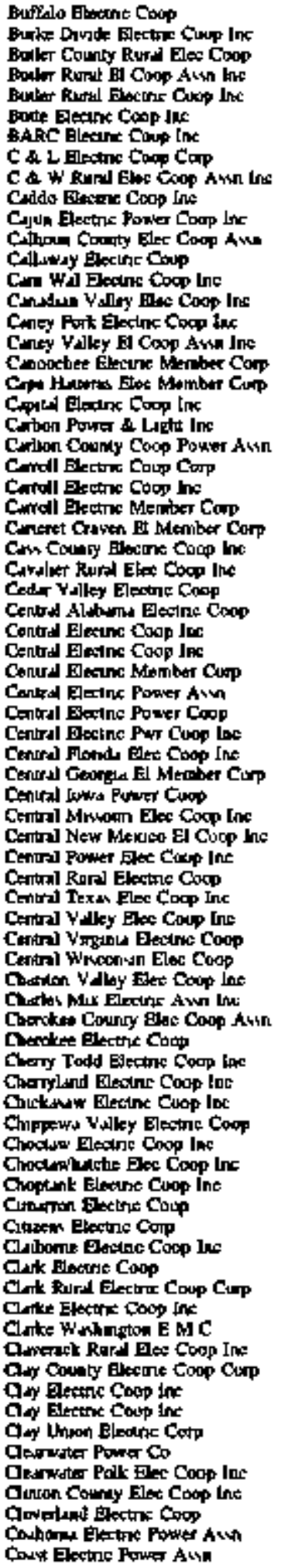 & 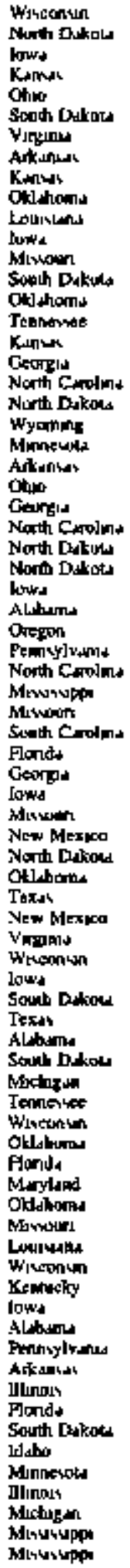 & 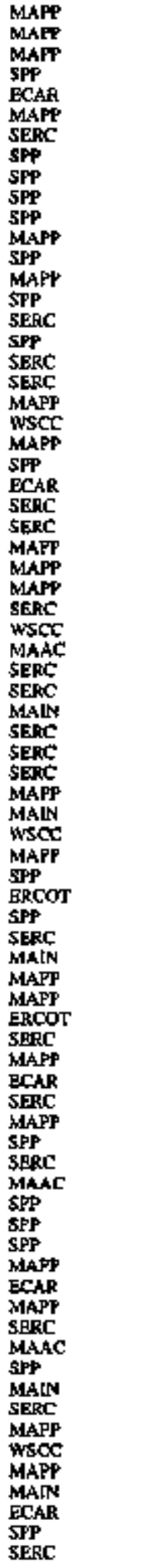 & 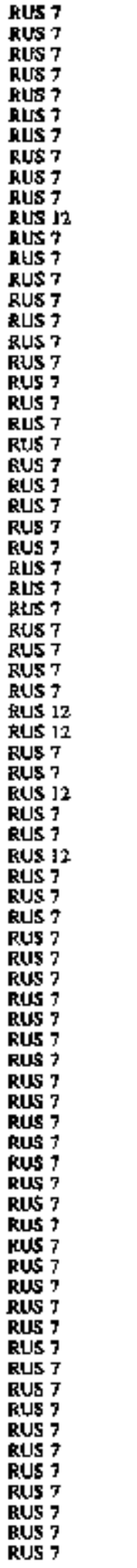 \\
\hline
\end{tabular}

Sec fooldoten at end of thble 
Tabk B5. Cooperative Borrowers, 1994 (Conlinued)

\begin{tabular}{|c|c|c|c|}
\hline Utuldyt & Stotel & NERC REJan ${ }^{2}$ & Grvey Parn't \\
\hline 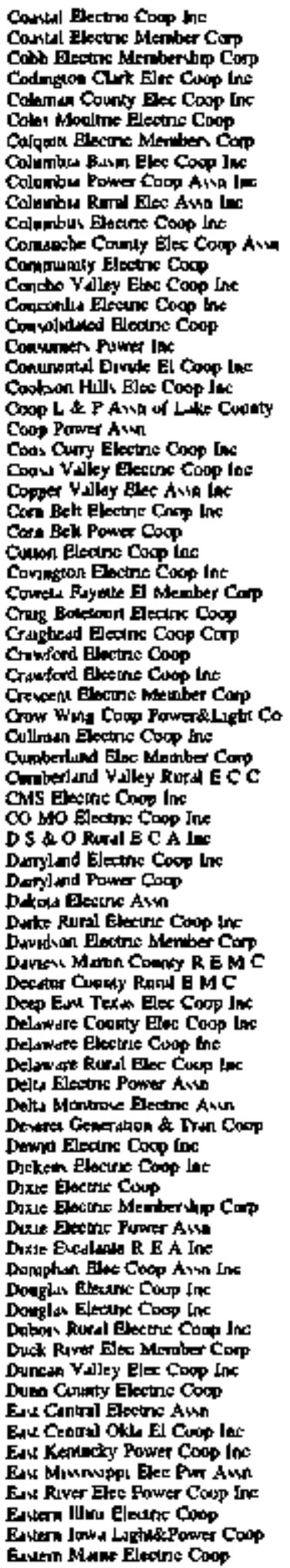 & 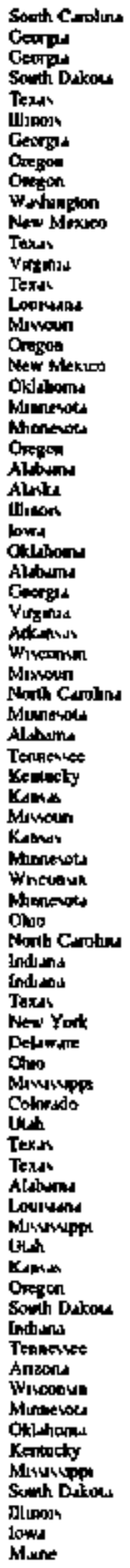 & 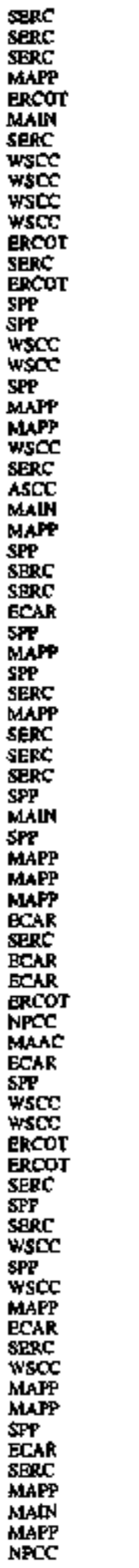 & 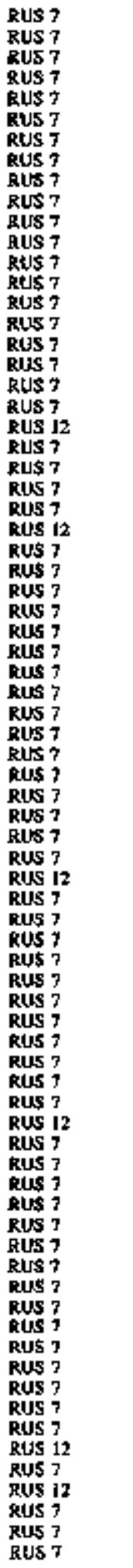 \\
\hline
\end{tabular}

See foompotes al ead of thble 
Table B5. Cooperative Barrowers, 1994 (Continued)

\begin{tabular}{|c|c|c|c|}
\hline Unyy & Stale] & NXPC Rsopa' & Survey Ferm ${ }^{3}$ \\
\hline 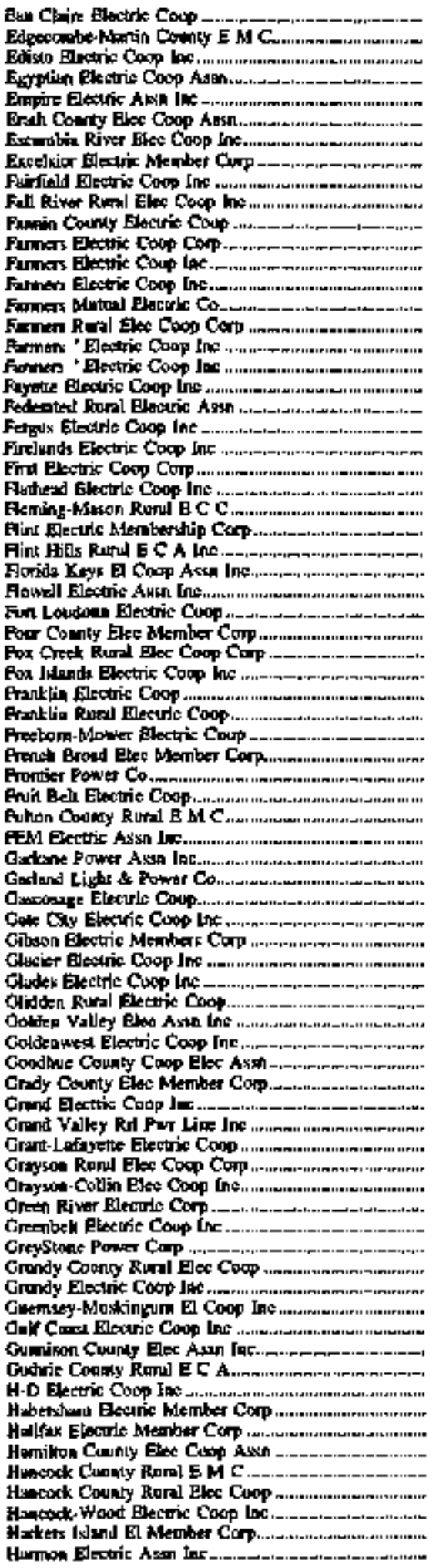 & 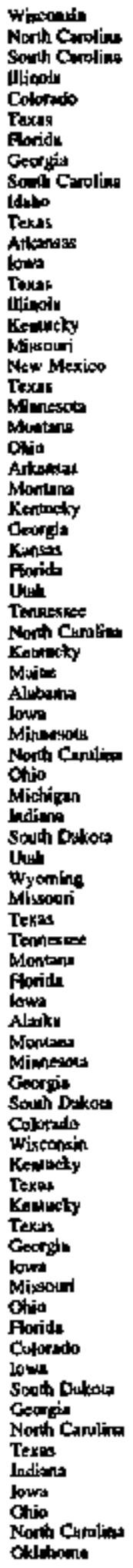 & 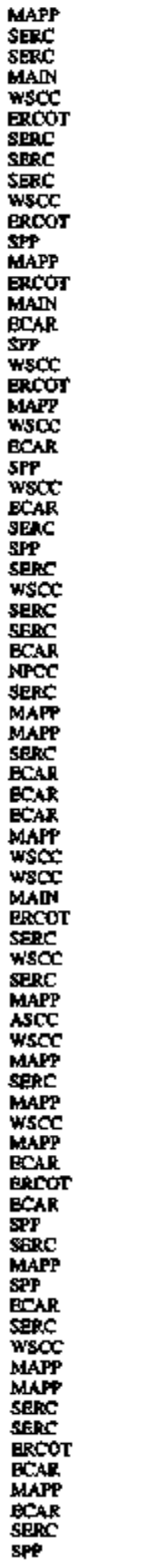 & 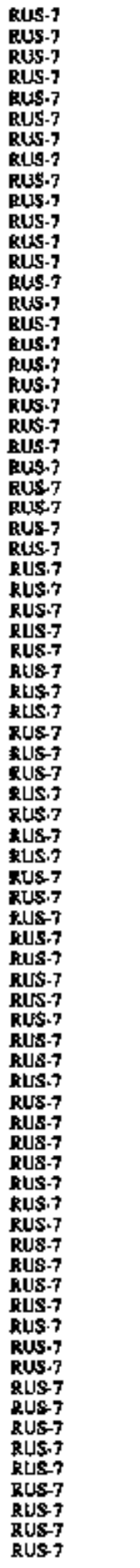 \\
\hline
\end{tabular}

See foctnotes at end of cable. 
Table B5. Cooperallve Borrowers, 1994 (Continned)

\begin{tabular}{|c|c|c|c|}
\hline Ut:ly & Stont 1 & NEAC Defioo? & Strity' Poem \\
\hline 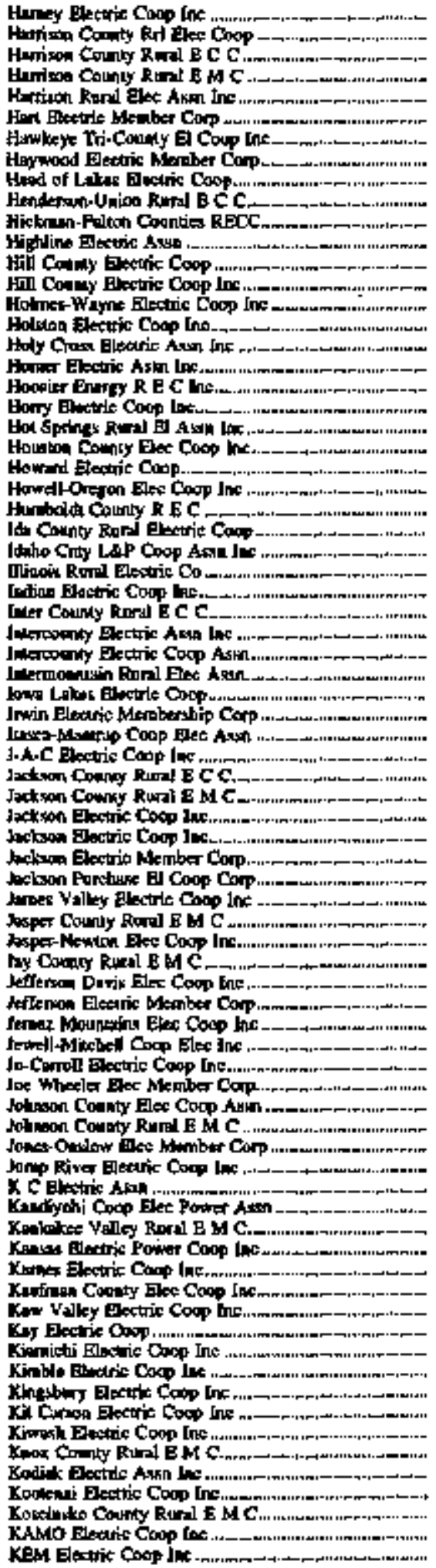 & 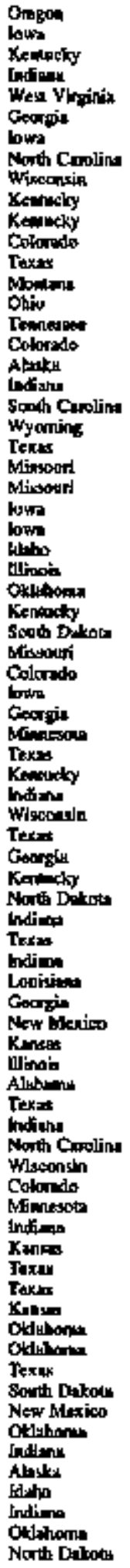 & 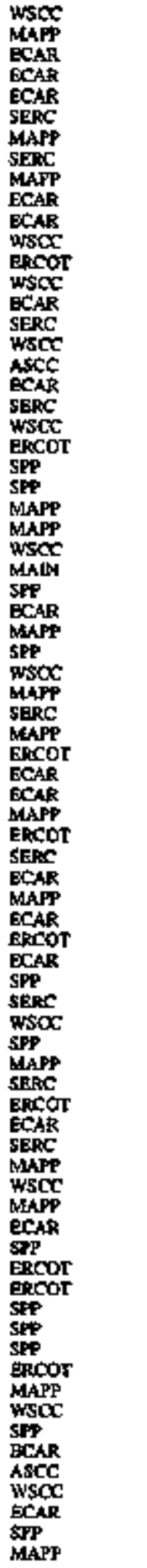 & 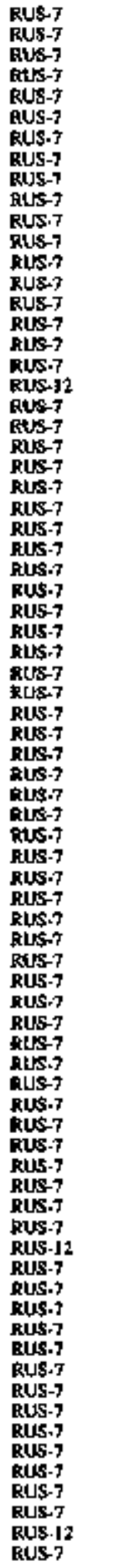 \\
\hline
\end{tabular}

Sec footonges it and of tobid. 
Takłe B5. Cooperative Boctowers, 1994 (Continned)

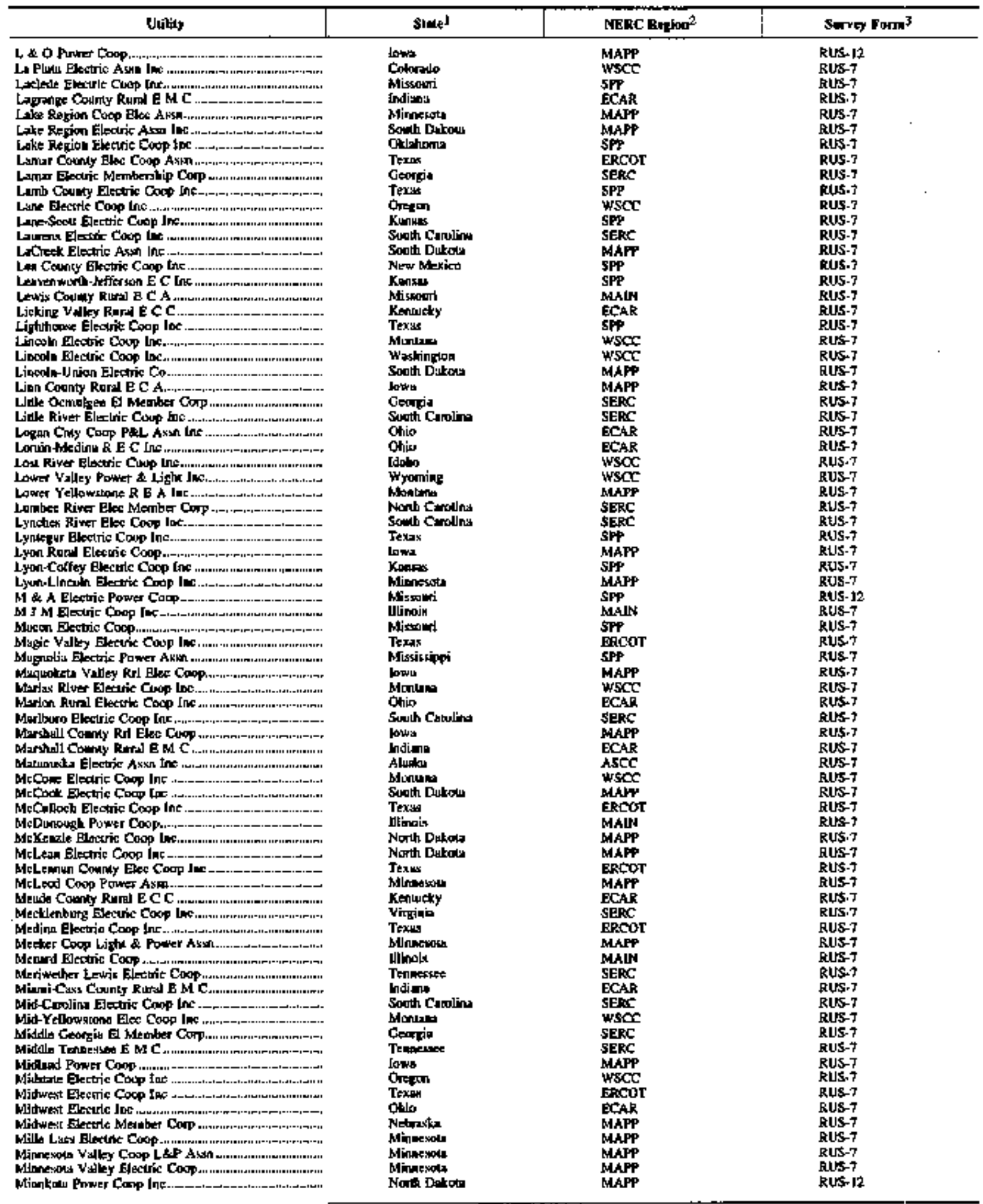

Set formotes an end of colls. 
Table B5. Cooperative Borrowers, 1994 (Continued)

\begin{tabular}{|c|c|c|c|}
\hline Uulty & Stral & MERC Requat? & Sucney Poxm? \\
\hline 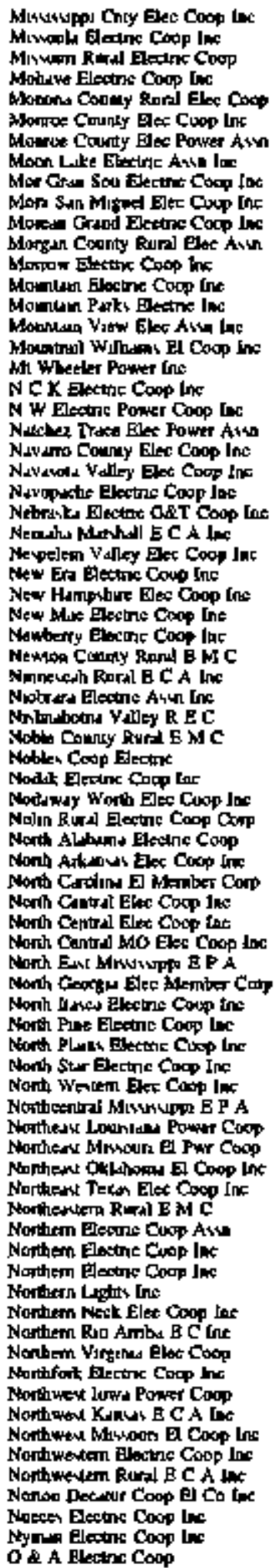 & 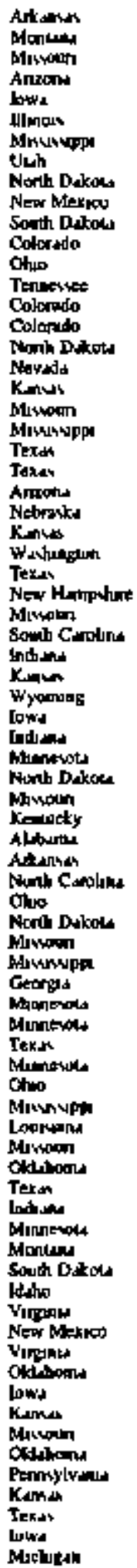 & 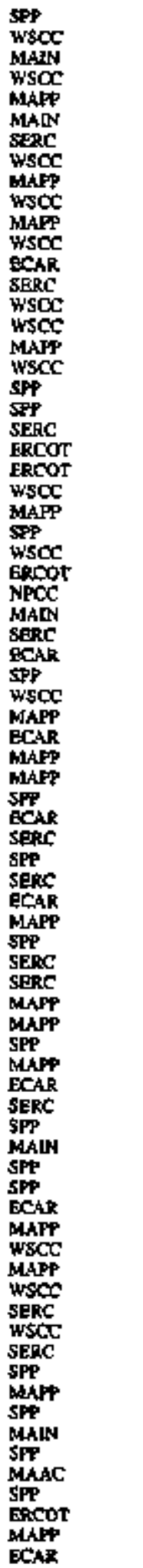 & 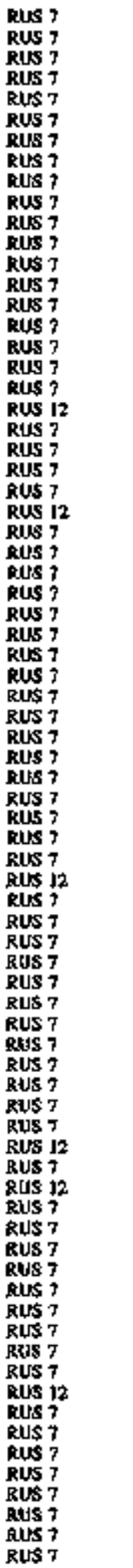 \\
\hline
\end{tabular}

Ser foothotev ta and of cuble 
Table BS. Cooperative Borrawers, 1994 (Continned)

\begin{tabular}{|c|c|c|c|}
\hline ditíty & gant & NLRC Rajiont & Sacky Ferm 3 \\
\hline 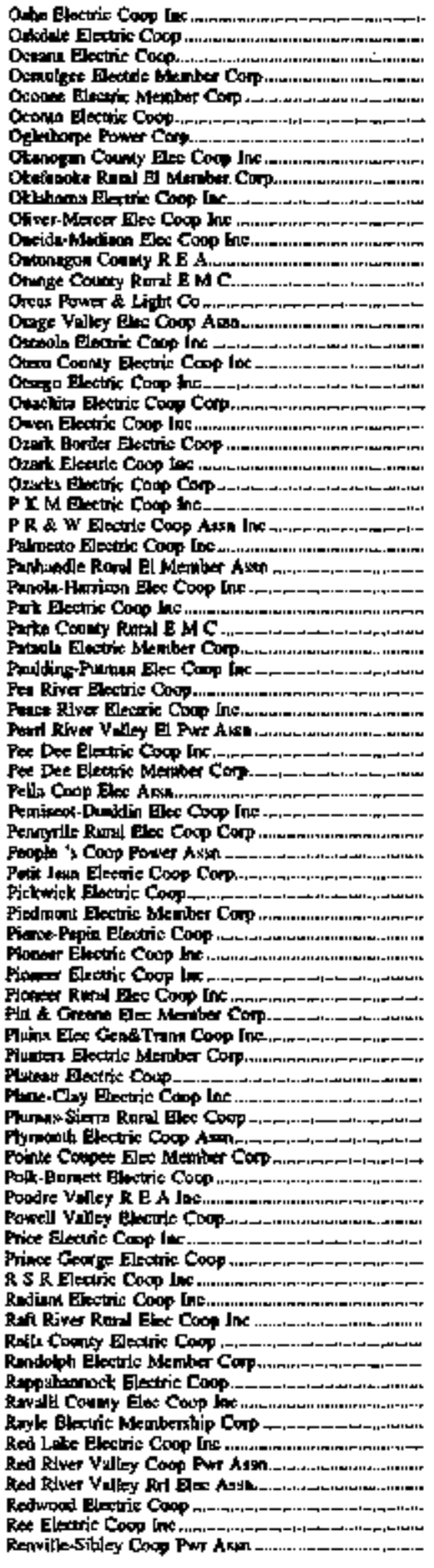 & 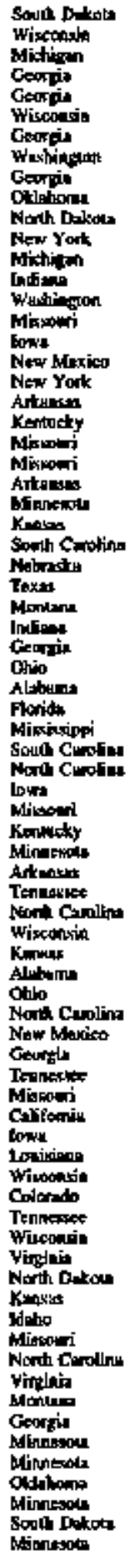 & 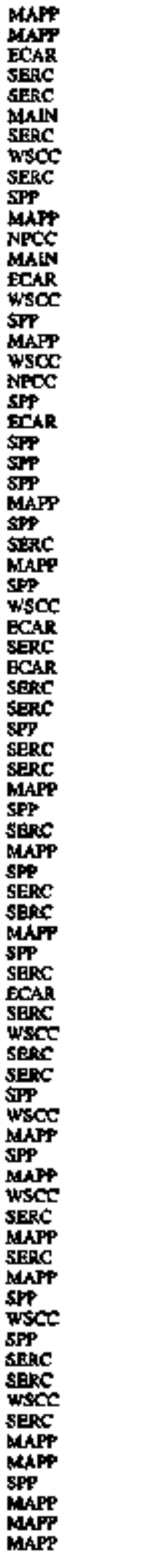 & 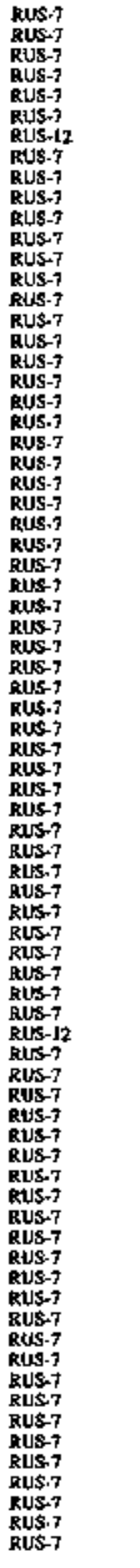 \\
\hline
\end{tabular}

Siec footnoter at ead af wble. 
Table B5. Cooperative Borrowers, 1994 (Contimed)

\begin{tabular}{|c|c|c|c|}
\hline पآा"ty & St=1.1 & 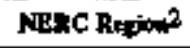 & Sarver Foe- \\
\hline 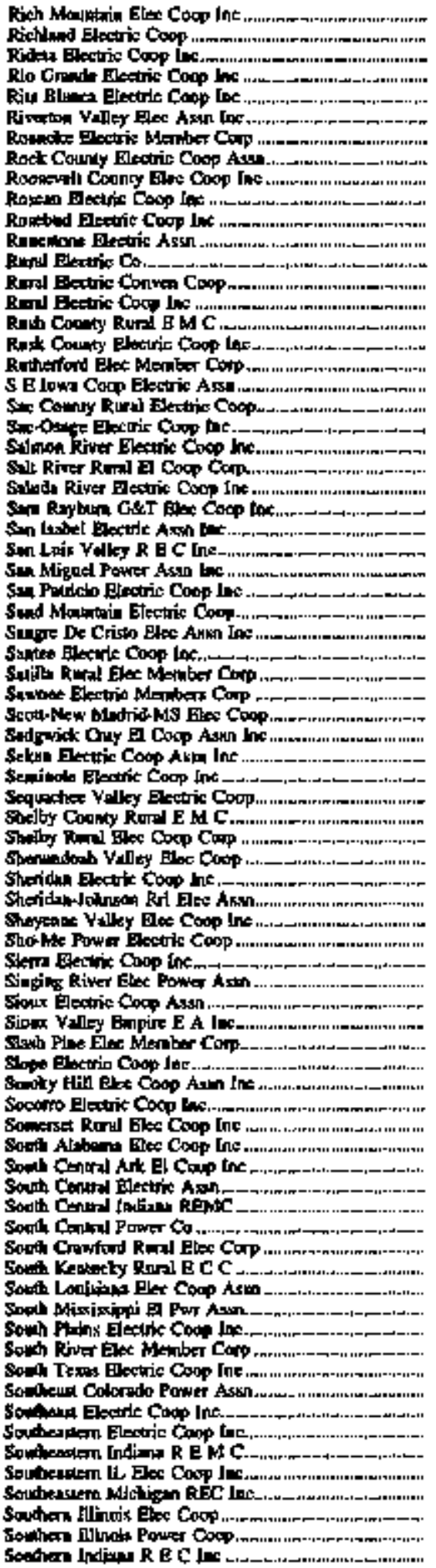 & 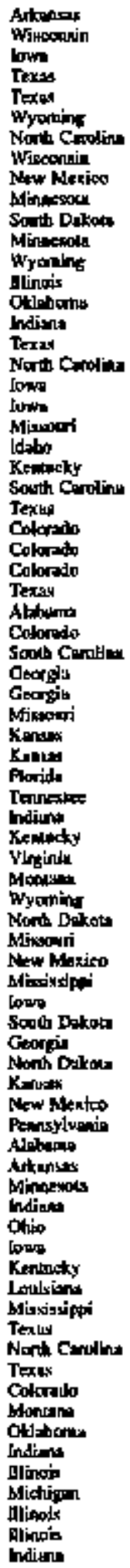 & 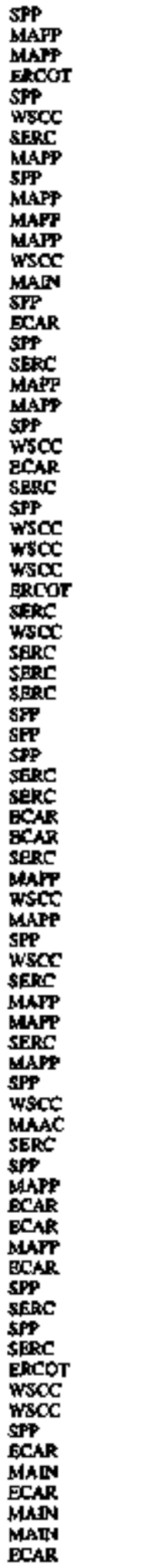 & 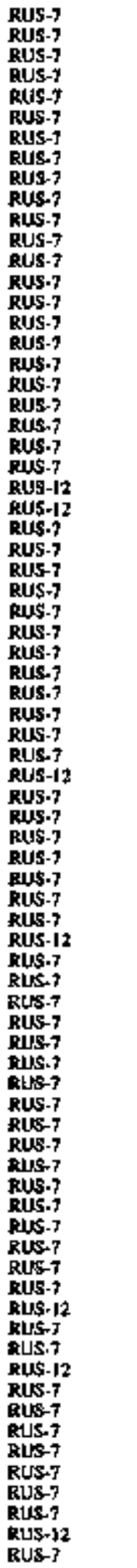 \\
\hline
\end{tabular}

See Jombinien at and of wbje 
Tahłe BS. Cooperative Borrowers, 1994 (Continued)

\begin{tabular}{|c|c|c|c|}
\hline Utit:ty & Sentel & NEAC Re:ph & Surwey Focin? \\
\hline 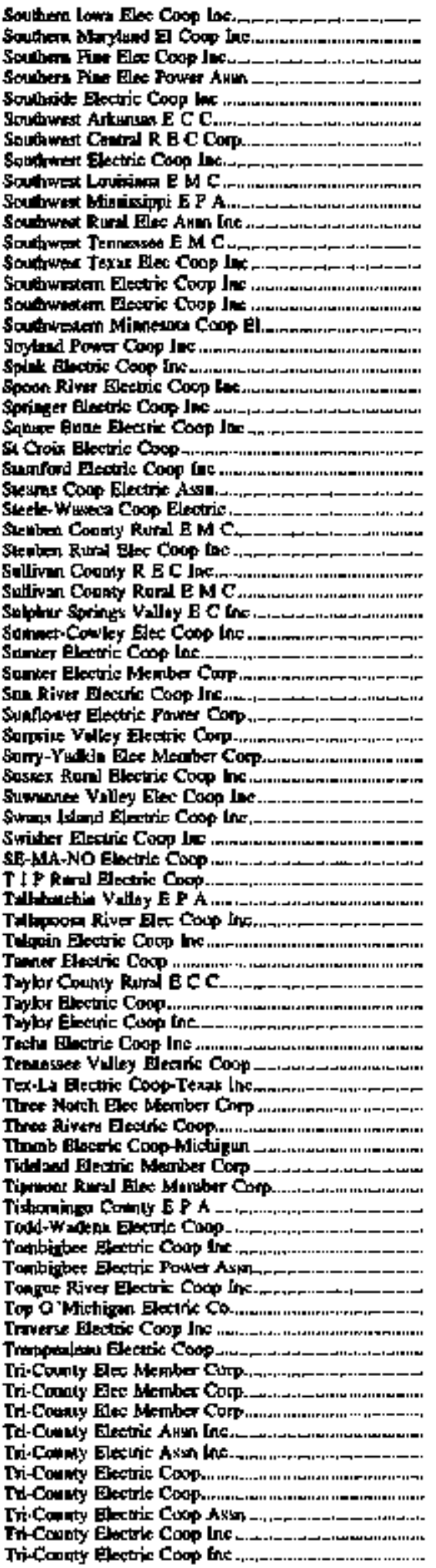 & 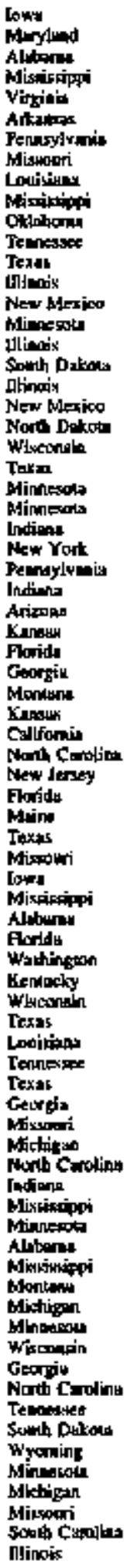 & 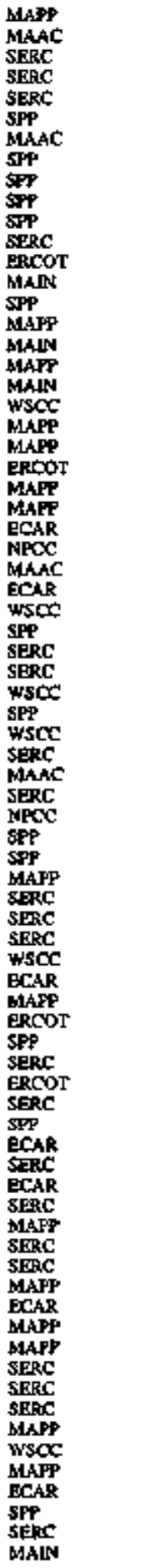 & 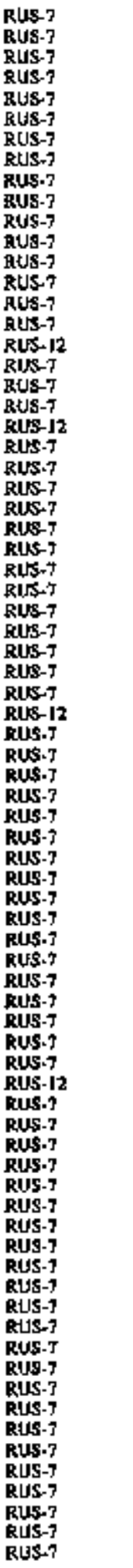 \\
\hline
\end{tabular}

Sed footnotes in and of tabit. 
Table BS. Cooperative Borrowers, 1994 (Continued)

\begin{tabular}{|c|c|c|c|}
\hline Uility & Sunel & NEIC Rathoses & Surney Fomb \\
\hline 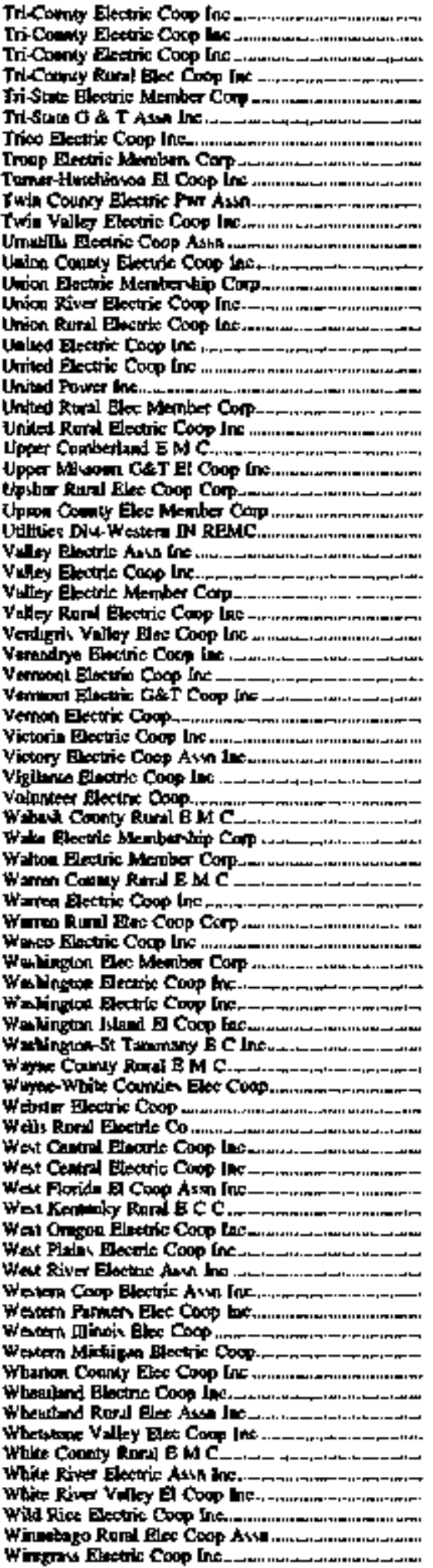 & 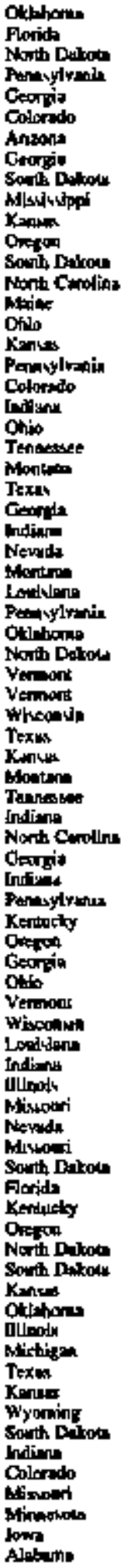 & 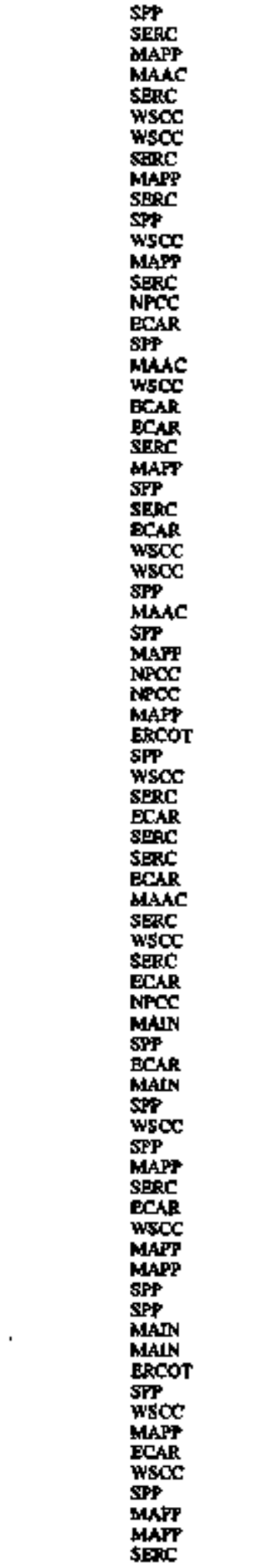 & 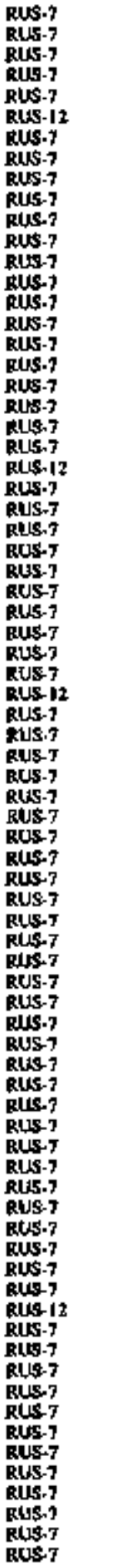 \\
\hline
\end{tabular}

Sec foounation at end of ute 
Table B5. Cooperative Borrowers, 1994 (Continued)

\begin{tabular}{|c|c|c|c|}
\hline ton:ty & Stitel & NLRC Rropen? & Survary Ponn' \\
\hline 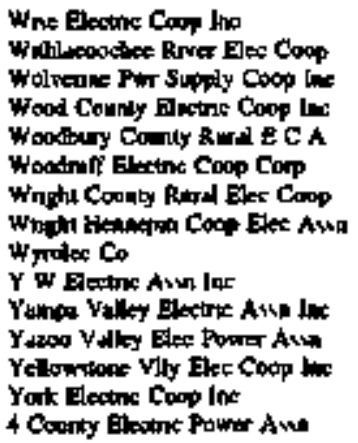 & 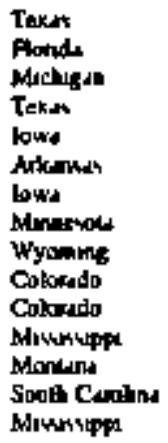 & $\begin{array}{l}\text { ERCOT } \\
\text { SERC } \\
\text { ECAR } \\
\text { SPP } \\
\text { MAPP } \\
\text { SPP } \\
\text { MAPP } \\
\text { MAPP } \\
\text { WSCC } \\
\text { HSCC } \\
\text { WSCC } \\
\text { SERC } \\
\text { WSCC } \\
\text { SERC } \\
\text { SERC }\end{array}$ & 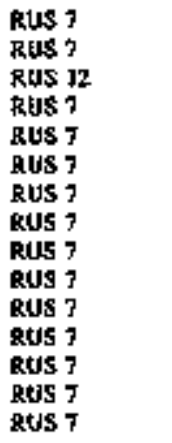 \\
\hline
\end{tabular}

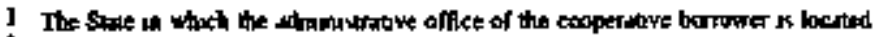

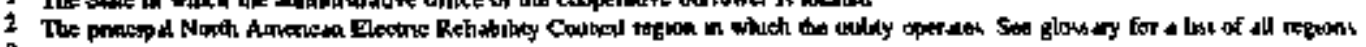

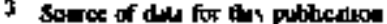

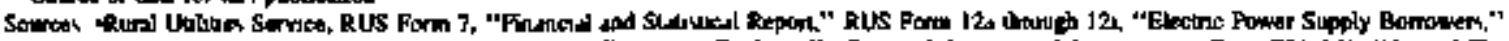

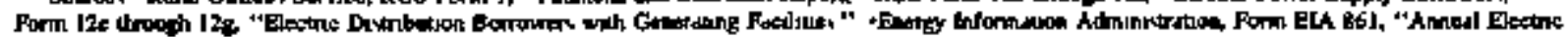
Uhliky Repert' 
Tabłe B6. Electric Utilities Not Reported Elsewhere in Survejs Reporting Detalled Transactions, 1994

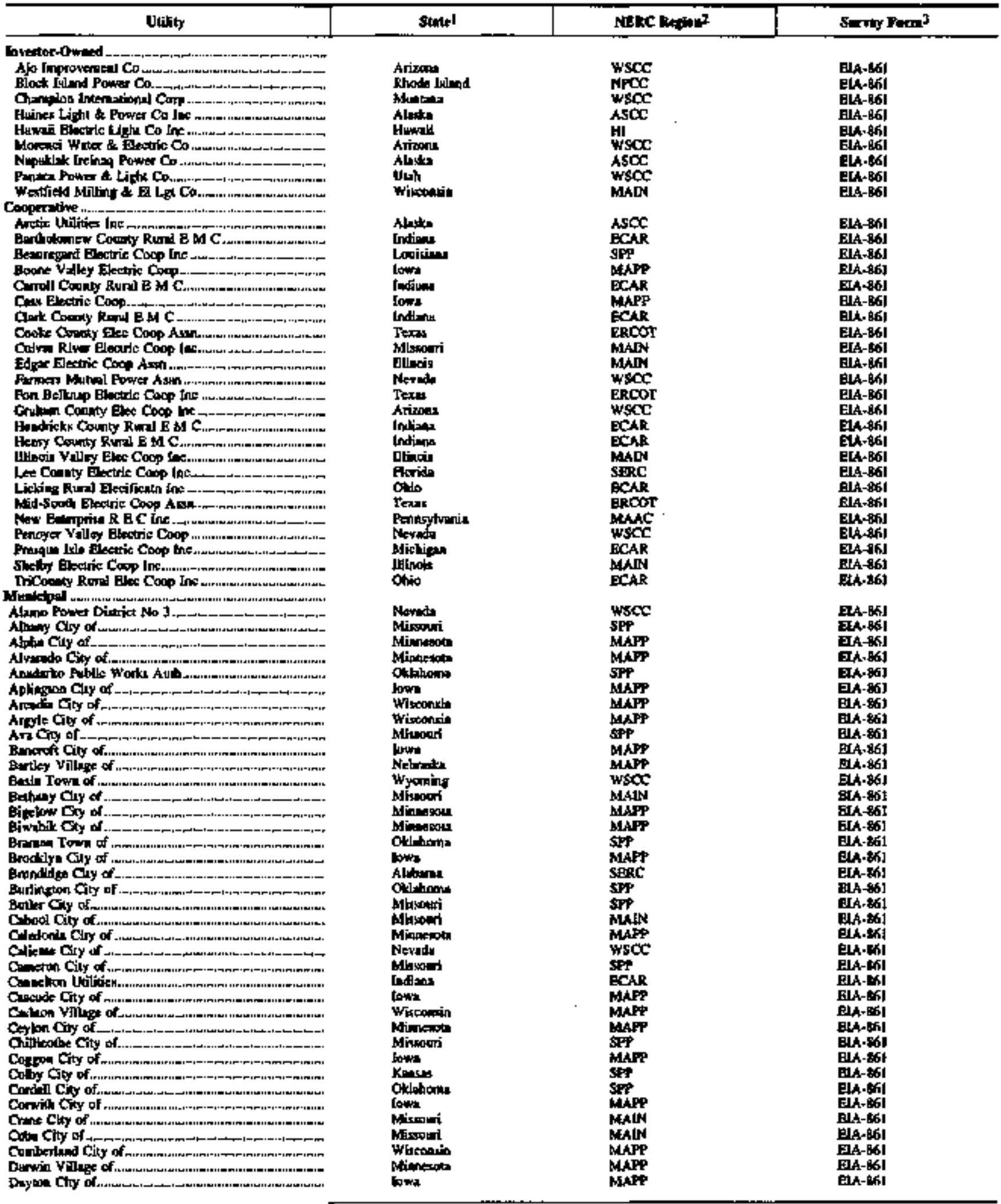

Sec fooungter in fad of thble. 
Table B6. Electric Ulitities Not Reparted Elsewhere in Surpeys Reporting Detailed Transactions, 1994 (Contimued)

\begin{tabular}{|c|c|c|c|c|}
\hline 14tily & Stote] & NRRC knderist & Surves Porm & \\
\hline 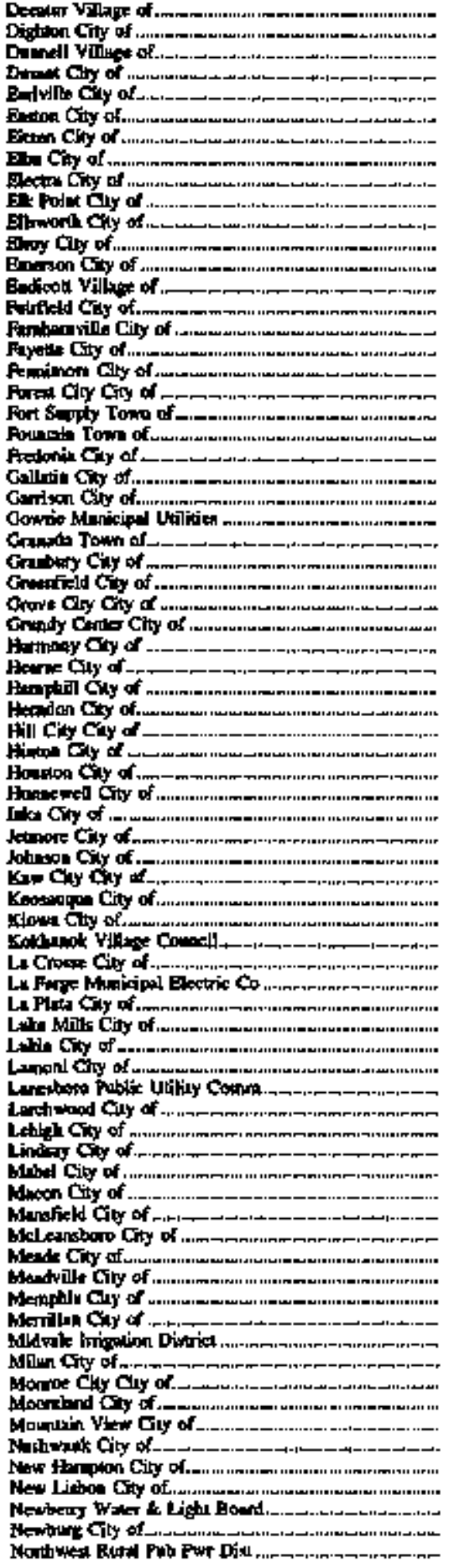 & 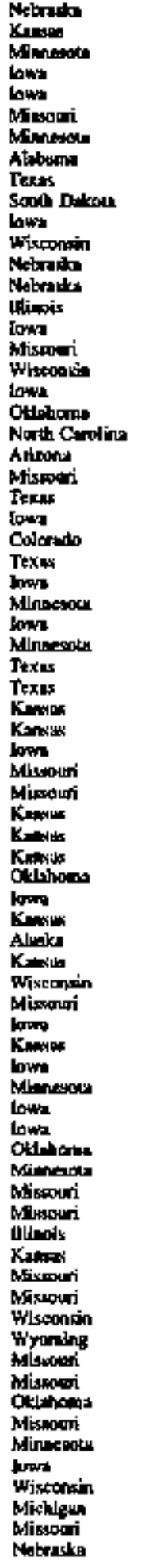 & 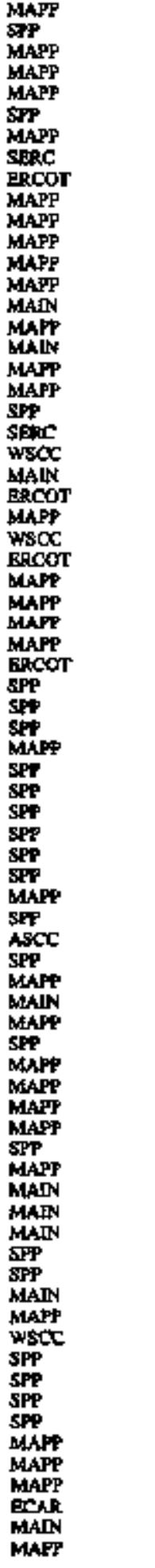 & 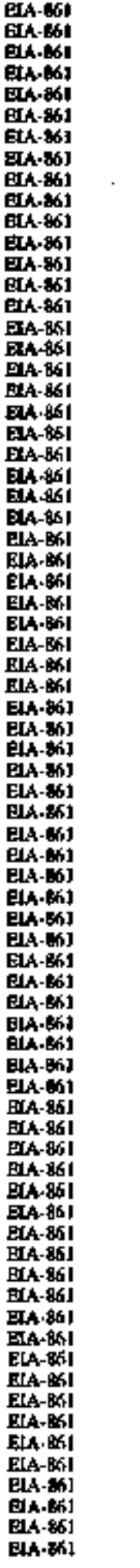 & $\begin{array}{r}\cdot \\
\end{array}$ \\
\hline
\end{tabular}

See foomotes w ead of lable. 
Table B6. Electric ULiftles Not Reported Elsewhere in Surveys Reporting Detalled Transactions, 1994 (Continued)

\begin{tabular}{|c|c|c|c|}
\hline Uunily & Sint & NIRC Rsuan? & Sarwey Farm ${ }^{3}$ \\
\hline 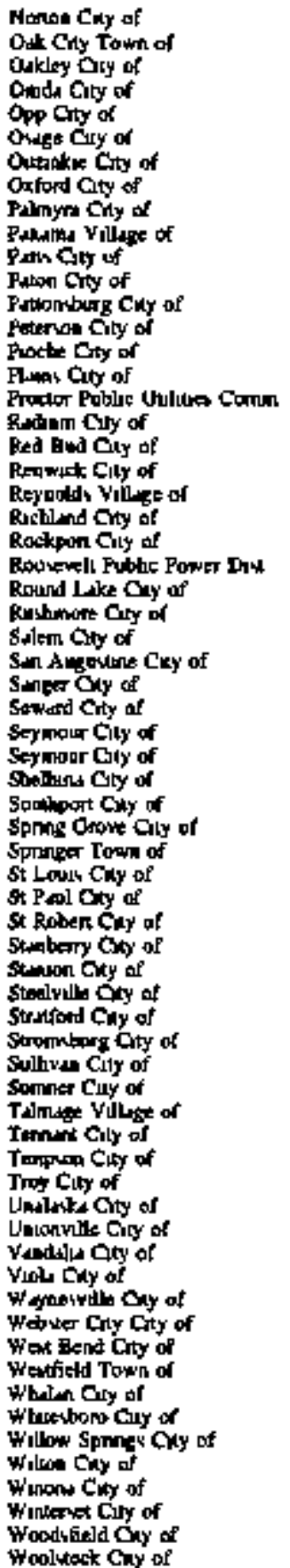 & 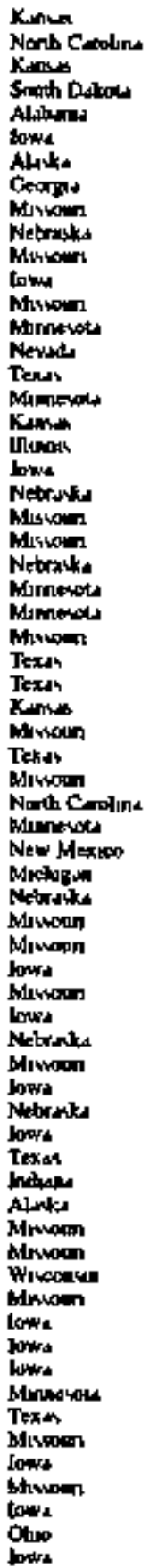 & 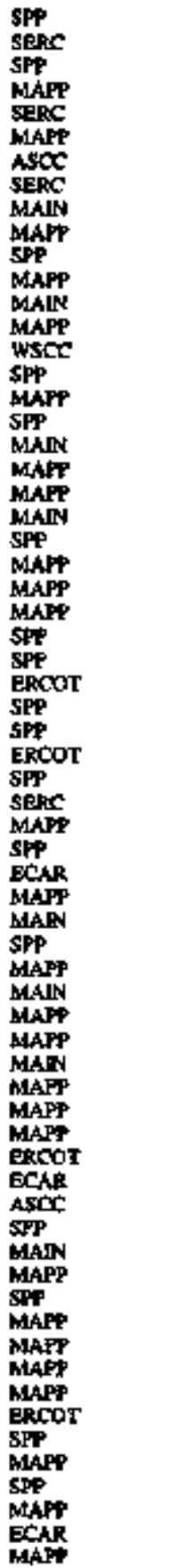 & 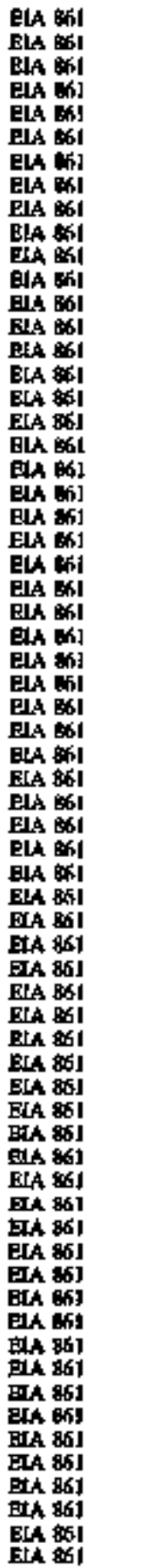 \\
\hline
\end{tabular}

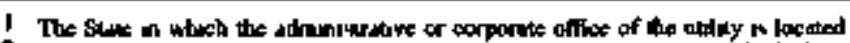

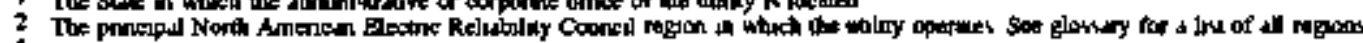

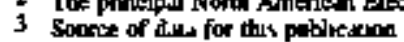

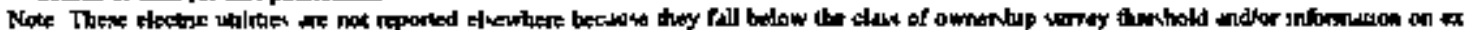

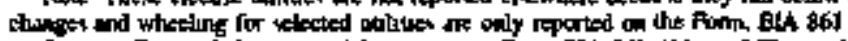

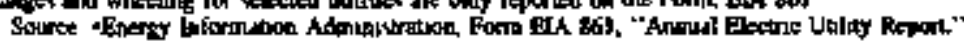


Table B7. Electric Power Marketers Authorined by FERC, as of Decenber 31, 1995

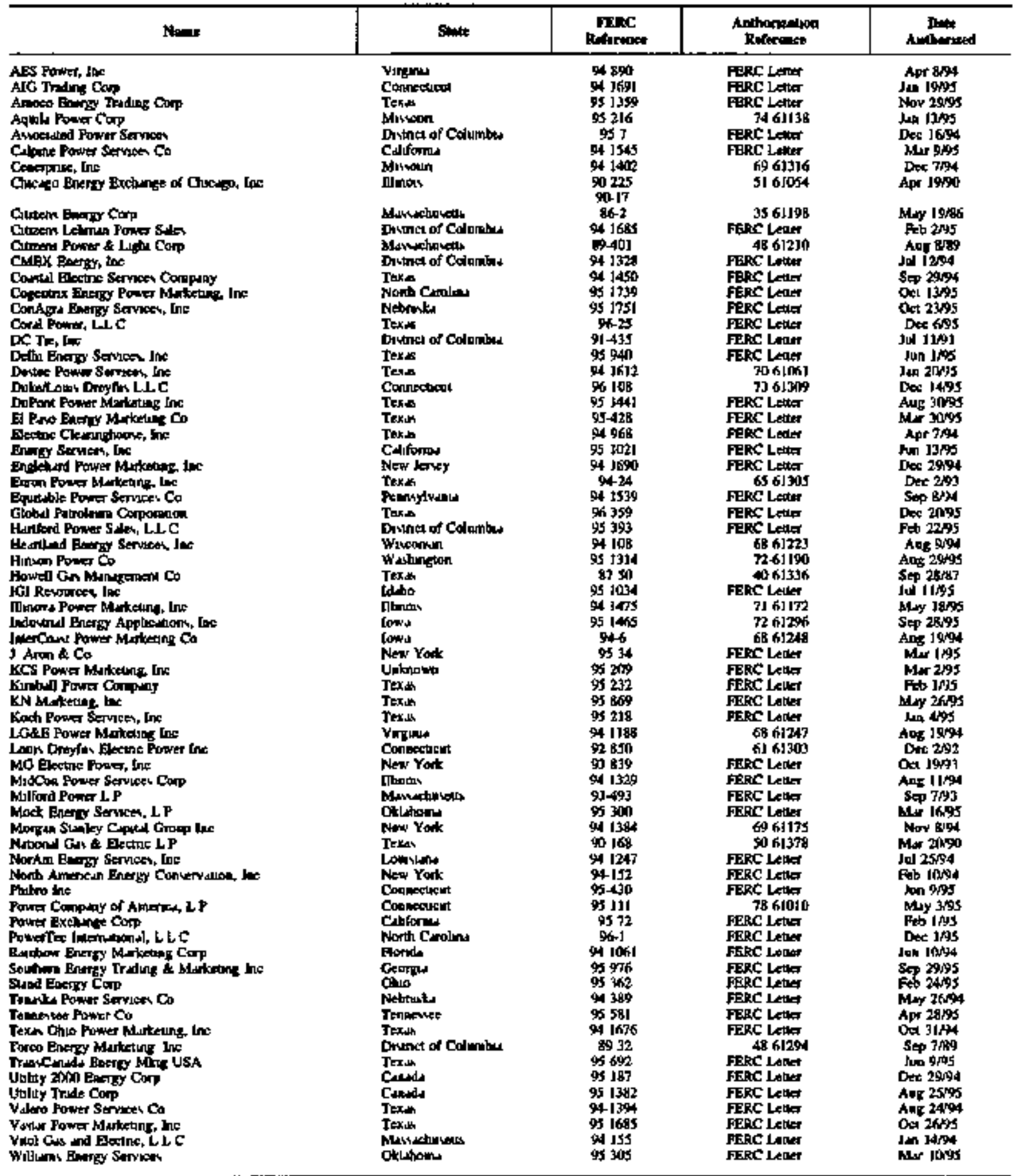

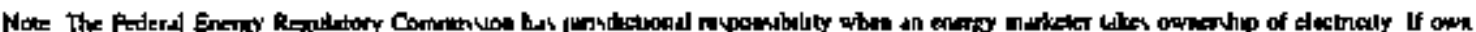

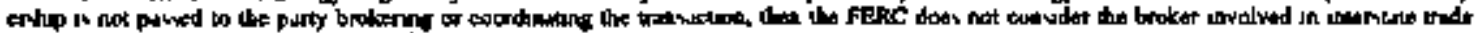

Sodre Fodasl Enata Regalstory Conmuswort 


\section{Appendix C}

Fundamentals

of Electric

Power

Transmission

and

Integrating

Nonutility

Generators 


\section{Fundamentals of Electric Power Transmission and Integrating Nonutility Generators}

\section{Fundamentals of the Electric Power Transmission System}

The tectric power system in the United States contains three interrelated elements: the generating facilities that produce the power; the transmission network that conducts the flow of power from the points of generation to the poinss of distribution; and the distributjon system that delivers the elecric power to the consumers. The transmission network is the integrating medium of the power supply system providing the electrical connections between the many geographically separated paris of the electric power generating and distribution systems.

The electric transmission network is unlike any other mode of trassportation. The flow of electricity is virtually instantaneous, changing magnitade and direction as conditions on the power system dictate." Electricity distributes itself along paths of least resistance that are determined by a complex electrical relationship involving the relarive size, location and distribution of generation resources, transmission line facilities, and centers of demand. All transmission paths share the power transfer, and the degree of sharing is determined by the relationship of the network components. The system consists of transmission limes, substations with voltage transformers, eircuit breakers, and other equipment required to transmit power safely from generation sources to ultimate customers. Transmission volcage levels have increased with improvements in technology and in kecping with the growing demand for electricity.

The transmission system performs several essential functions simultaneously: (1) it supplies the physica] means for delivering electricity from the generating sources to the load centers; (2) jt integrates generating sourtes and load centers into a flexible and resilient whole; and (3) it interconnects the physical facilities with those of peighboring systems. Although transmission lines are often added to the network inirially to meet a single specific requirement, once added they become an integrated part of the transmission network and thejr operation becomes interdependent with all the other elements of the network. Operating the system effectively requires significant planning and operational coordination of the gemerators and transmission facilities to: (1) achieve efficient use of all system facilities, (2) prevent overloading and failure, and (3) maintain adequate reserve transmission and gencration capacity to easure system reliability.

\section{The Need for Coordination of System Operation}

The curreat electrical system has developed in response to the regulations and economict of the electrical utility and nonutiliry sectors of the electric pawer industry, as well as to the changing technical factors that influence the generation, transmission, and djstribution of electricity. Since the different electrical systems operate as a unified power grid (there are three in the contiguous United States) and the effects of power flows are not confined to contractual paths or apparent direct paths, handling the ever changing flow of electricity is a critical activity for system operators of the power grids. Large power transfers, for example, ean change transmission line loadings bundreds of miles from the direct electrical path connecting the source and destination. Actions by individual utilities of NUGs can affoct the operation of all the others on the system.

\section{Controf and Operation of Electric Systems}

As clectrical energy itself cannot be stored (at the volrage and ampere levels in use on a power grid), power must be instantaneously available to end users at any time, in any amount at the proper voltage. As a result, severe demands are imposed on electrical equipment and the transmission network when meeking changing loads. Monitoring the flow of scheduled electricity, handling customer requirements, and eoordinating trade among utilities are the responsibility of the dispatch center. $A$ dispatch eenter

\footnotetext{
The frequency of electric pover supply iq the United Slates is almost entirely (00 hertz (formerly cycles per sec oad). The frequepcy of a nysien degreads entirely upon the speed at which the supply generalos ts rotated by its prime mover. James Robert Eaton, Eiciric Power

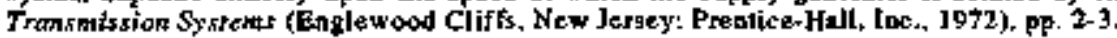


can be operated independently of other tectrical systems by a single utility; it can link two or more interconnected ntilities, or even unify several power systems with combined load requirements and maintenance programs.

The operators of dispatch centers must continually monitor load patterns to ensure that adequate electricity is avaifable at all times. For most dispatch cepters, it is the daily responsibility to (1) record the flow of electricicy at the customer load ceniers and the entering and exiting anounts on its transmission lines, (2) watch the transmission connecting points for each interconnected electrical system, and (3) monitor the power flow from each generation plant. The dispatch center determines the power available from its system, balances the unit-generation marginal costs witb buyor-sel] opportunities with other utilities, coordinates the bulk power transactions, examines what plants must be dispatched to avoid technical system problens or undue economic costs, and accounts for system power losses. It also projects demand requireenents in order to determine bow much genersing capacity will be reeded and when. These projections may be done bourly, dafly, wekly, or at longer intervals.

One electrical operating entity (power pool, electric ntility, State atthority, and/or Federal utility) within a group of interconnected electrical systems takes responsibility for maintaining system frequency for that electrical geographic area, monitors the toad, and ensures generation availability to meet load requirements. Some control centers within these control areas are highly compnterized, automatically loading the generating facilities as needed and maintajning the system at the correct operating frequency. This is important because deviations in the scheduled power llows or from the standard system frequency can automatically cause compensating chsnges in the outpat at the generating plonts. These deviations can mean there has been a loss or gain of a customer load, a plant or line has suffered a forced outege, or some plant or line has been returned to the system. Any of these clanges can require some revitw or action by these control cebters.

Stabilizing system frequency is made easier by coordination with other electric systems and by drawing from a larger base of on-line capability. Load changes are absorbed by all the electrical systems, and many of the increasing and decreasing load changes cancel out of offset each other, 80 that the effect on the entire interconnected electrical system is less than it would be on an isolated electric utility. Also, this integrated system frees each generating unit from the necessity to make continual large changes in production levels.

\section{Integrating Nonutility Generators with the Bulk Electric System}

Nonutility generation sources continue to be a growing portion of the U.S. electrical generation capacity. This role of NUGs reflects the changing structure of the electric supply system.

\section{Integraling Nonullity Generators}

NUGs present a challenge to the opejators of the power grids because of the increasing numbers and their growing contriburion to wholesale generation. Matching cuscomer losd and geveration for both daily operations and future planning activities is becoming increasingly complex with the growing NUG role and increasing use of the transmission system. Ejectrical reliabilicy coperas and the proper integration of NUGs into the supply system have become important issues.

The proper integration of NUGs into the electrical operations of interconnection and dispatching generation can be regarded as engineering problems for which technical solutions are available. However, there are institutional issues associated with the responsibility for serving customers and control of the electrical system. The increasing role of NUGs has altered the traditional view of participants in the electrical supply.

Utifities have three basic concerns involving the integration of NUGs with the bulk power \$ystem, relating primarily to the relationships of the NUGs, utilities, and customers:

- Utilkies, with the priacipal responsibility to operate the system, do not a]ways have full oper* ating control over the NUGs.

- The forces that dejve NUO development and opexation do not necessarily coincide with the obligations of the utilities to serve customer demands.

- The fulfillment of NUG development plans to support the futare generation requirements of the system ate not controlled by the atilities, which are obligated to provide sufficient capacity.

The investor-owned utility obligation to serve is part of what was once called the "regulatory compact," which tied the utility exelusivity to a service tetritory franchise and requires that the rate of remm and prices be set by a regulatory body. However, the obligation of NUGs to provide power tends to be contractual. This contractual obligation (power sales contract) must be satisfied to provide an adequate returk on investment asd to service febt.

The perspectives of some utility industry organizations and NUG participants on these obligations may differ. Some of these differences can be attributed to positions of the organizations in the market, with respect to their cost structures and existing capacity. 
Some utilities welcome the opportunity for potential cost savings and diversity of supply options offered by NUGs, others are more concerned about operations and overall system reliability.

In response to such concerns, the Nortb American Electric Reliability Counctl (NERC), which the utility industry charged to oversec the reliability of the bulk electric supply, has established goidelines on the minimum operating considerations that all utility and nonutility generators must follow to ensure the contioued reliability of the system. ${ }^{\text {to }}$

\section{Impsct of Nonutlity Generation on the Supply System}

The electric utility industry and nonutility industry have worked together to safely and relizbly intercon* nect NUG. Many utilities are increasingly relying on NUO power as an important source of power. Several factors which utitities may not control can influence the operation of the overall system. The overall level of increase in NUG capacity is just one element. The size of individual facilities has a direct bearing on the potential system impacts; small facilities are less likely to have the same impacts as large ones. At the sarne time, the Iocations of individual projecrs, even suisll ones, can be critical. Where a facility is sited can affect transmission line loadings and substation equipment operation. Similarly, the timing of power production from a NUG facility can affect the balance of power flows on the system. Moreover, the avallability and reljabiltty of NUG power can influence the operation of the system and the requirements for reserve capacity.

The electrict] supply system is operated within closely watched tolerances and can require comptex and real-time balancing of gencration and transmission facilities with fluctuating demand. The substantia] interrelationships of al! the system components--utility and nonutility--stggest that generation capacity that falls outside the direct control of system operators increases their operational and planning challenges, and may affect system retiability. The extent to which operation, size, locstion, timing, availability and reliabitity of NUG power production can be coordinated with system operators will determine the impacts of NUG integration on the bulk electricity supply system.

Technically, all of these factors exert both positive and negative influences on the electrical system, depending on site-specific conditions and timing of actions. For example, a NUG facility cotild be located specifically to belp a utility avoid a transmission or distribution bottleneck. Proper integration of NUGs into the daily operational control and management of the electrics] power grid is critical for capturing the benefits and minimizing the disadvantages for all entities connected to the grid.

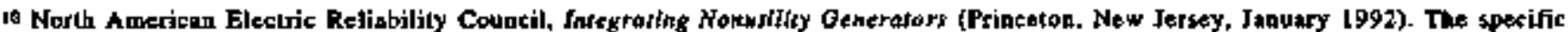

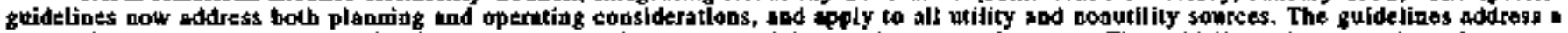
raoge of aeeds, from specifie dosign igsoss, to information needs, and data exchange requitementt. The guitelitues also cover how the geter-

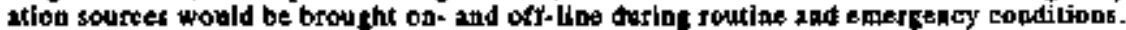




\section{Appendix D}

\section{Technical Notes}




\section{Technical Notes}

\section{Sources of Data}

The data sources used to profuce this publication include selected items from the following Federal Energy Regulatory Commjssion (FERC), Energy Information Administration (EIA), and Rural Electrification Administration (REA) anmual data co)lection forms:

- Form ELA-412, " Anmual Report of Public Electric Udilities"

- Form EIA-861, "Annual Electric Uriliry Report"

- Form FE-781R, "Annual Report of International Electrical Export/Import Data"

- FERC Form 1, "Annual Report of Major Electric Utilities, Licensees and Others"

- FERC Form 1-F, -Annual Report of Nonmajor Public Utilities and Licensees"

- REA Form 7, "Financial and Statistical Reports . Electric Distribution Borrowers"

- REA Fonm 12a through 12i, "Electric Power Supply Borrowers," and RUS Form 12c through 12g, "Elcetric Distribution Borrowers with Generating Facilitice"

Completion of these forms by electric urilities is mandatory. Individual forms are completed by all U.S. utilitics meeting specific ownership and size criteria. Sampling methads ase not used as part of these surveys except for minimum thresbold reporting; all qualifying utilities (that is, all utilities satisfying reporting criteria) are required to report anpualty.

Other 1994 data collected on these forms are also publisbed by EIA and RUS in the following reports: Financial Statistics of Selected Investor-Owned Eleciric Uilties 1994 (DOE/EIA-0437)/1, Financial Statistics of Selected Publicly Owned Electric Vitities 1994 (DOE/EIA-0437)/2, and Antwal Report of Energy Purchased by RUS Bormwers (REA).

\section{Form ElA-412, "Annual Report of Public Electric Utiltios"}

\section{Summay of Primary Purpose.}

Form EIA-412 is used to collect detailed accounting, financial, and operatiog date annualy from publicly owned electric utilities in the United States. For the fiscal or calendar year that ended December 31, 1994, new eriteria were used to select the 502 reporting respondtents for this form. Publicly owned electric utilities engaged in the generation, transmission, or distribution of electricity that had 120 million kilowatthours of sales to ulumate consumers or 120 million kitowatthour's of sales for resale for the 2 previous years as reported on Form ElA-86I, "Annual Electric Utility Repart," must subrit Form EIA-412.

\section{Instrument and Design History.}

Federal Power Commission (FPC) Form $1 \mathrm{M}$ was implemented as a mandatory survey by the FPC in 1961. It became the responsibility of the EIA to October 1977 when the FPC was merged into the DOE. Effective January 1980, the FPC Form IM was superseded by Fortr EIA-412, also a mandatory survey, used to eollect the same data as those previously reported on FPC Form IM.

\section{Date Processing}

The processing of dati reported on FERC Form $I$, Form EIA-412, and Form EIA-861 is the responsibility of the Data Systems Branch, Survey Management Division of the Office of Coal, Nuclear, Electric and Alternate Fuels. The normal processing of FERC Form 1-F is the Tesponsibility of the FERC. Automated systems are used to edit daca from the surveys. using deterministic and statistical checks. When these data have passed the editing process, they are aggregated into a master file, which is used as input to this publication's data base. 


\section{Form ElA-861, "Annual Electric Utility Aeport"}

\section{Summary of Primary Purpose.}

Form EIA-\$6I is a census of electric utitities in the United Scates. The mandatory survey js used to collect information on power production and sa]es data from approximately 3,232 electric utjlities. The data collected are used to maintain and update the ElA electric utility frame daca base. This data base smpports queries from the Executive Branch, Congress, other public agencies, and the general public. Summary data from Form EIA-\$6I are also contained in the Electric Power Honthly, the Electric Sales and Revenues 1994 , the Financial Statistics of Selected lnvestor-Owned Electric Utilities 1994, and the Financial Statistics of Selected Publicly Owned Electric Utilities 1994 These reports present totals for electric utilities on a national level, by State, and by ownership type.

\section{Instrument and Design History.}

Form ElA-861 was implemented in Janumy 1985 to collect data as of the end of 1984. The Federal Energy Adutinistration Act of 1974 (Public Law 93-275) defines the legislative authority to collect these data. Detailed comparisons and descriptions of conceptual problems affecting the quality of the data are discussed in the reporth An Assessment of the Quatity of Selected EIA Dala Series: Eleciric Power Data.

\section{Form FE-781R, "Annual Report of International Electrical Export/mport Data N}

\section{Summary of Ptimary Purpose.}

Form FE-781R is usced to collect on an annual basis, monthly international electricity trade information on the gross amovints of electrical energy imports and expotts and the costs and revenues associaled with these transactions. The use of the format contained in Form FE-78IR is optional for reporting purposes; however, submission of the data is mandatory

\section{Instrument and Design History.}

The authority to issue presidential permits pursuant to Executive Order Number 10485 was transferred to the Secretary of Energy by Executive Order Number 12038 (43 FR 4957 February 7, 1987). This responsibility was delegated by the Sectetary to the Exonomic Regulatory Administratiot (DOE Delegarjon Order Number 0204-04, October 1, 1977). The authority was redelegated (DOE Delegation Order Number 127) to the Office of Fuels Prograns, the Office of Fossil Energy (54 FR I1436 March 20, 1989). The survey ueniverse is defined under Title 10 of the Code of Federal Regulations, Sections 205.308 and 205.325 to include all public otilities or other entìies subject to the Department of Energy jurisdiction under Part II of the Federal Power Act, engaged in the export of electrical energy across the intemational borders of the
United States with Canada and Mexico, or, who own the physical facilities which are used to transmit power actoss the international border.

\section{Data Processing.}

Form FE-781R is mailed to the respondents to collect annually the monthy data for the preceding calendar year. The completed forms are to be returned to the DOE by February 15 . The receipts are manually edjted and the data used for the Presidential Permit Program are entered into a machine readable format.

\section{FEAC Form 1, "Annual Report of Major Electric Uithlies, Llcensees and Others"}

\section{Summary of Phimary Purpose.}

The Federal Energy Regulatory Commission (FERC) Form 1 is used to colleck detajled accounting, financial, and operating data annually from "major" investor-owned electric utilities having, in each of the last 3 consecurive years, sales or transmission service that exceeds any one of the following: 1 billtion kilowatthours of total sales, 100 thousand kilowatthours of sales for resale, 500 thousand kilowathours of gross interchange out, or 500 thou. sand kilowatrbonrs of wheeling for others (deliveries plus losses). The FERC Form 1-F is used to collect detajled accounting, financial, and operating data annually from "minor" investor-owned eloctric utilities that fall below the major category. For the year 1994, juristictional filings of the FERC Form 1 and 1-F totaled 198; though not all can be considered traditional electric utilities. See Table B1 (Appendix B) for those that qualified in 1994.

\section{instrument and Design History.}

FERC Form I was implemented in 1935 by the Federal Power Commission (FPC). In October 1977 , it became the responsibility of the EIA to publish energy data when the FPC was merged into the Department of Energy (DOE). This mandatory report is prepared in accotdance with the FERC's Uniform System of Accounts for private utilities and licensees. In 1990, the wholesale trade schedules were modified. The Summary of trterchange schedule was absorbed by the Purchased Power Schedule and the Transmission For and By Others schedule was changed from a narrative format into two separare tabular collection schedules. In addition to these schedule changes, the statistical classifications covering the data filed on these schedules were adjusted.

\section{Uniformity of Treatment.}

The uniformity of treatment accorded the utilities' statements in this report will generally permit ready comparison of items among the utilities included in the compilations. These statements were filed by all "major" investor-owned electric utilities subject to the FERC accounting jurisdiction. 
The Uniform System of Accounts prescribed for use by electric utilidjes under the FERC's jurisdiction was prepared in cooperation with the National Assonciation of Regulatory Utility Commissioners." In all material respects, the Uniform System of Accounts conforms with the Assaciation's approved system adopted by certain State commissions. The "major" investorowned electric utilities are required to follow the Uniform System of Accounts. In the presentation of individual utility data, the utilities are grouped according to the survey form and the class of owner. ship to which the utility belongs. The 502 perblicly owned electric utilities that filed the EIA-412 in 3994 are requested to follow the Uniform System of Accounts when making their filings.

No attempt has been made to consolidate (in the technical accounting sense) the accounts of the utilities included in the sumblary statement of this report. The combined totals represent the arithmetic sum of accounts as reported by the individual utilities. Consequently, duplications exist to a linited extent in the composite totals shown in several statements. Therefore, as a result of mergers, consolidations, and sales of properties to public authorities, as well as the introdoction of new utilities, the dollar and quantity figures presented in the report will not be representatjve of exactly the same properties froto year to year.

\section{FERC Form 1-F, "Annual Report of Nonmajor Publle Utlliles and Licensees"}

\section{Summary of Frimary Purpose.}

FERC Forts 1+F is used to collect limited accounting, financial, and operating data anpually from investorowned electric utilities thet are not classified as "major, and had total sales in each of the last 3 consecutive years of 10 million kilowatkhonss or more.

\section{Instrument and Design History.}

FERC Form 1-F was impleekented by the FPC regularions under the authority of the Federal Power Aet as FPC Form 1-F for the year 1961 and thereafter. In October 1977, the FPC was nierged into the DOE. This mandatory report is prepared in accordance with the FERC's Uniform System of Accounts for Private Udilittes and Licensees.

\section{Publication-fERC Form 1 and Form E/A-412}

Data on plant eonstruction cost, production expenses, generating equipment characteristics, and other retevant operating data are no longer published.

Financial data can be found in the Financial Sratistics of Selected Investor-Owned Electric Utilities 1994 and Financial Statistics of Selected Pkblicly Owned Electric Uitities 1994. These data include the following: incorne and earnings, taxes, depreciation and amortization, distribution of salaries and wages, electric utility operating revenues, electric utility operation and majntenance expenses, and generating plant statistics. Planted construction data, year-end bslance sheets, and general corporate information are also includert,

\section{Information Collected on FERC Form 1, Form EIA-412, and Form EIA-861}

Reported data selected from FERC Form 1 include sales for tessale, purchased power, exchanges, and wheeling, while the reported data selected from Form ElA-412 include only sales for resale and purchased power.

\begin{abstract}
"Electric Energy Account Summary data" for the municipals, cooperatives, Federal power marketing administrations, and the Tennessee Valley Authority were summarized from Form EIA-86]. Form ElA-861 is used to collect information for ELA on the statistics of electric vtilities and their generation, transmission and distribution of electric energy in the United States, its territories, or Puerto Rico. Form EJA-861 is mandatory under Public Law 93.275, the Federal Energy Administration Act of 1974.
\end{abstract}

\section{Rural Eloctrification Administration Cooperative Utiltles}

In an effort to broaden the coverage in this report of the electric utility industry, information has been included from the Cooperative Borrowers of the Rural Electrification Adrinistration (REA). These dara are based on financial and statistical information submitted to the RUS in 1994.

The operating data collected by the RUS furnish information needed for checking the security of govemimeat loans, the preparation of estimates osed in forecasting expenses for furure loans, and allow the RUS to tulful its reporting obligations. The reports prepared by the cooperative utilities must accurately reflect the financial data as shown by their books of account.

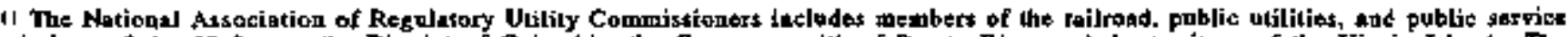
comodissions of the S0 Slates, the District of Colwabla. the Cominonwealth of Poerto Rdeo, and the territory of the Vurgin Islands. The

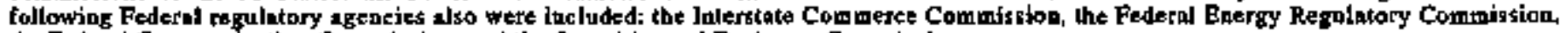
the Federtit Communication Commission, and the Securdies and Exchange Commis siob. 
Conflidentiality of the Data.

The data collected on the forms used for input to this report are not confidential.

\section{Quality of Data}

The Office of Coal, Nuclear, Electric and Alternste Fuels is responsible for routine data improvements and quality-assurance activities in accordance with standards established by ElA. These standards are the measuring rod necessary for quality statistics. Dataimprovement efforts include follow-op on nonrespondents, verification of data-keyed input by automatic computerized methods, and editing by subject-matter specialists.

Completed forms received by the Office of Cod, Nuclear, Electric and Altennate Fuels are sorted, screened for completion of reported information, and keyed onto computer tapes for storage and transfer to data bases on taidom access siorage devices for computer processing. The information coded on the computer tapes is spot-checked against the data reported on the forms to eertify accuracy of the tapes. Computerized respondent data files are checked to identify those who fail to respond to the survey. By law, nonrespondents may be fined or otherwise penalized for not filing an ElA data form as prescribed in the instructions. Before invoking the law, ElA tries to obtain the required information by encouraging coopertation of nourespondents.

The individual utility identification codes for new and existing entities are ediced prior to being sent for data entry. Additional edit checks of these tata are performed through compater prograns. The program edits include both detersinistic checks, in which records are checked for the presence of data in required fields, and statistical checks, in which the data are checked against a range of values baset on historical data values and for logical or mathematical consistency with data elements reported in the form. Discrepancies arising as a result of these checks are resolved either by the processing office or by a telephone call to the eompany concerned.

\section{Date Validation Procedures}

The methodology used to develop this report is based on procedures and techniques routinely employed it data requirement reviews and data valjdation projects conducted by and for EIA. The four-step process used consists of: (1) identifying patterns of respondent misreporting and inconsistencies in reporting between respondents; (2) analyzing each problem identified to develop probable causes; ( $\exists$ ) correcting the identified problems; and (4) modifying the forms and/or instructions to eliminate or mituimize futute occurrences. The objective of the process is to make improvements that will:

- Improve the teadability, acelracy, and meaningfulness of the data collected on wholesale electricity toade

- Reduce respondent burden by means of clarifying instructions, simplifying form layouk, and eliminating unnecessary calculations

- Effect data standardization among the various forms used to collect wholesale electricity trade data

- Facilitate the computerization of wholesale electricity trade data to be collected in the future.

A variety of methods were used to identìfy reporting problems inclutiding:

- Manual pte-edit of FERC Form 1 and Form ElA-412 to identify nonstandard responses that could not be computerized as submitted by the respondent

- Manual review of selected forms to characterize patterns of reporting and misreporting and the type of respondents responsible

- Automated statistical reports (such as frequency counts) summarizing responitent reporting regarding indjvitual data items

- Automated interform data validation to identify inconsistencies between aggregate level data reported by a respondent on Form EIA $\$ 61$ and FERC Form: ]

- Automated intraform data validation to identify reporting inconsistencies (such as, total does not equal the sum of the compogents)

- Autornated interforso data validation to identify inconsistencies betweot respondents reporting the same transaction (such as, the buyer and the seller of power reporting different quantities of esergy delivered and received)

- Follow-up telephome calls are made to respondepts (excluding those who filed FERC Forms) regarding confusing and/or inaccurato entries. Questions on the accuracy of filings made to the FERC are handled at the discretion of FERC staff.

The causes of misreporting and inconsistent reporting Wert identified principatly by studying in detail the form layouts and instructions, and jdeptifying pattems and trends in misreporting among all respondents. Considerable judgment was necessary in developing probable causes since all respondents could not be systematically contacted regarding trrors. As an alter. native, explanatory footnotes found in the completed forms, supplementary pages, and manual checks were used to confirm and validate the data. Once probable causes were identified, modification of the forms was straightforward. 


\section{Data Edits}

The data used for this publication are limited to those reported by respondent utilities. The following edit checks were used to identify missing or inaccurate data from the information that was initially reported by the respondent utilities.

- Energy--Sales for Resale and Purchased Power

- No single transaction could exceed 4,380 milinon kilowatthours.

- If sales or purchase transactions were reported, an energy recejpt or deljvery was required.

- Reported totals or subtotals had to eqaal the sum of energy transactions.

- Eaergy--Interchanges (Exchanges)

- No single transaction could exceed 4,380 million kitowathours.

- If interchange transactions were reported, an energy receipt or delivery was required.

- Net energy received for a transaction had to equal total energy received minus energy delivered.

- Reported totals or subtotals had to equal the sum of energy transactions.

- Revenue--Sales for Resale

- Total revenue for a transaction had to equal the sum of reported revenue-demand. revenue-energy, and revenue-other.

- Total revenue for a transaction divided by the kilowatthours sold for resale had to be in the range of 0.5 to 20 ceuts per kilowatthour.

- Reported revenue totals or subtotals had to equal the sum of revenue transactions.

- Cost+.Purchased Power

- Total cost for a transaction had to equal the sum of demand, energy, and other costs.

- Total cost of a transaction djvided by kilowatthours purchased had to be in the range of 0.5 to 20 cents per kilowattbour.

- Reported cost totals or subtotals bad to equal the sum of cost transactionts.

- Settlement--Interchanged Energy (Exchanges)

- For transactions involving monetary settlement, the setclement amount divided by net kilowatthours received or delivered was required to be in the range of 0.5 to 20 cents per kilowathour.

- For settlement transactions involving monetary settlement, settlement amount was reguired to be positive if more energy was recejved than delivered; negative if more energy was delivered than received.

- Reported totals and sabtotals had to equal the sum of settlement transactions.

\section{Adjustments}

Since the Federal forms used to edlect elecuricity trade data are essentially accounting forms, adjusiment data are rovtinely provided so that subnitted data will be consistent with the accounting systems of the utilities. Many respondents include adjustments, particularly those retaced to transactions occurring in the reporting year, as a component of the related trarsaction. However, adjustments to tronsactions occurring in previous years are aggregated with current year transactions or are listed separately (that is, rebates, overcharges, reclessifications, fuel cost adjustments, elc.).

\section{Salos for Resale and Purchased Power}

Sales for tesale data are collected on FERC Form 1. Form EIA-412, RUS Form 7, and RUS Form 12. The primary sources of this information are FERC Form 1 and a few of the Forth EIA-4]2's completed by Federal and State agencies. These respondents acconnt for most of the wholesale electricity trade in the United \$tates. The detajled data on sales for resale are not keyed jito a machine-readable format by the RUS; thesefore computer matching was not done.

Since sales for resale on Form ELA 412 is on a fiscalyear basis as opposed to calendar year, the Form ElA-412 dara are not consistent with data collected on the other wholesaje electricity trade schedules. Atso wholesale electricity trade sales cannot be aggregated for all Form E[A-412 respondents because the respondents use different fiscal years. This is also true for the purchased power data. In 1989 , the statistical classifications of the Form ELA-412 were modified to galber information on the rypes of electrical power and energy purchased and sold. The 1990 data reflect the collection of data using the enlarged statistical classification groupings.

The RUS Form 12, unlike FERC Form 1 and Form ElA-412, is not used to collect sales according to statistical elassification (that is, firm, dump, and other powery. The RUS does not process the sales for resale data reported on the RUS Forms 7 and 12. As soch, cross-checking between buyers and sellers is less comprebensive. It also should be noted that Form ElA-412 was used to collect statistical elassification data for both sales and purchased energy in 1994, while the RUS did not collect statistical classification data for either.

The purchssed power schedules on Form EIA-412 and RUS Fotms 7 and 12 does not include interchange (exchange) transactions. Forto ElA-412 respondents are asked to report exchanges on a gross basis on the Energy Account Balance schedule. The RUS Forms 7 and 12 do not specifically address juterchange (exchanges) transactions, but respondents are to report transactions on a net basis, if there is a cash payanent. 


\section{Interchange (Exchange)}

Both purtasse and intetchange costs fall under the Uniform System of Acconts No. 555, and were reported on two different schedules until 1990. In 1990 , the Summary of Interchange Schedule was deleted from the FERC Form 1 and the information collected on that schedule were shifted to the Puschased Power Schedule. The FERC has determined, for their regulatory accounting purposes, that the term, "interchange," means energy that is only exchanged in kind. Historically, however, "interchange" has been used by the industry to mean all energy transfers between utilities (sales, purchases, exchanges, and wheeling) and it has resulted in inconsistent reporting.

\section{Transmission For Others and By Others (Wheelling)}

Detailed data on the Transmission For or By Others are collected only on FERC Fom 1. Before 1990, respondents provided in a alrative format six basic elements of information for each whecling transaction:

- Name of company and description of service

- Points of origin and termination of service

- Megawarthours received and delivered

- Monetary settlement

- Nonmonetary settlement

- Other explanations needed to describe the trausactions.

The FERC Form 1 was modifjed for the $1990 \mathrm{col}$ lection year. The narrative schedule was changed to two separate tabular formats. The Transmission For Others schedule now collects additional information on: payments made by, energy received from; energy delivered to; statistical clessification; FERC rate schedute; disaggregation of monetary settlement into demand, energy, other, and total; and points of origin, termination of service, and nonmonetary settement were all deleted. Transmission By Others now collects information on name of company; megawatthours received and detivered; and expenses for transmission of electricity by others (e.g., demand, energy, other, and total).

\section{Difference between Publlcatton Methodologies}

The Federal Energy Regolatory Commission moditied the FERC Form 1 in 1990 in order to bandle better their responsibilities under the Federal Power Act. New information was requested that included statiscical classification caregories which collected data on power and energy rransactions based on the original contractual terims and conditions. Because of this new information, the firm, nonfirm, and miscelfaneous publication eategories used in prior years were adjusted to handle these new classifications.

In prior publications, firm trade was defined as any transaction in which the charges bad to include a demand charge. Firm trade data were then separated into two groutps. The first group included transactions with demand charges where no quantities of energy were delivered or received (possible under "talke or pay contracts"), and the second included quantities of entergy that were traded with an "Energy," and sometimes, "Other" charge. (The "Other" charge in the firm category covers associated payments made on utility crausactions for expenses associated with operationg andor renting physical facilities such as transmission lines and substations).

The differences between nonfirm electricity trade and the firm caregories is that no demand charges are involved in the nonfirm transactions. The energy charge plus any "Onher" charges associated with those transactions represented the value of these bonfirm transactions. The last grouping, "Other," includes transactions which did not include a dernand or energy charge. (The filing electric utilities usually did not provide descriptive explanations.) However, from the footnotes provided and the accoont descriptions, data in this category do include: parchasing or maintaining operatiog reserve expenses; stability and reliability standards expenses; transmission line and other facility rentals; and other system operating charges. ${ }^{* 2}$

For this publication, the revised FERC classificarions were used for inputs which helped define the firm, economy, and other electricity trade categories. Form redesigns implensented in reporting year 1990 for both FERC Form 1 and Form EIA-412 permitted respondent reported data to be nsed to differentiate between firm and nonfirm power trades.

The following list shows the type-of-trave categories reported by the FERC Form 1 and the Form EIA-412 respondents.

\section{- FERC Form 1}

- Requirements (RQ)

- Long-Term (LF)

- Intermediate Firm (IF)

- Short-Term Firm (SF)

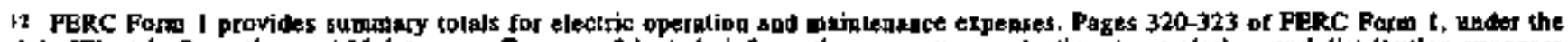

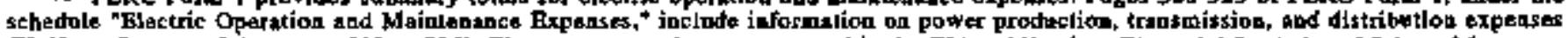
(Uaform Syttem of Accounts 500 to 935). These summary date are reported lo the ELA publication, Finepsial Staristics of Stilected ituvester. Owned Electric Utilities, DOE/EIA-0437(94). This informstion canot be linked to the individual transactious. 
- Long-Term

- Intermediate Firm (IF)

- Short-Term Firm (SF)

- Long-Term Unit (LU)

- Interrnediate Unit (IU)

- Exchange Power (EX)

- Other Service (OS)

- Adjustments (AD)

- Form ElA-412

- Firm Power (FP)

- Unit Power (UP)

- Economy Power (RP)

- Dump Power (DP)

- Majtenance/Emergency Power (ME)

- Operating Reserve (OR)

- Other Capacity/Energy (OT).

Appendix A provides the FERC Form 1 defintitions found on pages 310 and 326 . Form ELA-412 definitions are found on Schedules V and VI of the form.

Like previous years, 1994 transactions without energy receipts or deliveries but with demand charges are classified as firm, "demand charge only" trade. FERC Fotm 1 and Form ElA-4/2 respondents do not always report type of trade data, which results in missing data. Likewise, data is missing for cooperatives, since they are eot required to report the type-of-rtade on the Rural Utilities Service (RUS) Forms RUS-7 and RUS-12. Imputations for missing type-of-trade data are perforaned according to the following procedures:

- Purchased Power. If the respondent urility (the purchasing utility) reported no net generation on the 1994 Form EIA-861, then the trade is classjfied as firm. Otherwise the trade is classifjed as economy.

\section{Data Revisions}

The foregoing edits identified data errors. Data tnaccutacies resulting from transcription and typographical errors, reporting in the wrong units (for example, kilowatthours instead of megawatchours) and arithmetic errors were corrected after discussions with respopdents. Many suspected data errors were found to be correct as reported. Explanations provided by respondents included:

- The transaction was an energy or monetary adjustment to information previously reported. Adjustments reflected settlement of contract disputes, reimbursement of overcharges, or accounting errors and often were one-time bookkeeping corrections.

- The transaction involved large demand charges, but a smajl amovnt of energy was delivered.
- The transaction was a nonstandard operatjug condition sach as loop and inadvertent energy now, emergency power deliveries, or ssle of energy under a purchasso contract.

- The transaction was for access tights or the lease of egujpment used in the sale or purchase of energy.

- Tho transaction was between two associated ntiljties or a utility and a power pool reporting internal energy, revenue, or cost accourting prac. tices (for example, allocations).

\section{Conslstency Checks}

In additjon to edit checks, data consistency checks were perfortied to improve the quafity of the data presented in this publication. The consisrency checks inyolved comparing, when possible, Sales for Resale data (revenue and energy) reported by one respondent with the purchased power data (cost and energy) reported by the respective bayers of the energy. Discrepancies of more than 10 percent were discussed with both the setling and the buying utilities to determine if a reporting error had been made, A similar procedure was used to confirm data inconsistencies between utilities that interchanged energy.

Consistency checks identified data errors, but discussions with respondents determined that most of the data were correct as reported. Reasons provided included:

- A sale or purchase by one utility was considered an interchange by the other utifity in the transaction.

- Sales, purchases, and interchanges for noninvestor-owned utilities transacted late in the yoar can be teported in the year in which the transaction occurres or in the following year in which they are booked.

- Data submitted by 3 utility can cover differenz reporting petiods depending upon the requirements of the forms; Form ElA-412 is a report of the utility's fiseal year while the other forms are for a calendar year.

- Different accounting and reporting procedures are used for recording loop and inadvertent energy flow, equjpment and lease charges, service fees, fuel and other adjustments, and corrections to pre. viously reported information.

\section{Correctlons}

Corrections were made to the data when missing or insccurate data were provided by the respondent. Missing and insccurate information identified during data automation and report preparation were discussed with respondtents during the edit process either to correct the data supplied, or to confirm the values. In all cases, the forms were reviewed during the edit process with the respondent, and changes were made at the direction of the tespondent. 


\section{Explanatory Notes}

Data are reperted on FERC Forts 1 in accordance with the FERC's Uniform System of Accounts. Dats are reported on the RUS Forms 7 and 12 in accordance with the RUS Uniform System of Accounts (based on the FERC System, with minor modifications). Copies of the Uniform System of Accolents Prescribed for Both Privote and Cooperative Borrowing Electric Utilities are available from the U.S. Government Pripting office (GPO).

\section{General Information}

\section{Use of the Glossary}

The cerms in the glossary have been dofjned for general use. Restrictions on the definitions, as used in these data colfection systems, are included in each definition when necessary to define the terms as they are used in this report.

\section{Obtaining Copies of the Data}

This publication is availabte on a subscription basis from the Superintendent of Documents, U.S. Government Printing Ofice (GPO). Make check or monky order payable to the Superintendent of Documents. You may send your order to the GPO or the National Technical lnformation Center (NTIC). GPO and NTIC prices are subject to change without advance notice.

Superintendent of Documents

U.S. Government Printing Office

Washington, DC 20402

8 a.m. to 4 p.m. eastem tíne, M-F

(202) 783-3238

FAX (202) 5]2-2233

National Technical Information Service (NTIS)

Office of Data Base Services

U.S. Department of Commerce

5285 Port Royal Rosd

Springfit]d, VA 2216!

(703) $487-4650$ 


\section{Glossary}

Accounting System: A system of recording accounting data for a utility or company; or of supplying aceonting informtation for controlling, evaluating, planning, and decision-making.

Administrative and General Expenses: Expenses of an electric utility relating to the overall directions of its corporate offices and administrative affairs, as contrasted with expenses incurred for specialized functions. Examples include office salaries, office supplies, advertising, and other general expenses.

Ampere: The unit of measurenent of elcotrical current produced in a circuit by 1 volt acting througb a resistance of I ohm.

Associaled Company (Affillated): A company that is either directly or indirectly controlled by or owned by another firm ot holding company.

Automatic Generating Controk: The regulation of the power output of electric generators within a prescribed control area in response to changes in system frequency. The line loading or the relation of these to each other, so as to maintain the scheduled system frequency and/or establissh interchange with other areas within ptedetermined limits.

Available but not Needed Capability: Nel capability of main generating units that are operable but not considered necessary to carry load, and cannot be connected to load within 30 minutes.

Average Water Conditions: The amount and distribution of precipjtation withis a drainage basin, and the run-off conditions present, as determined by revitwing the area warer supply records over a long period of time.

Backup Power: Electric energy supplied by a utitity to replace power and energy lost during an unschefuled equipunenl outage.

Base Bill: A charge calculated by taking the rate from the appropriate electric rate schedule and applying it to the level of consumption.

Base Rate: A fixed amount charged each month for any of the classes of utility service provided to a cuslomkI.

Baseload: The minimum amount of eltctric power delivered or required over a given period of time at a steady state.
Baseload Capacity: The gentrating equipant normally operated to serve loads on a round-the-clock basis.

Baseload Plant: A plant, usually honsing highefficiency steam-electric onits, which is normally operated to take all or part of the minimum load of a system, and which consequently produces electricity at an esseptially constant rate and nuns continuously. These units are operated to maximize syscem mechanical and thermal efficiency and minimize system operating costs.

Black Start Capability: The ability of a generating unit or station to go from a shutdown confition to an operating condition and start delivering power without assistance from the power syster.

Block Rate Schednle: An electric rate schedule with a provision for charging a different unjt cost for various increasing blocks of demand or energy. A reduced price is charged on succeeding blocks.

Boller: A device for genterating stearn for power, processing, or heating purposes; or hot water for heating purposes or hot water supply. Heat from an extemal combustion source is transmitted to a flujd contained within the tubes found in the boiler shell. This fluid is delivered to an end-use at a desired pres. sure, temperature and quatity.

Boller Fuel: An energy source to produce heat that is transferred to the boiler vessel in order to generate steam or hot water. Fossil fuel is the primary energy source used to produce heat for boilers.

Borderline Customer: A customer located in the service area of one utility, but supplied by a neighboring utility through an arrangement between the utilities.

Bin (British thermal unit): A standard unit for messuring the quantity of beat energy equal to the quartity of heat required to raise the temperature of 1 pound of water by 1 degree Fahrenheit.

Balk Power System: The interconnected electrical systems comprising generation and transmission facilities on which faults on disturbances can have a significant effect outside the local area.

Bulk Power Transactions: The wholesale sale, purchase, exchange of electricity, and/or transmission services (wheeling) among electric utilities. Bulk power transactions are used by electric utilities for many djfferent aspects of electric utility operations, from maintaining load to reducing costs. 
Bus: Al electrical conductor which serves as a common connection for two or rnore electrical sircuits.

Capability: The maximum losd that a generating unit, generaring station, or other electrical apparatus can carry under specifted conditions for a given period of time without exceeding approved limits of iemperature and stress.

Capability Margin: The difference between net system capability and system peak load. Nationally, it is the difference between aggregate net system capability and the sum of system peak loads without altowance for time diversity between the loads of the individual systems. Regiotal compuratjous take into account the diversity betwcen peak loads of the systems that coordinate operations. This margin indicates the capability avilable to provide for scheduled maintenance, emergency outages, system operating requirements, and unforeseen loads.

Capability, Emergency Transfer: The amount of power transfer aljowed between areas or within an ares when operating to meet NERC emergency criteria contingencies.

Capability, Normal Transfer: The amouet of power transfer altowed between areas or within an area when operating to meet NERC normal criteria contingencies.

Capacify: The full-load continuous rating of a generator, prime mover, or other electric equipment usder specified conditions as designated by the manufacturer. It is usually indicated on a nameplate artached to the equipment as the foll-load rating.

Capacity (Nameplate): The amount of electric power delivered or required for which a generator, turbine, transformer, transnission circuit, station, of system is rated by the manufacturer.

Capacity Charge: An element in a two-part pricing method used in capacity transactions (energy charge is the other element). The capecity (demand) charge is assessed on the amount of capacity being purchased.

Capacity Pactor: The ratio of the average lond on the generatiog unit, generating plant, or other electrical apparatus during a specified period of time during its operations.

Capacity Transaction: The acquisition of a specified quantity of generating capacity from another ntility for a specified period of time. The utility selling the capaciry is obligated to make available to the buyer a specified quantity.

Certificate: A type of permit for public convenience and necessity issued by a utility commission, which tuthorizes a utillty or regulared company to engage in business, construct facilities, provide some services, of abandon service.

Circuit: A conductor or a system of conductors through which electric carrent flows.
Circult-Mile: The total length ja miles of separate circuits regardless of the number of conductors used per circuit.

Classes of Service: Customers grouped by similar characteristics in order to be identifjed for the purpose of setting a common rate for electric service. Usually classified into groups identified as residential, commercial, industrial, governmental, or other similar groupings within eacti onajor classifucation.

Code of Federal Regulations: A compilation of the general and permanent rules of the executive departments and agencies of the Federal Govemment as published in the Federal Register. The Code is divided into 50 titles that represent broad areas subject to Federal regulation. Title 18 contains the FERC's regulations.

Cogeneralion: The sequential or simultaneous process in which useful heat/steam is generated, used in a variety of process applitatjons, and then dirteted into * turbine to generate electricity and/or mechasical work from the useful thermal energy still available for use.

Cogenerator: A generating facility that produces electricity and another form of useful thermal energy (stech as heat or steam), used for industrial, commercial, heating, or cooling purposes.

Combined Cycle; A cogeneration technology in which additional electricity is produced sequentially from the otherwise lost waste heat exiting from one or nore gas-firced turbines. The exiting heat flow is routed to as exhaust-fired conventional boiler or to a heat recovery steam generator for vitization by a steam turbine in the production of electricity. This process increases the efficiency of an electric generating system by turning the rejected heat into thermal steam rather than discharging it into the atmosphere.

Commercial and Industrial: Classes of service supplied to a similar grouping of ctstomers. These customer groupings are usually subdjvided into stnaller segments by classifying such customers as commer. cial or industrial using the Federal Government's Office of Management and Budget's Standard Industrial Classification Guide and/or 2 scale of energy usage as yardsticks; other customers are actassified as commercial or industrial when their demands of annपal use oxceeds some specified limit. These limits are generally based on a utility's rate schedoles, except for those customers who are supplied under special contracts or agreements calling for particular services.

Commingling: The mixing of one utility's generated supply of electric energy with another vtility's gener. ated supply within a transmission system.

Commission: An agency of government usually composed of three or more members charged with specific duties, functions, and responsibilities. 
Conductor: Metal wires, cables, and bus-bar used for carrying electric current. Conductors may be solid or stranded, that is, built up by an assembly of smaller solid conductors.

Connected Load: The sum of the continuous patings or the capacities for a system, part of a system, or a customer's electric power consuming apparalus.

Contingency: The tnexpected event, usually the loss of one or more segments in the interconnected elecrrical power system.

Cooperative (electrlc utility): An electric utility legally established to be owned by and operated for the benefit of those nsing its services. The utility company will generate, transmit, and/or distribute supplies of electric energy to a specified area not being serviced by another utility. Such ventures are generally exempt from the Federal income tax laws. Most cooperatives have initially been financed by the U.S. Department of Agriculture's Rural Electrification Administration.

Coordination Service: Coordination service generally involves the sale, exchange, or transmission of electricity between two or more efectric utilities that cypically have sufficient generatjon and transmission capacity to supply their load requirements under normal couditions.

Condination Service Pricing: The typical price components of a bulk power coordination sale are an energy charge, a capacity, or reservation charge, and on adder. The price for a particular sale may embody some or all of these components. The energy charge is made on a per-kilowatthour basis and is intended to recover the seiler's systerd fncremental variable costs of making a sale. Since the nonfuct expenses are ustally bard to quantify and small relative to fuel expense, energy charges qwoted are usually based on fuel cost. A capacity charge is set at a certain level per kilowats and is normally paid whether or not energy is taken by the buyer. An adder is added to the energy charge to recover the hard-to-quantify nonfuel variable costs. There are three types of adders: percentage. fixed, and split-savings. A percentage adder increases the energy charge by a certain percentage. A fixed adder, adds a fixed amount per kilowatt-hour to the energy charge. Split-savings adders are used only in economy enetgy transactions. They split production cost savings between the selles and the buyer by adding one-hslf of the savings to the energy cost.

Cost: The amotnt paid to acquire resources such as plant and equipruent, fuel, or labor services. Fixed costs in the electric utility industry ate associated with respurces that cannot be changed easily daring a short time span (such as plant and equipment) and are independent of the level of generation. Variable costs are associated with resourees that can vary during a given time period (such as fuel or labor services) and are directly related to the level of generation.

Cost of Service: A ratemaking concept used for the design and development of rate schedules to ensure that the filed rate schedules recover only the cost of providing the electric service al issue. This concept attempts to cortelate the utility's costs and revenue with the service provided to each of the various cus. tomer classes.

Current: A flow of electrons in an electrical condvctor. The strength or rate of movement of the electricity is measured in amperes.

Deliveries (Electric): Energy generated by one system and delivered to another system through one or more transmission lines.

Demand (Electric): The rate at which electric energy is delivered to or by a system, part of a system, or piece of equipment, at a given instant or averaged over any designated period of time.

Demand Charge: That portion of the consumer's bill for electric service based on the consumer's maximum electric capacity usage and cajculated based on the billing demand charges onder the applicable rate schedule.

Demand Charge Credit: Compensation received by the buyer when the delivery rerras of the contract cannot be Inet by the seller.

Dermand Interval: The time period during which the flow of electricity is measured, usually in is-, 30-, or 60-minute increments.

Dependable Capacity: The load-carrying ability of a station or system under adverse conditions for a specjfied period of time.

Dlesej-Electrlc Plant: A generating station that uses diesel engines to drive its electric generators.

Direct Current (DC): An electric current that lows in a constant direction. The magnitude of the current does not vary or has a slight vatiation.

Dispatehing: The operating control of an integrated electric system involving operations such as (1) the assignment of load to specific generating stations and other soutces of supply to effect the most economical supply as the total ot the significant area loads rise or fall; (2) the control of operations and majntenance of high-voltage lines, substations, and equipment; (3) the operation of pripcipal tie lines and switching; (4) the scheduling of energy transactions with connecting electric atilitities.

Distribution system; The portion of the transmission lines and facilities of an electric system off the bulk power system that is dedicated to delivering electric energy to an end-oser.

Disturbance: Severe oscillations or sever step cbanges of current, voltage and/or frequency usually caused by fatilts.

Dlstarbance (System): An event resulting in widespread interruptions and characterized by one of more of the followiting phenomena: the loss of power system 
stability: cascading outages of circuits: abnormal ranges of frequency or voltage or botb.

Diversity: The electric utility system's load is made ap of many individual loads that make demands upon the system usually at different times of he day. The individual loads within the customer classes follow similar usage patterns, but these classes of service place different demands upon the facilities and the system grid. The service requirements of one electrical system can differ greatly from another by time of day usage, facility usage, and/or demands placed upon the system grid.

Diversity Erchange: An exchange of capacity or energy, or both, between systems whose peak loads occur at different times.

Diversity Factor: The ratio of the sum of the nowcoincident maximum demands for two or more losds to their coincjident maximum demand for the same tine period.

Docket: A formal record of a Federal Energy ReguJatory Commission proceeding. These records are arailable for inspection and copying by the public. Each indjuidual case proceeding is identified by an assigned number.

\section{DOE: Department of Energy.}

Donble-circuit Line: A transmission line having two separate circuits. Since each carries three-phase power, at least six conductors, three per circuit, ase required.

Doal-Fired Unit: A generating unit that can produce electricity using two or more input fuels. In some of these units only the primary fuel can be used continwously: the alternate fuel(s) can be used only as a start-up fucl or in emergencies.

Dump Energy: Energy gencrated in a bydroelectric plant by water that cannot be stored or conserved and which energy is in excess of the needs of the system producing the energy.

Economy Energy: Energy produced and supplied from a more economical source in one system, substiated for that being produced or capable of being produced by a less econtomical source in another system.

Economy of Scale: A proposition that relatively larger production facilities have lower unit costs than smatler facilities. Economy of scale toay exist for any of the phases of operarion: generation, transmission, or distribution.

ELA: The Energy Information Administration. An independent Bgency within the U.S. Department of Energy that develops surveys, collects energy data, and antyzes and moilets energy issues. The Agency must meet the requests of Congress, other elements within the Department of Energy, the Federal Energy Regulatory Commission, the Executive Branch, its own independent needs, and assist the genetal public. or otker interest graups, without taking a policy positiop.

Electric Current: The number of electrons per unit of time noving past a point in a conductor.

Electric Energy: The ability of an electric cunent to praduce work, heat. light or other forms of energy. It is measured in kilow atthours.

Electrle Expenses: The cost of labor, matorial, and expenses incurred in operating a facjlity's prime movers, generators, anxiliary apparatus, switching gear, and other electric equipment for each of the points where electricity enters the transmission or ifistribution grid.

Electric Plant (Financial): Assets comprising land, brilding, and equipment perusanently employed.

Electric Plant (Physical); A facility containing prime movers, electric generators, and auxiliary equipment for converting mechanical, chemical, and/or fission energy into electric energy.

Electric Power: The rate at which electric energy is generated, transinjtted, or consumed. Electic power is measured in watts or kilowatts.

Electric Power Industry: The public, private, and cooperative eloctric utility systems of the United States taken as a whole. This jncludes alt electric systems serving the public: regalated investor-owned electric utility companies; Federal power projects; State, monicipal, and other government-owned systems, including electric public utility districts: electric cooperatives, inciuding generation and transmission entities (G \& T"s); jointly owned electric utility facilities, and electric utility facilities owned by a lessor and leased to an olectric utility. Excloded from this list are the special purpose electric facilities or systems that do not offer service to the public.

Electric Power Sysiem: An individuad electric power entity-a company, an electric cooperative, a public electric supply corporation like the Tennessee Valley Authority, a similar Federal departanent or agency ljke the Bonneville Power Adminjstation, the Bureau of Reclamation or the Corps of Engineers, a municipally owned, electric department offering service to the public, or an electric public utility districr (a "PUD"); atso a jointly owned electric supply project such as the Keystone.

Electric Rate: The price set for a specified amount and type of electricity by class of service in an electric rate schedule or sales contract.

Electric Rate Schednle: A statement of the electric rate and the terms and conditions governing its application, including attendant contract terms and conditions that bave been accepted by a regulatory body with appropriare oversight authority.

Electric System Loss: Total electric energy loss frord alt causes for a electric utitity. 
Electric Utility: A corporation, person, agency, authority, or other legal entity or instrumentality that owns and/or operates facilities within the United States, its territories, or Puerto Rico for the genetation, transmission, distribution, or sale of elextric energy, primarily for use by the public and files forms listed in the Code of Feneral Regulations, Title 18, Part 14]. Facilities that qualify as cogenerators or small powet producers under the Public Utility ReguJatory Policies Act of 1978 and excmpt wholesale generators under the Energy Policy Act of 1992 are not considered electric utilitjes.

Electrical System Energy Losses: The anount of energy lost during generation, transmission, and distribution of electricity, including plant and unaccounted for use.

Electricity: A form of energy generated by friction, induction, or chemical change that is caused by the presence and motion of elementary charged particles of which matter consists.

Emergency: The failure of an electric power system to generate or deliver electric power as normally intended, resulting in the cutoff or curtailment of service.

Emergency Energy: Electric energy peovided for a limited duration, intended only for use during emer. gency condjuions.

Emergency, (Major): A situation usually accompabied by abnormal frequency, abnormal voltage and/or equipment overloads which might seriously affect the reliability of the bulk power system.

Emergency (System): An emergency is considered to exist in an area if firm load may have to be shed because sufficient power or energy is unavailable in that area or in a portion of it after due allowance for purchases.

Energy: The capacity for doing work as measured by the capability of doing work (potential energy) or the conversion of this capability to motion (kinetic energy). Energy has several forms, some of which are easily convertible and can be changed to another form useful for work. Most of the world's convertible energy comes from fossil fuels that are burned to produce hete that is then used as a transfer medium to mechanical or other means in order to accomplish tasks. Electrical entergy is usually measured in kilowatthours, while heat energy is usually measured in British thermal units.

Enerdy Broker System: lntroduced into Florida by the Public Service Commission, the energy broker system is a system for exchanging information that allows utilities to efficiently exchange bourly quotations of prices at which each is willing to buy and sell electric energy. For the broker system to operale, utiltty systems must have in plece bilateral agreements between all potevial parties and must have transmission arrangernents between all potential parties which allow the excbanges to take place.
Energy Charge: That portion of the charge for tlectric service based upon the electric energy (expressed in kilowatrhours) consumed or billed.

Energy Deliveries: Energy generated by one electric utility system and delivered to another system through one or more transinission lines.

Energy Loss (Power): The difference between energy input and output as a result of transfer of energy between two points. The loss changed to the equivalent power rating is sometimes referred to as capacity loss.

Energy Recelpts: Energy generated by one electric ntifity system and recejved by another system through one or mote transmission lines.

Energy Sonrce: The primary fuel or transfer modium that provides the heat/power that is converted to electricity through chemical, mechanical, or other meass. Energy sources include coal, petroteum and petroleum products, gas, water, urasium, wind, sublight, gtorbermal, and other sources.

End-Utser: The final consumer of electricity.

Exchange Energy: Electric energy received by an electric utility system osually in exchange for entrgy delivered to the other system at another time or place. Exchange energy is to be distinguished from a direct purchase or sale, although accumbiated energy balances are sometimes settled in cash.

Facilities Charge: An anount to be paid by the cus. tomer in a lomg surn, or periodically as reimbursement for facilities fumished. The charge may include operation and matntenance as well as tixed costs.

Facilify: A location at which prime movers, electric generators, and/or equipment for converting mechgnical, chemical, and/or nuclear energy into electric energy are situated. A facility may contain more than one rype of prime mover. For cogenerators, the facilicy includes the industrial or commercial process.

Failure or Hazard: Any electric power supply equipment or facility failure or other event that, in the judgment of the reporting entity, constitutes a hazard to maintaining the contibujty of the bulk electric power supply system such that a load reduction action may become necessary and a reportable outage may occur. The impact of this failure/hazard may require the imposition of a special operating procedure, the extended purchase of emergency power, and/or other bulk porrer system actions.

Federal Energy Reyulatory Commission (FERC): The independent Federal regulatory agency with jurisdiction over interstate electricity sales, wholesale electric rates, hydroelectric licensing. natural gas pricing, oil pipeline rates, and gas pipeline certification.

Federal Power Act: Enacted in 1920, and as amended in 1935, the Act consists of three parts. The first part incorporated the Federal Water Power Act 
administered by the former Federal Power Commission, whose activitjes were confjned almost entirely to ticensing non-Federal hydroelectric projects. Parts II and III were added with the passage of the Public Utility Act. These parts extended the Act's jurisdiction to include regulating the interstace transmission of electrical energy and rates for its sale as wholesale electricity in interstate commerce. The Federal Energy Regulatory Commission is now charged with the administration of this law.

Federal Power Commission: The predecessor agency of the Federal Epergy Regulatory Commission. The Federal Power Conmission was created by an Act of Congress under the Federat Warer Power Act on June 10, 1920. It was chatged originally with regulating the electric power and natural gas industries. It was abolished on Septenber 30, 1977, when the Department of Energy was created. Its functions were divided between the Department of Energy and the Federal Energy Regulatory Commission, an independent regulatory agency.

Feeder Line: An electrical line that extends radially from a distribution substation to supply ofectrical energy within an electric service area or sub-area.

\section{FERC: The Federal Energy Regulatory Commission.}

FERC Guidelines: A compilation of the Federa] Energy Regulatory Commission's enabling stantes, procedural and program regulations, and orders, opjn* ions and decisions.

File Rale Schedule: The rate for a particular electric service, including attendant contract terms and conditions, sccepted for filing by a reguiatory body with appropriate oversight authority.

Filing: Any written application, complaint, declaration, petition, protest, answer, rnotion, brief, exception, rate schedule, or other pleading. amendment to a pleadiog, cocument, or similar paper that is submitred to a utility commission.

Final Order: A final ruling by FERC that terminates sn action, decides some matter litigated by the petitioning parties, operates to sone right, or completely disposes of the subject matter.

Firm Power: Etectric power intended to meet the load requirement of a utility's customers: there is no planned interruption of services with this type of sale. Utilities may sell capacity for a limjted duration and time to other utilities and these types of contractual transactions are slso referred to as firm power.

Firm Service: The commitment of generation and/or rransutission service to a customer under a filed rate sehedule to which the parties to the service anticipate no planned interruption. The allocation of the utility's resources may be system wjde, or only for a named ubiz; the time of availability is usually prescribed as well.

Fiscal Year: A financial year based on a predetermined starting date. The Federal Government's 12-month finarcial yeas runs from October 1 through September 30.

Force Majeure Clanse: An occorrence generally beyond the control of the buyer or selfer in which relite is given by this clause to the parties involved in an agreement. This event, which results in nonper* formance of terms in a contract, reliability standards. or operating agreements, will typically relieve a buyer or seller of liability for damages. Some financial or other compensation relief may be avaitlable, if add:tional terms of conditions are written into the contract concerning unexpected problens beyond the control of the parties.

Forced Oucage: The shutdown of a generating anit, transmission line or other facility, for emergency reasons or a condition in which the generating equipment is unavailable for load becanse of unanticipated breakdown.

Frame: All units (i.e., electric utilities) that comprise the population of interest to a given survey. Subunits may in some instances only be represented in groups, but complete coverage is tmaintained.

Fuel Emergercy: An emergency that exists when supplies of fucls or bydroelectric storage for gener. ation are at a level or estimated to be at a level that would threaten the reliability or adequacy of the bulk electric power supply. The following factors should be raken into account to determine that a fuel emergency exists: (1) Fuel stock or hydroeleetric project water storage levels are 50 percent or less of normal for that particular time of the year and a continued downward trend in fuel stock or hydroelectric project water storage level are estimated; of (2) Unschedulted dispatch or emergency generation is causing an abnomal use of a particular fuel lype, such that the futare supply or stocks of that fuel could reach a level which threatens the reliability or adequacy of bolk electric power supplies.

Foll-Forced Ontage: The net capability of main generating units that are unavailable for load for emergency reasons.

Gas-Turbine Pant: A plant in which the prime mover is a gas torbine. A gas turbine consists typically of an axial-flow air compressor, one or more combustion chambers where liquid or gaseous fuel is borned and the hot gases are passed to the torbine; where the hot gases expand to drive the generator and then are used to num the compressor.

Generally Accepted Accounting Principles (GAAP): Defined as the conventions, rules, and procedures necessary to define accepted acconnting prac. ticc ar a particular time; includes both broad guidelines and relatively detailed practices and procedures.

Generathng Station: A station which consists of clectric generators and auxiliary equipment for converting mechanical, chemical or nuclear energy toto etectric epergy. 
Generating Unit: An electric generator together with its prime mover.

Generation: The process of producing elcctric energy by transforming other forms of energy; also, the amount of electric energy produced, expressed in kilowatthours.

Generation (Grass): The lotal amoun of electric energy produced by the generating units in a generating station or stations, measured at the genterakor tertninats.

Generation (Net): Gross genejalion less the electric enetgy consumed at the gencrating station for station use.

Generadion Rejection: The process of deliberately removing preselecied generation from a Power System in response to an abnormal condition to order to maiztain the integrity of the system; sometimes also called generator dropping.

Geothermal Energy: Energy from the internal hear of the earth which may be residual beat, friction beat, or a result of radioactive decay. The hest is found in rocks and flujds at various depths and can be extracted by drilling and/or pumping.

Geothermal Plant: A plant in which the prime mover is a stearn turbine. The turbine is driven either by steam produced from hot water or by narural steam that derives its energy fron beat found in rocks or fluids at various depths bencath the surface of the earth. The energy is extracted by drilling and/or putoping.

\section{Gigawatt (GW): One biltion watts.}

Giganatthour (GWh): One billion watthours.

Grid: The layout of an electrical system.

Gross Generation: The total amount of electric energy produced by a generating station or stations, measured at the generator terninals.

Gross Head: A dam's maximum allowed vertical distance between the upstream's surface water (headwater) fortbay clevarion and the downstream's surface water (tajlwater) elevation at the tajl-race for reaction wheel dans or the elevation of the jet at irnpulse wheel dams during specified operation and water conditions.

Head: The product of tho water's weight and a usable difference in elevation gives a measurement of the potentia] energy possessed by water.

Holding Company: A corporation (pareut company) that directly or indirectly owns a majority of all of the voting securities of one or more companics located in the same, contiguous States, or operating independent integrated public utility systems in noncontiguons States. A holding company is so structured to bring companies that can best be operated as part of an integrated public utility system under one jnanagement.
Many States do not pertuit or authorize a registered utility company to acquize ownership or operation of an electric or gas utility company serving substantially the same territory. The Securities and Exchange Commission, as administrator of the Public Utility Holding Company Act of 1935, defines a holding company as "a company which...owus, controls...10 percent or more of the outstanding voting securities of a public utility company."

Horsepower: A untt for measuring the rate of work (or power) equivalent to 33,000 foot-pounds per minute or 746 watts.

Hybrid Transmission Line: A double-circuit line which has one alternating eurreat circuit and one direct current circuit. The AC circuit usually serves local loads along the line.

Hydraulic Head: The distance between the respective slevarions of the upstream's water surface (headwater) above and the downstream surface water (tailwater) below a hydrotectric power plant.

Hydroelectric Energy: The production of electriclty from kinetic energy in flowing water.

Hydroelectric Plant: A plant in which the turbine generators are driven by falling water.

Hydroelectric Plant Capacify: This capacity figure is a function of fluid flow losses, hydraulic turbines, head, and water flow. The minimusn net head limits the firm capacity of the plant.

Hydroelectric Power: The harbessing of flowing water to produce mechanical or electrical energy.

Hydroelectric Project: The complete development of a hydroelectric power site. This thcludes datns, reservoirs, trapsmission jines and accessories needed for the maintenance and operation of the powerhouse and any other hydroelectric plant support facilities.

Impedance: The opposition to power flow in an AC circuil. Also, applicable to any device that jutroduces such opposition, in the form of resistance, reactance, or both. The impedance of a circuit or device is measured as the ratio of voltage to current, where a sinvsoidal voltage and curregt of the same frequency are used for the measurement; measured in ohms.

Jnadvertenl Power Exchange: An uninlended power exchange among utilities that is either not previously agreed opon or in an amount different from the amount agreed upon.

Jncremental Energy Costs: The additional cost of producing and/or transmitting electric energy above some previousty determined base cost.

Installed Nameplate Capacity: The full-Joad continuous rating of a generator, prime mover, or other electrical equipment under specified conditions as desiguated by the manufacturer. Installed nameplate capacity is usually indtcated on a nameplate atrached 
physically to the equipment. Installed station capacity does not inctude auxiliary or house units.

Instantaneous Peak Demand: The maximum demand at the instant of greatest load.

Insulator: A material that is a very poor conductor of electricity. The insulating material is usually a ceramic or fiberglass when used in the transmission line and is designed to support a conductor physically and to separate it electrically from other conductors and supporting material.

Integrated Demand: The summation of the continnously varying instantaneous demand averaged over a specified interval of time. The information is osualiy determined by examining a demand preter.

Integrated Gasilication-Combined Cycle Technology: Coal, water, and oxygen are fed to a gasifier, which produces synibetic natural gas (syngas). This medium Btu gas is cleaned (particulates and sulfur compounds removed) and is fod to a gas turbine. The hot exhaust of the gas turbine and heat recovered from the gasification process are routed through a heatrecovery generator to produce steam, which drives a steam turbine to produce electricity.

Interchange Energy: Killowathours delivered to or recejved by one electric utility or pooling system from snother. Settlement may be by payment, returned in tind at a later time or accumulated as energy balances until the end of the stated period.

Inlerconnected System: A system consisting of two or more individual power systems normally operating with connecting tie lines.

Interconnection: Two or more electric systems baving a common tratusmission line that permits a flow of energy between them. The physical connection of the electric power transmission facilities allows for the sale or exchange of energy.

Interdepartmental Sales: Includes amounts charged by the teceric department at tariff or other specifíed rates for electricity supplied by it to other utility departinents.

Interdepartmentol Service (Electric): Electricity supplied to departments of the electric utility company other than the elcctric generating department.

Interlocking Drectorates: The holding of a significant position in management or a position on the cosporate board of a utility, while simultaneously holding a comparable position with another utility, or with a firm doing business with the utility.

Intermediate Load (Electric System): The range from base load to a point between base load and peak. This point may be the midpoint, a percent of the peak load, or the load over a specified rime period.

Internal Combnstion Plant: A plant in which the prime mover is an internal combustion engine. An interal cornbustion engine has one or more cylinders in which the process of combustion take place, converting energy released from the rapid burning of a fuel-ajr mixture into mechanical energy. Diesel or gas-fired engines are the principal types nsed in electric plants. The plant is usually operated doring periods of high demand for electricity.

Interroptible Power: Power and usually the associated energy made avislable by one utility to another which is subject to curtailment or cessation of delivery by the supplier in accordance with a prior agreement with the other party or under specified conditions.

lntervenor: A person, institution, or organization admitted as a participant to a proceeding.

Investor-Owned Electric Utility: A class of utility whose stock is publicly traded and which is organized as a tax-paying business, usually financed by the sale of securities in the capital market. It is regulated and atthorized to achieve an allowed rate of return.

Island: A portion of a power system, or several power systems which is (are) electrically separated from the main grid.

Joint-Use Facility: A mutriple-purpose hydroelectric plant. An example is a dam that stores water for both flood control and power production.

JuristicUonal Utilttes: Utblities regulated by public laws.

Kilowatt (kW): One thousand watts.

Kllowatthour (kWh): One thousand watthours.

Line Lass: Electric enetgy lost because of the transmission of electricity. Mach of the loss is thermal in nacure.

Load: The power provided to custamers of a system. This may be further identified to more precisely indicate which portion of the system is being considered, (e.g., customer load, station auxiliary losd, etc.).

Load, Firm: Power provided to custorner's that is continuovsly available on demand and which is subject to interruption only under extreme circumstances.

Load, Jaterreptible: Those loads that, by contract, can be interrupted in the event of a deficiency on the supplying sysiem.

Laad (Electric): The amount of electric power delivered or required at any specific point or points on a system. The reguirement originates at the energyconsuming equipment of the customers.

Load Curve: The relationship of power supplied to the time of occurrence which illustrates the varying magnitude of the load during the period covered. 
Load Diversity: The tifference between the peak of coincident and noncoincident demands of two or more individual loads.

Lood Factor. The ratio of the average load to peak load during a specified time interval.

Load Following; Regulation of the power output of electric generators within a preseribed area in response to changes in system frequetcy, vieline loading, or the relation of these to each other, so as to maintain the scheduted system frequency andror the established interchange with other areas within prede. termined limits.

Losd Levelling: Any load control technique that dampens the cyclical daily load flows and increases baseload generation. Peak load prícing and time-of-day charges are two techniques that electric utilities use to reduce peak load and to maximize effocient generation of electricity.

Load Lass (3 bours): Any significant incident on an clectric utility system which results in a continuous outage of 3 hours or longer to over 50,000 customers or more than one balf of the rotal cuslomers being served immediately prior to the incident, whichever is less.

Load Management Technique: Utility demand management practices directed at reducing the maximum kilowatt demand on an electric system, and/or modjfying the coincident peak demand of one or mote clesses of service to better met the utility system capabitity for a given hotr, day, week, stason, or year.

Load Reduction Request: The issuance of any public or private request to any customer or the general public to reduce the use of electricity for reasons of majntaining the continuity of service of the reporting entity's electric power supply system. Requests to a custorner(s) served under provisions of an interruptible contract are not a reportable action unless the request is made for reasons of maint aining the continuity of service of the reporting entity's elec. tric power supply.

Load Reltef: Load reduction accomplished by voltage reduction and/or load shedding.

Lond Shedding: Deliberate discongcecion of customer load in response to an aboormal condition in order to maintain the integrity of the power system. Disconnection may be automatically or manualiy initiated. The routine use of load control equipment that reduces firm customer load is not considered to be a repostable action.

Loop Flow: The movement of electric power from generator to load by dividing along multiple parallel paths; it especially refers to power flow along an unimtended path that loops away from the most direct geographic path, or contract path.

Low Head: Vertical difference of 100 feet or less in the upstream surface water elevation (headwater) and the downstrean surface water elevation (tajlwater) at a dam.

Maximum Demand: The greatest of all demands of the load that has occurred within a specified period of time.

Maximnm Dependable Capacity, Net: The gross electrical output measured at the output terminats of the turbine generator(s) during the most restrictive seasonal condicions, tess the station service load.

Median Streamflow: The middle rate of flow of water past a given point for which there have been several greater and lesser rates of flow occurring during a specified period.

Medizn Water Condition: The middle precipitation and rug-off condition for a distribution of water conditions that have happened over a long period time; usually determined by examining water supply records of the periods in question.

Megawatt (MW): Onc million watts.

Megawatt-Electric (MW): One million watts of electric capacily.

Megawatthour (MWh): One miltion wathours.

Mill: A monetary cost and billing unit used by utilities; it is equal to $1 / 1000$ of the U.S. dollar (equivalent to $1 / 10$ of 1 cent?).

Minimam Streamflow: The minimum rate of flow of water past a given point during a specified period.

Maltipie Purpose Project: The development of hydroelectric facilitics to serve more than one function. Some of the uses include hydroelectric power, irrigation, water supply, water quality control, and/or ftsh and wildlife enhancement.

Maltiple Purpose Reservoir: Stored water and its usage govemed by advanced water resource conserva. tion practices to achieve more than one water control objective. Some of the objectives include flood control, bydro-electric power development, irrigation, recreation usage, and wilderness protection.

Manicipality: A city, county, distriet, or a political subdivision of a State comperent under the laws thereof to carry on the business of its charter.

National Association of Regulatory Utility Com. missioners: An affiliation of the public service com. missioners to promote the uniform teatment of members of the railroad, public utilities, and pablic service commissions of the 50 States, the District of Colunbia, the Commonwealth of Puerto Rico, and the territory of the Virgin Islands.

Natural Strenmflow: The rate of flow of water past a given point of an uncontrolled stream, or regulated streanflow adjusted to eliminate the effects of reservoir storage or upstream diversions at a set time interval. 
Ne1 Energy for Load: Net generation of main generating onits that are system-owned or system-operated plus energy recejpts minus energy deliveries.

Net Energy for System: The nct generation of a group of plants that are electrically interconnected and centrally controlled, plus systern receipts of any electrical energy from outside the system minus any system energy delivered to other electrical systems.

Net Generation: Gross generation less plant use, messured at the high-voltage terminals of a station's step-np transformer. The energy required for pumping at pumped-storage ptants is regarded as plant use and must be deducted from the gross generation.

Net Hewd: The gross head minus all hydraulic losses except those chargeable to the turbine.

Net Summer Capability: The steady hourly output which genterating equipment is expected to supply to system load exelusive of auxiliary power as dewionstrated by test at the time of summer peak demand.

Net System Capability; The generating station capa* bility of a system at a stated period of time (usually at the time of the systent's maximum load) plus capability avaitable at such time from other sources through fitm power contacts less firm power oblj. gations at such time to other companies or systems.

Ne1 Winter Capability: The steady hourly output which generating equipment is expected to supply to system load exclusive of auxitiary power as iemonstrated by test at the time of winter peak demand.

No-Load Loss: Power and energy lost by an electric system when not operating under demand.

Noncoincident Demand: Sura of two or more demands on individual systems that do not occar in the same demand interval.

Noncoincident Pesk Load: The sum of two or more peak loads on individual systems that do not occur in the same time interval. Meaningful only when considerimg loads within a limited period of time, suth as a day, week, month, a heating or cooling season, and usualty for not more than 1 year.

Nonfirm Power: Power or power-producing capacily supplied or available under a comrnitment having limited or no assured availability.

Nonspinning Reserve: The generating capacity not currently runting, but capable of being connected to the bus and load within a specified time.

Nonntility Generation: Electric generation by end users, or small power producers under the Publje Urility Regulatory Policies Act of 1978, to supply electric power for industrial, commercial, or sales to electric utilities.

Nonntility Power Producer: An enterprise that has electric generating capacity and is not an electric btility. Nonutilicy power producers include quatifying cogenerators, qualifying small power producers, and other nonutility generstors (including independent power producers) without a designated franchised service area, and which are not otherwise required to file forms listed in the Code of Federal Regulations, Title 18, Part 141.

North American Electric Reliablity Conncil (NERC): A conncil formed in 1968 by the electric utility industry to promote the reliability and adequacy of the bulk power stopply in the electric ntility systems of North America. NERC consists of ten regional reliability councils and encompasses essentially all the power systems of the contiguous United States, Canada, and some in Mexico. The data summarized by NERC regions in this publication are limited to that portion applicable to the United States, thereby excIuding that portion of NERC data applicable to Canada and Mexico. The NERC Regions are:

\section{ASCC--Alaska System Coordination Council \\ ECAR--East Central Area Reliability Coordi- nation Agreement}

$$
\begin{aligned}
& \text { ERCOT--Electric Retiability Council of Texas } \\
& \text { MAIN--Mid-America Interconnected Network } \\
& \text { MAAC--Mid-Atlantic Aren Council } \\
& \text { MAPP-Mid-Continent Area Power Pool } \\
& \text { NPCC--Northeast Power Coordinating Council } \\
& \text { SERC--Southeastern Electric Reliability Council } \\
& \text { SPP--Southwest Power Pool } \\
& \text { WSCC-Western Systems Coordinating Council }
\end{aligned}
$$

Nuclear Power Plant: A facility in which heat produced in a reactor by the fissioning of nuclear fuel is used to drive a steam turbint.

Ott-Peak: Period of relativaly low system demand. These periods often occur in daily, weekly, and seasonal patterns; these off-peak petiods differ for each individanl electric utility.

Ohm: The unit of measurement of electrical resistance. The resistance of a circuit in which 2 potential difference of 1 volt produces a cutrtent of 1 ampere.

On-Peak: Perjods of relatively bigh system demand. These periods often occur in daily, weekly, and seasonal patterns; these on-peak periods differ for each individnal electric utility.

Operating Limit: The maximum value of the most critical system operation parameter(s) which meets: precontingency criteria as deteruined by equipment loading capability and acceptable voltage conditions; transient performance criteria; and post contingency toading and voltage criteria.

Order: A ruling issued by a utility commission granting or denying an appljcation in whole or in part. The order explains the basis for the decision, noting eny dispute with the factual assertions of the applicant. Also applied to a final regulation of a ubility commission. 
Other Generation: Electricity originating from these sources: biomass, fuel cells, geothermal beat, soler power, waste, wind, and wood.

Other Sector: Electricity supplied to public street and highway lighting; other service to public authorities: service to railroads and railways: and interdepartuental service.

Other Service to Public Authorities: Electricity supplied to municipalities or divisions or agencies of Srate or Federal governments, under special contracts or agreements or servjce classifications applicable only to public authorities.

Other Unatailable Capability: Net eapability of main geverating units that are unavajlable for load for retasons other than full-fored outage or scheduled maintenance. Legal restrjetions or other cause make these units unavailable.

Outage: The period during which a generating wit, transmission line, or ober facility is out of service.

Oulput: The amount of power or energy produced by a generating unit, station, or system.

Peak Load: The maximum load during a specified period of time.

Pagk Load Plant: A plant usually housing old, lowefficiency steam umits, gas urbines, diesels, or pumped-storage hydroelectric equipment normally used during the peak-load periods.

Peaking Capacity: Capacity of generating equip. ment nomally operated during the hours of highest daily, reekly, or seasonat loads. Some generaring equipment may be operated at certain times is peaking capacity and at other times to serve loads on a 'rond-the-clock basis.

Percent Dillerence: The relative change in a quan. tity over a specified time period. It is calculated as follows: the current value nutaber has the prevjous value number subtracted from it, and this new number is divided by the absolute vatue of the previous value number, then this new number is multiplied by 100 .

Plant: A station at which are located prime movers. electric generators, and auxiliary equipment for converting mechanical, chemical, and/or nuclear energy into electric energy. A station may contain more than one type of prime mover. Electric utility plants exclude stations that satisfy the definition of quali. fying facility under the Public Utility Regulatory Policies Act of 1978.

Plant-Use Electrtctiy: The electric energy used in the operation of a plant. This energy total is subtracted from the gross encrgy production of the plant; for reporting purposes the plant enerigy production is then reported as a net figure. The energy required for punping at pumped-storage plants is by definition subtracted, and the energy production for these plants is then reported as a net figure.
Plant Hours Connected to Load: The number of hours the plant is synchronized to load pver a timt inter'sal usually of 1 year.

Pole-Mile: A unit of measuring the simple length of a transmission line carrying electric conduetors, without regard to the number of conductors carried.

Pondage: The amount of water stored behind a hydroelectric dan of relatively srall storage capacity; the dam is usually used for daity or weekly control of the flow of the tiver.

Power: The rate at which energy is transferred, usually measured in watts. Also used for a measurement of capacity.

Power (Electrical): An electric measurcment unit of power called a volrampere is equal to the product of 1 volt and 1 ampere. This is equivalent to 1 Watt for a direct current system and a unit of apparent power is separated into real and reactive power. Real power is the work-producing part of apparent power that measures the rate of supply of energy and is denoted as kilowatts (kW). Reactive power is the portion of apparent power that does no work and is referred to as kilovars; this type of power must be supplied to most types of magnetic equipment, such as motors, and is supplied by generator or by electrostatic equipment. Voltamperes ase usually divided by 1,000 and called kilovoltamperes (kVA). Energy is denoted by the product of real power and the length of itme utilized; this product is expressed as kilowattours.

Power Factor: The ratio of real power (kilowatc) to apparent power kilovoltampere for any given load and time.

Power Loss: The difference between power input and output as a resull of transter of energy between two points; sometimes referred to as capacity loss.

Power Pool: An association of two or phore interconnected electric systems to provide better system reliability and efficiencies. Depending upon the degree of control authorized by the member utilities, these pools many be run under a single system dispatch to supply power to meet combined load requirements and mintenasce programs, or just share the benefits of planned or hourly available wholesale sales of power and energy among the member utilities.

Power Production Plant: All the laad and land rights, structures and improvements, boiler or reactor vessel equipment, engines and engine-driven generator, tuktogenerator unirs, accessory electric equipment, and miscellaneous power plant expupment are groupes together for each individual facility.

Power System: A group of one or more generating sources and/or connecting transmission lines operated under common management of supervision to supply load.

Power Transfer Limit: The maximum power that can be transferred from one electric utility system to 
another without overloading any facility in either system.

Powerhonse: A structure at a hydroelectric plant site that contains the turbine and generator.

Preliminary Permit (Hydroelectric Power): A single site permit granted by the FERC, which gives the recjpient priority over anyone else to apply for a bydroelectric ficense. The preliminary permit enables the recipient to prepare a license application and conduct vartous studies for econoutic feasibitity and environmental impacts. The period for a preliminary permit may extend to 3 years.

Price: The amount of money or consideration-inkind for which a service is bought, sold, or offered for sale.

Price Squeeze: Discriminatory rates charged for wholesale electric power that impede competition for retail customers. A price squeeze occurs if an electric utillity's ptice for wholesale service is higher in relation to its wholesale cost than its price for retail service is in relation to its retail costs.

Prime Mover: The engine, turbine, water whet, ot similar machine that drives an electric generator.

Proposed Rates: New electric rate schedute pro* posed by an applicant to become effective at a future date.

Prudence: Carefulness, precaution, attentiveness, and good judgment, as appljed to action or conduct. A Jegal conecpt used by regulators to determine the costs to be allowed in a rate base.

Pablic Authorities: Electricity supplied to municipalities or divisions or ageacies of State and Federal governments, usually under special contracts or agreements that are applicable only to public authorities.

Pablic Utility: Enterprise providing essential public services, such as electric, gas, telephone, water, and sewer, under legally established monopoly conditions.

Public Utility District: Municipal copporations organized to provide eloctric service to beth fincorporated cities and sowns and unincorporated rural areas. Public urility distriets, sometimes called "People's Utility Districts" or "Public Power Districts, " operate in six States.

Pubicicly Owned Electric Utility: A class of ownership found in the electric power industry. This group includes those utilities operated by municipalities, and State and Federal power agencies.

Pumped-Storage Hydroelectric Plant: A plant that usually generates electric energy during peak-load periods by using water previously pamped into an elevated storage teservoir during off-peak periods when excess generating capacity is available to do so. When additional generating capacity is needed, the water can be released from the reservoir through a conduit to turbine generators located in a power plant at a lower level.

Purchased Power: Power purchased or available for purchase from a source outside the system.

Purchased Power Adjustment: A clause in a rate schedule that provides for adjustments to the bill when energy from other electric system is acquired and its cost varies from a specified unit base amount

Pure Punped-Storage Hydroelectric Plant: A plant that produces power only from water that has previously been pumped to an upper reseryoit.

Qualifying Facility: A cogeneration facilicy or small power production facility that meets certain ownership, operating, and efficiency criteria estsblished by the Federal Energy Commission pursuant to the Public Utilities Regulatory Policies Act of 1978 (See the Code of Federal Regulations, title 18, Part 282).

Rate Bast: The value of property opon which a utility is permitted to earn a specified rate of return ss established by a regulatory authority. The rate base generalty represents the value of property used by the utilicy in providing service and may be calculated by aay one or a combination of the following accounting methods: fair value, prudent investment, reprodaction cost, or origival cost. Depending on which methed is used, the rate base includes cash working capital, materials and supplies, and deductions for accumulated previsions for depreciation, contributions in aid of construction, customer adpances for construction, gccumulated deferred income taxes, and accumulated deferred investment tax credits.

Rate Case: A proceeding, vsually before a regulatory commission, invalving the rates to be charged for a public utility service.

Rate of Return: The ratio of net operating income earned by 2 utility is calculated as a percentage of its rate base.

Rate of Return on Rate Base: The ratio of net operating income to a specified rate base, expressed as a percentage.

Rate Schedule (Electric): A statement of the financial terms and conditions governing a class of classes of utility services provided to a customer. Approval of the schedule is given by the appropriate ratemaking authority.

Rates: The authorized charges per unit or level of consumption for a specified time period for any of the classes of utility services provided to a costomer.

Ratemaking Authority: A utility commission's legal authority to fix, modify, approve, or disapprove rates as determined by the powers given the commisstion by a State or Federal legislature.

Rating: A manufacturer's guaranteed performance of a machine, transmission line, of other electrical apparatus, based on design features and test data. The 
rating will specify such limits as load, voltage, temperature, and frequency. The rating is geterally printed on a nameplate attached to equipment and is commonly referred to as the nameplate rating, nameplate capacity, etc.

Reactance: A phenomenon associared with AC power characterized by the existence of a time difference between voltage and current variations.

Rectifier: A devjee for cọvverting alternating curтent to direct current.

Regulated \$treamnlow: The rate of flow past a given point during a specified period that is controlled by reservoir water release operations.

Regalatlon, Procedures, and Practices: A utility comnission carties out its regutlatory functions through sulemaking and adjudication. Under rulemaking, the ntility commission may propose a general role or regulation change. By law, it must jssue a notice of the proposed rule and a request for comments is also made; the Federal Energy Regulatory Commission publishes this in the Federal Register. The final decision must be published. A utility commission may also work on a case-by-case basis from submissions from regulated companies or others. Objections to a proposal may come from the commission or intervenors, in which case the proposal must be presented at a hearing presided over by an administrative law judge. The judge's docision may be adopted, modified, or reversed by the utility commissioners in which case those involved can petition for a rehearing, and may appeal a decision through the courts system to the U.S. Supteme Court.

Rellability (Electric System): A measure of the ability of the system to continue operation white some lines or generators are out of service. Reliability deals with the performance of the systern under stress.

Renewable Energy Source: An energy source that is regenerative or virtually inexhaustible. Typical examples are wind, geothermal and water powet.

Repowering: Replacement of the combustion techcology io an aging generating plant with a new com* bustion technology' usually resulting in better perfortance and greater capacity.

Required Rate of Retura: The minimam rate of retum necessary to induce investors to buy or hold a given security. It equals the risk-free rale of inierest, usually represented by the current yield on U.S. Treasury securities, growth potential, plas a risk pretninm-

Requirements Power: The firm service needs required by designated load plas losses from the points of supply.

Reserve Generating Capacity: Amount of generating capacity available to meet peak or abnomally high demands for power and to generate power during scheduled or unstheduted ontages.
Reserve Margin (Operating): The amount of unused available capabilicy of an electric power system at peak load for a utility system. This figure is calculated by adding ruoning and quick-start capability to the capability available but not needed and then subtracting peak load.

Reservoir: A body of water collected and stored in a batural or artificial lake.

Residentlal Sector: The residential sector includes private household establishments that consume energy primarily for space heating, water heating, air conditioning, lighting, refrigeration, cooking, and clothes drying. The classification of an individual consumer's account, where the use is both residential and commercial, is based on principal use. Apartment houses ase included.

Restoration Time: The time when the major portion of the interropted load has been restored and the emergency is considered to be ended. However, some of the loads interrupled may not have been restored because of local problems.

Retail Wheeling: An arrangement in which a utilicy transmits electricity from outside its service territory to a retail customer within irs customer service territory.

Retired Hydropower Plant Sites: The site of a plant that formerly produced electrical or mechanical power but is now out of service. Includes plants which have been abandoned, damaged by flood or fire, inundated by new reservoirs, or distrantled.

Reversible Turbine: A bydranlic cubine, normally installed in a pumped-storage plant, which can be used alternatively as a pamp or as an engine, turbine, water wheel, or other apparatus that drives an electrical generator.

Right-0t-Way: The land, and legal right to use and service the land along which a transmission line is located. Transmission tine right-of-way is ustality acquired in widths that vary with the kilovolt (kV) size of the line.

Rulemaking (Regulations): The anthority delegated to administrative agencies by Congress or State legislative bodies to make rules that have the force of law. Frequently, statutory laws that express broad tems of a policy are implemented more speciftcally by administrative rules, regurlations and practices.

Rnnning and Quick-Start Capability: The net capability of genetating units that carry load or have quick-start capability. In general, quick-start capabifity refers to generating units that can be avajlable for load within a 30-minute period.

Run-aff: That portion of the precipitation that flows over the land surface and uldimately reaches streams to complete the water eycle. Melting snow is an important source of this water as well as all atrounts of surface water that moves to streams or vivers through any given area of a drainage basin. 
Rural Electrification Administration (REA): A lending agency in the U.S. Department of Agriculture, RUS makes self-liquidation loans to qualified borrowers to finance electric and telephone service to rural areas. RUS fjpances the constriction and operation of generating plants, electric transrnission and distribution lines, or systems, for the furnishing of initial and continaed adequate electric services to persons in rural areas not receiving central station servlce.

Sales: The amount of kilowatthours sold in a given period of time; nsualiy grouped by classes of service such as residential, commercial, industrial, and other.

Sales for Resale: A type of wholesale sales covering energy supplied to other electric utilities, cooperatives, municipals, and Federal and State electric agencies for restle to ultimate consumers.

Schedule: A statement of the pricing format of electricily and the terms and conditions governing its applications.

Schednled Ontage: The shutdown of a generating unith transmission line, or other facility, for inspection or maintenance, in accordance with an advance schedule.

Service Ares: The territory in which a utility system or distributor is authorized to provide service to consumers.

Single-circnit Line: A transmission line with one elecuric circulit For three-phase supply, a single circuit requires at teast three conductors, one per phase.

Single-Parpose Projert: A hydroelectric project constructed onty to generate electricity.

Small Power Producer: Under the Public Utility Regulatory Policies Act, A smatl power prodnction facility (or small power producer) generates electricity using renewable biomass, conventional hydroelectric, solar, wind, and geothermal energy as a primary energy source. Fossil fuels can be used, but renewable resources must provjde at least 75 percent of the total energy input. (See Code of Federal Regulations, Title 18, Part 292.)

Spetial Contract Rate Sehedule: An electric nte schedule for an electric service agreement between a ukility and another party in addition to, or independent of any standard rate schedule.

Special Purpose Rate Schedule: An electric rate schedule limited in its application to some particular purpose or process within one, or more than one, type of industry or business.

Stability: The property of a system or element by virtue of which its output wili ultimately attain a steady state. The amount of power that can be transferred from one machine to another following a dis. torbance. The stability of a power system is its ability to develop restoring forces equal to of greater than the disturbing forces so as to maintain a state of equilib. rium.

Standby Charge: A charge for the potential use of a urility service, usually done by an agreeneat with another electric utility system. These services include system backup support, and other running and quickstart captbilities.

Standby Facility: A facility that is on call to support a utility system and is available to replace or supplement a facility bormally in service.

Standby Servicet Suppott service that is avaitable as needed to supplement a customer, a utility system, or to another utility if a schedule or an agreement anthorizes the transaction. The service is not regularly used.

State Severance Taxes: Any severance, production, or simitar tax, fes, or other levy imposed on the production of crude oil, natural gas, or coal by any State, tocal government acting under authority of State law, or by an Indian tribe recognized as eligible for services by the Secretary of the Interior.

Stution (Electric): A plant containing prime movers, electric generators, and auxiliary equipment for converting mechanical, chemical, and/or muclear energy into electric energy.

Station Ust: Energy that is used in the operation of an electric generating plant. It includes energy consumed for plant lighting, power, and auxiliary facilities, regardless of whether the energy is produced at the plant or comes from another source.

Steam-Electric Piant (Conventional): A plant in which the prime mover is a steam turbine. The steam used to atrive the turbitis is produced in a boiler where fossil fuels are butned.

Streamflow: The rate at which water passess a given point in a stream, usually expressed in cubic feet per second.

Sulustation: Facility equipment that switehes, changes, or regulates electric voltage.

Subtransmission: A set of transmission lines of voltages between transmission voltages and distribution voltages. Generally, lines in the voltage range of $69 \mathrm{kV}$ to $138 \mathrm{kV}$.

Supporting Structnre: The main supporting unit (usually a pole or tower) for trensmission line conductors, insulators, and other anxiliary line equipment.

Surplus Energy: Energy generated that is beyond the intmediate needs of the producing system. This energy may be supplied by spinning reserve and sold on an interruptible basis.

Suspended Rates: New rates that have been accepted for review by a utility commission. When these rates are suspended, they do not go into effect for a desiguated period of time, up to a maximum of 
five months for FERC. Charges under the new rate may be refunded after the resolution of the rate proeteding.

Switching Station: Facility equipment used to lie rogether two or more electric circuits through switches. The switches are selectively arranged to permit a circuit to be disconnected, or to change the electric connection between the circuits.

System (E]ectric): Physically connected generation, trassmission, and distribution facilities operated as an integrated unit under one central management, os operating supervision.

System Interconnection: A physical connection between two electric systems that permits the cransfer of electric esergy is either direction.

Tarift: A published volume of race schedules and genera] terms and condjions ander which a product or service will be supplied.

Terawatthour (TWh): One trillion $\left(10^{1 i}\right)$ watthours of electric energy.

Thermal Limit: The maximum amount of power a transmission line can carry without suffering hearrelated deterioration of line equipment, particularly conductors.

Three-party Wheeling: An arrangement in whicb a utility transmits electricity for two other utilities that are not physically connected, where the transmitting ntility neither buys nor sells the electricity.

Three-phase Power: Power generated, and transmitted from generator to load, on three conductors.

Tie Line: A Circuit connecting two or more power systems and/or a generating facility to the grid.

Transfer Capability: The overall capacity of inter. regional or international power lines, together with the associated electriegl system facilities, to transfer power and energy from one electrical system to anokher.

Transformer: An electrical device for changing the voltage of alternating current.

Transmission: The movement or transfer of electric energy over an interconnected group of lines and associated equipment between points of supply and points at which it is transformed for delivery to consumers, or is delivered to other electric systems. Transmission is considered to end when the energy is transformed for distribution to the consumer.

Transmission Circuit: A conductor used to transport electricity from gentrating stalions to load.

Transmission Line: A set of conductors, insulators, supporing stnictures, and associated equipment used to move large quantities of power at high voliage, tsually over long distances betwetn a generating or receiving point and major substations or delivery points.

Transmission Network: A system of transmission or distribution lines so cross-connected and operated as to permit multiple power supply to any principal point.

Transmission System, Electric: An interconnected group of electric transmission lines and associated equipment for moving or transferring electric energy in bulk between points of supply and points at which it is transformed for delivery over the distribution system lines to consumers, or is delivered to other electric systems.

Turbine: A machine for generating rotary mechanjeal power from the encrey in a stream of fluid (such as water, steam, or hot gas). Turbines convert the kinetic energy of fluids to mechanical energy through the principles of impulse and reaction, or a mixnure of the two.

Two-party Wheeling: An arrangement between two urilities in which one utility agrees to transmit electricity owned by the other.

Ultimate Consumer: A consumer thit purchases electricity for its own use and not for ressle.

Uniform System of Accounts: Prescribed financial rules and regulations established by the Federal Energy Regulatory Commission for utilities subject to its jutisdiction under the euthority granted by the Federal Power Act.

Unscheduled Outage Service: Power received by a system from another system to replace power from a generating unit forced out of service.

Used and Useful: A concept used by regulators to determine whether an asset shoutd be included in the utility's rate base. This concept requires that an asset curently provide or be capable of providing a needed service to customers.

Utility (Electric): Privately owned colmpanies and public agencies engaged in the generation, transmission, or distribution of electric power for public use. Public agencies include municipal electric utilities. Federal power projects, rural electriftcation cooperatives, power districts, agd State power authorities and projects.

Utility Genteration: Generation by electric systems engaged in selting electric energy to the public.

Volt: The unit of measurement of voltage, etectrical force, or pressire. The electrical force that, if steadily appijed to a circuit with a resistance of 1 obm, wilt produce a current of $t$ ampere.

Voltage: The difference is electrical potential between any two conductors or between a condutetor and ground. It is a measure of the electric epergy per clectron that electrons can acguire and/or give up as they move between the two conductors. 
Vollage Reduction: Any intentional reduction of system voltage by 3 percent or greater for reasons of maintaining the continuity of service of the reporting entity's electric power supply system.

Waterway: A river, chennel, canal, or other navigable body of water used for travel or transport.

Watt: The electrical unit of power. The rate of energy transfer equivalent to 1 ampere ftowing under a pressure of I volt at unity power factor.

Watthoar (Wb): An electrical energy unit of measure equal to 1 watc of power supplied to, or taken from, an electric citcuit steadily for ] hour.

Weir: A dam in a waterway over which water flows and that serves to raise the water level, or direct of regulate flow.

Wheeling: The wse of the transmission facilities of one entily to transmit electricity of and for anothes entity or entities. The most common type of wheeling involves one utility uansferring electricity generated by a second utility for sale to a third utitity.

Wheeling Charge: An annount charged by one electrical system to transtnit the energy of, and for, another system or systems.
Wheeling Service: The movement of electricity from one system to another over transmissjon facilities of intervening systems. Wheeling service contracts can be established between two or more systems.

Wholesale Sales: Energy supplied to other electric utilities, cooperatives, municipals, and Federal and State electric agencies for resale to ultimate consumers.

Wholesale Wheeling: An arrangement in which electricity is transmitted from a generator to a utility through the transmission facilities of an intervening system.

Wind Energy: Energy produced by harnessing the force of the wind. In a wind energy conversion system such as a windmill, the energy of wind is used to turn the shaft of a geaerstor, which in turn usually produces direct current. This direct current is usually converted to alternating current before being fed into a usility grif system.

Year to Dale; The cumulative sum of each month's value starting with January and ending with the current month of the data. 